
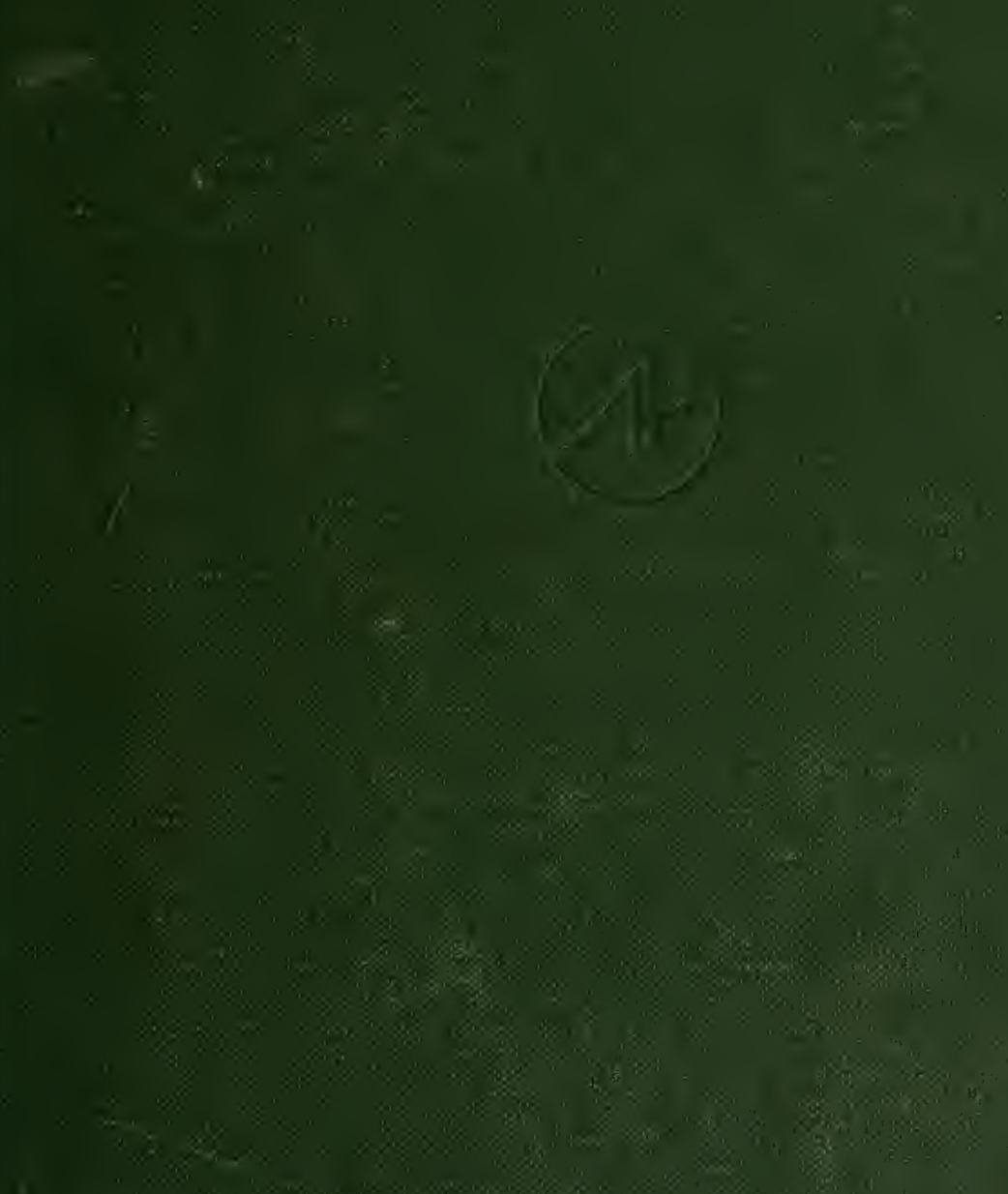


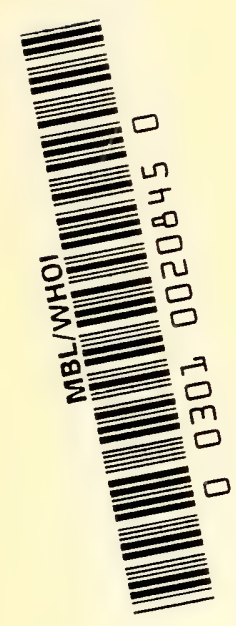





\title{
Enzyme and Metabolic Inhibitors
}

\author{
Volume I
}

General Principles of Inhibition 
Volume II Malonate

Structural Analogs

General Sulfhydryl Inhibitors

Iodoacetate and Iodoacetamide

Iodosobenzoate

Mercurials

Arsenicals

Quinones and Naphthoquinones

$N$-Ethylmaleimide

Maleic Acid

Dehydroacetate

Fluoride

Carbonyl Reagents

Volume III Uncouplers of Oxidative

Phosphorylation

Dinitrophenol

Arsenate

Cyanide

Carbon Monoxide

Azide

Sulfide

Antimycin

Fluoroacetate

p-Pyruvate

Urethanes

Chelating Agents

Volume IV Protein Group Reagents

Heavy Metals (Copper, Zinc,

Cadmium, Silver, etc.)

Dyes

Aldehydes

Dimercaprol

Antienzymes

Phlorizin

Selenite and Tellurite

Naturally Occurring Inhibitors

Cholinesterase Inhibitors

Monoamine Oxidase Inhibitors

Drugs as Inhibitors

Carbonic Anhydrase Inhibitors

Borate 


\title{
Enzyme
}

\section{and Metabolic}

\section{Inhibitors}

\author{
Volume I
}

General Principles of Inhibition

\section{J. LEYDEN WEBB}

School of Medicine

University of Southern California

Los Angeles, California

1963 
Copyright (1) 1963, by Academic Press Inc.

ALL RIGHTS RESERVED.

NO PART OF THIS BOOK MAY BE REPRODUCED IN ANY FORM, BY PHOTOSTAT, MICROFILM, OR ANY OTHER MEANS, WITHOUT WRITTEN PERMISSION FROM THE PUBLISHERS.

ACADEMIC PRESS INC.

111 Fifth Avenue, New York 3, New York

United Kingdom Edition published by

ACADEMIC PRESS INC. (LONDON) LTD.

Berkeley Square House, London W.1

Library of Congress Catalog Card Number: 62-13126

PRINTED IN THE UNITED STATES OF AMERICA 


\section{DEDICATION}

To those scientists who have in their publications on enzyme inhibitors written of their results clearly, who have provided the reader with all the pertinent conditions under which the work was done, who have taken the trouble to express their data in meaningful ways, and who have stimulated others to more quantitative and penetrating modes of thought. 



\section{FOREWORD}

The past fifty years have seen an astounding increase in our understanding of the nature of the physical world. Only fifty years ago was it discovered that an atom consists of a small heavy nuclens and some electrons surrounding the nucleus. A tremendous amount of knowledge has been gathered during this period of fifty years about the structure of atoms and of simple molecules.

It is now becoming possible to extend the experimental techniques that have been applied to simple molecules to the more complicated molecules that are found in living organisms. Some information has already been gathered about the structure of the simplest proteins, but the job of making a complete three-dimensional structure determination for a protein molecule has not yet been carried through to completion. Nevertheless, it is evident that this job will soon be done. During the past year great progress has been made by John Kendrew of Cambridge University in the deternination of the positions of the atoms in the molecule of myoglobin, and the techniques of X-ray diffraction that he and other investigators in the field of protein structure are now applying can be expected to lead in another decade or two to the complete determination of the structure of many globular proteins.

The enzymes constitute a class of protein molecules of extraordinary significance. Just as deoxyribonucleic acid may be said to be the substance that we need to understand if we are to know in its atomic detail the mechanism by means of which organisms reproduce themselves, so are the enzymes the molecules that we must understand to know how organisms carry on their metabolic activities.

No one knows the precise molecular structure of any enzyme and the exact way in which the enzyme carries on its characteristic function of catalyzing chemical reactions. During the next fifty years we can be assured that this knowledge will be gained, in large part probably through structure determinations made by the $\mathrm{X}$-ray diffraction techmique. Nevertheless, even though detailed structural information is still lacking, a very great deal has 
been discovered about enzymes, about the reactions that they catalyze, and about their interactions with inhibitors.

In this book, of which I have the honor to write the Foreword, John Webb has summarized our existing knowledge about enzymes and has contributed further to it by new methods of analysis. There are few men in the world who could have done this difficult job in such an effective way - few men with the background of knowledge and experience in the fields of mathematics, physics, and physical chemistry as well as biochemistry and biology sufficient to permit them to comprehend the great field of enzyme inhibition and to analyze it and discuss it in such a valuable way. In particular, the through discussion of intermolecular forces may be expected to be the basis for future discussion of enzyme inhibition for many years. I am confident that every scientist whose work brings him into contact with enzymes will find this book of value.

Linus Pauling 


\section{PREFACE}

Enzyme inhibitors have become valuable tools in many of the biological sciences and are used widely to study isolated enzymes and the various aspects of cellular metabolism, growth, and function in organisms ranging from protozoa to mammals and from bacteria to the higher plants, as the voluminous and disseminated modern hiterature shows. However, inhibitors can be more than tools. The study of the manifold actions of all substances that significantly distort cellular metabolism is one of the major provinces of molecular biology and its ramifications extend throughout all levels of biological investigation. My simple aims in writing these volumes are to present concisely the basic principles of inhibition, to describe the actions and mechanisms of the most important and interesting inhibitors, to correlate the actions at the enzyme level with the changes observed in cellular function, and to provide practical information on the uses of these inhibitors. It is hoped that these books will aid investigators in the various disciplines in discovering the fundamental relationships that occur ubiquitously in living matter and thus will advance in a small way the search for the elusive laws of cellular organization involved in the flow of energy that characterizes vital processes. There is another aim which is in one sense more personal. I believe that the subject of enzyme inhibition is fascinating in itself and I would like to think that my presentation here will inspire interest in others so that they will come to share my enthusiasm.

Every attempt has been made to place inhibition on a quantitative basis, inasmuch as I am convinced that only in this way can work with inhibitors develop significantly. Indeed, some of the theoretical and quantitative aspects have been developed here beyond our present experimental knowledge in the hope that this will stimulate both accurate experiments and better theories. I have not restricted the treatment to the enzyme and cellular metabolic levels but wherever possible have considered the actions of inhibitors on functioning organs and whole animals. Attention lias not been primarily focused on any form of life - microorganisms, plants, or animals - but instead the attempt has been made to treat all equally on the 
principle that the fundamental mechanisms and problems are the same and that workers in one field can learn a great deal from results obtained in other fields.

The writing of the first volume and the preparation for the succeeding volumes have involved the accumulation of approximately 16,000 references, and several thousand more will be added before the work is completed. A rigorous selection of the material and the examples to be presented is thus necessary. At the present time over 1,400 publications on enzyme and metabolic inhibition appear each year, and this figure must be increased if one includes the work on drugs and chemotherapentic agents that act in some manner by altering metabolism. The use of inhibitors has increased markedly since 1950. Not only are the older inhibitors applied more widely and intensively, but each year one sees with interest the development of new inhibitors. It is interesting to survey by decades the number of publications on the two common inhibitors, malonate and iodoacetate. The accompanying

\begin{tabular}{lcc}
\hline Decade & \multicolumn{2}{c}{ Number of publications } \\
\cline { 2 - 2 } $1880-1890$ & Nalonate & Iodoacetate \\
$1890-1900$ & I & 0 \\
$1900-1910$ & I & 0 \\
$1910-1920$ & 1 & 0 \\
$1920-1930$ & 3 & 0 \\
$1930-1940$ & 9 & 4 \\
$1940-1950$ & 47 & 2.27 \\
$1950-1960$ & 87 & 1.45 \\
\hline
\end{tabular}

table shows these figures taking into account only biologically oriented work. The large number of papers on iodoacetate between 1930 and 1940 was due to the demonstration of its interesting effects on muscle and yeast, and subsequently on the glycolytic pathway. Most enzyme inhibitors would provide similar histories. The marked upsurge in reports since 150 is evident and it is also clear that this acceleration has by no means ceased. Considering the tremendons amount of work that has been done with inhibitors and their importance in many fields, it is quite remarkable that no book on them has yet appeared; indeed, it is uncommon to find reviews or monographs on the individual inhibitors. 
This first volume will be concerned with the general principles of enzyme inhibition and specific inhibitors will be mentioned only for illustration of these principles. The second and third volumes will deal with the individual inhibitors and inhibitor groups, and there much that is discussed in the present volume will be applied.

Inasmuch as the Preface is the only place in a book where one can attend to personal questions, one may perhaps be anticipated. Some may justifiably question my qualifications for writing on such a subject and for so often suggesting the ways in which inhibition studies be done. I can only answer this by saying that I believe I realize my inadequacies more keenly than the reader does, having lived with them daily, but desire to contribute a little to the formulation of this field of inhibition, and perhaps stimulate others to more penetrating analyses and to better presentations in the future.

I would here like to express my gratitude to Professor Linus Pauling for perusing the mannscript and writing the Foreword, and also for what he has contributed to my understanding in the past.

J. Leydex WebB

\section{Department of Pharmacology}

School of Medicine

University of Southern California

January, 1963 


\section{CONVENTIONS}

The effects of inhibitors on enzyme activity, metabolism, or tissue function are expressed as fractional inhibitions, designated by the symbol $i$. This is the fraction of the total control activity that is inhibited under the conditions specified and thus may vary from zero to unity. The various ways in the literature of expressing inhibition have all been reduced to this convention and liberty has frequently been taken to cal. culate fractional inhibitions from untreated data. It is hoped that such a standard system will allow comparisons to be made readily. The kinetic equations are also ex pressed in terms of the fractional imhibition.

Rate constants are always expressed by the lower case $k$ and equilibrium constants by the upper case $K$. The subscripts for the rate constants are positive or negative depending on the direction of the reaction; thus, for the reaction $\mathrm{A} \rightleftharpoons \mathrm{B}, k_{1}$ is the rate constant for the reaction of $\mathrm{A}$ to $\mathrm{B}$ and $k_{-1}$ is the rate constant for the reverse reaction. The subscript number indicates the step in a sequence of reactions. $K$ always refers to a dissociation constant and thus is the inverse of the corresponding association constant; for example, for the reaction $\mathrm{A}+\mathrm{B} \rightleftharpoons \mathrm{AB}$, the value of $K$ is given by (A) (B)/(AB).

Concentrations of substrates and inhibitors are generally given as millimolar ( $\mathrm{m} M$ ), as are the dissociation constants, for the purpose of establishing a uniform system.

The enzyme nomenclature attempts to follow Table V. 1 of Dixon and Webb ("Enzymes," 1958) wherever possible.

\section{SYMBOLS}

The most commonly used symbols are defined in the following list. Other symbols of more restricted use are described in the text where they occur. Where the same symbol is applied to two or more different functions, there should be no confusion in the contexts in which the symbols appear.

$\begin{array}{ll}a & \text { fractional activity (enzymic) } \\ a & \text { activity (thermodynamic) } \\ \text { A } & \text { activator } \\ \text { A } & \text { agonist } \\ \text { A } & \text { antibody }\end{array}$

$\begin{array}{ll}\text { A } & \text { constant for attraction energy } \\ \text { AR } & \text { agonist-receptor complex } \\ B & \text { constant for repulsion energy } \\ d & \text { distance between interacting } \\ & \text { particles }\end{array}$


$d_{e}$ equilibrium distance between interacting particles

$d_{0}$ thermal equilibrium distance between interacting particles

$D \quad$ dielectric constant

e. electronic charge $\left(4.802 \times 10^{-10}\right.$ esu)

$\mathrm{E}$ enzyme (often apoenzyme)

$\mathrm{E}_{a} \quad$ catalytically active enzyme

$E_{\text {act }}$ Arrhenius activation energy

$\mathrm{E}_{d} \quad$ catalytically inactive enzyme (denatured)

$\mathrm{E}_{t} \quad$ total enzyme

EA enzyme-activator complex

$\mathrm{EH}$ protonated enzyme (activesite)

EI enzyme-inhibitor complex

EP enzyme-product complex

Es enzyme-substrate complex

EX* activated complex of enzyme with substance $\mathrm{X}$

$f$ force exerted on charged particle in electric field

$f_{b} \quad \mathrm{pH}$ function for base

$f_{h b} \quad \mathrm{pH}$ function for acid

$f_{h_{2} b} \quad \mathrm{pH}$ function for dibasic acid

$f_{r} \quad$ resistant fraction of rate

$f_{s} \quad$ sensitive fraction of rate

$F \quad$ electrical field strength

$\triangle F \quad$ change in free energy

$\Delta F^{*}$ activation free energy

$\Delta F_{e s}$ change in electrostatic free energy

$\Delta F_{n e s}$ change in nonelectrostatic free energy

$g \quad$ factor by which rate of branched reaction is changed

$h \quad$ Planck's constant $(6.624 \times$ $10^{-27}$ erg-sec)

$\mathrm{H}$ hapten

$\triangle H$ change in heat content (enthalpy)

$1 H^{*}$ activation enthalpy

$i \quad$ fractional inhibition

$i$ mean fractional inhibition

$i_{L} \quad$ inhibition required for lethal action

I inhibitor

$\mathrm{I}_{i} \quad$ inhibitor inside a compartment
Io inhibitor outside a compartment

$I_{1}, I_{2}$ ionization potentials

(I) ${ }_{L}$ lethal concentration

(I) $n$ minimal inhibitor concentration to produce effect

(I) $)_{50}$ concentration for $50 \%$ inhibition

$k$ rate or permeability constant

h. Boltzmann constant $(1.380 \times$ $10^{-16} \mathrm{erg} / \mathrm{deg}$-molecule)

$k_{m} \quad$ salting coefficient

$k_{n} \quad$ rate constant for a particular step $n$

$k_{-n} \quad$ rate constant for reverse reaction of step $n$

$K$ equilibrium (dissociation) constant $[=(A)(B) /(A B)]$

$K_{a} \quad$ activator constant

$K_{a}$ ionization constant

$K_{a s}$ dissociation constant for substrate-activator complex

$K_{i} \quad$ inhibitor constant

$K_{i}^{\prime}$ experimental or apparent inhibitor constant

$K_{2}{ }^{\circ} \quad$ inhibitor constant for isoelectric enzyme

$K_{m} \quad$ Hichaelis constant

$K_{0} \quad$ equilibrium constant between $S$ and $\mathrm{P}$

$K_{p} \quad$ Michaelis constant for reverse reaction (product reaction)

$k_{r}$ dissociation constant for inhibitor-reversor complex

$K_{s}$ dissociation constant for enzyme-substrate complex (substrate constant)

$K_{s}{ }^{\prime}$ experimental or apparent substrate constant

$K_{s i}$ dissociation constant for substrate-inhibitor complex

$K_{s p} \quad$ equilibrium constant between ES and EP

$l \quad$ length of dipole

$L \quad$ limiting rate coefficient

$n$ number of molecules of sub. strate or inhibitor binding to an active site 
$n \quad$ number of doses of an inhibitor

I Avogadro's number $(6.024 \times$ $10^{23}$ molecules $/$ mole)

$\mathrm{pH}_{i} \quad \mathrm{pH}$ inside a compartment

$\mathrm{pH}_{o} \quad \mathrm{pH}$ outside a compartment

$\mathrm{pH}_{\text {opt }}$ optimal $\mathrm{pH}$ for enzyme reaction

$\mathrm{pI} \quad-\log (\mathrm{I})$

$\mathrm{p} K_{i} \quad-\log K_{i}$

$\mathrm{pS}-\log (\mathrm{S})$

$\mathrm{P}$ product

P phosphate group

$\mathrm{P}_{\mathrm{i}} \quad$ inorganic orthophosphate

$P \quad$ pressure

$P \quad$ total polarization

$P_{a} \quad$ atomic polarization

$P_{e} \quad$ electronic polarization

$P_{i} \quad$ induced polarization

$P_{m} \quad$ orientation polarization

$q \quad$ magnitude of charge

$q_{d} \quad$ charge on a dipole

$r \quad$ ratios in general (e. g., of rates in branches of convergent chain)

$r_{0} \quad$ distance of closest approach of ionic atmosphere

$\mathrm{R}$ receptor

$\mathrm{R}$ reversor

$R$ gas constant $\left(8.31 \times 10^{7} \mathrm{ergs} /\right.$ (leg-mole $=1.99 \mathrm{cal} /$ (leg-mole)

$R_{0} \quad$ molar refraction

$\Delta R_{0} \quad$ refraction exaltation

$s \quad$ slope of an inhibition plot

$s \quad$ ionic strength

S substrate

$\mathrm{S}_{e} \quad$ endogenous substrate

$\mathrm{S}_{i} \quad$ substrate inside a compart. ment

$\mathrm{S}_{0} \quad$ substrate outside a compartment

$S_{t} \quad$ total substrate

$\triangle S \quad$ change in entropy

$\triangle S^{*} \quad$ activation entropy

$t \quad$ time

$t_{m} \quad$ minimal time in which effect is produced

$\Delta t \quad$ transition time between steady states

$T$ absolute temperature
T.N turnover number

$v \quad$ rate of reaction

$v_{f} \quad$ rate of forward reaction

$v_{f} \quad$ final rate of reaction

$v_{i} \quad$ rate of inhibited reaction

$v_{i} \quad$ initial rate of reaction

$v_{n} \quad$ rate of the $n^{\text {th }}$ step in multi-

enzyme system

$v_{\tau} \quad$ rate of reverse reaction

$v_{r} \quad$ rate of resistant reaction

$v_{s} \quad$ rate of sensitive reaction

$v_{s t} \quad$ steady-state rate of reaction

$v_{t} \quad$ total rate of reaction

w, IV water

$u \quad$ electrostatic factor

$\mathrm{X}$ transferred group

$\left(\mathrm{X}^{\prime}\right)$ specific concentration of $\mathrm{X}$

$z \quad$ valence of ion

$Z$ number of electrons in outer shell

$\alpha, \beta, \gamma, \ldots$ interaction constants giving the effects of the binding of one substance on the binding of another to the active site

$\alpha_{0} \quad$ molecular polarizability

$\beta$ constant characterizing the change in the rate of breakdown of the ES complex

$\delta$ molar depression of the dielectric constant

$\zeta \quad$ electrical potential between a particle and the medium

$\vartheta \quad$ angle between dipoles

* Debye-Hückel constant

$\mu \quad$ temperature characteristic

$\mu \quad$ dipole moment

$\bar{\mu}$ mean (statistical) dipole moment

$v \quad$ frequency of electrical oscillator

$\sigma \quad$ heterogeneity index

$\sigma \quad$ specificity of inhibition

$\sigma_{\max }$ maximal specificity of inhibition

$\varphi \quad$ inhibition index (of Kistiakowsky and Shaw)

$\varphi \quad$ potential energy

$\varphi_{e} \quad$ equilibrium potential energy of interacting particles 


\section{CONTENTS}

FOREWORD . . . . . . . . . . . . . . . . vii

Preface . . . . . . . . . . . . . . . ix

Conventions . . . . . . . . . . . . . . . . . . xiil

TABle of Symbols . . . . . . . . . . . . . . xiii

CHAPTER 1

Perspectives of Metabolic Inhibition . . . . . . . 1

CHAPTER 2

The Kinetics of Enzyme Reactions . . . . . . . 13

The Dependence of Enzyme Rate on the Substrate Concentration . . 14

Validity of the Michaelis-Menten Theory . . . . . . . . 17

Nature of the Intermediate Complexes with the Enzyme . . . . 27

Factors Determining the Rate of Enzyme Reactions . . . . . 31

Kinetics of Some Complex Enzyme Reaction Types . . . . . 3은

CHAPTER 3

The Kinetics of Enzyme Inhibition . . . . . . . 49

Reversible and Irreversible Types of Inhibition . . . . . . 52

Competitive and Noncompetitive Inhibitors . . . . . . . . 54

Generalized Kinetics of Reversible Inhibition . . . . . . . 55

Inhibition Kinetics in Mutual Depletion Systems . . . . . . . 66

Pseudo-Irreversible Inhibition . . . . . . . . . . 78

Inhibition by Reaction with Coenzyme or Activator Sites . . . . . 82

Inhibition by Reaction with Substrate. Coenzyme, or Activator . . . 85

The Enzyme Combines with More than One Molecule of Inhibitor . . 90

The Inhibitor Is Also a Substrate for the Enzyme . . . . . . . 96

Some Factors Occasionally Important in Inhibition . . . . 98

Expression and Interpretation of Inhibition . . . . . . . 104

CHAPTER 4

Substrate Inhibition and Product Inhibition . . . . 111

Substrate Inhibition . . . . . . . . . . . . 111

Product Inhibition . . . . . . . . . . . . . . . 140 


\section{CHAPTER 5}

\section{Determination: of the Mechanisms and Constants of In-} hibition

The Methods of Graphical Analysis . . . . . . . . . 150

Generalized Equations for Determination of Constants . . . . . 154

Specific Types of Inhibition . . . . . . . . . . . . . 157

Determination of Constants Other than $K_{i}$. . . . . . . 181

Isobolograms . . . . . . . . . . . . . . . . . . 183

Mutual Depletion Systems . . . . . . . . . . 184

Suggestions for Plotting and Determination of Constants . . . . . $\quad 188$

\section{CHAPTER 6}

\section{Interactions of Inhibitors with Enzymes}

The Nature of Enzyme Active Centers . . . . . . . . 194

Intermolecular Forces and Interaction Energy . _ . . . . . . 204

Relative Contributions of Different Interaction Forces . . . . . 242

Dielectric Constant . . . . . . . . . . . . 245

Hydration of Ions . . . . . . . . . . . . . . 250

Ionic Atmosphere . . . . . . . . . . . 260

Distortion Energy . . . . . . . . . . . . . 262

Inhibition Constants and Interaction Energy . . . . . . . . 264

Determination of the Equilibrium Distance $d_{e}$ from $K_{i} \cdot{ } \quad$. . . $\quad 270$

Interactions of Haptens with Antibodies . . . . . . . . 274

Interactions of Substrates and Inhibitors with Cholinesterase . . . $\quad \mathbf{2 8 4}$

Interaction Forces in Varions Enzyme Systems . . . . . . 292

Interactions of Aromatic Compounds . . . . . . . . . . . 297

Interactions of Hydrocarbon Chains . . . . . . . . . 300

Dependence of Interaction Energy upon Fit . . . . . . . 301

Effects of Alteration of Structure on Molecular Properties . . . . . 304

Interactions of Small Molecules with Proteins . . . . . . . 312

Future Problems in Molecnlar Interactions . . . . . . . 317

\section{CHAPTER 7}

\section{Inhibition in Multienzyme Systems . . . . . . . . . 319}

Concepts and Terminology . . . . . . . . . . . . . 320

Irreversible Monolinear Chains . . . . . . . . . . 322

Partially and Completely Reversible Monolinear Chains . . . . . 338

Branched Linear Chains . . . . . . . . . . 340

Polylinear (hains (Shunt System) . . . . . . . . . 345

Distributive Systems . . . . . . . . . . . . 346

Cyclic Systems . . . . . . . . . . . . . 348

Regenerative Systems . . . . . . . . . . . . 354

Self-Regulatory or Feedback Systems . . . . . . . . . . . 368

Network Systems . . . . . . . . . . . . . 372

General Considerations of Multienzyme Systems . . . . . $\quad 372$ 
Transition between Steady States . . . . . . . . . 383

The Future of the Study of Irultienzyme Systems . . . . . . 391

\section{CHAPTER 8}

\section{Distribution and Fate of Inhibitors in Living Organisms}

Distribution of Inhibitors in Animals . . . . . . . . . . 394

Distribution of Inhibitors in Plants . . . . . . . . . 405

Distribution of Inhibitors within Cells . . . . . . . . . . 410

Fate of Inhibitors in the Animal . . . . . . . . . . 413

Repeated Administration of an Inhibitor . . . . . . . . . 42]

Distribution and Effect of Inhibitors . . . . . . . . . 425

Chemical Composition of the Media . . . . . . . . . 428

Distribution and State of Enzymes in Cells . . . . . . . 432

\section{CHAPTER 9}

Inhibition in Cells and Tissues . . . . . . . . 427

Types of Inhibition Unique to Organized Systems . . . . . . . 436

The Problem of Endogenous Metabolism . . . . . . . . 441

Inhibitor-Stable Metabolism . . . . . . . . . . 447

Stimulation of Metabolism by Inhibitors . . . . . . . . 4.)

Intracellular Equilibria and Secondary Effects of Inhibitors . . . . 4.56

The Concept of Pacemakers in Metabolism . . . . . . . 458

Relations between Enzyme Inhibition and Changes in Cell Function . . 462

Quantitative Expression of Intracellular Enzyme Inhibition . . . . . 474

Inhibition in Tissue Slices and Isolated Tissue Preparations . . . . . 479

Differential Effects of Inhibitors in Whole Animals . . . . . . . . 481

Enzyme Variability . . . . . . . . . 485

\section{CHAPTER 10}

\section{Effects of More than One Inhibitor. . . . . . . 487}

Two Inhibitors Aeting on a Single Enzyme . . . . . . . 488

Two Inhibitors Acting on a Monolinear Chain . . . . . . . 498

Two Inhibitors Acting on Various Multienzyme Systems . . . . 500

Two lnhibitors Acting on Cellular and Tissue Metabolism . . . . 502

Antagonism, Summation, and Synergism . . . . . . . . 507

Specificity of Inhibition with Two Inhibitors . . . . . . . 510

Inhibition of Bioluminescence with Sulfanilamide and Urethane . . . 510

\section{CHAPTER 11}

Localization of the Site of Inhibition . . . . . . . . 513

Methods of Localization . . . . . . . . . . . 513

General Development of a Localization Program . . . . . . 527 
General Ways of Expressing the Rate of Inhibition . . . . . 536

Rates of Inhibition of Pure Enzymes . . . . . . . . . 541

Rates of Inhibition in Mitochondria, Cells, and Tissnes . . . . . 571

Rates of Lethal Action in a Cell Population . . . . . . . . 590

Concentration-Time Curves for Inhibition . . . . . . . . 595

\section{CHAPTER 13}

\section{Reversal of Inhibition}

Ways in Which Reversal of Inhibition May Be Achieved . . . . 606

Dissociation of the Enzyme-Inhibitor ('omplex . . . . . . . 607

Competitive Displacement of the Inhibitor by Substrate . . . . 612

Reversal of Inhibition by a Substance Binding the Inhibitor . . . . 615

Spontaneous Reactivation of Inhibited Enzymes . . . . . . 626

Induced Reactivation of Inhibited Enzymes . . . . . . . 630

Reversal of Inhibition by Destruetion of Inhibitor . . . . . . 637

Reversal of Inhibitions in Cells and Whole Animals . . . . . 642

\section{CHAPTER 14}

\section{Effects of pH on Enzyme Inhibition . . . . . . . 649}

Expressions for the pH-Functions of Weak Acids and Bases . . . 650

Variation of Enzyme Activity with pH . . . . . . . . 652

Variation of Enzrme Inhibition with $p \mathrm{H}$. . . . . . . . 667

Variation of Multienzyme Inhibition with $\mathrm{pH}$. . . . . . . 711

Variation of Intracellular Inhibition with $\mathrm{pH}$. . . . . . . 713

\section{CHAPTER 15}

Effects of Various Factors on Inhibition . . . . . . 747

Effects of Temperature: Enzymes . . . . . . . . . 747

Effects of Temperature: Multienzyme Systems. . . . . . . 768

Effects of Temperature: Cellular Systems . . . . . . . . 773

Effects of the Dielectric Constant . . . . . . . . . 799

Effects of Salt Concentration and Ionic Strength . . . . . . 815

Effects of Buffers . . . . . . . . . . . . . 839

Effects of Pressure . . . . . . . . . . 841

Effects of Extraneons Binding and Impurities . . . . . . 854

Effects of Enzyme or Metabolic Rate . . . . . . . . 857 
CHAPTER 17

\section{Suggestions for Planning and Reporting Inhibition}

Studies

Suggestions Particularly Applicable to Inhibitions of Purified Enzymes 879

Suggestions Particularly Applicable to Inhibitions in Cellular Preparations 882

Suggestions Applicable to Inhibitions of Both Purified Enzymes and Cells 883

Suggestions Relative to the Reporting of Inhibition Studies . . . . 885

Suggestions of a General Nature . . . . . . . . . . 886

References . . . . . . . . . . . . . . . . . 889

AUTHOR INDEX . . . . . . . . . . . . . . . 907

SUbJect INdex . . . . . . . . . . . . . . . . . . . . . . . 919 



\title{
Enzyme and Metabolic Inhibitors
}

\author{
Volume I
}

General Principles of Inhibition 



\section{PERSPECTIVES OF METABOLIC INHIBITION}

Living cells are characterized by a complex and beautifully organized pattern of chemical reactions mediated and directed by enzyme systems. These reactions provide the energy necessary for the various functions of the cell and support the synthesis of material upon which the cell depends for maintenance, growth, and multiplication. One basic way of expressing this pattern is to consider the flow of energy throngh the cell: the intake of the potential energy resident in prospective substrates. regulated by membrane permeability and occasionally by active processes; the release of this energy into utilizable forms during the oxidative degradation of these substances, wherein the energy is fractionated and distributed in smaller units; the transfer of this energy, perhaps through several steps, until it is manifested in terms of some evident cellular activity, such as secretion, active transport, impulse conduction, movement or emission of radiation; the partial flow of the energy into the complex compounds synthesized either for primarily structural purposes or for the metabolic and functional systems of the cell, for even the enzymes themselves must be assembled using the energy derived from their catalyzed reactions; and, finally, the inevitable release of a certain fraction of the energy as heat, since energy transfer and utilization cannot be completely efficient. If one were acquainted with the full pattern of this energy flow and the means by which it is organized, one could be said to understand the nature of life.

The application of enzyme inhibitors to cells or their aggregates is one of the ways in which the scientist attempts to penetrate into the nature of living material and its transformations. This approach pertains to that class of investigations in which a perturbation is induced in a complex system for the purpose of understanding better the initial normal state. Such a procedure must be quantitative to be fully effective. Also the primary perturbation produced should be as small as possible, consistent with the sensitivity of the methods of measurement, in order to reduce secondary phenomena to a minimum. The normal state of a biological system should not be conceived as a vague ensemble of simultaneous processes, but as a perfect balance of complex chemical and functional activities superimposed 
on an intricate structure, the possibility of life depending upon a proper and accurate coordination of the rates of these various processes. The introduction of an inhibitor into the system leads to an alteration of certain rates and a disturbance of this balance. The extent to which the imbalance will affect the total system will depend upon many factors. One can usually measure only the results of the imbalance and, hence, must always be aware of the factors that stand between the metabolic dislocation and what is measured. If the inhibitor shifts the balance to the detriment of the organism, it is generally classed as a poison; when the balance is shifted in favor of the survival of the total system, the inhibitor may be considered as a drug.

The actions of inhibitors upon living systems can often be most profitably visualized by interpreting the results in terms of a modification of the energy flow (Fig. 1-1). There are many ways of deviating the course of the flow

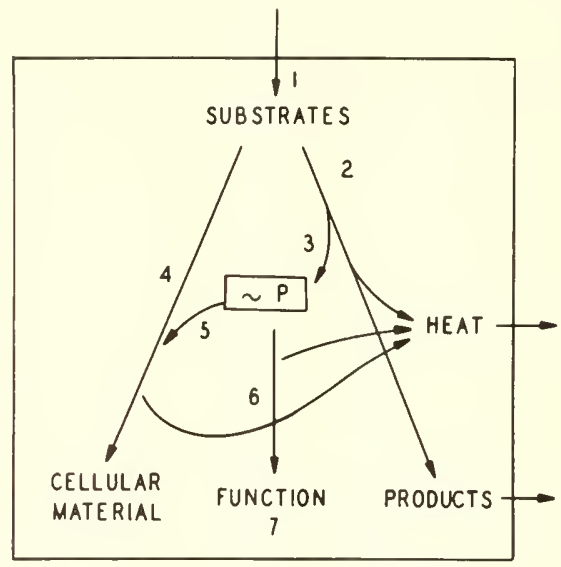

Fig. 1-1. Scheme of the basie energy flow through a cell.

of energy through cells and, therefore, in some mamer altering the biochemical morphology and the functional phenomena necessary for the life of the cell and for the cell's role in the life of the total organism. The principal pathways of energy flow are indicated in the diagram and it is possible in this scheme to designate some of the general loci at which inhibitors may act. An inhibitor may interfere with the entrance of the substrates into the cell (1) by altering permeability or depressing transport mechanisms; in this way the inhibitor can reduce the total potential energy available to the cell. The action may also be exerted on any of the metabolic pathways whereby energy and matter are distribnted, especially on the degradative reactions in which the substrates are broken down oxidatively to products 
(2), the formation of high-energy phosphate (3), the synthetic reactions for the formation of the protoplasmic components (4), or the utilization of the high-energy substances by these synthetic processes (5). With respect to the functional activity of the cell, the inhibitor may act either on the reactions involved in the utilization of energy (6) or directly on the functional systems themselves (7). It is necessary to realize that these intracellular processes are closely related so that, for example, a primary inhibition on the oxidation of substrate will depress to varying degrees the formation of high-energy substances, synthesis, function, and the appearance of products. Also it must always be borne in mind that the actions of many inhibitors are not localized to a single pathway inasmuch as inlibitors are seldom completely specific.

Directly distorting the pathways of enzymically directed reactions by the introduction of a chemical substance - blocking a pathway or diverting a sequence into a new channel, interfering with coupled processes or hindering the accumulation of energy - is one approach amongst others. It is an interesting and a useful way because it often provides a rather specific attack upon some particular reaction or phase of metabolism, and often one is able to relate certain aspects of cellular activity to this susceptible region in the metabolic network. In fact, it is often possible to isolate from the cell the special enzyme or enzyme system acted upon and to study the detailed mechanism of the interference, so that it becomes possible to postulate more accurately what occurs within the cell when the inhibitor exerts its effects. Many other ways of altering metabolic activity - for example, by changing the temperature or the $\mathrm{pH}$, by irradiation or high pressure - are nonspecific and seldom does one liave any ideas as to exactly what is occurring in the complex protoplasmic matrix. If one had to choose the most interesting and important characteristic of enzyme inhibitors, what it is that makes them one of the most powerful tools in so many fields of biological investigation, it would be their relative specificity, or at least the possibility of attaining greater and greater specificity as new inhibitors are discovered for attack upon reactions of interest. The question of specificity cannot be overemphasized. The more we know about the exact nature of the perturbation produced and the more selective this action can be made, the more likely it is that alear interrelationships will emerge and the goal of understanding the energetics of the cell be achieved.

Sometimes in using inhibitors one does not begin initially with a normal cellular system, but one in which something has gone astray in the complex pattern, in other words a group of pathological cells. If this aberration is basically metabolic, there is the possibility of restoring the cells towards the normal pattern by the use of substances effective in specifically altering appropriate reactions. It is probable that many of the most useful drugs act exactly in this manner. On the other hand, if the cells have become 
so abnormal as to resist our efforts at correction, we may desire to kill them in order that they will not disturb the normal cells and cause eventual dissolution of the organism, and again one looks towards a specific attack on those fundamental differences that distinguish pathological cells from normal ones. Recent work along these lines in carcinostasis would seem to justify this approach. Furthermore, foreign cells may enter the organism and endanger its survival, and here too one must examine the differences between these invading cells and the tissue cells, the different flows of energy, to progress intelligently in the development and use of agents designed to block the vulnerable processes in the intruders. Much of modern therapy depends upon such specific inhibition and almost every inhibitor group to be discussed has been used in some way in diseased states.

It may be worthwhile to outline briefly the importance of enzyme inhibitors in certain fields of investigation. The enzymologist attempts to understand in ever greater detail the intimate mechanisms of enzyme action so that he will have deeper insight into the energetic basis of living processes. Inhibitors have played a major role in the mapping of enzyme reactions in both time and space. Organic chemists use group-specific reagents to identify the groups in unknown compounds. Likewise, the use of substances reacting specifically with protein groups provides a means of characterizing the chemical pattern of the enzyme surface and, particularly if these substances interfere markedly with the catalyzed reactions, evidence is obtained that the reacted groups are part of the active center, that region of the enzyme primarily responsible for the activation of the substrate. We shall have ample opportunity to see how successful such procedures have been in ehcidating the topography of these regions and hence in aiding our understanding of the kinetic mechanisms by which biologically important compounds are transformed. The biochemist thus frequently applies inhibitors to the study of isolated and purified enzymes as part of the program of characterization, and certainly this is one of the most fascinating aspects of biology on the molecular level.

The study of molecular interactions in biological systems has been retarded by the deficiency of accurate data and by the inherent complexities of the forces that are involved, but the importance of this subject will certainly necessitate an increasingty vigorous combined attack on these problems by physicists, chemists, and biologists. The most significant interactions are between proteins and various substances - inorganic ions, hormones, lipids, drugs, nucleotides and nucleic acids, and dyes - or between different proteins. Almost all of cellular activity involves such interactions. There is much need for more work on the quantitative aspects of these interactions and for accurate studies of purified systems. The interactions of inhibitors with enzymes present one excellent system for such study and the results of inhibition often allow the over-all energies of the interac- 
tions to be calculated readily. Furthermore, these interactions are usually with specific regions of the protein and are not general adsorption phenomena. Comparison of structurally related inhibitors enables one to assign specific energy terms to certains groups. Not only are more accurate data needed but also more penetrating theoretical studies of the balance of forces between such interacting molecules, the roles of water and ions and the dielectric constant, the mapping of energy contours, and the configurations assumed by the interacting molecules. One can confidently look forward to the time when enough will be known about the patterns of enzyme active centers and the forces involved in complex formation to predict what types of substances will inhibit. just as drugs are now designed for special purposes on the basis of what is known of cellular function at a different level.

The biochemist also makes use of inhibitors in the investigation of the multiple enzyme systems in linear or cyclic metabolic sequences. It is often possible by using the proper inhibitors to determine the sequence in which the components of a catenary chain react. There are several classical examples of such analysis: the original assignment of the positions of the enzymes in the oxidative pathway by Keilin, the discovery of unknown components in specific loci in the electron-transport chain by Slater and Potter. the elucidation of the glycolytic pathway by Lohmam. Embden, and Heyerhof, and the more recent mapping of the sites of oxidative phosphorylation by Chance. In addition, the biochemist is occasionally able to isolate with an appropriate inhibitor certain metabolic reactions that are to be studied; relatively simple examples would be the use of malonate to block succinoxidase in studying the oxidation of $\alpha$-ketoglutarate in mitochondria, and the blocking of pyruvate entry into the tricarboxylic acid cycle by arsenicals in order to investigate the transformations of glycogen or glucose into pyruvate. Such techniques have been relatively little developed but hold much promise for the future study of metabolic pathways in tissues or whole organisms.

It may be noted incidentally that no manner of investigation so well demonstrates the inherent differences in the metabolism of various tissues or organisms as does that of enzyme inhibition. It is to be deduced from the markedly variable responses of cells or tissues to inhibitors that respiration and glycolysis, for example, are not the simple ubiquitously uniform processes usually presented in textbooks, but that each type of cell has its particular metabolic organization. that the enzymes catalyzing the same reaction in different cells are kinetically different, and that there are variations in the controlling factors governing the metabolic balance in varions cells. This is not to detract from the concept of the obvious and fundamental uniformity of enzyme types and general metabolic systems throughout living material, but it serves to emphasize that these units of 
activity can be arranged and controlled in a multiplicity of ways. Indeed, the specificity of the effects observed on certain tissues when the inhibitors are administered to whole animals is presumptive evidence for the metabolic differentiation of tissues.

The physiologist, whether interested in plants or animals, turns to enzyme inhibitors to establish relationships between the functional aspects of cellular activity and the metabolic systems supplying the energy and the materials necessary for these functions. Perhaps the most commonly used substances have been the uncouplers of oxidative phosphorylation in order to demonstrate the role of ligh-energy phosphate compounds in cellular processes. There are many other well-known studies in which inhibitors have aided our understanding of the metabolic basis of function: the investigations of nerve and muscle membrane potentials by Hodgkin, Shanes, and Gerard; the elucidation of various active transport or secretory mechanisms, in the kidney by Taggart and Hudge, in the gastric mucosa by Davenport, in the intestine by Visscher and Quastel, in the erythrocytes by Parpart, LeFevre, and Wilbrandt, and in plants by Lundegårdh; the investigation of muscle contraction by Lundsgaard; the studies of cell division by Rumuström, Clowes, and Krahl; and the determination of the role of acetylcholine in many tissues by Loewi and others. Despite the large amount of work done, only a modest degree of success has been achieved in accurately relating function to metabolism, due mainly to the complexity of the situation, but it is likely that this use of inhibitors will become increasingly important as the blocking can be made more selective.

The relationship between enzyme inhibition and pharmacology is so close that it would be very difficult (and useless) to draw a line between them. Some would prefer a broad definition wherein all substances acting on living matter are called drugs, so that inhibitors would then be considered as drugs and come under the field of pharmacology. Systems of classification here are not very important and we shall generally restrict the use of the term drug to substances currently useful in medicine. Substances such as carbon monoxide, which are important toxicologically and are discussed in the standard pharmacology texts, and yet are also classic enzyme inhibitors, will be considered for practical purposes as inhibitors. In some cases the decision must be arbitrary. Some organic mercurials are widely used as diuretics in edematous states and their actions are probably attributable to an interference witl renal metabolism in a manner similar to the actions of those mercurials, such as $p$-chloromercuribenzoate, used by biochemists to detect sulfhydryl groups on enzymes. Inhibitors of carbonic anhydrase (acetazoleamide, chlorothiazide, and the recent derivatives), cholinesterase (physostigmine, neostigmine, and the organophosphorus compounds), and monoamine oxidase (iproniazid and derivatives) are very commonly used in medicine, and it is likely that other enzyme inhibitor groups will be cli- 
nically applied in the future. It is indeed possible that many of the drugs whose actions are at present unknown are inhibitors of enzymes or in other ways disrupt metabolism. The recent classification and mathematical exposition of drug antagonisms and synergisms by Ariëns and his co-workers are simply enzyme inhibition kinetics applied to drug actions and his equations have their counterparts in the classical inhibition formulations. Enzyme inhibitors can, of course, be useful to the pharmacologist in the analysis of the site and manner of action of drugs, particularly when the drugs affect directly or indirectly some metabolic processes.

One of the most interesting facets of enzyme inhibition is the production in animals of metabolic blockades which become manifest as syndromes resembling certain diseased states. The pathologist is occasionally able to learn something about disease processes from the study of such artificially induced biochemical lesions resulting from the controlled use of enzyme inhibitors. There are numerous examples: the inflammation, vesication, and neuritis produced by the arsenicals, the induction of pseudocoeliac disease in rats with iodoacetate, the diabetic state from alloxan, the occurrence of obesity after the administration of the dithiocarbamates, the carcinogenicity of many inhibitors, the deficiency states brought about by analogs of essential coenzymes and by substances inactivating these coenzymes, and the abnormal mental states sometimes following the monoamine oxidase inhibitors. There is a wide field to be explored in the tracing of the progressive pathological changes occurring during chronic administration of substances disturbing metabolism in various ways. This would be the study of the sequence of cellular changes initiated by selective metabolic alteration in contrast to the observation of the immediate effects. There is also the possibility that naturally occurring inhibitors may be responsible for some diseases and many of such inhibitors are now known.

These relationships of enzyme inhibition to other disciplines point to the fact that the study of inhibition is one of the common meeting grounds of many diverse fields. This is also shown by the appearance of publications on inhibitors in journals of every type - chemical, biological, medical. and industrial. There is perhaps some need in present-day science to establish fields that cut across many others in order to combat the excessive compartmentalization that has inevitably developed. In the study of enzyme and metabolic inhibition one soon loses any conception of the artificial boundaries of these many fields and comes to look upon the study of living matter as the only subject to be pursued.

Inasmuch as there is no general account of the history of the use of inhibitors, it may not be out of place here to present a short summary of this development against the background of enzymological and biochemical history. The first recognition of an enzyme was by Payen and Persoz in 1830 when they observed the splitting of starch by a substance they called dias- 
tase, and this was soon followed by the enunciation of the principle of catalysis by Berzelius in 1835, who included diastase as a catalytic agent. The name " diastase" came to be used for all enzymes and the term "ferment" was also soon applied because of the relationship of enzyme action to fermentations. The name "enzyme" was introduced by Kühne in 1878 and simply means "in yeast" inasmuch as yeast was at that time the source of most of the known enzymes. A great advance was made by Emil Fischer in 1894 in the recognition of the specificity of enzymes, and the lock-and-key theory of the steric relationship between enzyme and substrate may be attributed to him. Serious attempts to isolate and purify enzymes did not begin until after 1920, most of the early work being done by Willstätter and his colleagues between 1922 and 1928. Urease was obtained in impure crystalline form by Sumner in 1926 and this was followed by the crystallization of certain proteolytic enzymes by Northrop and his students. The purification of intracellular enzymes did not begin until 1937 . These landmarks in enzymology are mentioned because the entire development of the study of inhibitors is intimately related to the progress in the knowledge of enzymes and metabolism. In one sense it is a tribute to the early workers to have advanced so far in the understanding of enzyme mechanisms and kineties long before enzymes had been isolated or purified. For example, the important concept of a complex between the enzyme and the substrate was put forward by A. Brown in 1902 and by 1904 Armstrong was able to represent this complex in quite modern terms. This picture of enzyme action enabled the correct kinetic expressions to be formulated, first by Henri in 1903 and in more detail by Michaelis and Menten in 1913. Inhibition mechanisms and kinetics have necessarily developed hand in hand with such concepts.

The early history of enzyme inhibition is amorphous. Some inhibitors have been known for hundreds of years as poisons. It might be said that classic toxicology led to the first studies of what are now known as enzyme inhibitors - cyanide, arsenic, hydrazine, mercury, and certain other heavy metals. Sporadic work on the effects of such substances on a variety of plants, small animals, and microorganisms was reported from 1850 to around 1890, and during the period from 1870 to 1900 there was increasing attention given to inhibitions of general metabolic processes, such as fermentation, glycolysis, and respiration, as well as to the actions of inhibitors on whole animal metabolism. It is actually very difficult to determine in this progressive clevelopment where the true study of inhibitors as inhibitors began. Certainly before 1910 these substances were not considered as primarily enzyme or metabolic inhibitors. Perliaps the modern study of enzyme inhibition may be said to have been initiated by the demonstrations of the depression of sea urchin egg respiration with cyanide by Warburg in 1910 and the inhibition of goose erythrocyte respiration with arsenite by 
Onaka in 1911. Although other inhibitions had been observed earlier, these respiratory inhibitions stimulated much further work on the mechanisms by which these substances acted. At this time the enzymes upon which such compounds acted were unknown and the components of the respiratory system were formulated only in very vague terms.

The kinetics of inhibition produced by a substance binding reversibly with the enzyme were developed in the original publication of Michaelis and Menten in 1913 but it required many years before this type of inhibition was characterized. Quastel and his co-workers in their studies of the inhibition of bacterial dehydrogenases by malonate between 1925 and 1928 were able to show that the inhibition was rather specific for succinate oxidation. The idea that there was a reversible competition between succinate and malonate for the enzyme site was mentioned but it was not until 1930 that Cook stated definitely that a competitive mechanism had been established. Michaelis with Rona and Pechstem in 1914 while studying maltase and invertase had recognized a second type of inhibition which they referred to as "inhibition without affinity." the inhibitor being said to decrease the rate of breakdown of the enzyme-substrate complex, but they did not develop a mathematical expression for this. Haldane in 1930 was the first to derive an equation for this type of inhibition and he gave it the name of "noncompetitive inhibition." Thus the formulation and experimental establishment of competitive and non-competitive inhibitions really must be dated as recently as 1930 .

The early theory of IVarburg that certain inhibitors, such as cyanide, combined with iron, or other metals involved in metabolism, was not established until around 1927 when the complexes of cyanide and carbon monoxide with the "Atmungsferment" (cytochrome oxidase) were demonstrated. The mechanism by which the arsenicals act was suggested by Voegtlin and his group in 1923 from their discovery that the inhibitions were combatted by sulfhydryl compounds. This, coupled with the contemporary interest in sulfhydryl substances (such as glutathione), stimulated by the work of Hopkins. led to the postulate that arsenicals inactivated necessary sulfhydryl compounds in the cell, but it was over 10 years later that enzyme inhibition on the basis of reaction with protein sulfhydryl groups was recognized by Hellerman through his study of heavy metals. It is interesting to note that in the "Allgemeine Chemie der Enzyme" of Haldane and Stern, published in 1932, sections are devoted to the following enzyme inhibitors: heavy metals, oxidants (hydrogen peroxide, iodine, and permanganate), amino group reagents, carbonyl reagents (phenylhydrazine), cyanide, sulfide, pyrophosphate, metal chelators, surfactants, finoricle, arsenicals, and antienzymes. Thus by this time the study of inhibitors had been recognized as a part of enzymology.

The decade of 1925-1935 might be appropriately termed the classic pe- 
riod in the history of enzyme inhibition. inasmuch as it was during this interval that the actions of many of the most important inhibitors were studied. It was also the period when inhibitors began to be used as tools to elucidate metabolic pathways and to probe the dependence of cell functions on metabolism. Before 1925 no exact sites and mechanisms of inhibition were known and, indeed, the concepts of metabolic blockade were vague. A cursory reading of the publications on enzymology and metabolism before 1925 clearly shows that this branch of enzymology was relatively undeveloped, despite the many reports of the actions of inhibiting substances on various systems. Actually, it required the isolation of enzymes and the mapping of metabolic pathways before inhibitors could be rationally studied. It is a well-known fact that the characterization of the components of several metabolic pathways was aided by the use of inhibitors whose sites of action were simultaneously established. Although there were hundreds of publications before 1925 on the miscellaneous effects of what we now recognize as enzyme inhibitors, the fundamental idea of a substance producing a relatively specific block in metabolism was not conceived as yet. Also the concept that inbibitors might be used to investigate the nature of enzymes was in its infancy.

Let us conclude this historical summary by outlining some of the major contributions made during this decade. The establishment of competitive inlibition, the importance of sulfhydryl groups, and the complexing of cyanide with iron have already been mentioned. The localization of the action of carbon monoxide to cytochrome oxidase and the demonstration of the light sensitivity of the complex by Warburg and Negelein in 1928 led to a more accurate characterization of this enzyme whose existence was known only from indirect evidence. Schwartz and Oschmann in 1925 reported that bromoacetate prevented the formation of lactate in muscle and this was followed in 1930 by the work of Lundsgaard with iodoacetate. These observations stimulated the subsequent work on glycolysis. Between 1931 and 1935 the glycolytic pathway was mapped and the sites of action of iodoacetate, fluoride, and arsenate were determined through the work of Dickens, Lipmann, Lohmann, Braunstein, Harden, and Meyerhof. The mechanism by which phlorizin induces a "diabetic" state of glucosuria was worked out in 1933 by Lundsgaard, who found that this inhibitor interfered with phosphorylations in the renal tubular cells. During 1934 and 1935 the action of dinitrophenol in stimulating respiration while simultaneously depressing function was noted and the study of the mechanisms involved was initiated. The localization of the site of arsenical action was carried a step forward by Lipmann in 1933 when he showed that a block was produced in the oxidation of the keto acids. The determination of inhibitor sites in the oxidative sequence by Quastel, using spectroscopic techniques, and Keilin's work on the actions of cyanide and azide on the cyto- 
chrome system during this period were the first investigations to utilize inhibitors to establish the order of activity in a metabolic sequence. This incomplete survey shows what sudden strides were made in this field during these years. More detailed accounts of this period and of the earlier developments will be given in the later volumes under the individual inhibitors.

Despite the importance of enzyme inhibitors as tools used by investigators in the various fields and the progressively greater application of inhibitors in industry and medicine, these inhibitors have an interest and fascination to some of us beyond their practical applications. The study of a new inhibitor, its intricacies of mechanism, and its manifold effects upon different types of cells, or the investigation of well-known inhibitors on new systems, is an exciting exploration involving concepts on all levels of biological organization and gives rewards of scientific experience over and above the satisfaction of having derived information that may be utilized in a more thorough understanding of the cell or of contributing to the alleviation of disease. 



\section{CHAPTER 2}

\section{THE KINETICS OF ENZYME REACTIONS}

An enzyme reaction occurs on the surface of a protein molecule, at a region where the arrangement of the polypeptide chains has placed particular groups in such a pattern that the substrate molecule is not only bound but altered in a manner conducive to reaction. This reaction may be intramolecular or, more commonly, with another molecule also bound to the enzyme, to form a new substance or substances. Groups other than of polypeptide origin and bound to the protein may, of course, participate in the catalysis in certain instances. The fundamental process is the formation of temporary complexes of the reacting molecules with the protein or its accessory groups at a special region or active center. One is interested in these complexes and the nature of the active center in inhibition studies because most enzyme inhibitors interfere with the formation or breakdown of such complexes by combining either with the active center directly or with adjacent regions of the enzyme. The over-all reaction catalyzed by a simple enzyme is made up of at least three phases: (a) the formation of the appropriate complex or complexes of enzyme and reactants, $(b)$ the intermolecular rearrangements to form complexes of the enzyme and the products, and $(c)$ the dissociation of these complexes to form the free enzyme and the products of the reaction. The rate at which the products are formed thus might depend on the rate of any one of these phases, if it is much slower than the others, or on any combination of the rates if they are of comparable magnitude; it may also depend on the rate at which the enzyme-substrate complex dissociates to form the original substrate and the free enzyme. The presence of an inhibitor modifies these rates so that a reduction in the formation of products results. A study of inhibition ideally involves a quantitative formulation of the rate changes so that some insight may be obtained into the mechanism. These quantitative aspects of inhibition are extensions of the general enzyme kinetics and thus it is necessary in this chapter to consider the basic concepts and formulations of enzyme catalysis. It will be particularly important to examine critically the interpretation of the mathematical expressions with regard to the actual molecular events occurring, inasmuch as true understanding implies at least a partial visual interpretation of the process. The excellent books of Dixon and Webb (1958) and Laidler (1958) 
are recommended for a more detailed exposition of fundamental enzyme kinetics. The mathematical approach to this subject is well presented by Reiner (1959).

\section{THE DEPENDENCE OF ENZYME RATE ON THE SUBSTRATE CONCENTRATION}

The rate of an enzyme-catalyzed reaction does not increase linearly with increase in substrate concentration, as wonld occur if the reaction were nonenzymic, but increases progressively more slowly in a hyperbolic manner to reach a level of maximal activity beyond which the rate is dependent only on the enzyme concentration (Fig. 2-1). This deviation from nonenzy-

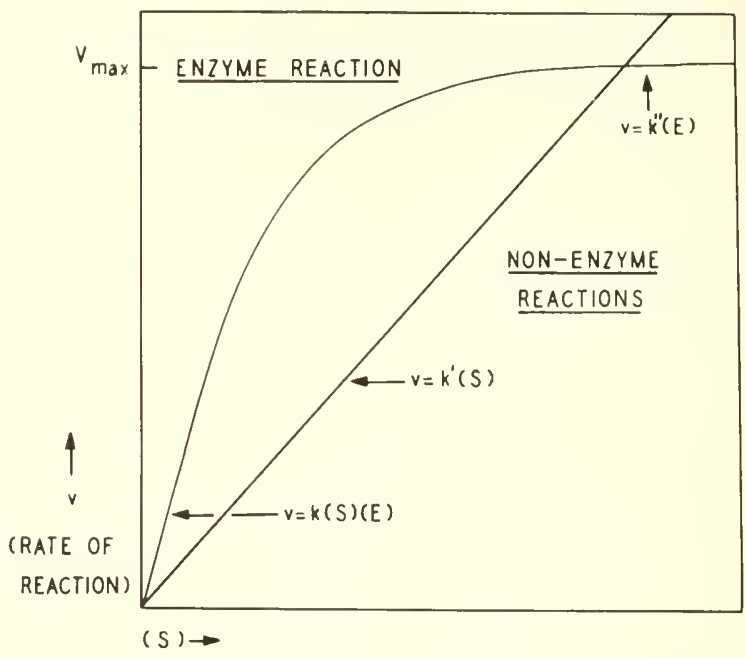

FIG. 2-1. Dependence of the rates of nonenzyme and enzyme reactions on the concentrations of the reactants.

mic kinetics in the case of the hydrolysis of sucrose by $\beta$-fructofuranosidase led Michaelis and Menten (1913) to assume that a complex was formed between the enzyme and the substrate, preliminary to the hydrolysis, and that the over-all rate of the reaction was dependent on the rate of breakdown of this complex into the products of the reaction, in this case glucose and fructose. The formation of this complex was assumed to be essentially instantaneous so that the concentration of the complex was maintained at a constant level dependent on the themodynamic equilibrium between enzyme, substrate, and complex. The rate expression on this basis can be 
derived in the following direct manner. The reaction sequence may be written as:

$$
\mathrm{E}+\mathrm{S} \underset{k_{-1}}{\stackrel{k_{1}}{\rightleftharpoons}} \mathrm{ES} \stackrel{k_{2}}{\rightarrow} \mathrm{E}+\mathrm{P}
$$

where $\mathrm{E}$ represents the enzyme, $\mathrm{S}$ the substrate, ES the enzyme-substrate complex, $\mathrm{P}$ the product or products, and $k_{1}, k_{-1}$, and $k_{2}$ are rate constants. The rate is assumed to be proportional to the concentration of the complex:

$$
v=\frac{d(\mathrm{P})}{d t}=k_{2}(\mathrm{ES})
$$

The equilibrium concentration of the complex may be obtained from the expression for the dissociation:

$$
K=\frac{k_{-1}}{k_{1}}=\frac{(\mathrm{E})(\mathrm{S})}{(\mathrm{ES})}
$$

and the conservation equation relating the forms of the enzyme existing during the reaction:

$$
\left(\mathrm{E}_{t}\right)=(\mathrm{E})+(\mathrm{ES})
$$

where $\left(\mathrm{E}_{t}\right)$ and $(\mathrm{E})$ represent the total and free enzyme concentrations respectively. Therefore:

$$
(\mathrm{ES})=\frac{\left(\mathrm{E}_{t}\right)(\mathrm{S})}{(\mathrm{S})+K}
$$

and substituting this value in Eq. 2-2:

$$
v=\frac{k_{2}\left(\mathrm{E}_{t}\right)(\mathrm{S})}{(\mathrm{S})+K}
$$

Since the maximal rate at high enzyme-saturating substrate concentration in equal to $k_{2}\left(\mathrm{E}_{t}\right)$, Eq. 2-6 may be rewritten in the usual form:

$$
v=\mathrm{V}_{m} \frac{(\mathrm{S})}{(\mathrm{S})+K}
$$

where $V_{m}$ is the maximal rate under the specified experimental conditions.

This equation gives the expected hyperbolic curve when $v$ is plotted against $(S)$ and a sigmoid curve when a logarithmic scale for $(S)$ is used (Fig. 2-2). The constant $K$ is commonly termed the "Michaelis constant" and, under the present assumptions, is the dissociation constant for the ES complex and an inverse measure of the affinity of the enzyme for the substrate. $V_{m}$ and $K$ for a particular enzyme reaction may be determined 
graphically by plotting $1 / v$ against $1 /(\mathrm{S})$ since the reciprocal form of the Michaelis-Menten equation:

$$
\frac{1}{v}=\frac{1}{V_{m}}+\frac{K}{V_{m}(\mathrm{~S})}
$$

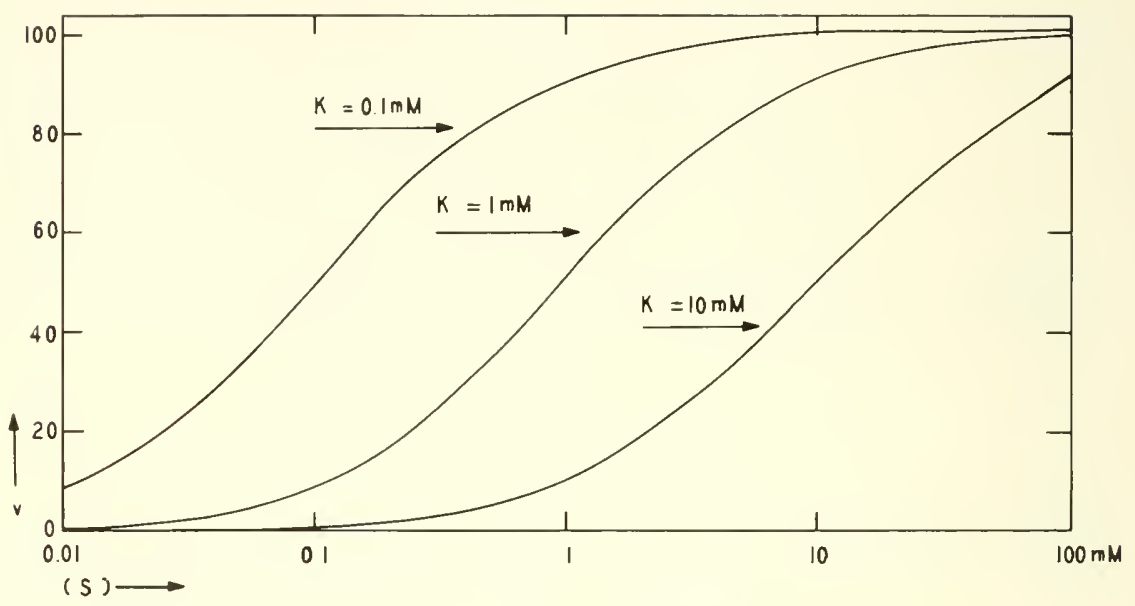

Fig. 2-2. Plots of Eq. 2-7 for different values of the Michaelis constant. $V_{m}=100$.

requires that such a plot will result in a straight line of slope $K / \boldsymbol{V}_{m}$ and will intersect the $1 / v^{\prime}$ axis at $1 / I_{m}$. The value of $K$ may be found directly by extending the plot to the intersection with the $1 /(\mathrm{S})$ axis, this being $1 / K$ (Dixon, 1953 a) (Fig. 2-3).

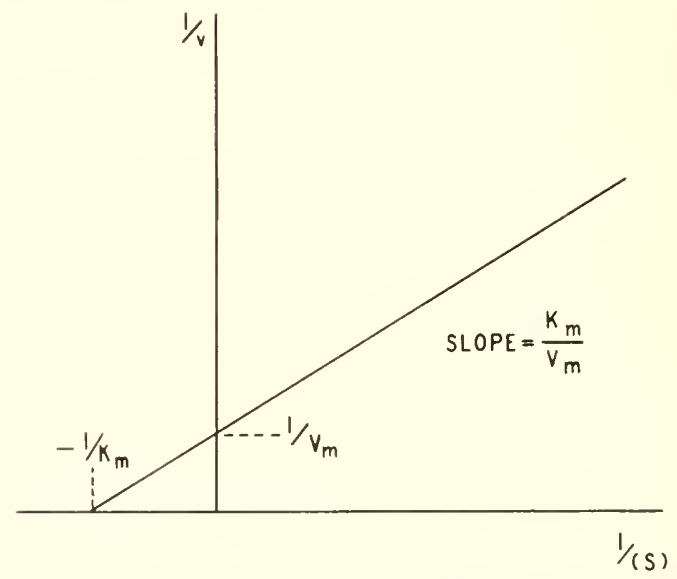

FIG. 2-3. Rieciprocal plot of the Michaclis-Menten equation for the purpose of determining $K_{m}$ and $V_{m}$. 
The constant $K$ as determined by a graphical procedure of this type may be conveniently called the Michaelis constant and hereafter will be designated by $K_{m}$. The true dissociation constant of the ES complex will be designated by $K_{s}$ and will be called the "substrate constant." According to the theory of Michaelis and Menten, $K_{m}=K_{s}$, but it is now known that the experimentally determined $K_{m}$ is not necessarily a dissociation constant and hence the distinction between the two constants must be carefully maintained. The differences in interpretation will be discussed in greater detail later.

Hany enzyme reactions have been found to be adequately described by this simple equation and the Michaelis constants are known for hundreds of enzymes. Selected values are shown in Table 2-1 to illustrate the range observed. A 150,000 -fold difference in $K_{m}$ is exhibited even in this limited table. The Michaelis constant has the dimensions of concentration and is actually the substrate concentration for which the rate is half the maximal value. It is a constant only under defined experimental conditions and generally varies with temperature, $\mathrm{pH}$. ionic strength, and other factors. The values of $k_{2}$, the rate constant for the breakdown of the ES complex into enzyme and products. vary similarly orer a wide range, usually lying between $10^{-3}$ and $10^{4} \mathrm{sec}^{-1}$.

It is, nevertheless, more and more apparent that many important enzyme reactions do not conform to this theory and it has become increasingly questionable if $K$ can be generally interpreted as the dissociation constant of the ES complex, even though the eqnation fits the experimental data. Furthermore, the conformation of the Michaelis-1Ienten equation to the data does not prove the validity of the mechanism postulated, inasmuch as similar or identical equations may be derived from other assumptions. Since much inhibition analysis involves the Michaelis-Menten formulation, it is necessary that we now consider critically the applicability of this treatment and the interpretation of the experimentally determined constants.

\section{VALIDITY OF THE MICHAELIS-MENTEN THEORY}

There are seven basic assumptions involved in the formulation and interpretation of the Michaelis-Menten Eq. 2-7 and these must be evaluated so that the range of applicability of the theory can be assessed.

\section{Assumption of an Enzyme-Substrate Complex}

The concept of intermediary complexes between enzyme and substrate is of the utmost importance in enzyme kinetics and the understanding of the mechanisms of inhibition. It should be mentioned that the idea of such complexes did not originate with Michaelis and Menten but was suggested by Henri (1902) and stated explicitly by Brown (1902) as follows: "Let 
Table 2-1

Substrate Michaelis Constants for a Few Exzymes

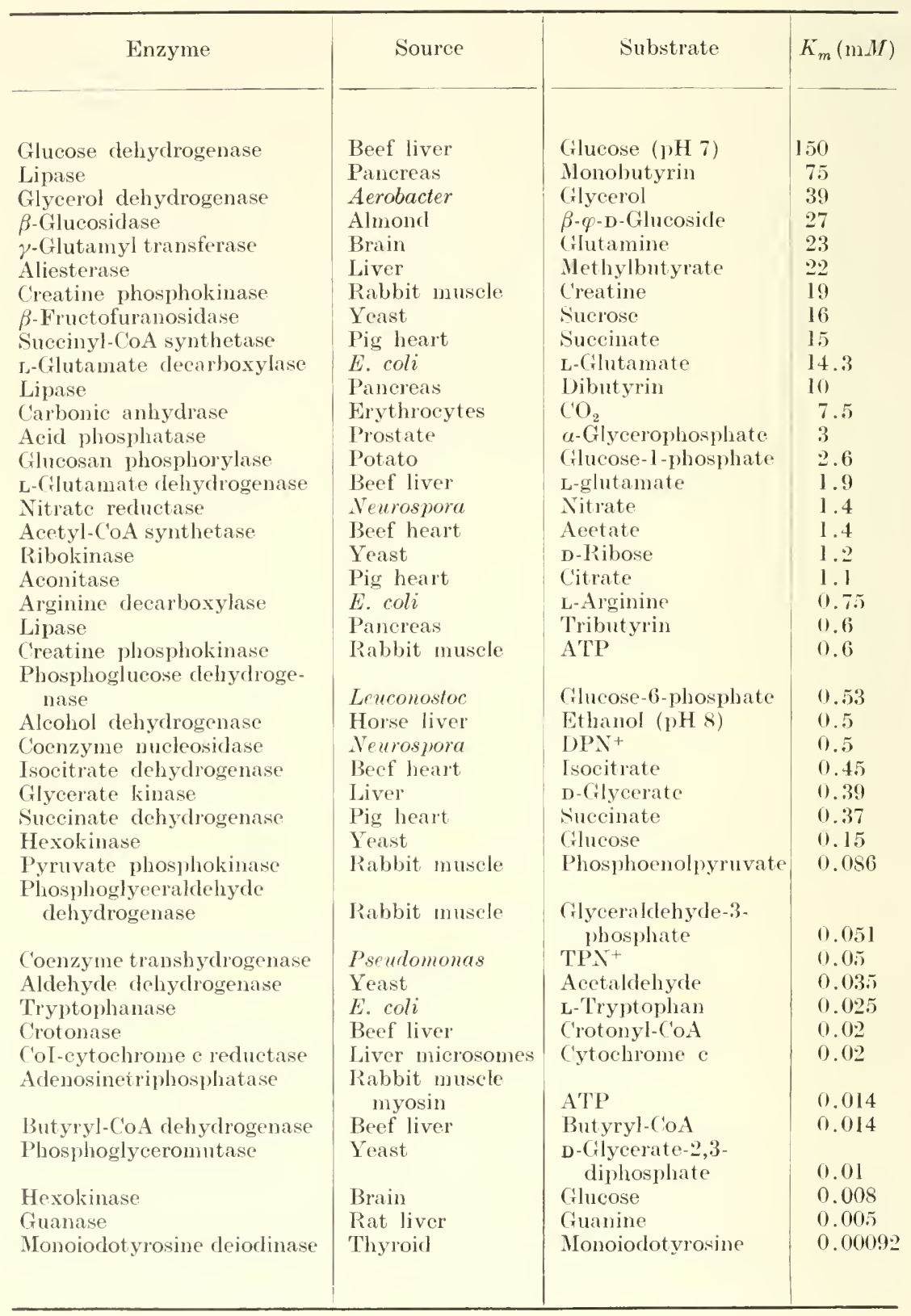


it be assumed, therefore, that one molecule of an enzyme combines with one molecule of a reacting substance, and that the compound molecule exists for a brief interval of time during the further actions which end in disruption and change." The rate-limiting breakdown of this complex was assumed in order to explain the kinetics of $\beta$-fructofuranosidase and it remained only for Michaelis and Menten to express this mechanism in mathematical form some 10 years later. The history of the ES complex concept is well told by Segal (1959).

The existence of ES complexes was generally accepted for many years on the basis of the success of the theory, since the demonstration of such a short-lived complex was technically difficult. Direct evidence for the complex was first obtained on peroxidase- $\mathrm{H}_{2} \mathrm{O}_{2}$ by Keilin and Mam (1937). Subsequent spectroscopic work in various laboratories has demonstrated complexes of catalase and $\mathrm{H}_{2} \mathrm{O}_{2}$ (Chance, 1947), catalase and alkylperoxides (Chance and Herbert. 1950), ribonuclease and ribonucleic acid (RNA) (Hakim, 1957), and aldolase and dihydroxyacetone phosphate (Topper, et al., 1957). The binding of the substrate $\mathrm{N}$-acetyl-3.5-dibromo-L-tyrosine to chymotrypsin has been demonstrated by equilibrium dialysis using an exchange reaction with $\mathrm{H}_{2} \mathrm{O}^{18}$ (Doherty and Vaslow, 1952) and a complex between pepsin and ovalbumin was shown by light-scattering measurements at low $\mathrm{pH}$ where the hydrolytic rate was slow (Yasnoff and Bull, 1953). Very stable complexes of the acyl derivatives of coenzyme A with acyl dehydrogenases have been isolated (Beinert and Steyn-Parvé, 1958; SteynParvé and Beinert, 1958). The complexing of DPN+ or DPNH to the following enzymes has been conclusively demonstrated: alcohol dehydrogenase (Theorell and Bonnichsen, 1951), lactic dehydrogenase (Chance and Neilands, 1952), phosphoglyceraldehyde dehydrogenase (Racker and Krimsky, 1952), and DPNH-peroxidase (Dolin. 1956). A number of different types of complexes between various dehydrogenases, $\mathrm{DPN}^{+}$, and nucleophilic agents has been observed (Eys et al., 1958). Indirect evidence for complexes is seen in the protection of enzymes from denaturation by their substrates and in the competitive inhibition of many enzymes by structural analogs of their substrates. There would thus seem to be sufficient evidence that the assumption of intermediary complexes is justified but the exact nature of the complex in most instances remains unknown. The assumption of a complex does not specify any particular lifetime, nor does it involve any designated structure for this complex, nor does it restrict in any way the nature of the interaction between enzyme and substrate.

\section{Assumption That $K$ Is the Dissociation Constant of the Complex}

The interpretation of $K$ as a dissociation constant depends on the assumption that the breakdown of the complex into the products is slower than the formation of the complex, limiting the rate of the over-all reaction, and slower 
than the dissociation of the complex into the enzyme and original substrate. A more general treatment was developed by Briggs and Haldane (1925) based on steady-state kinetics wherein the rates of formation and breakdown of the complex are assumed to be equal and the concentration of the complex remains constant during the experimental period. Assuming the same reaction sequence of Eq. 2-1:

$$
\frac{d(\mathrm{ES})}{d t}=k_{1}(\mathrm{E})(\mathrm{S})-k_{-1}(\mathrm{ES})-k_{2}(\mathrm{ES})=0
$$

Proceeding in the same manner as previously, the steady-state concentration of the complex is found to be:

$$
(\mathrm{ES})=\frac{k_{1}\left(\mathrm{E}_{t}\right)(\mathrm{S})}{k_{1}(\mathrm{~S})+k_{-1}+k_{2}}
$$

and the rate equation is identical in form to Eq. 2-7:

$$
v=V_{m} \frac{(\mathrm{S})}{(\mathrm{S})+\left[\left(k_{-1}+\bar{k}_{2}\right) / k_{1}\right]}=V_{m} \frac{(\mathrm{S})}{(\mathrm{S})+K_{m}}
$$

except that the Michaelis constant is no longer the simple dissociation constant of the complex, $k_{-1} / k_{1}$, but also involves $k_{2}$. Three general situations may be visualized.

(a) $\quad k_{-1} \approx k_{2} \quad K_{m}=\left(k_{-1}+k_{2}\right) / k_{1} \quad$ General Michaelis constant

(b) $\quad k_{-1} \gg k_{2} \quad K_{m}=K_{s}=k_{-1} / k_{1} \quad$ Substrate dissociation constant

(c) $k_{-1} \ll k_{2} \quad K_{m}=k_{2} / k_{1} \quad$ Kinetic constant

The constants derived from kinetic data may thus have different meanings and one must determine the values of the rate constants $k_{-1}$ and $k_{2}$ in order to be certain of the correct interpretation.

It is important to comment at this point on the symbolism chosen for the constants in the rate equation. The choice of $K_{m}$ to indicate the Michaelis constant as determined from kinetic data by graphical analysis, where the physical meaning is unknown, and of $K_{s}$ to represent the true dissociation constant of the ES complex or substrate constant is basically in agreement with Dixon and Webb (1958). In many of the rate equations throughout this volume, this constant will be written as $K_{s}$ because the derivations of the equations will assume equilibrium conditions, but in most cases $K_{s}$ may be replaced with $K_{m}$, where $K_{m}$, may be expressed in terms of the various rate constants from a steady-state treatment. In comparing affinities of enzymes for substrates and inhibitors, it is appropriate to use $K_{s}$ and $K_{i}$ (the dissociation constant of the EI complex), but in no case 
should $K_{m}$ be used in this connection when its interpretation is uncertain. With regard to the subscript numbers on the rate constants $\left(k_{1}, k_{-1}\right.$, and $k_{2}$ instead of the more commonly used $k_{1}, k_{2}$. and $k_{3}$ ). enzyme kinetics will be more and more concerned with longer multistep sequences and the use of the same number for a step, with a negative subscript referring to the reverse reaction, is clearer. For example, when one sees $k_{-3}$ it is immediately evident that it refers to the reverse reaction of the third step in the sequence.

The Michaelis constant $K_{m}$ is unquestionably an important and useful constant characterizing an enzyme even though its physical meaning is not known, since it specifies the quantitative dependence of the rate on the substrate concentration. Within the past few years, many enzymes have been subjected to exhaustive analysis to interpret $K_{m}$ and each of the three situations above has been observed; thus for trypsin and chymotrypsin, $K_{m}=K_{s}$ and Michaelis-Menten kinetics are followed. whereas for carboxypeptidase, peroxidase, alcohol dehydrogenase, and glucose oxidase, $K_{m}$ is a kinetic constant. The protease ficin is in the category where $K_{m}$ involves all the rate constants. The deviation of $K_{m}$ from $K_{s}$ increases as $k_{2}$ or $V_{m}$ increases in situations where $k_{2}$ can be varied by altering the conditions of the experiment (Slater and Bonner, 1952) since:

$$
K_{m}=K_{s}+\frac{V_{m}}{k_{1}\left(\mathrm{E}_{t}\right)}
$$

It is thus possible that the $K_{m}$ for a single enzyme may require different interpretations depending on the experimental conditions. Peroxidase under ordinary circumstances gives a $K_{m}$ that is essentially $k_{2} k_{1}$ but when the concentration of hydrogen donor (leucomalachite green) is reduced sufficiently $k_{2}$ can become comparable to $k_{-1}$ or even less (Chance, 1943). A similar situation holds for succinic dehydrogenase, where under the usual conditions $K_{m}$ is more likely to be a complex function that a dissociation constant (Slater and Bonner, 1952).

With regard to the relative values of the rate constants, it is illustrative to conceive of the course of an enzymic reaction in terms of the free energies of the molecular species and complexes. Several such energy contours are plotted in Fig. 2-4 for various possibilities in the relative values of the rate constants. Theoretically any of these pathways. or intermediate ones, might be followed by an enzyme reaction. However. in situations D, E, and $\mathrm{F}$ the $\mathrm{ES}$ complex is less stable than the reactants $\left(K_{s}>1 M\right)$ and this is seldom observed in enzyme reactions. Situation A represents the classic Michaelis formulation $\left(K_{m}=K_{s}=k_{-1} k_{1}\right)$ and $\mathrm{B}$ the case where $K_{m}$ is a kinetic constant $\left(K_{m}=k_{2} k_{1}\right)$; intermediate between these would be the situations where $K_{m}$ is a function of all three rate constants. In reaction $\mathrm{C}$ the rate would not be determined by the breakdown of the ES complex 
[instead $v=k_{1}(\mathrm{E})(\mathrm{S})$ ] and such reactions would appear to be rare. Thus A, B, and intermediates between them would represent the usually assumed situations, but the other four possibilities with certain enzymes or under particular experimental conditions should not be neglected.

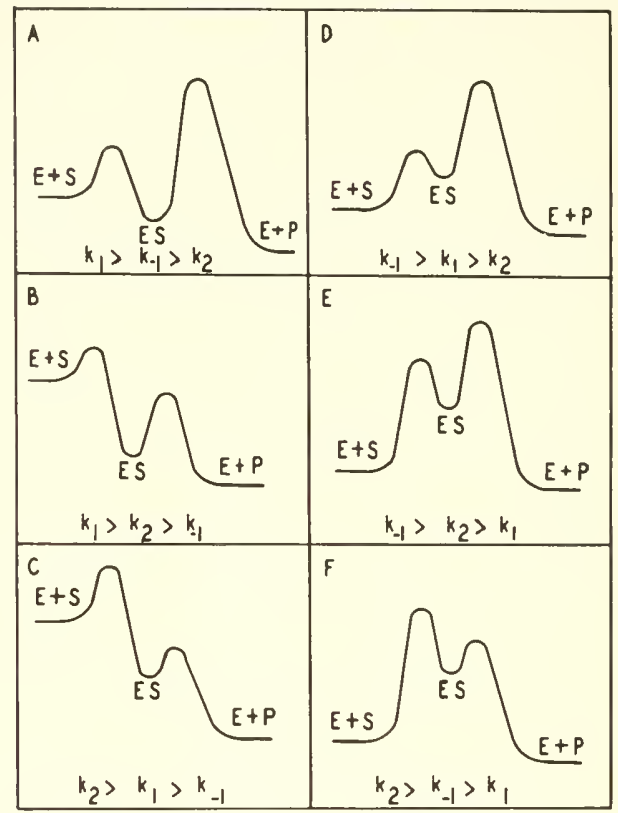

Fig. 2-4. Energy contours for simple enzyme reactions with various relative values of the rate constants. (From Laidler and Socquet, 1950.)

\section{Assumption That the Free Substrate Concentration Is Equivalent to the Substrate Added}

The substrate concentration is generally much higher than the enzyme concentration under experimental conditions and Michaelis and Menten therefore assumed that only the enzyme concentration, and not the substrate concentration, was reduced by the formation of the ES complex. Enzyme concentrations are probably within the range of $10^{-6}-10^{-2} \mathrm{~m} M$ in most studies, so that even if the enzyme were saturated with substrate, there would be no appreciable decrease in the substrate concentration, unless $K_{m}$ is very low and a low concentration of substrate is used. However, within the cell or in a limited region of the cell, such as the mitochondria, the concentrations of substrate and enzyme may be more comparable, especially in closed systems of condensed enzyme units where the substrate is being prodnced by another enzyme. When the formation of the ES complex re- 
duces the substrate concentration appreciably, the Michaelis formulation will not be obeyed and reciprocal plots may not be linear. in which case the treatment can be extended by putting:

$$
K_{s}=\frac{\left[\left(\mathrm{S}_{t}\right)\right]-(\mathrm{ES})\left[\left(\mathrm{E}_{t}\right)-(\mathrm{ES})\right]}{(\mathrm{ES})}
$$

where $\left(\mathrm{S}_{t}\right)$ is the total concentration of substrate. The expression for (ES) now is not simple but involves a quadratic equation and the actual determination of $(\mathrm{ES})$ to compare with $\left(\mathrm{S}_{\ell}\right)$ requires a knowlectge of the enzyme concentration (or specifically the concentration of enzyme active centers). The generalized treatment of Straus and Goldstein (1943) includes this possible reduction in substrate concentration and will be discussed in the following chapter.

It will suffice for the present to calculate the expected reduction in substrate concentration for a particular case. If one assumes that within the experimental reaction volume the enzyme protein is $10 \%$ and the molecular weight for a single reactive site is 62,000 , it is found that $\left(\mathrm{E}_{\ell}\right)$ is $1.6 \times 10^{-3} \mathrm{M}$. With a Michaelis constant of $3 \times 10^{-3} M$ and a substrate concentration of of $10^{-3} \mathrm{M}$, it may be calculated that the substrate concentration would be reduced by $30 \%$, a significant amount; if $K_{m}$ is $3 \times 10^{-4} M$, the reduction would be $74 \%$. It is particularly important that consideration be given to such effects when correlations are made between results on isolated enzyme systems in homogeneous solution and the same enzyme system within the cell.

Another reason for a decrease in substrate concentration under experimental conditions would be the utilization of the substrate in the reaction, but this difficulty can be avoided most simply by determining only the initial reaction rate before sufficient depletion of substrate has occurred.

\section{Assumption That the Products Are Released from the Enzyme Rapidly}

Michaelis and Menten postulated that the products formed from the reaction of the ES complex were essentially simultaneously set free from the enzyme. It is quite possible in some enzyme reactions that a relatively stable EP complex is formed which dissociates at a sufficiently slow rate to limit the over-all reaction. For this situation the following sequence can be formulated:

$$
\mathrm{E}+\mathrm{S} \underset{k_{-1}}{\stackrel{k_{1}}{\rightleftharpoons}} \mathrm{ES} \underset{k_{-2}}{\stackrel{k_{2}}{\rightleftharpoons}} \mathrm{EP} \stackrel{k_{3}}{\rightarrow} \mathrm{E}+\mathrm{P}
$$

and it is assumed that $v=k_{3}(\mathrm{EP})$. It is found that the rate equation is 
identical to the Michaelis-Menten Eq. 2-7 but the constants have different values:

$$
\begin{gathered}
V_{m}=\frac{k_{2} k_{3}\left(\mathrm{E}_{t}\right)}{k_{2}+k_{-2}+k_{3}} \\
K_{m}=\frac{k_{-1} k_{-2}+k_{-1} k_{3}+k_{2} k_{3}}{k_{1}\left(k_{2}+k_{-2}+k_{3}\right)}
\end{gathered}
$$

Reactions of this type cannot be distinguished from the Michaelis-Menten type by the usual steady-state kinetics and may be more common than smpposed. It may be noted that as $k_{3}$ becomes larger. $K_{m}$ more closely approaches $\left(k_{-1}+k_{2}\right) / k_{1}$; also if $k_{-2} \gg k_{2}$ and the enzyme complex is mainly ES, even though $k_{3}$ is small, $K_{m}$ becomes the substrate constant $K_{s}$. Peller and Alberty (1959) have presented a steady-state treatment of the kinetics followed by enzyme systems in which an indefinite number of intermediary complexes occur between a single substrate entering the reaction and a single product being formed. Reiner $(1959, \mathrm{p}, 46)$ has thoroughty analyzed a reversible enzyme system in which two intermediary complexes occur. In most cases, the number of such intermediary complexes is not known and it is very difficult to interpret $K_{m}$ or to determine the values of the rate constants.

\section{Assumption That the Over-All Reaction Is Irreversible}

The Michaelis-Menten treatment assumes that the breakdown of the ES complex to the products is irreversible; that is, that the reverse reaction whereby an EP complex would be formed does not occur. Enzymes, however, are known to catalyze reactions in both directions. From the practical standpoint, if the measurements are made early in the reaction before the concentration of products has risen or if the products are removed from the reaction as they are formed, this assumption of irreversibility is probably valid. For the situation where the reverse reaction is important, Haldane (1930) derived appropriate extensions to the Michaelis-Menten theory. If one assumes the reaction sequence:

$$
\mathrm{S}+\mathrm{E} \underset{k_{-1}}{\stackrel{k_{1}}{\rightleftharpoons}} \mathrm{ES} \underset{k_{-2}}{\stackrel{k_{2}}{\rightleftharpoons}} \mathrm{EP} \underset{k_{-3}}{\stackrel{k_{3}}{\rightleftharpoons}} \mathrm{E}+\mathrm{P}
$$

the rates of the forward and backward reactions will be given by typical Michaelis equations and the over-all rate by:

$$
v=\frac{V_{m}(\mathrm{~S}) K_{p}-V_{p}(\mathrm{P}) K_{m}}{K_{m} K_{p}+(\mathrm{S}) K_{p}+(\mathrm{P}) K_{m}}
$$

where $V_{m}$ and $V_{p}$ are the maximal rates for the forward and backward reac- 
tions and $K_{m}$ and $K_{p}$ are the corresponding Michaelis constants. These constants have the following values:

$$
\begin{gathered}
V_{m}=\frac{k_{2} k_{3}\left(\mathrm{E}_{t}\right)}{k_{2}+k_{-2}+k_{3}} \quad V_{p}=\frac{k_{-1} k_{-2}\left(\mathrm{E}_{t}\right)}{k_{-1}+k_{2}+k_{-2}} \\
K_{m}=\frac{k_{-1} k_{3}+k_{-1} k_{-2}+k_{2} k_{3}}{k_{1}\left(k_{2}+k_{-2}+k_{3}\right)} \quad k_{p}=\frac{k_{-1} k_{3}+k_{-1} k_{-2}+k_{2} k_{3}}{k_{-3}\left(k_{-1}+k_{2}+k_{-2}\right)}
\end{gathered}
$$

This reaction, started from either direction, will approach an equilibrium where the relative concentrations of $\mathrm{S}$ and $\mathrm{P}$ will be determined by the equilibrium constant of the over-all reaction $\mathrm{S} \rightleftharpoons \mathrm{P}$. It is interesting to note that this equilibrium constant is related to the kinetic terms in the following manner:

$$
K_{0}=\frac{(\mathrm{S})}{(\mathrm{P})}=\frac{k_{-1} k_{-2} k_{-3}}{k_{1} k_{2} k_{3}}=\frac{K_{s}}{K_{p}} K_{s p}
$$

where $K_{s p}$, as above, is the equilibrium constant (ES)/(EP). If an activator or inhibitor of the enzyme alters the affinity of the enzyme for the substrate (i.e. $K_{s}$ ), it is evident that the other dissociation constants must change appropriately since $K_{0}$ is unaffected by any effect on the enzyme.

\section{Assumption That One Substrate Molecule Is Bound At One Enzyme Site}

The assumption that only a single substrate molecule is bound to each enzyme active center, or that if there are more than one active center on the enzyme, the binding of substrate to each site is independent. is probably justified for most enzyme reactions but not for all. If the complex consists of more than one substrate molecule as in the sequence:

$$
n \mathrm{~S}+\mathrm{E} \underset{k_{-1}}{\stackrel{k_{1}}{\rightleftharpoons}} \mathrm{ES}_{n} \stackrel{k_{2}}{\rightarrow} \mathrm{E}+\mathrm{P}
$$

the rate is given by:

$$
v=V_{m} \frac{(\mathrm{S})^{n}}{(\mathrm{~S})^{n}+K_{m}}
$$

where $K_{m}=\left(k_{-1+} k_{2}\right) / k_{1}$ in the general case. The substrate concentration for half the maximal rate is $K_{m}^{1 / n}$ and the reciprocal plot of $1 / v$ against $1 /(\mathrm{S})$ will not be linear. Reactions of this type are uncommon and values of $n$ above two are very unlikely. If a plot of $1 / v$ against $1 /(\mathrm{S})^{2}$ gives a straight line, a reaction involving two substrate molecules may be suspected, but if non-integral values of $n$ are necessary, it is probable that the deviation from linearity is due to other factors. Situations where two different sub- 
strates react with the enzyme are discussed in a later section. The problem of interactions between adjacent substrate sites is also dealt with later.

\section{Assumption That Concentrations of Reactants May Be Used Instead of Activities}

This simplification is not mique to the Michaelis-Menten theory but has been used in most enzyme kinetic work for the reasons that either the deviations prodnced were considered negligibly small or that no aceurate estimation of the activity coefficients of the substances involved could be made. The activity coefficient $(\gamma)$ is the factor by which the concentration must be multiplied to give the activity or thermodynamic concentration, and it is this activity that ideally should be used in kinetic and equilibrium expressions. The activity is equivalent to the concentration only in ideal or very dilute solutions; solutions in which enzyme reactions are run are generally not ideal, nor is the intracellular medimm. The activity coefficient of an ion decreases with increasing charge on the ion and increasing ionie strength of the solntion. The ionic strengths of enzyme reaction media vary greatly and the exact intracellular ionic strength is not known. Examples of calenlated ionic strengths of possible reaction media are given in the tabulation below.

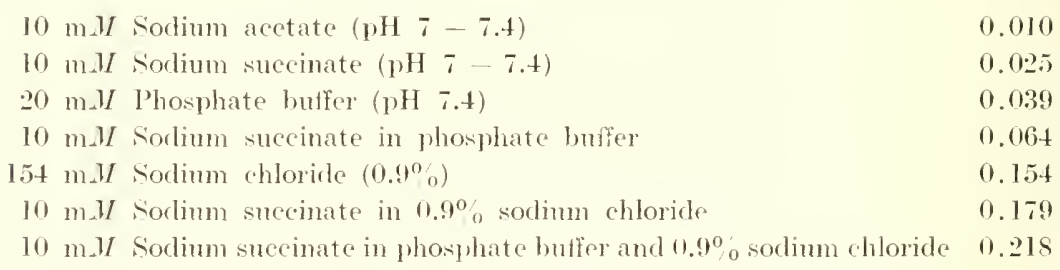

The ionic strength of Krebs-Ringer media is approximately 0.161 and it is probably safe to assume that the bulk cytoplasmic ionic strength is not very far from this figure. Using the Debye-Hückel eqnation, including the salting-ont effect. the activity coefficient of a single-charged ion at this ionic strength ean be ealculated to be approximately 0.76 and of a double-charged ion approximately 0.31 , values which eheck reasonably well with those determined experimentally. Even at an ionie strength of 0.01 , the activity coefficient of a uni-univalent salt is around 0.90 .

For an equilibrium of the type:

$$
K_{s}=\frac{(\mathrm{E})(\mathrm{S})}{\mid(\mathrm{ES})}
$$


the true or thermodynamic equilibrium constant $\left(K_{s}{ }^{\prime}\right)$ is related to the determined or classic equilibrium constant by the expression:

$$
K_{s}^{\prime}=\frac{a_{e} a_{s}}{a_{e s}}=\frac{\gamma_{e} \gamma_{s}}{\gamma_{e s}} \frac{(\mathrm{E})(\mathrm{S})}{(\mathrm{ES})}=\frac{\gamma_{e} \gamma_{s}}{\gamma_{e s}} K_{s}
$$

If free energy values are to be obtained from substrate constants, $K_{s}{ }^{\prime}$ should be used, and when the experimentally determined $K_{s}$ differs appreciably from $K_{s}^{\prime}$ these energy values may be in error. The ionic fractions of the enzyme active center are generally present at activities less than their concentrations and the factor $\gamma_{e} \gamma_{s} / \gamma_{e s}$ may deviate appreciably from unity.

\section{Additional Remarks Pertaining to the Validity of the Michaelis-Menten Treatment}

The foregoing evaluation of the assumptions of the original MichaelisMenten formulation make it apparent that although the fundamental concept of a substrate-enzyme complex is quite well established, the data obtained experimentally cannot usually be interpreted in a simple manner, particularly with regard to the Michaelis constant $K_{m}$. This applies to relatively simple enzyme reactions. Many important enzyme reactions are being found to be more complex than those systems upon which the theory was based, and the complications of activators, coenzymes, donors and acceptors, polycomplexes, and catenary reactions, make it evident that the basic Michaelis-Menten equation has only a limited range of applicability. Many instances might be cited where misinterpretation of the results might occur using the simple theory. A particularly illuminating example is provided by glucose oxidase (notatin), a relatively simple enzyme system, in the discussion of the conclusions of Slater by Dixon, Laidler, Ingraham, and Bernard (Slater, 1955, pp. 301-312). Certain of these more complex systems and the extensions that must be made to the original theory will be discussed briefly in a subsequent section.

\section{NATURE OF THE INTERMEDIATE COMPLEXES WITH THE ENZYME}

A brief discussion of the molecular mechanisms of enzyme catalysis will be presented in this section since it is only against such a background that the many types of inhibition may be visualized and interpreted. The binding of the substrate to the enzyme to form the ES complex provokes no unique problems, being one segment in the general field of interactions between various molecules and proteins; it is the subsequent reaction of the ES complex that distinguishes the enzymic event. The forces that bind substrates to enzyme active centers are those that are involved in molecular 
interactions generally: electrostatic attraction between ionic groups, dipole interactions, forces arising from polarization in local electric fields, dispersion forces, covalent bonds, and hydrogen bonds. Any one or a combination of these may be involved in the total interaction energy. The binding of inhibitors is quite comparable and a more detailed discussion of these interaction forces will be presented in Chapter 6 in connection with inhibition.

It is probable that most substrates are bound to the active center by two or more interaction loci or attachment points; i.e., several regions on the substrate molecule interact with the enzyme. This is attested to by the high specificity of most enzymes and is necessary in order that the substrate assume the proper configuration for reaction. In some cases it would appear that the entire substrate molecule, or at least one side of it, fits fairly accurately a complementary configuration on the enzyme, since the alteration of any group on the molecule changes the binding claracteristics. The situation here is probably not too different from the reaction between antigen, or hapten, and antibody, where conformity to within $1-2 \AA$ is requisite for maximal interaction. This multipoint attachment of substrate increases the possibilities of interference by inhibitors, since the blocking or modification of only one group out of several may be sufficient to reduce the reaction rate significantly or totally.

The problem of activation arises because it is generally stated that the substrate is activated upon being bound. The term activation has several meanings; it would be more satisfactory if its use were restricted to those states of high energy level and temporary abnormal electron configuration implied in transition state kinetics. If this view is maintained, it is evident that the usual ES complex does not represent an activated state, since its energy level is generally lower (it is really a more stable state of the enzyme and substrate considered themodynamically) and its atomic and electronic configuration is normal (its structure may be defined in terms of ordinary interatomic distances and electron positions). The ES complex, however, does pass through a truly activated state EX* in the course of the reaction, and sometimes perhaps throngh a series of such activated states (Fig. 2-5). In some reactions the products may be formed unbound to the enzyme from the transformation of the EX* complex, but in other cases the products form complexes with the enzyme that are comparable to the ES complexes.

Despite the fact that the substrate molecule may not be truly activated when bound in the ES complex, it is obvious that it is in a different state from that free in solution, inasmuch as binding implies forces that must alter the electronic configuration and over-all structure to some extent, increasing the probability of reaction with another molecule. It might be said that binding increases the probability of activation. There is also the 
restriction that is imposed on the movements of the molecule, as a whole or intramolecularly, resulting in a decrease of entropy that may play an important role in the energetics of activation. It would often be valuable to know in what configuration the substrate is bound in the ES complex when one is attempting to visualize the binding of inhibitors that interact with the active center in a way comparable to the substrate. Is the substrate

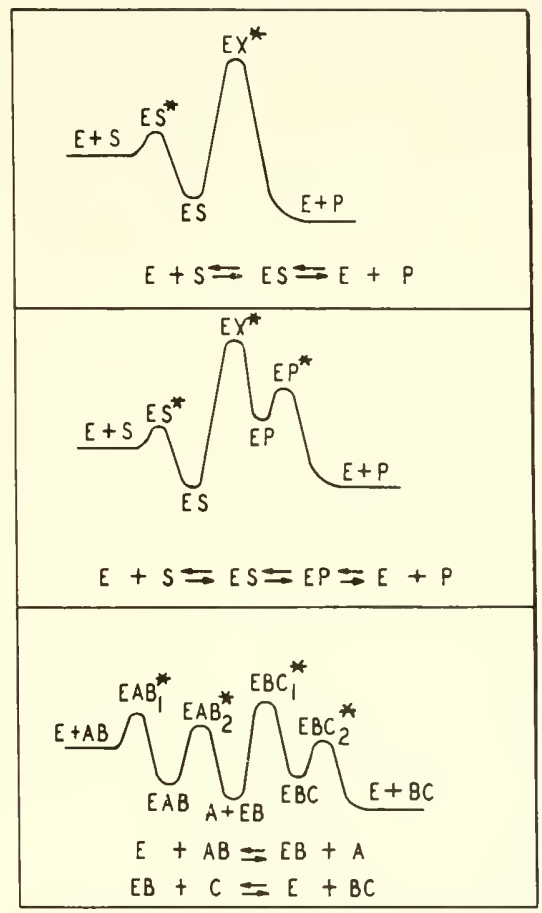

FIG. 2-5. Hypothetical energy contours for various types of enzyme reactions. The third situation might represent transfer reactions, such as transphosphorylation or transacetylation. The starred complexes indicate activated states.

bound in its normal most probable configuration or in a distorted configuration conducive to reaction? In the binding of succinate, for example, are the cationic groups on the dehydrogenase the same distance apart as the normal distance between the carboxylate groups on the succinate, or is the succinate bent or stretched into a form clifferent from the one it would statistically assume in solution? The interaction of competitively inhibiting dicarboxylic acids, such as malonate, could be interpreted more quantitatively if this question could be answered definitely. 
The most common mechanism by which enzymes accelerate reactions may well be the electron displacement induced in the substrate by which it is made more susceptible to attack by another substance, whether this be a second substrate, a coenzyme, a proton, or the solvent. Most organic reactions may be characterized as electrophilic or nucleophilic, depending on whether the attacking group possesses affinity for electrons or a tendency to lose them. The enzyme, by creating or modifying electron-rich and electron-poor regions in the substrate, may thus facilitate such attack. In other cases it may provide a pathway by which electrons or protons can move from one locus to another, these electrons or protons being directly donated or accepted by some enzyme group, the enzyme here truly participating in the reaction. The large number of charged groups on proteins gives them the ability not only to bind substrates but to polarize them. The binding groups may not play a role in the reaction but merely serve to anchor the substrate molecule so that appropriate groups can be polarized. In the large group of hydrolytic enzymes, acidic or basic catalysis may operate under more favorable conditions due to this polarization or the spatial apposition of the susceptible bond to acidic or basic groups on the active site. In the reduction of aldehyde catalyzed by the alcohol dehydrogenase:

$$
\mathrm{R}-\mathrm{CHO}+\mathrm{H}^{+}+\mathrm{DPNH} \rightarrow \mathrm{R}-\mathrm{CH}_{2} \mathrm{OH}+\mathrm{DPN}^{+}
$$

it has been shown that one hydrogen atom comes from the DPNH and one from an acidic group on the protein, and the importance of this acidic group for the binding indicates that a hydrogen bond may be formed in the ES complex, facilitating the hydrogen transfer (Sizer and Gierer, 1955). It has been postulated that both acidic and basic groups may participate in a so-called "push-pull" type of acid-base catalysis in certain enzymes, this being more efficient than either acidic or basic catalysis alone (Laidler, $1955 \mathrm{a})$. It is becoming more and more evident that in many reactions true chemical compounds, involving covalent bonds, are formed between substrate and enzyme at some stage of the reaction sequence and that the molecular transformations involving the activated complexes must inchude the active center groups as well as the reactants.

Reaction sequences or energy diagrams, as usually presented, make it appear that the total reaction occurs in a series of stages identified by various intermediate complexes and one comes to conceive of these reactions as actually proceeding through these discrete steps. It is possible, however, that on the molecular level such considerations are arbitrary and artificial, since the reaction may proceed quite smoothly without any distinguishable intermediate compounds or complexes. In fact, two or more steps that are written sequentially may occur simultaneously. The conventions of description should not too much limit our concepts of reality. It is perhaps ideal, whenever possible, to visualize an enzyme reaction in terms of the 
motions of electrons, protons, and atoms from the initial molecular configuration of the substrate to the final one of the products. Due to our lack of knowledge of the intimate mechanisms and kinetics of most enzyme reactions, this visualization is frequently difficult at the present time but it should be more and more realizable as progress in accurate investigation is made.

It is clear from the rough picture that has been developed of the enzyme complexes and the possible mechanisms of reaction that any phase of the total sequence may be affected by modification of the enzyme, either at the active center or at some distance from it. The effects of activators, inhibitors, $\mathrm{pH}$, temperature, ionic strength, and similar factors shonld be interpreted in such terms. An inhibitor may interfere with the binding of the substrate, this being the most common assumption, but it could also modify the formation of any of the subsequent complexes, hindering the necessary movements of electrons. protons, or groups either directly by effects exerted by the inhibitor molecule (electrical. chemical, or steric) or indirectly through the protein by modifying the active center groups involved in the reaction, perhaps by increasing or decreasing the electron or proton density in the critical regions. An inhibitor need not interfere with the reaction between two substrates to retard the process; in transphosphorylation or transacetylation reactions, for example, the inhibitor may hinder the phosphorylation or acetylation of the enzyme by modification of the acceptor group on the active center. It is the desire to present a comprehensive concept of inhibitor action that has prompted us to discuss in some detail the normal phases of the enzyme reaction and the participation of the active center.

\section{FACTORS DETERMINING THE RATE OF ENZYME REACTIONS}

Inlibitors of the simple enzymes reduce the rates at which the products are formed. It will facilitate later discussion if the factors that determine these rates are stated clearly and briefly. In Michaelis-Menten kinetics and in many extensions of this theory the rate is assumed to be expressed by $v=k_{2}$ (ES). The over-all rate is thus dependent on two basic factors: the concentration of the complex and the intrinsic rate at which the complex breaks down into the products. If one compares the rates with different substrates and a single enzyme, variation in rates may be due to different tightnesses of binding $\left(K_{s}\right)$ or different rates of breakdown of the corresponding complexes $\left(k_{2}\right)$. It is not necessary, as has sometimes been assumed, that any parallelism be exhibited between the affinities of the enzyme for a series of substrates and the rates observed, inasmuch as $k_{2}$ can vary independently. Some substances may be bound very tightly but react only slowly; in fact, most competitive inhibitors are substances binding reasonably tightly to the enzyme but for which $k_{2}$ is low or zero. If $k_{2}$ remains constant, then changes 
in binding may be reflected in the rate since the concentration of complex (ES) will be determined by this affinity, but otherwise no necessary relationship may be assumed. Thus an inhibitor by reducing the affinity of the enzyme for the substrate may slow the reaction by reducing (ES); on the other hand, it may retard the breakdown of the complex, with or without effect on $K_{s}$. A reduction in the maximal rate by an inhibitor does not, of course, reflect a decrease in $k_{2}$ but generally a reduction in total concentration of enzyme active centers, since $V_{m}=k_{2}\left(\mathrm{E}_{t}\right)$; an increase in $K_{m}$ does not necessarily imply a reduction in affinity unless it is certain that $K_{m}=$ $K_{s}$. Thus the usual kinetic analysis is not adequate generally to differentiate between action of an inhibitor on $K_{s}$ or $k_{2}$. For this purpose one of the special methods of independently determining $k_{2}$ or $K_{s}$ must be used and applied to both normal and inhibited systems. This lias been done only rarely. The more complex the reaction and the more steps it is assumed to include, the more possibilities exist for effects upon a variety of rate processes and equilibria.

The binding of the activated complex to the enzyme must also be considered since it is the energy and entropy of activation that determines the reaction rate constant. Comparing the over-all reactions:

$$
\begin{aligned}
\mathrm{A}+\mathrm{B} & \rightleftharpoons \mathrm{AB}^{*} \rightleftharpoons \mathrm{C}+\mathrm{D} \\
\mathrm{A}+\mathrm{B}+\mathrm{E} \rightleftharpoons \mathrm{EAB}^{*} & \rightleftharpoons \mathrm{E}+\mathrm{C}+\mathrm{D}
\end{aligned}
$$

the energy contributed by the binding in the EAB* complex may be responsible for the reduction in activation energy and the accelerated rate. Thus the possible effects of inhibitors on this binding of the activated complex must be considered, the inhibitor in this case raising the activation energy.

\section{KINETICS OF SOME COMPLEX ENZYME REACTION TYPES}

The purpose in presenting several more complex, but commonly observed, types of reaction is, first, to provicle kinetic rate equations that may be applied later in discussing the effects of inhibitors and, second, to emphasize the manifold possibilities in enzyme reactions in order to help disperse whatever illusions the reader may have concerning the uniformity of enzyme mechanisms and kinetics. Suecial and unique reactions will be dealt with in those chapters where the kinetics will be pertinent to inhibition.

\section{General Procedure For Derivation of Rate Equations}

Rate equations for any type of reaction - including those involving activators, coenzymes, hydrogen ions, inhibitors, multiple substrates, or interdependent active centers - may be derived assuming a steady 
state wherein the concentrations of all complexes remain constant. Although this derivation is often more laborious than the equilibrium procedure, it provides constants which are of general validity since equilibrinm conditions are not assumed.

First step: write out the equations for all the reactions that can occur in the system and assign rate constants to each forward and backward step.

Second step: write the equation giving the orer-all rate in terms of the appropriate complex or complexes, e.g. $v=k_{j}\left(\mathrm{EX}_{i}\right)$.

Third step: write the equation expressing the total enzyme concentration in terms of all the molecular species containing the enzyme, e.g.,

$$
\left(\mathrm{E}_{t}\right)=(\mathrm{E})+\left(\mathrm{EX}_{1}\right)+\left(\mathrm{EX}_{2}\right)+\ldots+\left(\mathrm{EX}_{n}\right)
$$

If the free concentrations of any other components of the system are appreciably less than the total concentrations, due to complex formation, similar equations for these components should be written.

Fourth step: write the steady-state equation for each complex in terms of the appropriate rate constants and concentrations, including all reactions whereby the complex is formed or broken down, and set the over-all rate equal to zero since the complex concentration is constant, e.g.,

$$
\frac{d(\mathrm{EX})}{d t}=k_{p} f_{p}(\mathrm{C})+k_{-q} f_{-q}(\mathrm{C})+\ldots-k_{-p} f_{-p}(\mathrm{C})-k_{q} f_{q}(\mathrm{C})-\ldots=0
$$

where $f(\mathrm{C})$ represents the concentrations pertinent to each reaction.

Fifth step: these steady-state equations in conjunction with the equations from step 3 provide a set of equations which may be solved for the concentration of the rate-determining complex $\left(\mathrm{EX}_{i}\right)$ and this expression can be substituted in the equation from step 2 .

Sixth step: if desired, the expression for the maximal rate $\left(V_{m}\right)$ may be determined by evahuating $v$ at ligh substrate concentration and $V_{m}$ inserted into the rate equation from step 5 .

The resulting rate equation may be modified by substitution of dissociation constants for the corresponding ratios of rate constants or by combination of rate constants into single constants to facilitate interpretation. Many equations derived in this manner are complex and unwieldy but occasionally some simplification can be introdnced by assuming appropriate relative values for the rate constants or concentrations of the substances involved, giving special cases that may be applicable under certain experimental conditions. 


\section{The Substrate Is Not Immediately Available to the Enzyme}

When the substrate used must penetrate a barrier to reach the enzyme (such barriers may occur in enzyme aggregates, or in mitochondrial and cell membranes) or requires chemical modification before it will react with the enzyme, the following equations describe the situation:

$$
\begin{gathered}
\mathrm{S} \underset{k_{-0}}{\stackrel{k_{0}}{\rightleftharpoons}} \mathrm{S}^{\prime} \\
\mathrm{S}^{\prime}+\mathrm{E} \underset{k_{-1}}{\stackrel{k_{1}}{\rightleftharpoons}} \mathrm{ES}^{\prime} \stackrel{k_{2}}{\rightarrow} \mathrm{E}+\mathrm{P}
\end{gathered}
$$

Four possible cases may be considered.

(I) Case 1. The formation of $\mathrm{S}^{\prime}$ from $\mathrm{S}$ is very slow and limits the overall rate. If $\mathrm{S}^{\prime}$ reacts as rapidly as it appears or if $k_{-0}$ is zero:

$$
v=k_{0}(\mathrm{~S})
$$

and the kinetics are not related to the enzyme reaction.

(II) Case 2. The formation of $\mathrm{S}^{\prime}$ limits the over-all rate but the back reaction of $\mathrm{S}^{\prime} \rightarrow \mathrm{S}$ cannot be ignored, in which case $v=k_{0}(\mathrm{~S})-k_{-0}\left(\mathrm{~S}^{\prime}\right)$. This reduces to $v=k_{0}\left[(\mathrm{~S})-\left(\mathrm{S}^{\prime}\right)\right]$ if $k_{0}$ is a permeability constant and diffusion occurs at equal rates in both directions. Best (1955 a) has equated this rate to the enzyme rate inside the barrier to give:

$$
v=V_{m}-\frac{k_{0} K_{m}}{\left(k_{0} / v\right)(\mathrm{S})-1}
$$

where $V_{m}$ is the maximal rate of the enzyme reaction, and has used this equation to obtain permeability constants for glucose and sucrose in yeast, but it is doubtful if this relationship holds except under very limited conditions.

(III) Case 3. The enzyme reaction limits the over-all rate in which case $\left(S^{\prime}\right)$ is in complete equilibrimm with $(\mathrm{S})$ and $\left(\mathrm{S}^{\prime}\right)=(\mathrm{S}) / K^{\prime}$ where $K^{\prime}=k_{-0} / k_{0}$. This value of $\left(\mathrm{S}^{\prime}\right)$ may be substituted in the Michaelis equation to give:

$$
v=\frac{V_{m}\left(\mathrm{~S}^{\prime}\right)}{\left(\mathrm{S}^{\prime}\right)+K_{m}}=\frac{V_{m}(\mathrm{~S})}{(\mathrm{S})+K^{\prime} K_{m}^{\prime}}
$$

so that in this case the determined Michaelis constant is not the true $K_{m}$ for the enzyme reaction, a fact of possible importance when such constants are determined in complex systems where the concentration of substrate inside the barrier is not necessarily the same as outside. 
(IV) Case 4. The over-all rate depends primarily on the enzyme reaction but the rate of formation of $\mathrm{S}^{\prime}$ is near enough to $k_{2}\left(\mathbf{E S}^{\prime}\right)$ to exert an effect. A steady-state treatment putting $d\left(\mathrm{~S}^{\prime}\right) / d t=d(\mathrm{ES}) / d t=0$ gives the following general equation:

$$
v=\frac{V_{m}^{\prime}(\mathrm{S})}{(\mathrm{S})+\left(1 / k_{0}\right)\left(V_{m}-v\right)+K^{\prime} K_{m}}
$$

where $K^{\prime}=k_{-0} / k_{0}$ and $K_{m}=\left(k_{-1}+k_{2}\right) / k_{1}$. This equation is similar, but not identical, to that of Lineweaver and Burk (1934) who did not include the back reaction from $S^{\prime} \rightarrow S$. They showed how the constants may be graphically evaluated by varying $V_{m}$ in certain ways.

When diffusion of substrate occurs through a membrane into a spherical or cylindrical region, additional complications arise due to the relations between volume and membrane area, as well as to possible lack of homogeneity in the system, and the rate equations are quite complex (Blum, 1956). Blum and Jenden (1957) have treated the situation where the concentration of substrate decreases with increasing distance from the boundary membrane due to utilization of the substrate by an homogeneously distributed enzyme. It is interesting that inhibitors competing with the substrate in such a system would inhibit to different degrees in various regions, the over-all inhibition being greater than expected from the external concentration of substrate and the linetics of the isolated enzyme.

\section{The Concentration of Enzyme Is Greater Than That of the Substrate}

This situation is rare in studies on isolated enzymes but intracellularly may be predominant over systems operating according to classic kinetics. For the present only the extreme case where $\left(\mathrm{S}_{t}\right)$ is so low that $(\mathrm{E})=\left(\mathrm{E}_{t}\right)$ will be considered; a more general treatment will be given in the next chapter. One may now put $\left(S_{t}\right)=(S)+(E S)$ and the resulting rate equation is:

$$
v=\frac{k_{2}\left(\mathrm{E}_{t}\right)\left(\mathrm{S}_{t}\right)}{\left(\mathrm{E}_{t}\right)+K_{m}}
$$

where $K_{m}=\left(k_{-1}+k_{2}\right) / k_{1}$. This expression is seen to be identical in form to Eq. 2-11 except that the rate is now linear with $\left(S_{l}\right)$ and hyperbolic with respect to $\left(\mathrm{E}_{\ell}\right) ; k_{2}\left(\mathrm{E}_{t}\right)$ is not equated with $V_{m}$ since under the conditions assumed the rate will not become maximal upon increasing $\left(\mathrm{S}_{t}\right)$. The reciprocal equation:

$$
\frac{1}{v}=\frac{1}{k_{2}\left(\mathrm{~S}_{t}\right)}+\frac{K_{m}}{k_{2}\left(\mathrm{E}_{t}\right)\left(\mathrm{S}_{t}\right)}
$$

shows that if $1 / v$ is plotted against $1 /\left(\mathrm{S}_{t}\right)$, a straight line that would pass through the origin would be obtained but from which no constants may be 
determined; however, if $1 / v$ is plotted against $1 /\left(\mathrm{E}_{t}\right)$, even though the absolute enzyme concentrations are not known, both $k_{2}$ and $K_{m}$ may be evaluated. Inhibition of such systems presents interesting possibilities to be apphed to intracellular studies.

\section{The Enzyme Contains Two Interdependent Active Centers}

An enzyme may possess two active centers that are sufficiently close together so that binding of substrate to one site will affect the properties of the other site, altering either the affinity for the substrate $\left(K_{s}\right)$ or the rate of breakdown of the ES complex $\left(k_{2}\right)$. The reactions may be written:

$$
\begin{gathered}
\mathrm{E}+\mathrm{S} \underset{k_{-1}}{\stackrel{k_{1}}{\rightleftharpoons}} \mathrm{ES} \stackrel{k_{2}}{\rightarrow} \mathrm{E}+\mathrm{P} \\
\mathrm{ES}+\mathrm{S} \underset{k_{-3}}{\stackrel{k_{3}}{\rightleftharpoons}} \mathrm{SES} \stackrel{\beta k_{2}}{\rightarrow} \mathrm{ES}+\mathrm{P}
\end{gathered}
$$

where $\beta$ characterizes any change in rate of ES breakdown brought about by binding of one substrate molecule. Actually SES conld break down with respect to either substrate molecule (forming $\mathrm{ES}+\mathrm{P}$ or $\mathrm{SE}+\mathrm{P}$ ) but the rates are identical for each reaction and we assume that the probability of both substrate molecules being involved simultaneonsly is negligible (i.e., the reaction $\mathrm{SES} \rightarrow \mathrm{E}+2 \mathrm{P}$ is ignored). One can now write:

$$
\begin{gathered}
\left(\mathrm{E}_{t}\right)=(\mathrm{E})+(\mathrm{ES})+(\mathrm{SES}) \\
\frac{d(\mathrm{ES})}{d t}=k_{1}(\mathrm{~S})(\mathrm{E})-k_{-1}(\mathrm{ES})-k_{2}(\mathrm{ES})=0 \\
\frac{d(\mathrm{SES})}{d t}=k_{3}(\mathrm{ES})(\mathrm{S})-k_{-3}(\mathrm{SES})-\beta k_{2}(\mathrm{SES})=0 \\
v=k_{2}(\mathrm{ES})+\beta k_{2}(\mathrm{SES})
\end{gathered}
$$

From these the following general rate expression may be derived:

$$
v=V_{m} \frac{\left[1+\beta(\mathrm{S}) / K_{m}^{\prime}\right](\mathrm{S})}{(\mathrm{S})+K_{m}+(\mathrm{S})^{2} / K_{m}^{\prime}}
$$

where $K_{m}=\left(k_{-1}+k_{2}\right) / k_{1}$ and $K_{m}{ }^{\prime}=\left(k_{-3}+\beta k_{2}\right) / k_{3}$, these being the Michaelis constants for Eqs. 2-31 and 2-32, respectively. If one now assumes that the $K_{m}{ }^{\prime}$ s are dissociation constants and that $K_{s}{ }^{\prime}=\alpha K_{s}$ :

$$
v=V_{m} \frac{\alpha K_{s}+\beta(\mathrm{S})}{(\mathrm{S})+\alpha K_{s}+\alpha K_{s}^{2} /(\mathrm{S})}
$$

where $\alpha$ characterizes any change in $K_{s}$ induced by the binding of one sub- 
strate molecule (e.g. if $\alpha>1$, the affinity for the second substrate molecule is less than for the first). If $\alpha$ is very high, the equation becomes the classic Michaelis Eq. 2-7, because the second substrate will be bound to a very small extent:

$$
v=V_{m} \frac{K_{s}+\beta(\mathrm{S}) / \alpha}{(\mathrm{S}) / \alpha+K_{s}+K_{s}{ }^{2} /(\mathrm{S})} \stackrel{\alpha \gg 1}{\rightarrow} V_{m} \frac{(\mathrm{S})}{(\mathrm{S})+K_{s}}
$$

If $\alpha=1$ (no effect on affinity) and $\beta=0$ (reaction 2-32 does not occur) the equation becomes:

$$
v=V_{m} \frac{(\mathrm{S})}{(\mathrm{S})+K_{s}+(\mathrm{S})^{2} / K_{s}}
$$

which represents one type of substrate inhibition, since at high (S) most of the enzyme will be in the form SES and this is inactive. If $\alpha=\beta=1$, no effect of the first substrate molecule on the binding or reaction of the second occurring, it may be noticed that the kimetics deviate from the simple Michaelis equation when (S) is near $K_{s}$ but follow the Michaelis equation when $(\mathrm{S})$ is greater than approximately $5 K_{\text {. }}$. The explanation of this deviation can be made as follows: (ES) + (SES) will not be equal to the value of (ES) when a single substrate only is involved and hence the rate of formation of $\mathrm{P}$ will differ from the simple case. When $(\mathrm{S})=K_{s}$, then $(\mathrm{ES}) /\left(\mathrm{E}_{t}\right)$ $=1 / 2$ for the simple one substrate reaction, but $[(\mathrm{ES})+(\mathrm{SES})] /\left(\mathrm{E}_{t}\right)=2 / 3$, for the reaction with two substrate molecules, the rate for the latter being greater than if only one substrate molecule reacts. Equation 2-39 is plotted in Fig. 2-6 to illustrate the effects of variations in $\alpha$ and $\beta$.

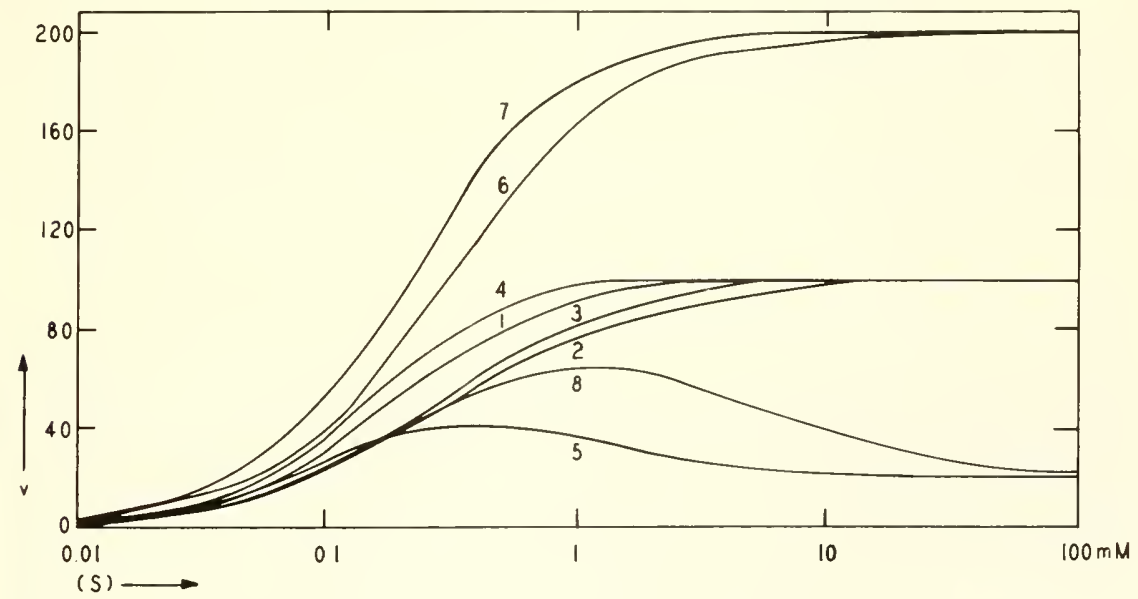

FIG. 2-6. Plots of Eq. 2-39 for different values of $\alpha$ and $\beta . K_{s}=0.3 \mathrm{~m} . M$ and $V_{m}=$ 100. Curve 1: $\alpha=1, \beta=1$; curve $2: \alpha=\infty, \beta=1$; curve $3: \alpha=10, \beta=1$; curve 4: $\alpha=0.5, \beta=1$; curve 5 : $\alpha=1, \beta=0.2$; curve 6 : $\alpha=1, \beta=2$; curve $7: \alpha=0.5$, $\beta=2$, curve $8: \alpha=10, \beta=0.2$. 
It is difficult to determine the constants $\alpha, \beta$, and $K_{s}$ from experimental data or even to prove that the mechanism is that given by Eq. 2-31 and 2-32 since other types of reaction can also lead to similar non-linearity in the reciprocal plots of $1 / v$ against $1 /(\mathrm{S})$ (Fig. 2-7). For example, the kinetics

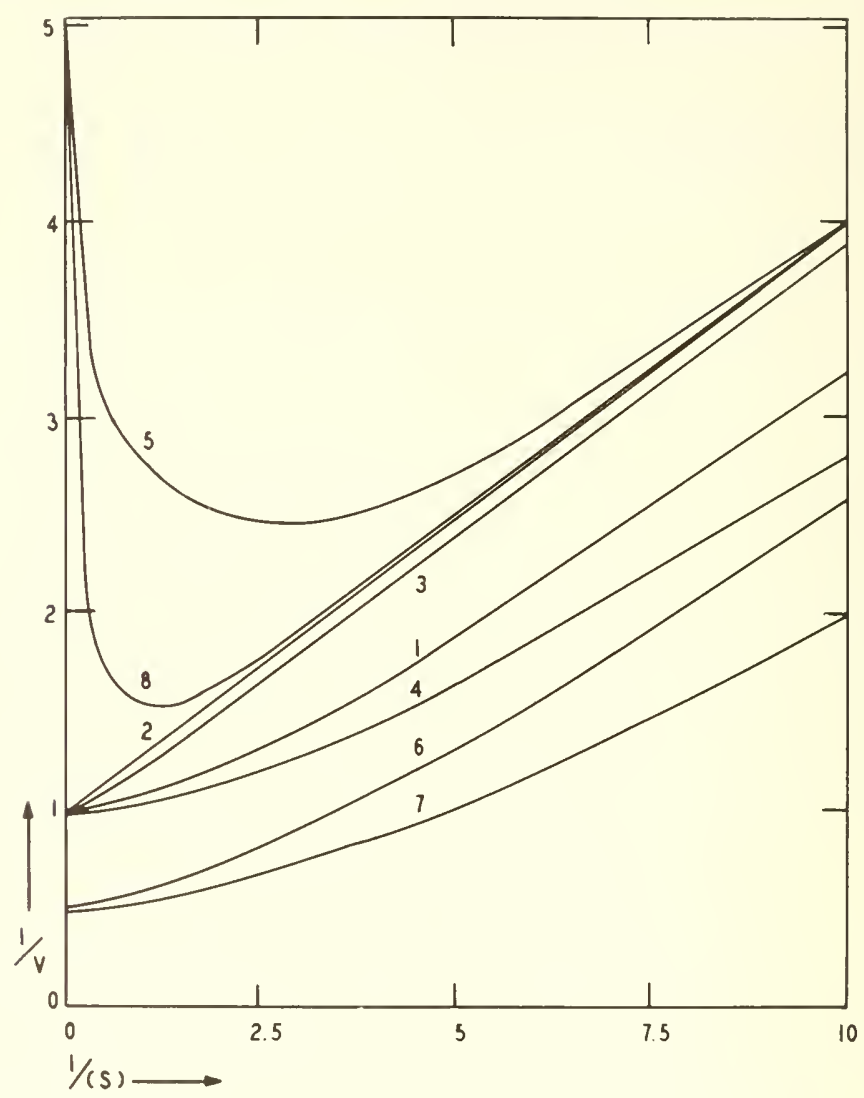

Fig. 2-7. Reciprocal plots for the curves shown in Fig. 2-6.

of urease indicated two active centers, but whether these were interacting sites or independent sites with different kinetic parameters conld not be established (Kistiakowsky and Rosenberg, 1952). However, it is seen from the reciprocal equation of $2-39$ with $\beta=1$ :

$$
\frac{1}{v}=\frac{1}{V_{m}^{r}}+\frac{1}{V_{m}} \frac{\alpha K_{s}{ }^{2}}{\alpha K_{s}(\mathrm{~S})+(\mathrm{S})^{2}}
$$

that if $1 / v$ is plotted against $1 /(\mathrm{S})^{2}$, the slope near the $1 / v$ axis (where substrate concentration is high) is $\alpha K_{s}{ }^{2} / V_{m}$ and the intercept $1 / V_{m}$. Since 
$\alpha K_{s}^{2}=\alpha K_{s}\left(K_{s}\right)=K_{s}{ }^{\prime} K_{s}$, the value of $\sqrt{ } \alpha K_{s}{ }^{2}$ will give only a mean value for the dissociation constant. In the case of two independent sites with different parameters, where:

$$
v^{v}=\frac{V_{m}(\mathrm{~S})}{(\mathrm{S})+K_{s}}+\frac{\beta \boldsymbol{V}_{m}(\mathrm{~S})}{(\mathrm{S})+\alpha \boldsymbol{K}_{s}}
$$

a plot of $1 / v$ against $1 /(\mathrm{S})$ will give a similarly shaped curve but the slope will approach zero near the $1 / v$ axis and approach $[\alpha /(1+\alpha)]\left(K_{s} / V_{m}\right)$ at low values of $(S)$ where the curve for interacting sites wonld have a slope of $K_{s} / V_{m}$. It is evident that the complexities become greater if more than two interacting active centers occur on the enzyme.

\section{Two Different Substrates Are Involved in the Reaction}

One of the most serious limitations of the Michaelis-Menten theory is that it assumes only one substrate enters into the reaction. whereas the majority of enzyme reactions involve two or more substrates. The common reactions involving two substrates fall into three categories:

$$
\begin{aligned}
& \mathrm{A}+\mathrm{B} \rightleftharpoons \mathrm{AB} \\
& \mathrm{A}+\mathrm{B} \rightleftharpoons \mathrm{A}+\mathrm{C} \\
& \mathrm{A}+\mathrm{B} \rightleftharpoons \mathrm{C}+\mathrm{C})
\end{aligned}
$$

but kinetically they may all be treated in a similar manner. Reaction $2-43$ represents an addition reaction; reaction 2-44 represents the situation where an activator is necessary; and reaction 2-45 represents transfer of groups between substrates. Most enzyme reactions are probably of type 2-45 inasmuch as this includes all reactions of group transfer:

$$
\mathrm{A}-\mathrm{X}+\mathrm{B} \rightleftharpoons \mathrm{A}+\mathrm{B}-\mathrm{X}
$$

where $\mathrm{X}$ may be hydrogen atoms as in oxidation-reduction, or chemical groups as in transmethylation. transamination. or transacylation, or that part of the substrate that is transferred to the hydroxyl group of water in hydrolytic reactions. It has been estimated that such transfer reactions may include over $90 \%$ of all enzyme reactions (Dixon and Webb, 1958, p. 184). All two-substrate reactions may he divided into two general categories: in the first, only one of the substrates forms an ES complex with the enzyme and the second substrate reacts directly with this, whereas in the second. both substrates form complexes with the enzyme and. these being adjacent, formation of the products occurs within this ternary complex. 
(a) Only One Substrate Forms a Complex With the Enzyme. When B reacts directly with the $\mathrm{EA}$ complex to form the products immediately, one may write:

$$
\begin{gathered}
\mathrm{E}+\mathrm{A} \underset{k_{-1}}{\stackrel{k_{1}}{\rightleftharpoons}} \mathrm{EA} \\
\mathrm{EA}+\mathrm{B} \stackrel{k_{2}}{\rightarrow} \mathrm{E}+\mathrm{C}+\mathrm{D}
\end{gathered}
$$

The rate equation is found to be:

$$
v=\frac{k_{2}\left(\mathrm{E}_{t}\right)(\mathrm{A})(\mathrm{B})}{(\mathrm{A})+\left[k_{-1}+k_{2}(\mathrm{~B})\right] / k_{1}}=V_{m} \frac{(\mathrm{A})(\mathrm{B})}{(\mathrm{A})+k_{\mathrm{A}}+\left(k_{2} / k_{1}\right)(\mathrm{B})}
$$

where $V_{m}$ is the maximal rate with respect to $\mathrm{A}$ and $K_{\mathrm{A}}$ is the dissociation constant of the EA complex. The expression is similar to the classic Michaelis-Menten formulation but both the maximal rate and the Michaelis constant now depend on the concentration of B. Since the reciprocal equation is:

$$
\frac{1}{v}=\frac{1}{V_{m}(\mathrm{~B})}+\frac{1}{V_{m}(\mathrm{~A})}\left[\frac{K_{\mathrm{A}}}{(\mathrm{B})}+\frac{k_{2}}{k_{1}}\right]
$$

plots of $1 / v$ against $1 /(\mathrm{A})$ at different concentrations of $\mathrm{B}$ will often allow evaluation of the constants.

If one of the products forms a complex with the enzyme, there are two binary complexes involved and the reactions may be written as:

$$
\begin{aligned}
& \mathrm{E}+\mathrm{A} \underset{k_{-1}}{\stackrel{k_{1}}{\rightleftharpoons}} \mathrm{EA} \\
& \mathrm{EA}+\mathrm{B} \stackrel{k_{2}}{\rightarrow} \mathrm{EC}+\mathrm{D} \\
& \mathrm{EC} \stackrel{\stackrel{k_{3}}{\rightarrow}}{\mathrm{E}}+\mathrm{C}
\end{aligned}
$$

The steps wherein products are formed are written in the forward direction only because we are considering initial rates before the products have accumulated sufficiently to make the back reactions significant. The rate equation is found to be:

$$
l=V_{m}^{\prime} \frac{(\mathrm{A})(\mathrm{B})}{(\mathrm{A})+K_{\mathrm{A}}+K^{\prime}(\mathrm{B})+K^{\prime \prime}(\mathrm{A})(\mathrm{B})}
$$

where $V_{m}=k_{2}\left(\mathrm{E}_{t}\right), K_{\mathrm{A}}=k_{-1} / k_{1}, K^{\prime}=k_{2} / k_{1}$, and $K^{\prime \prime}=k_{2} / k_{3}$. This is identical to Eq. 2-49 except for the additional term for $(A)(B)$ in the denominator, which alters the intercept but not the slope in the reciprocal plots. 
When a ternary EAB complex is formed, in which B is not necessarily attached to the enzyme, one can write:

$$
\begin{gathered}
\mathrm{E}+\mathrm{A} \underset{k_{-1}}{\stackrel{k_{1}}{\rightleftharpoons}} \mathrm{EA} \\
\mathrm{EA}+\mathrm{B} \underset{k_{-2}}{\stackrel{k_{2}}{\rightleftharpoons}} \mathrm{EAB} \stackrel{k_{3}}{\rightarrow} \mathrm{EC}+\mathrm{D} \\
\mathrm{EC} \stackrel{k_{4}}{\rightarrow} \mathrm{E}+\mathrm{C}
\end{gathered}
$$

and the rate equation is the same as Eq. 2-54 with $V_{m}=k_{2} k_{3}\left(\mathrm{E}_{\ell}\right)\left(k_{-2}+k_{3}\right)$, $K^{\prime}=k_{2} k_{3} / k_{1}\left(k_{-2}+k_{3}\right)$, and $K^{\prime \prime}=k_{2}\left(k_{3}+k_{4}\right) / k_{4}\left(k_{-2}+k_{3}\right)$.

It is obvious that these three mechanisms cannot be distinguished by the usual kinetic analysis and although it is often possible to evaluate the general constants, one is seldom able to interpret them in terms of rate constants or demonstrate a particular mechanism with certainty.

(b) Both Substrates Form Complexes With The Enzyme. The most general situation is where both substrates can form separate complexes with the enzyme and a ternary EAB complex is formed by either order of addition of the substrates:

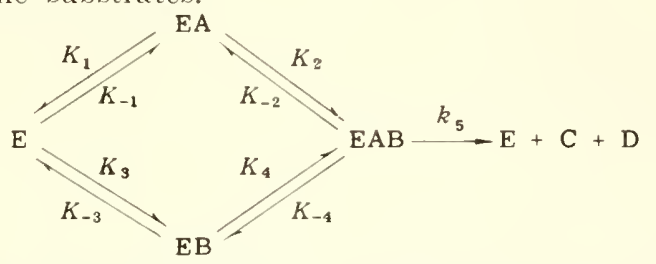

The steady-state treatment leads to a complex rate expression with numerous constants, each a function of several rate constants, which is not of the Michaelis-Menten form and does not give a linear reciprocal plot:

$$
v=\frac{K_{1}+K_{2}(\mathrm{~A})+K_{3}(\mathrm{~B})}{K_{4}+K_{5} \frac{1}{(\mathrm{~A})}+K_{6} \frac{1}{(\mathrm{~B})}+K_{7} \frac{(\mathrm{A})}{(\mathrm{B})}+K_{8} \frac{(\mathrm{B})}{(\mathrm{A})}+K_{9}(\mathrm{~A})+K_{10}(\mathrm{~B})+K_{11} \frac{1}{(\mathrm{~A})(\mathrm{B})}}
$$

where the constants have the following values:

$$
\begin{aligned}
& K_{1}=k_{1} k_{-3} k_{2} k_{5}\left(\mathrm{E}_{t}\right)+k_{-1} k_{3} k_{4} k_{5}\left(\mathrm{E}_{t}\right) \\
& K_{2}=k_{1} k_{4} k_{2} k_{5}\left(\mathrm{E}_{t}\right) \\
& K_{3}=k_{3} k_{4} k_{2} k_{5}\left(\mathrm{E}_{t}\right) \\
& K_{4}=k_{1} k_{-3} k_{2}+k_{1} k_{-4} k_{2}+k_{-1} k_{3} k_{4}+k_{3} k_{4} k_{-2} \\
& K_{5}=k_{-1} k_{3} k_{-4}+k_{-1} k_{3} k_{-2}+k_{-1} k_{3} k_{5}+k_{-3} k_{-4} k_{2}+k_{-3} k_{2} k_{5}
\end{aligned}
$$




$$
\begin{aligned}
& K_{6}=k_{1} k_{-3} k_{-4}+k_{1} k_{-3} k_{-2}+k_{1} k_{-3} k_{5}+k_{-1} k_{4} k_{-2}+k_{-1} k_{4} k_{5} \\
& K_{7}=k_{1} k_{4} k_{-2}+k_{1} k_{4} k_{5} \\
& K_{8}=k_{3} k_{-4} k_{2}+k_{3} k_{2} k_{5} \\
& K_{9}=k_{1} k_{4} k_{2} \\
& K_{10}=k_{3} k_{4} k_{2} \\
& K_{11}=k_{-1} k_{-3} k_{-4}+k_{-1} k_{-3} k_{-2}+k_{-1} k_{-3} k_{5}
\end{aligned}
$$

adapted from Dixon and Webb (1958, p. 99). This expression is presented here complete to emphasize the complexity of the general treatment of what is, probably, the most common reaction mechanism. It is clear why the majority of $K_{m}$ 's determined from simple reciprocal plots bear no necessary relationship to dissociation constants. Of course, many assumptions and simplifications may be introduced, but agreement of the resulting equation with the experimental data does not prove the particular mechanism assumed. One of the most common assumptions is that the concentrations of the complexes are determined by the usual equilibria, in which case the rate equation reduces to:

$$
v=V_{m} \frac{(\mathrm{A})(\mathrm{B})}{(\mathrm{A})(\mathrm{B})+K_{\mathrm{B}}{ }^{\prime}(\mathrm{A})+K_{\mathrm{A}}{ }^{\prime}(\mathrm{B})+\overline{K_{\mathrm{A}}} \overline{K_{\mathrm{B}}^{\prime}}{ }^{\prime}}
$$

where $K_{\mathrm{A}}=(\mathrm{E})(\mathrm{A}) /(\mathrm{EA}), \quad K_{\mathrm{A}}{ }^{\prime}=(\mathrm{EB})(\mathrm{A}) /(\mathrm{EAB})$ and $\quad K_{\mathrm{B}}{ }^{\prime}=(\mathrm{EA})(\mathrm{B}) /$ $(\mathrm{EAB})$, this applying to cases where binding of one substrate alters the binding of the second substrate. If the binding is independent $\left(K_{A}{ }^{\prime}=K_{A}\right.$ and $K_{\mathrm{B}}{ }^{\prime}=K_{\mathrm{B}}$ ), the following rate equation is obtained and this has been frequently applied to enzymic transfer reactions:

$$
v=V_{m} \frac{(\mathrm{A})(\mathrm{B})}{\left[(\mathrm{A})+K_{\mathrm{A}}\right]\left[(\mathrm{B})+K_{\mathrm{B}}\right]}
$$

It is seen from the reciprocal equation:

$$
\frac{1}{v}=\frac{1}{V_{m}}\left[1+\frac{K_{\mathrm{B}}}{(\mathrm{B})}\right]+\frac{K_{\mathrm{A}}}{V_{m}(\mathrm{~A})}\left[1+\frac{K_{\mathrm{B}}}{(\mathrm{B})}\right]
$$

that if $1 / v$ is plotted against $1 /(\mathrm{A})$, both the intercept and the slope will be changed by the factor $\left.1+K_{\mathrm{B}} / \mathrm{B}\right)$ compared to the case where $A$ is the only substrate; thus the determined $K_{m}$ is dependent on the concentration of the other substrate or coenzyme. However, by varying both $(A)$ and $(B)$, it is possible to determine the constants; for example, as (B) increases, the $K_{m}$ for A will decrease and will approach $K_{\mathrm{A}}$.

The most complete analysis of the kinetics of reactions involving coenzymes has been given by Alberty (1958). Following Dalziel (1957), he showed that all enzyme reactions in which two substrates (or a substrate and a 
coenzyme) participate, whatever the order or mechanism of complex formation, follow rate expressions of the general type:

$$
\frac{\left(\mathrm{E}_{t}\right)}{v}=\varphi_{0}+\frac{\varphi_{1}}{(\mathrm{~A})}+\frac{\varphi_{2}}{(\mathrm{~B})}+\frac{\varphi_{12}}{(\mathrm{~A})(\mathrm{B})}
$$

where (A) and (B) are the concentrations of the two substrates and the $\varphi$ 's are parameters which involve the rate constants and can be determined by the proper methods of plotting. This equation is equivalent to:

$$
r=r_{m} \frac{(\mathrm{A})(\mathrm{B})}{\varphi_{12}+\varphi_{2}(\mathrm{~A})+\varphi_{1}(\mathrm{~B})+\varphi_{0}(\mathrm{~A})(\mathrm{B})}
$$

with which the above expressions are seen to correspond.

\section{The Solvent Participates in the Reaction}

The role of water in enzyme reactions is frequently ignored. However, without directly entering into the reaction, water can contribute to the value of the Michaelis constant or substrate constant. The enzyme active center and the substrate may be hydrated and reaction between them to form the ES complex may involve displacement of water, according to the following equation:

$$
\mathrm{E}-\mathrm{W}+\mathrm{S}-\mathrm{W} \rightleftharpoons \mathrm{ES}+\mathrm{W}-\mathrm{W}
$$

The substrate constant, therefore, does not relate only to the affinity of the enzyme for the substrate but includes the relative affinities of both for water, as pointed out by Laurence (1955).

In hydrolytic reactions, water is one of the substrates but terms for it are usually omitted from the kinetic equations because its concentration is generally constant and very high. From Eq. 2-61, if B is water it is seen that the usual Michaelis-Menten expression is obtained since $\left(\mathrm{H}_{2} \mathrm{O}\right) \gg K_{\mathrm{H}_{2} \mathrm{O}}$ if an $\mathrm{E}_{-} \mathrm{H}_{2} \mathrm{O}$ complex exists. It is generally not known if a site exists on the enzyme for the binding of the water that enters hydrolytic reactions. It is known that the rate with $\beta$-fructofuranosidase is a function of the water concentration when this is altered by high concentrations of sucrose or alcohol (Nelson and Schubert, 1928) and a water-site on urease has been postulated to explain the inhibition by excess substrate (Laidler and Hoare, 1949). Two active centers were assumed to occur on urease, one for urea and one for water, and reaction occurs when the ternary complex is formed; however, urea can also compete with the water for the water-site, although such a complex is catalytically inactive. The rate expression derived for this situation was:

$$
v=\frac{l \cdot K_{2}\left(\mathrm{E}_{t}\right)(\mathrm{S})}{K_{1} K_{2}+\left(K_{1}+K_{2}\right)(\mathrm{S})+(\mathrm{S})^{2}}=V_{m} \frac{(\mathrm{S})}{(\mathrm{S})+K_{m}+(\mathrm{S})^{2} /\left(K_{1}+K_{2}\right)}
$$


where $k$ is the rate constant for the formation of products from the complex, $K_{1}$ and $K_{2}$ are dissociation constants for the complexes of substrate with its own site and the water-site respectively, $V_{m}=k\left(\mathrm{E}_{t}\right) K_{2} /\left(K_{1}+K_{2}\right)$ and $K_{m}=K_{1} K_{2} /\left(K_{1}+K_{2}\right)$. Such a mechanism leads to inhibition at high substrate concentrations since both sites are predominantly occupied by urea, and water camnot enter into the reaction. Equation 2-64 is plotted in Fig. 2-8 to illustrate how relative values of the $K$ 's affect the dependence of the rate on the substrate concentration. The degree of inhibition increases, of course, with the increasing affinity of the water-site for the urea.

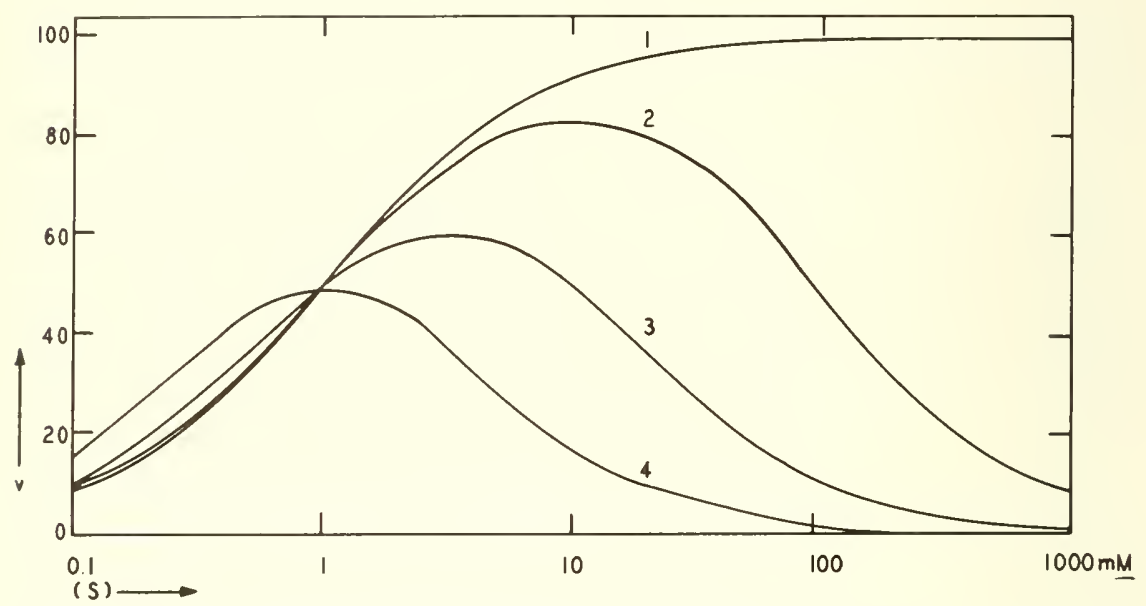

Fig. 2-8. Rate plots for an enzyme reaction involving two substrates, one of which is the solvent (Eq. 2-64). $V_{m}=100$. Curve 1: $K_{1}=1 \mathrm{~m} H, K_{2}=\infty$; curve $2: K_{1}=1$ $\mathrm{m} M, K_{2}=100 \mathrm{~m} H$; eurve $3: K_{1}=1 \mathrm{~m} M, K_{2}=10 \mathrm{~m} M$; curve $4: K_{1}=1 \mathrm{~m} M$, $K_{2}=1 \mathrm{~m} M$.

The most direct evidence for a specific water-site has come from a study of myosin ATPase where the activity of water relative to methanol in the splitting of adenosine triphosphate (ATP) is perhaps greater than 1000 . Since the activities in non-enzymic splitting are comparable, it is possible that an active center capable of binding water much more tightly than methanol exists on this enzyme; however, other explanations, such as a site for hydrated ATP, are tenable (Koshland and Herr, 1957). The general importance of a specific water-site for inhibition kineties lies in the possibility that certain inhibitors affect or compete for this site instead of the site binding the ordinarily considered substrate.

When water enters into a hydrolytic reaction sequence, it is necessary to include it in the kinetic expressions. For example, in the reactions pos- 
tulated for acetylcholinesterase (Wilson and Cabib, 1956):

$$
\begin{aligned}
& \mathrm{EH}+\mathrm{S} \underset{k_{-1}}{\stackrel{k_{1}}{\rightleftharpoons}} \mathrm{EHs} \stackrel{k_{2}}{\rightarrow} \mathrm{ES}^{\prime}+\mathrm{ROH} \\
& \mathrm{ES}^{\prime}+\mathrm{H}_{2} \mathrm{O} \stackrel{k_{3}}{\rightarrow} \mathrm{EH}+\mathrm{CH}_{3} \mathrm{COOH}
\end{aligned}
$$

the rate equation was found to be of the Michaelis form 2-11 with $V_{m}=$ $k_{2} k_{3}\left(k_{2}+k_{3}\right)$ and $K_{m}=k_{3}\left(k_{-1}+k_{2}\right) / k_{1}\left(k_{2}+k_{3}\right)$, i.e., the classic $V_{m}$ and $K_{m}$ are each altered by the factor $k_{3}\left(k_{2}+k_{3}\right)$. The constants for this reaction thus depend on the rate constant for the water reaction and an inhibitor could thus modify both $I_{m}$ and $K_{m}$ without interfering with the substrate acetylcholine in any way.

\section{The Enzyme Reaction Involves an Activator}

An activator is a substance, usually inorganic, that is not chemically altered in the reaction but, by its presence, increases the rate of the enzyme catalysis. In many cases the activator probably functions by participating in the binding of the substrates to the enzyme or by the stabilization of one or more of the complexes involved in the reaction sequence. In the simple situation where the presence of the activator on the enzyme is necessary for binding and reaction of the substrate:

$$
\begin{gathered}
\mathrm{E}+\mathrm{A} \underset{k_{-3}}{\stackrel{k_{3}}{\rightleftharpoons}} \mathrm{EA} \\
\mathrm{EA}+\mathrm{S} \underset{k_{-1}}{\stackrel{k_{1}}{\rightleftharpoons}} \mathrm{EAS} \stackrel{k_{2}}{\rightarrow} \mathrm{EA}+\mathrm{P}
\end{gathered}
$$

where $\mathrm{A}$ is the activator and $\mathrm{S}$ is the substrate, the rate equation is:

$$
v=V_{m}^{\prime} \frac{(\mathrm{A})(\mathrm{S})}{(\mathrm{A})(\mathrm{S})+K_{m}(\mathrm{~A})+K_{a} K_{m}}=V_{m}^{\prime} \frac{(\mathrm{S})}{(\mathrm{S})+K_{m}\left[1+K_{a} /(\mathrm{A})\right]}
$$

with $K_{a}=k_{-3} \mid k_{3}, K_{m}=\left(k_{-1}+k_{2}\right) k_{1}$, and $V_{m}$ the rate when all the enzyme is in the form EAS. It is interesting that the Nichaelis constant determined experimentally by reciprocal plotting depends on the activator concentration. In an enzyme preparation with an unrecognized activator (which may have been separated with the enzyme from the tissue or added in the reaction medium, and which may be present in variable concentration under different conditions), the Michaelis constant may vary unexpectedly. It is also evident that an inhibitor could slow the rate by increasing either $K_{s}$ or $K_{a}$. 
If the sirbstrate can bind to the enzyme in the absence of the activator (although the complex is not reactive) but the activator increases the affinity of the enzyme for the substrate, one may write:

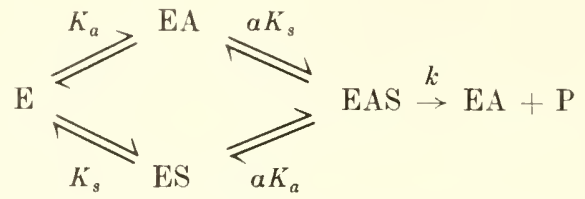

where $\alpha$ represents the change in substrate binding caused by the activator. Assuming equilibrium concentrations of the complexes for simplicity, the rate equation is found to be:

$$
v=V_{m}^{\prime \prime} \frac{(\mathrm{A})(\mathrm{S})}{(\mathrm{A})(\mathrm{S})+\alpha\left[K_{a} K_{s}+K_{s}(\mathrm{~A})+K_{a}(\mathrm{~S})\right]}=V_{m}^{\prime} \frac{(\mathrm{S})}{(\mathrm{S})+K_{m}^{\prime}}
$$

where $V_{m}=k\left(\mathrm{E}_{t}\right), V_{m}{ }^{\prime}=k\left(\mathrm{E}_{t}\right)(\mathrm{A}) /\left[(\mathrm{A})+\alpha K_{a}\right]$, and $K_{m}=K_{s} \alpha\left[(\mathrm{A})+K_{a}\right] /$ $\left[(\mathrm{A})+\alpha K_{a}\right]$. If $\alpha=1$, the rate equation simplifies to:

$$
v=V_{m}^{r}\left[\frac{(\mathrm{A})}{(\mathrm{A})+K_{a}}\right]\left[\frac{(\mathrm{S})}{(\mathrm{S})+K_{s}}\right]
$$

which is similar in form to Eq. 2-61 for transfer reactions. It is clear that often the kinetics of activator-dependent and two-substrate reactions will be very similar. However, generally $\alpha<1$ if $\mathrm{A}$ does facilitate the binding of $\mathrm{S}$; in the case of renal alkaline phosphatase, activated by $\mathrm{Zn}^{++}, \alpha$ was found to be about 0.1 (Gryder et al., 1955).

In the more general situation where the activator is not necessary for the reaction but accelerates it and where the activator can either modify the substrate binding or the rate of breakdown of the EAS complex relative to the $\mathrm{ES}$ complex:

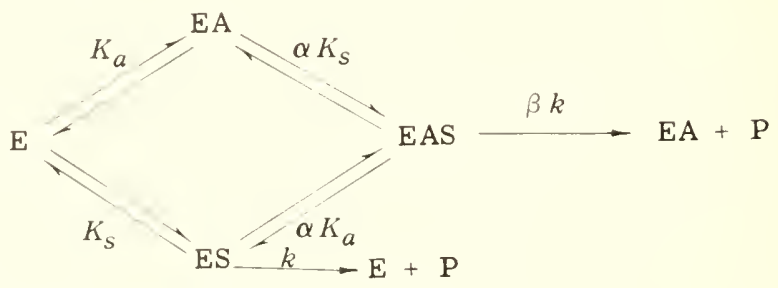

and $v=k(\mathrm{ES})+\beta k(\mathrm{EAS})$. The rate equation can be written in a MichaelisMenten form with $V_{m}=h\left(\mathrm{E}_{t}\right)\left[\beta(\mathrm{A})+\alpha K_{q}\right] /\left[(\mathrm{A})+\alpha K_{2}\right]$ and $K_{m}=$ $\alpha K_{s}\left[(\mathrm{~A})+K_{a}\right] /\left[(\mathrm{A})+\alpha \boldsymbol{K}_{a}\right]$. Several special cases can be derived from this 
general formulation, assuming appropriate relative concentrations and constants. One type of activation not included is that in which the activator combines with the substrate instead of the enzyme; the rate equations are complex and are discussed in some detail by Dixon and Webb (1958 p. 459).

\section{Reactions in Which the Enzyme Undergoes a Cyclic Change}

There are important reactions in which the enzyme is altered in one phase of the cycle and must be regenerated during another phase in order that the process proceed; oxidation-reduction reactions in which the enzyme, or a prosthetic group, is alternately in the oxidized and reduced states would be of this type.

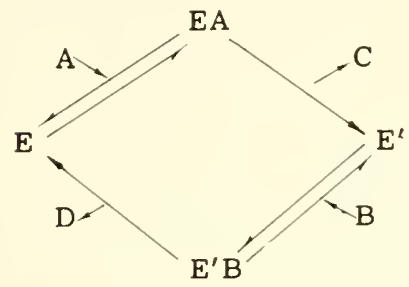

$$
\begin{aligned}
& \mathrm{E}+\mathrm{A} \frac{k_{1}}{k_{-1}} \mathrm{EA} \stackrel{k_{2}}{\longrightarrow} \mathrm{E}^{\prime}+\mathrm{C} \\
& \mathrm{E}^{\prime}+\mathrm{B} \frac{-k_{3}}{k_{-3}} \mathrm{E}^{\prime} \mathrm{B} \stackrel{k_{4}}{\longrightarrow} \mathrm{E}+\mathrm{D}
\end{aligned}
$$

The rate equation is of the type:

$$
v=V_{m} \frac{(\mathrm{A})(\mathrm{B})}{(\mathrm{A})(\mathrm{B})}+K_{\mathrm{A}}(\mathrm{B})+K_{\mathrm{B}}(\mathrm{A})
$$

where $V_{m}=k_{2} k_{4}\left(\mathrm{E}_{l}\right)\left(k_{2}+k_{4}\right), \quad K_{\mathrm{A}}=k_{4}\left(k_{-1}+k_{2}\right) k_{1}\left(k_{2}+k_{4}\right)$. and $K_{\mathrm{B}}=$ $k_{2}\left(k_{-3}+k_{4}\right) k_{3}\left(k_{2}+k_{4}\right)$. With respect to one substrate this may be rewritten:

$$
v=\boldsymbol{V}_{m}^{\prime} \frac{(\mathrm{A})}{(\mathrm{A})+K_{m}}
$$

where $V_{m}{ }^{\prime}=V_{m}(\mathrm{~B})\left[(\mathrm{B})+K_{\mathrm{B}}\right]$ and $K_{m}=K_{\mathrm{A}}(\mathrm{B})^{\prime}\left[(\mathrm{B})+K_{\mathrm{B}}\right]$. Such reactions thus conform to the Michaelis-Nenten formulation but the constants are complex and depend on the substance restoring the enzyme to its original condition.

The over-all equation for the above reactions is:

$$
\mathrm{A}+\mathrm{B} \rightleftharpoons \mathrm{C}+\mathrm{D}
$$

and is a transfer with the enzyme functioning as a carrier. In some cases the same substrate may participate in both reactions and the result is a dismutation:

$$
2 \mathrm{~A} \rightleftharpoons \mathrm{C}+\mathrm{D}
$$


for which the rate equation may be obtained by setting $(A)=(B)$ :

$$
v=V_{m} \frac{(\mathrm{A})}{(\mathrm{A})+K_{m}^{-}}
$$

where $V_{m}$ is the same as in Eq. 2-76 and $K_{m}=K_{\mathrm{A}}+K_{\mathrm{B}}$. It is worth noting that whereas from the over-all equation one might expect $(\mathrm{A})^{2}$ in the rate equation, this is not so if the two molecules of substrate react sequentially.

\section{Enzymic Reactions of Greater Complexity}

The few reaction mechanisms disenssed above do not by any means exhaust the possibilities of intricate patterns in enzyme catalysis. Many enzymes involve three or more reacting components in their mechanisms (e.g., a substrate, a coenzyme, and ATP) or catalyze several individual reactions on their surfaces before the product of interest is produced. The success of a simple kinetic analysis in some of these complex situations resides in the fact that occasionally only one of the component reactions controls the over-all rate, being relatively so slow that it limits the entire process. In such cases the kinetic data will apply only to this limiting reaction; however, it is not always easy to determine exaetly which of the multiple steps is limiting. Furthermore, different steps may be rate-limiting under different conditions and there is no guarantee that the same step is limiting in the cell as in the isolated preparation. From the practical point of view, it often minimizes the chance of misinterpretation in enzyme studies, with or withont inhibitors present, if each known component of the system is varied independently and in relation to every other component, and to cover a sufficient range of concentrations to allow valid kinetic analysis. 


\section{THE KINETICS OF ENZYME INHIBITION}

Inhibition of an enzyme implies a decrease in the rate of the particular reaction that is experimentally measured or metabolically important. An inhibitor generally will reduce the rate of enzyme catalysis whatever criterion is applied, whether it be utilization of substrate or formation of products. In some situations, however, where two or more products are formed, the inhibition may refer to only one product whereas the formation of the others may be unaffected or actually accelerated. In oxidative phosphorylation an uncoupling agent inhibits the formation of the biologically important ATP while the oxidation of substrate, uptake of oxygen, and formation of other products may be increased. Many so-called inhibitors are not truly enzyme inhibitors but alter the pattern of the over-all reaction to divert the course away from some product. Substances such as this, not specifically inhibiting some enzyme, might be termed metabolic inhibitors more appropriately because their final effect is to disturb or reduce the normal cellular flow of matter and energy. A substance that interferes metabolically with the function of a cell is an inhibitor. by whatever means it produces this effect.

A general definition of an inhibitor might be made in the terminology of Peters by stating that an inhibitor induces a biochemical lesion in a cell, providing we extend somewhat the concept of biochemical lesion. Originally the term was applied to the defect created in brain metabolism and function by a deficiency in vitamin $B_{1}$ (Gavrilesen and Peters, 1931) and was later extended to the effects produced by certain substances, such as the arsenicals, on cellular enzymes. The term might profitably include any clislocation of metabolism by agents acting directly on metabolic systems. The definition of an imhibitor could be made in a more straightforward mammer but the approach above introduces the idea of a biochemical lesion, comparable to other types of lesions in cells and tissues, an idea of some conceptual significance, and a term of use in designating the manifold perturbations that inhibitors can induce in cells. In the present chapter we shall limit the discussion to inhibitors that act directly on and inhibit individual enzymes, since the purpose here is an exposition of the kinetics of isolated enzymes. These results will be applied later to more complex systems and intracellular inhibitons. 
The mechanisms by which enzymes can be inhibited nay be classified kinetically in terms of the deviations produced in the normal kinetics as discussed in the previous chapter, or according to the exact sites and manner of action relative to the molecular events of the catalysis; the former is the most common method and the latter is the most difficult but preferable. The kinetic analysis, along with other pertinent data from various approaches, can often be a valuable aid to understanding the details of an inhibitory mechanism but usually should not be an end in itself. The most important thing to know about an inhibition is the exact site of action of the inlibitor, with what substance or enzyme group it interacts. Only then can one interpret the kinetic data meaningfully and derive quantitative parameters that characterize the inhibition. It is often stated that an inhibitor reacts with the active center of an enzyme. This is sometimes vague and ambiguous since on many enzymes there are two or more regions necessary for the catalysis of the entire reaction sequence, some pertaining to the substrates, some to coenzymes, and some to activators. It is better, when possible, to avoid the general term active center, unless the entire surface involved is referred to, and use a terminology indicative of more precise localization.

It is useful to designate the regions on the enzyme involved in the binding of the different components as substrate sites, coenzyme sites, and activator sites, where such distinctions may be made. These would together make up the active center, which would embrace all the areas on the enzyme involved in the catalysis. The substrate site may, of course, include the activator if the substrate is bound wholly or in part through the activator. The term apoenzyme will be used to designate the protein part of an enzyme and these varions sites may be considered as regions in the apoenzyme, or occasionally in prosthetic groups, under which are included nonprotein components, such as hemes, that are for practical purposes imdissociable from the apoenzyme and function as a permanent part of the enzyme.

All enzyme inhibitions are the result of an interaction of the inhibitor with some component of the enzyme system. A classification of the sites of inhibition can be based on the component primarily involved in the inhibition.

(I) Reaction of inhibitor with apoenzyme

A. Chemical reaction with specific protein groups: these groups, such as sulfhydryl, amino, or phenolic groups, may react irrespective of their position relative to the active center.

$B$. Specific reaction with sites on the apoenzyme: the specificity of interaction here resides in the spatial pattern of matter and charge over the site rather than in a simple chemical group.

(1) Substrate site

(2) Coenzyme site

(3) Activator site 
C. Generalized adsorption onto the protein surface: a relatively nonspecific and weak interaction by substances, frequently nonpolar or amphotropic compounds, that associate with the protein side-chains and may interfere with binding of any component.

D. Denaturation of the protein: an alteration of the basic protein structure, usually by substances reacting with those protein groups responsible for the bonds holding the polypeptide fabric in a specific orientation.

E. Hydrolysis of apoenzyme: the breaking of peptide bonds in the protein, generally by proteolytic enzymes, giving rise to fragments of the apoenzyme that may be partially or completely inactive.

(II) Reaction of inhibitor with substrate: the binding or the subsequent transformations of the substrate are hindered.

(III) Reaction of inhibitor with coenzyme: generally the ability of the coenzyme to participate in the reaction is reduced, although the affinity for the apoenzyme may also be decreased.

(IV) Reaction of inhibitor with activator

(V) Reaction of inhibitor with enzyme-complex: combination with the enzymesubstrate, enzyme-coenzyme, or enzyme-activator complex, although there is not necessarily a reaction with any of the indivirlual components.

(VI) Entry of inhibitor into the reaction sequence: the inhibitor may be acted upon by the enzyme so that it undergoes reactions similar to the substrate, this reducing the amount of substrate reacted or causing a subsequent block if a normal transfer reaction is slowed.

(VII) Reaction of inhibitor with linking components in an enzyme aggregate: dissociation of enzyme units in a complex system by interaction with substances, perhaps nonprotein in nature, functioning structurally in the spatial orientation of these units.

It may be noted that the words reaction and interaction as used here and elsewhere in this book do not imply any particular type of relationship between the molecules involved nor limit the forces that are operative. The reaction of an inhibitor with the sites and substances outlined above may be chemical in the sense that electron pair bonds are formed but it is generally not and the term reaction should not be construed as indicating such a chemical reaction. When two molecules enter into a relationship in which their mutual energy levels are altered, these molecules are said to react or interact; whether the relationship is one of greater or less stability depends on the direction of the energy change. The nature of the forces involved will be discussed in Chapter 6; we wish now only to emphasize that no specific forces are implied by the general terms used.

Visualization at the molecular level of the different types of inhibitor reactions at the enzyme surface is often helpful in distinguishing between 
the various possibilities and in understanding the mechanisms and kinetics of the inhibitions. Some of the numerous types of interference are represented schematically in Fig. 3-1 for one enzyme system. In the classification above, reaction with the substrate site was put under a single heading; it is obvious that there are various ways in which a substance can prevent the substrate molecule from reaching this site. It may combine with the entire site, much as the substrate does (A); or it may react with only part of the site (D); or it may react not with the site itself but combine at an adjacent region, sterically or electrostatically repelling the substrate $(\mathrm{F})$. Reactions of other types $(\mathrm{G}, \mathrm{H}, \mathrm{J}$, and $\mathrm{K}$ ) may also interfere with the formation of the ES complex. In fact, it is possible that varying degrees of interference may be produced and that inactivation of an enzyme may be partial or complete and not necessarily an all-or-none phenomenon. Chemical reaction with specific protein groups has been diagrammed only for the substrate site but it could occur at any or all sites. It must also be understood that the enzyme surface illustrated is only one of numerous possibilities; e.g., the activator may be involved in binding the coenzyme rather than the substrate, or the activator might link the substrate and coenzyme without reacting itself with the apoenzyme, or the spatial relations between the substrate and the coenzyme may be more intimate than indicated, the mutual configurations they assume in the activated complex being obligatory for reaction.

\section{REVERSIBLE AND IRREVERSIBLE TYPES OF INHIBITION}

It is of primary importance for the kinetics of inhibition to distinguish between inhibitors that react reversibly and irreversibly. In the equilibrium:

$$
\mathrm{E}+\mathrm{I} \underset{k_{-1}}{\stackrel{k_{1}}{\rightleftharpoons}} \mathrm{EI}
$$

with $K_{i}=k_{-1} / k_{1}=(\mathrm{E})(\mathrm{I}) /(\mathrm{EI})$, irreversibility may be rigorously defined by equating it with the sitnation in which $k_{-1}=0$. Since such complete irreversibility seldom occurs and since the $K_{i}$ 's of the so-called irreversible inhibitors are generally finite although small, it is evident that $k_{-1}$ is not zero in most cases and a more practical definition of irreversibility must be made. If the inhibited enzyme is placed in a solution not containing the inhibitor, or dialyzed against inhibitor-free solution, irreversibility may be characterized by the state in which restoration of enzyme activity is not significant over an interval commensurate with the periods involved in the kinetic experiments. Slow reversibility can be demonstrated with stable enzymes, and kinetic experiments over intervals of hours may be performed on this basis, but with very unstable enzymes many inhibitors must be considered to be irreversible with regard to the kinetics because insignificant 

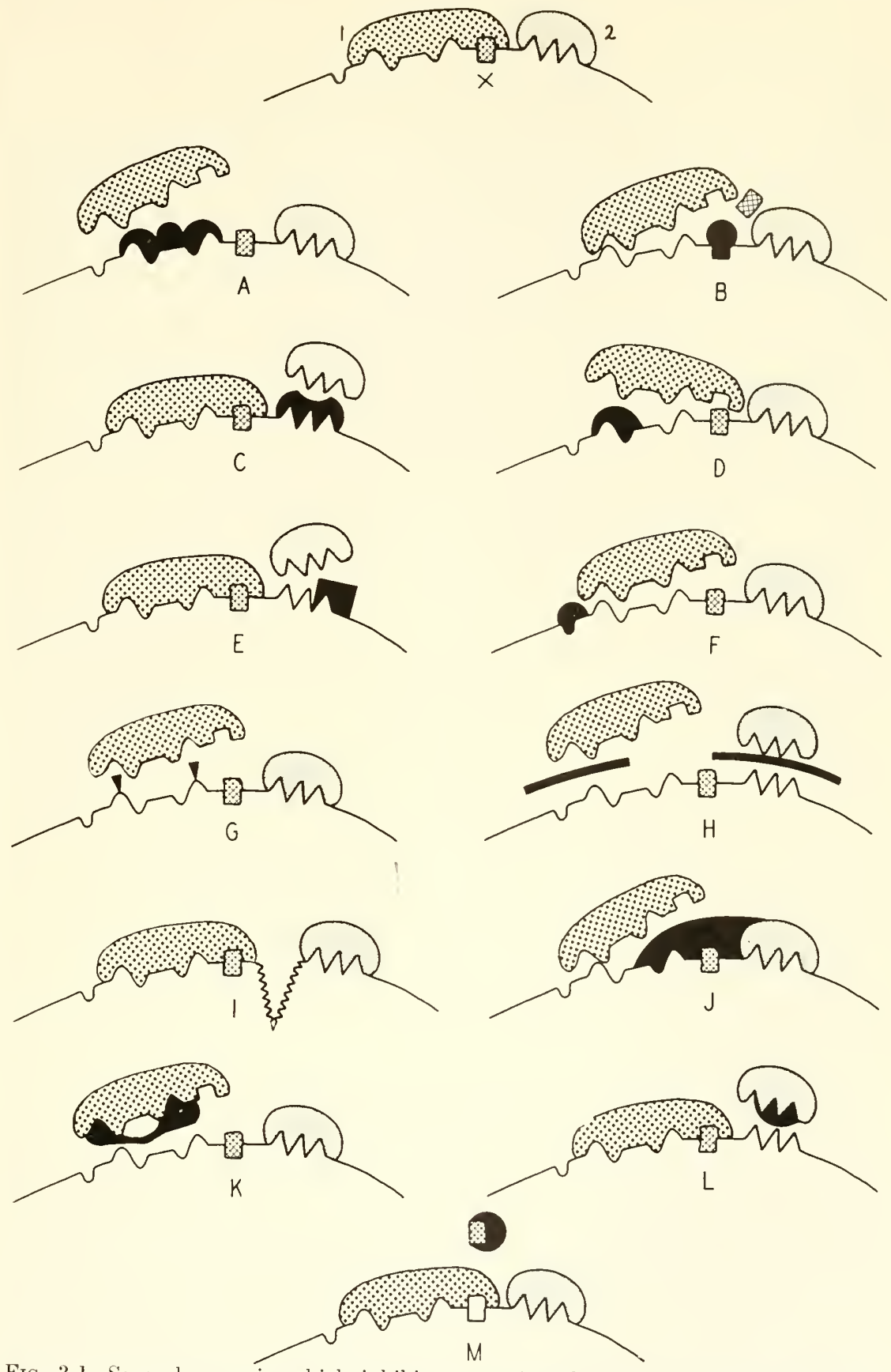

FIG. 3-1. Several ways in which inhibitors can interfere with an enzyme reaction. Molecules 1 and 2 are either two substrates or a substrate and coenzyme; $X$ is a cofactor, such as a metal ion. functioning in the binding of a substrate. The inhibitor in every case is represented by the solidly shaded molecule. The positions assumed by the reactants in the normal enzyme reaction are shown at the top. See the text for explanations. 
reversal can occur over periods of a few minutes and true equilibrium states cannot be achieved. Irreversible inhibitors are not necessarily characterized by very low $K_{i}$ 's, since $k_{1}$ may be small and comparable to $k_{-1}$. Most irreversible inhibitors react chemically with the enzyme, e.g., the arylation of amino groups with dinitrophenyl derivatives, the alkylation of thiol groups with iodoacetate, and the phosphorylation of serine residues with the phosphorofluoridates. Most irreversible imhibitors progressively inhibit an enzyme to completion if there is sufficient total inhibitor to react with all the enzyme groups (including other reactive groups that may be present in the preparation); the exceptions would be those inhibitors where $k_{1}$ and $k_{-1}$ are both small, since partial inhibition only may be achieved at equilibrium. The kinetics of irreversible inhibition are thus relatively simple since the inhibition increases with time as the active enzyme concentration is reduced. In fact, if the disappearance of active enzyme can be written as $-d(\mathrm{E}) / d t=k_{1}(\mathrm{E})(\mathrm{I})$, integration leads to an expression for the enzyme concentration at any time, nanrely $(\mathrm{E})=\left(\mathrm{E}_{0}\right) e^{-k_{1}(\mathrm{I}) t}$ where $\left(\mathrm{E}_{0}\right)$ is the initial concentration. The inhibited enzyme rate thus is $v_{i}=v e^{-k_{1}(\mathrm{I}) t}$ and the fractional inhibition $i=1-e^{-k_{1}(1) t}$. The reversal of inhibition by various means and the kinetics involved will be discussed in some detail in Chapter 13.

The usual formulations of inhibition kinetics apply only to reversible inhibitors where reaction $3-1$ is assumed to reach equilibrium. In many investigations the data have been analyzed withont adequate proof that equilibrium was reached under the experimental conditions. A comparison of several inhibitors on an enzyme using a constant experimental interval, a common procedure, may signify little, especially if the interval is short and the inhibitors are of different types, since some may not have reached equilibrium with the enzyme.

\section{COMPETITIVE AND NONCOMPETITIVE INHIBITORS}

A favorite classification of inhibitors is into competitive and noncompetitive types and it is necessary to consider the meaning of this distinction before presenting the kinetic equations. The usual meaning attached to competitive inhibition is that the inhibitor reacts reversibly with a site on the enzyme and thereby prevents combination of that component of the enzyme reaction that normally forms a complex at that site; thus an inhibitor may be competitive with substrate, coenzyme, or activator. In a competitive situation there are two complexes with the enzyme, ES and EI (assuming competition with substrate), one enzymatically active and the other inactive. The rate will depend as usual on the concentration of ES and the formation of EI will reduce this concentration. The relative concentrations of ES and EI depend on two factors - the relative concentra- 
tions of the substrate and inhibitor, and the relative affinities of the enzyme for each. Thus the degree of inhibition will depend on (S) and $K_{s}$.

Noncompetitive inhibition is assumed to involve reaction of the inhibitor with a region other than the active center so that combination of the substrate with the substrate site is unaffected but the breakdown of the ES complex is prevented. In this case the degree of inhibition will not depend on (S) or $K_{s}$ but only on (I) and $K_{i}$, the imhibitor dissociation constant.

In practice these two types of inhibition are distinguished by the characteristics of the various kinetic plots of experimental data, as explained later. It should be pointed out that there is often a discrepancy between the kinetic and the spatial concepts of competitive and noncompetitive inhibition. Many inhibitions designated as noncompetitive are actually not according to the definition above. In some cases an irreversible inhibition is claimed to be noncompetitive because it vields the appropriate plots, whereas this procedure is not applicable to irreversible inhibitors. In fact, such an inhibitor can react with the substrate site but competition will not be exhibited becanse of the failure to reach equilibrium. The substrate may, however, delay the development of the inhibition in this case because of the protection it affords its site. Occasionally an inhibitor is competitive with coenzyme or activator but if the data are analyzed $k i$ netically with respect to the substrate only, the inhibition will appear to be noncompetitive. Lastly, in many cases inhibitions have been called noncompetitive because the kinetics do not conform exactly to those of competitive inhibition, although they do not fit noncompetitive criteria either if examined carefully. Some of these inhibitions are partially competitive or of a mixed type. It would seem reasonable that pure noncompetitive inhibition must be rather uncommon, at least with simple enzymes, since it is unlikely that an inhibitor conld combine with the apoenzyme at a region close enough to the substrate site to prevent decomposition of the ES complex without hindering to some extent the binding of the substrate, thereby introducing a competitive component to the inhibition. Because of the difficulties, conclusions as to the site of inhibition from kinetic data alone must be made with caution. It cannot be overemphasized that for reliable kinetic interpretations, accurate quantitative data must be obtained under controlled conditions and a sufficient range of substrate and inhibitor concentrations must be utilized to allow accurate plotting of the data.

\section{GENERALIZED KINETICS OF REVERSIBLE INHIBITION}

No theory or inhibition equation can include all the types of inhibition outlined above but a generalized formulation may be applied to type (I) inhibitions where reaction is with the apoenzyme; the special types of inhibition, e.g. competitive and noncompetitive, can be derived from this 
by making the appropriate assumptions. The procedure is an extension of that presented by Friedenwald and Maengwyn-Davies (195t, p. 154). A discussion of the plotting of the resultant equations and the practical determination of the kinetic constants will be reserved for Chapter 5. It is convenient to use the equilibrium approach inasmuch as the steady-state treatment of generalized inhibition leads to equations and constants so complex that the basic concepts are obscured. Thus it will be assumed that all complexes with the enzyme are in equilibrium with their components and $K_{s}$ will be used instear of $K_{m}$; in the simplified equations, $K_{s}$ can generally be replaced by $K_{m}=\left(k_{-1}+k_{2}\right) / k_{1}$. This limitation will be discussed in more detail later. Initially it will be assumed that no coenzymes or activators are present and they will be introduced subsequently. It is assumed that the presence of the inhibitor can either alter the affinity of the enzyme for the substrate or affect the rate at which the substrate complex breaks down to products, according to the following equations:

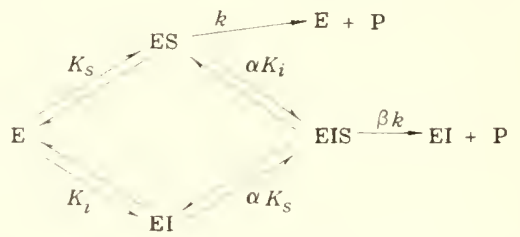

$$
\begin{aligned}
& \mathrm{E}+\mathrm{S} \rightleftharpoons \mathrm{ES} \stackrel{k}{\rightarrow} \mathrm{E}+\mathrm{P} \quad K_{s}=\frac{(\mathrm{E})(\mathrm{S})}{(\mathrm{ES})} \\
& \mathrm{E}+\mathrm{I} \rightleftharpoons \mathrm{EI} \quad K_{i}=\frac{(\mathrm{E})(\mathrm{I})}{(\mathrm{EI})} \\
& \mathrm{EI}+\mathrm{S} \rightleftharpoons \mathrm{EIS} \stackrel{\beta k}{-} \mathrm{EI}+\mathrm{P} \quad \alpha K_{s}=\frac{(\mathrm{EI})(S)}{(\mathrm{EIS})} \\
& \mathrm{ES}+\mathrm{I} \rightleftharpoons \mathrm{EIS} \stackrel{\beta k}{\rightarrow} \mathrm{EI}+\mathrm{P} \quad u K_{i}=\frac{(\mathrm{ES})(\mathrm{I})}{(\mathrm{EIS})}
\end{aligned}
$$

where $\alpha$ represents the change in affinity and $\beta$ the change in rate of complex decomposition induced by the inhibitor. It may be noted that this sitnation is similar to that ctiscussed for activators and formulated in Eq. 2-73; it will not be surprising that the resulting kinetic equations are similar. In fact, it may be assumed that $\mathrm{I}$ above is a modifier; the values of $\alpha$ and $\beta$ will determine whether it is an activator or inhibitor. The following relationships may be written:

$$
\begin{gathered}
\left(\mathrm{E}_{t}\right)=(\mathrm{E})+(\mathrm{ES})+(\mathrm{EI})+(\mathrm{EIS}) \\
\imath_{i}=k(\mathrm{ES})+\beta k(\mathrm{EIS})
\end{gathered}
$$


and combined with the equilibrium expressions 3-3 to 3-6 the rate equation is obtained:

$$
v_{i}=V_{m} \frac{(\mathrm{S})\left[\alpha K_{i}+\beta(\mathrm{I})\right]}{(\mathrm{S})(\mathrm{I})+\alpha\left[K_{i}(\mathrm{~S})+K_{s}(\mathrm{I})+K_{s} K_{i}\right]}
$$

where $v_{i}$ is the rate in the presence of inhibitor and $V_{m}=k\left(\mathrm{E}_{\imath}\right)$. This equation may be rewritten in the Michaelis form: $v_{i}=V_{m}{ }^{\prime}(\mathrm{S}) /\left[(\mathrm{S})+K_{m}{ }^{\prime}\right]$ where $V_{m}{ }^{\prime}=V_{m}\left[\alpha K_{i}+\beta(\mathrm{I})\right] /\left[\alpha K_{i}+(\mathrm{I})\right]$ and $K_{m}{ }^{\prime}=K_{s}\left[\alpha K_{i}+\alpha(\mathrm{I})\right] /$ $\left[\alpha K_{i}+\beta(\mathrm{I})\right]$. so that the kinetics with respect to substrate follow the classic Michaelis-Menten theory although the constants now depend on the inhibitor affinity and concentration. For inhibitors it is generally true that $\alpha>1$ and $\beta<1$, although this is not strictly necessary since the rate may be decreased even if $\alpha<1$ providing $\beta$ is sufficiently low.

The inhibition produced may be expressed in various ways; we shall uniformly use the fractional inhibition $i$. which may be defined as follows:

$$
i=1-a=1-\left(v_{i} / v\right)
$$

where $a$ is the fractional activity. Substituting expressions for $v$ and $v$, from Eqs. 2-7 and 3-9 into Eq. 3-10 gives:

$$
i=\frac{(\mathrm{I})\left[(\mathrm{S})(1-\beta)+K_{s}(\alpha-\beta)\right]}{(\mathrm{I})\left[(\mathrm{S})+\alpha K_{s}\right]+K_{i}\left[\alpha(\mathrm{S})+\alpha K_{s}\right]}
$$

The various types of inhibition are characterized by different values of $\alpha$ and $\beta$. Five characteristic types of inhibition may be considered on this basis.

(1) Completely competitive inhibition $(\alpha=\infty)$ : the inhibitor completely prevents the combination of substrate with the enzyme.

$$
\begin{gathered}
v_{i}=V_{m} \frac{(\mathrm{S})}{(\mathrm{S})+K_{s}\left[\mathrm{I}+(\mathrm{I}) / K_{i}\right]} \\
i=\frac{(\mathrm{I})}{(\mathrm{I})+K_{i}\left[1+(\mathrm{S}) / K_{s}\right]}
\end{gathered}
$$

(2) Partially competitive inhibition $(\infty>\mu>\mathrm{I}$ and $\beta=1)$ : the inhibitor only partially hinders binding of the substrate and does not affect the rate of breakdown of the substrate complex.

$$
\begin{gathered}
\iota_{i}=V_{m} \frac{(\mathrm{S})}{(\mathrm{S})+K_{s}\left\{[\alpha(\mathrm{I})+\alpha]_{i} /\left[(\mathrm{I})+\alpha K_{i}\right]\right\}} \\
i=\frac{(\mathrm{I})(\alpha-1)}{(\mathrm{I})\left[\alpha+(\mathrm{S}) / K_{s}\right]+\alpha K_{i}\left[1+(\mathrm{S}) / K_{s}\right]}
\end{gathered}
$$


(3) Completely noncompetitive inhibition $(\alpha=1$ and $\beta=0)$ : the inhibitor does not alter the binding of substrate but prevents its transformation into products.

$$
\begin{gathered}
v_{i}=V_{m} \frac{K_{i}}{(\mathrm{I})+K_{i}} \frac{(\mathrm{S})}{(\mathrm{S})+K_{s}} \\
i=\frac{(\mathrm{I})}{(\mathrm{I})+K_{i}}
\end{gathered}
$$

(4) Partially noncompetitive inhibition $(\alpha=1$ and $0<\beta<1)$ : the binding of substrate is not changed but the rate of breakdown of the EIS complex is slower than the ES complex.

$$
\begin{array}{r}
v_{i}=V_{m} \frac{\beta(\mathrm{I})+K_{i}}{(\mathrm{I})+K_{i}} \frac{(\mathrm{S})}{(\mathrm{S})+K_{s}} \\
i=(1-\beta) \frac{(\mathrm{I})}{(\mathrm{I})+K_{i}}
\end{array}
$$

(5) Mixed intibition $(\infty>\alpha>1$ and $\beta=0$ ): the affinity of the enzyme for the substrate is reduced by the inhibitor and the breakdown of the EIS complex is prevented.

$$
\begin{array}{r}
v_{i}=\mathrm{V}_{m} \frac{\alpha K_{i}}{(\mathrm{I})+\alpha K_{i}} \frac{(\mathrm{S})}{(\mathrm{S})+K_{s}\left\{\left[\alpha(\mathrm{I})+\alpha K_{i}\right] /\left[(\mathrm{I})+\alpha K_{i}\right]\right\}} \\
\quad i=\frac{(\mathrm{I})}{(\mathrm{I})+K_{i}\left\{\left[\alpha(\mathrm{S})+\alpha K_{s}\right] /\left[(\mathrm{S})+\alpha K_{s}\right]\right\}}
\end{array}
$$

The inhibitor in competitive inhibition is said to change the Michaelis constant (which we have assumed for convenience here to be $K_{s}$ ) withont affecting the maximal rate, as indicated by Eqs. 3-12 and 3-14; in noncompetitive inhibition it is the maximal rate that is changed and the $K_{s}$ is unaltered, as in Eqs. 3-16 and 3-18. The fifth type of inhibition is called mixed because both $V_{m}$ and $K_{s}$ are modified by the inhibitor and thus the inhibition partakes somewhat of the characteristics of both competitive and noncompetitive inhibition. Of course, mixed inhibition occurs if $\beta$ is less than 1 and $\alpha$ is greater than 1 (but not very large or infinity) and the relevant Eqs. are 3-9 and 3-11; one might designate it completely mixed inhibition when $\beta=0$ and partially mixed inhibition when $0<\beta<1$. It is necessary to understand that the term partially when applied to competitive and noncompetitive inhibition does not indicate mixture of inhibition types or impurity; it means that the inhibition is still purely competitive or noncompetitive, but the degree of inhibition is less than in the completely competitive or noncompetitive types, due to the fact that in the former the binding of substrate is not completely prevented or in the latter the breakdown of the EIS complex is not completely reduced to zero. 
Because it is frequently stated that in competitive inhibition the Michaelis constant is increased in the presence of the inhibitor, and because in reciprocal plotting the determined Michaelis constant can assume theoretically any value from $K_{s}$ to infinity, one must beware of assuming that the experimentally determined Michaelis constant bears any relation to this effect of the inhibitor on substrate affinity. To illustrate this, let us consider completely competitive inhibition: if (I) is kept constant and (S) is varied, the plotted results will provide a value for $K_{m}$, which from Eq. 3-12 is equal to $K_{s}$ multiplied by the factor $1+(\mathrm{I}) / K_{i}$ - however, this does not mean that the inhibitor has increased the dissociation constant $K_{s}$ by this amount, since actually the presence of the inhibitor on the enzyme raises $K_{s}$ to infinity, the substrate being entirely prevented from binding. The factor $1+\left[(\mathrm{I}) / K_{i}\right]$ is a statistical term indicating the number of enzyme molecules combined with inhibitor. In order to find the true effect of an inhibitor on substrate binding, the value of $a$ must be determined.

\section{Coupling or Uncompetitive Inhibition}

There is another possible type of inhibition that may be derived from the generalized equations above. This occurs if $u<1$, the inhibitor increasing the affinity of the enzyme for the substrate. If the breakdown of the substrate complex is maffected $(\beta=1)$ or accelerated $(\beta>1)$, this wonld result in activation rather than inhibition. However, if $\beta<1$, the over-all effect may be inhibition. If the situation where the EIS complex does not break down to products is considered $(\beta=0)$, increased binding of the substrate to form the inactive EIS complex will result in inhibition; if $\alpha$ is very low, approaching zero, the inhibition may be essentially complete since all of the enzyme will be in the inactive EIS form, inasmuch as in the present treatment it is assumed that $(\mathrm{S})$ and $(\mathrm{I})$ are greater than $\left(\mathrm{E}_{t}\right)$. Equations 3-20 and 3-21 express the kinetics for this situation, from which it may be seen that as $\alpha$ falls below one, the inhibition will increase, becoming complete when $\alpha$ is zero. This type of inhibition is really a special form of mixed inhibition. It was given the unsatisfactory name of uncompetitive inhibition by Ebersole et al. (1944), coupling inhibition (because the inhibitor increases the coupling of enzyme and substrate) by Friedenwald and Maengwyn-Davies (1954, p. 154), and anticompetitive intibition by Dodgson, et al. (1956). Friedenwald and Maengwyn-Davies developed the kinetic equations of coupling inhibition for the special case in which $\alpha=0$, assuming that the inhibitor combined only with the ES complex and not with the free enzyme.

$$
\begin{gathered}
\mathrm{E}+\mathrm{S} \rightleftharpoons \mathrm{ES} \rightarrow \mathrm{E}+\mathrm{P} \\
\mathrm{ES}+\mathrm{I} \rightleftharpoons \mathrm{EIS} \quad K_{i}{ }^{\prime}=\frac{(\mathrm{ES})(\mathrm{I})}{(\mathrm{EIS})}
\end{gathered}
$$


This leads to the following equations for the rate and inhibition:

$$
\begin{gathered}
v_{i}=V_{m}\left[\frac{K_{i}^{\prime}}{(\mathrm{I})} \frac{(\mathrm{S})}{+K_{i}^{\prime}}\right] \frac{(\mathrm{S})+K_{s}\left\{K_{i}^{\prime} /\left[(\mathrm{I})+K_{i}^{\prime}\right]\right\}}{(\mathrm{I})} \\
i=\frac{\left.K^{\prime}\right)+K_{i}^{\prime}\left[1+K_{s} /(\mathrm{S})\right]}{(1)}
\end{gathered}
$$

The rate expression shows that the inhibitor reduces both $V_{m}$ and $K_{s}$. Such inhibition is recognized by the family of parallel lines obtained by plotting $1 / v_{i}$ against $1 /(\mathrm{S})$ or $1 / v_{i}$ against (I) (see Chapter 5 ), but as Dixon and Webb (1958, p. 179) have pointed out, this method does not distinguish between this type of inhibition and the situation in which $K_{m}=k_{2} / k_{1}$ and the inhibitor in some manner decreases $k_{2}$.

Examples of compling inhibition are uncommon. The inhibition of cytochrome oxidase by azide (Winzler, 1943) and of pepsin by hydrazine (Schales et al., 1948) may possibly be of this type. The inhibitions of the arylsulfatase of Alcaligenes by cyanide and hydrazine (Dodgson et al., 1956) would appear to be the best documented examples of coupling inhibition. Not only were the $1 / v_{\imath}-1(\mathrm{~S})$ and $i / v_{i}-(\mathrm{I})$ plots clearly a family of parallel lines, but the $\mathrm{pH}$-dependence of the inhibitions showed a dissociating group present in the ES complex but absent in the free enzyme. It is likely that a similar mechanism must be assumed in the complexes formed between a variety of inhibitors (bisulfite, sulficle, cyanide, hydroxylamine, and various mercaptans) and the E-DPN* complexes of various dehydrogenases (Eys et al., 1958).

\section{Classification of Inhibitions by Reiner}

Reiner $(1959$, p. 148) has suggested a new terminology for the classification of enzyme inhibitions in order " to avoid the unnecessary connotations of the usual terminology." The correspondence between the two systems can be easily made:

$$
\begin{array}{ll}
\text { inclusive inhibition } & =\text { noncompetitive inhibition } \\
\text { exclusive } \mathrm{E} \text { inhibition } & =\text { competitive inhibition } \\
\text { exclusive } \mathrm{C} \text { inhibition } & =\text { coupling (uncompctitive) inhibition }
\end{array}
$$

These inhibitions were further subdivided into complete and partial and the use of these terms corresponds exactly to the similar terms in the treatment above. Reiner points out that the designation "competitive" suggests that the substrate and the inhibitor complex with the same active site on the enzyme and compete for this site, whereas there are numerons other mechanisms whereby substrate and inhibitor could interfere with each other's binding to the enzyme. Unfortunately, there are probably no terminologies that will fail to evoke some unnecessary connotations or erroneons interpre- 
tations. The usmal teminology will be followed in this book becanse it is so deeply implanted in the literature on enzyme inhibition and it is hoped that the terms will be understood in the most general way, so that they will not imply particular mechanisms on the molecular level.

\section{Inhibition of Enzyme Systems for Which Steady-State Kinetics Are Necessary}

The equations presented above (3-12 to 3-15) for competitive inhibition are valid for steady-state systems and $K_{s}$ may be replaced by $K_{m}$. However, for the non-competitive inhibition of steady-state systems, where $K_{m}=$ $\left(k_{-1}+k_{2}\right) k_{1}$ or depends on more complex combinations of the rate constants, the presence of the inhibitor will alter $K_{m}$ as well as $\mathrm{r}_{m}$. since the rate of breakdown of the critical complex will be a factor in both $K_{m}$ and $I_{m}$. and this rate will be modified by the inhibitor as pointed out by Dixon and Webb (1958, p. 179). Pure noncompetitive inhibition would thus be impossible in steady-state systems where $K_{m}$ does not represent the substrate constant. Noncompetitive inhibitors acting on such systems will therefore be classed as mixed from the forms of the reciprocal plots. This was stated in a somewhat different namer by Morales (1955). when he concluded that if inhibition kinetics are noncompetitive, the Michachs constant is an equilibriun constant.

\section{Dependence of Inhibition on Substrate and Inhibitor Concentrations}

The variations in rate and inhibition with substrate and inhibitor concentration are plotted in Figs. 3-2 to 3-5 for the common tyres of inhibition.

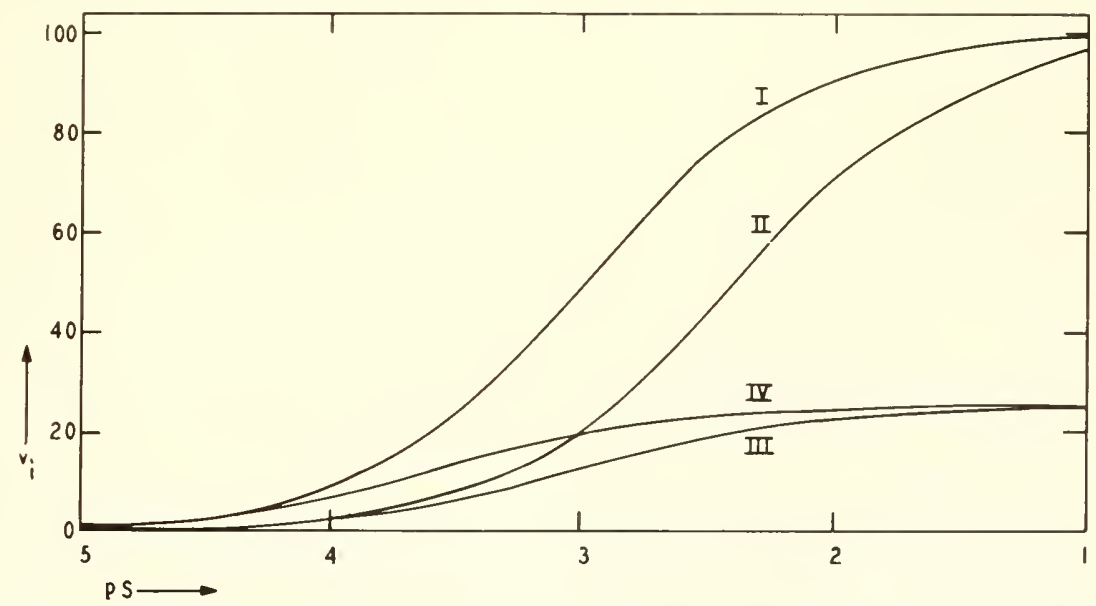

FIG. 3-2. Tariation of the rate with substrate concentration for different types of

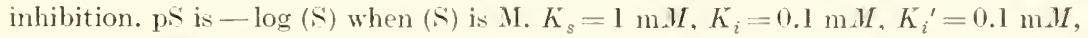
$(\mathrm{I})=0.3 \mathrm{~m} \mathrm{H}$, and $V_{m}=100$. Curve $\mathrm{I}$ : minhibited; curve II: competitive inhibition; curve III: noncompetitive inhibition; curve IV: coupling inhibition. 
and the dependence of inhibition on the constants $\alpha$ and $\beta$ is illustrated in Figs. 3-6 to 3-8. It is readily seen that the kinetic behavior is different in each type of inhibition. In Fig. 3-6 it may be observed that as $a$ becomes

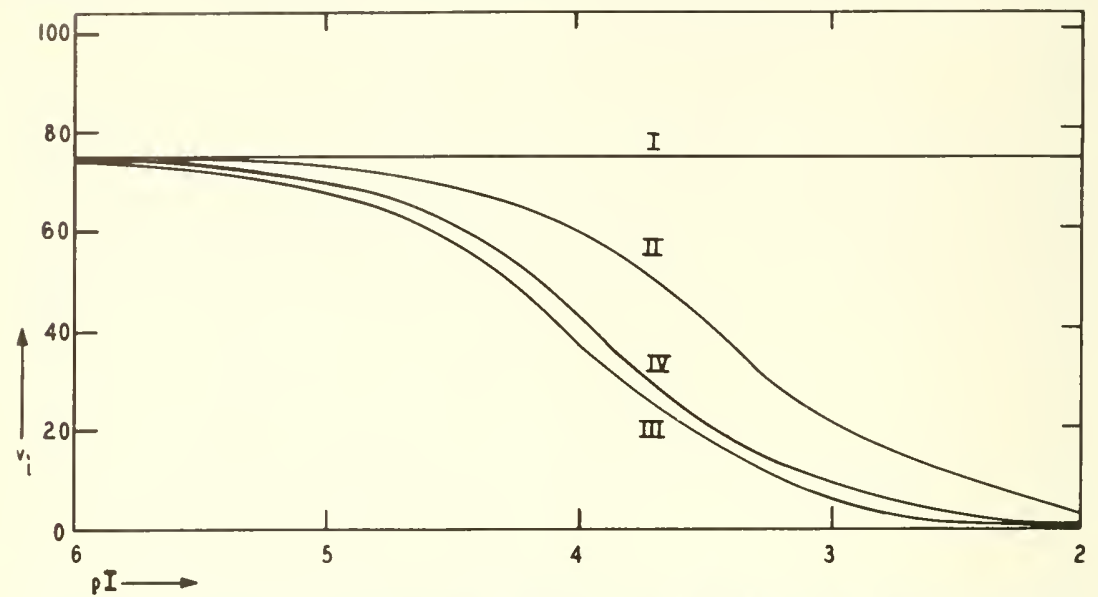

FIG. 3-3. Variation of the rate with inhibitor concentration for different types of inhibition. pI is - $\log$ (I) when (I) is $\mathrm{H} . K_{s}=1 \mathrm{~m} M, K_{i}=0.1 \mathrm{~m} M, K_{i}{ }^{\prime}=0.1 \mathrm{~m} \mathrm{H}$, $(\mathrm{S})=3 \mathrm{n} \mu$, and $V_{m}=100$. Curve $\mathrm{I}$ : uninhibited; curve II: competitive inhi-

bition; curve III: noncompetitive inhibition; curve IV: coupling inhibition.

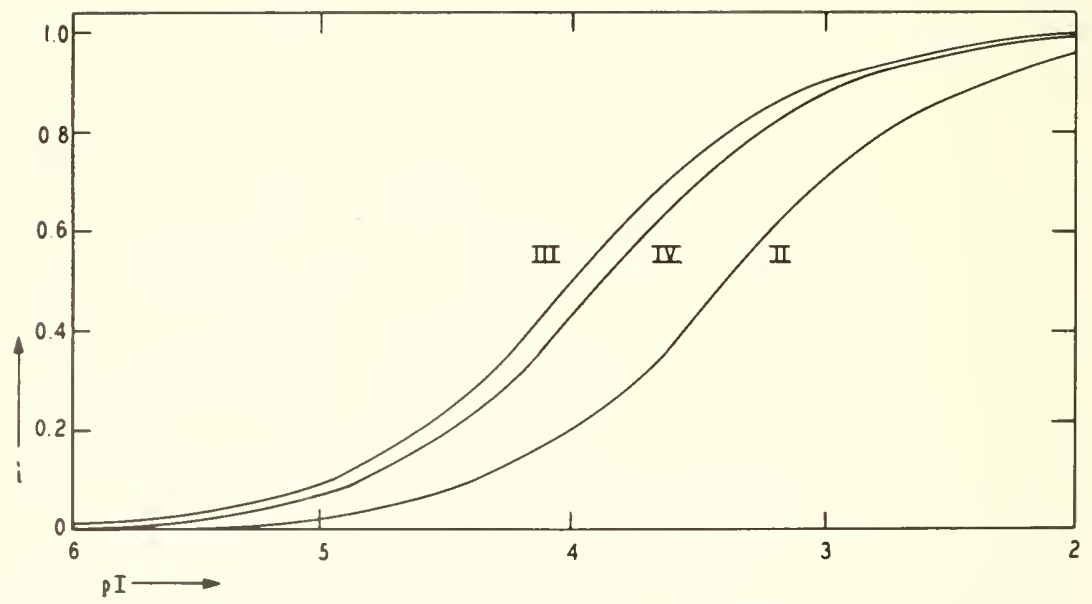

FIG. 3-4. Variation of the fractional inhibition with inhibitor concentration for different types of inhibition. Constants and curve designations as in Fig. 3-3.

larger, the inhibition approaches a constant level, whatever the value of $\beta$; when $\alpha$ is infinity the inhibition is completely competitive. It is interesting, however, that $\alpha$ must reach fairly high values, that is the substrate bind- 
ing must be reduced markedly, before the influence of changes in $\beta$ is negligible. In Figs. 3-7 and 3-8 it is evident that inhibition ceases and activation occurs where the lines cross the $\beta$-axis; for example. when $\alpha=30$ (i.e.,

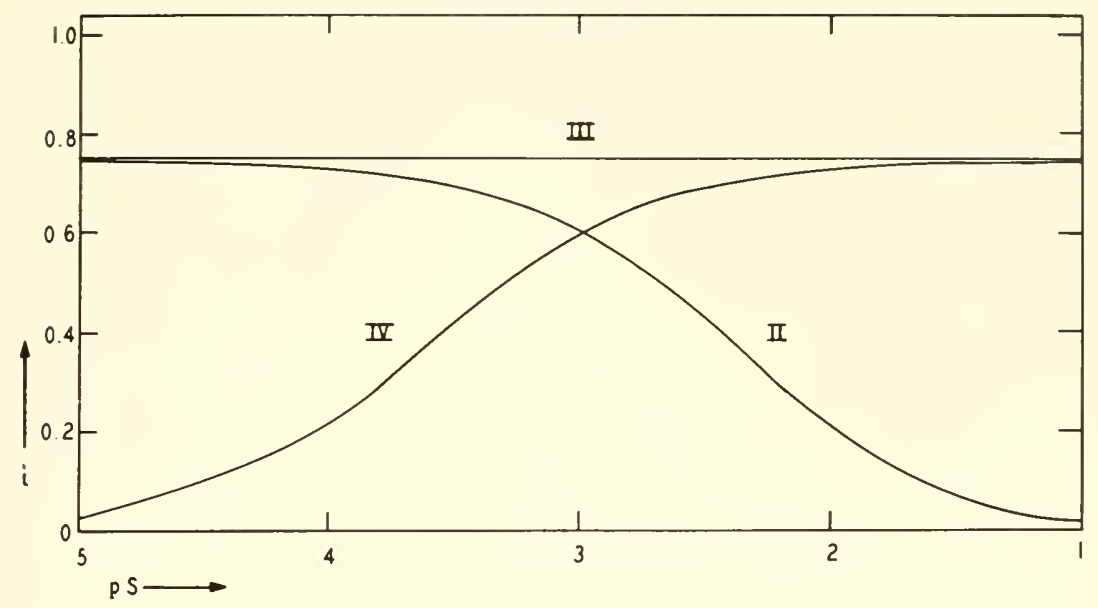

F1G. 3-5. Variation of the fractional inhibition with substrate concentration for different types of inhibition. Constants and curve designations as in Fig. 3-2.

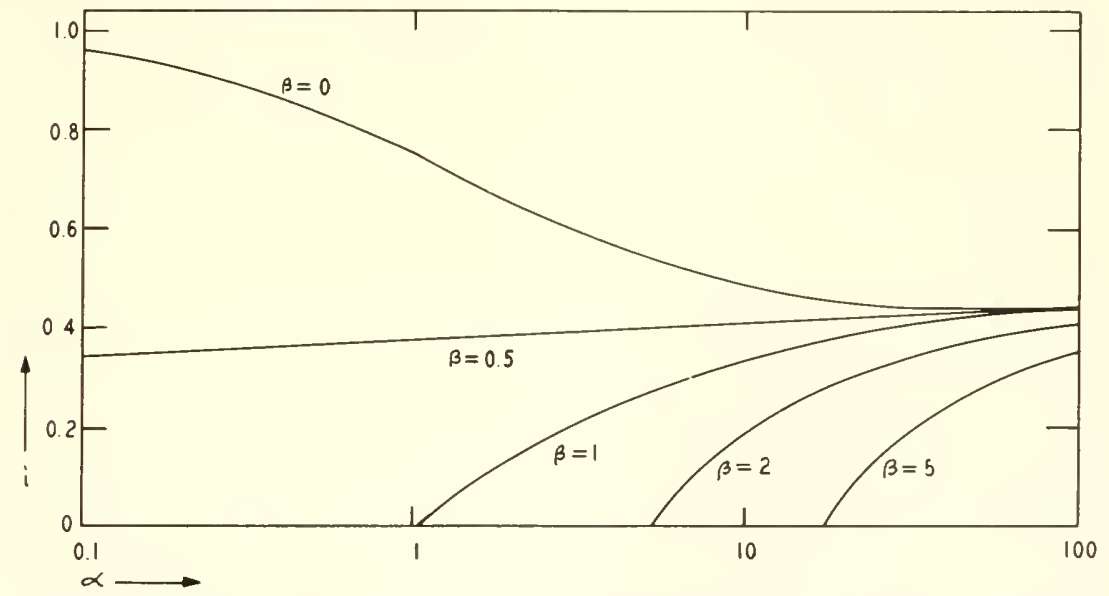

FIG. 3-6. Variation of the fractional inhibition with the interaction constant $\alpha$ for different values of $\beta . K_{s}=1 \mathrm{~m} . \mu, K_{i}=0.1 \mathrm{~m} \mu,(S)=3 \mathrm{~m} . \mu$, and $(\mathrm{I})=0.3 \mathrm{~m} . \mu$.

the $K_{s}$ is increased thirty-fold by the inhibitor). the rate of substrate complex breakdown must be accelerated over eight-fold for stimulation of the reaction to occur. These curves clearly show that the resulting inhibition 
depends always on the balance of the changes in both $K_{s}$ and $k$; it is probably too frequently assumed that $\beta$ is either unaffected or reduced to zero.

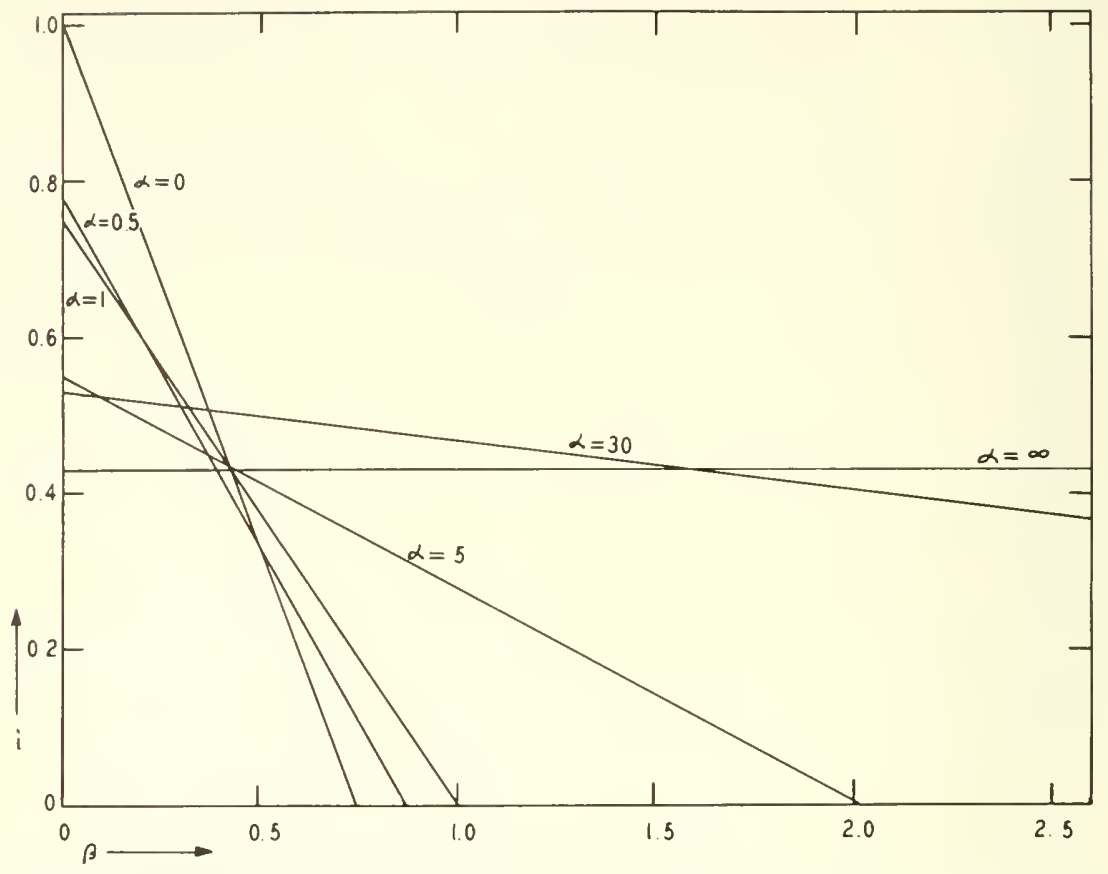

FIG. 3-7. Variation of the fractional inhibition with $\beta$ for different values of $\alpha$. Constants and concentrations as in Fig. 3-6.

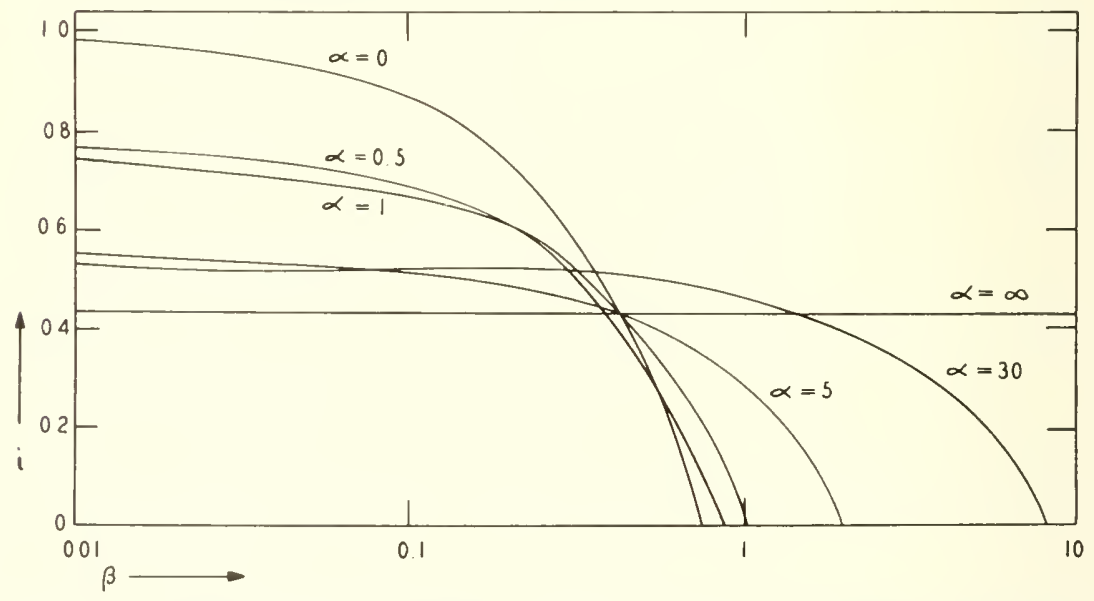

FIG. 3-8. Variation of the fractional inhibition with $\beta$ for different values of $\alpha$ when $\beta$ is on a logarithmic scale. Constants and concentrations as in Fig. 3-6. 


\section{Inhibition Equations in Terms of Specific Concentrations}

The rate of an enzyme reaction does not depend on the substrate concentration alone but on the concentration relative to the dissociation constant of the ES complex. This may be seen from rearrangement of the Michaelis equation:

$$
v=V_{m} \frac{(\mathrm{S})}{(\mathrm{S})+K_{s}}=V_{m} \frac{(\mathrm{S}) / K_{s}}{\left[(\mathrm{~S}) / K_{s}\right]+1}=V_{m} \frac{\left(\mathrm{S}^{\prime}\right)}{\left(\mathrm{S}^{\prime}\right)+1}
$$

where $\left(\mathrm{S}^{\prime}\right)=(\mathrm{S}) / K_{s}$. $\left(\mathrm{S}^{\prime}\right)$ was called the "specific concentration" of the substrate by Straus and Goldstein (1943) and is the quantity that determines the rate with respect to substrate. Likewise, the effect of an inhibitor depends on its concentration relative to $K_{i}$, i.e.. on (I) $/ K_{i}$ which can be designated $\left(I^{\prime}\right)$, the specific concentration of inhibitor. The same would also apply to coenzymes and activators. where the specific concentrations $\left(\mathrm{C}^{\prime}\right)$ and $\left(A^{\prime}\right)$ would determine their activity in the enzyme reaction. The inhibition equations may be rewritten in terms of specific concentrations:

$$
\begin{array}{lll}
\text { Generalized inhibition: } & i=\frac{\left(\mathrm{I}^{\prime}\right)\left[\left(\mathrm{S}^{\prime}\right)(1-\beta)+(\alpha-\beta)\right]}{\left(\mathrm{I}^{\prime}\right)\left[\left(\mathrm{S}^{\prime}\right)+\alpha\right]+\alpha\left[\left(\mathrm{S}^{\prime}\right)+1\right]} \\
\text { Competitive inhibition: } & i=\frac{\left(\mathrm{I}^{\prime}\right)}{\left(\mathrm{I}^{\prime}\right)+1+\left(\mathrm{S}^{\prime}\right)} \\
\text { Noncompetitive inhibition: } & i=\frac{\left(\mathrm{I}^{\prime}\right)}{\left(\mathrm{I}^{\prime}\right)+1} \\
\text { Coupling inhibition: } & i=\frac{\left(\mathrm{I}^{\prime}\right)}{\left(\mathrm{I}^{\prime}\right)+1+\left[1 /\left(\mathrm{S}^{\prime}\right)\right]}
\end{array}
$$

These equations demonstrate that the degree of inhibition depends only on the specific concentrations for any type. The fundamental reason for the importance of specific concentrations is that the activity of a component in an enzyme reaction depends on the concentration of its complex with the enzyme. and this depends on the component concentration relative to its affinity for the enzyme since $(\mathrm{EX}) /\left(\mathrm{E}_{t}\right)=\left(\mathrm{X}^{\prime}\right) /\left[\left(\mathrm{X}^{\prime}\right)+1\right]$.

The quantities here designated as specific concentrations are called "relative concentrations" by Dixon and Webb $(1958$, p. 76$)$. We retain the older term: it is felt that the use of the word "relative " may cause some confusion since it would usually be construed as indicating the concentration relative to another concentration or substance. 


\section{INHIBITION KINETICS IN MUTUAL DEPLETION SYSTEMS}

The Michaelis-Menten treatment of enzyme kinetics and the formulation of inhibition given above involve the assumption that the concentration of enzyme is so small that combination of either substrate or inhibitor with the enzyme does not appreciably reduce the concentrations of free substrate and inhibitor. However, under certain experimental conditions and frequently within the cell, the enzyme concentration may be sufficiently high relative to substrate or inhibitor so that the equations presented are not valid. In other situations the affinity of the enzyme for an inhibitor is so high that the concentration of free inhibitor is reshced due to formation of the EI complex, even though the enzyme concentration is low, for to obtain inhibitions in the usual range correspondingly low inhibitor concentrations must be used. The arklitional factor that must now be inchuded in the kinetics is the reduction in substrate or inhibitor concentrations in systems where the conditions lead to mutual depletion. These conditions are determined principally by the value of the specific concentration of enzyme $\left(\mathrm{E}_{t}{ }^{\prime}\right)$, since either a high enzyme concentration or a low dissociation constant favors mutual depletion in free concentrations.

Inasmuch as no general name has been given to enzyme systems of this type, it is suggested that they be called "mutual depletion systems," because there is of necessity a mutual reduction in concentrations of free enzyme and whatever components are involved. In situations where the concentration of free inhibitor is decreased, it is evident that there must also be a decrease in concentration of free enzyme if significant inhibition occurs. It should also be clear that this extension of the simple theory may apply to coenzymes and activators. In any system, composed of a number of substances involved in the reaction, depletion may occur in one, any number, or all of the components, depending on their relative dissociation constants with respect to the complexes they form with the enzyme. The specific concentration of the enzyme will usually be different with regard to each of the components. The specific concentration of enzrme with respect to substrate is $\left(\mathrm{E}_{t}\right) / K_{s}$, with respect to inhibitor is $\left(\mathrm{E}_{t}\right) / K_{i}$, and with respect to any component $\mathrm{X}$ is $\left(\mathrm{E}_{t}\right) / K_{x}$. We shall designate these different specific concentrations as $\left(\mathrm{E}_{s}{ }^{\prime}\right)$. $\left(\mathrm{E}_{i}{ }^{\prime}\right)$, and $\left(\mathrm{E}_{x}{ }^{\prime}\right)$. Since specific concentrations are more significant than ordinary concentrations in mutual depletion systems, it will be convenient to formulate the inhibition equations accordingly.

\section{Noncompetitive Inhibition in Mutual Depletion Systems}

No general formulation including the different kinds of inhibition has been made for systems of this type and, indeed, it woukl be mathematically complex. The basic concepts involved are most clearly illustrated in noncompetitive inhibition where only the inhibitor concentration is of impor- 
tance. Easson and Stedman (1936) were the first to consider this problem but did not develop the kinetics extensively because they were primarily concerned with the determination of enzyme concentration, a useful application of the basic kinetics that will be discussed later. The detailed presentation of the kinetics, with applications to cholinesterase, is due to Straus and Goldstein (1943) and Goldstein (1944).

Previously it has been assumed that $(\mathrm{I})=\left(\mathrm{I}_{t}\right)$ but now one must write:

$$
\left(\mathrm{I}_{t}\right)=(\mathrm{I})+(\mathrm{EI})
$$

where (EI) includes (EIS) because presence of substrate does not alter the binding of inhibitor to enzyme. The value of (I) may be obtained from the equilibrium expression $(\mathrm{I})(\mathrm{E}) /(\mathrm{EI})=K_{i}$, the fractional inhibition relationship $i=(\mathrm{EI}) /\left(\mathrm{E}_{t}\right)$, and the equation $\left(\mathrm{E}_{t}\right)=(\mathrm{E})+(\mathrm{EI})$ :

$$
\left(\mathrm{I}_{t}\right)=K_{i}\left[\frac{i}{1-i}\right]+i\left(\mathrm{E}_{t}\right)
$$

and this may be rewritten with specific concentrations as:

$$
\left(\mathrm{I}^{\prime}{ }^{\prime}\right)=\frac{i}{1-i}+i\left(\mathrm{E}^{\prime}{ }^{\prime}\right)
$$

Total inhibitor $=$ free inhibitor + combined inibitor

Three possible situations may be distinguished: the inhibitor is mainly free, or it is mainly combined, or it may occur significantly in both free and combined forms. Strans and Goldstein designated these three sitnations in terms of zones in the following manner:

Zone A: inhibitor mainly free. $\left(\mathrm{I}_{t}^{\prime}\right)=i /(1-i)$.

$$
i=\left(\mathrm{I}_{t}{ }^{\prime}\right) /\left[\left(\mathrm{I}_{t}{ }^{\prime}\right)+1\right]
$$

Zone B: inhibitor free and combined, $\left(\mathrm{I}_{t}{ }^{\prime}\right)=i /(1-i)+i\left(\mathrm{E}_{i}{ }^{\prime}\right)$.

$$
i=\frac{\left[\left(\mathrm{I}_{t}{ }^{\prime}\right)+\left(\mathrm{E}_{i}{ }^{\prime}\right)+1\right]-\sqrt{ }\left[\left(\mathrm{I}_{t}{ }^{\prime}\right)+\left(\mathrm{E}_{i}{ }^{\prime}\right)+1\right]^{2}-4\left(\mathrm{I}_{t}{ }^{\prime}\right)\left(\mathrm{E}_{i}{ }^{\prime}\right)}{2\left(\mathrm{E}_{i}{ }^{\prime}\right)}
$$

Zone C: inhibitor mainly combined, $\left(\mathrm{I}_{t}{ }^{\prime}\right)=i\left(\mathrm{E}_{i}{ }^{\prime}\right)$.

$$
i=\left(\mathrm{I}_{t}{ }^{\prime}\right) /\left(\mathrm{E}_{i}{ }^{\prime}\right)
$$

They were called zones becanse as $\left(\mathbf{E}_{i}^{\prime}\right)$ is increased, the system will pass from zone $\mathrm{A}$ through zone $\mathrm{B}$ into zone $\mathrm{C}$.

In Eq. 3-33 the designations of the form of the inhibitor do not indicate ordinary concentrations but specific concentrations; the actual concentra- 
tions are given in Eq. 3-32. We have retained the use of fractional inhibition $i$, rather than fractional activity $a$ as in the equations of Straus and Goldstein, to be in accord with our usual symbolism and because we shall be particularly concerned with the degree of inhibition.

When $n$ molecules of inhibitor react for each active center of the enzyme, Eq. 3-33 is modified to:

$$
\left(\mathrm{I}_{t}{ }^{\prime}\right)=\left[\frac{i}{1-i}\right]^{1 / n}+n i\left(\mathrm{E}_{i}{ }^{\prime}\right)
$$

where $\left(\mathrm{I}_{t}{ }^{\prime}\right)=\left(\mathrm{I}_{t}\right) /\left(\mathrm{K}_{i}\right)^{1 / n}$. The zones are defined similarly but the boundaries between the zones depend on the value of $n$ (Straus and Goldstein, 1943; Goldstein, 1944).

The variation of inhibition with inhibitor concentration depends upon the zone in which the system lies, as shown in Fig. 3-9, where the deviation

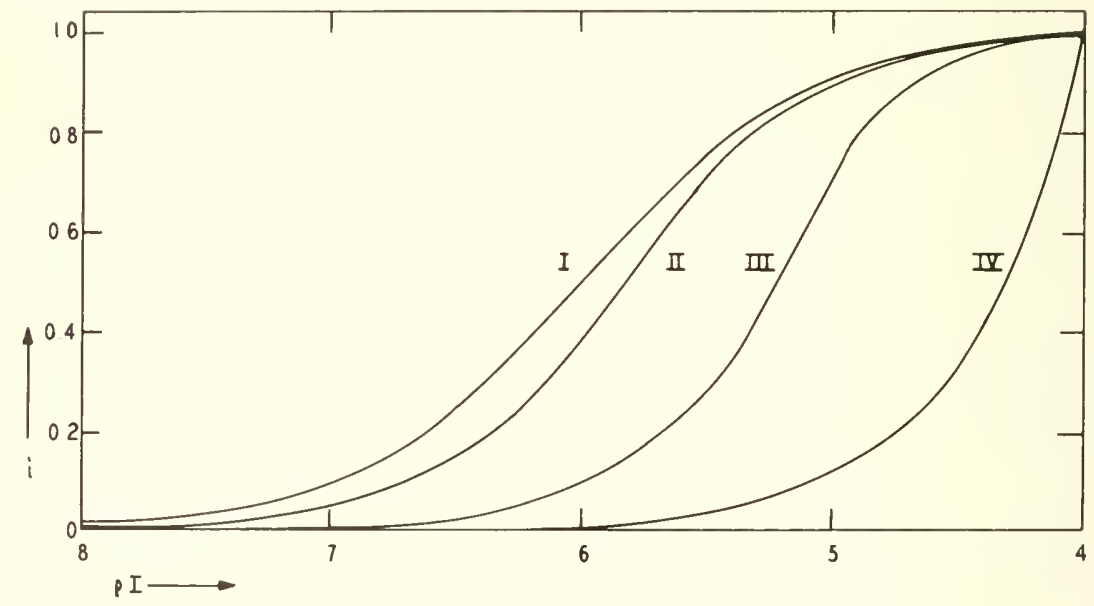

FIG. 3-9. Variation of the fractional inhibition with the inhibitor concentration at various concentrations of the enzyme in a mutual depletion system in different zones. $K_{i}=10^{-3} \mathrm{~m} H$. Curve I: $\left(\mathrm{E}_{t}{ }^{\prime}\right)=0.01$, zone A; curve II: $\left(\mathrm{E}_{t}{ }^{\prime}\right)=1$, zone $\mathrm{B}$; curve III: $\left(\mathrm{E}_{t}{ }^{\prime}\right)=10$, zone $\mathrm{B}$; eurve $\mathrm{IV}:\left(\mathrm{E}_{t}{ }^{\prime}\right)=100$, zone $\mathrm{C}$.

from zone A belavior as enzyme concentration rises manifests itself as a shift of the curves to the right and a change in shape. The decrease of inhibition at higher enzyme concentrations is due, of course, to the depletion of free inhibitor. In Fig. 3-10 the dependence of the inhibition on enzyme concentration is illustrated. It is clear that the lower $\left(\mathrm{I}_{t}\right)$, the more readily will increasing $\left(\mathrm{E}_{t}\right)$ reduce the inhibition; thus a system in which the inhibition is higl will tend to remain in zone $\mathrm{A}$ longer as $\left(\mathrm{E}_{t}\right)$ increases, compared to one in which the inhibition is low. 


\section{Boundary Conditions for Zone Behavior}

It is necessary now to determine for practical purposes the conditions under which zone $\mathrm{B}$ and $\mathrm{C}$ kinetics should be used, rather than the usual zone A equations. One may do this by establishing the boundaries between the three zones. These boundaries are, to some extent, arbitrary since they depend upon the accuracy desired in the treatment; i.e., zone A kinetics can be used over a wider range if the experiments do not require a rigorously quantitative expression. Straus and Goldstein (1943) determined the boundary conditions assuming certain vahes for the acceptable error in the degree of inhibition obtained from the various equations. They considered ralues of $\mathrm{Ai}$, the acceptable deviation in $i$; it might be more generally preferable to consider values of $1 i / i$, which would be a measure of the per cent error at any level of inhibition. The following treatment and the boundary curves obtained thus differ in this respect from those of Straus and Goldstein.

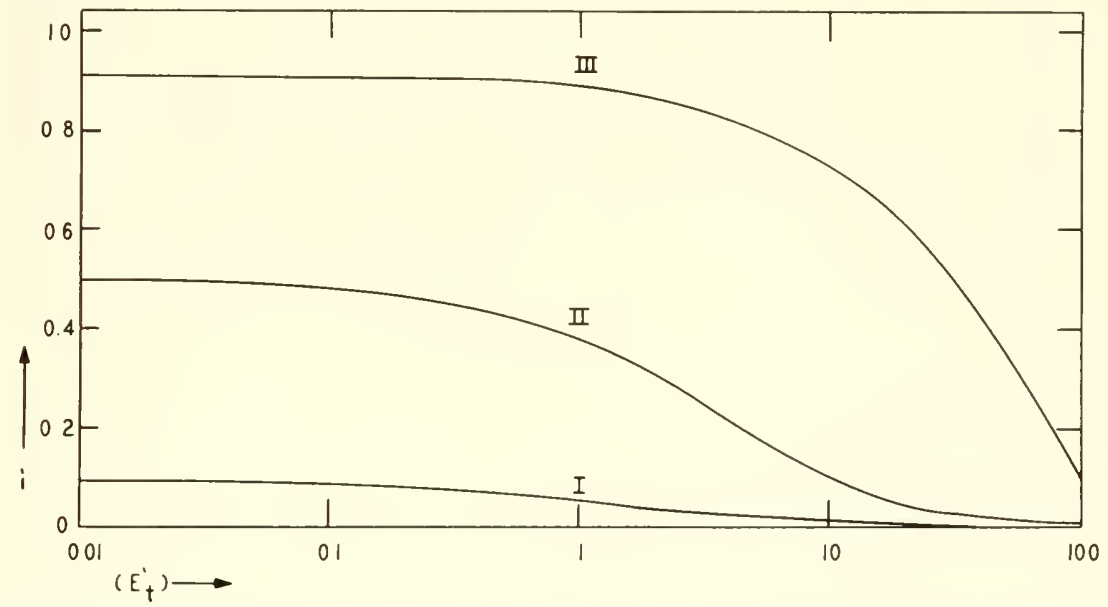

FIG. 3-10. Variation of the fractional inhibition with the specific enzyme concentration for different inhibitor concentrations. $K_{i}=10^{-3} \mathrm{~m} V$. C'urve $1: \mathrm{pI}=7$; curve II: $\mathrm{pI}=6$ : curve IIT: $\mathrm{pI}=5$.

The derivation of boundary equations may be done as follows. The general Eq. 3-33 is accurate and actually applies to all zones. Thus if one introduces the acceptable deviation $\mathrm{J} i$ into the special equations for zone $\mathrm{A}$ and zone $\mathrm{C}$ and equates these, since $\left(\mathrm{I}_{l}^{\prime}\right)$ is constant, with the general equation, one obtains:

Zone A - zone B: $\quad \frac{i}{1-i}+i\left(E_{i}{ }^{\prime}\right)=\frac{i+\Delta i}{1-i-4 i}$

Zone $\mathrm{B}$ - zone $\mathrm{C}: \quad \frac{i}{1-i}+i\left(\mathrm{E}_{i}{ }^{\prime}\right)=(i+\Delta i)\left(\mathrm{E}_{i}{ }^{\prime}\right)$ 
From these equations one may obtain expressions for $\left(\mathrm{E}_{i}{ }^{\prime}\right)$ which determine the boundaries:

$$
\begin{array}{ll}
\text { Zone A - zone B: } & \left(\mathrm{E}^{\prime}{ }^{\prime}\right)=\frac{1 i}{i} \frac{1}{(1-i-1 i)(1-i)} \\
\text { Zone B - zone C: } & \left(\mathrm{E}_{i}{ }^{\prime}\right)=\frac{i}{4 i} \frac{1}{1-i} i
\end{array}
$$

If we take the permissable error in the inhibition to be $10 \%$, i.e. $\Delta i / i=0.1$, the boundary curves in Fig. 3-11 are obtained. The boundaries are seen to depend not only on $\left(\mathrm{E}_{i}{ }^{\prime}\right)$ but on the extent of inhibition or $\left(\mathrm{I}_{t}{ }^{\prime}\right)$. The vertical lines are placed at $50 \%$ inhibition to indicate average values of $\left(\mathbf{E}_{i}{ }^{\prime}\right)$ that may be taken as the boundaries between zones in most experimental pro-

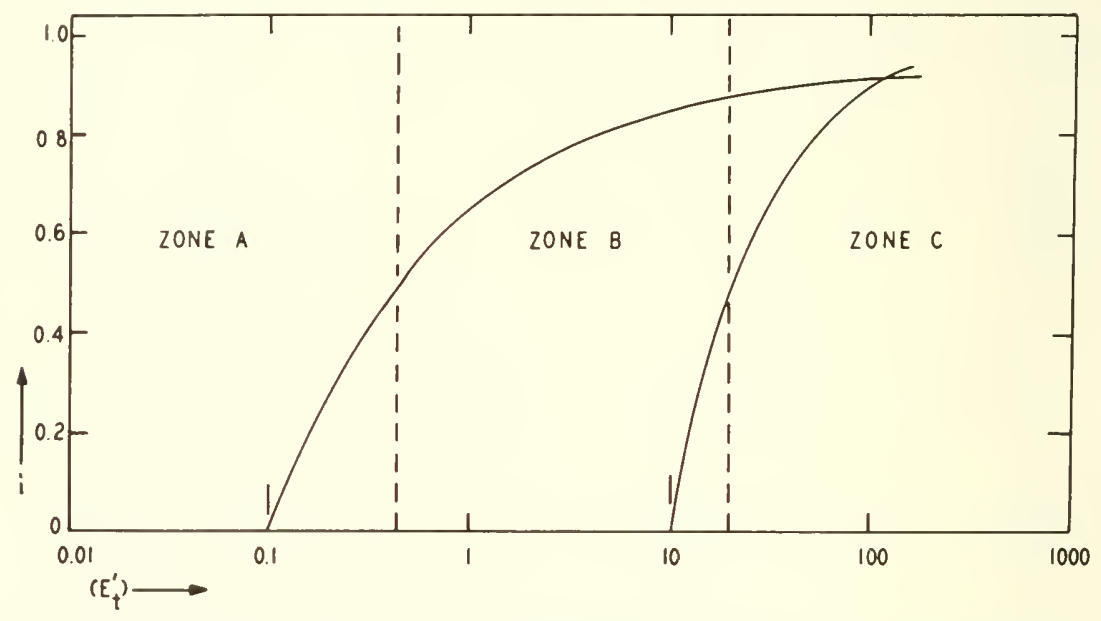

FIG. 3-11. Zone boundaries for noncompetitive inhibition in a mutual depletion system. $1 i / i=0.1$.

cedures. Thus when $\left(\mathrm{E}^{\prime},\right)$ reaches a value of 0.45 the system enters zone $\mathrm{B}$; at this point, if Michaelis-Menten equations are applied, the per cent error in the inhibition calculated will be $10 \%$. When $\left(\mathrm{E}_{\imath}^{\prime}\right)$ reaches 20 the system

\begin{tabular}{|c|c|c|}
\hline \multirow{2}{*}{ Acceptable error } & \multicolumn{2}{|c|}{$\left(\mathrm{E}_{i}^{\prime}\right)$} \\
\hline & Zone A - zone B & Zone B - zone C: \\
\hline $1 \%$ & 0.041 & 200 \\
\hline $5 \%$ & $0.2 \mathrm{I}$ & 40 \\
\hline $10 \%$ & 0.45 & 20 \\
\hline $20 \%$ & 1.0 & 10 \\
\hline $50 \%$ & 4.0 & 4 \\
\hline
\end{tabular}
will enter zone $\mathrm{C}$. The tabulation below gives the boundary values for 
$50 \%$ inhibition for various acceptable errors in the calculated inhibition. If one is quite critical regarding the acceptable error, zone B kinetics must be used over a much wider range than when only low accuracy is required.

Let us consider two examples applying these results. If an inhibitor with $K_{i}=10^{-7} M$ is being used (many inhibitors are known with $K_{i}$ 's as small as this or smaller) and the acceptable error is set at $5 \%$, zone $\mathrm{A}$ equations are no longer permissible (assuming inhibitions around $50 \%$ ) when $\left(\mathrm{E}_{i}{ }^{\prime}\right.$ ) is greater than 0.21 or $\left(\mathrm{E}_{\ell}\right)$ is greater than $2.1 \times 10^{-8} \boldsymbol{M}$. If the molecular weight of the enzyme is 50,000 per active center, this concentration of enzyme corresponds to $0.0001 \%$. Enzyme concentrations of this magnitude may be relatively common. In fact, in an homogenate, which is usually around $0.3 \%$ tissue, this enzyme concentration will occur if the concentration in the original tissue is $0.03 \%$. If one lias a reasonable estimate of the enzyme concentration, it is possible to decide which inhibitors will yield zone $B$ or $\mathrm{C}$ behavior on the basis of their $K_{i}$ 's. Thus the concentration of cholinesterase in human serum has been found to be $2.3 \times 10^{-9} M$ (Myers, 1952 a) and inhibitors with $K_{i}$ 's less than $1.1 \times 10^{-8} M$ would require zone $\mathrm{B}$ or if less than $5.7 \times 10^{-10} M$ would require zone $\mathrm{C}$ kinetics. Several inhibitors indeed possess $K_{i}$ 's in this range with respect to cholinesterase. The difficulty at the present time in applying such reasoning is the lack of knowledge of enzyme concentrations in either tissues or isolated preparations. A method for determining enzyme concentration in certain cases will be presented in the following section.

Finally, one may inquire as to what boundaries can be set for an investigation of enzyme inhibition where the range between $10 \%$ and $90 \%$ inhibition will be encountered. Approaching zone B from zone A, from Fig. 3-11 it may be seen that zone $\mathrm{B}$ kinetics should be used when $\left(\mathrm{E}_{i}^{\prime}\right)$ becomes greater than 0.12 ; approaching zone $\mathrm{B}$ from zone $\mathrm{C}$, the system enters zone $\mathrm{B}$ when $\left(\mathrm{E}_{i}{ }^{\prime}\right)$ becomes less than 100 . Thus when one includes a wide range of possible degrees of inhibition, the width of zone $B$ increases if the specified accuracy throughout is desired.

\section{Determination of Enzyme Concentration with Potent Noncompetitive Inhibitors}

It was pointed out by Easson and Stedman (1936) that if Eq. 3-32 is rewritten as:

$$
\frac{\left(\mathrm{I}_{t}\right)}{i}=\frac{K_{i}}{1-i}+\left(\mathrm{E}_{t}\right)
$$

a plot of $\left(I_{t}\right)_{i} i$ against $1 /(1-i)$ would show a slope of $K_{i}$ and an intercept with the $\left(\mathrm{I}_{t}\right) / i$ axis at $\left(\mathrm{E}_{t}\right)$. Thus $\left(\mathrm{E}_{t}\right)$ and $\left(\mathrm{E}_{i}{ }^{\prime}\right)$ can be determined and this was done for serum cholinesterase using miotine as the inhibitor. Another method, probably more accurate, was used by Myers (1952a) for serum 
cholinesterase with the very potent inhibitor Nu 683. Equation 3-32 may be written as:

$$
\left(\mathrm{I}_{t}\right)_{50}=K_{i}+0.5\left(\mathrm{E}_{t}\right)
$$

where $\left(I_{t}\right)_{50}$ is the inhibitor concentration producing $50 \%$ inhibition. If various dilutions of the enzyme are tested and the activities expressed in $\mu$ moles of substrate per minute, the activities will be equivalent to the turnover number $(\mathrm{TN}) \times\left(\mathrm{E}_{t}\right)$, so that:

$$
\left(\mathrm{I}_{t}\right)_{50}=K_{i}+\frac{0.5}{\mathrm{TN}} \mathrm{TN}\left(\mathrm{E}_{t}\right)
$$

A plot of $\left(I_{t}\right)_{50}$ against the relative activity of the enzyme will give a straight line with a slope of $0.5 / \mathrm{TN}$ and $\left(\mathrm{I}_{t}\right)_{50}$ intercept of $K_{i}$, from which $\left(\mathrm{E}_{t}\right)$ and $\left(\mathrm{E}_{t}^{\prime}\right)$ may be evaluated. Such methods may be applied to any enzyme providing an inhibitor potent enough to place the system in zone $\mathrm{B}$ is available. Competitive inhibitors may be used if their rate of displacement from the enzyme is slow and readings are made soon after addition of substrate to the inhibited system. Theoretical plots for the two methods are presented in Figs. 3-12 and 3-13.

\section{Competitive Inhibition in Mutual Depletion Systems}

When the substrate and inhibitor compete for binding to the active site, the general equation, corresponding to $3-33$, will be more complex:

$$
\left(\mathrm{I}^{\prime}{ }^{\prime}\right)=\left[\frac{i\left(\mathrm{~S}^{\prime}\right)+1}{(1-i)\left(\mathrm{S}^{\prime}\right)+1}\left\{\left(\mathrm{~S}^{\prime}\right)-\left(\mathrm{E}_{s}{ }^{\prime}\right)+1\right\}-1\right]
$$

Total inhibitor $=\quad$ free inhibitor

$$
\begin{gathered}
+\left[1-(1-i)\left\{\frac{\left(\mathrm{S}^{\prime}\right)}{\left(\mathrm{S}^{\prime}\right)+1}+\frac{1}{\left(\mathrm{~S}^{\prime}\right)-(1-i)\left(\mathrm{E}_{s^{\prime}}\right)+1}\right\}\right]\left(\mathrm{E}_{i^{\prime}}\right) \\
+\quad \text { combined inhibitor }
\end{gathered}
$$

where $\left(\mathrm{E}_{s}{ }^{\prime}\right)=\left(E_{\ell}\right) / K_{s}$ and $\left(\mathrm{E}_{i}{ }^{\prime}\right)=\left(\mathrm{E}_{t}\right) / K_{i}$, the specific concentrations of the enzyme with respect to substrate and inhibitor. This expression applies to the situation where the system is in zone B with respect to both substrate and inhibitor. Eqnation 3-45 is related to the equation derived by Goldstein (1944) but here is expressed in terms of fractional inhibition rather than fractional activity.

The general equation given by Goldstein is:

$$
\left(\mathrm{I}_{t}{ }^{\prime}\right)=\left[\left\{\left(\mathrm{S}^{\prime}\right)-a\left(\mathrm{E}_{s}{ }^{\prime}\right)\right\} \frac{1-a}{a}-1\right]+\left[1-a\left\{1+\frac{1}{\left(\mathrm{~S}^{\prime}\right)-a\left(\mathrm{E}_{s}{ }^{\prime}\right)}\right\}\right]\left(\mathrm{E}_{i^{\prime}}\right)
$$


where $a=$ fractional activity as defined by Goldstein. It is particularly important to note that this fractional activity is not equal to $1-i$ as it is usually defined. As used by Goldstein, $a=(\mathrm{ES})\left(\mathrm{E}_{t}\right)=v_{i} / V_{m}$ and hence re-

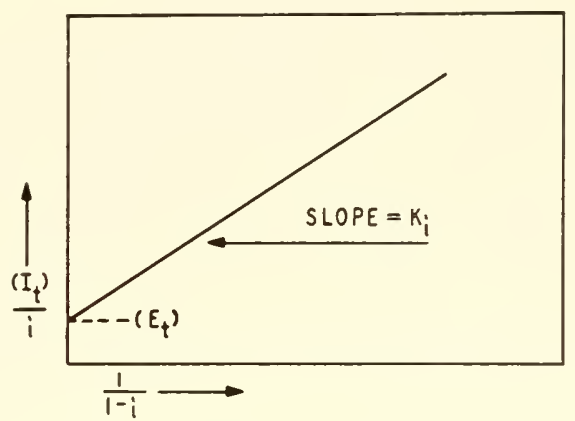

FIG. 3-12. Illustration of the method of Easson and Stedman (1936) for the determination of enzyme concentration from inhibition data. The plot is of Eq. 3-42.

presents the activity relative to the maximal rate, not relative to the actual rate in the absence of inhibitor, in which case the fractional activity would be equal to $v_{i} / v$. In other words, the fractional activity of Goldstein does

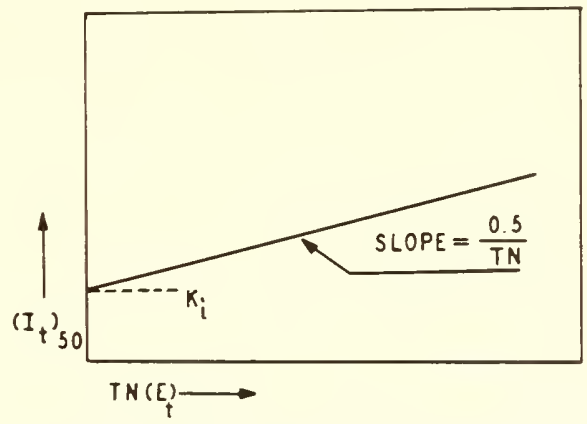

FIG. 3-13. Illustration of the method of Myers (1952 a) for the determination of enzyme concentration from inhibition data.

The plot is of Eq. 3-44.

not express the reduction of rate due to inhibitor alone. It is easy to show that:

$$
a=\frac{(1-i)\left(\mathrm{S}^{\prime}\right)}{\left(\mathrm{S}^{\prime}\right)+1}
$$


when used in the sense of Goldstein. He used this fractional activity because it is this value that is actually measured in enzyme experiments but, as stated above, here we are more concerned with the reduction in rate brought about by the inhibitor and hence shall express the equations in terms of $i$.

It will be convenient to designate the zone behavior with subscripts to indicate whether the substrate or inhibitor is referred to. Thus zone $\mathrm{A}_{s}$ refers to a system which is in zone $A$ with respect to substrate and zone $B_{i}$ to one that is in zone $\mathrm{B}$ with respect to inhibitor. A complete characterization of the system may be made by combining these designations: thus zone $\mathrm{A}_{s} \mathrm{~B}_{i}$ implies a system which is in zone $\mathrm{A}$ with respect to substrate and simultaneously in zone B with respect to inhibitor. There are therefore nine possible states in which a competitive inhibition system may exist.

Most enzyme systems will be in zone $\mathrm{A}_{\mathrm{s}}$ where $\left(\mathrm{E}_{s}{ }^{\prime}\right)$ is small (it must be less than 0.1) and this simplification leads to the following zone equations:

Zone $\mathrm{A}_{\mathrm{s}} \mathrm{A}_{i}$ : substrate and inhibitor mainly free

$$
\begin{aligned}
\left(\mathrm{I}_{t}^{\prime}\right) & =\frac{1}{1-i}\left[\left(\mathrm{~S}^{\prime}\right)+1\right] \\
i & =\frac{\left(\mathrm{I}_{t^{\prime}}\right)}{\left(\mathrm{I}_{t^{\prime}}\right)+\left(\mathrm{S}^{\prime}\right)+1}
\end{aligned}
$$

Zone $A_{s} B_{l}$ : substrate mainly free, inhibitor free and combined

$$
\begin{aligned}
& \left(\mathrm{I}^{\prime}\right)=\frac{i}{1-i}\left[\left(\mathrm{~S}^{\prime}\right)+1\right]+i\left(\mathrm{E}_{\imath}{ }^{\prime}\right)
\end{aligned}
$$

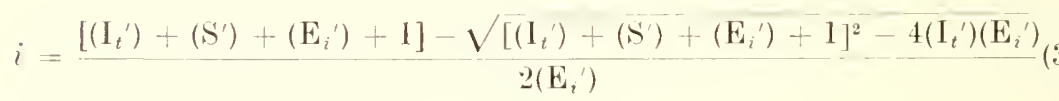

Zone $\mathrm{A}_{s} \mathrm{C}_{i}$ : substrate mainly free, inhibitor mainly combined

$$
\begin{gathered}
\left(\mathrm{I}_{t}{ }^{\prime}\right)=i\left(\mathrm{E}_{i}{ }^{\prime}\right) \\
i=\frac{\left(\mathrm{I}_{t}{ }^{\prime}\right)}{\left(\mathrm{E}_{i}{ }^{\prime}\right)}=\frac{\left(\mathrm{I}_{t}\right)}{\left(\mathrm{E}_{t}\right)}
\end{gathered}
$$

Equation 3-49 is the expected classic expression for competitive inhibition in systems uncomplicated by depletion of free inhibitor concentration; Eq. $3-51$ is identical with 3-35 with the addition of $\left(\mathrm{S}^{\prime}\right)$; and Eq. 3-53 is identical with 3-36 for noncompetitive inhibition. The last equivalence forces one to conclude, as did Goldstein, that competitive inhibition cannot occur in zone $\mathrm{C}_{i}$. When $\left(\mathrm{S}^{\prime}\right)$ becomes large enough so that competition can occur, it displaces the inhibitor from the enzyme, increasing the concentration of free inhibitor, and putting the system into zone $B_{i}$. 
The designation of zones and the choice of the appropriate equations to be used for a particular inhibition cannot generally be predicted but must be decided upon after sufficient data have been accumulated to characterize the behavior of the system.

\section{Boundary Conditions for Competitive Inhibition}

From Eq. 3-50 the boundary conditions corresponding to Eqs. 3-40 and 3-41 are obtained for competitive inhibition:

$$
\begin{aligned}
& \text { Zone } A_{i}-\text { zone } B_{i}: \frac{\left(\mathrm{E}_{i}{ }^{\prime}\right)}{\left(\mathrm{S}^{\prime}\right)+1}=\frac{1 i}{i} \frac{1}{(1-i-\Delta i)(1-i)} \\
& \text { Zone } \mathrm{B}_{i}-\text { zone } \mathrm{C}_{i}: \quad \frac{\left(\mathrm{E}_{i}{ }^{\prime}\right)}{\left(\mathrm{S}^{\prime}\right)+1}=\frac{i}{2 i} \frac{1}{1-i}
\end{aligned}
$$

so that the same boundaries exist as for noncompetitive inhibition (Fig. 3 -11) but they refer to $\left(\mathrm{E}_{i}\right) /\left[\left(\mathrm{S}^{\prime}\right)+1\right]$ instead of $\left(\mathrm{E}_{i}{ }^{\prime}\right)$. Since $\left(\mathrm{S}^{\prime}\right)+1$ will be greater than unity (sometimes much greater) and $\left(\mathrm{E}_{i}{ }^{\prime}\right.$ ) determines the zone. systems that would be in zone $B_{i}$ for noncompetitive inhibitors might well be translated into zone $\mathrm{A}$ for competitive inhibition. In the experiments of Goldstein on cholinesterase, for example, $\left(\mathrm{S}_{\ell}{ }^{\prime}\right)$ was usually 64.4 . It is reasonable that competitive systems, compared with noncompetitive, would less likely be in zone $B$. because binding of substrate to the enzyme reduces the amount of enzyme capable of binding the inhibitor and decreasing the concentration of its free form.

\section{Steady-State Kinetics of Mutual Depletion Systems}

Throughout the formulation it has been assumed that the Michaelis constant is a dissociation constant and the terms $\left(\mathrm{S}_{t}\right) / K_{s}$ and $\left(\mathrm{E}_{t}\right) / K_{s}$ have been used. However, if $K_{m}=\left(k_{-1}+k_{2}\right) / k_{1}$ it may be substituted for $K_{s}$ in the equations above, but the specific concentrations do not have their usual significance, since now, for example, $\left(\mathrm{S}_{l}^{\prime}\right)=\left(\mathbb{S}_{t}\right) / K_{m}$. Although this does not affect the validity of the theory or the equations, it is important in determinations of the individual rate constants from the kinetic data, just as in zone A systems. Indeed, the rate constants for cholinesterase calculated by Goldstein have been shown to be in error by Myers (1952 b) and this paper may be consulted for a detailed analysis of the problems involved in such determinations.

\section{Independence of Zone $C$ Inhibition on $\mathrm{K}_{i}$}

Straus and Goldstein pointed out the very interesting fact that in zone $\mathrm{C}$ all inhibitors. whatever their affinities for the enzyme, will produce the same effect. This follows from Eqs. 3-36 and 3-54 which may be written 
as $i=\left(\mathrm{I}_{t}\right) /\left(\mathrm{E}_{t}\right)$. As long as the system is in zone $\mathrm{C}$, most of the inhibitor is bound to the enzyme and the fraction of inactive enzyme is independent of the potency of the inhibitor. It is obvious that mo constants may be calculated from zone C kinetics.

\section{Dilution of Enzyme-Inhibitor Systems}

The characteristic changes in inhibition brought about by dilution were used by Straus and Goldstein as criteria for zone behavior. Two types of dilution must be distinguished: (a) only the enzyme is diluted and the inhibitor is added at the same concentration and $(b)$ the enzyme-inhibitor mixture is diluted. Enzyme dilution alone has no effect on the inhibition in zone $\mathrm{A}$ and increases inhibition in zones $\mathrm{B}$ and $\mathrm{C}$. or may translate the system from zone $\mathrm{C}$ into $\mathrm{B}$ or from zone $\mathrm{B}$ into $\mathrm{A}$; this is evident from the dependence of zone behavior on $\left(\mathrm{E}_{i}{ }^{\prime}\right)$. In the second situation where both enzyme and inhibitor are diluted (the "dilution effect" of Straus and Goldstein), equally simple relations are observed for noncompetitive inhibition. In zone A dilution leads to less inhibition simply because the inhibitor concentration becomes smaller and the enzyme concentration is of no importance; in zone $\mathrm{C}$ both $\left(\mathrm{I}_{i}{ }^{\prime}\right)$ and $\left(\mathrm{E}_{i}{ }^{\prime}\right)$ change equally and there is no effect on inhibition. In zone $\mathrm{B}$ the situation is somewhat more complex because both $\left(\mathrm{I}_{t}{ }^{\prime}\right)$ and $\left(\mathrm{E}_{i}{ }^{\prime}\right)$ are involved in determining the inhibition. Dilution will produce a decrease in inhibition, due to reduction of $\left(\mathrm{I}_{t}^{\prime}\right)$, but the decrease will be less than in zone $\mathrm{A}$ becanse the reduction of $\left(\mathrm{E}_{i}{ }^{\prime}\right)$ will tend to increase the inhibition somewhat; thus in zone $\mathrm{B}$ the change of inhibition on dilution depends on the change in $\left(\mathrm{E}_{i}{ }^{\prime}\right)$ whereas in zone $\mathrm{A}$ it does not. Dilution of competitively inhibited systems also involves decrease in $\left(\mathrm{S}_{t}{ }^{\prime}\right)$. In zone $\mathrm{A}_{s} \mathrm{~A}_{i}$ there will be no change in inhibition if the assumptions leading to Eq. 3-50 are valid, since both $\left(\mathrm{I}_{t}{ }^{\prime}\right)$ and $\left(\mathrm{S}_{t}{ }^{\prime}\right)$ change equally; however, from the more general Eq. 3-47 it is clear that inhibition will increase. In zones $\mathrm{A}_{s} \mathrm{~B}_{i}$ and $\mathrm{A}_{s} \mathrm{C}_{i}$ there will also be no change in inhibition on dilution if the simplifications giving Eqs. 3-52 and 3-54 are valid, but if the more general Eq. 3-48 must be used, there is a dilution effect which also depends on $\left(\mathrm{E}_{i}{ }^{\prime}\right)$ in zone $\mathrm{A}_{s} \mathbf{B}_{i}$. In case the system is in zones $\mathrm{B}_{s}$ or $\mathrm{C}_{s}$ the dilution effect is complex and depends generally on all four specific concentrations, $\left(\mathrm{S}_{t}{ }^{\prime}\right),\left(\mathrm{I}_{t}{ }^{\prime}\right),\left(\mathrm{E}_{s}{ }^{\prime}\right)$, and $\left(\mathrm{E}_{i}{ }^{\prime}\right)$, as is evident from $\mathrm{Eq} .3-45$. The complex equations for the magnitude of the dilution effect in noncompetitive systems are given by Straus and Goldstein and a graphical presentation for competitive systems by Goldstein, together with the effects of dilution on cholinesterase inhibition.

Noncellular preparations usually represent enzymes diluted from the concentration existing in the cell; this is dilution of the first type, i.e., of enzyme only. Sometimes a tissue is exposed to an inhibitor and the inhibited enzyme is subsequently extracted for the purpose of determining the extent of inhibition occurring in the tissue; this is dilution of the second type, 
since both enzyme and inhibitor are reduced in concentration. Care should be taken to distinguish between these two situations when comparing inhibitions in tissues and in extracts.

\section{Mutual Depletion Systems in the Cell}

The behavior of systems showing mutual depletion has been discussed in some detail because it is believed that they may be more common than previously supposed, particularly within cells, and that they will become more common as new inhibitors of high potency are applied to enzyme study. In a cell, or within subcellular structures, or in localized cellular regions, the concentration of enzyme may be very high, far greater than could be practically attained with the extracted enzyme, and the possibility suggests itself that zone $\mathrm{B}$ and $\mathrm{C}$ behavior would be observed. However, we must again distinguish between two situations: $(a)$ the substrate or inhibitor is added to the system and has a diffuse distribution, and $(b)$ the substrate or inhibitor is also localized in the same region as the enzyme, being formed in that region. Most experimental systems are of the former type and in this case the enzyme will bind no more substrate or inhibitor than if it were homogeneously distributed; mere concentration of enzyme in localized regions will not reduce the concentration of free substrate or inhibitor, which is what determines zone $\mathrm{B}$ and $\mathrm{C}$ behavior. A suspension of mitochondria, each containing a particular enzyme in high concentration, will not yield kinetic data different from a system in which the same amount of enzyme is dissolved uniformly in the same total volume. Only the effects of mutual depletion are being considered at this time; there are other possible factors which may alter the kinetic behavior when an enzyme is released from its localized condition. In the second type of situation zone behavior may be exhibited commonly, even with respect to substrate as well as inhibitor, since the total amount of substrate or inhibitor present is very small, even though the actual concentration may be comparable to that used in isolated preparations. It is easy to visualize that, within a mitochondrion for example, essentially all the substrate is bound to enzyme. If an inhibitor is formed from a precursor and this occurs in the localized region, it also may be mainly combined; examples of such inhibitors might be fluorocitrate or the various substrate and coenzyme analogs that must be phosphorylated or synthesized within the cell from simpler added substances. A simple example will illustrate this. Let us assume a mitochondrial suspension of $10 \mathrm{ml}$ containing an enzyme whose over-all concentration is $10^{-8} M$; an inhibitor is added at $10^{-5} M-$ no reduction of free inhibitor concentration occurs because there is a total of $10^{-10}$ moles of enzyme and $10^{-7}$ moles of inhibitor present. However, if the inhibitor is produced within the mitochondria and is localized there at the same concentration of $10^{-5} M$, the total amount in the system may be $1 / 1000$ of that previously or $10^{-10}$ 
moles, and it is likely that the system will be in zone $\mathrm{B}$ or $\mathrm{C}$ depending on the value of $K_{i}$. Prediction of competitive inhibition kinetics under such circumstances is extremely difficult since we never accurately know the localized substrate concentration; it is likely that competitive inhibition as it is usually formulated is uncommon in these cases.

\section{Role of Extraneous Substances in Mutual Depletion Systems}

In the systems so far discussed it has been assumed that one is dealing with a pure enzyme or, if not, that other material does not bind the inhibitor. In many enzyme preparations there are substances that can bind certain inhibitors as well, or even better, than the enzyme; protein impurities can usually bind heavy metals, arsenicals, and protein reactants extensively. Now, binding of inhibitors by such extra-enzyme material will cause the system to approach or enter zones B or C, because it makes no difference what binds the inhibitor and rednces its free concentration. Actually in systems containing binding components other than the enzyme, it is not the value of $\left(\mathrm{E}_{i}{ }^{\prime}\right)$ that determines the zone but a sum of such terms including each binding substance, or approximately considered the total concentration of binding sites divided by an average dissociation constant. This would apply particularly to impure enzyme preparations, mitochondria, homogenates, aud cell suspensions. If we designate the specific concentration of all binding sites by $\left(\mathrm{X}_{i}{ }^{\prime}\right)$ it is to this that the boundary conditions really apply and $\left(\mathrm{X}_{i}{ }^{\prime}\right)$ may well be 100-1000 times larger than $\left(\mathrm{E}_{i}{ }^{\prime}\right)$. Conversely, in such situatious, an estimation of enzyme concentration, as discussed above, may be erroneous. This will be taken up in more detail in Chapter 15 .

\section{PSEUDO-IRREVERSIBLE INHIBITION}

Ackermann and Potter (1949) defined pseudo-irreversible inhibitors as those that have such a high affinity for the enzyme that they give the appearance of irreversibility. The inhibition may be competitive or noncompetitive, but if it is truly the former, it may not be obvious since little change in enzyme activity will be observed upon adding the substrate to the inhibited enzyme, due to the slow dissociation of inhibitor, or the inbitor is bound so much more tightly to the enzyme than is the substrate that competition at equilibrium cannot be demonstrated. This is really another approach to systems in zones $\mathrm{B}$ or $\mathrm{C}$ and the equations given by Ackermann and Potter are identical to those of Straus and Goldstein for comparable situations; thus the expression for the inhibited rate given by Ackermann and Potter, $v_{i}=h\left(\mathrm{E}_{t}\right)-h\left(\mathrm{I}_{t}\right)$, is equivalent to an inhibition of $i=\left(\mathrm{I}_{\ell}\right) /\left(\mathrm{E}_{\ell}\right)$. since $v=h\left(\mathrm{E}_{\imath}\right)$. However, a short exposition of this approach will serve to introdnce some further practical applications for such inhibitors. 
If the inhibitor is specific for a certain enzyme, being bound to nothing else, it may be said to titiate the enzyme. in the sense that the amount of enzyme inactivated will be equivalent to the amount of inhibitor added. The rates of reaction are determined at different enzyme concentrations, with

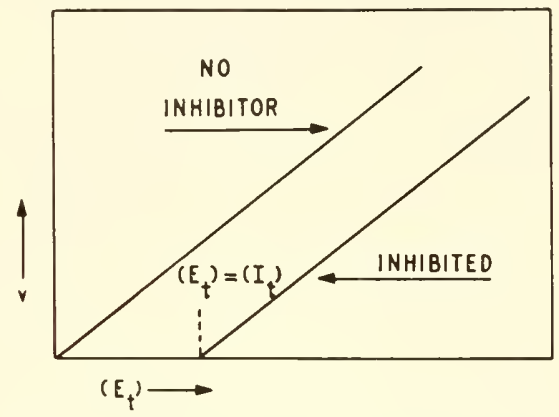

FIG. 3-14. Plot of rate against the enzyme concentration for pseudo-irreversible and irreversible inhibition.

and without inhibitor, and these rates are plotted against $\left(\mathrm{E}_{t}\right)$ in arbitrary units since the molar concentration of enzyme is generally not known. In the absence of inhibitor, one obtains a straight line passing through the origin, since $v=h\left(\mathrm{E}_{t}\right)$; in the presence of inhibitor a straight line of identical

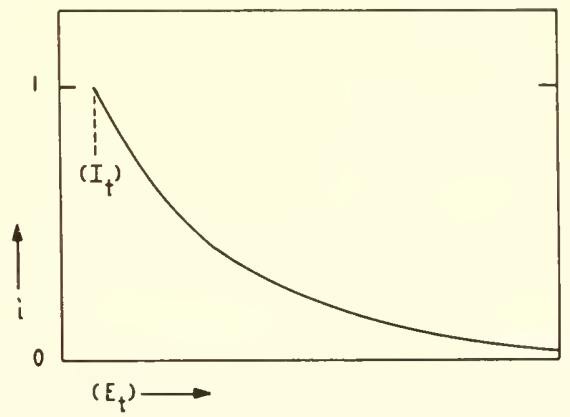

Fig. 3-15. Variation of the fractional inhibition with the enzyme concentration for pseudo-irreversible inhibition where $i=\left(\mathrm{I}_{t}\right) /\left(\mathrm{E}_{t}\right)$.

slope is obtained but intercepting the $\left(\mathrm{E}_{t}\right)$ axis at a point where $\left(\mathrm{E}_{t}\right)=\left(\mathrm{I}_{t}\right)$. Irreversible inhibitors will also yield the same results. Such a plot is shown in Fig. 3-14 and the hyperbolic curve when inhibition is plotted against $\left(\mathrm{E}_{t}\right)$ is illustrated in Fig. 3-15. 
Inhibitors of this type, if specific. can be used to estimate the relative amounts of enzymes in different preparations or tissues because using the same concentration of inhibitor, various tissues exhibit different degrees of inhibition if their enzyme content varies, according to Fig. 3-15. In fact, the inhibition is inversely proportional to the amount of enzyme present. The electron-transport inhibitor, antimycin A, has been used to estimate the relative amounts of succinoxidase in different tissues (Potter and Reif, 1952). The antimycin titer was defined as the $\gamma$ of antimycin per gram of tissue to give $50 \%$ inhibition: the values ran from around 0.5 for spleen, lung, and tumor, through 1.7 for liver and 3.2 for kidney, to 8.8 for heart.

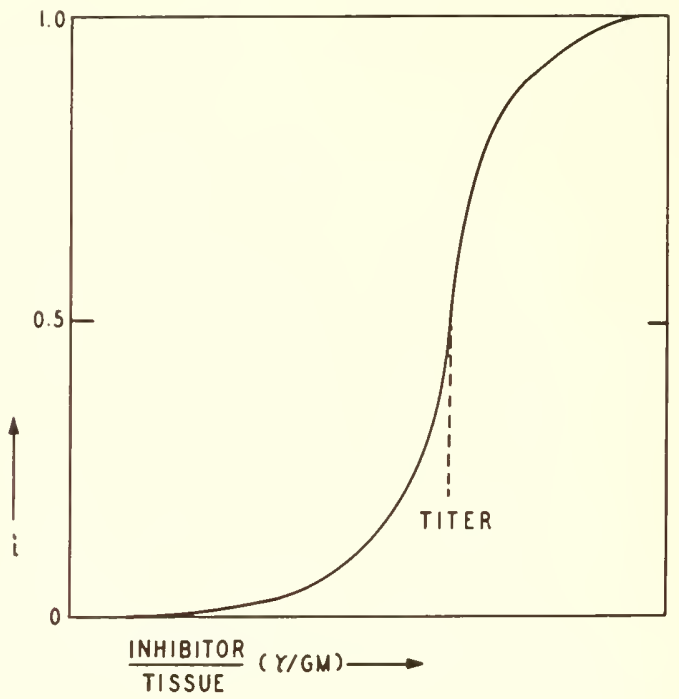

FIG. 3-16. Illustration of the determination of the inhibitor titer for a tissue preparation.

These vahues correspond quite well to those obtained manometrically on homogenates of the various tissues. Similar results were obtained using other cycle intermediates as substrates, since the step blocked by antimycin is common to many electron-transport systems. If such experiments were done on intact tissue and the inhibition of respiration determined, the antimycin titer would not, of course, refer only to succinoxidase, but to the amount of antimycin-sensitive component operating in the tissue. The value of the titer for a particular preparation is arrived at by plotting the inhibition against the ratio of inhibitor added to tissne weight; the midpoint (50\% inhibition) on the sigmoid curve represents the titer, just as in the usual chemical titrations (Fig. 3-16). 
When a pseudo-irreversible or irreversible inhibitor is introduced into an animal intravenously (to ensure relatively rapid and uniform distribution throughout the body). the susceptible enzyme will be inhibited to different degrees in the various tissues; those tissues possessing the smallest amount of enzyme will be inhibited most potently. It must be assumed that the inhibitor enters each tissue to approximately the same degree. This type of effect is in distinct contrast to the behavior of readily reversible inhibitors, where the inhibition in any tissue depends only on the concentration of inhibitor and not at all on the amount of enzyme present; if the inhibitor entered each tissue equally, the inhibition in each would be the same, assuming that the enzyme had the same properties and environment in every tissue. It was suggested by Potter et al., (1952) that the principle of enzyme titration might be applied to chemotherapy. Cells that contain the least amount of the susceptible enzyme are most readily inhibited and perhaps most easily killed; for example, tumor tissue succinoxidase was inhibited completely by an amount of antimycin that had no effect on heart succinoxidase, and only minimal effects on the enzyme from kidney. liver. brain, and muscle were observed, simply becanse there was less of the enzyme in the tumor tissue. Complications naturally arise: the effects of binding to blood components, the different blood supplies to varions tissues so that the inhibitor is not equally distributed. the different permeabilities of cells to the inhibitor. and the fact that an enzyme may exhibit somewhat different kinetic parameters in each tissue. However, it does represent one intelligent and directed approach to chemotherapy. which. if pursned intently, might well lead to the derelopment of useful drugs.

Finally, it may be remarked that figures given for the per cent inhibition of enzymes by inhibitors of this type mean very little. unless the amount of enzyme present is also stated (Ackermann and Potter, 1949). The inhibitions bear no necessary relation to the notency of the inhibitor or the $K_{i}$ and cannot be compared on any quantitative basis with inhibitions produced by readily dissociable inhibitors. For example, let us assume $\left(\mathrm{E}_{t}\right)=$ $10^{-5} . M$ and we compare four inhibitors with different $K_{i}$ s. The tabulation below shows the concentrations necessary to produce a standard inhibition and it is evident that these concentrations do not indicate quantitatively

\begin{tabular}{|c|c|c|c|c|c|}
\hline Inhibitor & $\mathrm{K}$, & $\begin{array}{l}\text { Relative } \\
\text { affinity }\end{array}$ & $\left(\mathrm{I}_{t}\right)$ & $i$ & Zone \\
\hline I & $10^{-4}$ & 1 & $270 \times 10^{-8}$ & 0.73 & A \\
\hline II & $10^{-5}$ & 10 & $34 \times 10^{-6}$ & 0.73 & B \\
\hline III & $10^{-6}$ & 100 & $10 \times 10^{-6}$ & 0.73 & $B$ \\
\hline IV & $10^{-9}$ & 100,000 & $7.3 \times 10^{-6}$ & 0.73 & C \\
\hline
\end{tabular}


the relative potency. It might be concluded that inhibitors III and IV are of comparable potency, although they actually differ by a factor of 1000 .

\section{INHIBITION BY REACTION WITH COENZYME OR ACTIVATOR SITES}

It has been assumed in the inhibitions discussed up to this point that the inhibitor interferes only with the binding or breakdown of substrate. The inhibitor may also interfere with the binding or functioning of the other possible components of the enzyme reaction. The general rate equation for an enzyme reaction in which an activator and an inhibitor are both present was developed by Friedenwald and Maengwyn-Davies (1954, p. 154) and the following is an application of this equation to varions special cases. The reactions that must be considered in the general case may be represented as:

$$
\mathrm{EA}+\mathrm{P}
$$

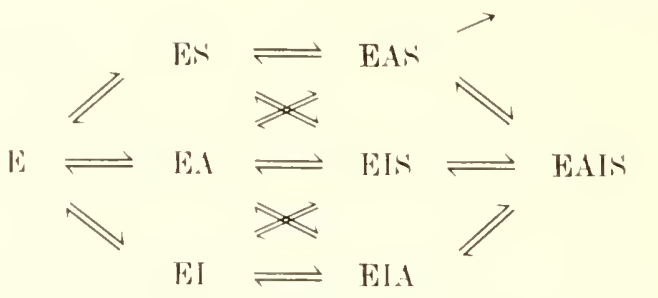

The rate equation can be witten in terms of either substrate or activator:

$$
\begin{aligned}
& v_{i}=\frac{V_{m}}{\beta}-\left(\mathrm{A}^{\prime}\right)\left[\frac{1}{\left(\mathrm{~S}^{\prime}\right)}+\frac{1}{\beta}\right]+\left(\mathrm{I}^{\prime}\right)\left[\frac{1}{\left(\mathrm{~S}^{\prime}\right)}+\frac{1}{\alpha}\right]+\left(\mathrm{A}^{\prime}\right)\left(\mathrm{I}^{\prime}\right)\left[\frac{1}{\gamma^{\prime}\left(\mathrm{S}^{\prime}\right)}+\frac{1}{\beta \mu}\right]+\left[\frac{1}{\left(\mathrm{~S}^{\prime}\right)}+1\right] \\
& v_{i}=\frac{V_{m}^{\prime}}{\beta} \\
& \left(\mathrm{S}^{\prime}\right)\left[\frac{1}{\left(\mathrm{~A}^{\prime}\right)}+\frac{1}{\beta}\right]+\left(\mathbf{I}^{\prime}\right)\left[\frac{1}{\left(\mathrm{~A}^{\prime}\right)}+\frac{1}{\gamma}\right]+\left(\mathrm{S}^{\prime}\right)\left(\mathrm{I}^{\prime}\right)\left[\frac{1}{\alpha\left(\mathrm{A}^{\prime}\right)}+\frac{1}{\beta \mu}\right]+\left[\frac{1}{\left(\mathrm{~A}^{\prime}\right)}+1\right]
\end{aligned}
$$

and they may be seen to be symmetrical with respect to substrate and activator. The interaction constants expressing the effects of a component on the binding of another may be defined as follows: $\alpha$ relates to $\mathrm{I}$ and $\mathrm{S}, \beta$ to $\mathrm{S}$ and $A, \gamma$ to $I$ and $A$, and $\mu$ to the effects of $A S$, IS, and IA on binding of $I$, A. and $\mathrm{S}$. That is, $K_{q}=(\mathrm{E})(\mathrm{A}) /(\mathrm{EA}), \gamma K_{a}=(\mathrm{EI})(\mathrm{A}) /(\mathrm{EIA}), \beta K_{a}=(\mathrm{ES})$ (A)/(EAS), and $\mu K_{a}=($ EIS $)(A) /($ EAIS $)$, with similar expressions for $K_{s}$ and $K_{i}$. These general equations include effects of the inhibitor on both substrate and activator: however, the formulation assumes that only the EAS complex breaks down into products, i.e., the activator is essential and the 
presence of inhibitor on the enzyme reduces activity to zero. If the rate equation with respect to substrate is written in the Michaelis-Menten form:

$$
K_{m}=K_{s} \frac{\left(\mathrm{A}^{\prime}\right)+\left(\mathrm{I}^{\prime}\right)+(1 / \gamma)\left(\mathrm{A}^{\prime}\right)\left(\mathrm{I}^{\prime}\right)+1}{(1 / \beta)\left(\mathrm{A}^{\prime}\right)+(1 / \alpha)\left(\mathrm{I}^{\prime}\right)+(1 / \beta \mu)\left(\mathrm{A}^{\prime}\right)\left(\mathrm{I}^{\prime}\right)+1}
$$

\section{Noncompetitive Inhibition with Respect to Activator}

When the inhibition is completely noncompetitive with the activator and the substrate, we may set $\alpha=\gamma=\mu=1$ and obtain the equation:

$$
v_{i}=V_{m}^{\prime} \frac{\left(\mathrm{S}^{\prime}\right)\left(\mathrm{A}^{\prime}\right)}{\left(\mathrm{S}^{\prime}\right)\left[\left(\mathrm{A}^{\prime}\right)+1\right]+\beta\left(\mathrm{A}^{\prime}\right)+1} \frac{1}{\left(\mathrm{I}^{\prime}\right)+1}
$$

and for the situation where activator doesn't influence substrate binding $(\beta=1)$ :

$$
V_{i}=V_{m}^{\prime} \frac{\left(\mathrm{S}^{\prime}\right)}{\left(\mathrm{S}^{\prime}\right)+1} \frac{\left(\mathrm{A}^{\prime}\right)}{\left(\mathrm{A}^{\prime}\right)+1} \frac{1}{\left(\mathrm{I}^{\prime}\right)+1}
$$

In the latter case one obtains the usual noncompetitive equation for the inhibition: $i=\left(\mathrm{I}^{\prime}\right) /\left[\left(\mathrm{I}^{\prime}\right)+1\right]$. However, in the more general case where $\beta \neq 1$, the inhibition will involve this factor: from Eqs. 2-71 and 3-60 one obtains:

$$
i=\frac{\left(\mathrm{I}^{\prime}\right)+\left[\left(\mathrm{S}^{\prime}\right)+1\right][1-\beta] /\left[\left(\mathrm{S}^{\prime}\right)\left(\mathrm{A}^{\prime}\right)+\left(\mathrm{S}^{\prime}\right)+\beta\left(\mathrm{A}^{\prime}\right)+1\right]}{\left(\mathrm{I}^{\prime}\right)+1}
$$

\section{Competitive Inhibition with Respect to Activator}

If the inhibitor competes with the activator for the activator site but does not affect substrate binding, we must set $\alpha=1$ and $\gamma=\mu=\infty$. The general rate equation is:

$$
v_{i}=V_{m} \frac{\left(\mathrm{S}^{\prime}\right)\left(\mathrm{A}^{\prime}\right)}{\left(\mathrm{S}^{\prime}\right)\left[\left(\mathrm{A}^{\prime}\right)+\beta\left(\mathrm{I}^{\prime}\right)+\beta\right]+\left[\left(\mathrm{A}^{\prime}\right)+\beta\left(\mathrm{I}^{\prime}\right)+1\right]}
$$

and for the special case where $\beta=1$ :

$$
v_{i}=V_{m} \frac{\left(\mathrm{S}^{\prime}\right)}{\left(\mathrm{S}^{\prime}\right)+1} \frac{\left(\mathrm{A}^{\prime}\right)}{\left(\mathrm{A}^{\prime}\right)+\left(\mathrm{I}^{\prime}\right)+1}
$$

From the latter we obtain an expression for the inhibition (relative to the rate in Eq. 2-72 for the uninhibited reaction):

$$
i=\frac{\left(\mathrm{I}^{\prime}\right)}{\left(\mathrm{I}^{\prime}\right)+\left(\mathrm{A}^{\prime}\right)+1}
$$


which is quite comparable to Eq. 3-28 applying to competition with substrate. Again, if $\beta \neq 1$, the inhibition is modified by this factor. The effects of the factor $\beta$ on determinations of the various dissociation constants will be discussed in Chapter 5 .

\section{Inhibition with Respect to a Coenzyme}

Since the equations for reaction rate are identical for activators, coenzymes, or second substrates (see Eqs. 2-61 and 2-72), when there is no effect of these on substrate binding, it is erident that similar equations are obtained for noncompetitive and competitive inhibition with respect to these components. In fact, under these conditions, competition with any component $\mathrm{X}$ will lead to an equation of the form:

$$
i=\frac{\left(\mathrm{I}^{\prime}\right)}{\left(\mathrm{I}^{\prime}\right)+\left(\mathrm{X}^{\prime}\right)+1}
$$

and with similar corrections when the substrate binding is modified by the presence of the component. Similarly, noncompetitive inhibition will always result in an equation $i=\left(\mathrm{I}^{\prime}\right) /\left[\left(\mathrm{I}^{\prime}\right)+1\right]$ which is independent of component concentration. It must be emphasized that this is not the case if substrate binding is altered by the presence of the other component.

An example of this type of mechanism for which the kinetics have been studied experimentally is the inhibition of yeast alcohol dehydrogenase by o-phenanthroline (Hoch et al.. 1958). The enzyme active center contains an atom of zine that is necessary for the binding of DPN+ and DPNH; the metal chelator, o-phenanthroline, inhibits the reaction by complexing with the zinc and competing with the coenzyme. The inhibitor, on the other hand, does not compete with either ethanol or acetaldehyde, and thus the zinc must be involved only in the binding of the coenzyme.

\section{Competitive Inhibition with Respect to an Activator Essential for Binding of Substrate}

When the primary function of the activator is the binding of the substrate to the enzyme, as it may often be in the case of metals, and the inhibitor blocks the activator from the enzyme, an interesting type of inhibition results. This situation may be represented as:

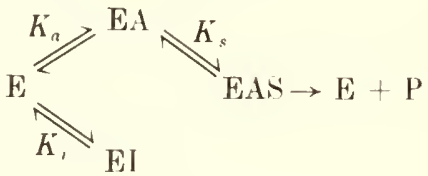


and the rate equation is:

$$
v_{i}=V_{m} \frac{\left(\mathrm{S}^{\prime}\right)\left(\mathrm{A}^{\prime}\right)}{\left(\mathrm{S}^{\prime}\right)\left(\mathrm{A}^{\prime}\right)+\left(\mathrm{A}^{\prime}\right)+\left(\mathrm{I}^{\prime}\right)+1}
$$

which compared to Eq. 2-69 gives the inhibition as:

$$
i=\frac{\left(\mathrm{I}^{\prime}\right)}{\left(\mathrm{I}^{\prime}\right)+\left(\mathrm{A}^{\prime}\right)+1+\left(\mathrm{S}^{\prime}\right)\left(\mathrm{A}^{\prime}\right)}
$$

The umexpected dependence of the inhibition on substrate concentration, although the mechanism is completely competitive with activator, is due to the fact that increasing $(\mathrm{S})$ and $(\mathrm{A})$ forms more EAS complex and reduces the amount of enzyme a vailable to combine with inhibitor. Reciprocal plots will remain linear but an error may be introduced into the calculation of $K_{i}$.

\section{INHIBITION BY REACTION WITH SUBSTRATE, COENZYME, OR ACTIVATOR}

The inhibitions previously discussed have all resulted from reaction of the inhibitor with the apoenzyme at various regions relative to the binding sites for the substrate or other components of the system. The situation in which the inhibitor reacts directly with the substrate. or with other dissociable components. will now be considered. The types of inhibition produced are frequently difficult to distinguish from those where the apoenzyme is attacked if the usual kinetic analysis is applied uncritically.

\section{Reaction of Inhibitor with Substrate}

When reaction of an inhibitor with the substrate prevents the binding of the substrate to the enzyme, the effective concentration of free substrate is reduced; only that fraction of the substrate that is uncombined with the inhibitor is free to undergo the enzymic reaction. From the dissociation constant for the substrate-inhibitor compound it is possible to calculate the concentration of free substrate (S) and use this value. instead of the total concentration $\left(\mathrm{S}_{l}\right)$, in the previous equations.

$$
\begin{gathered}
\mathrm{S}+\mathrm{I} \rightleftharpoons \mathrm{SI} \quad K_{s i}=\frac{(\mathrm{S})(\mathrm{I})}{(\mathrm{SI})} \\
\left(\mathrm{S}_{t}\right)=(\mathrm{S})+(\mathrm{SI}) \\
\left(\mathrm{I}_{t}\right)=(\mathrm{I})+(\mathrm{SI}) \\
(\mathrm{S})^{2}+\left[\left(\mathrm{I}_{t}\right)-\left(\mathrm{S}_{t}\right)+K_{s i}\right](\mathrm{S})-\left(\mathrm{S}_{t}\right) K_{s i}=0
\end{gathered}
$$


$(\mathrm{S})=\frac{1}{2} \sqrt{\left[\left(\mathrm{I}_{t}\right)-\left(\mathrm{S}_{t}\right)+K_{s i}\right]^{2}+4\left(\mathrm{~S}_{t}\right) K_{s i}}-\frac{1}{2}\left[\left(\mathrm{I}_{t}\right)-\left(\mathrm{S}_{t}\right)+K_{s i}\right]$

This value can then be used in the Michaelis-Menten equation $v_{i}=V_{m}(\mathrm{~S}) /$ $\left[(\mathrm{S})+K_{s}\right]$ and compared with the rate in the absence of inhibitor, given by the equation $v=V_{m}\left(\mathrm{~S}_{t}\right) /\left[\left(\mathrm{S}_{t}\right)+K_{s}\right]$, to determine the inlibition. The inhibition curves, plotted in Fig. 3-17 for two substrate concentrations, show an effect of substrate on the inhibition that superficially may resemble competitive inhibition. The inhibition curves rise more steeply in the region where $\left(\mathrm{I}_{t}\right)$ is comparable to $\left(\mathrm{S}_{t}\right)$ than for competitive inhibition; in fact, if

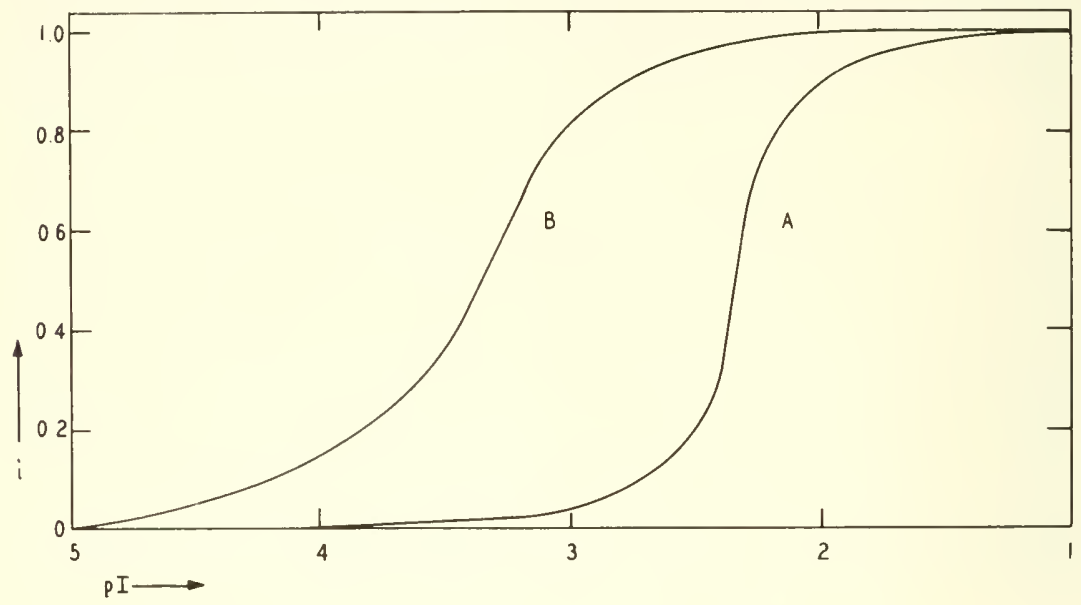

Frg. 3-17. Variation of the fractional inhibition with the inhibitor concentration when the inbibitor reacts with the substrate. $K_{s}=1 \mathrm{~m} M$ and $K_{s i}=0.1 \mathrm{mM}$. Curve A: $\left(\mathrm{S}_{t}\right)=5 \mathrm{~m} M$; curve $\mathrm{B}:\left(\mathrm{S}_{t}\right)=0.5 \mathrm{~m} M$.

the inflection is near this region it is often an indication of reaction with substrate although, of course, this is not necessary. A plot of the dependence of inhibition on substrate concentration (Fig. 3-18) shows that raising $\left(\mathrm{S}_{t}\right)$ will more readily overcome the inhibition caused by reaction with the substrate than competitive inhibition. It may be noticed that $\left(\mathrm{I}_{t}\right)_{90} /\left(\mathrm{I}_{t}\right)_{10}$ for competitive inlibition is usually around 100 while for reaction with substrate it is usually between 5 and 25 .

If inhibition by reaction with the substrate is established, it is possible to determine $K_{s i}$ from the kinetic data. The concentration of free substrate may be estimated from the degree of inhibition produced at a certain $\left(\mathrm{I}_{t}\right)$ :

$$
(\mathrm{S})=\frac{\left(\mathrm{S}_{t}\right) K_{s}(1-i)}{K_{s}+i\left(\mathrm{~S}_{t}\right)}
$$


and this value may be substituted in:

$$
K_{s i}=\frac{(\mathrm{S})\left[\left(\mathrm{I}_{t}\right)-\left(\mathrm{S}_{t}\right)+(\mathrm{S})\right]}{\left(\mathrm{S}_{t}\right)-(\mathrm{S})}
$$

which is obtained from Eq. 3-70.

Occasionally the inhibition may not be due to the simple depletion of free substrate but to the combination of the SI complex with the enzyme. In other words, the substance added and called the inhibitor actually does not interact with the enzyme but by complexing with the substrate pro-

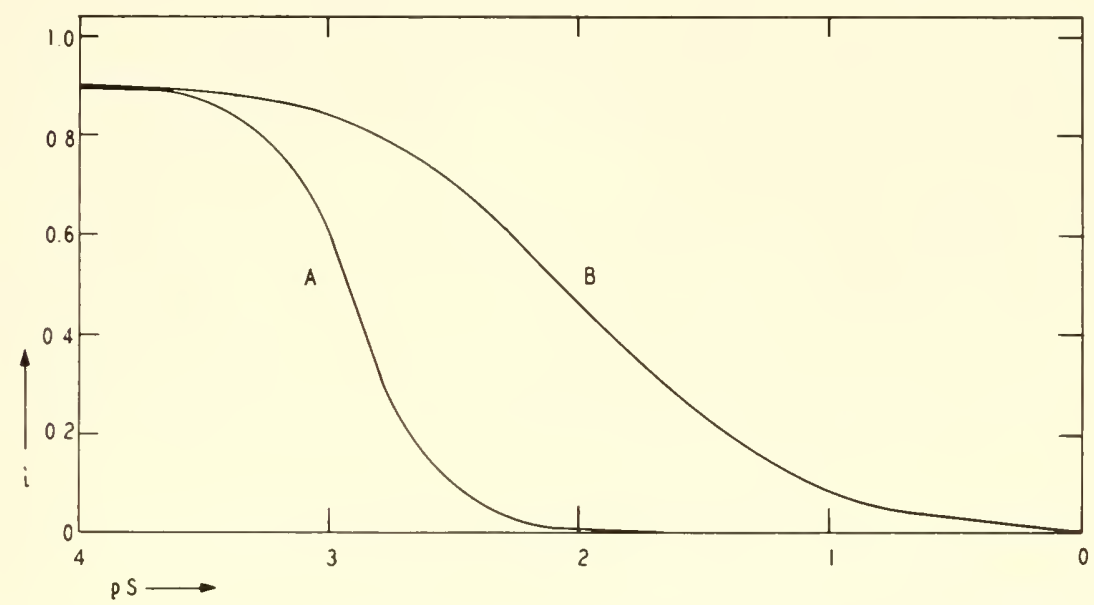

FIG. 3-18. Variation of the fractional inhibition with substrate concentration for competitive inhibition and for the situation where the inhibitor reacts with the substrate. $K_{s}=1 \mathrm{~m} H$. Curve A: $K_{s i}=0.1 \mathrm{~m} M,\left(I_{t}\right)=1 \mathrm{~m} M$; curve B: $K_{i}=0.1 \mathrm{~m} M$,

$$
\left(\mathrm{I}_{t}\right)=1 \mathrm{~m} M \text {. }
$$

duces an inhibitory compound. If the free substrate concentration is not significantly depressed and the inhibition is entirely due to the SI complex according to the following scheme:

$$
\begin{array}{ll}
\mathrm{E}+\mathrm{S} \rightleftharpoons \mathrm{ES} \rightarrow \mathrm{E}+\mathrm{P} & K_{s} \\
\mathrm{~S}+\mathrm{I} \rightleftharpoons \mathrm{SI} & K_{s i} \\
\mathrm{E}+\mathrm{SI} \rightleftharpoons \mathrm{ESI} &
\end{array}
$$

the expression for the concentration of SI complex:

$$
(\mathrm{SI})=\frac{\left(\mathrm{I}_{t}\right)\left(\mathrm{S}_{t}\right)}{\left(\mathrm{S}_{t}\right)+K_{s i}}
$$


may be substituted in the usual equations for competitive inhibition (3-12 and 3-13) as the concentration of the active inhibitor. If, in addition to the inhibition produced by SI, there is depletion of free substrate, the value of $(\mathrm{S})$ from $\mathrm{Eq}$. 3-72 must be used instead of $\left(\mathrm{S}_{\ell}\right)$. A characteristic of inhibitions exerted by SI is the increase in inhibitor concentration with increasing substrate concentration. Therefore, competition between $\mathrm{S}$ and I is not so evident as in those cases where $I$ is itself the inhibitor, at least over a certain range of substrate concentrations.

An interesting approach to inhibitions involving complexes of inhibitor with substrate has been formulated by Reiner (1959, p. 156). The plotting of $\left(I_{t}\right)$ against $i /(1-i)$ often hehs to distinguish between the different types of inhibition and occasionally allows the calculation of the constants. The equations for the three possible mechanisms may be written as follows:

(1) inhibition due to repletion of free substrate

$$
\left(\mathrm{I}_{t}\right)=\left[1+\left(\mathrm{S}_{t}\right) / K_{s}\right]\left[u K_{s i}+\frac{u\left(\mathrm{~S}_{t}\right)}{1+u\left[1+\left(\mathrm{S}_{t}\right) / \boldsymbol{K}_{s}\right]}\right]
$$

(2) inhibition due to the combination of SI with the enzyme

$$
\left(\mathrm{I}_{t}\right)=\left[\left(\mathrm{S}_{t}\right)+K_{s}\right]\left[\left(\mathrm{S}_{t}\right)+K_{s i}\right] \frac{K_{i} u}{\left(\mathrm{~S}_{t}\right) K_{s}}
$$

(3) inhibition due to both mechanisms

$$
\left(\mathrm{I}_{t}\right)=K_{i}\left[1+\left(\mathrm{S}_{t}\right) / K_{s}\right]\left\{\frac{u K_{s i}}{\left(\mathrm{~S}_{t}\right)+K_{i}}+\frac{u\left(\mathrm{~S}_{t}\right)}{\left(\mathrm{S}_{t}\right)+\overline{K_{i}}\left\{1+u\left[1+\overline{\left(\mathrm{S}_{t}\right) / K_{s}}\right]\right\}}\right\}
$$

The inhibition term, $i /(1-i)$, has been indicated by $u$ for convenience. Plots of $\left(I_{t}\right)$ against $u$ for the first and third situations lead to curves which are linear for low and high values of $u$, the slopes for these regions being easily evaluated by inspection of the equations. For example, for inhibition due to the depletion of substrate, the slope at low values of $u$ (i.e., low inhibitions) will be $\left[K_{s i}+\left(\mathrm{S}_{\ell}\right)\right]\left[1+\left(\mathrm{S}_{\ell}\right) / K_{s}\right]$ and for high vahes of $u$ the slope will be $K_{s i}\left[1+\left(S_{l}\right) / K_{s}\right]$. The curves will bend npwards or downwards, depending on the inhibition mechanism. However, the plot for inhibition by the complex SI will be a straight line passing throngh the origin. The slope will depend on $\left(S_{\ell}\right)$ in a characteristic way: it will be high for both low and high values of $\left(\mathrm{S}_{\ell}\right)$ and reach a minimum when $\left(\mathrm{S}_{\ell}\right)=\sqrt{K_{s} K_{s i}}$. Other features of this method of plotting will be found in the original presentation by Reiner. 


\section{Reaction of Inhibitor with Other Components of the Reaction}

When reaction of the inhibitor occurs with coenzyme, activator, or a second substrate, the same type of inhibition will result if the rate equations are symmetrical (e.g., Eqs. 2-61 and 2-72). However, if substrate binding is affected by these other components, this factor will complicate the inhibition. For the situation in which the activator is essential for the reaction (Eqs. 2-67 and 2-68), an equation similar to 3-72 will be obtained with $\left(\mathrm{A}_{t}\right)$ and $K_{a i}$ replacing $\left(\mathrm{S}_{t}\right)$ and $K_{s i}$. providing that the fraction of activator combined with enzyme is small, which was assumed in deriving the rate Eq. 2-69. The value of (A) can then be substituted in Eq. 2-69. When this is plotted, against either pI or pA, inhibition curves of form and slope much like those of competitive inhibition are obtained. The concentration of activator must be reduced below $K_{a}$ before appreciable inhibition occurs.

If the activator in the uninhibited system is mainly bound to the enzyme and (A) is negligible, addition of inhibitor will withdraw some of the activator from the enzyme and one can write $\left(A_{\ell}\right)=(E A)+(I A)$. The degree of inhibition produced will depend on the relative affinities of the enzyme and inhibitor for the activator and on the relative concentrations of enzyme and inhibitor. An expression for (EA) is quadratic:

$$
\begin{array}{r}
(\mathrm{EA})^{2}\left[K_{a}-K_{a i}\right]+(\mathrm{EA})\left[K_{a}\left\{\left(\mathrm{I}_{t}\right)-\left(\mathrm{A}_{t}\right)\right\}\right. \\
\left.-K_{a i}\left\{\left(\mathrm{~A}_{t}\right)+\left(\mathrm{E}_{t}\right)\right\}\right]-K_{a i}\left(\mathrm{~A}_{t}\right)\left(\mathrm{E}_{t}\right)=0
\end{array}
$$

and may be substituted in $i=1-\left[\left(\mathrm{EA}_{\mathrm{A}}\right) /\left(\mathrm{E}_{t}\right)\right]$, but it is seldom that the constants and the enzyme concentration are known. However, if the enzyme were saturated with activator and $\left(\mathrm{E}_{t}\right)=\left(\mathrm{A}_{t}\right)$, the enzyme concentration can be climinated from the equations. In the extreme case where $K_{a i} \ll K_{a}$, i.e., the activator is bound much more tightly to the inhibitor than to the enzyme, one may write $(E A)=\left(A_{t}\right)-\left(I_{t}\right)$ from which the inhibition is seen to be $i=\left(\mathrm{I}_{t}\right) /\left(\mathrm{A}_{t}\right)$, a linear relationship with inhibitor concentration. This is, of course. a titration type of inhibition and corresponds to zone $\mathrm{C}$ behavior with respect to inhibitor and activator.

The situation in which the activator may combine with both substrate and inhibitor, probably of frequent occurrence when the activator is a heavy metal, has been analyzed by Gryder et al. (1955), and the following rate equation derived:

$$
v_{i}=V_{m} \frac{(\mathrm{S})(\mathrm{A})(\mathrm{I})}{(\mathrm{S})(\mathrm{A})(\mathrm{I})+c\left[K_{s} K_{a s}(\mathrm{I})+K_{a} K_{a i}(\mathrm{~S})+\left(K_{s} K_{a} K_{a s} K_{a i}\right) /(\mathrm{A})\right]}
$$

where $K_{a s}=(\mathrm{A})(\mathrm{S}) /(\mathrm{AS}), K_{a i}=(\mathrm{A})(\mathrm{I}) /(\mathrm{AI})$, and $\alpha$ is the factor by which $K_{s}$ is changed by the presence of activator. This may be compared with the 
equation for the unimhibited reaction 2-71 and a rather complex expression for the inhibition may be obtained. This was applied to the inhibition by glycine of the hydrolysis of glycerophosphate by alkaline phosphatase using zine as the activator, and the constants were evaluated from the appropriate reciprocal plots. This treatment assumed that the concentration of free activator is negligible and that the activator is mainly present combined with substrate and inhibitor; if the affinity of the enzyme for the activator is high or the coneentration of activator is sufficient to form EA complex, the expressions become much more complex.

An interesting example of complieated inhibition kinetics involving a eoenzyme is provided by D-amino aeid oxidase (Yagi et al., 1959a,b). Certain inhibitors form complexes with flavin adenine dinucleotide (FAD) (phenol, salieylate, $m$-aminophenol, and chlortetracycline). The eomplex between FAD and chlortetracycline was demonstrated by the quenching of the flavin fluorescence and the shift in flavin absorption spectrum. Indeed, it was elaimed that chlortetracycline has a single inhibitory mechanism on this enzyme, the formation of the complex with the coenzyme. However, the phenols and certain other compounds (aniline, $p$-aminobenzoate, ete.) can compete with FAD for the eoenzyme site. Thus phenol, for example, inhibits by two mechanisms related to the coenzyme. Finally, some of these substanees can compete with the substrate. Salicylate inhibits by all three mechanisms and presents a good example of a situation that is probably more common than generally believer. It is too often assumed in interpreting the experimental data that a single meehanism of inhibition is operative and many of the deviations from the expeeted behavior may be due to multiple aetions.

\section{THE ENZYME COMBINES WITH MORE THAN ONE MOLECULE OF INHIBITOR}

It has been occasionally assumed that if $n$ moleenles of inhibitor react with the enzyme, noncompetitive inhibition is expressed by:

$$
i=\frac{(\mathrm{I})^{n}}{(\mathrm{I})^{n}+K_{i}{ }^{\prime}}
$$

and competitive inhibition by a similar equation with $K_{i}^{\prime}$ multiplied by $1+\left[(S) / K_{s}\right]$. However, it is doubtful if this type of equation ever corresponds to an aetual situation, since it assumes either that no intermediate complexes (EI, $\mathrm{EI}_{2}$ to $\mathrm{EI}_{n-1}$ ) are formed or, if they are formed, they do not modify the enzymie activity (i.e., the addition of the $n$th inhibitor molecule suddenly reduces the activity to zero). Furthermore, it is evident that $K_{i}^{\prime}$ is not a simple dissoeiation constant, since it equals $(\mathrm{E})(\mathrm{I})^{n} /\left(\mathrm{EI}_{n}\right)$; 
actually $K_{i}^{\prime}=K_{i 1} K_{i 2} \ldots K_{\text {in }}$ or, if each molecule is bound equally, $K_{i}{ }^{\prime}=$ $K_{i}^{n}$. Before presenting a general treatment of this problem, it will be well to examine the ways in which enzymes can bind several inhibitor molecules.

Several possible situations may be distinguished and the inhibition kinetics are in general different for each. In the following, $\mathrm{R}$ represents an active center while $\mathrm{B}$ is a nonenzymic or nonfunctional binding site.

(I) The enzyme possesses two or more active centers on each molecule

$A$. The centers are independent: in this case the inhibition kinetics are identical to those developed previously, since $\left(\mathrm{E}_{t}\right)$ refers to the concentration of active centers and not to enzyme molecules.

$$
\mathrm{ER}_{n}+n \mathrm{I} \rightleftharpoons \mathrm{ER}_{n} \mathrm{I}_{n} \quad K_{i} \text { same for each group }
$$

$B$. The centers have equal intrinsic affinities for the inhibitor but interact so that binding of each inhibitor molecule influences the binding of other molecules to adjacent sites.

$$
\mathrm{ER}_{n}+n \mathrm{I} \rightleftharpoons \mathrm{ER}_{n} \mathrm{I}_{n} \quad K_{i}, \alpha K_{i}, \beta K_{i} \ldots \text { for successive bindings }
$$

$C$. The centers have different intrinsic affinities for the inhibitor.

$\mathrm{ER}^{\prime} \mathrm{R}^{\prime \prime} \ldots \mathrm{R}_{n}+n \mathrm{I} \rightleftharpoons \mathrm{ER}^{\prime} \mathrm{IR}^{\prime \prime} \mathrm{I} \ldots \mathrm{R}_{n} \mathrm{I} \quad K_{i}^{\prime}, K_{i}{ }^{\prime \prime} \ldots$ for each site

(II) The enzyme possesses functional centers and nonfunctional binding sites

Only a certain fraction of the bound inhibitor exerts an inhibitory action. In this case there is no effect by the nonfunctional group binding on the inhibition unless the system is in zone $\mathrm{B}$ or $\mathrm{C}$ and thus this binding contributes to the reduction of free inbibitor concentration.

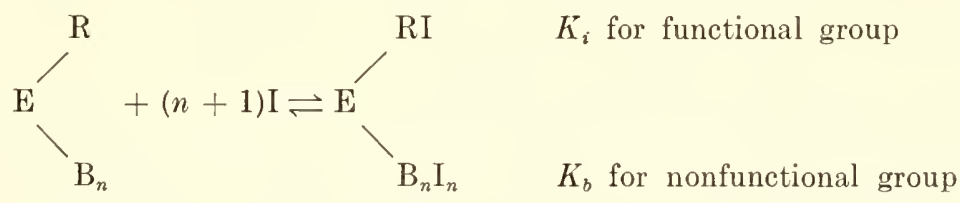

(III) Each active center binds more than one inhibitor molecule

$\mathrm{ER}+n \mathrm{I} \rightleftharpoons \mathrm{ERl}_{n} \quad K_{i}$ may be the same for each molecule of inhibitor or different if interaction occurs

There are thus three situations [(I) $B,(\mathrm{I}) C$, and (III)] which require extension of the previous formulation.

\section{Each Active Center Binds More Than One Inhibitor Molecule}

Inasmuch as binding of three or more inhibitor molecules to an active center is relatively rare, attention will be given to the situation where 
$n=2$; it is simple to extend this treatment to higher values of $n$. The most general pattern of reactions is the following:

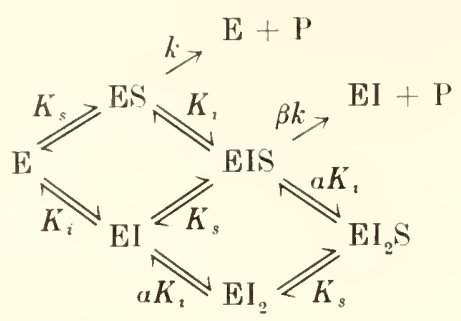

If noncompetitive inhibition is assumed (no effect on substrate binding), the following rate expression is obtained, using specific concentrations for convenience:

$$
v_{i}=V_{m} \frac{\left(\mathrm{S}^{\prime}\right)}{\left(\mathrm{S}^{\prime}\right)+1}\left[\frac{1+\beta\left(\mathrm{I}^{\prime}\right)}{1+\left(\mathrm{I}^{\prime}\right)+\left[\left(\mathrm{I}^{\prime}\right)^{2} / \alpha\right]}\right]
$$

where $\alpha$ is the factor expressing the change in inhibitor binding due to the attachment of the first inhibitor molecule and $\beta$ represents the reduction in $k$ produced by the binding of one molecule of inhibitor. The inhibition is then given by:

$$
i=\frac{(1-\beta)\left(\mathrm{I}^{\prime}\right)+\left[\left(\mathrm{I}^{\prime}\right)^{2} / \alpha\right]}{1+\left(\mathrm{I}^{\prime}\right)+\left[\left(\mathrm{I}^{\prime}\right)^{2} / \alpha\right]}
$$

Various special cases dependent upon the values of $\alpha$ and $\beta$ will now be considered. When $\beta=0$ the binding of the first inhibitor molecule prevents the breakdown of the substrate, but the inhibition is greater than in the case where only one inhibitor molecule combines because there are in addition to $\mathrm{EI}$ and EIS the complexes $\mathrm{EI}_{2}$ and $\mathrm{EI}_{2} \mathrm{~S}$, which further reduce the concentration of active centers. The inhibition is given by:

$$
i=\frac{\left(\mathrm{I}^{\prime}\right)+\left[\left(\mathrm{I}^{\prime}\right)^{2} / \alpha\right]}{1+\left(\mathrm{I}^{\prime}\right)+\left[\left(\mathrm{I}^{\prime}\right)^{2} / \alpha\right]} \quad \beta=0
$$

If $\alpha$ is very low (seccnd inhibitor molecule bound more tightly than first), the inhibition will increase and approach a situation where $\alpha K_{i}$ is the dominant constant:

$$
i=\frac{(\mathrm{I})^{2}}{(\mathrm{I})^{2}+\alpha K_{i}{ }^{2}} \quad \beta=0, \alpha \text { low }
$$

which is as close as the inhibition will come to Eq. 3-81, although $\alpha K_{i}{ }^{2}$ is quite different from $K_{i}{ }^{\prime}$. If $\alpha$ is very high (second inhibitor molecule bound less tightly than the first), the equation reduces to the usual one for noncompetitive inhibition:

$$
i=\frac{\left(\mathrm{I}^{\prime}\right)}{\left(\mathrm{I}^{\prime}\right)+1} \quad \beta=0, \alpha \text { high }
$$


Finally, if $\alpha$ has a value near unity, an intermediate situation occurs in which both inhibitor molecules play a role in the inhibition. Comparison of curve 4 with curve 1 in Fig. $3-19$ shows that when $\alpha=1$, the inhibition will be appreciably greater than for the case where $n=1$.

When $\beta=1$ there is no effect on the enzyme reaction produced by the binding of the first inhibitor molecule and in this case:

$$
i=\frac{\left(\mathrm{I}^{\prime}\right)^{2}}{\alpha+\alpha\left(\mathrm{I}^{\prime}\right)+\left(\mathrm{I}^{\prime}\right)^{2}} \quad \beta=1
$$

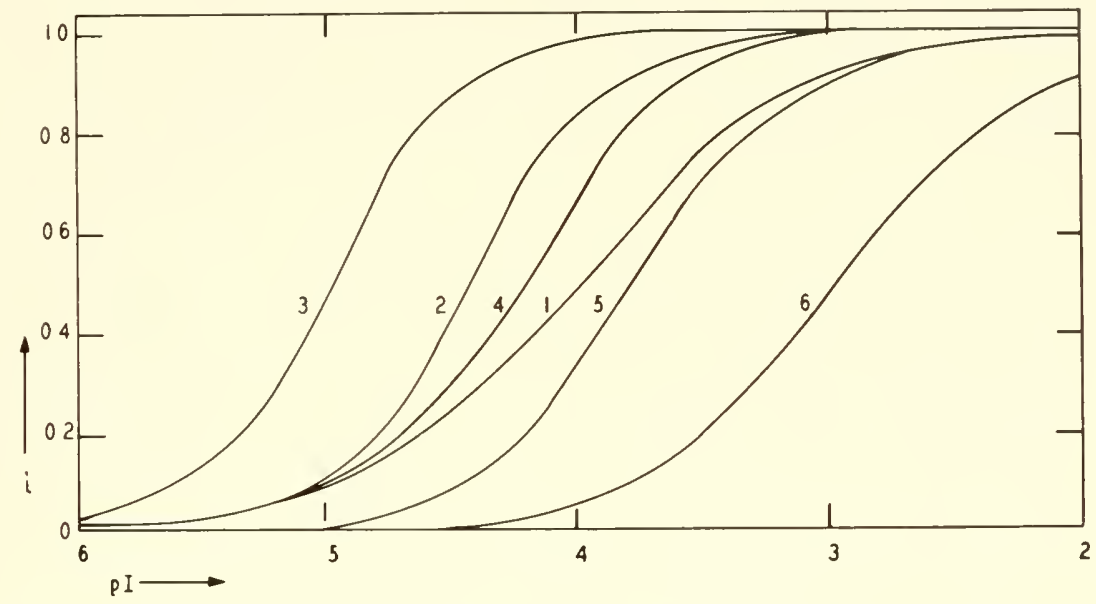

FIG. 3-19. Plots of Eq. 3-84 for different values of the constants $\alpha$ and $\beta$. Curve 1: $n=1$; curve 2 : $\alpha=0.1, \beta=1$; curve $3: \alpha=0.01, \beta=1$; curve $4: \alpha=1 ; \beta=0$; curve 5: $\alpha=1, \beta=1$; curve $6: \alpha=10 ; \beta=1$.

When $\alpha$ is rery low the inhibition becomes high whereas when $\alpha$ is high the inhibition is reduced: in the former case the inhibition is governed by the binding of the second inhibitor molecule and tends to follow an equation of the type:

$$
i=\frac{(\mathrm{I})}{(\mathrm{I})+\alpha \bar{K}_{i}} \quad \beta=1, \alpha \text { low }
$$

and in the latter case the binding of the second inhibitor molecule is so weak that little inbibition occurs. When $\alpha$ is around unity, the inhibition is generally less than when $n=1$ because only the binding of the second inhibitor molecule is inhibitory and it occurs to a lesser extent than the first molecule [the ratio of $(\mathrm{EI}) /\left(\mathrm{E}_{t}\right)$ is more than $\left(\mathrm{EI}_{2}\right) /\left(\mathrm{E}_{t}\right)$ when $\alpha=1$ ]. This may be seen by comparing curve 5 with curve 1 in Fig. 3 -19. The other curves given show the effect of $\alpha$ on the degree of inhibition. 
The most common values for $\alpha$ probably lie between 1 and 10 , since binding of one molecule usually decreases binding of a second, if there is any effect, and if $\alpha$ is much higher than 10 the inhibition generally behaves as if there were only one inhibitor molecule combining. The most likely values for $\beta$ are probably 0 or slightly above, since it is logical that binding of one molecule of inhibitor would decrease the enzymic rate, if it didn't abolish it entirely.

Noncompetitive inhibition with $n=1$ leads to $(\mathrm{I})_{0.5}=K_{i}$ and this concentration is often experimentally determined to calculate $K_{i}$. If $n=2$ or more, this no longer holds and $(\mathrm{I})_{0.5}$ varies over a wide range as can be observed in Fig. 3-19. It is of some interest to compare the values of $\left(\mathrm{I}^{\prime}\right)_{0.5}$ for various combinations of $\alpha$ and $\beta$, since this concentration is a good indication of the average degree of inhibition produced (see tabulation).

\begin{tabular}{l|cc|c}
\hline \multirow{2}{*}{ Values of $\alpha$} & \multicolumn{3}{|c}{$\left(\mathrm{I}^{\prime}\right)_{0.5}$} \\
\cline { 2 - 3 } & $\beta=0$ & $\beta=0.5$ & $\beta=1$ \\
\hline & & & \\
& & & \\
$\alpha=0.2$ & 0.23 & 0.32 & 0.43 \\
$\alpha=1$ & 0.37 & 0.71 & 1.37 \\
$\alpha=5$ & 0.46 & 1.58 & 5.45 \\
$\alpha=50$ & 0.50 & 5.00 & 50.5 \\
\hline
\end{tabular}

Uncritical equating of $(\mathrm{I})_{0.5}$ with $K_{i}$ is therefore apt to lead to error, even when the inhibition is of the pure noncompetitive type. The general equation for the inhibitor concentration to produce $50 \%$ imhibition is:

$$
\left(\mathrm{I}^{\prime}\right)_{0.5}=\frac{-\alpha(1-2 \beta)+\frac{\sqrt{\alpha^{2}(1-2 \beta)^{2}+2 \alpha}}{2}}{2}
$$

If $n$ is greater than 2 , the inhibition equation is of the general form:

$$
i=\frac{(1-\beta)\left(1^{\prime}\right)+\left(1-\beta^{\prime}\right) \frac{\left(\mathrm{I}^{\prime}\right)^{2}}{\alpha}+\left(1-\beta^{\prime \prime}\right) \frac{\left(\mathrm{I}^{\prime}\right)^{3}}{\alpha^{\prime}}+\ldots+\left(1-\beta_{n}\right) \frac{\left(\mathrm{I}^{\prime}\right)^{n}}{\alpha_{n}}}{1+\left(\mathrm{I}^{\prime}\right)+\frac{\left(\mathrm{I}^{\prime}\right)^{2}}{\alpha}+\frac{\left(\mathrm{I}^{\prime}\right)^{3}}{\alpha^{\prime}}+\ldots+\frac{\left(\mathrm{I}^{\prime}\right)^{n}}{\alpha_{n}}}
$$

where $\alpha, \alpha^{\prime} \ldots \alpha_{n}$ are the interaction constants for successive bindings and $\beta, \beta^{\prime} \ldots \beta_{n}$ represent the effects of successive inhibitor molecules on $k$. 
The Active Centers Have Different Intrinsic Affinities for the Inhibitor

If the enzyme possesses more than one substrate site and the inhibitor is bound with different affinities at or near these sites, the inhibition for each site is given by the simple equation:

$$
i_{j}=\frac{(\mathrm{I})}{(\mathrm{I})+K_{i_{j}}}
$$

but the total inhibition of the enzyme is not the sum of these separate inhibitions, i.e., $i \neq i_{1}+i_{2}+\ldots+i_{n}$. The inhibition is obtained by dividing the sum of the inhibitor-bound sites of each type by the total number of sites on the enzyme:

$$
i=\frac{1}{n}\left[\frac{n_{1}(\mathrm{I})}{(\mathrm{I})+K_{i_{1}}}+\frac{n_{2}(\mathrm{I})}{(\mathrm{I})+K_{i_{2}}}+\ldots+\frac{n_{p}(\mathrm{I})}{(\mathrm{I})+K_{i_{p}}}\right]
$$

if there are $p$ types of sites with different $K_{i}$ 's, if $n_{1}, n_{2} \ldots n_{p}$ are the numbers of sites of each type per molecule, and where $n=n_{1}+n_{2}+\ldots+n_{p}$. For competitive inhibition the $K_{i}$ s are simply multiplied by $1+\left[(\mathrm{S}) / K_{s}\right]$ if the $K_{s}$ is the same for each site. If there are only two different types of sites $(p=2)$ and if they are in equal number on the enzyme, $(\mathrm{I})_{0.5}=K_{i_{1}} K_{i_{2}}$, so that this may also introduce an error in equating $\left(\mathrm{I}_{\mathbf{0 . 5}}\right.$ with $K_{i}$. In this case also, the over-all inhibition will be one-half the sum of the individual inhibitions for the two sites.

\section{The Active Centers Are Not Independent}

When the enzyme possesses more than one active center, each with the same affinity for the inhibitor, so situated that the binding of inhibitor alters the affinity of adjacent groups for the inhibitor, the kinetics of inhibition deviate from those where the sites are independent. Let us first consider the situation in which there are two interactive centers on the enzyme. The problem is not inherently different from that for the dissociation of dicarboxylic acids, where association of an $\mathrm{H}^{+}$with one carboxylate ion leads to a change in the $K_{a}$ of the second group.

In the case of dibasic acids the macroscopic dissociation constants, obtained from titration data, always differ by a factor of 4 or more; i.e., $K_{n_{1}} K_{n_{2}}=4$ when there is no interaction between groups and more than 4 if there is interaction. This is a statistical factor and is due to the fact that the first hydrogen ion may dissociate from either carboxylic group and the second hydrogen ion may associate with either carboxylate ion. However, this factor does not enter into enzyme formulations since $\left(\mathrm{E}_{t}\right)$ is not the concentration of enzyme molecules but of active centers, whereas for dicarboxylic acids the concentration of acid refers to the entire molecule and not the individual groups. Thus the dissociation constants used here 
are really intrinsic or microscopic constants, although we designate them by $K_{i}$ rather than $k_{i}$ so that they will not be confused with rate constants. An excellent treatment of polyvalent molecules and the dissociation behavior of both independent and interactive groups is given by Edsall and Wyman (1958, p. 477).

The binding of one inhibitor molecule is assumed to change the dissociation constant of the second site to $\alpha K_{i}$. The situation is similar to that discussed above where there are two independent sites with constants $K_{i}$ and $\alpha K_{i}$. The inhibition is thus given by:

$$
i=\frac{1}{2}\left[\frac{(\mathrm{I})}{(\mathrm{I})+K_{i}}+\frac{(\mathrm{I})}{(\mathrm{I})+\alpha K_{i}}\right]
$$

The reactions involved may be represented as follows:

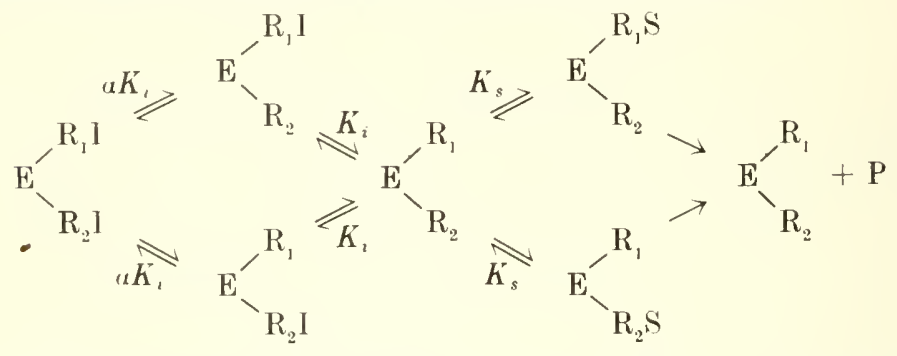

The complexes $\mathrm{ER}_{1} \mathrm{IS}$ and $\mathrm{ER}_{2} \mathrm{IS}$ can, of course, be formed since noncompetitive inhibition is assumed but, inasmuch as they are inactive, they will not alter the kinetics. Furthermore, any interaction between the substrate sites, altering $K_{s}$, will not influence this type of inhibition. If the inhibition is competitive the situation is expressed by Eq. 3-95, but when substrate site interaction occurs, an additional constant is introduced. When there are more than two sites an equation of the type 3-93 may be used.

The variation in inhibition with $\alpha$ in plotted in Fig. 3-20. Whatever the inhibition produced by a certain inhibitor concentration when the sites are independent $(\alpha=1)$, increasing the repulsion interaction $(\alpha>1)$ will reduce the inhibition progressively to one-half the initial value. The inhibitor concentration for $50 \%$ inhibition is given by $(\mathrm{I})_{0.5}=K_{i} \sqrt{\alpha}$ so that this interaction factor can also lead to significant deviation of $(\mathrm{I})_{0.5}$ from $K_{i}$.

\section{THE INHIBITOR IS ALSO A SUBSTRATE FOR THE ENZYME}

In certain inhibitions, usually competitive and with an inhibitor related structurally to the substrate, the inhibitor may be acted upon by the enzyme. If only one of these reactions is determined, Eqs. 3-12 and 3-13 for competitive inhibition are applicable since the inhibitor slows down the 
substrate reaction by reducing the available substrate sites. However, if both reactions are determined together (e.g., when the products measured are identical for each reaction or both products absorb similarly in a spectro-

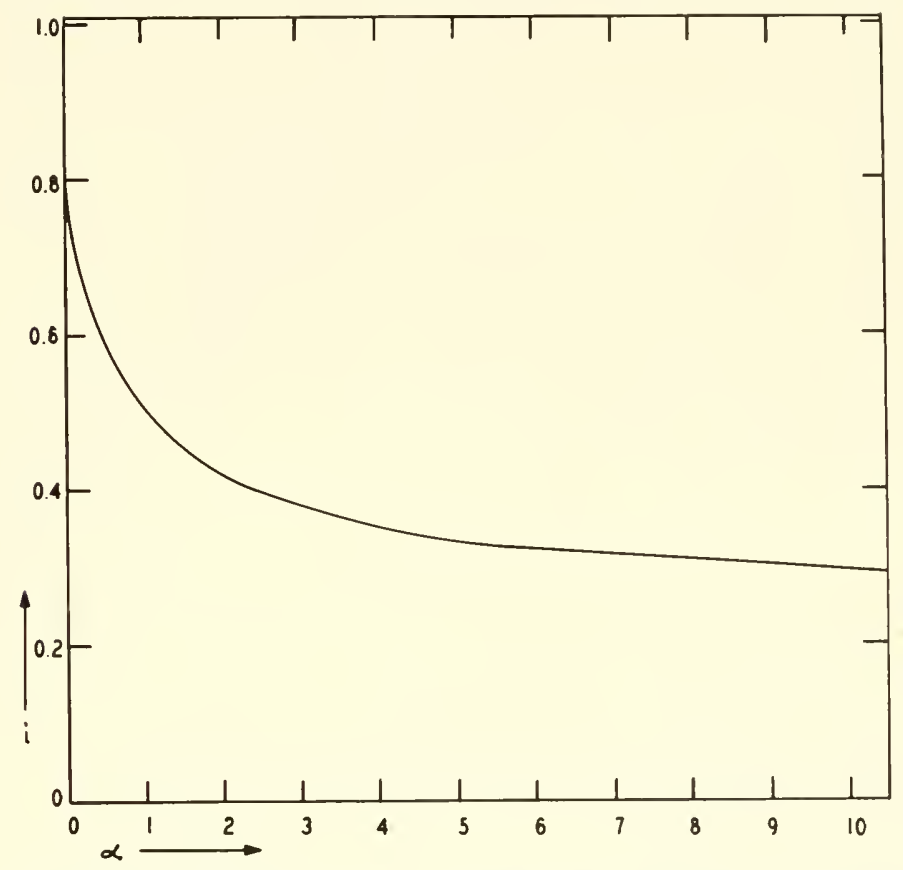

FIG. 3-20. Variation of the fractional inhibition with varying interaction of two adjacent active centers (Eq. 3-94). $K_{i}=0.1 \mathrm{~m} . \mathrm{I}$ and $(\mathrm{I})=0.1 \mathrm{~m} . \mathrm{I}$.

photometric determination) the inhibition is more complex. The reactions to be considered are:

$$
\begin{aligned}
& \mathrm{E}+\mathrm{S} \rightleftharpoons \mathrm{ES} \stackrel{k_{s}}{\rightarrow} \mathrm{E}+\mathrm{P}_{s} \\
& \mathrm{E}+\mathrm{I} \rightleftharpoons \mathrm{EI} \stackrel{k_{i}}{\rightarrow} \mathrm{E}+\mathrm{P}_{i}
\end{aligned}
$$

The over-all rate is the sum of the individual rates when both substrate and inhibitor are present:

$$
v_{t}=v_{s}+v_{i}=\frac{V_{s}\left(\mathrm{~S}^{\prime}\right)+V_{i}\left(\mathrm{I}^{\prime}\right)}{1+\left(\mathrm{S}^{\prime}\right)+\left(\mathrm{I}^{\prime}\right)}
$$

and the inhibition relative to the rate with substrate alone is:

$$
i=\frac{\left(\mathrm{I}^{\prime}\right)}{\left(\mathrm{I}^{\prime}\right)+1+\left(\mathrm{S}^{\prime}\right)}\left[1-\frac{V_{i}}{V_{s}\left(\mathrm{~S}^{\prime}\right)}-\frac{V_{i}}{V_{s}}\right]
$$


The inhibition is thus less than for the usual competitive situation where the inhibitor is stable and, since $V_{i} / V_{s}=k_{i} / k_{s}$, the rechetion factor in the numerator is $\left[1+1 /\left(\mathrm{S}^{\prime}\right)\right] k_{i} / k_{s}$. If the ratio $k_{i} / k_{s}$ is quite low, as it often is, the behavior approaches that of typical competitive inhibition. However, $k_{i}$ may be comparable to $k_{s}$ or even greater, in which case it is better to consider both reactants as substrates, since the inhibition Eq. 3-99 will not apply, the over-all rate being greater than for substrate. In fact,Eq. $3-99$ is valid only when $k_{i} / k_{s}<\left(\mathrm{S}^{\prime}\right) /\left[\left(\mathrm{S}^{\prime}\right)+1\right]$.

The ratio $K_{i} / K_{s}$ may sometimes be determined by measurements made on the maximal rates. If $(\mathrm{I})=(\mathrm{S})$, Eq. 3-98 leads to an expression for the maximal rate in the equimolar mixture:

$$
V_{m}=\left[V_{s} / K_{s}-V_{i} / K_{i}\right] /\left[1 / K_{s}+1 / K_{i}\right]
$$

from which it follows that:

$$
\frac{K_{i}}{K_{s}}=\frac{V_{m}-V_{i}}{V_{s}-V_{m}}
$$

and thus the ratio of the affinities may be calculated from determinations of the maximal rates with substrate alone, inhibitor alone, and both in equimolar proportions.

An interesting example of such inhibition is provided by sweet potato phosphatase where various combinations of glycerate-3-phosphate, pyrophosphate, phenyl-phosphate, ATP, and other phosphates give kinetics in accord with the treatment above (Ito et al.. 1955). All of these substances give values of $K_{s}$ or $K_{i}$ in the same range and compete with one another for the same active center.

\section{SOME FACTORS OCCASIONALLY IMPORTANT IN INHIBITION}

Many factors may modify the formulations of inhibition kinetics as they have been presented in this chapter. Of particular importance are $\mathrm{pH}$, ionic strength, buffer effects, temperature, purity of the enzyme, and similar factors; these will be considered in Chapters 14 and 15 . However, there are some aspects which may profitably be discussed at this point.

\section{The Enzyme Becomes Progressively Inactivated during the Reaction}

The determination of reaction rates and inhibition requires measurement of some quantity over an interval of time, occasionally only a few minutes but often an hour or longer. During this interval the enzyme may be progressively inactivated either spontaneously or as the result of secondary 
reactions of the inhibitor. This means that the concentration of active enzyme $\left(\mathrm{E}_{\ell}\right)$ is decreasing during the experimental interval, often resulting in significant deviation from inhibition kinetics in systems where the enzyme is stable. Certain isolated enzymes are unstable under the conditions for the determination of enzyme rate since they are operating in an environment abnormal compared to their intracellular state. Many enzymes may be reasonably stable at low temperatures but are inactivated at a rather rapid rate at $37-38^{\circ}$ when isolated. Inhibitors, especially those reacting chemically with protein groups, may induce a characteristic inhibition at or near the active center but in addition may alter the protein structure, or denature, which will secondarily affect the enzymic activity.

If the decrease in active enzyme concentration is linear, or approximately so over the experimental interval, there will be no effect on the inhibition measured for this interval if the enzyme inactivation is spontaneous and occurs at the same rate in the presence and absence of the inhibitor, since both uninhibited and inhibited rates will be similarly affected, but if the inactivation occurs only in the presence of the inhibitor, the inhibition is changed according to the following equation:

$$
i=\frac{\left(\mathrm{I}^{\prime}\right)+1 / 2\left[1-\left(V_{m^{\prime}} / V_{m}\right)\right]}{\left(\mathrm{I}^{\prime}\right)+1}
$$

where $V_{m}$ is the initial maximal rate and $V_{m}{ }^{\prime}$ is the maximal rate at the end of the experimental period. $V_{m}$ and $V_{m}{ }^{\prime}$ are, of course, proportional to the concentrations of active enzyme at the two times. Thus a noncompetitive inhibition of $50 \%$ by $\left(\mathrm{I}^{\prime}\right)$ would be raised to $62.5 \%$ if half the enzyme were inactivated during the experimental interval. Determination of $K_{i}$ may be incorrect under such circumstances. Indeed, $(\mathrm{I})_{0.5}=\left(V_{m}{ }^{\prime}\right)$ $\left.V_{m}\right) K_{i}$ so that the determined $K_{i}$ may be in error by the ratio of final to initial active enzyme concentration. If the decrease in $\left(\mathrm{E}_{\ell}\right)$ is nonlinear, the expression for the over-all inhibition is more complex; however, if the inactivation is first-order or logarithmic, it is clear that the effect on the inliibition will be even greater than for the linear inactivation.

It is well to bear such effects in mind in much inhibition study but it must be admitted that it is usually difficult to demonstrate such secondary inactivation if it is due to the enzyme, much less plot its time course. Ideally one would like to be able to determine the concentration of active enzyme at the termination of the experimental interval, but it is not possible to remove the inhibitor from the enzyme immediately, especially those inhibitors likely to produce secondary inactivation. However, if $1 / v_{i}$ is plotted against (I) (see Chapter 5), the true $K_{i}$ can be determined; substitution of this value into the usual inhibition equation (enzyme is stable) will demonstrate any deviation and enable $V_{m}{ }^{\prime} / V_{m}$ to be calculated approximately. Or the inhibitor concentration corresponding to the determined 
$K_{i}$ can be tested and deviation from $50 \%$ inhibition may indieate enzyme inactivation. Similar procedures may be undertaken for competitive inhibition.

\section{The Inhibitor Is Progressively Destroyed during the Reaction}

The sitnation here is relatively simple since the rate of destruction of the inhibitor can usually be determined, whether it is spontaneous or due to the enzyme preparation, and from this rate of destruction the inhibition at any time or over an experimental interval may be calculated. However, if a change in inhibitor concentration is not recognized, the kinetie analysis may be in error. In a plot of $1 / v$, against (I), for example, the slope will be changed (hecanse initial inhibitor coneentrations will be plotted whereas the actual concentrations will be lower than these) but the intercept will not, and therefore the calculated $K_{i}$ will be ineorrect. If the inhibitor concentration decreases at an aproximately linear rate, the inhibition over the experimental interval is given by:

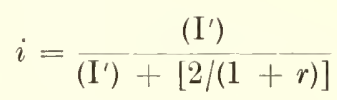

where $r=\left(\mathbf{I}^{\prime}\right)_{f} /\left(\mathbf{I}^{\prime}\right)_{0},\left(\mathbf{I}^{\prime}\right)_{0}$ and $\left(\mathbf{I}^{\prime}\right)_{f}$ being the initial and final inhibitor concentrations. (I $)_{0.5}$ is equal to $[2 /(1+r)] K_{i}$ for noneompetitive inhibition.

\section{Inhibition of Forward and Backward Reactions}

It might appear, on the basis of simple ehemical reactions, that inhibition would be produced equally on the forward and reverse reactions, since both forward and reverse rate constants must be ehanged equally if the over-all equilibrium is to be unaffected. However, in enzyme reactions the over-all equilibrium depends often on many rate constants and these may be ehanged in a great variety of ways withont altering the equilibrium. If either mechanism

or

$$
\mathrm{E}+\mathrm{S} \underset{k_{-1}}{\stackrel{k_{1}}{\rightleftharpoons}} \mathrm{EX} \underset{k_{-3}}{\stackrel{k_{3}}{\rightleftharpoons}} \mathrm{E}+\mathrm{P}
$$

$$
\mathrm{E}+\mathrm{S} \underset{k_{-1}}{\stackrel{k_{1}}{\rightleftharpoons}} \mathrm{ES} \underset{k_{-2}}{\stackrel{k_{2}}{\rightleftharpoons}} \mathrm{EP} \underset{k_{-3}}{\stackrel{k_{3}}{\rightleftharpoons}} \mathrm{E}+\mathrm{P}
$$

applies, the forward and reverse rates are given by:

$$
v_{f}=\frac{V_{s}(\mathrm{~S})}{(\mathrm{S})+K_{s}} \quad v_{r}=\frac{V_{p}(\mathrm{P})}{(\mathrm{P})+\mathrm{K}_{p}^{-}}
$$


where $K_{s}$ and $K_{p}$ refer to either dissociation or Michaelis constants depending on the conditions. The inhibition of forward and reverse reactions in the noncompetitive case would be:

$$
i_{f}=\frac{(\mathrm{I})}{(\mathrm{I})+K_{i_{f}}^{-}} \quad i_{r}=\frac{(\mathrm{I})}{(\mathrm{I})+K_{i_{r}}}
$$

and $i_{f}=i_{r}$ since the $K_{i}$ 's must be equal, the binding of the inhibitor being unaffected by the presence of either substrate or product. Thus noncompetitive inhibition is the same in both directions. Competitive inluibition is expressed by:

$$
i_{f}=\frac{(\mathrm{I})}{(\mathrm{I})+K_{i_{f}}\left[1+(\mathrm{S}) / K_{s}\right]} \quad i_{r}=\frac{(\mathrm{I})}{(\mathrm{I})+K_{i_{r}}\left[1+(\mathrm{P}) / K_{p}\right]}
$$

and it is evident that $i_{f}$ will generally not equal $i_{r}$ because competition of the inhibitor with substrate will usually be different from that with product. This applies to mechanism 3-105 where there may be different sites for binding of substrate and product; if they are bound at the same site, as in 3-104, $K_{i_{f}}=K_{i_{r}}$ but $K_{s}$ and $K_{p}$ may be different. In partially competitive inhibition or mixed inhibition $K_{i_{f}}$ may differ from $K_{i_{r}}$ also.

One may demonstrate varions differential degrees of inhibition by taking the general values of the constants for mechanism 3-105 (see Eq. 2-18) and assigning various values to the rate constants for the uninhibited and inhibited reactions, bearing in mind that Eq. 2-19 imposes certain restrictions if the over-all equilibrim is to remain unaffected. Some examples are given in Table 3-1 to illustrate possible types of inhibition. It is possible that the reaction in one direction is inhibited while that in the other direction is accelerated. There is little basis for prediction of the behavior in any system unless all the constants and the changes in them induced by the inhibitor are known.

If the inhibitor combines with either the substrate or the product, this naturally leads to a specific inhibition of one direction of the reaction. This may be a factor even though it is not the primary mechanism of the inhibition.

\section{Inhibition of Acceptor Reduction}

It is a common procedure to measure the rate of an oxidation reaction by a determination of the reduction rate of some coenzyme or dye. Much work on dehydrogenases has been done using methylene blue as a hydrogenacceptor and within the past few years a number of versatile acceptors have been introduced into enzymology. Certain problems may arise in connection with inhibitor study. The dye usually does not accept directly from the substrate but from one of the enzyme components in the transport se- 
TABLE 3-1

Effect of Inhibitors on the Forward and Reverse Reactions ${ }^{a}$

\begin{tabular}{|c|c|c|c|c|c|}
\hline & \multicolumn{2}{|c|}{ Mechanism 3.104 } & \multicolumn{3}{|c|}{ Mechanism 3-105 } \\
\hline & Uninhibited & Inlibited & Uninhibited & Inhibited & Inhibited \\
\hline$k_{1}$ & $10^{-3}$ & $10^{-4}$ & $10^{-4}$ & $10^{-4}$ & $10^{-4}$ \\
\hline$k_{-1}$ & $10^{-6}$ & $10^{-8}$ & $10^{-7}$ & $10^{-7}$ & $10^{-7}$ \\
\hline$k_{2}$ & - & - & $10^{-5}$ & $10^{-5}$ & $10^{-6}$ \\
\hline$k_{-2}$ & - & - & $10^{-6}$ & $10^{-6}$ & $10^{-5}$ \\
\hline$k_{3}$ & $10^{-9}$ & $10^{-10}$ & $10^{-7}$ & $10^{-8}$ & $10^{-6}$ \\
\hline$k_{-3}$ & $10^{-6}$ & $10^{-6}$ & $10^{-3}$ & $10^{-4}$ & $10^{-4}$ \\
\hline$K_{s}$ & $10^{-3}$ & $10^{-4}$ & $10^{-3}$ & $10^{-3}$ & $10^{-3}$ \\
\hline$K_{p}$ & $10^{-3}$ & $10^{-4}$ & $10^{-4}$ & $10^{-4}$ & $10^{-2}$ \\
\hline$K_{m_{s}}$ & $10^{-3}$ & $10^{-4}$ & $10^{-3}$ & $1.83 \times 10^{-4}$ & $1.75 \times 10^{-3}$ \\
\hline$K_{m_{p}}^{s}$ & 1 & $10^{-2}$ & $10^{-4}$ & $1.81 \times 10^{-4}$ & $1.89 \times 10^{-3}$ \\
\hline$i_{f}$ & - & 0.82 & - & 0.83 & 0.33 \\
\hline$i_{r}$ & - & 0.10 & - & 0.07 & -2.11 \\
\hline
\end{tabular}

$a K_{s}$ and $K_{p}$ are true dissociation constants whereas $K_{m_{s}}$ and $K_{m_{p}}$ are Michaelis constants. In all cases $(\mathrm{S})=(\mathrm{P})=10^{-3} M$. The negative value of $i_{r}$ in the last column indicates stimulation.

quence; different dyes may accept from different components, as illustrated in the following diagram where the hydrogen-atom or electron path is indicated by arrows:

$$
\mathrm{SH}_{2} \rightarrow \mathrm{X}_{1} \rightarrow \mathrm{X}_{2} \rightarrow \mathrm{X}_{3} \rightarrow \ldots \ldots \rightarrow \mathrm{X}_{n} \rightarrow \mathrm{O}_{2}
$$

The various components of the enzyme complex $\left(\mathrm{X}_{1}, \mathrm{X}_{2}\right.$, etc.) may be bound metal ions, flavine dinucleotides, nicotinamide dinucleotides, or any substance that is oxidized and reduced in the transport. An inhibitor may depress the rate markedly when one dye is used and scarcely at all with another dye; such would be the case if the inhibitor interfered with either component $\mathrm{X}_{2}$ or $\mathrm{X}_{3}$ above, reduction of $\mathrm{D}^{\prime}$ being inhibited without action on D reduction. Heavy metal chelating agents, such as 1,10-phenanthroline, inhibit potently the reduction of triphenyltetrazolium dyes by several dehydrogenase systems but have little effect on reduction of dichlorophenol-indophenol. Since a great deal of work has been published on "dehydrogenase" inhibition using the dye reduction technique, it is 
important to realize that the nature of the dehydrogenase has been defined by the dye used. Unfortunately it is not known from what components most dyes accept hydrogen-atoms or electrons.

The final transfer to the dye is generally a reaction that is nonbiological in the sense that it replaces another transfer reaction occurring in the intact system. An inhibitor may possibly interfere with the dye reduction and yet exert no action, or a different type of action, on the normal transfer sequence. That the final dye reduction step is involved in the kinetics and may often be limiting is indicated by the fact that the over-all rate is often dependent on dye concentration and that different dyes lead to greatly different over-all rates. The inhibitor may compete with the dye, in which case the dissociation constant of the enzyme-dye complex is involved. It is also possible that the flow of hydrogen-atoms or electrons is not exchnsively to the dye; in mechanism 3-107 the acceptor D competes with component $X_{2}$ and division of the flow will depend on several factors. Such a possibility is often circumvented by using anaerobic conditions or inhibiting the reaction to oxygen with cyanide. It is preferable, whenever possible, to determine the reduction of some normal component but, even here, the situation may not be simple; the reduction of added $\mathrm{DPN}^{+}$. for example, may be inhibited differently than the reduction of tightly-bound $\mathrm{DPN}^{+}$.

Calculation of the inhibition has often been made from an equation of the type:

$$
i=\frac{\left(1 / t_{s}\right)-\left(1 / t_{i s}\right)}{\left(1 / t_{s}\right)}
$$

where $t_{s}$ is the time required for a specified amount of dye reduction in the presence of the substrate and $t_{i s}$ is the time when both substrate and inhibitor are present. This equation involves two assumptions: $(a)$ that the rate of the reaction is inversely proportional to the time required to reach a certain end-point and $(b)$ that there is no endogenous activity. If the rate of dye reduction is linear or if reduced dye appears exponentially as the substrate is oxidized, according to the equation $\left(\mathrm{DH}_{2}\right)=\left(\mathrm{S}_{0}\right)\left(1-e^{-k t}\right)$ where $\left(\mathrm{S}_{0}\right)$ is the initial substrate concentration, $1 / t$ is proportional to the rate but this need not be so in all cases; this point should be verified under the experimental conditions employed. With regard to the second assumption. it is always necessary to introduce two other measurements, unless they can be shown to be unimportant. The correct equation for the inhibition is:

$$
i=\left[\frac{1}{t_{s}}-\frac{1}{t_{0}}\right]-\left[\frac{1}{t_{i s}}-\frac{1}{t_{i}}\right] /\left[\frac{1}{t_{s}}-\frac{1}{t_{0}}\right]
$$

where $t_{0}$ is the time required in the absence of substrate (endogenous) and $t_{i}$ is the time in the presence of only the inhibitor. These two terms correct for 
the endogenous rate and the effect of the inhibitor upon it. An example of the use of this equation is given by Euler (1942) in a study of competitive inhibition of dehydrogenases.

\section{EXPRESSION AND INTERPRETATION OF INHIBITION}

Recording of the inbibition merely as the fractional inhibition $i$ or the per cent inhibition is never illuminating with respect to the mechanism of the inhibition. Even the determination of a constant characteristic of the inhibition, such as $K_{i}$, is not generally indicative of the manner in which the inhibitor exerts its action. Various ways of expressing inhibition will be discussed in this section and a few aspects of the molecular interpretation of inhibition kinetics will be mentioned.

\section{The Ratio $K_{s} / K_{i}$ for Competitive Inhibition}

The clegree of inhibition in a competitive system does not depend only on $K_{i}$ but on the ratio of the substrate and inhibitor constants, i.e., the relative affinities for the enzyme. This may be seen by transposing the rate Eq. 3-12 for competitive inhibition to:

$$
v_{i}=V_{m} \frac{(\mathrm{S})}{(\mathrm{S})+K_{s}+(\mathrm{I})\left(K_{s} / K_{i}\right)}
$$

wherein the deviation from uninhibited Michaelis-Menten behavior is given by the term (I) $\left(K_{s} / K_{i}\right)$. In deriving Eq. 3-12 an equilibrium system was assumed but in general it is more correct to use $K_{m} / K_{i}$. Now, although it is $K_{m} / K_{i}$ that determines the inhibition at any inhibitor concentration, this ratio does not necessarily provide a measure of the relative affinities. It is often of interest to calculate the relative binding energies of substrate and inhibitor but this can be done only from $K_{s} / K_{i}$. When the proper reciprocal plots are made, the $K_{i}$ is derived are true dissociation constants, but the $K_{m}$ 's may not be. Uncritical comparison of $K_{i}$ with $K_{m}$ leads to no valid conclusions about molecular events or the energetics of the reaction. It is quite simple to determine $K_{m} / K_{i}$, either by determining each constant separately or directly (see Chapter 5), but in few studies of inhibition is one certain of the more important ratio. Sometimes it is not too difficult to determine the individual rate constants if $K_{m}=\left(k_{-1}+k_{2}\right) / k_{1}$ or adjust the conditions so that $K_{m}=K_{s}$. For example, if one can reduce $k_{2}$ by some means, it is possible by extrapolation to arrive at $k_{-1} / k_{1}$, as was done by Thorn (1953) for the malonate inhibition of succinic dehydrogenase. The $V_{m}$ was varied by using different concentrations of the dye acceptor, methylene blue, and this was plotted against $K_{m} / K_{i}$; the intercept on the 
$K_{m} / K_{i}$ axis was interpreted to indicate the value of $K_{s} / K_{i}$ since at this point $V$ and hence $k_{2}$ would be zero. A similar method was suggested by Slater (1955) and the ensuing discussions of this procedure and its limitations should be consulted (Slater, 1955, pp. 301-308). The important point is that $K_{m} / K_{i}$ is frequently meaningless and varies widely with the conditions of the experiment. The affinity ratio $K_{s} / K_{i}$ is however interpretable thermodynamically and from it can be calculated the difference in free energy of binding of substrate and inhibitor.

Reaction of the inhibitor with the substrate or with the enzyme-coenzyme complex leads to types of inhibition superficially competitive. If such mechanisms are not recognized, the determined $K_{i}$ 's will bear no physical relationship to the $K_{s}^{\prime}$ 's and the ratio $K_{s}^{\prime} K_{i}$ is meaningless. In partially competitive and mixed inhibitions, $K_{i}$ is altered by the binding of substrate to the same degree that $K_{s}$ is altered by inhibitor; the determined $K_{i}$ may thus not represent the true dissociation constant of the EI complex if certain methods of evaluating the constants are used (see Chapter 5).

\section{Variation of $K_{i}$ with the Nature of the Substrate in Competitive Inhibition}

According to the classic formulation of competitive inhibition, the value of $K_{i}$, as determined by the various graphical procedures, should be independent of the substrate used for a particular enzyme. However, Klein (1960) found that in the inhibition of pig kidney D-amino acid oxidase by benzoate, determinations of $K_{i}$ from $1 / v_{i}-1(\mathrm{~S})$ plots led to different values for each substrate (see tabulation). Furthermore, there appears to

\begin{tabular}{|c|c|c|c|}
\hline Substrate & Relative $V_{m}$ & $\begin{array}{c}K_{s} \\
(\mathrm{~m} \cdot)\end{array}$ & $\begin{array}{c}K_{i} \\
(\mathrm{~m} \cdot I)\end{array}$ \\
\hline $\begin{array}{l}\text { Alanine } \\
\text { Proline } \\
\text { Phenylalanine } \\
\text { Valine } \\
\text { Isoleucine } \\
\text { Methionine }\end{array}$ & $\begin{array}{l}1.00 \\
1.66 \\
1.39 \\
0.60 \\
0.79 \\
1.32\end{array}$ & $\begin{array}{r}6.3 \\
5.8 \\
14.0 \\
4.6 \\
4.1 \\
5.3\end{array}$ & $\begin{array}{l}0.059 \\
0.070 \\
0.092 \\
0.096 \\
0.104 \\
0.163\end{array}$ \\
\hline
\end{tabular}

be no correlation between $K_{i}$ and either $V_{m}$ or $K_{s}$. One possible explanation for this behavior was suggested. If the inhibitor reacts only with the ES complex to release $\mathrm{S}$ :

$$
\mathrm{ES}+\mathrm{I} \rightleftharpoons \mathrm{EI}+\mathrm{S} \quad K_{i}{ }^{\prime}=\frac{(\mathrm{ES})(\mathrm{I})}{(\mathrm{EI})(\mathrm{S})}
$$


the formation of EI would depend on the particular ES involved. The rate equation is the same but (I) $K_{s} / K_{i}$ is replaced with $(\mathrm{I}) / K_{i}{ }^{\prime}$, since $K_{i}{ }^{\prime}=$ $K_{i} / K_{s}$. However, inasmuch as the slopes of the $1 / v_{i}-1(\mathrm{~S})$ plots would be:

Classic competition:

Reaction with ES:

$$
s=\frac{K_{s}}{V_{m}(\mathrm{~S})}\left[1+\frac{(\mathrm{I})}{K_{i}}\right]
$$$$
s=\frac{K_{s}}{V_{m}(\mathrm{~S})}\left[1+\frac{(\mathrm{I})}{K_{i}{ }^{\prime} K_{s}^{\prime}}\right]
$$

and since $K_{i}^{\prime} K_{s}=K_{i}$, it would appear that $K_{i}$ is determined in either case. Also there is evidence that benzoate does combine with the free enzyme in the absence of substrate. We shall see there are many reasons why the experimentally determined inhibitor constant is not necessarily the true dissociation constant; the reason in the present case is unknown.

\section{The Inhibition Index}

This term is conducive to confusion since it has been defined according to two unrelated concepts. One definition arose in the field of competitive antagonism of metabolites as applied to growth inhibition in microorganisms. McIlwain (1942) defined the "antibacterial index" as (I)/(M) where (I) is the concentration of inhibitor that is just bacteriostatic in the presence of metabolite at a concentration (M), a metabolite here being either a substrate or cofactor. Shive and Hacow (1946) related this more specifically to competitive enzyme inhibition and defined the index as the ratio $(\mathrm{I}) /(\mathrm{S})$ when maximal inhibition occurs. Later workers have tended to call this ratio the "index of inhibition " (Albert, 1960, p. 50) or " inhibition index" (Ariens et al. 1955) and it has been generally assumed that this ratio remains constant for any degree of inhibition. Sometimes the inhibition index refers to the ratio (I)/(S) for which $50 \%$ inhibition is observed. Albert stated: "For each pair of substances there will be a unique index of inhibition, which is defined as the ratio of the number of molecules of analogne per molecule of metabolite required to give 50 per cent inhibition." One of the most frequent errors encountered in work on antagonism and enzyme inhibition is the belief that this ratio or inhibition index is constant. In fact, it is often stated that (I)/(S) $=K_{i} / K_{s}$. It is obvious that this is not generally so and that the true expressions are:

$$
\begin{gathered}
\frac{(\mathrm{I})}{(\mathrm{S})}=K_{i}\left[\frac{1}{K_{s}}+\frac{1}{(\mathrm{~S})}\right] \frac{i}{1-i} \\
\left\{\frac{(\mathrm{I})}{(\mathrm{S})}\right\}_{0.5}=\frac{K_{i}}{K_{s}}+\frac{K_{i}}{(\mathrm{~S})}
\end{gathered}
$$


This ratio will thus depend on (S) and is not a constant, as pointed out by Quesnel (1956), but will approach $K_{i} / K_{s}$ at high substrate concentrations (generally greater than $5 K_{s}$ ) when the enzyme would be mainly saturated, a fact pointed ont by Shive and Macow for the antibacterial index. Figure 3-21 illustrates the marked changes in (I)/(S) with substrate concentration. In fact, it is possible by plotting (I)/(S) against $1 /(\mathrm{S})$ for any chosen degree of inhibition to obtain values for $K_{i}$ and $K_{s}$, as can be seen from Eq. 3-114. It may also be noticed that even at higher substrate concentrations, (I)/(S) at $50 \%$ inhibition will equal $K_{i} / K_{m}$ in the general case and may not relate to the relative affinities of substrate and inhibitor for the enzyme.

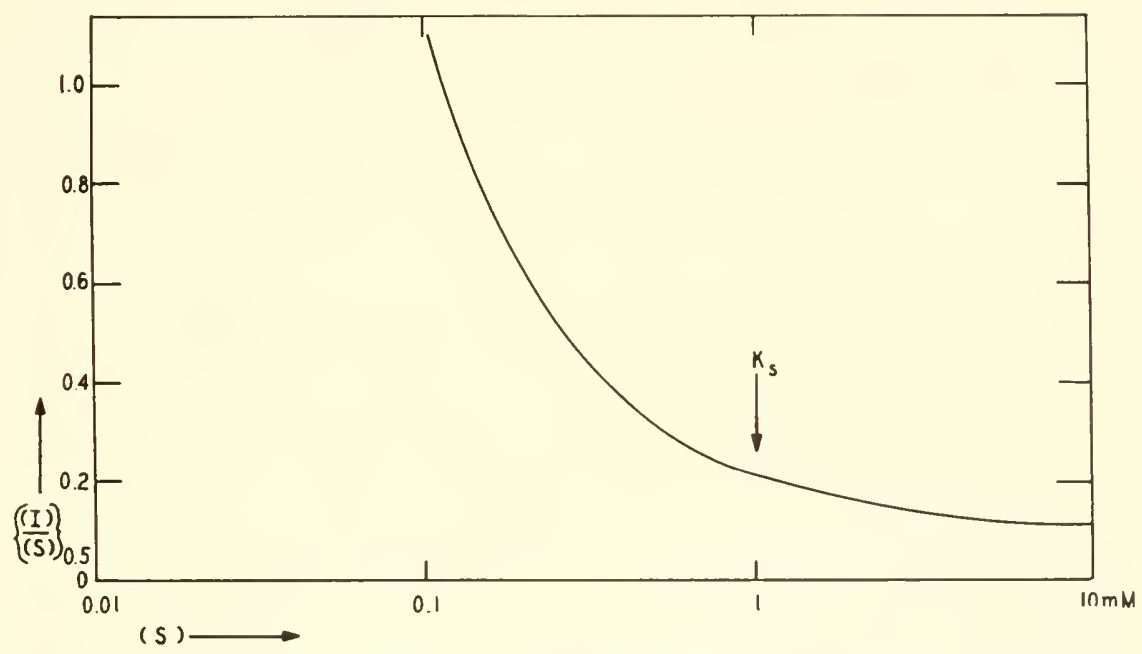

FIG. 3-21. Variation of $[(I) /(S)]_{0.5}$ with the substrate concentration for competitive inhibition. $K_{s}=1 \mathrm{~m} . /$ and $K_{i}=0.1 \mathrm{~m} . I$.

An entirely different definition of the inhibition index was made by Kistiakowsky and Shaw (1953 a) to facilitate expression of complex inhibition behavior.

$$
\varphi=\frac{v-v_{i}}{v_{i}}=\frac{i}{1-i}=\frac{i}{a}
$$

where $v$ is uninhibited rate, $v_{i}$ the inhibited rate, $i$ the fractional inhibition, and $a$ the fractional activity. The inhibition index here is not a constant but another way of expressing the degree of inhibition.

Noncompetitive inhibition: $\quad \varphi=(\mathrm{I}) / K_{i}=\left(\mathrm{I}^{\prime}\right)$

Competitive inhibition:

$$
\varphi=\frac{(\mathrm{I})}{K_{i}\left[1+(\mathrm{S}) / K_{s}\right]}=\frac{\left(\mathrm{I}^{\prime}\right)}{1+\left(\mathrm{S}^{\prime}\right)}
$$


The advantage of $q$ is that it is an additive function; if an inhibitor combines with two or more intermediates in an enzyme reaction or there are two or more noninteracting inhibitors, the total $q$ can be expressed as the sum of the individual $\varphi$ 's:

$$
\varphi_{\text {total }}=\sum_{i=1}^{n} \varphi_{i}
$$

when there are $n$ different contributions to the inhibition. The fractional inhibition, of course, is not additive. In certain complex inhibitions the treatment may thus be simplified, as in the inhibition of urease by thiourea which is complicated by inhibition by hydrogen ion and high concentrations of urea, as well as buffer effects. There is no particular advantage in using the inhibition index for simple inhibitions.

\section{Possible Heterogeneity of Active Centers}

It is generally assumed that enzyme molecules in a population are identical with respect to the molecular structure of their active centers and that the affinities for substrate and inhibitor do not vary from molecule to molecule. Heterogeneity however is not unlikely, at least in certain instances, since protein molecules may not be as rigidly uniform as more simple substances. Pauling et al. (1944) in their studies on hapten inhibition of antisera found that the results could be adequately explained on the basis of a statistical distribution of inhibitor constants among the antibody molecules. It is not suggested that this is evidence for heterogeneity of enzymes but the theoretical approach made to antibody group variation can be applied to enzyme inhibition if necessary. The distribution function is of the form:

$$
\frac{1}{\sigma \sqrt{\pi}} \exp \left[-\frac{\left(\ln K_{i} / K_{i_{0}}\right)^{2}}{\sigma_{2}}\right]
$$

where $K_{i}$ is the inhibitor constant for a particular enzyme, $K_{i_{0}}$ is the average inhibitor constant as determined experimentally, and $\sigma$ is the heterogeneity index. It is possible for different values of $\sigma$ to calculate the probability of a particular enzyme having its $K_{i}$ within a certain range. In the tabulation below $84 \%$ of the enzyme active centers will have $K_{i}$ 's within the range given. If $\sigma=2,84 \%$ of the active centers will have $K_{i}$ 's distributed throughout a range such that the highest $K_{i}$ is approximately fifty-seven fold greater than the lowest and the difference in binding energy of the inhibitor to the enzyme is $2.4 \mathrm{kcal} / \mathrm{mole}$ between these extremes in the given range; $16 \%$ of the active centers 


\begin{tabular}{c|c|c}
\hline$\sigma$ & Range of $K_{i}$ & Range of interaction free energy \\
\hline & & \\
\hline & $0.37 K_{i_{0}}-2.72 K_{i_{0}}$ & $1.2 \mathrm{kcal} / \mathrm{mole}$ \\
3 & $0.13 K_{i_{0}}-7.48 K_{i_{0}}$ & $2.4 \mathrm{kcal} / \mathrm{mole}$ \\
4 & $0.05 K_{i_{0}}-20.1 K_{i_{0}}$ & $3.6 \mathrm{kcal} / \mathrm{mole}$ \\
& $0.02 K_{i_{0}}-54.5 K_{i_{0}}$ & $4.8 \mathrm{kcal} / \mathrm{mole}$ \\
\hline
\end{tabular}

will have $K_{i}$ 's outside this range. The heterogeneity index $\sigma$ must be determined experimentally and in hapten-antibody reactions varied from 1.5 to 3 usually, with an average above 2 , indicating a marked degree of variation; there is no basis for estimating what the values of $\sigma$ may be for enzymes. It is possible that the interpretation of $K_{i}$ should be made on a statistical basis and that interaction energies calculated from them should be considered as average values. 



\section{CHAPTER 4}

\section{SUBSTRATE INHIBITION AND PRODUCT INHIBITION}

Many enzymes conform to Michaehis-Menten kinetics over a limited lower range of substrate concentration but deviate at higher substrate concentrations in that the rate progressively falls off, the expected maximal rate not being attained; indeed, at very high concentrations of substrate the rate may be reduced almost to zero. This phenomenon is termed substrate inibition and in the 50 years since it was initially observed it has become increasingly evident that several mechanisms may be responsible. Inhibition of the rate by a product of the reaction has also been reported for several enzymes and this is designated product inhibition. True product inhibition is not a matter of a shift in the thermodynamic equilibrium between substrate and product (i.e.. an increase in the reverse reaction) but a specific interference with the forward reaction. These types of inhibition will be discussed not only because of their inherent importance in enzyme kinetics and their possible regulation of intracellular metabolism, but also because systems which exhibit such phenomena demonstrate modified kinetics in inhibitions produced by other substances. Thus an instance of pure competitive inhibition may be obseured if increasing substrate not only displaces the inhibitor from the enzyme but inhibits the reaction itself.

\section{SUBSTRATE INHIBITION}

A variety of enzymes has been shown to be inhibited by substrate and the following partial list will indicate that this is a rather general phenomenon: xanthine oxidase (Dixon and Thurlow, 1924; Hofstee, 1955), fumarase (Alberty et al., 1954), lactic dehydrogenase (Hakala et al. 1956), $\beta$-hydroxysteroid dehydrogenase (Marcus and Talalay, 1955), acetylcholinesterase (Myers, 1952 c), enolase (Westhead and Malmström, 1957), ribonuclease (Dickman and Ring, 1958), urease (Laidler and Hoare, 1949; Kistiakowsky and Shaw, 1953a), carboxypeptidase (Lumry et al., 1951), lipases (Bamann and Schmeller, 1929; Murray, 1930), diamine oxiclase (Zeller et al., 1939), L-amino acid oxidase (Dixon and Webb. 1958, p. 86), $\beta$-fructofuranosidase (Nelson and Schubert, 1928), pyrophosphatase (Bailey 
and Webb, 1944; Heppel and Hilmoe, 1951; Bloch-Frankenthal, 1954). aminoacylase I (Mounter et al., 1958), sucrose transglucosylase (Neely, 1958), diphosphoglyceromutase (Joyce and Grisolia, 1959), and p-hydroxyphenylpyruvate oxidase (Zanmoni and La Du, 1959).

\section{Possible Mechanisms Involved}

Before considering the kinetics of substrate inhibition it is helpful to summarize the most important mechanisms by which this type of inhibition may be produced. These mechanisms are schematically illustrated in Fig. 4-1.

TYPE A
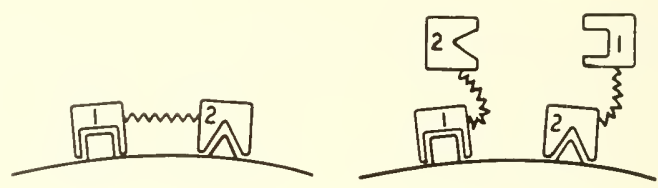

TYPE 8
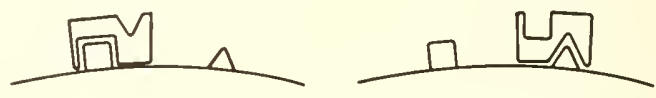

TYPE $C$
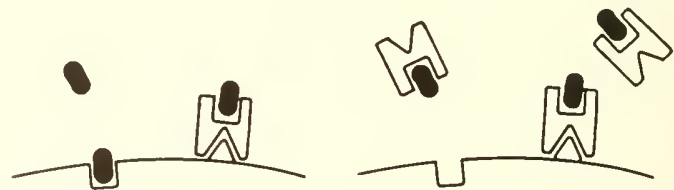

TYPE 0
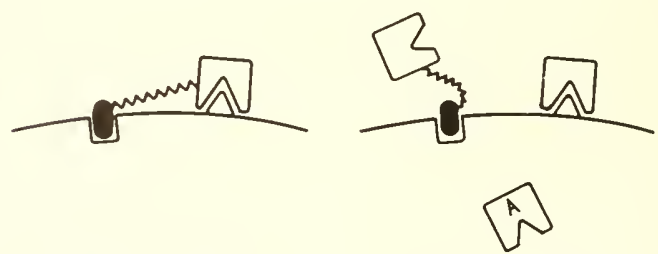

TYPE E
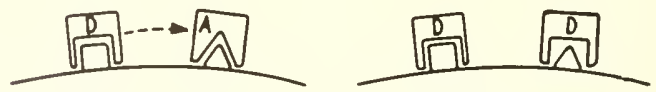

Fig. 4-1. Postulated mechanisms of substrate inhibition. In type $\mathrm{A}$ it may be noted that groups 1 and 2 may be identical. In type B the enzyme sites must be close enough so that interference occurs. In type $\mathbf{E}$ the binding of the second substrate molecule is generally less strong due to the poorer fit. See the text for explanation. 
$A$. The substrate in the active ES complex forms a multipoint attachment to the enzyme (i.e., a substrate molecule is bound to the active center by two or more enzyme groups) so at high substrate concentrations it is possible that two or more substrate molecules can be bound simultaneously at the active center. If multipoint attachment is necessary for reaction, binding of the substrate molecules at the single binding sites will lead to inactive complexes and a reduction of available enzyme.

$B$. The substrate may combine not only at the active substrate site but also at sites in various spatial relationships to the active site, interfering with either the binding of the substrate in an active ES complex or interfering with its reaction when so bound.

$C$. The substrate may react with an activator and at sufficiently high concentration deplete the system of the activator so that the enzyme becomes inactive.

$D$. The true substrate for the enzyme may be a complex of the added substrate with an activator, in which case the added substrate may compete with this complex for binding to the enzyme.

$E$. The substrate may interfere with the binding of a coenzyme or acceptor in transfer reactions.

$F$. The substrate at high concentrations may reduce the water concentration and slow the rate if water is one of the reactants.

$G$. Increasing substrate concentration may increase the ionic strength of the reaction mixture and this may modify the rate independently of any direct or specific effects of the substrate.

Finally, it should be noted that reduction of rate at high substrate concentrations may be due not to the substrate itself but to the ions simultaneously added. If the substrate is an anion, for example, it is possible that the sodium or potassium ions that accompany it may be responsible for the inhibition, in which case the inhibition should not be interpreted as true substrate inhibition. The kinetic behavior of the system in any case will, of course, depend on the mechanism that is involved.

The inhibition of dog liver $p$-hydroxyphenylpyruvate oxidase by excess substrate (Zannoni and La Du, 1959) may be used to illustrate what may be termed pseudosubstrate inhibition. Once the enzyme is inhibited by high substrate concentrations it cannot be reactivated by prolonged dialysis, but reducing agents such as 2,6-dichlorophenol-indophenol will bring about a restoration of the activity. The evidence pointed to an inhibitory substance formed from the substrate; the greater the substrate concentration, the more of this substance was formed. Preliminary extraction 
and isolation of the inhibitor was carried ont and a number of possible products tested for inhibitory activity. This is not true substrate inhibition but could easily be mistaken for it. If one is bent on demonstrating substrate inhibition, it might be well to bear in mind the possibility of an inhibitory product or intermediate, and to study carefully the rate of development of the inhibition.

\section{Inhibition by Partial Attachment to Substrate Sites (Type A)}

A general treatment of the kinetics, assuming that the substrate must biud at two points for reaction, will be presented and various special cases will then be considered as simplifications in this formulation. The reaction scheme may be represented as:

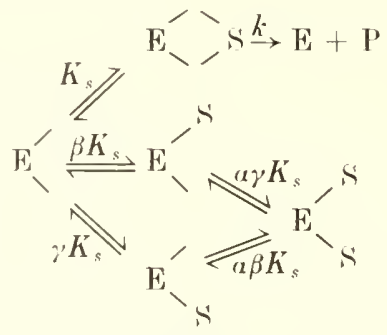

where $\beta$ and $\gamma$ represent the changes in binding constant when the substrate is bound by only one group and $\alpha$ is the interaction constant indicating the effect the first bound substrate molecule has on the binding of the second. It is assumed that reaction occurs only when the substrate is bound by both gromps. The general rate equation for this situation is:

$$
v=V_{m} \frac{\left(\mathrm{S}^{\prime}\right)}{1+\left(\mathrm{S}^{\prime}\right)[1+1 / \beta+1 / \gamma]+\left(\mathrm{S}^{\prime}\right)^{2} / \alpha \beta \gamma}
$$

where $V_{m}$ is the maximal rate that would be attained if the substrate did not bind to the single groups. Specific concentrations are used because it is somewhat easier to examine the effects of variation in the constants $\alpha$, $\beta$, and $\gamma$. The constants $\beta$ and $\gamma$ will generally be greater than unity since the substrate will probably be bound less tightly to one group than to two; $\alpha$ will also usmally be greater than unity because the two binding groups will be reasonably close on the enzyme surface and interaction between simmltaneously bound substrate molecules may be quite marked.

The variation in rate with substrate concentration is plotted in Figs. 4-2 and 4-3 for different values of $\alpha$. When $\alpha=1$ (noneompetitive inhibition by substrate) and the $\mathrm{ES}_{2}$ complex is readily formed, the inhibition is most marked. As a increases, substrate inhibition becomes less and less evident until $\alpha=\infty$ (competitive inhibition by substrate) when the $\mathrm{ES}_{2}$ 


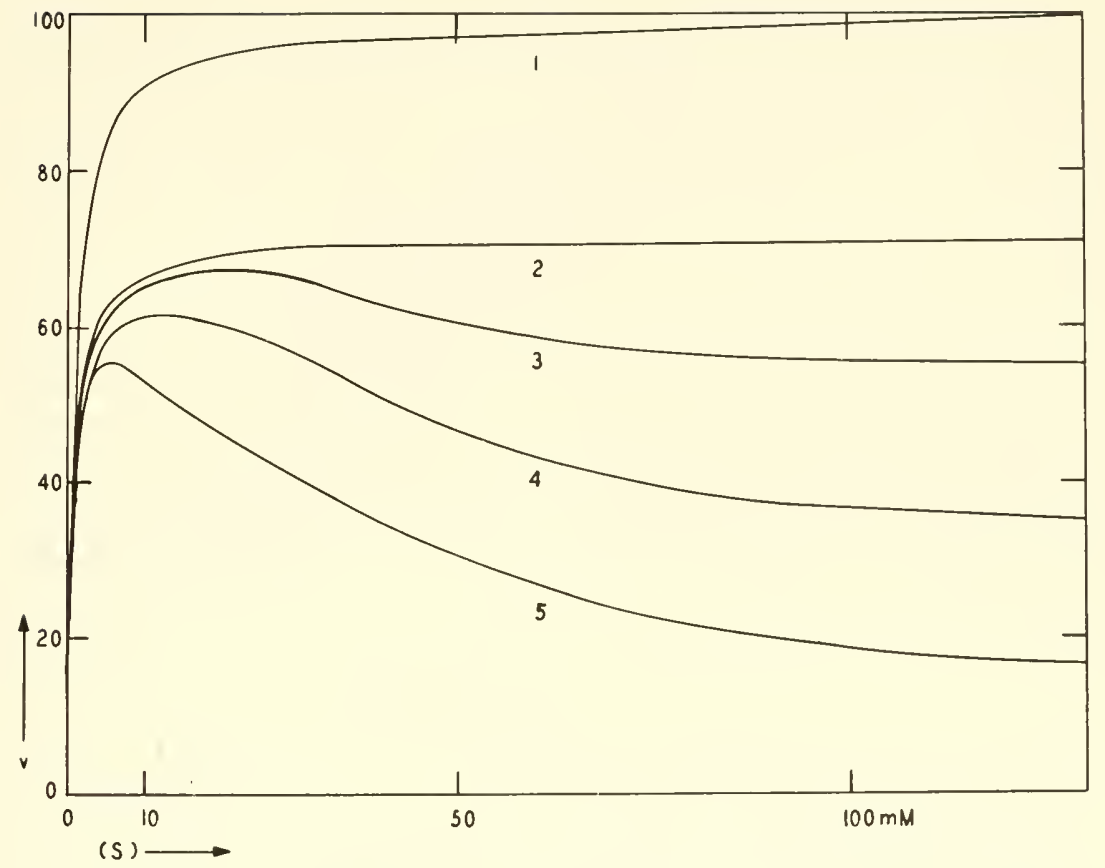

Fig. 4-2. Variation of the rate with the substrate concentration for type A substrate inhibition (Eq. 4-2). Curve 1: $\beta=x, \gamma=x$ (uninhibited); curve $2: \alpha=x, \beta=\gamma=5$; curve 3 : $\alpha=10, \beta=\gamma=5$; eurve $4: \alpha=3, \beta=\gamma=\tilde{5}$; curve 5 : $\alpha=1, \beta=\gamma=5$.

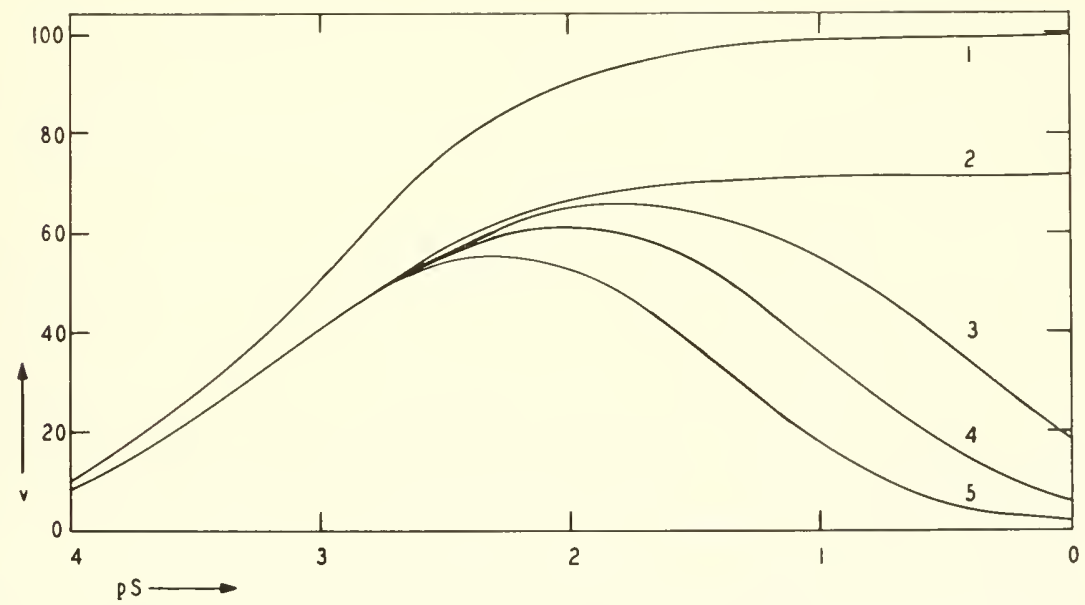

FIG. 4-3. Variation of the rate with the substrate concentration for type A substrate inhibition (Eq. 4-2) using the pS scate. The values for the eurves are the same as in Fig. 4-2. 
complex is not formed and no decrease in rate is found at high substrate concentrations.

When $u=\infty$ the rate may be less if the complexes with single groups are formed. In the curves shown, the rate in 2 is always less than in 1 because of the formation of the inactive complexes

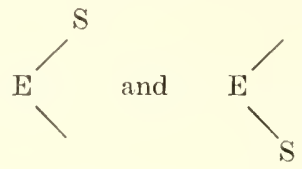

Practically such inhibition would not be recognized since the reciprocal plot of $1 / v$ against $I^{\prime}(\mathrm{S})$ will be linear as in the uninhibited situation and the apparent maximal rate determined from the intercept will be related to the true $V_{m}$ as follows:

$$
V_{m}^{\prime}=V_{m} \frac{1}{1+(1 / \beta)+(1 / \gamma)}
$$

However, the slope of the reciprocal plot is $K_{s} / V_{m}$ so that the use of $V_{m}^{\prime}$ will introduce an error in the determination of $K_{s}$. The apparent $K_{s}$ will actually be a mixed dissociation constant. Thus when complete competitive substrate inhibition occurs, it will not be recognized. The phenomenon termed substrate inhibition will be observed only when there is completely noncompetitive inhibition or mixed inhibition, i.e., when $\alpha=1$ or some finite value. There has been some confusion in terminology inasmuch as typical substrate inhibition (where the rate decreases at high substrate concentrations) has been called competitive (Dixon and Webb, 1958, p. 83) whereas by the usual criteria it is noncompetitive since the binding of the second substrate molecule is not prevented but the rate of substrate breakdown is reduced to zero.

In Figs. 4-2 and 4-3 the constants $\beta$ and $\gamma$ have been assigned arbitrary values of 5, indicating symmetrical binding and a reduction in affinity compared to the active complex. It may be noted that if the binding energy of the substrate to each single group is half that for the total binding in the active complex, $\beta=\gamma=1 / \sqrt{K_{s}}$. For $K_{s}=10^{-3} M$. as in the figures, it is seen that $\beta=\gamma=31.6$ and the inhibition would be less marked than shown. Theoretically these constants may have any values from less than unity to infinity and, of course, will differ when the binding sites are dissimilar.

When the rate is plotted against pS, as in Fig. 4-3, substrate inhibition of the type here considered is always represented by symmetrical bellshaped curves. Distortion of the symmetry indicates either that the inhibition is more complex than assmmed here (e.g., more than two substrate 
molecules bound) or occurs by a different mechanism. The peak of the rate curve is defined by the various constants as follows:

Substrate concentration for maximal rate: $\quad\left(\mathrm{S}_{0}{ }^{\prime}\right)=\sqrt{\alpha \beta \gamma}$

Maximal rate attained: $v_{0}=V_{m} \frac{1}{1+1 / \beta+1 / \gamma+(2 / \sqrt{\alpha \beta \gamma})}$

The value of $\left(\mathrm{S}_{0}{ }^{\prime}\right)$ is obtained by differentiating Eq. 4-2 and setting $d v / d\left(\mathrm{~S}^{\prime}\right)$ $=0$, and the peak rate in obtained by substituting this value in Eq. 4.2.

The usual formulation for this type of inhibition has been somewhat different from that given above. The treatment given originally by Haldane $(1930$, p. 84) and generally followed by others (Dixon and Webb, 1958, p. 81) assumes that a second substrate molecule is bound to the ES complex to inactivate:

$$
\begin{aligned}
\mathrm{E}+\mathrm{S} \rightleftharpoons \mathrm{ES} \stackrel{k}{\rightarrow} \mathrm{E}+\mathrm{P} \\
\mathrm{ES}+\mathrm{S} \rightleftharpoons \mathrm{ES}_{2}
\end{aligned}
$$

by which it is meant that the following reactions occur:

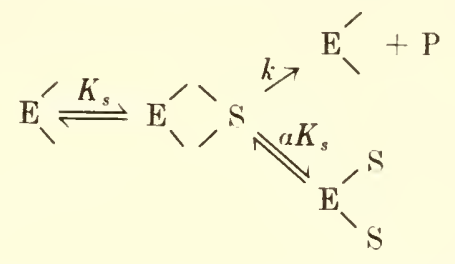

This differs basically from the previous treatment in that inactive complexes of the enzyme with single substrate molecules are ignored and the only inactive species is $\mathbf{E S}_{2}$. The rate equation is given by:

$$
v=V_{m} \frac{\left(\mathrm{S}^{\prime}\right)}{1+\left(\mathrm{S}^{\prime}\right)+\left(\mathrm{S}^{\prime}\right)^{2} / \alpha}=V_{m} \frac{1}{1+1 /\left(\mathrm{S}^{\prime}\right)+\left(\mathrm{S}^{\prime}\right) / \alpha}
$$

where the constant $\alpha$ corresponds to its previous meaning. representing the interference of one substrate molecule on the binding of the second. Since this equation is of the same general form as Eq. 4-2, it is impossible experimentally to distinguish between them and curves of similar form are predicted. The basic difference in these treatments is that Haldane's expresses the effect of the formation of $\mathrm{ES}_{2}$ only while the present formulation involves also the inactive ES complexes. In some respects, it depends on the choice of a normal baseline for the uninhibited system, but on the other hand the interpretation of the determined constants will depend on the pattern of reactions assumed. 
Inhibition by Attachment to Enzyme Groups Not Involved in the Active Complex (Type B)

This situation presents no special problems since the substrate interferes with the reaction in the same manner as the inhibitors discussed in Chapter 3 and the appropriate equations may be obtained by substitution of (S) for (I) in those equations and the replacement of $K$, by $\gamma K_{s}$ if desired (see below). An interesting formulation has been given by Friedenwald and Maengwyn-Davies (1954, p. 180) and a similar, bnt somewhat more general, treatment may be made assuming the following reaction scheme, in accordance with Eq. 3-2:

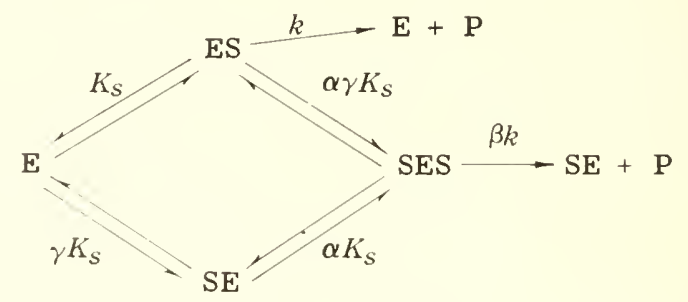

where ES is the reactive complex and SE the complex that inhibits either the formation or reactivity of ES. The rate equations for different types of interference are:

Completely competitive $(\alpha=\infty)$ :

$$
v=V_{m} \frac{1}{1+1 /\left(\mathrm{S}^{\prime}\right)+1 / \gamma}
$$

Partially competitive $(\infty>a>1, \beta=1)$ :

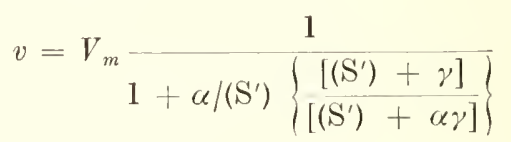

Completely noncompetitive $(\alpha=1, \beta=0)$ :

$$
v=V_{m} \frac{1}{1+1 /\left(\mathrm{S}^{\prime}\right)+1 / \gamma+\left(\mathrm{S}^{\prime}\right) / \gamma}
$$

Partially noncompetitive $(\alpha=1,0<\beta<1)$ :

$$
v=V_{m} \frac{1+(\beta / \gamma)\left(\mathrm{S}^{\prime}\right)}{1+1 /\left(\mathrm{S}^{\prime}\right)+1 / \gamma+\left(\mathrm{S}^{\prime}\right) / \gamma}
$$

Coupling (uncompetitive) $(\alpha=0)$ :

$$
v=V_{m} \frac{1}{1+1 /\left(\mathrm{S}^{\prime}\right)+\left(\mathrm{S}^{\prime}\right) / \alpha \gamma}
$$


It is likely that the majority of inhibitions will be noncompetitive inasmuch as secondary groups in the active center that would bind the substrate in inactive complexes must be uncommon. Noncompetitive and coupling inhibition lead to equations indistinguishable experimentally from those of type A inhibition (Eqs. 4-2 and 4-7), so that the interpretation of the determined constants is arbitrary unless additional evidence indicates the mechanism. It may be noted that the usual formulation of type A inhibition by Haldane is essentially identical to coupling inhibition of type B as far as kinetics are concerned. The rate-pS curves for the major types of inhibition are shown in Fig. 4-4 and the effect of variation in affinity for

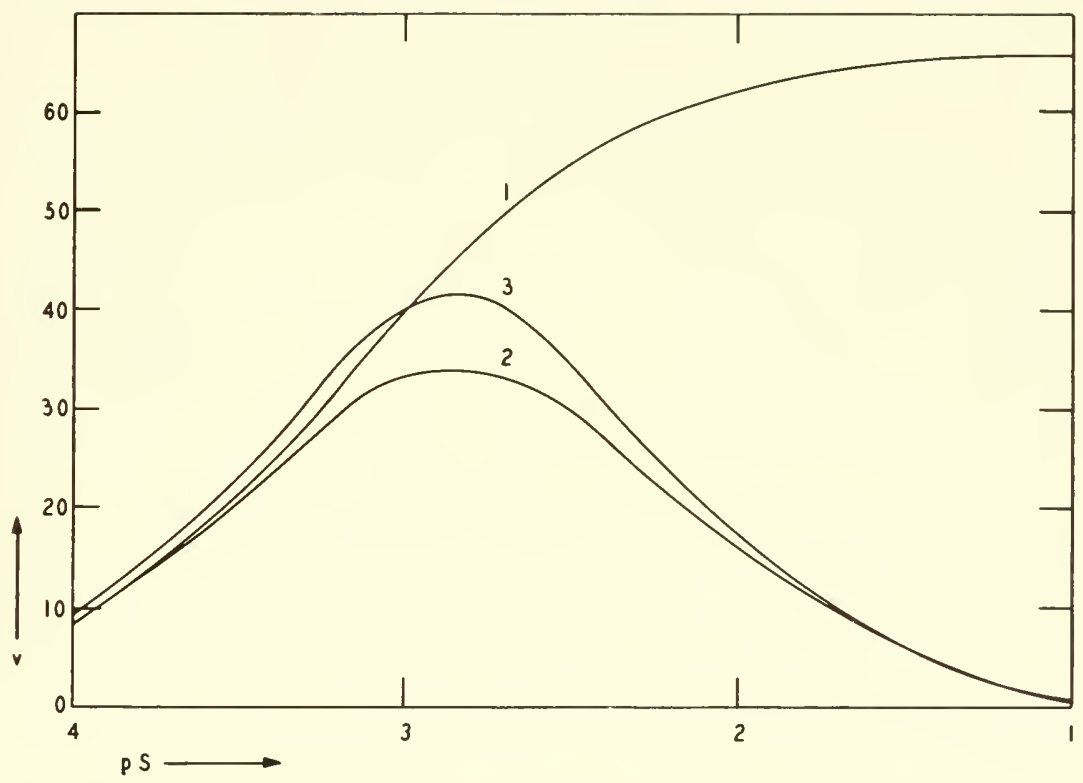

FIG. 4-4. Variation of the rate with the substrate concentration for type B substrate inhibition of different kinds (scheme 4-8). $V_{m}=100, K_{s}=1 \mathrm{~m} M$, and $\gamma=2$. Curve 1: competitive inhibition; curve 2: noncompetitive inhibition; curve 3: coupling inhibition.

the inhibiting substrate molecule is illustrated in Fig. 4-5 for noncompetitive inhibition. The shift in peak upwards and to higher substrate concentrations with decreasing affinity is evident. Inhibition has been considered only with respect to interference with substrate, but a second molecule of substrate can also, of course, interfere with the binding or reactivity of a coenzyme, activator, or acceptor. The appropriate rate equation can be obtained by simple substitutions in the equations given in the previous chapter.

If the rate at high substrate concentrations does not continue to fall 
towards zero but levels off at a finite value as the concentration of substrate is increased, this implies that the SES complex can break down to form the product and that the inhibition is partially noncompetitive. It may be seen from Eq. 4-12 that at high substrate concentrations, the rate is given by $v=\beta V_{m}$. This situation occurs, for example, in the case of carboxypeptidase (Lumry et al. 1951). Such systems have been discussed by Laidler (1958, p. 71) and Reiner (1959, p. 67).

Very few attempts have been made to distinguish between type $\mathrm{A}$ and type B mechanisms. The work of Hofstee (1955) is notable in this respect.

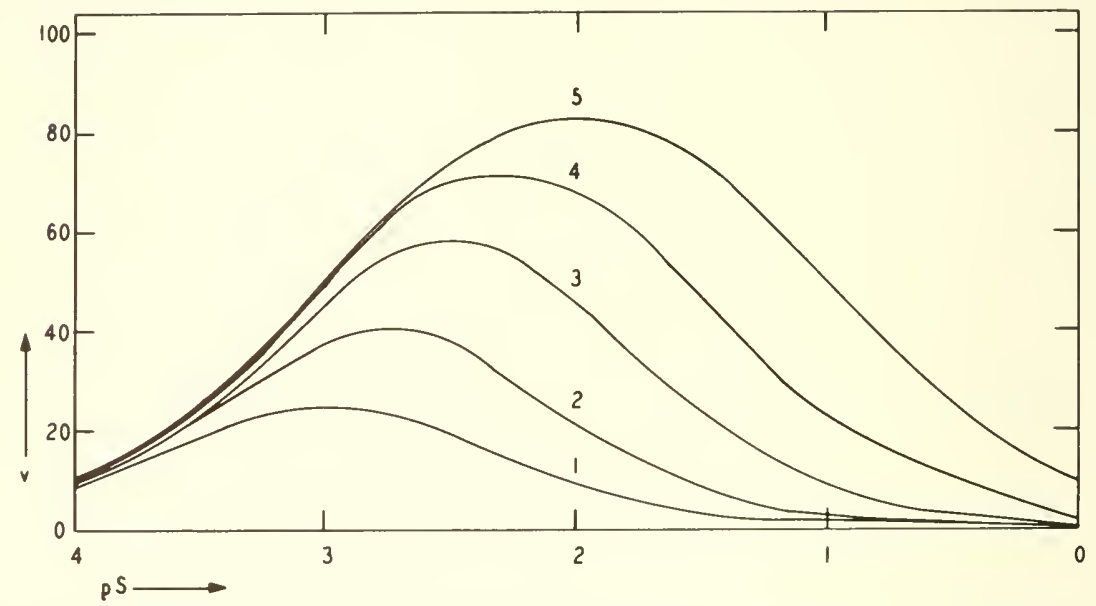

FIG. 4-5. Variation of the rate with the substrate concentration for completely noncompetitive type B substrate inhibition (Eq. 4-11). $K_{s}=1 \mathrm{~m} \cdot M$ and $V_{m}=100$. Curve 1: $\gamma=1$; curve $2: \gamma=3$; curve 3: $\gamma=10$; curve $4: \gamma=30$; curve 5: $\gamma=100$.

He showed for xanthine oxidase that kinetic data using substrate alone are insufficient to determine the nature of the inhibition. However, by the use of the competitive inhibitor isoxanthopterin he was able to distinguish between the two mechanisms (see p. 137). It was concluded that a noncompetitive type $\mathrm{B}$ inhibition was most likely, with $K_{\mathrm{s}}=2 \times 10^{-5} \mathrm{M}$, $\gamma=50, V_{m}=6.5$, and $v_{0}=5.1$. The binding of two xanthine molecules was believed to occur independently at distinet groups on the enzyme; one group was responsible for the binding and activation of the xanthine, while the other was necessary in the oxidation but not involved in the binding. If this is correct, $\gamma$ does not represent an interaction constant but merely the difference in binding between the two groups. Isoxanthopterin competes with xanthine for the active group but not for the anxiliary group; if the substrate inhibition had been of type $\mathrm{A}$, isoxanthopterin would probably compete with both substrate molecules. 
These conclusions are supported by the results of Fridovich and Handler (1958) who showed that substrate inhibition is marked when two-electron acceptors are used to measure the enzyme activity, but does not occur when the one-electron acceptor cytochrome $c$ is used. Furthermore, the substrate inhibition is reduced as the $\mathrm{pH}$ is raised and the enzyme nonheme iron becomes increasingly susceptible to auto-oxidation as is shown in the tabulation. It is postulated that the active center includes two mol-

\begin{tabular}{r|c}
\hline \multicolumn{1}{c|}{$\mathrm{pH}$} & Per cent substrate inhibition \\
\cline { 1 - 2 } 5.5 & 82 \\
7.7 & 71 \\
9.1 & 58 \\
10.1 & 23 \\
\hline
\end{tabular}

ecules of FAD bridged by a pair of ferric mercaptide groups; the flow of electrons occurs from the substrate to FAD-1 through the iron atoms to FAD-2 and hence to the two-electron acceptor dyes or oxygen. Cytochrome $c$, on the other hand, probably withdraws electrons from the non-heme iron directly and FAD-2 is not involved. The evidence thus points to the site of substrate inhibition as the FAD-2, which can presumably react with purines as does the FAD-1 site.

Several examples of substrate inhibition have been followed over a sufficiently wide range of substrate concentration to conclude that they obey type A-B kinetics, i.e., that they conform to an equation of the type:

$$
v=V_{m} \frac{1}{\mathrm{~A}+1 /\left(\mathrm{S}^{\prime}\right)+\left(\mathrm{S}^{\prime}\right) / \mathrm{B}}
$$

and give rate-pS curves that are symmetrical. It is not possible, however, to deduce the exact mechanism of the inhibition. Typical curves are illustrated in Figs. 4-6 to 4-9. It is likely that the esterase inhibition is of type A because of the probability that two binding groups are involved in the active complex; if this is so, the species differences in peak position indicate different degrees of binding interference between the substrate molecules. However, in the case of the $\beta$-hydroxysteroid dehydrogenase it is more difficult to visualize type A inhibition because of the large size of the substrate and the nature of the binding, since the evidence from substrate specificity points to the relatively high binding energy as being due to the summation of multiple dispersion forces between the steroid and the enzyme surface. For the lactic dehydrogenase it is possible that type A inhibition occurs and that the second pyruvate molecule is bound mainly by its aliphatic portion because of the $3 \mathrm{kcal} /$ mole difference between the 
binding in the active and inhibiting groups $(\alpha=139)$, indicating that pyruvate is bound differently in the two complexes. Nevertheless such reasoning in these cases is qualitative only and in the absence of pertinent data is only speculation. On the other hand, substrate inhibition in some systems, such as ribonuclease (Dickman and Ring, 1958), does not conform to these kinetics and cannot be mechanistically interpreted. The depression of substrate inhibition by increasing ionic strength, without a simul-

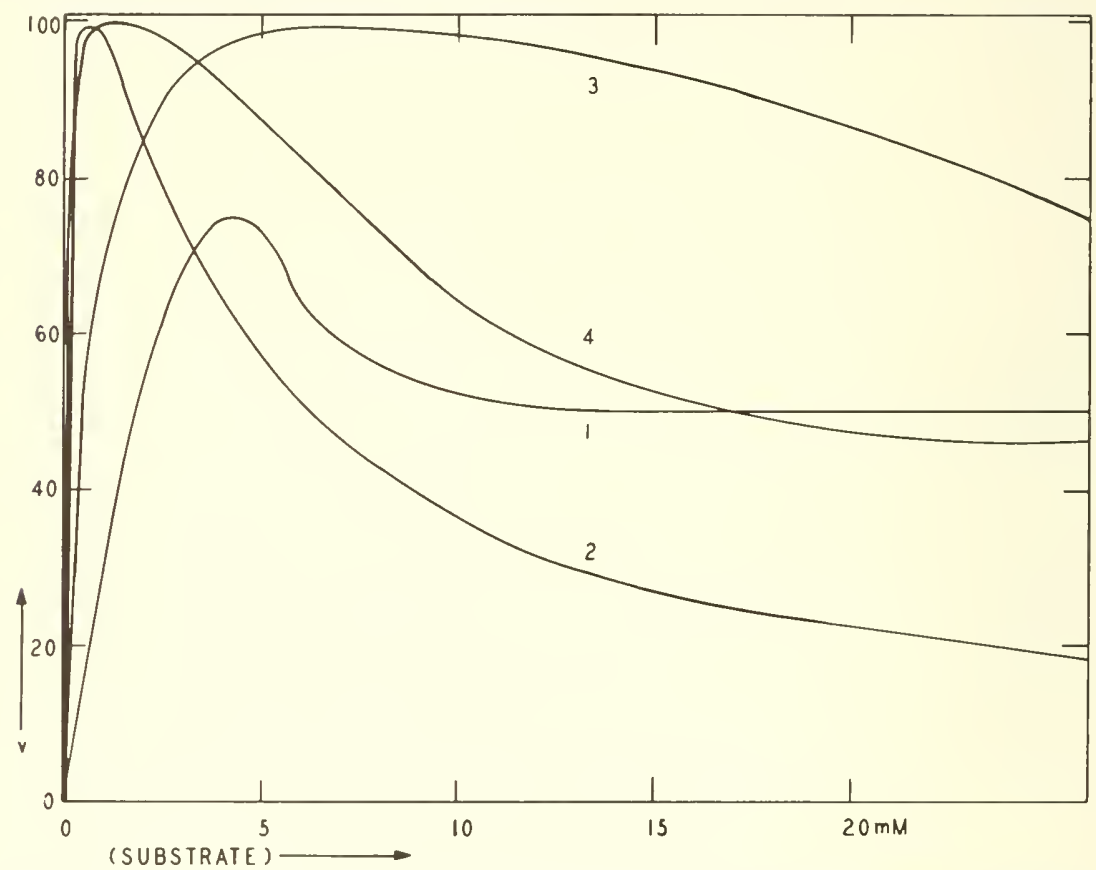

FIG. 4-6. Examples of substrate inhibition plotted on a linear substrate concentration scale. Curve 1: erythrocyte cholinesterase (Zeller and Bissegger, 1943). Curve 2: lactate dehydrogenase (Hakala et al. 1956). Curve 3: steroid dehydrogenase (Mareus and Talalay, 1955) (substrate concentration in $\mu . M$ ). ('urve 4: human erythrocyte cholinesterase (Myers, 1952 c).

taneous depression of ribonuclease activity, might indicate that the inhibition is due to relatively nonspecific adsorption through electrostatic interactions while the active complex involves a different type of binding.

The crystalline lactic deliydrogenase from heart catalyzes the reaction:

$$
\text { Pyruvate }+\mathrm{DPNH}+\mathrm{H}^{+} \rightleftharpoons \text { lactate }+\mathrm{DPN}^{+}
$$

which can be readily followed spectrophotometrically. Potent substrate inhibition is observed with pyruvate as shown in Fig. 4-6. The data conform 


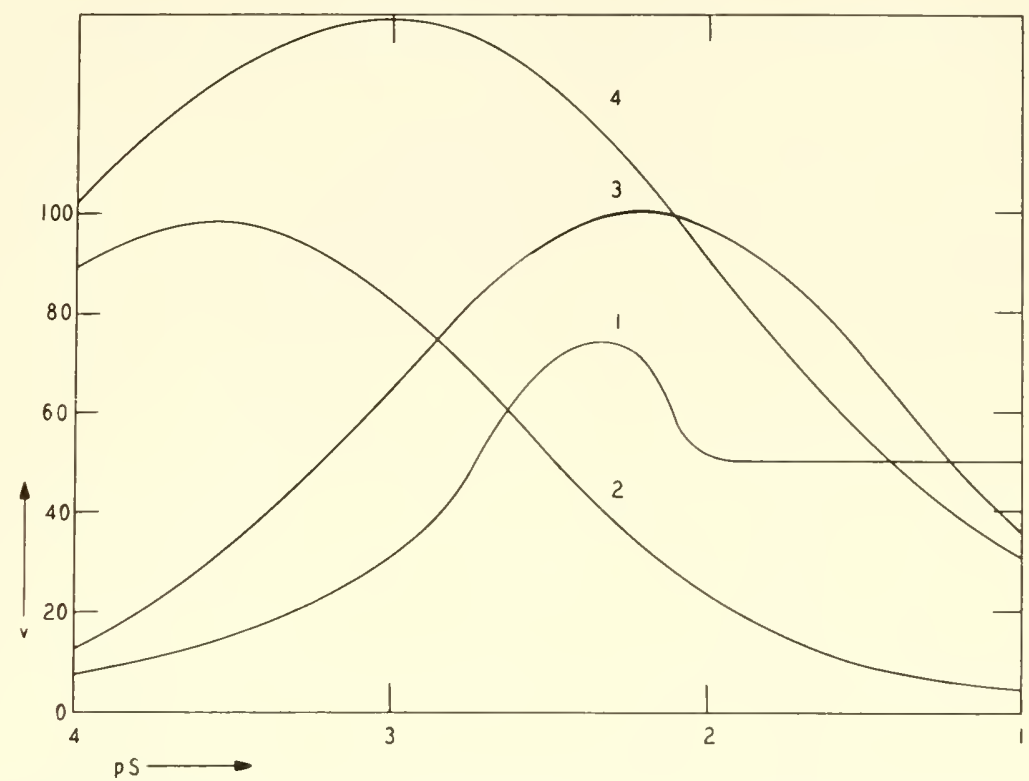

FIG 4-7. Examples of substrate inhibition plotted on a ps scale. Curves are the same as in Fig. 4-6. Curve 4 shifted vertically from previous figure.

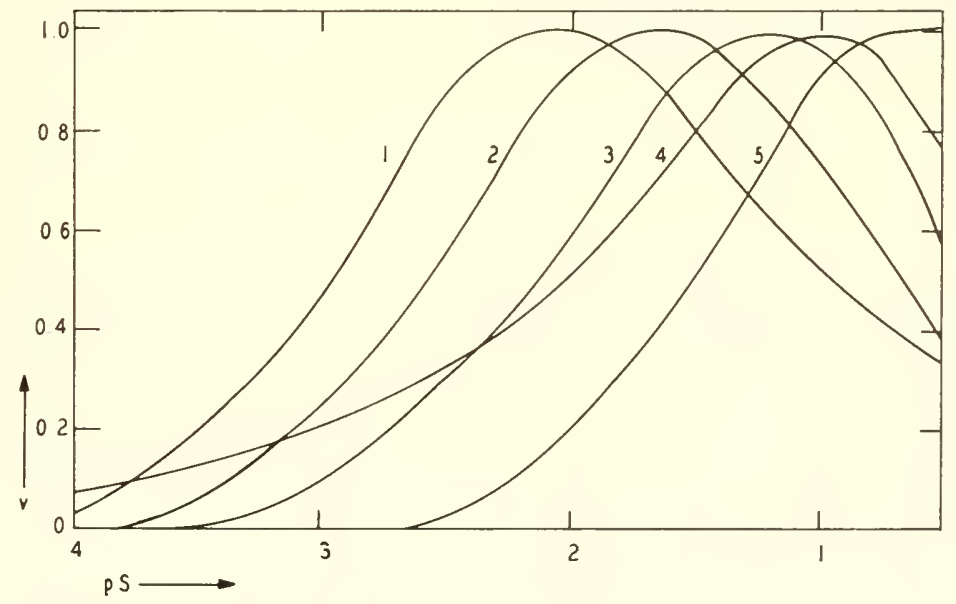

FIG. 4.8. Substrate inhibition exhibited by liver esterases. Curve 1: sheep; curve 2: dog; curves 3 and 4: human; curve 5: horse. (From Bamann and Schmeller, 1929.) 
to an equation such as $4-7$ with $K_{m}=0.018 m M$ and $\alpha=139$ at $\mathrm{pH} 6.8$. The active complex is probably E-DPNH-pyruvate and $K_{m}$ for pyruvate is a complex function of several rate constants, involving the binding of DPNH, so it is doubtful if a simple interpretation of $K_{m}$ can be made (Winer and Schwert, 1958). However, $K_{m}$ is equal to or greater than the dissociation constant for pyruvate in the active complex so that the second inhibiting pyruvate molecule is bound less tightly by at least $3 \mathrm{kcal} / \mathrm{mole}$.

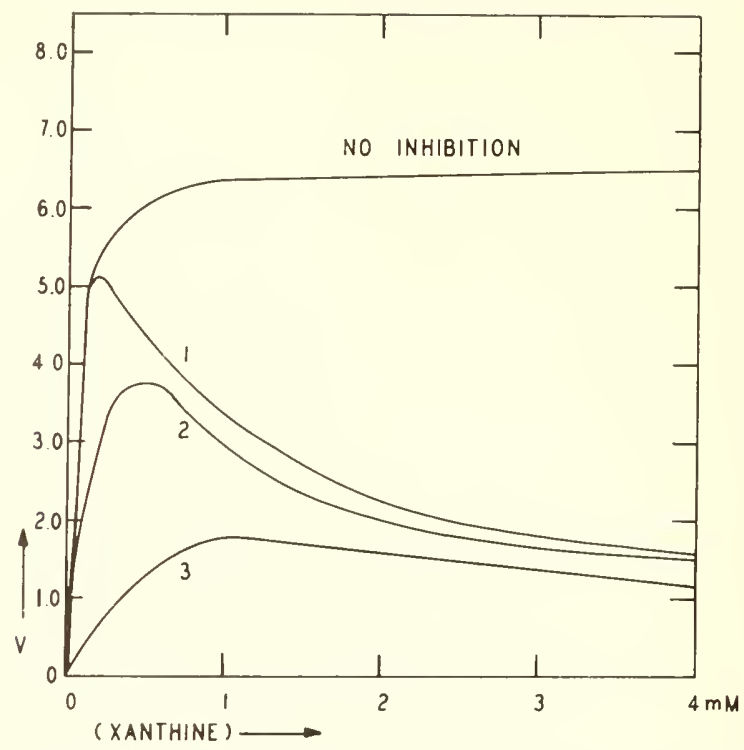

FIG. 4-9. Inhibition of xanthine oxidase by substrate and isoxanthopterin. (From Hofstee, 1955.) Curve 1: inhibition by substrate (xanthine) alone; curve 2: isoxanthopterin $(0.01 \mathrm{~m} M)$; curve 3 : isoxanthopterin $(0.1 \mathrm{~m} M)$.

The binding of both pyruvate molecules depends on $\mathrm{pH}$. The inhibition by pyruvate decreases with increase in $\mathrm{pH}$ as shown in Fig. 4-10; the constants used to construct these curves are given in the tabulation below.

\begin{tabular}{c|c|c|c}
\hline $\mathrm{pH}$ & $\begin{array}{c}K_{m} \\
(\mathrm{~m} M)\end{array}$ & $\begin{array}{c}\alpha K_{m} \\
(\mathrm{~m} M)\end{array}$ & $\alpha$ \\
\hline 6.11 & 0.08 & 5.2 & 65 \\
7.00 & 0.14 & 8.4 & 60 \\
8.02 & 0.46 & 24.0 & 52 \\
9.10 & 1.55 & 38.0 & 25 \\
9.95 & 6.7 & 113 & 17 \\
\hline
\end{tabular}


Rise in $\mathrm{pH}$ thus reduces affinity for both pyruvate molecules but has relatively less effect on the inhibitory binding. If the inhibition by pyruvate occurs when two pyruvate molecules are bound to two sites with which only one pyruvate molecule is bound actively, increase in $\alpha K_{m}$ with $\mathrm{pH}$ probably reflects in part the same decrease in affinity for pyruvate in the active complex. An imidazolium ring of histidine with a $\mathrm{p} K_{a}$ of about 7 may be involved in the binding and proton transfer, since there is evidence that

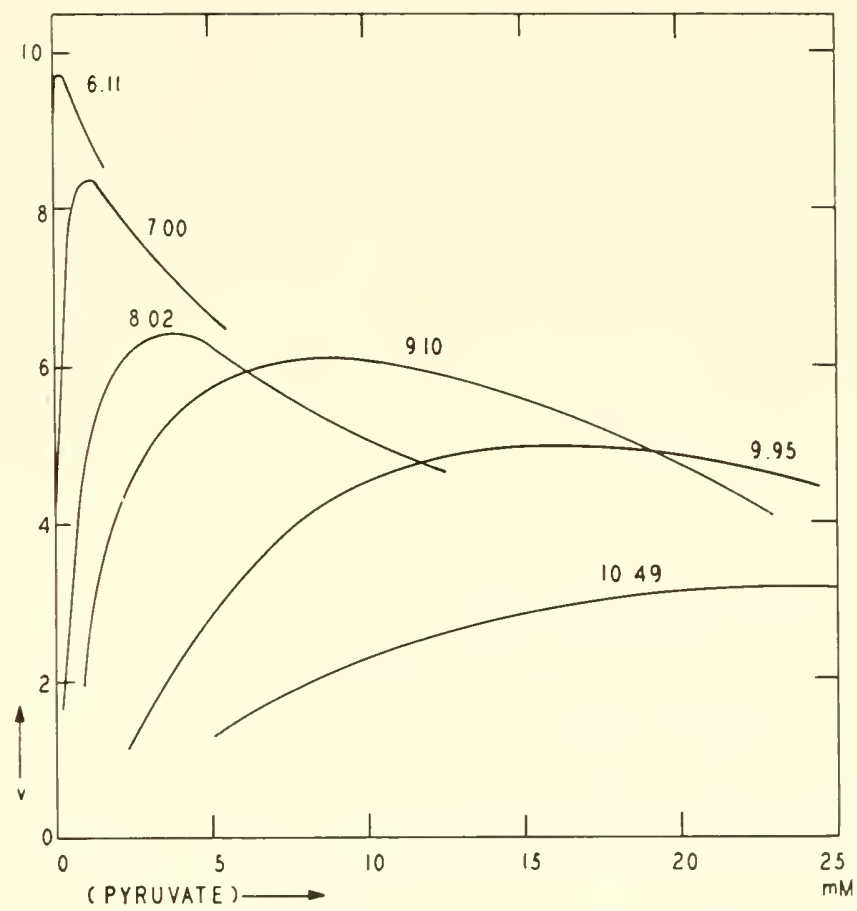

Frg. 4-10. Inhibition of the reverse reaction of lactate de. hydrogenase by pyruvate at different pH's. (From Winer and Schwert, 1958.)

the hydrogen ion does not come directly from the solution. Inhibition by higher concentrations of DPNH is usually not observed (Hakala et al., 1956; Nygaard, 1956; Winer and Schwert, 1958) but may occur if the pyruvate concentration is low, indicating a competitive situation.

Substrate inhibition of cholinesterase has been studied extensively and illustrates several interesting principles. Zeller and Bissegger (1943) postulated two binding sites in the active center, an anionic site with a negative charge to bind the $-\mathrm{N}\left(\mathrm{CH}_{3}\right)_{3}{ }^{+}$end of the acetylcholine molecule and an 
esteratic site to bind and hydrolyze the ester group. In conformity with the suggestion of Haldane, they explained substrate inhibition in terms of the binding of two molecules of acetylcholine in an inactive complex.
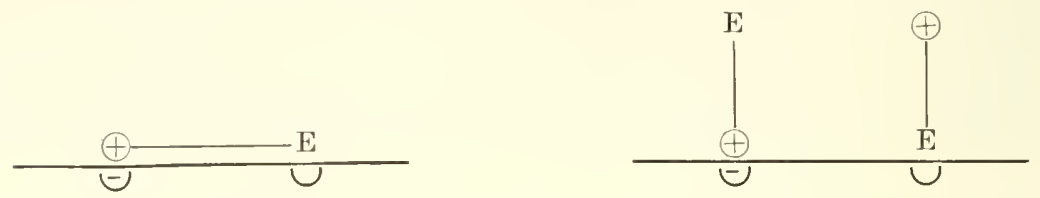

Such inhibition oceurs only with acetylcholinesterase (true cholinesterase) from erythrocytes and mammalian tissues, and not with the psendocholinesterases of serum (Alles and Hawes, 1940). Inasmuch as the rate-pS curves for human erythrocyte acetyleholinesterase are somewhat asymmetrical. Myers $(1952 \mathrm{c})$ concluded that the $\mathrm{ES}_{2}$ complex was partially active. Using an equation of the type 4-12 he showed that a value of $\beta=0.1$ accomted satisfactorily for the experimental data, i.e., the activity of the $\mathrm{ES}_{2}$ complex is one-tenth that of the ES complex.

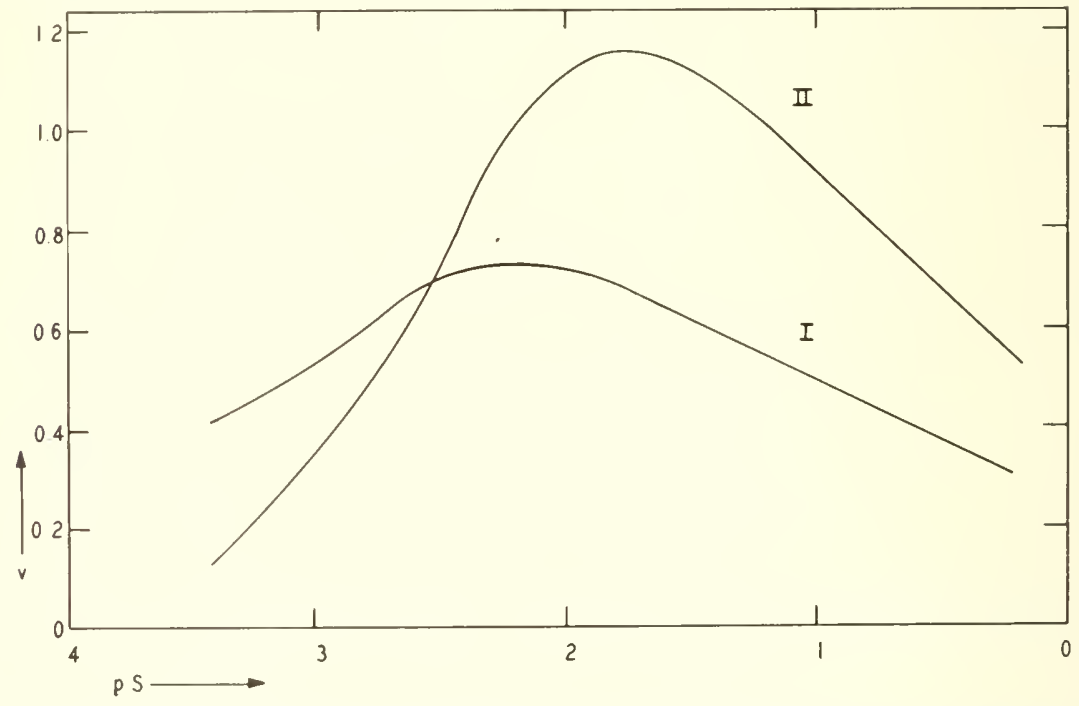

FIG. 4-11. Substrate inhibition of acetylcholinesterase at different salt concentrations. (From Alles and Hawes, 1940.) Curve I: $0.0059 . / \mathrm{NaCl}$; curve II: $0.145 . / I \mathrm{NaCl}$.

Increase of the ionic strength alters the form of the rate-pS curves in a characteristic way (Alles and Hawes, 1940). A change of $\mathrm{NaCl}$ eoncentration from $0.0059 . I$ to $0.145 M$ shifted the $s_{0}$ from 2.25 to 1.80 and led to an asymmetry as shown in Fig. 4-11. Inereasing the ionic strength slows the rate at low substrate concentrations and accelerates it at high substrate concen- 
trations. This salt effect was investigated more thoronghly by Myers (1952 c) who confirmed the earlier results and determined the effects of ionic strength on the varions constants, from which it is evident that increasing ionic strength decreases the affinity of the enzyme for acetylcholine but enhances the hydrolytic activity. That this effect is not entirely a nonspecific salt effect is inclicated by the fact that the doubly-charged $\mathrm{Ca}^{++}$ion exerts a stronger action than $\mathrm{Na}^{+}$, since $5 \mathrm{~m} .1 / \mathrm{CaCl}_{2}$ altered the rate-pS curve comparably to $200 \mathrm{~m} M \mathrm{NaCl}$. The effects of $\mathrm{NaCl}$ concentration on the reaction constants, based on Eq. 4-7, are shown in the tabulation below.

\begin{tabular}{|c|c|c|c|c|c|c|}
\hline $\begin{array}{l}\mathrm{NaCl} \\
(\mathrm{m} M)\end{array}$ & $\begin{array}{c}K_{s} \\
(m M)\end{array}$ & $\begin{array}{c}\alpha K_{s} \\
(\mathrm{~m} . M)\end{array}$ & $\alpha$ & $\begin{array}{c}\left(\mathrm{S}_{0}\right) \\
(\mathrm{m} \cdot I)\end{array}$ & $v_{0}$ & $V_{m}$ \\
\hline 0 & 0.055 & 11.0 & 200 & 0.78 & 140 & 157 \\
\hline 20 & 0.110 & 12.1 & 110 & 1.15 & 146 & 169 \\
\hline 50 & 0.19 & 13.5 & 70 & 1.60 & 156 & 190 \\
\hline 100 & 0.26 & 15.5 & 60 & 2.05 & 168 & 208 \\
\hline 200 & 0.37 & 18.0 & 50 & 2.70 & 175 & 221 \\
\hline 500 & 0.55 & 22.0 & 40 & 3.65 & 171 & $2 \cdot 21$ \\
\hline
\end{tabular}

In this formulation, $K_{s}$ is the constant for the active ES complex and $\alpha K_{s}$ for the relatively inactive $\mathrm{ES}_{2}$ complex (designated $K_{s_{1}}$ and $K_{s_{2}}$ by Myers). The interpretation of $\alpha \boldsymbol{K}_{s}$ as the dissociation constant for the second or inhibiting substrate molecule is probably doubtful. since actually in the $\mathrm{ES}_{2}$ complex there are two different dissociation constants and an interaction factor. In the more complete formulation 4-1. what is here designated as $\alpha$ would actually be $\alpha \beta \gamma$. If $\beta \boldsymbol{K}_{s}$ refers to the anionic site and $\gamma K_{s}$ to the esteratic site, it might be expected that $\beta$ would not differ greatly from unity, inasmuch as the major binding energy of the substrate in the active complex derives from interaction at this site. and that $\gamma$ would be reasonably large. It is difficult to estimate $\alpha$ but it should not be too large since the positive charges on the simultaneously bound molecules are maximally separated and from inhibitor studies the two sites would appear to be well separated. It is thus possible that a value of $\alpha \beta \gamma=200$ (in the absence of added $\mathrm{NaCl}$ ) is mainly dne to a high value of $\gamma$. The increase in $K_{s}$ with $\mathrm{NaCl}$ concentration would be expected on the basis of a reduced interaction with the anionic site due to increasing ionic strength or competition for the anionic site by $\mathrm{Na}^{+}$. It is difficult to interpret physically the changes in $\alpha$ or $\alpha \beta \gamma$ with $\mathrm{NaCl}$ concentration because in addition to the possible changes in the dissociation constants themselves, it it is likely that the acidic dissociation of groups at both sites on the enzyme is changed. If the above interpretation is correct, it would appear that the binding to the esteratic site is augmented by high $\mathrm{NaCl}$ concentration. In 
any event, the shift in $\left(\mathrm{S}_{0}\right)$ to higher values with increasing $\mathrm{NaCl}$ concentration wonld be due to the decreased affinity of the enzyme for the substrate. It might be possible to unravel the exact mechanism by a quantitative study of the effects of changes in $\mathrm{pH}$ on the inhibition, since the reactions at the two sites would respond differently. Addition of $\mathrm{CaCl}_{2}$ at 5 $\mathrm{m} M$ increases $K_{s}$ five fold without changing $\alpha K_{s}$; this would be expected if $\mathrm{Ca}^{++}$competed effectively with acetylcholine for the anionic site but did not alter binding at the esteratic site, upon which the vahe of $\alpha K_{s}$ mainly depends. The marked activation of the rate by $\mathrm{CaCl}_{2}$ would also imply an effect on the esteratic site, probably by combination with an adjacent group. It is odd that Angustinsson (1948) reported no effects of $\mathrm{CaCl}_{2}$ $(1-10 \mathrm{~m} M)$ and very little effect of $\mathrm{KCl}(100 \mathrm{~m} M)$ on the substrate inhibition of any type of cholinesterase. The $\mathrm{pS}_{0}$ was shifted only 0.1 unit on the average by $\mathrm{KCl}$.

Very few investigations have been made on the relation of substrate structmre to substrate inhibition, so that the results of Marcus and Talalay (1955) on the $\beta$-hydroxysteroid dehydrogenase are of great interest. The inhibition curves for nine steroids are shown in Fig. 4-12 and the reaction

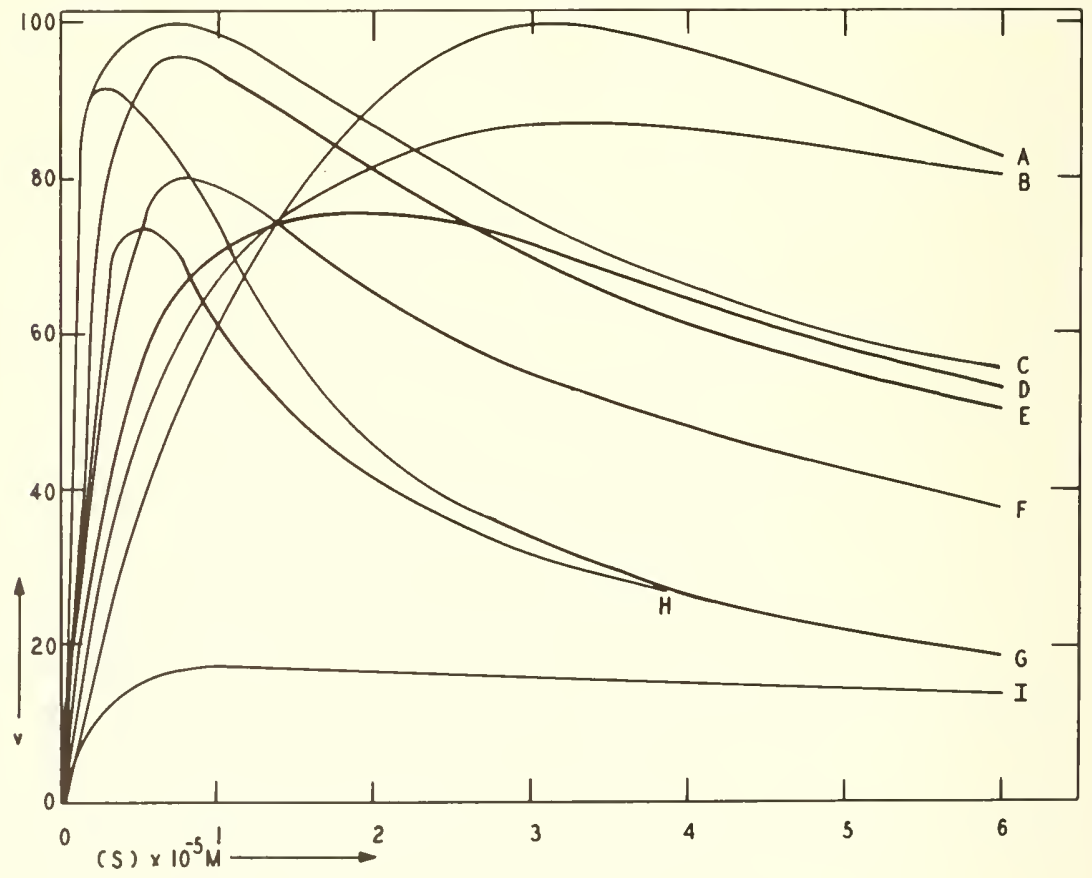

FIG. 4-12. Substrate inhibition of $\beta$-hydroxysteroid dehydrogenase by nine steroids whose structures and kinetic constants are given in the accompanying tabulation.

(From Marcus and Talalay, 1955.) 
Reaction Constants for Substrates Producing Inhibition ${ }^{a}$

\begin{tabular}{|c|c|c|c|c|}
\hline Substrate & $\begin{array}{c}K_{s} \\
(\mu M)\end{array}$ & $\begin{array}{l}\alpha K_{s} \\
(\mu M)\end{array}$ & $\alpha$ & $\begin{array}{c}\left(\mathrm{S}_{0}\right) \\
(\mu M)\end{array}$ \\
\hline $\mathrm{A}$ & 207 & 4.65 & 0.022 & 31 \\
\hline B & 6.86 & 179 & 26 & 35 \\
\hline $\mathrm{C}$ & 0.93 & 39 & 42 & 6 \\
\hline $\mathrm{D}$ & 5.33 & 48 & 9 & 16 \\
\hline $\mathrm{E}$ & 0.87 & 41.4 & 48 & 6 \\
\hline $\mathrm{F}$ & 1.76 & 25.5 & 14.5 & 6.7 \\
\hline G & 1.07 & 8.41 & 7.9 & 3 \\
\hline $\mathrm{H}$ & 1.82 & 8.81 & 4.8 & 4 \\
\hline I & 1.10 & 100 & 91 & 10.5 \\
\hline
\end{tabular}

a In the nomenclature of Marcus and Talalay, $K_{1}=K_{s}$ and $K_{2}=\alpha K_{s}$.<smiles>OC1CCC2C1CCC1c3ccccc3CCC12</smiles>

(A)<smiles>CC(C)C1CC2C3CCC(O)C3CCC2C2(C)CCC(=O)C=C12</smiles><smiles>O=C1CCC2C(CCC3C(O)CCC32)CC1O</smiles>

(B)<smiles>O=C1C=C2CCC3C(CCC4C(O)CCC43)C2CC1</smiles>

(F)<smiles>O=C1C=C2CCC3C(CCC4C(O)CCC43)C2CC1</smiles>

(C)<smiles>CC12CCC(O)CC1C1CCC3C(=O)CCC3C1CC2</smiles>

(D)<smiles>CC12CCC(=O)CC1CCC1C3CCC(O)C3CCC12</smiles>

(G)<smiles>O=C1CCC2C1CCC1C3CCC(O)CC3CCC21</smiles>

(H)<smiles>CC1CCC2C1CCC1C2CCC2C1CCC1C2CCC2C1CCC1C2CCC2CC(O)CCC21C</smiles>

(I)<smiles>O=C1C=C2CCC3C4CCC(O)C4CC(Br)C3C2CC1</smiles>

(2)<smiles>O=C1C=C2CCC3C4C(O)CCC4C(O)CC4(CC2CCC1O)C(O)CCC34</smiles>

(3)

(4)

constants are given in the accompanying tabulation. Four steroids not producing substrate inhibition are also shown for comparison. The remarkable specificity of interaction is apparent in the marked effects produced by minor structural changes: compare particularly $B$ and $G$, or $D$ and $H$, where the difference is only in the $\alpha$ or $\beta$ configuration. The most potent substrate inhibition is given by compounds $(a)$ with the ring system saturated, (b) with one $=\mathrm{O}$ and one $-\mathrm{OH}$ group at either the 3 - or 17-positions, and $(c)$ without substituent groups on the rings (an 11-OH group blocks the inhibition). It is interesting that the constants for $\mathrm{G}$ and $\mathrm{H}$ are very close and yet the molecules have opposite positions for the oxidized $\mathrm{OH}$ group; $\mathrm{B}$ and $\mathrm{D}$ do not show this similarity however. It may be that 
the inhibition is due to interference with $\mathrm{DPN}^{+}$binding since the steroid and $\mathrm{DPN}^{+}$must bind close together because of the stereospecificity in the hydrogen transfer.

Maximal rates for pig kidney aminoacylase were obtained at different concentrations with various substrates (Mounter et al., 1958). Although there are not enough substrates to make any detailed correlations with

\begin{tabular}{l|c}
\hline \multicolumn{1}{|c|}{ Substrate } & $\begin{array}{c}\left(\mathrm{S}_{0}\right) \\
(\mathrm{m} M)\end{array}$ \\
\hline$N$-Acetylglycine & \\
$N$-Propionylglycine & 40 \\
$N$-Butyrylglycine & 33 \\
$N$-Acetylmethionine & 12 \\
& 24 \\
\hline
\end{tabular}

structure, the fall in $\left(\mathrm{S}_{0}\right)$ with a lengthening of the hydrocarbon side-chain would indicate that this part of the molecule is at least involver in the binding to the site of inlibition (see tabulations).

\section{Combination of Substrate with an Activator (Type C)}

Enzymes requiring metal ion activators may show substrate inhibition since their substrates often have carboxylate or phosphate groups which can complex or chelate with the metal ion. Indeed, it is generally believed that metal ions function as activators becanse of their ability to bind the substrate, wholly or in part, to the enzyme. Whether inhibition of this type will or will not occur depends on the binding of the activator to the enzyme: if the activator is primarily bound to the substrate (i.e., if the true substrate is a complex with the activator) no inhibition will occur at high substrate concentrations due to activator depletion, but if the activator is attached primarily to the enzyme. such depletion may occur. These two situations may be represented as follows:

Type C inhibition absent

$$
\begin{gathered}
\mathrm{S}+\mathrm{A} \rightleftharpoons \mathrm{AS} \\
\mathrm{E}+\mathrm{AS} \rightleftharpoons \mathrm{EAS} \rightarrow \mathrm{E}+\mathrm{A}+\mathrm{P}
\end{gathered}
$$

Type $\mathrm{C}$ inhibition may occur

$$
\begin{gathered}
\mathrm{E}+\mathrm{A} \rightleftharpoons \mathrm{EA} \\
\mathrm{EA}+\mathrm{S} \rightleftharpoons \mathrm{EAS} \rightarrow \mathrm{EA}+\mathrm{P} \\
\mathrm{s}+\mathrm{A} \rightleftharpoons \mathrm{AS}
\end{gathered}
$$


The rate and inhibition equations are quite complex, involving a quadratic expression in the most general situation. However, if it is assumed that $\left(\mathrm{E}_{t}\right) \ll\left(\mathrm{A}_{t}\right)$ so that the activator exists mainly in the free and substratecombined forms, the expression for free activator:

$$
(\mathrm{A})=\frac{K_{a s}\left(\mathrm{~A}_{t}\right)}{(\mathrm{S})+K_{a s}}
$$

may be substituted in Eq. 2-69 to give the approximate rate. It would appear, contrary to expectations, that such a mechanism. although it may produce marked inhibition of the rate, cannot lead to a peak in the rate-(S) curves, and hence will not be recognized as typical substrate inhibition.

However, if the activation mechanism follows the reaction scheme 2-70, substitution of the vahue of (A) from Eq. 4-15 leads to an equation of the type:

$$
v=V_{m} \frac{1}{\mathrm{~A}+\mathrm{B} /(\mathrm{S})+(\mathrm{S}) / \mathrm{C}}
$$

where:

$$
\begin{aligned}
& \mathrm{A}=1+\frac{K_{a}}{\left(\mathrm{~A}_{t}\right)}\left[1+\frac{K_{s}}{K_{a s}}\right] \\
& \mathrm{B}=K_{s}\left[1+\frac{K_{a}}{\left(\mathrm{~A}_{t}\right)}\right] \\
& \mathrm{C}=\frac{K_{a s}\left(\mathrm{~A}_{t}\right)}{\left(K_{a}\right)}
\end{aligned}
$$

and the inhibition will lead to a decreasing rate at high substrate concentrations. Such inhibition may be distinguished from substrate inhibition of types $A$ and $B$ by varying the concentration of the activator. If the substrate inhibition is removed by increasing the activator concentration to above optimal levels, it is likely that the substrate is complexing with the activator and removing it from the system. This was done in the case of the inhibition of enolase by 2 -phosphoglycerate (Westhead and Malmström, 1957), where increasing the $\mathrm{Mg}^{++}$concentration from $1 \mathrm{~m} M$ to $10 \mathrm{~m} M$ abolished the inhibition. In such systems the buffer may also play a role, inasmuch as it often can complex with the activator and in the present case a difference was observed between Tris- $\mathrm{HCl}$ and phosphate buffers. The enolase system is also complicated by activator inhibition: high concentrations of $\mathrm{Ig}^{++}$reduce the rate and this is independent of the reduction in free substrate concentration. The kinetics for activator inhibition are worked out in detail by Friedenwald and Maengwyn-Davies (1954, p. 185). Needless to say, the degree of inhibition in type $\mathrm{C}$ systems depends on the value of $K_{a s}$ relative to $K_{a}$ and $K_{s}$, i.e., the relative affinities of activator and substrate for each other and for the enzyme. 


\section{Competition of Substrate with Substrate-Activator Complex (Type D)}

When the true substrate for the enzyme is a complex of the added substrate and an activator (as in the first reaction scheme in the previous section) it is possible that the reaction:

$$
\mathrm{E}+\mathrm{S} \rightleftharpoons \mathrm{ES}
$$

may also occur and block the AS complex from the enzyme. An example of this mechanism may be the inhibition of inorganic pyrophosphatase by excess substrate, first demonstrated by Bailey and Webb (1944). This inhibition was initially believed to be type $\mathrm{A}$, later type $\mathrm{C}$ with pyrophosphate complexing with and removing the activator $\mathrm{Ig}^{++}$(Heppel and Hilmoe, 1951), and most recently type D since evidence was obtained that the true substrate is magnesium pyrophosphate (Bloch-Frankenthal, 1954). One is mable to distinguish between types $\mathrm{C}$ and $\mathrm{D}$ inhibition by varying the concentrations of substrate and activator, since type D is also characterized by a symmetrical rate-pS curve most commonly and a rate equation identical to $4-16$ but with the following values for the constants:

$$
\begin{aligned}
& \mathrm{A}=1+\frac{K_{e a s}}{\left(\mathrm{~A}_{t}\right)}\left[1+\frac{K_{s}}{K_{a s}}\right] \\
& \mathrm{B}=\frac{K_{e a s} K_{a s}}{\left(\mathrm{~A}_{t}\right)} \\
& \mathrm{C}=\frac{K_{a s}\left(\mathrm{~A}_{t}\right)}{K_{e a s}} \\
& K_{e a s}=\frac{(\mathrm{E})(\mathrm{AS})}{(\mathrm{EAS})}
\end{aligned}
$$

Indeed, this type of inhibition is indistinguishable from types A and B if the activator is unrecognized and its concentration not varied. The conformity of most types of substrate inhibition to the same kinetic pattern has made it very easy for theorists to prove their particular mechanism.

\section{The Substrate Interferes with the Binding of an Acceptor (Type E)}

This represents the situation in transfer reactions where one substrate interferes with the bincling of the second substrate. In reactions involving a dye as an electron acceptor, the reduced substrate may hinder the reaction of the dye with the enzyme. The inhibition of xanthine dehydrogenase, using methylene bhe as an acceptor, by high concentrations of xanthine or hypoxanthine has been attributed to such a mechanism (Dixon and 
Thurlow, 1924) since the affinity of the methylene blue for the enzyme was shown to be markedly reduced. The oxidation of lencine by the L-amino acid oxidase of snake venom with methylene blue as an acceptor also shows substrate inhibition of this type (Dixon and Webb, 1958, p. 86). The rate-(S) curves for this mechanism may show a fairly broad platean, instead of a peak, if the affinity of the substrate for the acceptor site is much less than for the substrate site, since inhibition may not occur until the substrate concentration is substantially above that required to saturate the enzyme with respect to activity. By determining the course of substrate inhibition at different concentrations of acceptor, it is possible to identify this type of inhibition and obtain the various dissociation constants, as illustrated in the following chapter. Alberty (1958) has given a complete theoretical analysis of substrate inhibition for situations in which a coenzyme is involved in the reaction and he has included cases where the excess substrate may interfere with the binding of the coenzyme.

\section{The Substrate Reduces the Concentration of Water (Type F)}

When water is one of the reactants, reduction in its concentration may slow the rate, and this seems to be the mechanism for the inhibition by high sucrose concentrations of yeast $\beta$-fructofuranosidase (Nelson and Schubert, 1928). Inhibition was determined over a range of sucrose concentration from $0.17-2.0 M(6-70 \%)$ and the rate was proportional to the water concentration throughout. This type of inhibition is uncommon because concentrations of substrate sufficiently high to reduce water concentration appreciably are seldom used.

\section{The Substrate Inhibits by an lonic Strength Effect (Type G)}

This type of substrate inhibition has not been demonstrated conclusively but certainly is a possibility that cannot be neglected. Substrate inhibition of enolase depends on the ionic strength and disappears when $0.5 \nu_{i}^{r}$ $\mathrm{KCl}$ is present (Westhead and Malmström. 1957). Since at this concentration of $\mathrm{KCl}$, changes in ionic strength due to substrate would be negligible, this might be interpreted to mean that the inhibition is an ionic strength effect. However, as has been discussed, the inhibition has been shown to be due to removal of the activator $\mathrm{Mg}^{++}$by the 2-phosphoglycerate. The effect of increasing the ionic strength with $\mathrm{KCl}$ is probably to decrease the interaction between substrate and activator so that free activator is not reduced so readily. These results serve to illustrate the complexities that may be encountered by varying the ionic strength in enzyme systems with several components. 


\section{Inhibition of Substrate-Inhibited Enzymes by Other Inhibitors}

It might be thonght superficially that the inhibition produced by substrate and the inhibition produced by another inhibitor night be additive but this is never so. The presence of substrate inhibition will alter the kinetics formulated in the previous chapter in a characteristic manner. It would be expected that noncompetitive inhibition is unaffected by substrate inhibition, since the inhibitor does not alter substrate binding nor does substrate binding affect the inhibitor. On the other hand, a competitive inhibitor should modify the kinetics of substrate inhibition and be modified by the substrate inhibition in turn. It is easy to visualize this if one pictures the effects of increasing substrate concentration: there will be two simultaneous and opposing effects - the antagonism of the inliibition because of displacement of inhilsitor from the enzyme and the production of inhibition by whatever mechanism is involved.

We have seen that most types of substrate inhibition can be represented by rate equations of the following form:

$$
v=V_{m} \frac{1}{\mathrm{~A}+\mathrm{B} /(\mathrm{S})+(\mathrm{S}) / \mathrm{C}}
$$

It is easy to show that the inhibited rate equations are:

Completely competitive inhibition

$$
\begin{gathered}
v_{i}=V_{m} \frac{1}{\mathrm{~A}+\mathrm{B} /(\mathrm{S})\left[1+(\mathrm{I}) / K_{i}\right]+(\mathrm{S}) / \mathrm{C}} \\
i=\frac{\left(\mathrm{I}^{\prime}\right)}{\left(\mathrm{I}^{\prime}\right)+1+[(\mathrm{S}) / \mathrm{B}][\mathrm{A}+(\mathrm{S}) / \mathrm{C}]}
\end{gathered}
$$

Completely noncompetitive inhibition

$$
\begin{gathered}
v_{i}=V_{m}^{r_{m}}\left[\begin{array}{c}
1 \\
{\left[\mathrm{I}+\left(\mathrm{I}^{\prime}\right)\right][\mathrm{A}+\mathrm{B} /(\mathrm{S})+(\mathrm{S}) / \mathrm{C}]}
\end{array}\right. \\
i=\frac{\left(\mathrm{I}^{\prime}\right)}{\left(\mathrm{I}^{\prime}\right)+1}
\end{gathered}
$$

These expressions for the inhibition may be compared to Eqs. 3-28 and 3-29 to determine the effect of substrate inhibition. In agreement with expectations, the inhibition in noncompetitive systems is not altered; i.e., the inhibition produced by a noncompetitive inhibitor is neither altered by substrate concentration or substrate inhibition, nor will the presence of a noncompetitive inhibitor alter the rate-ps curves other than to depress them uniformly. On the other hand, a competitive inhibitor will shift the peak of the substrate inhibition curve: 
Substrate inhibition alone:

Presence of competitive inhibitor:

$$
\left(\mathrm{S}_{0}^{\prime}\right)=\sqrt{\mathrm{BC}}
$$

The peak will thus be shifted to higher substrate concentrations.

The simplest and least general representation of type A substrate inhibition as shown in reactions 4-6 may be modified for the presence of an inhibitor as follows:

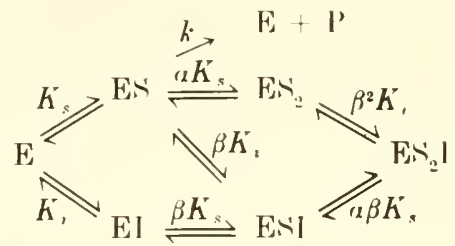

and the general rate equation written as:

$$
v_{\imath}=V_{m} \frac{1}{1+\frac{1}{\left(\mathrm{~S}^{\prime}\right)}\left[1+\left(\mathrm{I}^{\prime}\right)\right]+\frac{\left(\mathrm{S}^{\prime}\right)}{\alpha}\left[1+\frac{\left(\mathrm{I}^{\prime}\right)}{\beta^{2}}\right]+\frac{\left(\mathrm{I}^{\prime}\right)}{\beta}}
$$

from which the special cases of competitive and noncompetitive inhibition may be derived: completely competitive inhibition $(\beta=\infty)$,

$$
\begin{gathered}
v_{2}=V_{m} \frac{1}{1+\left[1 /\left(\mathrm{S}^{\prime}\right)\right]\left[1+\left(\mathrm{I}^{\prime}\right)\right]+\left(\mathrm{S}^{\prime}\right) / \alpha} \\
i=\frac{\left(\mathrm{I}^{\prime}\right)}{\left(\mathrm{I}^{\prime}\right)+1+\left(\mathrm{S}^{\prime}\right)\left[1+\left(\mathrm{S}^{\prime}\right) / \alpha\right]}
\end{gathered}
$$

and completely noncompetitive inhibition $(\beta=1)$.

$$
\begin{gathered}
v_{i}=V_{m} \frac{1}{\left[1+\left(\overline{\left.\left.I_{i}\right)\right]\left[1+1 /\left(\mathrm{S}^{\prime}\right)+\left(\mathrm{S}^{\prime}\right) / \alpha\right]}\right.\right.} \\
i=\frac{\left(\mathrm{I}^{\prime}\right)}{\left(\mathrm{I}^{\prime}\right)+1}
\end{gathered}
$$

The $\left(S_{0}{ }^{\prime}\right)$ will be shifted from $\sqrt{u}$ to $\sqrt{u\left[1+\left(I^{\prime}\right)\right]}$ in the presence of a competitive inhibitor. These equations are, of course. special cases of Eqs. 4-I8 to 4-21 and apply specifically to type $A$ inhibition. However, they are the most commonly used and the competitive rate equation was first derived by Mmray (1930) in connection with the inhibition of liver esterase by d-methyl-n-liexyl carbinol. the shift in $\left(\mathrm{S}_{0}\right)$ and the change in the form of the rate-ps curve being demonstrated. 
The most general situation in which there are two binding sites for the substrate can be represented as follows:

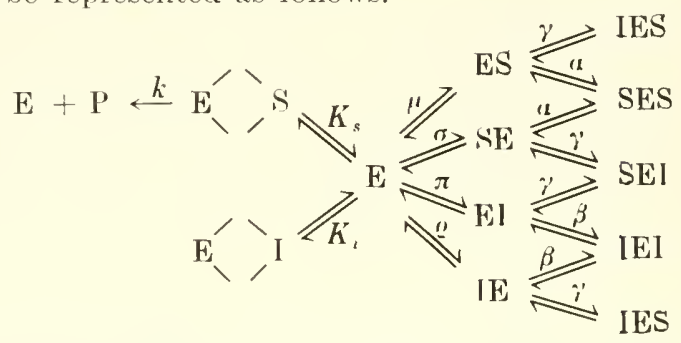

where the two binding sites are indicated on either side of the enzyme in the complexes to the right. The Greek letters are the factors by which either $K_{s}$ or $K_{i}$ must be multiplied for the designated reactions. Such a situation is not too unlikely in competitive inhibition by a substance structurally related to the substrate and able to combine similarly with the enzyme sites. The generalized rate equation may be written as:

$$
v_{i}=\frac{1+\frac{1}{\mu}+\frac{1}{\sigma}+\left(\mathrm{I}^{\prime}\right)\left[\frac{1}{\gamma \mu \varrho}+\frac{1}{\gamma \sigma \pi}\right]+\frac{1}{\left(\mathrm{~S}^{\prime}\right)}\left\{1+\left(\mathrm{I}^{\prime}\right)\left[1+\frac{1}{\pi}+\frac{1}{\varrho}\right]+\frac{\left(\mathrm{I}^{\prime}\right)^{2}}{\beta}\right\}+\frac{\left(\mathrm{S}^{\prime}\right)}{\alpha \mu \sigma}}{1+}
$$

The equation may be simplified by setting the constants for the reactions that are to be excluded equal to infinity.

The effects of different degrees of substrate inhibition on the inhibition produced by a competitive inhibitor are shown in Fig. 4-13. When the

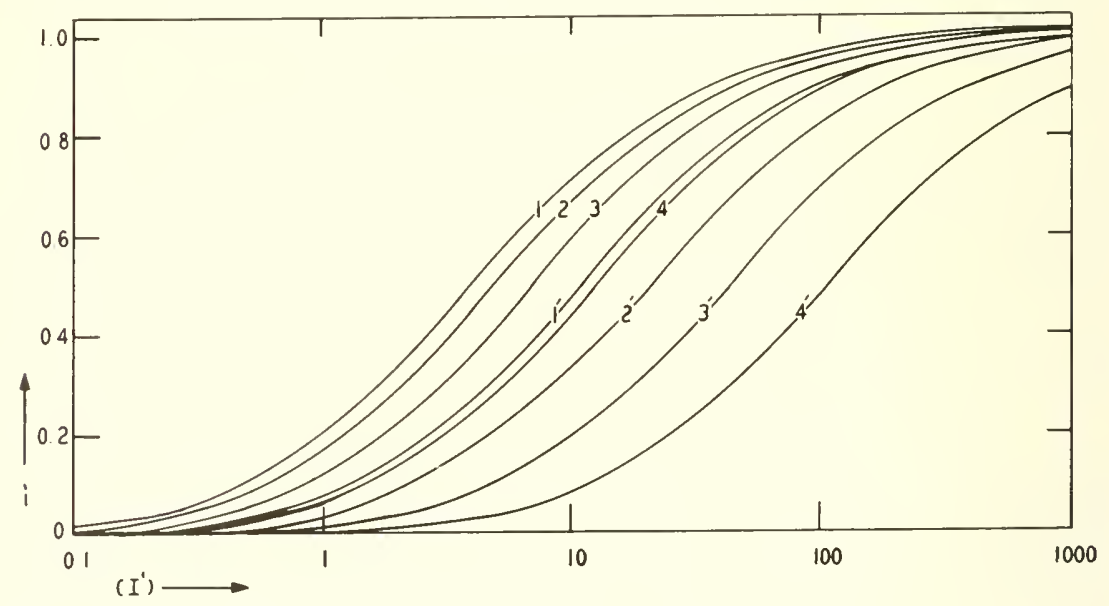

Fig. 4-13. Competitive inhibition of enzymes exhibiting substrate inhibition (Eq. 4-27). Curve 1: $\left(\mathbf{S}^{\prime}\right)=3, \alpha=\infty$ : curve $\mathrm{l}^{\prime}:\left(\mathbf{S}^{\prime}\right)=10, \alpha=\infty$. Curve $2:\left(\mathbf{S}^{\prime}\right)=3$, $\alpha=10$; curve $2^{\prime}:\left(S^{\prime}\right)=10, \alpha=10$. Curve 3: $\left(S^{\prime}\right)=3, \alpha=3$; curve $3^{\prime}:\left(S^{\prime}\right)=10$. $\alpha=3$. Curve $4:\left(\mathbf{S}^{\prime}\right)=3, \alpha=1$; curve $4^{\prime}:\left(\mathbf{S}^{\prime}\right)=10, \alpha=1$. 
binding of the second substrate molecule is approximately as tight as in the active complex, the inhibition may be reduced quite markedly. Competitive inhibition constants determined in the usual manner on such systems will be in error by an appreciable amount unless precautions are taken as discussed in the following chapter. The effects of increasing concentration of inhibitor on the rate-pS curves are evident from Fig. 4-14 - the

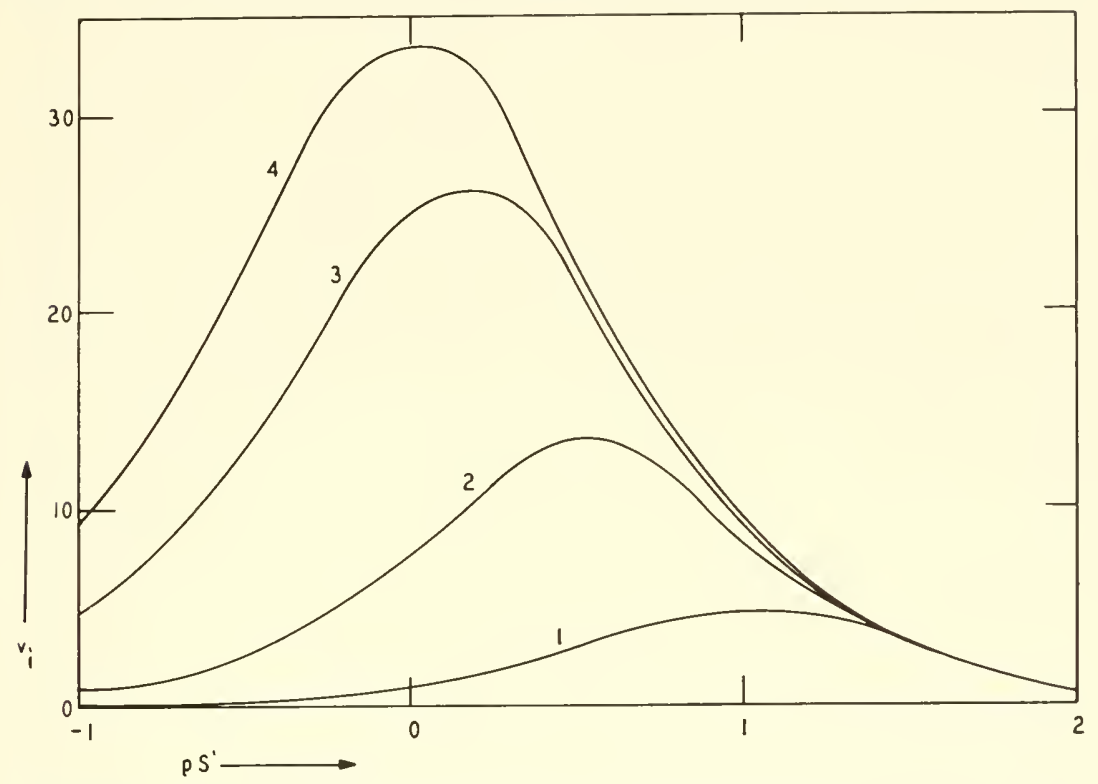

FIG. 4-14. Effects of a competitive inhibitor on the rate-pS' curves for substrate inhibition (Eq. 4-26). $\alpha=1$. Curve 1: $\left(I^{\prime}\right)=0,\left(S_{0}{ }^{\prime}\right)=1$; curve $2:\left(I^{\prime}\right)=1$; $\left(\mathrm{S}_{0}{ }^{\prime}\right)=1.41$; curve $3:\left(\mathrm{I}^{\prime}\right)=10,\left(\mathrm{~S}_{0}{ }^{\prime}\right)=3.32$; curve $4:\left(\mathrm{I}^{\prime}\right)=100,\left(\mathrm{~S}_{0}{ }^{\prime}\right)=10$,

decrease in $v_{0}$, the shift of $\left(\mathrm{S}_{0}\right)$ to higher concentrations, and the "pushing in " of the left side of the curves as noted by Murray (1930).

It was mentioned that Hofstee (1955) used the competitive inhibitor isoxanthopterin to determine the mechanism of substrate inhibition for xanthine oxidase. Inasmuch as this is a generally useful method of distinguishing between type $\mathrm{A}$ and type $\mathrm{B}$ noncompetitive mechanisms, the procedure will be outlined briefly. The equations Hofstee derived for the two situations are:

Type A substrate inhibition (Eqs. 4-7 and 4-26)

$$
\begin{gathered}
v=V_{m} \frac{1}{1+1 /\left(\mathrm{S}^{\prime}\right)+\left(\mathrm{S}^{\prime}\right) / \alpha} \\
v_{i}=V_{m} \frac{1}{1+\left[1 /\left(\mathrm{S}^{\prime}\right)\right]\left[1+\left(\mathrm{I}^{\prime}\right)\right]+\overline{\left(\mathrm{S}^{\prime}\right) / \alpha}}
\end{gathered}
$$


Type B noncompetitive substrate inhibition (Eq. 4-11)

$$
v=V_{m} \frac{1}{1+1 / \alpha+1 /\left(\mathrm{S}^{\prime}\right)+\left(\mathrm{S}^{\prime}\right) / \alpha}
$$

$$
\begin{gathered}
v_{i}=V_{m} \frac{1}{1+(1 / \alpha)\left[1+\left(\mathrm{I}^{\prime}\right)\right]+\left[1 /\left(\mathrm{S}^{\prime}\right)\right]\left[1+\left(\mathrm{I}^{\prime}\right)\right]+\left(\mathrm{S}^{\prime}\right) / \alpha} \\
v_{i}=V_{m} \frac{1}{1+1 / \alpha+\left(\mathrm{I}^{\prime}\right)+\left[1 /\left(\mathrm{S}^{\prime}\right)\right]\left[1+\left(\mathrm{I}^{\prime}\right)\right]+\left(\mathrm{S}^{\prime}\right) / \alpha}
\end{gathered}
$$

Equations 4-32 and 4-33 are special cases of 4-18. Mechanism (I) represents the sitnation in which the inhibitor combines with the active site and mechanism (II) in which it combines with an auxiliary group necessary for the reaction; it is assumed that the inhibition by substrate is due to its combination with this auxiliary group. The expressions for the maximal rate at the peak of the rate-(S) curves for the three possibilities are:

Type A:

$$
v_{0}=V_{m} \frac{1}{1+2 \sqrt{\left[1+\left(I^{\prime}\right)\right] / \alpha}}
$$

Type B (I):

$$
v_{0}=V_{m}^{\prime} \frac{1}{\left[1+\sqrt{\left[1+\left(I^{\prime}\right)\right] / \alpha}\right]^{2}}
$$

Type B (II):

$$
v_{0}=V_{m} \frac{1}{\left[\sqrt{1+\left(I^{\prime}\right)}+1^{\prime}(1 / \alpha)\right]^{2}}
$$

Since $\alpha$ could be calculated from substrate inhibition kinetics ( $\alpha$ was about 50 for xanthine oxidase), the values of $K_{i}$ could be determined from each of the Eqs. 4-34 to 4-36 using the measured values of $v_{0}$ at the two isoxanthopterin concentrations (see Fig. 4-9). The values of $K_{i}$ calculated for the two isoxanthopterin concentrations should be equal and this was the case only for mechanism $B(I)$, indicating that the inhibitory substrate molecule combined with the auxiliary gromp but that the isoxanthopterin did not. Combination of isoxanthopterin with both active site and auxiliary group was eliminated by the linearity of plots of $\left(v-v_{i}\right)$ against $\left(v-v_{i}\right) /(\mathrm{I})$.

A rather striking instance of competition in a substrate-inhibited system is given in the study of Austin and Berry (1953) on human erythrocyte acetylcholinesterase. The presence of the inhibitor $284 \mathrm{C} 51$ at $1.3 \times 10^{-3} \mathrm{mM}$ shifted the peak in the rate-ps curve from 2.65 to approximately 1.0 (a forty-five fold change in concentration) as shown in Fig. 4-15. Since $\left(\mathrm{S}_{0}\right)$ is shifted by a factor of $\sqrt{1+\left(\mathrm{I}^{\prime}\right)}(\mathrm{Eq} .4-23)$, the $K_{i}$ can be calculated to be roughly $6.4 \times 10^{-10} M$. Although the rate-pS maxima here could not be accurately evaluated, and hence this value of $K_{i}$ is only approxi- 
mate, it illustrates a method which could be quite accurate. These curves also show that over a certain range of substrate concentration, the rate of the control is decreased and the rate of the inhibited enzyme is increased by a rise in the substrate concentration, the inhibition by 284051 falling from $100 \%$ to less than $50 \%$. It is possible that in vivo at localized endplate or synaptic regions the acetylcholine reaches concentrations in this range and that the kinetics of inhibition follow the formulations presented in this section.

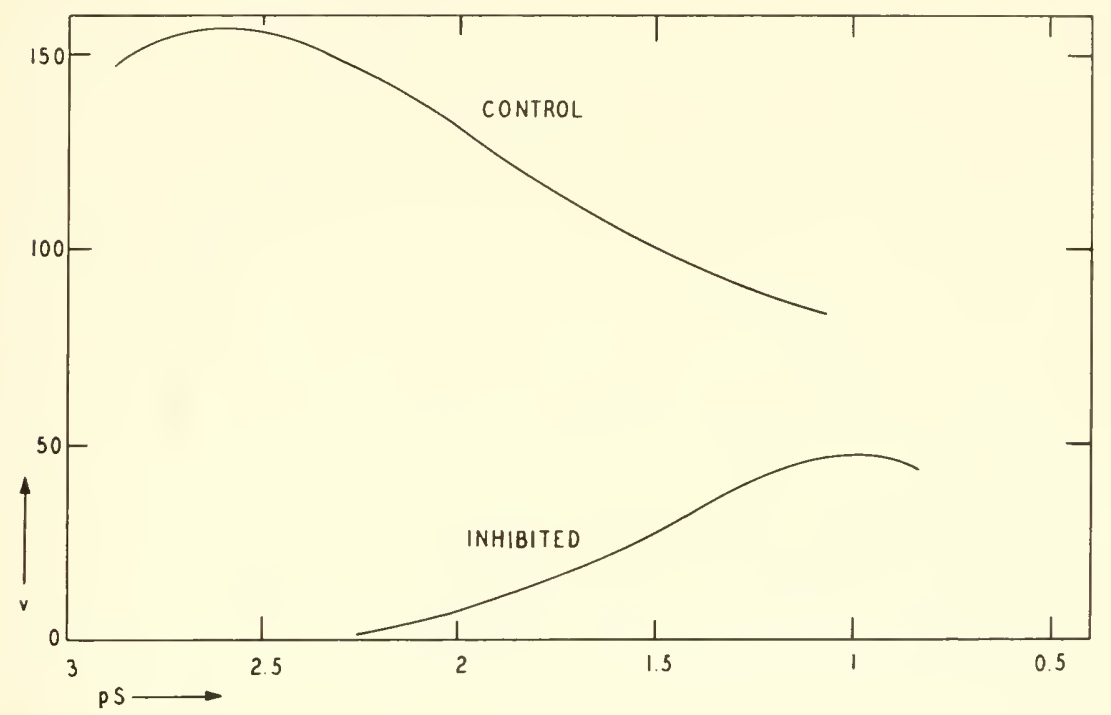

FIG. 4-15. Effect of the inhibitor $284 \mathrm{C} 5 \mathrm{l}$ on the rate-pS curve of substrate inhibition of human erythrocyte acetylcholinesterase. $(\mathrm{I})=1.3 \times 10^{-3} \mathrm{~m} . /$. (From Austin and Berry, 1953.)

Inhibition of various tissue cholinesterases by physostigmine $\left(3.63 \times 10^{-3}\right.$ $\mathrm{m} M I$ ) produced an average shift of $\mathrm{pS}_{0}$ from 2.57 to 1.40 and a depression in $v_{0}$ of $85 \%$. conforming to predictions. Another inhibitor, clupeine, of a less specific type, also reduced the $\mathrm{pS}_{0}$ to an average value of 1.92 but produced only a $5 \%$ depression of $\imath_{0}$ (Angustinsson. 1948). Gum arabic, which stimulates cholinesterase slightly, shifted the $\mathrm{pS}_{0}$ upwards to 2.75 . It should be noted that a marked shift in $\mathrm{pS}_{0}$ means that a substance inhibits a reaction in one range of substrate concentration and stimulates it in another range. The nature of the response to an inhibitor in such systems is strongly dependent on the substrate concentration. 


\section{PRODUCT INHIBITION}

The products of a reaction can slow down the forward rate in two general ways.

$A$. The reverse reaction, whereby the products are transformed into the substrate, competes with the forward reaction so that the measured rate of substrate disappearance or product formation is reduced. Inasmuch as most enzymes catalyze reversible reactions this type of product inhibition is theoretically very common; it is practically uncommon because in most reactions the equilibrium is far to one side and the reverse reaction is insignificant.

$B$. A product of the reaction can combine with the enzyme, or other component of the system, so that the forward rate is inhibited. In this case the product may inhibit by any of the mechanisms discussed in the previous chapter. The primary difference between these two mechanisms of inhibition is that in the former situation the product reacts with the enzyme in such a way as to form a reactive complex, i.e., it combines with the active center to form the same complex in the activated state as is formed from the substrate, whereas in the second type the complex of enzyme and product is inactive and may be located at the active center or vicinal to it.

When the reaction is started with only substrate present, product inhibition is seldom of importance if only initial rates are measured or obtained by extrapolation. However, if the equilibrium is in favor of the substrate or the product is a relatively potent inhibitor of type B, measurements made over arbitrary intervals of time may provide rates which are only average values over this interval and from which incorrect values for the constants of the enzyme reaction are obtained. These errors may be circumvented if it is first established that the rate is constant over the experimental interval employed.

\section{Inhibition by the Reverse Reaction}

The over-all forward rate for a reversible reaction is given by Eq. 2-18. This equation is valid whatever the number of complexes involved in the reaction, i.e., whether one assumes a single active complex ES, or one for both substrate ES and product EP as in reaction 2-17, or more as ES, $\mathrm{EX}_{1}, \mathrm{EX}_{2} \ldots \mathrm{EP}$. However, the constants $V_{m}, V_{p}, K_{m}$, and $K_{p}$ will be represented by different combinations of the rate constants. Equation 2-18 may be rewritten as:

$$
v=V_{m} \frac{(\mathrm{S})-(\mathrm{P})\left(V_{p} K_{m} / V_{m} K_{p}\right)}{(\mathrm{S})+K_{s}\left[1+(\mathrm{P}) / K_{p}\right]}
$$


The quantity $V_{p} K_{m} / V_{m} K_{p}=K_{0}$, the equilibrium constant for the over-all reaction and is equivalent to $(\mathrm{S}) /(\mathrm{P})$. Thus one may write:

$$
v=V_{m} \frac{(\mathrm{S})-(\mathrm{P}) K_{0}}{(\mathrm{~S})+K_{s}\left[1+(\mathrm{P}) / K_{p}\right]}
$$

which is similar to the equation derived by Sturtevant (1954, p. 210). When the equilibrium is far to the right, $K_{0}$ will be very small and (P) $K_{0}$ may be neglected relative to $(\mathrm{S})$ :

$$
v=V_{m} \frac{(\mathrm{S})}{(\mathrm{S})+K_{s}\left[1+(\mathrm{P}) / K_{p}\right]}
$$

which is identical with the rate equation for competitive inhibition. Thus

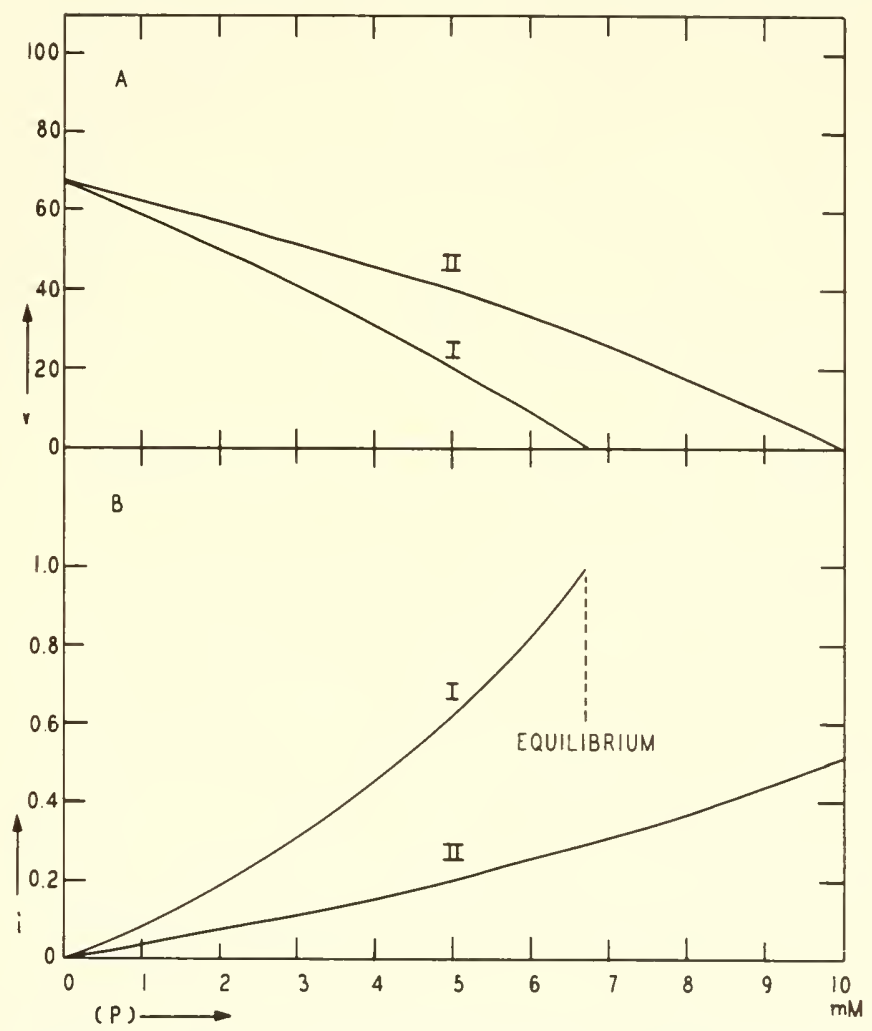

FIG. 4-16. Product inhibition by the reverse reaction. In the upper graph $A$ the rate is plotted against the product concentration (Eq. 4-37) and in the lower graph B the fractional inhibition is plotted against the product concentration (Eq. 4-40). $K_{s}=5 \mathrm{~m} \mu, K_{p}=10 \mathrm{~m} M$, and $\left(\mathrm{S}_{t}\right)=10 \mathrm{~m} . /$. Curves I: $V_{p} / V_{m}=1$; curves II: $V_{p} / V_{m}=\infty$ (Eq. 4-39). 
product inhibition can occur even in reactions where the equilibrium is markedly in favor of the product. This is due to binding of product to the sites involved in the reaction, even though it does not react. The inhibition produced by the product in the general case is:

$$
i=\frac{\left(\mathrm{P}^{\prime}\right)\left[1+\left(V_{p} / V_{m}\right)\left\{1+1 /\left(\mathrm{S}^{\prime}\right)\right\}\right]}{\left(\mathrm{P}^{\prime}\right)+1+\left(\mathrm{S}^{\prime}\right)}
$$

which is the same as the competitive inhibition expression 3-28 except for the factor by which $\left(\mathrm{P}^{\prime}\right)$ is multiplied. The ratio $V_{p} / V_{m}$ may be replaced by $K_{0} K_{p} / K_{m}$. If there is only substrate at the beginning of the reaction and the product is formed so that $(\mathrm{S})+(\mathrm{P})$ is constant, the inhibition will increase and the rate will fall as shown in Fig. 4-16. If the product is present

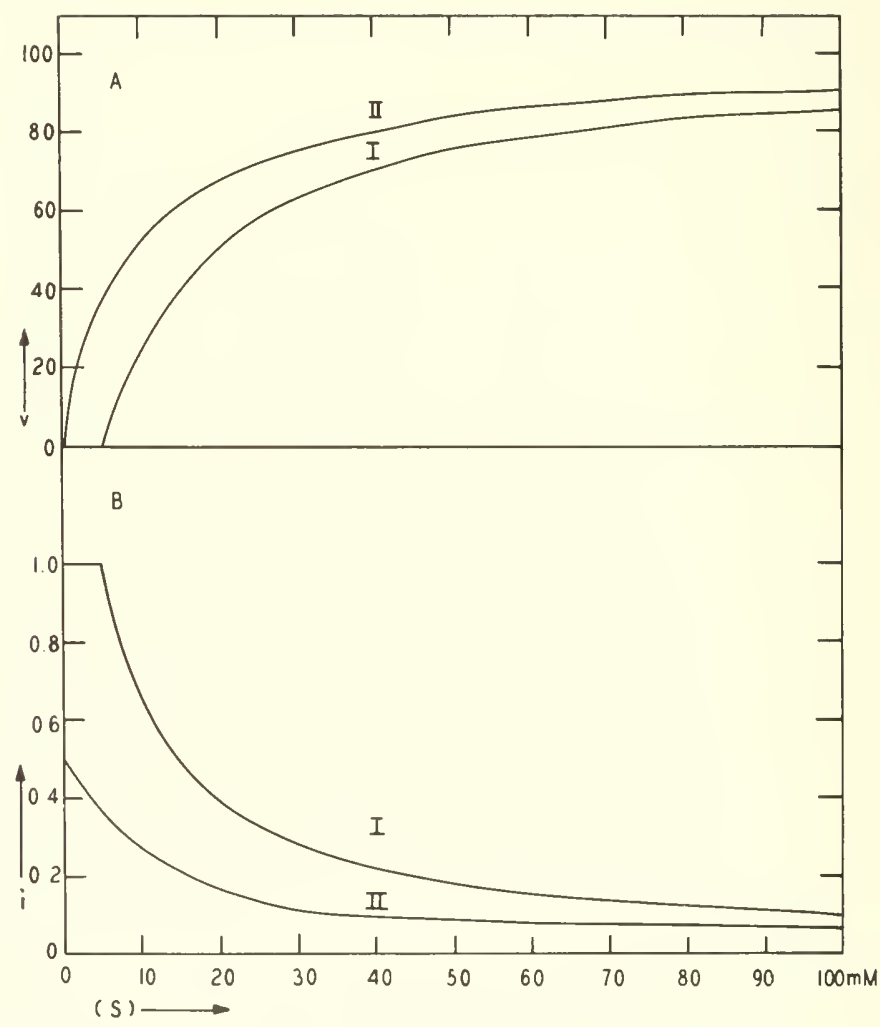

FIG. 4-17. Product inhibition by the reverse reaction. In the upper graph A the rate is plotted against the substrate concentration and in the lower graph B the fractional inhibition is plotted against the substrate concentration. $K_{s}=5 \mathrm{~m} M$ and $K_{p}=10 \mathrm{mH}$. The concentration of product is constant at 10 m.M. Cinrves I: $V_{p} / \mathrm{I}_{m}=1$; curves II: $V_{p} / V_{m}=\infty$. 
at the beginning, there is no reaction mntil the substrate concentration reaches a critical value and above this the rate increases as in Fig. 4-17. The differences between curves I and II represent the effect of the reverse reaction superimposed on the inhibition due to the binding of the product on the active center.

\section{Inhibition by Combination of the Product in an Inactive Complex}

The product can inhibit the forward reaction competitively, noncompetitively, or in any other way discussed in general inhibition. The general

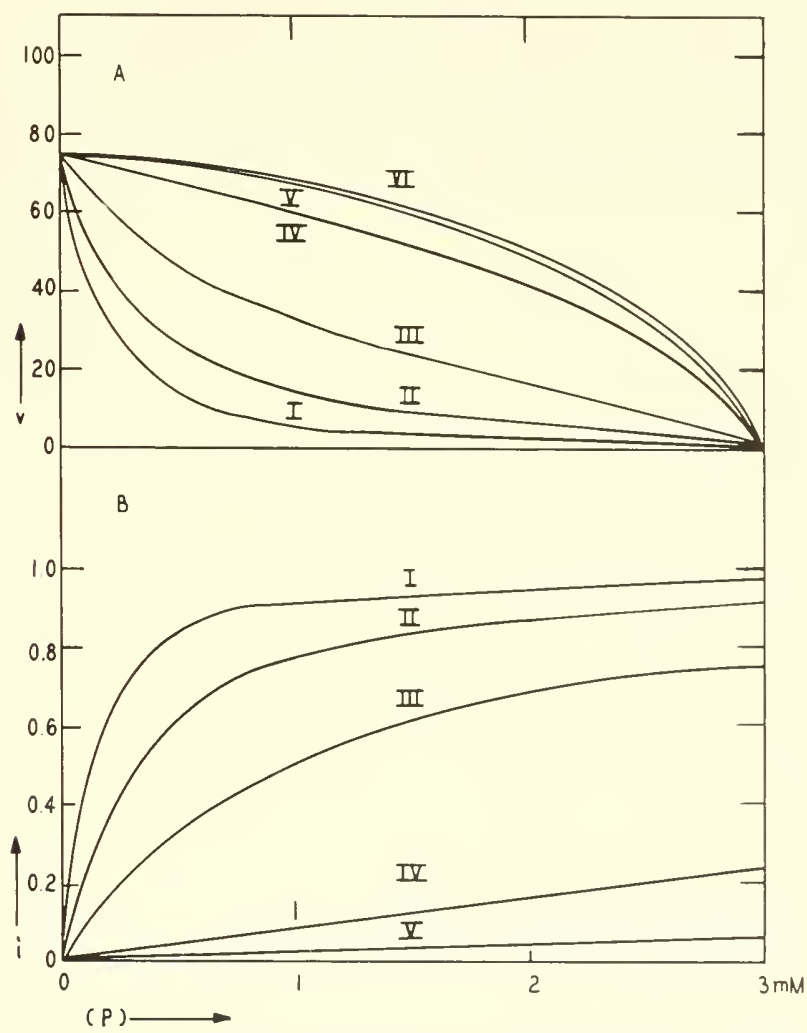

FIG, 4-18. Noncompetitive prodnct inhibition by the formation of an inactive complex between the product and the enzyme. In the upper graph $\mathrm{A}$ the rate is plotted against the product concentration and in the lower graph B the fractional inhibition is plotted against the product concentration. Curves I: $K_{p}=0.1 \mathrm{~m} . I$; curves II: $K_{p}=0.3 \mathrm{~m} . /$; curves III: $K_{p}=1 \mathrm{~m} . /$; curves IV: $K_{p}=10 \mathrm{~m} M$; curves $\mathrm{V}$ : $K_{p}=50 \mathrm{~m} . / 1$; curves VI: no inhibition. 
reaction scheme is similar to Eq. 3-2 and the rate and inhibition equations are similar to those from Eqs. 3-9 to 3-30 if one replaces (I) with $(\mathrm{P})$ and $K_{i}$ with $K_{p}$. If the produet is added at different concentrations and only initial rates are determined, the kinetics are identical with those for inhibition by substances unrelated to the reaction. However, if no product is present initially but is formed during the reaction, i.e., if $(\mathrm{S})+(\mathrm{P})$ is constant, it is interesting to observe, as in Fig. 4-18, that the course of the reaction depends markedly on $K_{p}$. If $K_{p}$ is small the rate may drop very rapidly after the reaction has started; e.g., if $K_{p}=10^{-4} M$ the inhibition reaches $50 \%$ after only $5 \%$ of the substrate has been transformed.

The products may also combine with an activator and inhibit by this mechanism although this situation has not been reported. Carboxylic and amino acids formed in hydrolytic reactions might complex with metal ions, the substrates having little or no affinity for these activators. Reuction of the products with coenzyme or acceptor sites is also possible and in all cases the kinetics will follow the corresponding equations in Chapter 3.

\section{Examples of Product Inhibition}

The hexokinases of brain and schistosomes are inhibited quite potently by the product glucose- 6 -phosphate, $50 \%$ inhibition occurring at a concentration of about $0.6 \mathrm{~m} . \mathrm{I} \mathrm{im}$ both cases (Crane and Sols, 1953; Bueding and MacKinnon, 1955) and this inhibition is noncompetitive with respect to either glucose or adenosine triphosphate (ATP) (Weil-Malherbe and Bone, 1951). On the other hand, yeast hexokinase is not inhibited by hexosemonophosphates (Colowick and Kalckar, 1943). The other product adenosine diphosphate (ADP) inhibits all of these enzymes but is competitive with ATP on the enzyme from brain (Sols and Crane. 1954) and noncompetitive in the case of hexokinase from yeast (Gamble and Najjar, 1955) and schistosomes (Bueding and MacKinnon, 1955); the inhibition in the latter case actually increases somewhat with increasing ATP concentration. The reaction rate falls off rapidly due to the aceumulation of these products in preparations of the purified enzymes. These inhibitions are not due to the occurrence of the reverse reaction but are of type B mechanism, as is evident from the free energy change of the reaction, the fact that either product alone is imhibitory (for the reverse reaction both products must be present), and the absence of glucose-6-phosphate inhibition of the yeast hexokinase. The inhibitions are fairly specific since other hexosephosphates inhibit less than glucose-6-phosphate or not at all, while adenosine-5-phosphate (AMP), pyrophosphate, and phosphate are inactive (Sols and Crane, 1954). The reverse reaction catalyzed by yeast hexokinase, measured by an exchange reaction between labeled glucose or glucose-6-phosphate, is inhibited by glucose, another example of product inhibition (Gamble and Najjar, 1955). 
Many phosphorylation reactions involving ATP are inhibited by ADP but in most cases the type of inhibition is not known. Pyridoxal phosphokinase is inhibited competitively by ADP (Hurwitz, 1953) as is glutathione synthetase (Yanari et al. . 1953); in the latter case ADP appears to be bound more tightly than ATP to the enzyme. Indeed, the ATPases from various sources are inhibited weakly by ADP (Kielley and Kielley, 1953; Blum, 1955).

Enzymes catalyzing the acylation of amino groups:

$$
\mathrm{R}-\cos \mathrm{CoA}+\mathrm{H}_{2} \mathrm{~N}-\mathrm{R}^{\prime} \rightarrow \mathrm{R}-\mathrm{CONH}-\mathrm{R}^{\prime}+\mathrm{CoASH}
$$

such as the arylamine transacetylase (Tabor et al., 1953) and glycine transacylase (Schachter and Taggart, 1954) of liver are inhibited by coenzyme A. The inhibition is not a function of the SH group because reaction of the CoASH with iodoacetate does not reduce the inhibitory activity. When benzoyl-CoA is used as the acyl donor, glycine transacylase catalyzes the formation of hippurate, which is also inhibitory; $50 \%$ inhibition is given by $0.58 \mathrm{~m} M$ hippurate and $0.65 \mathrm{~m} M \mathrm{CoASH}$. The free energy change for this reaction of approximately $-8 \mathrm{kcal} /$ mole indicates the inhibition if of type $\mathrm{B}$, as does the inhibition by single products. The inhibition is noncompetitive with respect to both benzoyl-CoA and glycine according to reciprocal plots. Finally, the inhibition by hippurate depends on the acyl donor, only about one-twentieth of the concentration required for $50 \%$ inhibition being necessary for acetyl-CoA compared to benzoyl-CoA. It is difficult to reconcile these data with any detailed mechanism unless it is assumed that the hippurate combines with a group adjacent to the active center and interferes in some manner with the transacylation process.

The inhibition of urease by $\mathrm{NH}^{+}{ }_{4}$ presents somewhat different problems (Hoare and Laidler, 1950). Here there are two sites at which urea can be bound although reaction occurs when urea is bound to one site and water to the other. The rate equations developed for the varions possibilities are:

Uninhibited reaction: $\quad v=V_{m} \frac{\left(\mathrm{S}^{\prime}\right)}{\left[1+\left(\mathrm{S}^{\prime}\right)\right]^{2}}$

Noncompetitive inhibition: $\quad v_{i}=V_{m} \frac{\left(\mathrm{S}^{\prime}\right)}{\left[1+\left(\mathrm{S}^{\prime}\right)\right]^{2}\left[1+\left(\mathrm{I}^{\prime}\right)\right]}$

Competitive inhibition:

Inhibition at one site:

$$
v_{i}=V_{m} \frac{\left(\mathrm{S}^{\prime}\right)}{\left[1+\left(\mathrm{S}^{\prime}\right)\right]\left[1+\left(\mathrm{S}^{\prime}\right)+\left(\mathrm{I}^{\prime}\right)\right]}
$$

Inhibition at both sites: $v_{i}=V_{m} \frac{\left(\mathrm{S}^{\prime}\right)}{\left[1+\left(\mathrm{S}^{\prime}\right)+\left(\mathrm{I}^{\prime}\right)\right]^{2}}$

By plotting $v / v_{i}$ against (I) it was shown that the inhibition was noncompetitive. Since deviations from the expected behavior did occur at 
high inhibitor concentrations, but were in the opposite direction from that predicted for competitive effects, it was conchuded that this simple treatment was not completely adequate. However, this example does illustrate that product inhibition can be complicated by deviations from the simple formulation of Michaelis and Menten and that multiple substrates and sites must be taken into account in elucidating specific mechanisms.

The inhibition of cholinesterase by choline was first demonstrated by Roepke (1937) in sermm and the competitive nature of the inhibition indicated by appropriate reciprocal plotting, the affinity for the choline being comparable to that for acetylcholine which would be expected if the principal binching energy is derived from the anionic site attachment. Similar results were obtained by Ziff et al. (1938) using a different procedure for the evaluation of the kineties and subsequent work has confirmed this picture of product inhibition for the serum enzyme. A more thorough investigation was made by Augustinsson (1948) and completely competitive inhibition was shown for horse serum cholinesterase using a variety of substrates. However, for the erythrocyte and brain enzymes the curves deviated from linearity, although a competitive type of inhibition was suggested by the fact that the curves all passed through a single point on the ordinate. The explanation is that for these tissue cholinesterases we have both substrate and product inhibition simultaneously. The presence of choline shifts the substrate concentration for maximal rate $\left(\mathrm{S}_{0}\right)$ to higher values as would any competitive inhibitor; a concentration of choline necessary for $44 \%$ inhibition increased $\left(\mathrm{S}_{0}\right)$ about ten-fold. It is interesting that the cholinesterase from Helix dart sac, which does not exhibit marked substrate inhibition, gave linear curves in the usual $1 / v-1 /(\mathrm{S})$ plot but these curves did not intersect on the $1 / v$ axis nor on the $1 /(\mathrm{S})$ axis. It is possible that this indicates mixed inhibition where the binding of choline does not completely prevent binding of acetylcholine; extrapolating the curves to their intersection indicates that choline increases the $K_{s}$ of acetylcholine some seven-fold (see Chapter 5).

The noncompetitive nature of much product inhibition has been surprising to some investigators because the usual structural similarity between product and substrate implies reaction at the same site. However, if it is assumed that there are two sites, a substrate site forming ES and a product site forming EP, and that ES is transformed into EP in the course of the reaction, the following scheme represents the situation:

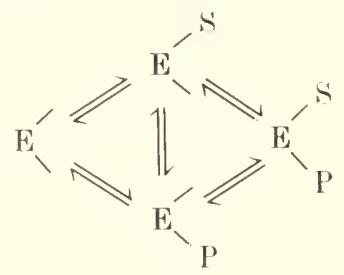


The binding of product to its site prevents the reaction $\mathrm{ES} \rightarrow \mathrm{EP}$ but does not necessarily prevent the binding of the substrate so that the complex ESP occurs. Noncompetitive kineties will be given by this system and it is possible that many of the instances of product inhibition fall into this category.

\section{Product Inhibition in Multienzyme Systems}

In a sequenee of reactions catalyzed by a series of enzymes, the product of any step may inhibit an enzyme earlier in the sequenee rather than the one responsible for its formation. Sueh a meehanism may exert a selfregulatory eontrol on the over-all rate. One of the final produets of pyrimidine synthesis is eytidylic acid and this inhibits the first reaction in the sequence (Pardee, 1959); thus the synthetic rate is controlled by the accumulation of this product. In systems of any degree of eomplexity, product inhibition may be a major factor in rate regulation and particularly within the cell this may eontribute to the metabolic organization.

The degree of inhibition exerted by a product depends on the level of coneentration reaehed and hence on both the rate of its formation and the rate of its disappearance. A produet may either diffuse away from the enzyme it inhibits or it may be further altered by another enzyme. In a steady state the product eoneentration will remain constant and exert a constant inhibition. If now another inhibitor is introduced there are two ways in which the system may be affected. If the inhibitor acts on the enzyme forming the product, the product coneentration will decrease and the inhibition exerted on this enzyme will be lessened; thus the inhibition caused by the added inhibitor will be less than expeeted on the basis of no product inhibition. Or the inhibitor may act on the enzyme that destroys the product, in whieh case the product concentration will rise and the inhibition will be greater than would have been exerted by the added inhibitor alone. The steady-state coneentration of product in the following system:

$$
\begin{array}{ll}
h_{s} & \mathrm{E}_{1}+\mathrm{S} \rightleftharpoons \mathrm{E}_{1} \mathrm{~S} \stackrel{k_{1}}{\rightarrow} \mathrm{E}_{1}+\mathrm{P} \\
K_{p} & \mathrm{E}_{1}+\mathrm{P} \rightleftharpoons \mathrm{E}_{1} \mathrm{P} \\
K_{\nu}^{\prime} & \mathrm{E}_{2}+\mathrm{P} \rightleftharpoons \mathrm{E}_{2} \mathrm{P} \stackrel{k_{2}}{\rightarrow} \mathrm{E}_{2}+\mathrm{Q}
\end{array}
$$

may be determined by the equation:

$$
\frac{d(\mathrm{P})}{d t}=k_{1}\left(\mathrm{E}_{1} \mathrm{~S}\right)-k_{2}\left(\mathrm{E}_{2} \mathrm{P}\right)=0
$$

The expression for $(\mathrm{P})$ will be quadratic and will depend on the type of product inhibiton. For competitive inhibition: 


$$
\begin{gathered}
(\mathrm{P})=\frac{1}{2} \sqrt{K_{p}^{2}\left[1+\frac{(\mathrm{S})}{K_{s}}-\frac{(\mathrm{S}) V_{m}}{K_{s} V_{m}{ }^{\prime}}\right]^{2}+\frac{4 K_{p} K_{p}{ }^{\prime}(\mathrm{S}) V_{m}}{K_{s} V_{m}^{\prime}}} \\
-\frac{K_{p}}{2}\left[1+\frac{(\mathrm{S})}{K_{s}}+\frac{(\mathrm{S}) V_{m}}{K_{s} V_{m}{ }^{\prime}}\right]
\end{gathered}
$$

where $V_{m}$ is the maximal rate for $\mathrm{E}_{1}$ and $V_{m}{ }^{\prime}$ for $\mathrm{E}_{2}$. The quantity $4 K_{p} K_{p}{ }^{\prime}(\mathrm{S}) V_{m} / K_{s} V_{m}{ }^{\prime}$ determines the level of (P). If the inhibition is on $\mathrm{E}_{1}$, either $K_{s}$ is increased or $V_{m}$ is decreased; in either case $(\mathrm{P})$ is seen to decrease. If $E_{2}$ is inhibited, either $K_{p}{ }^{\prime}$ is increased or $V_{m}{ }^{\prime}$ is decreased, resulting in increase in $(\mathrm{P})$. For noncompetitive inhibition, $K_{s} /(\mathrm{S})$ is replaced by $1+K_{s} /(\mathrm{S})$. If the product diffuses away at a rate proportional to $k^{\prime}(\mathrm{P})$, the corresponding quantity in the quadratic equation is $4(\mathrm{~S}) V_{m} /$ $k^{\prime} K_{s}$, from which a similar decrease in $(\mathrm{P})$ is seen to occur upon inhibition of the enzyme.

An example of such a mechanism occurs in the early stages of glycolysis:

$$
\text { Glucose } \stackrel{\mathrm{E}_{1}}{\rightleftharpoons} \text { glucose }-6-\mathrm{P} \stackrel{\mathrm{E}_{2}}{\rightleftharpoons} \text { fructose }-6-\mathrm{P} \stackrel{\mathrm{E}_{3}}{\rightleftharpoons} \text { fructose-1,6-diP }
$$

where $\mathrm{E}_{1}$ is hexokinase, $\mathrm{E}_{2}$ is phosphoglucose isomerase, and $\mathrm{E}_{3}$ is 6-phosphofructokinase. The second reaction is very rapid and the free energy change for it is very small (glucose-6-P/fructose-6-P $=2.3$ ); fructose-6-P also inhibits hexokinase but about half as potently as ghcose-6-P. Thus this second reaction is relatively mimportant and the third reaction may be considered as the principal step for the removal of inhibitory products. Crane and Sols (1953) found that addition of 6-phosphofructokinase did reduce the inhibition on hexolinase. Thus in the cell it is possible that these enzymes may form a steady-state system regulated in part by the level of hexose monophosphates. Both stimulation and inhibition of hexokinase will therefore be less effective than expected on the basis of studies of the purified enzyme. It is likely that many similar situations occur in the metabolic sequences and cycles within the cell. 


\section{DETERMINATION OF THE MECHANISMS AND CONSTANTS OF INHIBITION}

Information on the mechanism of an inhibition may be obtained from accurate kinetic data provided these data are subjected to the appropriate analyses. At the same time it is usually possible to determine the constants that quantitatively characterize the inhibition. A study of enzyme inhibition should ideally involve a determination of the important kinetic and equilibrium constants because the mechanism cannot be adequately expressed otherwise. A mechanism of inhibition in an isolated enzyme system cannot be arrived at without data and procedures capable of supplying these constants nor can the mechanism be applied to more complex situations without a quantitative delineation of the reactions involved. It is, for example, not sufficient to state that an inhibition is competitive without providing the appropriate constants, since if the data are not adequate for the evaluation of the constants they are usually not adequate to prove a competitive mechanism. The mere statement that an enzyme is inhibited to a certain degree by a single concentration of an inhibitor - and such reports are very common - may possibly provide some evidence of an enzyme group involved but alone indicates nothing of the mechanism nor of the behavior of the enzyme and inhibitor in the intact cell. The methods for the calculation of inhibition constants are straightforward in isolated enzyme systems and require only the proper data and a critical choice of the procedures used. The basic approach to such determinations was made by Lineweaver and Burk (1934) but other procedures have been developed which are more accurate and applicable in specific cases. True inhibition constants cannot usually be obtained directly from the simple plotting techniques because the constants derived are frequently not the dissociation constants of the enzyme-inhibitor complex but, as is the case with the Michaelis constant, contain other constants or depend on the concentrations of activators or coenzymes. However, it is generally possible to determine the true inhibition constant if the components of the enzyme are known, if their effects on the inhibition are investigated, and if these data are subjected to the proper graphical methods. It is equally easy to determine constants and to misinterpret them. If inhibition constants are to be useful in any way - whether applied to more complex cellular sys- 
tems or utilized for the accurate topological characterization of an active center - it is absolutely essential that the nature of the constants be understood. The pitfalls of interpretation increase with the complexity of the enzyme system and most of the important enzymes of cell metabolism are now realized to be quite complex.

The primary purpose of this chapter is to present several methods for the plotting of enzyme kinetic data and to indicate how misinterpretation of mechanisms and constants may be avoided. To facilitate explanation of the graphical techniques we shall illustrate each by simple competitive inhibition, then proceed to a generalized presentation, and finally give the plotted curves expected for the more important types of inhibition discussed in the previous two chapters. The different characteristics of these plots are more readily understood by directly examining the graphs rather than by inspection of the kinetic equations and hence emphasis has been placed on illustration; it is a simple matter to transform any of the previous rate or inhibition equations into the equation to be plotted but it is not always easy to visualize the results of the plotting.

\section{THE METHODS OF GRAPHICAL ANALYSIS}

The rate equation for completely competitive inhibition (Eq. 3-12):

$$
v_{i}=V_{m} \frac{(\mathrm{S})}{(\mathrm{S})+K_{s}\left[1+(\mathrm{I}) / K_{i}\right]}
$$

may be rearranged into four forms conducive to useful plotting.

\section{The Plot of $1 / v_{i}$ against $1 /(\mathrm{S})$ (Type $A$ )}

This method of plotting was ontlined by Lineweaver and Burk (1934) and the results are frequently referred to as Lineweaver-Burk plots or as double-reciprocal plots. The transformed equation is:

$$
\frac{1}{v_{i}}=\frac{1}{V_{m}}+\frac{K_{m}}{V_{m}(\mathrm{~S})}\left[1+\frac{(\mathrm{I})}{K_{i}}\right]
$$

Plotting $1 / v_{i}$ against $1 /(S)$ one obtains a straight line with a slope equal to $\left(K_{m} / V_{m}\right)\left[1+(\mathrm{I}) / K_{i}\right]$ and intercepting the $1 / v_{i}$ axis at $1 / V_{m}$. The comparable equation for the uninhibited system is:

$$
\frac{1}{i}=\frac{1}{V_{m}}+\frac{K_{m}}{V_{m}(S)}
$$

which gives a line of less slope but of the same intercept. This and the other methods of plotting are illnstrated for competitive inhibition in Fig. 5-1. 


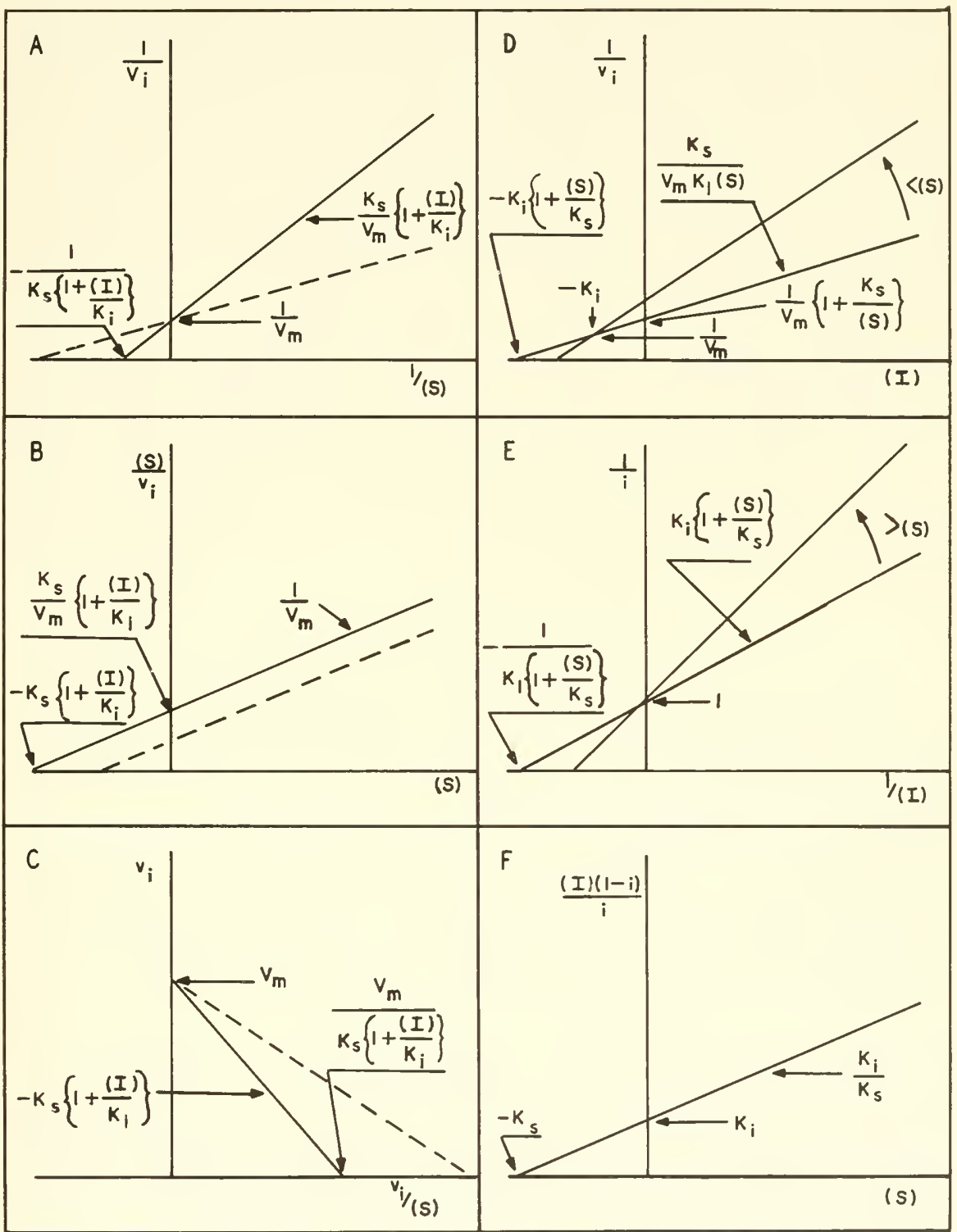

FIG. 5-1. The basic methods of plotting applied to competitive inhibition. The dotted lines represent the uninhibited reaction. 
The symbol $K_{s}$ is used in the graphs but it must be remembered that it is the Michaelis constant which is often determined by the graphical procedures. The difference between $K_{m}$ and $K_{s}$ has been discussed in Chapter 2 . In the interest of the correct historical attribution of these graphical procedures, it is necessary to point out that the plot of $1 / v$ against $1 /(\mathrm{S})$ was initially described in 1932 by Haldane and Stern in their book, " Allgemeine Chemie der Enzyme" (1932, p. 119) as the result of a suggestion by B. Woolf. The plots of $v /(\mathrm{S})$ against $v$ and $(\mathrm{S}) / v$ against (S) were also discussed. These methods were originally applied only to uninhibited enzyme reactions; however, Haldane and Stern distinguished between competitive and noncompetitive inhibition.

Thus a characteristic of these plots for competititive inhibition is that the slopes vary with inhibitor concentration but the $1 / v_{i}$ intercept remains constant. The intercept gives the value of $V_{m}$ and from the slope of the uninhibited reaction the value of $K_{m}$ may be determined; the slope from the inhibited reaction may then be used to calculate $K_{i}$. The value of $K_{i}$ should be independent of the inhibitor concentration used and it is advisable to plot at different inhibitor concentrations to confirm this and increase the accuracy in the determination of $K_{i}$.

\section{The Plot of $(\mathrm{S}) / v_{i}$ against $(\mathrm{S})$ (Type B)}

This procedure, also suggested by Lineweaver and Burk, provides parallel straight lines of constant slope $1 / V_{m}$, the constants $K_{m}$ and $K_{s}$ now being calculated from the $(\mathrm{S}) / v_{i}$ intercepts or the $(\mathrm{S})$ intercepts as indicated by the following equation:

$$
\frac{(\mathrm{S})}{v_{i}}=\frac{(\mathrm{S})}{V_{m}}+\frac{K_{m}}{V_{m}}\left[1+\frac{(\mathrm{I})}{K_{i}}\right]
$$

There are certain instances, as in substrate inhibition, where this method has advantages over the previous one.

\section{The Plot of $v_{i}$ against $v_{i} /(\mathrm{S})$ (Type C)}

This method was originally used by Augustinsson (1948) in his studies on cholinesterase and elaborated by Hofstee (1956) who claimed certain advantages which will be considered later. The equation:

$$
v_{i}=V_{m}-\frac{v_{i} K_{m}}{(\mathrm{~S})}\left[1+\frac{(\mathrm{I})}{K_{i}}\right]
$$

shows that straight lines of negative slope will be obtained, from which the constants may be calculated directly. 


\section{The Plot of $1 / v_{i}$ against (I) (Type D)}

One of the most useful methods, developed particularly by Dixon (1953 b), allows direct determination of $K_{i}$ and is based on the equation:

$$
\frac{\mathrm{l}}{v_{i}}=\frac{1}{V_{m}}\left[1+\frac{K_{m}}{(\mathrm{~S})}\right]+\frac{K_{m}(\mathrm{I})}{V_{m}(\mathrm{~S}) K_{i}}
$$

If plots are made for two different substrate concentrations, the intersection of these straight lines will occur at an ordinate of $-K_{i}$. When the constants for the uninhibited reaction are known, a plot for only one substrate concentration will provide means of calculating $K_{i}$, using either the slope or the (I) intercept. When the intersection method is used, it is evident that the two substrate concentrations should be as far apart as possible so that the intersection point can be accurately located.

\section{The Plot of $1 / i$ against $1 /(I)$ (Type $E$ )}

The imhibition equation for competitive inhibition (Eq. 3-13):

$$
i=\frac{(\mathrm{I})}{(\mathrm{I})+K_{i}\left[1+(\mathrm{S}) / K_{m}\right]}
$$

can be transformed into the reciprocal equation:

$$
\frac{1}{i}=\mathrm{I}+\frac{K_{i}}{(\mathrm{I})}\left[1+\frac{(\mathrm{S})}{K_{m}}\right]
$$

and when $1 / i$ is plotted against $1 /(\mathrm{I})$, straight lines intersecting on the $1 / i$ axis are obtained. This method will not provide $K_{i}$ unless $K_{m}$ is known but if the slopes at two different substrate concentrations are plotted against (S), the intercept will give $K_{i}$ since the slope approaches $K_{i}$ as the substrate concentration decreases.

\section{The Single-Curve Plot (Type F)}

This method was introduced by Hunter and Downs (1945) in connection with their studies on the inhibition of arginase by amino acids and is a very efficient and accurate procedure in certain instances. For competitive inhibition the equation used is of the form:

$$
\frac{\text { (I) }(1-i)}{i}=K_{i}+\frac{K_{i}(\mathrm{~S})}{K_{m}}
$$

and when $(\mathrm{I})(1-i) / i$ is plotted against $(\mathrm{S})$ a straight line is obtained which gives $-K_{m}$ on the (S) intercept and $K_{i}$ on the $(\mathrm{I})(1-i) / i$ intercept, the slope being the ratio $K_{i} / K_{m}$. 


\section{GENERALIZED EQUATIONS FOR DETERMINATION OF CONSTANTS}

Six types of plotting procedures have been outlined for the specific case of completely competitive inhibition. These may be applied to any mechanism of inhibition and before considering particular situations it will be profitable to indicate a general approach from which most cases of inhibition can be derived.

Let us assume that in addition to the substrate the enzyme binds other substances $\mathrm{X}_{1}, \mathrm{X}_{2}, \ldots \mathrm{X}_{n}$ which may be activators, inhibitors, cofactors, coenzymes, hydrogen ions, or any component of the total reaction. The general expression for the total enzyme concentration is thus:

$$
\left(\mathrm{E}_{t}\right)=(\mathrm{E})+(\mathrm{ES})+\left(\mathrm{EX}_{1}\right)+\ldots+\left(\mathrm{EX}_{n}\right)+\left(\mathrm{EX}_{1} \mathrm{~S}\right)+\ldots+\left(\mathrm{EX}_{n} \mathrm{~S}\right)
$$

If ES is the only active complex breaking down to the products, this equation may be rewritten in terms of (ES):

$$
\begin{gathered}
\left(\mathrm{E}_{t}\right)=\frac{K_{s}}{(\mathrm{~S})}(\mathrm{ES})+(\mathrm{ES})+\left[\frac{K_{s}\left(\mathrm{X}_{1}\right)}{(\mathrm{S}) K_{1}}+\ldots+\frac{K_{s}\left(\mathrm{X}_{n}\right)}{(\mathrm{S}) \boldsymbol{K}_{n}}\right](\mathrm{ES}) \\
+\left[\frac{\left(\mathrm{X}_{1}\right)}{\alpha K_{1}}+\ldots+\frac{\left(\mathrm{X}_{n}\right)}{v K_{n}}\right](\mathrm{ES})
\end{gathered}
$$

and the rate equation derived from $v=k(\mathrm{ES})$, in the donble-reciprocal (type A) form is given by:

$$
\frac{1}{v}=\frac{1}{\boldsymbol{V}_{m}}\left[1+\frac{\left(\mathrm{X}_{1}\right)}{a K_{1}}+\ldots+\frac{\left(\mathrm{X}_{n}\right)}{v K_{n}}\right]+\frac{K_{s}}{\boldsymbol{V}_{m}(\mathrm{~S})}\left[1+\frac{\left(\mathrm{X}_{1}\right)}{K_{1}}+\ldots+\frac{\left(\mathrm{X}_{n}\right)}{K_{n}}\right]
$$

where $\alpha \ldots v$ are the interaction constants:

$$
K_{1}=\frac{(\mathrm{E})\left(\mathrm{X}_{1}\right)}{\left(\mathrm{EX}_{1}\right)}, \quad \alpha K_{1}=\frac{(\mathrm{ES})\left(\mathrm{X}_{1}\right)}{\left(\mathrm{ESX}_{1}\right)} \text { etc. }
$$

indicating the effect of each component on the binding of substrate. A plot of $1 / v$ against $I /(S)$ will give a straight line with the characteristics:

$$
\begin{array}{r}
\text { Slope }=\frac{K_{s}}{V_{m}}\left[1+\frac{\left(\mathrm{X}_{1}\right)}{K_{1}}+\ldots+\frac{\left(\mathrm{X}_{n}\right)}{K_{n}}\right] \\
I / v \text { Intercept }=\frac{1}{V_{m}}\left[1+\frac{\left(\mathrm{X}_{1}\right)}{\alpha K_{1}}+\ldots+\frac{\left(\mathrm{X}_{n}\right)}{v K_{n}}\right]
\end{array}
$$

Thus the slope is dependent on the interaction constants while the intercept is not. If the inhibition is partially noncompetitive (or if any other 
components of the system merely modify the rate). complexes other than ES may be active and the rate expressions will be similar to that above but will involve additional terms for each active complex. If we take $\mathrm{X}_{1}$ to be an inhibitor, the six equations used in plotting may be written as:

Type A:

$\frac{1}{v_{i}}=\frac{1}{T_{m}}\left[1+\frac{(\mathrm{I})}{\alpha K_{i}}+\ldots+\frac{\left(\mathrm{X}_{n}\right)}{v K_{n}}\right]+\frac{K_{s}}{T_{m}(\mathrm{~S})}\left[1+\frac{(\mathrm{I})}{K_{i}}+\ldots+\frac{\left(\mathrm{X}_{n}\right)}{K_{n}}\right]$

Type B:

$\frac{1}{v_{\imath}}=\frac{K_{s}}{T_{n}}\left[1+\frac{(\mathrm{I})}{K_{i}}+\ldots+\frac{\left(\mathrm{X}_{n}\right)}{K_{n}}\right]+\frac{(\mathrm{S})}{V_{m}}\left[1+\frac{(\mathrm{I})}{u K_{i}}+\ldots+\frac{\left(\mathrm{X}_{n}\right)}{v K_{n}}\right]$

Type C:

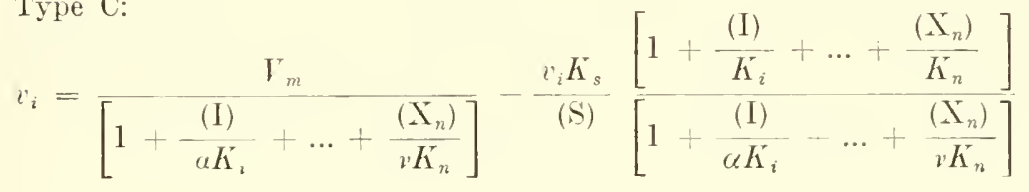

Type D:

$$
\begin{gathered}
\frac{1}{v_{i}}=\frac{1}{\Gamma_{m}}\left[1+\frac{\left(\mathrm{X}_{2}\right)}{\beta K_{2}}+\ldots+\frac{\left(\mathrm{X}_{n}\right)}{r K_{n}}\right]+\frac{K_{s}}{\Gamma_{m}(\mathrm{~S})}\left[1+\frac{\left(\mathrm{X}_{2}\right)}{K_{2}}+\ldots+\frac{\left(\mathrm{X}_{n}\right)}{K_{n}}\right] \\
+\frac{(\mathrm{I})}{Y_{m} K_{i}}\left[\frac{1}{\alpha}+\frac{K_{s}}{(\mathrm{~S})}\right]
\end{gathered}
$$

Type E:

$$
\frac{1}{i}=1+\frac{\alpha K_{i}}{(\mathrm{I})\left[\alpha+\frac{(S)}{K_{s}}\right]}\left\{1+\frac{\left(\mathrm{X}_{2}\right)}{K_{2}}+\ldots+\frac{\left(\mathrm{X}_{n}\right)}{K_{n}}+\frac{(S)}{K_{s}}\left[1+\frac{\left(\mathrm{X}_{2}\right)}{\beta K_{2}}+\ldots+\frac{\left(\mathrm{X}_{n}\right)}{v K_{n}}\right]\right\}
$$

Type F:

$$
\frac{(\mathrm{I})(\mathrm{I}-i)}{i}=\frac{\alpha K_{i}}{\alpha+\frac{(\mathrm{S})}{K_{s}}}\left\{1+\frac{\left(\mathrm{X}_{2}\right)}{K_{2}}+\ldots+\frac{\left(\mathrm{X}_{n}\right)}{K_{n}}+\frac{(\mathrm{S})}{K_{s}}\left[1+\frac{\left(\mathrm{X}_{2}\right)}{\beta K_{2}}+\ldots+\frac{\left(\mathrm{X}_{n}\right.}{v \cdot K_{n}}\right]\right\}
$$

When more than one substrate, inhibitor, or other component molecule reacts with the enzyme, the equations must be correspondingly modified 
but otherwise they provide the means for obtaining expressions for the most common types of enzyme systems and inhibitions.

The most important conclusion reached from inspection of these equations is that errors may be introduced into the determination of $K_{s}$ or $K_{i}$ by the presence of other compronents such as activators or coenzymes (or in enzyme systems where the hydrogen ion is involved in the active complex). Let us take one example to illustrate this. We shall assume that plotting the kinetic data gives curves indicating competitive inhibition as in Fig. 5-2. If an activator is present the following errors may be introduced.

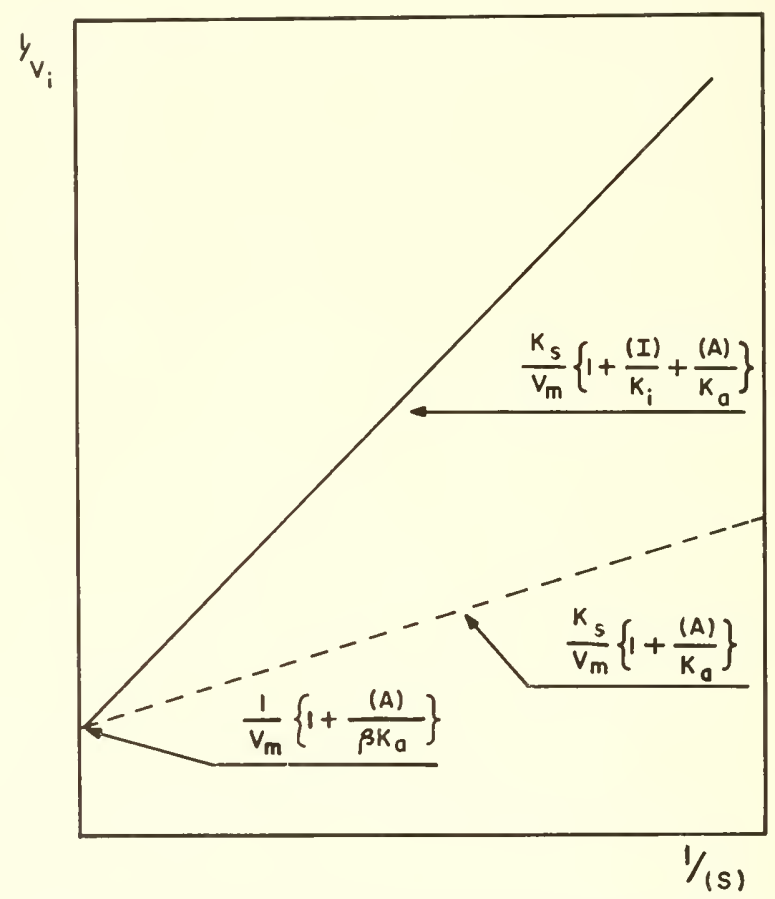

FIG. 5-2. Example to demonstrate the possible errors in the calculation of $K_{s}$ and $K_{i}$ in plots of type $\mathrm{A}$. It is assumed that an activator is present (Eq. 5-19).

To calculate $K_{s}$ the slope of the minhibited curve is divided by the $1 / v_{v}$ intercept; this will give the true $K_{s}$ (or the true $K_{m}$ in the general case) only when the presence of the activator on the enzyme does not alter the binding of substrate, i.e., when $\beta=1$.

$$
\frac{1}{v_{i}}=\frac{1}{V_{m}}\left[1+\frac{(\mathrm{A})}{\beta K_{a}}\right]+\frac{K_{s}}{V_{m}(\mathrm{~S})}\left[1+\frac{(\mathrm{I})}{K_{i}}+\frac{(\mathrm{A})}{K_{a}}\right]
$$


The substrate constant that is calculated would thus be:

$$
K_{s}^{\prime}=K_{s}\left[\frac{\beta K_{a}+\beta(A)}{\beta K_{a}+(A)}\right]
$$

Determination of $K_{i}$ would normally involve dividing the slope of the inhibited curve by the slope of the uninhibited curve:

$$
\text { slope }_{\text {inh }}=\mathrm{R} \text { slope }_{\text {uninh }}
$$

and $\mathrm{R}$ would be set equal to $1+(\mathrm{I}) / K_{i}$. However, when an activator occurs:

$$
\mathrm{R}=1+\frac{(\mathrm{I}) / K_{i}}{1+(\mathrm{A}) / K_{a}} \quad \text { and } \quad K_{i}{ }^{\prime}=K_{i} \frac{1}{1+(\mathrm{A}) / K_{a}}
$$

so that the calculated $K_{i}{ }^{\prime}$ will be less than the true $K_{i}$ by a factor that may be appreciable. Of course, if the occurrence of the activator is recognized and its effects are determined, it is possible to calculate the true $K_{i}$. It may be observed that if the inhibitor or enzyme active site has groups that dissociate within the experimental $\mathrm{pH}$ range, the calculated $K_{i}^{\prime}$ will include a factor in $\left(\mathrm{H}^{+}\right)$and thus depend on the $\mathrm{pH}$.

\section{SPECIFIC TYPES OF INHIBITION}

The basic types of inhibition on simple systems - completely and partially competitive and noncompetitive, and mixed - give the curves shown in Fig. 5-1 and 5-3 to 5-6. The marked differences between the curves in completely competitive and noncompetitive systems make these two types of inhibition easy to distinguish if the data and plotting are accurate, whatever system of plotting is used. However, a problem arises if the inhibition is partially competitive or noncompetitive, because in general the curves will be of the same basic nature but with modified slopes or intercepts. If this is not realized it will lead to errors in the calculation of the constants. As an example we shall take a case of partial competitive inhibition where the inhibitor does not prevent binding of substrate $(\alpha=\infty)$ but only increases the dissociation constant five-fold $(\alpha=5)$. The ratio $\mathrm{R}$ between the slopes of the inhibited and uninhibited curves in a type A plot would normally be expected to be equal to $1+$ (I) $/ K_{i}$ but when $\alpha \neq \infty$ its value is given by:

$$
\mathrm{R}=\frac{\alpha K_{i}+\alpha(\mathrm{I})}{\alpha K_{i}+(\mathrm{I})}
$$

Thus the calculated $K_{i}^{\prime}$ is not the true $K_{i}$ but:

$$
K_{i}^{\prime}=K_{i}\left[1-\frac{\mathrm{R}}{\alpha}\right]
$$




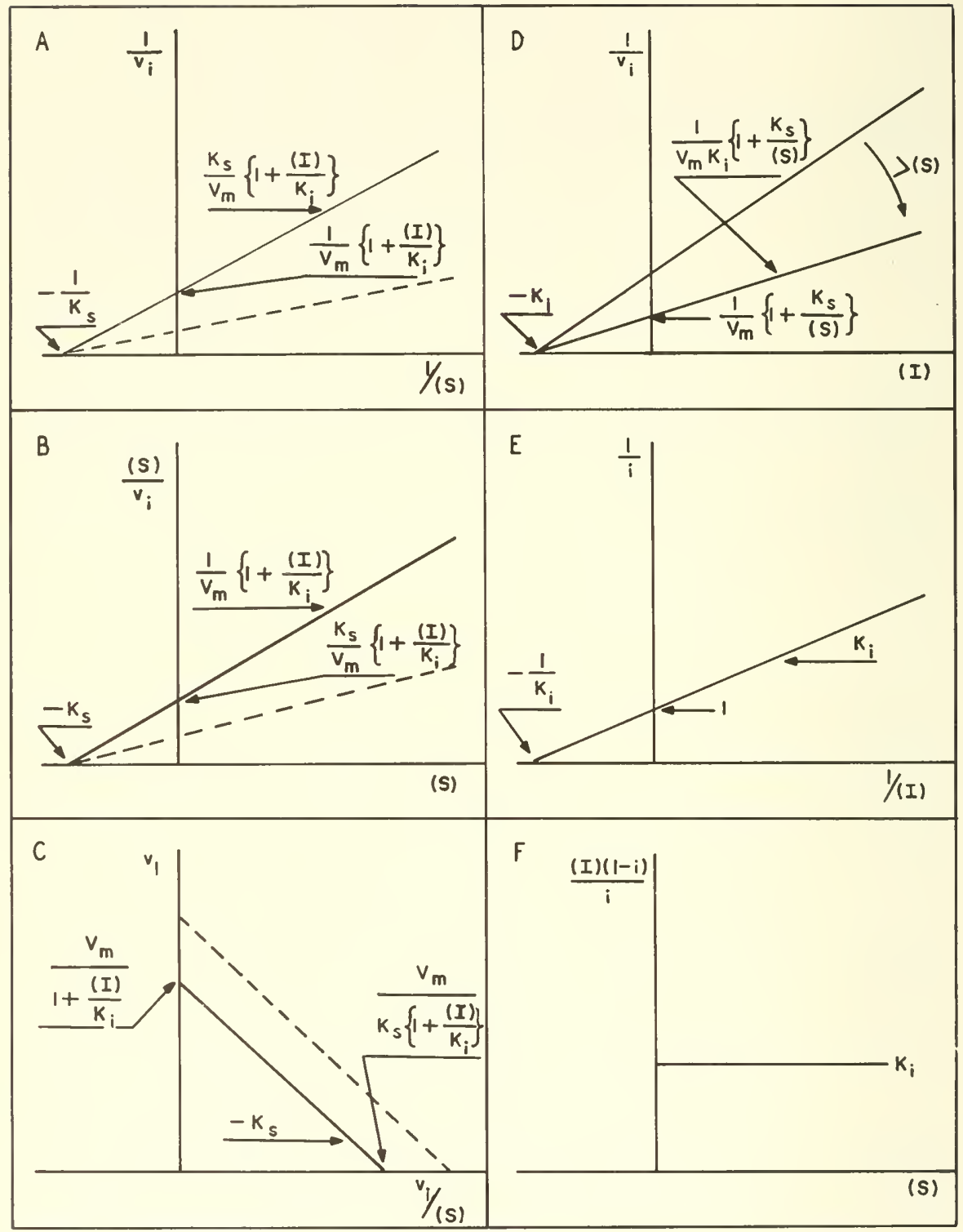

Fra. 5-3. The basie methods of plotting applied to noncompetitive inhibition. The dotted lines represent the uninhibited reaction. 


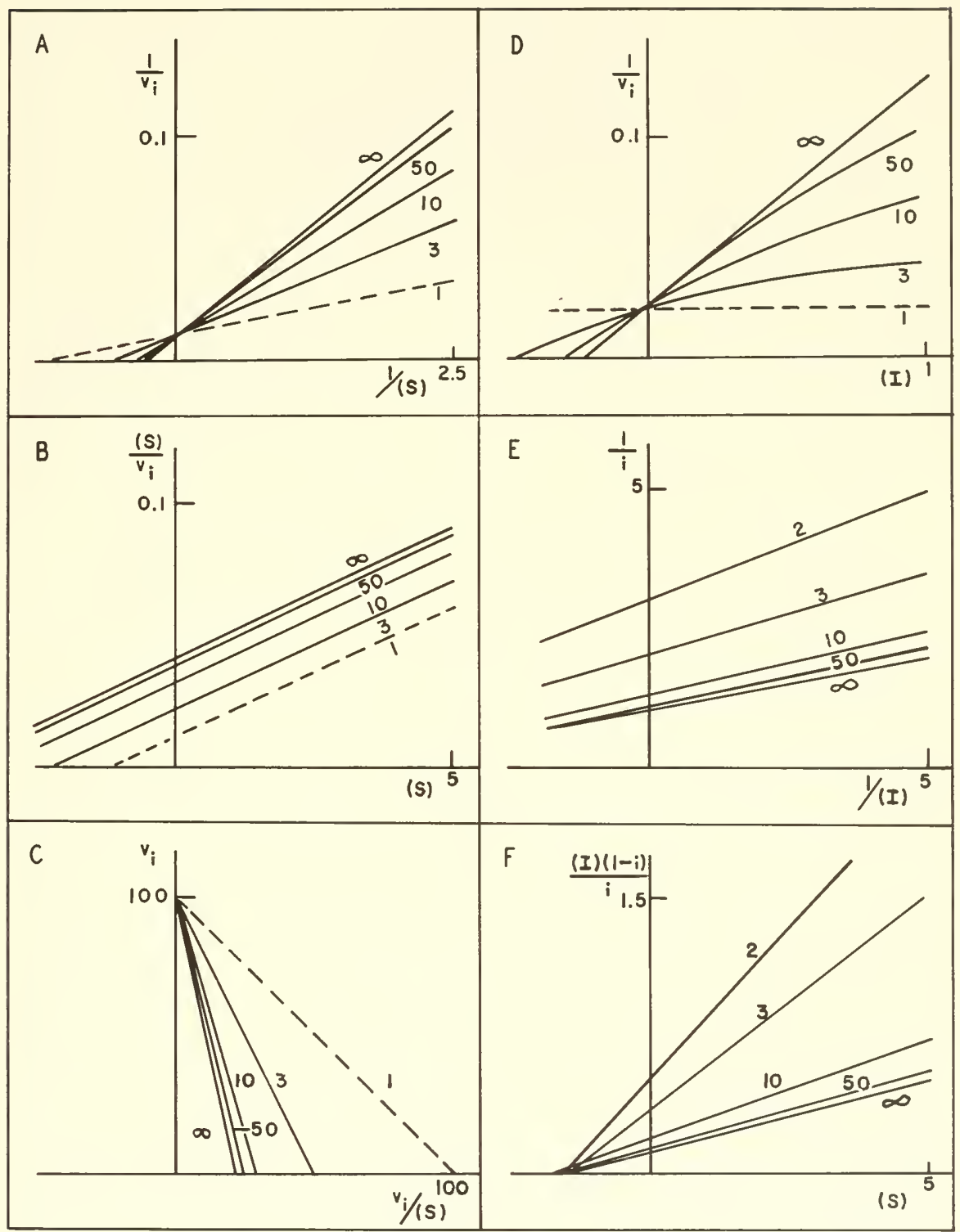

FIG. 5-4. Partially competitive inhibition. The numbers on the curves are the values of the substrate-inhibitor interaction constant $\alpha$. 
If we make the reasonable assumption that the slope of the inhibited curve is twice that of the unimhibited $(\mathrm{R}=2)$, we find that $K_{i}{ }^{\prime}=0.6 K_{i}$. When $\alpha$ has some value between 2 and 50, carefully plotted type D curves will be seen to be curved concavely downward (Fig. 5-4 D) and this may possibly give a hint of partially competitive inhibition. Nevertheless this is a modification of simple kinetics that is difficult to detect experimentally. A rather cumbersome method may be suggested. If type A plots are made for different concentrations of inhibitor and the values of $\mathrm{R}$ obtained from each are plotted against $K_{i}^{\prime}$, the true $K_{i}$ is indicated by the $K_{i}^{\prime}$-intercept. Perhaps a simpler procedure is to examine the $1 / i$ intercepts of type $\mathrm{E}$ plots; almost all types of inhibition give intercepts of unity but partial inhibitions are characterized by higher intercepts (Fig. 5-4 E and 5-5 E). The values of $\alpha$ or $\beta$ may be calculated if the type (competitive or noncompetitive) of inhibition is established by other plots; the $1 / i$ intercepts are:

Partially competitive:

Partially noncompetitive:

$$
\frac{\alpha+(\mathrm{S}) / K_{s}}{\alpha-1}
$$

$$
\frac{1}{1-\beta} \text {. }
$$

A situation which has seldom been considered, but yet may be of considerable importance, is mixed inhibition. In this case the presence of the inhibitor on the enzyme prevents the breakdown of the active complex but also interferes to some extent with the binding of substrate. The intersections of type A-D curves do not fall on either axis but at points from which the extent of deviation from noncompetitive inhibition may be determined. The literature contains many examples of type A plots where the curves, although linear, do not meet at the axes; usually the inhibition is stated to be competitive or noncompetitive depending upon the axis to which the intersection is closer. It is quite possible that these represent mixed inhibition. The imhibition in such cases will be intermediate between competitive and noncompetitive for any specific concentration of inhibitor. From Fig. 5-6 A it may be observed that the intersection is at $-1 / \alpha K_{s}$ on the abscissa and thus the value of $\alpha$ may be easily calculated. It is interesting, however, that the slope does not depend on $\alpha$ and hence the usual method of calculating $K_{i}$ from the slope ratio leads to no error. The other methods of plotting can yield similar results. If $\alpha$ is not too high, a plot of type $\mathrm{F}$ can be indicative of mixed inhibition due to the characteristic turning downward of the curve (Fig. 5-6 F).

\section{Coupling Inhibition (Uncompetitive or Anticompetitive)}

Although this type of inhibition may be relatively uncommon, there should certainly be no difficulty in detecting it inasmuch as the various 


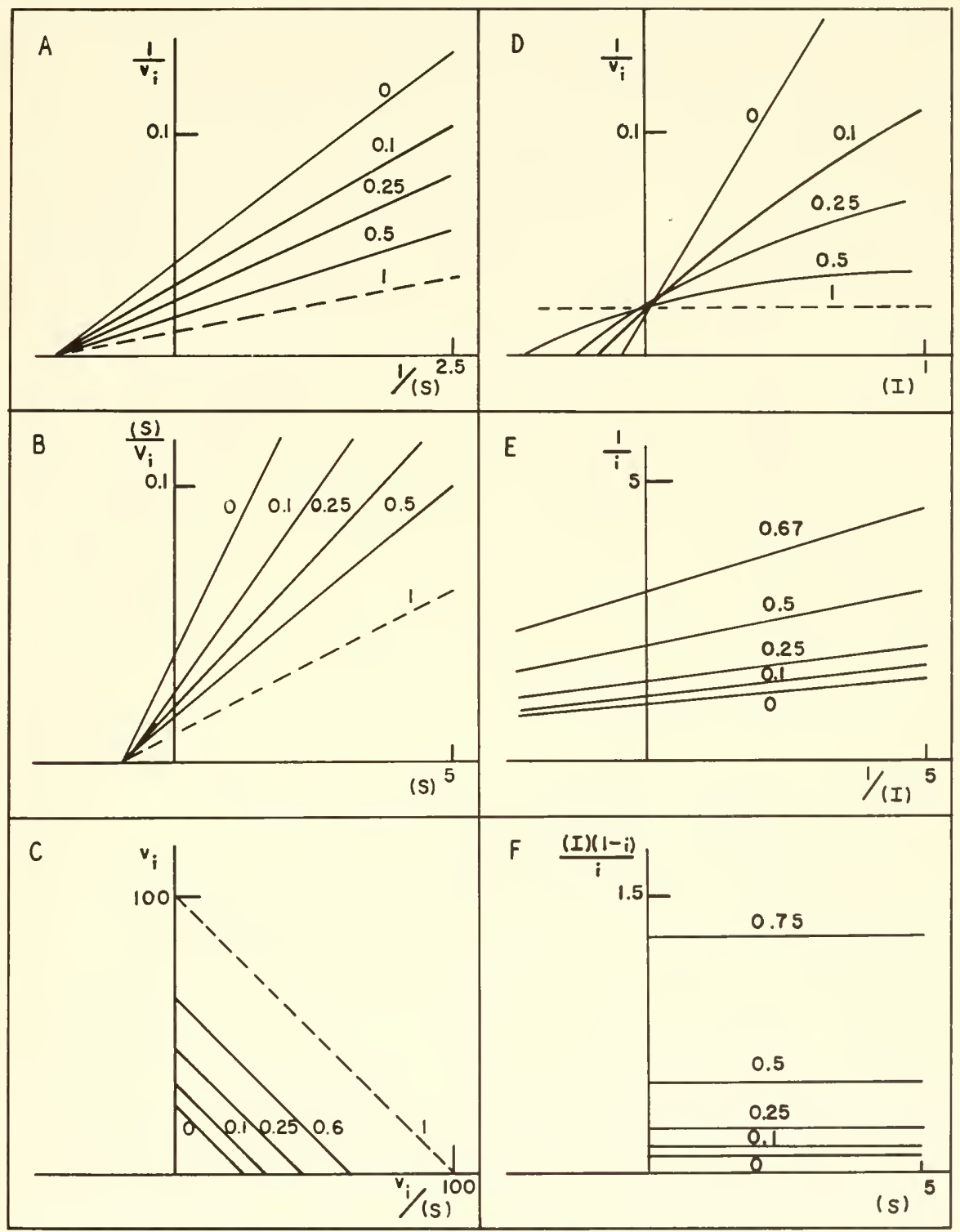

Fig. 5-5. Partially noncompetitive inhibition. The numbers on the curves are the values of the constant $\beta$. 


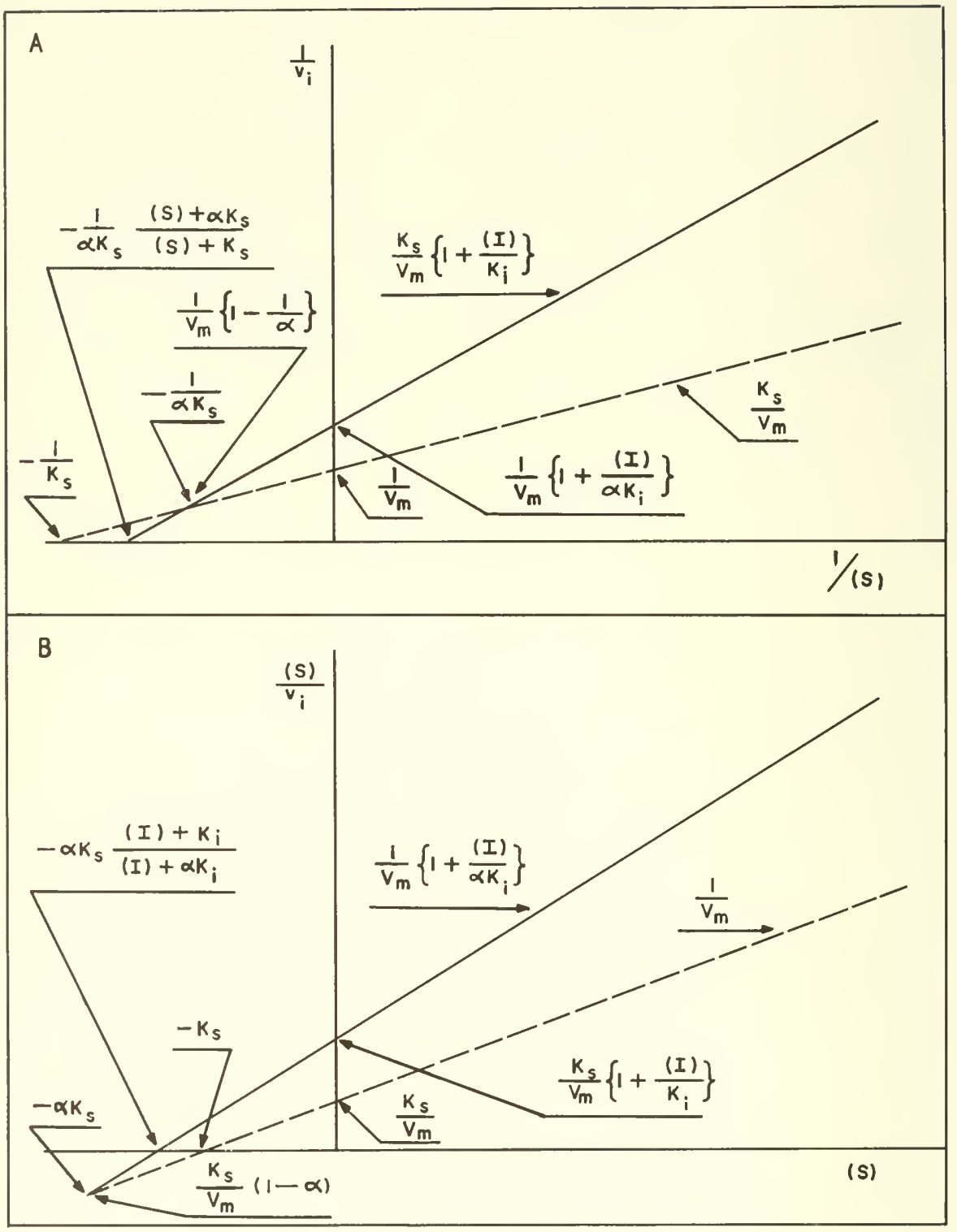

FIG. 5-6. Hixed inhibition. The dotted lines represent the uninhibited reaction. 


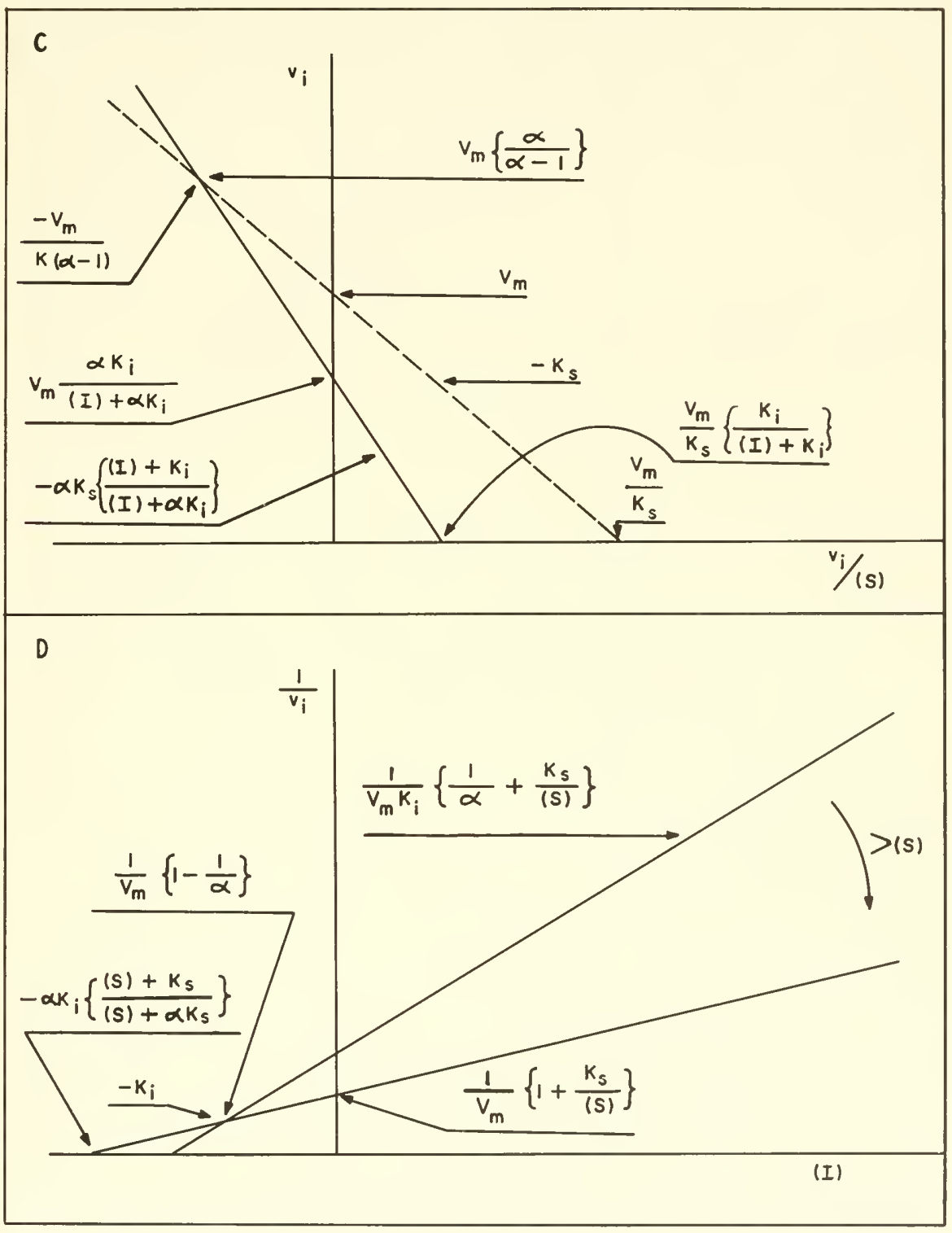

FIG. 5-6 (continued) 


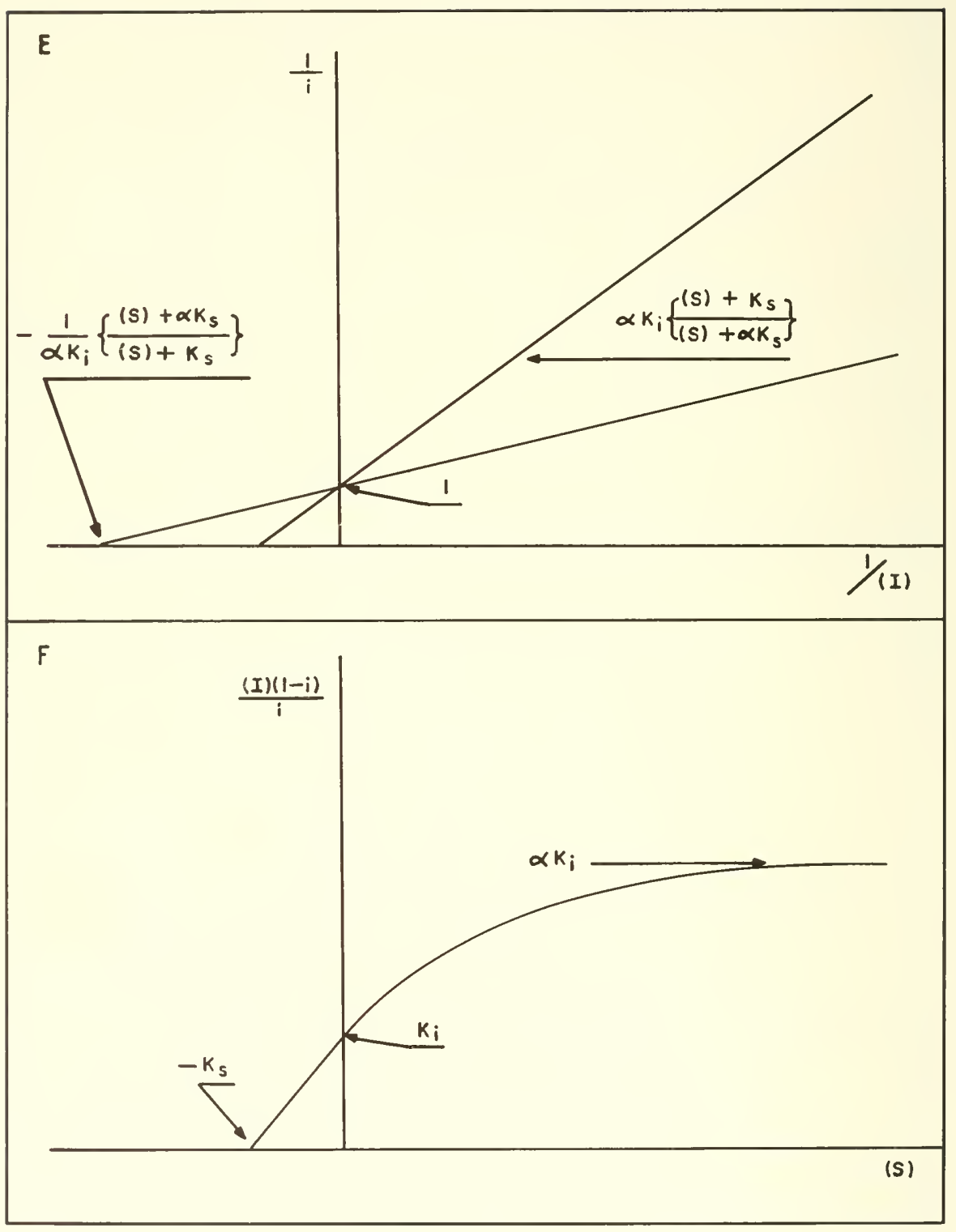

FIG. 5-6 (continued) 
plotting procedures give curves markedly different from any type of usnal inhibition (Fig. 5-7). The inhibition constant $K_{i}{ }^{\prime}$, representing the dissociation constant of the EIS complex into ES and I, may be readily calculated by most of the graphical methods. It may be noticed that this is the only type of inhibition considered which gives a hyperbolic-type curve in type $\mathrm{F}$ plotting (Fig. 5-7 F).

\section{Inhibition in Systems Involving an Activator}

When the inhibition is noncompetitive with respect to both substrate and activator, the curves obtained by all the above-described methods are identical in behavior with those for noncompetitive inhibition in systems without an activator (compare Fig. 5-3 and 5-8); however the values of the slopes and intercepts are different. A more interesting situation arises in inhibition that is competitive with the activator but not with substrate. It is evident that plotting by the methods outlined will lead to curves of a noncompetitive type because they have been plotted with respect to the substrate. However, if the activator is essential for the binding of the substrate, the curves will be of a competitive type (compare Fig. 5-9 and 5-10) because in such a system the inhibitor is essentially competing with both activator and substrate, although with the latter indirectly. The finding of competitive type curves can then mean that the inhibitor is primarily reacting with the activator site and not with the substrate site. If this is not realized, the calculations of constants will be in error.

The true situation and accurate evaluation of constants can be attained by plotting with respect to activator as in Fig. 5-11 to 5-13. The most straightforward method is perhaps the single-curve plotting of type $\mathrm{F}$ because the constants are directly obtainable but it has been rarely, if ever, utilized. The method of plotting $1 / v_{i}$ against $1 /(\mathrm{S})(\mathrm{A})$ has been illnstrated for it has been used occasionally but it presents no obvious advantages. As pointed out in Chapter 3 , the situation involving coenzymes or donor-acceptor reactions follows activator kinetics and the plotting procedures are similar. In any case, where there is a second component in the enzyme system, it is well to investigate the effects upon the inhibition of varying its concentration, plotting the results with respect to this component as well as substrate.

\section{Reaction of Inhibitor with Substrate}

The usual methods of plotting in this case lead to deviations from linearity (Fig. 5-14). It is not difficult to detect such inhibition but the constant $K_{s i}$ for the dissociation of the SI complex is not readily obtamable from the curves. A procedure suggested in Chapter 3 is perhaps more practical (see Eqs. 3-73 and 3-74). 


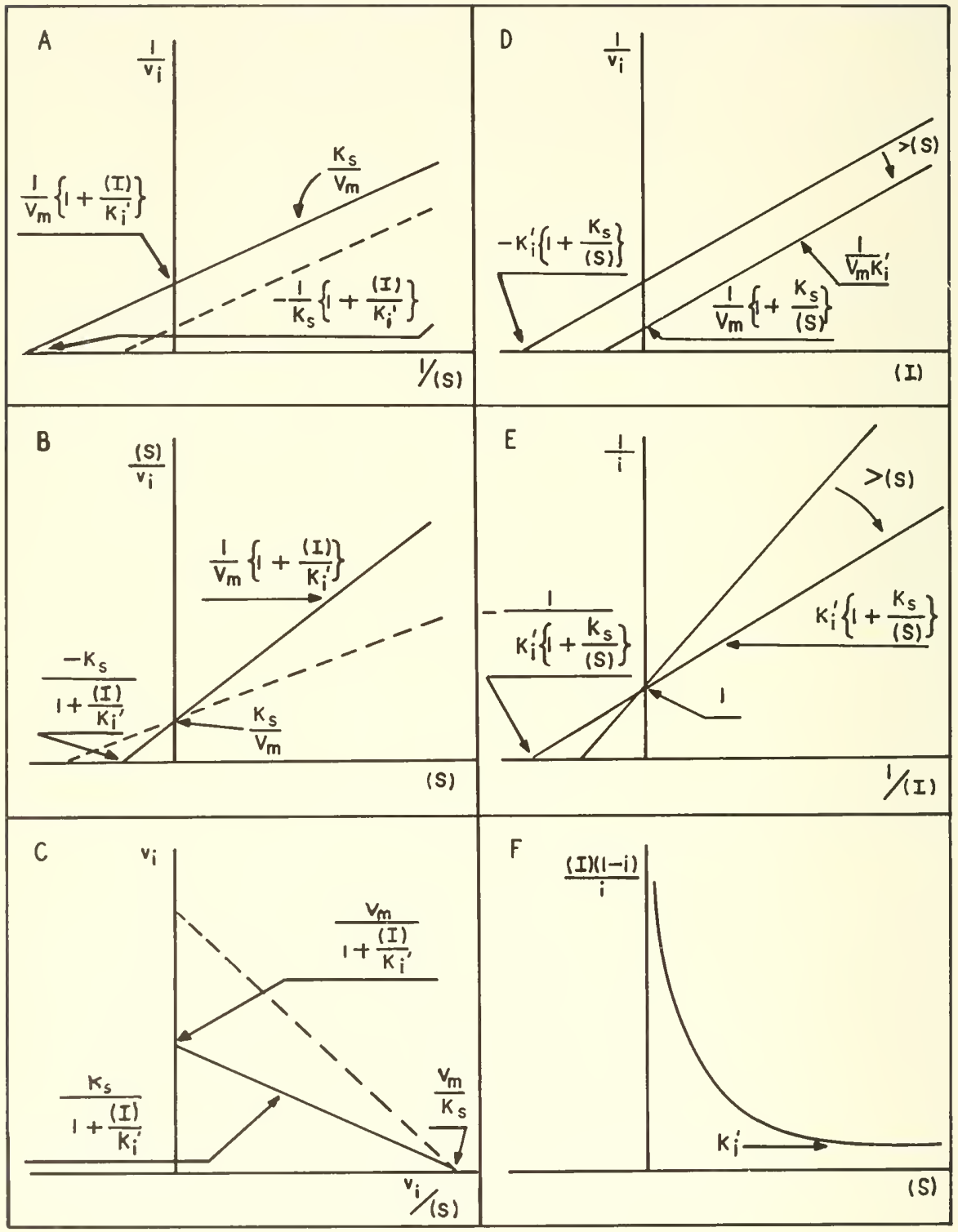

FIG. 5-7. Coupling or uncompetitive inhibition. The dotted lines represent the uninhibited reaction. 


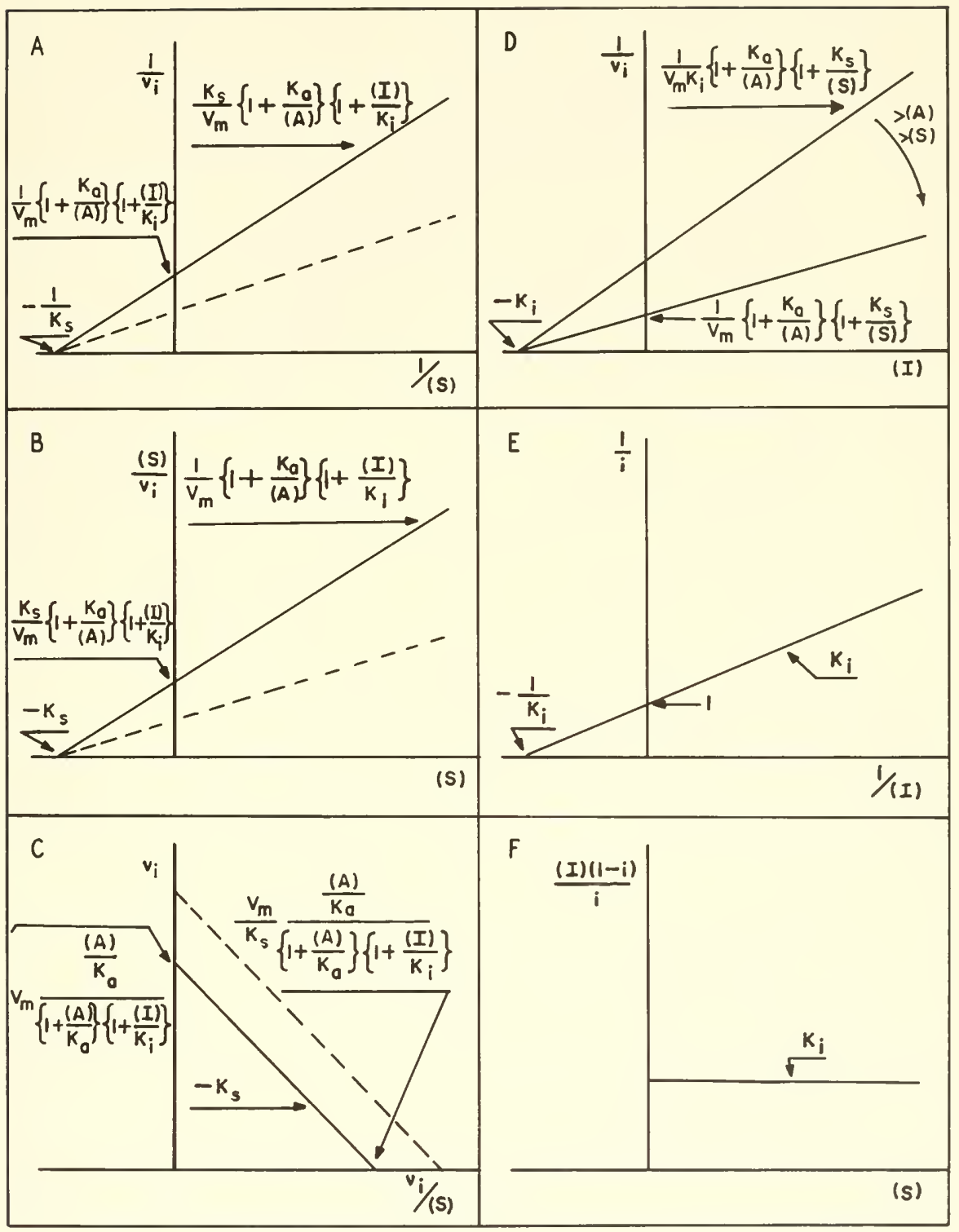

FIG. 5-8. Noncompetitive inhibition with respect to both the substrate and an activator. The dotted lines represent the uninhibited reaction. 


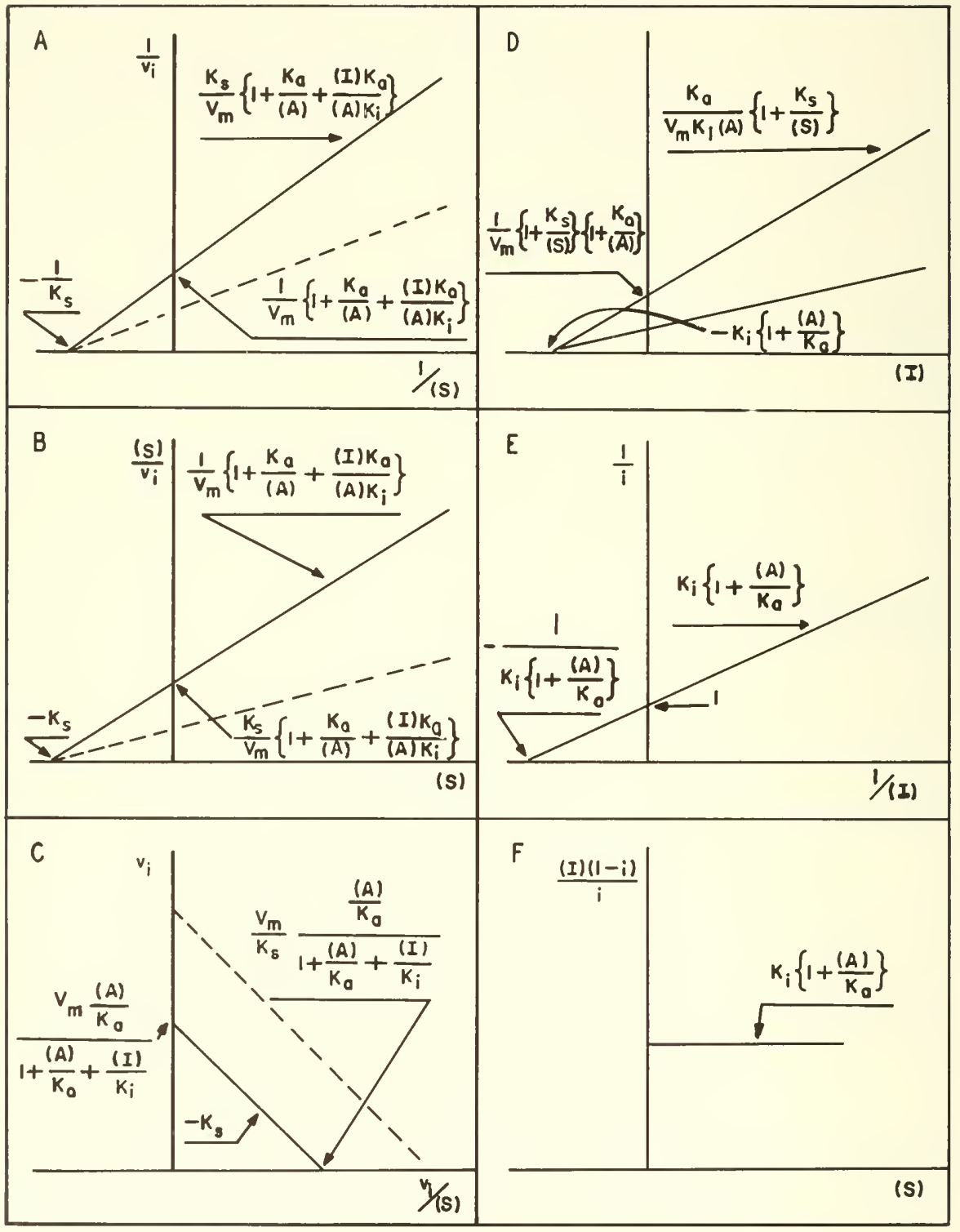

FIG. 5-9. Competitive inhibition with respect to an activator. The dotted lines represent the uninhibited reaction. 


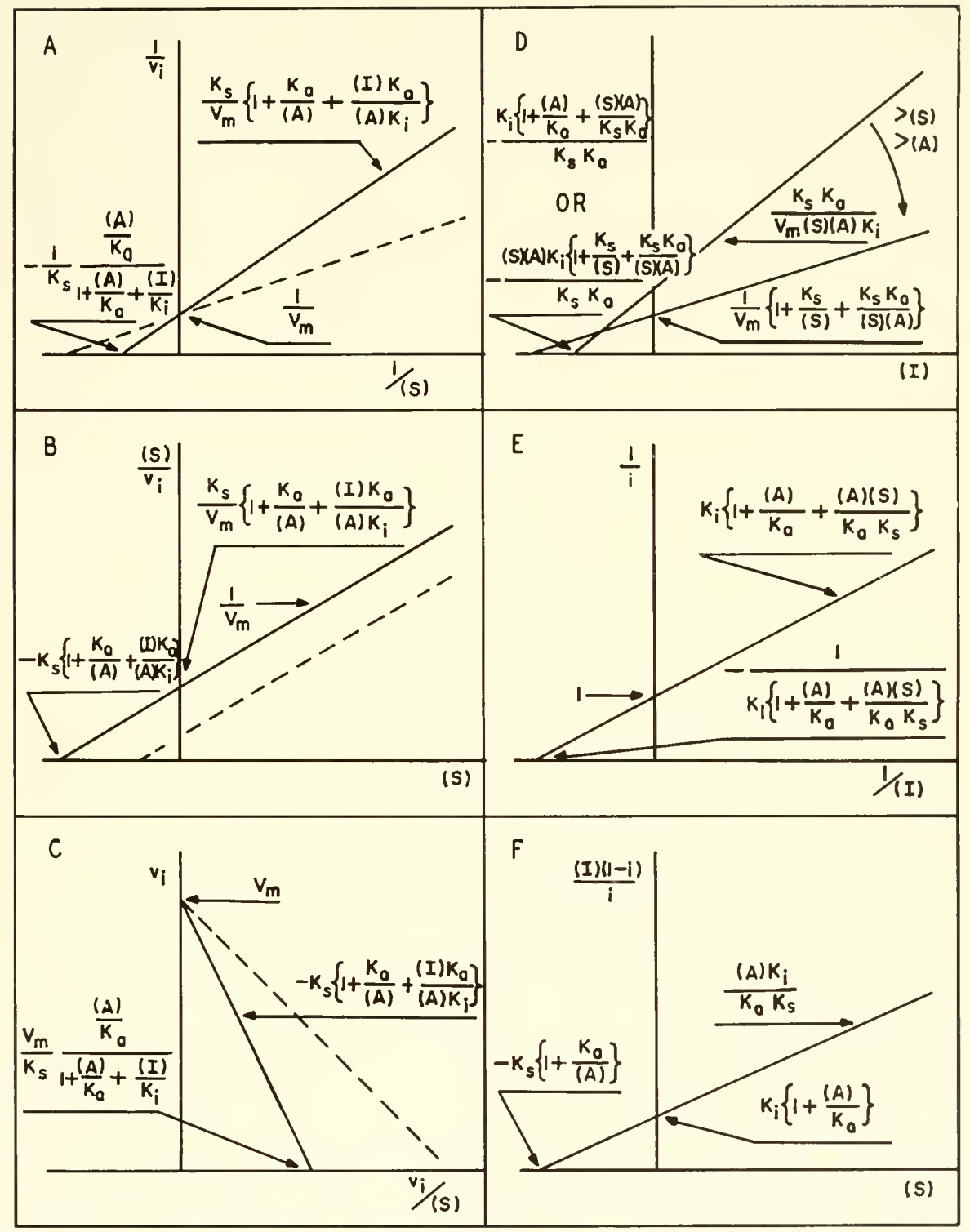

FIG. 5-10. Competitive inhibition with respect to an activator that is essential for the binding of the substrate. The dotted lines represent the uninhibited reaction. 


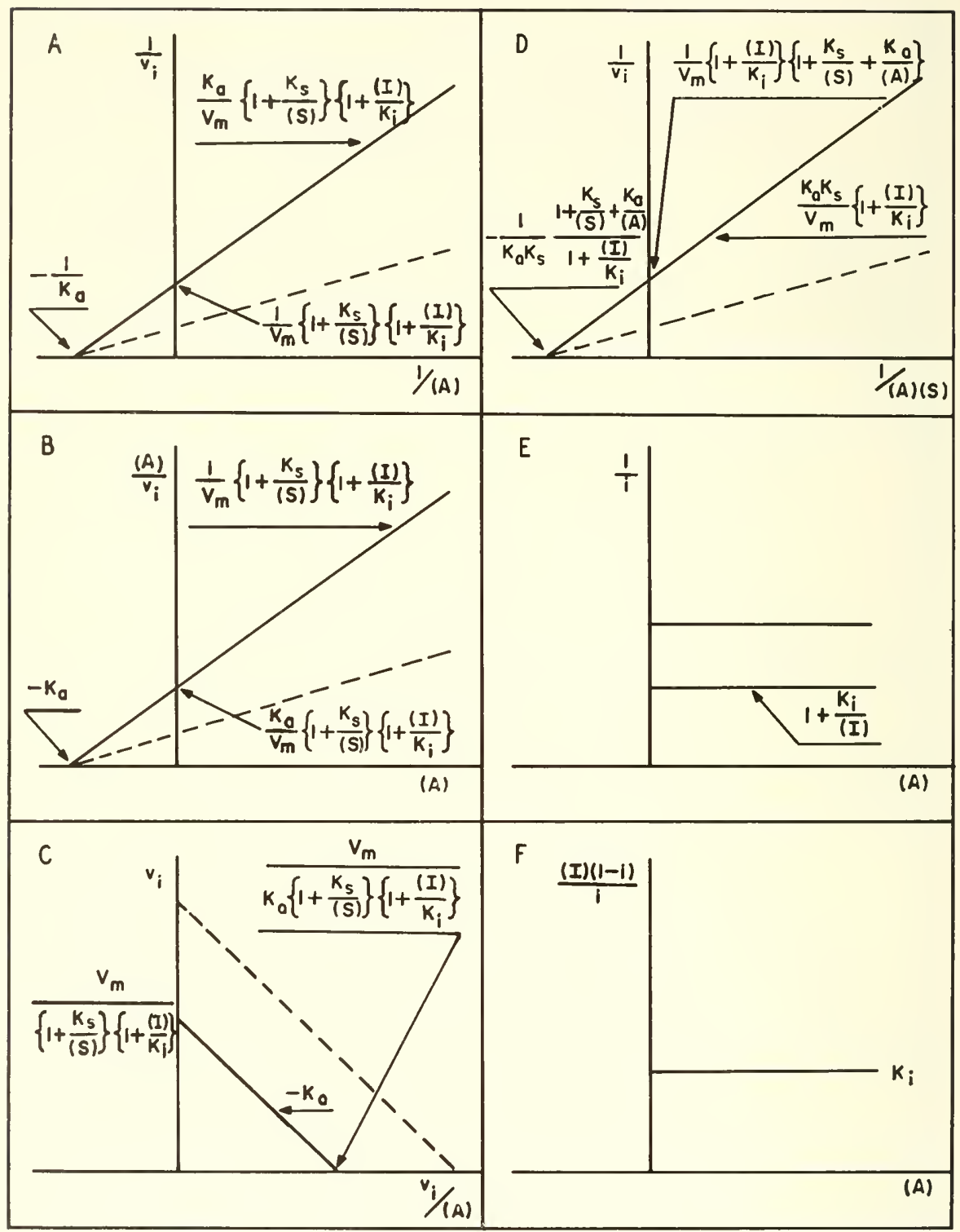

Fig. 5-11. Noneompetitive inhibition with respect to both the substrate and an activator. The plots are made using the concentration of the activator rather than the substrate. The dotted lines represent the uninhibited reaction. See Eq. 3-61. 


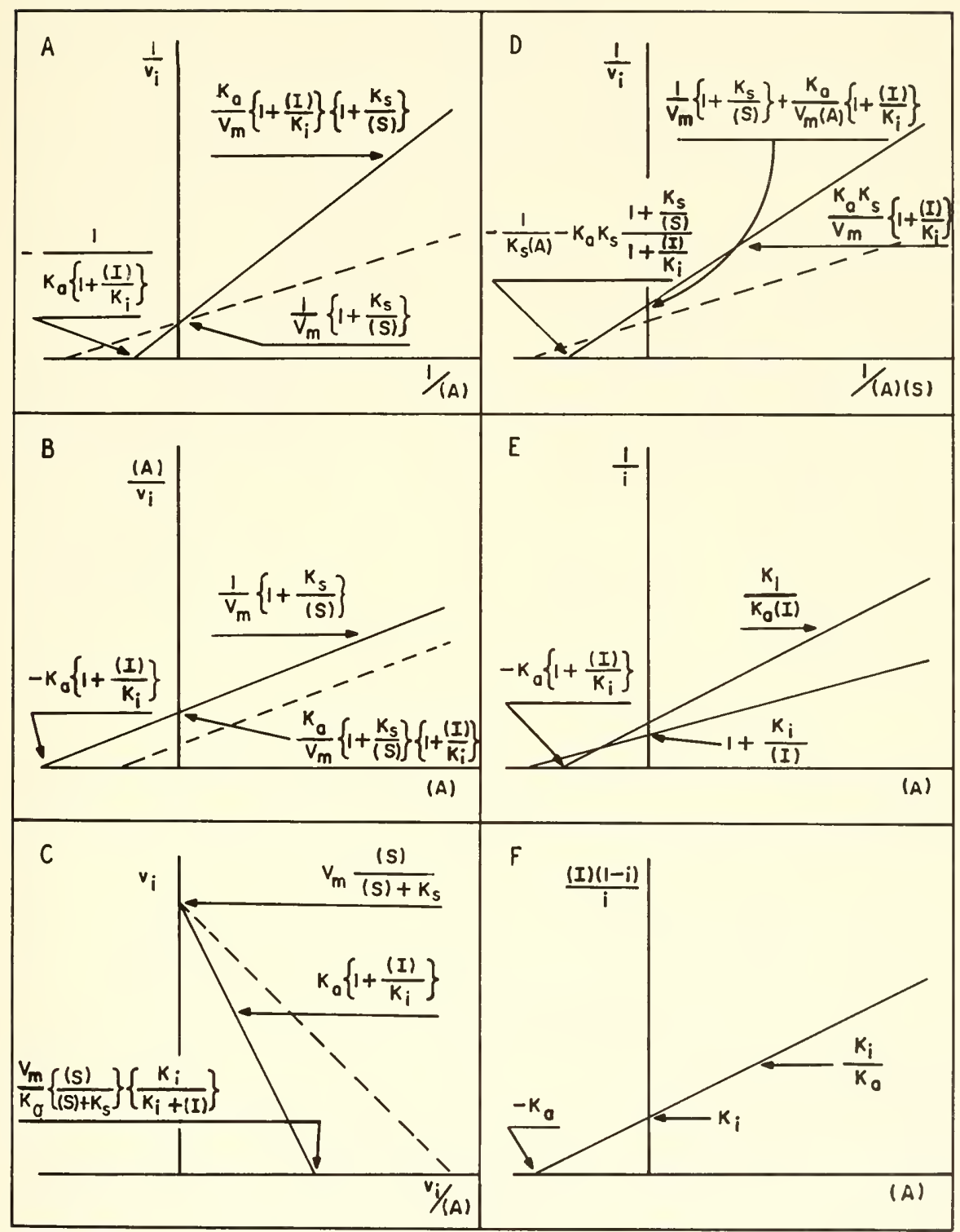

FIG. 5-12. Competitive inhibition with respect to an activator. The plots are made using the concentration of the activator rather than the substrate. The dotted lines represent the uninhibited reaction. See Eq. 3-6t. 


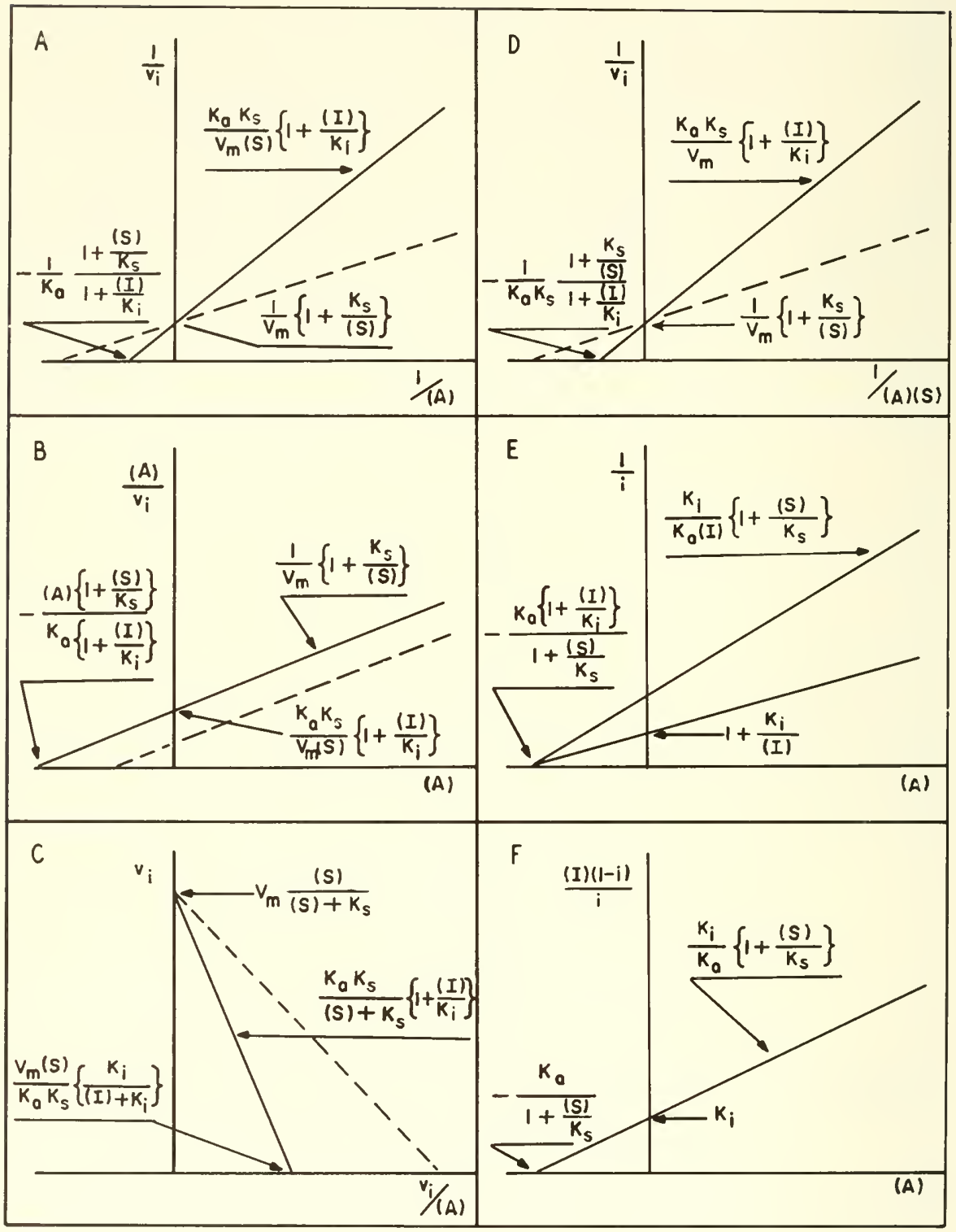

FIG. 5-13. Competitive inhibition with respect to an aetivator that is essential for the binding of the substrate. The plots are made using the eoncentration of the activator rather than the substrate. The dotted lines represent the uninhibited reaetion. See Eq. 3-68. 


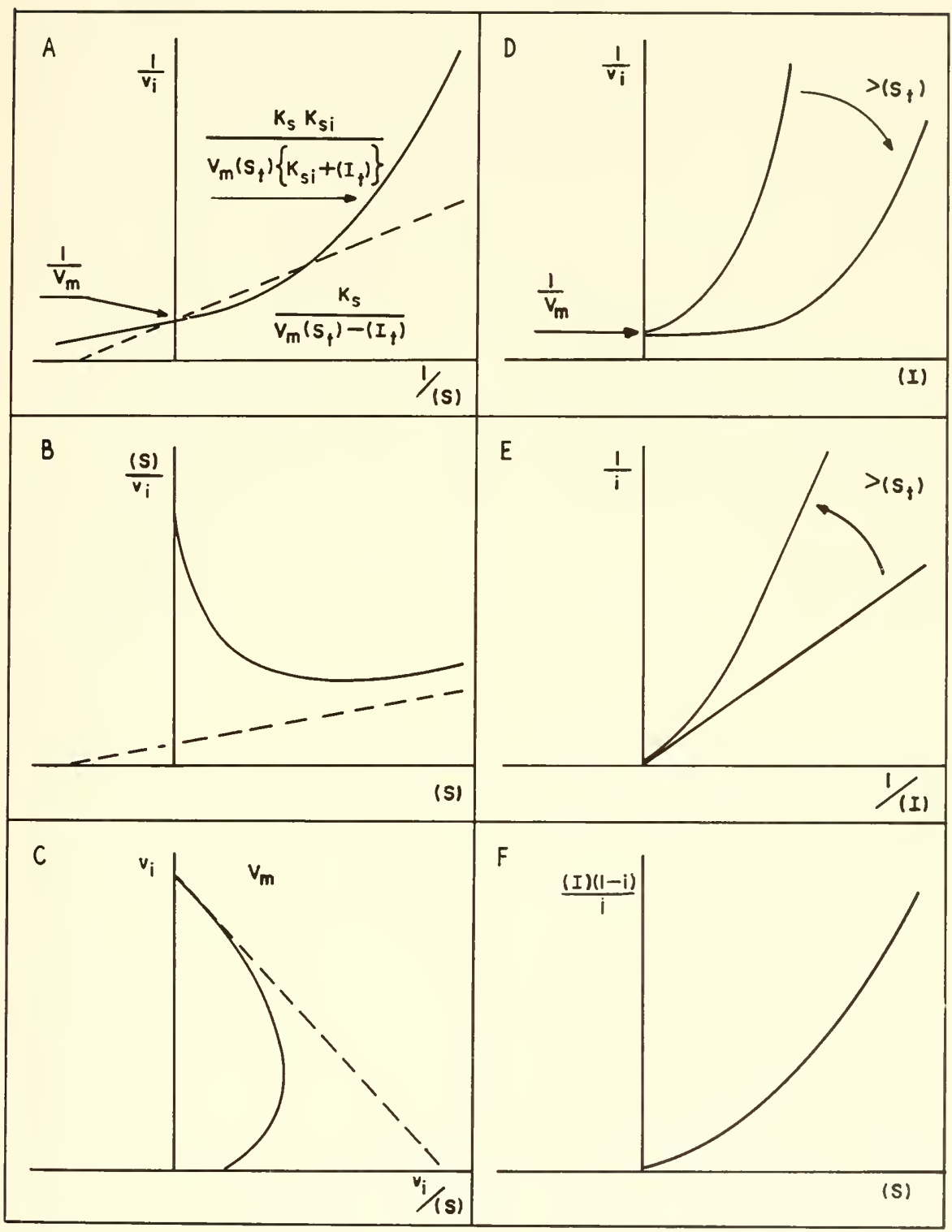

FIG. 5-14. The inhibitor reacts with the substrate, I $+S \rightleftharpoons$ IS. The dotted lines represent the uninhibited reaction. 


\section{Two Inhibitor Molecules Are Bound to the Enzyme}

There are many possibilities of behavior depending upon the exact mechanism and only two eases have been illustrated in Figs. 5-15 and 5-16. It is seen that noncompetitive inhibition yields the same type curves as for the simple case of a single inhibitor molecule, but again the slopes and intercepts are different and more complex. The exceptions are types D and $\mathrm{E}$ where deviations from linearity may occur. If only the double-reciprocal plot type $\mathrm{A}$ is made and simple competitive inhibition is assumed, the determination of $K_{i}$ is subject to error. If an inhibitor concentration of 0.3 $\mathrm{m} M$ increases the slope two-fold, $K_{i}$ would be found to be $0.3 \mathrm{~m} M$ assuming only one inhibitor molecule is reacting; however, $K_{i}$ would range from 0.186 to $0.486 \mathrm{~m} M$ if two inhibitor molecules are involved, depending on the value of $\beta$. It is possible, but laborious, to determine $\beta$ from several plots of type A-C at different inhibitor concentrations. The difficulty is in determining the values of both $\alpha$ and $\beta$ if one is not known; in fact it is unexpectedly difficult to distinguish between inhibitions with one and two inhibitor molecules, and cases where there are multiple sites with interaction or intrinsically different $K_{i}$ 's are impossible to deal with by the ordinary procedures. Suffice to say that in such eases, even when the kinetics follow simple patterns, the determined inhibition constants may correspond to no actual constants.

\section{Substrate Inhibition}

If the eoncentration of substrate has not been increased to where inhibition is obvious by a definite drop in the rate, this type of inhibition is usually detected in plots of type $\mathrm{A}$ by a rise in the curve as it approaches the $1 / v$ axis. It is not difficult to calculate a constant for the inhibiting substrate molecule but, as pointed out in the previous chapter, it is not easy to interpret this constant in the general ease. Values for $V_{m}$ and $K_{s}$ may be obtained from the slope at lower substrate concentrations and the intercept of the prolongation of the linear part of the curve on the $1 / v$ axis. This $V_{m}$ is the theoretical maximal rate if substrate inhibition did not occur; the actual maximal rate $v_{0}$ can only be determined experimentally. The value of $\alpha$ in the simplified scheme 4-6 may be calculated from the peak in the rate curve which occurs at $\left(\mathrm{S}_{0}\right)=K_{s} \sqrt{a}$. Another method involves a plot of $1 / v$ against (S), for from the reciproeal of Eq. 4-7:

$$
\frac{1}{v}=\frac{1}{V_{m}}+\frac{(\mathrm{S})}{\alpha V_{m} K_{s}}+\frac{K_{s}}{V_{m}(\mathrm{~S})}
$$

it is seen that when $(S)$ is high the last term is negligible, the slope at high (S) being $1 / \alpha V_{m} K_{s}$ and the intercept of this line prolonged on the 


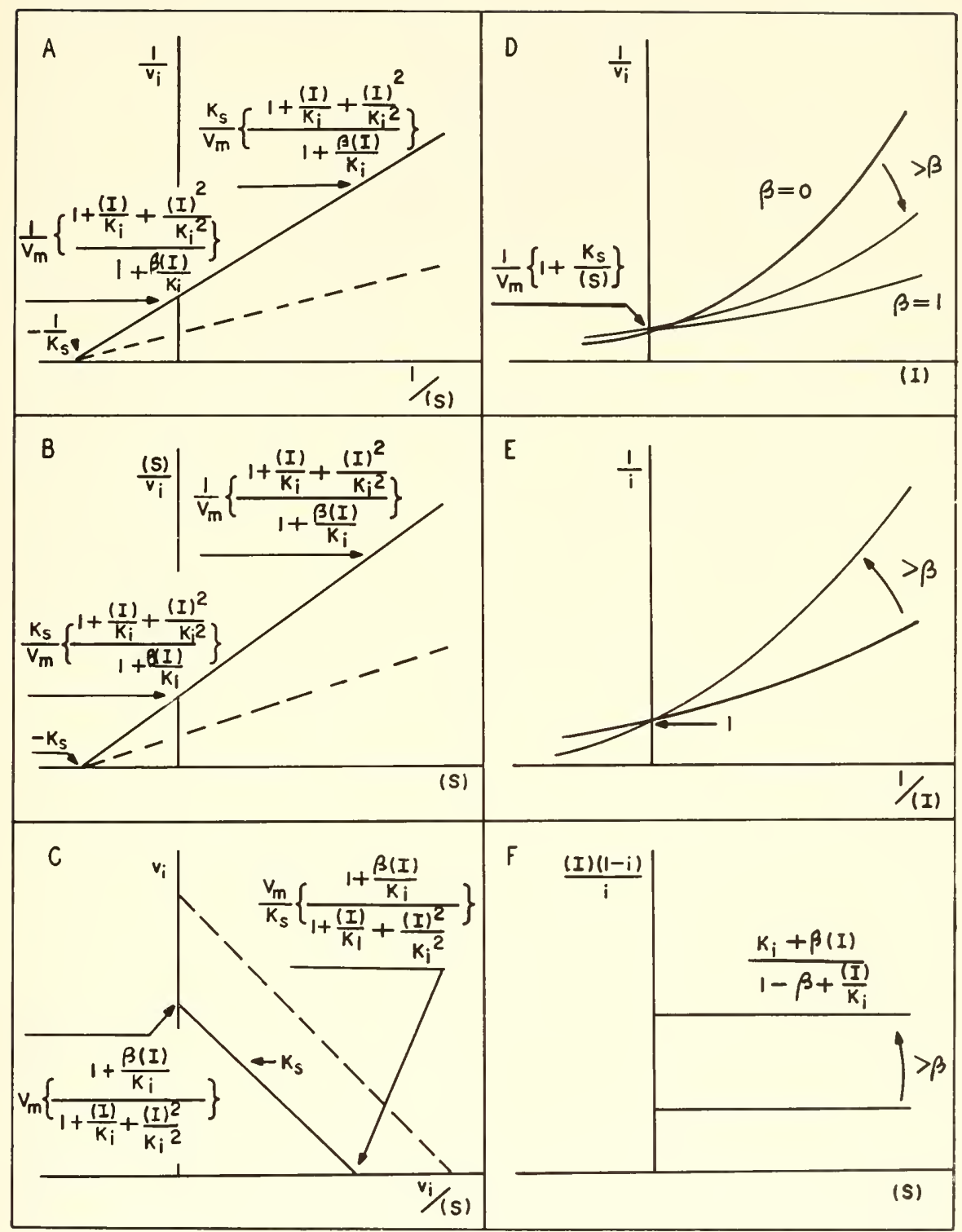

FIG. 5-15. Toncompetitive inhibition in which two inhibitor molecules are bound to the enzyme $(\alpha=1)$. The dotted lines represent the uninhibited reaction. See Eqs. 3-83 and 3-84. 


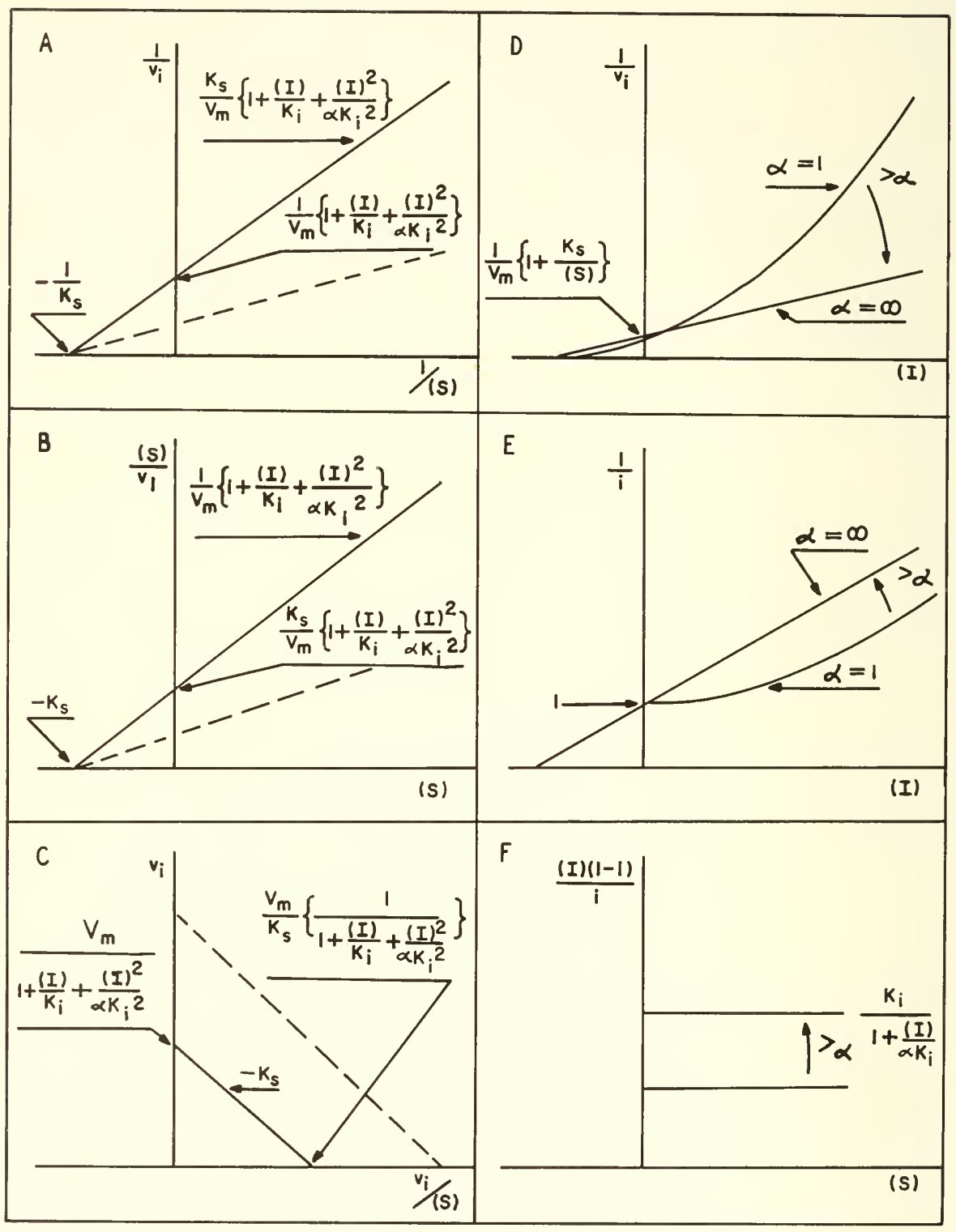

FIG. 5-16. Inhibition in which two inhibitor molecules are bound to the enzyme and $\beta=0$. The dotted lines represent the uninhibited reaction. 
$1 / v$ axis $1 / V_{m}$. The general characteristics of the standard plots are shown in Fig. 5-17. The meaning of $\alpha$ or the calculated inhibition constant will vary with the choice of binding situation or mechanism; it usually is not the dissociation constant of the $\mathrm{ES}_{1} \mathrm{~S}_{2}$ complex into $\mathrm{ES}_{1}$ and $\mathrm{S}_{2}$.

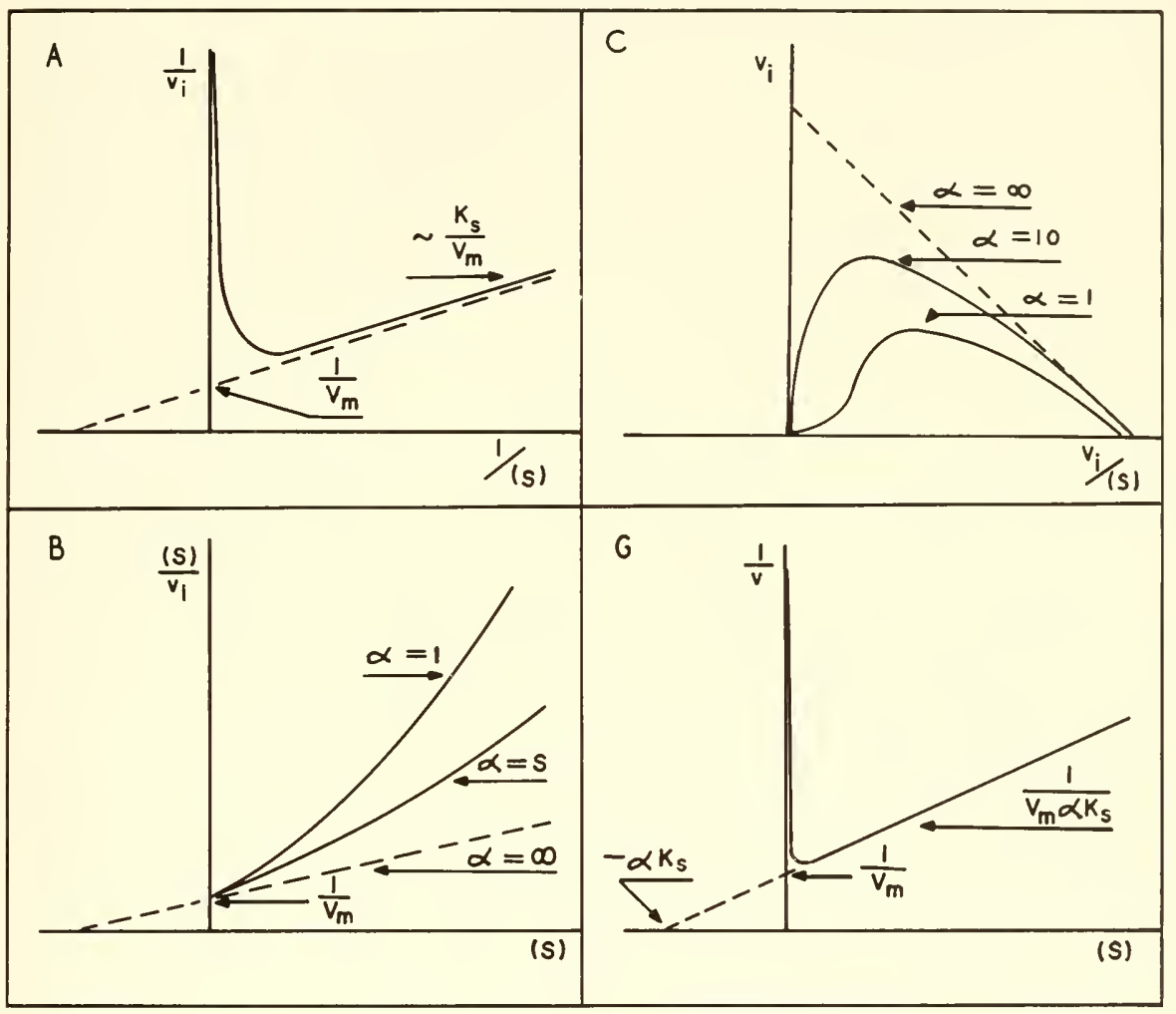

FIG. 5-17. Substrate inhibition of type A. The dotted lines represent the uninhibited reaction. See Eq. 4-7.

Inhibition of enzyme systems in which substrate inhibition occurs does not present serious problems as long as the substrate inhibition is recognized and the proper graphical methods are used. Figures 5-18 and 5-19 give the plots for noncompetitive and competitive inhibition in such systems and the characteristics of those graphs involving (S) are dominated by the substrate inhibition; in fact, for noncompetitive inhibition, where the degree of inhibition doesn't depend on (S), the curves are merely raised or lowered a constant fraction at any inhibitor concentration. The plots of types $\mathrm{E}$ and $\mathrm{F}$ are the most valuable here and the inhibition constant may be obtained directly. 


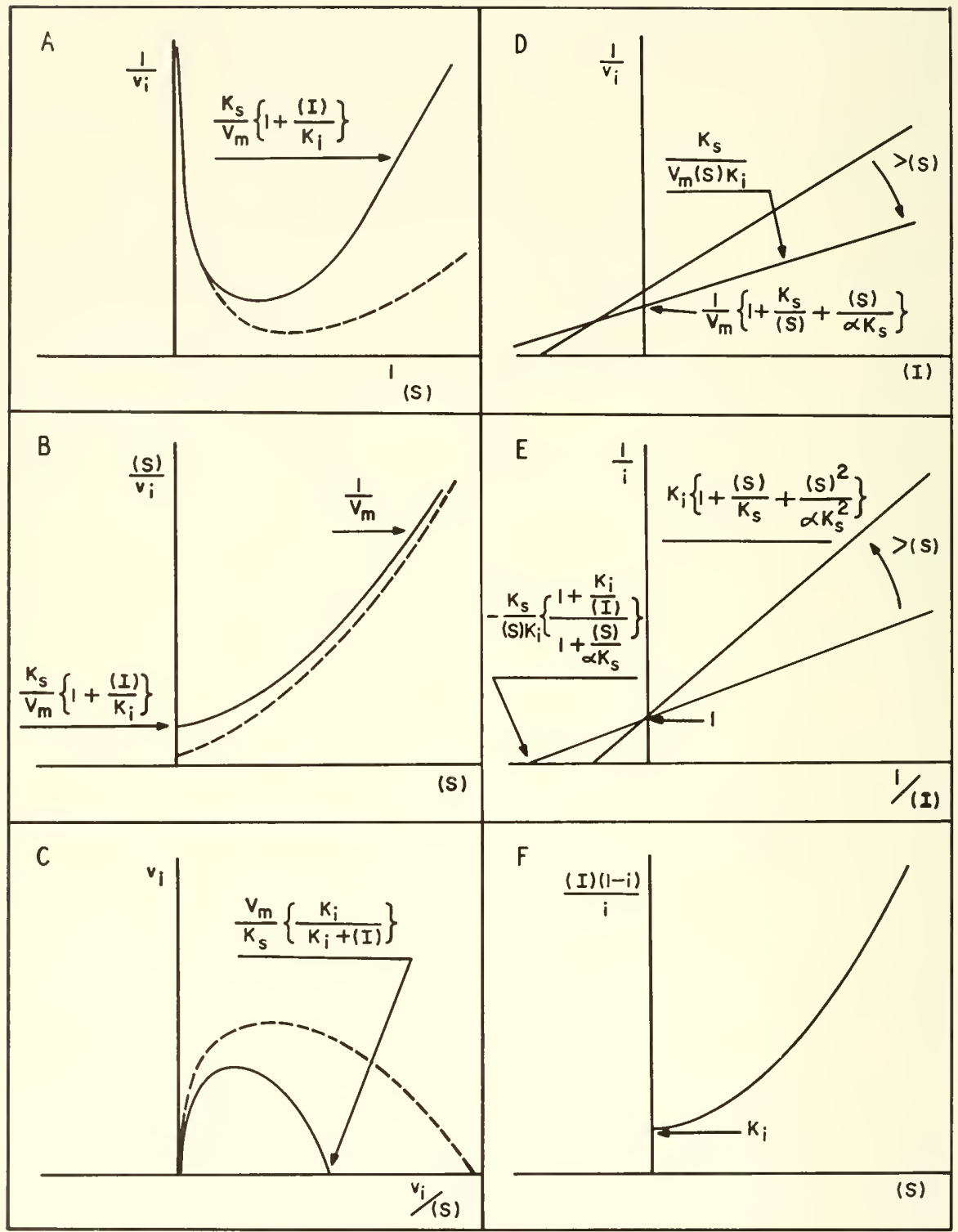

FIG. 5-18. Competitive inhibition of enzyme exhibiting substrate inhibition $(\alpha=5)$. The dotted lines represent the uninhibited reaction. See Eqs. 4-26 and 4-27. 


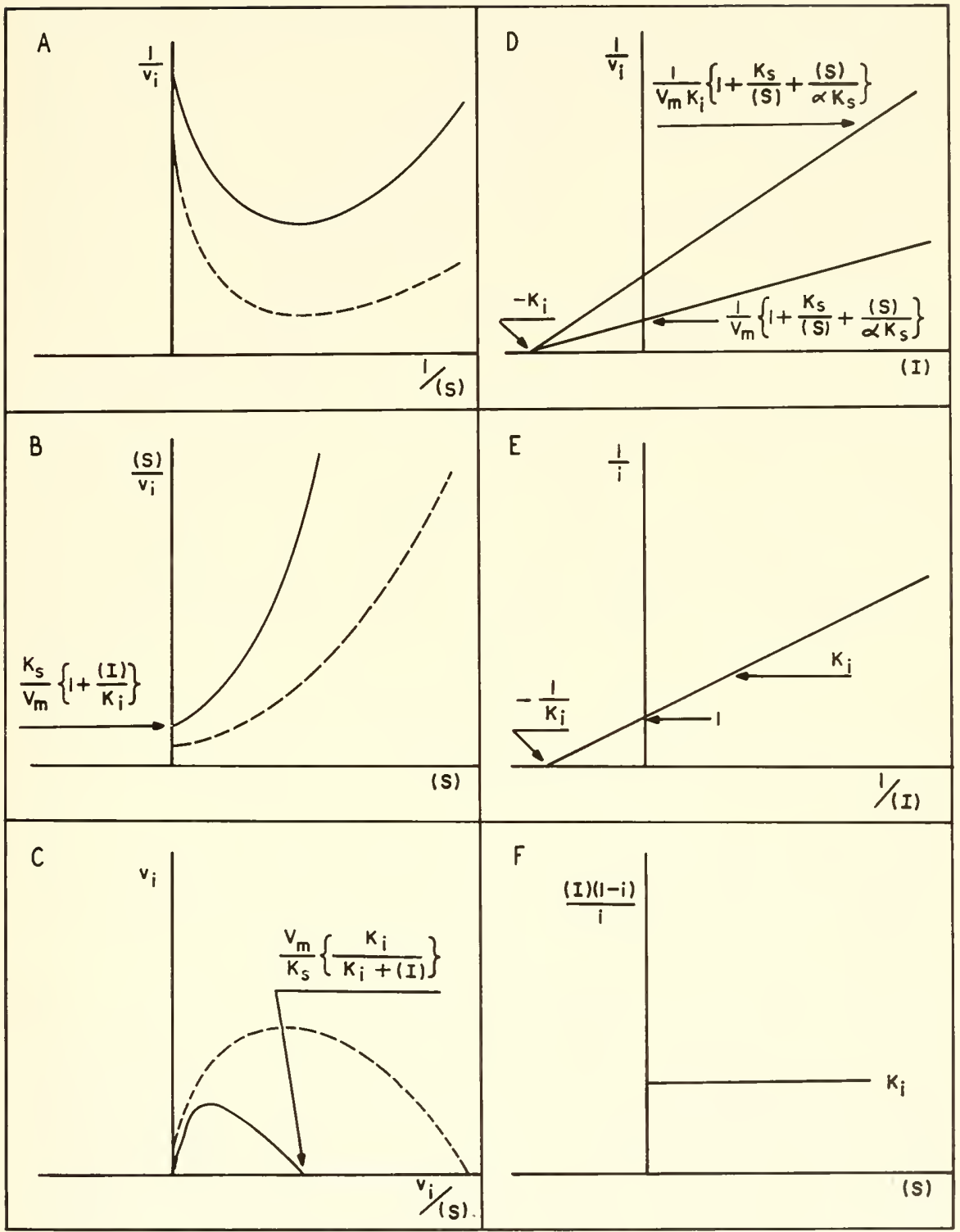

Fig. 5-19. Noncompetitive inhibition of enzymes exhibiting substrate inhibition. The dotted lines represent the uninhibited reaction. See Eqs. 4-28 and 4-29. 


\section{Product Inhibition}

When the product inhibits the forward reaction by combining with the enzyme or an activator in a manner similar to that of an inhibitor by any of the possible mechanisms, the kinetics and the plots derived from them will be quite comparable to those exhibited by the inhibitions outlined above. The only unique situation is where the back reaction is occurring and the deviation from nomal kinetics will depend on the over-all equilibrium constant as indicated in Fig. 5-20. It may be noticed that even when

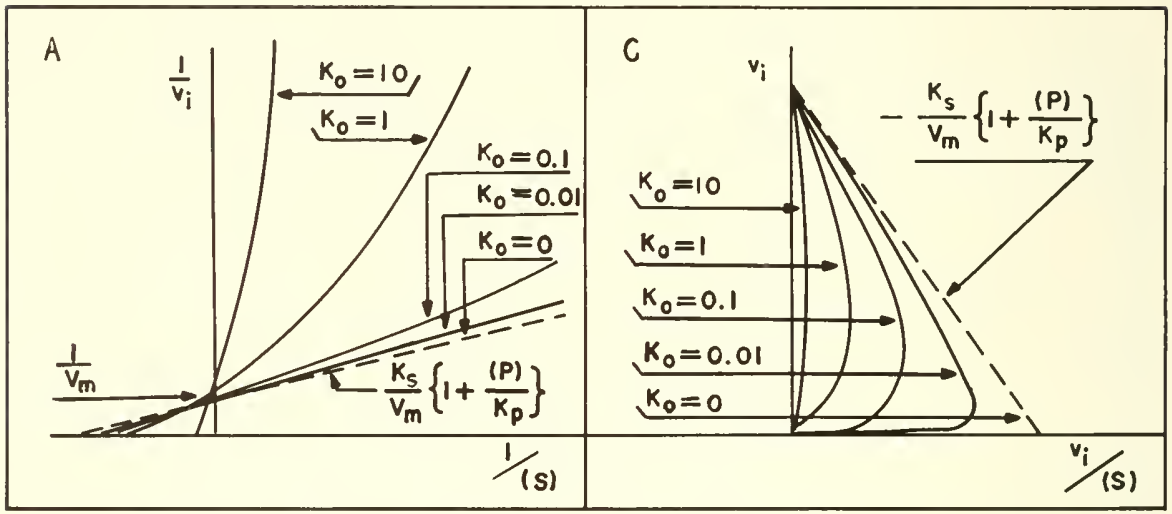

FIG. 5-20. Product inhibition at different values of the over-all equilibrium constant $K_{0}$. The dotted lines represent the uninhibited reaction. See Eq. 4-38.

the equilibrium is strongly in favor of the product. the slope of the type A plot is dependent on $(\mathrm{P}) / K_{p}$; in this case the product is behaving as a competitive inhibitor and $K_{p}$ in easily obtained. The $K_{p}$ thus determined will be a true dissociation constant and will not necessarily represent the Michaelis constant for the back reaction.

The inhibition of yeast pyruvate decarboxylase by acetaldehyde was analyzed by Gruber and Wassenaar (1960) and designated as noncompetitive from a plot of $1 / v_{i}$ against (I). It must be emphasized that from a single plot of this type (i.e., at one substrate concentration), the mechanism of the inhibition cannot be immediately derived. However, from a single curve it is possible to calculate $K_{i}$ from either the slope or the intercept on the (I) axis, assuming competitive or noncompetitive inhibition, and these values for $K_{i}$ should be identical if the assumed mechanism is correct. Nevertheless, it is preferable to plot several curves for different substrate concentrations and determine whether they do or do not meet at one point on the (I) axis. 


\section{DETERMINATION OF CONSTANTS OTHER THAN $K_{i}$}

Most inhibitions may be adequately characterized by two types of constants: dissociation constants indicating the binding energy and interaction constants describing the effects of the bound inhibitor on either the binding of the components of the reaction or the rate at which the active complex breaks down to products. It is obvious that the inhibition is not quantitatively expressed by $K_{i}$ alone but that the effect of the inhibitor on the reaction must also be evaluated. These effects and the values of the interaction constants are implied when the inhibition is stated to be completely competitive or noncompetitive but insufficient attention has been paid to situations where the inhibition is partial, mixed, or more complex.

\section{Determination of Interaction Constants}

The general reaction scheme 3-2 for an enzyme not involving activators or coenzymes presents an inhibition characterized by three constants $-\alpha, \beta$ and $K_{i}$ - and is dependent also on the uninhibited enzyme constants $V_{m}$ and $K_{s}$. If the inhibition is not of the simple types where $\alpha=1$ or $\infty$ or where $\beta=1$ or 0 , it is possible to determine these constants by the usual graphical procedures. In the general case, the slopes and intercepts of the type A double-reciprocal plot are given by:

$$
\begin{aligned}
& \text { Slope }=s=\frac{\alpha K_{s}}{V_{m}}\left[\frac{(\mathrm{I})+K_{i}}{\beta(\mathrm{I})+\alpha K_{i}}\right] \\
& 1 / v_{i} \text { Intercept }=t=\frac{1}{V_{m}}\left[\frac{(\mathrm{I})+\alpha K_{i}}{\beta(\mathrm{I})+\alpha K_{i}}\right] \\
& 1 /(\mathrm{S}) \text { Intercept }=u=-\frac{1}{\alpha K_{s}}\left[\frac{(\mathrm{I})+\alpha K_{i}}{(\mathrm{I})+K_{i}}\right] \\
& \frac{s}{t}=\alpha K_{s}\left[\frac{(\mathrm{I})+K_{i}}{(\mathrm{I})+\alpha K_{i}}\right]
\end{aligned}
$$

From these equations, or comparable ones from other types of plotting, or from combinations of different plots, it is possible to determine $\alpha, \beta$, and $K_{i}$. For example, determination of the slopes and $1 / v_{i}$ intercepts for two different concentrations of inhibitor will lead to a value for $\alpha$ :

$$
\alpha=\frac{1}{K_{s}}\left[\frac{s_{1}\left(\mathrm{I}_{1}\right)\left(s_{2}-t_{2} K_{s}\right)-s_{2}\left(\mathrm{I}_{2}\right)\left(s_{1}-t_{1} K_{s}\right)}{t_{1}\left(\mathrm{I}_{1}\right)\left(s_{2}-t_{2} K_{s}\right)-t_{2}\left(\mathrm{I}_{2}\right)\left(s_{1}-t_{1} K_{s}\right)}\right]
$$

from which $\beta$ and $K_{i}$ may also be calculated. If the inhibition is obviously of a type in which either $\alpha$ or $\beta$ assume extreme values, the calculations 
are simplified. Even for partially competitive or partially noncompetitive inhibitions, however, calculations must be based on plots made at different inhibitor concentrations, or results from two or more types of plotting. It has already been pointed out that the type $E$ plot of $1 / i$ against $1 /(\mathrm{I})$ is particularly useful for calculation of $\alpha$ or $\beta$ in cases of partially competitive or noncompetitive inhibitions since the intercepts involve only $\alpha$ or $\beta$ and known quantities.

\section{Determination of $K_{s} / K_{i}$}

It is frequently of interest, especially in competitive inhibition, to know the relative affinities of the enzyme for substrate and inhibitor, inasmuch as any estimation of comparative binding energies, to relate with the structure or topography of the active site, depends upon an accurate determination of the ratio $K_{s} / K_{i}$ as discussect in Chapter 3 . It is usually easy to determine the ratio $K_{m} / K_{i}^{\prime}$ where $K_{i}{ }^{\prime}$ represents a calculated inhibition constant that need not correspond to the true dissocition constant of the EI complex. Thorn (1953) has outlined three methods applicable to competitive inhibition.

(a) The values of $K_{m}$ and $K_{i}^{\prime}$ may be determined separately by the plotting procedures previously described and the ratio of them secondarily calculated.

(b) The ratio may be determined directly by rearranging the rate Eq. 3-12 for competitive inhibition to:

$$
\frac{V_{m}}{v_{i}}=1+\frac{K_{m}}{(\mathrm{~S})}+\frac{K_{m}}{K_{i}} \frac{(\mathrm{I})}{(\mathrm{S})}
$$

and plotting $V_{m} / v_{i}$ against (I) at constant substrate concentration, from the slope of which the ratio of the constants may be obtained. This is essentially type D plotting as described above (Fig. 5-1 D).

(c) An approximate method involves transformation of Eq. 5-30 as follows:

$$
\frac{1}{v_{i}}=\frac{1}{V_{m}}\left[1+\frac{K_{m}}{(\mathrm{~S})}\right]+\frac{K_{m}(\mathrm{I})}{V_{m} K_{\imath}(\mathrm{S})}=\frac{1}{v}+\frac{K_{m}(\mathrm{I})}{V_{m} K_{i}(\mathrm{~S})}
$$

where $v$ is the rate in the absence of inhibitor. The ratio is thus found to be:

$$
\frac{K_{m}}{K_{i}}=\frac{(\mathrm{S})}{(\mathrm{I})}\left[\frac{v}{v_{i}}-1\right]
$$

when (S) is sufficiently high so that $v$ is approximately equivalent to $V_{m}$. This can also be written as:

$$
\frac{K_{m}}{K_{i}}=\frac{\text { (S) }}{\text { (I) }}\left[\frac{i}{1-i}\right]
$$


or in the form:

$$
\frac{(\mathrm{I})(1-i)}{i}=\frac{K_{i}(\mathrm{~S})}{K_{m}}
$$

which is similar to the Eq. 5-7 for single-curve plots, but neglects the $K_{i}$ term on the right side, thus indicating the error introduced by this approximate method.

The difficulty lies usually in relating this ratio to the ratio of the true dissociation constants, since neither $K_{m}$ nor $K_{i}{ }^{\prime}$ may be such. The calculated inlibition constant $K_{i}{ }^{\prime}$ will equal $K_{i}$ in simple systems where no other components are involved, but it has already been pointed out that $K_{i}{ }^{\prime}$ may include the concentrations and constants of activators, coenzymes, hydrogen ions, and other possible components when determined by the standard methods without recognizing the role such components may play in the reaction. One common source of error appears when the rate and inhibition depend on $\mathrm{pH}$, for, as will be shown in greater detail in Chapter 14 , the $K_{i}$ found for a particular $\mathrm{pH}$ will generally not correspond to the $K_{i}$ for the EI complex. If the $\mathrm{pH}$ is not varied and the $\left(\mathrm{H}^{+}\right)$factor is not taken into consideration in the calculation of $K_{i}$, considerable error can arise. The best example of the determination of a true $K_{s} / K_{i}$ ratio is probably that of Thorn (1953) for the inhibition of succinic dehydrogenase by malonate. It was found here that $K_{m} / K_{i}$ varied from 4.7 to 60 depending on the nature and concentration of the hydrogen acceptors used, since $k_{2}$ is dependent on these; extrapolation to $k_{2}=0$ led to a value of near 3 for $K_{s} / K_{i}$. Such a low value indicates that succinate and malonate are bound to comparable degrees (about $0.67 \mathrm{kcal} /$ mole difference in binding energy) whereas earlier values (which were really $K_{m}\left(K_{i}\right)$ wonld have indicated a $2-3 \mathrm{kcal} /$ mole difference in binding. Interpretation of the spatial relations of the cationic sites on the succinic dehydrogenase is correspondingly modified.

\section{ISOBOLOGRAMS}

A method of plotting that has not been used for enzyme inlibition but was developed for the study of drug antagonism (Loewe and Muischnek, 1926) involves the construction of isobols or curves of constant activity on a graph that has substrate and inhibitor concentrations as the abscissa

and ordinate. A specific degree of inhibition is chosen and the various pairs of $(\mathrm{S})$ and (I) giving this inhibition are plotted so that each point on an isobol represents the same activity of the enzyme ( $\sigma \circ \varsigma_{\varsigma}=$ same $+\beta \circ \lambda \cdot$ ' $=$ stroke, effect, or activity). It is, of course, only of interest when substrate concentration affects the inhibition, as in competitive cases or where the inhibitor reacts with the substrate. Isobols plotted in the usual way and 
in $\log -\log$ coordinates are given for competitive inhibition in Figs. 5-21 and 5-22 using the following rearrangement of the usual inhibition Eq. 3-13:

$$
\text { (I) }=K_{i}\left[\frac{i}{1-i}\right]+\frac{K_{i}}{K_{s}}\left[\frac{i}{1-i}\right](\mathrm{S})
$$

This is seen to be a variation of the single-curve plot of Eq. 5-7 with the terms in $i$ transposed. Thus it has no advantage as a method for the determination of constants but can be used for visually demonstrating the combinations of substrate and inhibitor concentrations to produce specific degrees of inhibition. When the inhibitor reacts with the substrate, straight lines are obtained in the log-log plot, which may help to distinguish this type of inhibition from truly competitive situations, as indicated in Fig. 5-23.

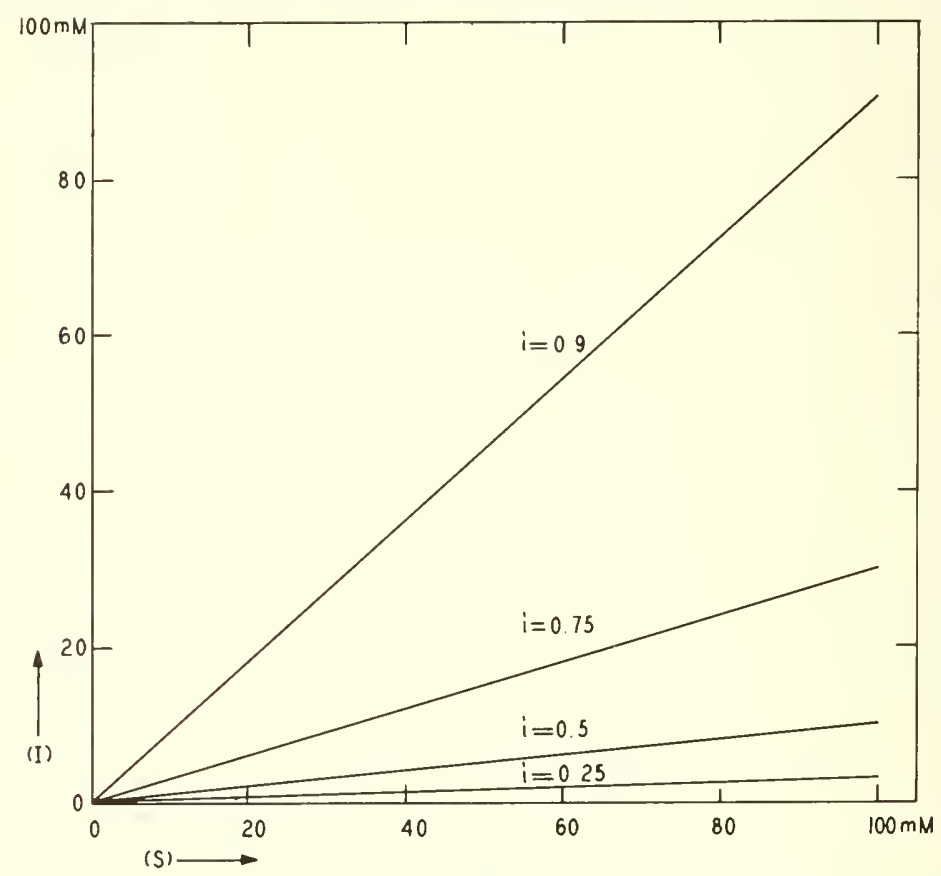

FIG. 5-21. Competitive inhibition isobologram (Eq. 5-35) plotted on linear scales. $K_{s}=1 \mathrm{~m} \nu$ and $K_{i}=0.1 \mathrm{~m} M$.

\section{MUTUAL DEPLETION SYSTEMS}

When the concentration of free inhibitor is reduced by combination of the inhibitor with the enzyme, the characteristics of the curves plotted by the usual procedures are altered in most cases. We have considered only systems in zone $A_{i}$ and some comment on zone $C_{i}$ kinetics is needed. Inas- 
much as in zone $C_{i}$, competitive and noncompetitive inhibitions are expressed by identical equations $(3-36$ and $3-53)-i=\left(\mathrm{I}_{t}\right) /\left(\mathrm{E}_{\ell}\right)-$ it is evident that one cannot distingnish between them; as we have seen, however, it is unlikely that a competitive system can exist in zone $\mathrm{C}_{i}$. Zone $\mathrm{C}_{i}$ inhibition is plotted in Fig. 5-24 and these curves may be compared to those in Fig. 5-3 to illustrate the differences between zone $A_{i}$ and zone $C_{i}$ be-

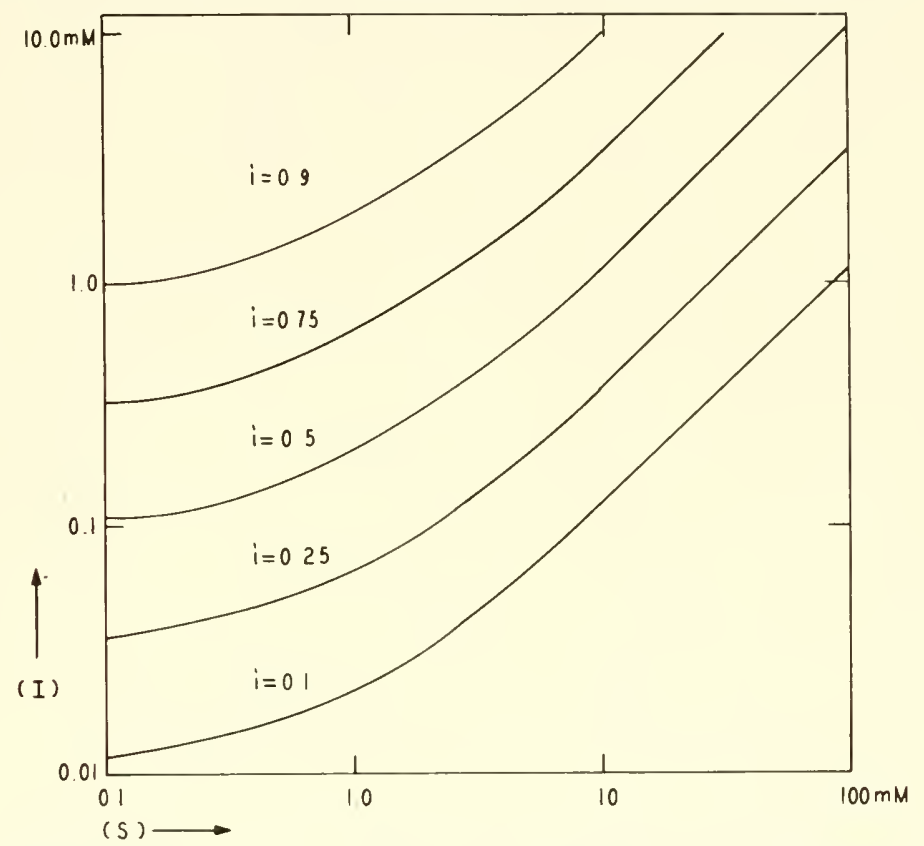

FIG. 5-22. Competitive inhibition isobologram (Eq. 5-35) plotted on logarithmic scales. $K_{s}=1 \mathrm{~m} M$ and $K_{i}=0.1 \mathrm{~m} M$.

havior. The curves plotted by methods $\mathrm{A}, \mathrm{B}, \mathrm{C}$, and $\mathrm{F}$ are similar for both zones but the slopes and intercepts have different values, dependent on the relative concentrations of enzyme and inhibitor and not on $K_{i}$. If zone $C_{i}$ behavior is not recognized (and it may not be in plots of types $\mathrm{A}-\mathrm{C}$ and $\mathrm{F}$ ), calculation of $K_{i}$ in the usual manner gives a constant without physical meaning. Zone $C_{i}$ kinetics may be detected by plots of type $\mathrm{D}$ and $\mathrm{E}$; particularly if a plot of $1 / i$ against $1 /\left(I_{t}\right)$ results in a straight line whose extension passes through the origin, zone $C_{i}$ behavior is evident, and from curves of this type an estimate of the enzyme concentration may be made (see Chapter 3 ). The rate equation for noncompetitive inhibition in zone $C_{i}$ is given by:

$$
v_{i}=V_{m} \frac{(\mathrm{S})}{(\mathrm{S})+\bar{K}_{s}}\left[1+\frac{\left(\mathrm{I}_{t}\right)}{\left(\mathrm{E}_{t}\right)}\right]
$$


from which the plotting equations may be derived. The rate equations for inhibition in zone $B_{i}$ are too complex to be useful. We have already given equations from which $K_{i}$ may be determined for noncompetitive systems (Eqs. 3-42 and 3-44) and it is also possible, but more laborious, to do so for competitive cases. If at constant substrate concentration one determines

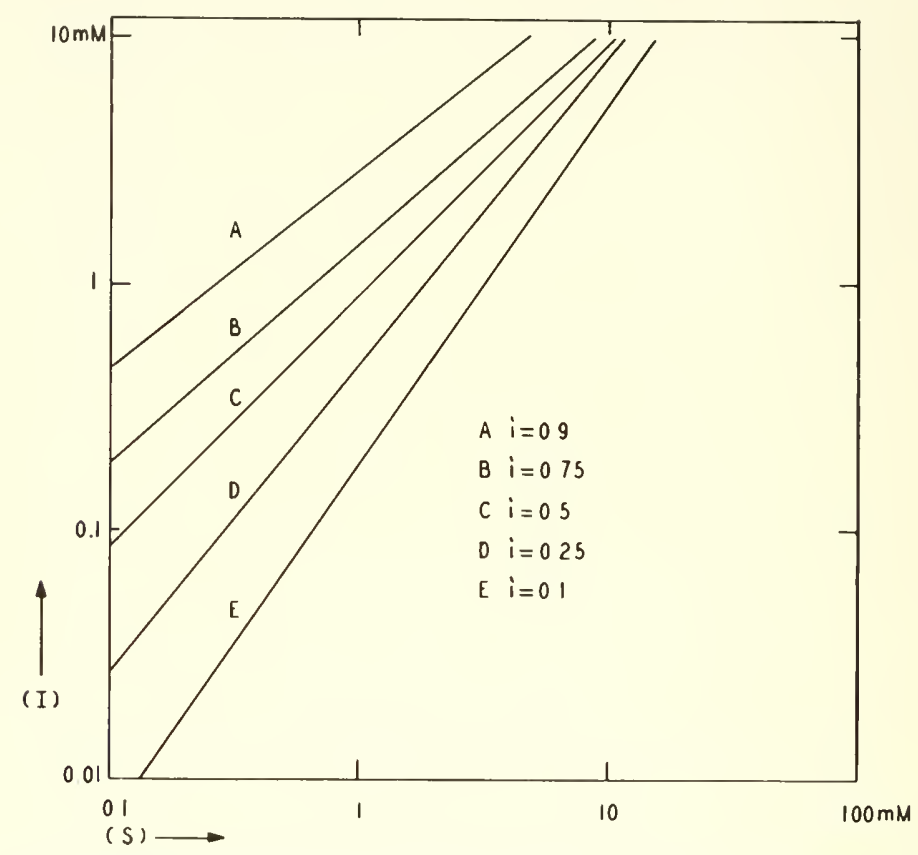

F1G. 5-23. Isobologram for the situation in which the inhibitor reacts with the substrate.

accurately the degree of inhibition at two inhibitor concentrations (as different as is practically possible), the value of $K_{i}$ may be found from:

$$
K_{i}=\frac{i_{2}\left(\mathrm{I}_{t}\right)_{1}-i_{1}\left(\mathrm{I}_{t}\right)_{2}}{i_{1} i_{2}\left[1 /\left(1-i_{1}\right)-1 /\left(1-i_{2}\right)\right]\left[1+(\mathrm{S}) / K_{s}\right]}
$$

A potent inhibitor may either form a simple complex with the enzyme or give rise to an EI complex by a chemical reaction associated with the splitting off of a part of the inhibitor molecule. Krupka and Laidler (1959) treated these situations kinetically and showed how they might be distinguished. The equations may be written as follows:

$$
\begin{aligned}
& \mathrm{E}+\mathrm{I} \rightleftharpoons \mathrm{EI}: \\
& \qquad\left(\mathrm{I}_{t}\right)-i\left(\mathrm{E}_{t}\right)=\frac{i}{1-i}\left[1+\frac{(\mathrm{S})}{K_{s}}\right] K_{i}
\end{aligned}
$$


$\mathrm{E}+\mathrm{I} \rightarrow \mathrm{EI}+\mathrm{X}:$

$$
i=\frac{1}{K_{i}\left[1+(\mathrm{S}) / K_{s}\right]}\left[\frac{\left(\mathrm{I}_{t}\right)(1-i)}{i\left(\mathrm{E}_{t}\right)}+i-1\right]
$$

These equations are modified from those of Krupka and Laidler merely by substituting $i$ for $1-v_{i} / v$. Equation $5-38$ is seen to be the same as $3-50$ for zone $A_{s} B_{i}$ inhibition. In the first case, $\left(I_{t}\right)-i\left(E_{t}\right)$ is plotted against $i /(1-i)$, and in the second case, $i$ is plotted against the expression in the square brackets. In both cases, $K_{i}$ may be readily evaluated. They applied these kinetics to the inhibition of $\alpha$-chymotrypsin by diisopropylfluorophosphate (DFP), and found that at low inhibitor concentrations the first

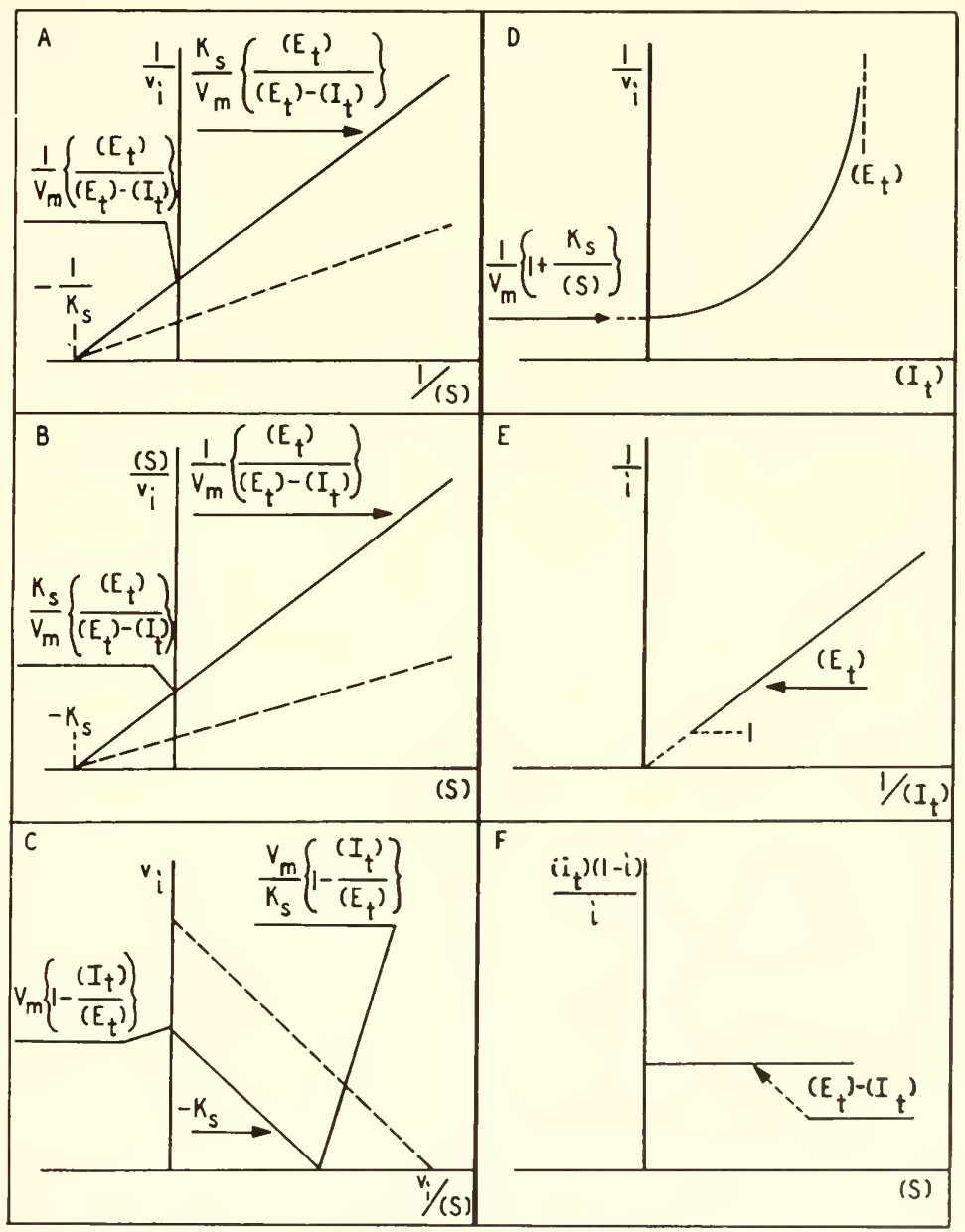

FIa. 5-24. Mutual depletion system in zone $C_{i}$. 
mechanism was involved with $K_{i}=2.04 \times 10^{-6} M$. This is surprising since a simple EI complex is not formed in this case, so that the general validity of this approach is still in doubt. The plot is shown in Fig. 5-25.

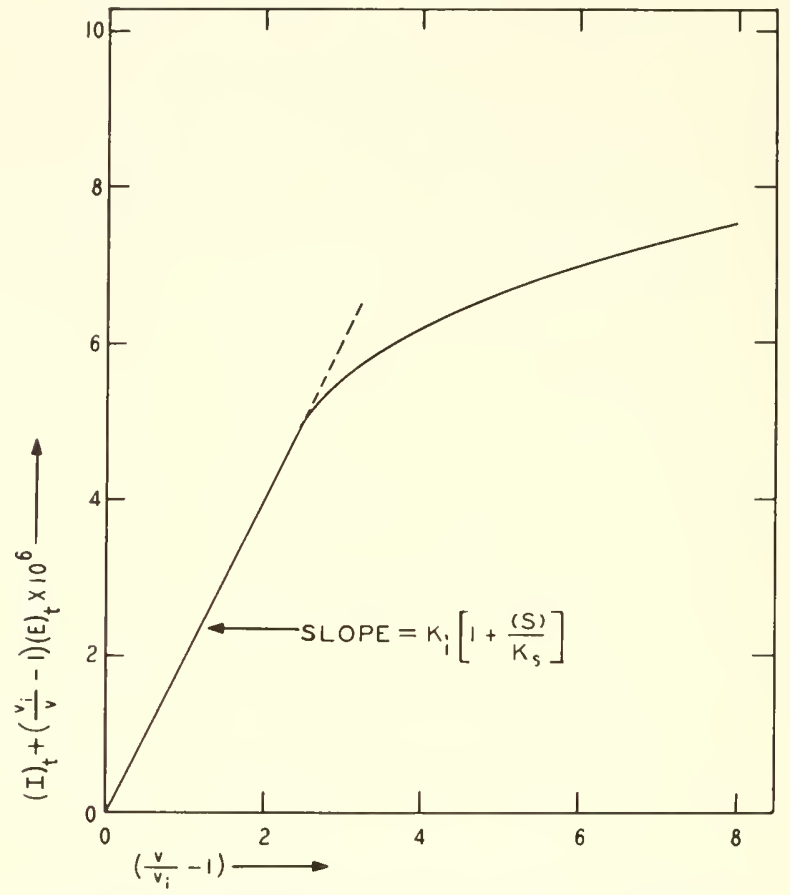

FIg. 5-25. Krupka-Laidler plot for the inhibition of chymotrypsin by DFP at $\mathrm{pH}$ 8.1. (From Krupka and Laidler, 1959.)

\section{SUGGESTIONS FOR PLOTTING AND DETERMINATION OF CONSTANTS}

It will perhaps be useful to conclude this chapter with some general suggestions concerning the types of experimental data desirable, the approach to different methods of plotting, and the interpretation of the results. It is evident without discussion that the reliability of mechanistic interpretations and the accuracy of the calculated constants depend directly on the accuracy of the experimental data. In much quantitative scientific investigation it is required that multiple runs be performed so that mean values and their significance can be determined. However, in enzyme work it has been the rule that plots are made and constants calculated from a single experimental series. Most of the methods used for enzyme rate determination involve appreciable errors and greater accu- 
racy could certainly be achieved by using mean values from several experiments.

The following suggestions are meant to be only a guide to the quantitative study of enzyme inhibition and are of a quite general nature. It is likely that many investigators in the past have not fully realized the complexity of most enzyme systems and for this reason their interpretations and constants are suspect. It is better to anticipate a degree of complexity and then experimentally reduce the system to its simplest terms than to assume simplicity at the beginning.

\section{(A) Experimental Procedure}

(1) Attempt to measure initial reaction rates or determine them by extrapolation in order to avoid effects of reduction in substrate concentration, rise in product concentration, or spontaneous loss of activity of the enzyme.

(2) Use the proper controls so that the rates measured can be attributed specifically to the enzyme studied. The occurrence of a nonenzymic reaction should be investigated as well as the possibility of a nonsubstrate (endogenous) reaction.

(3) Vary the concentrations of all the known components of the enzyme system, including $\left(\mathrm{H}^{+}\right)$, independently. If a system contains a coenzyme or activator, its effect upon the rate in the presence and absence of the inhibitor may be just as important as the effect of substrate.

(4) Utilize as wide a range of concentrations as possible so that accuracy in plotting may be increased and deviations from linearity may be more readily detected. Care must be taken in using high concentrations because the rate may be reduced by secondary or nonspecific mechanisms, such as ionic strength effects. For example, it is probable that all experimental curves on a plot of $1 / v$ against $1 /(\mathbf{S})$ will turn up sharply as the $1 / v$ axis is approached due to these mechanisms.

(5) Give consideration to the time factors in the inhibition, i.e., the rates at which inhibition is achieved or disappears. A system that is truly competitive may be interpreted as noncompetitive if the displacement of the inhibitor from the enzyme by substrate is low, i.e., if equilibrium is not reached. The equations that have been presented in previous chapters are derived on the basis of equilibrium states.

\section{(B) Plotting Procedure}

(1) Plot the data in several different ways, e.g., by the six different methods discussed above or other methods applicable to special situations. This is important for two reasons. In the first place, errors in interpreta- 
tion may be avoided; there is no single method of plotting which can distinguish between all the various possible mechanisms of inhibition. Secondly, the constants obtained by the different methods may be compared and more accurate values reached. The best types of plots will vary with the system studied so that no general statement can be made as to the relative values of the various techniques. Deviations from linearity may be more obvious in certain types of plots; e.g., type C plotting provides somewhat more accurate interpretation at low substrate concentrations and nonlinear behavior in this range is more readily detected, while type $\mathrm{F}$ plotting often gives a more direct determination of the constants. Nevertheless, it is advisable always to utilize different methods as is evident from inspection of the sample plots presented in this chapter.

(2) Plot the data with respect to the concentrations of all the known components of the system. For example, in type A plotting it is necessary to construct graphs of $1 / v_{i}$ against $1 /(\mathrm{A})$ and similarly for coenzymes or hydrogen ions. The results may indicate noncompetitive inhibition from a plot of $1 / v_{i}$ against $1 /(\mathrm{S})$ and yet the inhibition may be due to competition with another component. Type $\mathrm{F}$ plots may be made with the concentration of activator or coenzyme as the abscissa instead of substrate concentration. If such plots are not made, errors both in interpretation and determination of constants are possible. The slopes and intercepts usually have values dependent on the concentrations and dissociation constants of the other components of the reaction.

(3) It is generally better to plot as many curves as feasible for each type of graph. For type A plotting, several curves for different inhibitor concentrations, and for type E plotting several curves at different substrate concentrations, will increase the accuracy and reliability of the methods.

(4) Do not use logarithmic coordinates in the methods of plotting described above; this seems obvious but examples from the literature can be cited. Although the intercepts may be easily interpretable, the slopes are not; in fact, linearity would be only fortuitous, for when a plot of A against $\mathrm{B}$ is linear, plots of $\mathrm{A}$ against $\log \mathrm{B}$ or of $\log \mathrm{A}$ against $\log \mathrm{B}$ are not.

\section{(C) Interpretation}

(1) Note deviations from linearity carefully; a straight line drawn through points that are obvionsly not linear may simplify the problem a good deal but provide meaningless results. Any deviation can be indicative of a different mechanism from the one assumed on the basis of a straight line. The deviations may not relate directly to the inhibition but be due to extraneous factors, but this must be proved. 
(2) Utilize all the intercepts and intersections available in the plots because accuracy in the determination of constants is increased and the calculation often made more direct. For example. Dixon (1953 b) showed that $K_{i}$ may be immediately calculated from the intersection of lines in the plot of $\mathrm{l} / v_{i}$ against (I) for competitive inhibition, which may be more aceurate than determinations from the slope or (I) intercept.

(3) It is obvions from what has been said above that consideration be given to the other components of the enzyme system with respect to the effects they have on the slopes and intercepts; in other words it is important to calculate the true $K_{i}$ and not a physically meaningless constant which at best only characterizes the behavior of the inhibition.

(4) Consider all of the possible mechanisms which may be implied by the curves obtained. It is frequently easy to decide upon a mechanism as a matter of simplicity or prejudice without realizing that the data conld equally well indicate other mechanisms. It is usually possible to eliminate most of the suggested mechanisms if it is realized that there are several possibilities.

(5) Although problems of inhibition in tissues, cells, and subcellular fractions have not been discussed, it is well to point out that special care must be exercised in the interpretation of plots made from data on such systems. Particularly when the measurements involve responses to enzyme inhibition that are far removed from the enzyme, one should put little reliance on the techniques of plotting devised for relatively simple systems. Permeability factors alone may distort the basic inhibition kinetics beyond ready interpretation.

The following list of papers and works on kinetics and the plotting of enzyme reactions, both uninhibited and inhibited, is recommended for further refence: Lineweaver and Burk (1934), Hunter and Downs (1945), Wilson (1949, p. 16), Dixon (1953 b). Friedenwald and Maengwyn-Davies (1954, pp. 154, 180), Slater (1955), Alberty (1956 b), Hofstee (1956), Dixon and Webb (1958), Neilands and Stumpf (1958), Laidler (1958), Hearon et al. (1959, p. 49), and Reiner (1959). 



\section{INTERACTIONS OF INHIBITORS WITH ENZYMES}

Inhibition of an enzyme reaction is most frequently due to the binding of the inhibitor at certain regions on the enzyme protein. The present chapter will be mainly concerned with the forces and energies involved in these interactions and the relationships between enzyme inhibition and the structural characteristics of inhibitor and enzyme. Reaction of the inhibitor with the enzyme may be chemical in nature (with the formation of covalent bonds) or it may involve the less specific types of attractive forces that exist between molecules. Attention will be primarily directed to these nonchemical interactions of relatively lower energy and the instances of specific chemical reaction will be discussed in comnection with the inhibitors where they occur. It is believed that enzymology in the future will be much concemed with enzyme surfaces, particularly with the active centers, and the forces between these regions and other molecules surrounding the enzyme in solution. This is a conceptually new micro-world with new dimensions for our thinking, in which it is necessary to visualize complex fields of electrical intensity in addition to the blurred contonrs of the molecules, where every atom or group reacts in some manner with every other atom or group as molecules approach one another and where the most stable position of minimum potential energy is determined by the balance of the various attractive and repulsive forces.

Knowledge and theory at this molecular level are not adequate at present to form the basis for a comprehensive approach to an accurate evaluation of intermolecular forces between complex molecules in solution. But enough has been accomplished so that a tentative beginning can be made, from which both experimental procedure and theory may proceed simultaneously to provide more concrete and reliable data and concepts than are now possible. The presentation in this chapter is always approximate, usually crude, and often naive. It is hoped that it will contain some stimulation for a quantitative approach to intermolecular interactions in biological systems and assist in the evolntion of exciting and fruitful cooperation between the physical and biological disciplines. If this is accomplished, the inadequacies of the present treatment may be forgiven. Con- 
sideration will first be given to the structures and properties of active centers; the various types of intermolecular interactions will then be discussed; the application of such interactions to biologically important substances and groups will be outlined; finally, the role of these interactions in enzyme inhibition will be presented.

\section{THE NATURE OF ENZYME ACTIVE CENTERS}

Accurate visualization of the interaction between an inhibitor molecule and an enzyme depends in part upon a spatial concept of the properties of the surface of the enzyme and especially some idea of the topography of the active centers where catalysis occurs. The active region should, however, not be thought of solely in terms of molecular configuration; beyond the electronic contours of the groups there are complex and often strong electrical fields emanating from the atoms and ions composing the active center. The interaction and orientation of an imhibitor molecule with respect to an enzyme are primarily dependent on the field pattern.

\section{Composition of the Active Center}

The active center comprises that region of the enzyme surface where the reactants are bound, interact, and are chemically altered. It may be made up of several specific sites where the individual components of the reaction are bound, e.g., substrate site, coenzyme site, and activator site. An active site may be very small and simple (perhaps a single group) or it may extend over a molecularly large region of the enzyme and itself contain many interacting groups and multiple binding points. There are basically three types of active site: $(a)$ polypeptide chains only, $(b)$ a complex of a metal ion with protein, and $(c)$ a nonpeptide prosthetic group bound firmly to the protein. Enzymic catalysis is most intimately related to the polypeptide composition of the active center.

The active region is usually only a small fraction of the total enzyme protein and it has been stated that the remainder of the protein is without importance in the catalysis. Indeed the irradiation of trypsin led Augenstine (1959) to conclucle that the active surface area was $2.5 \%$ of the total area. Since the total area is about $4,050 \AA^{2}$ (on the basis of a molecular weight of 20,000 ), the active center would be approximately $100 \AA^{2}$ in area, which would implicate four surface amino acids. However, in living cells it is likely that few enzymes are free in solution and it is possible that much of the protein is involved in the organization of the enzymes into functionally efficient structures; the noncatalytic portion of the enzyme could thus be important in the operation of more complex metabolic sequences or cycles. Furthermore, those regions of the enzyme surrounding 
or underlying the active center, although not directly concerned in the reaction, can influence the activity of the center through steric or electrostatic effects. The total protein, nevertheless, is apparently not necessary for activity becanse active fragments have been obtained from several proteins (aldolase, papain, ribonuclease, trypsin, and pepsin) and the intact structure of the total protein is at least sometimes of little significance since cross-linkages between polypeptide chains have been broken without loss of activity (e.g., pepsin S-S bridges), The behavior of ribonuclease is particularly interesting inasmuch as pepsin inactivates it rapidly by removal of a tetrapeptide asp-ala-ser-val whereas carboxypeptidase removes the tripeptide ala-ser-val without inactivation, from which it is apparent that the aspartate residue is involved in the active site which is near the $C$-terminal end of the polypeptide chain (Anfinsen, 1956). The most extensive degradation of an enzyme without loss of activity is the removal of two-thirds of the 180 anino acid residues from papain by leucine aminopeptidase (Hill and Smith, 1956).

Most enzymes investigated by end-group analysis contain only one polypeptide chain although some undoubtedly possess more. The configuration of the chain in enzymes, particularly at the active center, is generally unknown. Parts of the polypeptide chain are probably hydrogen-bonded into helices, but not all because both $\mathrm{X}$-ray and optical dispersion data in the few cases studied have indicated that only a fraction of the protein is helical; in ribonuclease only $15 \%$ of the protein is helical (Yang and Doty, 1957). The nonhelical portions are not randomly oriented but are undoubtedly in specific configurations determined to a great extent by the looping of the polypeptide chains through cross-linkages, such as the disulfide R-S-S-R' (ribonuclease has four), phosphate R-O-P-OR' (pepsin has one), or pyrophosphate R-O-P-O-P-O-R'. Hydrogen bonding can also occur in nonhelical structures and the nonpolar cohesive forces between hydrocarbon side-chains are often stronger than generally realized.

The presence of certain amino acids at an active site can be demonstrated or inferred by a variety of techniques: the reaction of ammo acid side-chains with group-specific reagents, a reduction or loss of activity indicating that the particular group is associated with the active site, but not necessarily proving it; the isolation of amino acid or peptide fragments containing a stable inhibitor; the subjection of the enzyme to proteolysis; and the determination of the ionization constants of active site groups from the variation of enzymic activity with $\mathrm{pH}$. Sulfhydryl groups are recognized as present in many active sites because of the marked inhibition by substances reacting quite specifically with such groups. The use of fluorophosphonates, such as DFP, has led to the isolation of phosphoserine peptides from trypsin and chymotrypsin; the amino acid sequence for short distances on either side of serine can be determined, although the relationship of 
these amino acids to the active site is obscure. It is possible that histidine is involved in the reaction of DFP with serine and hence occurs at the active site but in another portion of the polypeptide chain. The group $\mathrm{p} K_{a}$ 's of the active sites of a variety of enzymes have allowed tentative identification of histidine as a component. It is probable that histidine is an important constituent of many enzyme active sites since it may function efficiently in acid-base catalysis, due to its $\mathrm{p} K_{a}$ being near neutrality. Indirect evidence for the participation of histidine in enzyme catalysis comes from studies on the photo-oxidation with visible light in the presence of methylene blue of the imidazole ring in enzymes, this treatment selectively destroying the histidine residues and producing a progressive fall in activity (Weil and Buchert, 1952; Neely, 1959). The ability of the imidazole ring to accelerate the nonenzymic hydrolysis of phenyl acetates (Bender and Turnquest, 1957; Brnice and Schmir. 1957) and to complex with the pyridine nucleotides (Eys, 1958) may be indicative of the role histidine plays in certain enzyme reactions. An excellent summary of determinations of the groups and amino acids in enzyme active centers is in the book by Dixon and Webb (1958, p. 492).

It is possible that some groups participating in catalysis are present at the active center in especially reactive states. The SH group at the substrate site of papain appears to be not an ordinary SH group but one that is themodynamically at a higher energy level and kinetically more reactive (Smith, 1958). It was postulated that this SH group forms a "high-energy" bond with an adjacent carboxyl gromp, this being made possible by the folded configuration of the native protein, and that these two groups function as follows in the hydrolysis of peptide bonds:
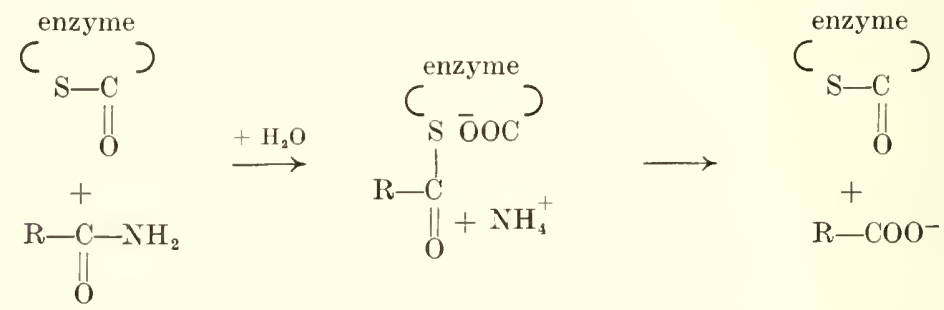

The behavior of gromps in special states with respect to $\mathrm{pH}$ or to reaction with group-specific reagents may deviate from the.predicted on the basis of the same groups on amino acids.

Another interesting approach, applicable to enzymes that occur in an inactive or zymogen form, shonld be mentioned at this point. The formation of active trypsin from trypsinogen involves the splitting off of a hexapeptide val-(asp)-lys to leave a terminal isoleucine group; thus the breaking of a single peptide bond can result in the appearance of the active site. 
The chymotrypsinogen $\rightarrow$ chymotrypsin transformation is similar in that initially a single peptide is removed; in this case there is evidence that there is a rather radical alteration in the polypeptide configuation as a result of this splitting, more of the chain assuming a helical configuration. The bonds split or the peptides removed may be considered as impeding the development of the configuration necessary for the active site (Neurath, 1957). One can also anticipate results pertinent to the composition of active sites in the modifications of enzyme synthesis that will soon be possible.

Finally, one must consider that the active site includes the water molecules that are associated with the protein groups and the atmosphere of ions that surrounds the charged groups on the site. During reaction of a substrate or inhibitor with the active site, water and ions must often be displaced and thus contribute to the final energy of interaction and the kinetics. If they are not displaced they will modify the configuration of the active site and the electrical fields emanating from the protein groups.

\section{Topography of Active Centers}

Most active sites are regions in which the sequence and characteristic configurations of the polypeptide chain form a pattern of amino acid residues that allows a participant in the enzyme reaction to be bound and undergo electronic distortion. These sites may be more or less closely associated with water, specific metal ions. or nonspecific ions of the environment. The configuration of the site. both spatially and electrically, provides the close fit between substrate and enzyme that is usually necessary for activation and confers the high degree of specificity that is exhibited by most enzymes. It is not necessary that the site be complementary to the entire substrate molecule; certain groups or regions only may interact with the enzyme, as is evident from specificity and inhibitor data, and it is the corresponding pattern of groups on the active site that is pertinent. It has been postulated by Pauling (1948) that the active site may not be complementary to the normal state of the substrate but to the substrate in the activated complex, thereby reducing the activation energy and accelerating the reaction. This concept is of particular interest in connection with the interactions of molecules structurally related to the substrate. Furthermore, variations in activity towards various substrates should not be interpreted solely in terms of binding but also with respect to the ability to form the appropriate activated complex; in other words, there may be differences in both the concentration of ES and in the rate constant $k_{2}$.

There is evidence that most substrates must be bound to the active site at two or more points for reaction to occur (Levine, $1954 \mathrm{a}, \mathrm{b}$ ) and that frequently there are two or more groups involved in the displacements 
occurring during the reaction (Laidler, 1955 a). Thus enzymic hydrolysis can occur following the multipoint binding of the substrate by a "pushpull " acid-base catalysis exerted by two groups, one acidic and one basic. That there are binding groups determining the orientation and specificity and also groups activating a labile bond has been postulated for chymotrypsin on the basis of the entropy differences observed between optical isomers of the substrates (Vaslow and Doherty, 1953). This concept is inherent in the mechanisms ascribed to several enzymes, such as succinic dehydrogenase, fumarase, acetylcholinesterase, carboxypeptidase, prolidase, and others. In more complex reactions, two or more of such sites may cooperate, especially in transfer processes; such is evident in many oxidation-reduction reactions but may also occur in transfer associated with phosphatases and other hydrolytic enzymes, the acceptor being either water for hydrolysis or some other molecule for transfer, either acceptor being bound to a specific site adjacent to the donor site (Morton, 1955). A metal ion, complexed to the protein, may serve as a site for either binding or reaction or both but full catalytic effectiveness is only achieved in conjunction with other sites. The transphosphorylation ATP $+\mathrm{P}^{32} \rightarrow \mathrm{ADP}$ $+\mathrm{PP}^{32}$ is catalyzed by various metal ions $\left(\mathrm{Mn}^{++}, \mathrm{Ca}^{++}, \mathrm{Cd}^{++}\right)$in the absence of any enzyme, but the concentration of substrates must be about fifty-fold greater than for the enzymic reaction, indicating that the additional groups on the enzyme increase the efficiency of the reaction by orientation in the proper position (Lowenstein, 1958). Spatial coordination of groups within active sites, and of sites within an active center, is often necessary for the catalysis; as a consequence, disturbances in this structural pattern can inhibit such enzymes.

Despite the fact that most enzymes are globular or ellipsoidal, the surface should not be considered as even approximately smooth but, in the most interesting regions, perhaps more like a holly bush. At least the various protrusions, indentations, and crevices that undoubtedly occur are occasionally important in the configuration of the active site according to several recent speculations on the ES complex as in Figs. 6-1 and 6-2. The advantage to the "action in a hole" concept is that more groups can be brought to bear on the reactants and attack can be made from opposite sides. It must be admitted that there is essentially no direct evidence for such sites but in peroxidase and catalase the presence of the heme in a protein crevice is made likely by inhibition and ionization studies (George and Lyster, 1957). Such crevices may exist between helices. An active site may be situated in a helix but it is more likely that it comprises portions of the polypeptide chain in more extended configurations, or combinations of the two strnctures; in any event it is evident that tertiary protein structure is of significance in enzymes and that alterations of this structure. even at some distance from the active site, can modify the catalysis. 


\section{Flexibility of Protein Structure}

It is certain that proteins are not rigid structures despite their specific configurations; what one calls the specific configuration may be the end result of the adaptation of the protein to the interacting molecule. The polypeptide chain and the amino acid side chains may assume those con-

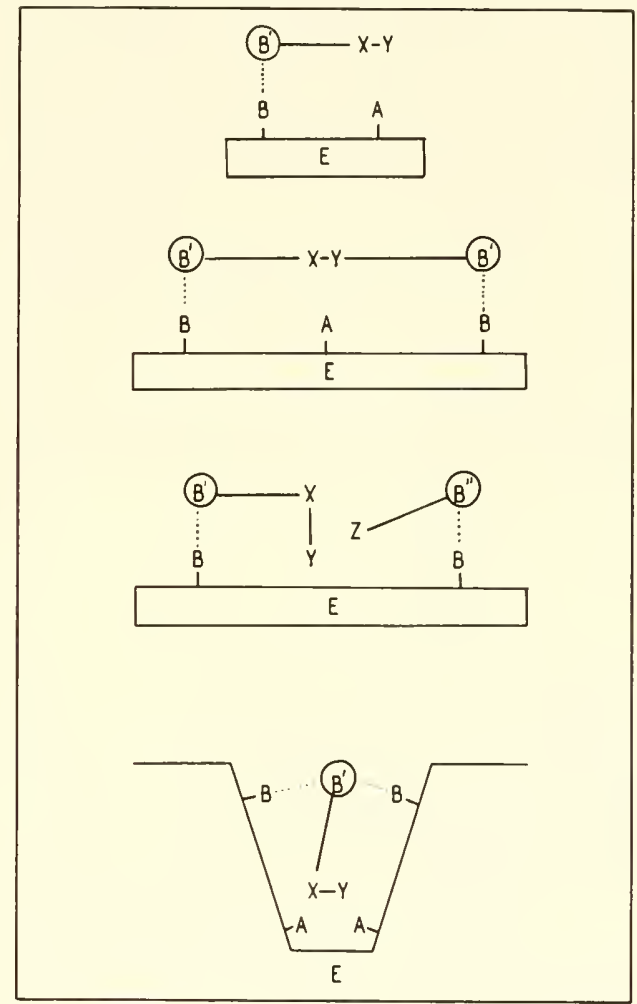

FIG. 6-1. Schematic representations of some possible types of active centers. The A groups are responsible for the catalytic reaction while the $B$ groups serve to bind the substrate to the enzyme. B may be a single chemical group or may extend over a region of the surface. In each case a reaction at the $\mathrm{X}-\mathrm{Y}$ bond takes place.

figurations along or around the substrate or inhibitor that provide the maximal interaction energy. In other cases the specificity towards molecules with respect to distances between binding groups indicates a fairly rigid structure of that portion of the enzyme. If a substance fits into a cavity 


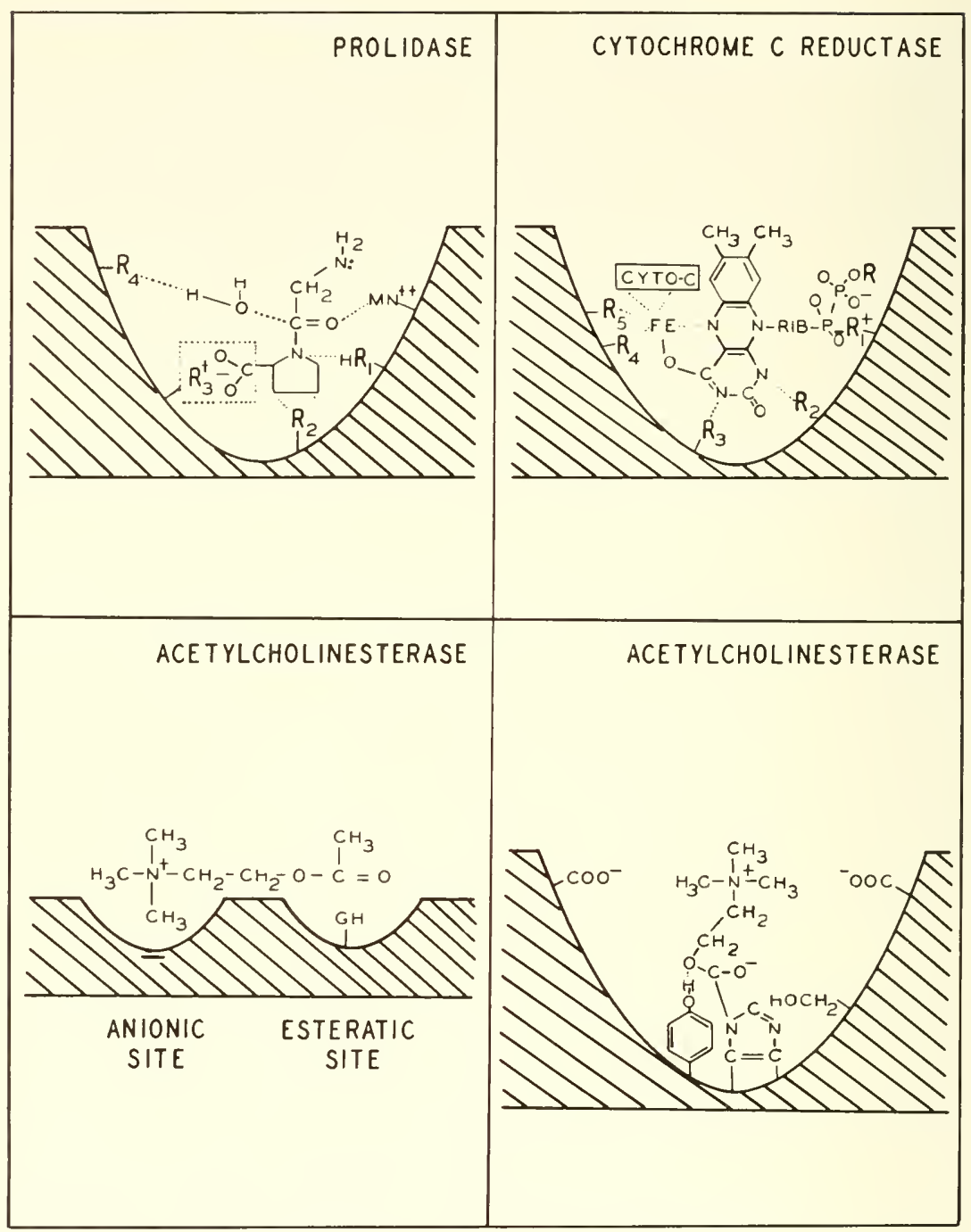

Fic. 6-2. Examples of some active centers and reaction mechanisms postulated for different types of enzymes. In all of these cases it may be observed that two more or enzyme groups are involved in the catalysis.

Prolidase: The manganese ion chelates with the substrate which is also held by four binding groups: $R_{1}$ donates a proton during the transition period; $R_{2}$ interaets through van der Waals' forces with the $-\mathrm{C}^{\prime} \mathrm{H}_{2}-\mathrm{CH}_{2}-$ part of the ring; $\mathrm{R}_{3}$ interacts electrostatically with the carboxylate group; and $\mathrm{R}_{4}$ eatalyzes the hydrolysis and aceepts a proton. (Rabin, 1958, p. 21.)

Cytochrome c reductase: This illustrates interaction between two components of the 


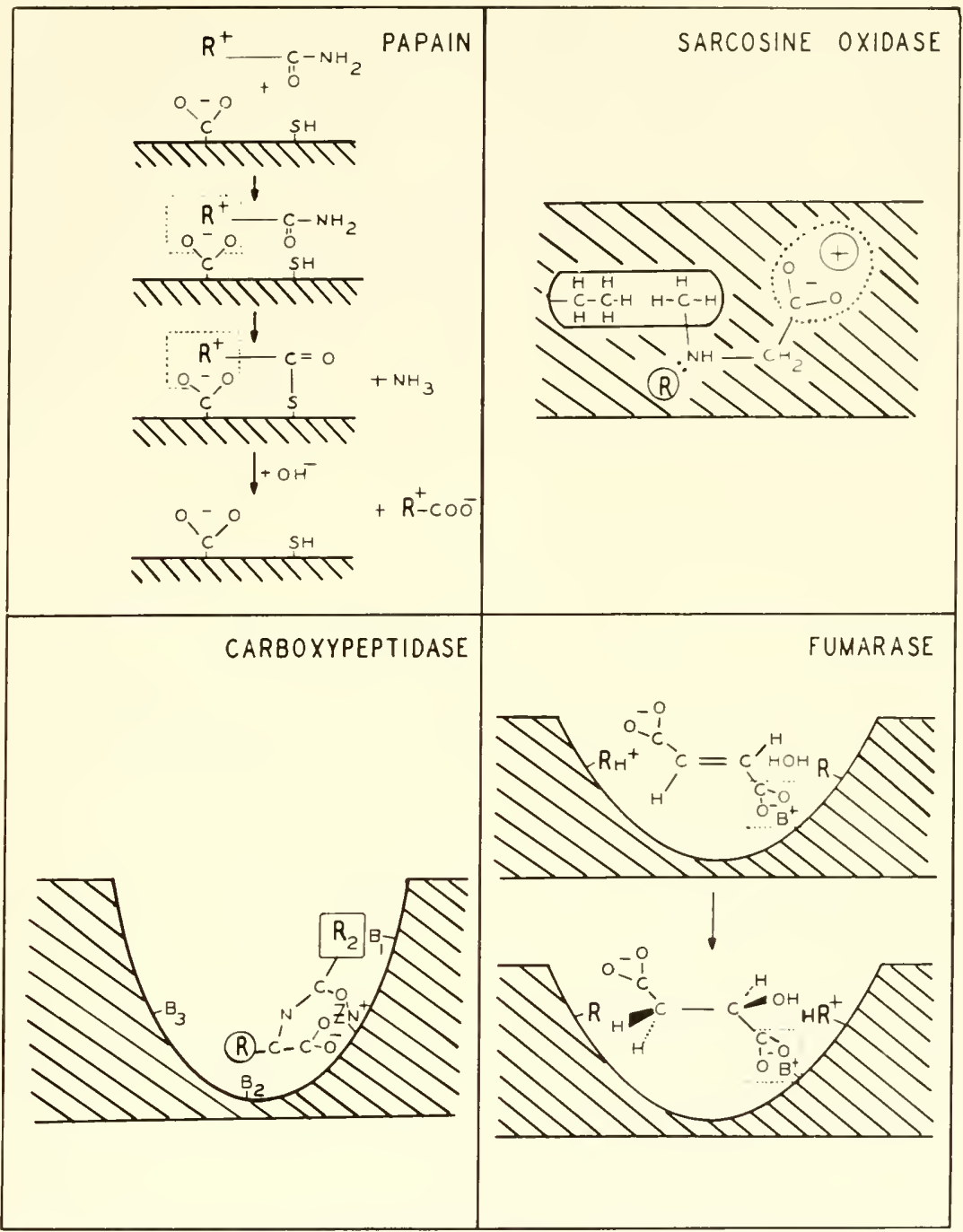

FIG. 6-2. (continued)

electron-transport system. The FAD is bound to the protein electrostatically through the phosphate groups at $R_{1}$ and to electrophilic groups through the ring at $R_{2}$ and $R_{3}$, while interaction with a non heme iron occurs by which the electrons are transferred to cytochrome $c$, the iron ion being bound to the protein at $R_{4}$ and $R_{5}$. (Mahler and Elowe, 1954.)

Acetylcholinesterase: The substrate is bound electrostatically to the anionic site (probably a carboxylate group) and the ester link is subjected to the esteratic site 
or crevice of the protein, the over-all binding energy will depend on the fit and the possible modification in the size of the invagination. If a cavity has to expand slightly to achieve maximal mutual contact with the reactant, the energy involved in this dilatation must be considered. Indeed Pauling and Pressman (1945) have calculated the amount of dilatation necessary to accommodate various hapten groups in their interaction with antibodies. Even the helix structure is not completely stable since it depends on hydrogen bonding and the electrostatic forces between the polypeptide chains. Although the energy required to break such a hydrogen bond may be 8 $\mathrm{kcal} / \mathrm{mole}$, in water where hydrogen bonds are formed between the water molecules and the polypeptide chain when the helix is disrupted, the overall energy required may be only $1.5 \mathrm{kcal} / \mathrm{mole}$ (Edsall and Wyman, 1958.

(continued legend for Figure 6-2.)

and the basic group: GH. Van der Waals' interactions probably also occur with the methyl groups of the choline portion. (Wilson, 1954.)

Acetylcholinesterase: This represents another concept of the active site wherein the cationic head is held electrostatically by the surrounding carboxylate groups and the ester link is subjected to tyrosine and histidine residues, the presence of a serine residue at the active site being indicated by $-\mathrm{CH}_{2} \mathrm{OH}$. Again, van der Waals' forces are probably involved in the binding. (Bergmann, 1958.)

Papain: The substrate is held mainly by electrostatic attraction and the susceptible bond is placed spatially to the $\mathrm{SH}$ group so that an acyl intermediate is formed. (Kimmel and Smith, 1957.)

Sarcosine oxidase: Three binding sites occur: (1) the interaction of the methyl group with a hydrocarbon residue; $(2)$ the interaction of the nitrogen atom with an electrophilic group R; and (3) the electrostatic interaction of the earboxylate with a positively charged protein group. The sarcosine is thus held in a compact configuration. (Frisell and Nackenzic, 195.5.)

Carboxypeptidase: The substrate is initially bound to the zinc ion as a chelate with three subsidiary binding groups, $B_{1}$ interacting with the $R_{2}$ and the acyl groups, $B_{2}$ interacting with the $R_{1}$ group, and $B_{3}$ which interacts with $P_{1}$ later in the reaction when the carboxylate group has shifted. (Lumry and Snith, 1955.)

Fumarase: The substrate is anchored mainly by one carboxylate group and the molecule is exposed to the influence of two acidic groups that can donate and accept $\mathrm{H}^{+}$, this being a type of acid-base catalysis. (Alberty, 1957.)

In all cases the original representations of the active sites were intender to be only indicative of the reaction mechanism and the known groups involved, and were not meant to be accurate pictures of the true sites. Holes or crevices in the enzyme are not necessary in all cases. In some cases the sites have been redrawn to conform to a uniform presentation. 
p. 123). Alteration of the ionic strength, $\mathrm{pH}$, or dielectric constant and the introduction of substances (e.g. urea) that can form hydrogen bonds with the polypeptide chain may influence the helical structure or the fraction of the protein in this configuration.

It is interesting to admit the possibility that protein flexibility is involved in interactions with some molecules and that a rigid configuration is by no means necessary. The ability of serum albumin to bind many types of substance has been attributed to a high degree of flexibility of the protein structure (Klotz and Urquhart, 1949a). When an inhibitor approaches an enzyme surface it is possible that occasionally there is a mutual orientation produced by the interaction forces so that the final state will be maximally stable.

\section{Relationships of Active Site Structure to Inhibition}

The importance of some of the concepts discussed in this section to enzyme inhibition may conveniently be summarized.

(A) Since an active center may contain several individual sites on which dissociable acceptors, activators, or coenzymes are bound, it is possible for an inhibitor to interfere in the binding of any component or to disturb the coordination between the sites.

$(B)$ The fact that an individual site can be reasonably complex and comprise numerous groups makes it possible for the inhibitor to block the reaction by several mechanisms; one might state that the more complex the site, the greater will be the opportunity for inhibition.

(C) In considering the forces and energies of interaction between an enzyme and an inhibitor, the presence of multiple groups makes it necessary to take into account a variety of interactions of different types. Simplification of the picture is often mandatory for progress but the approximate nature of the treatment should never be forgotten.

(D) In those situations where an accurate fit of the substrate to the active site is necessary over an extended area, an inhibitor related structurally to the substrate usually must conform fairly closely to this pattern and deviations from the substrate structure will result in much reduced binding. However, where only a portion of the substrate molecule need fit the active site, or the intermolecular forces are sufficiently strong to obviate the necessity of close fit, substances related to the substrate in various ways will be more likely to be bound and produce inhibition.

$(E)$ Flexibility of the protein at the active site will allow interaction with a greater range of inhibitor molecules and the assignment of rigid active site structures cannot be made so readily. 
(F) The fact that most active sites comprise amino acid side-chains with acidic or basic groups makes it necessary to consider the effects of $\mathrm{pH}$ on the constants of inhibition and the interaction energy.

$(G)$ Since the active site may bind water or ions, the effects of these on the topography and electrical fields of the site may be important in the reaction with an inhibitor. Also the energy of displacement of these substances must be included in the total interaction energy.

(H) The possibility that some active sites may be within holes or crevices introduces an additional factor into the question of the specificity of inhibition because steric or electrostatic repulsion may prevent the inhibitor from entering such spaces, even though the inhibitor could react readily with the groups within the cavity.

(I) The region surrounding the active site, although not catalytically involved, can be of importance in inhibition inasmuch as an inhibitor might react outside the site and yet produce steric or electrostatic influence on the binding and reaction of the substrate. The inhibitor does not have to react at the active site and groups that might be indicated by this inhibition do not have to participate in the catalysis.

$(J)$ The fact that the active site might include sections of a folded polypeptide chain or two or three turns in a helix or might involve two adjacent helices makes it possible for substances that can alter the tertiary protein structure, the configuration of the polypeptide chain in space, to modify enzyme activity. Reactions of inhibitors with hydrogen bonds or the various types of cross-linkages may occur and distort the active site, so that again inhibition does not necessarily imply reaction at the active site.

A variety of interaction forces, both attractive and repulsive, are usually involved in the binding of an inhibitor to an enzyme and to evaluate these it is necessary to know the chemical nature and configuration of the enzyme surface. The knowledge of inhibition mechanisms and the accuracy in the calculation of the interaction energies involved will progress with the advance in active site delineation. Indeed, the use of inhibitors is one of the most powerful tools in the elucidation of the nature of the active site.

\section{INTERMOLECULAR FORCES AND INTERACTION ENERGY}

The forces binding inhibitor molecules to enzymes are those recognized by physicists as occurring generally between various types of molecules. That is, all inhibitor interactions can be adequately explained on the basic of a few fundamental well-known forces and there is no necessity to postulate any unique effects inherent only in protein or enzyme reactions. The only difference between enzyme-inhibitor interactions and interactions 
of simple molecules in a vacuum is one of degree only: the former are generally more complex because of the multiple groups involved, the different types of forces simultaneonsly operative, and the presence of the solvent water. The various forces involved will be discussed briefly, following which certain applications to situations of importance in protein interactions will be made. These possible forces between enzymes and inhibitors may be classified in the following manner, although it should be emphasized that at the ultimate level all forces are electrostatic in nature.

I. Covalent: bond formed by the sharing of an electron pair between two atoms

II. Electrostatic: interaction between atoms or groups that possess permanent electric charges
A. Ion-ion interaction
B. Ion-dipole interaction
C. Dipole-dipole interaction
D. Hydrogen bonds

III. Induced: interaction resulting from the induction of a dipole in a group subjected to the electric field of a permanently charged group
A. Ion-induced dipole interaction
B. Dipole-induced dipole interaction

IV. Electrokinetic: interaction between neutral nompolar groups due to mutual induction of fluctuating dipoles - also called dispersion or London forces

I. Short-range repulsive: due to the electrostatic repulsion between electrons in the overlapping of the electron clonds of two atoms

These forces differ from one another principally in their directional character and the influence of orientation, their variation with the distance between the interacting groups. and the amount of energy they involve at the usual interaction distances. In some cases of inhibition, one type of force dominates, but in others the binding is due to the simultaneous operation of several forces, each of which contibutes to the total interaction energy.

\section{The Covalent Bond}

A normal covalent bond exists between atoms when two electrons of opposite spin moment are shared between them. The bond energy may be attributed to the resonance of the electrons between the atoms. The electron density in a covalent bond is thus greatest in the region between the atoms. Such bonds have three important characteristics: $(a)$ they are directed so that when two or more bonds are associated with an atom they tend to assume a fixed angle to one another, $(b)$ they, of all types of inter- 
actions, lead to the shortest distances between atoms, nsmally 1-2 $\AA$, and (c) they involve high binding energies of the order of 50-100 kcal/mole. Covalent bonds are, however, not necessarily electrically symmetrical and when they join two unlike atoms it is probahle that the bond will possess some ionic character, i.e., the electrons will be attracted more to one of the atoms (the relative ability of atoms to attract electrons is termed their electronegativity), resulting in a fractional negative charge on the more electronegative atom and an equal positive charge on the other atom. This gives such a bond a dipolar character which is of great importance in intermolecular interactions. Although in many instances covalent bonds are formed between enzymes and inhibitors (generally called a chemical reaction of the inhibitor with the enzyme), these situations present no special problems here and will be discussed in connection with the inhibitors reacting in this manner. The following examples may be mentioned: the formation of mercaptide bonds between mercurials or arsenicals and the sulfhydryl groups of the enzyme, the alkylation of sulfhydryl groups by iodoacetate, the acetylation of amino or hydroxyl groups on the enzyme, the reaction of amino groups with fluorodinitrobenzene, and the phosphorylation of serine by the organophosphorus compounds.

\section{Ion-Ion-Interactions}

A point charge in a homogeneous medium possesses an electrical field of radial symmetry, the intensity of which decreases with distance from the charge according to the expression:

$$
\text { Electrical field strength }=F=\frac{q}{d^{2} D}=\frac{z e}{d^{2} \bar{D}}
$$

where $q$ is the magnitude of the charge, $d$ is the distance from the charge to the point in question, $z$ is the valence of the ion, and $e$ is the electronic charge. The dielectric constant $D$ depends on the medium in which the charge is immersed and particularly on the polar nature of the molecules making up this medium: for a vacumm $D=1$ and for water at $37.5^{\circ} D=74.1$. The important question of the dielectric constant will be discussed in some detail following the presentation of the interaction types and for the present the interactions are assumed to take place in a vacuum. Ions of finite dimensions are considered here to have their total charge concentrated at a central point. When the charge is in electrostatic units (esu) and the distance in centimeters, the field strength is given in dynes/esu. At a distance of $5 \AA$ from an electron in a vacuum the field strength would be $-4.8 \times 10^{-10}$ $\mathrm{esu} /\left(5 \times 10^{-8} \mathrm{~cm}\right)^{2}=-1.92 \times 10^{5}$ dynes/esu, the field strength being of the same sign as the charge from which it originates. Since 1 dyne/esu $=$ 300 volts $/ \mathrm{cm}$, the field strength can also be written as $-1.92 \times 10^{5} \times 300=$ 
$-5.76 \times 10^{7}$ volts $/ \mathrm{cm}$. This illustrates the very intense fields that occur in the vicinity of ions, and the effects snch ions have on neighboring molecules must be considered in terms of these strong fields.

The electrical field strength is the force that is exerted on a unit charge at the point under consideration. Hence the force exerted on an electron by another electron at a distance of $5 \AA$ would be $-1.92 \times 10^{5}$ dynes/esu $\times-4.8 \times 10^{-10}$ esu $=9.2 \times 10^{-5}$ dynes since:

$$
\text { Force }=f=q F=\frac{q_{1} q_{2}}{d^{2} D}=\frac{z_{1} z_{2} e^{2}}{d^{2} D}
$$

The electrical potential in the neighborhood of a point charge is the work required to bring a unit charge from infinity to the point at which the potential is evaluated and is given by:

$$
\text { Electrical potential }=\mathrm{V}=\frac{q}{d D}=\frac{z e}{d \bar{D}}
$$

The electrical potential $5 \AA$ from an electron wonld thus be $-4.8 \times 10^{-10} \mathrm{esu} /$ $5 \times 10^{-8} \mathrm{~cm}=-9.6 \times 10^{-3}$ ergs/esu $=-2.88$ volts.

Finally, the most important quantity for our purposes, the mutual potential energy of two separated charges, is the work required to bring the charges from infinite separation to the distance under consideration. This energy can be evaluated by integration of the force between the charges from infinity to the separation distance $d$ :

$$
\text { Potential energy }=\varphi=-\int_{\infty}^{d} f d x=\frac{q_{1} q_{2}}{d D}=\frac{z_{1} z_{2} e^{2}}{d D}
$$

It is also the product of the electrical potential at the distance $d$ from the first charge $\left(V_{1}\right)$ by the magnitude of the second charge $\left(q_{2}\right)$, since $V_{1}$ is the work involved in taking unit charge over the same path. The potential energy of two electrons at $5 \AA$ distance would then be $-9.6 \times 10^{-3}$ ergs $/$ esu $\times-4.8 \times 10^{-10} \mathrm{esu}=4.6 \times 10^{-12} \mathrm{ergs}=1.1 \times 10^{-22} \mathrm{kcal}$. The potential energy is positive if the charges are of the same sign (repulsion) and negative if the charges are of opposite sign (attraction). A univalent cation separated by $5 \AA$ from a univalent anion would possess $-1.1 \times 10^{-22}$ kcal potential energy. In such cases it is most convenient to express the energy in molar terms. Thus a mole of such ion-pairs would have a potential energy of $-1.1 \times 10^{-22} \mathrm{kcal} /$ ion pair $\times 6.024 \times 10^{23}$ ion pairs $/ \mathrm{mole}=$ $-66.3 \mathrm{kcal} / \mathrm{mole}$. In fact. Eq. $6-4$ may be rewritten in the convenient form:

$$
\varphi=332 \frac{z_{1} z_{2}}{d D} \mathrm{kcal} / \mathrm{mole}
$$


when $d$ is in $\AA$. This electrostatic interaction between ions is the most important, but not the only, contribution to the total energy; this will be apparent in the following sections. The interaction between ions in solution depends on the dielectric constant in the region of the ions, the hydration of the ions, and the ionic atmosphere, and will be much less than calculated above (see page 249).

\section{Ion-Dipole Interactions}

An electrical dipole results when two equal charges, $q_{d}$, of opposite sign are held at a distance, $l$, apart and the magnitude of this dipole is given by:

$$
\text { Dipole moment }=\mu=q_{d} l
$$

The electrostatic interaction between a dipole and an ion is the sum of the interactions of the ion with each of the charges on the dipole. From Fig. 6-3 it is seen that the potential energy is given by:

$$
\varphi=\frac{q_{d} q}{D}\left[\frac{1}{d_{1}}-\frac{1}{d_{2}}\right]
$$

When the dipolar distance, $l$, is less than the distance, $d$, between the ion and the center of the dipole, as is usually the case, this equation may be rewritten in the approximate form:

$$
\varphi=\frac{q \mu \cos \theta}{d^{2} D}\left[1-\frac{3 l^{2}}{8 d^{2}}\right]
$$

The term in brackets approaches unity as the distance of the ion from the dipole increases: it is 0.91 when $d^{\prime} l=2,0.96$ when $d l=3$, and 0.98 when $d / l=4$. Thus the further approximation may often be used with minor error since $l$ is often $1-2 \AA$ and the interaction distances 5-10 $\AA$ :

$$
\varphi=\frac{q \mu \cos \theta}{d^{2} D}
$$

The dependency of the potential energy on orientation is also shown in Fig. 6.3. If the dipole were rapidly and uniformly rotating, the time average of the potential energy would be zero. However, due to the field of the ion, the dipole will be statistically oriented towards the position of maximal attraction or minimal potential energy. The degree of orientation will depend on the relative magnitudes of the interaction energy and the thermal rotational energy $k T$. This is the orientation polarization of a permanent dipole in an electric field whereby a statistical dipole is produced with a moment:

$$
\bar{\mu}=\frac{\mu^{2} q}{3 k T d^{2} D}
$$


The potential energy of the ion and this dipole is thus:

$$
\varphi=-\frac{q^{2} \mu^{2}}{3 k T d^{4} D^{2}}
$$

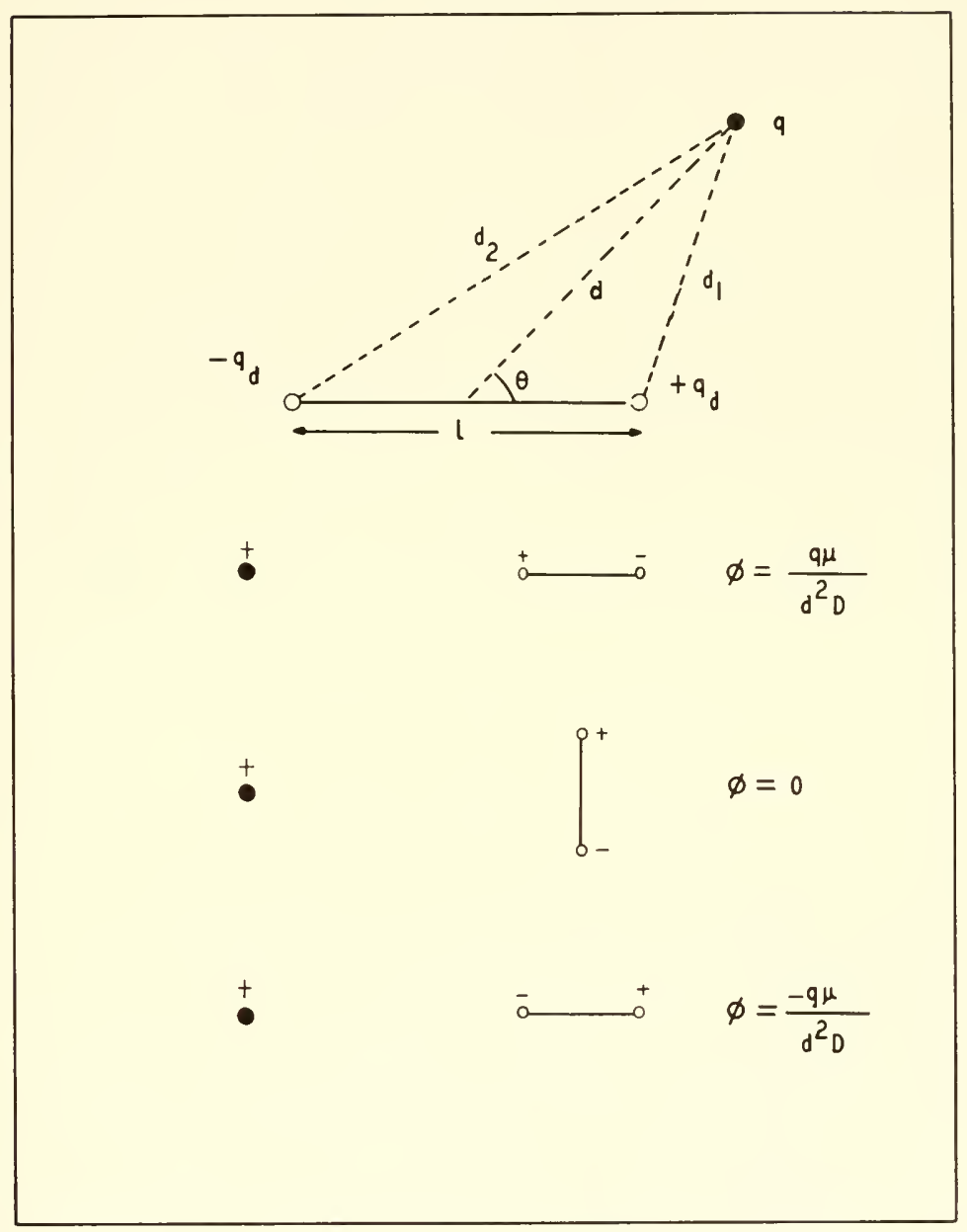

Fig. 6-3. Interaction of an ion with a dipole.

This applies particularly to situations where the electrostatic potential energy is small compared to $k T$; when it is larger than $k T$ the dipole will be essentially oriented in the position of minimal energy and the potential energy will be given approximately by $-q \mu^{\prime} d^{2} D$.

Dipole moments are usually expressed in electrostatic units. Thus if each of the charges in a dipole is half an electron charge and the distance 
between them is $2 \AA, \mu=2.4 \times 10^{-10} \times 2 \times 10^{-8}=4.8 \times 10^{-18}$ esu. The moments of most bonds and small molecules are of the order of magnitude of $10^{-18}$ esu and this has been called a debye. In the case above, the dipole moment would be 4.8 debyes. The potential energy will be given in ergs/ molecule when $q$ and $\mu$ are in electrostatic units and $d$ is in centimeters. Thus a dipole of 1 debye at a distance of $10 \AA$ from a univalent ion at $37.5^{\circ}$ would give a potential energy due to orientation polarization in the ion field of:

$$
\varphi=-\frac{\left(4.8 \times 10^{-10}\right)^{2}\left(10^{-18}\right)^{2}}{(3)\left(1.38 \times 10^{-16}\right)(310.7)\left(10^{-7}\right)^{4}}=-1.87 \times 10^{-14} \mathrm{ergs} / \mathrm{molecule}
$$

which is equivalent to $-0.269 \mathrm{kcal} / \mathrm{mole}$. Since at $37.5^{\circ}$ the thermal energy is $4.29 \times 10^{-14} \mathrm{ergs} / \mathrm{molecule}$ or $0.617 \mathrm{kcal} / \mathrm{mole}$ and over twice the magnitude of the interaction energy, it is evident that the dipole will not be fixed but rotating with preferential orientation towards the ion. It is interesting to note that a rigidly fixed dipole in the position of maximal attraction to the ion would give a potential energy of $-0.691 \mathrm{kcal} / \mathrm{mole}$; the electrostatic interaction when the dipole is free is thus about $40 \%$ of that when the dipole is fixed. Equations 6-9 and 6-11 may be rewritten as:

$$
\begin{aligned}
& \varphi=69.1 \frac{z \mu}{d^{2} D} \cos \theta \mathrm{kcal} / \mathrm{mole} \\
& \varphi=-2693 \frac{z^{2} \mu^{2}}{d^{4} D^{2}} \mathrm{kcal} / \mathrm{mole}
\end{aligned}
$$

where $z$ is the valence of the ion, $\mu$ is in debyes, and $d$ is in $\AA$.

The dipole moment of a molecule in solution may be different from that in the gas phase as pointed out by Kirkwood (1939). Let us represent the classic moment of the isolated molecule in the gas phase by $\mu_{0}$. When this molecule is surrounded by water molecules, its moment may be altered by the electrostatic displacement effects of the water dipoles. The moment of the molecule in solution, designated by $\mu$, is usually somewhat larger than $\mu_{0}$. Finally, this dipole will exert a slight orienting effect on the surrounding water molecules and partially restrict their rotation. The electric field at some distance from this molecule will depend not only on its intrinsic moment is solution, $\mu$. but on the vector sum of the moments of all the water molecules influenced by the central molecule; this moment of the molecule with its solvent cluster may be designated $\bar{\mu}$. The relationship between these may be written:

$$
g \mu_{0}^{2}=\mu \cdot \bar{\mu}
$$

where $g$ is a correlation parameter. In Kirkwood's theory of polar liquids, 
the quantity $\sqrt{\mu \cdot \bar{\mu}}$ might be said to be the effective moment and replaces $\mu_{0}$ in the older formulations. The value of $g$ is generally unknown and may be less or greater than unity. For water it is around 2.65 (Oster and Kirkwood, 1943) but the high value is in part due to hydrogen bonding. The effective dipole moment of a molecule in aqueons solution may thus be different from the usually given value. Such deviation may be of some significance in the interaction of small molecules with proteins and important in future refinements of the treatment of intermolecular forces.

\section{Dipole-Dipole Interactions}

The potential energy of two dipoles depends not only on the distance between them but on their relative orientations. The interactions of both fixed dipoles and freely rotating dipoles must be considered. If two dipoles are fixed relative to one another as in Fig. 6-4(a) and the distance, $d$.

(a)
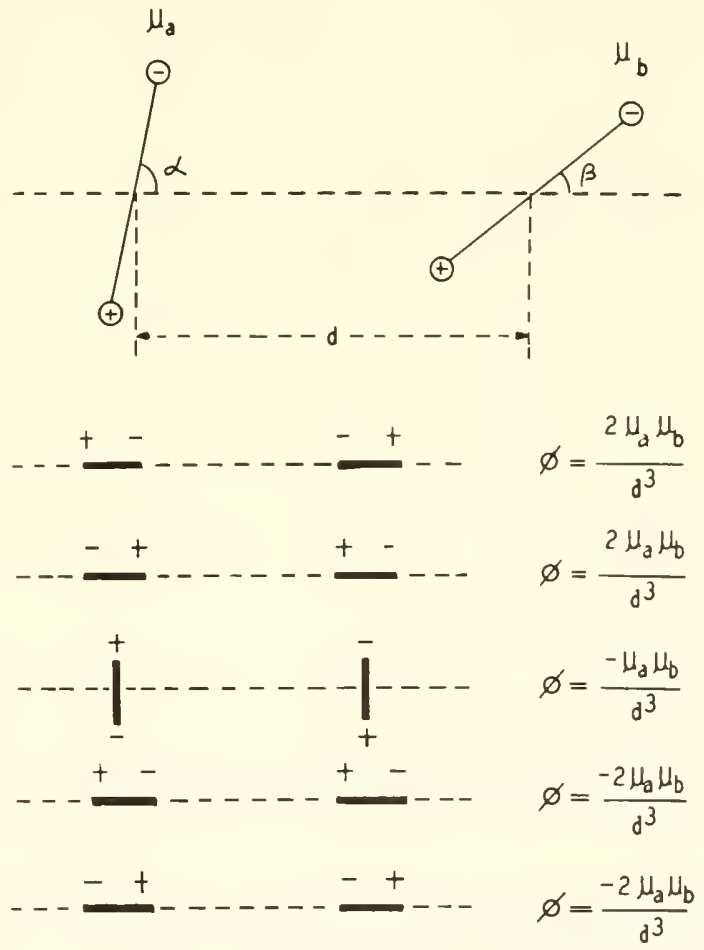

(b)

FIG. 6-4. Interaction of two dipoles. 
between them is much greater than the lengths of the dipoles, the potential energy is given by (Moelwyn-Hughes, 1957, p. 301):

$$
\varphi=-\frac{\mu_{a} \mu_{b}}{d^{3} D}(2 \cos \alpha \cos \beta-\sin \alpha \sin \beta)
$$

The potential energies for certain configurations are shown in Fig. 6-4 (b), from which it is seen that the maximal attraction occurs when the dipoles are oriented in a head-to-tail position.

Interactions of substrates and inhibitors with enzymes must often occur at distances which are not markedly greater than the dipolar lengths. It is thus necessary to enquire into the energies involved at these shorter distances. The two configurations shown in Fig. 6-5 lead to the following expressions for the potential energy:

$$
\text { Parallel: } \quad \varphi_{a}=\frac{2 \mu_{a} \mu_{b}}{l^{2} D}\left[\frac{1}{\sqrt{l^{2}+d^{2}}}-\frac{1}{d}\right]
$$

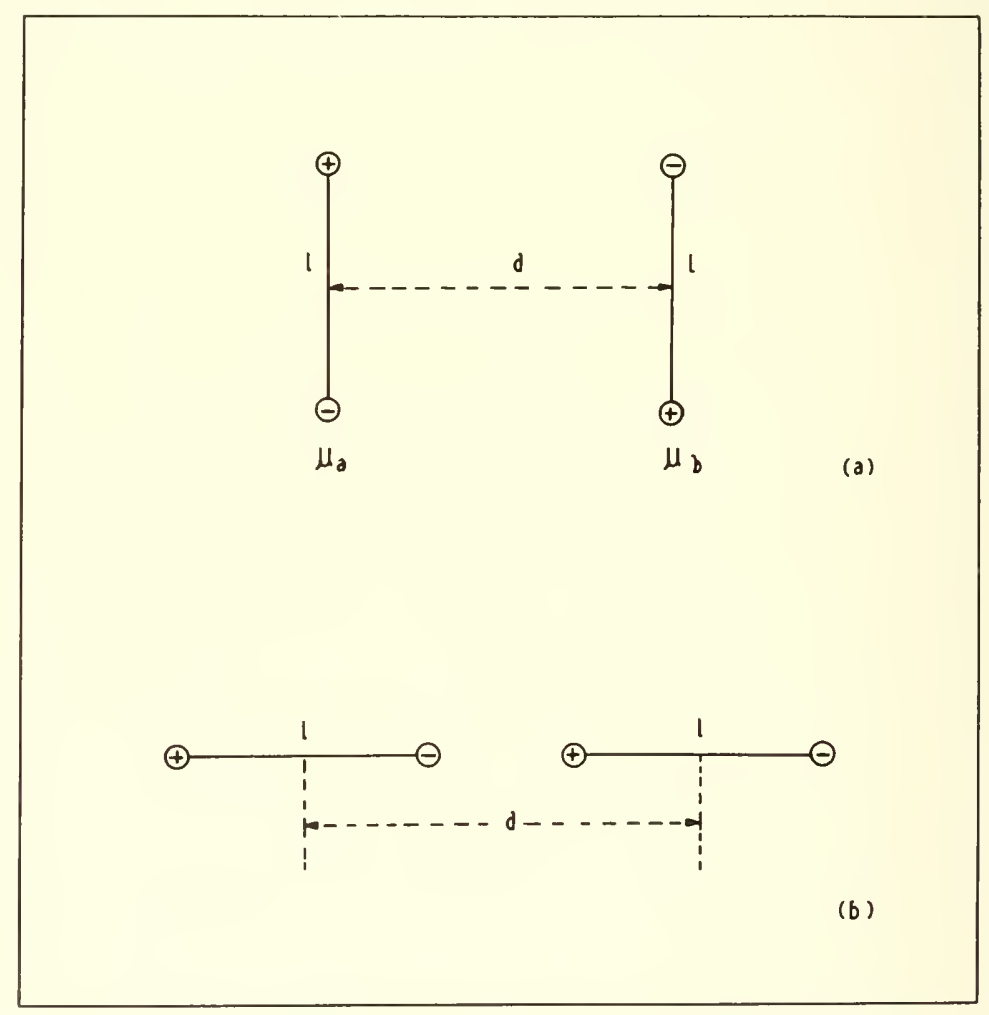

FIG. 6-5. Interactions of two dipoles at small distances. 


$$
\text { Head-to-tail: } \quad q_{b}=\frac{\mu_{a} \mu_{b}}{l^{2} D}\left[\frac{2}{d}-\frac{1}{d-l}-\frac{1}{d+l}\right]
$$

Taking different ratios of $d$ to $l$ leads to the following values of the potential energy (see tabulation below). As $d$ becomes much larger than $l$ the potential energies, of course, approach $\varphi_{a}=-\mu_{a} \mu_{b} / d^{3}$ and $\varphi_{b}=-2 \mu_{a} \mu_{b} / d^{3}$. However, when $d / l$ becomes less than 5 the usual expressions for dipolar interaction are progressively more inaccurate. When the dipoles are parallel, the potential energy upon close approach becomes less than expected on the basis of Eq. 6-15 (i.e., less absolute potential energy), while when the dipoles are head-to-tail the interaction energy becomes greater than ex-

\begin{tabular}{l|c|c}
\hline$d$ & $\varphi_{a}$ & $\varphi_{b}$ \\
\hline$l$ & $-0.586 \mu_{a} \mu_{b} / d^{3}$ & - \\
$2 l$ & $-0.845 \mu_{a} \mu_{b} / d^{3}$ & $-2.67 \mu_{a} \mu_{b} / d^{3}$ \\
$3 l$ & $-0.914 \mu_{a} \mu_{b} / d^{3}$ & $-2.25 \mu_{a} \mu_{b} / d^{3}$ \\
$4 l$ & $-0.962 \mu_{a} \mu_{b} / d^{3}$ & $-2.13 \mu_{a} \mu_{b} / d^{3}$ \\
\hline
\end{tabular}

pected. These deviations may be of considerable importance in enzyme interactions.

Equations of the type $\varphi=\mathrm{A} \mu_{a} \mu_{b} / d^{3} D$, such as 6-15 to 6-17, can be written in the following convenient form:

$$
\varphi=-14.4 \mathrm{~A} \frac{\mu_{a} \mu_{b}}{d^{3} D} \mathrm{kcal} / \mathrm{mole}
$$

when the $\mu$ 's are in debyes and $d$ in $\AA$. The energy involved in such dipole pairs is usually not very large: two dipoles of $\mu=2$ debyes at a distance of $5 \AA$ in orientation for maximal interaction possess a potential energy of approximately $\mathrm{I} \mathrm{kcal} / \mathrm{mole}$ in a vacuum, much less than for ionic interactions over the same distance.

The problem of freely rotating dipoles must now be considered. There are two possible situations of interest. The first is where dipole $\mathrm{A}$ is fixed and dipole $\mathrm{B}$ is free, as might occur in the interaction of a small molecule in solution with a dipole on the enzyme surface, in which case the potential energy is given by:

$$
\varphi=-\frac{\mu_{a}^{2} \mu_{b}^{2}}{3 k T d^{6} D}\left(1+3 \cos ^{2} \theta\right)
$$

where $\theta$ is the angle the line of centers makes with the axis of dipole A. The second is where both dipoles are free and their interaction is due to 
the mutual orientation polarization they exert, in which case the potential energy is:

$$
\varphi=-\frac{2 \mu_{a}^{2} \mu_{b}{ }^{2}}{3 k T d^{6} D^{2}}
$$

These expressions may be derived from the electrical field around a dipole, the orientation such a field produces on a freely rotating dipole, and the interaction of the field with the average moment induced. The energy is always negative since orientation tends towards the most stable state. Equations of type $\gamma=-A \mu_{a}^{2} \mu_{b}^{2} / k T d^{6} D^{2}$, such as 6-19 and 6-20, may be written:

$$
\varphi=-350 \mathrm{~A} \frac{\mu_{a}^{2} \mu_{b}^{2}}{d^{6} D^{2}} \mathrm{kcal} / \mathrm{mole}
$$

when the $\mu$ 's are in debyes and $d$ in $\AA$. Thus the potential energy of two freely rotating dipoles of $\mu=2$ debyes at a distance of $5 \AA$ in a vacum wonld be $-0.24 \mathrm{kcal} /$ mole. For this treatment to be valid, the potential energy must be less than the thermal energy $k T$, for otherwise the interaction would be strong enough to orient the dipoles in relatively fixed positions and equations of type 6-18 would be applicable. The dependence of the potential energy of two dipoles upon distance will thus be related to the magnitudes of the moments and the ability to approach close enough to produce a partially rigid configmation.

\section{Hydrogen Bonding}

Hydrogen bonds are said to occur when electronegative atoms are bonded by a hydrogen atom between them. The bond energy is less than that involved in covalent bonding and is usually of the order of $2-10 \mathrm{kcal} / \mathrm{mole}$. Such a bond between nitrogen and oxygen is usually written as $\mathrm{O}-\mathrm{H} \cdots \mathrm{N}$ where the $\mathrm{O}-\mathrm{H}$ bond is essentially covalent and the dots indicate the hydrogen bond. Hydrogen cannot form two covalent bonds and it is not a matter of resonance of the hydrogen atoms between the two electronegative atoms because the bonds are asymmetrical. The explanation is probably to be sought in the electrostatic interaction of bond dipoles. If $\mathrm{A}$ and $\mathrm{B}$ are electronegative atoms, they will usually possess a fractional negative charge when bonded to other atoms, due to the ability of such atoms to distort the electronic configuration. Thus the bond $\mathrm{A}-\mathrm{H}$ is a dipole and may be written $\mathrm{A}^{-}-\mathrm{H}^{+}$. these charges being fractions of electronic and protonic charges. The bond $\mathrm{B}-\mathrm{R}$ will similarly be a dipole and may be written $\mathrm{B}^{-}-\mathrm{R}^{+}$. The interaction of these two bonds in the appropriate head-to-tail position can be represented as follows:

$$
\mathrm{A}^{-}-\mathrm{H}^{+} \cdots \cdots \mathrm{B}^{-}-\mathrm{R}^{+}
$$


and here the dots represent the dipolar interaction. If this is the correct mechanism, the bond energy should be given approximately by a calculation of this dipolar interaction. Let us take the $\mathrm{O}-\mathrm{H} \cdots \mathrm{O}$ bond as it occurs in water: the bond distance is $2.76 \AA$ and the distance between dipole centers is therefore $2.40 \AA$ (taking into account the configuration of the water molecules), the dipole moment of the $\mathrm{O}-\mathrm{H}$ bond is 1.51 debyes and of the water molecule 1.83 debyes, and the $\mathrm{O}-\mathrm{H}$ bond distance is $0.955 \mathrm{\AA}$. Using Eq. 6-17 the potential energy is found to be -6.61 kcal mole: this must be corrected for repulsion interaction (see page 2:31) by a reduction of approximately $35 \%$ and the final calculated energy is therefore -4.29 hcal/ mole. The experimental bond energy is - $4.5 \mathrm{kcal}$ mole and the agreement is satisfactory; there are. of comrse, other interactions of less importance, such as induction effects. which will increase the stability slightly. One can therefore interpret the hydrogen bond simply as an ordinary dipoledipole interaction. Such bonds are formed more readily with hydrogen than with other atoms becanse the small size of the hydrogen atom allows close approach of the interacting electronegative atoms.

An interesting discussion of the various theories of the hydrogen bond has been presented by Pimentel and McClellan (1960. p. 2206). A critical evaluation of the electrostatic theory was given and it was concluded that it does not account for all the phenomena observed in hydrogen bond formation. They believe that a molecular orbital treatment, involving both bonding and nonbonding orbitals, might be preferable, but at the present time such a description cannot be quantitative. Another approach is to interpret the stability of the hydrogen bond as the result of resonance between structures such as the following:

$\begin{array}{lrr}\text { A }-\mathrm{H} & & \text { :B } \\ \text { A: } & \mathrm{H}^{+} & : B \\ \text { A: } & & \mathrm{H}-\mathrm{B}\end{array}$

where the dashes indicate covalent bonds (Pauling. 1960, p. 419; Wheland, $1955, p .47)$. These and other theories are usually not as incompatible as has often been assumed.

The strength of the hydrogen bond will depend in part on the magnitude of the dipole moments involved and thus on the degree of electronegativity of the atoms bonded. The electronegativities for biologically important atoms are (Gordy, 1946): F. 3.95: 0. 3.45: X. 2.98; Cl. 2.97; Br. 2.75; C, 2.55; S. 2.53; I, 2.45; H, 2.13; and P, 2.10. In Table $6-1$ the bond moments and electronegativity differences are given for some $\mathrm{X}-\mathrm{H}$ bonds of possible significance in inhibitor interactions. A reasonable correlation may be observed. It should be pointed out that these bond moments are average values only and that the actual moment in a molecule will depend on the 
TABLE 6-1

Bond Moments and Electronegativity Differences ${ }^{a}$

\begin{tabular}{c|c|c}
\hline Bond & Dipole moment & $\begin{array}{c}\text { Electronegativity } \\
\text { difference }\end{array}$ \\
$\mathrm{F}-\mathrm{H}$ & 1.94 & 1.82 \\
$\mathrm{O}-\mathrm{H}$ & 1.51 & 1.32 \\
$\mathrm{~N}-\mathrm{H}$ & 1.31 & 0.85 \\
$\mathrm{Cl}-\mathrm{H}$ & 1.08 & 0.84 \\
$\mathrm{Br}-\mathrm{H}$ & 0.78 & 0.62 \\
$\mathrm{~S}-\mathrm{H}$ & 0.68 & 0.40 \\
$\mathrm{C}-\mathrm{H}$ & 0.40 & 0.42 \\
$\mathrm{I}-\mathrm{H}$ & 0.38 & 0.32 \\
$\mathrm{P}-\mathrm{H}$ & 0.36 & -0.03 \\
\hline
\end{tabular}

a The dipole moments were taken from Smyth (1955, p. 244) with the positive pole being the hydrogen atom in each case. Exact correlation with the electronegativity difference is not to be expected because of size differences between the atoms and the oceurrence of orbital hybridization.

groups attached to the atoms. The important $\mathrm{C}-\mathrm{H}$ bond may be cited as an example: althongh its moment is generally given as 0.4 with the direction $\mathrm{C}^{-}-\mathrm{H}^{+}$, there is good evidence that, at least in some cases, the dipole is reversed as $\mathrm{C}^{+}-\mathrm{H}^{-}$(Wheland, 1955, p. 207; Smyth, 1955, p. 240). The reason for this direction, unexpected from the relative electronegativities, is probably the $s p$-hybridization in the bond and the unequal distribution of electrons aromd the carbon atom; the resultant moment will depend on the groups attached to the carbon atom. This sitnation is mentioned because it is wished to emphasize the variability in bond monents. Carbon can participate in hydrogen bonding (hydrogen cyanide and acetaldehyde) so in these cases the hydrogen atom must be positive.

The energy of the $\mathrm{O}-\mathrm{H} \cdots \mathrm{O}$ bond probably ranges from 4-6 kcal $/ \mathrm{mole}$ and other biologically common hydrogen bonds are weaker: for example, the $\mathrm{N}-\mathrm{H} \cdots \mathrm{O}$ bond may be in the range $2-3 \mathrm{keal} /$ mole and the $\mathrm{N}-\mathrm{H} \cdots \mathrm{N}$ bond between 1-2 kcal/mole. Nitrogen is less electronegative than oxygen and has a greater covalent radius; thus the moments are smaller and the distance between the dipoles somewhat larger when nitrogen forms a hydrogen bond, both of which would decrease the bond energy. It is evident from the electrostatic nature of the hydrogen bond that it will vary in stability with changes in $\mathrm{pH}$ or ionie strength; stability is generally reduced by decrease in $\mathrm{pH}$ or increase in ionic strength. The energy of activation for the formation or rupture of hydrogen bonds is quite low and thus they show high mobility. Since the formation of hydrogen bonds between a 
substance and a protein involves breaking of the previous hydrogen bonds between protein and water and between the substance and water, this mobility may be of some significance in the rates of such reactions.

The entropy change resulting from the formation of a single hydrogen bond is usually between -10 and $-20 \mathrm{cal} / \mathrm{mole} /$ degree in the gas or liquid phases. However, in aqueous solution the IS and $\mathbf{I H}$ for hydrogen bond formation may be quite different from the values that are generally given for the pure state. Let us assume that two molecules, A and B, associate through a hydrogen bond. Before this bond is formed, each molecule in solution has, to some extent, formed a hydrogen bond with a neighboring water molecule; following the association of $\mathrm{A}$ and $\mathrm{B}$, the water molecules released will tend to form hydrogen bonds with other water molecules in the aqueous structure. Thus the over all reaction may be written as:

$$
\mathrm{A}-\mathrm{H}-w+\mathrm{B}-\mathrm{H}-w \rightarrow \mathrm{A}-\mathrm{H}-\mathrm{B}+w-\mathrm{H}-w
$$

where $w$ represents either $\mathrm{H}_{2} \mathrm{O}$ or $\mathrm{OH}$, depending on the origin of the hydrogen atom in the bond. The over-all thermodynamic changes are, therefore, not as great as in the pure phase. There is probably still a small decrease in the entropy during hydrogen bond formation in aqueous solution (Lumry, 1959, p. 157).

\section{Ion-Induced Dipole Interactions}

All molecules in an electric field become polarized and this results in a dipole whose moment is dependent on the strength of the field and the properties of the molecule. In the most general case the total polarization is given by:

$$
P=P_{e}+P_{a}+P_{m}
$$

$P_{e}$ is the electronic polarization which is due to the displacement of the electrons in the field. $P_{a}$ is the atomic polarization and is due to the displacement of atoms or groups in the molecule. $P_{m}$ is the orientation polarization and results from the preferential orientation of a molecule with a permanent dipole in the field. In a molecule without a permanent dipole the polarization induced by the field is $P_{e}+P_{a}$ which may be called the induced polarization $P_{i}$. It is a polarization that is dependent on the inherent flexibility of movement of the electrons and atoms in a molecule, that is, the polarizability of the molecule. Such induction will, of course, occur in a molecule with a permanent dipole. but in this section we shall be concerned only with electronic and atomic polarizations; the polarization of a permanent dipole in the field of an ion has been discussed in a previous section. The distinction between $P_{e}$ and $P_{a}$ is important only in alternating fields, with which we are not concerned, and therefore the resultant induced 
polarization $P_{i}$ will be the important quantity for our present purpose. The dipole induced in a molecule by the field of an ion will always lead to attraction between the ion and the molecule and thus will contribute to the energy of interaction in favor of stability.

The induced dipole moment will be proportional to the field strength:

$$
\mu_{i}=\alpha_{0} F
$$

and oriented with the field; in the field of an ion the dipole will be aligned radially to the ion. The proportionality constant $\alpha_{0}$ is called the polarizability of the molecule; in conformity with previous usage, the total polarizability, inchuding the orientation of a permanent dipole, will be designated by $\alpha$. Since the potential energy of a dipole in an electrical field is given by $q=-\mu F \cos \theta$ and here $\theta=0^{\circ}$ :

$$
p_{i}=-\alpha_{0} F^{2}
$$

However, it requires energy to induce the dipole and this is $\alpha_{0} F^{2} / 2$. Thus the potential energy of the dipole in the ion field is:

$$
\text { i }=\frac{\alpha_{0} F^{2}}{2}-\alpha_{0} F^{2}=-\frac{\alpha_{0} F^{2}}{2}
$$

The field of an ion is given by $F=q / d^{2} D$, where $q=z e$, so that:

$$
\varphi=-\frac{\alpha_{0} z^{2} e^{2}}{2 d^{4} D^{2}}=-166 \frac{\alpha_{0} z^{2}}{d^{4} D^{2}} \mathrm{kcal} / \mathrm{mole}
$$

where the energy is given in kcal/mole if $\alpha_{\mathbf{0}}$ is in units of $10^{-24} \mathrm{ml} / \mathrm{molecule}$ and $d$ is in $\AA$. The potential energy of a water molecule $\left(\alpha_{0}=1.444\right)$ in the field of a univalent ion at a distance of $5 \AA$ in vacuum, due only to inductive effects, is thus $-0.384 \mathrm{kcal} / \mathrm{mole}$. This contribution would be small compared to the interaction of the ion with the permanent dipole of the water molecule, which would be around $-5 \mathrm{kcal} / \mathrm{mole}$. The potential energy due to an induced dipole is dominant only in interactions between ions and nonpolar molecules.

The values of the polarizability $\alpha_{0}$ are determined by measurements of the optical refraction since the latter depends upon the clectronic and atomic distortions induced by the alternating electrical field of the radiation. The refraction varies with the wavelength of the light used; by determinations at two or more wavelengths it is possible to calculate the refraction at zero frequency where the effect of a constant ficld is obtained. The molar refraction at zero frequency is related to the polarizability:

$$
R_{0}=\frac{4 \pi \mathrm{N} \alpha_{0}}{3}
$$


and thus the expression for the potential energy may be rewritten as:

$$
\varphi=-\frac{3 R_{0} z^{2} e^{2}}{8 \pi \mathrm{N} d^{4} D^{2}}=-65.8 \frac{R_{0} z^{2}}{d^{4} D^{2}} \mathrm{kcal} / \mathrm{mole}
$$

The potential energy is given in $\mathrm{kcal} /$ mole when $R_{0}$ is in $\mathrm{ml} /$ mole and $d$ in $\AA$. The molar refraction of a substance is approximately equal to the sum of the atomic refractions of the constituent atoms, as proposed by Landolt in 1862. The usually accepted values for atomic refractions are given in the tabulation below.

\begin{tabular}{c|c|c|c}
\hline Atom & $R_{0}$ & Atom & $R_{\mathbf{v}}$ \\
\hline $\mathrm{H}$ & 1.07 & $\mathrm{~F}$ & 1.06 \\
$\mathrm{C}$ & 2.35 & $\mathrm{Cl}$ & 5.79 \\
$\mathrm{O}$ (carbonyl) & 2.15 & $\mathrm{Br}$ & 8.60 \\
$O$ (hydroxyl) & 1.48 & $\mathrm{I}$ & 13.5 \\
O (ether) & 1.59 & $\mathrm{~S}$ & 7.15 \\
N (primary amine) & 2.25 & $\mathrm{P}$ & 8.15 \\
N (secondary amine) & 2.43 & Double bond & 1.68 \\
N (tertiary amine) & 2.75 & Triple bond & 2.33 \\
\hline
\end{tabular}

A somewhat more accurate approach is to consider the molar refraction as the sum of bond refractions, inasmuch as the polarizability is mainly due to the bonding electrons. Bond refractions and polarizabilities are presented in Table 6-2. Double bond conjugation leads to refractions greater than expected on the basis of the atomic or bond refractions; this is called exaltation and values for certain conjugated systems are shown in Table 6-3. Some approximate ionic and group refractions are given in Tables 6-4 and 6-5. The molar refractions of compounds may also be calculated from the indices of refraction by the Lorentz-Lorenz equation (indices of refraction for many compounds are given in the International Critical Tables, 1930, Vol. 7. and in Timmermans" "Physico-Chemical Constants of Pure Organic Compounds" 1950). The induced polarization involves principally the electrons in the outer shell of an atom or ion, including those in bonding orbitals, and the contribution from atomic polarization is only $1-5 \%$ of the electronic contribution.

The polarizability $\alpha_{0}$ is a spherical average but actually most bonds and molecules are asymmetric with respect to their polarizability. Bonds generally exhibit a much greater polarizability along the bond axis than transversely, and the polarizability of benzene is about twice as great in the plane of the ring as perpendicular to it. This refraction anisotropy may 
TABLE 6-2

Properties of Biologically Important Bonds ${ }^{a}$

\begin{tabular}{|c|c|c|c|c|c|c|}
\hline Bond & $\begin{array}{c}\text { Length } \\
(\AA)\end{array}$ & $\begin{array}{l}\text { Dipole } \\
\text { moment } \mu \\
\text { (debyes) }\end{array}$ & $\begin{array}{l}\text { Atomic } \\
\text { charge } \\
\text { (electron } \\
\text { charge) }\end{array}$ & $\begin{array}{c}\text { Molar } \\
\text { refraction } R_{0} \\
(\mathrm{ml} / \text { mole })\end{array}$ & $\begin{array}{c}\text { Polari- } \\
\text { zability } \alpha_{0} \\
\left(\times 10^{24} \mathrm{ml} /\right. \\
\text { molecule })\end{array}$ & $\begin{array}{c}\text { Bond } \\
\text { energy } \\
\text { (kcal/mole) }\end{array}$ \\
\hline $\mathrm{C}-\mathrm{C}$ & 1.54 & 0 & 0 & 1.20 & 0.475 & 81 \\
\hline $\mathrm{C}-\mathrm{H}$ & 1.09 & 0.4 & 0.08 & 1.65 & 0.655 & 99 \\
\hline $\mathrm{C}-\mathrm{O}$ & 1.42 & 1.5 & 0.22 & 1.41 & 0.559 & 80 \\
\hline $\mathrm{C}-\mathrm{N}$ & 1.46 & 1.15 & 0.16 & 1.51 & 0.598 & 66 \\
\hline$C-S$ & 1.81 & 1.73 & 0.20 & 4.43 & 1.75 & 61 \\
\hline$C-F$ & 1.38 & 2.24 & 0.34 & 1.78 & 0.705 & 102 \\
\hline $\mathrm{C}-\mathrm{Cl}$ & 1.76 & 2.3 & 0.27 & 6.38 & 2.53 & 76 \\
\hline $\mathrm{C}-\mathrm{Br}$ & 1.93 & 2.2 & 0.24 & 9.19 & 3.64 & 63 \\
\hline$C-I$ & 2.13 & 2.0 & 0.19 & 14.08 & 5.57 & 47 \\
\hline $\mathrm{N}-\mathrm{N}$ & 1.44 & 0 & 0 & 1.52 & 0.602 & 37 \\
\hline $\mathrm{N}-\mathrm{H}$ & 1.01 & 1.01 & 0.27 & 1.82 & 0.721 & 92 \\
\hline $\mathrm{O}-\mathrm{O}$ & 1.32 & 0 & 0 & 1.62 & 0.641 & 34 \\
\hline $\mathrm{O}-\mathrm{H}$ & 0.96 & 1.51 & 0.33 & 1.85 & 0.733 & 105 \\
\hline$S-S$ & 2.08 & 0 & 0 & 7.41 & 2.93 & 66 \\
\hline $\mathrm{S}-\mathrm{H}$ & 1.34 & 0.68 & 0.11 & 4.62 & 1. 83 & 87 \\
\hline $\mathrm{C}=\mathrm{C}$ & 1.35 & 0 & 0 & 4.02 & 1.59 & 140 \\
\hline $\mathrm{C} \equiv \mathrm{C}$ & 1.20 & 0 & 0 & 5.83 & 2.31 & 193 \\
\hline $\mathrm{C}=\mathrm{O}$ & 1.22 & 3.12 & 0.53 & 3.32 & 1.31 & 178 \\
\hline $\mathrm{C} \equiv \mathrm{N}$ & 1.15 & 3.5 & 0.63 & 4.69 & 1.86 & 211 \\
\hline $\mathrm{N}=\mathrm{O}$ & 1.16 & 2.0 & 0.36 & 3.34 & 1.32 & 104 \\
\hline
\end{tabular}

a These are eclectic values from several recent sources. The clipole moments of some bonds have been corrected for the reversal of the earlier $\mathrm{C}-\mathrm{H}$ bond moment (Wheland, 1955. p. 209); according to the classic direction the moments are $\mathrm{C}-\mathrm{N}$, $0.22 ; \mathrm{C}-\mathrm{O}, 0.74 ; \mathrm{C}-\mathrm{Cl}, 1.46 ; \mathrm{C}-\mathrm{Br}, 1.38 ; \mathrm{C}-\mathrm{I}, 1.19$ (Smyth, 1955, p. 244). The atomic charge has beer calculated from the bond distance and the dipole moment and is given in electron charge units. In some cases $R_{0}$ has been calculated from $R_{D}$, the refraction at the sodium $D$ line. All values are subject to variation with different groups on the bonded atoms.

be important in situations in which the polarizing charge is in a fixed configuration with respect to the molecule polarized.

In the evaluation of the interaction energy between an ion and the induced dipoles in a complex molecule, it is necessary to integrate the interaction over the atoms and bonds involved. In a large molecule it is not necessarily the total molar refraction that is important; in a uniform field the total refraction or polarizability has significance but in the decremental field of an ion it is more accurate to consider the contributions of each bond or group at its distance from the ion. 
TABLE 6-3

Refraction Exaltations Due to Conjugation ${ }^{a}$

\begin{tabular}{l|c} 
Compound & $\begin{array}{c}\text { Exaltation }\left(1 R_{0}\right) \\
(\mathrm{ml} / \text { mole })\end{array}$ \\
\hline $\mathrm{CH}_{2}=\mathrm{CH}_{2}-\mathrm{CH}_{2}=\mathrm{CH}_{2}$ & +1.42 \\
$\mathrm{CH}_{2}=\mathrm{CH}_{2}-\mathrm{CH}_{2}=\mathrm{CH}_{2}-\mathrm{CH}_{2}=\mathrm{CH}_{2}$ & +2.07 \\
$\varphi-\mathrm{CH}=\mathrm{CH}_{2}-\mathrm{CH}_{2}=\mathrm{CH}_{2}$ & +3.66 \\
$\varphi-\mathrm{CH}=\mathrm{CH}_{2}$ & +1.20 \\
$\varphi-\mathrm{CHO}$ & +1.18 \\
$\varphi-\mathrm{NO} \mathrm{O}_{2}$ & +0.93 \\
$\varphi-\mathrm{NH}$ & +0.93 \\
$\varphi-\mathrm{SH}$ & +0.49 \\
$\varphi-\mathrm{OH}$ & +0.24 \\
Naphthalene & +2.55 \\
Anthracene & +8.17 \\
\hline
\end{tabular}

a From Smyth (1955).

TABLE 6-4

Ionic Refractions ${ }^{a}$

\begin{tabular}{l|c} 
Ion & $\begin{array}{c}R_{D} \\
(\mathrm{ml} / \mathrm{mole})\end{array}$ \\
& \\
$\mathrm{Na}^{+}$ & 0.50 \\
$\mathrm{~K}^{+}$ & 2.2 \\
$\mathrm{Ig}^{++}$ & 0.29 \\
$\mathrm{Ca}^{++}$ & 1.35 \\
$\mathrm{Zn}^{++}$ & 0.3 \\
$\mathrm{Cd}^{++}$ & 2.4 \\
$\mathrm{~F}^{-}$ & 2.5 \\
$\mathrm{Cl}^{-}$ & 8.7 \\
$\mathrm{Br}^{-}$ & 12.2 \\
$\mathrm{I}^{-}$ & 18.5 \\
\hline
\end{tabular}

a From Simyth (1955, p. 407). 
TABLE $6-5$

Molecular Group Refractions axd Dipole Momexts a

\begin{tabular}{|c|c|c|c|c|}
\hline \multirow{2}{*}{ Group } & \multirow{2}{*}{$\begin{array}{c}R_{0} \\
\text { (calculated) }\end{array}$} & \multicolumn{2}{|c|}{ Dipole moment $\mu$} & \multirow{2}{*}{$\begin{array}{l}\text { Dipole } \\
\text { Angle }\end{array}$} \\
\hline & & $\varphi-$ & $\mathrm{Et}-$ & \\
\hline$-\mathrm{CH}_{3}$ & 6.15 & 0.4 & 0 & $180^{\circ}$ \\
\hline$-\mathrm{CH}_{2} \mathrm{CH}_{3}$ & 10.65 & 0.4 & 0 & $180^{\circ}$ \\
\hline$-\mathrm{OH}$ & 3.26 & 1.6 & 1.7 & 620 \\
\hline$-\mathrm{OCH}_{3}$ & 7.77 & 1.25 & 1.25 & $55^{\circ}$ \\
\hline$-\mathrm{SH}$ & 9.05 & 1.3 & 1.4 & $\sim 60^{\circ}$ \\
\hline$-\mathrm{NH}_{2}$ & 5.15 & 1.53 & 1.38 & $\begin{array}{l}100^{\circ} \\
1420\end{array}$ \\
\hline$-\mathrm{NH}_{3}{ }^{+}$ & 6.97 & - & - & - \\
\hline$-\mathrm{NO}_{2}$ & 6.42 & 3.98 & 3.3 & $0^{\circ}$ \\
\hline$-\mathrm{COOH}$ & 7.78 & 1.64 & 1.68 & $74^{\circ}$ \\
\hline$-\mathrm{COO}^{-}$ & 5.93 & - & - & - \\
\hline$-\mathrm{COCH}_{3}$ & 10.67 & 2.89 & 2.7 & $57^{\circ}$ \\
\hline$-\mathrm{CHO}$ & 6.17 & 2.76 & 2.5 & $55^{\circ}$ \\
\hline$-C N$ & 5.89 & 4.0 & 3.57 & $0^{\circ}$ \\
\hline
\end{tabular}

a The molar refractions were calculated from the bond refractions in Table 6-2 assuming that the group is bonded to a carbon atom. Thus to obtain the change in refraction upon substitution of these groups for a hydrogen atom, the refraction of the $\mathrm{C}-\mathrm{H}$ bond (1.65) must be subtracted from the values given in the table. The group moments for both phenyl and ethyl derivatives were obtained mainly from Smyth (1955, p. 253) as were the angles that the group dipoles form with the $\mathrm{C}$-group boud axis. The two figures given for the dipole angle of the amino compounds are for the aliphatic and aromatic sitnations respectively.

\section{Dipole-Induced Dipole Interactions}

A permanent dipole can also polarize a neighboring molecule. The potential energy of a polarizable molecule in an electrical field is $q=-\alpha_{0} F^{2} / 2$ (Eq. 6-25). The field strength of a permanent dipole is given by:

$$
F=\frac{\mu}{d^{3} D} \sqrt{1+3 \cos ^{2} \theta}
$$

where $\theta$ is defined in Fig. 6-3. The potential energy is thus:

$$
r=-\frac{\alpha_{0} \mu^{2}}{2 d^{6} D^{2}}\left[1+3 \cos ^{2} \theta\right]
$$

When the polarized molecule lies on the dipole axis, $q=-2 \alpha_{0} \mu^{2} / d^{6} D^{2}$, and when it lies on a lime perpendicular to the dipole and passing through 
the dipole center, $\varphi=-\mu_{0} \mu^{2} / 2 d^{6} D^{2}$. When the molecules are freely rotating one may take the average field of the dipole $\left(F=\sqrt{2} \mu / d^{3}\right)$, from which the arerage potential energy dne to the induced moment is $q=$ $-\alpha_{0} \mu^{2} / d^{6} D^{2}$. Two identical dipoles will mutually polarize each other and the potential energy will then be $\varphi=-2 \alpha_{0} \mu^{2} / d^{6} D^{2}$. The type equation $\varphi=-A \alpha_{0} \mu^{2} / d^{6} D^{2}$ may be written in terms of $\mathrm{kcal} / \mathrm{mole}$ :

$$
\begin{aligned}
& \varphi=-14.4 \mathrm{~A} \frac{\alpha_{0} \mu^{2}}{d^{6} D^{2}} \mathrm{kcal} / \mathrm{mole} \\
& \varphi=-5.71 \mathrm{~A} \frac{R_{0} \mu^{2}}{d^{6} D^{2}} \mathrm{kcal} / \mathrm{mole}
\end{aligned}
$$

when $\alpha_{0}$ is in units of $10^{-24} \mathrm{ml} /$ molecule, $R_{0}$ is in $\mathrm{ml} / \mathrm{mole}, \mu$ is in debyes, and $d$ is in $\AA$. Such energies are usually quite small unless the dipole is very near the molecule that is polarized. Two water molecules at a distance apart of $5 \AA$ would involve an energy due to mutual induction of only - $0.009 \mathrm{kcal}$ mole, which may be compared to the $-0.384 \mathrm{kcal} / \mathrm{mole}$ previously calculated for the potential energy of a water molecule at the same distance from an ion. Even at $3 \AA$ separation in a mutually fixed configuration the energy would be less than $0.1 \mathrm{kcal} / \mathrm{mole}$.

\section{Electrokinetic Interactions (Dispersion or London Forces)}

Neutral nonpolar molecules or groups attract one another despite the apparent lack of any charge localization. Such forces have been recognized for a long time and often are the major contribution to van der Waal's interaction. However, the theories of classic electrostatics were unable to explain such attraction and the nature of such forces was unknown until London in 1930 pointed out the relationship to optical dispersion and derived expressions for these interactions on a quantum mechanical basis (London, 1930, 1937). A symmetrical molecule will at any instant possess a dipole moment due to fluctuations in the relative positions of the nuclei and outer electrons; this instantaneous dipole will create a field that will polarize an adjacent molecule. Likewise, the second molecule will possess a fluctuating dipole which will polarize the first molecule. There will thus arise a coupling between the oscillations of the electrons of the two molecules so that the statistical distribution of electrons will continuously favor attraction. There is not a single dipole in a molecule but many oscillating clipoles; these dipoles of different moments must be integrated for each molecnle to arrive at the interaction energy. Since the London formulation has been used in estimating interactions in protein and enzyme systems, it will be presented first. following which some more recent suggestions for improvement will be considered. 
The potential energy of two neutral nonpolar molecules was given by London as:

$$
P=-\frac{3 \alpha_{1} \alpha_{2}\left(h v_{i}\right)_{1}\left(h v_{i}\right)_{2}}{2 d^{6}\left[\left(h v_{i}\right)_{1}+\left(h v_{i}\right)_{2}\right]}
$$

$\alpha$ is the molecular polarizability (for convenience we shall drop the subscript in $\left.\alpha_{0}\right) . h$ is Planck's constant $\left(6.624 \times 10^{-27}\right.$ erg-sec $), r_{i}$ is the frequency of the electrical oscillator responsible for the polarizability, and the subscripts 1 and 2 refer to the two molecules interacting. London simplified the equation by equating $h r_{i}$ to the ionization potential of the molecule. Thus Eq. 6-33 may be rewritten as:

$$
p=-\frac{3 \alpha_{1} \alpha_{2}}{2 d^{6}} \frac{I_{1} I_{2}}{I_{1}+I_{2}}
$$

where $I$ is the ionization potential. Since $\alpha=3 R / 4 \pi N$ :

$$
\varphi=-\frac{27 R_{1} R_{2}}{32 \pi^{2} N^{2} d^{6}} \frac{I_{1} I_{2}}{I_{1}+I_{2}}
$$

Since molar refractions and ionization potentials are experimental quantities, this equation may be used to calculate interaction energies for any specified distance between the molecules. In terms of kcal/mole:

$$
\begin{gathered}
\varphi=-1.5 \frac{\alpha_{1} \alpha_{2}}{d^{6}} \frac{I_{1} I_{2}}{I_{1}+I_{2}} \mathrm{kcal} / \mathrm{mole} \\
\varphi=-0.236 \frac{R_{1} R_{2}}{d^{6}} \frac{I_{1} I_{2}}{I_{1}+I_{2}} \mathrm{kcal} / \mathrm{mole}
\end{gathered}
$$

when $\alpha$ is in units of $10^{-24} \mathrm{ml} /$ molecule, $R$ is in $\mathrm{ml} / \mathrm{mole}, I$ is in kcal $/$ mole, and $d$ is in $\AA$. When $I$ is in electron volts (ev) each equation must be multiplied by 23.06. Pauling and Pressman (1945) assumed an average ionization energy of $14 \mathrm{ev}$ and applied the equation:

$$
\varphi=-38.1 \frac{R_{1} R_{2}}{d^{6}} \mathrm{kcal} / \mathrm{mole}
$$

to protein interactions.

The London treatment gives values for the interaction energy that are less than the experimental values. An improvement was made by Slater and Kirkwood (1931), who introduced the factor $Z$, the number of electrons in the onter shells of the molecule (the bonding and unbonded elec- 
tron pairs), and a new vibrational frequency $v_{e}$, this being dependent on the mass and charge of the electron and the polarizability of the molecule:

$$
p=-\frac{3 \alpha_{1} \alpha_{2}\left(Z_{1} Z_{2}\right)^{1 / 4}\left(h v_{e}\right)_{1}\left(h v_{e}\right)_{2}}{2 d^{6}\left[\left(h v_{e}\right)_{1}+\left(h v_{e}\right)_{2}\right]}
$$

which for the interaction between identical molecules reduces to:

$$
\varphi=-\frac{3 \alpha^{2} \sqrt{Z}\left(h v_{e}\right)}{4 d^{6}}
$$

The quantity $h w_{e}$ is no longer the ionization energy. Values of $v_{e}$ and $Z$ for some common molecules are given in Table 6-6. Actually this treatment still does not provide energy values sufficiently high and further improvement may be made by using another frequency ${ }^{\prime}{ }$ " which is obtained from dispersion data and is related to $v_{e}$ by the equation $v_{r}=\sqrt{s} v_{e}$ :

$$
\varphi=-\frac{3 \alpha_{1} \alpha_{2}\left(Z_{1} Z_{2}\right)^{1 / 4}\left(h v_{v}\right)_{1}\left(h v_{v}\right)_{2}}{2 d^{6}\left[\left(h v_{v}\right)_{1}+\left(h v_{v}\right)_{2}\right]}
$$

\begin{tabular}{|c|c|c|c|c|c|c|c|}
\hline Molecule & $\begin{array}{c}v_{e} \times 10^{-15} \\
\left(\sec ^{-1}\right)\end{array}$ & $\begin{array}{c}v_{v}^{\prime} \times 10^{15} \\
\left(\sec ^{-1}\right)\end{array}$ & $\begin{array}{c}h v_{v} \times 10^{12} \\
\quad(\mathrm{ergs})\end{array}$ & $Z$ & $s$ & $\begin{array}{c}\alpha_{0} \times 10^{24} \\
(\mathrm{ml})\end{array}$ & $\begin{array}{c}P_{0} \\
\text { ( } \mathrm{ml} \text { mole })\end{array}$ \\
\hline $\mathrm{H}_{2}$ & 2.82 & 3.52 & 23.3 & $\underline{2}$ & 1.56 & 0.802 & 2.04 \\
\hline $\mathrm{O}_{2}$ & 2.02 & 3.58 & 23.7 & 12 & 3.13 & 1.56 & 3.96 \\
\hline$N_{2}$ & 1.92 & 4.13 & $2 \pi .4$ & 10 & 4.64 & 1.73 & 4.40 \\
\hline $\mathrm{Cl}_{2}$ & 1.19 & 3.10 & 20.5 & 14 & 6.74 & 4.50 & 11.43 \\
\hline $\mathrm{CO}$ & 1.82 & 3.35 & 22.2 & 10 & 5.37 & 1.93 & 4.89 \\
\hline NO & 1.94 & 3.49 & 23.1 & 11 & 3.25 & 1.69 & 4.31 \\
\hline $\mathrm{H}_{2} \mathrm{O}$ & 2.10 & 3.27 & 21.6 & 8 & 2.42 & 1.44 & 3.67 \\
\hline $\mathrm{H}_{2} \mathrm{~S}$ & 1.32 & -2.79 & 18.5 & 8 & 4.45 & 3.64 & 9.25 \\
\hline $\mathrm{N}_{2} \mathrm{O}$ & 1.48 & 3.38 & 22.4 & 16 & 5.22 & 2.92 & 7.42 \\
\hline $\mathrm{CO}_{2}$ & 1.57 & 3.74 & 24.8 & 16 & 5.71 & 2.59 & 6.59 \\
\hline $\mathrm{NH}_{3}$ & 1.72 & 2.85 & 18.9 & 8 & 2.73 & 2.14 & 5.45 \\
\hline $\mathrm{HCN}$ & 1.60 & 3.06 & 20.3 & 10 & 3.65 & 2.49 & 6.32 \\
\hline $\mathrm{C}^{1} \mathrm{H}_{4}$ & 1.54 & 3.42 & 2.2 .6 & 8 & 4.83 & 2.70 & 6.85 \\
\hline $\mathrm{C}_{2} \mathrm{H}_{6}$ & 1.21 & 3.11 & 20.6 & 14 & 6.55 & 4.33 & 10.99 \\
\hline $\mathrm{C}_{6} \mathrm{H}_{6}$ & 0.803 & 2.54 & 16.8 & 30 & 10.03 & 9.89 & 25.11 \\
\hline $\mathrm{CH}_{3} \mathrm{C} N$ & 1.22 & 3.02 & 20.0 & 16 & 6.10 & 4.27 & 10.85 \\
\hline
\end{tabular}

TABLE $6-6$

Optical Coxstayts for simple Molecules a

a Taken in part from Noelwyn-Hughes (1957, p. 373) and includes only molecules of possible interest in biological interactions. 
Values of ${ }^{\prime}{ }^{\prime}$ are also given in Table 6-6. The use of this equation provides satisfactory values for the interaction energy in those cases of simple molecules that have been investigated. The factor $s$ relating $v$, and $v_{e}$ is the effective number of dispersion electrons and valnes are given in Table 6-6. Although Eq. 6-11 is admittedly only an approximation, it will be nsed in the present book for calculating dispersion energies. When possible. the values of $h r$, and $Z$ should be used. but in cases where the interaction involves many gromps or the groups interacting are not known (as is the situation usually in binding to enzymes). it is useful to derive an expression employing the most probable values for molecules of the type encountered in enzyme studies. The values of ${ }^{\prime}{ }_{2}$, for molecules composed of C, O, H, N and S are reasonably constant (Table 6-6) and an average value of $3.34 \mathrm{sec}^{-1}$ may be assumed leading to an average value for $h r$ of $22.1 \times 10^{-12}$ ergs molecule. Taking $Z$ to be approximately the same in each of the interacting molecules or groups:

$$
\begin{aligned}
& q=-238.6 \sqrt{Z} \frac{\alpha_{1} \alpha_{2}}{d^{6}} \mathrm{kcal} / \text { mole } \\
& q=-37.5 \sqrt{Z \frac{R_{1} R_{2}}{d^{6}}} \mathrm{kcal} / \text { mole }
\end{aligned}
$$

The difference between this result and Eq. 6-38 of Pauling and Pressman is mainly in the $\sqrt{ } Z$ factor. Since $Z$ is usually between 8 to 16 for interactting atoms, bonds and small groups, the energies of interaction will be about three to four fold greater than in the Pauling and Pressman expression. The potential energy due to dispersion forces between two methyl groups at a separation of $5 \AA$ from values in Table $6-6$ and $E q .6-41$ is -0.322 keal mole and from Eq. 6-13 is - 0.318 keal mole. Although single group interactions exhibit small energies due to dispersion forces over several $\AA$, the strong dependency on distance leads to apnreciable energies when the groups are in contact; also in larger molecules these dispersion forces may occur over an extensive surface of contact and the total energy may be fairly large.

The London expressions for the interaction of mutually induced dipoles are inaccurate for short distances where the atomic wave functions overlap and at long distances due to the phenomenon of retardation. It was pointed out by Casimir and Polder (1948) that the dispersion energy should fall off faster than predicted by London when the distance between the atoms or molecules becomes comparable to the wavelength conresponding to the electronic frequencies involverl (actually the chief absorption wavelength of the interacting atom). The effect of the movement of a charge on another charge is not instantaneous but progresses at the velocity of light. As long as the mutually oscillating dipoles are close together, the interaction 
between them occurs so rapidly that the movements are synchronized but as the separation becomes greater. a point is reached where the electrodynamic effects cannot pass between the atoms sufficiently rapidly and retardation of the mutual interaction occurs. This factor may be included as a correction factor in the London expressions:

$$
\varphi=f \varphi_{L}
$$

where $\varphi_{L}$ is the usual London interaction energy and $f$ is the retardation correction. At short distances, $f=1$. but as the separation increases $f$ becomes proportional to $1 / d$. From plots of $f$ made by Casimir and Polder, it may be seen that it equals about 0.5 when the separation is one-fifth the appropriate wavelength and about 0.25 when the separation is one-half the wavelength. At distances near or greater than the critical wavelength, $f$ thus varies as $1 / d^{7}$ for two interacting spheres, rather than as $1 / d^{6}$. It is interesting that the importance of retardation has been experimentally demonstrated recently by Derjaguin et al. (1956), direct measurements of the molecular forces between solids separated by narrow gaps (around $1000 \AA$ ) following the Casimir-Polder law rather than the London law. In the usual enzyme interactions, the distances are. of course, small enough so that retardation is probably umimportant, but there are biological situations. some perhaps connected with enzymes and the control of their activity, in which retardation effects might be significant. The technical advances through which such intermolecular forces can be measured directly make it likely that in the future it will be possible to determine dispersion interactions of importance to enzymologists and place the theoretical treatments on a firmer basis.

Experimental values for the dispersion energy are almost confined to situations in which the interacting molecules are identical and little accurate data are available for reactions between unlike molecules. For the latter one may use the expressions given above or recourse may be made to relations between the interaction constants for each substance. If the potential energy dne to dispersion forces between two unlike molecules is given by:

$$
\eta=-\frac{B_{12}}{d^{6}}
$$

the constant $B_{12}$ can be related to $B_{11}$ and $B_{22}$, the interaction constants for each substance, by making varions assumptions. Equation 6-41 taken in connection with:

$$
\begin{aligned}
& B_{11}=\frac{3 \alpha_{1}^{2} \sqrt{Z_{1}}\left(h v_{v}\right)_{1}}{4} \\
& B_{22}=\frac{3 \alpha_{2}{ }^{2} \sqrt{Z_{2}}\left(h v_{t}\right)_{2}}{4}
\end{aligned}
$$


if it is assumed that $\left(h v_{1}\right)_{1}=\left(h v^{\prime}\right)_{2}$, which from Table $6-6$ is seen to be not unreasonable as an approximation, leads to:

$$
B_{12}=\sqrt{B_{11} B_{22}}
$$

As an approximation, therefore, it is possible to evaluate $B_{12}$ and hence $\varphi$ from the interaction constants for the individual molecules.

The expression for the energy, $\varphi=-B / d^{6}$, is the first term and the most important one in a series:

$$
\varphi=-\frac{B}{d^{6}}-\frac{B^{\prime}}{d^{8}}-\frac{B^{\prime \prime}}{d^{10}}-\ldots
$$

Values for $B, B^{\prime}$, and $B^{\prime \prime}$ are calculated by Margenau (1939) for various molecules and, although they are probably too low, they provide an estimate of the contribution of these higher terms. For most molecules, $B^{\prime}$ is about $10 \%$ of $B$ and $B^{\prime \prime}$ is less than $1 \%$ of $B$, with respect to the contributed energy. Thus for greater accuracy, the energy may be considered to be at least $10 \%$ greater than calculated on the basis of the first $-B / d^{6}$ only.

It is frequently stated that these dispersion forces are nonspecific, and they are in the sense that they operate between all molecules, but it should be recognized that some specificity can be manifested. Since dispersion forces result from the interaction of fluctuating dipoles, the spatial orientation and fundamental oscillatory frequencies of these dipoles in the molecules will detcrmine the attraction to some extent. It is likely that identical macromolecules interact more strongly than unlike macromolecules (Yos et al., 1957). Some directional dependence also may oceur due to the anisotropy of the polarizability.

\section{Multipoles}

When the distribution of charged regions in a molecule shows a higher symmetry than is the case with dipoles and yet is not spherically symmetrical. an electrical field is induced around the molecule; this field is said to arise from quadrupoles, octupoles, or higher multipoles. One simple and probably common situation occurs when two dipoles are opposed:
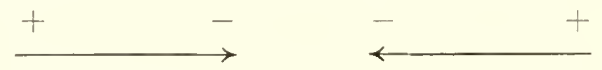

and another when the dipoles are oppositely aligned within the molecule:

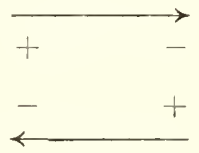


Such multipoles cannot be determined experimentally but our knowledge of molecular structure and boud moments make it likely that they occur frequently and play a subsidiary role in intermolecular interactions. They are no longer considered to be the explanation of attraction between neutral nondipolar molecules; rather they probably contribute to a minor degree in certain cases and are to be thought of in addition to the dispersion forces. Whereas the field strength of a dipole is proportional to $1 / d^{3}$, that of a quadrupole is proportional to $1 / d^{4}$ and that of an octupole to $1 / d^{5}$. Thus dipole-quadrupole and quadrupole-quadrupole interactions are of significance only when the molecules are quite close. There are also induced multipoles and the $B^{\prime}$ and $B^{\prime \prime}$ terms in Eq. 6-49 are due to such effects. Even in interactions between hydrogen atoms the contribution of quadrupole terms is appreciable in the total dispersion energy. An excellent discussion of quadrupoles and their interactions with ions, dipoles, and other quadrupoles has been given by Buckingham (1959). The quadrupole moments of some simple molecules are given in units of $10^{-26}$ esu: $\mathrm{H}_{2}$, $0.63 ; \mathrm{N}_{2}, \pm 1.5 ; \mathrm{CO},-3 ; \mathrm{H}_{2} \mathrm{O}, 2 ; \mathrm{NH}_{3}, 1 ;$ and $\mathrm{C}_{2} \mathrm{H}_{4}, \pm 1$.

\section{Short-Range Repulsive Interactions}

Having discussed the various attractive forces between molecules, it is now necessary to treat the repulsive forces that maintain a separation between atoms and molecules. Repulsion can, of course, arise from ion-ion, ion-dipole, and dipole-dipole interactions, and these are undoubtedly of importance in certain enzyme reactions. However, the purpose of the present section is to sketch the nature of the general nonspecific repulsive forces that occur whenever atoms or molecules come into contact. When negatively charged clouds of electron pairs, such as surround all molecules, begin to interpenetrate, repulsion occurs. It has not yet been possible to treat this problem in a satisfactory manner theoretically but the repulsion constants can often be evaluated. It will be satisfactory for our purpose to represent the energy of repulsion as $\phi=A / d^{a}$. We may thus write for the total potential energy of a pair of interacting structures:

$$
\varphi_{\text {total }}=q_{\text {repul sion }}+q_{\text {attraction }}=A / d^{a}-B / d^{b}
$$

when one type of attractive force is dominant; $A$ and $B$ are constants representing the magnitudes of repulsion and attraction energies and $a$ and $b$ indicate the dependence on the distance of separation. The exponent $a$ is usually high (often between 9 and 15) and is difficult to evaluate accurately; it also varies with the electronic nature of the atoms and molecules involved. The product $a b$ and the sum $a+b$ can be approximated by various experimental procedures (Moelwyn-Hughes, 1957, p. 314); usually one knows fairly accurately the value of $b$, and hence $a$ can be derived. 
However, the variation in results between the different methods and the variation between substances in a particular method make it impossible to assign a reliable value to $a$ in a specified case. For groups and molecules of the type here of interest, we shall arbitrarily assume $a=12$; this value is indicated by data from compressibility and heat of vaporization but it should be considered only as a reasonable approximation.

The importance of the repulsion constants for our purpose is in the determination of the equilibrium distances and energies of interacting molecules. The interaction energy of the greatest significance is that at the separation distance corresponding to the stable configuration, i.e., where attractive and repulsive forces are equal. This is illustrated on the energy-distance curve in Fig. 6-6: the equilibrim energy $\varphi_{e}$ is the

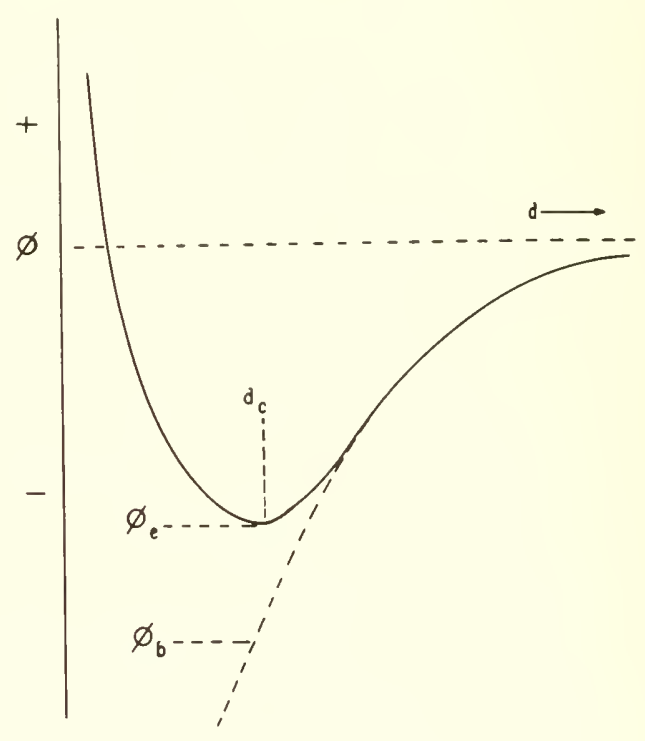

Fic. 6-6. Energy curve for a generalized interaction involving attraction and short-range repulsion. The dotted curve represents the energy from the attraction alone. $\varphi_{e}$ is the energy of interaction at the equilibrium distance $d_{6}$. 
minimum energy of interaction and this occurs at the equilibrimm distance $d_{e}$. The equilibrium values may be obtained from Eq. 6-50 by setting $(d \varphi, d d)_{d=d_{e}}=0$.

$$
\begin{gathered}
d_{e}=[a A / b B]^{1 /(a-b)} \\
\varphi_{e}=-\frac{B}{d_{e}^{b}}\left[1-\frac{b}{a}\right]
\end{gathered}
$$

\section{Repulsion Correction Factors for the Interaction Energy}

In Fig. 6-6 it may be seen that the equilibrium interaction energy is not that which would be calculated from the attraction equations previously given in this chapter; the value so calculated is indicated by $\varphi_{b}$. The relation between $\varphi_{e}$ and $\varphi_{b}$ may be given by:

$$
\varphi_{e}=r \varphi_{b}
$$

where $r$ may be termed the correction factor by which the calculated energy must be multiplied to give the equilibrium energy. Since:

$$
\varphi_{b}=-B / d_{e}^{b}
$$

the correction factor is given by:

$$
r=1-\frac{b}{a}
$$

The values for $r$ assuming that $a=12$ are given in Table 6-7 for the types of interaction previously discussed; the corrected energy equations are also presented for convenience in reference.

These correction factors are applicable only to equilibrium distances. At greater distances the necessary correction decreases rapidly. We shall, however, be mainly concerned with $q_{e}$ and for estimating this interaction energy the equations in Table 6- 7 will be used. Despite the admittedly approximate nature of these equations, they may be useful as a basis for more accurate formulations.

When two types of attraction must be considered:

$$
\varphi=\frac{A}{d^{a}}-\frac{B}{d^{b}}-\frac{C}{d^{0}}
$$

and the equilibrium energy is given by:

$$
\varphi_{e}=-\frac{B}{d_{e}^{b}}\left[1-\frac{b}{a}\right]-\frac{C}{d_{e}^{c}}\left[1-\frac{c}{a}\right]
$$

Thus $q_{e}$ may be obtained from the attraction energy $q_{b c}$ by multiplying each of the terms by the respective correction factor given in Table 6-7. 
TABLE 6-7

Correction Factors and Corrected Energy Equations a

\begin{tabular}{|c|c|c|c|c|}
\hline Interaetion & $r$ & Corrected equation & $\begin{array}{l}\text { Equation } \\
\text { corrected }\end{array}$ & $\begin{array}{l}\text { Present } \\
\text { number }\end{array}$ \\
\hline Ion-ion & 0.92 & $q_{e}=305 \frac{z_{1} z_{2}}{d_{e} D}$ & $6-5$ & $6-56$ \\
\hline Ion-dipole & 0.67 & $\begin{array}{l}\varphi_{e}=57.4 \frac{z \mu}{d_{e}^{2} D} \cos \theta \\
\varphi_{e}=-1804 \frac{z^{2} \mu^{2}}{d_{e}^{4} D^{2}}\end{array}$ & $6-12$ & $\begin{array}{l}6-57 \\
6-58\end{array}$ \\
\hline Dipole-dipole & $\begin{array}{l}0.75 \\
0.50\end{array}$ & $\begin{array}{l}\varphi_{e}=-10.8 \mathrm{~A} \frac{\mu_{a} \mu_{b}}{d_{e}{ }^{3} D} \\
\varphi_{e}=-175 \mathrm{~A} \frac{\mu_{a}{ }^{2} \mu_{b}{ }^{2}}{d_{e}{ }^{6} D^{2}}\end{array}$ & $6-18$ & $6-59$ \\
\hline $\begin{array}{l}\text { Ion-induced } \\
\text { dipole }\end{array}$ & 0.67 & $\begin{array}{l}\varphi_{e}=-111 \frac{\alpha_{0} z^{2}}{d_{e}{ }^{4} D^{2}} \\
\varphi_{e}=-44.1 \frac{R_{0} z^{2}}{d_{e}{ }^{4} D^{2}}\end{array}$ & $6-25$ & $6-61$ \\
\hline $\begin{array}{l}\text { Dipole-induced } \\
\text { dipole }\end{array}$ & 0.50 & $\begin{array}{l}\varphi_{e}=-7.2 A \frac{\alpha_{0} \mu^{2}}{d_{e}{ }^{6} D^{2}} \\
\varphi_{e}=-2.85 A \frac{R_{0} \mu^{2}}{d_{e}{ }^{6} D^{2}}\end{array}$ & $6-31$ & $6-63$ \\
\hline Dispersion & $\begin{array}{l}0.50 \\
0.50\end{array}$ & $\begin{array}{l}\varphi_{e}=-0.75 \frac{\alpha_{1} \alpha_{2}}{d_{e}{ }^{6}} \frac{I_{1} I_{2}}{I_{1}+I_{2}} \\
\varphi_{e}=-0.118 \frac{R_{1} R_{2}}{d_{e}{ }^{8}} \frac{I_{1} I_{2}}{I_{1}+I_{2}} \\
\varphi_{e}=-19.0 \frac{R_{1} R_{2}}{d_{e}{ }^{8}} \\
\varphi_{e}=-119 \sqrt{Z} \frac{\alpha_{1} \alpha_{2}}{d_{e}{ }^{8}} \\
\varphi_{e}=-18.7 \sqrt{\frac{7}{Z} \frac{R_{1} R_{2}}{d_{e}{ }^{6}}}\end{array}$ & $6-4 z$ & $6-67$ \\
\hline
\end{tabular}

a The interaction energy is given in keal/mole when $\mu$ is in debyes, $\alpha$ is in units of $10^{-24} \mathrm{ml} / \mathrm{molecule}, R$ is in $\mathrm{ml} / \mathrm{mole}, I$ is in keal/mole, and $d$ is in $\AA$. 


\section{Equilibrium Distances between Molecules}

It is at present impossible to determine the equilibrium distances between interacting molecules in solution. However, sufficient data from diffraction studies in solids are available to estimate distances of separation of molecules held together by only van der Waals' forces. One may define van der Waals' forces as including all the forces responsible for the constant $a$ in van der Waals' equation. These would comprise dipole-dipole, dipole-induced dipole, and dispersion interactions; when the molecules do not possess permanent dipoles, only dispersion forces are involved. Van der Waals' radii of atoms or molecules are half the distance separating two identical atoms or molecules at equilibrium in the solid state. Table 6-8 presents both van der Waals' and covalent radii for important atoms. Since

TABLE $6-8$

Covalext and van der $W_{\text {aAls' }}^{+}$Radil of Atoms ${ }^{a}$

\begin{tabular}{l|c|c|c}
\hline \multirow{2}{*}{ Atom } & \multicolumn{2}{|c|}{ Covalent radius } & Van der Waals' radius \\
& $\begin{array}{c}\text { Single bond } \\
(\AA)\end{array}$ & $\begin{array}{c}\text { Double bond } \\
(\AA)\end{array}$ & $(\AA)$ \\
\hline $\mathrm{H}$ & 0.30 & - & 1.2 \\
$\mathrm{C}$ & 0.77 & 0.67 & 1.57 \\
$\mathrm{~N}$ & 0.70 & 0.61 & 1.5 \\
$\mathrm{O}$ & 0.66 & 0.57 & 1.4 \\
$\mathrm{~S}$ & 1.04 & 0.95 & 1.85 \\
$\mathrm{P}$ & 1.10 & 1.00 & 1.9 \\
$\mathrm{As}$ & 1.21 & 1.11 & 2.0 \\
$\mathrm{~F}$ & 0.64 & 0.55 & 1.35 \\
$\mathrm{Cl}$ & 0.99 & 0.90 & 1.80 \\
$\mathrm{Br}$ & 1.14 & 1.05 & 1.95 \\
$\mathrm{I}$ & 1.33 & 1.25 & 2.15 \\
\hline
\end{tabular}

a The van der Waals' radius for the carbon atom has been estimated on the basis that van der Waals' radii are approximately $0.8 \AA$ greater than the single bond covalent radii for most atoms. The values for the van der Waals' radii are to be taken as approximate and appreciable variation may occur in different combinations of the atoms.

van der Waals' radii have been used to determine the interaction distance of molecules forming complexes in solution, it is necessary to enquire into the reliability of such a procedure. In the first place, the reasonable assumption has been made that van der Waals' radii are additive when different atoms interact; however, the accuracy of this has never been experimentally assessed. Secondly, there is little assurance that the internuclear distances occurring when two molecules interact in solution are the same as in a compact lattice structure; it might be anticipated that the 
interaction distances in solution would be somewhat greater. Lastly, in interactions of molecules with proteins, it is likely that all of the groups are not able to come into close contact, due to other types of forces or sterie factors; parts of the molecule would thus be at greater distances from the protein groups than predicted on the basis of equilibrium distances and would eontribute less than expeeted to the interaction energy. The van der Waals' radii should probably be taken as the valnes for the closest approach of atoms assuming the attractive forces here considered. Approximate van der Waals' radii for some important groups are given in Table 6-9. It is evident that these radii are not spherically symmetrical and that the radius used must depend on the assumed configuration of the interacting molecules (Fig. 6-7).

\section{TABLE 6-9}

Van der WaAls' Radil of Groups and Molecules ${ }^{a}$

\begin{tabular}{|c|c|c|}
\hline Group & $\begin{array}{l}\text { Vand der Waals' radius } \\
\qquad(\AA)\end{array}$ & Remarks \\
\hline$-\mathrm{OH}$ & $\begin{array}{l}1.40 \\
2.16\end{array}$ & $\begin{array}{l}\text { From } \mathrm{O} \text {, head-on } \\
\text { From } \mathrm{O} \text {, along } \mathrm{O}-\mathrm{H}\end{array}$ \\
\hline$-\mathrm{SH}$ & $\begin{array}{l}1.85 \\
2.54\end{array}$ & $\begin{array}{l}\text { From } \mathrm{s} \text {, head-on } \\
\text { From } \mathrm{S} \text {, along } \mathrm{S}-\mathrm{H}\end{array}$ \\
\hline$-\mathrm{CH}_{3}$ & $\begin{array}{l}1.57 \\
2.27\end{array}$ & $\begin{array}{l}\text { From } \mathrm{C} \text {, head-on } \\
\text { From } \mathrm{C} \text {, along } \mathrm{C}-\mathrm{H}\end{array}$ \\
\hline$-\mathrm{NH}_{2}$ & $\begin{array}{l}1.50 \\
2.20\end{array}$ & $\begin{array}{l}\text { From } \mathrm{N} \text {, head }- \text { and side-on } \\
\text { From } \mathrm{N} \text {, along } \mathrm{N}-\mathrm{H}\end{array}$ \\
\hline$-\mathrm{NH}_{3}{ }^{+}$ & $\begin{array}{l}1.70 \\
1.98\end{array}$ & $\begin{array}{l}\text { From N, head-on } \\
\text { From N, side-on }\end{array}$ \\
\hline$-\mathrm{COO}^{-}$ & $\begin{array}{l}1.40 \\
2.15 \\
2.36\end{array}$ & $\begin{array}{l}\text { From } \mathrm{N} \text {, along } \mathrm{N}-\mathrm{H} \\
\text { From } \mathrm{O} \\
\text { From } \mathrm{C} \text {, head-on } \\
\text { From } \mathrm{C} \text {, along } \mathrm{C}-\mathrm{O}\end{array}$ \\
\hline$-\mathrm{N}\left(\mathrm{C}^{\top} \mathrm{H}_{3}\right)_{3}{ }^{+}$ & $\begin{array}{l}3.17 \\
3.74\end{array}$ & $\begin{array}{l}\text { From } \mathrm{N} \text {, head-on } \\
\text { From } \mathrm{N} \text {, along } \mathrm{N}-\mathrm{CH}_{3}\end{array}$ \\
\hline$-\mathrm{CH}_{2}-$ & $2.10 \times 2.23$ & Half-thicknesses \\
\hline Benzene & 1.85 & Half-thickness \\
\hline Water & $\begin{array}{l}1.40 \\
2.16\end{array}$ & $\begin{array}{l}\text { From O, elosest approach } \\
\text { From O, along } \mathrm{O}-\mathrm{H}\end{array}$ \\
\hline
\end{tabular}

a These radii are estimated from the atomic van der Waals' radii in Table 6-8 and the group configurations shown in Fig. 6-7. They should be considered as minimal valnes and the interaction distances calculated from them must be taken as the measure of elosest approach, the actual interaction distances, for various reasons, often being greater. The designation "head-on "refer's to a direetion directly opposite to that of the bond between the group and the rest of the moleenle; " sirle-on" refers to the distance of elosest approach from the side of the group; " along X-Y " refers to the direction of the bond indicated. 

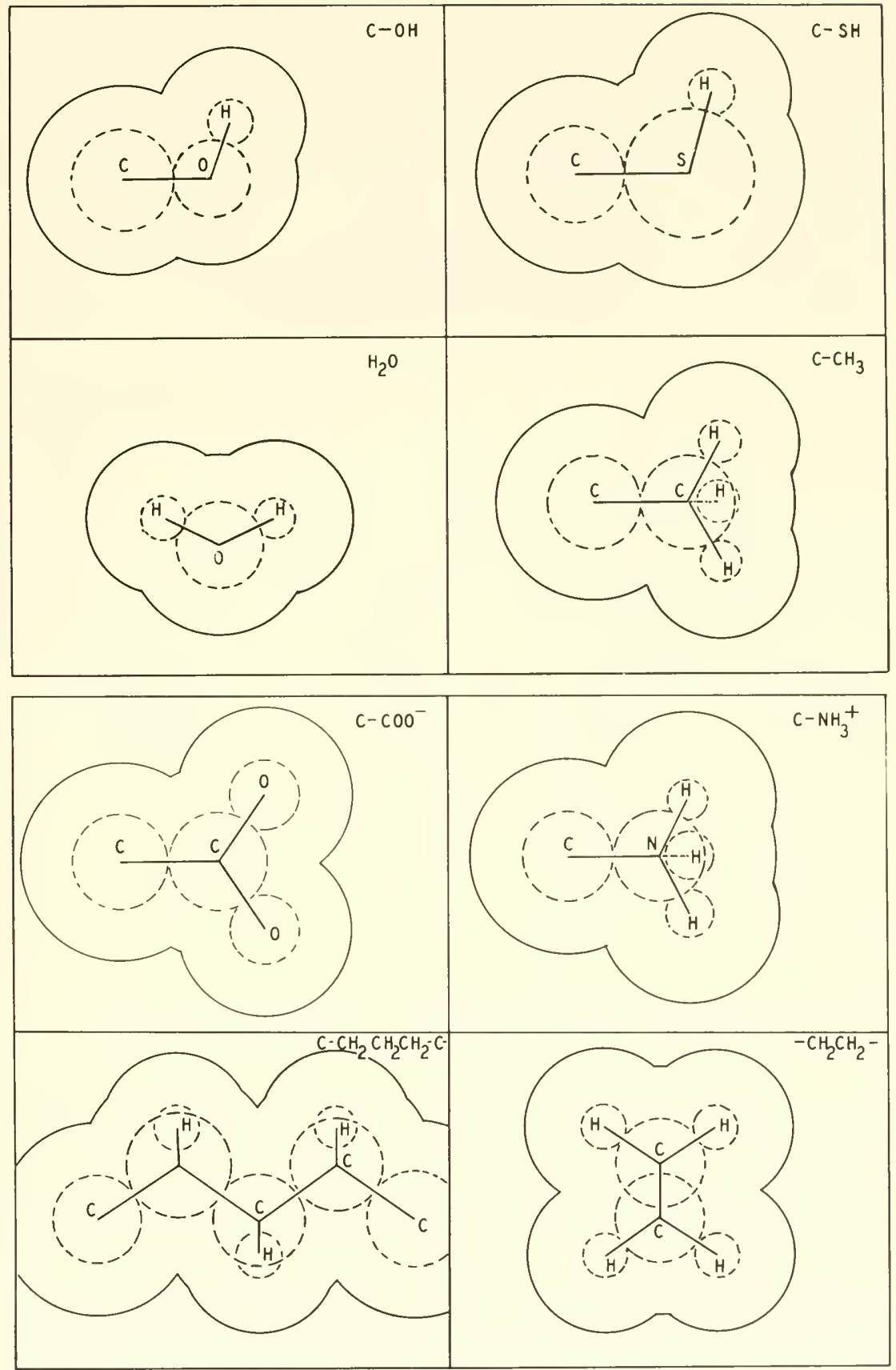

Fig. 6-7. Seale drawings of the ran der Waals' contours of some important groups and molecules to indieate as clearly as possible in two dimensions the configurations that must be considered in interactions. The dashed circles represent the covalent radii of the atoms. 
The interactions between four types of nonpolar molecules are shown in Fig. 6-8 and Table 6-10; the data and constants from which these curves were calculated are given in Tables 6-11,6-12, and 6-13. These molecules have equilibrium interaction distances that are very similar and yet the potential energies at these equilibrium distances are quite different, showing a twenty-four fold range from $\mathrm{H}_{2}$ to $\mathrm{CO}_{2}$. This demonstrates the de-

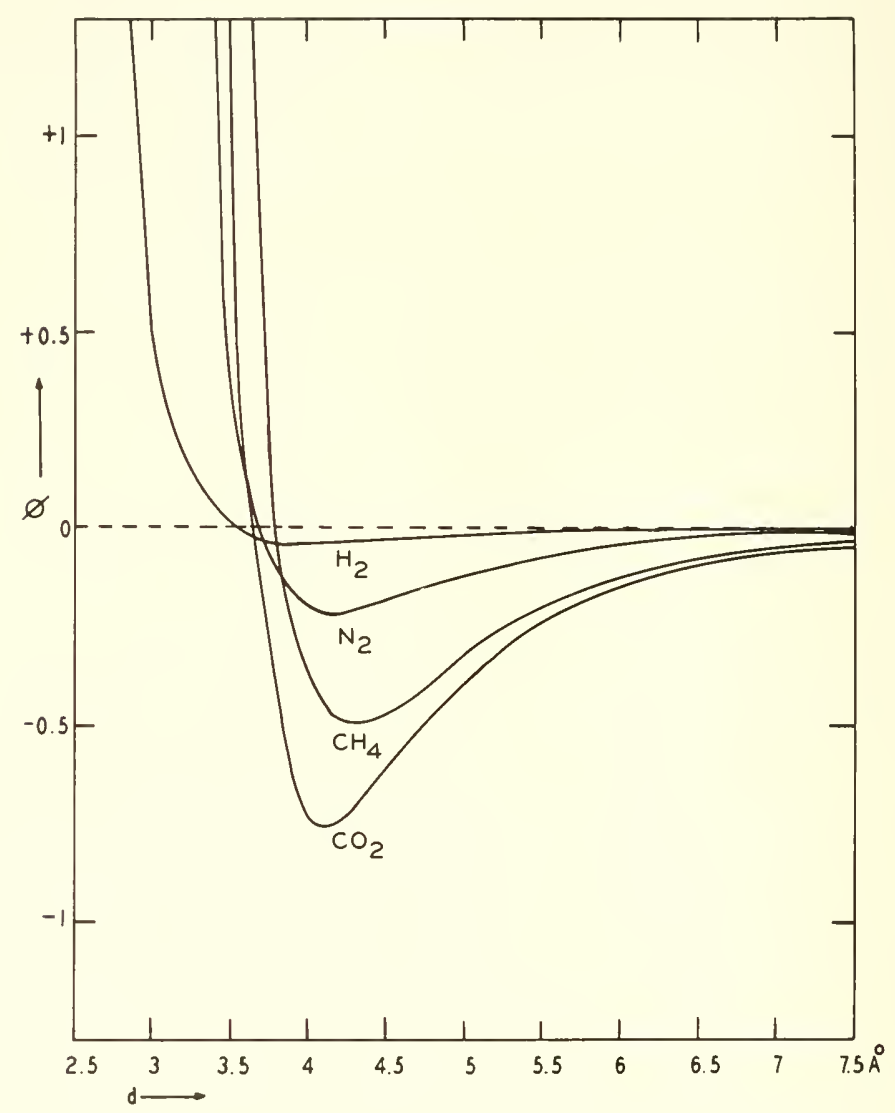

Fig. 6-8. Energy curves for the interactions between four types of nonpolar molecules. $\varphi$ is in keal/mole. See Tables 6-10 and 6-11.

pendence of the interaction energy on the number of polarizable electrons involved. It may be observed that the repulsion forces increase rapidly below the equilibrium distance; in each case the interaction energy is zero when the molecules are around $0.4 \AA$ closer than the equilibrium distance. The appreciable amount of energy resulting from dispersion interactions may also be remarked. 
TABLE $6-10$

Yariation of Potextel Exergy with Distaxce betweex Molecules

\begin{tabular}{c|c|cc|c}
\hline \multirow{2}{*}{$\begin{array}{c}\text { Distance }(d) \\
(\AA)\end{array}$} & \multicolumn{4}{|c}{ Interaction energy } \\
\cline { 2 - 4 } & \multicolumn{1}{|c|}{$\mathrm{H}_{2}$} & $\mathrm{~N}_{2}$ & $\mathrm{CO}_{2}$ & $\mathrm{CH}_{4}$ \\
\hline 2.0 & 103 & 1299 & 3860 & 4445 \\
2.5 & 6.42 & 82.9 & 244 & 288 \\
3.0 & 0.504 & 1.95 & 21.0 & 26.6 \\
3.5 & 0.002 & 0.44 & 0.97 & 2.20 \\
4.0 & -0.0312 & -0.205 & -0.75 & -0.375 \\
4.5 & -0.0219 & -0.183 & -0.612 & -0.472 \\
5.0 & -0.0132 & -0.117 & -0.383 & -0.313 \\
5.5 & -0.0070 & -0.0714 & -0.233 & -0.196 \\
6.0 & -0.0048 & -0.044 & -0.144 & -0.121 \\
7.0 & -0.0020 & -0.0184 & -0.059 & -0.050 \\
\hline
\end{tabular}

a The interaction energy is given in keal mole and was calculated from the following equations:

$$
\begin{aligned}
& \mathrm{H}_{2}: \quad \varphi=\frac{4.39 \times 10^{5}}{d^{12}}-\frac{2.347 \times 10^{2}}{d^{6}} \\
& \mathrm{~N}_{2}: \quad \varphi=\frac{5.46 \times 10^{6}}{d^{12}}-\frac{2.174 \times 10^{3}}{d^{6}} \\
& \mathrm{CO}_{2}: \quad q=\frac{1.625 \times 10^{7}}{d^{12}}-\frac{7.042 \times 10^{3}}{d^{6}} \\
& \mathrm{CH}_{4}: \quad \varphi=\frac{1.86 \times 10^{7}}{d^{12}}-\frac{6.077 \times 10^{3}}{d^{6}}
\end{aligned}
$$

\begin{tabular}{|c|c|c|c|c|c|c|}
\hline \multirow[b]{2}{*}{ Molecule } & \multicolumn{2}{|c|}{ From lattice constants } & \multicolumn{2}{|c|}{ Calculated } & \multirow{2}{*}{$\begin{array}{l}d_{e} \\
(\AA)\end{array}$} & \multirow{2}{*}{$\begin{array}{c}\varphi_{e} \\
\text { (kcal/mole) }\end{array}$} \\
\hline & $\begin{array}{c}A \\
\left(\text { keal-A } \AA^{12}\right)\end{array}$ & $\begin{array}{c}B \\
\left(\text { kcal- } \AA^{6}\right)\end{array}$ & $\begin{array}{c}A \\
\left(\text { kcal- }^{12}\right)\end{array}$ & $\begin{array}{c}B \\
\left(\text { kcal- } \AA^{6}\right)\end{array}$ & & \\
\hline $\mathrm{H}_{2}$ & $4.39 \times 10^{5}$ & 234.7 & $4.06 \times 10^{5}$ & 217.1 & 3.94 & -0.0314 \\
\hline $\mathrm{N}_{2}$ & $5.46 \times 10^{6}$ & 2174 & $5.72 \times 10^{6}$ & 2278 & 4.14 & -0.216 \\
\hline $\mathrm{C}^{\prime} \mathrm{H}_{4}$ & $1.86 \times 10^{7}$ & 6077 & $1.51 \times 10^{7}$ & 4923 & 4.28 & -0.495 \\
\hline $\mathrm{CO}_{2}$ & $1.63 \times 10^{7}$ & $704:$ & $1.48 \times 10^{7}$ & 6402 & 4.08 & -0.763 \\
\hline
\end{tabular}

TABLE 6-II

Exergy Coxstants for Noxpolar Molecules ${ }^{a}$

a The values of $A$ and $B$ obtained from diffraction data are given in the first two columns and are used for the calculation of the total interaction energy $p_{e}$. The calculated values for $A$ and $B$ were obtained from Eq. 6-4.? and the relationship $A=$ $B d_{e}{ }^{6} / 2$ when $a=12$ and $b=6$ and are shown to illustrate the accuracy of the approximate equation. 
TABLe 6-12

Coyalent Bond Lengths ${ }^{a}$

\begin{tabular}{|c|c|c|c|c|}
\hline & Bond & Length $(\AA)$ & Bond & Length $(\AA)$ \\
\hline $\mathrm{C}-\mathrm{C}$ & & 1.54 & C-I (aromatic) & 2.03 \\
\hline $\mathrm{C}-\mathrm{C}$ & (butadiene) & 1.47 & $\mathrm{C}-\mathrm{Hg}$ & 2.07 \\
\hline $\mathrm{C}-\mathrm{C}$ & (benzene) & 1.39 & $\mathrm{C}-\mathrm{As}$ & 1.94 \\
\hline $\mathrm{C}=\mathrm{C}$ & & 1.35 & & \\
\hline $\mathrm{C} \equiv \mathrm{C}$ & & 1.20 & $N-N$ & 1.46 \\
\hline $\mathrm{C}-\mathrm{H}$ & & 1.09 & $\mathrm{~N}-\mathrm{H}$ & 1.01 \\
\hline $\mathrm{C}-\mathrm{N}$ & & 1.47 & $\mathrm{~N}-\mathrm{O}$ & 1.21 \\
\hline $\mathrm{C}=\mathrm{N}$ & & 1.28 & $\mathrm{~N}=\mathrm{O}$ & 1.16 \\
\hline $\mathrm{C} \equiv \mathrm{N}$ & & 1.15 & & \\
\hline$C-O$ & (aliphatic) & 1.42 & $\mathrm{O}-\mathrm{O}$ & 1.32 \\
\hline $\mathrm{C}-\mathrm{O}$ & (carboxyl) & 1.32 & $\mathrm{O}=\mathrm{O} \quad$ (oxygen $)$ & 1.21 \\
\hline $\mathrm{C}-\mathrm{O}$ & (aromatic) & 1.36 & $\mathrm{O}-\mathrm{H}$ & 0.96 \\
\hline $\mathrm{C}-\mathrm{O}$ & (urea) & 1.26 & $\mathrm{O}-\mathrm{P} \quad$ (briclge) & 1.64 \\
\hline $\mathrm{C}=\mathrm{O}$ & & 1.22 & $\mathrm{O}-\mathrm{P} \quad$ (apical) & 1.39 \\
\hline $\mathrm{C}=\mathrm{O}$ & (quinone) & 1.16 & $\mathrm{O}-$ As (bridge) & 1.79 \\
\hline $\mathrm{C}-\mathrm{S}$ & & 1.81 & $\mathrm{O}-\mathrm{As}$ (apical) & 1.52 \\
\hline$C=S$ & & 1.55 & $\mathrm{O}-\mathrm{B}$ & 1.38 \\
\hline $\mathrm{C}-\mathrm{B}$ & & 1.56 & & \\
\hline $\mathrm{C}-\mathrm{F}$ & (aliphatic) & 1.36 & $S-S$ & 2.05 \\
\hline $\mathrm{C}-\mathrm{F}$ & (aromatic) & 1.31 & $\mathrm{~S}-\mathrm{H}$ & 1.34 \\
\hline $\mathrm{C}-\mathrm{Cl}$ & (aliphatic) & 1.76 & $\mathrm{~S}=\mathrm{O}$ & 1.43 \\
\hline $\mathrm{C}-\mathrm{Cl}$ & (aromatic) & 1.71 & & \\
\hline $\mathrm{C}-\mathrm{Br}$ & (aliphatic) & 1.93 & $\mathrm{~B}-\mathrm{H}$ & 1.20 \\
\hline $\mathrm{C}^{\prime}-\mathrm{Bl}^{\prime}$ & (aromatic) & 1.87 & $\mathrm{Hg}-\mathrm{Cl}$ & 2.26 \\
\hline$(-I$ & (aliphatic) & 2.13 & & \\
\hline
\end{tabular}

a These values have been selected from several sourees, mainly by averaging the bond lengths of a ppropriate compounds in the table in Wheland (1955, p. 696). There is a variation of $0.03-0.10 \mathrm{~A}$ for most bonds depending on the compound of which they are a part.

The actual distance between interacting molecules is not $d_{e}$ because the molecules possess kinetic energy. The average kinetic energy of harmonic oseillation is $R T\left(0.617 \mathrm{keal}\right.$ mole at $\left.37.5^{\circ}\right)$ and thus at equilibrium the total energy of the interacting molecules is $f_{e}+R T$, represented by the line $d_{1} d_{2}$ in Fig. 6-9. The molecules, due to this thermal kinetic energy, will oscillate between $d_{1}$ and $d_{2}$. Since this oscillation is asymmetric, the mean time-averaged distance between the molecules will he some value such as $d_{0}$, greater than $d_{\ell}$.

The kinetic energy $R T$ is, of course, the average energy and the distribution of energy is expressed by the Maxwell-Boltzmann law. Thus indi- 
vidual pairs of interacting molecules may have widely different linetic energies; a certain fraction will possess kinetic energy greater than $\varphi_{e}$ and the molecules will not be associated. The average energy of interaction, or the decrease in energy involved in bringing the molecules from infinite distance apart to their equilibrium separation, will however be $p_{e}$ since the total kinetic energy does not change. The average equilibrium distance between the molecules, $d_{0}$, will differ from $d_{e}$ by an amount dependent on

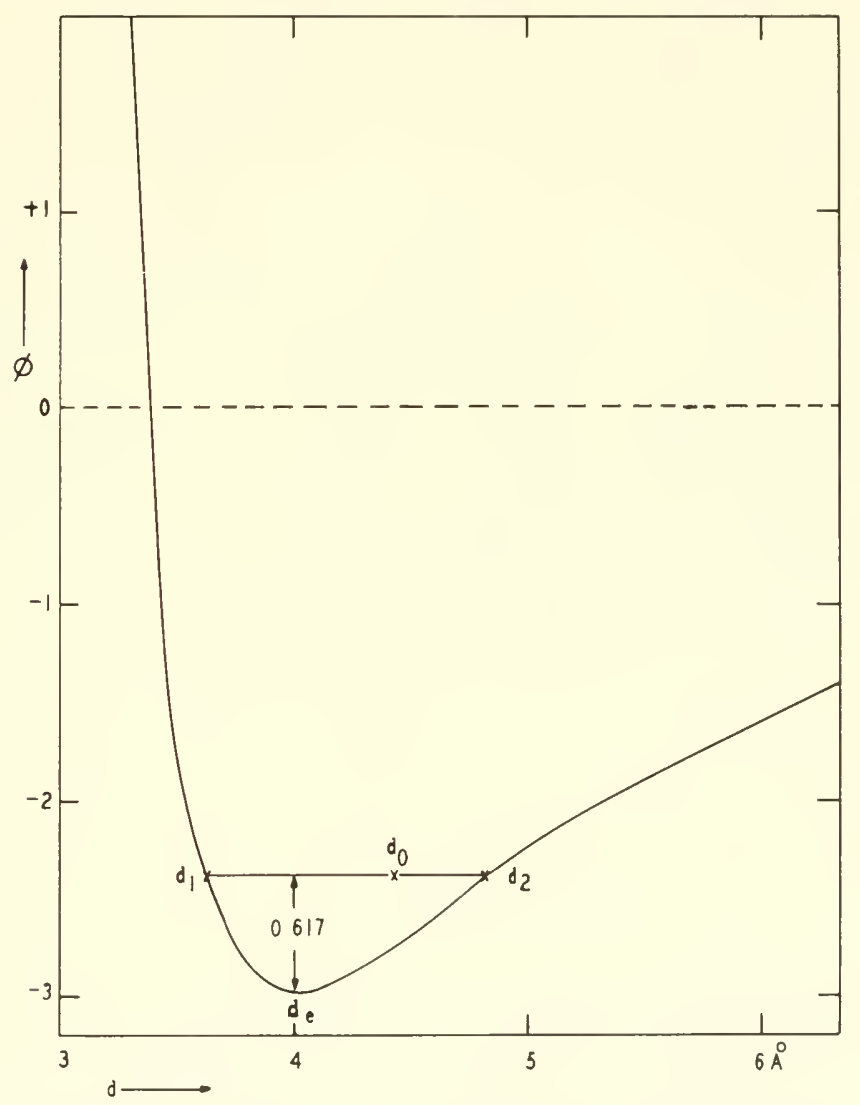

Fig. 6-9. Generalized energy curve to illustrate the mean timeaveraged distance between interacting groups due to thermal kinetic energy. For the interaction between an ion and a dipole obeving the equation:

$$
\varphi=10^{7} / d^{12}-57.4 / d^{2}
$$

the distances may be calculated to be: $d_{1}=3.63 \AA, d_{e}=4.00 \AA$, $d_{0}=4.43 \AA, d_{2}=4.82 \AA$, and $d_{2}-d_{1}=1.19 \AA$. 
the relative values of $a$ and $b$ (Eq. 6-50) and the potential energy $q_{e}$. An empirical approximation of this difference is:

$$
d_{0}=d_{e}+\left(0.5 / \sqrt{p_{e}-1}\right)
$$

based on the relative velocities of oscillatory movement at different points on the path $d_{1} d_{2}$. In general, $d_{0}-d_{e}$ will be between $0.2-0.4 \AA$ for most interactions of interest in enzymology.

TABLE 6-13

Bond Axgles

\begin{tabular}{|c|c|c|c|c|}
\hline & Bonds & Angle & Bonds & Angle \\
\hline $\mathrm{H}-\mathrm{C}-\mathrm{H}$ & (aliphatic) & $110.4^{\circ}$ & $\mathrm{O}-\mathrm{C}=\mathrm{O} \quad($ carboxyl $)$ & $122.4^{\circ}$ \\
\hline $\mathrm{H}-\mathrm{O}-\mathrm{H}$ & (water) & $104.5^{\circ}$ & $\mathrm{O}-\mathrm{C}^{-} \mathrm{O}^{-}$(carboxylate) & $125.8^{\circ}$ \\
\hline $\mathrm{C}-\mathrm{C}-\mathrm{C}$ & (aliphatic) & $111.7^{\circ}$ & $\mathrm{O}-\mathrm{C}-\mathrm{O} \quad$ (esters) & $116.4^{\circ}$ \\
\hline $\mathrm{H}-\mathrm{O}-\mathrm{C}$ & (alcohols) & $104.7^{\circ}$ & $N-N \equiv N$ & $180^{\circ}$ \\
\hline $\mathrm{O}-\mathrm{C}-\mathrm{C}$ & (alcohols) & $110.0^{\circ}$ & $\mathrm{C}-\mathrm{C} \equiv \mathrm{N}$ & $180^{\circ}$ \\
\hline $\mathrm{H}-\mathrm{S}-\mathrm{C}$ & (mercaptals) & $100.3^{\circ}$ & $\mathrm{N}-\mathrm{C}=\mathrm{O}$ & $123^{\circ}$ \\
\hline $\mathrm{S}-\mathrm{C}-\mathrm{C}$ & (mercaptals) & $113.0^{\circ}$ & $\mathrm{O}-\mathrm{N}-\mathrm{O} \quad($ nitro $)$ & $124.4^{\circ}$ \\
\hline $\mathrm{C}-\mathrm{O}-\mathrm{C}:$ & (ethers) & $109.5^{\circ}$ & $\mathrm{C}-\mathrm{Hg}-\mathrm{Cl}$ & $180^{\circ}$ \\
\hline $\mathrm{H}-\mathrm{C}=\mathrm{O}$ & (alclehydes) & $119.3^{\circ}$ & $\mathrm{C}-\mathrm{As}-\mathrm{Cl}$ & $100^{\circ}$ \\
\hline $\mathrm{C}-\mathrm{C}=\mathrm{O}$ & (ketones) & $122.7^{\circ}$ & As $-\mathrm{C}-\mathrm{C}$ & $122^{\circ}$ \\
\hline
\end{tabular}

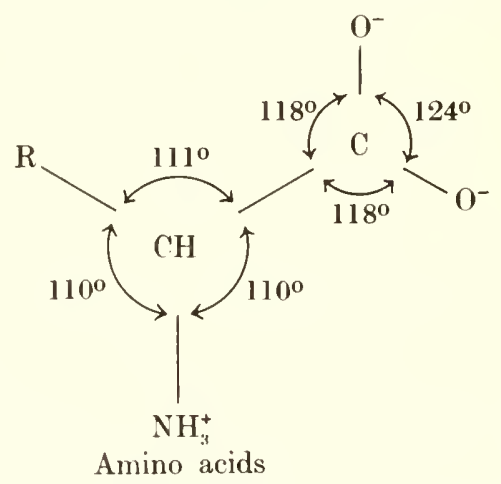

Another approach to this problem is to assume that a molecule creates a cavity, due to the intermal pressure arising from its thermal motion, the radius of this cavity being greater than the molecular radius. Equating this outward pressure with the electrical pressure. which is related to the energy of charging of an ion in the cavity and is directed inward, the cavity radii for ions in water have been estimated (Benson et al., 1957). Figure 6-10 presents the relation between ion size and cavity size; for most 
common ions the cavity radius is about $30-35 \%$ greater than the ionic radins. The accepted values of ionic radii and van der Waals' radii, determined by diffraction studies, include this thermal increase in interaction distance; thus the true radii are somewhat smaller. However, the cavity size in the solid state may not be the same as in the liquid state or in solution.

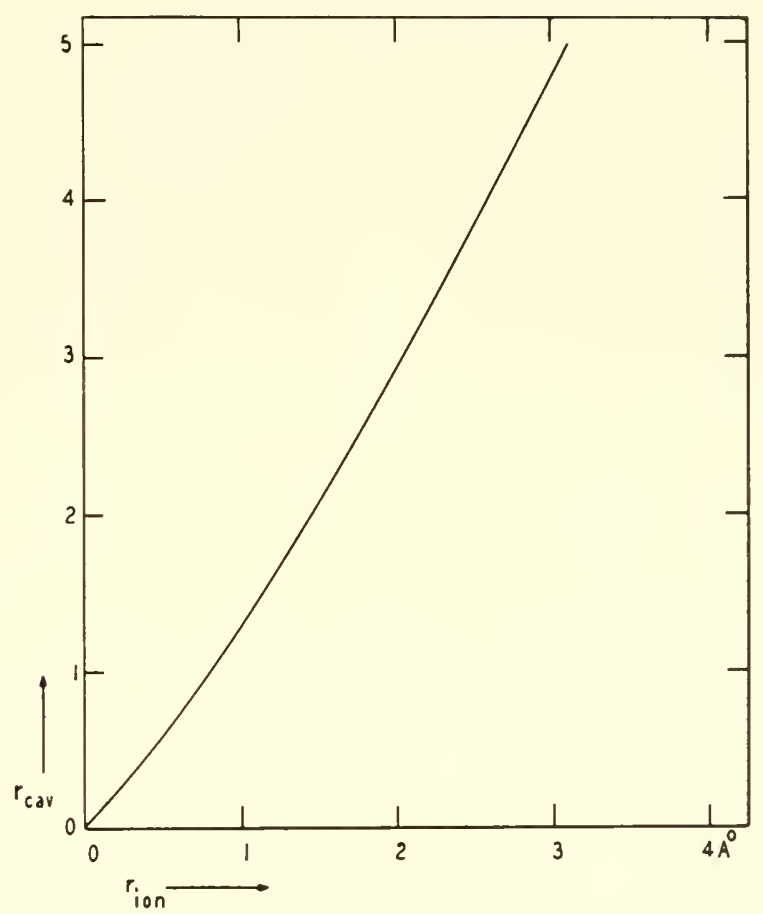

FIG. 6-10. Relationship between the thermal cavity size and the ionic size in water.

\section{Orientation Factors in Repulsion Forces}

The orientation of groups or molecules relative to one another is an important factor in determining the magnitude of the repulsion energy, just as it is a factor to be considered in evaluating the attraction energy. For example, we have seen that two dipoles will attract one another with forces dependent on their relative orientations; likewise the repulsion will vary with the orientation. Hence the equilibrium energy $\varphi_{e}$ and distance $d_{e}$ will depend on orientation. The importance of this factor is indicated in the results of Mason and Hirschfelder (1957) for the interaction of hydrogen molecules (Table 6-14), where it is seen that the repulsion energy is more sensitive than the attraction (dispersion) energy to orientation. Although 
more complex situations have not been treated, it is obvious that repulsion forces will play a large role in the orientation of two interacting molecules in the position of maximal stability.

TABLE 6-14

Effect of Orientation on Interaction Energy in $\mathrm{H}_{2}{ }^{a}$

\begin{tabular}{|c|c|c|c|c|c|c|c|c|c|}
\hline \multirow{2}{*}{\multicolumn{2}{|c|}{ Configuration }} & \multicolumn{3}{|c|}{ Distance $=2.27 \AA$} & \multicolumn{3}{|c|}{ Distance $=-3.18 \mathrm{~A}$} & \multirow{2}{*}{$d_{e}$} & \multirow{2}{*}{$\varphi_{e}$} \\
\hline & & $\varphi_{a}$ & $\varphi_{b}$ & $f_{t}$ & $f_{a}$ & $\varphi_{b}$ & $q_{t}$ & & \\
\hline -0 & 6 & 13.54 & -2.04 & 11.50 & 0.417 & -0.230 & 0.187 & 3.86 & -0.035 \\
\hline \multirow[t]{3}{*}{0} & & 6.64 & -1.87 & 4.77 & $0.2 \cdot 2 \cdot 2$ & -0.211 & 0.011 & 3.58 & -0.057 \\
\hline & & 3.68 & -1.63 & 2.05 & 0.143 & -0.175 & -0.032 & 3.40 & -0.050 \\
\hline & & 3.43 & -1.61 & 1.82 & 0.131 & -0.172 & -0.041 & 3.38 & -0.054 \\
\hline
\end{tabular}

a Data from Mason and Hirschfelder (1957). The values of $d_{e}$ and $q_{e}$ were calculated from energy-distance curves. The treatment was based on the eonsideration of interacting and overlapping orbitals and the evaluation of the various exehange integrals was involved. It is an elegant, but laborious. method that at present eannot be applied to more complex interactions.

\section{RELATIVE CONTRIBUTIONS OF DIFFERENT INTERACTION FORCES}

Interactions between groups or molecules usually involve more than a single attractive force and occasionally more than one repulsive force. It is now necessary to consider in general terms contributions made by different attractive forces to the total equilibrium potential energy. In calculating either interaction energies or equilibrim distances from experimentally determined energies, it is necessary to take into account all the forms of attraction that contribute significantly. The contributions will depend on the distance between the interacting groups or molecules because the respective potential energies vary with different powers of the distance. This is illustrated in Table 6-15 where the relative contributions are calculated for two distances of separation. There is no change for the dipoledipole interaction because all energy terms involved contain $1 / d^{6}$; however, for the other three examples it may be observed that one energy term is more likely to be dominant at greater distances. Dispersion energies par- 
CONTRIBUTIONS OF DIFFERENT INTERACTION FORCES

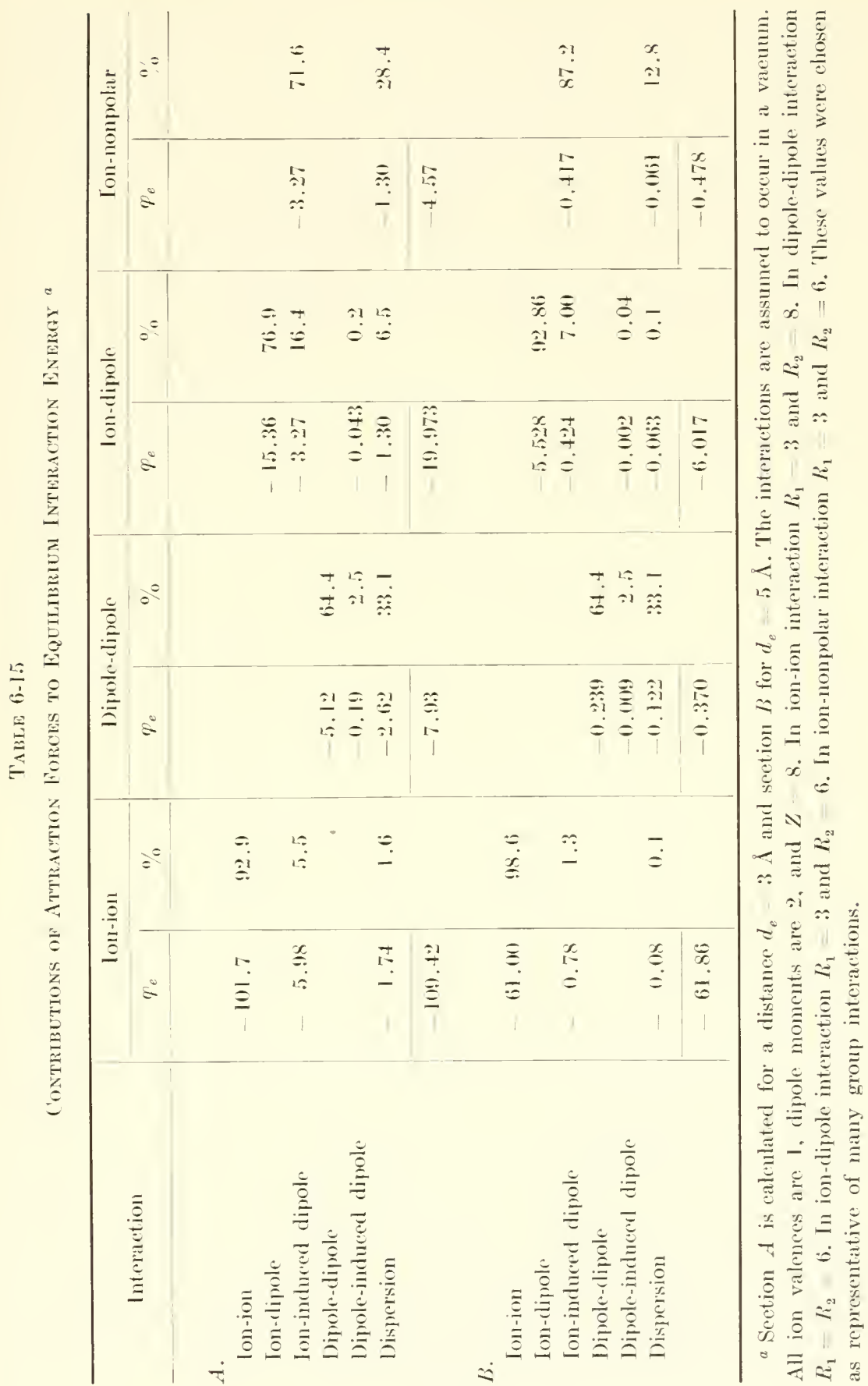


ticularly become relatively mimportant at distances greater than $5 \AA$, when other forces are operative and the surfaces of interaction are not extensive. At the usual distances of separation at equilibrium, dispersion forces generally contribute significantly. It is also instructive to compare the magnitudes of the total potential energy for the four different interactions and the way in which they each vary with distance. However, it should be noted that these relations are changed somewhat when interaction in a dielectric occurs (see page 248).

In dipole-dipole interactions, the relative contributions of induction and dispersion forces will depend on the magnitude of the dipole moment and the polarizability. Table 6-16 gives the estimated contributions for several

TABLE 6.16

Interaction Exergy Contributions for Dipolar Molecules ${ }^{a}$

\begin{tabular}{l|c|c|c|c}
\hline \multirow{2}{*}{ Molecule } & $\begin{array}{c}\text { Dipole } \\
\text { moment }\end{array}$ & $\begin{array}{c}\text { Dipole-dipole } \\
\text { (alignment) }\end{array}$ & $\begin{array}{c}\text { Dipole-induced } \\
\text { dipole }\end{array}$ & Dispersion \\
\hline & 0.12 & 0.005 & 0.085 & 99.91 \\
\hline $\mathrm{CO}$ & 0.38 & 0.09 & 0.43 & 99.48 \\
$\mathrm{HI}$ & 0.78 & 3.33 & 2.18 & 94.49 \\
$\mathrm{HBr}$ & 1.03 & 14.4 & 4.2 & 81.4 \\
$\mathrm{HCl}$ & 1.50 & 44.9 & 5.3 & 49.8 \\
$\mathrm{NH}_{3}$ & 1.62 & 63.4 & 14.4 & 22.2 \\
$\mathrm{CH}_{3} \mathrm{OH}$ & 1.66 & 55.0 & 12.6 & 32.4 \\
$\mathrm{C}_{2} \mathrm{H}_{5} \mathrm{OH}$ & 1.83 & 77.0 & 4.0 & 19.0 \\
$\mathrm{H}_{2} \mathrm{O}$ & & & & \\
\hline
\end{tabular}

a Calculated from Margenau (1939) and Syrkin and Dyatkina (1950, p. 266).

types of molecule. The contribution from alignment of dipoles increases with the dipole moment, as does the induction effect, but the dispersion contribution becomes relatively less although it may be in absolute magnitude somewhat greater. For nompolar molecules the dispersion energy may be small but it is always the sole contribution to the total potential energy. The greater dispersion contribution in ethanol compared with methanol is due, of course, to the greater polarizability of the former, i.e., the increase in the nompolar part of the molecule.

A great deal of theoretical and experimental material applicable to enzyme interactions may be found in the literature of colloid chemistry and heterogeneous catalysis. One of the clearest and most comprehensive reviews of intermolecular forces is that of de Boer (1950). He considers particularly interactions of molecules with ionic or higly polarizable surfaces and estimates the contibutions of the various forces involved. Enzyme surfaces may be occasionally rather diffuse ionic surfaces because of the 
many ionic groups distributed over the molecule and thus such considerations are not as irrelevant as might appear at first glance. The induction of the adsorbed molecule is shown to be an important contribution in interactions with an ionic surface and the same probably holds for many molecules bound to enzymes.

The importance of dispersion energies in ion-ion interactions is evident in the binding of the $-\mathrm{N}\left(\mathrm{CH}_{3}\right)_{3}{ }^{+}$to the active site on acetylcholinesterase. Although an important contribution is made by the interaction of this cationic group with an anionic group on the protein, the contribution of the methyl groups is indicated by the decrease in binding energy as the methyl groups are removed from the nitrogen atom. Cations such as $\mathrm{K}^{+}, \mathrm{Na}^{+}$, or $\mathrm{NH}_{4}^{+}$also do not interact with the enzyme anionic site as readily as the $-\mathrm{N}\left(\mathrm{CH}_{3}\right)_{3}{ }^{+}$group, whereas on the basis of purely coulombic interaction one might expect the smaller cations to be bound more tightly due to their smaller radius. Hydration of these ionic gromps introduces another factor and a more detailed discussion of such interactions will be left until the role of the solvent is considered.

\section{DIELECTRIC CONSTANT}

The ratio of the force between two charged particles in a vacuum to the force between them when they are immersed in some medium is the dielectric constant of that medinm. It is also the ratio of the potential energies in a vacuum and the medium (Eqs. 6-2 and 6-4). Heretofore interactions in a vacuum have been assumed and it is now necessary to consider the situation when the interacting molecules are dissolved in water. The decrease in force or potential energy between two charges when placed in a medium is due to the polarization of the molecules of the medium. In solvents composed of dipolar molecules the most important factor is the orientation or alignment of the solvent molecules in the electrical field of the charges. An ion or charged group will orient the water molecules around it and this alters the electrical field due to the charge. The bulk dielectric constant $D$ of water at $37.5^{\circ}$ is 74.1 . Charges separated by large distances (above at east $20 \AA$ ) will have their potential energy reduced by this factor. However, as the charges approach one another, the effective dielectric constant decreases until it reaches a low value when the charged species are in contact.

The dielectric constant for water varies with temperature according to the equation:

$$
D=D_{0} e^{-T / \theta}
$$

where $D_{0}=305.7$ and $\theta=219$ (Gurney, 1953). Thus $D=87.9$ at $0^{\circ}$, 78.2 at $25^{\circ}$, and 55.7 at $100^{\circ}$. In this book, body temperature will be assumed in the formulation but when experiments are performed at other temperatures the appropriate correction should be made. 
One of the most important and most difficult problems is the evaluation of the dielectric constant applicable to a particular separation of charges. A reliable estimation of intermolecular forces, particularly those involving ionic groups, can be made only with an accurate assignment of the dielectric constant. At the present time this is impossible but an approach to at least reasonable values has been made.

\section{Variation of Dielectric Constant with Distance}

The dielectric constant of the medium near an ion may be estimated theoretically by assuming certain models of water orientation in the electrical field of the ion or evaluated indirectly from experimental dissociation constants of dicarboxylic acids (the electrostatic effect of one carboxylate ion on the $\mathrm{p} K_{a}$ of the other acilic group being dependent on the dielectric constant). Calculations of the former type often involve the assumption of one rigidly oriented layer of water molecules around the ion and a honogeneous dielectric outside this. Since it is likely that there are partially oriented water molecules beyond the first bound layer and since the structure of the hydrated ion is not certain, it is felt that this procedure is not satisfactory. Figure 6-11 presents the variation of the dielectric

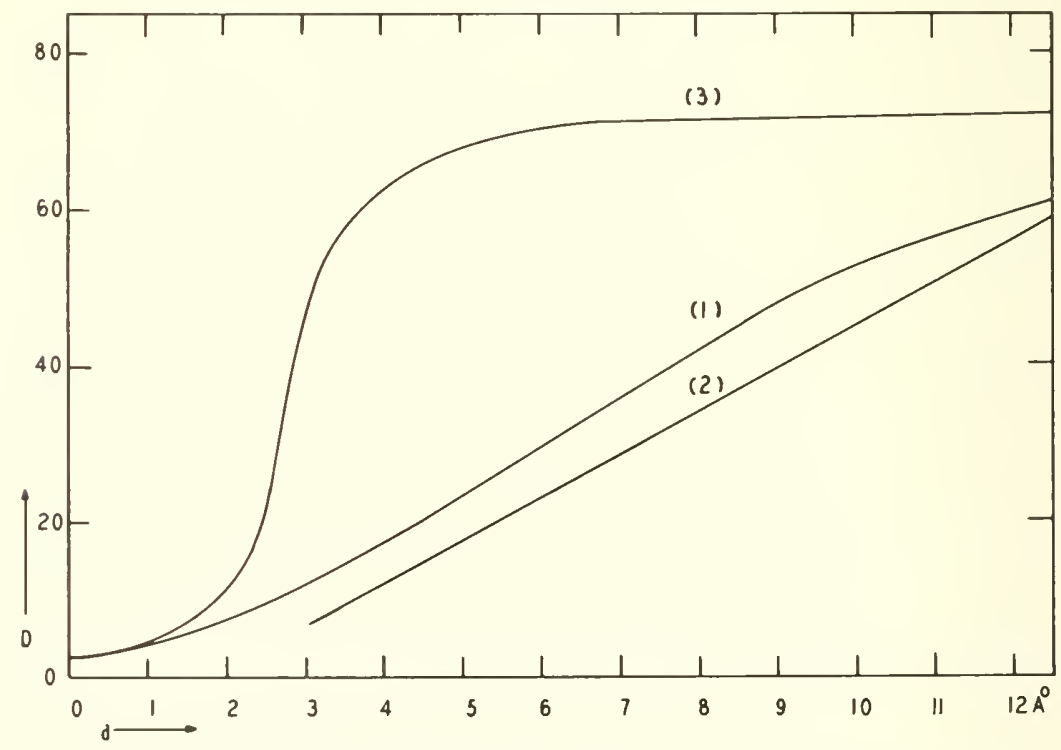

FIG. 6-11. Variation of the dielectric constant with the distance from a univalent ion at $37.5^{\circ}$ as calculated by different methods. Curve (1) from Conway et al. (1951), curve (2) from Schwarzenbach (1936), and curve (3) from Grahame

(1950) and Ritson and Hasted (1948). 
constant with distance from a univalent ion as calculated by different mothods. Curve (1) shows the most reasonable values as determined by Conway et al. (1951) from several previons estimations. This curve can be quite accurately expressed by the equation:

$$
D=6 d-7
$$

for distances between 3-10 $\AA$ and this relationship will be adopted as a basis for calculation of interaction energies between ions. Curve (2) shows the familiar Schwarzenbach (1936) variation as used by Pressman et al. (1946) and others; this is a plot of $D=6 d-11$ corrected to $37.5^{\circ}$. Curve (3) shows typical results of the theoretical procedure (Ritson and Hasted, 1948; Grahame, 1950) which minimizes the effects of the ion field on the dielectric constant beyond the first bound layer of water.

Several points in connection with the evaluation of $D$ may be brought up. (a) For interactions involving di- and trivalent ions, lower values of $D$ should be used inasmuch as the field strength of such ions is greater. Figure 6-12 shows the variation of $D$ with field strength, curve (1) giving values in a vacuum and curve (2) the actual values in the dielectric. As an approximation, $D$ should be reduced by $10-15$ for divalent ions and by

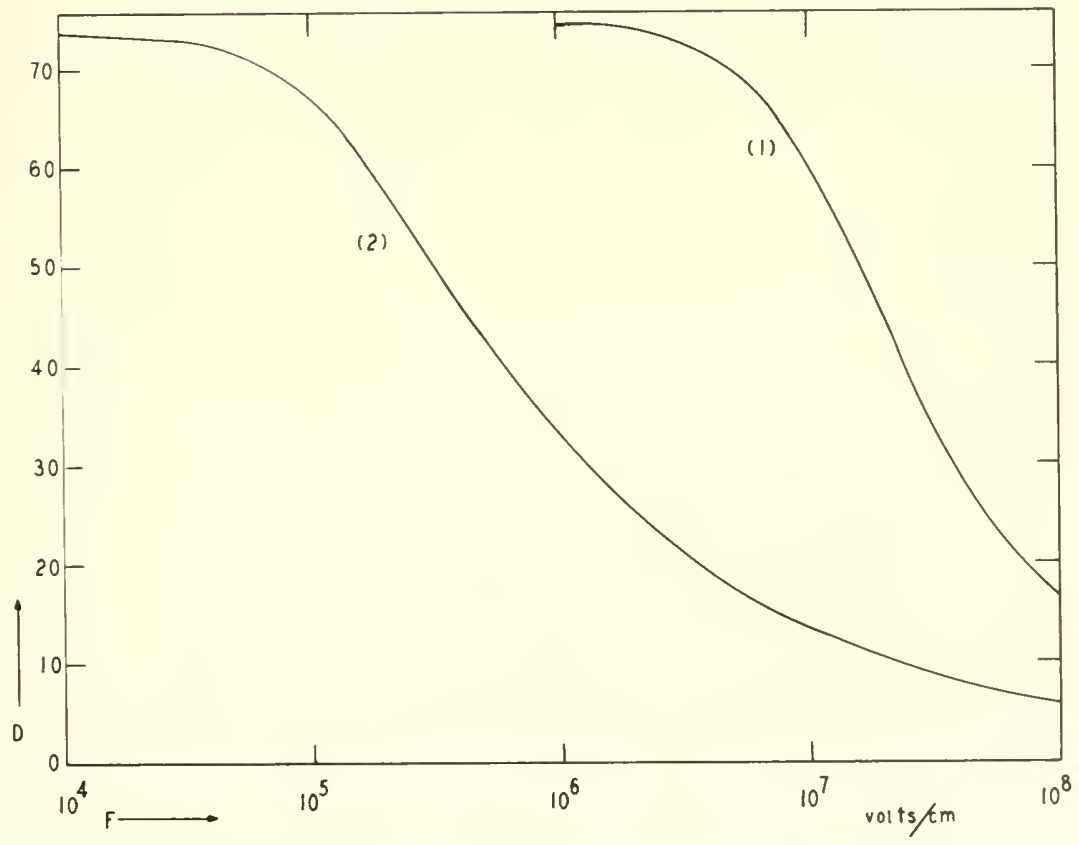

FIG. 6-12. Variation of the dielectric constant with the electrical field strength, curve (2) giving the actual values in the dielectric water. 
20-25 for trivalent ions. (b) The above treatment assumes that ions are point charges and takes no account of ionic radii. It is probable that the greater the ionic size, the smaller will be the required value of $D$. There are no means for estimating the magnitude of this correction but it is probably small. (c) Most treatments assume a mimimum value for $D$ of around 3 at very small distances. This is based on the dielectric constants of solids at low temperatures (ice, 3.1 ; dodecane, 2.2; hydrogen sulfide, 3 ; hydrogen chloride, 3.1; octadecanol, 3; monopalmitin, 2.4; isobutyl chloride, 2.8; etc.), these values being related to the electronic polarizabilities since the molecules are rigidly fixed and orientation polarization does not occur. However, the validity of this assumption may be questioned. When ions are in contact (that is, with no water molecules between them), the presence of water dipoles oriented around the ions outside the area of contact will reduce the interaction; however, if the ionic groups are bonded to molecules it may be that water is excluded from direct interaction with the ions and this reduction does not occur. The problem of hydration will be considered in more detail in the next section.

\section{Variation of Potential Energy with Distance in Ion-Ion Interactions}

Since the dielectric constant varies with interionic distance, the potential energy of two ions will no longer be inversely proportional to the distance as in a vacuum. Combining Eqs. 6-5 and 6-74 we have:

$$
\varphi=332 \frac{z_{1} z_{2}}{d(6 d-7)} \mathrm{kcal} / \mathrm{mole}
$$

Figure 6-13 is an energy-distance diagram for the interaction of two univalent ions, assuming a reasonable repulsion constant. The effect of the water on the interaction is given by the change from curve $\mathrm{B}$ to curve A; the equilibrium potential energy is decreased markedly and the equilibrium distance is increased by almost $0.5 \AA$. The energy involved in displacement of bound water was ignored in the calculation of these curves.

\section{Dielectric Constant for Different Types of Interaction}

It is probable that the estimations of $D$ given above apply to any type of interaction involving an ion, including ion-dipole and ion-induced dipole. The dielectric constant does not enter into the dispersion energy because the oscillating fields are both small and of such a high frequency that no effect on the solvent molecules would be expected. A problem, however, arises in dipole-dipole interactions. The electrical field around a dipole is not negligible. A dipole of moment $\mu=1.5$ possesses a mean electrical field strength in a vacuum of $22 \times 10^{6}$ volts $/ \mathrm{cm}$ at $3 \AA, 9.8 \times 10^{6}$ volts $/ \mathrm{cm}$ at 
$4 \AA$, and $5.0 \times 10^{6}$ volts $/ \mathrm{cm}$ at $5 \AA$. Using curve (1) of Fig. 6 - 12 it is seen that beyond $5 \AA$ distance no significant effect on $D$ would be expected but at shorter distances from the dipole, $D$ would be reduced. It is likely that in most enzyme dipole-dipole interactions of importance, the dipoles are

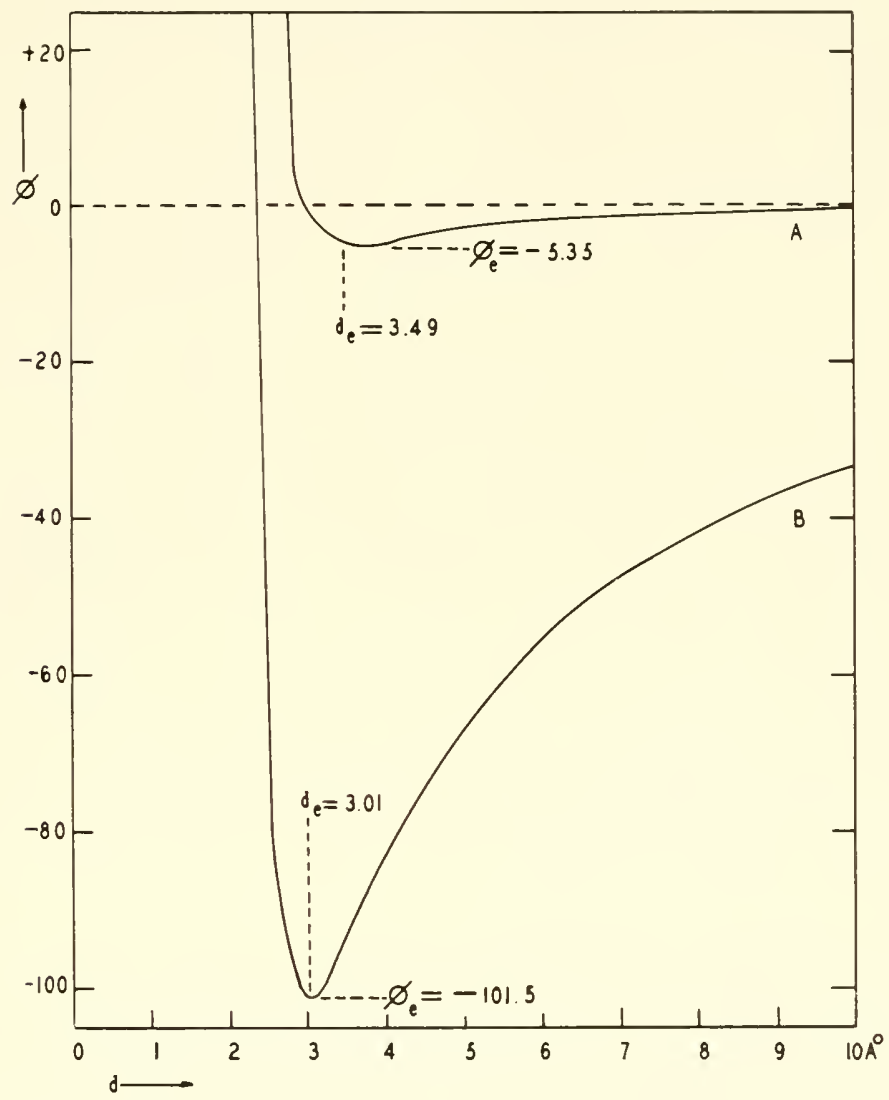

FIg. 6-13. Energy-distance curves for the interaction of two univalent ions. Curve $B$ represents the interaction in a vacuum and curve $\mathrm{A}$ in water. The repulsion constant (Eq. 6-50) was assumed to be $A=5 \times 10^{6} \mathrm{kcal} / \AA^{12}$. $\varphi$ is in $\mathrm{kcal} / \mathrm{mole}$.

quite close together and uninfluenced by water molecules; in such cases it is probably legitimate to use a value of $D=1$. If two dipoles are immersed in water and are several $\AA$ apart, the potential energy is negligible. Amis (1953) has presented experimental evidence that the dielectric constant influences the rate of dipole-dipole interactions. 


\section{HYDRATION OF IONS}

Ions exist in aqueous solution surrounded by water molecules in various degrees of orientation and restricted movement. Primary hydration refers to water molecules near an ion which have lost their translational degrees of freedom and move as an entity with the ion during Brownian movement; secondary hydration refers to those water molecules not included in the above which undergo electrostatic interaction with the primarily hydrated ion sufficient to affect certain thermodynamic quantities (Bockris, 1949). Considerations of interactions between ions or ionic groups in solution must take into account not only the ions but the water molecules associated with them. Let us consider two ions of opposite charge approaching one another. Below a certain separation distance. water molecules interacting with the ions must be displaced from the region between the ions and when the ions have reached their equilibrium position an appreciable amount of energy may have been expended on this displacement. If the ions come to rest with their primary water layers intact, then only displacement of secondary hydration has occurred; if the ions approach more closely, water molecules in the primary layers must also be displaced. It is difficult to determine if ions in solntion lose their primary hydration layer upon interaction and it may well be that the minimum distance of approach is often when the ions are separated by one or two water layers. The water molecules that are displaced associate by hydrogen bonding (or dipole-dipole interaction) with other water molecules and the energy involved in this process also enters into the total interaction. The total potential energy of two interacting ions in solution is thus:

$$
q_{t}=q_{i-1}+q_{i-w}+q_{w-w}
$$

where $q_{i-i}$ is the potential energy resulting from the interaction of the two ions (as calculated previonsly and including induction and dispersion terms), $p_{i-w}$ is the energy associated with the displacement of water from the ion and $\gamma_{w-w}$ is the energy of the water-water interaction. For ions of opposite charge, $p_{i-,}$ and $p_{w-w}$, will be negative and $q_{i-\vartheta}$ will be positive. The crystallization of a salt from solution involves the same terns but in that case much more water is displaced from the ions.

The molar hydration energy is the change in energy when one mole of ions in the gaseous state passes into an infinitely dilute solntion (or $N$ times the energy involved in a single ion being transferred from the gas phase to an aqueous phase). Attending this process there are large decreases in heat content, free energy, and entropy; these values and the primary hydration numbers are given in Table 6-17. The relative contributions from primary and secondary hydration are unknown but it is likely that well over $95 \%$ of the hydration energy results from the primary water layer. The energy 
values are seen to correlate with ionic charge and radius; thus they may be of use in estimating liydration magnitudes for ionic groups of biological importance. Unfortunately. little is known experimentally of the liydration characteristies of groups such as $\mathrm{R}-\mathrm{NH}_{3}{ }^{+}, \mathrm{R}=\mathrm{NH}_{2}{ }^{+}, \varphi-\mathrm{O}^{-}$, and $\mathrm{R}-\mathrm{COO}^{-}$but there is no reason to expect marked deviation from the inorganic ions, except for the steric factors introduced by the part of the molecule to which the ionic group is bonded. Taking into account ionic size and steric factor, the primary hydration numbers for $\mathrm{R}-\mathrm{NH}_{3}{ }^{+}$and $\mathrm{R}-\mathrm{COO}^{-}$ may be assumed to be approximately 3 .

TABLE $6-17$

Ionic Radi, Primary Hydration Numbers, and Hydration Exergies for SOME Commox Ioxs a

\begin{tabular}{|c|c|c|c|c|c|}
\hline Ion & $-\Delta F$ & $-\Delta H$ & $-\Delta S$ & $\begin{array}{l}\text { Ionic } \\
\text { radius }\end{array}$ & $\begin{array}{l}\text { Primary } \\
\text { hydration } \\
\text { number }\end{array}$ \\
\hline $\mathrm{H}^{+}$ & 214 & 223 & 31.0 & - & 5 \\
\hline $\mathrm{H}_{3} \mathrm{O}^{+}$ & 73 & 76 & 10.6 & 1.64 & 4 \\
\hline $\mathrm{Li}^{+}$ & 111 & 121 & 32.1 & 0.76 & 6 \\
\hline $\mathrm{Na}^{+}$ & 87 & 95 & 26.3 & 1.01 & 6 \\
\hline $\mathrm{K}^{+}$ & 70 & 76 & 17.7 & I. 34 & 6 \\
\hline $\mathrm{NH}_{4}{ }^{+}$ & 66 & 70 & 14.9 & 1.48 & 4 \\
\hline $\mathrm{C}^{\prime} \mathrm{1}^{++}$ & 473 & 496 & 75.0 & 0.41 & 12 \\
\hline $\mathrm{Zn}^{++}$ & 462 & 484 & 74.2 & 0.57 & 12 \\
\hline $\mathrm{Mg}^{++}$ & 432 & 456 & 77.1 & 0.78 & 12 \\
\hline $\mathrm{Ca}^{++}$ & 352 & 370 & 58.4 & 1.05 & 10 \\
\hline $\mathrm{Ba}^{++}$ & 291 & 306 & 48.4 & 1.39 & 8 \\
\hline $\mathrm{Al}^{+++}$ & 888 & 926 & 126.8 & 0.55 & 24 \\
\hline $\mathrm{F}^{-}$ & 113 & 123 & 31.8 & 1.29 & 5 \\
\hline $\mathrm{Cl}^{-}$ & 84 & 89 & 18.2 & 1.81 & 2 \\
\hline $\mathrm{Br}^{-}$ & 78 & 81 & 14.4 & 1.97 & 2 \\
\hline $\mathrm{I}^{-}$ & 89 & 72 & 10.1 & 2.23 & 1 \\
\hline $\mathrm{OH}^{-}$ & 81 & 91 & 32.6 & 1.33 & 5 \\
\hline
\end{tabular}

${ }^{a}$ The experimentally estimated values for $\Delta H$ and $\triangle S$ were selected from Latimer et al. (1939), Bockris and Conway (1954), and Mloelwyn-Hughes (1957) mainly; $\triangle F$ was ealculated from these. The ionic radii were taken from Moelwyn-Hughes (1957) and the primary hydration number estimated from values obtained by different methods in Bockris and Conway (1954). There is a good deal of variation in all these values for individual ions and the figures in the table must be taken as only approximate; the attempt has been made to present the most reasonable values on the basis of recent data. 
The best presentation of the modern concepts of ion-solvent interactions is to be found in the symposium published in the Discussions of the Faraday Society in 1957 (No. 24). The work of Platzman and Franck (1954), Brady (1958) and George (1959) may also be mentioned as particularly pertinent to the problems of ionic hydration in the interactions of proteins with inorganic ions and small molecules.

\section{Calculation of Hydration Energy from Interaction Energies}

It is interesting to calculate the hydration energy of an ion on the basis of the interaction equations proposed in order to illustrate the complexities of such systems and to obtain a rough estimation of the relative contributions of the various types of interaction. We shall consider the potential energy of an ion surrounded by its primary hydration layer; the specific calculations will refer to the $\mathrm{K}^{+}$ion for which a primary hydration number of six is assumed. There are the following contributions to the total potential energy.

Ion-dipole interaction:

$$
p_{e}=-57.4 \frac{n z_{i} \mu_{w}}{\left(d_{e}+d_{0}\right)^{2}} \cos \theta \quad-63.1 \mathrm{kcal} / \mathrm{mole}
$$

Ion-induced dipole interaction:

$$
p_{e}=-111 \frac{n \alpha_{w} z_{i}{ }^{2}}{d_{e}{ }^{4}} \quad-13.8 \mathrm{kcal} / \mathrm{mole}
$$

Dispersion (ion-water and water-water):

$$
p_{e}=-119 \frac{n \sqrt{Z} \alpha_{w}}{d_{e}{ }^{6}}\left[\alpha_{i}+\frac{\alpha_{w}}{4}\right] \quad-6.03 \mathrm{kcal} / \mathrm{mole}
$$

Dipole-dipole interaction (water-water):

$$
\begin{aligned}
\varphi_{e}=+14.4 \frac{n \mu_{w}{ }^{2}}{2 \sqrt{2} d_{e}{ }^{3}} & +4.24 \mathrm{kcal} / \mathrm{mole} \\
\text { Total interaction energy: } & -78.69 \mathrm{kcal} / \mathrm{mole}
\end{aligned}
$$

The primary hydration number $n=6, z_{i}=1, \mu_{u}=1.83$ debyes, $\alpha_{i}=$ 0.87, $\alpha_{w}=1.44, Z=8,0=0^{\circ}$, and $d_{e}=2.891 \AA$. The value of $d_{e}$ was obtained by summing the ionic radins of $\mathrm{K}^{+}$and the van der Waals' radius of water; $d_{0}$ is the distance of the water dipole center from the molecular center $(0.274 \AA)$. Two further energy terms must be included to 
calculate the hydration energy: the interaction energy of the ion with water molecules outside the primary layer and the energy required to disrupt the water structure - these are difficult to estimate accurately. From the expression derived by Moelwyn-Hughes (1949), assigning $D=3$, the interaction of the ion with water molecules outside the primary layer results in a potential energy of $-5.6 \mathrm{kcal}$ mole; using Eq. 6-58 and assuming interaction with ten water molecules outside the primary layer results in $-7.0 \mathrm{kcal} /$ mole. If it is assumed that the six water molecules in the primary layer have each lost one of their interactions with neighboring water molecules, the energy required would be $+13.3 \mathrm{kcal} / \mathrm{mole}$. Thus approximately $+7.0 \mathrm{kcal} /$ mole must be added to the sum obtained from individual interactions, giving $-71.7 \mathrm{kcal} /$ mole for the over-all potential energy. If this can be equated with the experimental hydration energy of $-75.8 \mathrm{kcal}$ mole, it may be concluded that this treatment has led to at least the right magnitude and indicates the relative importance of the different interactions. Moelwyn-Hughes (1949) has calculated hydration energies of several ions by a similar method, neglecting dispersion energy and disruption of water structure and assuming that the repulsion energy varies with $d_{e}^{-9}$, and has arrived at values close to the experimental ones. It may be observed that the calculations within the primary water layer assumed a value of $D=1$; the correspondence between the results and the experimental values indicates that this assumption is valid for interactions at small distances. Perhaps the principal value of such calculations lies in the resulting appreciation of the inadequacies of our knowledge and the stimulus this gives for theoretical and experimental advances in interactions at the molecular level.

It has been stated that the $\mathrm{K}^{+}$ion is close to the size of the water molecule and henee probably enters substitutionally into the water structure without disturbing the strueture (Brady and Krause, 1957). However it is not the size of the ion that is most important but its charge and the orienting effect the ionic field has on the surrounding water molecules. The alteration in water structure brought about by such ions is attested by electrostriction.

Two other treatments of the hydration energy will be mentioned because they illustrate the relative contributions made by the various interactions and also show how different approaches and assumptions can lead to similar results. Buckingham (1957) assumed a hydration number of four for the univalent ions and an orientation of the water wherein the dipole is on a line with the center of the ion. The total interaction energy was written as the sum of a variety of terms representing the different forces involved:

$$
\varphi=p_{d i p}+p_{\text {quad }}+p_{i}+p_{d}+p_{l}+p_{0}+p_{h}+\varphi_{r}
$$

The subscripts refer to the following: $\varphi_{\text {dip }}$ to the ion-dipole interactions, 
$\varphi_{q u a d}$ to quadrupole interactions, $\varphi_{i}$ to induced dipoles and multipoles, $\varphi_{d}$ to dispersion forces, $\varphi_{l}$ to lateral interactions between the tetrahedral water molecules, $\gamma_{0}$ to the interactions with water molecules ontside the hydration layer, $\varphi_{h}$ to the energy required to make a hole in the water structure, and $p_{r}$ to the repulsion energy arising from atomic overlap. These individual values were calculated for several ions and compared to the experimental hydration energies; the treatment generally overestimates the energy by $12-15 \mathrm{kcal} / \mathrm{mole}$, and this was attributed to the rigidity of the assumed water structure. However, the neglect of the repulsion correction may account for this difference also, inasmuch as it was assumed that $p_{r}$ is zero unless the interacting surfaces are in contact; actually the repulsion forces do contribute appreciably in hydration. This factor was included in the equations used in the previous calculation of the hydration energy.

\begin{tabular}{l|r|r}
\hline & \multicolumn{1}{|c|}{$\mathrm{K}^{+}$} & \multicolumn{1}{c}{$\mathrm{Br}^{-}$} \\
\hline$\varphi_{\text {dip }}$ & -67.7 & -44.7 \\
$\varphi_{\text {quad }}$ & 26.4 & -14.1 \\
$\varphi_{i}$ & -17.9 & -7.7 \\
$\varphi_{d}$ & -3.7 & -1.9 \\
$\varphi_{l}$ & 1.4 & 4.1 \\
$\varphi_{0}$ & -38.8 & -33.6 \\
$\varphi_{h}$ & 10.0 & 10.0 \\
$\varphi$ & -90.3 & -87.9 \\
\hline
\end{tabular}

Brady (1958) calculated the hydration energy for $\mathrm{K}^{+}$assuming four water molecules oriented with their two centers of negative charge towards the ion. The existence of two such negative centers in water is probably questionable. The hydration energy was written as:

$$
\varphi=\frac{z^{2} e^{2}}{2 r}\left[1-\frac{1}{D}\right]+n \mathrm{P}-\alpha
$$

The first term is the Born expression for the energy required to charge a sphere of radius $r=r_{i o n}+2 r_{\text {water }}$ in a medium of dielectric constant $D$. The sccond term is the product of the number of water nolecules by the potential energy of each in the hydration layer, $\mathrm{P}$ being given by:

$$
P=\Sigma \frac{\beta e^{2}}{r}-\frac{G \mu^{2}}{\left(r_{\text {ion }}+r_{\text {water }}\right)^{2}}
$$

which represents the coulombic and the dipolar interactions, $\beta e$ being the fractional charge on the $H$ or $\mathrm{O}$ atoms and $G$ a geometrical factor dependent on the hydration number. The third term takes account of the energy lost 
by the water molecules when they are reoriented from their water structure. For the $\mathrm{K}^{+}$ion the three terms were calculated to be:

$$
\varphi=65.2+40-28=77.2 \mathrm{kcal} / \mathrm{mole}
$$

which is quite close to the experimental value.

\section{Hydration Differences between Cations and Anions}

There has been considerable confusion about the hydration of anions; in fact, it was believed for some time that only cations were appreciably hydrated. However, the hydration energies of anions (Table 6-17) are greater than for cations of comparable radii; for example, $\mathrm{K}^{+}$and $\mathrm{F}^{-}$are approximately the same size and yet the hydration energy of the latter is - $47 \mathrm{kcal} / \mathrm{mole}$ greater. The reason for this difference is that the dipole center of water can approach an anion more closely than a cation. The distance from the nuclens of a $\mathrm{K}^{+}$ion to the water dipole center is $3.16 \AA$ whereas an anion of the same size would approach to within $2.55 \AA$, using the accepted ionic radius and configuration of the water molecule. It might be asked why the primary hydration number of anions is usually given as less than for cations. In fact, the significance of a primary hydration of one or two might be questioned. The fact that the hydration energies of $\mathrm{Cl}^{-}, \mathrm{Br}^{-}$, and $\mathrm{I}^{-}$are of the same magnitude as $\mathrm{K}^{+}$. as are the entropy changes, would indicate that there is about the same degree of interaction with the water molecules and the same disruption of water structure. The larger anionic radius is balanced by the closer approach to the water dipole; in other words, a $\mathrm{Br}^{-}$ion would have about the same interaction distance with water as a $\mathrm{K}^{+}$ion, and a similar primary hydration of six would be expected. In the case of the small $\mathrm{F}^{-}$ion it is possible that steric factors allow only four water molecules to surround the ion, but for the larger anions this is not a restriction. It may well be that such anions should be considered as having as complete a hydration layer as the cations, since the repulsion energy (dipole-dipole) of the bound water is relatively small compared to the ion-dipole energy. It is likely that the $\mathrm{R}-\mathrm{COO}^{-}$group is hydrated but due to its asymmetry it is possible there is a primary hydration of only three. The $\mathrm{R}-\mathrm{NH}_{3}{ }^{+}$group probably also has a primary hydration of three. These values will be accepted in future calculations as the most reasonable.

\section{The Structure of Water and the Effects of Solutes on this Structure}

The interactions of molecules in aqueous solution involve not only the hydration water associated with ions or ionic groups but also the structure of water in the bulk phase and surrounding nonpolar molecules or parts 
of molecules. The association energy depends on the enthalpy and entropy changes occurring during modification of this water structure. Pure water possesses structure in the statistical sense; that is, at any time a certain fraction of the water molecules will be bound to neighboring molecules to form small regions of tetrahedral orientation as in ice. Varions terms have been used in discussing water structure: the temporary structural aggregates may be called "frozen regions," "ice-bergs," or " microdonains of ice," while the amount of structure in water under specified conditions has been termed the "icelikeness" or " degree of polymerization." When water molecules form icelike struetures around small nonpolar molecules or groups, these are frequently referred to as "cages." The association of water molecules involves mainly hydrogen bonding, with other dipolar interactions and small dispersion forces contributing to a lesser degree. Water thus contains "flickering clusters" of various sizes and shapes, the half-lives of these structures being perhaps of the order of $10^{-10}$ to $10^{-11} \mathrm{sec}$ (Frank and Wen, 1957). The fraction of the total water that is present in these icelike clusters depends strongly on the temperature and this is one factor that must be considered in the effects of temperature on interactions in water. It was postulated that when two water molecules associate, there is a tendency for neighboring molecules to become bonded and participate in the structure, in a manner similar to erystallization. Conversely, if the bond between two water molecules is broken in a formed cluster, there will be a tendency for the clnster to break down or melt. This provides a means by which localized effects on orientation can be propagated for some distance in the solvent.

When solnte molecules are introduced into water, various effects may be exerted on the water structure in the sense of altering the fraction of the water that is in the icelike state or of changing the size and configuration of the association clusters. The over-all effect may be an increase or a decrease in the water structure. Substances that produce an increase in the structure have generally been called structure formers and those that cause a decrease in the structure have been called structure breakers. It has been possible by studies on viscosity and entropy changes to elassify the common ions into these two categories (Frank and Wen, 1957). Thus $\mathrm{F}^{-}, \mathrm{OH}^{-}, \mathrm{Ca}^{++}$, and $\mathrm{Mg}^{++}$are elassed as structure formers and $\mathrm{Cl}^{-}, \mathrm{Br}^{-}$, $\mathrm{I}^{-}, \mathrm{NO}_{3}^{-}, \mathrm{SO}_{4}{ }^{-}$, and $\mathrm{K}^{+}$as structure breakers $\left(\mathrm{K}^{+}\right.$exerting a very slight effect). It is believed that multiply-charged cations, such as $\mathrm{Mg}^{++}$, produce a long-range ordering of the water structure, i.e., beyond the primary hydration layer (Kaminsky, 1957), this being shown by studies on infrared spectra, entropy changes, apparent molal heat capacities, and other properties. One might consider hydrated ions as providing nuclei for the formation of icelike clusters, but the orientation of the water molecules around the ions is usually not such as to fit into the usual water structure. An in- 
teresting approach to this problem has been made by Samoilov (1957) who considered the rate of exchange of water molecules between the bulk phase and the hydration layers; if this rate is low, hydration must be strong. The activation energies for this exchange were calculated for the common ions, based on certain assumptions whose validity may be questioned, and the following values in $\mathrm{kcal} /$ mole were obtained: $\mathrm{Mg}^{++}, 2.61 ; \mathrm{Li}^{+}, 0.73$; $\mathrm{Ca}^{++}, 0.45 ; \mathrm{Na}^{+}, 0.25 ; \mathrm{K}^{+},-0.25 ; \mathrm{Cl}^{-},-0.27 ; \mathrm{Br}^{-},-0.29$, and $\mathrm{I}^{-}$, -0.32 . The negative values would indicate that certain water molecules near the hydrated ions have greater freedom than in pure water. This was termed "negative hydration" but is probably merely an expression of the structure-breaking activity of these ions and should not be construed as indicating a lack of hydration.

A nonpolar molecule introduced into water will affect the structure in at least two ways. In the first place, a hole must be made in the water and this requires appreciable energy, which may be estimated roughly from the latent heat of water (about $10 \mathrm{kcal} /$ mole). In the second place, a pure nonpolar molecule will interact so little with the surrounding water molecules that icelike clusters tend to form at its surface, and thus these nonpolar solutes are structure formers to some extent. This is experimentally shown by the longer dielectric relaxation times observed in solutions of nonpolar substances (Frank and Wen, 1957). If the solute possesses groups that may participate in hydrogen bonding, these may act as nuclei for icelike clusters and further increase the structural component.

Most molecules of interest in enzyme inhibition contain both polar and nonpolar parts and it is of importance to consider the disposition of water around such molecules. Fatty acids, for example, exist either as $\mathrm{R}-\mathrm{COOH}$ or $\mathrm{R}-\mathrm{COO}^{-}$. In the former the water will be affected by the hydrocarbon chain as described in the preceding paragraph; in addition there will be some hydrogen bonding with the carboxyl group. When ionization occurs, the anionic carboxylate group will become hydrated and there will probably be a structure-breaking effect. Everett (1957) has presented evidence that an anionic group in such molecules can exert an effect on the surrounding water structure to a distance of at least $5 \AA$. Thus in short-chain fatty acids (such as formic or acetic acids), the noncarboxylate part of the molecule will be of no or little importance since the water surrounding it will be under the influence of the ionic group, whereas in long-chain fatty acids (probably past butyric acid) only a fraction of the water lying along the lyydrocarbon chain will be influenced by the ionic group. Situations of this nature are very important in considerations of the interactions of small molecules with enzymes, because the effective configuration of the hydrated molecules and the changes that occur in the water structure upon binding are factors of fundamental significance.

One final word should be said concerning the orientation of water mole- 
cules around ions. There has been no general agreement as to the directions in which the water molecnles around an anion lie. It has been often assumed that a water molecule takes up a position wherein a hydrogen atom lies on a line between the ion and the oxygen atom, but as Buckingam (1957) pointed out, this means that the water dipole is oriented about $52^{\circ}$ from the ionic electric force field, as in orientation (A). Buckingahm prefers

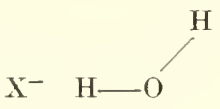

(A)

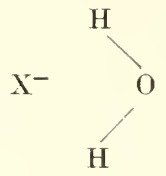

(B)

the orientation (B) in which the maximal interaction with the water dipole can oceur. The hydrogen ion presents a special case because a proton is probably associated with one water molecule as $\mathrm{H}_{3} \mathrm{O}^{+}$and it is this ion that is hydrated, not only because of the electrical field but as the result of hydrogen bonding. Also the orientation of water molecules around an hydroxyl ion is probably asymmetrical because of the different positions the water molecules must assume to form hydrogen bonds (Ackermamn, 1957).

\section{Hydration of Proteins}

Water is associated with protein molecules in solution in a variety of ways inasmuch as the protein surface is made up of regions of differing properties. There are both structure-forming and structure-breaking regions, and there are groups that will be hydrated because of their ionic nature and others because of their ability to form hydrogen bonds with the water. In summary, the water near the protein or enzyme surface will be in a different state than the water in the bulk phase. The water structure around a protein will depend particularly upon the amino acid composition and the pH because these will determine the ionic state of the protein. Experimental evidence for an oriented water structure around proteins has been obtained by Klotz (1958) who attached the ionizing azomercmial group, $-\mathrm{Hg}-\boldsymbol{\varphi}-\mathrm{N}=\mathrm{N}-\boldsymbol{\varphi}-\mathrm{N}\left(\mathrm{CH}_{3}\right)_{2}$, to several proteins and found that the ionization was decreased (i.e., it was more difficult for a proton to reach the $-\mathrm{N}\left(\mathrm{CH}_{3}\right)_{2}$ group to produce a positively charged group). He attributed this to the icelike structure of the water smrounding the proteins. In the case of sermm albumin, this cage structure becomes especially important below a $\mathrm{pH}$ of 4.2 , the extent and rigidity of the icelike structure increasing. It is obvious that the interaction of any molecules with enzymes must be determined to some extent by this water envelope and that the ability of a molecule to bind to an enzyme may depend on whether the molecule can disrupt the water structure. 


\section{Importance of Hydration in Enzyme Interactions}

It was pointed out earlier that ionic interactions involve displacement of water. To varying extents this is true for all molecular interactions in solntion. The reaction of an inhibitor with an enzyme is usually written as $\mathrm{E}+\mathrm{I} \rightarrow \mathrm{EI}$ but from the standpoint of the over-all interaction energy it should be:

$$
E-w_{x}+\mathrm{I}-w_{y} \rightarrow \mathrm{E}-\mathrm{I}-w_{z}+(x+y-z) w
$$

where $w$ represents a boumd water molecule and $x, y$, and $z$ the number of water molecules associated with each species; the number of waters displaced wonld then be $x+y-z$ and these will interact with free water molecules. The interaction of water with enzyme or inhibitor may be of any type (ion-dipole, dipole-dipole, or dispersion) and thus complexes such as $\mathrm{E}-w_{x}$ do not represent necessarily stable hydration layers but indicate all energetically significant interactions of the molecules with water. Even nonpolar molecules or groups, such as hydrocarbon sidechains, exhibit some dispersion interaction with surrounding water molecules, and when two nonpolar groups interact this water is displaced. In such a case where dispersion forces only are important, $x=y$ because the interaction surface between the groups is the same as that for interaction with the water, and $z=0$ since all the water must be displaced for significant interaction between the groups:

$$
\mathrm{E}-w_{x}+\mathrm{I}-w_{x} \rightarrow \mathrm{E}-\mathrm{I}+2 x w
$$

Since each dispersion interaction involves the polarizabilities of the substances we may write $p_{e}=-x K \alpha_{1} \alpha_{2}$ for the potential energy of each group with its surrounding water. The over-all interaction energy is the difference between the final and initial states.

$$
\begin{array}{ll}
\text { Inital state: } & \psi_{e}=-x K \alpha_{e} \alpha_{w}-x K \alpha_{i} \alpha_{w} \\
\text { Final state: } & \tilde{q}_{e}=-x K \alpha_{w}{ }^{2}-x K \alpha_{e} \alpha_{i}
\end{array}
$$

The over-all dispersion interaction energy is thus:

$$
r_{e}=-x \boldsymbol{K}\left(\alpha_{w}-\alpha_{i}\right)\left(\alpha_{w}-\alpha_{e}\right)
$$

and not $-x K \alpha_{e} \alpha_{i}$ as often assumed. The disruption of the water structure and the entropy factors involved will be discussed later. The role of such water dispersion forces in protein reactions has been considered by Pauling and Pressman (1945) in the interactions of haptenic groups with antibody. 


\section{IONIC ATMOSPHERE}

The energy involved in ionic interactions in solution must be further corrected for the ionic atmosphere that surrounds each ion. Each ion in the solution attracts ions of opposite charge and, although the ions retain their mobility, there is established an ion distribution the characteristics of which can be predicted by statistical theory. The presence of this ionic atmosphere around an ion alters the electrical field of the central ion and hence the interaction energy. The ionic atmosphere will be contributed to mainly by small ions such as $\mathrm{Na}^{+}, \mathrm{K}^{+}, \mathrm{Cl}^{-}$, and others. It is evident that the greater the concentration of these ions, the smaller will be the interaction energy between ionic groups, such as on an enzyme or inhibitor. This situation may be conceived as a competition between the numerous small inorganic ions and the inhibitor ionic group for the enzyme ionic group; as the inhibitor approaches the enzyme, energy is required to displace part or all of the ionic atmosphere.

The electrical potential in the neighborhood of an ion can be shown to be:

$$
V=\frac{z e}{d D} \frac{e^{\varkappa\left(r_{0}-d\right)}}{1+\varkappa r_{0}}
$$

where $d$ is the distance from the ion to the point where the potential is considered and $r_{0}$ is the closest approach of the ionic atmosphere to the ion. The Debye-Hückel constant $\varkappa$ is given by:

$$
\varkappa=\sqrt{\frac{4 \pi e^{2}}{D k T} \Sigma n_{i} z_{i}^{2}}=\sqrt{\frac{8 \pi N e^{2} s}{1000 D k T}}
$$

where $n_{i}$ is the number of ions per $\mathrm{ml}, z_{i}$ is their charge, and $s$ is the ionic strength. The potential in Eq. 6-85 is actually made up of two separate potentials, one due to the central ion and one to the atmosphere. The potential of an ion will be recluced by the ionic atmosphere and the magnitude of this reduction will depend primarily on the ionic radii and the ionic strength. The potential energy of two interacting ions will now be given by:

$$
q=\frac{z_{1} z_{2} e^{2}}{d D} \frac{e^{\varkappa\left(r_{0}-d\right)}}{1+x r_{0}}
$$

The evaluation of the potential energy requires an estimation of $x$ and $r_{0}$. At $37.5^{\circ}$ and assuming $D=74.1$ :

$$
x=0.332 \sqrt{ } \bar{s} \AA^{-1}
$$

In most enzyme studies under physiological conditions, the ionic strength $s=0.16$, in which case $x=0.133 \AA^{-1}$. The radius of the ionic atmosphere, 
usually expressed as $1 / \%$, would be approximately $7.5 \AA$. If the distance of closest approach $r_{0}$ is taken as $3.0 \AA$ (radius of enzyme or inhibitor ionic group $=1.7 \AA$ and radius of atmosphere ion $=1.3 \AA$ ) the potential energy at equilibrium, correcting for repulsion, would be:

$$
\varphi_{e}=183 \frac{z_{1} z_{2}}{d_{e} D} e^{0.133\left(3-d_{e}\right)} \mathrm{kcal} / \mathrm{mole}
$$

If the ions retain their primary hydration, $r_{0}$ would be approximately $10.2 \AA$ (diameter of two water molecules $=7.2 \AA$ ) and the potential energy would be:

$$
\varphi_{e}=165 \frac{z_{1} z_{2}}{d_{e} D} e^{0.0834\left(10.2-d_{e}\right)} \mathrm{kcal} / \mathrm{mole}
$$

When it is reasonable to assume that the interacting ions reach an equilibrium distance equivalent to the closest approach of the atmosphere ions, the exponential factor disappears; when this is not the case, the variation of the exponential factor with distance may be estimated from Fig. 6-14. It

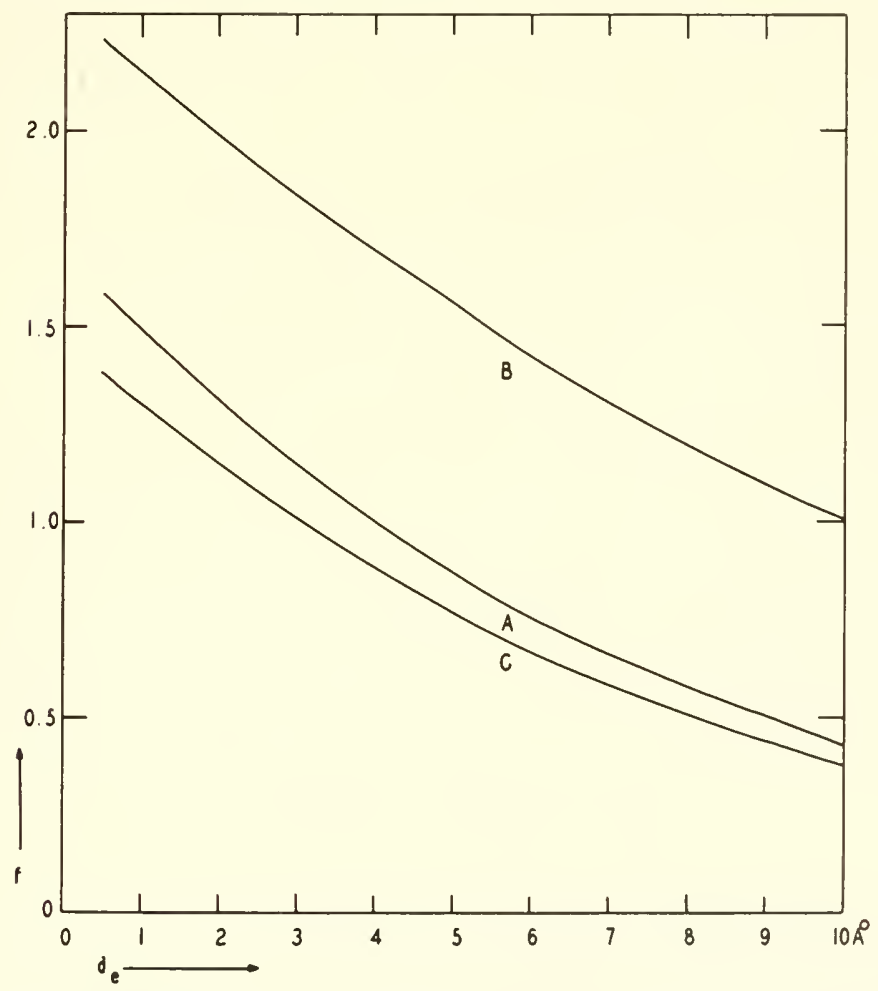

FIG. 6-14. Variation of the exponential factor with distance. Curve A: $f=e^{0.133\left(4-d_{e}\right)}$; curve B: $f=e^{0.0834\left(10.2-d_{e}\right)}$; curve C: $f=e^{0.133\left(3-d_{e}\right)}$. 
is possible that $d$ may be less than $r_{0}$ in the second case above if the interacting ions lose their primary hydration. Within the cell, $r_{0}$ for positive ions is probably greater than given above due to the larger size of the anions present, and the ionic strength and $x$ may be less than assumed.

When the ionic atmosphere is displaced by any type of interaction, a rough estimate of the energy involved may be made from the value of the ionic energy in the Debye-Hückel treatment. The potential energy due to the ionic atmosphere at $37.5^{\circ}$ and at an ionic strength of 0.16 is about $-0.3 \mathrm{kcal} / \mathrm{mole}$. The energy is probably somewhat less than this for ionic gromps on molecules due to steric factors. When ionic groups interact in enzyme inhibition it is likely that the entire ionic atmosphere of each is displaced, so that approximately $0.4 \mathrm{kcal} / \mathrm{mole}$ must be expended on this. Thus this represents only a minor correction factor in such interactions.

The binding of ions to some proteins is more complex than indicated by the treatment above, as studies on myosin have demonstrated (Lewis and Saroff, 1957). Myosin A binds maximally about $210 \mathrm{~K}^{+}$or $\mathrm{Na}^{+}$ions per molecule (assuming a molecular weight of 420,000 ) and myosin $\mathrm{B}$ about half this number. The effects of $\mathrm{pH}$ on this binding indicate the participation of cationic gromps and a "chelation" type of binding:

$$
-\mathrm{COO}^{-} \ldots{ }^{+} \mathrm{H}_{3} \mathrm{~N}-+\mathrm{K}^{+} \rightarrow-\mathrm{COO}^{-} \ldots \mathrm{K}+\cdots \mathrm{H}_{2} \mathrm{~N}-+\mathrm{H}^{+}
$$

There are two types of binding sites, one involving the imidazole group of histidine and one an amino group. The thermodynamic constants for $\mathrm{K}^{+}$are:

\begin{tabular}{c|c|c|c}
\hline Sites & Number/molecule & $K$ & $1 F$ \\
\hline Imidazole & 63 & $1.25 \times 10^{-3}$ & -3.98 \\
Amino & 147 & $5 \times 10^{-3}$ & -2.33 \\
\hline
\end{tabular}

where $K$ is the dissociation constant. The high binding energy would also indicate some type of binding other than simple ion-ion interaction. The binding is, of course, markedly dependent on $\mathrm{pH}$; there is essentially a competition between $\mathrm{K}^{+}$and $\mathrm{H}^{+}$ions for the nitrogen group. That appropriate conjunctions of anionic and cationic groups on a protein is necessary for such binding is shown by the failure of actin and seralbumin to bind either $\mathrm{K}^{+}$or $\mathrm{Na}^{+}$.

\section{DISTORTION ENERGY}

The binding of an inhibitor to an enzyme may involve modifications in the structure of either or both components and such changes from the normal state will require expenditure of energy. These energy changes should be incorporated in the expression for the over-all energy of interaction but it is generally impossible to evalnate the magnitude of the contributions. 


\section{Distortion of Enzyme Structure}

When the inhibitor fits into a cavity or crevice in the enzyme sone expansion of the protein structure may oceur. Dilatation of antibody protein to aecommodate various haptenic groups has been diseussed by Pauling and Pressman (1945) although the energy involved could not be calculated. Entropy data indicate a "loosening " of the enzyme structure on forming the enzyme-substrate eomplex (Laidler, 1955a). Little is known of inhibitor-induced structural changes but a denaturation-like effeet is postulated to oecur in the inhibition of biolumineseent enzymes by urethanes and sulfonamides (Johnson et al., 1954).

\section{Distortion of Inhibitor Structure}

The bound inhibitor may exist in a strained state. that is in a configuration different from that assumed in solution. Energy may be required to alter bond angles or bring eharged groups closer together. It is possible that in the inhibition of succinic dehydrogenase by malonate the carboxylate groups are closer when the malonate is attached to the enzyme; some bond strain might also ocenr if the enzyme cationie groups have a separation distance different from the inter-carboxylate distanee in malonate.

\section{Distortion of Enzyme Side-Chains}

The amino acid side-chains at the surface of an enzyme probably possess a degree of flexibility. In such eases they may assume positions around the inhibitor to provide maximal contact. As an inhibitor molecule approaches the enzyme surface these side-ehains may swing around to equilibrium configurations in which the potential energy is minimal. Some energy, although usually quite small. may be necessary for the orientation, since in the uninhibited enzyme the side-chains are in the most stable positions. The flexibility of these side-chains may in part be determined by the content of hydroxy amino aeids, these forming hydrogen bonds with amino or earboxyl groups and stabilizing their configuration. The absence of such hydroxy amino acids has been postulated as explaining the ability of seralbumin to bind many substanees, this protein being more flexible than most (Klotz and Urquhart, 1949b).

\section{Distortion of Enzyme Charge Distribution}

It was pointed out by Kirkwood and Shumaker (1952) that the protein surface possesses many groups capable of combining with protons and that the distribution of charged groups can therefore flnctuate and will depend on a number of faetors. The protein surface may be considered as having many possible charge distributions differing slightly in energy. 
When a substrate or inhibitor approaches the enzyme surface it will modify the charge distribution if it possesses ionic groups; a positively-charged inhibitor will create a region of relative negativity on the enzyme and the attraction will be increased. One might think of the enzyme as being polarized or of the ionic inhibitor evoking image forces (as in the adsorption of ions on metal or ionic surfaces). An inhibitor bound to an enzyme site will experience forces of attraction arising from the fluctuating charges on the surface vicinal to the site (Kirkwood, 1955). The potential energy involved will depend on $1 / d^{4}$ where $d$ is the distance from the interacting ion to the fluctuating ions and, since the forces are electrostatic, will depend on the ionic strength (Kirkwood, 1954, p. 4). Hill (1956) has considered such interactions on a quantitative basis and pointed out that the increased stability would be opposed by the entropy changes. In any event, the distortion of the normal charge distribution by the inhibitor introduces a small energy term into the total interaction. This effect is closely related to the alteration in proton affinity of acidic or basic groups induced by the proximity of an ion and the resultant change in $\mathrm{p} K_{a}$; this aspect will be considered in more detail in Chapter 14.

\section{INHIBITION CONSTANTS AND INTERACTION ENERGY}

The various types of interactions and energy contributions involved in the binding of inhibitors to enzymes have now been discussed. We shall next relate the interaction energy to the experimentally determined inhibition constant $K_{i}$. If $K_{i}$ is known, it is possible to calculate the over-all change in free encrgy attending the binding of an inhibitor to the enzyme from the equation:

$$
\Delta F=R T \ln K_{i}
$$

which at $37.5^{\circ}$ may be written as:

$$
\lrcorner F=1.422 \log K_{i} \mathrm{kcal} / \mathrm{mole}
$$

The interaction anergy values discussed in the previous sections generally refer to the free energy changes in the process of bringing the interacting molecules from infinite distance to the equilibrium separation $d_{e}$. The changes in free encrgy, enthalpy, and entropy are related by the equation:

$$
\Delta F=\Delta H-T \Delta S
$$

The relative contributions of $\triangle H$ and $T \angle S$ to the free energy change depend on the nature of the interaction and the medium in which it occurs. It is possible to determine experimentally both $\Delta H$ and $\triangle S$ and these often give useful information with respect to the reaction mechanism. 


\section{Determination of Enthalpy and Entropy Changes}

Substitution of $\Delta F$ from Eq. 6-91 in:

$$
\left(\frac{\partial \Delta F}{\partial T}\right)_{p}=-\Delta S
$$

leads to the following expression for the change in enthalpy:

$$
\Delta H=2.303 R T^{2} \frac{d \mathrm{p} K_{i}}{d T}=441.8 \cdot \frac{d \mathrm{p} K_{i}}{d T} \mathrm{kcal} / \mathrm{mole}\left(\text { at } 37.5^{\circ}\right)
$$

Thus the slope of the plot of $\mathrm{p} K_{i}$ against temperature will provide the change in enthalpy and the entropy change may then be estimated from Eq. 6-93 which may be written for $37.5^{\circ}$ as:

$$
\Delta S=1422 \frac{d \mathrm{p} K_{i}}{d T}+4.58 \mathrm{p} K_{i}
$$

where $A S$ is given in cal $/$ mole/degree as is customary.

For interactions involving ionic groups the following method leads to the entropy change directly (Laidler and Ethier, 1953). If the electrostatic ion-ion interaction energy is given by $J F=305 z_{1} z_{2} / d_{e} D$ from Eq. 6-56. substitution of this in Eq. 6-94 leads to:

$$
\Lambda S=305 \frac{z_{1} z_{2}}{d_{e} D^{2}}\left(\frac{\partial D}{\partial T}\right)_{p}
$$

From Eq. 6-73, $(\partial D / \partial T)_{p}$ is found to be -0.338 at $37.5^{\circ}$, and if $D$ is taken as 74.1:

$$
\Delta S=-18.8 \frac{z_{1} z_{2}}{d_{e}} \mathrm{cal} / \mathrm{mole} / \text { degree }
$$

However, if $D$ varies with the interaction distance and Eq. 6-74 is used, it is seen that the entropy change will depend on $d_{e}$; for separations of 5-7 $\AA, \Delta S$ would be 8.7-4.4-fold greater than in Eq. 6-98, which is more in accord with experimental values in ionic reactions. If we now assume that the total free energy change is made up of electrostatic and nonelectrostatic contributions:

$$
\Delta F=\lrcorner F_{n e s}+\Delta F_{e s}
$$

one may write:

$$
\ln K_{i}=\frac{\Delta F_{n e s}}{R T}+\frac{305 z_{1} z_{2}}{R T d_{e} D}
$$

and from a plot of $\ln K_{i}$ against $1 / D$ the value of $z_{1} z_{2} / d_{e}$ may be obtained. 
Finally, $1 S$ may be calculated from Eq. 6-98. The dielectric: constant may be varied by employing different mixtures of aqueous medium and a miscible organic liquid (such as dioxane or methanol): direct effects of such organic substances on the enzyme or inhibitor must be ruled out.

Although a beginning has been made in the determination of entropy changes in protein binding and the formation of the enzyme-substrate complex, unfortunately there is little work with enzyme inhibitors (see Chapter 15). However, one may take as typical the entropy of formation of the ES complex (Laidler, 1955 a). Values in the range between -50 and $+50 \mathrm{cal} / \mathrm{mole} /$ degree have been found and indeed in some cases the entropy change is the principal driving force in the formation of the complex. Such would be the case in the binding of the hapten ${ }^{-} \mathrm{HO}_{3} \mathrm{As}-\boldsymbol{\varphi}-\mathrm{NHCO}-$ $\varphi-\mathrm{CONH}-\boldsymbol{\varphi}-\mathrm{AsO}_{3} \mathrm{H}^{-}$to antibody protein, in which the thermodynamic constants were found to be $\mid F=-7.4 \mathrm{kcal} / \mathrm{mole}, \angle H=-0.8 \mathrm{kcal} /$ mole, and $\angle \mathrm{I} S=22 \mathrm{cal} / \mathrm{mole} /$ degree (Epstein et al., 1956). In the binding of $\mathrm{Ca}^{++}$ions to seralbumin, there was little effect of temperature on the binding and hence $1 H$ was assumed to be zero, in which case $1 S=-\Delta F / T$ or around $14 \mathrm{cal} /$ mole/degree (Katz and Klotz, 1953). The binding of $\mathrm{Cu}^{++}$ions is similar and gave a value of $1 \mathrm{~S}=28 \mathrm{cal} / \mathrm{mole} /$ degree (Klotz, 1952, p. 427). It is evident that the role of entropy in protein interactions is very important.

\section{Components of the Entropy Change in Protein Interactions}

The over-all entropy change in the reaction:

$$
\mathrm{E}-w_{x}+\mathrm{I}-w_{y} \rightarrow \mathrm{EI}-w_{z}+(x+y-z) w
$$

may be considered to be made up of three parts in the general case. $(a)$ The rigidity imposed in the molecules by the formation of the EI complex which would result in a negative $1 . S$. (b) Structural modifications in the enzyme or substrate consequent to binding, the IS for which could be either positive or negative. (c) The liberation of water molecules with an increase in their rotational degrees of freedom resulting in a positive $1 S$. It is probable that the last is the most important contribution in reactions involving ionic groups. In fact when $\mathcal{L S}$ is definitely positive, it is evidence that oppositely charged groups are interacting and displacing water of hydration as pointed out by Laidler (1955 a). Enzymes such as pepsin, trypsin, and ATPase (which act on ionic substrates) show a $+1 S$ for formation of the ES complex, while chymotrypsin and urease (which act on uncharged molecules) show a $-1 S$. Similar $+1 S$ values are obtained for the ionic interactions of the arsenical hapten with antibody and for $\mathrm{Ca}^{++}$and $\mathrm{Cu}^{++}$ with seralbumin. 
The entropy change from the liberation of water in ionic interactions may be quite large. In the reaction of the $-\mathrm{HO}_{3} \mathrm{As}-\varphi-\mathrm{NHCO}-\varphi-$ $\mathrm{CONH}-\varphi-\mathrm{AsO}_{3} \mathrm{H}^{-}$lapten with antibody, it was calculated that the $\triangle S$ for association (with loss of translational and rotational freedom) would be approximately - $100 \mathrm{cal}$ mole/degree. Thus the $1 S$ for water liberation would be $+122 \mathrm{cal} /$ mole degree. Since the melting of ice involves an entropy change of $1 S=+5$ cal mole degree. it was concluded that around 24 molecules were displaced (Epstein et al., 1956). In the reaction of ATP with myosin ATPase the calculations showed that the electrostatic interactions contributed only part of the entropy change (Laidler and Ethier, 1953). It is possible that the remainder was due to the structural changes in the myosin which are known to occur on reaction with ATP. The negative values of $1 S$ found for nonionic enzyme interactions have their origin mainly in the association of enzyme and substrate, but it is possible that here there is also a small entropy term for the displacement of water.

The importance of water and entropy in protein interactions is indicated by the fact that the formation of the mercury dimer of seralbumin (Kirkwood, 1954, p. 16) and the combination of trypsin with soybean trypsin inhibitor (Sturtevant. 1954. p. 17) both show a negligible change in enthalpy. Yet there are large negative changes in free energy which must mean that $\triangle S$ is positive and that water molecules must be displaced from between the interacting protein molecules.

\section{Relation of Inhibition to Interaction Energy}

Since $K_{i}=e^{i F / R T}$, the relation between fractional inhibition and the interaction energy may be written as:

$$
i=\frac{(\mathrm{I})}{(\mathrm{l})+x e^{\overline{A F / R T}}}
$$

where $x$ depends on the type of inhibition and on the value of $\alpha$, tha factor by which the binding of inhibitor is changed in the presence of bound substrate (see Eq. 3-21); for noncompetitive inhibition $x=1$ and for competitive $x=1+\left[(\mathrm{S}) / K_{s}\right]$. Equation 6-102 is plotted in Fig. 6-15 to show the nature of the dependence of inhibition on the interaction energy. In the midrange of the curves, the inhibition is quite sensitive to small changes in the interaction energy; a change in $1 F$ of $0.25 \mathrm{kcal} / \mathrm{mole}$ will increase or decrease the inhibition by $10 \%$. When a series of related inhibitors is tested at the same concentration, the relative interaction energies may be read off the appropriate curve. 


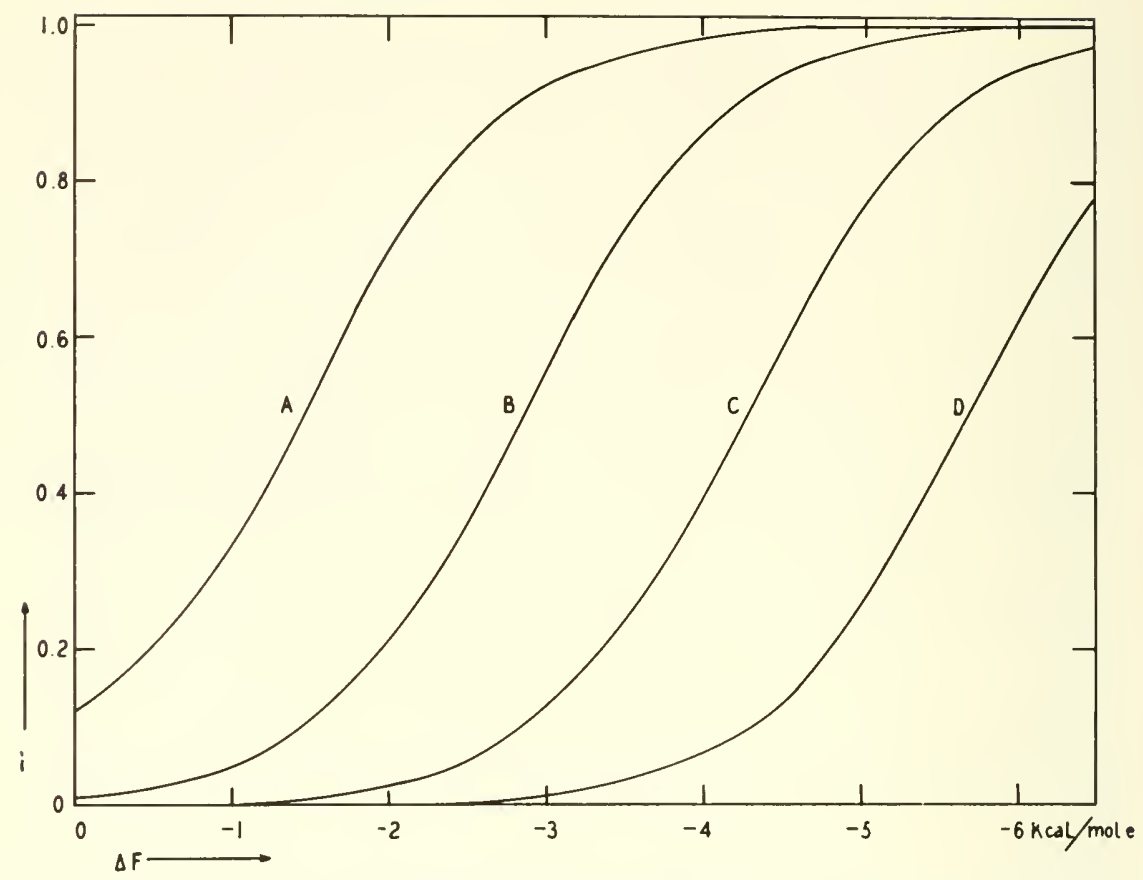

FIG. 6-15. Variation of the fractional inhibition with the free energy of interaction between the enzyme and the inhibitor (Eq. 6-102). Curve $\mathrm{A}$ : $(\mathrm{I}) / x=0.1$; eurve $\mathrm{B}$ : $(\mathrm{I}) / x=0.01$; curve $\mathrm{C}:(\mathrm{I}) / x=0.001$; curve $\mathrm{D}:(\mathrm{I}) / x=0.0001$.

\section{Determination of Relative Interaction Energies}

The over-all free energy change may be calculated from the value of $K_{i}$ but this usually includes energy terms other than for the direct interaction of enzyme and inhibitor. These other terms, such as for the displacement of water or ionic atmosphere, are difficult to evaluate accurately. Thus it is generally impossible to derive a value for the enzyme-inhibitor interaction alone. However, it is often this energy that is of the most significance in deriving accurate spatial relations of the binding and evaluating the specific contributions of various groups on the inhibitor. Although it is not always possible to obtain an absolute value for this interaction energy, one can occasionally determine with sufficient accuracy the relative binding energies of two or more related inhibitors and attribute these differences to variations in the properties of the inhibitors.

Let us consider two structurally related competitive inhibitors. From the relations:

$$
\begin{aligned}
\Delta F_{1} & =1.422 \log K_{i_{1}} & \Delta F_{2} & =1.422 \log K_{i_{2}} \\
K_{i_{1}} & =\frac{\left(\mathrm{I}_{1}\right)\left(1-i_{1}\right)}{x i_{1}} & K_{i_{2}} & =\frac{\left(\mathrm{I}_{2}\right)\left(1-i_{2}\right)}{x i_{2}}
\end{aligned}
$$


where $x=1+\left[(\mathrm{S}) / K_{s}\right]$, the difference in binding energy is given by:

$$
\begin{aligned}
& \Delta F_{1}-\Delta F_{2}=1.422 \log \frac{K_{i_{1}}}{K_{i_{2}}} \\
& \Delta F_{1}-\Delta F_{2}=1.422 \log \frac{\left(\mathrm{I}_{1}\right)\left(1-i_{1}\right) i_{2}}{\left(\mathrm{I}_{2}\right)\left(1-i_{2}\right) i_{1}}
\end{aligned}
$$

If $50 \%$ inhibition is taken for both inhibitors:

$$
\Delta F_{1}-\Delta F_{2}=1.422 \log \frac{\left(\mathrm{I}_{1}\right)_{0.5}}{\left(\mathrm{I}_{2}\right)_{0.5}}
$$

where $(\mathrm{I})_{0.5}$ is the concentration of inhibitor inhibiting $50 \%$. If the same concentration is used for each inhibitor, $\left(\mathrm{I}_{1}\right)=\left(\mathrm{I}_{2}\right)$, and:

$$
\Delta F_{1}-\Delta F_{2}=1.422 \log \frac{i_{2}\left(1-i_{1}\right)}{i_{1}\left(1-i_{2}\right)}
$$

Knowing the concentration of each inhibitor and the degree of inhibition, is thus possible to calculate the difference in interaction energy; the accuracy of the experimental values will determine the reliability of the energy difference - with reasonable care it is possible to determine the value of $J F_{1}-J F_{2}$ to an accuracy of $0.01 \mathrm{kcal} / \mathrm{mole}$ or better.

If the two inhibitors are reasonably alike in properties and size, this difference in binding energy will be independent of factors such as changes in water structure or effects of the ionic atmosphere. If one inhibitor is $\mathrm{R}-\mathrm{A}$ and the other R-B, the difference in binding energy may be related to the different interactions of the groups $\mathrm{A}$ and B provided these groups are similar. For example, if $\mathrm{A}$ is a nonpolar group and $\mathrm{B}$ is an ionic group, the energy difference will not be an accurate expression of the binding difference becanse $J F$ for the ionic group will include terms that do not occur in that for the nonpolar group. However, such factors are corrected for in expressions for the interaction energy (Eq. 6-90) and hence the experimentally determined $\Delta F_{1}-\Delta F_{2}$ may be equated with such a corrected $\varphi_{e}$.

Although this procedure is the most reliable for the estimation of interaction energies of single groups or parts of inhibitor molecules, some precautions must be observed in the interpretation. When a group is altered, or replaced by another group, or when a group is added, the changes are not necessarily localized to the region of the group but may extend to adjacent parts of the molecule. Thus the placing of a group on a benzene ring not only changes that position on the ring but alters the electronic distribution and polarizability of the ring. The binding energy difference for $\varphi$ and $\varphi$-A will not be due entirely to the forces exerted by the group A 
on the enzyme but also to changes in the benzene ring and its interaction with the enzyme. Also it is possible that adding a group to a molecule may sterically prevent the rest of the molecule from approaching the enzyme surface as closely as before; the difference in binding energy will then not reflect only interactions of the added group.

\section{DETERMINATION OF THE EQUILIBRIUM DISTANCE $d_{e}$ FROM $K_{i}$}

The interaction energy clerived from $K_{i}$ may be used to estimate the equilibrium distance between the inhibitor and the enzyme. This applies, of course, to specific gromps whose interaction can be ascertained and not usually to the entire inhibitor molecule and the total interaction energy. When $\varphi_{e}$ is made up of several contributions, with different dependencies on separation distance $d_{e}$, calculation of the equilibrium distance is difficult and graphical methods must be used. If ionic interaction is considered, it might be thought that one could simply use the expression $d_{e}=305 z_{1} z_{2} / \varphi_{e} D$ obtained from Eq. 6-56. In the first place, one must decide upon what value of $D$ to nse. Since $D$ cannot be taken as nuity (as in a vacuum) or as 74.1, the bulk dielectric constant, but depends upon the separation distance, its value cannot be assigned without knowing $d_{e}$. Thus an equation such as 6-75 must be used which results in a quadratic expression for $d_{\ell}$. In addition, the effect of the ionic atmosphere, which also depends on the distance, should be included, according to Eq. 6-89 or 6-90. Finally, the contributions to $q_{e}$ from induced dipole and dispersion interactions should be included.

One of the most important interactions in inhibitions is that between an amino group and a carboxyl group in the ionized state. Let us assume that the value of . $I F$ for this interaction has been determined experimentally for a particular inhibitor and we wish to calculate the equilibrim separation distance between these ionic groups. The expression for the total potential energy may be written as:

$$
\begin{aligned}
& \varphi_{e}=-\frac{305 e^{\varkappa\left(r_{0}-d_{e}\right)}}{d_{e} D\left(1+x r_{0}\right)}-\frac{111\left(\alpha_{1}+\alpha_{2}\right) e^{\varkappa\left(r_{0}-d_{e}\right)}}{d_{e}{ }^{4} D^{2}\left(1+x r_{0}\right)}-\frac{119 \sqrt{Z} \alpha_{2} \alpha_{2}}{d_{e}{ }^{6}} \\
& \text { Ion-ion Ion-induced dipole Dispersion }
\end{aligned}
$$

using the equations derived in the previous sections. The following values may be used: $\alpha_{1}$ for the carboxyl group $=$ I.87, $\alpha_{2}$ for the amino gromp $=2.14 ; Z_{1}$ for the carboxyl group $=16$, and $Z_{2}$ for the amino group $=8$, whence $\sqrt{Z}=11.3$ since $\sqrt{Z}=Z_{1} Z_{2}$, and $D=6 l_{c}-7$. With regard to $r_{0}$

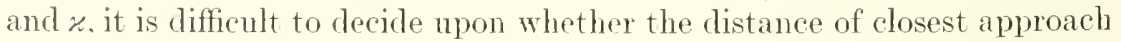
should involve the primary hydration layers or not. Calculations will be 
made for three cases: ( $a$ ) assuming the water shell does not enter into the closest approach, in which case $r_{0}=3.0 \AA$ and $\psi$ may be taken as 0.133 as derived above; $(b)$ assuming the atmosphere ions and the involved ions do not lose their primary hydration, in which case $r_{0}=10.2 \AA$ (diameter of two water molecules being $7.2 \AA$ ) and $\%$ can be taken as 12 , since it must be somewhat larger than $r_{0}$; and $(c)$ neglecting the ionic atmosphere entirely, which would apply when the ionic strength is very low or when the charged group is within a large inhibitor molecule and uninfluenced by the free ions in the solution. The energy equation for the first case may be written as:

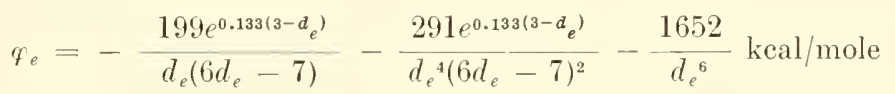

and similar equations may be written for the other situations. The energy contributed by each type of interaction at various distances for the three cases considered are shown in Table 6-18 and the total interaction energy is calculated. Figure $6-16$ shows the variation of $q_{e}$ or $\angle F$ with the equilibrium distance. This is not a potential energy-distance plot in the ordinary sense, but gives the conditions only at equilibrium separation. From these curves one may determine $d_{e}$ from the experimental value of $\_F$. It may be seen that whichever assumption is made regarding the elosest approach of the ionic atmosphere, there is not a great difference in the results, except at low interaction energies when the equilibrium distances will differ by as much as $1 \AA$. Preference here will be put on curve $\mathrm{B}$ since it is likely that the atmosphere ions approach the interacting ions at their closest with two layers of water molecules between them. The $-\mathrm{NH}_{3}^{+}$and $-\mathrm{COO}^{-}$ ionic groups, however. can lose their hydration layers upon interaction. These curves will be applied to experimentally determined interaction energies in the following sections.

George (1959) suggested that there are two types of association between ions in aqueous solntion. In the first type there is a loss of the hydration water in the association process, in which case the entropy change is related to the entropy of hydration. This appears to apply to associations of cations with singly-charged anions, such as $\mathrm{Cl}^{-}, \mathrm{Br}^{-}, \mathrm{F}^{-}, \mathrm{OH}^{-}$, and $\mathrm{HCO}_{3}{ }^{-}$. In the second type the association does not involve a loss of the hydration water and the various entropy and energy terms that are related to the hydration are not so important. This would apply more particularly to the doubly charged cations and anions, such as the sulfates of $\mathrm{Mg}^{++}, \mathrm{Ca}^{++}$, $\mathrm{Ba}^{++}$, and $\mathrm{Pb}^{++}$. It was felt that a simple coulombic treatment might be satisfactory for associations of the second type, but that the disturbances in water structure occurring in associations of the first type would complicate the situation. Yet Eigen (1957) from the relaxation spectra of 2:2 electrolytes in aqueous solution had concluded that specific ionic interactions 


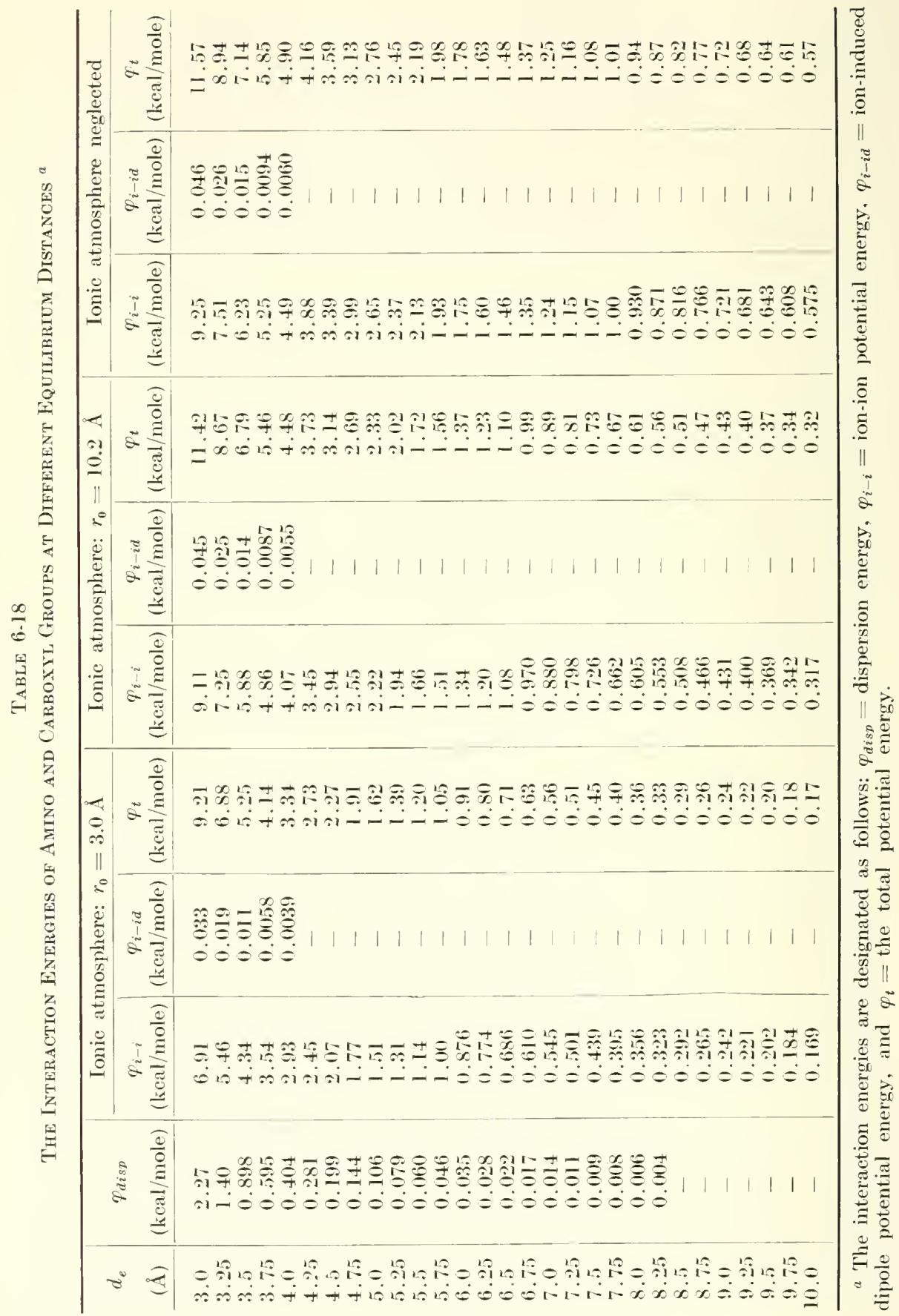


occur when the ions approach within a distance of three water layers, and that this interaction energy could not be described adequately by a coulombic formulation, whether one used a macroscopic dielectric constant or one that decreases with approach of the ions. The problem of hydration water displacement in ion-ion interactions is certainly not solved at the present time.

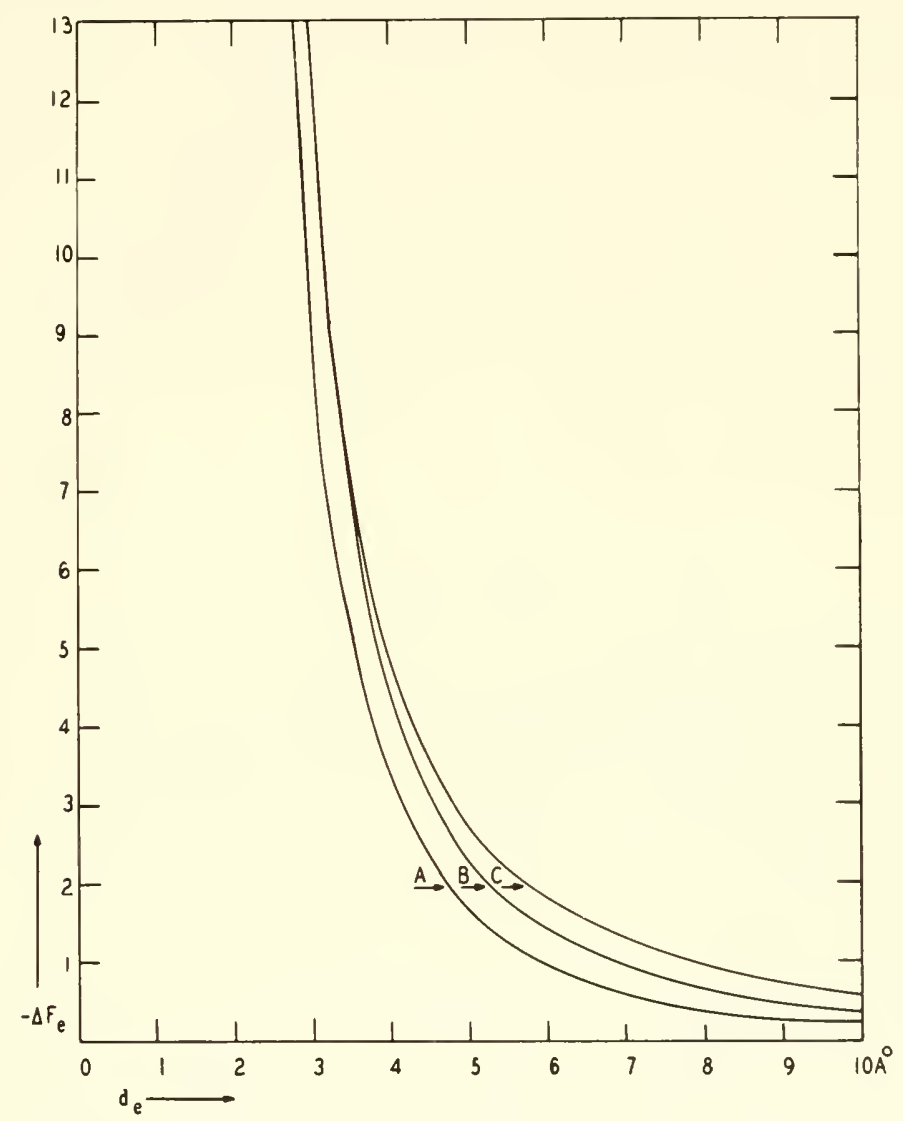

FIG. 6-16. Dependence of the interaction energy on the equilibrium distance between $-\mathrm{NH}_{3}{ }^{+}$and $-\mathrm{COO}^{-}$groups (Eq. 6-109). Curve A: $r_{0}=4 \AA$; curve $\mathrm{B}: r_{0}=10.2 \AA$; curve C: ionic atmosphere neglected.

Many different approaches to the problem of the association of ions in aqueous solution have been used and all of this work is intimately related to the interactions between ionic groups on enzymes and inhibitors. Fuoss (1958) has assumed the solvent to be a continum and thus there are no 
factors in his expressions for the ion-solvent interactions. His expression for the association constant of an ion pair must therefore be considered as very approximate.

$$
K=2.524 \times 10^{-3} a^{3} e^{b}
$$

where $a=$ the ionic diameter in $\AA$ and $b=e^{2} / a D k T$. A much more complex, but probably more accurate, treatment has been given by Levine and Wrigley (1957) for the interaction energy of two univalent ions in water at small separations. The region around an ion is divided into two portions: a primary hydration shell and the rest of the water (which is assumed to be a continuous dielectric medimm). The dipole moments that are induced in the hydration water molecules by the ion are calculated and taken into account. The energy of two hydrated ions in a vacumm is calculated and then to this is added the energy required to introduce the dielectric medium. The deviation from the simple conlombic expression is given by a correction factor, $\varphi_{c}$ :

$$
q^{\prime}=\left(e^{2} / d D\right)+p_{c}
$$

The correction factor takes the form:

$$
q_{c}=\frac{C_{4}}{d^{4}}+\frac{C_{6}}{d^{6}}+\frac{C_{7}}{d^{7}}
$$

where the $C$ 's are complex functions of the ionic polarizability, dielectric constant, and other factors. Calculations were male for potassium fluoride and it was found that at ion separations of $6-8 \AA$ (contact of hydration shells) $\varphi_{c}$ was about $+8 \%$ of the conlombic term. It is also interesting that the effective dielectric constant for the hydrated ions was found to be close to 2.5 .

\section{INTERACTIONS OF HAPTENS WITH ANTIBODIES}

Much of the quantitative work on antibody-hapten binding reported during the past 15 years, especially by Panling, Pressman, and their associates. can be applied to interactions of inhibitors with enzymes. In both cases one is dealing with the binding of relatively small nolecules to proteins, the sane types of interaction forces are involved, there are similar orientation factors, and in both there is usually a specifically patterned site on the protein where the binding occurs. The energies for the interactions of varions simple groups with proteins have been rerived from hapten inhibition studies and these values are useful in estimating energy contributions from enzyme inhibitor groups, as well as providing an experimental test of the theoretical interaction equations derived above. 
The basic procedure is to produce antibodies in rabbits by injecting sheep serum coupled with some substance such as diazotized $p$-arsanilic acid and then precipitate these antibodies by mixing with simple polyhaptenic substances possessing $p$-diazophenylarsonate groups. When various substituted monohaptenic phenylarsonates are present, the precipitation is inhibited due to these substances competing with the polyhaptens for the specific binding sites. From the degree of inhibition produced, the binding relative to a standard hapten can be determined and expressed as a combining constant $K_{0}{ }^{\prime}$; the standard hapten, such as phenylarsonate, is assigned a $K_{0}{ }^{\prime}=1$. These constants then are not absolute and do not relate directly to the free energy of binding. However. from them may be calculated the difference in binding energy between any two compounds using Eq. 6-105 (with a numerical factor of 1.273 since equilibrium is usually at 50 ). It is usually convenient to compare the substituted phenylarsonate haptens with the standard unsubstituted compound, in which case the energy differences may be correlated with the substituent group.

$$
\text { protein }-\mathrm{N}=\mathrm{N}-
$$

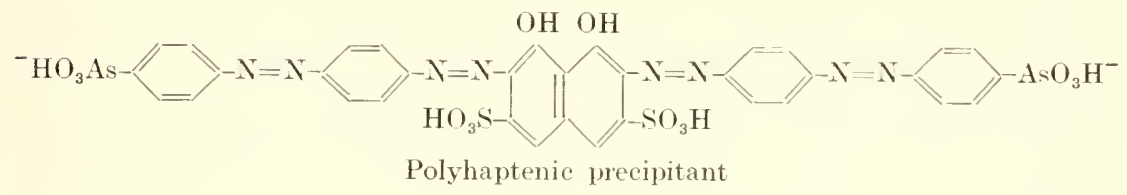

$$
\begin{aligned}
& =\mathrm{AsO}_{3} \mathrm{H}^{-} \\
& \text {Standard inhibitor }
\end{aligned}
$$

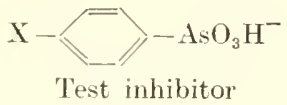

\section{Determination of Group Interaction Energies}

The binding of the inhibitor hapten $\mathrm{X}-\boldsymbol{\varphi}-\mathrm{AsO}_{3} \mathrm{H}^{-}$to the antibody protein would result from the following interactions: (1) the electrostatic attraction between the negative arsonate group and a positive charge on the protein; (2) dipole-dipole interactions between the arsonate group and the protein; (3) the dispersion attraction between the arsonate group and the benzene ring for the protein; (4) the dispersion attraction between the group $\mathrm{X}$ and the protein; and (5) other interactions between the group $\mathrm{X}$ and the protein, depending on the nature of $\mathrm{X}$. Comparing the inhibitor 
haptens, interactions (1), (2), and (3) may be assumed to remain constant, so that differences in binding energy may be attributed to interactions of the group $\mathrm{X}$ with the protein, specifically that part of the protein adjacent to the $-\mathrm{N}=\mathrm{N}-$ group in the normal antigen-antibody reaction. Let us first consider the binding energy difference due only to dispersion forces between the group $\mathrm{X}$ and the protein. The displacement of water originally adjacent to both hapten and antibody must also be considered. Furthermore, the substitution of the group $\mathrm{X}$ on the benzene ring replaces a hydrogen atom, the interaction of which must be included. The reactions may now be written:

$$
\begin{array}{ll}
\text { Unsubstituted hapten: } & \mathrm{H}(\mathrm{W})+\mathrm{A}(\mathrm{W}) \rightarrow \mathrm{HA}+\mathrm{W} \\
\text { Substituted hapten: } & \mathrm{N}(\mathrm{W})+\mathrm{A}(\mathrm{W}) \rightarrow \mathrm{XA}+\mathrm{W}
\end{array}
$$

where $\mathrm{H}$ is phenylarsonate, $\mathrm{X}$ is $\mathrm{X}$-phenylarsonate. $\mathrm{A}$ is antibody, and W represents an unspecified number of water molecules. The dispersion energy changes for the reactions are given by:

$$
\begin{aligned}
& \varphi_{H}=\varphi_{H-A}+\varphi_{W-W}-\varphi_{H-W}-\varphi_{A-W} \\
& \varphi_{X}=\varphi_{X-A}+\varphi_{W-W}-\varphi_{X-W}-\varphi_{A-W}
\end{aligned}
$$

and the difference in dispersion energy is:

$$
\Delta \varphi_{d i s p}=\varphi_{X}-\varphi_{H}=\varphi_{X-A}-\varphi_{X-W}-\varphi_{H-A}+\varphi_{H-W}
$$

From Eq. 6-69 the individual dispersion terms may be written as:

leading to:

$$
\begin{aligned}
& \eta_{X-A}=-18.7 \sqrt{Z_{1}} R_{X} R_{A} / d_{e}{ }^{8} \\
& \varphi_{X-W}=-18.7 n \sqrt{Z_{2}} R_{X} R_{W} / d_{e}{ }^{8} \\
& \varphi_{H-A}=-18.7 \sqrt{Z_{3}} R_{H} R_{A} / d_{e}{ }^{8} \\
& \varphi_{H-W}=-18.7 n \sqrt{Z_{4}} R_{H} R_{W} / d_{e}{ }^{6}
\end{aligned}
$$

$\varphi_{d i s p}=-\frac{18.7}{d_{e}{ }^{6}}\left[\sqrt{Z_{1}} R_{X} R_{A}-n \sqrt{Z_{2}} R_{X} R_{W}-\sqrt{Z_{3}} R_{H} R_{A}+n \sqrt{Z_{4}} R_{H} R_{W}\right]$

where $Z_{1}=\sqrt{Z_{X} Z_{A}}, Z_{2}=\sqrt{Z_{X} Z_{W}^{-}}, Z_{3}=\sqrt{Z_{I I} Z_{A}}, Z_{4}=\sqrt{Z_{I I} Z_{W}}, n=$ the number of water molecules adjacent to hapten group, and where the equilibrium distance $d_{e}$ is assumed to be the same for each interaction. From the molar refractions, electron numbers and van der Waals' radii of the 
various components, the values of $\Delta \varphi_{d i s p}$ may be calculated and compared with the experimental values derived from the combining constants $K_{0}{ }^{\prime}$.

The equilibrium distance $d_{e}$ may be estimated by adding the group van der Waals' radius to an average protein group radius. The latter was assigned a value of $2.0 \AA$ by Panling and Pressman (1945) but it is felt that this figure is a little large. Perusal of Table 6-9 for groups common in proteins, such as amino, hydroxyl, phenyl, hydrocarbon, and peptide, and considering that these groups will often be flexible and adjust to nearminimal distance, leads one to conclude that the average protein group radius is nearer $1.8 \AA$. Next, one must assume some reasonable figure for the number of water molecules adjacent to the hapten groups and antibody site. Pauling and Pressman assumed $n=8$ but it is likely, since these groups usually will not disrupt the water structure and pack closely about the group, that this value is high. If one assumes that the hapten groups are not greatly different in size from a water molecule, it will be seen that $n=3$ will be reasonable (the fourth water molecule that would surround the isolated group is replaced by the bond). Finally, the molar refraction of the antibody, which refers to the integrated refractions of the protein groups over the surface in contact with the hapten group, must be estimated. Pauling and Pressman calculated a value of $R_{A}=5.90 \mathrm{ml}$ for a portion of the protein equivalent to one water molecule, using the index of refraction of squash seed globulin. From the summary of the indices of refraction of eight wa :r-free proteins by Doty and Geiduschek (1953), the average value would be 1.604 , from which $R_{A}=5.28 \mathrm{ml}$, and this value, as probably more accurate, will be used here. It must be noted that $R_{A}$ will differ in different regions of the protein and that the figure assumed above is approximate only.

Equation 6-116 may now be rewritten nsing $n=3$ and reasonable values for the electron number $Z$ :

$$
\varphi_{d i s p}=-\frac{18.7}{d_{e}^{6}}\left[2.83 R_{X}-2 R_{H}\right]\left[3 R_{A}-3 R_{W}\right]
$$

for all the groups considered except $\mathrm{NO}_{2}$ and $\mathrm{COO}^{-}$, for which the numerical factor of $R_{X}$ is 3.46. Calculations based on this equation are presented in Table 6-19 and compared with the experimental values for the smaller hapten groups. The combining constants for the two antibodies often differ due to the different patterns presented by the regions adjacent to the groups; it is hoped that by averaging the energy differences, forces others than dispersion, such as hydrogen bonding, may be partly eliminated. The agreement between the experimental over-all free energy changes and the calculated dispersion energy is quite good considering that the equilibrium distances can only be approximately estimated. It would appear that dispersion forces are mainly responsible for the interaction of these groups 
with the antibody, but it is probable that dipole-dipole effects do occnr in individnal instances. The deviations for $-\mathrm{OH},-\mathrm{NH}_{2}$, and $-\mathrm{NO}_{2}$ indicate that on the p-azophenylarsonate antibody there is a dipole with positive end lirected toward the surface of contact, whereas in the $p$-phenylazobenzoate antibody there is a dipole with the opposite orientation.

TABLE 6-19

Group Dispersion Energies in Interactions of Haptens with Antibodies to p-Azophenylarsonate and p-Phexylazobenzoate ${ }^{a}$

\begin{tabular}{|c|c|c|c|c|c|c|c|}
\hline \multirow{2}{*}{ Group } & \multirow{2}{*}{$\begin{array}{c}\text { Molar } \\
\text { refraction } \\
R_{X} \\
\text { (ml/mole) }\end{array}$} & \multirow{2}{*}{$\begin{array}{c}\text { Group } \\
\text { radius } \\
r_{X} \\
(\AA)\end{array}$} & \multirow{2}{*}{$\begin{array}{c}\text { Separation } \\
\text { distance } \\
d_{e} \\
(\mathrm{~A})\end{array}$} & \multirow{2}{*}{$\begin{array}{c}\text { Calculated } \\
\text { energy } \\
\text { difference } \\
\text { If } \text { disp } \\
\text { (kcal/mole) }\end{array}$} & \multicolumn{2}{|c|}{$\begin{array}{l}\text { Combining } \\
\text { constants }\end{array}$} & \multirow{2}{*}{$\begin{array}{c}\text { Average } \\
\text { exptl. } \\
\text { energy } \\
\text { difference } \\
\triangle \Delta F \\
\text { (kcal/mole) }\end{array}$} \\
\hline & & & & & $\begin{array}{l}K_{0}{ }^{\prime} \\
\text { (1) }\end{array}$ & $\begin{array}{l}K_{0}^{\prime} \\
(2)\end{array}$ & \\
\hline $\begin{array}{l}-\mathrm{CH}_{3} \\
-\mathrm{Cl} \\
-\mathrm{Br} \\
-\mathrm{I} \\
-\mathrm{OH} \\
-\mathrm{NH}_{2} \\
-\mathrm{NO}_{2} \\
-\mathrm{COO}^{-}\end{array}$ & $\begin{array}{r}6.15 \\
6.35 \\
9.19 \\
14.08 \\
3.26 \\
5.15 \\
6.42 \\
5.93\end{array}$ & $\begin{array}{l}1.92 \\
1.80 \\
1.95 \\
2.15 \\
1.60 \\
1.85 \\
2.62 \\
2.10\end{array}$ & $\begin{array}{l}3.72 \\
3.60 \\
3.75 \\
3.95 \\
3.40 \\
3.65 \\
3.82 \\
3.90\end{array}$ & $\begin{array}{l}-0.58 \\
-0.74 \\
-0.89 \\
-1.05 \\
-0.42 \\
-0.52 \\
-0.66 \\
-0.53\end{array}$ & $\begin{array}{l}3.30 \\
4.15 \\
4.63 \\
5.97 \\
1.73 \\
1.36 \\
9.92 \\
1.26\end{array}$ & $\begin{array}{l}1.80 \\
5.30 \\
5.40 \\
9.00 \\
4.70 \\
2.10 \\
1.80 \\
5.30\end{array}$ & $\begin{array}{l}-0.49 \\
-0.85 \\
-0.89 \\
-1.10 \\
-0.57 \\
-0.29 \\
-0.79 \\
-0.52\end{array}$ \\
\hline
\end{tabular}

a The following molar refractions were used: $R_{W}=3.67, R_{A}=15.84$, and $R_{H}=$ 1.65. The electron numbers were estimated to be: $Z_{H}=2, Z_{A}=8 . Z_{W}=8$, and $Z_{X}=18$ for $\mathrm{NO}_{2}$ and $\mathrm{COO}()^{-}$, and 8 for the rest, leading to $Z_{1}=Z_{2}=1.2$ for $\mathrm{NO}_{2}$ ane $\mathrm{COO}^{-}$ and 8 for the rest, and $Z_{3}=Z_{4}=4$. The average radius of antiloody groups was taken as $1.8 \AA$. Interaction of the hapten group with three water molecules was assumed. The combining constants, $K_{o}^{\prime}(1)$ were averages obtained from Pressman and Siegel (1953) and were corrected for nonspecific binding to serum: the $K_{o}{ }^{\prime}(2)$ 's were obtained from Nisonoff and Pressman (1957). $K_{0}^{\prime}(1)$ 's are for antiborly to $p$-azophenylarsonate and $K_{o}^{\prime}(2)$ 's are for antibody to $p$-phenylazobenzoate.

The procedure used in these calculations was developed by Panling and Pressman (1915) and the modifications introduced here were made to incorporate more accurate values of $K_{0}^{\prime}$ (Pressman and Siegel, 1953) and $R_{A}$, as well as to apply the extended equations derived in the previous sections. The agreement with experiment is gratifying and provides a certain amount of confidence in the equations and the assumptions upon which the treatment is based. 


\section{Determination of Electrostatic Interactions of Ionic Groups}

Antibody to $p$-azophenyltrimethylammoninm ion

$$
-\mathrm{N}=\mathrm{N}-\varphi-\mathrm{N}\left(\mathrm{CH}_{3}\right)_{3}{ }^{+}
$$

was reacted with two haptens that were identical except one contained the trimethylammonium ionic group and the other a neutral tertiary butyl group.

The former
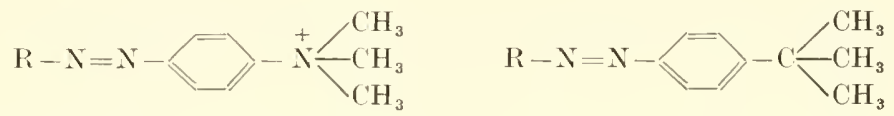

was bound more tightly and this difference may be attributed to the electrostatic attraction between the positively charged hapten and a negative antibody group. The ratio of the combining constants is 8.0 and hence the binding energy difference is $1.15 \mathrm{kcal} /$ mole (Pressman et al., 1946; Pressman and Siegel, 1953). The distance between charge centers was calculated to be $8 \AA$, leaving approximately $3 \AA$ separation between the van der Waals' surfaces of the ionic grouns, sufficient space to accomodate a molecule of water. However, since the reaction occurred in a physiological medimm, the effect of the ion atmosphere should be taken into. account. Using Eq. 6-90, an equilibrium distance $d_{e}$ between charge centers of $6.35 \AA$ is obtained. If the groups interact head-on, $0.6 \AA$ must be added to this as the distance from the charge center to the carbon-atom of the carboxylate group, giving $6.95 \AA$ as the distance between group centers. The plot of Eq. 6.90 is shown in Fig. 6-17 to facilitate such calenlations of $d_{e}$. The distance of closest head-on approach would be $5.32 \AA$, using the van der Waals' radii in Table 6-9; if interaction were along the $\mathrm{C}-\mathrm{O}$ and $\mathrm{N}-\mathrm{C}$ bond directions, the distance wonld be $5.10 \AA$, this being the maximal distance when the groups are in contact. There is, however, an energy term that has been omitted; the $-\mathrm{N}\left(\mathrm{CH}_{3}\right)_{3}^{+}$group will attract water molecules more strongly than the $-\mathrm{C}\left(\mathrm{CH}_{3}\right)_{3}$ group, and some energy may be necessary to displace these. If this displacement energy amounted to only $0.7 \mathrm{kcal} /$ mole, making the true ion-ion interaction energy $1.85 \mathrm{kcal} / \mathrm{mole}$, the calculated separation would be that for head-on contact. It seems likely that these ionic groups interact directly without interposed water molecules.

A similar determination was made with antibody to $p$-( $p^{\prime}$-azophenylazo)benzoate and haptens that were identical except that one had a terminal negatively charged benzoate group and the other a terminal nitrobenzene group; the former was hound very tightly with a $K_{0}{ }^{\prime}=89$ and the latter was bound so weakly that the $K_{0}{ }^{\prime}$ could not be determined but was less than 0.01 . The difference in binding energy is thus at least 5.0 kcal/mole and possibly more. Reference to Fig. 6-17 shows that this indicates a charge 
separation of $3.70 \AA$ or a group center separation of approximately $4.30 \AA$. Van der Waals' radii give minimal separation of $3.85 \AA$ and maximal separation of $4.56 \AA$, depending on the orientation of the groups, so that

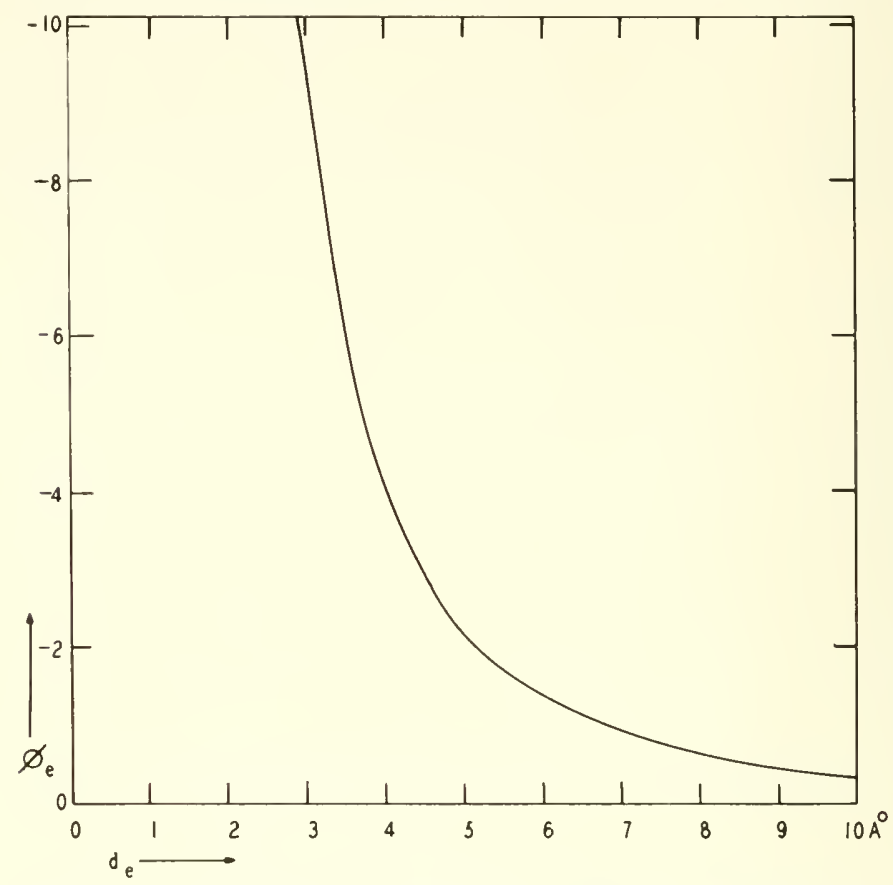

Fia. 6-17. Plot of Eq. 6-90 for ion-ion interactions, assuming that $\mu=0.16$, for the purpose of estimating equilibrium distances from experimental interaction energies.

the calculated value is in very satisfactory agreement assuming direct group contact. These results are very similar to those calculated by Nisonoff and Pressman (1957).

\section{Specificity of the lonic Group}

Antibody to the $p$-azophenylarsonate group will react equally with the corresponding phosphonate group, but will not react with sulfonate, carboxylate, methylarsonate, or stibonate groups (Pressman et al., 1945). Antibodies to the benzoate group will similarly not combine with the corresponding sulfonate or arsonate (Nisonoff and Pressman, 1957). In the former case the specificity is surprising inasmuch as the sulfonate and carboxylate groups are smaller than the arsonate group and wouk be expected to fit in satisfactorily. The difference in binding energy between 
these groups cannot be estimated (since $K_{0}{ }^{\prime}$ for sulfonate and carboxylate is given as 0 ), but it is certainly greater than $2.5 \mathrm{kcal} /$ mole. Since steric factors reducing the interaction of sulfonate and carboxylate cannot be important, there must be forces other than ion-ion attraction to stabilize the arsonate complex. Hydrogen bonds between the arsonate<smiles>[3H][As]([3H])(O)(O)[18OH]</smiles>

or phosphonate<smiles>[3H]P1([3H])([3H])OCO1</smiles>

groups with an adjacent : $\mathrm{X}$ group on the antibody surface might account for part of this difference but not all. In any event, it is a very interesting example of ionic specificity and similar situations will be found in enzymeinhibitor interactions.

\section{Effect of the Ionic Group Size}

The antibody to $p$-azophenyltrimethylammonium combines more weakly with haptens as the size of the charged group is increased (Pressman et al., 1946; Pressman, 1957). The experimental combining constants, $K_{0}{ }^{\prime}$, are given in Table 6-20 and calculations based on head-on interaction with a carboxylate ion are given, using Eq. 6-90 to account for the effect of the ion atmosphere. It is seen that the predicted $K_{0}$ 's are quite in accord with

TABLE $6-20$

Effect of Ionic Group Size or Haptex-Antibody Ixteraction a

\begin{tabular}{|c|c|c|c|c|c|}
\hline Group & $\begin{array}{c}\text { Radius } \\
\text { (A) }\end{array}$ & $\begin{array}{l}d_{e} \\
(\AA)\end{array}$ & $\begin{array}{c}p_{e} \\
\text { (kcal/mole) }\end{array}$ & $\begin{array}{c}\text { Calculated } \\
K_{o}^{\prime}\end{array}$ & $\begin{array}{c}\text { Experimental } \\
\qquad K_{0}^{\prime}\end{array}$ \\
\hline$+\stackrel{+}{-} \underset{\mathrm{CH}_{3}}{\mathrm{CH}_{3}}$ & 3.17 & 5.32 & 1.86 & 1.00 & 1.00 \\
\hline$-\mathrm{As}=-\begin{array}{l}-\mathrm{CH}_{3} \\
\mathrm{CH}_{3}\end{array}$ & 3.68 & 5.83 & 1.45 & 0.48 & 0.49 \\
\hline$-\mathrm{N}-\mathrm{CH}_{2} \mathrm{CH}_{3}$ & 4.17 & 6.32 & 1.17 & 0.29 & 0.2 .2 \\
\hline
\end{tabular}

"The ionic group radii were calculated on the basis of head-on approach. The combining constant $K_{0}^{\prime}$ for the trimethylammonium ion is assumed to be unity in both cases. 
the experimental values, from which it is probable that the reduced interaction energy is indeed dne to the increasing separation between the charge centers.

\section{Effect of Group Position on Interaction Energy}

The antibody binding site for the antigenic gromp must be patterned quite closely to the van der Waals' surface of this group because replacement of the small hydrogen atom on a benzene ring by a large group rednces the interaction energy. The energy decrease attending the substitution of a chlorine atom is pictured in Fig. 6-18 for a typical hapten-antibody interaction. The fact that different energy decreases are observed for the

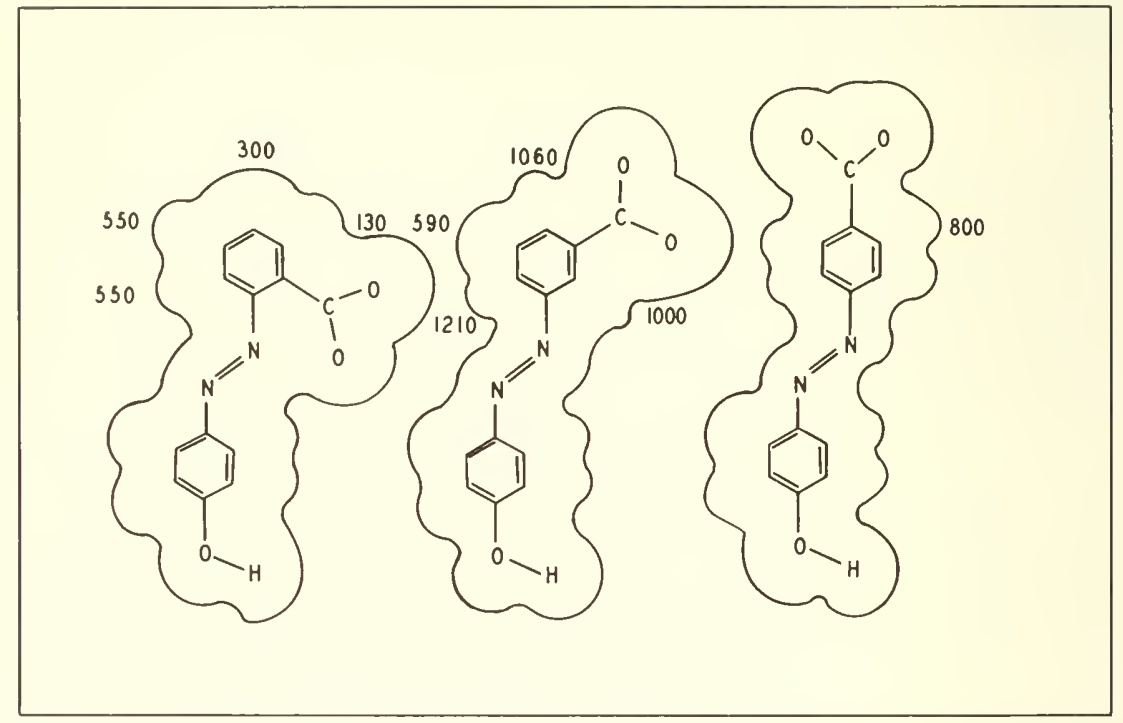

FIG. 6-18. The steric repulsion energies for chloro-substituted haptens reacting with antibodies to $o-, m$-, and $p$-azophenylazobenzoate. The numbers show the decrease in over-all binding energy when chlorine atoms are substituted in the positions indicated; the values are in eal/mole and have been recaleulated from the combining constants given by Pressman t al. (1954). The outlines give approximately the van der Waals' contours of the antigenic groups.

various ring positions means that the eloseness of fit of the antibody around the hapten is not the same everywhere. It is generally true that ortho substitution produces the greatest energy change, indicating that the fit is closer here than in the meta position, as is secn in Table 6-21 where the results with substituted benzoates are given. Para substitution results in increased binding, of course, because the group then fits in the region formed around the $-\mathrm{N}=\mathrm{N}$ - group. An increase in size from the hydrogen atom to the 
chlorine atom $(0.6 \AA)$ results in $1.22 \mathrm{kcal} /$ mole less bincting energy in the $o$-position and the larger groups, such as $-\mathrm{I}$ or $-\mathrm{NO}_{2}$. produce an even greater effect. The origin of this effect may be sought in three possible contributions: (1) the substituent group may prevent the adjacent parts of the hapten molecule from approaching as closely to the antibody as with the unsubstituted compound, (2) the substituent group may tilt the plane of the benzene ring so that it and the planar carboxylate group are not oriented for maximal interaction, and (3) the substituent group may dilate that region of the enclosing antibody, a process requiring energy. The important conclusion is that binding will be increased by a substitnent group only when there is space for it to oceupy. Such large steric energies

TABLE 6-21

The Effect of Substituents ox Benzonte Interaction with the Antibody TO $p$ - $\left(p^{\prime}\right.$-AZOPHENYLAZO)-BENZOATE ${ }^{a}$

\begin{tabular}{|c|c|c|c|}
\hline \multirow[b]{2}{*}{ Group } & \multicolumn{3}{|c|}{ Change in free energy } \\
\hline & $\begin{array}{c}\text { Ortho } \\
\text { (kcal/mole) }\end{array}$ & $\begin{array}{c}\text { Meta } \\
\text { (kcal/mole) }\end{array}$ & $\begin{array}{c}\text { Para } \\
\text { (kcal/mole) }\end{array}$ \\
\hline $\begin{array}{l}-\mathrm{CH}_{3} \\
-\mathrm{Cl} \\
-\mathrm{I} \\
-\mathrm{NO}_{2} \\
-\mathrm{COO}^{-}\end{array}$ & $\begin{array}{r}+1.94 \\
+1.22 \\
+2.40 \\
>+2.50 \\
>+2.50\end{array}$ & $\begin{array}{r}+0.86 \\
+0.46 \\
+0.69 \\
+1.17 \\
+0.06\end{array}$ & $\begin{array}{l}-0.32 \\
-0.92 \\
-1.21 \\
-0.32 \\
-0.92\end{array}$ \\
\hline
\end{tabular}

${ }^{a}$ From Nisonoff and Pressman (1957).

indicate that hapten-antiboly interactions must generally occur within cavities or trenches. The effects of substitution on interactions with a relatively flat protein surface would be expected to be of lesser magnitude.

With respect to position effects, it is worthwhile mentioning that separation of the benzene ring from the charged group by one $-\mathrm{CH}_{2}-$ group results in loss of binding. Thus $\varphi-\mathrm{CH}_{2}-\mathrm{AsO}_{3} \mathrm{H}^{-}$is not bound to the antibody for $p$-azophenylarsonate, whereas $\varphi-\mathrm{AsO}_{3} \mathrm{H}^{-}$is readily bound (Pressman, 1957). Likewise, $\varphi-\mathrm{CH}_{2}-\mathrm{COO}^{-}$is not bound to antibody for $p$-( $p^{\prime}$-azophenylazo)-benzoate, while $\varphi-\mathrm{COO}^{-}$is bound (Nisonoff and Pressman, 1957). Such increased separation would result in displacement of the benzene ring or the charged group or both from the closely fitting antibody region. The benzene ring is necessary for hapten binding, since $K_{0}{ }^{\prime}$ is zero for both $\mathrm{CH}_{3}-\mathrm{AsO}_{3} \mathrm{H}^{-}$and $\mathrm{CH}_{3}-\mathrm{COO}^{-}$, indicating that the ring contributes at least $2.5 \mathrm{kcal} /$ mole to the total binding energy. The antibody to the $p$-azophenyltrimethylammonium group reacts well with 
$\varphi-{ }^{+} \mathrm{N}\left(\mathrm{CH}_{3}\right)_{3}$ but not with $\mathrm{CH}_{3}-{ }^{+} \mathrm{N}\left(\mathrm{CH}_{3}\right)_{3}$, the difference in binding energy being $4.7 \mathrm{kcal} / \mathrm{mole}$ (Pressman et al., 1946). Induction forces involving the benzene ring are of primary importance in the binding of these haptens.

\section{Effect of Hydration on Hapten-Antibody Binding}

We noted previously that the free energy for binding of the divalent hapten ${ }^{-} \mathrm{HO}_{3} \mathrm{As}-\boldsymbol{\varphi}-\mathrm{NHCO}-\boldsymbol{\varphi}-\mathrm{CONH}-\boldsymbol{\varphi}-\mathrm{AsO}_{3} \mathrm{H}^{-}$to antibody for $p$-azophenylarsonate is mainly derived from the entropy change due to water displacement, and such must occur to a greater or lesser extent in all combinations of hapten and antibody. However, when water is bound tightly to a group on the hapten, so that it is not displaced upon interaction, it alters the contour of the molecule much as a substituent group would do. This is clearly seen when a pyridine ring replaces the benzene ring of haptens reacting with antibody to the azobenzoates (Pressman and Siegel, 1957). The pyridine carboxylates, for example, are bound with 1.2-1.5 $\mathrm{kcal} / \mathrm{mole}$ less energy than benzoate to the antibody for 0 -azobenzoate. These effects are greater than occur upon substitution of a chlorine atom on the benzene ring, indicating that the pyridine nitrogen atom has a greater effective size than the chlorine atom, which could result only from the hydration of the pyridine. It is known that pyridine is hydrated in aqueous solution from the fact that it is miscible with water whereas benzene is only slightly soluble; the heat of hydration of pyridine is $12 \mathrm{kcal} /$ mole. Thus one cannot always consider just the contour of the hapten molecule itself but the spatial modifications produced by hydration.

\section{INTERACTION OF SUBSTRATES AND INHIBITORS WITH CHOLINESTERASE}

The quantitative approach to the treatment of hapten-antibody interactions was presented by Pauling in lectures at Oxford in 1948. He pointed out that this method was applicable to enzymes and Adams and Whittaker published from Oxford in 1950 the original treatment of enzyme complexes in terms of intermolecular forces. The enzyme cholinesterase was convenient to use for this purpose and since that time several investigations have been directed toward the elucidation of the active site and the forces it exerts on both substrates and inhibitors.

\section{Electrostatic Interaction of Ionic Groups}

The true erythrocyte cholinesterase, which splits acetylcholine and other choline esters at a very rapid rate, would appear to possess within its active site an anionic group, presumably a carboxylate ion, which reacts with the 
cationic group of the substrate. The evidence lies mainly in the different bindings of ionic and nonionic substrates and inhibitors to the enzyme, and in the effects of $\mathrm{pH}$ and ionic strength on the binding. There is some disagreement as to whether there are one or two anionic groups on the true cholinesterase: Wilson (1957) believes that the results are compatible with a single anionic site while Bergmann (1958) has brought forth reasons for assuming that two such groups interact with a single cationic substrate or inhibitor. Before discussing this problem further, let us consider the results obtained.

Three general methods have been used to estimate the magnitude of the ion-ion interaction: (1) the comparison of cationic substrates or inhibitors with the uncharged analogs, (2) the determination of the difference in binding at two pH's sufficiently apart so that the substrate is charged at one $\mathrm{pH}$ and uncharged at the other, and (3) the comparison of inhibition of true and pseudocholinesterase by a cationic inhibitor, inasmuch as the latter enzyme does not have an anionic group (or has one less than the true cholinesterase). The results of such study are summarized in Table 6-22 and calculations of the equilibrium interaction distance are given for assumptions of either one or two anionic sites. The average charge separation, assuming one anionic group at the active site, is $5.55 \AA$; this wonld imply an approximate distance of $6.15 \AA$ between the $\mathrm{N}^{+}$and the carbon atom of the carboxylate group, which is very close to the separation at contact of the van der Waals' surfaces. If two anionic groups are assumed on true cholinesterase, the calculated separation of $7.18+0.6=7.78 \AA$ would not allow the interposition of a water molecule and it must be concluded that the data in Table 6-22 point to a single anionic group. The pseudocholinesterase would then possess no anionic group immediately adjacent to the interacting cationic group. The calculations of $d_{e}$ from the $K_{i}$ of tetramethylammonium ion, obtained by extrapolation from higher analogs, made by Bergmann (1958) are probably not valid since no account was taken of interactions other than ion-ion. Bergmann stated that van der Waals' forces camnot make an important contribution to these interactions, but this is certainly incorrect, especially when the displacement of water from the groups is considered.

\section{Interactions of Alkylammonium lons with Cholinesterase}

The possibility of determining the dispersion contribution to the interaction energy of the $-\mathrm{N}\left(\mathrm{CH}_{3}\right)_{3}{ }^{+}$end of the acetylcholine molecule led Wilson (1952 a) to examine the relative inhibitory potencies of varionsly substituted ammonium ions. The results are shown in Table 6-23. Removal of one methyl group from the tetramethyl compounds does not reduce the binding, which indicates that the entire group does not fit into a cavity but that one methyl group is directed away from the surface. Removal of 
further methyl groups reduces the binding energy; the average reduction per methyl group is $1.14 \mathrm{kcal} /$ mole. Wilson interpreted this energy difference as due to dispersion forces between the methyl groups and the protein. However, Bemhard (1955) calculated that this is about $0.7 \mathrm{kcal} / \mathrm{mole}$ more than expected from dispersion forces. Using Eq. 6-17, the dispersion energy for interaction of a methyl group with protein is $-0.58 \mathrm{kcal} / \mathrm{mole}$,

TABLE 6-2:2

Ion-Ion Interactions for True (holinesterase ${ }^{a}$

\begin{tabular}{|c|c|c|c|c|c|c|}
\hline \multirow{3}{*}{ Compounds tested } & \multirow{3}{*}{$K_{0} / K_{+}$} & \multirow{3}{*}{$\begin{array}{c}\triangle F \\
\text { (keal/ } \\
\text { mole) }\end{array}$} & \multicolumn{3}{|c|}{$\begin{array}{l}\text { Calculated equilibrium } \\
\text { distance }\end{array}$} & \multirow{3}{*}{ Ref. ${ }^{b}$} \\
\hline & & & Values in & From & 1. $6-85$ & \\
\hline & & & $\begin{array}{c}\text { literature } \\
\text { (A) }\end{array}$ & $\begin{array}{c}n=1 \\
(A)\end{array}$ & $\begin{array}{c}n=2 \\
(\AA)\end{array}$ & \\
\hline $\begin{array}{l}\text { (1) Nicotinamide and } \\
N \text {-methylnicotinamide } \\
\text { (2) Isoamyl aleohol and } \\
\text { dimethylethanolammonimm } \\
\text { (3) } 3,3 \text {-Dimethyllyutyl aeetate } \\
\text { and acetyleholine } \\
\text { (4) Physostigmine at } \mathrm{pH} 6 \\
\text { and pH } 10 \\
\text { (5) Dimethylaminoethyl acetate } \\
\text { at } \mathrm{pH} 6 \text { and } \mathrm{pH} 10 \\
\text { (6) Choline on true and } \\
\text { pseudocholinesterase } \\
\text { (7) Tetramethylammonium ion } \\
\text { on true and psendocholin- } \\
\text { esterase } \\
\text { (8) Tetraalkylammonimm ions } \\
\text { extrapolated to tetra- } \\
\text { methylammonium ion }\end{array}$ & $\begin{array}{r}8 \\
30 \\
31 \\
8 \\
8 \\
20 \\
16 \\
20 \\
20\end{array}$ & $\begin{array}{l}-1.23 \\
-2.01 \\
-2.10 \\
-1.23 \\
-1.2 \\
-1.77 \\
-1.64 \\
-1.77 \\
-1.99\end{array}$ & $\begin{array}{l}5.8 \\
5.0 \\
5.6 \\
6.6 \\
6.6 \\
6.5 \\
5.4 \\
6.5 \\
\end{array}$ & $\begin{array}{l}6.15 \\
5.15 \\
6.15 \\
5.38 \\
5.55\end{array}$ & $\begin{array}{l}7.83 \\
6.65 \\
7.83 \\
7.00 \\
7.17 \\
7.00 \\
6.67\end{array}$ & $\begin{array}{l}(a) \\
(b) \\
(c) \\
(b) \\
(c) \\
(d) \\
(a) \\
(d) \\
(e)\end{array}$ \\
\hline
\end{tabular}

${ }^{a} K_{o} / K_{+}$is the ratio of the dissociation constants of the substrates or inhibitors used and $n$ is the number of anionie groups assumed on the enzyme site. The values of $d_{e}$ from the literature include correction for the ion atmosphere except for those in parentheses. The values of $d_{e}$ in the last two colnmns were estimated from Fig. 6-17. The $J F \mathrm{in}(8)$ is for one anionie group, inasmuch as the total interaetion energy was determined and a value of $n=2$ was assumed.

b References: (a) Wilson (1957), (b) Wilson (1952-a), (c) Bernhard (195.5), (d) Wilson and Bergmann (1950), (e) Adams and Whittaker (1950), (f) Bergmann (1958). 
TABLE $6-23$

Ixhibitiox of Eel Electric Orgax Cholmanterase by Alkylanmoxich axd Alkylethasolamoximi Ions ${ }^{a}$

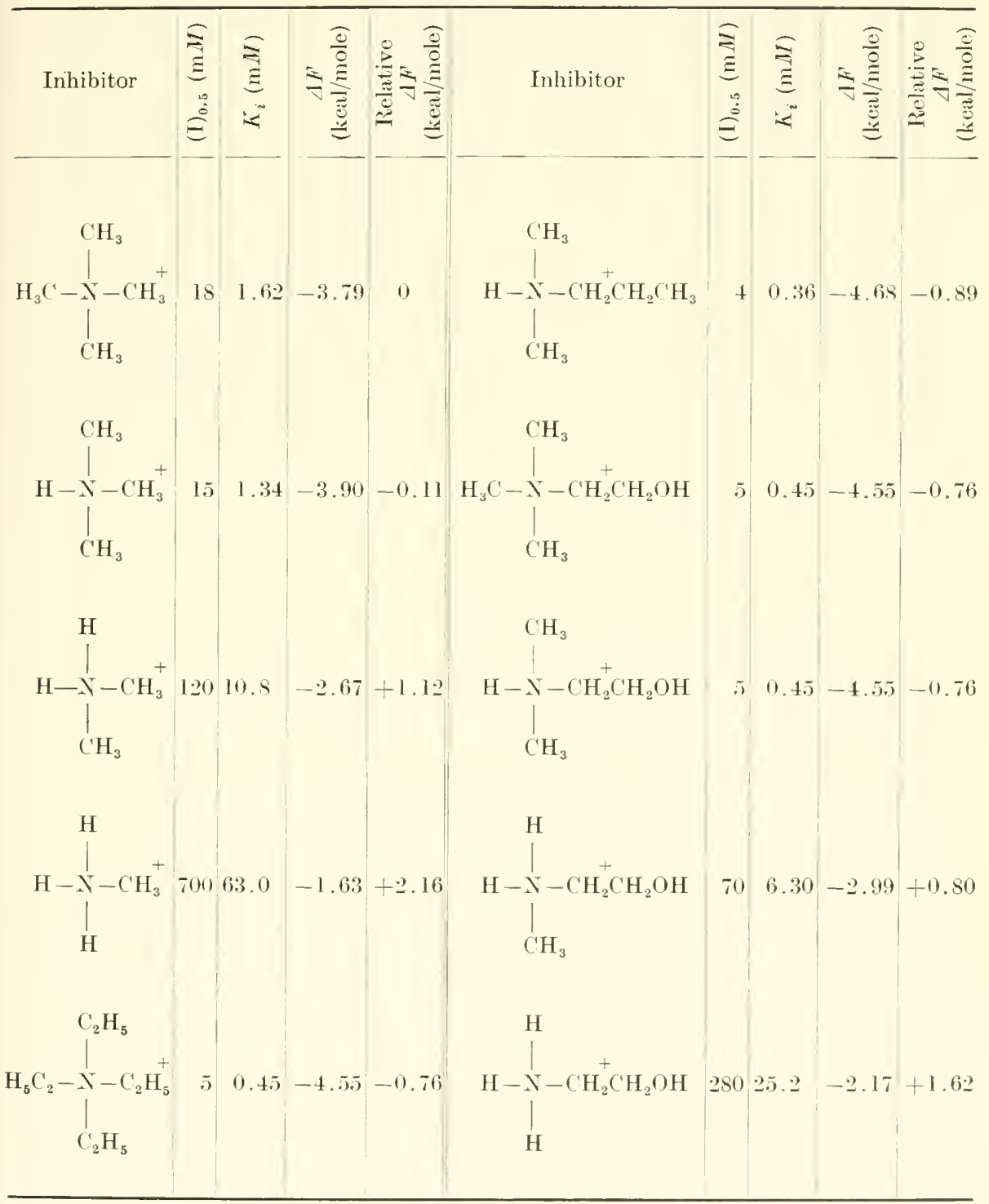

${ }^{a}$ Data from Wilson (1952- a). The relative free energy values were all calculated on the basis of tetramethylammonium ion as zero. 
which leads to a discrepancy of $0.56 \mathrm{kcal} / \mathrm{mole}$, close to the value given by Bernhard. Bergmann (1958) considered the energy rednction in removing a methyl gromp to result from the greater hydration of the cationic group. It was felt that the methylated nonhydrated ammonium ion could approach the anionic site more closely than the hydrated forms. It is likely that distance of approach is not the only factor but the effect of hydration must be important and is perhaps responsible for the $0.56-0.7 \mathrm{kcal} / \mathrm{mole}$ deficit noted above.

The increased binding of $\mathrm{N}\left(\mathrm{C}_{2} \mathrm{H}_{5}\right)_{4}{ }^{+}$over $\mathrm{N}\left(\mathrm{CH}_{3}\right)_{4}{ }^{+}$must be accounted for on the basis of greater dispersion energy. Since the radius of the former ion is greater, the ion-ion interaction energy must be somewhat less; calculation leads to $0.25 \mathrm{kcal} /$ mole less based on a $1 \AA$ increase in radius. This must be added to the relative $1 F$, giving $-1.01 \mathrm{kcal} /$ mole for the extra dispersion energy, which is equivalent to $-0.34 \mathrm{kcal} / \mathrm{mole}$ for each $-\mathrm{CH}_{2}-$ group, a very reasonable value. The increased binding of the dimethylpropyl over the trimethylammonimm ion should be due to the dispersion energy from $-\mathrm{CH}_{2} \mathrm{CH}_{2}$-, which would be predicted to be $2 \times 0.34=$ $0.68 \mathrm{kcal} / \mathrm{mole}$, which compares with the experimental value of $0.78 \mathrm{kcal} /$ mole. The failure of the ethanol group to be bound more tightly than the propyl group indicates that no hydrogen bond is formed by the hydroxyl group with the protein.

\section{Dispersion Interaction of Substituted Halogen Groups}

If the plasma cholinesterase contains no anionic gromp at the active site, attraction between the enzyme and substrate must be due to dipolar and dispersion forces, and the magnitude of these forces will depend on the dipole moments and polarizabilities of the substrates. In order to test this, Adams and Whittaker (1950) compared the affinities of the enzyme for esters of chloroacetate and bromoacetate with similar esters of propionate.

$$
\mathrm{R}-\mathrm{O}-\mathrm{CO}-\mathrm{C}_{2}-\mathrm{CH}_{3} \quad \mathrm{R}-\mathrm{O}-\mathrm{CO}-\mathrm{CH}_{2}-\mathrm{Cl} \quad \mathrm{R}-\mathrm{O}-\mathrm{CO}-\mathrm{CH}_{2}-\mathrm{Br}
$$

The results are shown in Table $6-24$. Their calculations show that dispersion forces can account for the different binding encrgies with the plasma cholinesterase. The calculated energy valıes, obtained from Table 6-19 agree almost exactly with the experimental figures.

The energy differences for the erythrocyte enzyme are more difficult to interpret. Adams and Whittaker assumed that the increased energy difference over the plasma enzyme was dne to the fact that the erythrocyte enzyme possessed an anionic group which would interact with dipoles and induced dipoles in the substituted gromps. However, the relative changes for the $\mathrm{Cl}$ and $\mathrm{Br}$ gromps are in the opposite direction from that expected 
TABLE 6-24

Interaction Energies of Substituent Halogen Groups with Plasma and Erythrocyte Cholinesterase a

\begin{tabular}{|c|c|c|}
\hline & $\begin{array}{c}\text { Plasnıa } \\
\text { cholinesterase }\end{array}$ & $\begin{array}{l}\text { Erythrocyte } \\
\text { cholinesterase }\end{array}$ \\
\hline $\begin{array}{l}K_{\mathrm{Cl}} / K_{\mathrm{CH}_{3}} \\
\text { Experimental } \Delta F_{\mathrm{Cl}}-\Delta F_{\mathrm{CH}_{3}} \\
\text { Calculated } \Delta F_{\mathrm{Cl}}-\angle F_{\mathrm{CH}_{3}}\end{array}$ & $\begin{array}{r}1.31 \\
-0.16 \\
-0.16\end{array}$ & $\begin{array}{r}3.80 \\
-0.79 \\
-0.63\end{array}$ \\
\hline $\begin{array}{l}K_{\mathrm{Br}} / K_{\mathrm{CH}_{3}} \\
\text { Experimental } \Delta F_{\mathrm{Br}}-\Delta F_{\mathrm{CH}_{3}} \\
\text { Calculated }\lrcorner F_{\mathrm{Br}}-\Delta F_{\mathrm{CH}_{3}}\end{array}$ & $\begin{array}{r}1.76 \\
-0.33 \\
-0.31\end{array}$ & $\begin{array}{r}2.26 \\
-0.48 \\
-0.74\end{array}$ \\
\hline
\end{tabular}

a The subscripts refer to the compounds compared and specifically to the substituted group on the acetate radical: both butyl- and isoamylesters were averaged from the data of Adams and Whittaker (1950). The calculated differences in free energy between the groups for the plasma enzyme were obtained from Table 6-19. The calculations for the erythrocyte enzyme assume maximal ion-dipole interaction: Eq. 6.57 was used with the following values $-\mu_{\mathrm{C}-\mathrm{Cl}}=2.3, \mu_{\mathrm{C}-\mathrm{Br}}=2.2, d_{e}=4.43 \AA$ for $\mathrm{C}-\mathrm{Cl}$ and $4.52 \mathrm{~A}$ for $\mathrm{C}-\mathrm{Br}$, and $\mathrm{D}=14.3$. The dipole moment of the $\mathrm{C}-\mathrm{CH}_{3}$ bond was considered negligible for this interaction. The configuration assumed was:

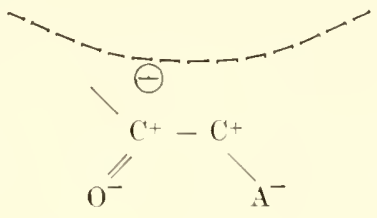

on the basis of their polarizabilities; the ion-dipole energies for the $\mathrm{C}-\mathrm{Cl}$ and $\mathrm{C}-\mathrm{Br}$ dipoles would be roughly the same inasmuch as the dipole moments are similar. Furthermore, if these substances are bound like the acetylcholine molecule, the anionic site would be expected to be at some distance removed from these substituted groups. If they are not bound in this manner, the anionic site could interact with both the

$$
\overrightarrow{\mathrm{O}}=\stackrel{+}{\mathrm{C}}
$$

group and the terminal dipole, increasing the stability of the complexes, the chlorine derivative being somewhat more stable than the bromine derivative due to its slightly higher dipole moment. In this position, the substituent groups might not interact with the protein and the dispersion contribution from them might be neglected. The calculation for the ion- 
dipole contributions is approximate since the dielectric constant is difficult to estimate in this situation and provides over-all energy differences which conld not be satisfactory for both chlorine and bromine derivatives. Some factor, other than ion-dipole interaction, must be involved here.

\section{Dispersion Energy of Benzene Ring and Cholinesterase}

The inhibition constant $K_{i}$ for $\mathrm{H}-\mathrm{N}\left(\mathrm{CH}_{3}\right)_{3}{ }^{+}$is $1.34 \mathrm{~m} M$ (Wilson, 1952a) and for $\varphi-\mathrm{N}\left(\mathrm{CH}_{3}\right)_{3}{ }^{+}$is $0.038 \mathrm{~m} . I$ (Wilson and Quan, 1958). This corresponds to an energy difference of $2.10 \mathrm{kcal}$ mole which probably can be attributed to dispersion forces between the benzene ring and the adjacent protein. Calculation of the average interaction distance can be made using Eq. 6-116 and assuming the following values: $R_{W_{F}}=3.67$, $R_{\text {benz }}=25.11, R_{I I}=1.65, R_{\text {prot }}=21.12, Z_{\mathrm{W}}=8, Z_{\text {benz }}=24, Z_{i 1}=2$, $Z_{\text {rol }}=8$, and $n=4$. It is probable that the benzene ring lies flat on the protein surface and thus interacts with one side only. The distance $d_{e}$ is found to be $4.16 \mathrm{~A}$. which is reasonable, inasmuch as the closest approach wonld be $3.65 \AA$ and it is unlikely that there is exact fit between the benzene ring and the protein.

\section{Effect of Substituent Position on Interaction Energy}

The inhibition constants for various substituted phenyltrimethylammonium ions were determined by Wilson and Quan (1958) and are shown in Table 6-25 with the calculated free energy differences using the unsubstituted ion as a standard. The introduction of an hydroxyl group in the 3 -position increases the binding energy by $2.84 \mathrm{kcal}$ mole, indicating the formation of a hydrogen bond between this hydroxyl group and some adjacent gromp on the protein. That this is a strong hydrogen bond rather than a dispersion interaction is suggested by the magnitude of the energy difference, the fact that the corresponding methyl and methoxy compounds are much less tightly bound, and the poorer binding of the $2-\mathrm{OH}$ and 4-OH derivatives. The hydrogen bond could be formed with the hydroxyl hydrogen atom in one of the following two positions:

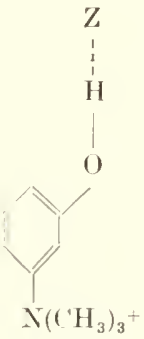

(I)

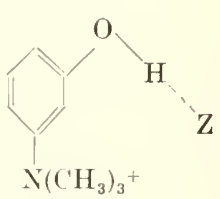

(II) 
The second is considered more likely because of the stronger binding of the 2-OH compared to the $4-\mathrm{OH}$ derivative; the hydroxyl group in the 2-position conld form a weaker hydrogen bond with the $\mathrm{Z}$ group but the liydroxyl in the 4-position could not. This indicates that one factor in the relative binding of differently substituted derivatives is the orientation of the group with respect to certain protein groups with which it may interact.

\section{TABLE 6-9.5}

Bindixg of Phexyltrinethylamionium Derivatives to Cholinesterase ${ }^{a}$

\begin{tabular}{|c|c|c|}
\hline Compound & $\begin{array}{c}K_{i} \\
(m . M)\end{array}$ & $\begin{array}{c}\text { Relative } \\
\text { binding energy } \\
\text { (kcal mole) }\end{array}$ \\
\hline 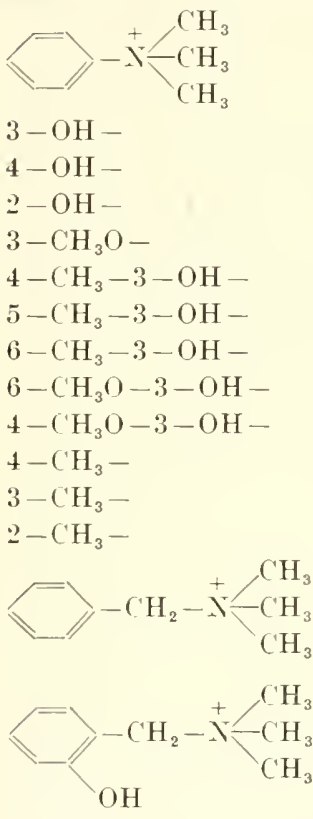 & $\begin{array}{l}0.038 \\
0.00031 \\
0.023 \\
0.0052 \\
0.0075 \\
0.000099 \\
0.00062 \\
0.0009 \\
0.00052 \\
0.00117 \\
0.029 \\
0.012 \\
0.041 \\
0.091\end{array}$ & $\begin{array}{c}- \\
-.2 .84 \\
-0.29 \\
-1.17 \\
-0.96 \\
-3.51 \\
-2.42 \\
-2.21 \\
-2.53 \\
-2.06 \\
-0.16 \\
-0.63 \\
+0.04 \\
+0.51 \\
-0.32\end{array}$ \\
\hline
\end{tabular}

a Data taken from Wilson and Quan (195s). The relative binding energies were caleulated with the phenyltrimethylammonium ion as the standard, and the derivatives given, with the exception of the last two, are all of this compound. The $K_{i}$ 's were obtained from inhibition studies on eel electric organ acetyleholinesterase.

Introduction of further groups into the $3-\mathrm{OH}$ derivative generally reduces the binding energy, pointing to steric interference with the approach of the ring to the protein surface. However, the $4-\mathrm{CH}_{3}-3-\mathrm{OH}$ derivative is bound more tightly than the $3-\mathrm{OH}$ derivative by $0.67 \mathrm{kcal} /$ mole. Since 
the $4-\mathrm{CH}_{3}$ derivative is only $0.16 \mathrm{kcal} / \mathrm{mole}$ more stable than the unsubstituted ion, it may be concluded that $0.51 \mathrm{kcal} /$ mole originates from the steric effeet the $4-\mathrm{CH}_{3}$ group has on the $3-\mathrm{OH}$ gromp, making configuration (II) above statistically more probable and more favorable to hydrogen bond formation.

Separation of the benzene ring from the trimethylammonium ionic group by one $-\mathrm{CH}_{2}-$ group leads to a reduction in binding as it did in the hapten-antibody interactions discussed previously. However, the fit here on the enzyme is not as eritical and only $0.68 \mathrm{kcal} / \mathrm{mole}$ is lost from the binding energy by this separation.

\section{INTERACTION FORCES IN VARIOUS ENZYME SYSTEMS}

Four more enzyme systems will be briefly discussed to illustrate the various types of interactions and the problems involved in the calculations of the binding energies.

\section{Inhibitors of Carboxypeptidase}

Ion-ion type interaction is known to be important in the binding of substrates and inhibitors to peptidases but Smith et al. (1951) have presented evidence that van der Waals' forees are also involved. The inhibition constants and calculated binding energies for a number of compounds with crystalline pancreatic carboxypeptidase are shown in Table 6-26. Carbobenzoxyglycol amino acids are hydrolyzed by the enzyme as follows:

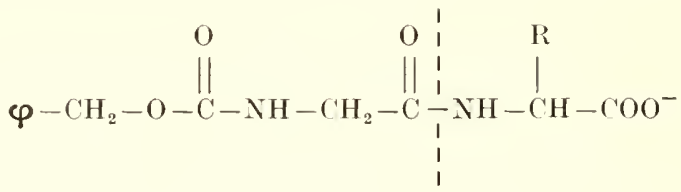

Splitting occurs at the dotted line and the nature of the R-chain is important in determining the rate of the reaction. Interactions of the terminal - $\mathrm{COO}^{-}$ and the peptide carbonyl group probably occur, but van der Waals' interaction of the $\mathrm{R}$-chain provides a significant contribution to the total binding energy. Some insight into these interactions may be obtained from a study of the inhibitors in Table 6-26.

The differences in the binding energies of these inhibitors may be attributed mainly to van der Waals' forces, and particularly to dispersion forces. A side-chain of sufficient size on the $-\mathrm{COO}^{-}$group is necessary for binding, inasmuch as acetate is scarcely bound. In the aliphatic acid series, the addition of a $-\mathrm{CH}_{2}-$ group results in about 1.5 kcal/mole more binding energy from acetate to butyrate, but further additions have little effect. One might conclude that there is a region on the enzyme, about 6 -8 $\AA$ from 
TABLE $6-26$

Relative Binding Energies of Inhibitors to Carboxypeptidase ${ }^{a}$

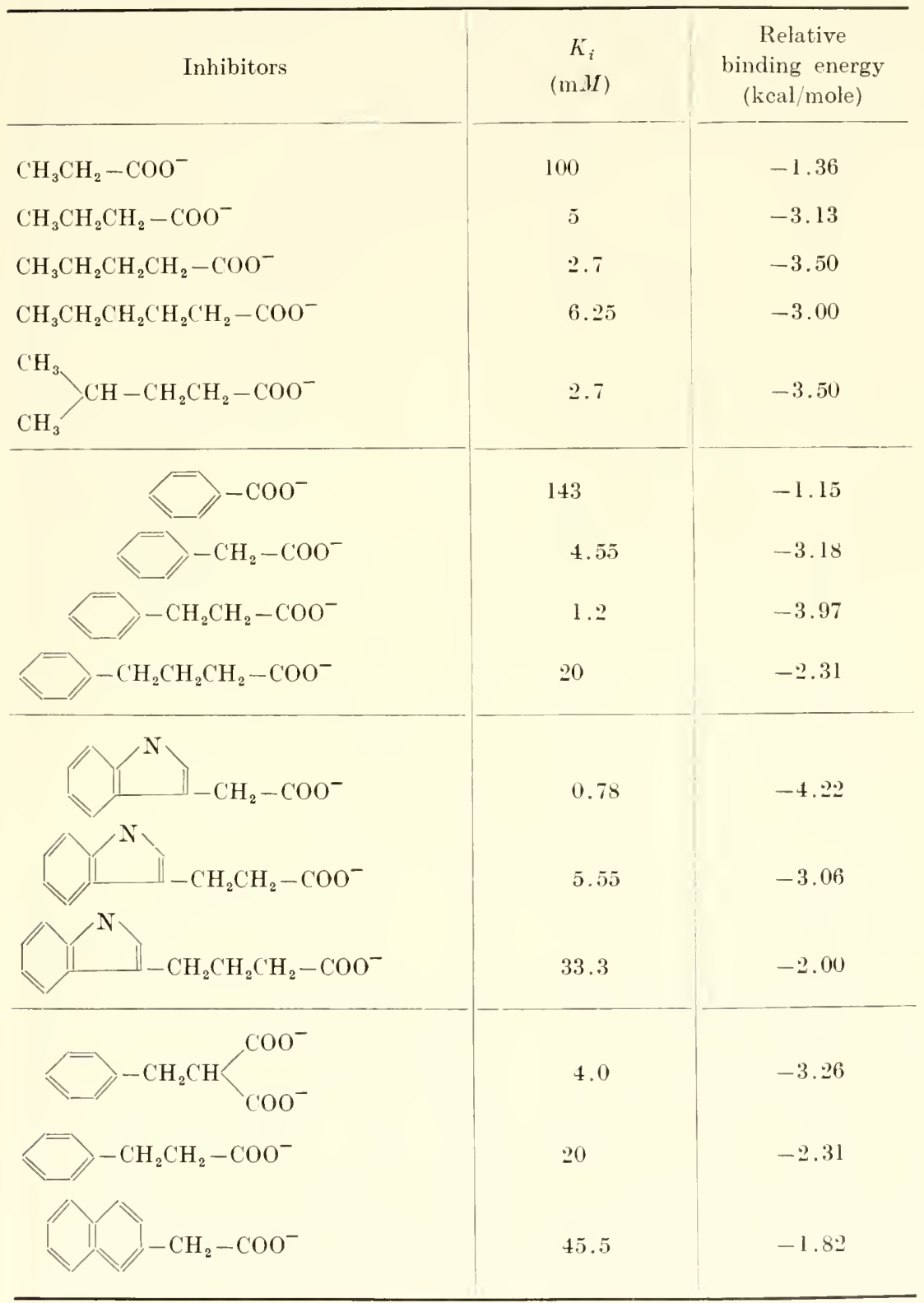

a Data from Smith et al. (1951). The binding energies were calculated from the values of $K_{i}$. 
the eationic group, that is capable of interacting with the nonpolar part of the inhibitors. Essentially the same is observed in the $\varphi-\left(\mathrm{CH}_{2}\right)_{n}-\mathrm{COO}^{-}$ series; when the benzene ling is at the proper distance from the $-\mathrm{COO}^{-}$ group, the interaction is maximal. In the indole- $\left(\mathrm{CH}_{2}\right)_{n}-\mathrm{COO}^{-}$series the benzene ring is already at near optimal distance in indole acetate and further interposition of $-\mathrm{CH}_{2}-$ groups only decreases the binding energy. Comparing phenylacetate with propionate, the benzene ring is bound more tightly than a methyl group by $1.82 \mathrm{kcal} /$ mole. This is a reasonable value as calculation of the dispersion energy of a benzene ring interacting on one side with a protein surface leads to $2.35 \mathrm{kcal} /$ mole (the experimental value from eholinesterase is $2.1 \mathrm{kcal} / \mathrm{molo}$ ), and the methyl group dispersion energy amounts to approximately $0.3 \mathrm{kcal} / \mathrm{mole}$ for side-on interaction with the protein (see Table 6-19).

The phenyl group would seem to be bound by 1.66 keal/mole more tightly than the cyclolexyl group, comparing the respective propionates. The latter will probably be at a somewhat greater listance from the protein, due to the nomplanar hyllogen atoms, and this could well account for the difference. If the latf-thickness of the benzene ring is taken as $1.85 \AA$ (Table 6-9), the eyclohexane ring ean be calenlated to be $2.19 \AA$; the molar refractions are $R_{\text {benz }}=25.1$ and $R_{\text {cyclo }}=27.0 \mathrm{ml} /$ mole. Using these values, it is found that the phenyl gromp should be bound $0.88 \mathrm{keal} / \mathrm{mole}$ more tightly; if these rings fit into a slit or cavity, this value would be approximately doubled and would be of the same orter of magnitude as the experimental difference. The ring certainly must be oriented properly beeause cis- and truns-cimnamate do not inlibit, the double bond here restricting the rotation of the ring and holding it at an angle.

\section{Binding of ATP to ATPase}

Laidler and Ethier (1953) determined the entropy change on binding of ATP to muscle ATPase by varying the dielectrie constant of the medium with methanol and lioxane, using an equation similar to 6-95, and found a values of $25 \mathrm{cal} / \mathrm{mole} /$ degree for the ion-ion entribution. From Eq. $6-98$, the value of the interaction distance is thus given by $d_{e}=0.75 z_{1} z_{2}$. If this procedure is valid, it is evirlent that multiple charges are involved; if two phosphate groups interact with two eationie grouns on the enzyme, the $d_{e}$ would be $3.0 \mathrm{~A}$ and if three groups on each interactant were involved, $d_{e}$ would be $6.75 \AA$. The clata are only approximate because the bulk dielectric constant was assmmed and the ion atmosphere was ignored; taking these into account, it would seem most likely that only two ionic gromps are involved in the linding, or possibly even two phosphate groups and one cationic enzyme group.

Knowledge of substrate and inhibition constants for ATP, ADP, and AMP would provide some information on these electrostatic forces, but these 
constants are not accurately known, and furthermore they depend strongly on the concentrations of ions, particularly $\mathrm{Ca}^{++}$and $\mathrm{Ig}^{++}$. Blum (1955) determined the value of $K_{\text {ATP }}$ for rabbit myosin ATPase in the presence of $1 \mathrm{~m} M \mathrm{Ca}^{++}$to be $6.25 \times 10^{-3} \mathrm{~m} . \mathrm{I}$; from inhibition experiments the $\mathrm{K}_{\mathrm{ADP}}$ under the same conditions was calculated to be $1.64 \times 10^{-1} \mathrm{~m} . I$. This corresponds to a difference in binding energy of $1.93 \mathrm{kcal} /$ mole. Since AMP inhibits much more weakly than ADP, its binding energy must be lower than for ADP and probably of a similar order of magnitude to the ATPADP difference. This would imply that the two terminal phosphate groups participate in the binding; nothing can be concluded about the proximal phosphate group.

\section{Interaction Energies of Sarcosine Oxidase Inhibitors}

Sarcosine is oxidized to formaldehyde and glycine in a suspension of liver mitochondria; this reaction is competitively inhibited by methoxyacetate, the oxygen analog of sarcosine, and many related compounds (Frisell and Mackenzie, 1955). In Table 6-27 is shown the inhibition produced by $20.8 \mathrm{~m} . M$ inhibitor when sarcosine is $8.33 \mathrm{~m} . M$. The relative binding energy is calculated from the inhibition constants obtained from Eq. 3-13:

$$
K_{i}=\frac{(1)(1-i)}{\left[1+(\mathrm{S}) / K_{s}\right] i}
$$

Since the $\mathrm{pH}$ was 7.8 , the carboxyl groups are ionized and it is likely that the amino groups and $-{ }^{+} \mathrm{NH}_{2}-$ group of sarcosine are protonated. The active site of sareosine oxidase is pictured in Fig. 6-2; a three-point attachment of sarcosine was suggested by Frisell and Mackenzie, the carboxylate group interacting with a cationic protein group, the terminal methyl group being attracted to a protein hydrocarbon residue by dispersion forces, and the $-{ }^{+} \mathrm{NH}_{2}-$ group reacting with some electrophilic radical on the protein.

Comparing acetate and ethanol, methoxyacetate and methoxyethanol, and sarcosine with sarcosine amide, the ion-ion carboxylate binding energy would be about $1.8 \mathrm{kcal} / \mathrm{mole}$, implying an equilibrium distance of $5.3 \mathrm{~A}$, similar to values obtained on other enzymes for such interactions. Comparison of sarcosine with butyrate (assuming that the $K_{m}$ for sarcosine represents the true dissociation constant) indicates that the replacement of a $-\mathrm{CH}_{2}-$ group by a $-{ }^{+} \mathrm{NH}_{2}-$ group leads to $1.59 \mathrm{kcal} /$ mole greater binding energy; the groups $-\mathrm{O}-$ and $-\mathrm{S}$ - also increase the binding but not so much. It would appear likely that hydrogen bonding is responsible; this would argue for the nitrogen group in sarcosine being uncharged as - $\mathrm{NH}-$, which would be substantiated by the small binding of glycine, but the $K_{a}$ for this group is given as 10.01 (Cohn and Edsall, 1943, p. 85) 
TABLE 6-27

Interaction ENergies of Sarcosine Oxidase Inhibitors a

\begin{tabular}{|c|c|c|c|}
\hline \multicolumn{2}{|c|}{ Inhibitor } & $\begin{array}{c}\% \\
\text { Inhibition }\end{array}$ & $\begin{array}{c}\text { Relative } \\
\text { binding energy } \\
(\mathrm{kcal} / \mathrm{mole})\end{array}$ \\
\hline Formate & $\mathrm{H}-\mathrm{COO}^{-}$ & $\tilde{5}$ & -1.76 \\
\hline Acetate & $\mathrm{CH}_{3}-\mathrm{COO}^{-}$ & 48 & -3.52 \\
\hline Fluoroacetate & $\mathrm{F}-\mathrm{CH}_{2}-\mathrm{COO}^{-}$ & 34 & -3.16 \\
\hline Chloroacetate & $\mathrm{Cl}-\mathrm{CH}_{2}-\mathrm{COO}^{-}$ & 31 & -3.08 \\
\hline Bromoacetate & $\mathrm{Br}-\mathrm{CH}_{2}-\mathrm{COO}^{-}$ & 28 & -2.99 \\
\hline Iodoacetate & $\mathrm{I}-\mathrm{CH}_{2}-\mathrm{COO}^{-}$ & 22 & -2.79 \\
\hline Propionate & $\mathrm{CH}_{3}-\mathrm{CH}_{2}-\mathrm{COO}^{-}$ & 36 & -3.22 \\
\hline Butyrate & $\mathrm{CH}_{3} \mathrm{CH}_{2} \mathrm{CH}_{2}-\mathrm{COO}^{-}$ & 14 & -2.45 \\
\hline Valerate & $\mathrm{CH}_{3} \mathrm{CH}_{2} \mathrm{CH}_{2} \mathrm{CH}_{2}-\mathrm{COO}^{-}$ & 6 & -1.88 \\
\hline Acrylate & $\mathrm{CH}_{2}=\mathrm{CH}-\mathrm{COO}^{-}$ & 33 & -3.13 \\
\hline$\beta$-Chloropropionate & $\mathrm{Cl}-\mathrm{CH}_{2} \mathrm{CH}_{2}-\mathrm{COO}^{-}$ & 11 & -2.28 \\
\hline Glycine & $+\mathrm{NH}_{3}-\mathrm{CH}_{2}-\mathrm{COO}^{-}$ & $\underline{2}$ & -1.18 \\
\hline Sarcosine & $\begin{array}{l}\mathrm{CH}_{3}-{ }^{+} \mathrm{NH}_{2}-\mathrm{CH}_{2}-\mathrm{COO}^{-} \\
\mathrm{CH}_{3}\end{array}$ & - & -4.04 \\
\hline$N$-methylsarcosine & $\mathrm{CH}_{3} \stackrel{+}{\mathrm{N}} \mathrm{H}-\mathrm{CH}_{2}-\mathrm{COO}^{-}$ & 0 & - \\
\hline$\beta$-Alanine & $\begin{array}{l}+\mathrm{NH}_{3}-\mathrm{CH}_{2} \mathrm{CH}_{2}-\mathrm{COO}^{-} \\
+\mathrm{NH}_{3}\end{array}$ & 4 & -1.62 \\
\hline$\alpha$-Alanine & $\mathrm{CH}_{3}>\mathrm{CH}-\mathrm{COO}^{-}$ & 0 & - \\
\hline Methoxyacetate & $\mathrm{CH}_{3}-\mathrm{O}-\mathrm{CH}_{2}-\mathrm{COO}^{-}$ & 64 & -3.92 \\
\hline$S$-methylthioglycolate & $\mathrm{CH}_{3}-\mathrm{S}-\mathrm{CH}_{2}-\mathrm{COO}^{-}$ & 39 & -3.30 \\
\hline Acetaldehyde & $\mathrm{CH}_{3}-\mathrm{CHO}$ & 28 & -2.99 \\
\hline Ethanol & $\mathrm{CH}_{3}-\mathrm{CH}_{2}-\mathrm{OH}$ & 5 & -1.76 \\
\hline Acetamide & $\mathrm{CH}_{3}-\mathrm{CONH}_{2}$ & 0 & - \\
\hline Methoxyethanol & $\mathrm{CH}_{3}-\mathrm{O}-\mathrm{CH}_{2} \mathrm{CH}_{2}+\mathrm{OH}$ & 5 & -1.76 \\
\hline Methoxyacetamide & $\mathrm{CH}_{3}-\mathrm{O}-\mathrm{CH}_{2}-\mathrm{CONH}_{2}$ & 0 & - \\
\hline Sarcosinamide & $\mathrm{H}_{3}-{ }^{+} \mathrm{NH}_{2}-\mathrm{CH}_{2}-\mathrm{CONH}_{2}$ & 15 & -2.51 \\
\hline
\end{tabular}

a Inhibition data from Frisell and Mackenzie (1955). Relative binding energy calculated as described in the text. Value for sarcosine obtained from $K_{m}$, assuming it is $K_{8}$. 
so that at $\mathrm{pH} 7.8$ it should be in the ionized state. It is strange that the replacement of the hydrogen atom of acetate by almost any group (except in sarcosine and methoxyacetate) leads to poorer binding. Halogen atoms decrease the binding in proportion to their size and this is not likely to be due to decreased dispersion forces, but to steric factors or dipolar interactions. It may well be that the acetate series fits onto the surface in a different configuration than the sarcosine-methoxyacetate group, and certainly importance must be attached to the terminal methyl group in these latter compounds, which could fit tightly into a carity as suggested, contributing 1-2 kcal/mole and serving to orient the entire molecule. Various comparisons point to the following provisional assignments of binding contributions for sarcosine; $-\mathrm{COO}^{-}, 1.8 \mathrm{kcal} / \mathrm{mole} ;-\mathrm{CH}_{2}-, 0.2 \mathrm{kcal} /$ mole; $-\mathrm{NH}_{2}{ }^{+}-, 1.1 \mathrm{kcal} / \mathrm{mole}$, and $-\mathrm{CH}_{3}, 1.0 \mathrm{kcal} / \mathrm{mole}$. Further relationships will be discussed in Volume II, Chapter 2, which will be concerned with inhibition produced by analogs of substrates and coenzymes.

\section{Interaction of Substrate Analogs with Yeast Lactate Dehydrogenase}

All of the substrates or inhibitors for this enzyme have anionic carboxylate groups so it is likely that the active center contains a positively charged cationic group. Dikstein (1959) determined the concentrations required for $50 \%$ inhibition for a number of analogs and for the fatty acid series he plotted $\mathrm{pI}_{0.5}$ against the number of methylene groups. The slope of the resulting straight line allowed the calculation of the free energy of transfer of a methylene group from the solvent phase to the surface of the enzyme; this was about $0.5 \mathrm{kcal} /$ mole at $25^{\circ}$ and represents the contribution to the binding made by each methylene group. The intercept of the line with the $\mathrm{pI}_{0.5}$ axis allowed the calculation of the ion-ion interaction energy, from which the equilibrium separation (assuming $D=30$ ) was determined to be about $11 \AA$ if a single cationic group is involved. The interaction of the hydrocarbon chain with the enzyme surface in this series is thus of major importance in determining the inhibitory potency. The enzyme cationic group is most likely a $\delta$-guanidino group of arginine.

\section{INTERACTIONS OF AROMATIC COMPOUNDS}

Due to the relatively high polarizability of aromatic ring systems, there are often strong interactions with similar ring systems or polarizing groups, particularly when the mutual orientation is such as to allow a close approach over an appreciable surface of the molecules. Thus highly resonating molecules, composed of several aromatic rings with mobile electrons, tend to form complexes in which the molecules lie in parallel planes, this being the orientation consonant with the greatest dispersion interaction. It is 
possible that some enzyme inhibitions involve simiłar positions of aromatic rings and hence it is of interest to determine the effect of orientation on interaction energy.

This problem was considered by de Boer (1936) who compared the dispersion energy of two benzene rings that were $3.5 \AA$ apart when lying in the same plane (A) or lying parallel (B), and found it to be four times greater in position (B). He also constructed distance-energy curves and concluded that $3.5 \AA$ would be the equilibrium distance for maximal attraction; calculations, using the equations derived earlier in this chapter, lead to the somewhat greater distance of $3.7 \AA$ for position (B). The dispersion energy at this latter separation would be about $24.6 \mathrm{kcal} / \mathrm{mole}$. For benzene the heat of fusion is $2.35 \mathrm{kcal} / \mathrm{mole}$ and the heat of vaporization is $7.37 \mathrm{kcal} / \mathrm{mole}$, so that the heat of sublimation is $9.72 \mathrm{kcal} / \mathrm{mole}$; however, in benzene crystals the rings are not parallel throughout but inclined at angles in alternate rows and displaced along their axes. The average interaction energy of a molecule with its neighbors in a crystal would be about $19.4 \mathrm{kcal} /$ mole so that the vahe calculated above is not unreasonable. The high dispersion energies possible with aromatic rings may be important in binding substrates and inhibitors to enzymes, for even at a separation of $5 \AA$ the attraction energy may amount to 4 kcal/ mole.

The polarization of a benzene ring by an ionic group can under certain circumstances give rise to appreciable interaction energy. An amino or carboxylate group on a line perpendicular to the plane of the ring and at the distance of closest approach $(3.55 \AA)$ would be attracted to the induced ring dipole with an energy of approximately $7 \mathrm{kcal} / \mathrm{mole}$ (calculated from Eq. 6-62). However, if water were present and a single hydration layer around the ionic group remained, the energy would drop to only $0.2 \mathrm{kcal} /$ mole (assuming $D=3$ ). Such ion-induced forces could contribute strongly if the interaction of inhibitor with enzyme protein occurred in a cavity from which water is exchuded or if the other forces binding the inhibitor were strong enough to force the water from between the reactants. Needless to say, the attraction between a constellation of protein charges and a polycyclic aromatic compound could provide the high affinity sometimes observed between derivatives of naphthalene and anthracene with enzymes, if the contours and patterns of the contact surface were satisfactory.

An excellent example of the energies involved in such interactions is given by Munck et al. (1957), who studied the complexes formed between steroids and adenine nucleotides. These 1:1 complexes were shown to be attributable to dispersion forces acting over the relatively large surfaces of these molecules. The reaction may be written as: 
for the case of DPN or TPN where W represents the total number of water molecules in contact with the reactants. When $40 \AA^{2}$ was taken as the area of interaction, the following energy values were calculated:

$$
\begin{aligned}
& \text { steroid-W } \rightarrow \text { steroid }+\mathrm{W} \\
& \text { coenzyme- } \mathrm{IH}^{+} \rightarrow \text { coenzyme }+\mathrm{W}+7.6 \\
& \text { steroid }+ \text { coenzyme } \rightarrow \text { steroid-coenzyme }-5.7 \\
& \mathrm{~W}+\mathrm{W} \rightarrow \mathrm{W}-\mathrm{W} \quad-13.4 \\
& -6.2 \mathrm{kcal} / \mathrm{mole}
\end{aligned}
$$

Values around $-6 \mathrm{kcal} /$ mole were obtained experimentally but they point out that the close agreement is probably fortuitous inasmuch as the energy calculations are only approximate. However, two important conclusions may be drawn from these results. In the first place, it would indicate that the major contribution to such complexes is dispersion with regard to the forces between the reactants but that the largest factor is the recombination of the displaced water molecules. Secondly, it points out the necessity of taking interactions between inhibitor and coenzyme into account in the inhibitions by steroids of the $\beta$-hydroxysteroid dehydrogenase reported by Marcus and Talalay (1955), since this enzyme is DPNdependent.

The dispersion interaction between the benzene ring and varions alkyl groups has been treated by T. L. Brown (1959) who calculated the energies when the groups were attached to the ring (see tabulation below).

\begin{tabular}{l|c|c}
\hline Group & $\begin{array}{c}\text { Polarizability } \\
(\mathrm{ml})\end{array}$ & $\begin{array}{c}\varphi \\
(\mathrm{kcal} / \mathrm{mole})\end{array}$ \\
\hline Methyl & $2.7 \times 10^{-24}$ & 2.2 \\
Ethyl & $\pm .6 \times 10^{-24}$ & 3.7 \\
Isopropyl & $6.5 \times 10^{-24}$ & 5.2 \\
tert-Butyl & $8.4 \times 10^{-24}$ & 6.8 \\
\hline
\end{tabular}

The polarizability of the benzene ring normal to its axis was taken as $12.3 \times$ $10^{-24} \mathrm{ml}$. The amount of dispersion energy is thus quite appreciable and this must play a major role in inductive effects on the ring. When these aromatic molecules are in solution, it would be expected that the solvent would reduce somewhat the interaction energy. 


\section{INTERACTIONS OF HYDROCARBON CHAINS}

The interactions of hydrocarbon groups on the substrate or inhibitor with hydrocarbon or nonpolar residues on the enzyme may occasionally contribute a significant fraction of the total binding energy. Dispersion forces only need be considered here. Calculation of the dispersion energies between $\left(-\mathrm{CH}_{2}-\right)_{n}$ chains in paraffin crystals was reported by Müller (1936) and satisfactory agreement with the heats of sublimation was obtained. However, the orientation of the chains in these crystals is unlikely to be that found in enzyme interactions and thus it will be of interest to present some tentative calculations based on reasonable models. It is relatively easy to determine the dispersion energy of two adjacent hydrocarbon chains in any assumed configuration, but the energy terms for water displacement present some difficulties since the changes in water structure during the interaction are not clearly undestood.

Neglecting the water temporarily, a simple calculation of the dispersion energy assuming two parallel $\left(-\mathrm{CH}_{2}-\right)_{n}$ chains separated by their van der Waals' radii ( $4.2 \AA$ between chain axes) gives $-0.17 \mathrm{kcal} / \mathrm{mole}$ for each $-\mathrm{CH}_{2}-$ unit $\left(\mathrm{Z}=6, R_{0}=4.50 \mathrm{ml} / \mathrm{mole}\right.$ in Eq. 6-69). If the two chains are assumed to be oriented for closest fit, with each $-\mathrm{CH}_{2}-$ midway between those on the other chain (see Fig. 6-7), and the interactions of each $-\mathrm{CH}_{2}-$ with all others on the adjacent chain are considered, the dispersion energy may be as high as $-0.76 \mathrm{kcal} / \mathrm{mole}$, but it is likely that such perfect orientation would be achieved only under very special conditions. The change in dispersion energy involved in the displacement of water molecules may be calculated to be $-0.19 \mathrm{kcal} / \mathrm{mole}$ for each $-\mathrm{CH}_{2}-$ unit, assuming that an average of two water molecules are in contact with each mit before reaction. Thus the total interaction energy for a single $-\mathrm{CH}_{2}-$ unit would be around $0.36-0.95 \mathrm{kcal} /$ mole, depending on the degree of fit, taking into account the dispersion energy only. If the formation of hydrogen bonds between water molecules occurs as the result of the displacement, the over-all interaction energy might be greater than these figures. The binding energies for some aliphatic sulfates with serum albumin are: octyl sulfate, $-5.01 \mathrm{kcal} / \mathrm{mole}$; decyl sulfate, $-6.03 \mathrm{kcal} /$ mole, and dodecyl sulfate. $-7.22 \mathrm{kcal} / \mathrm{mole}$ (Klotz, 1949). The interaction energy per $-\mathrm{CH}_{2}-$ unit would thus be about $0.55 \mathrm{kcal} / \mathrm{mole}$, which is within the calculated range. In any event it is evident that such hydrocarbon chains, if oriented properly for interaction with nonpolar regions of the enzyme, can contribute appreciably to the binding energy. Particularly high energy values might result from the position of a hydrocarbon chain within a slit on the protein or if the mobility of the protein side-chains allowed partial or complete enclosure of the chain. 


\section{DEPENDENCE OF INTERACTION ENERGY UPON FIT}

It would be superfluous to emphasize further the importance of proper fit for the binding of many substances to proteins after what has been presented already in this chapter, but certain aspects of this problem may be summarized at this time. In discussing the fit of a molecule to a protein surface it is well to remember that one must sometimes take into account the contour of the molecules with water of hydration included, since undoubtedly interactions occur where the water is not displaced.

Generally speaking there are three different, but closely related, types of fit. (1) Most inhibitor molecules possess two or more attachment points by which they are bound to the enzyme, the binding energy and hence the inhibition depending upon the degree to which these attachment points correspond to appropriate groups on the enzyme. Thus molecules of the type:

$$
\mathrm{R}_{1}-\mathrm{X}-\mathrm{R}_{2} \quad \mathrm{R}_{1}-\mathrm{X}<\mathrm{R}_{\mathrm{R}_{3}}^{\mathrm{R}_{2}}
$$

for which the attachment points are represented by $\mathrm{R}$ and the remainder of the molecules determining the spatial relations of these points by $\mathrm{X}$, would be bound maximally to enzymes only if these R-groups can all come into intimate contact with enzyme groups with which they have affinity. The actual distances between these $\mathrm{R}$-groups and the flexibility of the molecules will be the major factors in determining the efficacy of the inhibitor and there are many examples where alteration of the distance by addition or subtraction of intervening units causes marked changes in inhibitory potency. (2) In certain instances there would seem to be no dominant attachment points in the bound molecule and the over-all interaction energy is dependent upon the more or less continuous fit of the molecule to the protein surface, adequate binding arising from the summation of many small van der Waals' type forces. Such would be the case in the binding of steroids to proteins. Here the most important factor is the total surface contour with respect to regions on the protein that are spatially complementary to the bound molecule. The introduction, for example, of even a small group on the side of the molecule which interacts with the protein may reduce the fit sufficiently to inhibit binding. (3) Finally there are the situations where fit within an invagination of the protein surface occurs and these cases perhaps present the most stringent requirements for fit. An energy-distance curve, such as is shown in Fig. 6-19 for a methyl group within a protein carity, demonstrates what will happen as either the group or the cavity alters in size. In such cases, there is an optimal size for both group and cavity, deviations from which will result in a lessening of the interaction energy.

It would be convenient if the requirement for fit in any particular in- 
stance could be expressed in an exact and quantitative manner. Let us take as a measure for this the change in interaction energy occurring when the separation distance is altered by a small amount; thus the greater the energy change upon displacement, the greater is the requirement for proper fit. The valne of $d \gamma / d d$ can be evaluated if the dependence of energy on distance is known. In the general case where $q=A / d^{a}-B / d^{b}$ (Eq. 6-50), differentiation leads to:

$$
\frac{d \varphi}{d d}=-\frac{a A}{d^{a+1}}+\frac{b B}{d^{b+1}}
$$

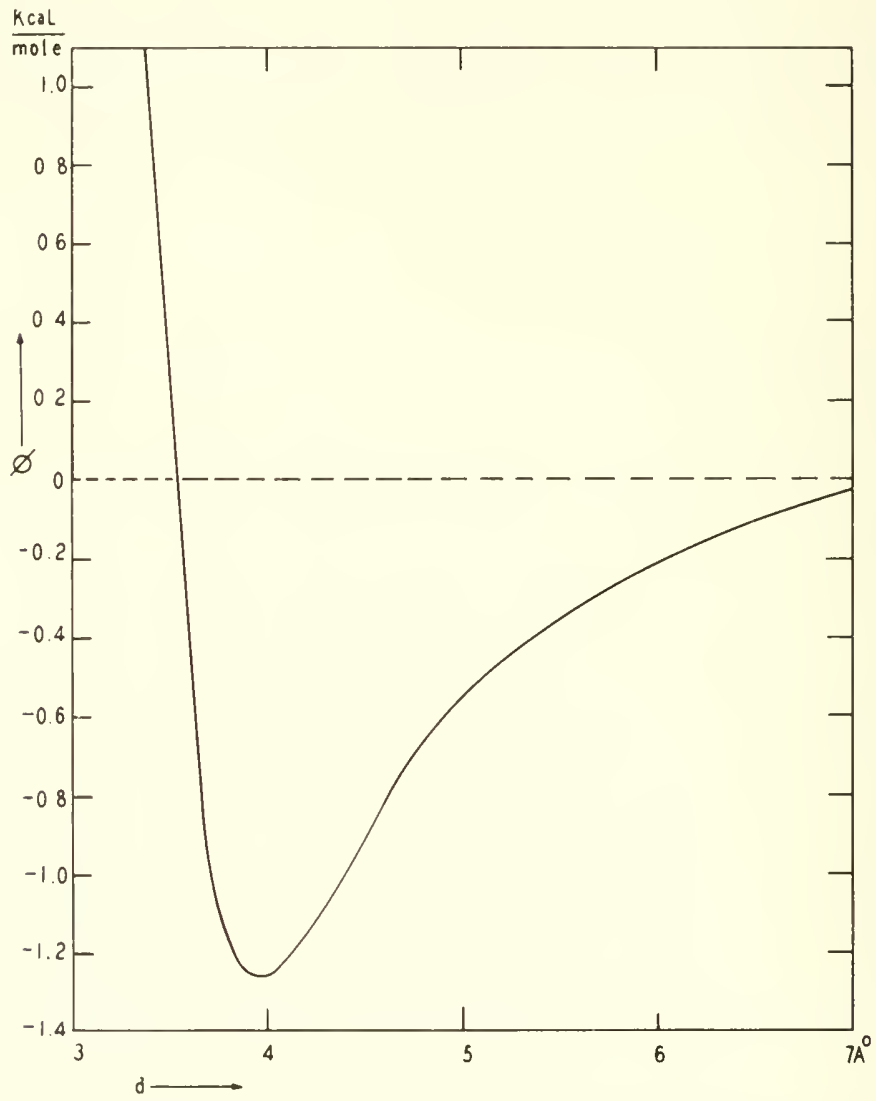

FIa, 6-19. Energy-distance curve for the interaction of a methyl group within a protein cavity. The equation used is:

$$
p=\frac{1.86 \times 10^{7}}{d^{12}}-\frac{9.73 \times 10^{3}}{d^{6}}
$$

Displacement of two water molecules is assumed. $\varphi_{e}=-1.26$ $\mathrm{kcal} /$ mole and $d_{\mathrm{s}}=3.95 \AA$. 
which may be called the "equation of fit" since it represents the dependence of interaction energy on distance at any chosen separation. It may be convenient to call $d f / d d$ the "index of fit." The indices of fit have been plotted for two types of interaction in Fig. 6-20 from which it may be noted that the importance of accurate fit varies with the separation between the gronps, naturally rising very rapidly as the groups approach clos-

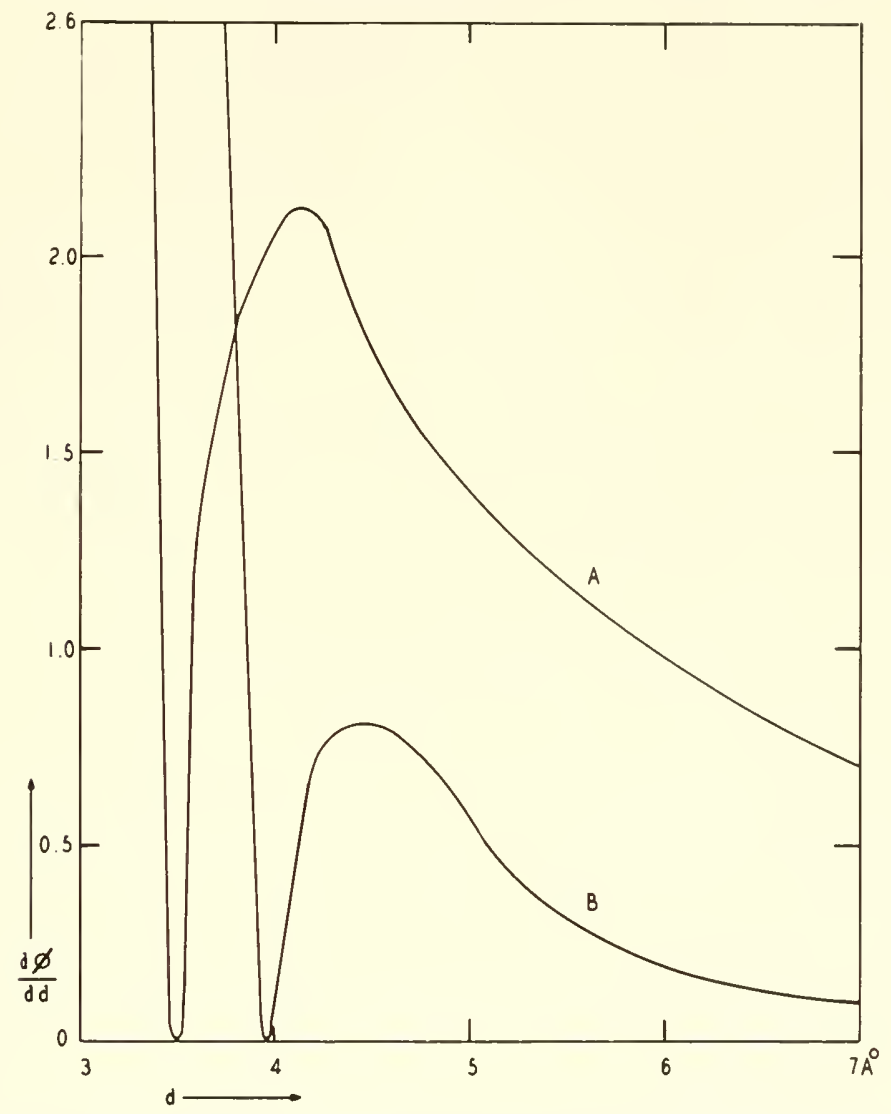

FIg. 6-20. Variation of the index of fit with distance. Curve A is for an ion-ion interaction (same characteristics as in Fig. 6-13) and curve $B$ is for the interaction of a methyl group within a protein cavity (as in Fig. 6-19).

er than the equilibrium distance. due to repulsion forces, and showing a maximum at a separation distance of approximately $0.5 \AA$ greater than the equilibrium distance. Near the equilibrium distance the fit is not so acnte because of the relative flatness of the energy-distance curves. 
It has been frequently stated that accuracy of fit is particularly important in dispersion interactions, since the energy varies with $d^{-6}$, whereas in ion-ion interactions, for example, where the energy varies with $d^{-\mathbf{1}}$, the fit would not be so important. This has only an element of truth in it. In the first place, such considerations have often neglected the effect of the repulsion forces in modifying the slope of the energy-distance curve; for dispersion interactions the repulsion forces the curve to flatten out at relatively lower values of the energy (in absolute figures) than for ion-ion interactions, and thus the slope predicted at the equilibrium distance without repulsion effects is not applicable. Secondly, the index of fit may be larger for ion-ion interactions than for dispersion, which may be seen in Fig. 6-20. It is true that in the ion-ion interaction a greater equilibrium energy is involved and that, considering the peaks in the curves, $(d p)$ $d d)_{\max } / \varphi_{e}=0.40$ for the ion-ion case whereas $(d \varphi / d d)_{\max } / \varphi_{e}=0.64$ for dispersion, but the importance for inhibition usually lies in the total energy change upon variation of separation distance, not the change relative to the maximal interaction energy. Actually the index of fit will depend on a number of factors, as indicated in Eq. 6-119, including the values of $A$ and $B$ as well as the power dependencies on the distance. Thus the index of fit will be greater for a hexyl chain lying in a slit than for a single methyl group, since the value of $B$ will be greater in the former.

\section{EFFECTS OF ALTERATION OF STRUCTURE ON MOLECULAR PROPERTIES}

It is a very common practice in metabolic and enzyme studies to alter the structure of a substrate or known inhibitor to determine the modification in the behavior. Thus a useful way of finding speeifie inhibitors is to modify a substrate in different ways. with the aim to produce a substance which retains the ability to interact with the active site but which will not be catalytically acted upon. Determination of the topography of the active site often involves the use of series of related compounds in order to characterize and localize reactive or binding groups on the enzyme surface. Some insight can often be obtained into the mechanism of an inhibition and of the forces oeeurring in the binding by the use of inhibitors differing from one another only by discrete groups or structures. It is therefore of considerable importance to inquire into the changes in molecular properties that may occur upon such structural modifieation and the relationships of these changes to the interpretation of inhibition studies.

\section{Possible Ways in Which Structural Modification May Be Made}

The changes in structure most commonly used in inhibition work may be conveniently classified in the following three types. 
I. Change in position of a group: a single group is moved from one position in a molecule to another position.<smiles>[R]CCC(I)C([R])I</smiles>

II. Replacement of a group: a single group is removed and another group put in the same position.<smiles>[R]CC(I)C[3H]</smiles><smiles>O=[N+]([O-])c1ccc(O)c([N+](=O)[O-])c1</smiles><smiles>[R]CC([B])C[3H]</smiles><smiles>Nc1cc([N+](=O)[O-])ccc1O</smiles>

III. Addition or removal of a group: a group is removed from the molecule (usually replaced by $\mathrm{H}$ ) or a new group is added (usually replacing an $\mathrm{H})$.

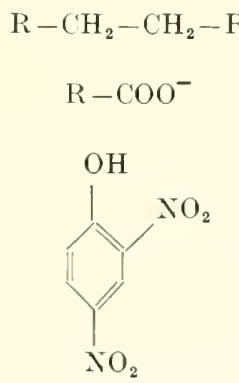

$$
\mathrm{R}-\mathrm{CH}_{2}-\mathrm{R}^{\prime}
$$

It is our immediate problem now to determine what physical and chemical properties of molecules may be modified by such changes in structure. 


\section{Classification of the Possible Changes in Molecular Properties}

A fact of major importance that has frequently been neglected is that when a particular group is altered or eliminated or added, the changes in the molecule are not confined to this group. Any change of one part of a molecule generally produces changes either throughout the molecule or at least in those regions adjacent to the changed part. We must therefore primarily divide molecular modifications into those attributable to the group itself and those occurring in the rest of the molecule. The following classification of possible changes may be helpful although it is not exhaustive.

(1) Molecular changes attributable to the modified group itself

(A) Introduction into the molecule of an ionically charged or dipolar group, or the elimination or modification of such.

(B) Introduction into the molecule of a group that is chemically reactive with protein groups, or the elimination or modification of such reactivity.

(C) Altcration of the contour of the molecule at the region of the group, which will either enable the molecule to interact more completely with the enzyme or sterically to interfere with the interaction.

(2) Molecular changes induced by the modified group

(A) Changes in the dissociation constants of acidic or basic groups.

$(B)$ Changes in the oxidation-reduction potential.

(C) Changes in the charge distribution or dipole moments throughout the molecule.

(D) C'hanges in the solubility of the molecules.

(E) Changes in the position or freeclom of rotation or movement of other groups due to steric or electrostatic effects.

(F) Changes in stability or reactivity of the molecule.

Most of the changes observed in molecules resulting from the modification of a particular group can be attributed to mechanisms which are termed inductive, in which there is a shift in the average electron positions dne to the dipole moment of the group; electrostatic. in which the properties of the various regions of the molecule are altered by the electrical field of a charged group; or resonance, in which the electrons of the group are capable of resonating with those in adjacent bonds. These mechanisms are often difficult to distinguish and frequently overlap. Before presenting examples of such changes, let us enumerate some of the ways in which the changes in molecular properties could influence the behavior of an inhibitor in a metabolic system. For the sake of clarity we may assume that a group has been added to an established inhibitor, $\mathrm{I} \rightarrow \mathrm{I}-\mathrm{A}$, and we inquire into the 
reasons why the new substance behaves differently, quantitatively or qualitatively, from the original inhibitor.

(1) The penetrability of the inhibitor into cells may be altered due to changes in size, charge, or solubility; thus the new substance may reach the enzyme in greater or lesser amounts than did the original inhibitor.

(2) The new substance may be more or less readily inactivated, either spontaneously or enzymically, as occurs with certain barbiturates or substituted naphthoquinones upon lengthening the side-chain; the concentration of the new substance may thus rary with time in a different manner than the original inhibitor.

(3) The ability of the substance to alter another enzyme system may differ from the parent inhibitor and if this other enzyme system is involved in what is meas. ured a modified action will be observed.

(4) The addition of the group may provide increased surface for interaction, reactivity with sone enzyme group, a favorable charge or dipole, or may sterically or electrostatically interfere with the binding to the enzyme; these effects may be attributed directly to the additional group.

(5) The binding of the new substance may be different from the initial inhibitor because of the changes enumerated above in the rest of the molecule as produced by the presence of the added group.

(6) The binding to the enzyme may not be significantly changed but the ability to interfere with the enzyme reaction may be changed, such as would occur if the added group were not involved in binding but sterically blocked the substrate from the active site.

It is frequently very difficult to establish the mechanism whereby a change in molecular structure modifies an inhibitory effect because in most cases many properties of the substance are changed simultaneously; for example, the molecular size and configuration, the distribution of charges, the penetrability through membranes. and the solubility may all be changed along with an altered ability to bind to an enzyme. When the data from an homologous series of inhibitory compounds on cell respiration are immediately interpreted in terms of differences in oil-water distribution coefficient, or any other single factor, without further evidence, a certain degree of naivety is implied. Indeed, the attribution of specific binding energies to particular groups, as has been done previonsly in this chapter for some enzymes, is an unsophisticated approach, justified here only by the fact that isolated enzymes are at least independent of membrane permeability factors and other complications present in all tissue work, and by the desire to illustrate the forces of binding in as straightforward a manner as possible. However, in more critical calculations, it is well to consider all the possibilities of molecular change upon altering a group before assigning the binding energy difference to the groups involved. 


\section{Examples of Group Changes and Inhibitions by Related Compounds}

In order to demonstrate some of the inherent difficulties in interpreting the effects of group modification, the following examples are presented as typical of sitnations often found in inhibition studies.

(a) Molecular polarizations by groups and the resulting dipole moments. The total dipole moment of a molecule cannot be accurately estimated from the moments of the groups present because inductive or resonance effects alter electronic distributions over considerable distances. Many inhibition studies involve the addition or elimination of simple groups on an aliphatic chain or aromatic nucleus. The dipole moments of some alkyl and phenyl halogens are given in Table 6-28. In $\mathrm{CH}_{3} \mathrm{Cl}$ the moment is less

TABLE 6-2S

Dipole Moments of Halogen Derivative ${ }^{a}$

\begin{tabular}{l|c|c|c}
\hline & \multicolumn{3}{|c}{ Dipole moment (debyes) } \\
\cline { 2 - 3 } & $\mathrm{Cl}$ & $\mathrm{Br}$ & $\mathrm{I}$ \\
\hline Mroup & 1.87 & 1.78 & 1.59 \\
Ethyl & 2.05 & 2.02 & 1.90 \\
Propyl & 2.10 & 2.15 & 2.01 \\
Butyl & 2.09 & 2.15 & 2.08 \\
& & & 1.50 \\
\hline
\end{tabular}

${ }^{a}$ From Wheland (1955, p. 213).

that the $\mathrm{C}-\mathrm{Cl}$ moment (2.3) because of the oppositely directed three $\mathrm{C}-\mathrm{H}$ moments (0.4), but is greater than would be expected due to the polarization of the methyl group by the $\mathrm{C}-\mathrm{Cl}$ bond. $\mathrm{In}_{\mathrm{CH}_{3}} \mathrm{CH}_{2} \mathrm{Cl}$ the moment is higher because the ethyl group has a greater polarizability than the methyl group, and the propyl compound has a still higher moment. However, beyond this there is essentially no change. This indicates that such inductive effects can extend along an aliphatic chain about three carbon atoms with decrement. One might expect phenyl-Cl to have a moment even higher than the $\mathrm{C}-\mathrm{Cl}$ bond because the benzene ring is quite polarizable, but actually the moments are lower than the alkyl compounds. The explanation is that resonance occurs between the following structures:

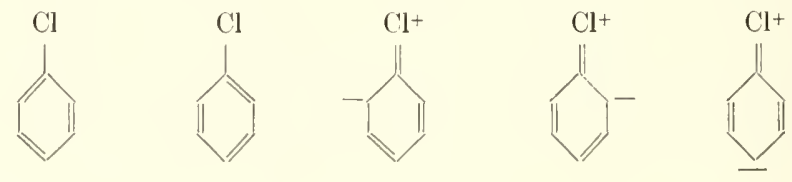


Although the last three structures do not contribute greatly in terms of molecular species, their dipole moments are high and they counteract the $\mathrm{C}-\mathrm{Cl}$ moment effectively. A charge of around 0.05 that of an electron charge would be sufficient to account for this reversal if this fractional charge is located on the ortho or para positions. Similar resonance forms contribute to the over-all moments of nitro-, amino-, and carboxy-substituted benzenes (Wheland, 1955, p. 213). Thus the total dipole moment of a simple molecule and its changes when groups are altered are not simply referable to the groups only; when binding to an enzyme involves such moments, the inhibition will depend upon inductive and resonance effects within the molecules.

(b) Distance of separation between assumed binding groups. Inhibitors that contain two distinct groups separated by relatively unreactive regions of the molecules, $\mathrm{R}-\mathrm{X}-\mathrm{R}$, are often assumed to inhibit differently when the size of $\mathrm{X}$ is varied due to changes in separation of the groups. An excellent example of such variation in enzyme inhibition may be taken from the study of Blaschko and Himms (1955) on the amine oxidases from various tissues, the results of which are plotted in Fig. 6-21. Compounds of the series

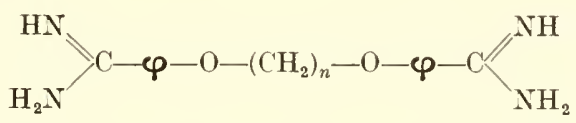

were tested for their ability to inhibit the oxidation of tyramine and in most cases an optimal chain length was found for maximal imhibition. These results might be interpreted as follows: on the enzyme there are two sites capable of reacting with the terminal amidine groups and these sites are a constant distance apart, the degree of inhibition depending on the distance between the amidine groups and how well this corresponds to the enzyme site distance (it was not so interpreted by Blaschko and Himms). This may be so but there are other explanations that are justifiable. For example, the $-\left(\mathrm{CH}_{2}\right)_{n}-$ chain may also interact with the enzyme surface and the variations in binding attributed to differing dispersion and steric effects resulting from the change in $n$. Similar variations are often observed when a hydrocarbon chain length is altered when there is only one possibly reactive group in the molecule.

(c) Effect of optical isomerism on group effects. The hydrolysis of substituted $\mathrm{L}$-tyrosinamides by $\alpha$-chymotrypsin is inhibited by the corresponding D-isomers (Manning and Niemann, 1958) and the dissociation constants are shown in Table 6-29. The D-isomers are bound as tightly as the L-isomers but presumably possess a configuration in which the electronic distortion leading to hydrolysis camnot occur. These results illustrate not only the 
marked dependence of catalytic activity upon the spatial configuration and the effects of various groups on the strength of binding, but also show that the effect of a certain group on the energy of interaction varies with its orientation with respect to the rest of the molecule. For example, introducing three fluorine atoms into the acetyl group of $\alpha$-N-acetyltyrosinamide increases somewhat the affinity of the enzyme for the L-isomer substrate but decreases the affinity for the D-isomer inhibitor. In establishing the effects of such group alterations one must thus take the total configuration into account.

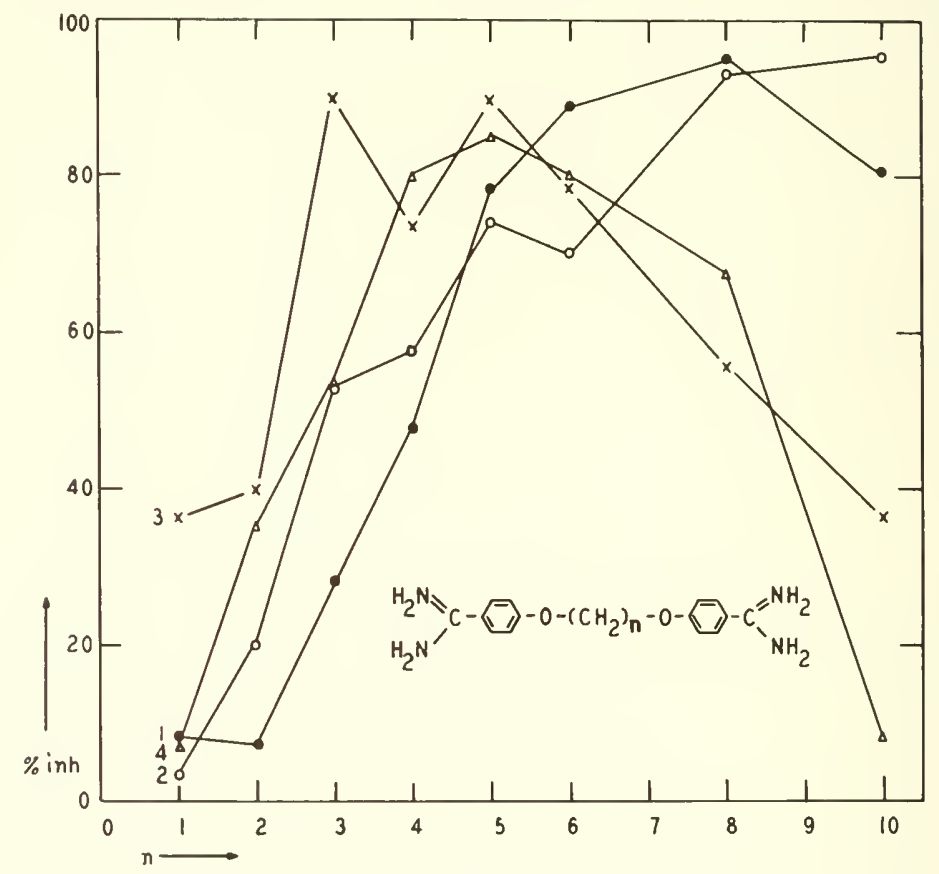

FIG. 6-21. Inhibition of amine oxidases by diamidines as inflnenced by the number of $-\mathrm{CH}_{2}-$ groups in the inhibitor. (From Blaschko and Himms, 1955.) Curve l: guinea pig liver, tyramine, (I) $=1 \mathrm{~m} .1 \mathrm{I}$; curve 2: pig liver, tyramine, $(\mathrm{I})=0.2 \mathrm{mM}$; curve 3 : cat liver, tyramine,

(I) $=0.2 \mathrm{~m} M$; curve 4 : ox serum, spermine, $(\mathrm{I})=0.005 \mathrm{~m} M$.

(d) Effect of group changes on type of action. When simple gronp additions or replacements are made in an inhibitor, it may not be certain that the observed change in inhibition is due to an altered effect on a single enzyme system. Determination of the respiratory inlibition produced by the halogen-acetates illustrates this well. The change in inhibition characteristics when a fluorine atom replaces an iodine or bromine atom might 
TABLE 6-29

Dissochation Constants of Substrates and Inhibitors of $\alpha$-Chymotrypsin ${ }^{a}$

\begin{tabular}{|c|c|c|c|}
\hline \multirow[b]{2}{*}{ Compound } & \multicolumn{2}{|c|}{ Dissociation constant } & \multirow[b]{2}{*}{$\begin{array}{c}\text { Ratio } \\
\left(K_{s} / K_{i}\right)\end{array}$} \\
\hline & $\begin{array}{l}\mathrm{L}-\mathrm{Isomer} \\
K_{s}(\mathrm{~m} \|)\end{array}$ & $\begin{array}{l}\mathrm{D}-\mathrm{Isomer} \\
K_{i}(\mathrm{~m} M)\end{array}$ & \\
\hline$\alpha-N$-carbethoxy tyrosinamide & 6.4 & 21 & 0.3 \\
\hline$\alpha-\lambda$-nicotinyltyrosinamide & 12 & 9 & 1.3 \\
\hline$\alpha-N$-trifluoroacet yltyrosinamide & 26 & 20 & 1.3 \\
\hline$\alpha-N$-acetyltyrosinamide & 32 & 12 & 2.7 \\
\hline$\alpha-N$-chloroacetyltyrosinamide & 27 & 6.5 & 4.1 \\
\hline
\end{tabular}

a From Manning and Niemann (1958).

be attributed solely to a change in the inhibition of the enzymes sensitive to iodoacetate or bromoacetate, whereas it actually reflects a new type of action on the tricarboxylic acid cycle. Another example would be in the testing of group substitutions in phenols on the inhibition of some enzyme. The addition of most groups onto the ring would merely reflect different interactions with the enzyme although with the same inhibition mechanism whereas the introduction of a $p$-hydroxyl group would perhaps allow inhibition by a new mechanism. namely the reaction with enzyme sulfhydryl groups through the formation of a quinone.

\section{General Suggestions with Regard to Comparison of Members of an Inhibitor Series}

Summarizing the principles of this section, it must be emphasized that great care be exercised in the interpretation of results on inhibition produced by homologous series and chemically related substances. The following points are believed to be particularly important for consideration in work of this type.

(a) Comparison of inhibition potencies must be made on a molar basis and not on a weight/volume or dilution basis since it is only on the molar basis that true relative activities and binding energy differences may be obtained.

(b) In most systems it is advisable to determine the effects of the various members on the measured rate in the absence of the substrate, in order to eliminate the possibility that some of the inhibitors are contributing to the measurements by being acted upon by the enzyme in question or by another enzyme in the preparation. This also serves to establish control determinations of the effects on any endogenous activity. 
(c) The stability of the inhibitors in the preparation used should often be checked so that differences in rates of concentration decrease over the period of measurement will not be interpreted as due to different degrees of inherent inhibitory potency.

(d) The possibility of different rates of penetration into cellular or tissue preparations should be considered before attributing differences in inhibition to actions upon an enzyme system.

(e) The attempt should be made to determine if different inhibition mechanisms may be involved with different members of a series. The establishing of competitive inhibition, for example, with one member does not ensme that this mechanism holds for all the nembers.

( $f$ ) When gromps are added, eliminated, or modified in an inhibitor, great care should be taken not to attribute the differences observed solely to the group itself, but the effects of this group change on the rest of the molecule should be considered.

\section{INTERACTIONS OF SMALL MOLECULES WITH PROTEINS}

A great deal of very fine work in recent years has been done on the binding of molecules of all types to proteins, but the results and general principles that have emerged have been little applied to the problems of enzyme inhibition. It has often been felt that the types of binding are distinct; that measurements of over-all binding to proteins (such as would result from equilibrim dialysis studies) refer to a nonspecific interaction, while inhibition studies relate to specific interactions. It is true that competitive inhibition on substrate sites by substances closely related structurally to the substrates is probably quite specific; on the other hand, many inhibitions are certainly not specific for any active site. Likewise, over-all protein binding can be quite specific or relatively nonspecific. The inhibition of enzymes by heavy metal ions is generally not specific in the sense that these metals combine only with active sites; they bind at many sites on the protein and incidentally at or near the active sites - a study of the binding by physical or chenical methods can be very informative and applicable in many respects to enzyme inhibition. The basic principles involved and the types of forces operative are certainly the same in both cases. In fact, the binding energies of inhibitors and small molecules generally cover very much the same range. Unfortunately, the subject of the general binding of substances to proteins is so large that it conld not be adequately treated in the present work. It may be worthwhile, however. to mention several of the concepts that have been derived from such binding studies and which bring some insight into the mechanisms and forces involved in enzyme inhibition. For more detailed information on the bind- 
ing of small molecules to proteins, the following general references may be consulted: Goldstein (1949). Klotz (1949, 1953), Gurd and Wilcox (1956), Scatchard et al. (1954), and Edsall and Wyman (1958, p. 591).

\section{Competition between Ligands for Binding Sites}

It was pointed ont earlier in this chapter that the binding of an inhibitor to an enzyme does not occur in a vacuum but that various types of molecules may have to be displaced from both the site and the ligand. In the absence of the inhibitor, the enzyme sites are not free but may be associated to a greater or lesser degree with water, physiological ions including $\mathrm{H}^{+}$, or other substances in the medium used. The over-all binding energy must inchude the energies required for displacement of these substances upon interaction of the inhibitor with the enzyme sites. These relationships have been studied most carefully in the binding of small molecules to proteins or to simpler components of the proteins, such as peptides or amino acids, and it is upon studies of this kind that one must base reasonable estimates of the magnitude of the effects occurring in enzyme inhibition.

In the formation of ammonia complexes of the metal ions, such as $\mathrm{Cu}^{++}$, the displacement of water molecules from the ions must be considered. Since the activity of the water remains relatively constant in such systems it has been customary to include it in the dissociation constants, but in calculating the interaction energy of ammonia with metal ions it must be remembered that the dissociation constant does not represent this alone. Likewise, the dissociation constant of oxyhemoglobin includes the displacement of the water molecule that is bound to the iron atom in the absence of oxygen. In the same manner, substrate and inhibitor constants include such displacement reactions. The effects of $\mathrm{pH}$ on binding may often be attributed to a competition between the $\mathrm{H}^{+}$and the cation whose interaction one is measuring; this has been studied particularly in the binding of $\mathrm{Ca}^{++}$and $\mathrm{Mg}^{++}$to proteins (Carr and Woods, 1955). We have considered the effects of the ionic atmosphere on binding but in many cases the common ions of the medium are bound more tightly and more specifically to protein sites. $\mathrm{K}^{+}$and $\mathrm{Na}^{+}$ions are bound to some proteins and not to others (Carr, 1956) and with greater affinity than would be expected, leading to the postulate that a chelation mechanism is responsible (Saroff, 1957). If this is true, it would imply a fair degree of specificity since the carboxylate groups of glutamate and asparate must be close enough to the imidazole groups of histidine for the chelation to occur. Serum albumin can bind several simple cations and as much as forty $\mathrm{Cl}^{-}$ions per molecule and it is likely that many enzyme proteins woukl show similar binding properties.

The effects of different buffers and ionic strengths are perhaps to be ascribed in part to such associations. We shall see that the nature of the buffer may be important in enzyme inhibition (Chapter 15) and it is per- 
tinent now to point ont that the binding of the dye methyl orange to serum albumin has been studied quantitatively in this regard (Klotz, 1949). The buffer anion can compete with the dye anion for binding sites and the effect is thus dependent upon the concentration of the buffer anion. Even the $\mathrm{Cl}^{-}$ion can interfere with the dye binding by this mechanism.

\section{Multiple Binding Sites on One Protein Molecule}

In the expressions of enzyme kinetics, (E) refers to the concentration of active sites, not necessarily enzyme molecules, and hence the number of sites on a single enzyme molecule is of no consequence in these formulations unless the sites can interact with one another. Interactions between sites on enzymes have not been adequately studied but situations analogous to hemoglobin, where the four heme sites are interdependent, may well oceur. The kinetics of multiple interactive sites have been presented (Chapter 3 ) and the forces responsible for such interaction may now be attributed to a direct influence (eleetrostatic or steric) or an indirect influence mediated through the protein. In the binding of small ions or molecules to proteins, it has been found that several sites are usually present on a molecule. For serum albumin the following number of sites have been demonstrated: $\mathrm{Cu}^{++}, 16$; $\mathrm{Ca}^{++}$and $\mathrm{Mg}^{++}$, 8; dodeeyl sulfate. 14; phenylbutyrate, 24; methyl orange, 22; and testosterone, 9. Interactions between such sites have been found.

The binding of $\mathrm{Cu}^{++}$to serum albumin at $25^{\circ}$ is characterized by a reduction in binding energy with each $\mathrm{Cu}^{++}$ion bound. The first $\mathrm{Cu}^{++}$ion is bound with $\mathrm{IF}=-5.91 \mathrm{keal} /$ mole whereas the last or sixteenth $\mathrm{Cu}^{++}$ ion is bound with $1 F=-1.72 \mathrm{kcal} / \mathrm{mole}$, a reduction of $0.26 \mathrm{kcal} / \mathrm{mole}$ for each $\mathrm{Cu}^{++}$bound on the average. This effect is due to two factors: the statistical effect, whereby the progressive occupancy of sites restricts the probability for a $\mathrm{Cu}^{++}$ion finding an unoccupied site, and an electrostatic effect resulting from the altered electrical field of the protein upon binding of the $\mathrm{Cu}^{++}$ions (Klotz, 1949). Simpler systems are presented by the complexes of metal ions with nitrogenous substances, such as $\mathrm{Zn}\left(\mathrm{NH}_{3}\right)_{4}{ }^{++}$, $\mathrm{Ni}\left(\mathrm{NH}_{3}\right)_{6}{ }^{++}$, and $\mathrm{Cu}(\text { imidazole })_{4}{ }^{++}$, where the binding of each successive ammonia or imidazole is altered by the previously bound molecules. The complexes of 4-methylimidazole have been studied recently by Nozaki et $a l$. (1957) and the successive p $K$ 's (the $K$ is here the intrinsic dissociation constant in order to eliminate the statistical factor) were found to be:

\begin{tabular}{c|c|c}
\hline & $\mathrm{Cu}^{++}$complexes & $\mathrm{Zn}^{++}$complexes \\
\hline $\mathrm{p} K_{1}$ & 3.53 & 1.84 \\
$\mathrm{p} K_{2}$ & 3.31 & 2.35 \\
$\mathrm{p} K_{3}$ & 3.05 & 2.82 \\
$\mathrm{p} K_{4}$ & 2.56 & 2.98 \\
\hline
\end{tabular}


The $\mathrm{Zn}^{++}$and $\mathrm{Cu}^{++}$thus behave differently: the binding of 4-methylimidazole to $\mathrm{Cu}^{++}$decreases the affinity for succeeding molecules, whereas binding to $\mathrm{Zn}^{++}$increases the affinity. The explanation probably lies in the planar configuration of the $\mathrm{Cu}-\mathrm{N}$ bonds and the tetrahedral arrangement of the $\mathrm{Zn}-\mathrm{N}$ bonds. The evaluation of successive constants was first treated thoroughly by Bjerrum (1941) and a very comprehensive presentation is given by Edsall and Wyman (1958, p. 591).

Similar interactions between binding sites may well occur on enzymes and in such cases the determined $K_{s}$ or $K_{i}$ may not relate to any specific binding but represent only the mean value over the range studied. For positive interactions (where the binding of a molecule helps the binding of another molecule) the determined $K_{i}$ might be smaller than for the mncomplicated binding of the first inhibitor molecnle, whereas for negative interactions (where the binding of a molecule hinders further binding) it wonld be larger.

It is probable that is some cases the total number of binding sites for an inhibitor is greater than the number of enzymically active sites; that is, the inhibitors may be bound to both active and inactive sites. Binding to inactive sites would not necessarily affect the active sites with respect to enzyme rate but might affect the binding of other inhibitor molecules to the active sites and thus modify the determined $K_{i}$. Although this effect is not generally very significant, it may well be important in mutual depletion systems (psendoirreversible or titration inhibition), inasmuch as the characteristic behavior of such systems depends on the reduction in inlibitor concentration, and it is of no importance where the inhibitor is bound. It is very difficult, for example, to estimate the number of active sites from such titration inhibition data, even with pure enzymes, due to possible binding with other groups. This problem has been referred to in Chapter 3 and will be taken up in more detail in Chapter 15 .

\section{Effect of Total Protein Charge on Binding}

Except at their isoelectric points, proteins possess a net charge and it has been frequently assumed that this net charge determines whether ions will be bound or not. However, there are many, instances where ions are bound to proteins although the signs of the charges are the same. $\mathrm{Mg}^{++}$ is bound to lysozyme at $\mathrm{pH}$ 7.4. although at this $\mathrm{pH}$ the protein is positively charged (Carr and Woods, 1955) and the anion of methyl orange is bound to serum albumin thronghont a $\mathrm{pH}$ range above the isoelectric point when the protein is negatively charged. Undoubtedly a net protein charge must influence many interactions electrostatically, and probably prevents some from occurring, but it is not necessarily the deciding factor. Presumably on a protein that has a net negative charge, there are regions where a 
positive charge dominates and where binding of a negative ion can occur. Thus the net charge on an enzyme should not be considered to be as important as the regional charge or electrical field at the active sites, with respect to the interaction of ionic inhibitors.

One method of determining the nature of the protein groups involved in the binding of a particular ligand is to react certain of the protein groups and note the effect this has on the binding. A decreased binding following acetylation of free amino groups on the protein might be considered as indicating the participation of these amino groups at the binding sites, but, as has been pointed out by Levedahl and Bernstein (1951), such acetylation also results in a reduction of positively charged groups and may change the net charge of the protein from positive to negative. This could have a nonspecific effect of repelling anions and be the explanation for the decrease in binding. Modification of charged groups on proteins must then be considered from this point of view also in attempts to determine the groups with which inhibitors react.

\section{Effect of Temperature on Binding}

The binding of various substances to serum albumin is not markedly affected by temperature changes (Klotz, 1949; 1953) and in some cases it has been difficult to determine accurately the change in dissociation constant over a range of $20^{\circ}$ to $30^{\circ}$. Since $d \ln K / d T=1 H R T^{2}$, it would appear that the enthalpy of binding is low in these cases and thus that the affinity for the protein is mainly dependent on entropy changes. It is true that the substances studied were mainly anions so that entropy changes due to water displacement would be anticipated, but it is likely that binding of nonionic substances often involves appreciable changes in the water structure and that temperature coefficients will be relatively low for these. In conformity with these results, we shall find that enzyme inhibition is frequently little dependent on temperature and probably for the same reasons. Of course there are types of inhibition which represent more than mere binding to the enzyme and for which high temperature coefficients have been found.

\section{Specificity in Simple Protein Binding}

That protein complexes with small molecules are not instances of simple surface adsorption or nonspecific binding is indicated in several ways. The number of binding sites is often small relative to the number of molecules that could cover the protein surface and the data support the hypothesis that the affinity for these sites (the intrinsic dissociation constant) is constant for a substance, except where interactions between sites occur. 
By group-specific reactants it has been demonstrated that certain protein side-chains may be necessary for the binding. The binding of testosterone to serum albumin is especially interesting in this connection because the substance is neutral and changes in total protein charge would not be expected to be of much importance. The binding energy is approximately - $6 \mathrm{kcal}$ /mole and the maximal number of testosterone molecules bound is 5 or 6 per molecule of protein (Levedahl and Perlmutter, 1956). Two tyrosine groups in the serum albumin seem to be involved in the binding of one testosterone molecule since the effect of $\mathrm{pH}$ on binding indicates that the testosterone is released when the phenolic groups become ionized. Although iodination of the tyrosine rings does not alter binding significantly, ketenization of the phenolic groups prevents binding (Oyakawa and Levedahl, 1958). It was postulated that the testosterone molecule lies parallel to the tyrosine rings and that the hydroxyl or ketone groups on the sterol react with the phenolic groups. In this instance the specificity of binding seems to be rather high and might be extended to other sterols, such as cortisone and estradiol (Eik-nes et al., 1954). A rery interesting discussion of structural specificity in sterol binding has been given by Turner (1954).

When enzyme inhibitors are optically active, it is generally found that differences in potency are observed between the isomers, so that the demonstration by Karush (1952) that optically isomeric dyes are bound differently to serum albumin is important in illustrating such specificity. The relative positions of the phenyl ring in

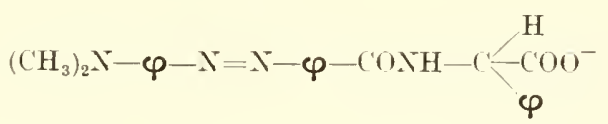

must be important in the affinity of this dye for the protein. indicating a specific configuration at the hinding sites.

Finally, the concept of specific binding is strengthened by the fact that for a particular compound, binding may occur to only certain proteins and not to others. even though the over-all net charge on the proteins may be the same. Thus with methyl orange, only serum albumin and $\beta$-lactoglobulin of the some dozen proteins studied were found to present adequate binding sites (Klotz and Urquhart, 1919 b). Proteins with similar physical and chemical characteristics often exhibit preferential binding for small molecules and ions. indicating that certain group configurations on the protein surface are necessary.

\section{FUTURE PROBLEMS IN MOLECULAR INTERACTIONS}

A cycloramic approach to the subject of molecular forces in enzyme inhibition has been presented and it is to be regretted that in most cases the real objects in the picture are slight in comparison with the painted back- 
ground. It is not advisable that the inherent complexities of intermolecular reactions be minimized for the problems must be faced and progressively solved. The development with respect to enzyme inhibition must progress through the interplay of two principal lines of investigation: the theoretical and experimental study of intermolecular forces between simple molecules of biological significance on the one hand, and the accumulation and interpretation of much quantitative data from enzyme inhibitions on the other. Only by the full utilization of the results of both approaches can a more accurate and meaningful understanding on the molecular level be attained. Many investigations are concerned only with whether an inhibitor acts on the system or not and such studies contribute nothing to the problem of mechanisms. It is somewhat laborious to vary concentrations and various factors, to obtain accurate data and to submit these data to a thorough analysis, bearing in mind all of the possibilities in mechanism and the many types of forces that must be considered as contributing to the total binding energy, but once the interest of the investigator is attuned to molecular levels it is very rewarding. The study of enzyme inhibition is, of course, only one aspect of the general problem of the interactions with proteins and advances in the general ficld must be applied to the special phenomenon of inhibition to achieve maximal progress. As in any scientific field, the greater our knowledge of the basic mechanisms, the more readily we can predict interactions and develop enzyme inhibitors of high potency and specificity for use in both research and medicine.

It must be admitted that the state of knowledge of enzyme interactions is relatively rulimentary, as it is in the field of molecular interactions in general. The author has probably exceeded the limits of certainty in some cases and stated some cloubtful points with more conviction than is justified. This has been done in the interests of clarity but apologies must be made not only for such overstatements but also for the sins of omission, because of which important reports have been murecognized or here unrecorded. If the inaccuracies stimulate others to any profitable emotion or to more rigorous solutions of the problems, we shall be indebted to these inaccuracies, although I regret them. 


\section{INHIBITION IN MULTIENZYME SYSTEMS}

The previous chapters have been concerned with the kinetics, interactions, and inhibitions of single enzymes. However, most enzymes participate in metabolic pathways in the cell and are related functionally to one another by the intermediates that arise in the individual reactions. Such a group of interrelated enzymes. catalyzing the various steps in a metabolic sequence. may be termed a multienzyme system. The enzymes involved in these systems may be bound together or confined within small regions of the cell; on the other hand they may exist in homogeneous solution. Spatial orientation of enzymes relative to one another may accelerate the over-all rate, since the diffusion time between enzymes is reduced; the concentrations of intermediates may be maintained at relatively high levels; and utilization of unstable intermediates is made possible. Such organization is not necessary in multienzyme systems and many reactions of many steps occur readily when the enzymes are separate and freely diffusible. For the purpose of the present chapter, the organization of the enzyme system will not be of importance in most cases and this question will be considered more fully in Chapter 9. Inhibition in multienzyme systems is often quantitatively and sometimes qualitatively different from inhibitions on single enzymes. We shall be principally concerned with the effects of inhibition of a single enzyme unit of a multienzyme system on the overall rate of this system and the concentrations of the intermediates. Very little quantitative work has been done on these aspects of multienzyme systems, nor have the rate equations and inhibition kinetics been rigorously developed. The systems to be discussed in this chapter are much simpler than many of those occurring in cells, but illustrate certain principles that may be extended to the more complex metabolic patterns that cannot be treated mathematically with ease. Such complex systems will be taken up in Chapter 9 in connection with inhibition within cells and tissues. General considerations and examples of multienzyme systems may be found in Dixon (1949), Greenberg (1956), Hinshelwood (1916, Chapt. IV), Mehler (1957, Chapt. IX), and Reiner (1959, Chapt. XI). 


\section{CONCEPTS AND TERMINOLOGY}

A multienzyme system may be said to be in a steady stute when the concentrations of all components (enzymes, substrates, intermediates, and products) and the rates of the individual reactions remain constant with time. Such a state differs from equilibrium in that there is a constant flow of material through the system and a constant flux energy characteristic of the state (Burton, 1939). In the simple linear sequence:

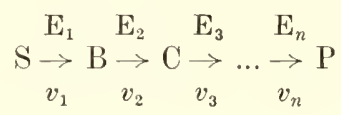

a steady state implies that $v_{1}=v_{2}=v_{3}=v_{n}$ and that (S), (B), (C), and (P) remain constant. The concentrations of substrate and product can be kept constant by diffusion of $\mathrm{S}$ into and of $\mathrm{P}$ out of the system, and these diffusion rates will then be equal to the enzyme rates $v_{1}, v_{2}, \ldots v_{n}$. The multienzyme system above conld be represented within the cell as follows:

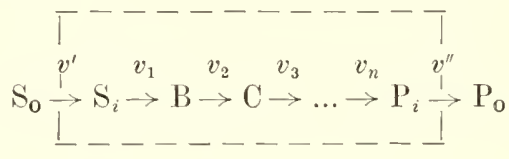

where $v^{\prime}$ and $v^{\prime \prime}$ are the rates of diffusion through the cell membrane and where $v^{\prime}=v^{\prime \prime}=v_{1}=v_{2}=v_{3}=v_{n}$. Such a situation is designated as an open system since material enters and leaves the system under consideration. In a closed system, on the other hand, material is not exchanged with the enviromment and a true steady state is not possible (Bertalanffy, 1950). A further classification of some importance in cellular metabolism may be made with respect to the uniformity of the system. In a uniform system the enzymes are distributed homogeneously whereas in a nomuiform system they may be aggregated or fixed within certain regions or structures (Bierman. 1954). Finally, a step in a multienzyme system is said to be a limiting reaction when the over-all rate of the system is determined primarily by the rate of this reaction; more precise definitions will be proposed later.

Biological systems and metabolic preparations are usually not in a true steady state for several reasons. (1) Enzyme experiments run in reaction vessels of finite size represent closed systems, the concentration of substrate decreasing and the concentration of product increasing with time. However, it is possible that the reaction will sinmlate a steady state if the initial substrate concentration is greater than that required to saturate its enzyme; negligible changes in rate will occur until the substrate concentration falls below this level. (2) Many preparations of mitochon- 
dria, cells, or tissues are unstable and tend to lose various substances from the cells and pick up others from the medium. These substances may not be involved directly in the multienzyme system but may influence its behavior; the common inorganic ions may be placed in this category. (3) The concentrations of the component enzymes may change. Inactivation of enzymes in metabolic preparations usually occurs and leakage of enzymes or coenzymes from cellular or subcellular structures is well known. Furthermore, the enzyme concentration may fluctuate with the concentration of its substrate in the manner of adaptive enzymes. (4) Cell populations are seldom static and are generally either increasing by growth or decreasing by various processes of degeneration or death. Such processes would have direct and indirect effects on any multienzyme system being studied. For practical purposes, therefore, one must usually be satisfied with a system approaching steady state kinetics. Such an approximation can often be accomplished by restricting the experimental time interval to a period during which negligible changes have occurred in the rates and concentrations. It would be more correct to speak of such systems as in a pseudosteady state. In such situations there is a continuous succession of steady states with time, the transitions between them being smooth and continuous.

The more important multienzyme systems to be discussed may be classified in the following manner.

I. Monolinear chains

$\mathrm{A} \rightarrow \mathrm{B} \rightarrow \ldots \rightarrow \mathrm{P}$

\section{Branched chains}

A. Convergent<smiles>[10BH]C=CC</smiles>

$B$. Divergent

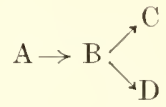

III. Polylinear chains (shunts)

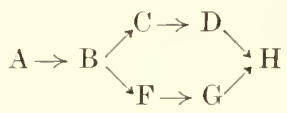

IV. Distributive systems

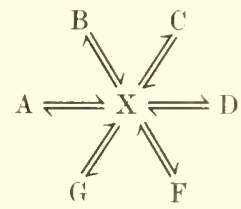

V. Cyclic systems

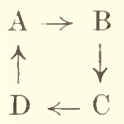


VI. Regenerative systems

(The substance $\mathrm{X}$ is regenerated during the reactions.) $\mathrm{A}+\mathrm{X} \rightarrow \mathrm{B}+\mathrm{Y}$ $\mathrm{B} \rightarrow \mathrm{C}$

$\mathrm{C}^{\prime}+\mathrm{Y} \rightarrow \mathrm{D}+\mathrm{X}$

VII. Self-regulatory system (feedback)

(D affects the rate of $\mathrm{A} \rightarrow \mathrm{B}$.)

$\stackrel{\downarrow}{\rightarrow} \mathrm{B} \rightarrow \mathrm{C} \rightarrow \mathrm{D}$

VIII. Network systems

(Electrons flow from A to B through

a grid of metal ions.)

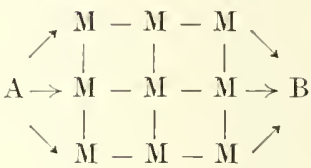

Any or all of the reactions indicated may, of course, be reversible. Many reactions in multienzyme systems are truly reversible but for practical purposes may be considered as irreversible if the back-reaction under the experimental circumstances is negligible.

Each enzyme of a multienzyme system catalyzes a reaction which may be represented in a steady state, as described in Chapter 2 , since for the simplest enzyme reaction there are two steps. the formation of the ES complex and its breakdown. Thus each single reaction in the schemes above is in reality nswally complex kinetically and the steady state of a multienzyme system implies the steady state of each component reaction.

\section{IRREVERSIBLE MONOLINEAR CHAINS}

The simplest multienzyme system may be represented by:

$$
\stackrel{\mathrm{E}_{1}}{\rightarrow} \underset{\mathrm{A}}{\mathrm{E}} \stackrel{\mathrm{E}_{2}}{\rightarrow} \mathrm{C}
$$

where each reaction is essentially irreversible. The kinetics and inhibi tion of this system can be readily extended to chains of greater length. Actually there are probably relatively few simple monolinear chains in the cell becanse most systems depend in some manner on other reactions or sequences, or are parts of more complex metabolic patterns. However, in isolated preparations or reconstructed systems, monolinear chains are more common. Under the appropriate conditions the following may behave as monolinear chains: ethanol $\rightarrow$ acetaldehyde $\rightarrow$ acetate; epinephrine $\rightarrow$ $o$-quinone form $\rightarrow$ adrenochrome: $\mathrm{ATP} \rightarrow \mathrm{ADP} \rightarrow \mathrm{AMP}$; phenylalanine $\rightarrow$ hydroxyphenylalanine $\rightarrow$ hydroxyphenylpyruvate $\rightarrow$ homogentisate; and succinate $\rightarrow$ fumarate $\rightarrow$ malate. If reaction $7-1$ is in a steady state and some mechanism is provided whereby the concentrations of $\mathrm{A}$ and $\mathrm{C}$ do not change, the rate of each step is the same, i.e., $v_{1}=v_{2}=v_{s t}$ where 
$v_{s t}$ shall designate the steady-state rate of the system as a whole, usually experimentally determined by the formation of the product $\mathrm{C}$.

\section{Concentration of the Intermediate B}

If Michaelis-Menten kinetics hold for each enzyme, the individual rates are given by:

$$
v_{1}=\frac{V_{1}(\mathrm{~A})}{(\mathrm{A})+K_{1}} \quad v_{2}=\frac{V_{2}(\mathrm{~B})}{(\mathrm{B})+K_{2}}
$$

where $K_{1}$ and $K_{2}$ are the Michaelis constants and $V_{1}$ and $V_{2}$ are the maximal rates for reactions 1 and 2 , respectively. Setting $v_{1}=v_{2}$ and solving for $(B)$, the steady-state concentration is found to be:

$$
(\mathrm{B})=\frac{V_{1} K_{2}(\mathrm{~A})}{(\mathrm{A})\left(V_{2}-V_{1}\right)+V_{2} K_{1}}
$$

Since $V_{1}=k_{1}\left(E_{t}\right)_{1}$ and $V_{2}=k_{2}\left(E_{t}\right)_{2}$, where $k_{1}$ and $k_{2}$ are the rate constants for the breakdown of the ES complexes, (B) depends on the Michaelis constants, rate constants, and enzyme concentrations of both reactions. It may be noted, however, that $(B)$ depends on the ratio $V_{1} T_{2}$ and not on the absolute values. The variation of $(B)$ with the concentration of substrate $\mathrm{A}$ as it depends on $V_{1} / T_{2}$ is shown in Fig. 7-1. The value of $(\mathrm{B})$ can theoretically be greater or smaller than (A) in the steady state. However, limits to which (B) can rise are generally imposed by the nature of the system. In reactions run in homogeneous closed systems (as with enzyme solutions in reaction vessels), (B) must always be a small fraction of (A), since otherwise it would imply that an appreciable amount of $\mathrm{A}$ had been reacted and its concentration reduced from the initial value. In open systems where (A) can be maintained constant from an outside source, or in nonuniform systems where B can be trapped, (B) can rise to values higher than (A). The level to which (B) can rise often determines whether the system will be in a steady state or not, and this will be considered in more detail later. It may also be noted that (B) is not necessarily linearly related to (A), except when $V_{1}=V_{2}$.

\section{The Over-All Steady-State Rate, $v_{\text {st }}$}

When the system is in a steady state, the rate of disappearance of the substrate, $-d(\mathrm{~A}) / d t$, is equal to the rate of formation of the product. $d(C) / d t$, and this rate will be determined by the first reaction. Thus $v_{s t}$ will always be $V_{1}(\mathrm{~A}) /\left[(\mathrm{A})+K_{1}\right]$ in the steady state. However. if the system in not in a steady state and $B$ is being formed faster than it can be utilized, $d(\mathrm{C}) / d t$ will not be equal to - $d(\mathrm{~A}) / d t$ but will depend on (B) and will not 


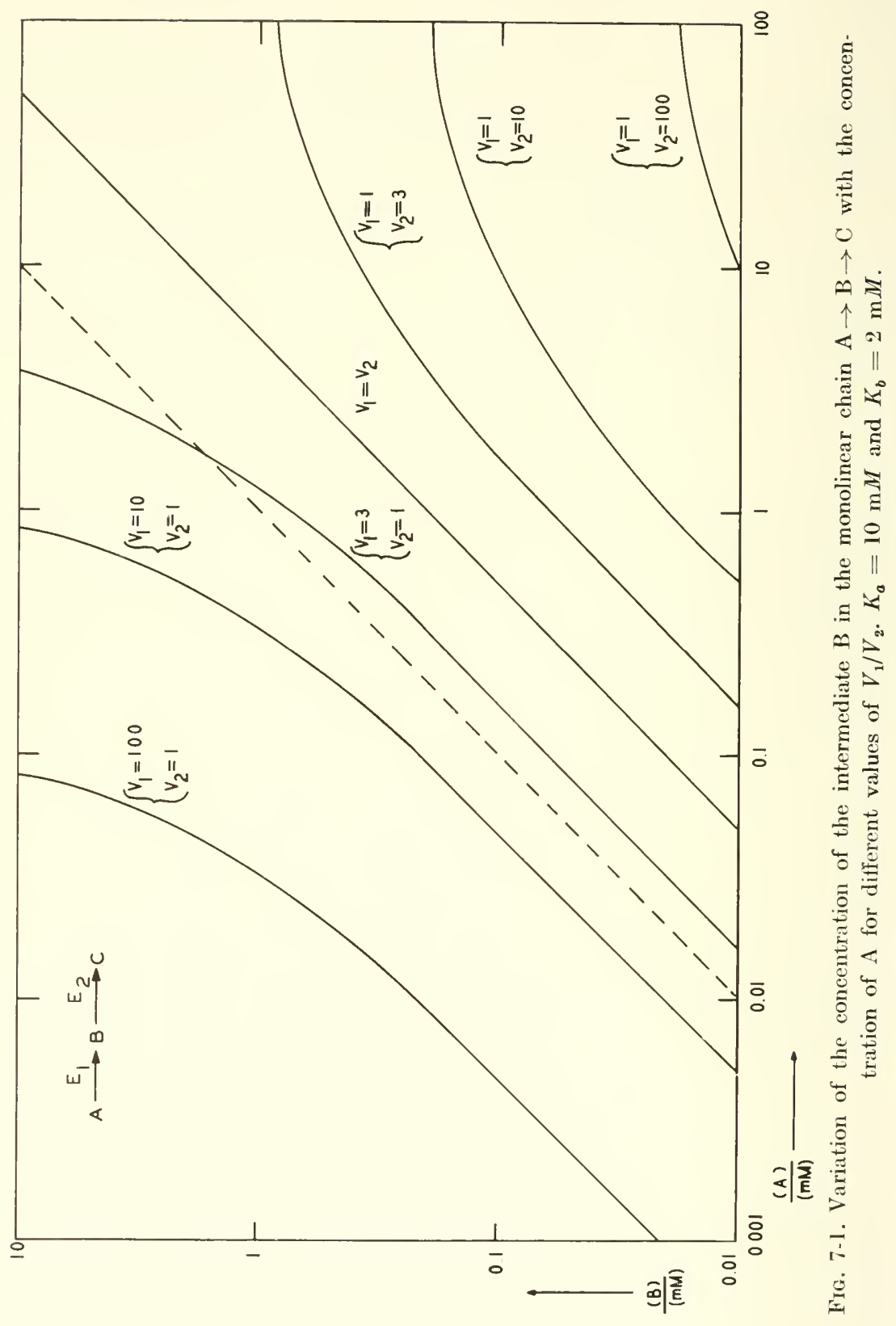


be constant because (B) is probably increasing with time. The results of inhibition on such systems will depend on whether the system is in a steady or nonsteady state.

\section{Graphical Representation of Sequential Reaction Chains}

It is convenient to visualize the relationships in multienzyme systems by inspection of the rate curves for each enzyme reaction involved. Such curves are plotted for three values of $V_{1} / V_{2}$ in Figs. 7-2, 3, and 4. When

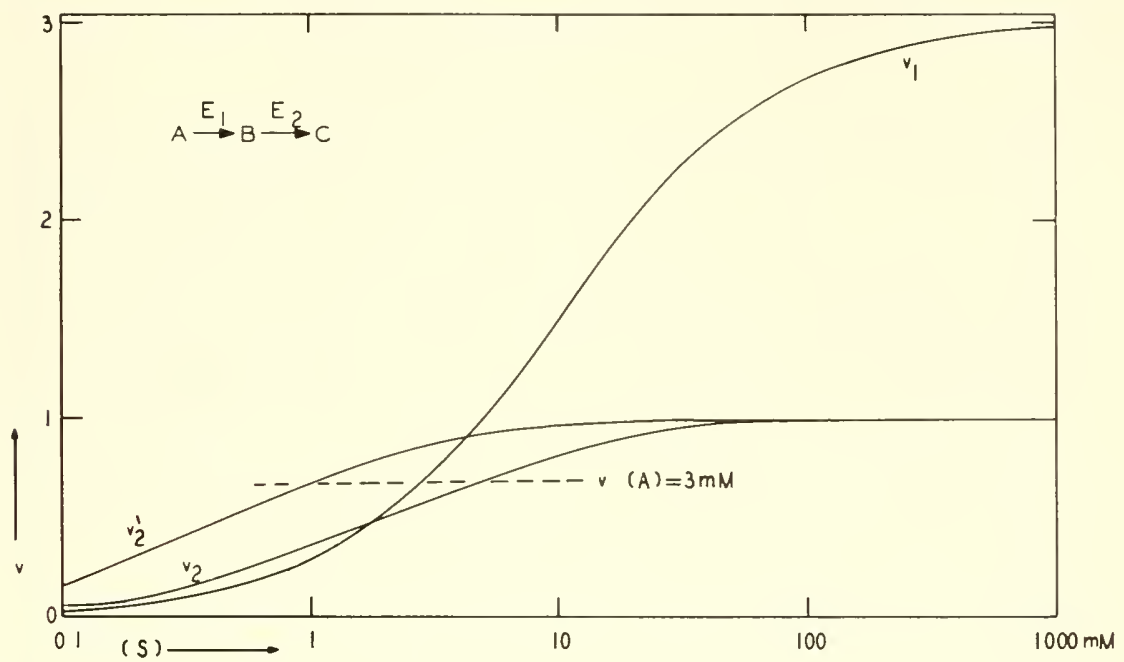

FIG. 7-2. Rate curves for a monolinear chain. Curve $v_{1}$ shows the rate for reaction 1 alone: $V_{1}=3$ and $K_{1}=10 \mathrm{~m} . H$. Curve $v_{2}$ shows the rate for reaction 2 alone: $V_{2}=1$ and $K_{2}=2 \mathrm{~m} \cdot I$. Curve $v_{2}{ }^{\prime}$ shows the rate for reaction 2 alone: $V_{2}=1$ and $K_{2}{ }^{\prime}=0.5$ $\mathrm{m} M$. Dashed lines cut the curves $v_{2}$ and $v_{2}{ }^{\prime}$ at points indicating the concentration of B necessary for the steady state when $(\mathrm{A})=3 \mathrm{mM}$.

$V_{1} / V_{2}=3$ it is seen that if $v_{1}$ is greater than $V_{2}$ the system cannot be in a steady state, for $\mathrm{B}$ cannot be reacted at a rate faster than $V_{2}$ whatever (B). In the present case, this puts a limit on (A) of $5 \mathrm{~m} M$ for a steady state. When $V_{1} / V_{2}=1$ or 0.33 the system can always be in a steady state at any value of $(\mathrm{A})$, since $v_{1}$ can never be greater than $V_{2}$, but restrictions may limit the level that $(\mathrm{B})$ can attain and hence there may be a maximal (A) beyond which the steady state will not occur.

From these curves the value of (B) for any substrate concentration can be estimated when the system is in a steady state. A horizontal line is drawn at the rate of reaction $I$ for the specified concentration of $\mathrm{A}$ and the intersection with the rate curve for reaction 2 gives (B). Thus in the 


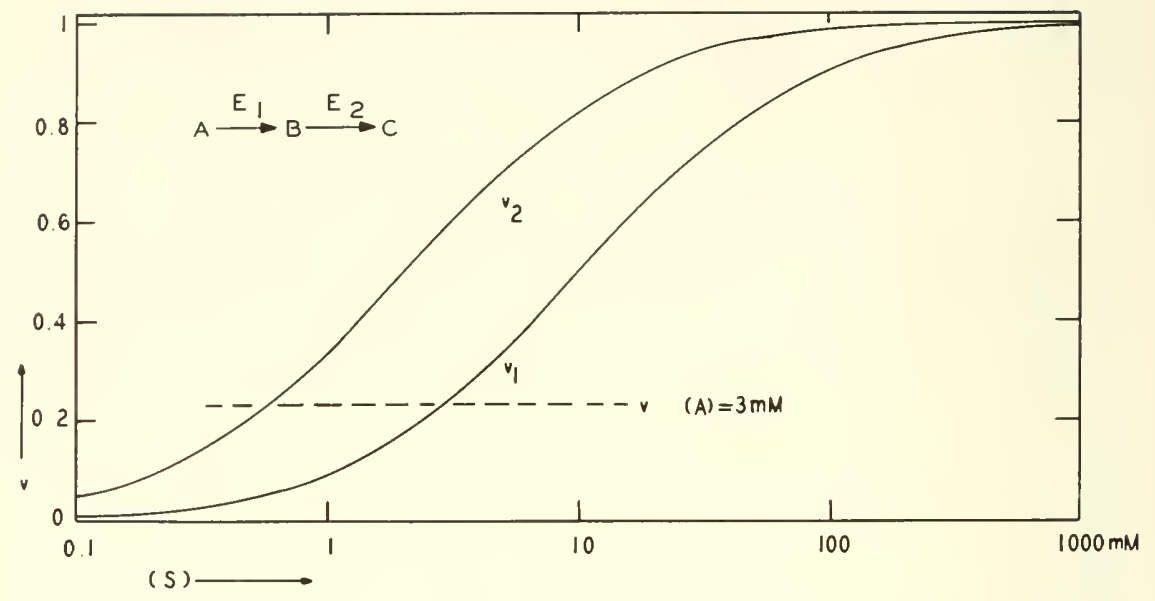

FIg. 7-3. Rate curves for a monolinear chain. Curve $v_{1}$ shows the rate for reaction 1 alone: $V_{1}=1$ and $K_{1}=10 \mathrm{~m} . H$. Curve $v_{2}$ shows the rate for reaction 2 alone: $V_{2}=1$ and $K_{2}=2 \mathrm{~m} M$. Dashed line cuts curve $v_{2}$ at the concentration of B necessary for the steady state when $(\mathrm{A})=3 \mathrm{~m} . \boldsymbol{H}$.

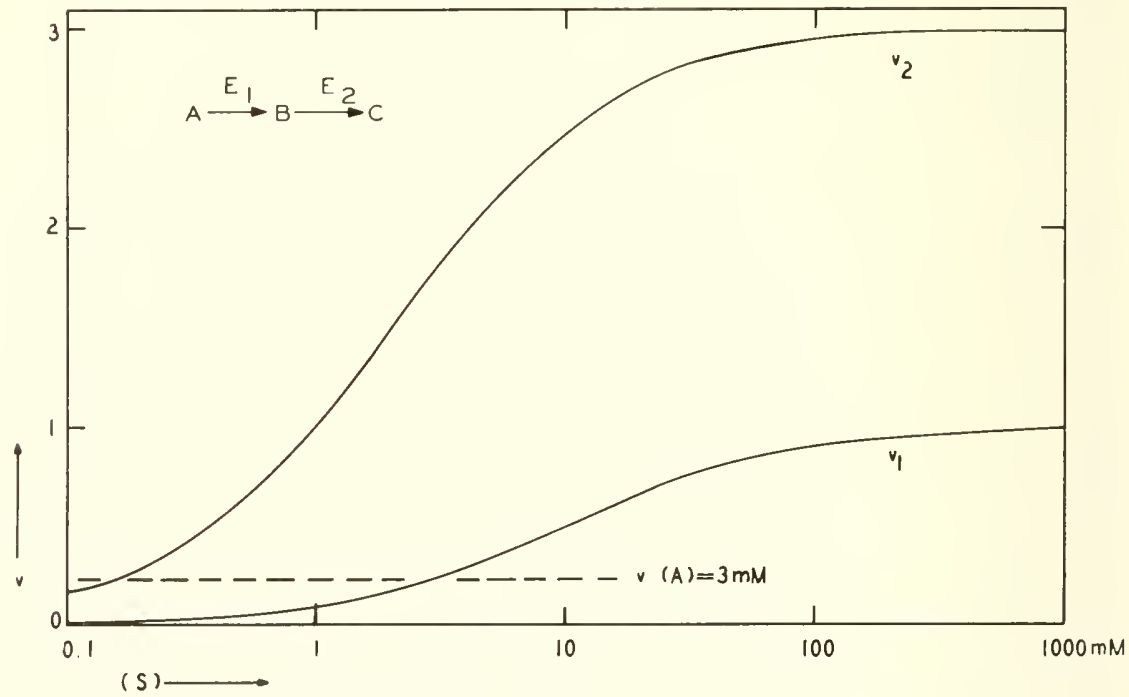

Fig. 7-4. Rate curves for a monolinear chain. Curve $v_{1}$ shows the rate for reaction 1 alone: $V_{1}=1$ and $K_{1}=10 \mathrm{~m} . H$. Curve $v_{2}$ show the rate for reaction 2 alone: $V_{2}=3$ and $K_{2}=2 \mathrm{~m} H$. The dashed line cuts curve $v_{2}$ at the concentration of $\mathrm{B}$ necessary for the steady state when $(\mathrm{A})=3 \mathrm{~m} . \mathrm{K}$. 
situations plotted in Figs. 7-2, 3, and 4, lines have been drawn for $(\mathrm{A})=$ $3 \mathrm{~m} U$, from which it can be seen that (B) must reach the following levels to achieve a steady state:

\begin{tabular}{|c|c|}
\hline$V_{1} / V_{2}$ & $\begin{array}{c}(\mathrm{B}) \\
(\mathrm{m} M)\end{array}$ \\
\hline 3 & 4.5 \\
\hline l & 0.6 \\
\hline 0.33 & 0.17 \\
\hline
\end{tabular}

The third curve for $v_{2}^{\prime}$ in Fig. $7-2$ shows that reduction in $K_{2}$ leads to a marked reduction in (B) for the steady state; low values of $K_{2}$ thus favor the possibility of a steady state.

\section{Inhibition of Monolinear Chains}

The nature of the response of a multienzyme system to inhibition depends on three things: (1) the reaction step inhibited (i.e., $E_{1}$ or $\mathrm{E}_{2}$ in system 7-1), (2) the type of inhibition (e.g.. competitive or noncompetitive), and (3) the state of the system previous to inhibition (which is determined by the concentrations of substrate and enzymes, and the various constants of the system). Inhibition may shift the steady-state rate of the system to a new level, or take the system out of a steady state, or put the system into a steady state from a nonsteady one. The concentrations of intermediates may go up or down and this may have effects on cellular processes other than the simple system considered. The accumulation of citrate during the fluorocitrate block of aconitase is responsible for some of the toxic effects observed. The striking effects of alcohol following disulfiram (Antabuse) are due to the accumulation of acetaldehyde in the tissues as the result of the inhibition of the aldehyde dehydrogenase, catalyzing the second reaction in the sequence ethanol $\rightarrow$ acetaldehyde $\rightarrow$ acetate.

(A) Noncompetitive inhibition. The maximal rate, $V_{m}$, of the enzyme noncompetitively inhibited will be decreased (see Eq. 3-16) and thus the primary result of such inhibition on the simple system treated here will be a change in the $V_{1} / V_{2}$ ratio. The effects of inhibitions of $E_{1}$ and $E_{2}$ on various systems are shown graphically in Fig. 7-5 (I to VI). Since the over-all steady-state rate, $v_{s t}$, depends on the rate of reaction 1 , inhibition of $\mathrm{E}_{1}$ will result in the same reduction of $v_{s t}$ as of $v_{1}$. However, if the system is originally not in a steady state, inhibition of $E_{1}$ may not affect the formation of $\mathrm{C}$ until reaction 1 has been reduced sufficiently to limit the overall rate. The level of $(\mathrm{B})$ will be decreased in all cases in which $\mathrm{E}_{1}$ is inhibited. 

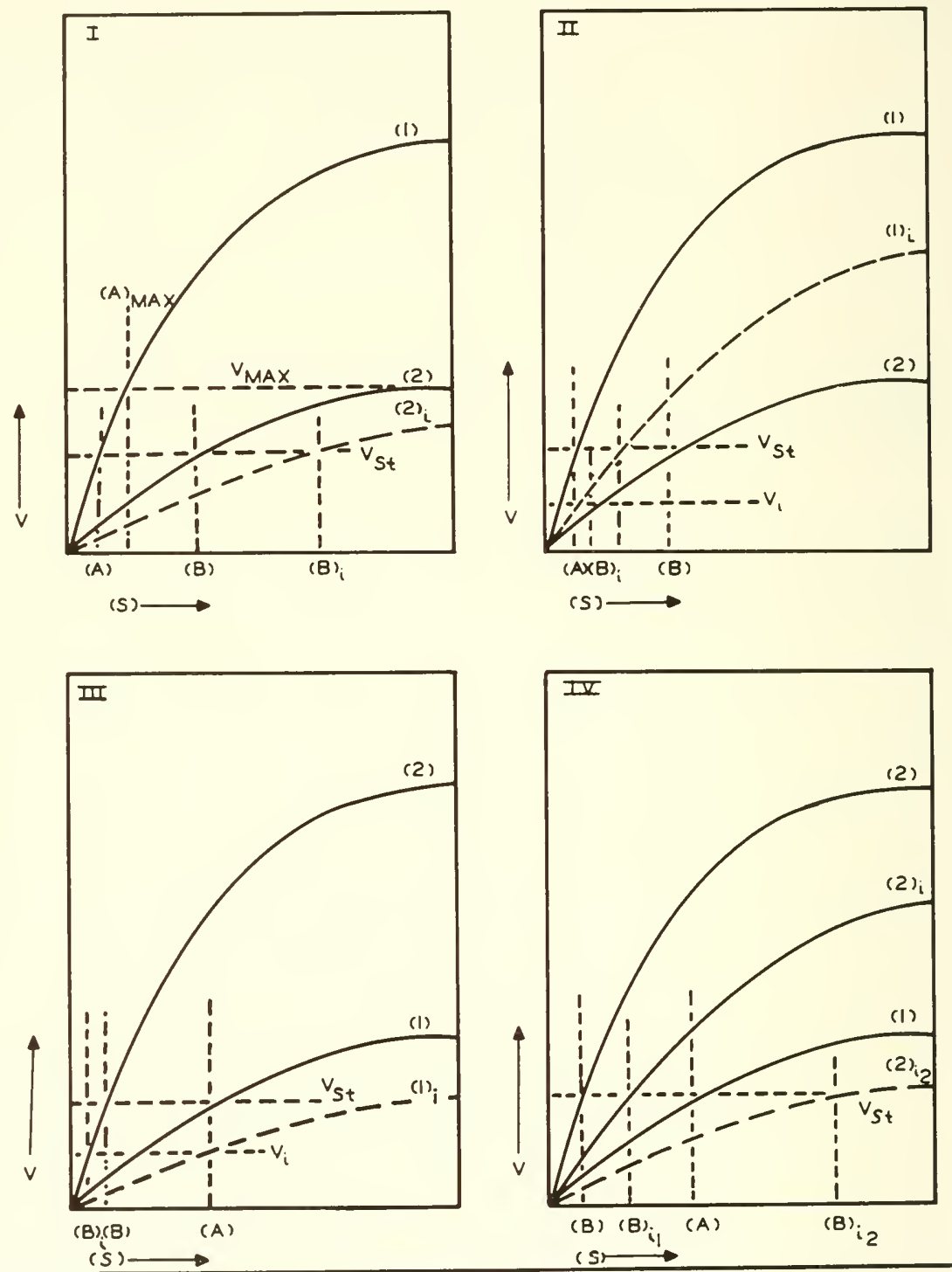

FIG. 7-5. Effects of noncompetitive inhibition on a monolinear chain $\mathrm{A} \rightarrow \mathrm{B} \rightarrow \mathrm{C}$. Curves 1 and 2 in each case show the rates of reaction 1 and reaction 2 , respectively. The dashed curves, labeled either $1_{i}$ or $2_{i}$, show the rates of these reactions following inhibition. The horizontal dashed line labeled $v_{s t}$ indicates the steady-state rate and cuts the curves at the concentrations of $\mathrm{A}$ and $\mathrm{B}$ in the steady-state. When reaction 1 is inhibited this line is lowered to that indicated 

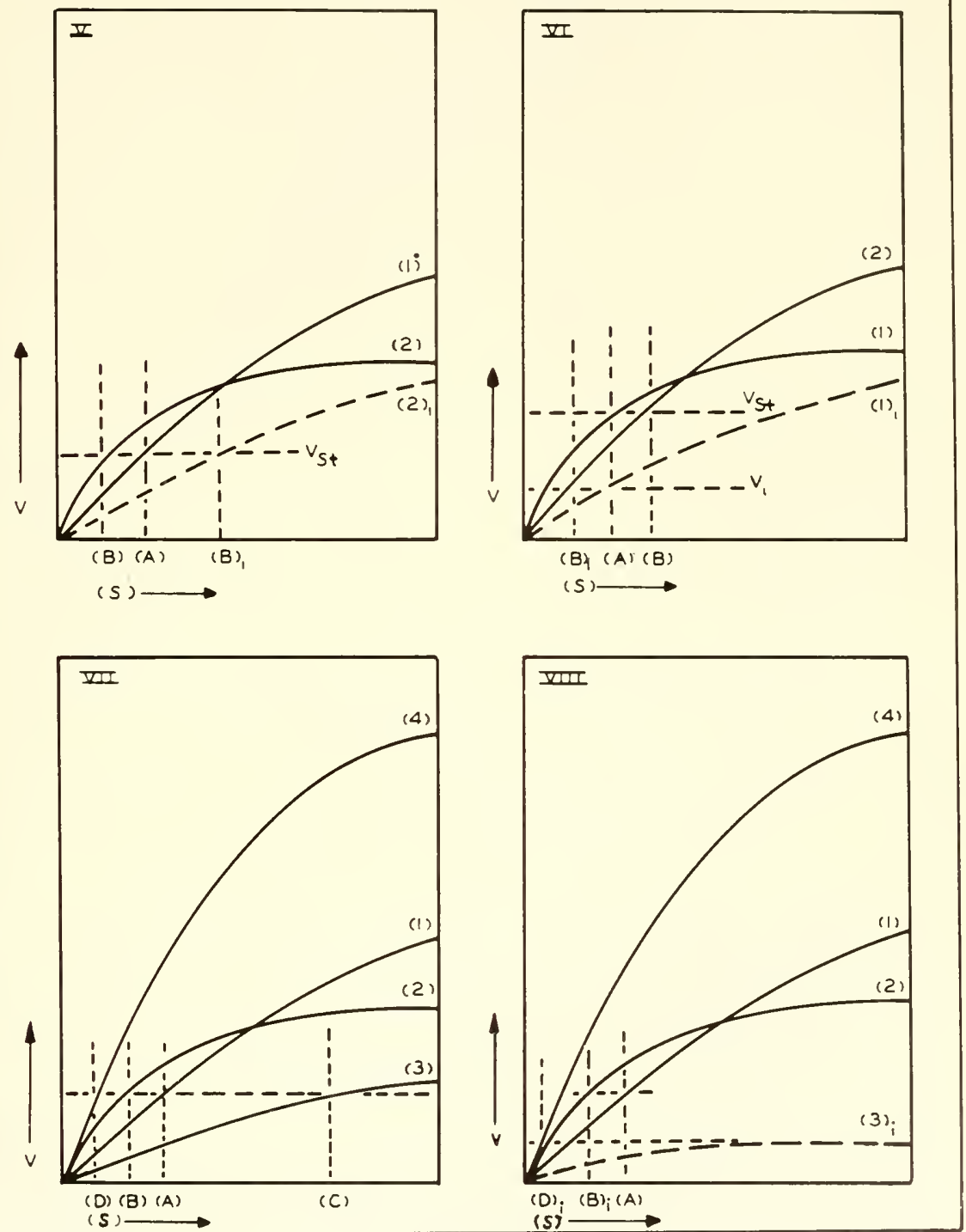

FIG. 7.5 (continued)

by $v_{i}$. The vertical dashed lines indicate the concentrations of $\mathrm{A}$ and $\mathrm{B}$ and are labeled below. For example, in case I the concentrations are (A) and (B) initially and the system is operating in the steady state at a rate $v_{s t}$. The addition of an inhibitor of $\mathrm{E}_{2}$ leads to a depression of the rate curve for this reaction and the concentration of the intermediate rises from $(\mathrm{B})$ to $(\mathrm{B})_{i}$. 
Inhibition of $\mathrm{E}_{2}$ will increase $(\mathrm{B})$ and the rate of formation of $\mathrm{C}$ will not be reduced as long as the system can remain in a steady state. It is evident that if $V_{2}$ is decreased below $v_{1}$, the steady state cannot be maintained and the formation of $\mathrm{C}$ will be depressed. The rise in (B) upon inhibition depends on the relative maximal rates of the two reactions $\left(\mathrm{V}_{1} / V_{2}\right)$ as shown in Fig. 7-6. When the system is barely in the steady state, as in

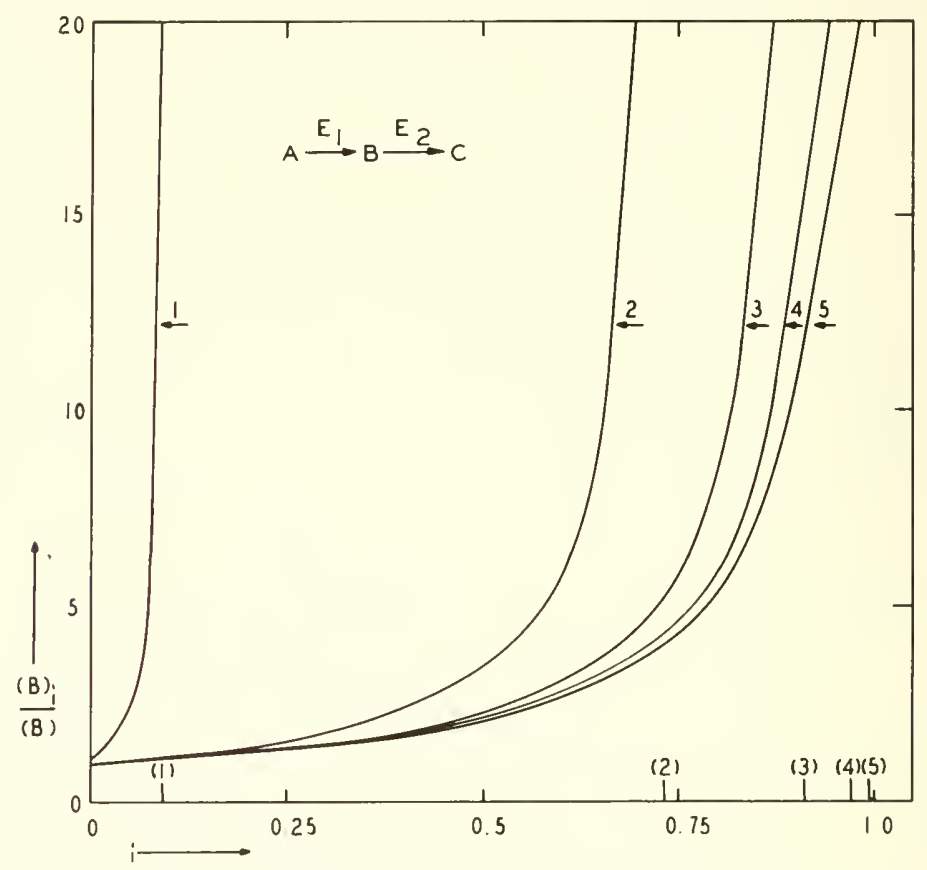

Fig. 7-6. Curves showing the rise in (B) following inhibition of $\mathrm{E}_{2}$ in a monolinear chain. $K_{1}=10 \mathrm{~m} \nu, K_{2}=2 \mathrm{~m} \nu$, and $(\mathrm{A})=1 \mathrm{~m} \mu$. The numbered lines on the $i$ axis show the maximal inhibitions which may be exerted before the systems leave the steady state. See Eq. 7-4. Curve 1: $V_{1} / V_{2}=10$; curve 2: $V_{1} / I_{2}=3$; eurve 3 : $V_{1} / V_{2}=1$; eurve $4: V_{1} / V_{2}=0.33$; curve $5: V_{1} / V_{2}=0.1$.

curve 1 where $V_{1} / V_{2}=10$, it requires very little inhibition to increase (B) and take the system out of the steady state; in fact, for the system plotted, only $9 \%$ inhibition will make the steady state impossible. Of course, the high values of (B) necessary for the steady state may not be able to be achieved and the system will leave the steady state sooner. As the maximal rate of reaction 2 becomes greater relative to reaction 1 , it requires more and more inhibition to increase (B). An expression for the change in (B) may be derived as follows. If it is assumed that the maximal 
rate of reaction 2 in the presence of the inhibitor, $V_{2 i}=(1-i) V_{2}$, and we apply Eq. 7-3:

$$
\frac{(\mathrm{B})_{i}}{(\mathrm{~B})}=\frac{V_{2}\left[(\mathrm{~A})+K_{1}\right]-(\mathrm{A}) V_{1}}{(1-i) V_{2}\left[(\mathrm{~A})+K_{1}\right]-(\mathrm{A}) V_{1}}
$$

where $(B)_{i}$ is the concentration of $\mathrm{B}$ in the inhibited system, and it is this equation that is plotted in Fig. 7-6. Since the denominator on the right side must be greater than zero if the nmmerator is positive and less than zero if the numerator is negative, the maximal inhibition allowable for the system to remain in a steady state is:

$$
i_{\max }=1-\frac{V_{1}}{V_{2}}\left[\frac{(\mathrm{A})}{(\mathrm{A})+K_{1}}\right]
$$

One of the most important general properties of multienzyme systems may be illustrated with this simple sequence. The inhibition of the rate of formation of product, in this case $d(\mathrm{C}) d t$, will often be less than the inhibition of the single enzyme affected. In some cases, reaction 2 must be almost completely blocked before reduction of $d(\mathrm{C}) / d t$ is observed. This ability to resist inhibition might be thought of as the buffer capacity of the system against inhibition. It depends for one thing on the capacity of the system for (B) to increase until reaction 2 again becomes as rapid as reaction 1 , or at least to increase to a level satmrating $\mathrm{E}_{2}$. Since the mimhibited steady state $d(\mathrm{C}) / d t=V_{1}(\mathrm{~A}) /\left[(\mathrm{A})+K_{1}\right]$ and the inhibited $d(\mathrm{C}) / d t=V_{2 i}=(1-$ i) $V_{2}$, if (B) can rise to saturate $\mathrm{E}_{2}$, the inhibition exerted on the formation of $\mathrm{C}$ is given by:

$$
i_{t}=1-(1-i) \frac{\mathrm{V}_{2}}{V_{1}}\left[\frac{(\mathrm{A})+K_{1}}{(\mathrm{~A})}\right]
$$

Plotting $i_{l}$ against $i$ gives a straight line with a slope of $V_{2}\left[(\mathrm{~A})+K_{i}\right] / V_{1}(\mathrm{~A})$, as shown in Fig. 7-7 for the systems plotted in Fig. 7-6. This form of plotting shows particularly well the buffering capacity of the system. If $V_{1} / V_{2}$ is much greater than ten in the system assumed, a steady state would not exist previous to inhibition and $i$, will always equal $i$. A third manner of plotting such phenomena is shown in Fig. 7-8, where $i_{i}$ is related to the inhibitor concentration. In systems with adequate buffer capacity, no effect will be noted on the formation of $\mathrm{C}$ until a critical concentration of inhibitor is reached, at which point the inhibition will increase rapidly.

The buffer capacity of a system, as defined by $d i / i_{t}$, is the change in the inhibition of a single enzyme necessary to produce the change $d i$ in the inhibition of prockuct formation. For the two-step monolinear chain, the buffer capacity may be obtained from Eq. 7-6:

$$
\frac{d i}{d i_{t}}=\frac{V_{1}}{\mathbf{J}_{2}}\left[\frac{(\mathrm{A})}{(\mathrm{A})+K_{1}}\right]=\frac{v_{s t}}{V_{2}}
$$


and thus depends not only on $V_{1} / V_{2}$ but also on the concentration of the initial substrate, $\mathrm{A}$, and the Michaelis constant of $\mathrm{E}_{1}$. In the examples plotted, (A) was assumed to be $1 \mathrm{~m} . M$; if this were increased to $10 \mathrm{~m} M$, the buffer capacity would be increased 5.5 -fold (from $V_{1} / 11 V_{2}$ to $V_{1} / 2 V_{2}$ ). The relation between $i_{t}$ and $i$ in most multienzyme systems will not be linear as it is here, and $d i / d i_{t}$ will change with the degree of inhibition. The

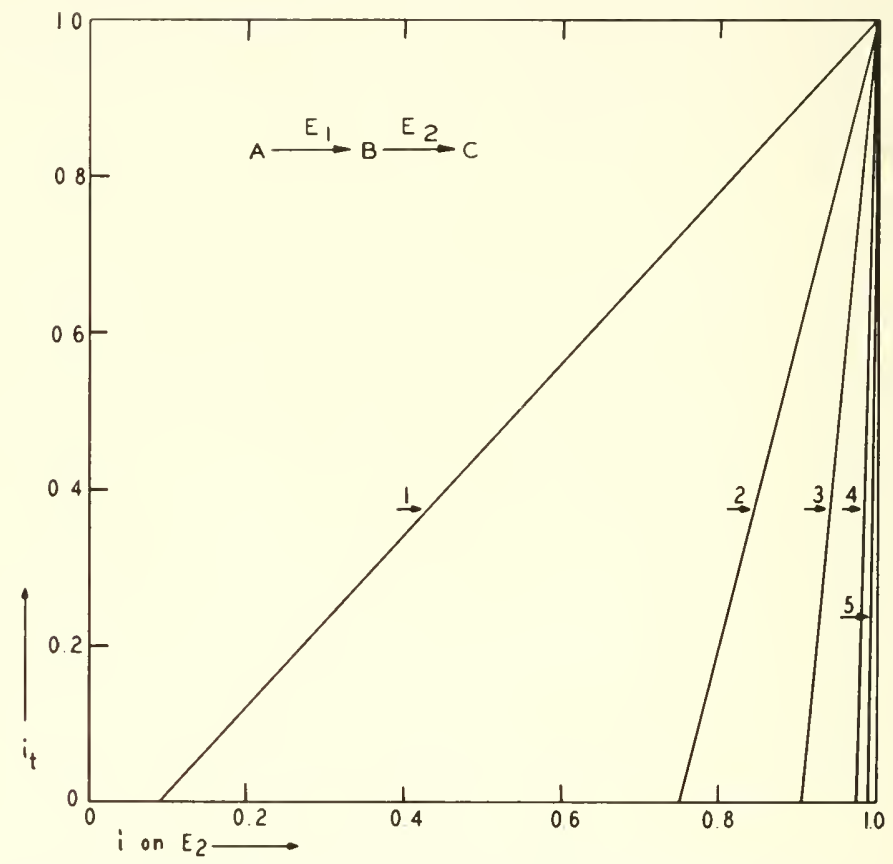

FIG. 7-7. Relationship between the over-all inhibition and the inhibition on $\mathrm{E}_{2}$ in a monolinear chain (Eq. 7-6). Curve 1: $V_{1} / V_{2}=10$; curve $2: V_{1} / V_{2}=3$; curve $3: V_{1} / V_{2}=1$; curve $4: V_{1} / V_{2}=0.33$; curve 5: $V_{1} / V_{2}=0.1$.

buffer capacity might also be defined as $d(\mathrm{I}) / d i_{t}$, which is more comparable to the definition of buffer capacity in acid-base systems, but it is felt that

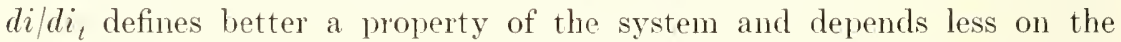
characteristics of the inhibition, thus being of greater value in comparing different multienzyme systems.

(B) Competitive inhibition. The response of the monolinear system to competitive inhibition of $E_{1}$ will be similar to that of the single enzyme since (A) is assumed to remain constant. Competitive inhibition of $E_{2}$, however, introduces an additional factor because the rise in (B) will reduce the inhibition on $\mathrm{E}_{2}$. Inasmuch as competitive inhibition implies an in- 
crease of $K_{2}$ by the factor $1+$ (I) $/ K_{i}$ (see Eq. 3-12), from Eq. 7-3 it is found that the increase in $(B)$ due to inhibition is simply given by:

$$
\frac{(\mathrm{B})_{i}}{(\mathrm{~B})}=1+\frac{(\mathrm{I})}{K_{i}}
$$

and hence, in contrast to noncompetitive inhibition, is not dependent on any of the parameters of the uninhibited enzyme system. In terms of the inhibition of $\mathrm{E}_{2}$ :

$$
(\mathrm{B})_{i}=\frac{(\mathrm{B})}{1-i}\left[1+\frac{i(\mathrm{~B})}{K_{1}}\right]
$$

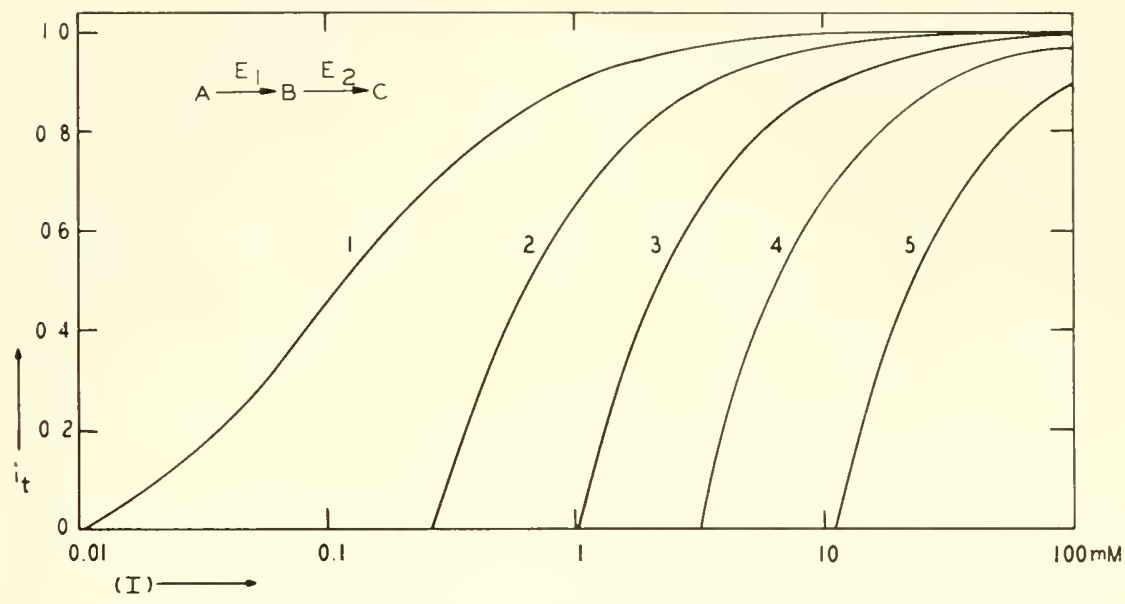

FIG. 7.8. Variation of the over-all inhibition with the concentration of an inhibitor of $E_{2}$ in a monolinear chain. Curve 1: $V_{1} / V_{2}=10$; curve $2: V_{1} / V_{2}=3$; curve $3: V_{1} / V_{2}$ $=1$; curve $4: r_{1} / I_{2}=0.33$; curve $5: r_{1} / V_{2}=0.1$.

into which the value of (B) from Eq. 7-3 may be substituted. In Fig. 7-9 it is seen that $(B)_{i}$ rises more slowly with increasing inhibition for competitive inhibition than for noncompetitive inhibition. More interesting is the fact that the system does not pass out of the steady state whatever the inhibition on $\mathrm{E}_{2}$ or the inhibitor concentration. Thus theoretically a competitive inhibitor acting on $\mathrm{E}_{2}$ will never reduce the rate of formation of $\mathrm{C}$. This may also be seen from the fact that the rate of reaction 2 is:

$$
\frac{d(\mathrm{C})}{d t}=\frac{V_{2}(\mathrm{~B})_{i}}{(\mathrm{~B})_{i}+K_{2}\left[1+\frac{(\mathrm{I})}{K_{i}}\right]}
$$

and substitution of $(\mathrm{B})_{i}$ from Eq. $7-8$ shows $d(\mathrm{C}) / d t$ to be unchanged from the uninhibited state. The reason for this is that $(B)_{i}$ can rise to sufficient 
levels to overcome the inhibition on $\mathrm{E}_{2}$. However, in actual systems, there will be a limit imposed on the level to which $(\mathrm{B})_{i}$ can rise. It is important to emphasize that competitive inhibition of any enzyme after the first in an irreversible monolinear chain will alter the rate of formation of the product by a degree primarily dependent on the physical state of the system, and particularly on the presence and nature of compartmentalization of the reactions in the system.

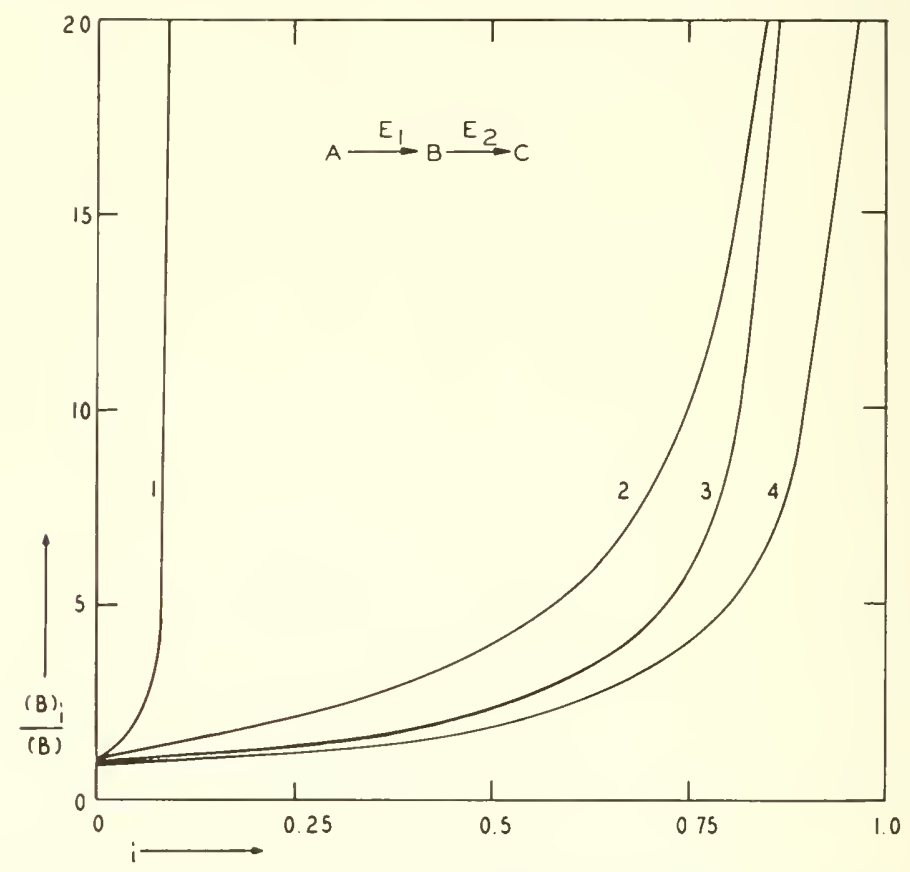

FIG. 7-9. Comparison of noncompetitive and competitive inhibitions of $\mathrm{E}_{2}$ in a monolinear chain. $K_{1}=10 \mathrm{~m} . \mathrm{H}, K_{2}=2 \mathrm{~m} . \mathrm{H}$, and $(\mathrm{A})=1 \mathrm{~m}, \mathrm{~L}$. Curve $\mathrm{I}: V_{1} / V_{2}=10$, noncompetitive; curve 2 : $V_{1} / V_{2}=10$, competitive; curve 3: $V_{1} / T_{2}=1$, noncompetitive; curve 4: $V_{1} / V_{2}=1$; competitive.

$(C)$ Reaction of the inhibitor with the intermediate $B$. A substance that reacts with $B$ according to the equilibrium expression $I+B \rightleftharpoons I B$ will reduce the concentration of free $B$ and hence the rate of reaction 2. Since there is no effect on reaction 1 . B will be formed more rapidly than it is transformed into $\mathrm{C}$. Whether the rate of reaction 2 will recover towards the initial level will depend on the equilibrium constant $K_{i b}$ and the amount of I relative to $B$. If ( $I$ ) is large, (B) may be kept close to the equilibrium value and the formation of $\mathrm{C}$ may be depressed to a level that is stable, 
at least during the interval of measurement. An interesting situation might arise if $K_{i b}$ were very small and the amount of I not much greater than $B$. since the rate would be decreased to near zero and would remain there until enough $\mathrm{B}$ was formed from reaction 1 to combine with all the I present, at which time (B) would rise and the formation of $\mathrm{C}$ would recommence.

\section{Diffusion-Limited Monolinear Chain}

The diffusion of $\mathrm{A}$ into the multienzyme region may be so slow that the concentration of A inside cannot be maintamed equal to that outside and in this sense the diffusion may be considered to limit the formation of $\mathrm{C}$.

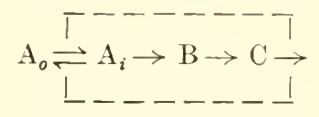

This restriction of diffusion may be imposed by a membrane surrounding the multienzyme system. The rate of inward diffusion will be $v_{d}=k\left[\left(\mathrm{~A}_{0}\right)-\right.$ $\left(\mathrm{A}_{i}\right)$ ], where $k$ is the diffusion constant, and equating this to $v_{1}$ in the steady state, the value of $\left(A_{i}\right)$ is found to be given by a quadratic equation:

$$
\left(\mathrm{A}_{i}\right)^{2}+\left[\frac{V_{1}}{k}+K_{1}-\left(\mathrm{A}_{0}\right)\right]\left(\mathrm{A}_{i}\right)-K_{1}\left(\mathrm{~A}_{0}\right)=0
$$

From $\left(A_{i}\right)$ may be determined the steady-state rate of the enzyme chain. If $\mathrm{E}_{1}$ is now inhibited. $\left(A_{i}\right)$ will rise but it will not rise to a level where the enzyme rates have retumed to the uminhibited state because the inward diffusion of $A$ will decrease as $\left(A_{i}\right)$ increases. The relationship of the inhibition on the formation of $\mathrm{C}$ to the imbibition on $\mathrm{E}_{1}$ is shown in Fig. 7-10. At first, as inhibition on $\mathrm{E}_{1}$ is increased, there is a resistance to inhibition of the over-all rate. but this is eventually lost and at high inhibitions of $\mathrm{E}_{1}$ the system is quite sensitive. In terms of buffer capacity, $d i_{i} d i_{t}$ is large at small inhibitions of $\mathrm{E}_{1}$ and decreases with inhibition: in the case plotted, di/dit is 6.8 between $i=0$ to 0.25 and decreases to 0.24 between $i=0.9$ to 1.0. The buffer capacity thus changes constantly and markedly in such a system. It may be pointed out that working in the middle range of inhibition may obscure this effect; for example, in the present case if inhibitions of $40 \%$ and $90 \%$ were tested, di/dit would be very close to one, although $i$, would be definitely less than $i$. Determination of buffer capacity with many levels of inhibition over as wide a range as possible may be useful in elucidation of mechanisms in multienzyme systems. The occurrence of a diffusion-limited step, for example. might be detected, because for the monolinear chain the $i-i$, curves are quite lifferent from those in which diffusion is not a factor.

The enzymic oxidation of ascorbate, catalyzed by peroxidase, was studied 
under open steady-state conditions by Passynsky (1958) by enclosing the enzyme between two membranes. The substrate diffused in through one membrane and the product diffused out through the other against running water. The total reaction was written as:

$$
\mathrm{A}_{0} \stackrel{k_{0}}{\rightleftharpoons} \mathrm{A}_{i} \underset{k_{-1}}{\stackrel{k_{1}}{\rightleftharpoons}} \stackrel{k_{2}}{\rightleftharpoons} \mathrm{P}_{i} \rightleftharpoons \mathrm{P}_{0}
$$

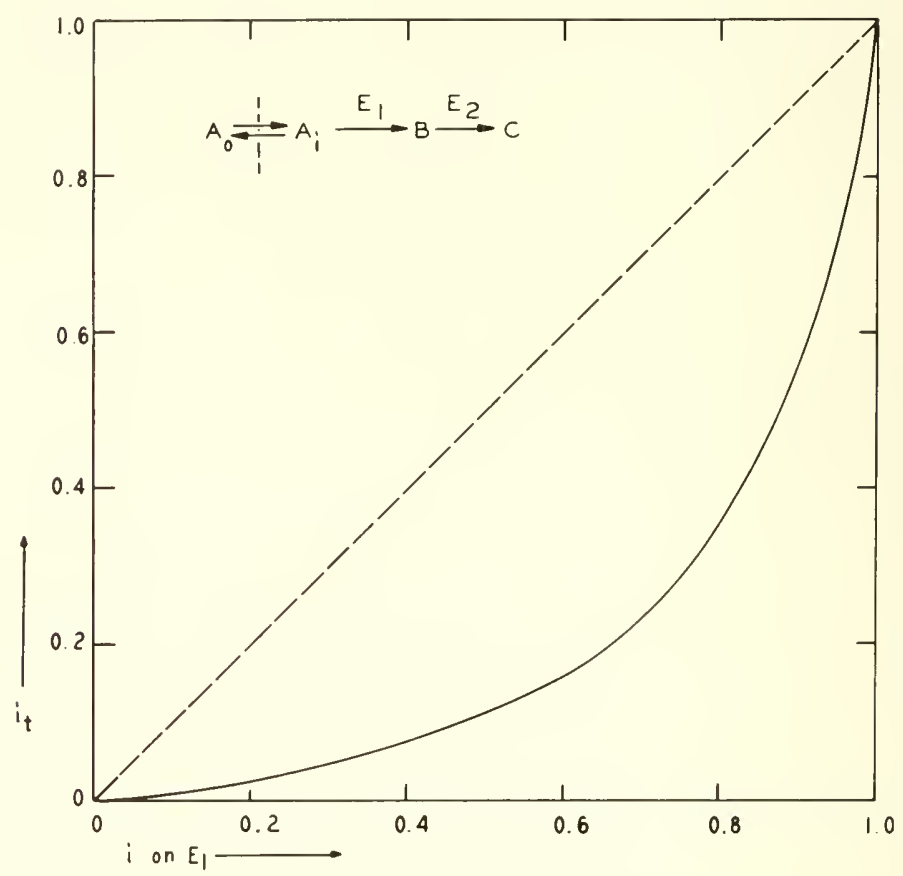

FIx. 7-10. Relationship between the over-all inhibition and the inhibition on $\mathrm{E}_{1}$ in a diffusion-limited monolinear chain. $V_{1}=1$, $K_{1}=10 \mathrm{~m} M, k=0.01$, and $\left(\mathrm{A}_{0}\right)=10 \mathrm{~m} \mu$. The dashed line simply shows the behavior to be expected if diffusion is not a faetor.

The complete solution for $\left(\mathrm{A}_{i}\right)$, assuming Fick's law of diffusion and firstorder reactions, is given by:

$$
\left(\mathrm{A}_{i}\right)=\frac{k_{0}\left(k_{-1}+k_{2}\right)\left(\mathrm{A}_{0}\right)+k_{-1} k_{2}\left(\mathrm{P}_{0}\right)}{k_{0} k_{2}+k_{0} k_{-1}+k_{1} k_{2}}
$$

Under the experimental conditions, $\left(\mathbf{P}_{0}\right)=0$, and the reaction was essentially irreversible, so that $k_{-1}=0$. Thus:

$$
\left(A_{i}\right)=\frac{\left(A_{o}\right)}{1+\left(k_{1} / k_{0}\right)}
$$


Activity-time curves are plotted for varions additions of enzyme or ascorbate and the transitions from one steady state to another are easily demonstrated. It was possible by changing the areas of the membranes to show that the attaimment of a steady state depends on the diffusion capacity of the system relative to the enzymic activity.

\section{Extension to Longer Monolinear Chains}

The kinetics developed above can be easily extended to chains of any length:

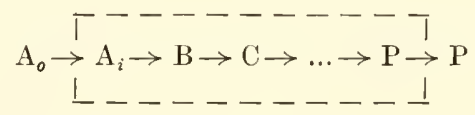

and it will suffice to enumerate the laws governing such systems.

(1) The rate of formation of product from an enzyme chain of any length in a steady state is determined by the rate of the first reaction; when a steady state does not occur, the rate is determined by that reaction with the lowest maximal rate.

(2) The concentration of any intermediate will depend on the rate constant, Michaelis constant, and concentration of the enzyme acting upon it; for the intermediate $J$ it is given by

$$
(\mathrm{J})=\frac{V_{1} K_{j}(\mathrm{~A})}{(\mathrm{A})\left(V_{j}-V_{1}\right)+V_{j} K_{1}}
$$

The relative concentrations may be visualized on $v$-(S) curves, as shown for a four-enzyme system in Fig. 7-5 (VII-VIII).

(3) The noncompetitive inhibition of any enzyme after the first, when the system is initially in a steady state, will have no effect on the formation of product until the inhibition has reduced the maximal rate of that enzyme to a level lower than the initial uninhibited rate (see Fig. 7-5, VIII).

(4) When a system is in a steady state, competitive inhibition of any enzyme after the first will have no effect on the formation of product, unless the intermediate which is the substrate for that enzyme cannot increase in concentration sufficiently to antagonize the inhibition and maintain the rate. The effect on product formation will thus depend on the spatial organization or physical state of the system. 


\section{PARTIALLY AND COMPLETELY REVERSIBLE MONOLINEAR CHAINS}

Either the first, the second, or both steps in a two-enzyme chain may be reversible. For the system:

$$
\mathrm{A} \stackrel{\mathrm{E}_{1}}{\rightleftharpoons} \stackrel{\mathrm{E}_{2}}{\rightleftharpoons} \mathrm{B} \rightarrow \mathrm{C}
$$

the rates of the individual reactions are given by:

$$
\begin{aligned}
& v_{1}=\frac{V_{1} K_{-b}(\mathrm{~A})-V_{-1} K_{a}(\mathrm{~B})}{K_{a} K_{-b}+K_{-b}(\mathrm{~A})+K_{a}(\mathrm{~B})} \\
& v_{2}=\frac{V_{2}(\mathrm{~B})}{(\mathrm{B})+K_{b}^{-}}
\end{aligned}
$$

where the negative sign of a subscript refers to the reverse reaction from $\mathrm{B} \rightarrow \mathrm{A}$. Solution of these equations gives a quadratic expression for (B); from (B) the rate of formation of $\mathrm{C}$ may be calculated using Eq. 7-15.

$$
\begin{gathered}
K_{a}\left(V_{-1}+V_{2}\right)(\mathrm{B})^{2}+\left[V_{2} K_{-b} K_{a}+V_{2} K_{-b}(\mathrm{~A})+V_{-1} K_{a} K_{b}-V_{1} K_{-b}(\mathrm{~A})\right](\mathrm{B}) \\
-V_{1} K_{-b} K_{b}(\mathrm{~A})=0
\end{gathered}
$$

The reduction in (B) and the steady-state rate as the reverse reaction increases is shown in Fig. 7-11. Such a system will more often be in a steady state, compared to a similar irreversible system, because a slow second reaction, by increasing $(B)$, will slow down the first reaction.

Noncompetitive inhibition of $\mathrm{E}_{2}$ will have a different effect on the overall system compared to the irreversible system 7-1. As $V_{2}$ is decreased, the rate of formation of $\mathrm{C}$ will be reduced (Fig. 7-12) more readily because the increase in (B) will slow down the over-all forward rate of reaction 1. The sensitivity of the system to inhibition will depend on the initial level of $V_{2}$; when it is high relative to $V_{1}$, the inhibition on $\mathrm{C}$ formation will be slight, but when it is lower than $V_{1}$ the inhibition may be appreciable. The buffer capacity of this partially reversible system is thus less than that of the irreversible chain.

The system:

$$
\mathrm{A} \rightarrow \mathrm{E} \rightarrow \stackrel{\mathrm{E}_{2}}{\rightleftharpoons} \mathrm{C}
$$

under the conditions specified [that $(\mathrm{C})$ is kept constant by some diffusion process] does not behave differently to inhibition compared to the irreversible chain because the reverse reaction remains constant. However, it is more difficult to maintain the steady state since the over-all forward rate 
of reaction 2 is less than in the irreversible case. The steady-state level of (B) will of course be different. The individual rates are:

$$
\begin{aligned}
v_{1} & =\frac{V_{1}(\mathrm{~A})}{(\mathrm{A})+K_{a}} \\
v_{2} & =\frac{V_{2} K_{-c}(\mathrm{~B})-V_{-2} K_{b}(\mathrm{C})}{K_{-c} K_{b}+K_{-c}(\mathrm{~B})+K_{b}(\mathrm{C})}
\end{aligned}
$$

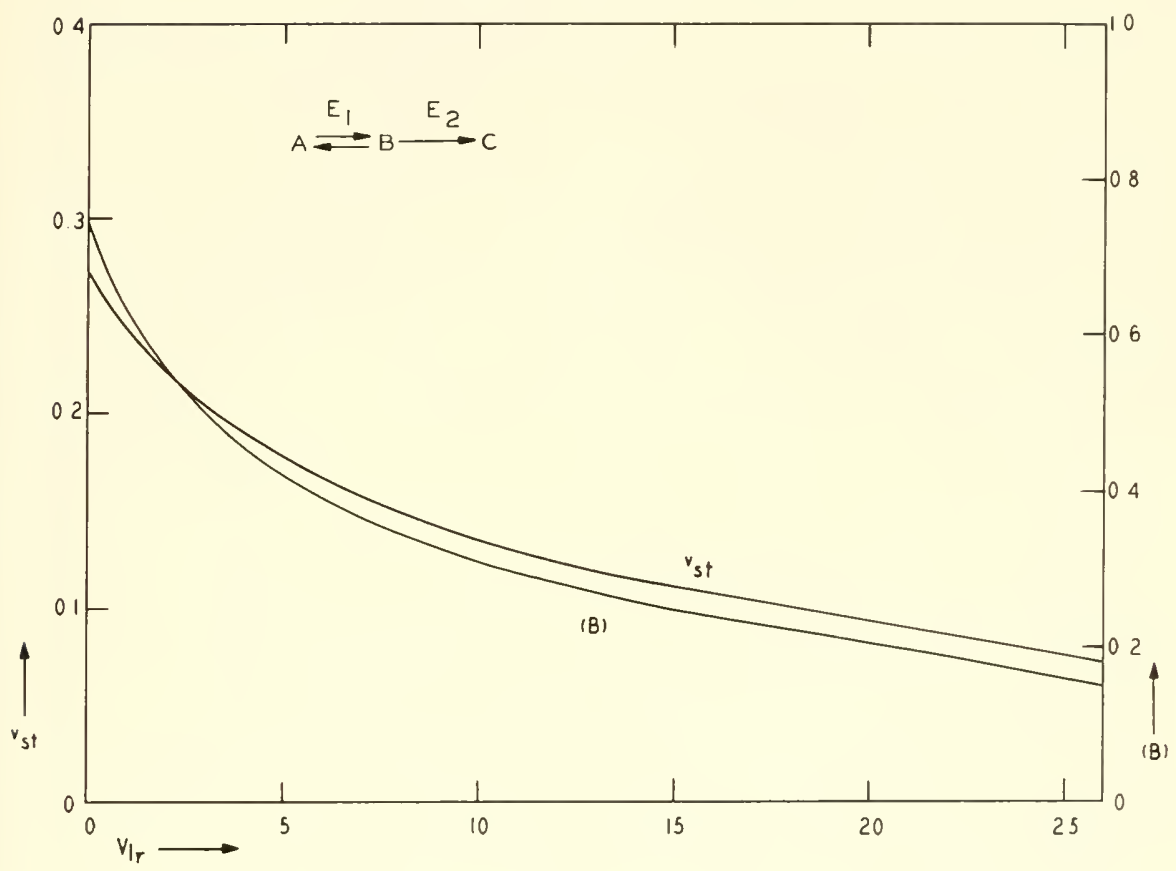

FIG. 7-11. Effect of the reverse reaction on the steady-state rate and intermediate concentration in a partially reversible monolinear chain. $V_{1_{f}}=3, V_{2}=1, K_{a}=10$ $\mathrm{m} . H, K_{b}=1 \mathrm{~m} . U, K_{-b}=20 \mathrm{~m} . \mu$, and $(\mathrm{A}) \stackrel{1}{=} 1 \mathrm{~m} . \mu$.

from which the level of $(B)$ is given by:

$$
(\mathrm{B})=\frac{K_{b}}{K_{-c}} \frac{\left.V_{-2}(\mathrm{C})\left[(\mathrm{A})+K_{a}\right)\right]+V_{1}(\mathrm{~A})\left[(\mathrm{C})+K_{-c}\right]}{V_{2}\left[(\mathrm{~A})+K_{a}\right]-V_{1}(\mathrm{~A})}
$$

which reduces to Eq. 7-3 when the reverse reaction is zero.

Finally, the system:

$$
\mathrm{A} \stackrel{\mathrm{E}_{1}}{\rightleftarrows} \mathrm{B} \stackrel{\mathrm{E}_{2}}{\rightleftarrows} \mathrm{C}
$$

behaves similarly to system $7-13$, (C) being kept constant, but (B) will be different. The individual rates are given by Eqs. $7-14$ and $7-19$. The response 
to inhibition will be essentially the same as in system 7-13. In these connections it must be remembered that noncompetitive inhibition of a reversible step implies equal inhibitions on both the forward and the reverse reactions (see Chapter 3 under system 3-105). However, competitive inhibition is not necessarily the same on the rates in forward and reverse directions and some complication is introduced into the quantitative response to such inhibition.

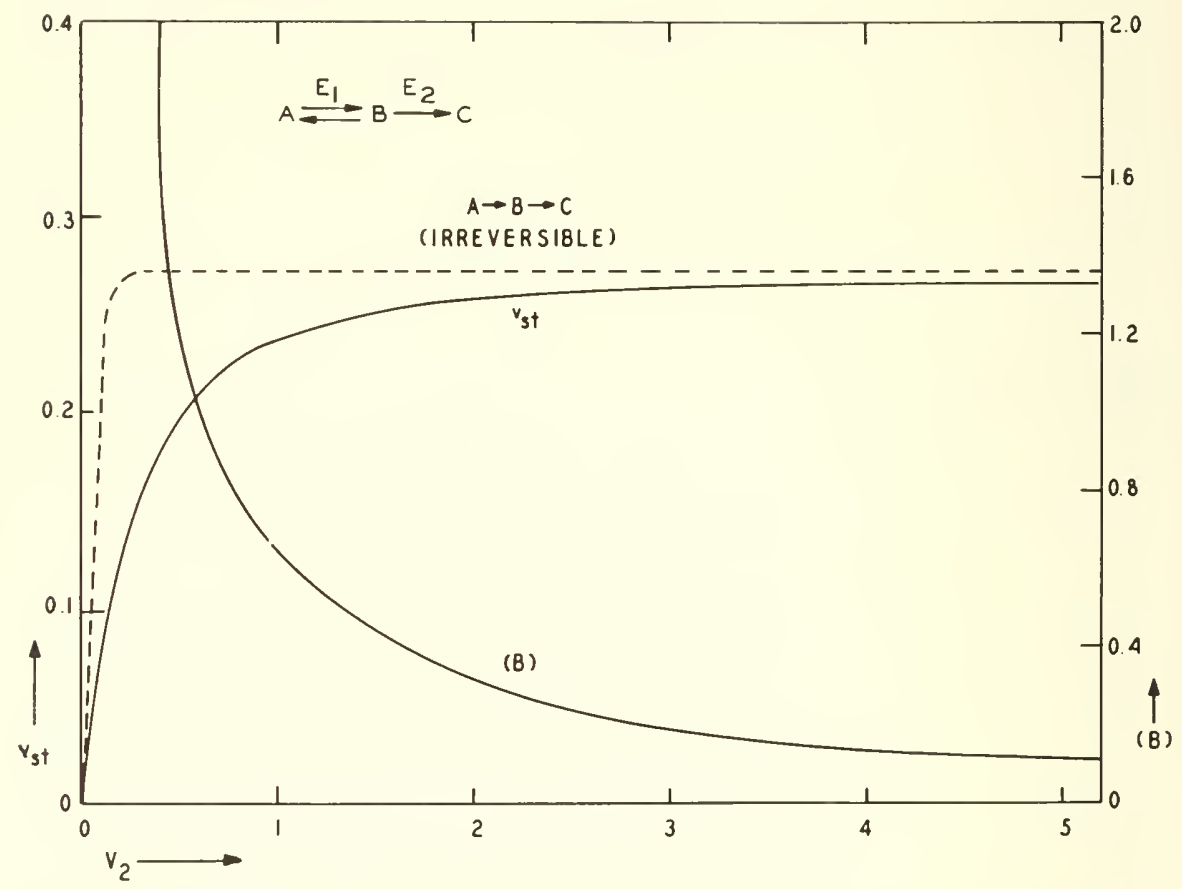

FIG. 7-12. Noncompetitive inhibition of $\mathrm{E}_{2}$ in a partially reversible monolinear chain. $V_{1}=3, I_{-1}^{r}=1, K_{a}=10 \mathrm{~m} . \mu, K_{b}=2 \mathrm{~m} . I, K_{-b}=20 \mathrm{~m} . \mu$, and $(\mathrm{A})=1 \mathrm{~m} . \mu$. The dashed curve shows the change in $v_{s t}$ in an irreversible system.

\section{BRANCHED LINEAR CHAINS}

Branched chains may be of two general types: (1) convergent where two or more substrate systems feed into the chain, or (2) dicergent where two or more products are produced by a monolinear chain.

\section{Convergent Chains}

Many examples of convergent enzyme sequences in metabolism have been reported: the formation of ghcose-6-phosphate from both glucose 
and glycogen in a purely glycolytic system, or the impingement of hychrogen atoms from two or more dehydrogenases or substrates on a common electron-transport system, would be obvious examples. For simplicity we may represent such a system as:

$$
\underset{\mathrm{E}_{3}}{\mathrm{E}} \stackrel{\mathrm{E}_{1}}{\mathrm{E} \rightarrow \mathrm{C}}
$$

where during the steady state, $v_{1}+v_{3}=v_{2}=d(\mathrm{C}) / d t$. The steady-state level of (B) is given by:

$$
\text { (B) }=\frac{K_{2}\left(v_{1}+v_{3}\right)}{V_{2}-v_{1}-v_{3}}
$$

where $v_{1}$ and $v_{3}$ are equal to their ordinary Michaelis expressions. It is evident that inhibition of either $E_{1}$ or $E_{3}$ will reduce $d(C) / d t$, but that the reduction of product formation will be less than the inhibition on the single enzyme, and that the inhibition of $d(C) / d t$ can never be complete. Since the response of the system depends on the relative rates of reaction 1 and reaction 3 , let us define this by putting $v_{3}=v_{1}$, in which case it may be easily shown that:

$$
i_{t}=\frac{i_{1}}{1+r}
$$

where $i_{t}$ is the inhibition of $d(\mathrm{C}) / d t$ and $i_{1}$ is the inhibition on $\mathrm{E}_{1}$. The maxinum inhibition on the formation of $\mathrm{C}$ would thus be $1 /(1+r)$.

Inhibition of $\mathrm{E}_{2}$ will not affect $d(\mathrm{C}) / d t$ until $V_{2}$ is reduced below $v_{1}+v_{3}$ or until for some reason (B) cannot rise sufficiently to maintain the rate, the system behaving similarly to the unbranched system 7 - 1 .

\section{Divergent Chains}

Metabolic processes in which a substance formed can react in two or more different pathways are common. The oxidation of pyruvate formed from glycolysis to acetyl-CoA or its condensation with $\mathrm{CO}_{2}$ to form dicarboxylic acid (Freedman and Graff 1958) and the diversion of glucose-6-phosphate formed from glucose into glycogen or the glycolytic patluway would be important examples. Such a system may be represented by:

$$
\mathrm{A} \rightarrow \mathrm{B}
$$

where the steady state implies that $v_{1}=v_{2}+v_{3}$ and the individual rates are:

$$
v_{1}=\frac{V_{1}(\mathrm{~A})}{(\mathrm{A})+K_{1}} \quad v_{2}=\frac{V_{2}(\mathrm{~B})}{(\mathrm{B})+K_{2}} \quad v_{3}=\frac{\Gamma_{3}(\mathrm{~B})}{(\mathrm{B})+K_{3}}
$$


The concentration of $\mathrm{B}$ is given by:

$$
\begin{aligned}
\left\{(\mathrm{A}) V_{1}\right. & \left.-\left(V_{2}+V_{3}\right)\left[(\mathrm{A})+K_{1}\right]\right\}(\mathrm{B})^{2} \\
& \left.+\left\{(\mathrm{A}) V_{1}\left(K_{2}+K_{3}\right)-\left(V_{2} K_{3}+V_{3} K_{2}\right)\left[(\mathrm{A})+K_{1}\right)\right]\right\}(\mathrm{B}) \\
& +V_{1} K_{2} K_{3}(\mathrm{~A})=0
\end{aligned}
$$

If we assume that the formation of $\mathrm{C}$ is being measured, the addition of reaction 3 will reduce this rate by reduction in $(B)$. There will not be a constant ratio of $d(\mathrm{C}) / d t$ to $d(\mathrm{D}) / d t$ but this will vary with $(\mathrm{B})$ and hence with the rate of reaction 1 . For this reason, inhibition of $E_{1}$ will reduce $v_{2}+v_{3}$ to the same degree as $v_{1}$, but need not reduce $v_{2}$ and $v_{3}$ equally. In fact, it is possible that only $d(\mathrm{C}) / d t$ will be reduced and $d(\mathrm{D}) / d t$ remain unaffected if the steady-state level of $(B)$ is such as to saturate $\mathrm{E}_{3}$, as shown graphically in Fig. 7-13. Conversely, only the formation of $D$ may be inhibited.

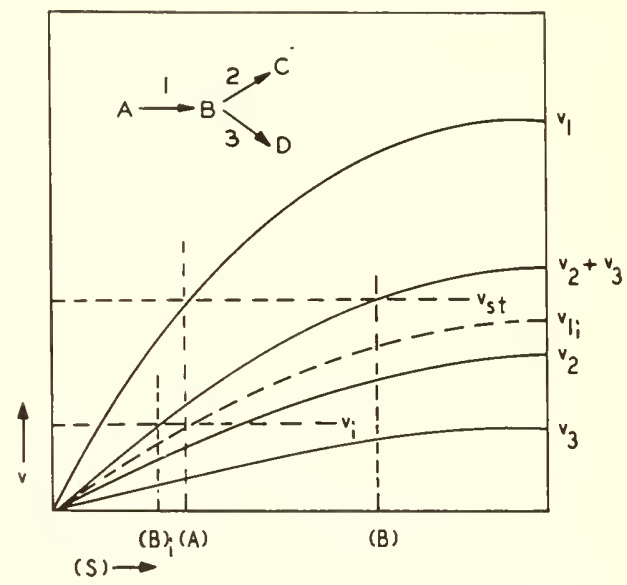

Fig. 7-13. Rate curves for a divergent chain. The curves labeled $v_{1}, v_{2}$, and $v_{3}$ show the variation of the rates with substrate concentration for each of the reactions alone; curve $v_{2}+v_{3}$ shows the summed rates of $v_{2}$ and $v_{3}$. Neither reaction 2 nor reaction 3 could maintain a steady state alone when the concentration of A is (A), but together they can $\left(v_{s t}\right)$. If $\mathrm{E}_{1}$ is inhibited the rate curve is decreased to $v_{1}$, the steady-state rate is depressed to $v_{i}$, and the concentration of the intermediate falls from $(\mathrm{B})$ to $(\mathrm{B})_{i}$. However, $v_{i}$ is still greater than $V_{3}$ so that the fall

in (B) has not changed the rate of reaction 3 ; thus only $d(\mathrm{C}) / d t$ is depressed.

Inhibition of $\mathrm{E}_{2}$ may or may not reduce $d(\mathrm{C}) / d t$ depending on the state of the system before inhibition. If (B) is high enongh to saturate $\mathrm{E}_{3}$, inhibition of $\mathrm{E}_{2}$ will cause $(\mathrm{B})$ to rise and $d(\mathrm{C}) / d t$ will increase to maintain the steady state as in the monolinear chain $\mathrm{A} \rightarrow \mathrm{B} \rightarrow \mathrm{C}$. However, if $\mathrm{E}_{3}$ is 
not saturated, inhibition of $\mathrm{E}_{2}$ will produce a rise in (B) which will increase the rate of reaction 3 . Since $v_{2}+v_{3}$ must remain constant in the steady state, an increase in $v_{3}$ implies a decrease in $v_{2}$. This divergent chain thus has less buffer capacity generally than the monolinear system.

The effects of inhibition of $E_{3}$ are particularly interesting since the resulting rise in (B) may increase $d(\mathrm{C}) / d t$ and such a mechanism may be one of the explanations for the stimulation frequently observed with enzyme inhibitors. The variation in $d(\mathrm{C}) / d t$ is plotted against $V_{3}$ in Fig. 7-14 and from such a curve the degree of inhibition of $d(\mathrm{C}) / d t$ by reduction in $V_{3}$ through noncompetitive inhibition may be calculated. The amount of stimulation will depend on the relative values of $v_{2}$ and $v_{3}$ in the uninhibited system. For the particular system plotted, the stimulation of $d(\mathrm{C}) / d t$ for different initial values of $V_{3}$ at an inhibition of $E_{3}$ of $90 \%$ is as follows:

\begin{tabular}{c|c}
\hline Initial $V_{3}$ & $\%$ Stimulation of $d(\mathrm{C}) / d t$ \\
\cline { 2 - 2 } & 528 \\
100 & 147 \\
10 & 25 \\
1 & 3 \\
0.1 & \\
\hline
\end{tabular}

Another type of divergent chain may occur when an intermediate spontaneously decomposes nonenzymically into a product other than the enzymically catalyzed one.

$$
\underset{\mathrm{A} \rightarrow \mathrm{B}}{\mathrm{E}_{1}} \stackrel{\mathrm{E}}{\longrightarrow}_{\mathrm{D}}^{\mathrm{E}}
$$

The reaction of $\mathrm{B} \rightarrow \mathrm{D}$ may be assumed to be monomolecular and $v_{3}=$ $k$ (B) rather than the value in Eq. 7-26 above. The essential difference between this system and the three-enzyme system is that reaction 3 cannot now become saturated and the rate will always be linear with (B), which is given by:

$$
k\left[(\mathrm{~A})+K_{i}\right](\mathrm{B})^{2}+\left\{\left(V_{2}+k K_{2}\right)\left[(\mathrm{A})+K_{1}\right]-(\mathrm{A}) V_{1}\right\}(\mathrm{B})-V_{1} K_{2}(\mathrm{~A})=0
$$

Thus if $\mathrm{E}_{2}$ is inhibited, (B) will rise slightly but never sufficiently to maintain the initial $d(\mathrm{C}) / d t$; if $V_{2}$ is much smaller than $k K_{2}$. as seen from Eq. 7-28, changes in $V_{2}$ will scarcely affect (B) and no buffer capacity will be observed. It may also be noted that the system will always be in a steady state whatever the concentration of $\mathrm{A}$. The spontaneous reactions of unstable intermediates, such as free radicals, may be important in several metabolic pathways. 
Another multienzyme system of common occurrence involves the transformation of an intermediate back to the substrate:

$$
\mathrm{A} \underset{\mathrm{E}_{3}}{\stackrel{\mathrm{E}_{2}}{\rightleftarrows}} \mathrm{B} \stackrel{\mathrm{E}_{3}}{\longrightarrow} \mathrm{C}
$$

Phosphatases and deacylases hydrolyzing phosphorylated or acylated intermediates respectively often are responsible for such systems. An espe-

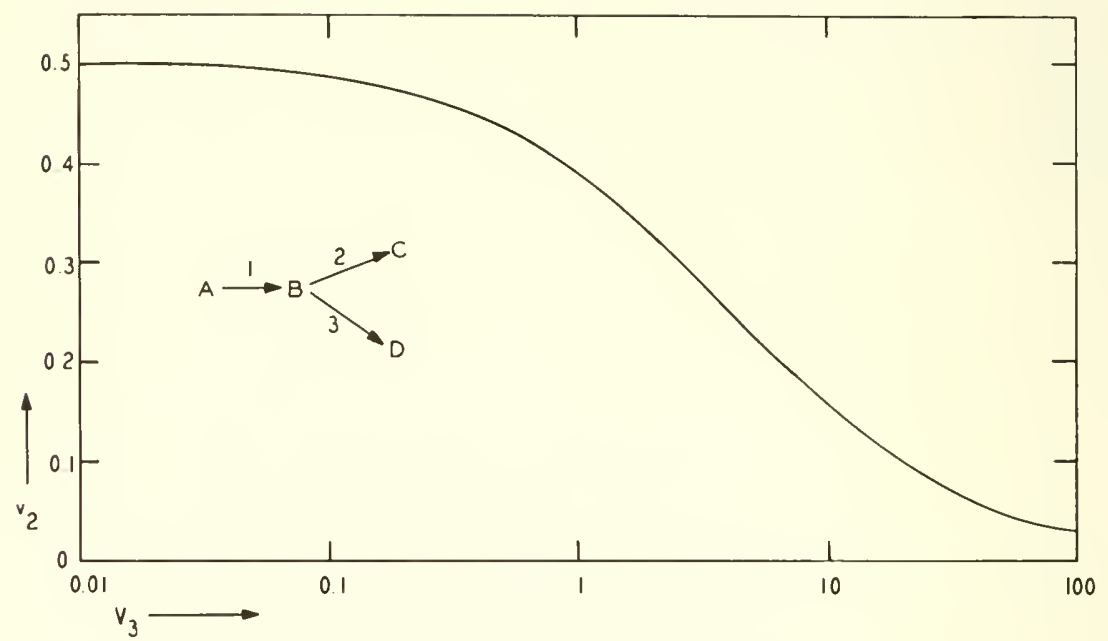

FIG. 7-14. Noncompetitive inhibition of $\mathrm{E}_{3}$ in a divergent chain. The variation of $v_{2}$ with $\mathrm{J}_{3}$ is indicated. Inhibition of $\mathrm{E}_{3}$ will reduce $V_{3}$ and $d(\mathrm{C}) / d t$ will increase, illustrating the stimulation of one branch by the inhibition of the other branch. $I_{1}=1$, $V_{2}=1, K_{1}=5 \mathrm{~m} M, K_{2}=2 \mathrm{~m} H, K_{3}=10 \mathrm{~m} . /$, and $(\mathrm{A})=5 \mathrm{~m} M$.

cially important instance is the formation of glucose-6-phosphate catalyzed by glucokinase and the hydrolysis of glucose-6-phosphate back to glucose by a phosphatase. Actually the system formulated above is a type of divergent chain and follows kinetics identical to those of the divergent chain 7-25, inasmuch as the individual rate expressions are the same. Inhibition and activation of the enzymes involved may be of great importance in the regulation of the metabolism as related to the needs of the tissue (Cahill et al., 1959). Systems of this type involving phosporylation from ATP can act as ATPases, since at each round of the cycle an ATP is split, as pointed out by Krebs et al., (1958) for the

2 phosphorylase b phosphorylase a 
transformation, where reaction 1 is catalyzed by phosphorylase kinase and reaction 2 by a phosphoprotein phosphatase, 4 ATP molecules being required for the formation of one molecule of phosphorylase a. Such a system operating as an ATPase wonld have complex kinetics and quite different inhibition behavior compared to the single enzyme ATPase.

\section{POLYLINEAR CHAINS (SHUNT SYSTEMS)}

A multienzyme system wherein a substrate can be transformed to some product by two or more different pathways may be termed a polylinear chain. Many examples are known: electron-transport systems in which the electrons may flow through different channels to oxygen (Mahler, 1956; Singer et al., 1957); the two pathways of pyruvate $\rightarrow$ acetyl-CoA in yeast, one by pyruvic oxidase and the other through pyruvic decarboxylase, aldehyde dehydrogenase, and aceto-CoA-kinase (Holzer and Goedde, 1957); the two rontes by which coenzyme $\mathrm{A}$ is formed from pantothenic acid, one involving pantetheine, and the other phosphopantothenic acid (Brown, 1959); and the well-known hexose monophosphate shunt. The kinetics will depend on whether the chains branch from the substrate or from an intermediate whose concentration can vary. The former system may be represented as:

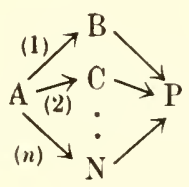

where the over-all rate of formation of $\mathrm{P}, v=v_{1}+v_{2}+\ldots+v_{n}$. Inhibition of the first enzyme of any sequence will reduce $d(\mathrm{P}) / d t$ but inhibition of the succeeding enzymes may not because the intermediate can increase in concentration. If an inhibitor is assumed to exert an inhibition of $i_{1}$ on pathway (1), $i_{2}$ on pathway (2), etc., the inhibition of the over-all rate will be:

$$
i_{t}=\frac{i_{1} v_{1}+i_{2} v_{2}+\ldots+i_{n} v_{n}}{v_{1}+v_{2}+\ldots+v_{n}}
$$

so that the inhibition on the over-all rate will always be less than on the individual enzymes. Such polylinear chains are perhaps responsible for many instances of inhibitor-resistant metabolism in the cell. When the sequences are irroversible, each sequence operates independently of the others and the effect that some modification of a single sequence will have on the 
over-all rate will depend simply on the relative contribution it makes to the over-all rate.

A somewhat different situation exists when the branching occurs from a substance formed from the original substrate, as in the system:

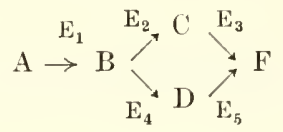

where $v_{1}=v_{2}+v_{4}=v_{3}+v_{5}$ in the steady state. The concentration of $\mathrm{B}$ will be given by an expression similar to that for system 7-25, and the concentrations of $\mathrm{C}$ and $\mathrm{D}$ may be determined from (B), since $v_{2}=v_{3}$ and $v_{4}=v_{5}$. Inhibition of $\mathrm{E}_{2}$ will result in a rise of $(\mathrm{B})$ which will increase the rates of reactions 4 and $5, v_{2}$ and $v_{3}$ decreasing to an equal degree, so that $d(\mathrm{~F}) / d t$ remains constant while (C) falls and (D) rises. Such systems can also contribute to inhibitor-resistant metabolism but never demonstrate the phenomenon of stimulation of the over-all rate observed with the simple divergent chain. Huzisige (1954) has postulated a system such as 7-32 to explain inhibition of photosynthesis, although only two of the steps were assumed to be enzymic. However. like many others, he did not use enzyme kinetics in his formulation and the expressions for inhibition are much more complex than those he derived.

The scheme 7-32 may represent in part the electron-transport systems in mitochondria, where two or more substrates can fumnel into A. The choline and succinate respiratory chains are not compartmentalized in liver mitochondria but appear to be linked somewhere above the site of the oxidation of cytochrome $c_{1}$ (Kimura and Singer, 1959); this linkage is probably not cytochrome $b$, the components of which seem to be on different pathways. The use of antimycin $A$ to block the transfer from cytochrome $b$ to cytochrome $c_{1}$ could not produce a complete inhibition of $\mathrm{cy}$ tochrome c reduction due to the "leakage" from cytochrome b in the choline chain. Amytal block, however, was complete and no alternate pathway was evident, indicating somewhat different sites of action of these two inhibitors. The response to inhibition by most respiratory chain inhibitors will depend on the presence of such multiple pathways and the sites at which they link.

\section{DISTRIBUTIVE SYSTEMS}

Transferring enzymes may form multienzyme systems around single compounds which are capable of donating groups to a variety of substances. Various distributive systems have been discussed by Dixon (1949, p. 45; 
Dixon and Webb, 1958, p. 565). A generalized system may be represented as:

$$
\underset{\mathrm{G}}{\rightarrow} \stackrel{\mathrm{II}}{\mathrm{B}}-\underset{\mathrm{X}}{\rightarrow} \mathrm{C}
$$

where A, B, C, D, F, and $\mathrm{G}$ are substrates that can accept the group $\mathrm{X}$ from the central molecule MI-X, each substrate requiring a different transferring enzyme. Any step may be reversible or essentially irreversible. In the latter case no satisfactory thermodynamic equilibrium of $\mathrm{X}$ between the various substrates will be achieved but the system may be in a steady state, M-X being formed by one of the reactions and utilized by the rest, at least one of the products, such as $\mathrm{C}-\mathrm{X}$, reacting further outside the system. It is unlikely that distribution of $\mathrm{X}$ will be commonly determined by the equilibrium constants of the individual reactions, but rather by the relative rates, since systems in the cell are usually not static. The central compound and the enzymes associated with it in some important systems

\begin{tabular}{l|l|l}
\hline \multicolumn{1}{c|}{$\mathrm{M}-\mathrm{X}$} & \multicolumn{1}{c|}{$\mathrm{X}$} & \multicolumn{1}{c}{ Type of enzyme } \\
\cline { 2 - 3 } & & \\
\cline { 2 - 3 } DPNH & $\mathrm{H}+\mathrm{e}^{-}$ & Dehydrogenases \\
ATP & $-\mathrm{PO}_{4}$ & Phosphokinases \\
Glutamate & $-\mathrm{NH}_{2}$ & Transaminases \\
Acetyl-CoA & $-\mathrm{COCH}_{3}$ & Transacylases \\
Adenosylmethionine & $-\mathrm{CH}_{3}$ & Transmethylases \\
& & \\
\hline
\end{tabular}

are given in the tabulation (following Dixon and Webb). With regard to kinetics, these systems are really multibranched chains when II-X is formed at a constant rate and the effects of inhibitors as discussed above apply.

Inhibition of a single enzyme in such a system would cause the distribution of $\mathrm{X}$ to become abnormal and thus may modify the pattern of metabolic flow in the cell. The steady-state concentration of M-X will be more or less stabilized, in the sense that alterations of enzyme activity will have relatively little effect on it. For this reason it is often difficult to relate levels of ATP in the cell to inhibitions of enzymes involved in its formation or utilization, the concentration of ATP being rather unresponsive to such inhibition over an appreciable range.

The inhibition of enzymes in distributive systems may have complex effects on the over-all metabolism. When one of the acceptors can serve as a reserve of $\mathrm{X}$, which, upon cessation of the formation of MI-X by the usual 
pathways, allows the supply of M-X to be maintained for an interval, inhibition of this reaction would have no effect on the system in the steady state but if the formation of M-X stopped, transfer of $\mathrm{X}$ would fail suddenly. Such inhibition would be observed only when the normal state of the system was disturbed sufficiently. An example would be the equilibrium between ATP and creatine. Inhibition of creatine phosphokinase wonld have no effect on the flow of phosphate from oxidative phosphorylation to the various phosphorylated acceptors, but if the formation of ATP were depressed, as by an uncoupler, the supply of high-energy phosphate in creatine phosphate would no longer be available for utilization. This may be simply represented as:

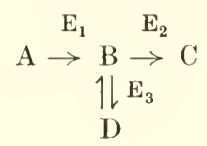

where $(\mathrm{B})$ is given by Eq. 7-3 and $(\mathrm{D})=(\mathrm{B}) / \boldsymbol{K}_{e q}$. When $\mathrm{E}_{\mathbf{1}}$ is inhibited, (B) falls and the reaction of $\mathrm{D} \rightarrow \mathrm{B}$ occurs unti! equilibrium is again established, and thus the formation of $\mathrm{C}$ is not so suddenly depressed. However, inhibition of $E_{3}$ will slow down or prevent this compensatory mechanism. For this effect to be marked, (D) must be appreciably greater than the steady-state (B).

\section{CYCLIC SYSTEMS}

Many metabolic pathways involve cyclic systems whereby one of the reactants is regenerated during the process. The tricarboxylic acid cycle, the urea cycle, and the glyoxylate cycle would be recognized examples but there are undoubtedly many more operative in the cell. A simple cycle for the development of inhibition kinetics may be taken as:

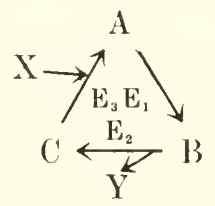

where $\mathrm{X}$ is taken into the cycle by reaction with $\mathrm{C}$, and $\mathrm{Y}$ is formed in the breakdown of $\mathrm{B}$, the over-all reaction being $\mathrm{X} \rightarrow \mathrm{Y}$, the substances $\mathrm{A}$, $\mathrm{B}$, and $\mathrm{C}$ being termed cycle intermediates. In the stearly state $v_{1}=v_{2}=v_{3}$ and the individual reaction rates are given by:

$v_{1}=\frac{V_{1}(\mathrm{~A})}{(\mathrm{A})+K_{1}} \quad v_{2}=\frac{V_{2}(\mathrm{~B})}{(\mathrm{B})+K_{2}} \quad v_{3}=\frac{V_{3}(\mathrm{C})(\mathrm{X})}{\left[(\mathrm{C})+K_{c}\right]\left[(\mathrm{X})+K_{x}\right]}$

In addition there is the requirement that the total intermediate concentration remains constant, i.e., $(\mathrm{A})+(\mathrm{B})+(\mathrm{C})=(\mathrm{M})_{t}$. Since the rate of for- 
mation of $\mathrm{Y}$ may be calculated from (B), the rate characteristics of the system may be derived from an expression for (B) in terms of (X), (M) and the constants of the cycle enzymes. The steady-state values for (A) and $(\mathrm{C})$ may be easily shown to be:

$$
\begin{gathered}
(\mathrm{A})=\frac{V_{2} K_{1}(\mathrm{~B})}{V_{1}\left[(\mathrm{~B})+K_{2}\right]-(\mathrm{B}) I_{2}^{r}} \\
(\mathrm{C})=\frac{V_{2} K_{c}(\mathrm{~B})\left[(\mathrm{X})+K_{x}\right]}{V_{3}(\mathrm{X})\left[(\mathrm{B})+K_{2}\right]-(\mathrm{B}) V_{2}\left[(\mathrm{X})+K_{x}\right]}
\end{gathered}
$$

and by substitution of these in the conservation equation, an expression for (B) may be obtained. This expression is a cubic equation with coefficients of many terms, the solution of which is arduous. However, it is possible to obtain values for $(\mathrm{A}),(\mathrm{B})$, and $(\mathrm{C})$ by graphical methods when the constants of the system are known.

The graphical method used consists of the following. Accurate $r^{-}(\mathrm{S})$ curves are plotted for each reaction of the cycle; precision is often increased by the use of semilog paper. A complete solution is obtained by finding a horizontal line that intersects the curves so that $(\mathrm{A})+(\mathrm{B})+(\mathrm{C})$ is equal to the assumed total concentration of intermediates. (II) $t$. The height of this line gives the steady-state rate (Fig 7-15). The effects of inhibition of any

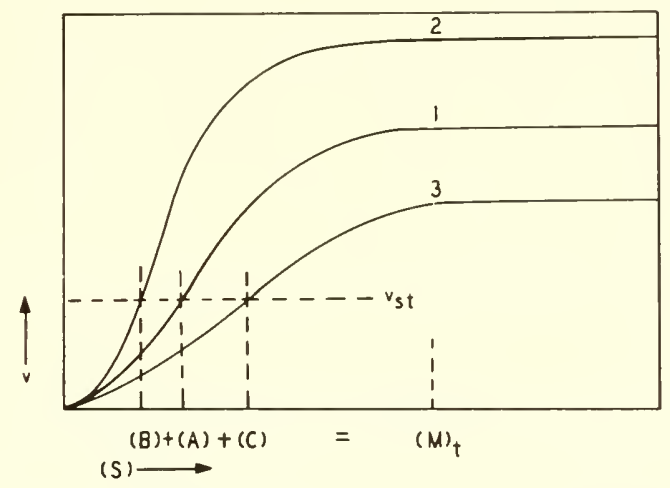

FIG. 7-15. Illustration of the graphical method for the determination of steady-state rates and concentrations in eyclic systems. See text for explanation.

enzyme in the cycle may be determined by the same procedure using the appropriately modified $v$-(S) curve for that enzyme. This method is believed to be generally better than the simplification of the complete equations by assuming the relative unimportance of certain factors, inasmuch 
as cyclic systems are frequently misleading when their characteristics are obtained by general inspection.

One of the characteristic features of cyclic systems is the constancy of the total concentration of intermediates, which is not a restriction that applies to linear sequences. As a consequence, a change in one intermediate necessarily brings about changes in the others, with consequent changes in the rates of those reactions. It is not, however, obligatory to assume such constancy in $(\mathrm{MI})_{t}$ since one or more of the intermediates may be formed or utilized in reactions outside the cycle. as is often the situation for the tricarboxylic acid cycle, in which case the kinetics presented here must be extended. Before describing the effects of imhibition, it is of interest to inquire into the changes brought about by alterations in (X) and (M) ${ }_{t}$.

\section{Effect of Substrate Concentration $(X)$}

The effects of varying $(\mathrm{X})$ on the steady-state rate and concentrations of cycle intermediates are shown in Fig. 7-16 for a system of arbitrarily chosen constants and assumed (II). As (X) increases, (C) falls and this re-

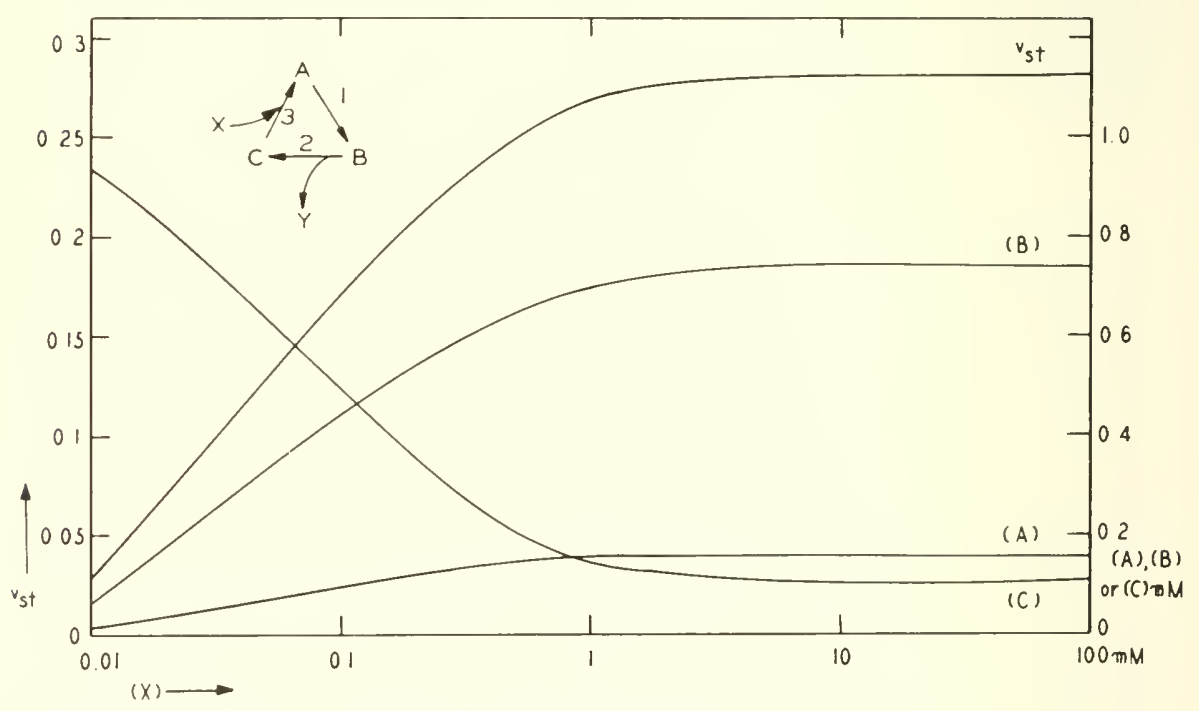

FIG. 7-16. Effect of the substrate concentration on the steady-state rate and concentrations of the intermediates in a cyclic system. $V_{1}=2, V_{2}=1, V_{3}=3, K_{1}=1$ $\mathrm{m} M, K_{2}=2 \mathrm{~m} M, K_{c}=1 \mathrm{~m} H, K_{x}=0.5 \mathrm{~m} H$, and $(M)_{t}=1 \mathrm{~m} M$.

duces the rate of reaction 3 compared to a single enzyme system; i.e., the rate does not rise as rapidly with $(\mathrm{X})$ in the cyclic system as it would with the isolated $\mathrm{E}_{3}$ where $(\mathrm{C})$ would remain constant. Simultaneously, (A) and 
(B) rise so that reactions 1 and 2 keep pace with the entry of $(\mathrm{X})$. Above a certain value of $(\mathrm{X})$, here about $1 \mathrm{~m} \cdot \boldsymbol{M}$, the system may be said to be saturated and little further change is brought about by increasing $(\mathrm{X})$. It may be noted that this is far below the maximal rates for any of the reactions. The rate of formation of $\mathrm{Y}$ is thus limited by the total concentration of cycle intermediates.

\section{Effect of Total Concentration of Cycle Intermediates $\left(M_{t}\right)$}

The steady-state rate is shown to be quite dependent on (M) ${ }_{t}$ in Fig. 7-17 and the maximal rate reached at high values of $(\mathrm{MI})_{t}$ is seen to be equal to the lowest maximal rate of the individual reactions, in this case reaction 2

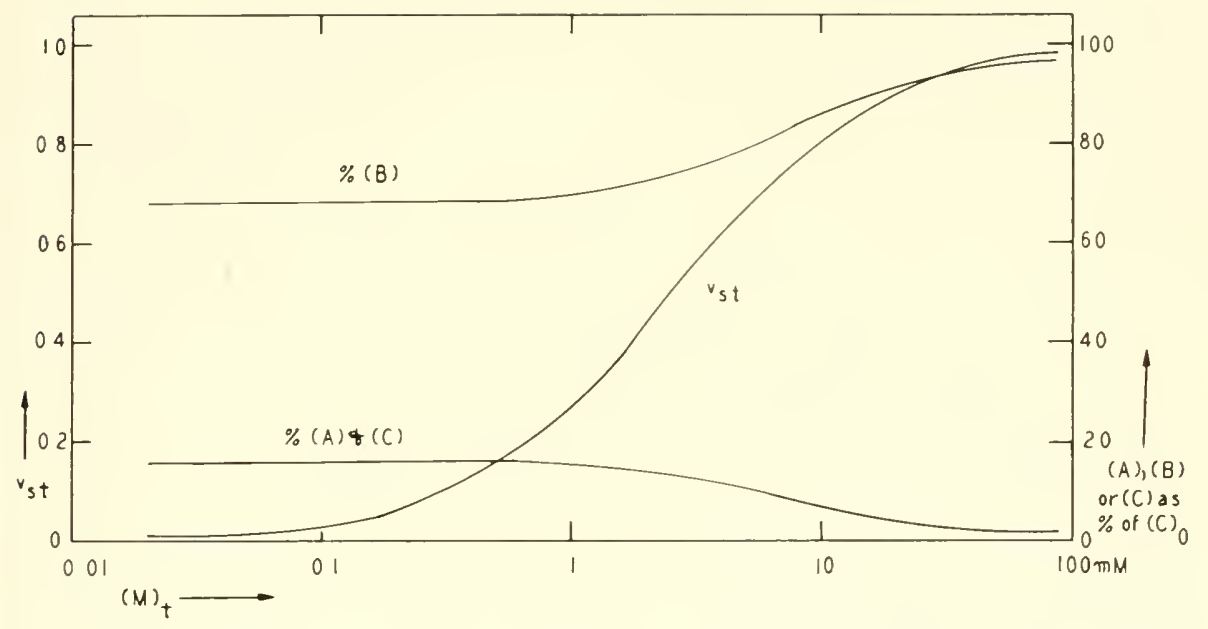

FIG. 7-17. Effect of the total concentration of cycle intermediates on the steadystate rate and concentrations of the individual intermediates in the cyclic system whose characteristics are given in Fig. $7-16 .(\mathrm{X})=1 \mathrm{~m} H$ and $v_{s t}=d(\mathrm{Y}) / d t$.

It is also important to note that as $(\mathrm{MI})_{t}$ changes. the relative concentrations of the cycle intermediates change. in the present case (B) increasing as reaction 2 becomes limiting. Any factors that change (M) $)_{t}$. such as reactions of the intermediates outside the cycle or reaction of an inhibitor with one of the intermediates. may alter the steady-state rate appreciably.

\section{Effects of Inhibition of the Cycle Enzymes}

The changes occurring upon noncompetitive inhibition of $\mathrm{E}_{1}, \mathrm{E}_{2}$, and $\mathrm{E}_{3}$ are shown in Figs. 7-18 to 7-28. Curves are given for three levels of $(\mathrm{M})_{t}$ in each case, inasmuch as the total concentration of intermediates is of importance in the quantitative changes occurring during inhibition. The 
inhibitions of the steady-state rate of the cycle are also given in terms of the inhibitions on the individual enzymes. Finally, in Fig. 7-29 are given the inhibitions of the cycle rate in terms of inhibitor concentration and they are compared with the inhibition of the single enzyme. For each inhibition there are characteristic changes in the relative concentrations of the cycle intermediates. The dependency of the cycle inhibition on the

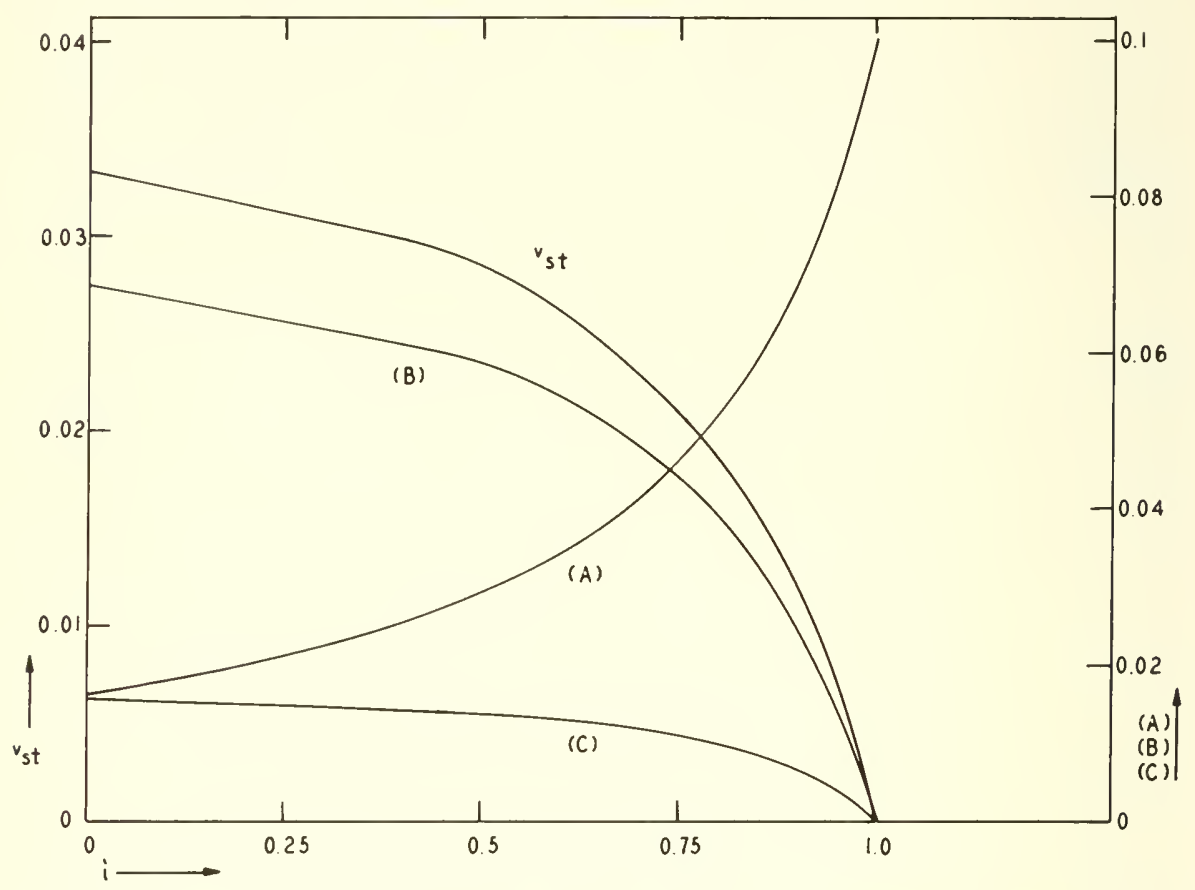

FIG. 7-18. Noncompetitive inhibition of $\mathrm{E}_{1}$ in the cyclic system shown in Fig. 7-16. $(\mathrm{X})=1 \mathrm{~m} . M$ and $(\mathrm{MI})_{t}=0.1 \mathrm{~m} M$.

enzyme inhibition varies with the particular enzyme inhibited and the total concentration of cycle intermediates.

Inspection of the curves will reveal in each case the behavior of the system but a few general remarks may be made concerning the response of such a cycle to inhibition.

(1) A cyclic system generally possesses buffer capacity, in that the inhibition of the cycle rate will usually be less than that of the enzyme that is affected. However, in contrast to a linear chain. there will be no range of absolute buffer capacity; any inhibition of a component enzyme will depress the cycle rate to some extent. 
(2) The buffer capacity at lower inhibitions depends on (M) and be-

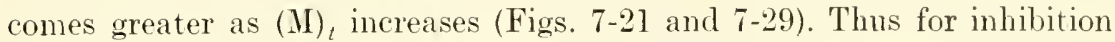
on $\mathrm{E}_{1}$ or $\mathrm{E}_{3}$, di dit has the following approximate values for inhibitions up to $40 \%$ : when (M) $t_{t}=0.1 \mathrm{~m} M$ it is 4 , when $(\mathrm{II})_{t}=1 \mathrm{~m} M$ it is 5.3 . and when $(\mathrm{MI})_{t}=10 \mathrm{~m} M$ it is 16 . The reason for this is that at high levels of (MI) ${ }_{t}$. the high concentration of $\mathrm{B}$ is approaching saturation of $\mathrm{E}_{2}$ and it requires a fair degree of inhibition to reduce (B) so that the rate falls.

(3) Inhibition of either $\mathrm{E}_{1}$ or $\mathrm{E}_{3}$ affects the cycle rate identically but the changes in the concentrations of $\mathrm{A}$ and $\mathrm{C}$ are reversed. Inhibition of $\mathrm{E}_{2}$ alters the cycle rate readily and little buffer capacity is evident, since

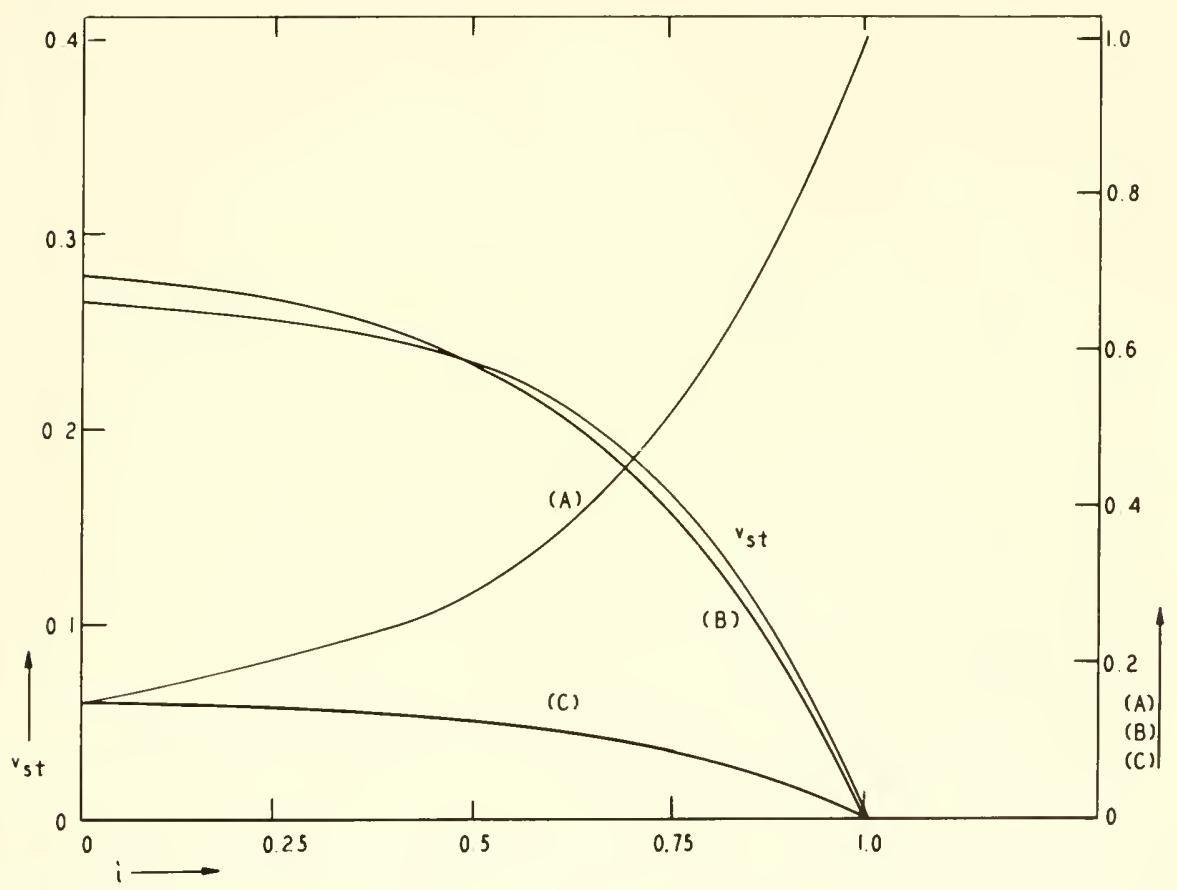

FIG. 7-19. Noncompetitive inhibition of $\mathrm{E}_{1}$ in the cyclic system shown in Fig. 7-16. $(\mathrm{M})_{t}=1 \mathrm{mH}$.

this is the reaction that forms $\mathrm{Y}$. Although inhibition of $\mathrm{E}_{2}$ causes a rise in (B), this in turn causes a fall in (A) and (C) so that the cycle slows down, and this finally prevents the rise in (B) from being as marked as it would be in a linear chain.

(4) When the inhibition on a component enzyme passes beyond a certain point (in the present case around $50 \%$ inhibition), the buffer capacity, 
as given by $d i / d i_{t}$ becomes less than nnity and the cycle rate is sensitive to inhibition (Fig. 7-21).

(5) If the concentration of a particular cycle intermediate is important, as in determining the rate of some side reaction, inhibition can often alter this concentration markedly and produce changes in pathways outside the cycle. For example, inhibition of certain steps in the tricarboxylic acid cycle may influence amino acid metabolism through transaminations involving the keto acid intermediates.

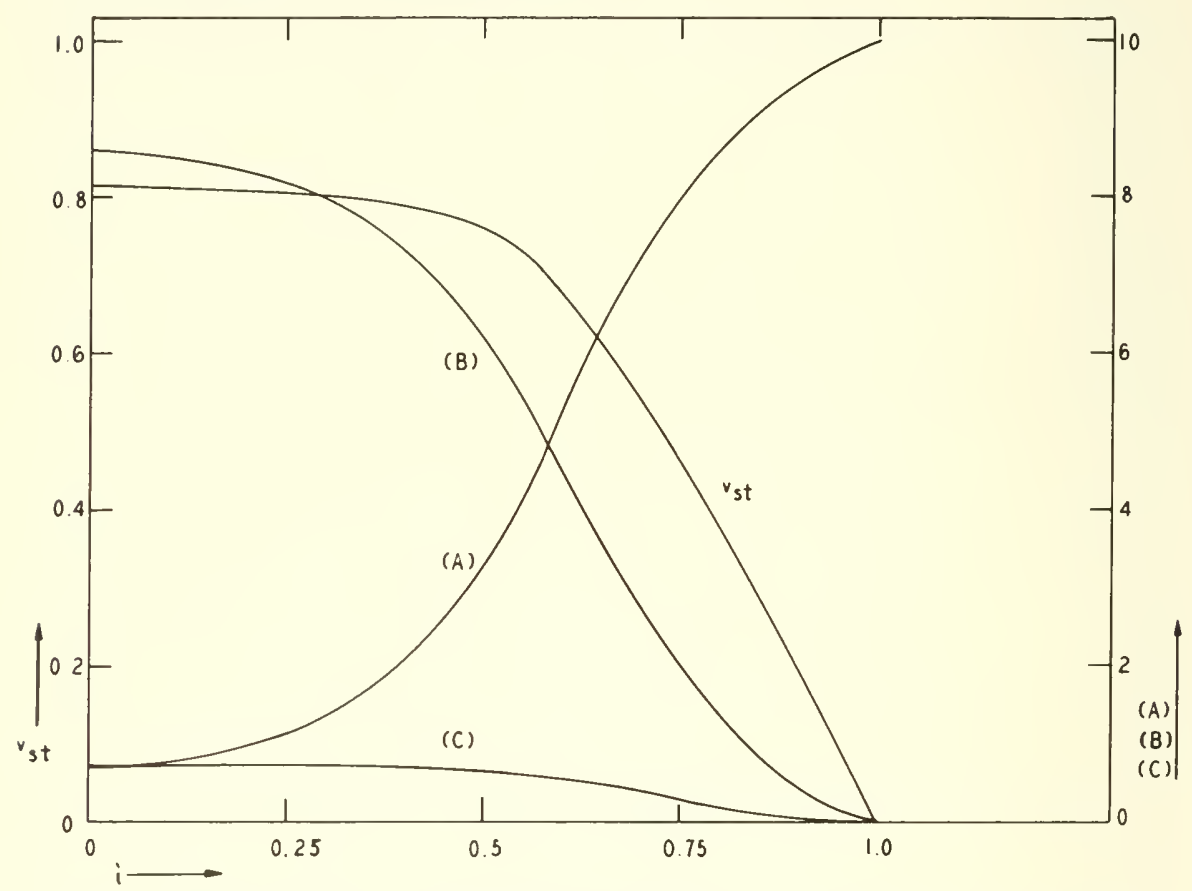

FIG. 7-20. Noncompetitive inhibition of $E_{1}$ in the cyclic system shown in Fig. 7-16. $(\mathrm{MI})_{t}=10 \mathrm{~mL}$.

(6) Competitive inhibition in a cyclic system will have effects which differ little from noncompetitive inhibition, due to the fact that the substrate for the enzyme inhibiter cannot increase in concentration sufficiently to antagonize the inhibition greatly.

\section{REGENERATIVE SYSTEMS}

An enzyme sequence catalyzing reactions in which one or more substances serve as acceptors and donors, alternately gaining and losing groups, 
will be termed a "regenerative system" because these intermediate substances are not transformed into one another to move along the chain, but shuttle back and forth as some group is transferred along the chain. Oxidation-reduction chains and systems transferring phosphate or other

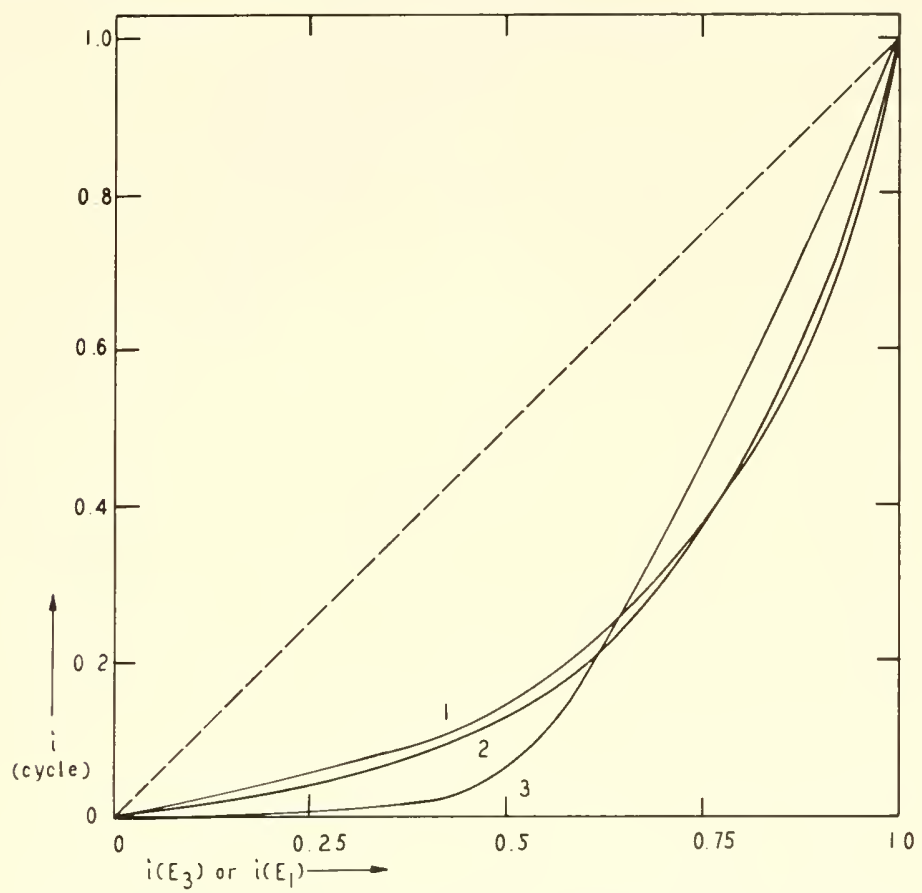

FIG. 7-21. Noncompetitive inhibition of either $\mathrm{E}_{1}$ or $\mathrm{E}_{3}$ in the cyclic system shown in Fig. 7-16. The variation of the over-all cycle inhibition with the individual inhibitions is shown. Curve $1:(\mathrm{MI})_{t}=0.1$ $\mathrm{m} . \mathrm{H}$; curve 2: $(\mathrm{M})_{t}=1 \mathrm{~m} H$; curve $3:(M)_{t}=10 \mathrm{~m} . /$.

groups would be regenerative systems. The simplest representation of such reactions would be:

$$
\begin{aligned}
& \mathrm{A}-\mathrm{X}+\mathrm{B} \rightarrow \mathrm{B}-\mathrm{C}+\mathrm{A} \\
& \mathrm{B}-\mathrm{C}+\mathrm{C} \rightarrow \mathrm{C}-\mathrm{X}+\mathrm{B} \\
& \mathrm{A}-\mathrm{C}+\mathrm{C} \rightarrow \mathrm{C}-\mathrm{X}+\mathrm{A}
\end{aligned}
$$

where $\mathrm{X}$ is the group transferred from $\mathrm{A}$ to $\mathrm{C}$, in which $\mathrm{B}$ functions as a link between the two reactions, as pointed out by Dixon (1949, p. 8). and is regenerated continuously as the reaction proceeds, being part of the catalytic mechanism and often termed a cofactor or coenzyme. For 
oxidation-reduction reactions, $\mathrm{X}$ represents hydrogen atoms or electrons, and for transphosphorylations, $\mathrm{X}$ represents phosplate. The kinetics of these systems and their response to inhibition are very interesting and of great importance in understanding cellular metabolic pathways and their modiflcation.

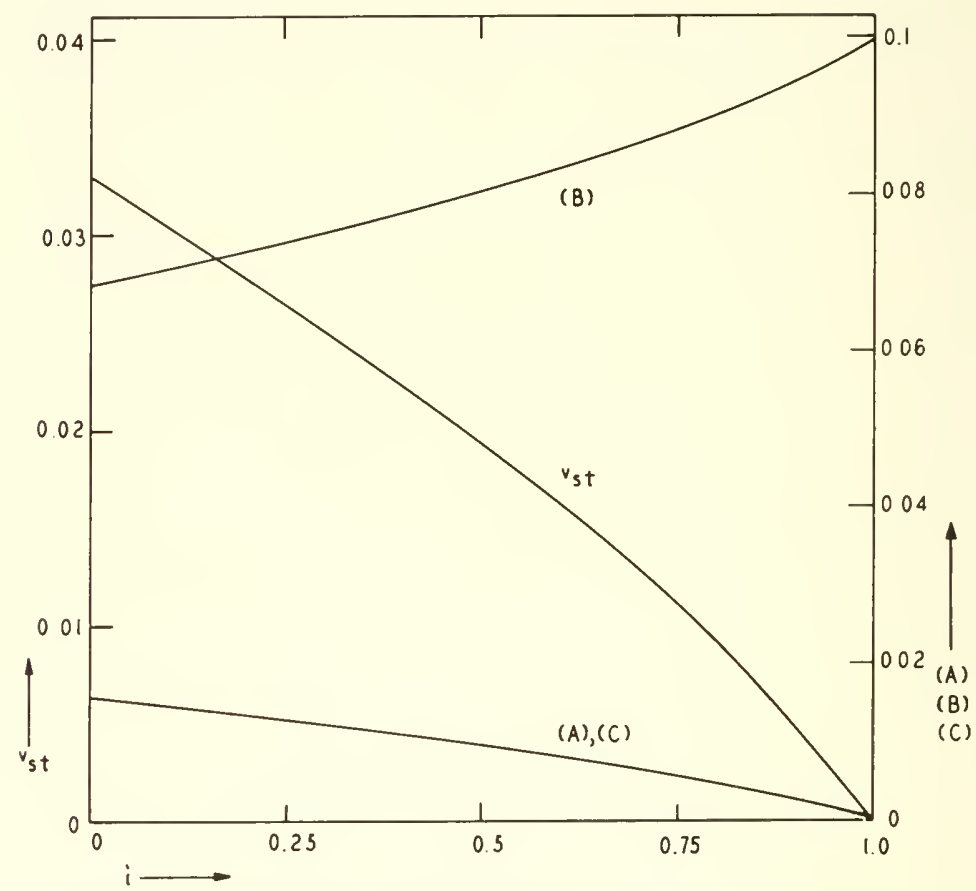

FIG. 7-22. Noncompetitive inhibition of $\mathrm{E}_{2}$ in the cyclic system shown in Fig. $7-16 .(\mathrm{MI})_{t}=0.1 \mathrm{~m} H$.

In the steady state $v_{1}=v_{2}$ where these rates are given by:

$$
\begin{aligned}
& v_{1}=\frac{V_{1}(\mathrm{AX})(\mathrm{B})}{\left[(\mathrm{AX})+K_{a x}\right]\left[(\mathrm{B})+K_{b}\right]} \\
& v_{2}=\frac{V_{2}(\mathrm{BX})(\mathrm{C})}{\left[(\mathrm{BX})+K_{b x}\right]\left[(\mathrm{C})+K_{r}\right]}
\end{aligned}
$$

where $\mathrm{B}$ is a dissociable linking cofactor. Solving for (BX):

$$
(\mathrm{BX})=\frac{V_{1} K_{b x}}{r_{2}\left[1+\frac{K_{b}}{(\mathrm{~B})}\right]\left[1+\frac{K_{a x}}{(\mathrm{AX})}\right] \frac{(\mathrm{C})}{(\mathrm{C})+K_{c}}-V_{1}}
$$


Since the total concentration of cofactor remains constant:

$$
(\mathrm{B})_{t}=(\mathrm{B})+(\mathrm{BX})
$$

the following quadratic expression may be derived for (BX):

$$
(1-R)(\mathrm{BX})^{2}+\left[(\mathrm{B})_{t}(\mathrm{R}-1)+R K_{b}+K_{b x}\right](\mathrm{BX})-(\mathrm{B})_{t} K_{b x}=0
$$

where:

$$
R=\frac{V_{2}}{V_{1}}\left[1+\frac{K_{a x}}{(\mathrm{AX})}\right] \frac{(\mathrm{C})}{(\mathrm{C})+K_{c}}
$$

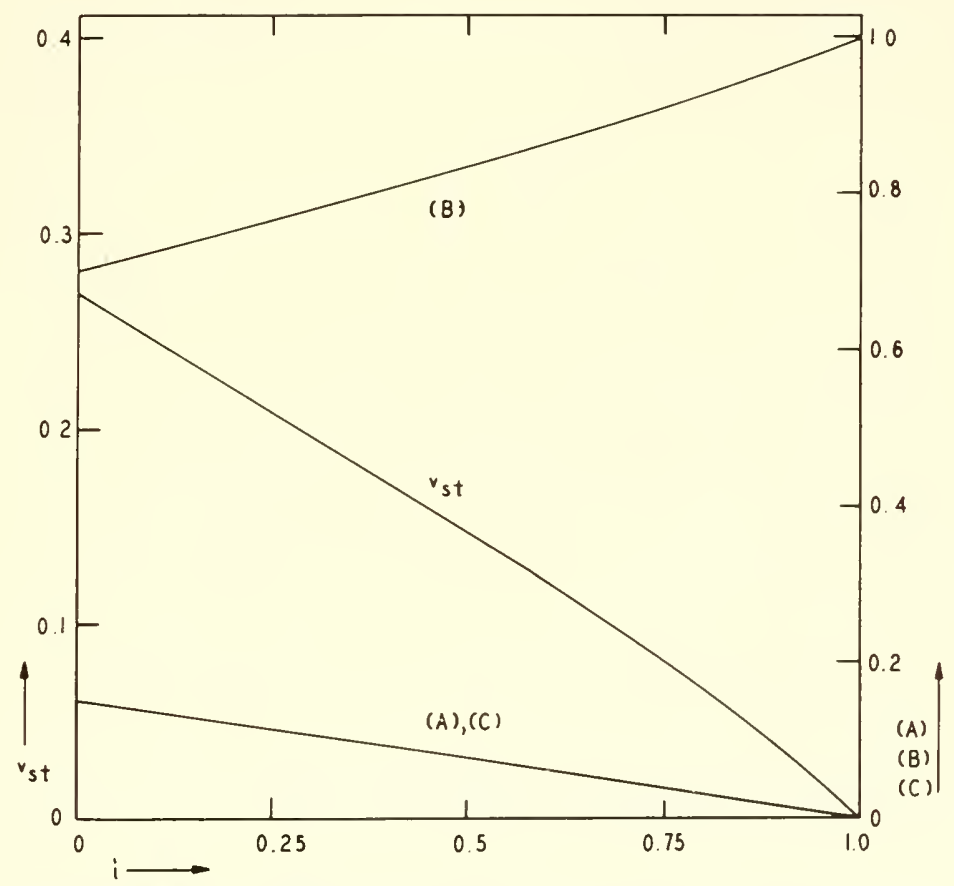

FIG. 7-23. Noncompetitive inhibition of $E_{2}$ in the cyclic system shown in Fig. 7-16. $(\mathrm{M})_{t}=1 \mathrm{~m} M$.

The concentration of BX thus depends on the concentrations of all the components of the system, including the enzymes, and all the constants of the individual reactions. In order to make the results more general, the ratio $(\mathrm{BX}) /(\mathrm{B})_{t}$ will be used to express the state of the regenerated substance. which will be called the "regenerant" for convenience. 


\section{Effects of the Concentrations of Reactants and Regenerant}

In an irreversible monolinear chain, the steady-state rate is always determined by the first reaction, but the coupling between the reactions in a regenerative system gives the second reaction some importance also. The steady-state rate is plotted as a function of the substrate AX in Fig. 7-30 (curve 2) where it may be compared to the rate in the absence of the second reaction (curve 1). The depression caused by the second reaction is due

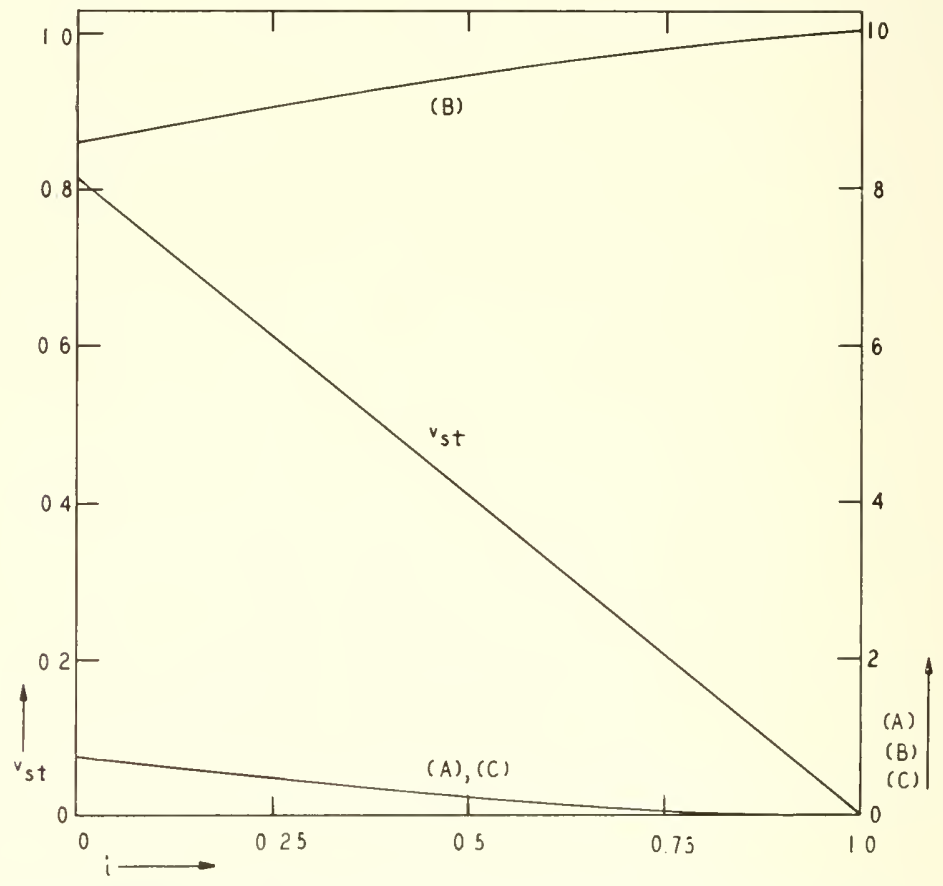

FIG. 7-24. Noncompetitive inhibition of $\mathrm{E}_{2}$ in the cyclic systen shown in Fig. 7.16. $(\mathrm{MI})_{t}=10 \mathrm{mM}$.

to the reduction in (B) that occurs in the steady state. part of it now being in the form BX. Reaction 2 thus plays a role in the control of the rate by its effect on the concentration of regenerant accepting the group $\mathrm{X}$ in reaction 1 . On the other hand. if there is a limited amount of regenerant, the restoration carried out by reaction 2 allows the reaction to proceed for a longer time than if no regeneration occurred.

The changes in the ratio $(\mathrm{BX}) /(\mathrm{B})$, brought about by changes in the concentrations of substrate $(A X)$, total regenerant $(B)_{\iota}$, and final acceptor (C), are shown in Fig. 7-31. As expected, the ratio increases with rise in 
(AX) and decreases with rise in (C). It would not be so easy to predict that the ratio would slowly fall with increasing $(B)_{t}$. Such changes may be of importance in experiments where $(\mathrm{B})$ or $(\mathrm{BX})$ is determined during the steady state, as in the spectrophotometric measurement of DPNH in oxidation-reduction sequences.

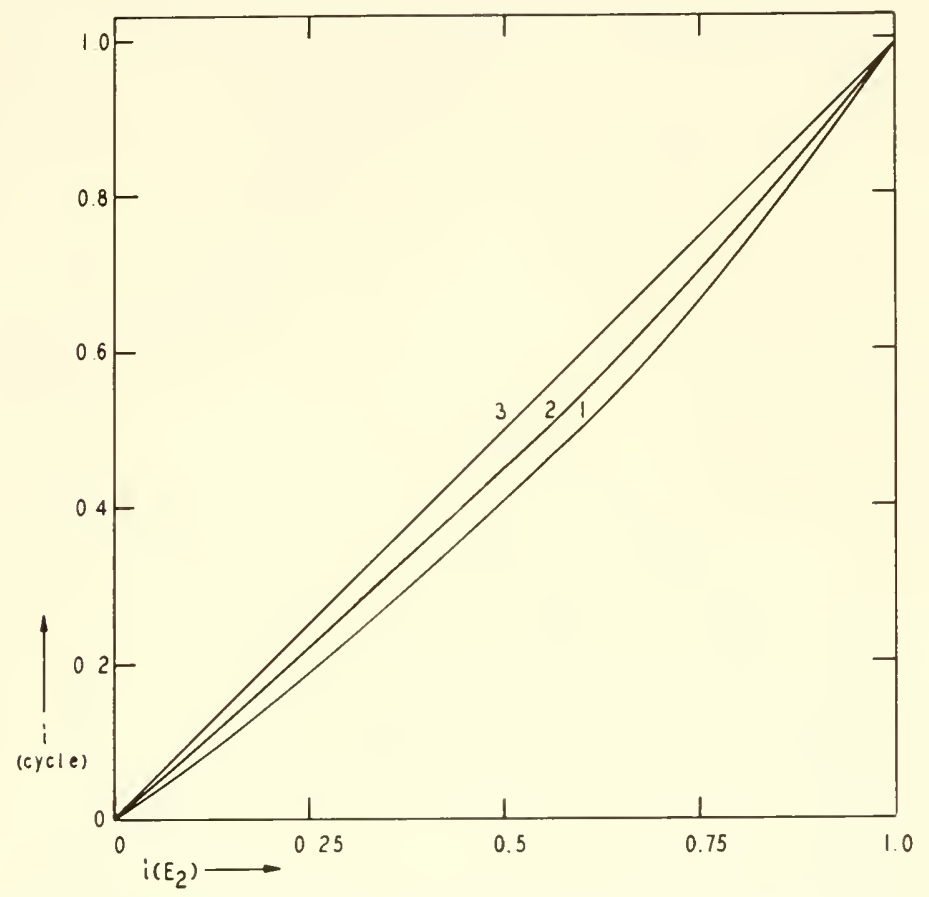

F1G. 7-25. Noncompetitive inhibition of $\mathrm{E}_{2}$ in the cyclic system shown in Fig. 7-16. The variation of the over-all cycle inhibition with the individual inhibition is shown. Curve 1: $(\mathrm{MI})_{t}=0.1 \mathrm{~m} . /$; curve 2 : $(\mathrm{MI})_{t}=1 \mathrm{~m} M$; curve $3:(\mathrm{MI})_{t}=10 \mathrm{~m} M$.

\section{Conditions for the Steady-State and Maximal Rates}

A regenerative system can always assume a steady state whatever the concentrations of substrate, regenerant. or final acceptor. This is because the ratio $(\mathrm{BX}) /(\mathrm{B})_{t}$ will adjust in any case to a valne so that $v_{1}=v_{2}$. This can be shown rigorously in the following manner. An expression for (B) is derived from Eq. 7-40 and for (BX) from Eq. 7-41; the addition of these will give $(\mathrm{B})_{i}$; the rates $v_{1}$ and $v_{2}$ for a steady state are equal and represented by $v_{s t}$; the equation for $(\mathrm{B})_{t}$ is solved for $v_{s t}$. 


$$
\begin{aligned}
& {\left[(\mathrm{B})_{t}+K_{b}+K_{b x}\right]\left[(\mathrm{AX})+K_{a x}\right]\left[(\mathrm{C})+K_{c}\right] v^{2}{ }_{s t} } \\
- & \left\{V_{1}(\mathrm{AX})\left[(\mathrm{B})_{t}+K_{b x}\right]\left[(\mathrm{C})+K_{c}\right]+V_{2}(\mathrm{C})\left[(\mathrm{B})_{t}+K_{b}\right]\left[(\mathrm{AX})+K_{a x}\right]\right\} v_{s t} \\
+ & (\mathrm{B})_{t}(\mathrm{AX})(\mathrm{C}) V_{1} V_{2}=0
\end{aligned}
$$

The nature of this quadratic equation is such that $v_{s t}$ will always have a real and physically compatible value whatever the concentrations or constants. It also illustrates that the steady-state rate is dependent on both reactions and not only on the first. From this equation the value of $v_{s t}$ may be determined for any assumed system and its changes calculated when the concentrations are altered or inhibition is imposed.

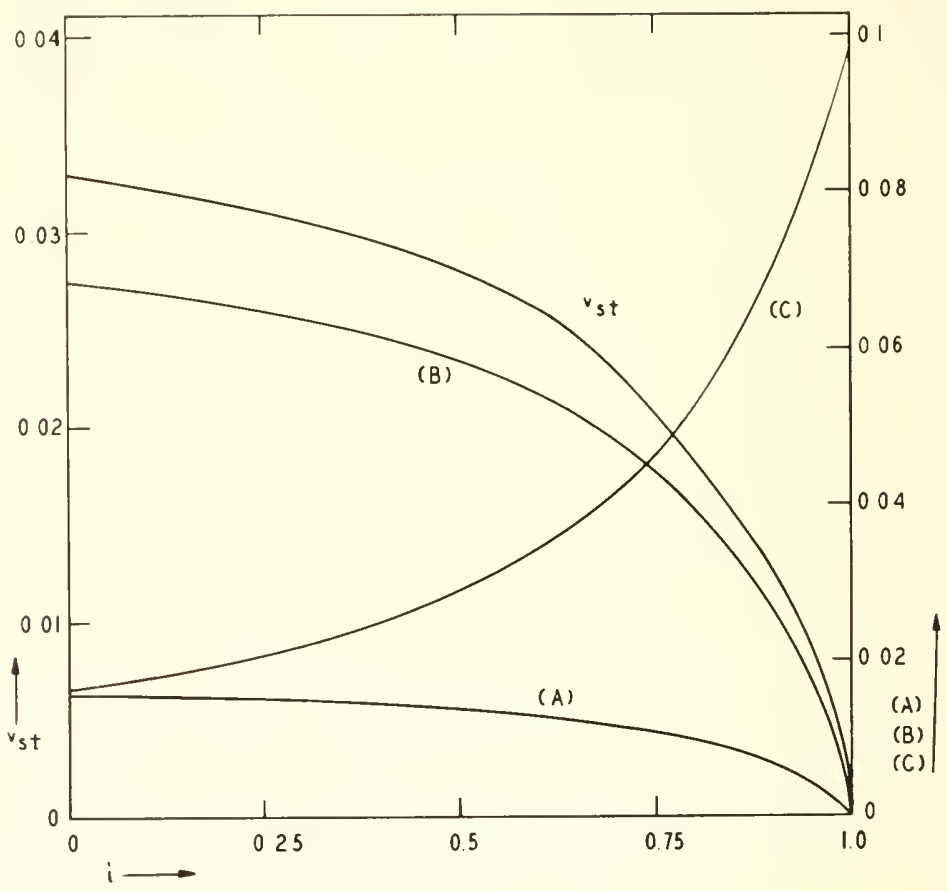

FIG. 7-26. Noncompetitive inhibition of $E_{3}$ in the cyclic system shown in Fig. 7-16. $(\mathrm{MI})_{t}=0.1 \mathrm{mM}$.

\section{Inhibition of Regenerative Systems}

The steady-state rate of a regenerative system may be more sensitive to inhibition of either $\mathrm{E}_{1}$ or $\mathrm{E}_{2}$, depending upon the initial uninhibited state of the system. Inspection of Eq. 7-45 shows that when the two terms in the coefficient of $v_{s t}$ are equal, identical reductions of either $V_{1}$ or $V_{2}$ will have the same effect on $v_{s t}$; such a system would be equally sensitive 
to inhibition of $\mathrm{E}_{1}$ or $\mathrm{E}_{2}$. When the first term in the coefficient is much greater than the second, the steady-state rate will be more sensitive to inhibition of $\mathrm{E}_{2}$, and when it is much less than the second term, the rate will be more sensitive to inhibition of $\mathrm{E}_{1}$. Figures 7-32 and 7-33 show the effects of inhibition in a system where the second term is greater than the first, i.e., where the second reaction is potentially faster than the first, and a marked degree of buffer capacity is observed when $\mathrm{E}_{2}$ is inhibited, espe-

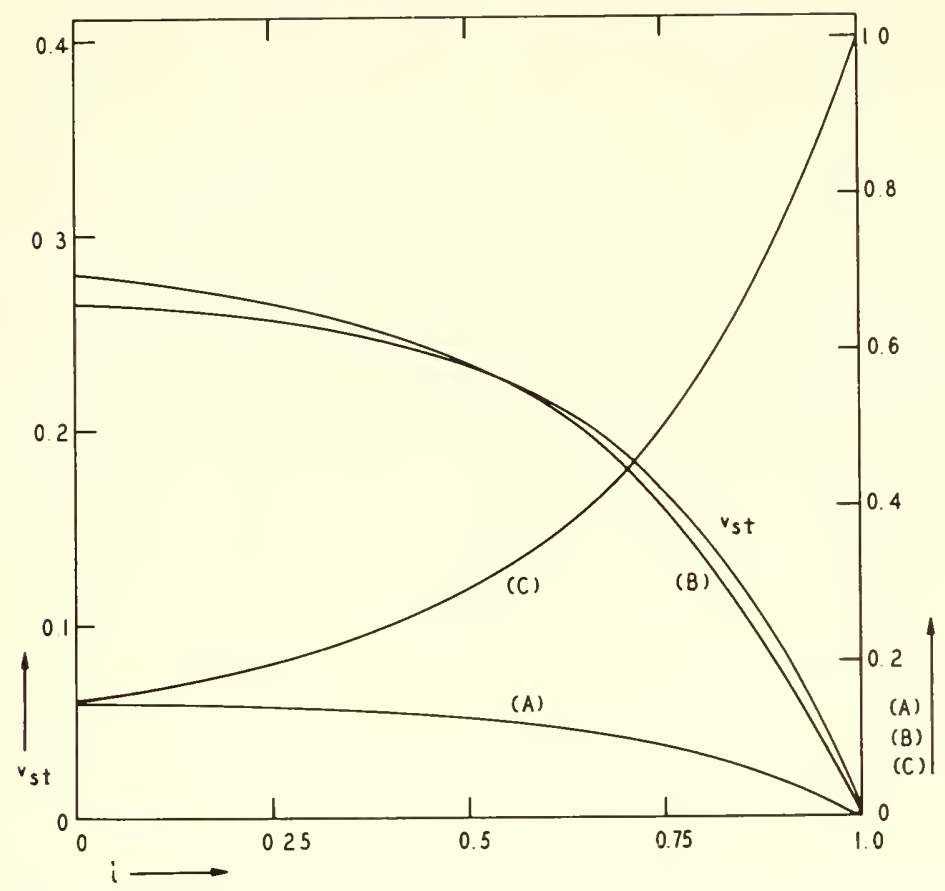

Fic. 7-27. Noncompetitive inhibition of $\mathrm{E}_{3}$ in the cyclic system shown in Fig. 7-16. $(\mathrm{M})_{\ell}=1 \mathrm{mM}$.

cially when the concentration of the regenerated coupler B is high. Regenerative reactions thus have the important property of being particularly sensitive to inhibition on that enzyme which is potentially the slowest, this being determined by the relative values of the constants $K_{m}$ and $k$ and the concentration of enzyme $\left(\mathrm{E}_{t}\right)$. The reason for this characteristic behavior is the constancy of $(\mathrm{B})_{t}$. When $\mathrm{E}_{2}$ is inhibited, (BX) rises, but this requires a necessary reduction in (B) so that the first reaction is slowed, the degree of slowing being dependent on the change brought about in the ratio $(\mathrm{BX}) /(\mathrm{B})_{t}$. 


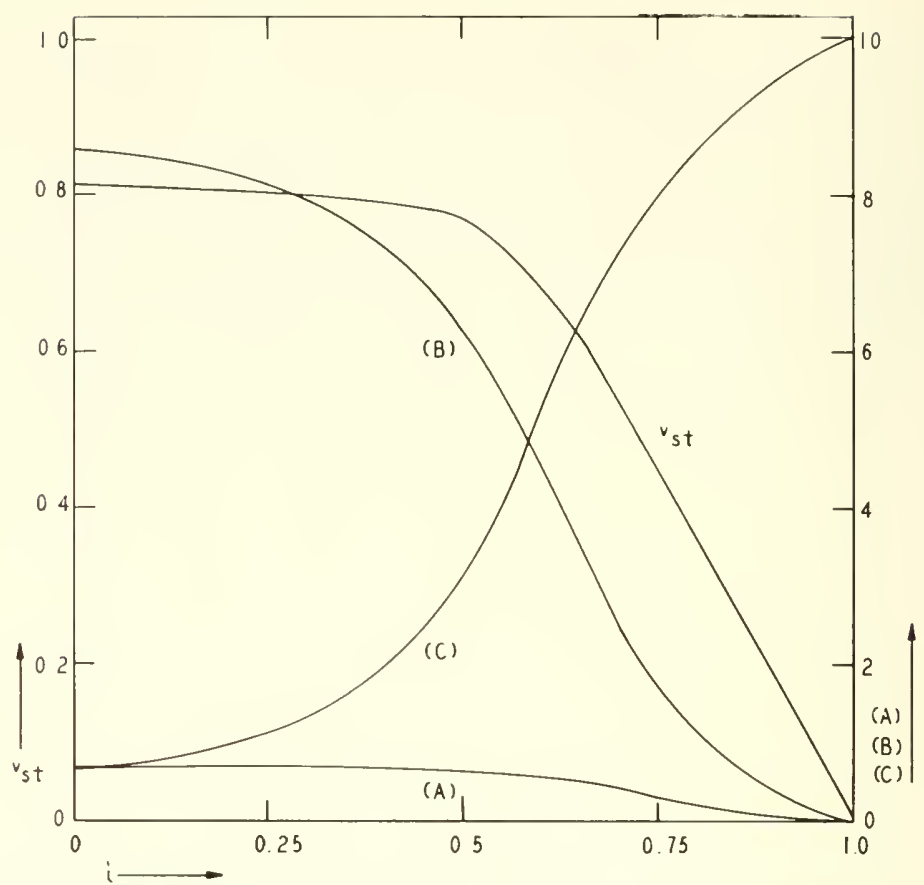

Fig. 7-28. Noncompetitive inhibition of $\mathrm{E}_{3}$ in the cyclic system shown in Fig. 7-16. $(\mathrm{M})_{t}=10 \mathrm{~m} M$.

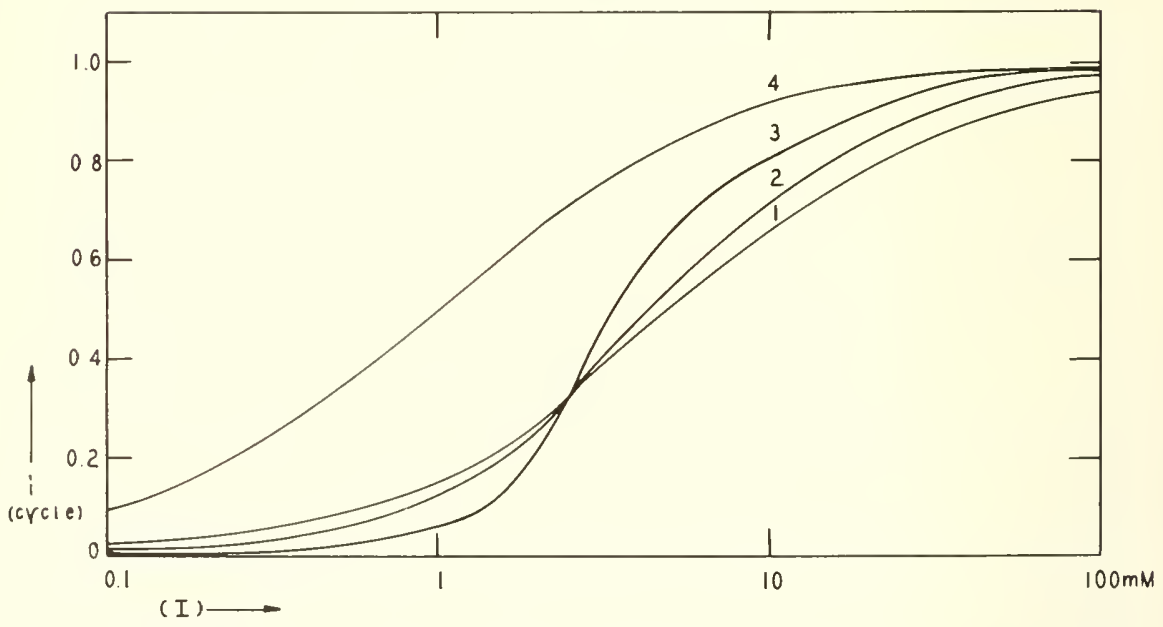

FIG. 7-29. Variation of the over-all cycle inhibition with inhibitor concentration. The inhibition is on $\mathrm{E}_{1}$ in the cyclic system shown in Fig. $7-16 .(\mathrm{X})=1 \mathrm{~m} .1 \mathrm{l}$. Curve 1: $(\mathrm{M})_{t}=0.1 \mathrm{~m} M$; curve 2: $(\mathrm{M})_{t}=1 \mathrm{~m} \boldsymbol{M}$; curve $3:(\mathrm{I})_{t}=10 \mathrm{~m} \boldsymbol{L}$; curve 4: inhibition exerted on $\mathrm{E}_{1}$. 


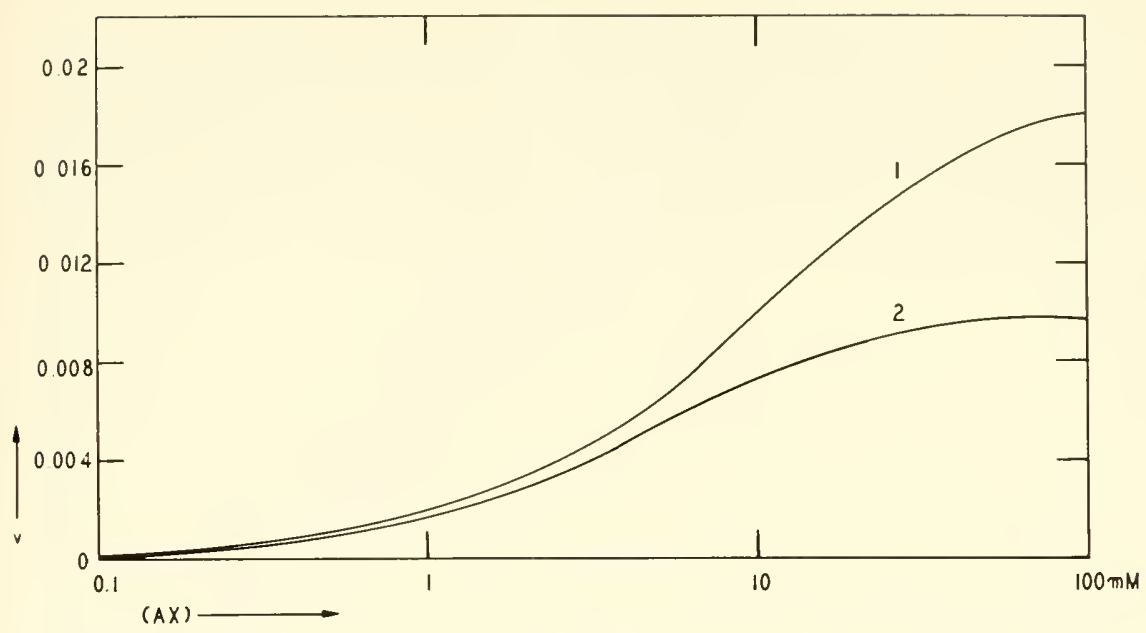

Fig. 7-30. Variation of the rate in a regenerative system $(7-39)$ with the concen. tration of AX. $V_{1}=1, V_{2}=1, K_{a x}=10 \mathrm{~m} . \mu, K_{b}=5 \mathrm{~m} . H, K_{b x}=1 \mathrm{~m} H, K_{c}=2$ $\mathrm{m} M,(\mathrm{~B})_{t}=0.1 \mathrm{~m} M$, and $(\mathrm{C})=1 \mathrm{~m} . \mu$. Curve 1 : assuming only the first reaction; curve 2: regenerative system.

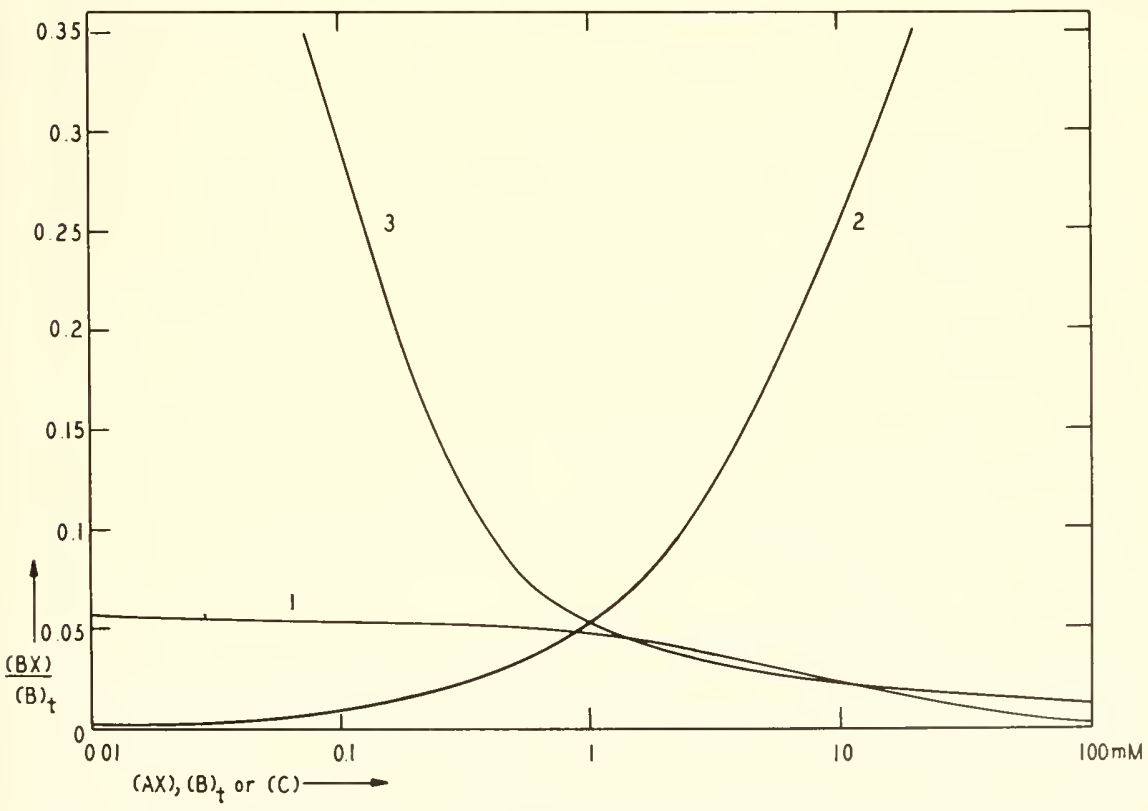

Fig. 7-31. Variation of the ratio $(\mathrm{BX}) /(\mathrm{B})_{t}$ with the concentrations of substrate $(\mathrm{AX})$ (curve 2), total regenerant $(\mathrm{B})_{t}$ (curve 1), and final acceptor $(\mathrm{C})$ (curve 3 ) in a regenerative system $(\overline{7}-39)$. 


\section{Regenerative Systems of Greater Complexity}

In some cases the coupling is not as close as in scheme 7-39 and two or more reactions may be interposed before regeneration of the intermediate is achieved. An oxidation-reduction system involving hydrogen transfer may utilize some metabolic product of the substrate for regeneration:

$$
\begin{aligned}
& \mathrm{AH}_{2}+\mathrm{Co} \stackrel{\mathrm{E}_{1}}{\rightarrow} \mathrm{A}+\mathrm{CoH}_{2} \\
& \mathrm{~A} \underset{\mathrm{E}_{2}}{\rightarrow} \mathrm{B} \\
& \stackrel{\mathrm{E}_{3}}{\mathrm{~B}+\mathrm{CoH}_{2}} \rightarrow \mathrm{BH}_{2}+\mathrm{Co} \\
& \hline \mathrm{AH}_{2} \rightarrow \mathrm{BH}_{2}
\end{aligned}
$$

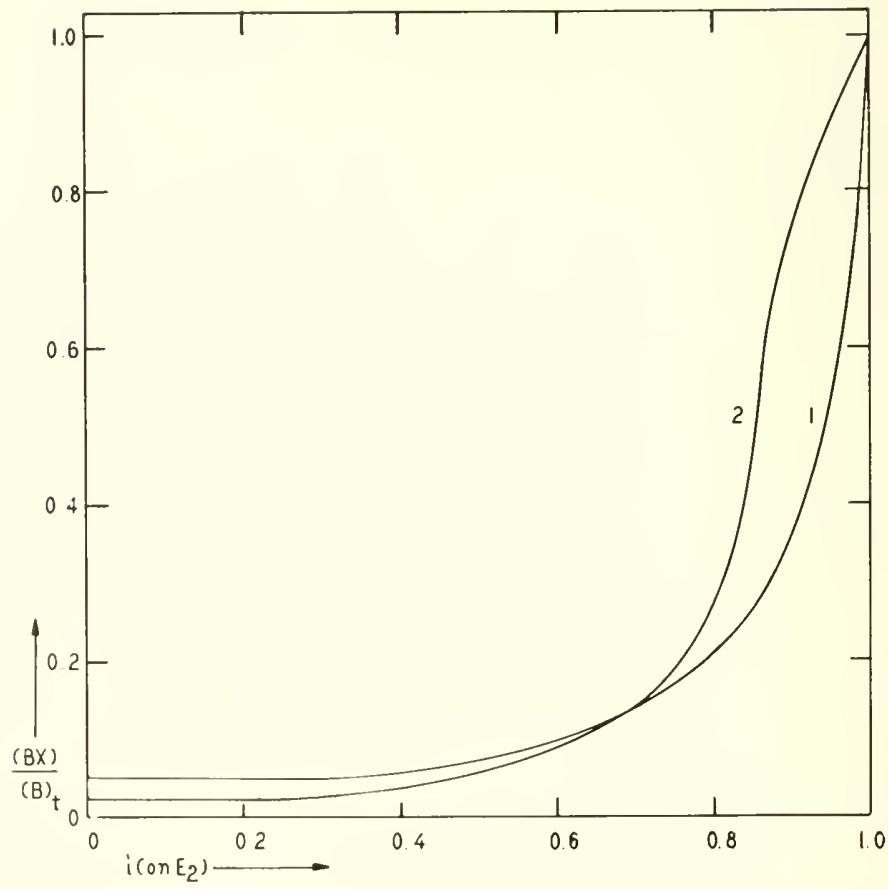

FIG. 7-32. Effect of noncompetitive inhibition of $\mathrm{E}_{2}$ on the ratio $(\mathrm{BX}) /(\mathrm{B})_{t}$ in a regenerative system (7-39). Curve $1:(\mathrm{B})_{t}=0.1 \mathrm{~m} M$; curve 2: $(\mathrm{B})_{t}=10 \mathrm{mM}$.

Co is a coenzyme which transfers hydrogen atoms and is regenerated. The anaerobic formation of lactate by the glycolytic pathway occurs in this way: $\mathrm{AH}_{2}$ is glyceraldehyde- 3 -phosphate, $\mathrm{B}$ is pyruvate, and Co is $\mathrm{DPN}^{+}$, omitting the phosphorylation. In this case the reaction $\mathrm{A} \rightarrow \mathrm{B}$ is not a 
single step but a monolinear chain involving three enzymes. Inhibition of $\mathrm{E}_{2}$ (or any enzyme in a chain taking $\mathrm{A}$ to $\mathrm{B}$ ) will not reduce the rate of formation of $\mathrm{BH}_{2}$ as long as (A) can rise to maintain the initial rate. However, inhibition of $\mathrm{E}_{3}$ will depress the rate to some degree since the rise in $\left(\mathrm{CoH}_{2}\right)$ will decrease $(\mathrm{Co})$, unless $\mathrm{Co}$ is initially at a concentration more than required to saturate $\mathrm{E}_{1}$. As in scheme 7-39, the effect will depend on the relative constants of $\mathrm{E}_{1}$ and $\mathrm{E}_{3}$, so that imhibition of $\mathrm{E}_{3}$ may reduce the formation of $\mathrm{BH}_{2}$ to a lesser degree than expected on the basis of the inhibition of the isolated enzyme. In the example cited above, the rate of lactate formation will be reduced to different degrees by inhibition of the glyceraldehyde-3-phosphate dehydrogenase, enolase, or lactic dehydrogenase when the inhibitors are present at concentrations depressing the isolated enzymes identically.

Certain multienzyme systems involving phosphate transfer can also be represented as regenerative sequences. If the substrate must be phosphorylated for further reaction, regeneration of the phosphate donor may occur:

$$
\begin{aligned}
\mathrm{A}+\mathrm{ATP} \underset{\mathrm{E}_{2}}{\rightarrow} \mathrm{AP}+\mathrm{ADP} \\
\mathrm{AP} \underset{\mathrm{E}_{3}}{\rightarrow} \mathrm{BP} \\
\mathrm{BP}+\mathrm{ADP} \rightarrow \mathrm{C}+\mathrm{ATP}
\end{aligned}
$$

This system will behave kinetically and in response to inhibition in an identical manner to the previons sequence $7-46$. On the other hand, such a system may serve to form ATP if reaction 3 is an oxidation coupled with the synthesis of more than one high energy phosphate bond:

$$
\begin{gathered}
\mathrm{A}+\mathrm{ATP} \underset{\mathrm{E}_{2}}{\rightarrow} \mathrm{AP}+\mathrm{ADP} \\
\mathrm{AP} \underset{\mathrm{E}_{3}}{\rightarrow} \mathrm{B}+\mathrm{P} \\
\mathrm{B}+n \mathrm{ADP}+n \mathrm{P} \rightarrow \mathrm{C}+n \mathrm{ATP} \\
\mathrm{A}+(n-1) \mathrm{ADP}+(n-1) \mathrm{P} \rightarrow \mathrm{C}+(n-\mathrm{I}) \mathrm{ATP}
\end{gathered}
$$

During transformation of $\mathrm{A} \rightarrow \mathrm{C}$ there is the over-all production of ATP, such as occurs. for example. during glycolysis. An interesting feature of this system is that adequate concentrations of both ADP and ATP must be present for the rate to be appreciable. Since one may assume (ADP) + (ATP) to be constant. any condition which makes the ratio (ADP)/(ATP) very high or very low will inhibit the over-all rate. Such regulation may operate in glycolysis, which may be depressed by either low (ADP) or low (ATP) (Lynen, 1958), although there is some evidence that the gly- 
colytic system may be compartmentalized in that the ATP formed by the oxidation of glyceraldehyde-3-phosphate may not be immediately available to glucokinase (Chance and Hess, 1959).

The detailed mechanism of oxidative phosphorylation is not sufficiently understood to represent the various steps accurately, but the following steady-state system will serve to illustrate certain aspects of the behavior.

$$
\begin{aligned}
\mathrm{A}+\mathrm{P} \underset{\mathrm{E}_{2}}{\rightarrow} \mathrm{B}+\sim \mathrm{P} \\
\sim \mathrm{P}+\mathrm{ADP} \underset{\mathrm{E}_{3}}{\rightarrow} \mathrm{ATP} \\
\mathrm{ATP} \rightarrow \mathrm{ADP}+\mathrm{P} \\
\mathrm{A} \rightarrow \mathrm{B}
\end{aligned}
$$

Reaction 1 establishes a high energy bond, $\sim \mathrm{P}$, which may involve a group on the enzyme, reaction 2 transfers the high energy phosphate to $\mathrm{ADP}$, and reaction 3 is a means of regenerating ADP so that reaction 2 may proceed in a steady state. Reaction 3 may be the splitting of ATP

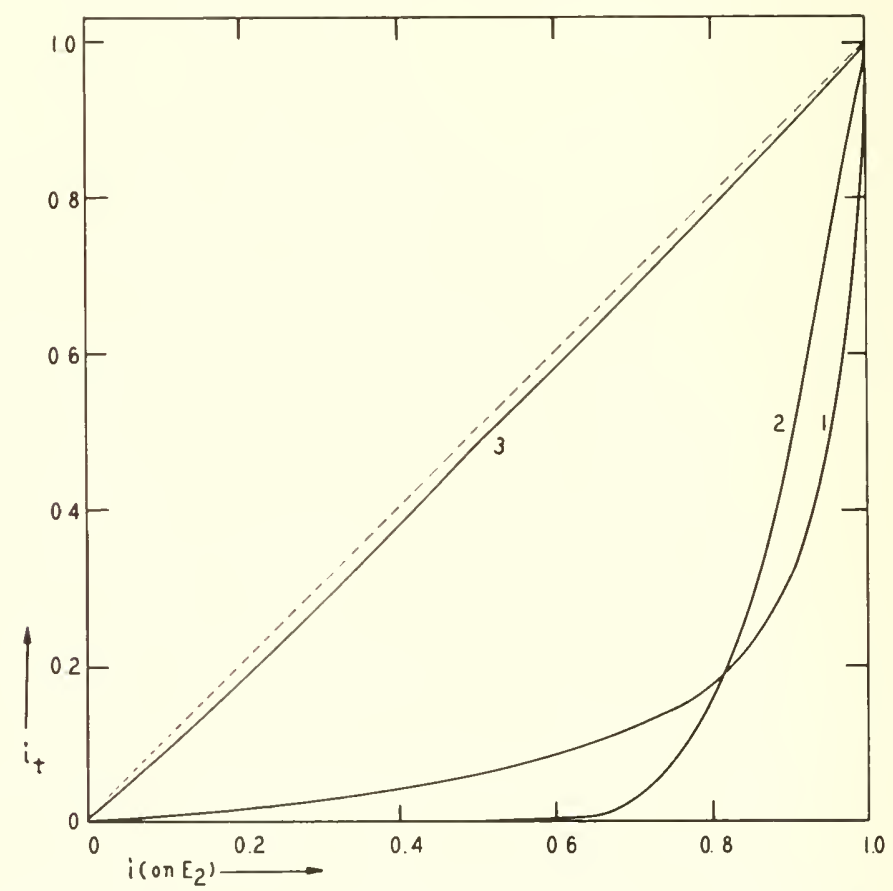

FIG. 7-33. Effect of noncompetitive inhibition of $\mathrm{E}_{2}$ on the over-all inhibition of a regenerative system (7-39). Curve $1:(B)_{t}=0.1 \mathrm{~m} .1$; curve 2: $(\mathrm{B})_{t}=10 \mathrm{~m} \boldsymbol{M}$; curve 3: inhibition of $\mathrm{E}_{1}$ for comparison. 
by an ATPase or the utilization of ATP by synthetic or functional systems in the cell. The transformation of $\mathrm{A} \rightarrow \mathrm{B}$ may be said to be obligatorily coupled to the formation of ATP if $\sim \mathrm{P}$ is present in very small concentration, as it would be if the bond were to some enzyme gronp, because the reaction will rapidly stop if the $\sim \mathrm{P}$ is not removed by ADP. The concentration of ADP may limit the over-all reaction and hence the formation of $\mathrm{B}$ will depend on the rate of the regenerative reaction 3, as has been demonstrated in the electron-transport sequence (Chance and Williams, 1956). Such systems may be involved in the regulation of cellular metabolism, since the rate of utilization of $\mathrm{A}$ will be determined in part by the rate at which ATP is used in synthetic and functional processes. If an uncoupling agent, such as 2,4-dinitrophenol, is added, it may be assumed that $\sim \mathrm{P}$ is split from the enzyme as inorganic phosphate:

$$
\sim \mathrm{P} \rightarrow \mathrm{P}
$$

This will decrease the formation of ATP but may increase the formation of $\mathrm{B}$ if reaction 2 has been limiting. If reaction $7-50$ becomes much more rapid than the formation of ATP, the system will be almost completely uncoupled. At the present time it is not possible to treat this system exactly because of the uncertainties in mechanism and the possibility that other reactions may be involved. Another complexity is that most uncoupling agents inhibit the electron-transport in some manner, so that the experimental stimulation of $\mathrm{A} \rightarrow \mathrm{B}$ is not as great as would be expected on the basis of scheme 7-49. Alteration of the rate of reaction 3, either by effects on $\mathrm{E}_{3}$ or on systems removing ATP, may also change the rate of formation of $\mathrm{B}$. Thus inhibition of an ATPase might slow down $\mathrm{A} \rightarrow \mathrm{B}$ since it could reduce (ADP), so that removal of $\sim \mathrm{P}$ from the enzyme is reduced; likewise, activation of such an ATPase could accelerate $A \rightarrow B$. It may be pointed out that regenerative systems can usually be represented in a cyclic form; thus the sequence $7-49$ is equally a cyclic system and obeys the kinetics of cyclic systems.

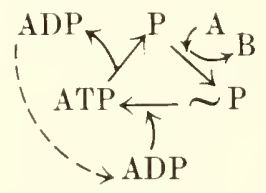

If the ATP were in equilibrium with a store of creatine phosphate this would provide some buffer to the system with respect to inhibition and would in some instances supply a further means for regeneration of ADP.

Another type of uncoupling effect may be mentioned. A substance may be capable of accepting the $\sim \mathrm{P}$ from the enzyme and competing with ADP:

$$
\mathrm{I}+\sim \mathrm{P} \rightarrow \mathrm{I} \sim \mathrm{P}
$$


If $\mathrm{I} \sim \mathrm{P}$ is stable, the presence of I will drain off a fraction of the high energy phosphate and the formation of ATP will be decreased. If $\mathrm{I} \sim \mathrm{P}$ is unstable and breaks down rapidly to $I$ and $P$, a very small amount of $I$ may be sufficient to uncouple becanse it will be constantly regenerated. Such cycles or regenerative systems are not limited to phosphate transfer; in any cycle a substance may compete with or combine with an intermediate so that inhibition may be observed with respect to some product, although the initial substrate wonld be utilized more rapidly.

\section{SELF-REGULATORY OR FEEDBACK SYSTEMS}

When one of the intermediates or products of a reaction sequence affects the activity of an enzyme catalyzing one of the previons steps, a self-regulatory action on the rate may be exerted and such may be termed "feedback systems." Feedback may be positice, if the effect on the enzyme is activation, or negative, if it is inhibition. It is quite possible that this is an important mechanism in the regulation of cellular metabolism, particularly synthetic pathways, althongh as yet only a few feedback systems have been demonstrated. The action on the enzyme may be either direct or upon its synthesis, as is illnstrated in Fig. 7-34. Some examples of direct negative

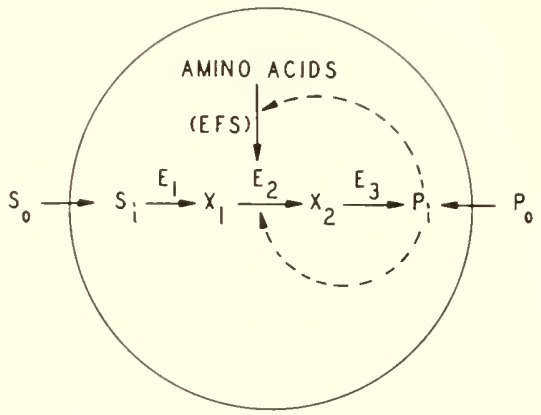

Fig. 7-34. Feedback mechanisms. A substrate, $\mathrm{S}$, is taken into the cell and metabolized progressively through the intermediates, $X_{1}$ and $X_{2}$. to a product $P$. This product is able to affect the activity of $\mathrm{E}_{2}$ either directly (lower dashed arrow) or by altering the rate of its synthesis (upper dashed arrow) from amino acids in the enzyme-forming system (EFS). If the product is added to the medium outside the cell, $\mathrm{P}_{0}$, it may enter and exert the same action as the endogenously formed product. (Adapted from Umbarger and Brown, 1958.)

feedback may be cited: the inhibition of L-threonine deaminase in Escherichia coli, by the product L-isoleucine, preventing the accumulation of its own precursor, since this enzyme catalyzes the initial step in the formation of L-isolencine from L-threonine (Umbarger and Brown, 1958); 
the inhibition by histidine of the formation of compound III, an intermediate in histidine biosynthesis (Moyed and Friedman, 1959); the inhibition by uridine diphosphate of the reaction orotidylic acid $\rightarrow$ uridine monophosphate in pyrimidine biosynthesis of rat liver (Potter and Blair, 1959); the inhibition of deoxycytidylate deaminase by two products subsequent to deoxycytidylate, namely deoxyuridylate and deoxythymidylate, in rat liver (Maley and Maley, 1959); and the inhibition by tryptophan of the synthesis of its precursor, anthranilate, in Aerobacter aerogenes (Doy and Pittard, 1960). An interesting situation occurs in certain mutants of $E$. coli where 5-amino-4-imidazolecarboxamide riboside accumulates because no transformylase is present (Gots and Gollub, 1959). The accumulation of this compound ceases when any purine that can support growth is added. The accumulation is also depressed when purine analogs are added to the medium. These analogs sufficiently resemble the natural purines to act as feedback inhibitors. Since these analogs may also interfere with the metabolism in the later stages of purine nucleotide biosynthesis, they may inhibit at two sites. An example of negative feedback on enzyme synthesis is the suppression of ornithine transcarbamylase by the final product arginine (Ennis and Gorini, 1959). The kinetic behavior of such systems and their response to inhibition are interesting and justify more than the superficial treatment presented below.

\section{Dependence of Rate on the Concentration of Substrate}

A simple negative feedback system may be be represented as:

$$
\mathrm{A} \stackrel{\mathrm{E}_{1}}{\rightarrow} \mathrm{B} \stackrel{\mathrm{E}_{3}}{\rightarrow} \mathrm{C} \stackrel{\mathrm{E}_{3}}{\rightarrow} \mathrm{D}
$$

where the intermediate $\mathrm{C}$ inhibits $\mathrm{E}_{1}$ competitively. Competitive inhibition is assumed because it is likely that most feedback inhibition will be of this nature, due to the structural similarity of $\mathrm{A}$ and $\mathrm{C}$. Reaction 3 is included so that (C) may be kept constant in a steady state. The individual rate expressions are:

$$
\begin{aligned}
& v_{1}=\frac{V_{1}(\mathrm{~A})}{(\mathrm{A})+K_{1}\left[1+\frac{(\mathrm{C})}{K_{\mathrm{c}}}\right]} \\
& v_{2}=\frac{V_{2}(\mathrm{~B})}{(\mathrm{B})+K_{2}} \\
& v_{3}=\frac{V_{3}(\mathrm{C})}{(\mathrm{C})+K_{3}}
\end{aligned}
$$


where $K_{c}$ is the inhibition constant for $\mathrm{C}$ on $\mathrm{E}_{1}$. Equating $v_{1}$ and $v_{3}$, the following quadratic espression for (C) in the steady state is obtained:

$$
(\mathrm{C})^{2}+K_{c}\left[1+\frac{(\mathrm{A})}{K_{1}}\left(1-\frac{V_{1}}{V_{3}}\right)\right] \text { (C) }-\frac{V_{1} K_{c} K_{3}(\mathrm{~A})}{V_{3} K_{1}}=0
$$

From $(C)$ the steady-state rate may be obtained from Eq. 7-56. Results for an arbitrarily chosen system are shown in Fig. 7-35. The steady-state rate is, of course, less than if no feedback existed, and the fractional reduction due to feedback is shown in curve 3. However, the feedback helps

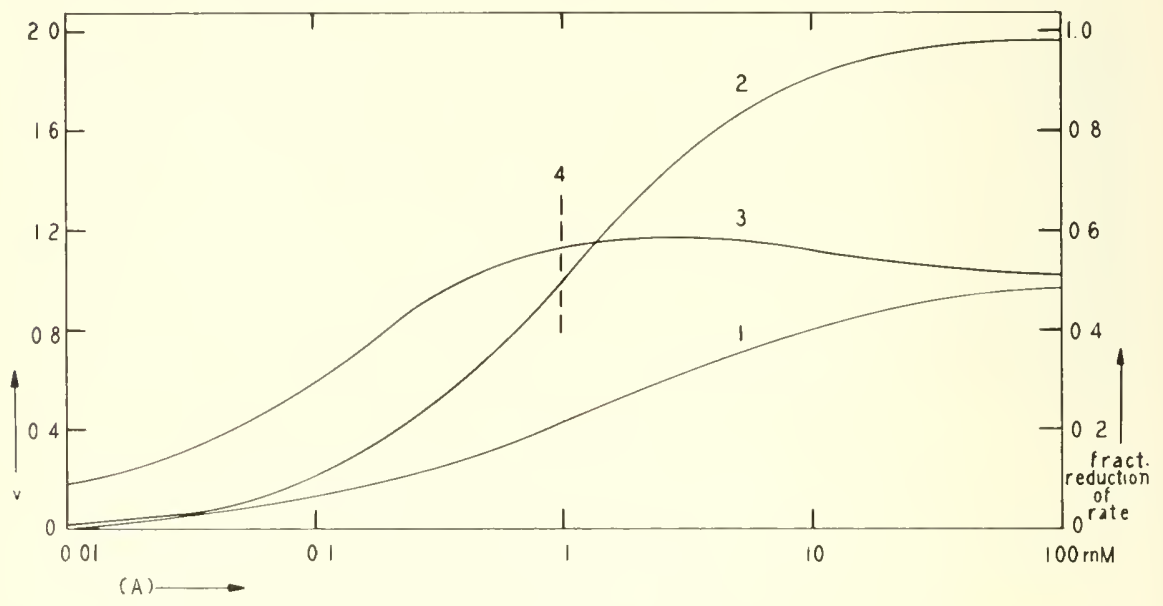

FIG. 7-35. Variation of the rate with substrate concentration in a feedbaek system (7-53). $V_{1}=2, V_{2}=10, V_{3}=1, K_{3}=1 \mathrm{~m} . H, K_{c}=0.3 \mathrm{~m} M$, and $K_{3}=1 \mathrm{~m} M$. Curve 1: steady-state rate $v_{s t}$. Curve 2 ; rate of reaction 1 assuming no feedback. Curve 3: fraetional reduetion of the rate by feedback. The dashed line 4 indicates the maximal (A) for a steady state if there is no feedbaek.

to maintain the system in a steady state; without the feedback, (A) could not be greater than $1 \mathrm{~m} M$, as indicated by line 4 , but with the feedback, (A) can be any value and a steady state may be established. The reason for this is that as (A) increases. (C) increases and exerts more inhibition on $\mathrm{E}_{1}$ so that reaction 1 slows down; an equilibrimm is reached when $(\mathrm{C})$ is at that level which will inhibit $\mathrm{E}_{1}$ sufficiently to maintain (C).

\section{Inhibition of Feedback Systems}

Inhibition of either $\mathrm{E}_{1}$ or $\mathrm{E}_{3}$ will alter the steady-state concentration of $\mathrm{C}$, according to $\mathrm{Eq} .7-57$, and when the resultant over-all rates are calculated it is found that there is relatively little buffer capacity, as shown 
in Fig. 7-36. Furthermore, the same effect on over-all rate is produced by inhibition on either enzyme. A simple monolinear chain without feedback would behave quite differently; there would be buffer capacity upon inhibition of $\mathrm{E}_{1}$ and generally quite marked buffer capacity upon inhibition of $\mathrm{E}_{3}$. Reaction 1 is no longer the sole determinant of the rate. The results

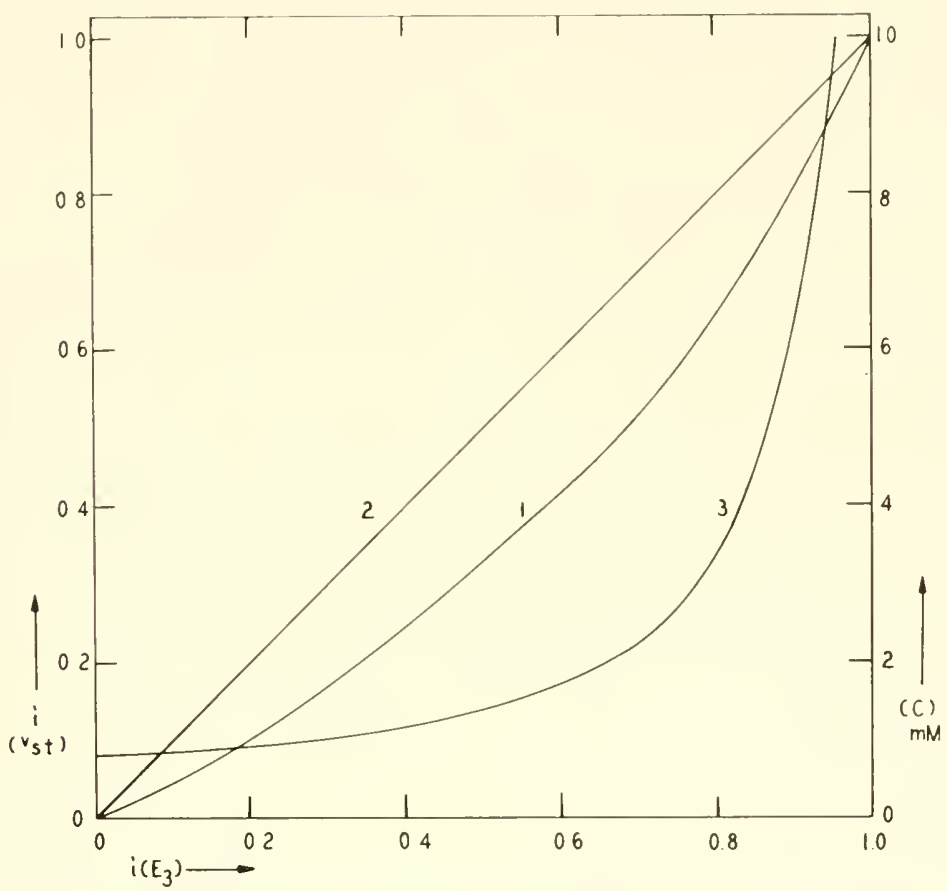

FIG. 7-36. Effect of noncompetitive inhibition of $\mathrm{E}_{3}$ on the over-all inhibition of a feedback system (Eq. 7-53) and on the concentration of the inhibiting product. Same system as in Fig. 7-35. Curve 1: inhibition of over-all rate. Curve 2: inhibition of $\mathrm{E}_{3}$. Curve 3: concentration of $\mathrm{C}$.

may be understood to arise in the following ways. When $\mathrm{E}_{1}$ is inhibited by an added inlibitor, the rate of formation of $\mathrm{C}$ is reduced so that $(\mathrm{C})$ falls, which releases $\mathrm{E}_{1}$ from some of the inhibition previously exerted on it by $\mathrm{C}$. Thus the over-all rate will not be inhibited proportionately to the inhibition on $\mathrm{E}_{1}$. Likewise, when $\mathrm{E}_{3}$ is inhibited there is a rise in $(\mathrm{C})$ which will slow down reaction 1 through the feedback inhibition, but (C) cannot rise to a sufficient level to maintain the initial rate because its rate of formation is reduced. A feedback system thus has less buffer capacity than a monolinear chain without feedback when an enzyme after the first is inhibited, but greater buffer capacity when the initial enzyme in the chain is inhibited. 
On the whole, although feedback systems operate more slowly than comparable linear chains, they possess a wider degree of stability in resisting inhibition.

\section{NETWORK SYSTEMS}

If grids of transferring sites actually exist on complex enzymes or in multienzyme systems, as has been postulated for the succinic and DPNH dehydrogenases by Green (1956), the kinetics may be quite different than those of ordinary enzyme reactions. In an electron-transferring grid, rates cannot be formulated in terms of Wichaelis-Menten kinetics, unless the conductance is so high that electron movement through the grid does not contribute to the over-all rate behavior. The mechanism of conduction here, of course, is not the same as through metals but involves cyclic oxidation and reduction of the grid components. It is quite probable that such electron transfers would not be limiting under normal conditions, but might become so in the presence of certain inhibitors, for example those chelating metal ions in the grid. The response to inhibition would have to be expressed in terms of the statistical distribution of inhibitor molecules throughout the grid. but in the present state of ignorance of the organization of such systems, a quantitative treatment wonld be meaningless. One could predict, in general, that as the concentration of inhibitor was increased, there would be little effect on the conductance or rate until a relatively large fraction of the sites was combined, since some pathways would remain functional despite a scattering of inactive sites. However, at higher inhibitor concentrations the rate will drop rapidly, so that an inhibition-concentration curve will be very steep. It may also be noted that combination of a grid site with an inhibitor need not block electron flow through that site, but may alter the oxidation-reduction potential or merely slow the rate of movement of electrons through that site. A spectrophotometric study of the changes in the steady-state conditions of the respiratory enzymes in wheat roots and yeast by Lundegårdh (1959) led him to postulate a spatial organization of the respiratory system in which the individual enzymes form multimolecular units. Cytochrome $b$ and FAD occupy one complex grid system and cytochrome $c$ and cytochrome oxidase occupy another mixed grid, the flow of electrons occurring within a network in each case.

\section{GENERAL CONSIDERATIONS OF MULTIENZYME SYSTEMS}

There are certain general properties of multienzyme systems and several concepts applicable to all types of system that will be discussed briefly in this section. 


\section{Transit Time}

Dixon (1949, p. 15; Dixon and Webb, 1958, p. 566) has pointed out that in multienzyme systems there may be a factor additional to the rates of the component enzymes that may play a role in determining the over-all rate, namely the diffusion of an intermediate from one enzyme to the next. The transit time is the average time required for the diffusion between enzymes to occur. If an irreversible monolinear chain is in a steady state, this implies that the rates of the diffusion processes are identical with the rate of the initial step. If diffusion is slower than this rate, intermediates may progressively accumulate and the rate of formation of the product may be less than the rate of utilization of the initial substrate, in which case the diffusion may be said to be limiting. When diffusion is limiting, the response of the system to inhibition may be different than when the rate is determined by an enzyme reaction. Diffusion is apt to be more important in compartmentalized systems and this will be discussed in the following section.

The transit time will depend on (1) the distance over which diffusion takes place, (2) the diffusion constant of the intermediate, which is related to its molecular size and shape, and (3) the presence of any barriers to diffusion between the enzymes. The modifications in the kinetics of multienzyme systems introduced by a diffusion process will depend on the structural properties, that is, the spatial relations of the enzymes. In heterogeneous catalysis. the problem is usually one of diffusion of the reactant from the body of the solution to a surface containing the active sites; the kinetics of diffusion and of diffusion-limited catalysis have been formulated for various types of systems (Lee. 1953. p. 125; Moelwyn-Hughes, 1957, p. 1167; Crank, 1956).

Let us first consider the simple situation where a substance, B, diffuses from a plane, at which the concentration of $B$ remains constant, to another plane at which $\mathrm{B}$ is adsorbed and reacted catalytically (Fig. $7-37$ ). The rate at which the substance arrives at the active sites is given by:

$$
\frac{d B}{d t}=\frac{a D}{d}\left[(\mathrm{~B})_{1}-(\mathrm{B})_{2}\right]
$$

where $a$ is the area of the planes under consideration, $D$ is the diffusion coefficient, $(\mathrm{B})_{1}$ is the concentration at the first plane and $(\mathrm{B})_{2}$ is the concentration at the catalytic plane. The rate at which $B$ is reacted if the mechanism is enzymic is:

$$
\frac{d \mathrm{~B}}{d t}=\frac{a V_{b}(\mathrm{~B})_{2}}{(\mathrm{~B})_{2}+K_{b}}
$$

since the rate will also be proportional to the area over which the reaction nccurs. In the steady state, these two rates must be equal, and $(B)_{2}$ may be 
obtained in terms of $(B)_{1}$ by equating 7-58 and 7-59 and solving for $(B)_{2}$, leading to the quadratic equation:

$$
(\mathrm{B})_{2}^{2}+\left[\frac{V_{b} d}{D}+K_{b}-(\mathrm{B})_{1}\right](\mathrm{B})_{2}-K_{b}(\mathrm{~B})_{1}=0
$$

From $(B)_{2}$ the steady-state rate of the reaction may be calculated. Inspection of Eq. 7-60 shows that the greater the distance between the planes, the less $(B)_{2}$ will be and the slower the reaction rate will be. Also the greater the diffusion coefficient, the higher $(B)_{2}$ will be and the more rapid the reaction rate; thus, in the general case, the rate of formation of $\mathrm{C}$ will be dependent upon the diffusion process, both with respect to the rate of diffusion of $\mathrm{B}$ and the distance to the reactive sites. Of course, when $K_{b}-$ $(B)_{1}$ is much larger than $V_{b} d / D$, changes in either $d$ or $D$ will not alter $(B)_{2}$

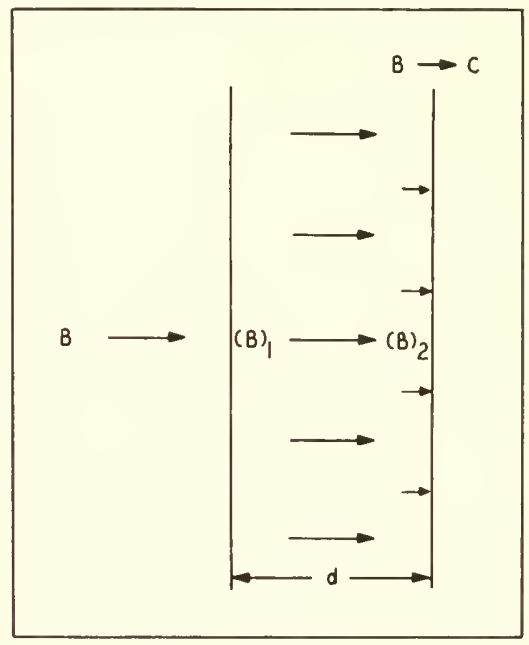

Frg. 7-37. Diagram illustrating diffusion of substance, B, from plane 1 to catalytic plane 2 where it is reacted to form $\mathrm{C}$.

or the rate appreciably. If the enzyme is inhibited, $(B)_{2}$ will rise in the general situation and comteract the inhibition to maintain the steady rate within limits; however, in almost all cases there will be some inhibition on the rate of formation of $\mathrm{C}$, although generally less than on the enzyme in the presence of constant substrate concentration. When $V_{b} d / D$ is small compared to $K_{b}-(B)_{1}$, that is, when the enzyme rate is inherently slow, the distance is small, or the diffusion is rapid, inhibition of the enzyme will produce identical inhibition in the formation of $\mathrm{C}$; these would be conditions in which diffusion is not limiting and $(B)_{2}$ is approximately equal to $(B)_{1}$. 
This system nay now be extended to the situation where $\mathrm{B}$ is being formed enzymically at a constant rate from $\mathrm{A}$ :

$$
\mathrm{A} \stackrel{\mathrm{E}_{1}}{\rightarrow} \mathrm{B}_{1} \rightleftharpoons B_{2} \stackrel{\mathrm{E}_{2}}{\rightarrow} \mathrm{C}
$$

In the steady state:

$$
\frac{V_{a}(\mathrm{~A})}{(\mathrm{A})+K_{a}}=\frac{V_{b}(\mathrm{~B})_{2}}{(\mathrm{~B})_{2}+K_{b}}
$$

from which the value of $(B)_{2}$ may be obtained in terms of $(A)$. Since the rate is also that given in Eq. 7-58, the value of $(B)_{1}$ may be calculated from $(\mathrm{B})_{2}$ :

$$
(\mathrm{B})_{1}=(\mathrm{B})_{2}\left[\frac{d}{D} \frac{\mathrm{I}_{b}}{(\mathrm{~B})_{2}}+K_{b}+1\right]
$$

It is quite possible, however, that the rate of reaction 1 may be too rapid to allow diffusion of $\mathrm{B}$ at a rate necessary to maintain reaction 2 in a steady state with reaction 1 , since, although $B$ will accumulate, in many systems $(B)_{1}$ cannot rise above a limited value. In such diffusion-limited multienzyme systems, inhibition of $\mathrm{E}_{1}$ will not affect the formation of $\mathrm{C}$ to any extent until the rate of reaction 1 has been reduced to a level comparable to the diffusion process. Inhibition of $\mathrm{E}_{2}$ will generally depress formation of $\mathrm{C}$ but the rate will progressively increase as $\mathrm{B}$ accumulates.

It is likely that in the cell, where distances between enzymes are small, diffusion is seldom a limiting factor in metabolic rates, unless the consecutive enzymes are separated by some barrier membrane slowing diffusion. However, in isolated enzyme preparations, diffusion may well be an important rate-determining process, the enzymes usually being diluted and relatively far apart. The opportunity for diffusion to become important is increased as the size of the diffusing intermediate molecule is increased. Many interenzyme diffusing molecules are quite large, such as ATP or DPNH, and in isolated preparations carriers as large as cytochrome c may be compelled to diffuse.

The diffusion law applied above (Eq. 7-58) may not be valid for systems where the distances involved are of molecular dimensions. A molecule does not diffuse in a straight line but along a complex path determined by collisions with other molecules. The average speed along a single linear path between collisions is high but the rate of diffusion is much slower due to the circuitous path taken. If the active sites of two consecutive enzymes are separated by distances of the order of $10-200 \AA$ and in a cavity where no water molecules are present, the time required for transfer of an intermediate between sites may be exceedingly brief. The mean speed of a molecule along a linear path is given by $\bar{v}=(8 R T / \pi \mathrm{M})^{1 / 2}$ and for a molecule of molecular weight of $100, \bar{v}=2.6 \times 10^{4} \mathrm{~cm} / \mathrm{sec}$ at $37.5^{\circ}$. It would thus 
require only $3.9 \times 10^{-11} \sec$ for a molecule to go $100 \AA$ on the average. Even if the molecule had to bounce around within the cavity many times before it collided appropriately with the active site. an extremely short time would be necessary and such conld not possibly exert a limiting action on the rate.

However, most instances of transfer involve diffusion through the aqueous medium and this requires displacement of water molecules, many collisions, and a nonlinear path. The time required for a mean displacement, $\bar{d}$, of a diffusing molecule is given by the approximate equation, $t=\bar{d}^{2} / 2 D$, where $D$ is the diffusion coefficient and applies to a unit concentration gradient. We may take $D=1 \times 10^{-5} \mathrm{~cm}^{2} / \mathrm{sec}$ for a molecule of molecular weight of 100. Actually a good deal of variation in $D$ occurs between molecules of the same molecular weight, because hydration of ionic groups or hydrogen bonding to the solvent water may slow diffusion significantly. Since the diffusion coefficient varies inversely with the cube root of the molecular weight, approximate values for $D$ may be assigned for any size molecule. It is also evident that the shape of a molecule will be a modifying factor in the rate of diffusion. Since here we are interested only in rough values for comparative purposes, the valnes assigned $D$ in Table 7-1 are adequate and correspond well with experimental results. The table gives the times required for molecules of various sizes to diffuse over the distances indicated. These figures may be compared with the times required to catalyze the reaction of one molecule in a multi-enzyme system. The turnover numbers of enzymes vary over a wide range but for many important enzymes occurring in sequences (such as those in glycolysis,

Table $7-1$

Transit Times of Molecules Diffusing through Aqueous Medium ${ }^{a}$

\begin{tabular}{lcccc}
\hline \multirow{2}{*}{$d .(\AA)$} & \multicolumn{4}{c}{ Transit times (msee) } \\
\cline { 2 - 6 } & Mol. wt. 100 & 500 & 10,000 & 50.000 \\
& $D: 1 \times 10^{-5}$ & $0.5-10^{-5}$ & $0.2 \times 10^{-5}$ & $0.07 \times 10^{-5}$ \\
\hline $50 \AA$ & & & & \\
$100 \AA$ & $1.25 \times 10^{-5}$ & $2.5 \times 10^{-5}$ & $6.2 \times 10^{-5}$ & $1.8 \times 10^{-4}$ \\
$1000 \AA$ & $5.10^{-5}$ & $1 \times 10^{-4}$ & $2.5 \times 10^{-4}$ & $7.1 \times 10^{-4}$ \\
$10,000 \AA(1 \mu)$ & $5 \times 10^{-3}$ & $1 \times 10^{-2}$ & $2.5 \times 10^{-2}$ & $7.1 \times 10^{-2}$ \\
$100.000 \AA(10 \mu)$ & 0.5 & 1 & 2.5 & 7.1 \\
& 50 & 100 & 250 & 710 \\
\hline
\end{tabular}

a The transit times given are the average intervals in msee reyuired for spherieal molecules of the moleeular weight indieated to diffuse the distances shown in the lefthand column. The figures are only approximate and give merely the general order of magnitude to be expeeted. 
the tricarboxylic acid cycle, electron transport, and phosphate transfer) the values generally fall between 2000 and 25,000 molecules of substrate reacting per enzyme site per minute under optimal conditions. Since the enzymes of a sequence are seldom saturated with substrate and inasmuch as the over-all rate relates particularly to the slowest step, a turnover number of around 5000 may be selected as representing an active multienzyme system. At this rate, one molecule of substrate and each intermediate is reacted per enzyme site each $12 \mathrm{msec}$. If molecules are to be transferred from enzyme to enzyme as rapidly as reacted, so that no marked accumulation occurs, the transit time must be less than this value. Table 7-1 shows that this will be the case, even with molecules of protein size, when the distance between enzymes is less than $10,000 \AA$ or $1 \mu$. Within the cell the distances would certainly be less, so that diffusion would not be limiting unless permeability barriers between the enzymes existed. In an isolated homogeneous enzyme preparation, where each enzyme of the sequence is present at $10^{-7} M$, the average distance between consecutive enzymes would be $0.25 \mu$. Thus it is unlikely that diffusion will be limiting even in these preparations unless the turnover number of an enzyme is very high and the diffusing intermediate is large, or the enzyme concentration is much less that $10^{-7} \mathrm{M}$.

\section{Compartmentalization}

The effect of restriction of multienzyme sequences to limited regions of the system on the maintenance of the steady state during inhibition has been discussed. However, there are many possible types of compartmentalization in cells and it is probable that this is often an important factor in the response to inhibition. Recent studies of cell structure indicate that there may be more compartmentalization than previously believed. The demonstration of a system of membranes within the cytoplasm (endoplasmic reticulum). the probability of two or more distinct regions in mitochondria, and the unsuspected double nature of most membranes all point to the likelihood that the cell consists of a very complex structure of different compartments separated by barriers with different properties. Evidence is accumulating that metabolic systems exist in these various regions and can, within limits, operate independently.

The subject of compartmentalization with all its ramifications is much too extensive and complex to be taken up here in any detail, but it may be useful to outline in a qualitative fashion the changes brought about by two basic types of compartmentalization (Fig. 7-38). Each of the three systems - the homogeneous (I), the single compartment (II), and the double compartment (III) - contains the same total amount of enzymes and substrate, the only difference being the restriction of the enzymes to certain regions. Two general situations may be distinguished: the compartment membranes 
either do or do not restrict diffusion sufficiently to make this a factor in the over-all rate. When diffusion is not limited, $d(\mathrm{~A}) / d t$ will be the same in each case because the spatial distribution of $\mathbf{E}_{1}$ does not affect the rate, but $d(\mathrm{C}) / d t$ is not necessarily the same. Compartmentalization may allow (B) to rise to ligher levels than in a homogeneons system and aids maintenance of the steady state in (II); however, in (III), where B must diffuse between compartments. the situation will not differ from (I). System (II) will thus be more resistant to inhibition of $\mathrm{E}_{2}$ with respect to $d(\mathrm{C}) / d t$. When the membranes suppress diffusion. $d(\mathrm{~A}) / d t$ may be reduced in (II) and (III), and the reduction of $d(\mathrm{C}) / d t$ is apt to be greater in (III). It may be pointed out that the kinetics of such systems are the same as those of the simple diffusion case treated above (Eqs. 7-58 and 7-59) with $D$ replaced

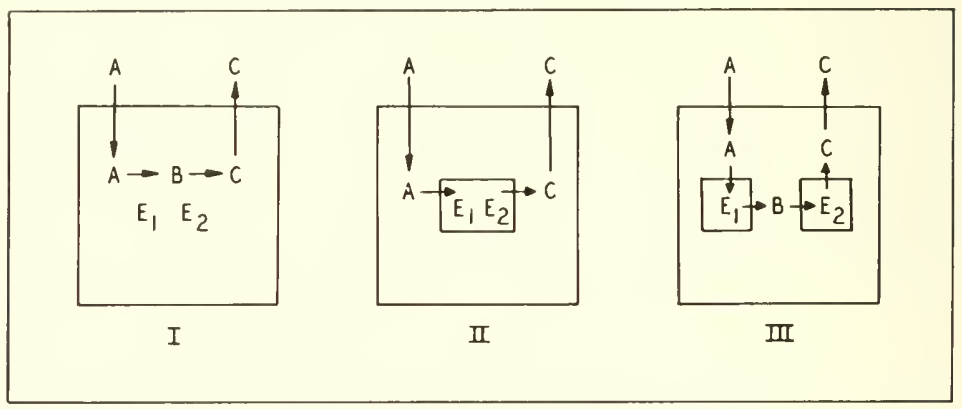

FIG. 7-38. Illustration of the two basic types of compartmentalization. System (I) is homogeneous, system (II) has a single compartment, and system (III) has two compartments.

by a permeability constant. It is evident that inhibition of enzymes in a system where permeability determines the metabolic rate will not produce an effect on this rate until the enzyme activity is rednced sufficiently so that the permeability is no longer the limiting factor. The total number of diffusion steps in a two-enzyme reaction within a cell may thus be large: five for (I), nine for (II) and thirteen for (III). One or more of these steps may be important in the rate of formation of $\mathrm{C}$.

Hearon (1949 b) and Bierman (1954) have discussed metabolic systems limited by diffusion. Bierman particularly has presented a detailed analysis of several types of systems. Unfortunately, the kinetics were derived on the basis of a linear relationship between substrate concentration and rate, and are not applicable generally to metabolic systems. The approximation, $v=V_{m}(\mathrm{~A})$, is valid only when (A) is much less than $K_{m}$; an appreciable error is introduced if $(\mathrm{A})$ is greater than $K_{m} / 10$. One may say that the steady-state concentrations of intermediates are usually low in most multienzyme systems, but it is not the concentration only, it being 
rather the specific concentration $(\mathrm{A})^{\prime}$, that is important. The over-all concentration of an intermediate in a reaction mixture may be low but the level within a compartment may be much higher also. Nevertheless, some of the general conchsions on the effects of compartmentalization brought out by Hearon and Bierman are qualitatively valid.

Holzer (1959) has pointed out well that it is difficult to determine the behavior of multienzyme systems, especially branched chains, because the intracellular concentrations of the substrates and intermediates are generally not known. The concentration of inorganic phosphate within the cell as calculated from the equilibrium reaction catalyzed by phosphorylase is much lower than that based on the analysis of total inorganic phosphate. This must be interpreted to mean that phosphate is very unequally distributed within the cell. Certainly the rates of many intracellular enzyme reactions must depend on the particular region in which the enzymes occur and on the concentrations of the involved substrates in these regions.

\section{Structural Disruption of Multienzyme Systems}

When multienzyme systems are spatially organized, either by aggregation of enzymes into complexes or localization into specific regions, inhibition of the metabolic rate may be brought abont by the inhibitor disturbing in some manner this organization rather than by a direct action on the enzyme. This possibility must always be kept in mind when interpreting the results of the action of an inhibitor on such complex systems. The types of structural disturbance to which a system is sensitive will depend on the way in which the component enzymes are organized. If the enzymes are held together in a complex, a substance conld alter the distance between active sites or actually split the complex by reacting with the enzyme protein or with some cementing material. Compounds which react avidly with protein groups, such as the sufhydryl reagents, or form hydrogen bonds readily, such as urea. or are adsorbed at water-protein interfaces, such as a detergent, would be most likely to interfere in this manner. If the consecutive enzymes interact by rotation, as has been postulated by Chance (1956) for electron transport, a substance conld hinder rotation withont necessarily splitting apart the enzymes. Restriction of rotation could be achieved sterically by simply reacting with certain protein groups. Finally, if the enzymes are compartmentalized by membranes, a substance could decrease the permeability to the substrate or intermediates, or possibly disrupt the membrane so that organization and the steady state are abolished. In these and other ways, nonenzymic effects (that is, those that would not be observed with the isolated enzyme) may depress sequential reactions in cells and some of the effects observed in mitochondrial suspensions may be attributable to changes in the surrounding membrane, leading to loss of coenzymes and disorganization of structure from swelling. 


\section{The Concept of the Limiting Rate}

The belief that in any type of sequential reaction there is a step whose rate is slower than that of any other step and hence is limiting the over-all rate has been very common amongst biochemists and biologists for many years. It has been stated in various ways that the over-all kinetics will depend only on those of the limiting step, that effects upon the system will be observed only when the limiting step is altered, that indeed everything from growth rates to temperature coefficients depend predominantly on some limiting step. It is a dangerous concept, unless properly applied, because it is one of the primary means of introducing simplification into a complex system, which, although a laudable goal. is to be deplored if the assumption is incorrect. Warnings have been issued: e.g.. Greenberg (1956) stated "It is apparent ... that in a sequence of enzyme reactions the step with the lowest velocity at saturation with its substrate may not be the limiting reaction in a steady state," and Hinshelwood (1946) emphasized that "The velocity of a series of consecutive reactions is certainly not governed in general by that of the slowest." It is important to understand when this concept is valid and when it is merely an unjustified simplification.

One of the sonrces of confusion is the failure to differentiate between the inherent or potential rate of an enzyme and its actual rate under the experimental conditions. If each enzyme of a multienzyme system is isolated and its maximal rate determined, it is not necessary when these enzymes are operating seqnentially that the enzyme with the lowest maximal late will be limiting, because the concentrations of the initial substrate and the intermediates will determine to a great extent the over-all rate and which enzyme might be considered to be limiting. Thus the first enzyme in a monolinear chain will often be limiting, whatever its potential rate, due to a low concentration of its substrate, and likewise the inability of a particular intermediate to rise sufficiently may impose limitation on an active enzyme. The presence of compartmentalization will thus determine in some cases what step is rate-controlling.

Another difficulty in thinking about limiting rates has been the lack of a quantitative expression for the degree to which a particular step is controlling the formation of the product. The response to enzyme inhibition often depends markedly upon this and thus a simple approach to a quantitative formulation will be presented. Let us assume that in the monolinear chain. $\mathrm{A} \rightarrow \mathrm{B} \rightarrow \mathrm{C}$, the concentration of $\mathrm{A}$ is such that reaction 1 is proceeding at a rate greater than the maximal rate of reaction $2, V_{2}$. It is evident that $\mathrm{E}_{1}$ must be inhibited so that $v_{1}$ falls below $V_{2}$ before any depression of $v_{2}$ occurs. The initial $v_{1}=V_{1}(\mathrm{~A}) /\left[(\mathrm{A})+K_{1}\right]$ and this must be 
brought down to $V_{2}$ to inhibit reaction 2 : the degree of inhibition to reduce $v_{1}$ just to $V_{2}$ is then:

$$
i_{0}=1-\frac{V_{2}}{V_{1}^{-}}\left[1+\frac{K_{1}}{(\mathrm{~A})}\right]=1-\frac{V_{2}}{v_{1}}
$$

The limiting rate coefficient for reaction 2 may be defined as $V_{2} / v_{1}$ and designated by $L_{2}$. Thus:

$$
i_{0}=1-L_{2}
$$

When $L_{2}$ is unity or greater, reaction 2 is not limiting and inhibition of $E_{1}$ will always depress $v_{2}$; when $L_{2}$ is less than unity. a certain amount of inhibition, $i_{0}$, must be exerted on $\mathrm{E}_{1}$ before $v_{2}$ is altered, and the smaller $L_{2}$ is, the greater must this inhibition be. We may next inquire into the inhibition exerted on $v_{2}$ by any inhibition of $v_{1}$. The inhibition of $v_{1}$ is $i_{1}$ and the consequent inhibition of $i_{2} i_{2} i_{2}$ and the relation between them is:

$$
i_{2}=\frac{i_{1}-i_{0}}{1-i_{0}}
$$

which can be shown to reduce to:

$$
i_{2}=1-\frac{1-i_{1}}{L_{2}}
$$

after substitution from Eq. 7-65. The inhibition of $v_{2}$, or the formation of product C. is thus relater quantitatively to the limiting rate coefficient $L_{2}$. This may be extended to a chain of any number of steps and to primary inhibition of any nonlimiting reaction in that sequence:

$$
\begin{gathered}
i_{0}=1-L_{j} \\
i_{j}=1-\frac{1-i_{k}}{L_{j}}
\end{gathered}
$$

where $j$ refers to the step that is limiting and $k$ to the step that is acted upon by the inhibitor. $L_{j}$ would be given by $V_{j} / v_{k}$. In certain cases, $i_{0}$ might be greater than indicated by Eq. $7-68$ because the concentration of the intermediate may not be able to rise to a level saturating the enzyme, so that $i_{0}$ is actually the minimal degree of inhibition that must be exerted before the formation of product is depressed.

This simple formulation indicates the danger inherent in such statements as "the over-all rate can be depressed only by inhibition on the reaction that is limiting." for it will depend on how great the inhibition is and the degree to which the reaction is limiting. that is. on $i_{k}$ and $L_{j}$. If $L_{j}$ is not much less than unity, although reaction $j$ is truly limiting the rate of formation of the product, inhibition on another enzyme may quite readily depress the over-all rate. Determination of limiting rate coefficients is 
not difficult if the enzymes can be isolated or studied independently and they would provide a better understanding of many multienzyme systems.

When more complex multienzyme systems are considered, it is clear that the concept of limiting rate is even more vague and often inapplicable. In cyclic or regenerative systems, although one step may be said to be more important in controlling the rate than any other step, no one step can be the sole determinant, as indicated by the fact that inhibition on any step will depress the over-all rate to some extent. Furthermore, occasionally the controlling role must be attributed to more than one reaction. For example, under certain circumstances it is believed that the triose-P dehydrogenase step is the limiting reaction in glycolysis. Since this may be so because of a low concentration of ADP, the formation of pyruvate of lactate may be said to be limited by some reaction forming ADP from ATP; inhibition of either the triose-P dehydrogenase or the ATPase will depress glycolysis. In very complex metabolic systems there may be several reactions sensitive to inhibition with respect to the rate of formation of some product.

The danger of assuming a certain reaction step to be limiting a complex metabolic process is illustrated in the fine study of metabolic regulatory mechanisms by $W_{n}$ (1959). Although the rate of lactate formation in HeLa cells was the same under either aerobic or anaerobic conditions, the lactate formed from endogenons carbohydrate showed a pronounced Pasteur effect. It was demonstrated that the rate-limiting step in endogenous glycogenolysis is glncosan phosphorylase. On the other hand, the conversion of glucose to lactate in cells grown in horse serum appears to be limited by phosphofructokinase, whereas in cells grown in hmman serum, the limiting factor is the inorganic phosphate entering into the phosphoglyceraldehyde dehydrogenase reaction. Thus the same cells can show at least three different rate-limiting steps under various conditions. Such results make the assignment of liniting steps in the cells or tissue being studied, based only on work with other types of cells or different species, completely unreliable.

Within the cell it is probable that the evolution of metabolic pathways has resulted in systems in which the component reactions are balanced so that no one step is predominantly limiting, at least under all conditions. The varions processes controlling metabolic rates. such as adaptive enzyme formation, also tend to lessen the occurrence of markedly limiting reactions. In any event, one is on safer ground in assuming the absence of limiting reactions until they are proved to occur, and even then it is well to remember that a limiting state may exist only within a certain range of conditions.

\section{Determination of Substrate and Inhibitor Constants}

Determination of the constants, $K_{m}$ and $K_{i}$, by reciprocal plotting or related methods will often not be possible in multienzyme systems. The variation in rate with either (S) or (I) has been shown above to deviate 
appreciably from that in single enzyme systems. It is even impossible in many cases to establish the type of inhibition - competititive or noncompetitive - in multienzyme systems, becanse the special kinetics of these systems tend to obscure the behavior of the single enzyme. A ralid determination of $K_{i}$ in a multienzyme system depends on the assumption that the step inhibited is truly limiting the rate measured under the conditions of the experiment, and in the previous section the dangers of such assumptions have been pointed out. Even the simple equating of $K_{i}$ to $(\mathrm{I})_{i=\mathbf{0 . 5}}$ for noncompetitive inhibition is generally unjustified in multienzyme systems.

\section{TRANSITION BETWEEN STEADY STATES}

Inhibition of an enzyme in a reaction sequence may induce the system to change into a new steady state. The transition from one steady state to another does not occur instantaneously because a certain interval of time is required for the concentrations of the intermediates to reach their new values. This interval will be called the transition time. Since this depends

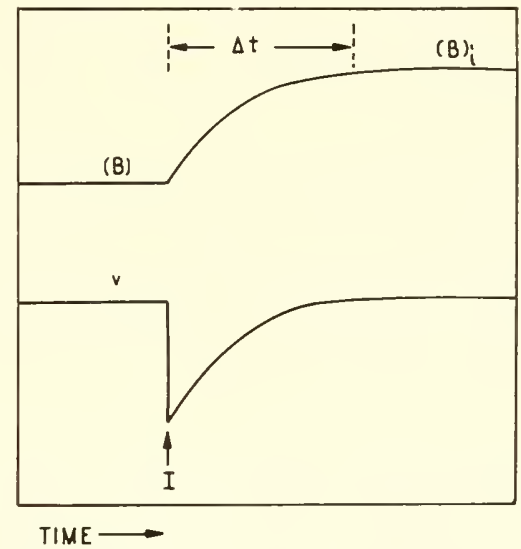

FIG. 7-39. Changes in the rate and concentration of intermediate, $\mathrm{B}$, with time following inhibition of $\mathrm{E}_{2}$ in a monolinear chain, $\mathrm{A} \rightarrow \mathrm{B} \rightarrow \mathrm{C}$. The interval, $\Delta t$ required for the steady state to be resumed is the transition time. The arrow at I indicates the addition of the inhibitor.

on the rate of change of intermediate concentration, it will be determined by the relative rates of the reactions, the degree of inhibition, and the physical state of the system. The situation for the simple monolinear chain, $\mathrm{A} \rightarrow \mathrm{B} \rightarrow \mathrm{C}$, is shown in Fig. 7-39, where inhibition of $\mathrm{E}_{2}$ requires a rise in (B) for the steady state to be maintained. The importance of this in multienzyme systems was first pointed out by Burton (1939). If the tran- 
sition time is long, it may lead to a changing rate during measurement and erroneous conclusions about the kinetics of the system. In the cell, a prolonged transition time may make possible a marked effect on the cell by the inhibition and can even secondarily prevent the re-establishment of the new steady state. It is thus of some interest to look into the methods for estimation of the transition time and present the factors upon which it depends.

Calculation of the transition time for inhibition in a monolinear chain requires an expression for the time necessary for $(B)$, the initial concentration in the uninhibited steady state, to rise to $(B)_{i}$. the concentration that makes it possible for the steady state to be resumed in the inhibited system. It is clear that $d(\mathbf{B}) / d t=v_{1}-v_{2}$ and becomes zero when the rates are equal. The exact solution requires the integration of:

$$
\frac{d(\mathrm{~B})}{d t}=\frac{V_{1}(\mathrm{~A})}{(\mathrm{A})+K_{1}}-\frac{(1-i) V_{2}(\mathrm{~B})}{(\mathrm{B})+K_{2}}
$$

between $(\mathrm{B})$ and $(\mathrm{B})_{i}$, which gives for the transition time:

$$
\Delta t=\frac{(\mathrm{B})_{i}-(\mathrm{B})}{v_{1}-(1-i) V_{2}}-\frac{(1-i) V_{2} K_{2}}{\left[v_{1}-(1-i) V_{2}\right]^{2}} \ln \frac{v_{1} K_{2}+\left[v_{1}-(1-i) V_{2}\right](\mathrm{B})_{i}}{v_{1} K_{2}+\left[v_{1}-(1-i) V_{2}\right](\mathrm{B})}
$$

where $v_{1}=(\mathrm{A}) V_{1} /\left[(\mathrm{A})+K_{1}\right]$, the rate of reaction 1 . Since substitution of the expression for $(\mathrm{B})_{i}$ [from Eq. $7-3$ with the maximal rate as $(1-i) V_{2}$ for noncompetitive inhibition] leads to a value of zero for the numerator of the fraction after $\ln$, we see that theoretically it requires infinite time to achieve a new steady state. Thus it will be convenient to calculate the transition time for (B) to rise $90 \%$ of its way to the fural steady state $(B)_{i}$. This is given by:

$$
\Delta t_{0.9}=\frac{0.9\left[(\mathrm{~B})_{i}-(\mathrm{B})\right]}{v_{1}-(1-i) V_{2}}+\frac{2.3(1-i) V_{2} K_{2}}{\left[u_{1}-(1-i) V_{2}\right]^{2}}
$$

It is interesting that the logarithmic term is unity under these specified conditions and depends only on the assumed percentage rise towards $(B)_{i}$. Although this is not the full transition time, it provides a practical measure of the rate of change from one steady state to another.

The variation of transition time with the degree of inhibition in a typical monolinear chain is shown in Table $7-2$. As expected there is a rapid rise in $\Delta t_{0.9}$ as the inhibition approaches completeness. The units of $\Delta t_{0.9}$ will depend on those taken for $v_{1}$ and $v_{2}$; for example, if the rates are in moles/minute. (B) and $(\mathrm{B})_{i}$ must be in molar concentration and $\Delta t_{0.9}$ will be expressed in minutes. The last column in Table 7-2 gives transition times 
calculated from a simplified equation based on the assumption that the concentration of the intermediate is so low that $v_{2}$ is proportional to (B). The error introduced by this assumption is seen to be appreciable, especially at high inhibitions.

TABLE $7-2$

Variation of Transition Time with Inhibition ${ }^{a}$

\begin{tabular}{|c|c|c|c|c|c|}
\hline $\begin{array}{l}\text { Inhibition } \\
\text { (i) }\end{array}$ & $\begin{array}{c}\text { (B) } \\
(m, I)\end{array}$ & $\begin{array}{r}(\mathrm{B})_{i} \\
(\mathrm{~m} M)\end{array}$ & $\begin{array}{c}(\mathrm{B})_{i}-(\mathrm{B}) \\
(\mathrm{m} L)\end{array}$ & $\begin{array}{c}\text { Exact } \\
\Delta t_{0.9} \\
\text { (Eq. } 7-72)\end{array}$ & $\begin{array}{c}\text { Approx. } \\
\Delta t_{0.9} \\
\text { (Eq. } 7-74)\end{array}$ \\
\hline 0.1 & 0.238 & 0.266 & 0.028 & 0.75 & 0.65 \\
\hline 0.25 & 0.238 & 0.323 & 0.085 & 2.32 & 1.95 \\
\hline 0.5 & 0.238 & 0.500 & 0.262 & 7.38 & 5.75 \\
\hline 0.75 & 0.238 & 1.11 & 0.872 & 25.7 & 17.3 \\
\hline 0.9 & 0.238 & 4.16 & 3.922 & 147.1 & 51.8 \\
\hline
\end{tabular}

a Noncompetitive inhibition of $\mathrm{E}_{2}$. The transition times have been corrected for the zero inhibition artifact resulting from the assumption that (B) rises only $90 \%$ of the way to $(B)_{i}$, which, of course, cannot be applied to the uninhibited system. $V_{1}=1, V_{2}=2, K_{1}=10 \mathrm{~m} M, K_{2}=5 \mathrm{~m} . H$, and $(\mathrm{A})=1 \mathrm{~m} . H$.

When the intermediate concentration is low so that $(\mathrm{B}) \ll K_{2}$, the integrated expression for the transition time is:

$$
\Delta t=\frac{K_{2}}{(1-i) V_{2}} \ln \frac{v_{1}-v_{2}}{v_{1}-\left(v_{2}\right)_{i}}
$$

where $v_{2}$ is the unimhibited rate and $\left(v_{2}\right)_{i}$ the inhibited rate. Substitution of $(\mathrm{B})$ and $(\mathrm{B})_{i}$ and making the same assumption as previously, that $(\mathrm{B})$ rises $90 \%$ of the way to $(\mathrm{B})_{i}$, gives:

$$
\Delta t_{0.9}=\frac{2.3 K_{2}}{(1-i) V_{2}}
$$

In the particular example chosen. (B) is about one-twentieth of $K_{2}$, so that the approximation is not too inaccurate until the inhibition on $\mathrm{E}_{2}$ requires a relatively high $(\mathrm{B})_{1}$ to maintain the rate.

Variation of the transition time with (A) is shown in Table 7-3. One might expect that the higher $(A)$ and the faster reaction 1 , the less would be the transition time, but the opposite is seen to occur. The reason for this is 
that $(B)_{i}-(B)$ is much greater when $(A)$ is high; thus $(B)$ must rise more and reaction 2 is correspondingly increased in rate. There is also an increase in $\Delta t_{0.9}$ when $V_{1}$ is increased relative to $V_{2}$.

TABLE $7-3$

Variation of Transition Time with Substrate Concentration ${ }^{a}$

\begin{tabular}{|c|c|c|c|c|c|}
\hline $\begin{array}{l}(\mathrm{A}) \\
(\mathrm{m} H)\end{array}$ & $v_{1}$ & $\begin{array}{c}(\mathrm{B}) \\
(\mathrm{m}, H)\end{array}$ & $\begin{array}{c}(\mathrm{B})_{i} \\
(\mathrm{~m} H)\end{array}$ & $\begin{array}{c}(\mathrm{B})_{i}-(\mathrm{B}) \\
(\mathrm{m}, W)\end{array}$ & $\Delta t_{0.0}$ \\
\hline 0.1 & 0.010 & 0.025 & 0.05 & 0.025 & 5.94 \\
\hline 0.4 & 0.038 & 0.098 & 0.20 & 0.102 & 6.39 \\
\hline 1 & 0.091 & 0.238 & 0.50 & 0.262 & 7.40 \\
\hline 4 & 0.286 & 0.833 & 2.00 & 1.167 & 13.25 \\
\hline 10 & 0.500 & 1.667 & 5.00 & 3.333 & 29.8 \\
\hline 40 & 0.800 & 3.333 & 20.00 & 16.667 & 196 \\
\hline
\end{tabular}

${ }^{a}$ Noncompetitive inhibition of $\mathrm{E}_{2}$ with $i=0.5$. Constants as in Table $7 \cdot 2$.

Finally, one may inquire into the change in transition time when both $V_{1}$ and $V_{2}$ are increased or decreased equally, $V_{1} / V_{2}$ remaining constant It may be shown that $(B)$ and $(B)_{i}$ remain the same, since they depend only on $V_{1} / V_{2}$, and that if $V_{1}$ and $V_{2}$ are changed $x$-fold. $y_{0,9}$ will be changed by a factor of $1 / x$, inasmuch as the denominators in both terms of Eq. 7-72 will be multiplied by $x$. Hence, as expected, the more rapid the rates of the enzymes composing a monolinear chain. the shorter will be the time necessary to change from one steady state to another.

\section{Fluctuation and Overshoot}

In more complex multienzyme systems, the transition between steady states may not follow the simple logarithmic course. When, following inhibition, the rate falls to a level below the final steady state and then rises to it, the time variation of the rate may not be as simple as previously described. The rate may not drop suddenly as in Fig. 7-39. In the threestep monolinear chain, $\mathrm{A} \rightarrow \mathrm{B} \rightarrow \mathrm{C} \rightarrow \mathrm{D}$, inhibition of $\mathrm{E}_{2}$ will usually not depress $d(\mathrm{D}) / d t$ immediately because it will require a finite time for (C) to decrease. When there are more steps past the point of inhibition or when the system is properly compartmentalized, there may be a lag period before any effect on $d(D) / d t$ can be measured. Simultaneonsly, (B) will increase and the drop in (C) will be progressively counteracted until the final steady 
state is reached. The transition time, as defined above, will not be altered but the rate during this period may never reach as low a level as the inhibition on $\mathrm{E}_{2}$ would imply. Such behavior is illustrated in Fig. 7-40.

Deviating behavior of the concentration of intermediate may be described in the terminology of Denbigh et al. (1948). When the initial change in an intermediate concentration exceeds that of the final steady-state

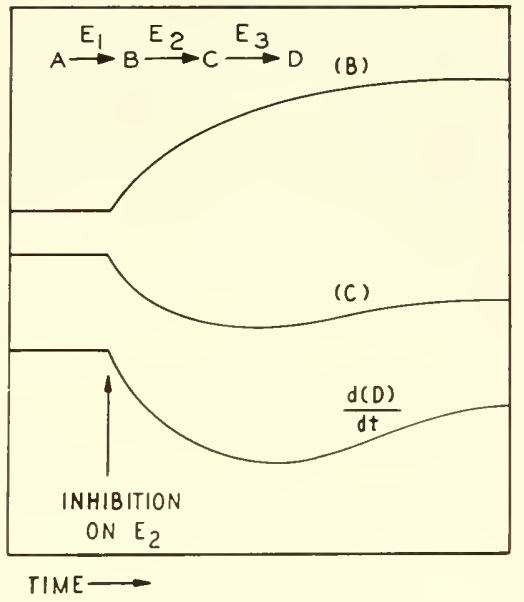

FIG. 7-40. Illustration of the changes in the concentrations of the intermediates and in the rate of formation of $\mathrm{D}$ in a monolinear chain following inhibition of $\mathrm{E}_{2}$.

concentration, the system may be said to exhibit overshoot (Fig. $7-41 \mathrm{~A}$ ) and if the concentration oscillates before reaching a final level, such behavior may be termed fluctuation (Fig. $i-41 B$ ). In some systems the concentration may initially change in an opposite direction to its final change and the system is said to make a false start (Fig. 7-41C). Although firstorder kinetics were assumed (Denbigh et $a l ., 1948$ ), the treatment is qualitatively applicable to enzyme reactions. The rate of formation of product may or may not follow such changes in intermediate concentration but the same terminology may be applied to rate changes. The origin of these phenomena is to be sought in the varying rates at which the different steps in a multienzyme system can adapt to inhibition. The greater the complexity of the system, the more oportunity there is for these deviations. Cyclic, regenerative, and feedback systems are particularly prone to overshoot and fluctuation. Such behavior may be of importance not only in kinetic measurements of inhibition but may be reflected in the functional response of cells and tissues to an inhibitor. A simple specific inhibition may thus 
induce a complex pattern of response when the metabolic system involved is composed of multiple interdependent reactions.

Although enzyme systems are much more complex, the simple formulation for first-order kinetics (Denbigh et al., 1948) is useful in expressing these complex changes in mathematical form After perturbation of the system, the concentration of any intermediate may be written as:

$$
(\mathrm{B})=(\mathrm{B})_{0}+\alpha_{1} e^{-\beta_{1} t}+\alpha_{2} e^{-\beta_{2} t}+\ldots+\alpha_{n} e^{-\beta_{n} t}
$$

where $(\mathrm{B})_{0}$ is the initial steady-state concentration The constants, $\beta_{1} \ldots \beta_{n}$, depend only on the kinetic parameters of the enzymes but $\alpha_{1} . . \alpha_{n}$ may depend in addition on the intermediate concentration. Each exponential term gives the effect of one component of the system on (B). The $\alpha$ 's may

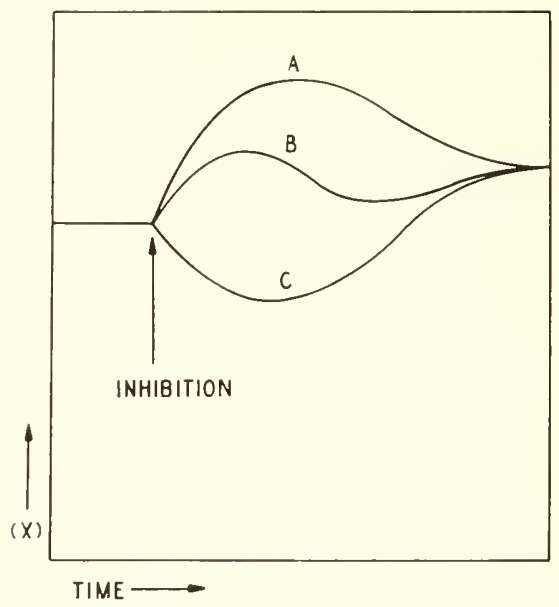

FIG. 7-41. Illustration of overshoot (curve A), fluctuation (curve B), and false start (curve C) following inhibition in a multienzyme system. (X) represents the concentration of any intermediate.

be either positive or negative depending on whether the effect is to increase or decrease (B). Various combinations of values of the $\alpha$ 's and $\beta$ 's will give all the possible types of overshoot. false start, and fluctuation. When multienzyme systems are more rigorously characterized, the evaluation of these parameters, or the more complex ones resulting from enzyme kinetics, may be worthwhile but at the present would be premature. 


\section{Examples of Transition between Steady States}

In most instances it is difficult to determine the transition time or the exact pattern of change with time, not only because such changes are often very rapid, but also due to the fact that the rate at which the inhibitor exerts its effect is generally unknown. If the inhibitor acts slowly, due to either permeability or kinetic factors, the meaning of transition time loses its significance. However, there are in the literature many reports of the establishment of a new steady state that is certainly slower than the rate at which inhibition is induced, although exact determinations of these rates are uncommon. Perhaps the best demonstration of the time variation in intermediate concentrations is provided by the work of Chance (Chance, 1954; Chance and Williams, 1956) on the regenerative type electron-transport systems, where the state of the electron acceptors fluctuates characteristically following inhibition. It is probable that adaptive phenomena are often examples of such a transition, expressed at the cellular level in functional or growth changes. Hinshelwood (1946) has interpreted bacterial adaptation to inhibitors, such as proflavine, in terms of steady-state processes, wherein, however, the concentrations of enzymes may also change. The best examples of overshoot are not from inhibitor studies but in the rapid changes resulting from a sudden increase in pressure. The rate of beating of frog heart cells in tissue culture (Landan and Marsland, 1952) and of Mytilus gill cilia (Pease and Kitching, 1939) is increased by a sudden pressure rise but rapidly returns to normal; in the former case. pressures of 10,000 psi can be achieved in steps with the rate returning to normal in each case, and this can best be understood in terms of transitions between steady states, even though we are ignorant of the fundamental reactions that are involved. A similar example, more directly related to enzyme systems, would be bioluminescence: a high intensity "flash" is caused by a sudden rise in pressure while a "black-ont" results from rapid pressure release (Fig. 7-42) (Brown et al., 1942). There is a need for a more quantitative kinetic approach to the responses of enzyme systems and cells to inhibitors because the over-all effects of inhibition must depend in many cases on the rates at which metabolic activity is altered and new steady states reached. A common example of metabolic transition is the Pasteur reaction and useful information as to the exact mechanism might be provided by determinations of the rates at which glycolysis changes in response to $\mathrm{pO}_{2}$ alterations or the action of cyanide. Such an approach has been initiated in the excellent work of Lynen (1958), where the time variation of the hexosephosphates, adenine nucleotides, and inorganic phosphate following inhibition by cyanide and 2,4-dinitrophenol in yeast has been determined and the establishment of new steady states demonstrated. The oxygen uptake and ATP levels of Chlorella vary in a very interesting fashion when glucose is suddenly added or the metabolic conditions are 
changed in various ways (Syrett, 1958). Similar results have been obtained in yeast (Holzer, 1958). The phenomenon of overshoot is clearly seen and in all cases the final steady-state rates are achieved only after eircuitous pathways. Such studies, with or without inhibitors, are frequently valuable in determining whether certain cellular processes are related or not; much more can be found out about this from the variations of these parameters with time than from the final levels reached.

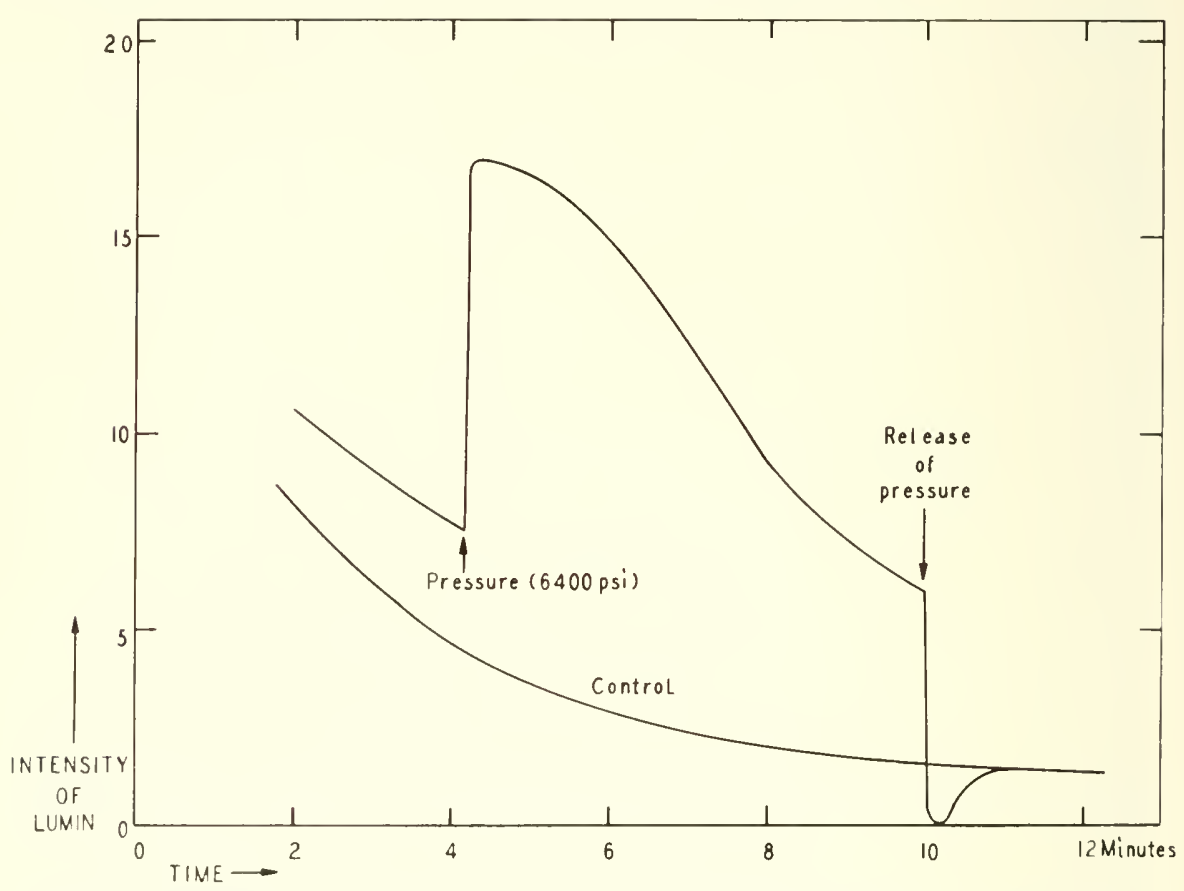

FIG. 7-42. Example of transition between steady states. The effects of sudden high pressure and the release of this pressure on bioluminescence. A "flash " results from an increase in the pressure and a "blackout" from the drop in the pressure. (From Brown et al., 1942.)

\section{Metabolic Transitions within Cells}

A number of factors may influence transition rates in the complex system of the cell. Some of these will be mentioned here and they will be taken up in greater detail in the following chapter. We have assumed that the concentrations of the various enzymes in the multienzyme system remain constant in the treatment above, but in the cell this is not necessarily so, since the ability of the enzyme-controlling systems to adlapt to changes in substrate concentration is well known. This may lead to very long tran- 
sition times because the loss or synthesis of the enzymes is usually not a rapid process; transition times of several hours might be expected not to be uncommon.

Another important factor is compartmentalization of all or parts of the multienzyme system. This may either accelerate or slow the transition between steady states following inhibition. If the establishing of a new steady state involves only the simple rise or fall in the concentration of some intermediate, this will proceed much more rapidly when this occurs in localized regions than in a homogeneous state. On the other hand, when diffusion is an important factor, especially in situations where membranes surround the compartments [as in Figure 7-38 (III)], the rate of achievement of the new steady state may be impeded.

Lastly, a wide variety of secondary reactions and effects may occur in the cell that will modify the behavior of the system studied. The accumulation of some intermediate may exert an action that in its usual low conconcentration is negligible; the possible effect of citrate as it accumulates during the block by fluoroacetate might be cited. The disturbance in the metabolism may alter the $\mathrm{pH}$, either locally or generally, and this may exert a number of effects on the rate at which a steady state can be reached. Structural and permeability changes may further influence these processes. It is only to emphasize the complexity of the situation that these factors are mentioned. One must, as always, exercise great caution in applying the results of steady-state studies on isolated enzyme systems to the intact living cell. And yet it is necessary to surmount the difficulties eventually because the rates at which transitions between states in cells occur is of primary importance in the metabolism and functioning of the cell, not only in inhibition of enzymes but in the responses of the cell to all stimuli.

\section{THE FUTURE OF THE STUDY OF MULTIENZYME SYSTEMS}

It is perhaps pertinent at this point to indicate the state of our present knowledge and predict some of the ways in which progress in this field may occur, because it is likely that biochemistry now is itself in a state of transition between simple enzyme kinetics and the application of this material to the complex metabolic pathways present in the cell. We are, with respect to multienzyme systems, in a perior that wonld be comparable to the earliest phases in the study of single enzymes. Advances can be made by the simultaneons development of equations and laws to express the behavior of multienzyme systems and by quantitative kinetic investigation of the systems experimentally, both isolated and in the cell. Some may even object to the relatively simple mathematical treatment given previously in this chapter, on the basis that our knowledge of such systems is as yet too inadequate, but it is only upon such a preliminary approach 
that the experimental data may be rationally obtained. Only the experimental results will demonstrate whether the treatment is correct or point out the modifications that must be made in it. The progress made in single enzyme kinetics, where theory and experiment for the past several decades have gone hand in hand, will be duplicated in these more complex systems. It is hoped that simple mathematical treatments will often indicate what crucial experiments are to be done and, particularly, what is to be measured. The techniques required for this will arise in response to the necessity for more accurate characterization of these systems. 


\section{DISTRIBUTION AND FATE OF INHIBITORS IN LIVING ORGANISMS}

The subject of multienzyme systems leads logically to the problems of inhibition within cells and whole animals. However, the action of an inhibitor in a complex living system depends on the concentrations of the inhibitor in the various regions in which the susceptible enzyme is located. The present chapter, therefore, will discuss briefly the distributions of inhibitors within living organisms and the ultimate fate of these inhibitors as they are chemically altered or excreted. When an inhibitor is introduced into a system of cells (whether a multicellular plant or animal, isolated organ, tissue slice, or cell suspension), the inhibitor concentration in each type of cell and in each region within the cells rises and falls with characteristic time variations that are best expressed by a family of concentration-time curves. The individual curves may differ in several respects: the rate of rise to the maximal concentration and the rate of loss of the inhibitor. the maximal level reached, and the over-all configuration of the curve. The primary aim will be to present some of the factors that determine the nature of such curves.

The most difficult correlations in the study of inhibition are those one wishes to make between the primary action of an inhibitor on an enzyme and the effects that are exerted on whole animals when the inhibitor is introduced into the systemic circulation. One of the most important factors is the distribution of the inhibitor and without knowledge of this an accurate correlation between fundamental and final actions is impossible. It must be admitted that this is one aspect of inhibition which has not been intensively studied and unfortmately in most instances we lack quantitative information on the behavior of inhibitors in organisms. Nevertheless, an appreciation of the factors that are involved can be useful and may eventually stimulate accurate experimental investigation. The importance of this subject becomes greater each year as the possibility of using specific inhibitors in disease becomes more evident. The specificity of action on invasive or abnormal cells depends on the characteristic distribution of the inhibitor as well as the specificity of the primary enzyme attack. 


\section{DISTRIBUTION OF INHIBITORS IN ANIMALS}

The relative concentrations of inhibitor in the various tissues depend on several factors but before discussing these it is necessary to present some examples of inhibitor distribution to establish clearly the fact of differential patterns of concentration. The many recent studies of drug distribution in the body have demonstrated (1) that drugs are essentially never homogeneously partitioned between the body tissues and (2) that marked differences in distribution are observed for drugs of different types. These two basic findings undoubtedly apply equally to inhibitors. One must always anticipate, in the absence of experimental data, that an inhibitor will be selectively picked up by certain tissues and rejected by others. It will also be clear that prediction of the distribution of an inhibitor must be highy speculative since the behavior of the substance in the body depends strongly on the physical and chemical characteristics of the molecule. We know virtually nothing of the distributions of the most commonly used inhibitors and for examples in this chapter it will be necessary to refer to results on drugs whose mechanism of action is likely to be enzyme inhibition. Table $8-1$ shows the distribution of certain arbitrarily chosen substances in various animals in both absolute amounts and relative to the concentrations in plasma. Unfortunately, all the inhibitors for which adequate data are available are those reacting with or bound tightly to tissue, and thus the results on these should not be taken to represent inhibitors in general. The purpose in presenting this table is to illustrate the marked differences in concentrations in the various tissues for any one inhibitor and between the inhibitor's for any one tissue; species variation is demonstrated in the results with Paludrine.

The methods of expressing tissue inhibitor concentration call for some comment at this point. In Table $8-1$ all data are given in terms of wet weight of tissue and one may very well question the significance of the ratios to the concentration in plasma (which is about $8.7 \%$ solids compared to $18-25 \%$ solids in most tissues). Especially in the case of inhibitors which react with or are bound to proteins, the expression of concentrations in terms of dry weight may well be preferable, since insignificant amounts of the inhibitors would be present in the fluid component. In this case the ratios would be roughly one-tenth those shown in the table. The units of concentration chosen should depend on the type of inhibitor and the use to which the values are put. Ideally, one wonld often wish to know the actual concentration of inhibitor acting on the cellular enzymes during the development of inhibition. The closest approximation to this might be analyses made soon after application of the inhibitor and the expression of the results in terms of intracellular water. The data in Table 8-1 show differential uptake but actually are not particularly pertinent to the subject of enzyme inhibition for at least two reasons. In the first place, over-all tissue con- 


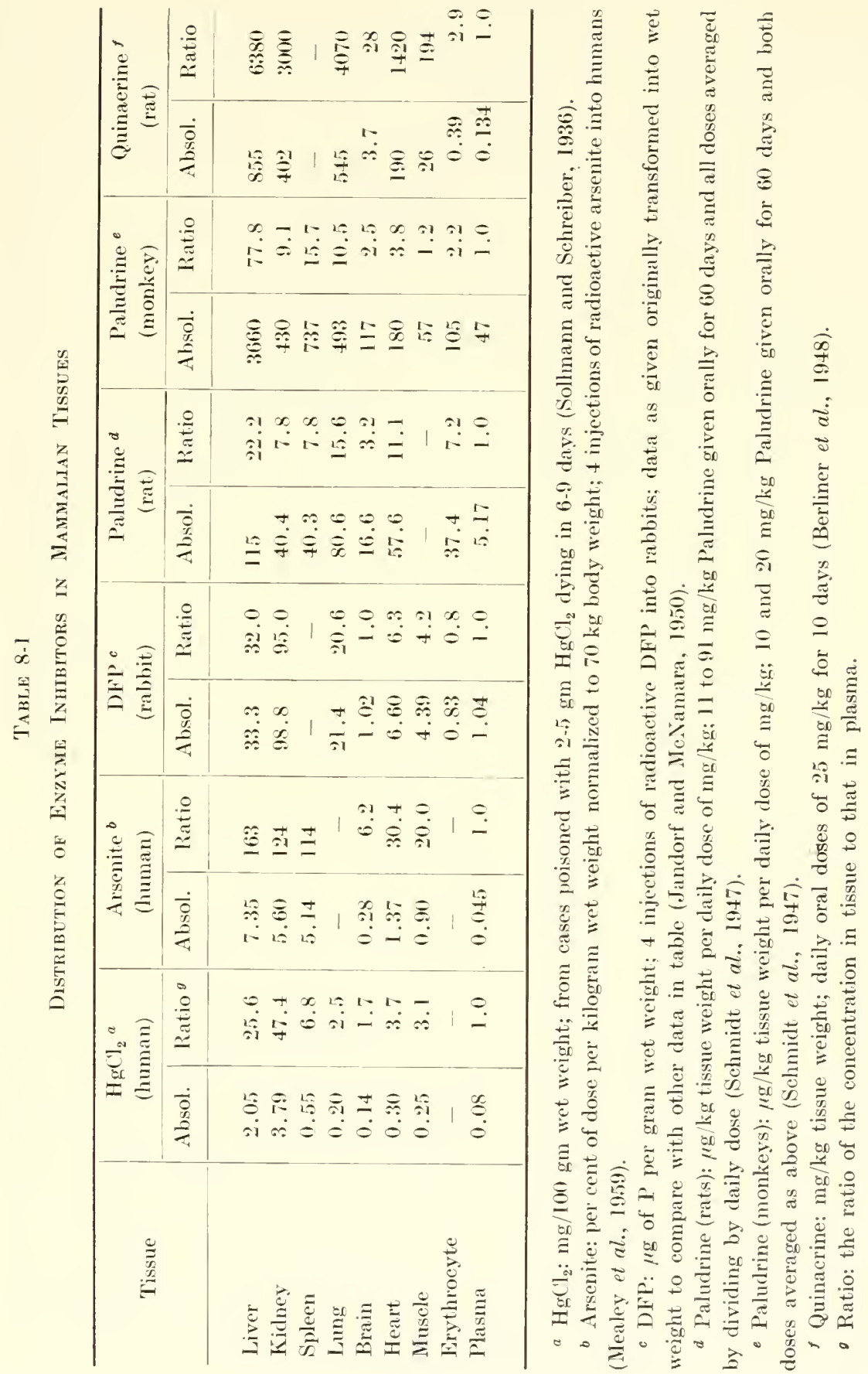


centrations do not reflect the probably marked variations in concentration between different cells of a tissue or different parts of a cell; in other words, the concentration of inhibitor in the enzyme region may be quite unrelated to the results of gross analysis. In the second place, most of the inhibitor present after long exposures is not combined with enyzme but with many other components of the cells. The figures are really not concentrations in the sense in which (I) is used in kinetic equations. The reason a high tissue uptake of an inhibitor occurs may be unrelated to any enzymic or metabolic factors; cells containing much high-sulfhydryl protein might well accumulate inhibitors such as arsenite and yet the concentration of inhibitor acting on a particular enzyme would be the same, or even less, than in other cells. To illustrate this in another way, the kidney/plasma concentration ratio for $\mathrm{Hg}^{203}$, administered to rats as the diuretic chlormerodrin, increases steadily: 53 at 1-2 hr, 351 at $3-6 \mathrm{hr}, 470$ at $12 \mathrm{hr}$, and 975 at 18-24 hr (Giebisch and Dorman 1958). This does not imply that the concentration of mercurial, active against enzymes, has risen during this

TABLE 8-2

Distribution of Enzyme Inimbitors in tile ('entral Nervous System

\begin{tabular}{|c|c|c|c|c|c|c|c|c|}
\hline \multirow[t]{2}{*}{ Region } & \multicolumn{3}{|c|}{$\begin{array}{c}\text { Isoniazid-('14 } a \\
\text { (counts/mg/min) }\end{array}$} & \multicolumn{3}{|c|}{$\begin{array}{c}\text { Diamox-\$35 } \\
\text { (counts/mg/min) }\end{array}$} & \multicolumn{2}{|c|}{$\begin{array}{l}\text { Concentration } \\
\text { Relative to } \\
\text { Spinal cord }\end{array}$} \\
\hline & $\mathrm{I} \mathrm{hr}$ & $4 \mathrm{hr}$ & $12 \mathrm{hr}$ & $\mathrm{l} \mathrm{hr}$ & $4 \mathrm{hr}$ & $12 \mathrm{hr}$ & Isoniazid & Diamox \\
\hline Cerebral cortex & 302 & 193 & so & 75 & 78 & 29 & 2.52 & 2.27 \\
\hline Cerebellar cortex & 292 & 183 & 92 & 48 & 89 & 34 & 2.43 & 1.46 \\
\hline Hippocampus & 277 & 208 & 100 & 60 & 161 & 57 & 2.31 & 1.82 \\
\hline Hypothalamus & 251 & 2018 & 88 & 93 & 234 & 51 & 2.09 & 2.82 \\
\hline Caudate nuclens & 241 & 147 & 75 & 64 & 145 & 66 & 2.00 & 1.94 \\
\hline Thalamus & 240 & 180 & 90 & 35 & 81 & 46 & 2.00 & 1.06 \\
\hline Lateral geniculate & 226 & 96 & - & 27 & 52 & 33 & 1.88 & 0.81 \\
\hline Inferior colliculus & 188 & 137 & 66 & 36 & 80 & 27 & 1.56 & 1.09 \\
\hline Medulla & 155 & 117 & 69 & 10 & 56 & 35 & 1.29 & 0.30 \\
\hline Globus pallidus & 156 & 118 & 64 & 30 & 50 & 33 & 1.30 & 0.90 \\
\hline Cerebral white matter & 153 & 128 & 58 & 5 & 4.5 & 25 & 1.27 & 0.15 \\
\hline Pyramids & 123 & 90 & 52 & 35 & 56 & 24 & 1.02 & 1.06 \\
\hline Spinal cord & 120 & 109 & 50 & 33 & 60 & 38 & 1.00 & 1.00 \\
\hline
\end{tabular}

a Isoniazid-('14: intraperitoneal injection of $10 \mathrm{mg} / \mathrm{kg}$ in cats. From Barlow et al. (1957).

$b$ Diamox $-\mathrm{S}^{35}$ (acetazolamide): intravenous injection of $150 \mathrm{mg} / \mathrm{kg}$ in cats. From Roth et al., (1959). When the region borders on the ventricles, analyses for the central portion are given inasmuch as the relatively high concentration in the cerebrospinal fluid introduces inhibitor into the ventricular surfaces. 
time. The changing is due mainly to the drop in plasma mercurial and the minor increase in tissue mercurial from 2 to $24 \mathrm{hr}$ is certainly not indicative of a rising concentration of free mercurial. The data do show that renal tissue does accumulate mercurial well; if this is due to exposure to a higher concentration than for other tissues, it may be indicative of a higher initial free concentration and relatively greater enzymic inhibition.

A more detailed pattern of inhibitor distribution has been established within the central nervous system for the amine oxidase inhibitor, isoniazid (Barlow, et al., 1957), and the carbonic anhydrase inhibitor, acetazolamide (Roth. et al., 1959). The results in Table 8-2 show that the distributions for these two inhibitors are far from uniform and Fig. 8-1 demonstrates the changes in distribution with time for acetazolamide. Autoradiograms of brain sections also clearly showed the unequal patterns of distribution. Thus even within a single type of tissue, an inhibitor may enter certain cells at a greater rate, and it is likely that, if smaller region could be analyzed,

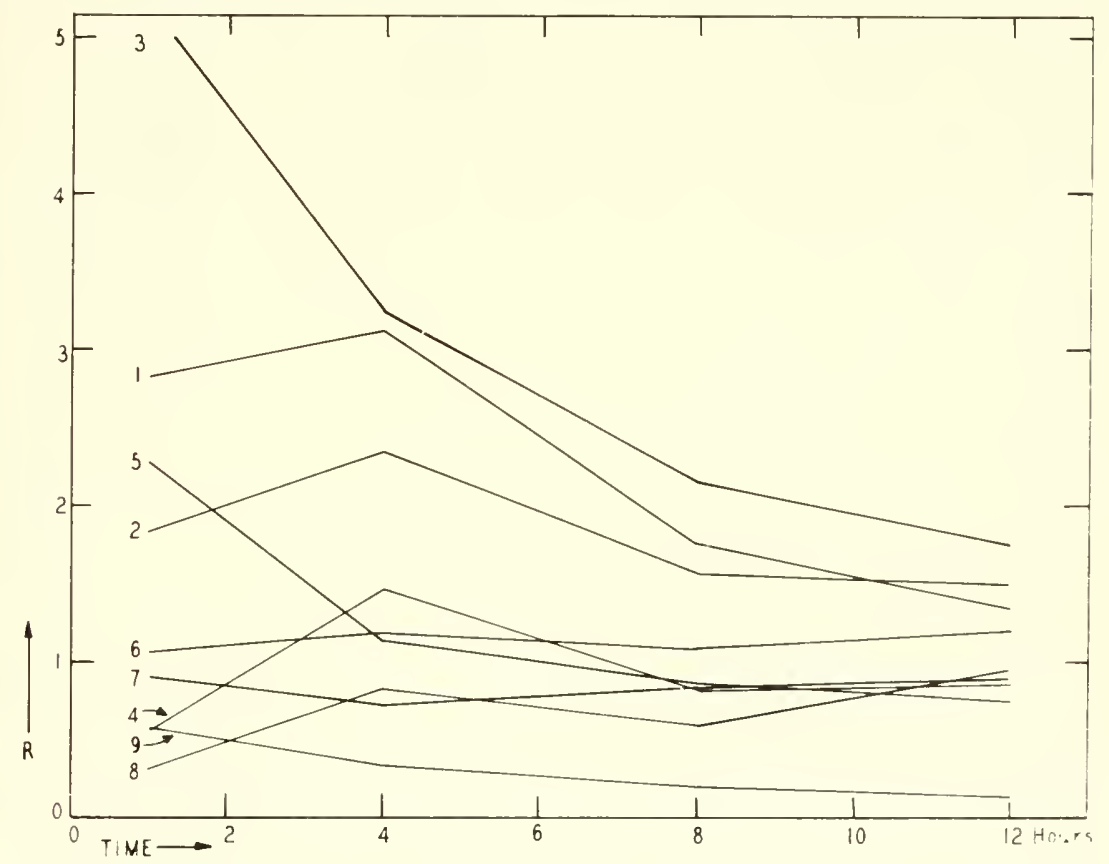

FIg. 8-1. Concentrations of acetazolamide (Diamox) in varions regions of the cat brain following intravenons injection of $150 \mathrm{mg} / \mathrm{kg}$. $\mathrm{R}$ is the ratio of the concentration in the region specified to the concentration in the spinal cord. (From Roth et al., 1959.) Curve 1: hypothalamus; curve 2: hippocampus; curve 3: caudate nucleus (ventral surface); curve 4: cerebral white matter (ventral surface): curve 5: cortex; curve 6: thalanus; curve 7: globus pallidus; curve 8: medulla (centrum); curve 9: cerebrospinal fluid. 
greater selectivity could be demonstrated. An analysis of the total brain, as is often made, is then only a weighted average of the component regions. The distribution of acetazolamide in the ocular tissues of the rabbit may also be mentioned (Ballintine and Maren, 1955). After $10-20 \mathrm{mg} / \mathrm{kg}$ of acetazolamide were given intravenously, the concentrations in micrograms per gram of tissue or fluid from 20-120 min were roughly: aqueous humor, 0.64 ; lens, 0.73 ; iris, 1.4 ; ciliary process, 4.1 ; and plasma, 13.4 . This distribution is of interest in understanding the mechanism by which acetazolamide alters the formation of aqueous humor and the intraocular pressure. It is now necessary to discuss some of the factors that are involved in producing the inhomogeneous patterns of distribution described.

\section{Route of Administration of the Inhibitor}

The manner in which the inhibitor is introduced into the animal can influence the distribution in three ways. An inhibitor cannot be simultaneously presented in equal concentration to all the cells of a complex animal but enters into the body through specific channels. This may simply be called the spatial factor. When an inhibitor is given intravenously, it will be picked up by the cells in those tissues through which the blood flows and thus there will be usually a progressive decline in blood concentration of inhibitor within the circulation. In some cases, the inhibitor may be almost completely extracted from the blood stream before it reaches certain tissues. Thus the initial distribution will depend on the site of injection and the rate of the injection. This factor has been taken advantage of in the use of certain drugs, where an action limited to a particular tissue is desired (the slow injection of a nitrogen mustard into an artery supplying the organ containing a tumor or the injection of vasodilators into the limb arteries in peripheral vascular disease). It is possible that such a technique might often be useful in inhibitor studies, especially combined with temporary occlusion of the vessels through which the blood leaves the tissue. The slow injection of a mercurial into one renal artery, producing diuresis only in that kidney, would be an example of the successful use of this technique. Similar concepts apply to all other routes of administration, with the additional factor that some inhibitor may be taken up before it enters into the circulation. Intramuscular or subcutaneous injection often leads to marked uptake and effects locally, while oral administration can lead to high concentrations of the inhibitor in the intestinal wall. An inhibitor that is absorbed from the intestine passes through the liver immediately and may be accumulated there initially, either to affect the liver or to be metabolized by the multitudinous hepatic enzyme systems.

The rates at which inhibitors enter the systemic circulation vary with the route of administration and the distribution may depend on such rates. This may be called the temporal factor. If an inhibitor is suddenly intro- 
duced into the circulation by intravenous injection, the initial distribution will depend in part on the blood supply to the tissues; when slow absorption occurs, there may be time for other factors to become important. This is illustrated best by a drug, such as thiopental, which, when given intravenously, is present in the brain in relatively high concentrations so that anesthesia is produced, but which is slowly redistributed in the body, as the result of its high lipid solubility, the brain concentration decreasing with time. A very slow intravenous administration would not produce the characteristic initial distribution seen on more rapid injection and much more would have to be present in the body to achieve a comparable central depression. The variations in time of the tissue concentrations of isoniazid and acetazolamide described above may be due to such redistribution factors.

Finally, the distribution of an inhibitor may depend on the concentration present in the circulating blood, especially in situations in which the inhibitor is producing differential effects in the tissues. This may be termed the concentration factor. The plasma concentration of inhibitor will, of course, vary with the route of administration. This factor is related to the effect of dosage on the distribution.

\section{Dose of the Inhibitor}

If penetration of an inhibitor into tissues were a simple matter of diffusion dependent on concentration gradients, one might expect that the distribution pattern would not vary with the dosage within limits. Although this may be true in some instances, there is much evidence that more complex situations are common. There is little experimental evidence from inhibitors, but several drug studies have shown that changing distribution occurs with increasing dosage. Analysis of the data from studies of Paludrine (chlorguanide) in rat tissues (Schmidt et al., 1947), although suffering from a limited number of animals, shows that the differences between tissues became progressively greater as the dose was increased. The liver/ brain ratio rose progressively from 1.4 to 30 as the dose was increased from 11 to $91 \mathrm{mg} / \mathrm{kg}$.

The fact that the concentration of drug or inhibitor in a tissue is not linearly proportional to the dose also indicates complexities in the behavior of these substances in the body. When acetazolamide was given orally to mice and the concentrations in brain determined (Millichap et al., 1955), as the dose was increased from 25 to $400 \mathrm{mg} / \mathrm{kg}$, the concentration in the brain rose more slowly, so that on the average for every doubling of the dose, the brain concentration increased by a factor of 1.21.

The reasons for these deviations are to be found in the saturation of binding sites for inhibitors, the different rates of inactivation or chemical modification of the inhibitor at different dosage levels, active processes in inhibitor uptake, and the failure of the concentration of free drug 
or inhibitor in the plasma to follow the dosage linearly, due to the binding of the substance by the plasma proteins or the erythrocytes. Inhibitors may be bound to different cell components with varying affinities; at low doses, relatively more will be takeu up, but as the high-affinity binding sites disappear, the uptake will fall off as the dose rises. It is conceivable that in the case of acetazolamicle above, a tissue with a relatively large amount of carbonic anhydrase, such as brain, wonld bind a large fraction from a small dose, but when the enzyme molecules have become saturated with inhibitor, any further increase in concentration would be due to nonspecific binding or merely the inhibitor in solution.

\section{Vascularity or Blood Supply of the Tissues}

The initial distribution of an injected inhibitor will often depend primarily on the blood flow through the various organs. The concentration of the inhibitor in the tissue is related, not to the total blood supply, but to the flow volume per mit weight per unit time. In Table 8-3 the blood flow in human tissues is given in milliliters/100 $\mathrm{gm}$ of tissue/minute and these values are nore pertineut than the total flow in milliliters per minute. However, the total amount of imhibitor in the varioms organs is related to the total flow. To what degree this initial distribution will determine eventual distribution will depend for one thing on the affunity of the inhibitor for the tissues. An inhibitor with little affinity will rapidly become equilibrated throughout the body and the final distribution may bear little relationship to the relative blood flows, whereas an inhibitor reacting rapidly and strongly with the tissue components, as wonld arsenicals or DFP, will tend to remain fixed in the initial pattem.

The fractionation of the cardiac ontput between several organs has been determined in the rat by intravenous injections of $K^{42}$ and analysis of the tissues for radioactivity during the first few minutes (Sapirstein. 1956). Immediately following injection the clearance of $K^{42}$ from the blood is essentially complete and during the first minute the tissue concentrations remain constant, following which the $\mathrm{K}^{42}$ may leave a tissue and redistribution occur. The arerage results for the first mimute in terms of the per cent of total cardiac output are given in the tabulation.

\begin{tabular}{l|c|c}
\hline Organ & $\%$ of cardiac output & $\begin{array}{c}\text { Blood flow } \\
(\mathrm{ml} / 100 \mathrm{gm} / \mathrm{min})\end{array}$ \\
\hline Liver & 20.5 & \\
Arterial & 6.5 & \\
Portal & 14.0 & \\
Kidney & 19.3 & 470 \\
Heart & 2.6 & 205 \\
Brain & 0.23 & 10 \\
Skin & 6.3 & \\
\hline
\end{tabular}


These results are of interest because the blood flow distribution in the smaller animals is not well known. However, these animals were anesthetized with pentobarbital and the blood flow through some of the tissues, such as the brain and skin, miglnt have been quite different from the normal animal.

Potassium is a substance which diffuses rapidly from the vessels, is negligibly bound to plasma proteins, and equilibrates readily with the intracellular compartment. In such a case, removal of a small amount of a substance from the blood may be essentially complete during one passage through an organ. The amount of substance in the tissue will depend only on the volume of blood entering it; it will not depend on the rate of flow or the

TABLE $\quad 8-3$

Composrtion and Blood Flow of Tissues

\begin{tabular}{|c|c|c|c|c|c|c|c|c|}
\hline \multirow{4}{*}{ Organ } & \multicolumn{3}{|c|}{ Organ Weight ${ }^{a}$} & \multirow{4}{*}{$\begin{array}{l}\text { Water } \\
\text { content } \\
(\%)\end{array}$} & \multirow{4}{*}{$\begin{array}{c}\text { Lipid } \\
\text { content } \\
(\%)\end{array}$} & \multicolumn{3}{|c|}{ Blood Flow (human) } \\
\hline & \multicolumn{2}{|c|}{ Human } & \multirow{3}{*}{$\begin{array}{c}\text { Small } \\
\text { animals } \\
\% \text { of } \\
\text { total }\end{array}$} & & & \multirow{3}{*}{$\begin{array}{c}\mathrm{ml} / 100 \\
\mathrm{gm} / \mathrm{min}\end{array}$} & \multirow{3}{*}{$\mathrm{mI} / \mathrm{min}$} & \multirow{3}{*}{$\begin{array}{l}{ }_{0}^{0} \text { of } \\
\text { cardiac } \\
\text { output }\end{array}$} \\
\hline & & & & & & & & \\
\hline & grams & totaI & & & & & & \\
\hline Liver & 2.10 & 3.2 & 3.7 & 71.9 & 5.2 & $66^{b}$ & 1390 & 26 \\
\hline Kidneys & 0.32 & 0.49 & 0.77 & 78.1 & 3.3 & 410 & 1310 & 25 \\
\hline Spleen & 0.18 & 0.28 & 0.30 & 78.11 & 2.2 & 58 & 104 & $\stackrel{2}{2}$ \\
\hline Intestine & 1.45 & 2.2 & 7.6 & 76.6 & 5.4 & 59 & 850 & 16 \\
\hline Muscle & 29.8 & 45.9 & 41 & 75.0 & 3.6 & 3 & 894 & 17 \\
\hline Heart & 0.33 & $0.5]$ & 0.48 & 78.4 & 4.8 & 92 & 304 & 6 \\
\hline Brain & 1.41 & 2.2 & 0.76 & 76.7 & $13.7 \mathrm{c}$ & 54 & 761 & 15 \\
\hline Spinal cord & 0.16 & 0.25 & 0.3 & 69.4 & 18.0 & 47 & 75 & 1 \\
\hline Lungs & 1.10 & 1.7 & 0.83 & 78.6 & $\stackrel{2.6}{0}$ & 477 & 5.250 & - \\
\hline Skin & 4.20 & 6.5 & 9.5 & 69.5 & $1.4^{d}$ & 13 & 545 & 10 \\
\hline Blood & 4.55 & 6.9 & 6.5 & 83.3 & 0.8 & - & - & - \\
\hline Serum & 2.75 & 4.2 & - & 91.3 & 1.0 & - & - & - \\
\hline Erythrocytes & 1.80 & 2.7 & - & 66.0 & 0.6 & - & - & - \\
\hline
\end{tabular}

a Organ weights for small animals a veraged from cat, rat, dog, guinea pig, and rabbit. Water content averaged from man, rat, dog, cat, and rabbit. Lipid content averaged mainly from man, rabbit, and rat. These values have been colfected from a variety of sources, including unpublished data from Dr. Alfin-slater (Dept. of Blochemistry, University of Southern (alifornia) and our laboratory. There is remarkable variation in data reported in the literature and the above values are to be taken as reasonable approximations only.

${ }^{b}$ The arterial blood flow through the liver is about one-third the total flow and the portal two-thirds.

c Gray matter is about $6.9^{\circ}$ o lipid and white matter $20.3 \%$.

d This value is for dermis and does not include the more lipid subcutancous tissne. 
surface area of the capillaries. However, most inhibitors would not necessarily follow this simple scheme because of the restriction in diffusion brought about by the binding to plasma proteins and the possibly slow penetration into the tissues. In this case, the capillary surface within a tissue may be important, the rate of absorption being directly proportional to the diffusion surface. If it is assumed that the capillaries are all of approximately the same diameter, the volume of blood flowing through a tissne in unit time will be proportional to the number of capillaries and thus the surface for diffusion. Hence, the rate of absorption of an inhibitor and the tissue concentration will be proportional to the tissue blood flow. The surface for diffusion, however, may also depend on the length of the capillaries in a tissue; a tissue with capillaries twice as long as another tissue might pick up twice as much of an inhibitor in unit time with a comparable blood flow through each tissue. Finally, it must be remembered that the inhibitor can alter the blood flow by a dilating or constricting effect on the blood vessels.

The fractionation of the blood flow through the various tissues is by no means constant but may be altered markedly by a number of factors. The activity of a tissue is perhaps the most important variable. The blood flow through skeletal muscles may increase as much as fifteen-fold during intense activity and the coronary flow increase eight-fold upon stimulation of the heart. The blood flow through the central nervous system also depends on the state of functional activity. Anesthesia will alter blood flow by several mechanisms. principally by elimination of the normal control of the vascular tone. The skin vessels are particularly sensitive to nervous control and environmental temperature. A frightened animal will have a different pattern of blood flow than a normal animal due to nervous factors and the release of epinephrine.

The role of vascularity in the distribution of isoniazid in the central nervous system would appear to be of some importance, since there is a progressive decrease in vascularity from the cerebral cortex (2.22) to the globus pallidus (1.18) (See Table 8-2) (Barlow et al., 1957). However, the hippocampus was unusual in that it showed a relatively high concentration of the inhibitor and yet has a low vascularity: also it retained the isoniazid for a longer time than the other tissues.

\section{Penetration of Inhibitors into Tissues}

An inhibitor may enter tissue cells either by simple diffusion or by an active transport process. The latter is uncommon and mainly confined to the analogs of actively transported substrates or metabolites. The permeability properties of the plasma membrane are important in determining the degree of inhibition in some cases but usually only affect the rate at which inhibition develops. Malonate, occurring mainly in the doubly-charged ionic 
form at physiological pH's, often has very little or no effect on the metabolism or function of intact tissues at concentrations capable of almost complete inhibition of succinic dehydrogenase and blocking of the tricarboxylic acid cycle. Of course, the various tissues may differ appreciably in their permeabilities to inhibitors, and it is this that may be a factor in the distribution of inhibitors in the body. Unfortunately very few adequate studies have been made on the permeabilities of different cells to the important enzyme inhibitors. There is general opinion that certain inhibitors penetrate into cells rapidly (cyanide, carbon monoxide, dimercaprol, DFP, bipyridine, dinitrophenol), some moderately well (fluoroacetate, idoacetate, fluoride, arsenite), and some poorly [malonate, parapyruvate, ethylenediaminetetraacetate (EDTA)], but quantitative data are lacking and our knowledge of differences between tissues or cells is meager. Permeability, in isolated instances, has been shown to be a primary factor in inhibition. The actions of organic arsenicals on protozoa depend on the degree of penetration and the development of resistance to the arsenicals often involves a limitation on the uptake of the inhibitor. The problems of permeability will be discussed wherever possible when the individual inhibitors are treated in later chapters. The effects of $\mathrm{pH}$ on penetrability into cells will be discussed in Chapter 14.

The kidney requires special consideration because the tubular cells are not only in contact with arterial blood but also with the progressively changing glomerular filtrate. Unless the inhibitor is resorbed as rapidly as water, its concentration will rise in the tubular fluid; at the maximum it is possible for the concentration to rise approximately a hundred-fold over the plasma value. The urinary concentration of many drugs and inhibitors is constantly higher than the level in plasma. Thus one side of the tubular cell, especially in the distal segment, may be exposed to higher concentrations of inhibitor than any other tissue. In addition to this, the large amount of fluid continually passing through the tubular cells may carry with it the inhibitor, so that even though the concentration in the tubular fluid does not rise, the cells will be presented with much more inhibitor than are other cells. An inhibitor that is picked up readily by the cells will be accumulated there. Many inhibitors, such as the mercurials, exert their actions primarily on the kidney and their concentration there is undoubtedly the important factor, rather than any special sensibility of the renal cells.

There has been much discussion of penetration into the central nervous system and the slow rates of entry often observed have given rise to the concept of the blood-brain barrier, which is supposed to exist at or near the vascular wall. Since the brain has an excellent vascular supply, this hypothetical barrier must be very impermeable to certain substances to limit their entry into the brain cells; it must also be very specific, since many 
compounds enter and affect the brain very rapidly. It is probable that some phenomena attributed to the blood-brain barrier are the result of other factors. For example, the brain is a tissue with a relatively small extracellular free fluid space and the nerve cells possess membranes specialized for the control of ionic movements and the conduction of impulses. These membranes exhibit somewhat unique permeability properties. Furthermore, the myelin sheaths can also impede the movement of small nolecules, particularly when they are charged. This combination of circumstances makes it difficult for some substances to enter the brain cells in appreciable concentration and no special barrier need be assumed. There is, of course, a "barrier" of living cells between the blood and the cerebrospinal fluid, so that charged drugs and inhibitors usually enter this space slowly.

\section{Chemical Composition of the Tissues}

Tissues vary in their lipid contents (Table 8-3). When equilibrium of the inhibitor in the body has been reached, a lipid-soluble inhibitor will be found in relatively high concentrations in the lipid-rich organs. However, this probably has little bearing on the inhibition of enzymes, since the enzymes are not located in the lipid material. Much of the lipid analyzed in tissues is extracellular and if pure cells could be worked with, it would be likely that little difference in lipid content would be observed. Of course, there are cells that contain many fat droplets but these are spatially separated from the enzymically active regions. The distribution coefficient of an inhibitor between lipid and water may be of importance in determining the total amount of inhibitor in a tissue but is probably of little significance in enzyme inhibition. As mentioned previously. the water content of a tissue (Table 8-3) may be a factor in the tissue concentration of an inhibitor if the inhibitor is tightly bound to proteins or other cell components. Some tissues possess $50 \%$ more solids than other tissues and an increased concentration of the inhibitor in the former may relate to this rather than to any more specific mechanism of uptake. However, the water content is of no importance in enzyme inhibition.

\section{Binding of the Inhibitor to Plasma Proteins}

Many inhibitors, just as drugs, are probably bound to plasma proteins with varying degrees of affinity. This effectively reduces the concentration of the free inhibitor in the blood and thus slows down penetration into the tissues and delays excretion. Such binding will not alter the distribution of the inhibitor among the tissues to a large extent because only the available concentration is reduced. But it may alter the initial distribution in favor of an equilibrium distribution, just as slow absorption into the blood stream does. Protein hinding may also be a factor in deviating the relation between the tissue concentration and the dose from linearity. 


\section{Correlation of Enzyme Inhibition with Tissue Distribution}

In some cases a specific effect on an enzyme in a particular tissue may be correlated with a high concentration of the inhibitor in that tissue. The inhibition of renal succinic dehydrogenase by mercurials, without alteration of this enzyme in other tissues, can certainly be related to the specific accumulation of mercurial in the kidney tubular cells (Handley and Lavik, 1950). When chlormerodrin- $\mathrm{Hg}^{203}$ is administered to rats or dogs, the kidney/plasma ratios rum from 50-1300 while in all other tissues examined the tissue/plasma ratio is less than unity (Giebisch and Dorman, 1958). Indeed, there may be a correlation between the mercurial concentration in the proximal and distal segments, and the degree of inhibition of succinic dehydrogenase in these regions (Cafruny and Farah, 1956; Cafruny et al., 1955).

The inhibition of cholinesterase by diisopropylfluorophosphate in various tissues parallels the concentration of the inhibitor as determined by $\mathrm{P}^{32}$ analysis (Jandorf and IcNamara, 1950). The time curve for the inhibition of erythrocyte cholinesterase follows closely the curve for $\mathrm{P}^{32}$ concentration over 3 weeks, but in the plasma the $\mathrm{P}^{32}$ decreases rapidly although the cholinesterase remains inhibited for some time (this is probalby due to the very small amount of $\mathrm{P}^{32}$ attached to plasma cholinesterase, the larger nonbound $\mathrm{P}^{32}$ having left the blood rapidly). A similar situation has been reported for the inhibition of monoamine oxidase by iproniazid in rats (Hess et al., 1958). The i.hibitor enters brain, muscle, and liver rapidly and the monoamine oxidase is inhibited completely almost at once. However, the iproniazid concentration in these tissues falls off rapidly (within $20 \mathrm{hr}$ ) but the inhibition persists for 3 to 6 days or longer. The results for liver are shown in Fig. 8-2. The explanations suggested were: (1) the enzyme may be irreversibly destroyed by the inhibitor and activity does not return until new enzyme is synthesized, or (2) the inhibitor may be irreversibly incorporated into the enzyme (the enzyme concentration in the tissue being so low that attached inhibitor would not be detected), or (3) the continuing inhibition may be due to some degradation product of the inhibitor. For iproniazid the explanation is not known but DFP is partially incorporated into the cholinesterase molecule. Reversible monoamine oxidase inhibitors, such as harmaline, show a more normal behavior, in that enzyme inhibition ceases with the disappearance of the inhibitor from the tissue.

\section{DISTRIBUTION OF INHIBITORS IN PLANTS}

Certain aspects of inhibitor distribution are quite similar for both the higher plants and animals but special factors of importance only to plants must be considered. There is regrettably little information from analyses 
of inhibitors in plant tissues but recently much work has been done on the movements and distribution of herbicides, such as 2,4-dichlorophenoxyacetate (2,4-D), in the higher plants, and the results probably are often applicable to the more common enzyme inhibitors when they are applied to

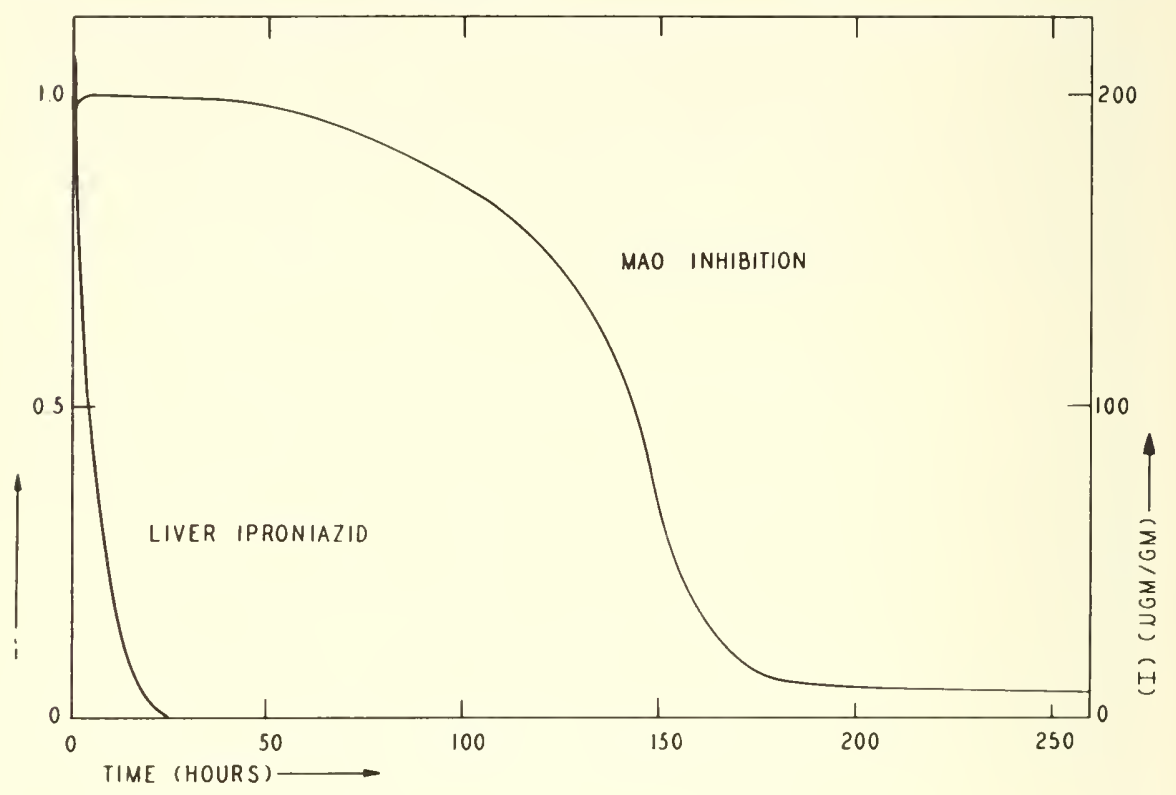

Fig. 8-2. Tissue concentration of iproniazid and the inhibition of monoamine oxidase following intraperitoneal injection of $195 \mathrm{mg} / \mathrm{kg}$ in rats. The eurves are for the liver but brain and muscle are similar. Curve 1: tissue concentration ( $\mu \mathrm{g} / \mathrm{gm})$; curve 2: monoamine oxidase inhibition (MAO). (From Hess et al., 1958.)

plants in the same ways as the herbicides. The distribution, as in animals, depends on the manner of administration, the absorption through certain tissues, and the circulation within the plant.

\section{Route of Administration of the Inhibitor}

There are two general ways in which inhibitors may be applied to intact plants: in the soil or solution for uptake by the roots or on the upper leafy parts of the plant. In either case the inhibitor may spread throngh the circulatory pathways from the site of absorption. The factors involved in penetration into the roots are little known but the process may well be complex since active transport for ions is well established and the flow rate of water is often dependent on transpiration conditions in the leaves. Inasmuch as absorption into the roots depends on metabolic energy, an inhibitor can 
immediately and directly affect its own absorption, and absorption in general, as soon as it gains access to the root cells. If inhibitor distribution throughout the plant is desired, the concentration of the inhibitor surrounding the roots must be below that level where the roots are damaged. To gain entrance to the conducting system. the inhibitor must pass through at least two plasma membranes and it is likely that penetration of ionic inhibitors will be relatively slow because of the specialization for uptake of certain physiologically important ions.

An inhibitor applied to the surface of a leaf meets with four possible barriers before entering an epidermal cell. From the cell outwards there is the plasma membrane, the epidermal cell wall, the cuticular layer, and the extruded waxy deposit on the surface. The cell wall probably seldom presents any barrier to diffusion and the plasma membrane behaves typically and shows selective permeabilities just as in animal cells. It is the outer layers which present some unique problems. In the first place, penetration is generally not through the stomata because of capillary forces preventing access of the solution; if surfactants are included with the inhibitor, stomatal penetration is possible. This is quite similar to absorption of substances through the hair follicles and sebaceous glands of the skin. The waxy material is deposited in various ways characteristic of the plant and presents a barrier to diffusion mainly by preventing adequate contact between the surface and aqueous solutions. The wax is, however, often spongy and may swell after contact with water, the penetration rate of a dissolved substance increasing. The herbicide 2,4-D is absorbed very slowly from ordinary aqueous solutions when applied to leaves; a surfactant increases penetration fivefifteenfold. The absorption and distribution of 2.4-D is discussed thoroughly by Loomis (1955). The cuticle is composed of pectin and the fatty material, cutin, in various proportions, possibly in some cases associated with cellulose. The permeability properties of the cuticle are poorly understood and its role in inhibitor penetration can only be surnised. In any event, penetration of large molecules or ionic substances into epidermal cells is slow, although lipid-soluble substances may enter readily. The entrance of 2,4-D into phloem tissue occurs at a rate of $304 \mu / \mathrm{hr}$ in bean leaves but once within the phloem, transport is much more rapid and may amount to 10-100 cm/hr (Day, 1952).

\section{Translocation of Substances in Plants}

Many substances necessary for plant growth must be transported between tissues. The salts and nitrogenous material absorbed by the roots must be transported upwards into the growing regions of shoot and leaf, while the organic substances synthesized in the leaves are transported to the roots. It is probably along these normal pathways of movement that inhibitors are transported. The excellent reviews of translocation processes by 
Crafts (1951) and van Overbeek (1956) are recommended. The following is a cursory discussion of those aspects of absorption and translocation pertinent to the distribution of inhibitors; the attempt has been made to incorporate recent work with radioactive substances, herbicides, and antibiotics.

The upward movement of an inhibitor that is absorbed by the root system would occur mainly in the xylem with the transpiration stream. The xylem is basically dead tissue, the unit cell being the long hollow tracheid, communication between adjacent tracheids being made through perforations in the walls. The rise of fluid is due mainly to the loss of water from the leaves by transpiration and to a lesser extent to root pressure, the active movement of fluid by the root cells (evident in the flow of liquid from the cut surface of a stem). The rate of the transpiration stream through the xylem thus depends on many factors, both in leaves and roots; some of these processes can be affected by inhibitors as they move through the plant. $\mathrm{A}$ substance can be distributed to all the tissues of a plant by this means. Thus when $\mathrm{P}^{32}$ is applied to the roots in the form of inorganic phosphate it is absorbed and moves upward rapidly, being found in the growing tips within an hour, indicating rates of $50-100 \mathrm{~cm} / \mathrm{hr}$ (Frazier et al., 1956). As pointed out many years ago (Stout and Hoagland, 1939), the diffusion out of the xylem into adjacent living tissue is very rapid during translocation of phosphate. Little is known about the differential uptake by the various tissues but differences do occur as shown in radioautographs of wheat kernels during uptake of $\mathrm{P}^{32}$. Large ionic molecules, such as penicillin and streptomycin, are distributed throughout plants much more slowly, due probably to the slow uptake by the roots, although it has been postulated that a negative charge on the tracheid walls might account in part for the very slow translocation of substances such as streptomycin and $\mathrm{Mn}^{++}$ (Pramer, 1954; Single, 1958). Within 19 hr after application of chloramphenicol to the roots of bean plants, the antibiotic was distributed throughout the plant, but it required several days for the distribution to become uniform, whereas after $18 \mathrm{hr}$ in streptomycin there was antibiotic only in the lower parts of the plant. Indication that uptake by roots is limiting here was shown by the more rapid accumulation of these antibiotics in shoots from which the roots had been cut (Pramer, 1954).

The downward movement of carbohydrates, amino acids, auxins, and herbicides from the leaves is mainly in the phloem tissue and it is likely that this is the ronte taken after application of inhibitors. This type of translocation is complex because phloem is a living tissue and movement of a substance occurs through protoplasm; indeed, the mechanisms involved are not at present well understood. The unit cell is the sieve tube and the protoplasm of each sieve cell is in contact with the protoplasm of adjacent sieve cells through the perforations in the walls. It is not known if the pro- 
toplasm is continuous or separated by plasma membranes. There is some evidence that both situations occur. In the first place, the movement of many substances in phloem is much more rapid than could be accounted for by simple diffusion; a factor of 40,000 may be observed (Biddulph and Cory, 1957). If many membranes had to be passed, it is evident that they could not possess the usual permeability properties of the plasma membrane; instead, they would have to participate actively in the transport. Furthermore, various substances move at different rates indicating that neither simple diffusion nor mass flow of fluid is the primary mechanism. Metabolism is necessary for normal transport through the phloem. Part of this energy may be utilized for protoplasmic streaming (cyclosis) which has been thought by some to be an important factor in accelerating the movement of dissolved substances: however, cyclosis camıot account for either specificity of transport or the rapid rates observed. Gross novement of fluid through phloem can occur, perhaps as the result of active processes, but dissolved substances usually move more slowly than the water, as in a chromatogram. The observations of polarized transport (i.e., movement of a substance in one direction from the site of application) would imply active participation of membranes. Studies with fluorescein have demonstrated the complexity of the transport. The rates of movement are different in various regions of the plant and the polarity may also differ; for example, application of the dye to stems results in upward movement into leaves or fruits, while application to a basal region results in movement downwards into the roots (Crafts, 1951).

Foliar painting or spraying of a substance may result in the distribution of the substance throughout the plant. This may be seen most clearly with dyes. If a potato plant is dug up, the apex of a leaf excised, and the cut surface immediately immersed in eosin solution. within an hour all the leaf veins are stained and some dye has penetrated into the roots; in several hours the dye has entered the tuber itself. Similar results have been obtained with $\mathrm{P}^{32}$, $\mathrm{C}^{14}$-labeled photosynthesates, $\mathrm{S}^{35}$, tritiated water, and some herbicides. When $\mathrm{P}^{32}$ is applied to a single unifoliate leaf of a bean plant for $2 \mathrm{hr}$ and the plant is then allowed to grow for a week, radioactivity is demonstrated throughout the plant (Barrier and Loomis, 1957). Many factors have been shown to influence absorption and translocation: the air humidity, light intensity, age of the leaf, wetting of the leaf, and whether absorption is from upper or lower surface of the leaf (Thorne, 1958). The influence of light on translocation may be through the sugars produced, since application of sugar with the transportable substance will usually allow normal translocation to occur. The application of $\mathrm{C}^{14}-\alpha$-methoxyphenylacetate to leaves led to diffusion of this substance from the roots into a bathing solution within $5 \mathrm{hr}$ : this increased steadily for 4 days and then declined until by the tenth day exudation was negligible (Linder, et al., 
1957). It is interesting that reducing the oxygen tension of the roots slowed down the loss of $\alpha$-methoxyphenylacetate, indicating an active process in the movement. The translocation of the herbicide 2,4-D from leaves is also dependent on either photosynthesis or added sugar. Although the bearing of these results on inhibitor translocation may be tenuous, the author believes that similar mechanisms will be operative and identical pathways demonstrable when experiments with inhibitors are done.

There is, however, one complication in the transport of inhibitors. In phloem tissue the inhibitor may depress the metabolic components of the process and thereby suppress the mechanism for its own movement and dissemination. Destruction of phloem is a characteristic result of the application of 2,4-D and if the applied concentration is too high, it camnot reach other parts of the plant because the transport system is inactivated. If enzyme inhibitors are to be distributed throughout a plant by foliar application, their concentration in the phloem cells must be below the level required to produce appreciable inhibition of exergonic metabolism or to otherwise interfere with the translocation mechanisms.

It is likely that some upward transport can occur in the phloem and that occasionally downward movement may be observed in the xylem. The importance of the varions conducting systems depends on the type of plant, the condition of the plant, the substance under study, and the region of the plant. Circulation of a substance may also occur in the following way. The material passes out of the leaf into the stem phloem and as it descends it diffuses out into the xylem and is transported upwards. This has been shown with $\mathrm{P}^{32}$ in cotton plants (Biddulph and Markle, 1944). Such a circulation ensures more rapid and complete distribution of the substance. For our present purpose, the important aspect of the problem is that the distribution of an inhibitor in various plant tissues depends on quite complex mechanisms of transport, processes which can easily be affected by the inhibitor. The amount of uptake of an inhibitor by various tissues will vary, just as in animals, with the concentrations presented to the tissues, and this will depend on transport pathways. Accurate analyses of inhibitors in plant tissues are much needed for a better understanding of the differential effects produced.

\section{DISTRIBUTION OF INHIBITORS WITHIN CELLS}

There are two primary problems: the total concentration of the inhibitor in the cell relative to the concentration outside, and the distribution of the intracellular inhibitor between the various structures or compartments making up the cell. One would particularly like to know in any case the concentration of inhibitor at the site of the enzyme it is acting upon. We have seen that analyses of organs for inhibitors are not always reliable as 
an indication of the true concentrations in the enzyme region; the same situation occurs, of course, in the single cell because it is mlikely that any substance will be homogeneously distributed throughout the entire cell.

A term such as "intracellular concentration" of an inhibitor is usually glibly used, both for convenience and to mask out ignorance of the true sitnation, and the value is generally arrived at by analysis of a group of cells. One may often ask, "concentration in what?" The most common way of expressing such concentrations is in milligrams per gram or similar weight of inhibitor per weight of cell units. This provides very little information on the concentration in intracellular water. It is easy to correct for the solid content of the cells but one cannot assume that all the inhibitor is free in solution. It is clear that the concentration of the inhibitor in solution may be negligible when the total amount in the cell is large. Plant cells provide a further complication because of the presence of a large vacuole. Since the vacuole can occupy $80-90 \%$ of the cell volume, analysis of a plant cell for inhibitor could give very erroneous ideas of the concentration in the protoplasm if the vacuole either accumulates or excludes the inhibitor. Ideally, there should be another word to designate the total amount of a substance in a cell, irrespective of the location or state of the substance. Instead, we shall, where necessary, designate by the term effective concentration the true concentration of a substance in solution in the region where it is exerting its effect. It is the effective concentration of an inhibitor we should know but which is not usually provided by gross analyses. Very often the variation of effective concentration with time will be in the opposite direction to that of the over-all concentration.

The ratio of the effective concentration of an inhibitor in a cell to the concentration outside may not be unity. Deviations from unity have usually been discussed relative to permeability. However, there are several other factors that may play a role and these will be mentioned only briefly because there is little experimental evidence directly bearing on inhibitors. If the inhibitor is an ion, its penetration into a cell implies either the entrance of an oppositely charged ion or the exchange for a similarly charged intracellular ion. Anions particularly are distributed extracellularly for the most part. It is likely that fluoride would behave similarly to chloride and bromide, that is that it would be mainly excluded from cells because of the stable anion content of the cells. There may also be reactions within the cell by which the effective concentration of inhibitor is reduced; these could be metabolic degradation, binding to nonenzymic cell components or entry into cell material in which the inhibitor is soluble. In such cases, the effective concentration may be determined by the balance between rates of entry and disappearance, at least over a particular time interval. The possibility of active transport of the inhibitor into the cell in some cases cannot be neglected. Lastly, the effective concentration in the cell is not 
necessarly linearly proportional to the outside concentration and this factor often makes impossible a quantitative kinetic treatment of inhibition in living material.

The distribution of inhibitors within cells is as yet an almost totally unexplored field. In the previous chapter some consideration was given to the problems of compartmentalization of enzyme systems. In such systems the possibilities for inhomogeneous inhibitor distribution must also be present. There are more types of membrane in the cell than classically conceived but as to how far these membranes impede the diffusion of inhibitors there is no information. It would appear that mitochondrial membranes are more generally permeable than plasma membranes; at least isolated mitochondria are more readily susceptible to inhibitors such as malonate than intact cells and it is likely that large molecules, e.g., diphosphopyridine nucleotide (DPN) and ATP, can penetrate into mitochondria. Whether mitochondria within the living cell are so readily permeable is not known. Lacking evidence one way or the other, one would expect the endoplasmic reticular membranes not to present serious barriers to the diffusion of low molecular weight substances, although one must admit the possibility in some cells of a relatively extensive "extracellular" space invaginated within the cell. At present, it must be concluded that the most important, hindrance to the diffusion of inhibitors into the cell, or through the cell, is the outer plasma membrane. When inhibitors are unevenly distributed within cells, it is more likely that binding and solubility factors are operative. Some studies recently have attempted to demonstrate inhomogenous distribution of drugs within cells, although such work is subject to great technical difficulties. Interesting results were obtained with quinacrine by analysis of liver cell fractions (Reiner and Gellhorn, 1956). Equilibration of quinacrine with isolated nuclei resulted in a concentration ratio of 200 in favour of the nuclei and it is reasonable that the quinacrine was extensively bound to nucleic acids. When the quinacrine was administered by various rontes to mice at different dosage levels, the distribution between nuclei, mitochondria. and cytoplasmic supernate was quite variable and indicative of relatively nonspecific binding. The binding could not be attributed to deoxyribonucleic acid (DNA) entirely nor to any one protein. However, these results, although of importance in understanding the meaning of cell analyses, probably have very little relevance to the metabolic disturbance induced by quinacrine, since all that could be measured was quinacrine bound to mainly nonenzymic material. Since quinacrine is bound quite avidly by cell components, one can only conclude that the concentration of free quinacrine in solution must have been quite low, since saturation of binding sites was not reached. 


\section{FATE OF INHIBITORS IN THE ANIMAL}

Following administration of an inhibitor, and either during or after its distribution throughout the body, there is disappearance of the inhibitor. This may take place by a metabolic degradation or inactivation of the inhibitor, or by excretion from the body, or by both. The curve representing the variation in inhibitor concentration with time in a tissue will rise to a maximal value, at a rate depending on the factors previously discussed, and will then fall according to principles to be considered in this section. Some inhibitors that are tightly bound to the tissues will remain in the body for many days or weeks and it may be that the rate of removal from the body is dependent on the rate of dissociation, rather than on processes of destruction or excretion.

\section{Variation of Intracellular Inhibitor Concentration with Time: Simplified} System

The usplal representation of the fate of a substance in the body is:

$$
\mathrm{I}_{0} \stackrel{k_{1}}{\rightarrow} \mathrm{I}_{i} \stackrel{k_{2}}{\rightarrow} \mathrm{X}
$$

where $I_{o}$ is the inhibitor outside the region considered, $I_{i}$ is the inhibitor inside this region, and $\mathrm{X}$ is either a metabolic product or represents the excretion of the inhibitor from this region. This system implies a one-way passage of inhibitor into the region and its application would be limited to certain cases where loss from the region by this ronte is negligible. There are two situations to be considered: either $\left(I_{0}\right)$ remains constant or it decreases as the inhibitor is taken up. The outside concentration of the inhibitor may be considered practically constant over an interval when the amount of uptake is negligible compared to the supply. Tissue slices or cell suspensions in large volumes of medium or with relatively high concentrations of inhibitor might be examples. Also, constant intravenous infusion can maintain the concentration in the plasma constant, the plasma in this case being the outside phase relative to the tissue. Where $\left(I_{0}\right)$ remains constant, the differential equation:

$$
\frac{d\left(\mathrm{I}_{i}\right)}{d t}=k_{1}\left(\mathrm{I}_{0}\right)-k_{2}\left(\mathrm{I}_{i}\right)
$$

may be directly integrated to give the internal inhibitor concentration at any time:

$$
\left(\mathrm{I}_{i}\right)=\left(\mathrm{I}_{0}\right) \frac{k_{1}}{k_{1}+k_{2}}\left[1-e^{-\left(k_{1}+k_{2}\right) t}\right]
$$

Equilibrium will be reached theoretically when $\left(\mathrm{I}_{i}\right)=k_{1}\left(\mathrm{I}_{0}\right) /\left(k_{1}+k_{2}\right)$ since 
the exponential factor will disapper at large values of $t$. When $\left(\mathrm{I}_{o}\right)$ does not remain constant, integration of $d\left(\mathrm{I}_{0}\right) / d t=-k_{1}\left(\mathrm{I}_{0}\right)$ gives:

$$
\left(\mathrm{I}_{0}\right)=\left(\mathrm{I}_{0}\right)_{0} e^{-k_{1} t}
$$

where $\left(\mathrm{I}_{o}\right)_{0}$ is the initial external concentration. For very approximate results, this value may be substitued in Eq. 8-3. Such expression have been applied to drugs but they are too inaccurate for further consideration and it is necessary to turn to a more realistic system.

\section{Variation of Intracellular Inhibitor Concentration with Time: Complete System}

In most cases of interest, the entrance of the inhibitor into the region of interest is reversible and the external inhibitor concentration is not constant.

$$
\mathrm{I}_{0} \underset{k_{-1}}{\stackrel{k_{1}}{\rightleftharpoons}} \mathrm{I}_{i} \stackrel{k_{2}}{\rightarrow} \mathrm{X}
$$

The differential equations for this situation are:

$$
\begin{aligned}
& \frac{d\left(\mathrm{I}_{o}\right)}{d t}=k_{-1}\left(\mathrm{I}_{i}\right)-k_{1}\left(\mathrm{I}_{o}\right) \\
& \frac{d\left(\mathrm{I}_{i}\right)}{d t}=k_{1}\left(\mathrm{I}_{o}\right)-k_{1}\left(\mathrm{I}_{i}\right)-k_{2}\left(\mathrm{I}_{i}\right)
\end{aligned}
$$

These simultaneous equations may be solved to give both external and internal inhibitor concentrations at any time.

$$
\begin{aligned}
& \left(\mathrm{I}_{0}\right)=\left(\mathrm{I}_{0}\right)_{0} \frac{1}{\alpha-\beta}\left[\left(k_{1}+\alpha\right) e^{\beta t}-\left(k_{1}+\beta\right) e^{\alpha t}\right] \\
& \left(\mathrm{I}_{i}\right)=\left(\mathrm{I}_{0}\right)_{0} \frac{k_{1}}{\alpha-\beta}\left[e^{\alpha t}-e^{\beta t}\right]
\end{aligned}
$$

where $\left(\mathrm{I}_{o}\right)_{0}$ is the initial concentration of external inhibitor and $\alpha$ and $\beta$ have the values:

$$
\begin{aligned}
& \alpha=1 / 2\left[-\left(2 k_{1}+k_{2}\right)-\sqrt{4 k_{1}^{2}+k_{2}^{2}}\right] \\
& \beta=1 / 2\left[-\left(2 k_{1}+k_{2}\right)+\sqrt{4 k_{1}^{2}+k_{2}^{2}}\right]
\end{aligned}
$$

It has been assumed here that $k_{-1}=k_{1}$ since in most instances the entrance of an inhibitor into a cell is a diffusion process with equivalent rates in each direction. 
The derivation of the equations above may be briefly indicated. Equations 8-6 and 8-7 may be written in operational form:

$$
\begin{aligned}
\left(D+k_{1}\right)\left(\mathrm{I}_{o}\right) & =k_{1}\left(\mathrm{I}_{i}\right) \\
\left(D+k_{1}+k_{2}\right)\left(\mathrm{I}_{i}\right) & =k_{1}\left(\mathrm{I}_{o}\right)
\end{aligned}
$$

and solved to give:

$$
\left[D^{2}+\left(2 k_{1}+k_{2}\right) D+k_{1} k_{2}\right]\left(I_{i}\right)=0
$$

from which the values of $\alpha$ and $\beta$ can be determined for the general solution:

$$
\begin{aligned}
& \left(\mathrm{I}_{o}\right)=C_{1} e^{\alpha t}+C_{2} e^{\beta t} \\
& \left(\mathrm{I}_{i}\right)=C_{3} e^{\alpha t}+C_{4} e^{\beta t}
\end{aligned}
$$

The constants $\mathrm{C}_{1} \ldots \mathrm{C}_{4}$ may be related by differentiating these expressions and substituting back in Eq. 8-6 or 8-7, and may finally be evaluated by imposing the conditions when $t=0$, where $\left(\mathrm{I}_{o}\right)=\left(\mathrm{I}_{0}\right)_{0}$ and $\left(\mathrm{I}_{i}\right)=0$.

The behavior of this system may be illustrated for the special case in which the rate of entrance is equal to the rate of disappearance of the inhibitor $\left(k_{1}=k_{2}\right.$ ). Equations $8-8$ and $8-9$ are plotted.in Fig. 8-3, from which it may be seen that as $\left(\mathrm{I}_{0}\right)$ falls, $\left(\mathrm{I}_{i}\right)$ rises in the cell, reaches a maximum and then decreases, as expected. The inhibition exerted on a hypothetical enzyme in the cell is also shown in the figure, assuming the inhibition to be rapidly reversible. In most cases when inhibitors are administered to animals, the enzyme inhibitions in the various tissues will rise and fall in some such manner. The deviations observed when $k_{1}$ does not equal $k_{2}$ are shown in Fig. 8-4. The maximal $\left(I_{2}\right)$ reached depends on the relative values of $k_{1}$ and $k_{2}$; the greater $k_{1}$ is relative to $k_{2}$, the higher will be $\left(\mathrm{I}_{i}\right)_{\max }$. The maximal level of $\left(\mathrm{I}_{l}\right)$ and the time required to reach this may be obtained by differentiation of Eq. 8-9 and setting it equal to zero; for the special case when $k_{1}=k_{2}$ :

$$
\begin{aligned}
\left(\mathrm{I}_{i}\right)_{\max } & =0.275\left(\mathrm{I}_{o}\right)_{0} \\
t_{\max } & =0.861 / k
\end{aligned}
$$

where $k=k_{1}=k_{2}$.

It has been assumed that disappearance of the inhibitor in the cell is first-order. If the inactivation is enzymic, it may be necessary to modify the treatment by introduction of the appropriate rate expressions in Eq. 8-6 and 8-7. The difference in rates of disappearance for the two types of reaction is shown in Fig. 8-5; the enzymic reaction is initially slower and eventually faster than the first-order reaction, when the over-all rates of the two are comparable. However, the modification of the above treat- 
ment due to an enzymic reaction would be relatively slight unless $\left(\mathrm{I}_{i}\right)$ attained near-saturating concentrations, which must be uncommon. The curve for an enzymic reaction was obtained by integration of the MichaelisMenten equation, from which:

$$
\left[(\mathrm{I})_{0}-(\mathrm{I})\right]-2.3 K_{m} \log (\mathrm{I}) /(\mathrm{I})_{0}=V_{m} t
$$

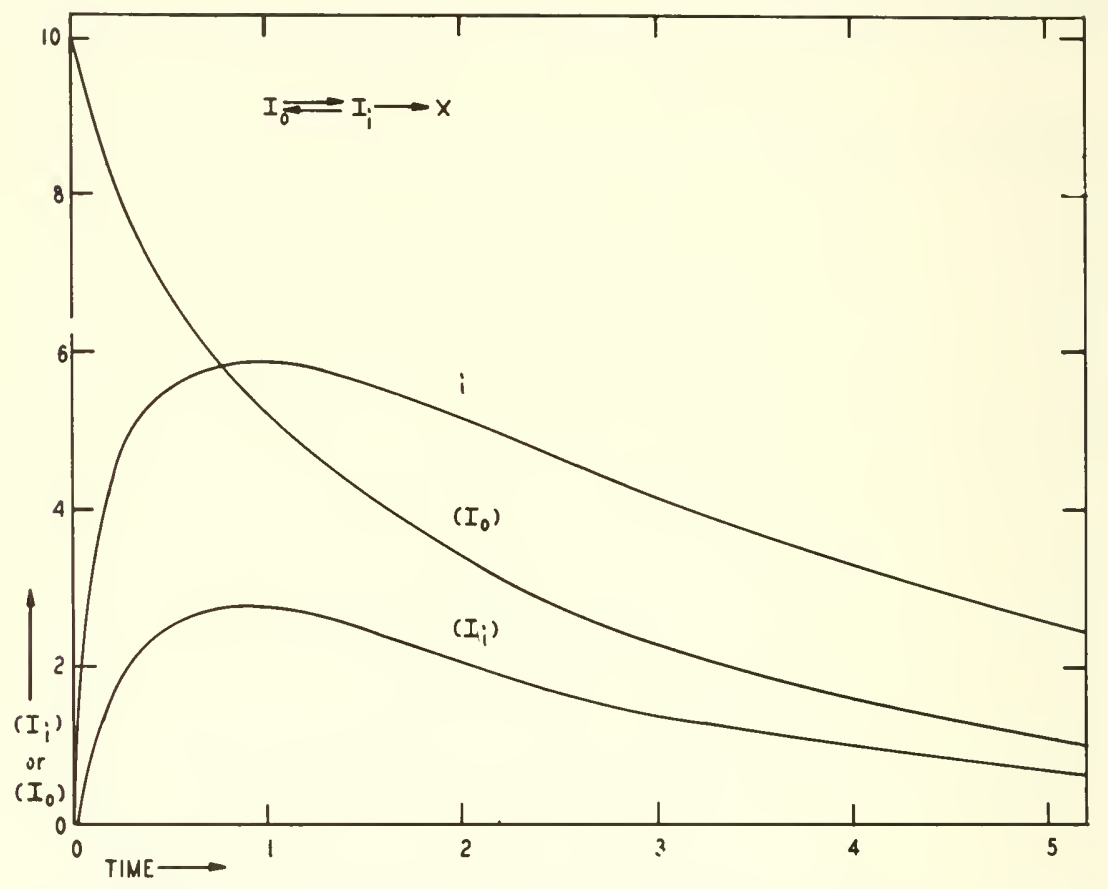

FIG. 8-3. Variation of intracellular inhibitor concentration and inhibition with time (Eq. 8-5). It is assumed that $k_{1}=k_{-1}=k_{2}$. Equations 8-8 and 8-9 then take the following forms when $\left(\mathrm{I}_{o}\right)_{0}=10 \mathrm{~m} .1 /$ and the $k$ 's are unity:

$$
\begin{aligned}
& \left(\mathrm{I}_{\omega}\right)=7.24\left(0.382 e^{-2.618 t}+e^{-0.382 t}\right) \\
& \left(\mathrm{I}_{i}\right)=4.47\left(e^{-0.382 t}-e^{-2.618 t}\right)
\end{aligned}
$$

The inhibition is assumed to be noncompetitive with $K_{i}=2 \mathrm{~m} \boldsymbol{H}$.

A situation of even more common occurrence in the administration of inhibitors to animals may be represented as:

$$
\mathrm{Y} \stackrel{k_{0}}{\leftarrow} \mathrm{I}_{o} \underset{k_{-1}}{\stackrel{k_{1}}{\rightleftharpoons}} \mathrm{I}_{i} \stackrel{k_{2}}{\rightarrow} \mathrm{X}
$$

where $\mathrm{Y}$ designates excretion from the blood stream through the kidney: the inhibitor introduced into the circulation can thus either enter the tissues 
and be inactivated or be lost by renal excretion. both usually occurring simultaneously. The equations are similar to those for the simpler case:

$$
\begin{aligned}
& \left(\mathrm{I}_{0}\right)=\left(\mathrm{I}_{0}\right)_{0} \frac{1}{\alpha-\beta}\left[\left(k_{1}+k_{0}+\alpha\right) e^{\beta t}-\left(k_{1}+k_{0}+\beta\right) e^{\alpha t}\right] \\
& \left(\mathrm{I}_{i}\right)=\left(\mathrm{I}_{0}\right)_{0} \frac{k_{1}}{\alpha-\beta}\left[e^{\alpha t}-e^{\beta t}\right]
\end{aligned}
$$

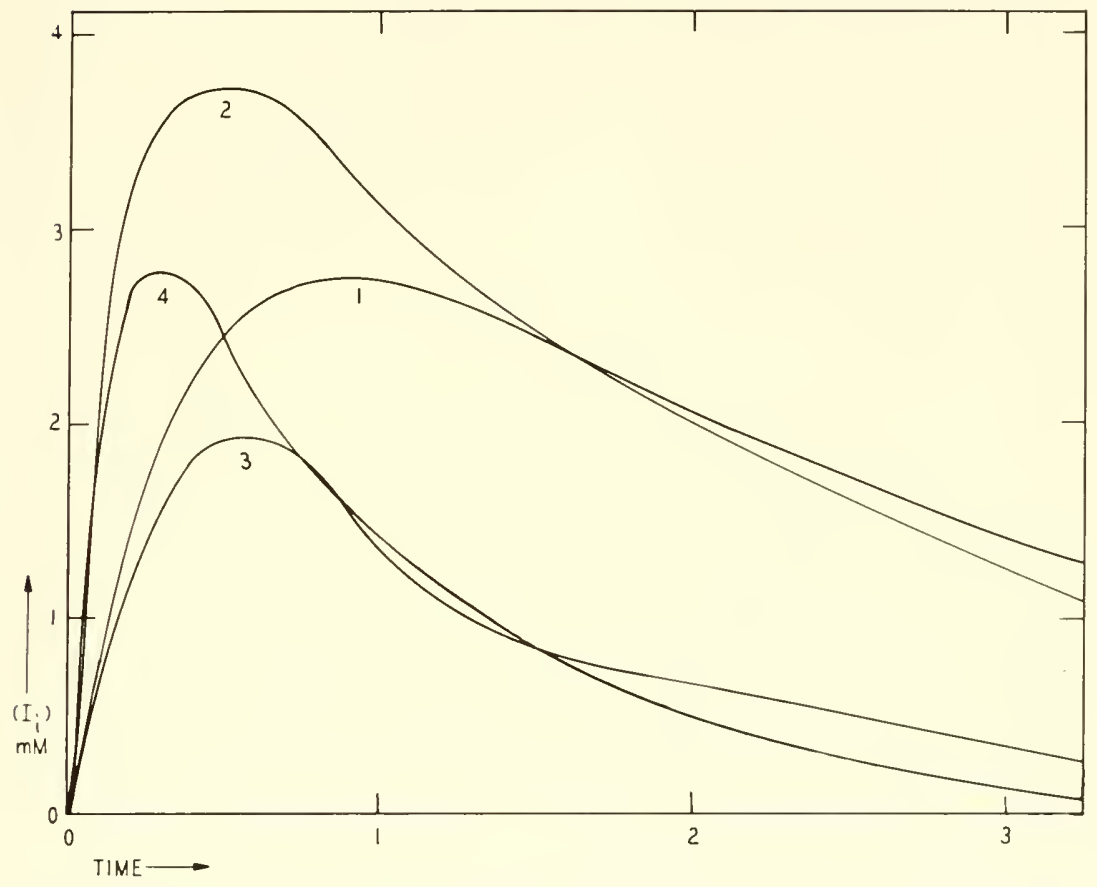

FIG. 8-4. Variation of intracellular inhibitor concentration with time for different values of the rate constants. $\left(\mathrm{I}_{0}\right)_{0}=10 \mathrm{~m} M$. Curve $1: k_{1}=1 . k_{2}=1$; curve $2: k_{1}=3$, $k_{2}=1$; curve $3: k_{1}=1 . k_{2}=3$; curve $4: k_{1}=3, k_{2}=3$.

where the constants have the following values:

$$
\begin{aligned}
& \alpha=1 / 2\left[-\left(2 k_{1}+k_{2}+k_{0}\right)-\sqrt{4 k_{1}^{2}+k_{2}^{2}+k_{0}^{2}-2 k_{0} k_{2}}\right] \\
& \beta=1 / 2\left[-\left(2 k_{1}+k_{2}+k_{0}\right)+\sqrt{4 k_{1}^{2}+k_{2}^{2}+k_{0}^{2}-2 k_{0} k_{2}}\right]
\end{aligned}
$$

It may be seen by inspection of these equations that $\left(\mathrm{I}_{i}\right)$ will reach lower values than in circumstances where no excretion occurs and that its rate of decrease will be greater. If, in these equations, $k_{2}$ is set equal to zero, 
the situation represented will be that in which no inactivation of the inhibitor occurs in the tissues and only excretion is responsible for loss of the inhibitor. The effects of excretion on the intracellular inhibitor concentration are shown in Fig. 8-6; as the excretion becomes relatively larger, the maximal intracellular level reached is less, although the early rate of entry into the cells is approximately the same in each case. The marked influence such excretion may have on the degree of inhibition produced is evident.

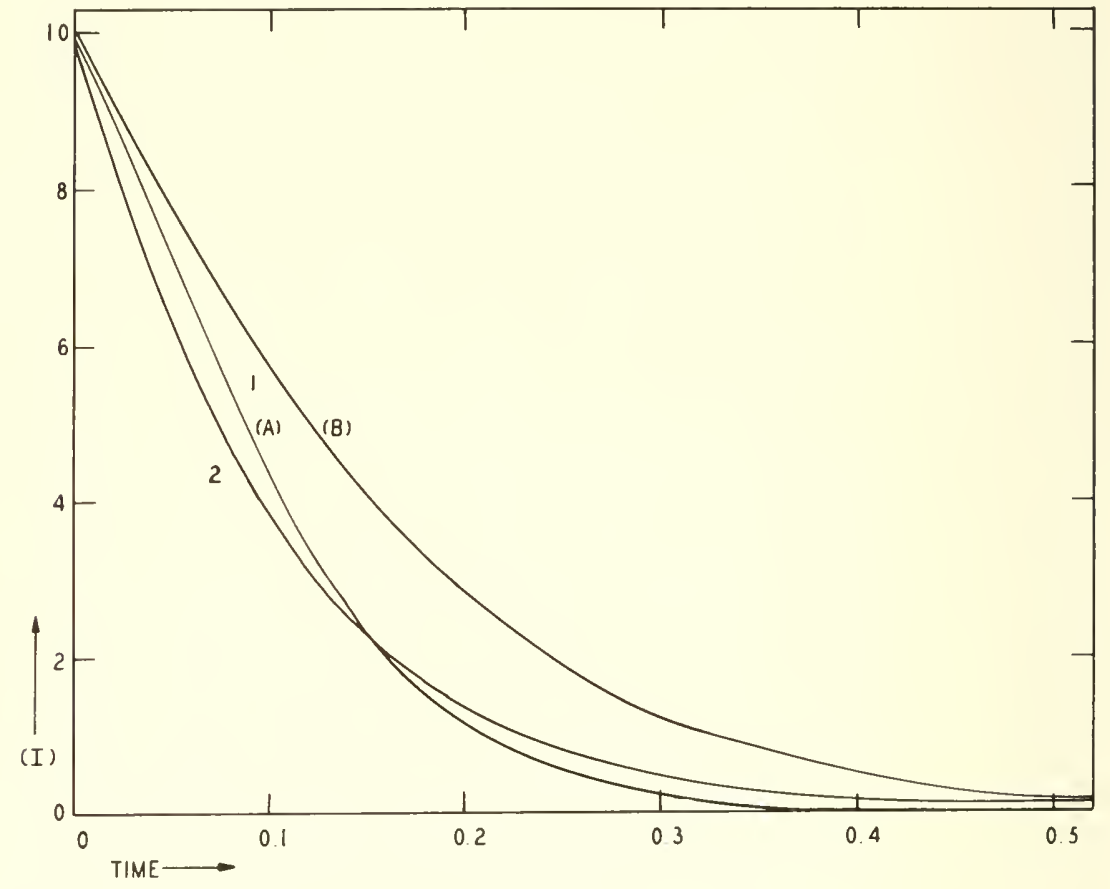

FIG. 8-5. Comparison of the rates of disappearance of an inhibitor being destroyed enzymically and by a first-order reaction. Curve 1: enzymic (Eq. 8-13), $\left(\mathrm{I}_{o}\right)_{0}=10$ $\mathrm{m} M$ and $Y_{m}=100 ;(\mathrm{A}), K_{m}=5 \mathrm{~m} M$; (B), $K_{m}=10 \mathrm{~m} M$. Curve 2: nonenzymic (Eq. 8-4); $k_{1}=10$.

It is scarcely necessary to point out that there are several factors which may modify this idealized behavior. The rate of entry of the inhibitor into the cell may not be linearly proportional to the external concentration throughout the experimental range; cells may become saturated with inhibitor or active membrane processes may occur. Likewise, the excretion may not be proportional to $\left(I_{o}\right)$, especially if tubular secretion or resorption of the inhibitor takes place. The binding or trapping of inhibitor within the tis- 
sues will delay the eventual inactivation or excretion. Finally, the enzyme inhibition itself need not follow the level of $\left(I_{i}\right)$, since the rate of combination of the inhibitor with the enzyme may be slow or the reversibility of the inhibition may be the limiting factor. However, these and other factors, when known, can be superimposed on the basic belavior expressed by the equations derived here. The important concepts to be gained by a study of these equations relate to the dependence of intracellular inhibitor concentration on the relative rates of the fundamental processes

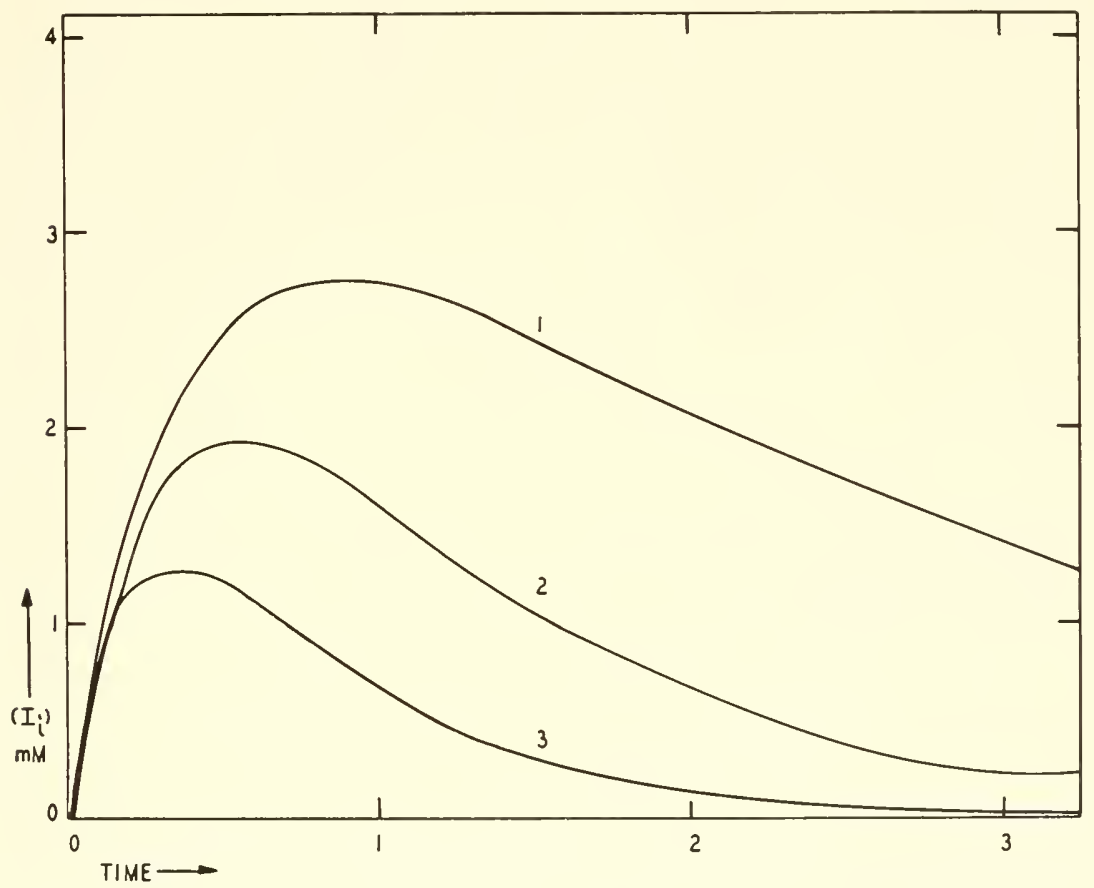

FIG. 8-6. Variation of intracellular inhibitor concentration with time in an organism in which the inhibitor is excreted as well as destroyed in the tissues. $\left(\mathrm{I}_{0}\right)_{0}=10$ $\mathrm{m} M$ and $k_{1}=k_{2}=1$. Curve 1: no excretion (Eq. 8-9); curve 2: excretion with $k_{o}=1$ (Eq. 8-16); curve 3: excretion with $k_{o}=3$ (Eq. 8-16).

that are involved, namely the rate of uptake, the rate of inactivation, and the rate of excretion.

If the inhibitor is metabolized to another compound in the body and both the inhibitor and its metabolic product are exereted from the body, the concentration of each is determined by the rate constants of the metabolic process and the excretion. This problem was treated by Nelson and O' Reilly (1960) and applied to the behavior of sulfisoxazole in the human. 
This drug is acetylated in the tissues and both forms are excreted throngh the kidneys. This sitnation may be represented as:

$$
\mathrm{I}_{e} \stackrel{k_{3}}{\leftarrow} \mathrm{I}_{i} \stackrel{k_{1}}{\rightarrow} \mathrm{I}_{m} \stackrel{k_{2}}{\rightarrow} \mathrm{I}_{m e}
$$

where $I_{i}$ is the inhibitor within the body, $I_{e}$ is the excreted inhibitor, $I_{m}$ is the metabolic product, and $\mathrm{I}_{m e}$ is the excreted metabolic product. It may be pointed ont that either $I_{i}$ or $I_{m}$ may be the active inhibitory form, or that both may participate in the inhibition to varying extents. The following differential equations may be written:

$$
\begin{gathered}
\frac{d\left(\mathbf{I}_{i}\right)}{d t}=-\left(k_{1}+k_{3}\right)\left(\mathrm{I}_{i}\right) \\
\frac{d\left(\mathrm{I}_{m}\right)}{d t}=k_{1}\left(\mathrm{I}_{i}\right)-k_{2}\left(\mathrm{I}_{m}\right) \\
\frac{d\left(\mathrm{I}_{m e}\right)}{d t}=k_{2}\left(\mathrm{I}_{m}\right) \\
\frac{d\left(\mathrm{I}_{e}\right)}{d t}=k_{3}\left(\mathrm{I}_{i}\right)
\end{gathered}
$$

If we set $k_{1}+k_{3}=k$ (representing the over-all rate of removal of the inhibitor from the body) and let $f$ be the fraction of the inhibitor that is excreted unchanged:

$$
\begin{aligned}
& \frac{d\left(\mathrm{I}_{i}\right)}{d t}=-k\left(\mathrm{I}_{i}\right) \\
& \frac{d\left(\mathrm{I}_{e}\right)}{d t}=f k\left(\mathrm{I}_{i}\right)
\end{aligned}
$$

Expressions for the concentrations of the forms of the inhibitor and the amounts excreted may be obtained by integration and combination of these equations.

$$
\begin{aligned}
\left(\mathrm{I}_{i}\right) & =\left(\mathrm{I}_{i}\right)_{0} e^{-k t} \\
\left(\mathrm{I}_{e}\right) & =f\left(\mathrm{I}_{i}\right)_{0}\left[1-e^{-k t}\right] \\
\left(\mathrm{I}_{m}\right) & =\left[\left(\mathrm{I}_{m}\right)_{0}+\frac{k_{1}\left(\mathrm{I}_{i}\right)_{0}}{k-k_{2}}\right] e^{-k_{2} t}-\left[\frac{k_{1}\left(\mathrm{I}_{i}\right)_{0}}{k-k_{2}}\right] e^{-k t} \\
\left(\mathrm{I}_{m e}\right) & =\frac{1}{k-k_{2}}\left\{\left[k\left(\mathrm{I}_{m}\right)_{0}-k_{2}\left(\mathrm{I}_{m}\right)_{0}+k_{1}\left(\mathrm{I}_{i}\right)_{0}\right]\left[1-e^{-k_{2} t}\right]\right. \\
& \left.-\left[\frac{k_{1} k_{2}\left(\mathrm{I}_{i}\right)_{0}}{k}\right]\left[1-e^{-k t}\right]\right\}
\end{aligned}
$$


where $\left(I_{i}\right)_{0}$ and $\left(I_{m}\right)_{0}$ are the initial concentrations of inhibitor and its metabolic product in the body. The curves derived from these equations fit quite accurately the experimental data and the various rate constants were calculated for sulfisoxazole. Although there are many factors that may modify these simple relationships, such an approach is valuable in providing a base from which more complex behavior may be derived.

\section{REPEATED ADMINISTRATION OF AN INHIBITOR}

Occasionally it is important to know the inhibitor concentration in the animal body when the inhibitor is administered repetitively. Accumulation of the inhibitor will occur to varying extents when the intervals between administrations is shorter than the time required for complete disappearance of the inhibitor from the body. Repeated dosage is one way in which the inhibitor concentration in a tissue may be built up to high levels, withont the acute effects sometimes occurring when the total dose of the inhibitor is given all at once. Such considerations are particularly important when an inhibitor is being used to suppress a particular type of cell, as in the growth inhibition of neoplastic cells. There are two basic questions: (1) what will be the final concentration achieved upon repeated administration? and (2) how long will it take to achieve this level or any particular concentration?

The simplest situation is when the inhibitor is introduced suddenly and disappears at a rate proportional to its concentration, the concentration at any time after the administration being given by $(\mathrm{I})=(\mathrm{I})_{0} e^{-k t}$ as shown in Fig. 8-7. If a second administration of inhibitor is made while the concentration is falling, the maximal concentration reached will be the sum of that already present and the added inhibitor; for example, if the second dose is given at time 10 in the case chosen, the concentration will immediately rise to $13.7 \mathrm{~m} . \boldsymbol{I}$, and if a third dose is given at time 20 , the level reached will be $15.05 \mathrm{~m} . \mathrm{H}$. Each additional dose will produce a progressively smaller increment to this maximal concentration until. for practical purposes, a constant level is reached. This is illustrated in Fig. 8-8, where in the case chosen the maximal concentration of inhibitor is essentially reached after the fifth dose. If the administration is stopped at this point, the time taken for the concentration to fall to some designated low level will be greater than after a single dose.

It may easily be shown that the concentration level reached following the $n$th dose will be $(\mathrm{I})_{n}=(\mathrm{I})_{p}\left[1+e^{-k \cdot 1 t}+e^{-2 k \cdot t t}+\ldots+e^{-(n-1) k \cdot 1 t}\right]$. This geometrical series may be written in its equivalent form:

$$
(\mathrm{I})_{n}=(\mathrm{I})_{p} \frac{1-e^{-n k \Delta t}}{1-e^{-k \cdot 1 t}}
$$


where $1 t=$ the interval between doses and $(\mathrm{I})_{p}$ is the concentration peak produced by a single dose. The maximal constant level attained will be:

$$
(1)_{\max }=(\mathrm{I})_{\rho} \frac{1}{1-e^{-k .1 t}}
$$

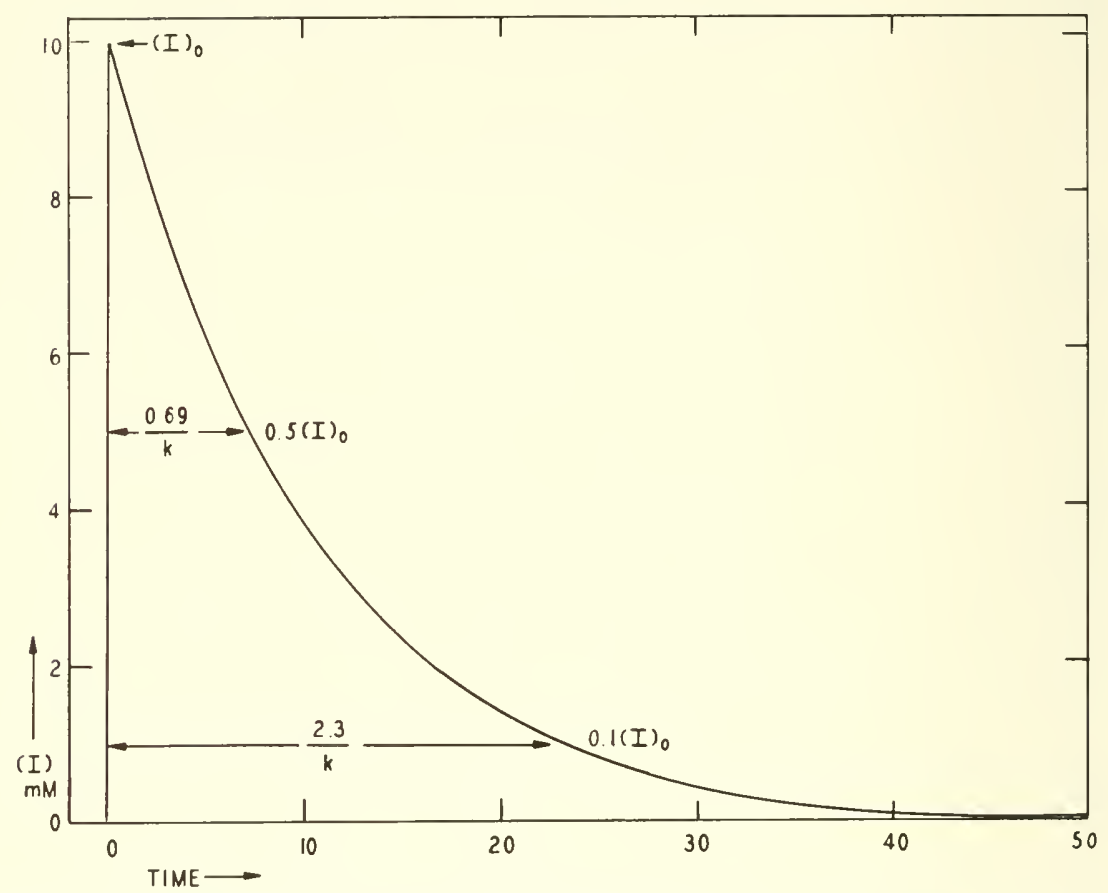

FIG. 8-7. Variation of the inhibitor concentration with time following a single administration assuming $(\mathrm{I})=(\mathrm{I})_{0} e^{-k t}$. $(\mathrm{I})_{0}=10 \mathrm{~m} M$ and $k=0.1$. The time required for the inhibitor concentration to fall to one-half its initial value is $0.69 / k$ and to one-tenth is $2.3 / k$.

The time required to reach this maximal level would be infinite theoretically but for pratical purposes we can calculate the time required to reach to within $90 \%$ of this final concentration [i.e., $90 \%$ of the way between (I) and $(\mathrm{I})_{\max }$ ]. Substituting $0.1(\mathrm{I})_{n}+0.9(\mathrm{I})_{\max }$ for $(\mathrm{I})_{n}$ in Eq. 8-19, the following general relationship is obtained:

$$
(n-1) k: 1 t=2.3
$$

Since the interval between the first dose and the $n$th dose is $(n-1)<1$, the time for the concentration to rise to the $90 \%$ level is:

$$
t_{0.8}=2.3 / k
$$


This is the same time as is required for the concentration after a single dose to fall to $10 \%$ of $(\mathrm{I})_{0}$. It is interesting that $t_{0.9}$ does not depend on how frequently the inhibitor is administered; the shorter the interval between doses, the more must be given, but the total time required is the same. The rise in concentration with the number of administrations and with

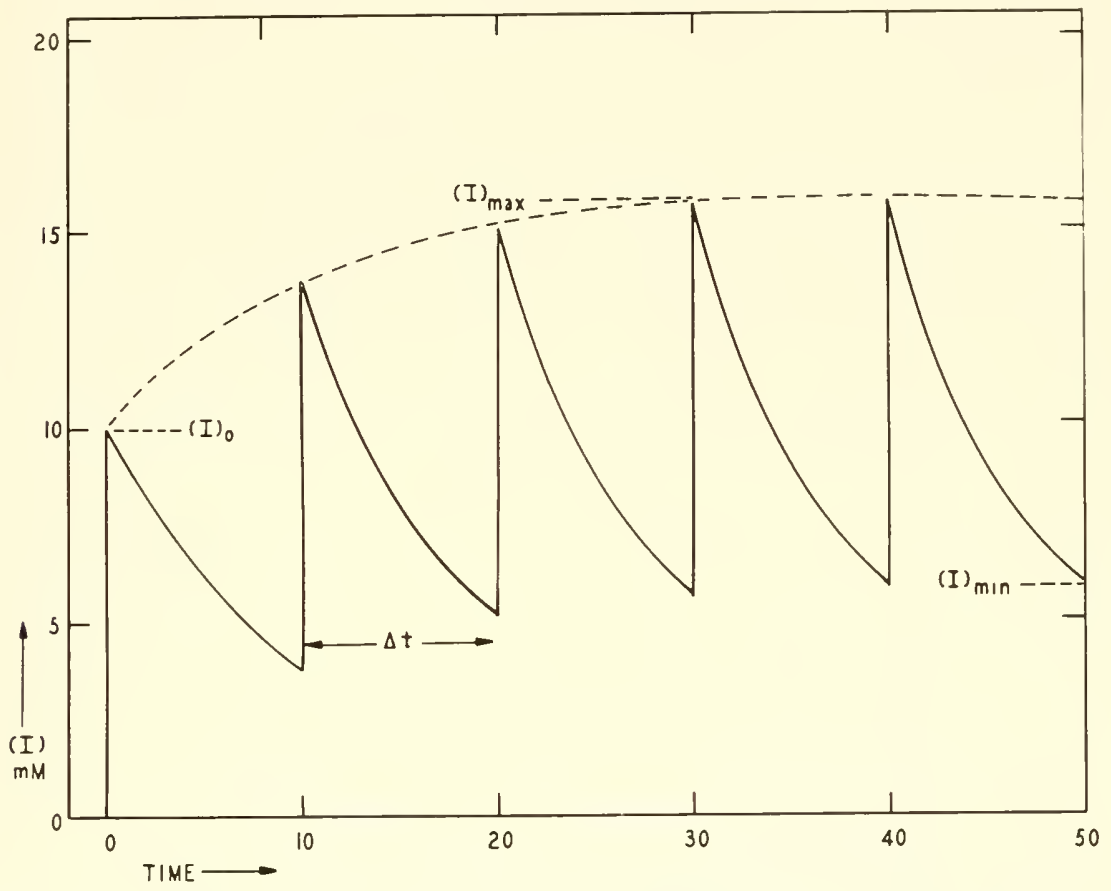

FIG. 8-8. Changes in the intemal inhibitor concentration during repeated intravenous administrations in an animal. $\Delta t=10$ and $k=0.1$. The final concentrations reached after approximately five administrations are $(\mathrm{I})_{\min }=5.81 \mathrm{m.H}$ and $(\mathrm{I})_{\max }=15.81 \mathrm{~m} / /$.

the time is plotted in Fig. 8-9 for different intervals between doses. The very high inhibitor concentrations reached with frequent administrations are evident. Likewise, for a constant dosage interval, the slower the disappearance of the inhibitor (the smaller $k$ ). the ligher the final level reached. If the inhibitor is tightly bound to the tissues, continuous administration can result in very high total concentrations. but the concentration of free inhibitor in solution may not rise greatly and the inhibition on a particular enzyme need not follow the total concentration of inhibitor.

The value of such a quantitative formulation of accumulation is that it can aid in the rational planning of experimental procedures. If a constant 
inhibitor concentration in a tissue is desired over a period of time, instead of a continuously changing concentration as after a single dose, it is possible to determine the dosage and interval to achieve this on the basis of very little basic data (the concentration or effect produced by a certain dose

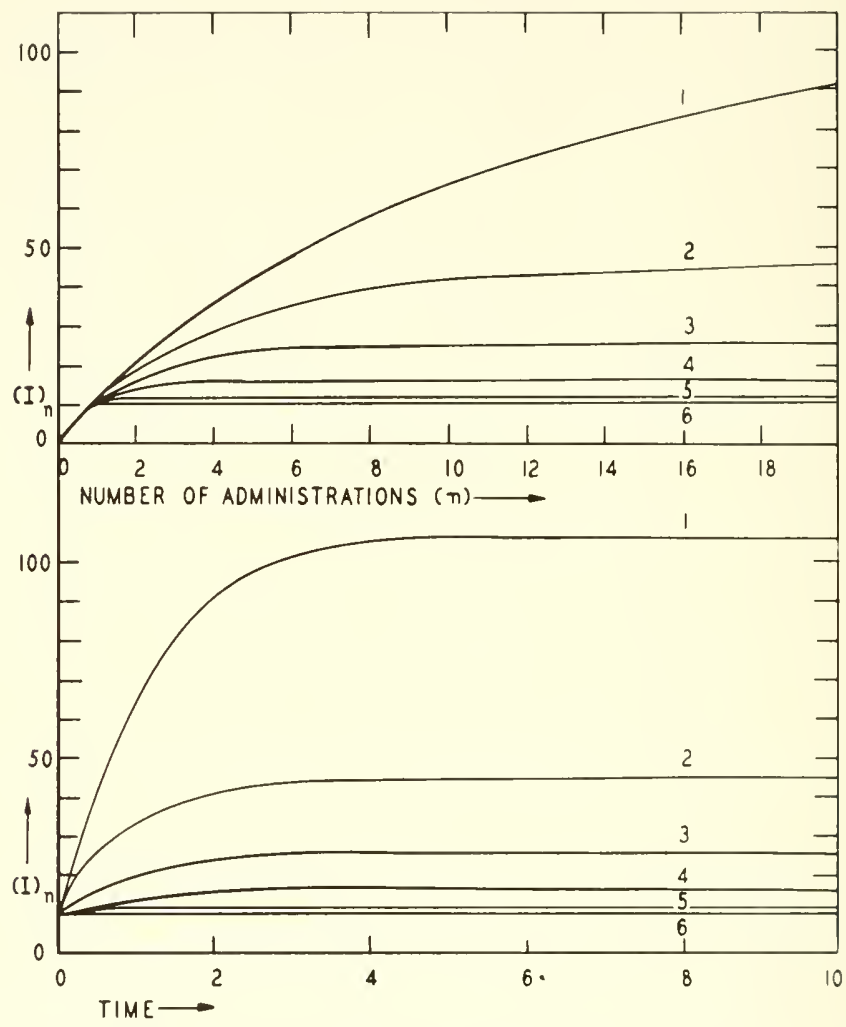

FIg. 8-9. Increases in the internal inhibitor concentration with the number of administrations and with the time for different intervals between the doses (Eq. 8-30). ( $\mathrm{I})_{0}=10 \mathrm{~m} . M$ and $k=1$. Curve 1: $\Delta t=0.1$; curve $2: \Delta t=0.25$; curve $3: \Delta t=0.5$; curve 4: $\Delta t=1$; curve $5: \Delta t=2$; curve $6: \Delta t=3$.

and the rate of disappearance of the inhibitor). Although many factors may modify the above simple treatment, whatever the mechanisms involved in the time course of inhibitor effect, the principle of superposition of curves and final constant levels is valirl. One important complication may, however, be mentioned. When the peak concentration from a single administration is not reached immediately, but follows a curve such as discussed in the previons section, it might seem that the behavior of the system would 
differ appreciably from that of the system assumed in this treatment. Actually, the time course of the concentration maxima is described quite accurately by Eqs. 8-19 and 8-20, providing the costant $k$ is evaluated properly. The determination of $k$ in any case is best done from a plot of the log concentration (or log total amount in body or tissne) against time, since in most cases an approximately straight line will be obtained. In the simple case (Eq. 8-4), the slope will be $-k$, and in the more complex situation of intracellular inhibitor (Eq. 8-9). the slope will be simply $\beta$ over most of the range of falling concentration. It might also be pointed out in this comnection that the approximate valnes of $k_{1}$ and $k_{2}$ may be found if the behavior is similar to that in Fig. 8-4. The value of $\beta$ is determined as just described and from the peak concentration reached (where $\alpha e^{\alpha t}=\beta e^{\beta t}$ ), the value of $\alpha$ is obtained. Since the intercept on the $\ln \left(I_{i}\right)$ axis is $k_{1}\left(I_{o}\right)_{0} /$ $(\beta-\alpha)$, the value of $k_{1}$ and hence $k_{2}$ may be found.

\section{DISTRIBUTION AND EFFECT OF INHIBITORS}

Occasionally there may be a good correlation between the distribution of an inhibitor in the tissues or cells of the body and the degree of inhibition that is produced. However, when one is not determining the effective concentration of inhibitor, a lack of correlation can be expected. It is especially important to realize that the relative effects in the different tissues need not correspond to differences in distribution. The rate of accumulation of citrate following the aconitase block induced by the administration of fluoracetate is not necessarily proportional to the concentration of the inhibitor present in the tissue, but is perhaps more closely related to the rate of cycle operation, that is the rate of citrate formation. Similarly, the degree to which an inhibitor affects the functioning of the various tissues is even less likely to be related to the distribution of the inhibitor for, as will be discussed in the following chapter, the dependency of functional activity on metabolism varies markedly from tissue to tissue. The distribution of an inhibitor throughout the body can only be determined by direct analyses of the tissues and little reliance can be placed on the differential effects on metabolism or function. Inasmuch as the specific actions of certain inhibitors endow them with the possibility of being useful either as experimental tools or in the treatment of disease, it would seem that some quantitative studies of the correlation (or lack of it) between distribution and inhibition would be quite valuable, not only with single administrations but with repeated doses of the important inhibitors. Such relationships will be discussed wherever possible in the chapters on specific inhibitors but a deficiency will always be evident due to the lack of comprehensive experimental data. 



\section{INHIBITION IN CELLS AND TISSUES}

The previous chapters have been primarily concerned with inhibition of enzymes isolated from cells and studied in artificial media. When attention is turned to inhibition in intact living cells or whole animals, many new problems are presented as a result of the increased organization and complexity of the metabolic systems acted upon. Information obtained on isolated enzymes is valuable at the enzyme level and inhibition studies have contributed to our knowledge of the nature of active sites and enzyme mechanisms. However, the most important aspects of enzyme inhibition are the elucidation of the metabolic events and pathways in cells and organisms, the determination of the relationship between cell function and metabolism, and the use of inhibitors to alter specifically certain processes or depress selectively the growth of cells invading the organism. The full importance of inhibitors as tools in biochemical, physiological, and clinical research is manifested only when the inhibitors are applied to living systems. The basic knowledge gained from isolated enzymes must be extended to these enzymes when they are integral units of the organized metabolic complex. The basic question immediately arises: what is the relation between inhibition observed on an isolated enzyme and the inhibition of that enzyme in the cell? When the characteristics of a particular inhibition are known from studies of the pure enzyme - the effect of inhibitor concentration on the degree of inhibition, the type of inhibition, the rate of inhibition, the reversibility, the various constants - one may inquire as to how reliable these characteristics are when applied to the living cell. Many important correlations and conclusions from the use of inhibitors depend on the assumption that extra- and intracellular inhibitions are similar or indeed identical. One would prefer to believe that what happens outside the cell may actually happen within, but the matter is more complex than has been generally assumed and there are now reasons for suggesting that a well-balanced pessimism is not inappropriate. The statement by Davenport (1956) well expresses the modern more realistic attitude: "Only the most fortunate combination of circumstances will allow one to relate quantitatively the action of an enzyme in an extract and its activity in the intact cell." 
The activity and susceptibility to inhibition of an enzyme depend on the state of the enzyme and since there are several possible reasons why the states of isolated and intact enzymes should differ, it is reasonable to anticipate that an exact correlation between the behavior of an enzyme in the cell and the behavior of the same enzyme in an extract or in a purified state would seldom be possible. Some of the factors responsible for these different states of an enzyme may be outlined.

(I) Different chemical compositions of the media

A. Inorganic ions (including $\mathrm{pH}$ )

$B$. Organic substances (both low molecular weight compounds, such as glutathione, and high molecular weight compounds, such as proteins and nucleic acids).

(II) Different physical states of the enzyme.

$A$. Complexes of the enzyme with other substances, such as lipids, in the cell, or a variety of possible compounds released from their normal sites during extraction

$B$. Presence of the enzyme at an interphase or in a molecular layer

(III) Different temperatures

(IV) Different states or concentrations of the enzyme's substrate

A. Enzyme may be part of an organized multienzyme system so that its substrate may be immediately available in a high local concentration or activated form

B. Enzyme may be separated from its substrate by membranes that reduce the substrate concentration in the region of the enzyme

(V) Different concentrations of the inhibitor used

The last two categories have been discussed in the previous chapters. The effects of $\mathrm{pH}$ on enzyme inhibition will be treated in Chapter 14 and the effects of temperature, ions. ionic strength, and buffers in Chapter 15; for the present we may assume that these factors are often of significance. Some inquiry must now be made into the differences in enzyme environment within the cell and in the media generally used for the investigation of isolated enzymes.

\section{CHEMICAL COMPOSITION OF THE MEDIA}

It is, of course, impossible at the present time to duplicate exactly intracellular conditions in the study of isolated enzymes, since our understanding of the normal enzyme environment in the cell is limited. The usual media employed in the characterization of enzymes contain only a buffer in ad- 
dition to the enzyme and substrate; when a requirement for a coenzyme or cofactor is known, such may also be added. The $\mathrm{pH}$ may be chosen arbitrarily or as that giving maximal enzyme activity, but is seldom selected with any reference to the intracellular $\mathrm{pH}$. It must be admitted that, although much attention has been given to the designing of appropriate extracellular media, a minimal effort has been expended in the attempt to test enzymes under reasonably normal conditions. The enzymologist has been more interested in characterizing an enzyme in chemical or catalytic terms than in determining its behavior under physiological conditions. In attempting to extend inhibition studies on isolated enzymes to cellular levels, it must be realized that most enzyme media used are grossly unphysiological.

A cursory examination of some five dozen studies on enzymes isolated from mammalian tissues (in the 1959 issues of the Joumal of Biological Chemistry) showed that in $60 \%$ only a buffer was used, in $35 \%$ a cofactor was added (such as $\mathrm{Mg}^{++}$), and in the remaining $5 \%$ some stabilizer, such as glutathione, cysteine. or EDTA, was present. No attempt was made to create a medium ionically physiological. In some cases sodium buffers were used rather than potassium buffers, despite the preponderance of potassium over sodium in the cell. A similar impression may be obtained by perusing the standard testing conditions for enzymes in "Methods in Enzymology" (Colowick and Kaplan, 1955. Vols. I and II). In the papers examined above, the $\mathrm{pH}$ chosen was anywhere from 5 to 10 , with most between 7.4 and 7.8 ; the $\mathrm{pH}$ was above $7 \mathrm{in} 82 \%$ despite the fact that the intracellular $\mathrm{pH}$ is generally somewhat less than 7 . A variety of buffers was used - potassium phosphate. sodium acetate. glycine, glycylglycine, sodium pyrophosphate, sodium citrate, tris(hydroxymethyl)aminomethane (Tris), barbital. or combinations of these - and these were in concentrations from 7 to $160 \mathrm{~m} . M$. There is little rationale in the nse of an odd assortment of unnatural buffers and if experiments were made nearer the physiological $\mathrm{pH}$ there would be no need for buffers effective outside this range. The testing of enzyme activity in several buffers and choosing the one giving the highest activity also is not conducive to physiological results. It seems reasonable to suggest that the lowest concentration of buffer, commensurate with controlling the $\mathrm{pH}$. should be selected; indeed, in some reactions it is not necessary to have a buffer, at least over the interval measured. $\mathrm{Mg}^{++}$, when used, varied between 2.8 and $25 \mathrm{~m} \boldsymbol{M}$. Temperatures ranged from $23^{\circ}$ to $38^{\circ}$ (in $10 \%$ of the reports it was not specified) and in approximately half the cases it was physiological $\left(37^{\circ}-38^{\circ}\right)$. The balance between $\mathrm{K}^{+}$and $\mathrm{Na}^{+}$ions was seldom considered.

The over-all concentrations of the common physiological inorganic ions in vertebrate cells are reasonably well known and selected values are given in Table 9-1. It is evident that $\mathrm{K}^{+}$is usually at a much higher concentra- 
tion than $\mathrm{Na}^{+}$, with the exception of the kidney. For this reason $\mathrm{K}^{+}$salts should be used as the major source of cation in most work on isolated enzymes. The following ionic concentrations might be considered as approaching a reasonable intracellular enviromment: $135 \mathrm{mM} \mathrm{K}+20 \mathrm{mM} \mathrm{Na} \mathrm{Na}^{+}, 20$ $\mathrm{m} M \mathrm{Mg}^{++}, 1 \mathrm{~m} M \mathrm{Ca}^{++}, 20 \mathrm{~m} M \mathrm{HCO}_{3}^{-}, 10 \mathrm{~m} M$ inorganic phosphate, and a $\mathrm{pH}$ of $6.7-6.8$. The difficulty is in providing sufficient anion to balance the required cation concentrations, since in the cell this anion deficit is supplied by various organic substances. It is likely that it is generally safer to use $\mathrm{KCl}$ rather than high concentrations of phospluate, since most enzymes are not affected markedly by $\mathrm{Cl}^{-}$concentration. The excellent review of Caldwell (1956) may be consulted for the intracellular pH's of the individual tissues but it is clear that most mammalian tissues have a $\mathrm{pH}$ slightly below neutrality when determined by the best techniques. Organic ions make up a major fraction of the total in most cells; in frog muscle the values have been given as amino acids $8.8 \mathrm{mM}$, phosphocreatine 35.2 $\mathrm{m} \boldsymbol{M}$, carnosine $14.7 \mathrm{~m} \boldsymbol{M}$, creatine $7.4 \mathrm{~m} \boldsymbol{M}$, lactate $3.9 \mathrm{~m} \boldsymbol{M}$, ATP $4.0 \mathrm{~m} \boldsymbol{M}$, hexose monophosphates $2.5 \mathrm{~m} M$ (Conway, 1957), and there are many other

TABLe $9 \cdot 1$

Sodium and Potassium Concentrations in Vertebrate Tissues ${ }^{a}$

\begin{tabular}{|c|c|c|c|}
\hline Tissue & Species & $\begin{array}{c}\text { Potassimm } \\
(\mathrm{m} H \text { in cell water) }\end{array}$ & $\begin{array}{l}\text { Sodium } \\
(\mathrm{m} M \text { in cell water) }\end{array}$ \\
\hline \multirow[t]{3}{*}{ Skeletal muscle } & Frog & 136 & 16 \\
\hline & Rat & 171 & 8 \\
\hline & Dog & 140 & 12 \\
\hline \multirow[t]{2}{*}{ Ventricle muscle } & Rat & 143 & 21 \\
\hline & Dog & 125 & 8 \\
\hline \multirow[t]{2}{*}{ Nerve } & Frog & 140 & 37 \\
\hline & Toad & 135 & 42 \\
\hline Liver & Rat & 170 & 87 \\
\hline Kidney & Guinea pig & 98 & 81 \\
\hline \multirow[t]{2}{*}{ Erythrocytes } & Rat & 142 & 23 \\
\hline & Human & 143 & 17 \\
\hline
\end{tabular}

${ }^{a}$ Relatively less data are available for the other physiological ions: $\mathrm{Ig}^{++}=14 \mathrm{mM}$ in frog muscle, $15 \mathrm{~m} . I \mathrm{in}$ rat ventricle, $36 \mathrm{~m} . M$ in rat liver, $11 \mathrm{~m} . U$ in rat erythrocytes; $\mathrm{Cl}^{-}=1.5 \mathrm{~m} M$ in frog muscle, $8 \mathrm{~m} . \mathrm{H}$ in rat ventricle, $27 \mathrm{~m} . M$ in toad nerve, $18 \mathrm{~m} M$ in rat liver, $82 \mathrm{~m} . \mathrm{I}$ in rat erythrocytes; $\mathrm{HCO}_{3}^{-}=10 \mathrm{~m} . \mathrm{H}$ in rat muscle, $14 \mathrm{~m} . U$ in rat ventricle, $20 \mathrm{~m} . I \mathrm{in}$ rat liver, $28 \mathrm{~m} . / \mathrm{in}$ rat erythrocytes, $12.4 \mathrm{~m} M$ in frog muscle; phosphate $=7.3 \mathrm{~m} . I \mathrm{in}$ frog muscle; total phosphates $=143 \mathrm{~m} . I \mathrm{in}$ rat muscle, 100 $\mathrm{m} . U$ in rat ventricle. $123 \mathrm{~m} . \mathrm{I}$ in rat liver. The values have been summarized from numerous references and represent only approximate concentrations, which may vary rather widely with the age or the state of the animal. 
ions present (particularly the various carboxylates, the proteins, and nucleotides). Finally, it may be noted that different regions or cells of an organ may possess different ionic contents, as has been well shown for heart (Davies et al., 1952; Kühns, 1954). It is even possible that within an apparently homogeneous cell population there occur variations between the individual cells (Webb, 1956; Conway, 1957).

A further difficulty in duplicating an intracellular enviromment for an enzyme arises from the fact that the distribution of the ions within the cell is unknown; even when the over-all concentration is known, one cannot be certain that this is the concentration in the enzyme region. In the first place, a fraction of each ion population may be bound. This applies particularly to ions such as $\mathrm{Mg}^{++}$or $\mathrm{Ca}^{++}$, the actual concentration of the free ions possibly being very low, but also to $\mathrm{K}^{+}$and $\mathrm{Na}^{+}$. Myosins $\mathrm{A}$ and $\mathrm{B}$ bind $\mathrm{K}^{+}$and $\mathrm{Na}^{+}$on the imidazole and amino groups (Lewis and Saroff, 1957). A total of around fffty ions may be bound per $10^{5} \mathrm{gm}$ of protein on a molar basis and the dissociation constants for myosin $\mathrm{A}$ are $1.25 \times 10^{-3} \mathrm{M}$ for $\mathrm{K}^{+}$and $6.25 \times 10^{-4} \mathrm{M}$ for $\mathrm{Na}^{+}$with respect to the imidazole sites. Thus appreciable amounts of these ions may be removed from solution in muscle cells. In the second place, there is evidence that mitochondria can possess different ionic concentrations than the external medium (Macfarlane and Spencer, 1953; Davies, 1954; Ulrich, 1960). Apparently both $\mathrm{K}^{+}$and $\mathrm{Na}^{+}$ may be at higher concentrations within the mitochondria, although how much of this is due to binding is not known. Analysis of nuclear, mitochondrial, microsomal, and supernate fractions of liver cells for $\mathrm{K}^{+}, \mathrm{Na}^{+}$, $\mathrm{Ca}^{++}$, and $\mathrm{Mg}^{++}$has shown definite differential distribution (Griswold and Pace, 1956). While $\mathrm{K}^{+}$and $\mathrm{Na}^{+}$are mainly in the supernate $(53 \%$ and $66 \%$ respectively), $\mathrm{Ca}^{++}$is highest in the mitochondria, and $\mathrm{Mg}^{++}$is highest in the microsomes. Studies of the exchangeability of intracellular ions and the rates of efflux of radioactive ions from cells have usually indicated different compartments, fractions of the total ion content having different mobilities. In erythrocytes the $\mathrm{K}^{+}$is divided between at least two compartments (Solomon and Gold, 1955) while in muscle the $\mathrm{Na}^{+}$is not homogeneonsly distributed (Conway, 1954). Similar results have been obtained with these and other ions on a variety of tissues. Summarizing this evidence, it must be admitted that the concentration of an ion in the enzyme region may be either higher or lower than indicated by the over-all concentration.

The effects of the organic compounds of the cell on enzyme properties are mostly obscure. Certain substances, such as ghtathione and ascorbic acid, may regulate activity through redox equilibria with the enzyme $\mathrm{SH}$-groups, or they may exert a protective action against inhibition, and their absence in reaction media may modify the enzyme appreciably. On the other hand, the use of certain substances in media to increase the activity or to stabilize enzyme preparations may create abnormal con- 
ditions. Thus the common incorporation of sucrose (usually $250 \mathrm{mM}$ ) into mitochondrial media may help maintain a steadier metabolic rate but may also alter the biochemical properties of the component enzymes. Likewise, the use of EDTA in mitochondrial suspensions should probably be discontinued because it not only removes calcium and other metal ions from solution, but exerts more direct effects to disturb the normal electron transport and phosphorylation (Yang, 1960).

The purpose of this section is to emphasize the striking discrepancies between conditions within the cell and in the artificial media used for enzyme study. Some of these differences are inherent in the technical impossibility of reproducing complex intracellular conditions, but some are also due to the general lack of interest in investigating enzymes physiologically. This state of affairs introduces uncertainty into the interpretations of inhibition in the cell on the basis of work on isolated enzymes. Uncertainty of this sort was expressed long ago by Linderstrøm-Lang and Holter (1931) but the problen still remains unsolved. In later chapters, correlations between in vitro and in vieo results will be made because at present there is no alternative, but it should always be remembered that the conchusions must be provisional until data from more physiological studies are available.

\section{DISTRIBUTION AND STATE OF ENZYMES IN CELLS}

Enzymes are not distributed homogeneously throughout a cell but are concentrated in certain compartments or subcellular structures. This may be of great importance in cellular inhibition since the accessibility of the inhibitor to the enzyme can determine the effect produced. The manifold reactions of the cell could not be carried out simultaneously in an organized manner without a heterogeneous arrangement of enzymes. Cori (1956) has stressed the role of metabolic compartments in controlling many enzymic reactions. There are many instances known where a substrate and enzyme exist in the cell without reaction and, in some cases at least, this is probably due to spatial separation, which may be broken down when the cell is activated functionally. If enzymes are unavailable to their substrates, it is likely that they are protected from certain inhibitors. Compartmentalization, in addition, can modify enzyme linetics by introducing diffusion factors, and hence alter the response of these systems to inhibitors as already discussed in Chapter 7. Finally, one should be cognizant of the possibility of an inhibitor directly or indirectly affecting the intercompartmental barriers and in this way changing metabolism without any necessary effect on the enzymes involved. 


\section{Distribution of Enzymes in Cell Fractions}

Enzyme analyses of cell components separated from homogenates by centrifugation have invariably shown differential distribution. It has been known for some time that nuclei contain very little or no oxidizing enzymes, but do have enzymes involved in protein and nucleic acid metabolism, such as proteinases, cathepsin, dipeptidases, and ribonuclease (Siebert and Smellie, 1957). The enzymes for the tricarboxylic acid cycle are mainly or entirely located in the mitochondria or related structures, although recently the problem of different types of mitochondria, with varying composition, has arisen (Novikoff, 1957). The different concentrations of enzymes (succinic dehydrogenase, uricase, DPN-cytochrome c reductase, and acid phosphatase) in differently sedimentable fractions from liver indicate a degree of complexity previously umappreciated (Kuff and $\mathrm{Ho}_{\mathrm{o}}$ geboom. 1956). The usual relegation of the glycolytic enzymes to the supernate fraction has been questioned in brain where these enzymes have been found associated in part with the mitochondria (Abood et al., 1959). Although localization of enzymes in the cell undoubtedly occurs, care must be taken not to assume the segregation between cell fractions to be complete. Although much progress has been made in this field, it is evident that the fractionations are still rather crude and errors due to redistribution of enzymes during the preparation are inevitable. Histochemical analyses have also shown characteristic distributions of enzymes in cells but at present the techniques have not advanced far enough to allow detailed localizations, although these methods may eventually provide the desired answers. Results on the distribution of inhibitors within the cell are not of much value unless we also know the enzyme pattern.

\section{Localization of Enzymes Relative to the Plasma Membrane}

Since the plasma membrane is the major barrier to the entrance of inhibitors into the cell, it is of primary importance to determine if the involved enzymes are in the cell, within the membrane, or outside. A great deal of indirect evidence has been presented that some enzymes are at or near the cell surface and are susceptible to influences that do not extend within the cell. If an enzyme is both at the membrane and inside the cell, a specific inhibition of the membrane enzyme may be achieved when the inhibitor does not penetrate into the cell, and a specific functional change in the membrane may be produced.

Yeast $\beta$-fructofuranosidase (invertase) in extracts and suspensions of intact cells is affected equally by changes in $\mathrm{pH}$; it was therefore suggested nearly 30 years ago that this enzyme must be in the outer region of the cell where the $\mathrm{pH}$ follows the external $\mathrm{pH}$ (Wilkes and Palmer, 1932). Comparison of $\mathrm{pH}$ effects on the hydrolysis and fermentation of sucrose 
also pointed to $\beta$-fructofuranosidase being external, while the glycolytic enzymes are within the cell (Myrbäck and Vasseur, 1943). These conclusions have been supported by kinetic studies on $\beta$-fructofuranosidase and hexokinase in intact yeast (Best, $1955 \mathrm{c}$ ). The results indicated that $\beta$-fructofuranosidase was external to the major diffusion barrier and perhaps associated with the polysaccharide of the capsule in the outer space of Conway and Downey (1950). However, some barrier to diffusion seems to exist outside the $\beta$-fructofuranosidase region. Finally, the differential inactivation of $\beta$-fructofuranosidase by bombardment of yeast cells with low voltage electrons of differing penetration powers localized the enzyme to a zone lying between depths of 500 and $1000 \AA$ from the outer surface (Preiss, 1958). Yeast phosphatase also seems to be at the cell surface because, although antibodies inhibitory to hexokinase and pyruvic decarboxylase showed no effect in the intact cell, antiphosphatase was able to inhibit in yeast suspensions (Derrick et al., 1953). Mention may also be made of the evidence for transport enzymes at the yeast cell membrane from studies on uranium inhibition (Rothstein, 1951). The postulated membrane transport enzymes, permease (Monod, 1956; Gale, 1957) and translocase (Mitchell, 1957), must be situated somewhere within the membrane in bacteria but exact localization and susceptibility to inhibition have not been determined. In micrococci the following enzymes appear to be loealized in the membrane: succinic dehydrogenase, lactic dehydrogenase, malic dehydrogenase, formic dehydrogenase, acid phosphatase, and the cytochrome system (Nitchell and Moyle, 1956). Enzyme activity of the erythrocyte stroma has been established, especially for the synthesis of ATP (Prankerd, 1956) but most of the evidence for membrane enzymes in mammalian cells is very indirect. It wonld be reasonable to expect the enzymes involved in active transport and ionic pumps to be situated in or near the membrane. Inhibitor's exert actions on cardiac cell membrane potentials most readily-interpreted by assuming that the enzymes attacked are in the membrane (Webb and Hollander, 1959). The effects of $\mathrm{Ca}^{++}$on brain glycolysis, as related to ehanges in $\mathrm{pH}$, have led to the suggestion that the neuronal surface is an important site o :glucose metabolism (Adams and Quastel, 1956). Finally, it would appear that there are two glycolytic systems capable of forming lactate in diaphragm muscle, one intracellular and able to form glycogen and the other at the membrane and unable to form glycogen (Shaw and Stadie, 1959). Only the former pathway is dependent on insulin, inasmuch as insulin is involved in the inward transport of glucose and the membrane system requires no such transport.

The problems of enzyme localization have been made more complex by the demonstrations of double membranes throughout the cell (Robertson, 1957). The classic plasma membrane seems to consist generally of 
two membranes, each about $100 \AA$ thick and separated by a space that varies in thickness from 100 to $500 \AA$. In addition, certain cells may possess dense regions a few hundred angstroms thick on the inner or outer sides of these membranes. Indirect evidence has been adduced for two membranes of different conductivities at the nerve surface. so that an ion, such as $\mathbf{K}^{+}$, leaving the cell may be temporarily retained between the two membranes (Frankenhaeuser and Hodgkin, 1955). The implications of such double membranes for enzyme inhibition in the cell have never been discussed but it is clear that the rates of penetration into the cell, the localization of the inhibitor, and the specificity of effect upon the cell may all be dependent on these structures. One must now abandon the simple concept of "outside" or "inside" enzymes, since the enzyme may be situated in a variety of regions all near the cell surface. Actually, nine different zones may be imagined in the general case, as illustrated in Fig. 9-1.

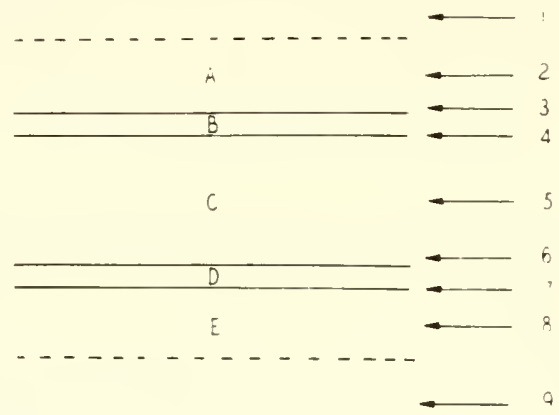

Fig. 9-1. Possible locations of an enzyme near the surface of a cell. B and D represent the double membrane with a space, $\mathrm{C}$, between, while $\mathrm{A}$ and $\mathrm{E}$ are the special regions adjacent to these membranes and are not neeessarily present in every cell. The enzyme may be at: 1 , the extreme outer surface; 2 , in the outer differentiated layer; 3 , at the outer surface of the outer membrane; 4 , at the inner surface of the outer membrane; 5 , in the space between the membranes; 6 , at the outer surface of the inner nembrane; 7 , at the inner surface of the inner membrane; 8 , in the inner differentiated layer; or 9 , in the interior of the cell.

\section{State of Intracellular Enzymes}

Some instances have been noted where enzymes within the living cells seem to have different properties from the isolated enzymes. Thus aconitase, with respect to its inhibition by fluorocitrate, behaves differently when isolated than in intact mitochondria (Peters, 1955), and tryptophanase isolated from $E$. coli has an apparent Michaelis constant some twenty times greater than the enzyme in the cells (Rydon, 1955). The latter observation would indicate a much greater affinity of the enzyme for its substrate within the cell and if this is a common phenomenon it would 
have important bearings on competitive inhibition studies. It is actually not known if these differences are due to the altered environment of the enzyme when isolated or whether it is a true structural variation between the enzymes in the two states. One factor of importance is probably the existence of the enzyme at one of the numerous cell interphases. Catalase can be adsorbed onto artificial interphases to give states from complete activity to complete inactivity (Fraser et al., 1955). It has been suggested that catalase in normal yeast cells exists in a partially unfolded condition at some oil/water boundary, since extraction could increase the activity manyfold. On the other hand, in some cases, extraction from the cell could lead to partial unfolding or denaturation by contact of the enzyme with substances from which it is normally separated. It is also conceivable that an inhibitor within the cell could either directly or secondarily alter these structural conditions so that the activity of an enzyme might change without a direct attack being made upon it. The lipoprotein nature of some enzymes within the cell may favor the stability of active structures and any dissociation of such complexes would change the properties of the enzyme.

\section{TYPES OF INHIBITION UNIQUE TO ORGAINIZED SYSTEMS}

Inhibitors may alter cell metabolism or function in a variety of ways not possible in the simple isolated enzyme preparation. Sometimes the whole cell is not necessary for these more complex types of inhibition, but in all cases it requires the participation of several enzymes or a structurally organized metabolic system. It is not profitable at the present time to treat such inhibitions by a completely quantitative approach because too many unjustified assumptions must be made, but a semiquantitative formulation may be useful in showing what experimental data are needed for a more rigorous treatment.

\section{The True Enzyme Inhibitor Is Formed Metabolically from the Added Substance}

The substance presented to the cells may not itself be an enzyme inhibitor but is chemically transformed through the mediation of the cell enzymes into an inhibitor. The substance from which the inhibitor is formed will be called the precursor. This phenomenon is commonly designated as lethal synthesis, an expression coined by Peters for the metabolism of fluoroacetate into fluorocitrate. Although the result may not be lethal, this terminology is convenient and generally accepted. An excellent discussion of the more general aspects of lethal synthesis has been given by 
Markham (1958). We shall restrict the discussion to cases in which an enzyme inhibitor is formed and omit the incorporation of unnatural analogs (such as the analogs of amino acids, purines, or pyrimidines) into proteins or nucleic acids. since these interfere with metabolism indirectly. Some examples of lethal synthesis in this restricted sense would be: the formation of fluorocitrate from fluoroacetate, resulting in the inhibition of aconitase; the transformation of deoxypyridoxine to deoxypyridoxine phosphate, which interferes with enzymes utilizing pyridoxal phosphate; the formation of DPN and TPN analogs from the acetylpyridines, resulting in disturbances of electron transport: and the formation of 5-fluoronicotinamide from 5-fluoronicotinic acid. blocking the phosphorylation of nicotinamide riboside.

The precursor is usually chemically related to the normal substrate of the enzyme that forms the inhibitor since otherwise it is mnlikely that it would combine with the enzyme. The precursor may differ from the substrate in a single group; the following substitutions are frequently effective, as pointed out by Markham $-\mathrm{Cl}, \mathrm{Br}$, or $\mathrm{I}$ for a $\mathrm{CH}_{3}$ group; $\mathrm{F}$ for $\mathrm{H}$; $\mathrm{SH}$ for $\mathrm{OH}$ or $=\mathrm{S}$ for $=\mathrm{O}$; n-propyl for isopropyl; $-\mathrm{N}=$ for $-\mathrm{CH}=$; $-\mathrm{S}-$ for $-\mathrm{CH}=\mathrm{CH}-$; Se for $\mathrm{S}$ or As for P. It is impossible to predict if the analog will directly inhibit the enzyme or if it will serve as an abnormal substrate. The advantage of an inhibitor formed by lethal synthesis is that it is often quite specific and directed efforts to discover effective precursors are now common.

The kinetics of inhibition resulting from lethal synthesis will depend on the relationship of the enzyme inhibited to the the enzyme forming the inhibitor. If the inhibited enzyme bears no direct relationship to the lethal synthesis, it is evident that the inhibition will be progressive with time, in that the inhibitor will increase in concentration, unless there is a limited supply of the precursor or the inhibitor is eliminated by some mechanism as it is formed. The rise in inhibition will depend, too, on the spatial configuration or compartmentalization of the system because the inhibitor concentration will increase more rapidly if the inhibitor is restricted to the region where it is formed. The great potency of fluoroacetate is in part dne to the accumulation of the fluorocitrate in the mitochondria.

Two simple cases of lethal synthesis may be taken as illustrative of the more general properties of such inhibition. If the inhibition is exerted on the enzyme forming the inhibitor:

$$
\begin{array}{ll}
\mathrm{S} \stackrel{\mathrm{E}}{\rightarrow} \mathrm{P} & v_{s}=\frac{V_{s}(\mathrm{~S})}{(\mathrm{S})+K_{s}} \\
\mathrm{X} \stackrel{\mathrm{E}}{\rightarrow} \mathrm{I} & v_{x}=\frac{V_{x}(\mathrm{X})}{(\mathrm{X})+K_{x}}
\end{array}
$$


where $\mathrm{S}$ is the normal substrate, $\mathrm{P}$ its product, $\mathrm{X}$ the precursor, and $\mathrm{I}$ the inhibitor formed. When I inhibits $\mathrm{E}$ (a form of product inhibition actually), both reactions will, of course, be depressed, and progressively so as (I) increases. The development of the inhibition is thus slowed relative to the situation in which the inlribition is on another enzyme. However, a steady state will not be reached unless $\mathrm{X}$ is limited or I diffuses away. If the inhibition is on a succeeding enzyme in a sequence:

$$
\begin{aligned}
& \stackrel{\mathrm{E}_{1}}{\rightarrow} \mathrm{P}_{1} \stackrel{\mathrm{E}_{2}}{\rightarrow} \mathrm{P}_{2} \\
& \mathrm{X} \stackrel{\mathrm{E}_{1}}{\rightarrow} \mathrm{I} \stackrel{\mathrm{E}_{2}}{\rightarrow} \mathrm{Y}
\end{aligned}
$$

that is, where $I$ inhibits $E_{2}$, the kineties are different than in the previous case. The rate of formation of $\mathrm{P}_{2}$ will be progressively depressed as (I) rises. The level of $(\mathrm{I})$, as in any monolinear chain, will depend on the relative rates of $\mathrm{X} \rightarrow \mathrm{I}$ and $\mathrm{I} \rightarrow \mathrm{Y}$; if the latter is rapid. (I) may reach only low levels and inhibition on $\mathrm{E}_{2}$ will be small. However, inasmuch as $\mathrm{I}$ slows down its own disappearance, its concentration will slowly rise and the inhibition will become greater and greater. If step $\mathrm{I} \rightarrow \mathrm{Y}$ does not occur and $\mathrm{I}$ accumulates, the formation of $\mathrm{P}_{2}$ is progressively depressed but $\left(\mathrm{P}_{1}\right)$ may rise. If $\mathrm{X}$ is limited in amount, after it has been used up the inhibition on the formation of $\mathrm{P}_{2}$ will be the same as if one had added $\mathrm{I}$ in the initial concentration of $\mathrm{X}$, providing the inhibitor can gain access to the enzyme. In the case of fluoroacetate added in small amounts, the inhibition will reach a constant level when the precursor has been utilized, but the inhibition will not be the same as if an equivalent concentration of fluorocitrate were added, because fluorocitrate cannot penetrate readily to the mitochondrial aconitase.

This example illustrates one of the interesting aspects of lethal synthesis. If an inhibitor is incapable of entering a cell, or any region of a cell, a precursor that can penetrate will allow the inhibition to be exerted. The useful group of inhibitors comprising the phosphorylated substrate and coenzyme analogs is usually unable to penetrate into the cell, but the nonphosphorylated precursors may enter and be phosphorylated.

\section{Inhibition of Transport Systems in the Membrane}

The substrate for an intracellular reaction may be obtained from outside the cell and its uptake regulated or limited by the activity of certain proteins or enzymes in the plasma membrane. Two general situations may be recognized. The transport may be active and involve energy derived metabolically, in which case the uptake rate will depend on a number of enzymes as well as the specific transport mechanisms. On the other hand, 
the transport may be facilitated by a combination with certain proteins in the membrane, and no energy other than for maintenance is required. It is claimed that these transport proteins may function similarly to enzymes in that they combine specifically and reversibly with the substrate; however, they act in movement of the substrate from one region to another rather than altering it chemically. These proteins facilitating entry into the cell have been called permeases or translocases.

The terms "permease" and "translocase" are actually misleading when applied to these membrane proteins, since the suffix "-ase" implies that they are enzymes, which they are not in the usually accepted sense, since do not catalyze a chemical reaction; even activation of the substrate probably does not occur. If a true alteration of the substrate occurs during its passage through the membrane and restoration of its original structure occurs before release into the cell, it would be justifiable to call the catalyzing proteins enzymes, but no evidence for such a mechanism has been presented. If the substrate is altered at the membrane and released into the cell in this altered form, it is simply a matter of one enzyme in a sequence being situated in the membrane and there is no need for a special name. The new nomenclature scarcely improves the long-accepted concept of the carrier and introduces nothing unique with respect to specificity. It must also be admitted that there is no direct evidence for specific combinations of substrate with membrane proteins leading to facilitated diffusion.

Whatever the exact nature of these membrane systems may be, it is becoming increasingly evident that cellular metabolism can be readily altered as a result of interference with substrate uptake. An inhibitor acting in this manner could (1) compete with the substrate for the transport system, (2) inactivate one of the components of the membrane, (3) inhibit an enzyme involved in the energy supply for an active process, or (4) depress the synthesis of one of the proteins or enzymes interacting with the substrate (the permease system is inducible and any inhibition of protein synthesis might prevent its formation or reduce its concentration in the membrane). In each case the result will be a reduction in the supply of substrate to some intracellular enzyme. A decrease in substrate utilization or of any metabolic reaction will not be evident unless the rate of uptake into the cell is limiting (or is made to limit) the over-all process. This in turn will depend on the over-all rate and perhaps the level of activity of the cell. When the substrate uptake is determining the rate, the kinetics of the inhibition may be reasonably simple. Competition by the inhibitor in the membrane will provide the same inhibitor-substrate relationships as if the competition were for a single enzyme; indeed, it is more apt to follow the classic competitive laws than for competition on an enzyme within a cell, because concentration gradients are not as likely to occur. On the 
other hand, the other possible mechanisms enumerated above will generally lead to very complex kinetic behavior, although superficially it may appear to be simple.

A discrepancy between the imhibition on a metabolic process in intact cells and the same series of reactions when isolated from the cell may be an indication that transport systems are being affected, but is not proof. The only generally reliable method for demonstrating a selective inhibition on uptake is analysis for the substrate in the cell, and this must usually be done with labeled material since the intracellular concentration may in any case be quite low. If the transport is inhibited, the intracellular concentration of substrate will usually fall, but if an enzyme in the cell is inhibited, the substrate concentration may rise. Mechanisms (3) and (4) above are not likely to produce a specific inhibition of transport because the same systems for formation of energy and synthesis of proteins presumably are used throughout the cell.

\section{Inhibition of Enzyme Synthesis}

Many types of inhibitor can depress the synthesis of enzymes. This is particularly well seen in the case of adaptive or inducible enzymes. Protein synthesis is particularly susceptible to inhibition because it depends on so many phases of cell metabolism. Aside from the complex series of steps that are directly related to formation of protein - the activation of amino acids, the transfer to soluble RNA, the transfer to ribonucleoprotein, and the linking of peptide bonds - there are the ATP-supplying reactions of oxidative phosphorylation, and in some cases the reactions forming one or more of the amino acids required. Whenever an experiment is run over an interval of time during which enzyme synthesis is important, the secondary inhibition of this process should be taken into account, although it seldom is. To take one example, the uncoupling of oxidative phosphorylation by 2,4-dinitrophenol will not only produce immediate effects on the metabolism but will secondarily interfere with protein synthesis and eventually disturb various enzyme systems upon which it has no direct action. Indeed, a "vicious cycle" may be readily set up, since failure in the synthesis of transport proteins or enzymes can further limit the amount of energy available for this synthesis.

Enzyme synthesis may be modified by inhibitors in other ways. An amino acid analog may be incorporated into an enzyme as it is synthetized and, as a result, the catalytic activity may be lost. This has been demonstrated with $p$-fluorophenylalanine and tryptazan. The analog here is not truly an enzyme inhibitor but its introduction can lead to a decrease in enzyme activity. Inasmuch as enzymes, like other proteins, are presumably formed on a template, the binding or interaction of a substance with this 
template may disturb the synthetic process. Some polypeptide antibiotics may possibly inhibit bacterial growth by this mechanism. With respect to enzyme inhibitors, particular attention must be given to those capable of chemically reacting with protein or nucleic acids (such as the arsenicals, mercurials, heavy metals, alkylating agents, and phosphorylating agents). Our ignorance of the exact nature of the template surface prevents more rational predictions as to the compounds most likely to react there.

\section{THE PROBLEM OF ENDOGENOUS METABOLISM}

When an unpurified enzyme is being examined, it is common to find that some enzymic or metabolic activity is exhibited in the absence of added substrate. If the effect of an inhibitor on the metabolism of the added substrate is desired, some correction must be applied to the inhibition observed on the total system. If the respiration of a tissue slice preparation, for example, is being determined in the presence of an added substrate, the inhibition observed upon adding some substance cannot be quantitatively equated with the inhibition on the oxidation of this substrate, mless the proper endogenous controls are also run. In many reports this correction has not been made and frequently one finds no mention of endogenons metabolism in situations where it wonld be expected to occur. Let us consider three examples to illustrate the errors in interpretation that are possible (see tabulation).

\begin{tabular}{|c|c|c|c|}
\hline & Case 1 & Case 2 & Case 3 \\
\hline Endogenous & 6 & 15 & 15 \\
\hline Endogenous + inhibitor & 6 & 3 & 3 \\
\hline Substrate & 30 & 27 & 27 \\
\hline Substrate + inhibitor & 16 & 15 & 20 \\
\hline
\end{tabular}

In the first case, if the endogenous contribution were neglected, the inhibition would be calculated as $46.7 \%$. However, the addition of substrate increases the metabolism by 24 in the absence of the inhibitor and by 10 when the inhibitor is present; thus the inhibition of substrate metabolism is actually $58.4 \%$. This example also shows that even though there is no inhibition of the endogenons metabolism, a correction is necessary. In the second case, the uncorrected inhibition is $44.4 \%$. However, addition of substrate increases the rate by 12 whether inhibitor is present or not; thus there is actually no inhibition of the metabolism of the added substrate. In the third case, an inhibition of around $26 \%$ would be calculated, and yet the substrate produces a greater rise in rate when the inhibitor is present than in the uninhibited preparation. 
When correction for the endogenous factor is made, it is usually done in the manner just described, that is by determining the relative effects of adding substrate in the absence and presence of the inhibitor. This may not be justified in some instances. It has been assumed, for example, that the addition of substrate does not alter the endogenous rate, so that the exogenous and endogenous rates are additive, but many instances where this is not true can be conceived. It is, however, very difficult to determine if a change in endogenous rate does occur, unless the endogenons reaction is so different from the exogenous one that they can be measured separately by some means.

\section{Relations between Endogenous and Exogenous Metabolism}

Let us now consider in more detail the possible effects that an added substrate may have. There are four cases which must be analyzed.

Case 1: one enzyme and one substrate (the same enzyme and the same substrate are responsible for both endogenous and exogenous rates).

$$
\begin{aligned}
& \mathrm{S}_{e}+\mathrm{E} \rightleftharpoons \mathrm{ES}_{e} \rightarrow \mathrm{E}+\mathrm{P} \\
& \mathrm{s}+\mathrm{E} \rightleftharpoons \mathrm{ES} \rightarrow \mathrm{E}+\mathrm{P}
\end{aligned}
$$

(The subscript $e$ will be used to designate whatever is endogenous; in this case, $S_{e}$ and $S$ are the same substrate but the former occurs with the enzyme and the latter is added.) The endogenous rate will be $v_{e}=V\left(\mathrm{~S}_{e}\right) /$ $\left[\left(\mathrm{S}_{e}\right)+K_{m}\right]$ and the rate after the substrate is added will be $v=V\left[\left(\mathrm{~S}_{e}\right)+\right.$ $(\mathrm{S})] /\left[\left(\mathrm{S}_{e}\right)+(\mathrm{S})+K_{m}\right]$. If no endogenous reaction were occurring, the rate due to the added substrate would be $V(\mathrm{~S}) /\left[(\mathrm{S})+K_{m}\right]$, which is clearly not $v-v_{e}$.

Case 2: one enzyme and two substrates (the endogenous substrate is different from that added).

$$
\begin{aligned}
& \mathrm{S}_{e}+\mathrm{E} \rightleftharpoons \mathrm{ES}_{e} \rightarrow \mathrm{E}+\mathrm{P} \\
& \mathrm{S}^{\prime}+\mathrm{E} \rightleftharpoons \mathrm{ES}^{\prime} \rightarrow \mathrm{E}+\mathrm{P}
\end{aligned}
$$

The individual rates would be given by the usual Michaelis-Menten expressions but the actual rate when $S^{\prime}$ is added will be:

$$
v=\frac{V_{1}\left(\mathrm{~S}_{e}\right)}{\left(\mathrm{S}_{e}\right)+K_{1}+\left(K_{1} / K_{2}\right)\left(\mathrm{S}^{\prime}\right)}+\frac{V_{2}\left(\mathrm{~S}^{\prime}\right)}{\left(\mathrm{S}^{\prime}\right)+K_{2}+\left(K_{2} / K_{1}\right)\left(\mathrm{S}_{e}\right)}
$$

Here also the rates are not additive because the two substrates may be considered as competing for the same enzyme. 
Case 3: two enzymes and one substrate (the enzyme responsible for the endogenous rate is different from the enzyme which acts on the added substrate).

$$
\begin{aligned}
& \mathrm{S}_{e}+\mathrm{E}_{1} \rightleftharpoons \mathrm{E}_{1} \mathrm{~S}_{e} \rightarrow \mathrm{E}+\mathrm{P} \\
& \mathrm{S}+\mathrm{E}_{2} \rightleftharpoons \mathrm{E}_{2} \mathrm{~S} \rightarrow \mathrm{E}+\mathrm{P}
\end{aligned}
$$

Each rate is given by the characteristic expression and they are additive to give the total rate when the substrate is added. This situation could occur only if the two enzymes are so separated that the substrate from different sources could reach either one or the other.

Case 4: two enzymes and two substrates (the endogenous rate is due to one substrate and its enzyme, the exogenous rate to another substrate and another enzyme).

$$
\begin{aligned}
& \mathrm{S}_{e}+\mathrm{E}_{1} \rightleftharpoons \mathrm{E}_{1} \mathrm{~S}_{e} \rightarrow \mathrm{E}_{1}+\mathrm{P} \\
& \mathrm{S}^{\prime}+\mathrm{E}_{2} \rightleftharpoons \mathrm{E}_{2} \mathrm{~S}^{\prime} \rightarrow \mathrm{E}_{2}+\mathrm{P}
\end{aligned}
$$

The individual rates, as in case 3 , are additive.

The results will be interpreted differently depending on what is measured in the enzyme reaction. It has been assumed above that a common product is determined. If the two substrates involved are two phosphate esters, one can measure the inorganic phosphate released during hydrolysis, or one can determine the formation of the different dephosphorylated fragments. The latter method gives more accurate information but usually cannot be applied. In the determination of respiration it is generally impossible to fractionate the total oxygen uptake into the various reactions proceeding.

A rule for these simple systems is seen to emerge from the treatment of the four cases above. The individual rates are additive when two enzymes are involved and are not additive when a single enzyme is responsible for both exogenous and endogenous reactions. In practice it is often difficult to know if one or more enzymes are participating. Reiner (1959, p. 89) has presented methods for determining if one or more than one substrate or enzyme is involved in such cases. This is the only quantitative approach to the problem of endogenous metabolism known to the author.

\section{Effect of Endogenous Metabolism on Inhibition Kinetics}

The reciprocal plot of $1 / v$ against $1 /(\mathrm{S})$, where $\mathrm{S}$ is the exogenous substrate, will no longer be linear when endogenous metabolism occurs, and this is true whether the endogenous and exogenous rates are additive or not. 
The reciprocal equation for case 1 is:

$$
\frac{1}{v}=\frac{1}{V}+\frac{K_{m}}{V(\mathrm{~S})} \frac{1}{1+\left(\mathrm{S}_{e}\right) /(\mathrm{S})}
$$

the curve for which is given in Fig. 9-2. At low substrate concentrations the curve falls off from linearity, as pointed out by Reiner (1959, p. 89).

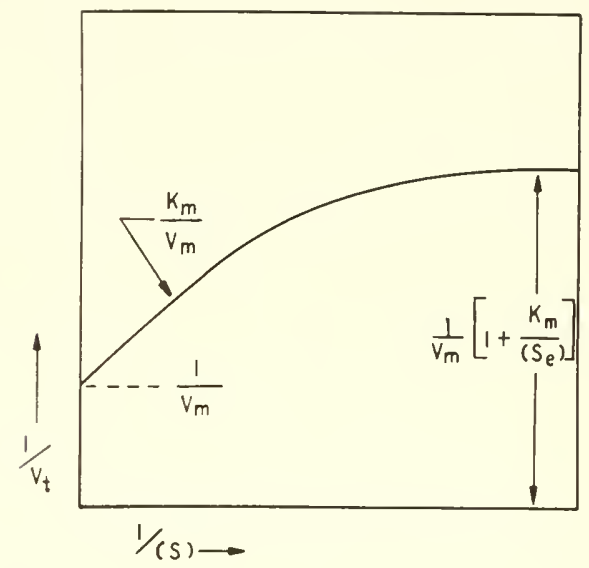

FIG. 9-2. Effect of endogenous metabolism on the determination of the enzyme constants in the double reciprocal plot (Eq. $9-2) .\left(S_{e}\right)$ is the concentration of the endogenous substrate.

From Eq. 9-1, it is seen that case 2 will also not provide a straight line. Finally, in cases 3 and 4 , where the total rate may be written in the form:

$$
n=C+\frac{\mathrm{I}^{\prime}(\mathrm{S})}{(\mathrm{S})+K_{m}}
$$

the constant endogenous rate being represented by $C, 1 / v$ is also not linear with $1 /(\mathrm{S})$.

When an inhibitor is used, the interpretation of the usnal plots must also be made with caution if there is an endogenons factor. This can best be illustrated by discussing certain asprects for each of the four possible cases described above. There are basically two questions: (1) what is the relation of the total inhibition to the individual inhibitions, and (2) how does endogenous metabolism affect the characteristics of the common analytical plots? 


\section{(a) Case 1: one enzyme and one substrate}

For noncompetitive inhibition. both factors in Eq. 9-2 are multiplied by $1+\left[(\mathrm{I}) / K_{i}\right]$; hence the intercept and slope will be increased by this term and the curves will appear as in Fig. 9-3. This presents no particular problem mless the nonlinearity is interpreted as arising from another mech-

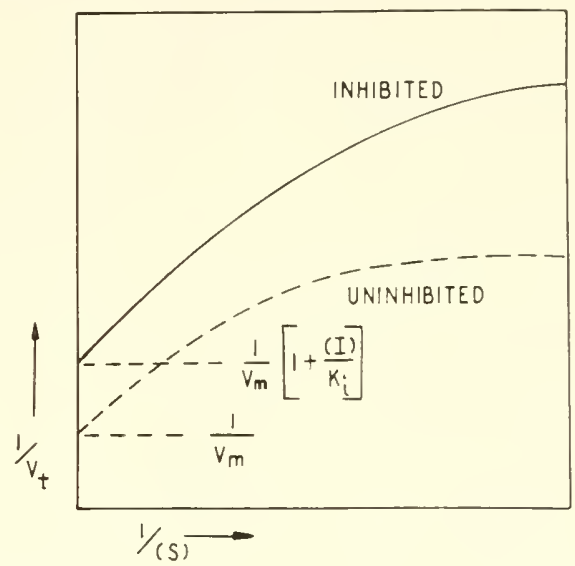

Fis. 9-3. Effect of endogenous metabolism on the determination of the inhibitor constant. The intercepts of both the uninhibited and the inhibited reaction curves are unchanged but the slopes are altered; it is thus important that sufficiently high substrate concentrations be used in order to determine these intercepts.

anism. On the other hand, the plot of $I^{\prime} \ell^{\prime}$ against (I) will remain a straight line, but the intercept and slope will each contain the term $\left(S_{\epsilon}\right)+(S)$ in place of (S) (see Fig. 5-3D). The fractional inhibition will be the same as the inhibition on either the endogenons reaction or the exogenous reaction alone, since each rate expression will be multiplied by $K_{i}\left[(\mathrm{I})+K_{i}\right]$ (Eq. 3-16). Thus any plots using $i$ will not be altered by the endogenons factor. The fractional inhibition for a competitive inhibitor, however, will not be the same for each individual reaction and the total reaction, because it depends on the substrate concentration. That is, the inhibition found on the total reaction will be less than would have ocenrred if only the added substrate were present. It may also be noted that if only the slope and intercept values are used in the $1 / x$ against (I) plot, an incorrect value for $K_{i}$ may be calculated when only $(\mathrm{S})$ is used instead of $\left(\mathrm{S}_{\epsilon}\right)+(\mathrm{S})$ (see Fig. 5-1D). 


\section{(b) Case 2: one enzyme and two substrates}

If a noncompetitive inhibitor affects the rate for each substrate equally, which is likely but not necessary, the inhibition on the total reaction will be the same as for the individual reactions, but for competitive inhibition the problem is more complex because competition with the two substrates may be different. Also the same effect noted in case 1, the effect of total substrate concentration, will occur here. The situation actually involves competition for an enzyme by two substrates and an inhibitor. In this case not even the $1 / v_{i}$ against (I) plot will be linear since:

$$
v_{i}=\frac{V_{1}\left(\mathrm{~S}_{e}\right)}{\left(\mathrm{S}_{e}\right)+K_{1}\left[1+\frac{\left(\mathrm{S}^{\prime}\right)}{K_{2}}\right]\left[1+\frac{(\mathrm{I})}{K_{i}}\right]}+\frac{V_{2}\left(\mathrm{~S}^{\prime}\right)}{\left(\mathrm{S}^{\prime}\right)+K_{2}\left[1+\frac{\left(\mathrm{S}_{e}\right)}{K_{1}}\right]\left[1+\frac{(\mathrm{I})}{K_{i}}\right]}
$$

\section{(c) Case 3: two enzymes and one substrate}

When only one of these enzymes is inhibited noncompetitively to the extent $i$, the inhibition on the total system will be $i_{t}=i\left(v_{e} / v_{t}\right)$ in case the endogenously active enzyme is affected and $i_{t}=i\left(v / v_{t}\right)$ when it is the enzyme acting on the added substrate that is affected. The inhibition on the enzyme is thus quite easy to calculate if the endogenous controls are run. The plots of $1 / v_{t}$ against either $1 /(\mathrm{S})$ or $(\mathrm{I})$ will be nonlinear, but plots with $1 / v$ (where $v$ is obtained by correction for the endogenous metabolism) wil] be linear. Similar conchusions apply to competitive inhibition. It may be noted that when the inhibitor acts on both enzymes, $i_{1}$ on the enzyme responsible for the endogenous activity and $i_{2}$ on the enzyme acting on the added substrate, the total inhibition is given by:

$$
i_{t}=\frac{i_{1} v_{e}+i_{2} v^{\prime}}{v_{t}}
$$

where $v_{e}$ is the endogenous rate and $v$ is the rate due to the added substrate.

\section{(d) Case 4: two enzymes and two substrates}

Since the rates of the separate reactions are additive, what has been said about case 3 applies equally here. There is perhaps more opportunity for a specific inhibition of one enzyme than in the previous case.

It is important to examine the effects of the substrate concentration on inhibition in systems with two or more enzymes. The total inhibition of the system when the enzyme depressed is the one acting on the added substrate may be written as:

$$
i_{t}=\left[\frac{v}{v_{e}+v}\right]\left[\frac{(\mathrm{I})}{(\mathrm{I})+K_{i}}\right]
$$


The fraction $v /\left(v_{e}+v\right)$ may vary with the substrate concentration and hence the inhibition depends on (S), even though the in hibition is noncompetitive. The total inhibition may either rise or fall as the substrate concentration is increased, depending on the relative values of $V_{m}$ and $K_{s}$ for the two enzymes. It is quite possible that noncompetitive inhibition might be interpreted as competitive under certain circumstances if correction for the endogenous metabolism is not made.

\section{INHIBITOR-STABLE METABOLISM}

An inhibitor will frequently depress a metabolic process in cells or tissues incompletely, even though the concentration of the inhibitor is raised to reasonably high levels, at least sufficiently high to produce nearly complete inhibition of the enzyme system attacked when that system is isolated from the cell. The interpretation of the inhibitor-stable fraction and the factors that determine its magnitude are often of importance in inhibition studies. The most informative discussion of this phenomenon is that of Commoner (1940) although he was concerned only with cyanide. He pointed out that changes in the cyanide-stable fraction of respiration during embryogenesis and cellular activation could be used to elucidate the metabolic mechanisms and he proposed a simple formulation, which was applied with success to respiratory inhibition in many tissues. The total respiration was assumed to be made up of two fractions, one sensitive and one resistant to cyanide, the latter relatively constant:

$$
\underset{\text { (total) }}{v_{t}}=\underset{\text { (resistant) }}{v_{r}}+\underset{\text { (sensitive) }}{v_{s}}
$$

Alteration of substrate concentration or changes in tissue function have marked effects on $v_{s}$ without modifying $v_{r}$ appreciably. The total respiration thus depends primarily on the level of $v_{s}$ and so the degree of inhibition produced by cyanide also depends on the total rate of respiration. This may be seen by plotting $v_{t}$ against $v_{s}$ (Fig. 9-4) where a straight line of $45^{\circ}$ inclination intersecting the $v_{l}$ axis at $v_{r}$ is obtained. It may be shown that the inhibition of respiration is given by:

$$
i_{t}=i\left(v_{s} / v_{t}\right)
$$

where $i$ is the inhibition of cytochrome oxidase in the case of cyanide. If the inhibition of the oxidase is complete and $v_{r}$ is assumed to be 10 , the inhibition of the total respiration is $9 \%$ when $v_{s}=1,33 \%$ when $v_{s}=$ $5,67 \%$ when $v_{s}=20$, and $83 \%$ when $v_{s}=50$. As a consequence, respiratory inhibition need not give a quantitative measure of the inhibition on the enzyme attacked. One must determine the true inhibitor-stable frac- 
tion if the enzyme inhibition is to be obtained, and $\mathrm{Eq} .9-8$ be rewritten to show how this enzyme inhibition is related to the measmred inhibition:

$$
i=i_{t}\left[1+\left(i_{r} / v_{s}\right)\right]
$$

Since $v_{s}$ depends on the substrate concentration in many eases - and on the particular substrate used also - the degree of inhibition will likewise

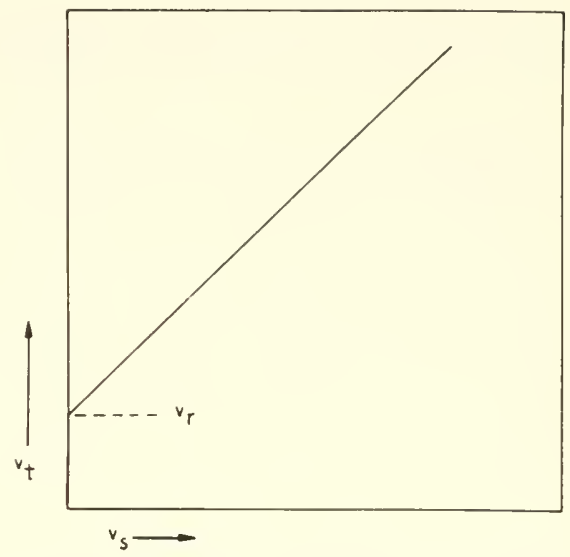

Fig. 9-4. Graphical representation of inhibitor-resistant metabolism. As the sensitive fraction of the metabolism is inhibited, the total metabolism will decrease as indicated by this line. See Eq. 9-7.

vary with the metabolic state of the cells and the availability of both endogenous and exogenous substrates. The increased sensitivity to cyanide of cells that are functionally activated (as during fertilization of marine eggs, development of insect embryos, or germination of Neurospore spores) may be related merely to the increased respiratory rate, due possibly to greater availability of the substrate.

The assumptions of this treatment shonld be borne in mind. The constancy of the inhibitor-stable respiration. " $r$ under varions conditions, is probably approximate only. Examination of the graphs presented by Commoner shows that deviation of the experimental points from the icleal straight lines is marked in some eases. In determining accurately the inhibitor-stable fraction, it is necessary that the enzyme inhibited be completely blocked and since the top portion of the inhibition-concentration curves is quite flat, total inhibition is sometimes not easy to obtain. For example, in simple noncompetitive inhibition, if $K_{i}=0.1 \mathrm{~m} M$, although $1 \mathrm{~m} / \boldsymbol{U}$ inhibitor will depress $91 \%$, it requires $10 \mathrm{~m} M$ to inhibit $99 \%$. Fur- 
thermore, the intracellular inhibitor concentration is often less than in the medinm and a selected concentration. based on nearly complete inhibition of an isolated enzyme, will not necessarily achieve the desired effect in the cell. For this reason, it is advisable to plot a curve of the metabolic rate against the inhibitor concentration to determine the level necessary to induce maximal inhibition, rather than to rely on isolated enzyme studies. Finally, it is unfortumate that this formulation has not been extended to other inhibitors to see if or when it is applicable. There are certainly some forms of inhibitor-stable metabolism to which this simple treatment would not be valid, as will be described below.

Some of the conditions responsible for an inhibitor-stable fraction of the metabolism will now be discussed.

I. Presence of branched, polylinear, or parallel chains. When there is more than one pathway for the utilization of a substrate or the formation of a product, inhibition of an enzyme in only one of these paths. even thongh it is complete, will generally not reduce the total process completely. The total respiration of a cell is usually made up of several pathways. some of which are branched and interrelated, some of which are separate, often utilizing different substrates, so that the inhibition of any particular enzyme will seldom block every pathway. There are many examples which may be cited. In a tissue possessing an active hexose monophosphate shunt, the action of an inhibitor such as iodoacetate (which here fairly selectively blocks the triosephosphate step in the Embden-Ileyerhof pathway) will not completely depress glucose utilization or oxygen uptake. In some tissues the arsenicals, by blocking the pyruvic oxidase, may essentially completely prevent the utilization of pyruvate, but in others, especially microorganisms. there are other pathways of pyruvate metabolism not susceptible to the arsenicals. Again. if formation of ATP is being determined, 2,4dinitrophenol will not always reduce this to zero because the uncoupling action is not exerted on phosphorylation linked with substrate level transfers. There is evidence that cytochrome $\mathrm{c}$ may be oxidized not only by the cytochrome $a$ and $a_{3}$ systems but by a peroxidase (Chance and Williams, 1956), so that an inhibitor acting on cytochrome $a_{3}$. but relatively less on peroxidase, could not completely abolish the uptake of oxygen. Conditions such as these are perhaps the most important in explaining inhibitorstable metabolism.

II. Reactions proceed ahead of the step blocked in a chain. If in a monolinear chain such as $\mathrm{A} \rightarrow \mathrm{B} \rightarrow \mathrm{C} \rightarrow \mathrm{D}$, the last step is blocked, the earlier reactions may continue. A complete block at a single enzyme in a sequence of oxidations will allow oxygen to be taken up in all the steps occurring before the block. This may also occur in cyclic systems. When the succinic dehydrogenase in the tricarboxylic acid cycle is inhibited, the cycle 
must stop if it is operating under self-regenerating conditions, but if there is a supply of oxalacetate (or fumarate or malate), pyruvate will continue to enter the cycle and the oxygen uptake will be depressed to a limited degree. The cycle blocked at aconitase by fluorocitrate will continue to incorporate pyruvate and take up oxygen as long as some source of oxalacetate is available, which in this case conld even be glutamate.

\section{Maintenance of the steady state in multienzyme systems. In Chapter 7} it has been shown that many types of multienzyme systems can be resistant towards inhibition, principally because of the adjustment that is possible in the coneentrations of the intermediates. This is particularly true for competitive inhibition where the accumulation of the substrate for the inhibited enzyme counteracts this inhibition. One of the reasons for the failure of malonate to depress significantly the respiration of many tissues may be the increase in the succinate concentration resulting from the inhibition.

IV. Shift in metabolic pathway. Initially the total rate may be due to a single pathway or enzyme but upon inhibition another pathway becomes available. The increase in one branch of a divergent chain as a result of the depression of the other branch has been discussed, but one may note here that under normal conditions one pathway may be almost inactive due to kinetic factors and yet may contribute to the inhibitor-stable metabolism when the sullstrate concentration rises. Another type of adjustment that can take place is a true adaptation of the cell in the synthesis of enzymes catalyzing a new pathway; this, however. is not so much a mechanism to explain the stable metabolism as a means of achieving this condition.

V. Failure to inhibit the enzyme or pathway completely. Although the external concentration of inhibitor may be high enough to inhibit an enzyme completely when it is isolated from the cell, only partial inhibition of the intracellular enzyme may oceur for reasons discussed earlier in this chapter, namely the failure of the inhibitor to enter the cell at the external level or the fact that the environment of the intracellular enzyme is different. When the metabolism is stable to an inhibitor simply because it does not enter the cell readily, it is perhaps best not to consider this as inhibitorstable metabolism in the sense of this section, beeanse basically it is not the metabolism that is stable. However, this factor can lead to errors in interpretation and in most cases it is difficult to determine the extent of inhibition within the cell.

VI. A reserve store of intermediate past the block. In any sequence of enzyme reactions, complete block of a step need not necessarily stop the formation of final product immediately since a supply of the intermediates 
may be available. Although this is not common, it does occur occasionally. When formation of ATP is suddenly stopped, phosphorylation reactions will proceed until the ATP is exhausted, and in this case a reserve source of high-energy phosphate is often present as creatine phosphate which can be used to maintain the ATP for an interval.

It is now clear that the original formulation of the total metabolism as being made up of two parts, a sensitive and a resistant fraction, is not valid for all cases; the resistant pathway may not even be operative in the uninhibited cell and its eventual magnitude may depend on a number of factors. It is also evident that it is not justifiable to conclude without evidence that the resistant fraction of the metabolism is different qualitatively from the sensitive fraction. In the early speculations on cyanide-stable respiration it was often believed that this originated from noncarbohydrate substrates, simply because the respiration resulting from added sugars was mainly sensitive to cyanide, but this differentiation is no longer necessary. The relationship of the stable fraction to cell function is important and will be taken up in a later section.

\section{STIMULATION OF METABOLISM BY INHIBITORS}

It might seem paradoxical for an enzyme inlibitor to increase the rate of some phase of metabolism but actually such stimulation is not at all uncommon. It is probably justifiable to say that the greater the number of interrelated enzymes in the total system, and the greater the complexity and organization of the metabolic pathways, the more likely will it be that stimulation can occur. Although this stimulation is generally of cell metabolism or of functions related to metabolism, it has also been observed on single enzymes.

There may be some doubt in the mind of the reader as to the advisability of designating substances that stimulate as inhibitors. Some explanation of what is implied is necessary. In the first place, we are concerned in these volumes with substances that are generally considered as inhibitors of enzymes or metabolic processes. Now, although these substances may be inhibitory to many enzymes (or to one or a few specifically). they can in some cases exert a stimulatory action on other enzymes. These latter actions must be taken into account in any treatment of the effects these substances produce in living material. One must speak not only of the beneficial therapeutic actions of drugs but also of their toxic effects; likewise, one cannot consider only the inhibitory aspect of compounds such as cyanide, iodoacetate, or arsenite, and it is necessary to include every possible effect they may have on cells and organisms. Secondly. a substance may produce a stimulatory effect by a primary inhibitory action on an enzyme; the response of the particular rate measured need not be identical with the 
fundamental change brought about by the inhibitor. Possible mechanisms for such behavior will be discussed presently. Lastly, a substance may exhibit a biphasic action involving stimulation at lower concentrations and inhibition at higher concentrations. The primary action of interest may be the inhibition, for which the substance is being used, but unfortunately the substance exerts both actions despite the purpose of the investigation.

Although many examples of various types of stimulation brought about by enzyme inhibitors will be given in the chapters on the inhibitors, a few illustrations of the phenomenon may serve to make the presentation more concrete. Arsenite, although a general enzyme inhibitor, has been found to stimulate papain (Bersin, 1934), malic dehydrogenase (Green, 1936), uricase (Mahler et al., 1955), hexose diphosphatase (Peters et al., 1946), and the $\mathrm{P}_{i}$-ATP exchange enzyme (Plant, 1957). Likewise, it has been shown to stimulate respiration of spermatozoa (Barron et al., $1948 \mathrm{~b}$; Humphrey, 1950), diatoms (Lewin. 1955), Trichomonas (Ryley, 1955), and yeast (Shacter, 1953), despite the fact that in most cells or tissues a marked inhibition is observed. Some of the quinones, although very inhibitory to enzymes such as papain, proteinases, pyruvic decarboxylase. urease, and lipase, are known to stimulate deoxyribonuclease (Hoffmann-Ostenhof and FrischNiggemeyer, 1952); and, although inhibitory to the respiration of many tissues, they may stimulate that of Arbacia eggs (Anfinsen, 1947), spermatozoa (Lardy and Phillips, 1943), mammalian erythrocytes (Friedheim, 1934; Supniewski et al., 1936), and mouse liver and muscle (Supniewski et al., 1936). Phenylmercuric compounds inhibit succinoxidase (Cleland, 1949) and muscle ATPase (Polis and Meyerhof, 1947) almost completely at $1 \mathrm{~m} M$ but both enzymes are stimulated by concentrations around $0.01 \mathrm{mM}$. An interesting example of selective stimulation has been found in yeast (Lundsgaard, 1930 b; Nilsson et al., 1931), where iodoacetate at a concentration almost completely inhibiting fermentation may have a stimulating effect on respiration. The ability of malonate to increase the utilization of substrates such as $\beta$-ketoglutarate in heart mitochondria has been reported (Slater and Holton, 1954) and we have noted a 15-30\% increase in pyruvate incorporation in such mitochondria when low concentrations of malonate are used. The biphasic action is particularly well shown in the actions of fluoride and azide on the endogenous respiration of yeast (Borei, 1942). In the case of fluoride, a stimulation greater than $100 \%$ may occur at $20 \mathrm{~m} M$, while at $30 \mathrm{~m} M$ a $70 \%$ inhibition talies its place. These are only a few randomly chosen examples of stimulation but they are perhaps suffeient to indicate the widespread occurrence of this effect and the different ways in which it may be manifested.

There are many possible mechanisms which may be hypothesized to explain these instances of stimulation but there is little experimental evidence to implicate a particular mechanism in any situation. Some of the 
more obvious ways in which stimulation could be produced will now be considered briefly.

I. Removal or inactivation of some inhibiting substance. This type of stimulation probably is more frequently observed in disintegrated cell preparations and extracts because the breakdown of the normal organization may expose an enzyme to substances from which it is usually separated. The only well-established cases involve the removal of an inhibitory metal ion. Thus chelating agents, such as EDTA or o-phenanthroline, or substances forming complexes with metal ions, such as cyanide, have been shown to increase the activity of certain enzymes by removing traces of heavy metal ions from the medium. Yet, because of this same ability to combine with metal ions, these agents can simultaneously inhibit other enzymes or eventually even the enzyme activated. Ions such as $\mathrm{Ca}^{++}$and $\mathrm{Mg}^{++}$ may also be involved and the effects of inhibitors such as EDTA or malonate can sometimes be partially attributed to the reduction of the concentration of these ions. It is easy to stimulate ATPase activity with malonate when either $\mathrm{Ca}^{++}$or $\mathrm{Mg}^{++}$is unfavorably high.

The conditions necessary for stimulation when only one enzyme is present are given by Reiner (1959, p. 173). The reactions are:

$$
\begin{aligned}
& \mathrm{S}+\mathrm{E} \rightleftharpoons \mathrm{ES} \rightarrow \mathrm{E}+\mathrm{P} \quad K_{s} \\
& \mathrm{I}_{1}+\mathrm{E} \rightleftharpoons \mathrm{EI}_{1} \quad K_{1} \\
& \mathrm{I}_{2}+\mathrm{E} \rightleftharpoons \mathrm{EI}_{2} \quad \mathrm{H}_{2} \\
& \mathrm{I}_{1}+\mathrm{I}_{2} \rightleftharpoons \mathrm{I}_{1} \mathrm{I}_{2} \quad K_{12}
\end{aligned}
$$

If the amount of either inhibitor bound to enzyme is neglected, the reaction rate is given by:

$$
v_{i}=\frac{V_{m}(\mathrm{~S})}{(\mathrm{S})+K_{s}\left[1+\frac{\left(\mathrm{I}_{1}\right)}{K_{1}}+\frac{\left(\mathrm{I}_{2}\right)}{K_{2}}\right]}
$$

where the free inhibitor concentrations are:

$$
\begin{aligned}
\left(\mathrm{I}_{1}\right) & =\frac{\left(I_{1}\right)_{t}}{1+\left[\left(\mathrm{I}_{2}\right) / K_{12}\right]} \\
\left(\mathrm{I}_{2}\right) & =\frac{1}{2}\left\{-\left[\left(\mathrm{I}_{i}\right)_{t}-\left(\mathrm{I}_{2}\right)_{t}+K_{12}\right]\right. \\
& \left.+\sqrt{\left[\left(\mathrm{I}_{1}\right)_{t}-\left(\mathrm{I}_{2}\right)_{t}+K_{12}\right]^{2}+4 K_{12}\left(\mathrm{I}_{2}\right)_{t}}\right\}
\end{aligned}
$$

$I_{1}$ will be assumed to be the inhibiting substance present with the enzyme and $I_{2}$ the inhibitor added; it is the possible stimulation by $I_{2}$ we are now 
concerned with. When $\left(\mathrm{I}_{2}\right)=0$ the enzyme will be inhibited by $\mathrm{I}_{1}$ to a certain degree, and when $\left(I_{2}\right)$ is very high the rate will be zero. To determine if a maximum in the rate curve occurs as $\left(\mathrm{I}_{2}\right)$ is increased, the derivative $d v_{i} / d\left(\mathrm{I}_{2}\right)$ may be determined; it is simpler to take the derivative of the denominator of Eq. 9-10 and equate it to zero since a minimum in this denominator implies a maximum in the rate.

$$
\left(\mathrm{I}_{2}\right)_{\max }=\sqrt{K_{2} K_{12}\left(\mathrm{I}_{1}\right)_{t} / \overline{K_{1}}}-K_{12}
$$

where $\left(\mathrm{I}_{2}\right)_{\max }$ is the inhibitor concentration giving the maximal rate. If $K_{2}\left(\mathrm{I}_{1}\right)_{t} / K_{1}$ is greater than $K_{12},\left(\mathrm{I}_{2}\right)_{\max }$ will have a positive value. Thus the possibility of stimulation depends on the concentration of the original inhibitor, the relative affinities of the two inhibitors for the enzyme and the dissociation constant of the $\mathrm{I}_{1} \mathrm{I}_{2}$ complex. When these factors are favorable, a plot of Eq. 9-10 will show a biphasic effect as $\left(I_{2}\right)$ is increased. The relationships may be a great deal more complex, however, if the initial inhibitor is a metal ion that can function both as an activator and inhibitor, as $\mathrm{Mg}^{++}$and $\mathrm{Ca}^{++}$with ATPase.

II. Alteration of metabolic flow in multienzyme systems. Some examples of this mechanism have already been discussed in Chapter 7 . It is usually exhibited in divergent or polylinear chains when one pathway is inhibited. The over-all flow rate may not be altered but the activity in a particular pathway may be increased. Thus the appearance of stimulation will depend on what aspect of the total system one is examining. For example, a branched chain of the following type:

$$
\mathrm{A} \rightarrow \mathrm{B} \longrightarrow \mathrm{D}
$$

where B can either be oxidized or enter into the synthesis of D, will, upon inhibition of $\mathrm{B} \rightarrow \mathrm{C}$, appear to be inhibited if oxygen uptake is being measured, to be stimulated if synthesis of $\mathrm{D}$ is determined, or to be unaffected if utilization of $\mathrm{A}$ is the criterion of rate. This mechanism is actually a diversion of metabolism rather than a true stimulation, but nevertheless it manifests itself as stimulation and in most cases it is difficult to distinguish it from a direct effect. Electron transport systems apparently have phosphorylating and nonphosphorylating pathways and a shift from one to the other, brought about by specific inhibition, could increase or decrease the rate of formation of ATP and all the reactions dependent upon ATP. The inhibition of cytochrome oxidase with cyanide increases the diversion of hydrogen atoms to methylene blue or other acceptor dyes; without knowledge of the situation here, one might conclude that cyanide stimulated the oxidation of the substrate. These examples show that care 
must be exercised in the interpretation of a stimulatory response to an inhibitor.

III. Reduction in enzyme concentration leads to an increased rate. In certain instances where a dissociable coenzyme or activator is required, increase in the concentration of enzyme will augment the rate only up to a point and then the rate will fall off. This is shown very well by lactic dehydrogenase (Straub, 1940) and probably is a general phenomenon for such enzymes. If in the cell the enzyme concentration is in the range of decreasing rate, the decrease in enzyme resulting from the action of an inhibitor would actually stimulate. It is quite possible that such high concentrations of enzyme occur in the cell, or at least in limited regions, and so this type of stimulation should not be ignored although at present there is no evidence that it is the mechanism in any particular case.

IV. Reversal of a dynamic equilibrium. In growing cells there is a constant incorporation of amino acids and their synthesis into proteins with the energy provided by ATP:

Amino acids $+\mathrm{ATP} \rightarrow$ protein

Simultaneously the proteins are broken down, presmmably by proteolytic enzymes in the cell:

\section{Protein $\rightarrow$ amino acids}

This type of dynamic equilibrium can be disturbed by an inhibitor of either reaction. If the formation of ATP is depressed, as with 2,4-dinitrophenol, the over-all process may be one of protein breakdown; from limited observations it might be concluded that 2,4-dinitrophenol stimulated proteolysis. In any equilibrium of this nature, inhibition of one reaction will tend to increase the other one relatively.

V. Depression of a reaction controlling metabolism. The ability of a living cell to adjust its metabolism according to its functional activity makes it necessary for the principal metabolic pathways to be under some sort of controlling or regulating mechanism. Thus many metabolic reactions do not operate at the maximal rate in the normal resting cell. The removal of the restricting control will manifest itself as stimulation. One of the chief regulatory mechanisms involves the adenine mucleotides. The obligatory coupling of oxidation with phosphorylation in some electron transport chains limits the rate of electron flow, so that any uncoupling agent may accelerate oxidation. Another very important metabolic control is the Pasteur-Crabtree relationship between glycolysis and oxidative processes, whereby the concentrations of the mucleotides and inorganic phosphate both control and are determined by the rates of the various reactions. It 
is thus possible for inhibitors of oxidation, such as cyanide, to stimulate the utilization of ghcose, and for other inhibitors, acting at different loci, to modify the rates of reactions secondarily.

There are many other mechanisms which may be postnlated and it will suffice to mention a few of these. The inhibition of an enzyme that destroys some important metabolic substance may increase the steady-state level of this substance and accelerate reactions with which it is concerned. Inhibition of ATPase, for example, can secondarily influence many reactions dependent on ATP. The inhibitor may also increase permeability in some manner and thus stimulate reactions whose rates are limited by access of substrate to the enzyme, or it may either directly or indirectly damage the cell so that the structural disorganization will allow reactions to be released from their normal control, which may be simply a spatial separation of reactants. Any change in functional activity induced by an inhibitor can in turn alter metabolism; the temporary stimulation often observed in nerve and muscle following the application of an inlibitor conld be related to the partial depolarization of the membrane, with consequent flow of ions and activation of pump mechanisms. Some attention has been given to the protection of the enzyme against denaturation, due to combination with the inhibitor and stabilization of the active structure (Spiegelman and Reiner, 1945; Reiner, 1959, p. 171), but it seems unlikely that denaturation within the cell would be of significance in determining metabolic rate, and it must also be very uncommon for a substance to combine with an enzyme in such a way that it stabilizes its structure and simultaneously does not interfere with its catalytic activity. Lastly, the possibility that the inhibitor is simply an activator or substrate for enzymes other than those inhibited must be kept in mind. The designation of a substance as an enzyme inhibitor must not prevent us from considering the substance as capable of a great variety of actions on a system so complex as the living cell.

\section{INTRACELLULAR EQUILIBRIA AND SECONDARY EFFECTS OF INHIBITORS}

A multitude of enzymically catalyzed reactions proceed simultaneously within the organized structure of the protoplasm of the living cell. Many of these reactions are interrelated: some form substances used by other reactions, some provide energy required by endergonic processes, some are involved in the synthesis of material necessary for the catalysis of other reactions, while others may be important in the disposal or modification of the products of metabolism. Protoplasm must take up the material and generate the energy to synthesize its own substance, inchuding the enzymes and coenzymes which are necessary for these very reactions; it is a system 
of unstable catalysts kept in existence by the occurrence of reactions which they catalyze. The equilibria here are clearly different from the statically balanced systems of physics and chemistry, a distinction emphasized by Dixon (1949), who termed these equilibria "floating." These very complex equilibria of living material include the steady-state systems discussed in Chapter 7 , but are dependent on a higher order of organization. A certain flow of substance and energy must enter the system for maintenance; an additional amount is required for the performance of function or growth. It is this that imposes instability in living material. in the sense that disturbances in the metabolism, as produced by inhibitors, can undermine the organized material basis for the metabolism. This instability is generally tempered by the plasticity or adaptability of the cellular metabolism, characteristics that have been evolved to protect the cell against periods of adverse conditions, such as deficiencies in substrate or oxygen. Since many inhibitors induce states in cells approximating these deficiencies, the same adaptive processes often come into play, and the dynamic equilibria are not so readily dismpted as might be expected. Nevertheless, once the capacity of the cell to adapt has been exceeded by the metabolic disturbance, there will be a progressive disintegration of the complex equilibria and eventual dissolution of the structural basis of life.

Let us consider briefly a very simple illustration of this sort of equilibrium and the effect of inhibitors upon it. Many oxidations require coenzymes that are synthesized in reactious utilizing ATP, which in turn is generated by the oxidations. When an inhibitor depresses the oxidative reaction,

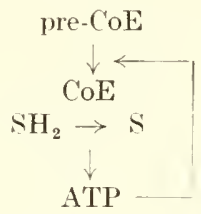

less ATP will be formed, and consequently less CoE will be available, this further decreasing the oxidative rate; under certain conditions this may proceed until the entire system ceases to function. An inhibitor acting on any other reaction in this equilibrium would have the same end result. Much of the damage to cells resulting from exposure to inhibitors probably results from phenomena of this nature. The instability of mitochondria to anoxia, lack of substrate, or inhibition may be due in part to loss of coenzymes or other metabolically important factors.

Cells and tissue vary a great deal in their resistance to such disturbances. Anoxia or lack of ghucose produces differential damage to cells of the central nervous system, some surviving for hours and others only for a few minutes (van Liere, 1942), and it is well known that anoxia can induce differential damage to cells lying in the metabolic gradients of the embryo 
or various invertebrates. Liver tissue made anoxic by clamping the hepatic artery for short times shows a reduced respiration and ability to deaminate amino acids (Wilhelmi et al., $1944 \mathrm{a}, \mathrm{b}$ ). A period of $5 \mathrm{~min}$ of anoxia depressed the respiration $10 \%$ and $10 \mathrm{~min}$ of anoxia $\mathrm{r}$. duced it $58 \%$; reduction of deamination and uptake of amino acids occurred simultaneously. Brain cortex slices (Wilhelmi et al., 1944c) and heart slices (Webb et al., 1949 a) subjected for short periods to anoxia show progressive failure of respiration. The mechanisms involved in these effects are not known, but it is established that chemical and structural changes - alterations in ionic or nucleotide concentrations, swelling, vacuolization, and chromatolysis - can occur rapidly in cells deprived of oxygen. What is to be emphasized is that in oxygen deficiency we have evidence of the disturbances that may occur in dynamic equilibria and visible proof that energy is required for cell maintenance, as well as for growth and function, and in the case of anoxia there is no question but that the primary effect is metabolic depression, whereas inhibitors could be supposed to act in other nonmetabolic ways. Furthermore, we see that in many cells, particularly the most active, this dynamic equilibrium does not require much time to be irreparably damaged. The possibility of such changes taking place during the action of an inhibitor on cells should be kept in mind at all times, since in experiments of duration exceeding a few minutes one may be measuring secondary and tertiary effects. In cells, and especially in whole organisms, the primary action of an inhibitor may progressively produce a series of secondary changes, radiating out to encompass eventually processes and tissues not even in contact with the inhibitor. A heavy metal is able by acting specifically on the enzymes involved in renal tubular transport to produce biochemical and functional changes in the brain mediated through the altered composition of the blood. Such phenomena are obvious but there are many relationships more subtle and less understood. Each time an inhibitor acts on a living cell there is the possibility that in some way protein synthesis is depressed and that the resultant alteration in intracellular enzyme concentration will derange all manner of metabolic processes. These phenomena are not so much related to the properties of an inhibitor as to the inherently complex characteristics of cells and organisms. The type of response will, to some extent, depend on the nature of the inhibitor; it will depend even more, in most cases, on the potentialities of the living system to respond.

\section{THE CONCEPT OF PACEMAKERS IN METABOLISM}

The concentrations of the varions metabolic intermediates in the normal cell are generally low and this lack of accumulation indicates that in most instances the rates are limited not by enzyme activity but by the availability of substrate. Some enzymes are known to be present in excess of the 
amount needed for their ordinary functioning; fumarase in some cells might be cited as an example. Inhibition of such enzymes will not produce a marked effect on metabolism until their activity has been reduced to the point where they are limiting. The enzymes of the tricarboxylic acid cycle are often given as an illustration of a metabolic system in which the intermediates are handled rapidly and readily and little accumulation occurs. However, it has recently been emphasized that this does not apply to all enzymes in the cell and that those enzymes that do limit the rate are the "pacemakers of metabolism" (Krebs, 1957, 1958). According to this concept, there are certain key enzymes or reactions that mainly determine the over-all metabolic rates and it is these that are most susceptible to the action of inhibitors. In general, those reactions initiating the degradation of the primary substrates - glucose. amino acids, fatty acids, and oxygen are the pacemakers, but other pacemaking reactions may occur after a certain amount of degradation has occurred, particularly at branching points which determine the directions of metabolic flow. On this basis Krebs divides inhibitors of respiration into three classes:

I. Inhibition by interference with utilization of oxygen: cyanide, CO, azide, and sulfide.

II. Inhibition by interference with utilization of substrate, i.e., by action on the dehydrogenases: malonate and arsenite.

III. Inhibition by interference with reactions at the branching points of metabolism, diverting metabolism abnormally: agents that may reduce the supply of oxalacetate, such as malonate or ammonium chloride.

With respect to glycolysis, there are two principal pacemakers, the hexokinase and the triose phosphate dehydrogenase, and most inhibitors of glycolysis, such as iodoacetate and glyceraldehyde, act at these loci. A very crucial branching point is often in the systems for the disposition of acetyl-CoA. The number of pathways available for this compound depends upon the type of cell. but in all cells it is a central substance in the utilization and synthesis of carbohydrate and fatty acids. This is also a vulnerable point for attack by inhibitors and the whole pattern of metabolism may be readily altered by changing the ratios acetyl- $\mathrm{CoA} / \mathrm{CoA}$, reduced flavoprotein /oxidized flavoprotein, and DPNH/DPN. According to Krebs, then, these pacemaker reactions are those to which we must turn our attention in considering ways and means of potently inhibiting cellular metabolism.

Without denying the importance of this concept. I believe that a too ready acceptance of it may oversimplify a very complex problem and lead to errors in interpretation. If one jumps to the conclusion that only a few vital reactions or enzymes in the cell are of much importance in the action of inhibitors, that the effects of inhibition can be entirely explained on the basis of these few pacemaker reactions, this conclusion will be quite er- 
roneous and an unjustifiable extension of the original concept. For this reason it may be well to mention some of the mitigating circumstances that restrict the complete applicabilty of this concept. (1) Reactions which are pacemakers in certain states of a cell may not be when conditions are changed. The concentration of substrates in the medium outside the cell may determine which step is limiting and it is evident that the type of substrate that is available may be of primary importance. Alterations of intracellular ion concentrations or $\mathrm{pH}$ can likewise shift a pacemaker; it is possible that in magnesium deficiency, enolase would be a limiting step in glycolysis. (2) The ability of multienzyme systems to maintain their steady state in the presence of an imhibitor is to a great extent dependent on the possibility of intermediates rising sufficiently in concentration. This is not always possible for a number of reasons: the intermediate may be metabolized along another pathway when its concentration begins to rise, it may diffuse away from the reaction region. or it may begin to inhibit some other enzyme at a higher concentration. Thus inhibition of respiration by fluoride can be quite marked, despite the fact that enolase is normally not a pacemaker in glycolysis, but it may well be that phosphoenolpyruvate cannot accumulate to maintain the original rate. (3) We have seen in Chapter 7 that there are many types of multienzyme systems in which buffering against inhibition is present but far from complete. In cyclic and feedback systems, inhibition on any step usually produces some inhibition of the over-all process, and in regenerative systems, such as electron transport, the constancy of total regenerant concentration imposes a restriction on the adaptability to inhibition. Therefore, for inhibition to be exhibited the inhibitor need not attack the rate-determining enzyme, if such exists. (4) Sometimes a metabolic pathway is considered as if isolated from the other reactions to which, in the living cell, it is closely related. The over-all rate of glycolysis may well depend on reactions outside the main sequence. For example, inasmuch as the glycolytic rate does depend on the availability of ATP, ADP. and inorganic phosphate, enzymes, such as ATPase, can indeed be factors in determining the rate, and inhibition of ATPase, by elevating ATP and reducing ADP and $\mathrm{P}_{i}$ levels, might possibly modify glucose utilization. One does not know as yet the kinetic importance of such enzymes in the over-all pattern of metabolism, so often it is somewhat premature to designate pacemakers. (5) The factor of time must also be considered. If inhibition is suddenly achieved with respect to a single step in metabolism, it may require some time for the system to regain a steady state, assuming it can (see Chapter 7 ). Thus during this period of temporary inhibition the over-all rate will be suppressed, even though the enzyme attacked is not a pacemaker, and during this interval secondary changes may take place so that a return to the original state is made impossible. 
It is possible to cite many examples of metabolic inhibition in which the primary reaction affected is not a pacemaker. The inhibition of glycolysis and respiration by fluoride acting on enolase has been mentioned. A good example is the very potent inhibition induced by fluoroacetate. inasmuch as aconitase would certainly not be considered a limiting enzyme in respiration. Also, respiration can be readily inhibited by substances acting on enzymes other than eytochrome oxidase or the dehydrogenases in the electron transport pathways; antimycin $A$. dimercaprol, and certain naphthoquinones all act on some intermediate transfer. Reasons such as those presented above and experimental evidence of this nature do not by any means invalidate the basic concept but only suggest caution in its application.

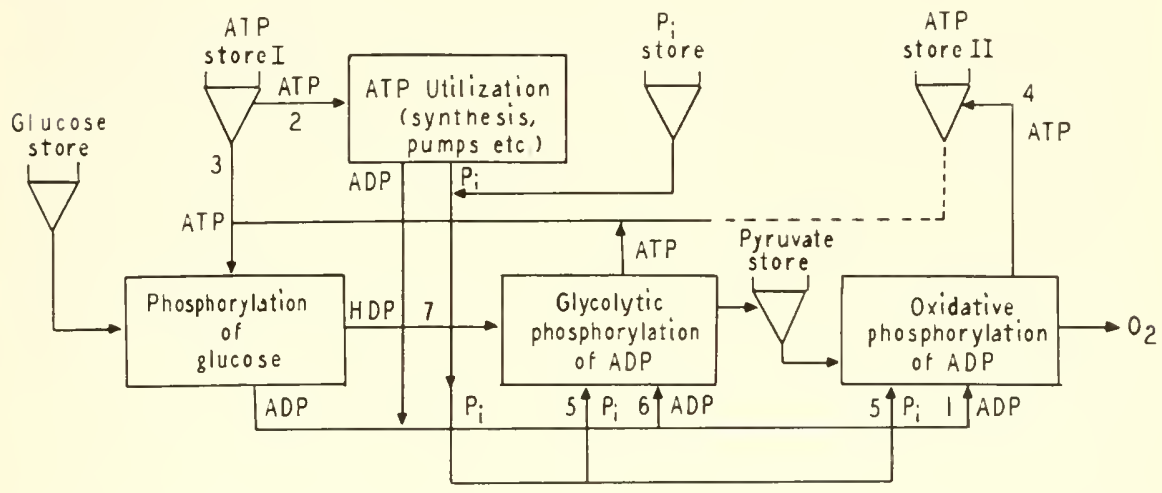

Fig. 9-5. Possible sites for metabolic control in ascites tumor cells. (1) control of respiration by ADP concentration. ( 2 ) control of ATP utilization in endogenous processes by ATP concentration. (3) control of glucose phosphorylation by the ATP level. (4) reservoir of ATP formed in mitochondrial oxidations not readily available for gheose phosphorylation. (5) control of glycolytic or oxidative phosphorylation by the inorganic phosphate level. (6) control of glycolytic phosphorylation by the ADP level. ( 7 ) control of glycolytic phosphorylation by the substrate (hexose diphosphate) level. (From Chance and Hess, 1959).

Another concept of great importance. and related closely to the elucidation of pacemaker reactions. is that of certain controlling processes in the cell. In any complex metabolic system, there are specific loci where various influences can reartily control the over-all rate. This has been expressed particularly clearly by Chance and Hess (1959) whose suectroscopic studies of glucose utilization by ascites tmmol cells have proviched several such loci. Figure 9-5, showing possible controlling points for this particular type of cell, summarizes their conclusions. It is very interesting to work out the effects of inhibition at rarious sites in such a scheme and 
to determine how these inhibitory responses will be modified by changes in the availability of substrate, the depletion of oxygen supply, or the process of active growth.

\section{RELATIONS BETWEEN ENZYME INHIBITION AND CHANGES IN CELL FUNCTION}

Frequently it is desired to determine in what manner an inhibitor can influence a particular functional activity of a cell or organ - such as cell division, movement, impulse conduction, secretion, or active transport and thereby learn something about the enzymic processes upon which this function is dependent. The tricky problem of correlating the observed functional changes with the metabolic inbibition thus arises. All of these cellular activities require energy, either directly expended on function or indirectly for the synthesis of the functional components. The use of inhibitors that presumably will specifically depress certain enzymes in the metabolism involved in energy formation and utilization will usually produce some measmable modification of the function. But there are many other mechanisms by which function can be altered by inhibitors and an effect need not be interpreted as che to the interference with the energy supply. It has been pointed out that for several reasons it is not always possible to assume that within the cell the inhibitor is duplicating the inhibition observed on isolated systems. There are many instances where a fair degree of inhibition is exerted on the cellular metabolism without marked effects on function and there are also cases where function can be essentially abolished without detectable distmbance in metabolism. Such complexities often make accurate correlation very difficult. Of all the aspects of the problem, however, the most important is the specificity of the inhibitor, the degree to which we can be certain that a particular enzyme or metabolic pathway is being blocked without significant action of other systems. Specificity will be discnssed in detail in Chapter 17 and only the most pertinent points will be mentioned here.

The depression of function by an inhibitor is generally interpreted in terms of a rednction in the energy supply for the process but it should be borne in mind that there are other mechanisms that may be operative. Let us assume that cardiac or skeletal muscle contraction has been found to be altered by $p$-chloromercuribenzoate. Some possible mechanisms for this action would be: an effect directly on the contractile elements, a modification of the membrane leading to changes in ionic permeability and electrical behavior, a depression of the coupling between excitation and contraction, an interference with the formation or destruction of acetylcholine, or an inhibition of the transfer or high-energy phosphate as well as a suppression of its generation. In some cases, a direct or indirect change 
in intracellular $\mathrm{pH}$ or ionic composition, brought about by the inhibition, might affect the activity of the cell.

\section{Nonenzymic Effects of Inhibitors on Cell Function}

An inhibitor that has been found to be quite specific metabolically, acting only on a particular enzyme or group of enzymes, may still possess the ability to react in some manner with the components of the functional systems in the cell or to modify function in other nonenzymic ways. Many tissues have an electric potential across the cell membrane; the functional activity and metabolism will depend upon and vary with the fluctuations in this potential. This would apply particularly to muscle and nerve but the membrane processes may also be important in kidney, marine invertebrate eggs, plant cells, and various microorganisms. These membrane potentials may be modified by inhibitors either by action on enzymes (both within the cell and at the surface of the cell) or nonenzymically. Many inhibitors are ions and can change the potential directly, especially if used in relatively high concentrations (usually above $10 \mathrm{~m} M$, although the permeability to the ion is of importance). The ion that accompanies the inhibitor ion must also be considered. The $\mathrm{Na}^{+}$with inlibitors such as malonate of fluoride may have a measurable effect on membrane potentials in concentrations above $10 \mathrm{~m} \boldsymbol{M}$; such effects of $\mathrm{Na}^{+}$have been demonstrated on rat atrial potentials. The potassium salts of inhibitors should be avoided in work with intact cells for this reason, since $\mathrm{K}^{+}$usually has a marked effect on the resting potential.

All inhibitors that react with groups on proteins must be assumed to attack not only the enzymes but also the various proteins of the cell. These proteins may be in the plasma membrane and their modification induce permeability changes that will secondarily affect metabolic systems. It is likely that many of the effects observed with heavy metal ions and organic metal compounds are due to such actions; e.g., the rapid cytolysis sometimes seen is probably murclated to any enzyme action and is indicative of the effects on membrane structure. The inhibition of metabolism in yeast by uranium salts would appear to be the result of a decrease in the permeability to various substrates and there is no evidence that this is an effect on enzymes (Barron et al., 1948a). Inhibitors that are lipid-soluble or possess polycyclic structures could interfere with the interactions of cell membrane components; the bipyridines and phenanthrolines may act in this way, in addition to their chelation of metal ions.

It is usually difficult and often impossible to distinguish between the enzymic and nonenzymic actions of inhibitors. It is doubtful if any type of kinetic analysis would be useful. In some cases a variation of the inhibitor structure may be informative. For instance in the example of the metal chelating agents, given above, the testing of bipyridines or phenan- 
throlines that are not chelating agents, or of compounds structurally related in other ways, may allow establishing a correlation between the effect on the cell and the chelating activity. The lack of a demonstrable effect on the over-all metabolism of the cell or tissue is not sufficient evidence for a nonenzymic effect because the action may be localized and that fraction of the total metabolism affected could be insignificant. Also one camot be certain that he is measuring the right thing to show the metabolic effect if it does exist.

\section{Dependency of the Functional Activity on the Level of High-Energy Bonds}

The cell function may require an available source of energy in the form of ATP or other high-energy nucleotides. These nucleotides, furthermore, are often in equilibrium with a store of creatine phosplate. The function may remain relatively unchanged mitil the ATP drops below a certain level due to an inhibitor and some ATP may be furnished throngh phosphorylation of ADP by the creatime phosphate. A cell function requiring little energy can thus continue for some time after the fundamental energysupplying reactions are blocked or uncoupled. The function may also be dependent on conditions established by ATP or other energy sources. These conditions may be fairly stable and persist for a long time after ATP formation is stopped. The conduction of nerve impulses or ameboid movement during periods of metabolic failure would be examples. In the former case, it it basically due to the fact that the ion pumps have established concentration differences and potential differences at the membrane; each impulse requires so little ion movement that the potential drops only slowly when the pumps cease activity. The degree of functional inhibition in these cases clearly bears no relationship to the interference with metabolism.

The more actively a cell functions, the more rapidly will it deplete its store of energy and suffer depression from an inhibitor. Thus nerve tissue or a heart will fail more rapidly in the presence of an inhibitor if it is stimulated rapidly so that impulses or contractions are more frequent. The functional activity is therefore dependent not only on the rate at which energy is supplied but also on the rate at which it is utilized. Most cells perform several functions (we may consider syntheses and growth as functions here for convenience) and some may require more energy than others. In this case, an inhibitor can more or less selectively depress certain functions. Generally the function requiring the most energy will be depressed more readily than the rest. It might be erroneously conchuled that this function only was dependent on the particular metabolic phase inhibited, whereas all the processes might depend on the same enzymic reactions but to different degrees. Let us for the moment assume that all the functions draw energy from a common pool and are not specifically related to indi- 
vidual metabolic pathways. If a cell performs three functions $-F_{1}, F_{2}$, and $F_{3}-$ the energy required for the normal maintenance of each function will be $E_{1}, E_{2}$, and $E_{3}$. Assuming, for simplicity, that these make up the total cell activity, the energy requirement for the cell is $E_{t}=E_{1}+E_{2}+$ $E_{3}$. The energy-supplying reactions provide energy equivalent or in excess of this, but in the presence of an inhibitor the energy supply may drop below $E_{t}$ as illustrated in Fig. 9-6. If the inhibition lowers the energy pro-

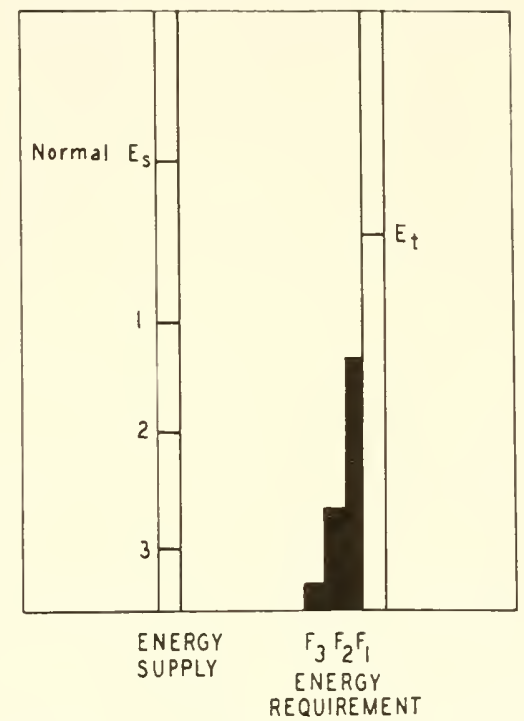

FIG. 9-6. Relationship between metabolic energy supply and functional energy requirement. The heights of the solid bars represent the energy requirements for the three different functions and $E_{t}$ is the total requirement. The normal energy supply is $E_{s}$ and the reductions by inhibition are indicated by the numbers. See text for detailed explanation.

duction to level 1 , it is possible that all the functions will be depressed somewhat. or. if one function has preference over the others (that is, if a function is able to obtain the energy available more efficiently), it will remain unchanged at the expense of the others. But when the energy supply is lowered to level 2. it is evident that $F_{1}$ could not continue normally, although $F_{2}$ and $F_{3}$ may still do so. At level 3 , only $F_{3}$ could remain normal and then only if it had preference. In such cases the results may be thought 
of in terms of competition between functional processes for energy and the relative depressions will reflect to some extent the competitive abilities. Of course, in the living cell, although this simplified picture may be valid generally, it is probable that some functions are specifically and spatially related to enzyme sytems of different types. One of the most interesting problems in nerve and muscle physiology is whether the excitatory events at the membrane are dependent on enzyme reactions within the membrane, as opposed to the total cell metabolism, and if inhibitors can modify the electrical behavior of the membrane, and secondarily the cell function, by acting on these enzymes of the membrane. It might be expected that all types of oxidative or phosphorylative inhibition would produce the same effects upon membrane behavior if this behavior were dependent only on a source of ATP from the bulk of the cell or from the mitochondria. However, the effects of different inhibitors are not the same on resting and action potentials and this might be taken as indirect evidence for enzymic processes at the membrane (Webb and Hollander, 1959).

\section{Inhibition of an Enzyme System Forming an Agonist}

An enzyme inhibitor may alter tissue function by reducing the formation or inactivation of some substance that is produced by the tissue, this substance itself controlling the tissue function. Such a substance may be termed an agonist, in conformity with pharmacological nomenclature (Furchgott, 1955), which implies that the substance acts on the responsive cells, either to stimulate or depress them. The agonist could be formed by the cells under investigation or it could arise in other types of cells present in the preparation. In the latter situation, the agonist may either produce its effect by reacting with receptors at the surface of the responding cells, or it may have to enter into the membrane to react, or it may have to pass through the membrane into the interior of the cell. The enzyme inactivating the agonist may likewise be situated spatially either ontsicle, within the membrane, or inside the cell. Finally, the agonist may simply diffuse away from the region where it is produced and where it acts. These processes may be represented diagramatically as:

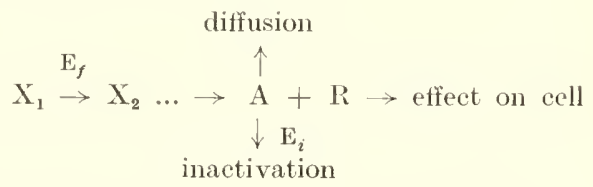

where $\mathrm{X}_{1}$ and $\mathrm{X}_{2}$ are precursors of the agonist, $\mathrm{E}_{f}$ represents the enzymes involved in its formation, $\mathrm{R}$ is the receptor group with which it must react to affect the cell, and $\mathrm{E}_{i}$ is the enzyme inactivating the agonist. The action of an enzyme inhibitor on either $\mathrm{E}_{f}, \mathrm{E}_{i}$, or $\mathrm{R}$ will modify the functional ac- 
tivity of the cells indirectly, since the effect of the agonist will depend primarily on the concentration present in the region of the receptors and the ability of the receptors to react with the agonist. The reaction with the receptors is usually represented simply as:

$$
\mathrm{A}+\mathrm{R} \rightleftharpoons \mathrm{AR} \quad K_{a}=\frac{(\mathrm{A})_{r}(\mathrm{R})}{(\mathrm{AR})}
$$

and the effect on the tissue is assumed to be proportional to $(\mathrm{AR}) /\left(\mathrm{R}_{t}\right)$, the fraction of the total receptors combined with agonist:

$$
\frac{(\mathrm{AR})}{\left(\mathrm{R}_{t}\right)}=\frac{(\mathrm{A})_{\tau}}{(\mathrm{A})_{\tau}+K_{a}}
$$

where $(A)_{r}$ is the concentration of agonist in the receptor region. Some common agonists would be acetylcholine, epinephrine, norepinephrine, histamine, 5-hydroxytryptamine, and related substances, but the concept could be extended to any substances produced in the preparation and having an effect on the cellular activity. Even in isolated tissues, such substances may control the functional level. Isolated cardiac tissue or intestine are under the influence of acetylcholine in most cases, as may be readily shown by the effects of the specific antagonist atropine. Thus these tissues are susceptible to effects of inhibitors on any of the reactions determining the concentration of agonist.

The simplest system in which the agonist is formed and is active and inactivated in a discrete region (diffusion either through membranes or away from the region being umimportant), is actually represented by a monolinear chain (Eq. 7-1) and the concentration of agonist is given by the expressions developed for multienzyme systems. Inhibition of the formation reaction would, of course, lead to a decrease in agonist concentration and a changed level of tissue activity. The agonist is usually formed, not by a single reaction but by a series of enzymically catalyzed steps. The synthesis of acetylcholine, for example, requires choline and an acetyl donor (probably acetyl-CoA); hence, the enzymes involved in the formation of the acetyl donor are possible sites for inhibition as well as the choline transacylase, the enzyme catalyzing the final condensation. Inhibitorinduced functional failure in synaptic networks most likely results from depression in the formation of acetylcholine or some other transmitter.

\section{Inhibition of an Enzyme Inactivating an Agonist}

The potent effects on tissue function of the inhibitors of enzymes inactivating acetylcholine or the sympathomimetic amines are well known. These actions are due to the rise in agonist concentration at the receptor region. The normal balance between formation and inactivation is disturbed. 
Whether a new steady state will be attained will depend not only on the enzymic inactivation but possibly on the diffusion of the agonist from the region. It will also depend on the rate at which the agonist is formed; when it is synthesized in and released by nerve endings, its production will naturally depend on the frequency of impulses in the nerve. In some isolated preparations, the rate of formation is apparently steady and inhibition of the inactivating enzyme will cause a progressive aceumulation of the agonist, as in isolated atria (Wel)b, 1950 b).

The kineties of such a multienzyme system will depend on the spatial arrangement of the three basic elements - the receptor groups and the loci for formation and inactivation of the agonist. These are probably never distribnted homogeneously within a region since all are intimately related to certain cell structures. The agonist usually arises ontside the cell it affects and must diffuse to the receptors, which can be on the surface of the cell, in the membrane. or inside the cell; the inactivating enzyme can likewise be located in any of these regions. The effect of inhibiting the inactivating enzyme will be much greater if this enzyme is so located that the agonist must diffuse throngh a "screen" of it before it can react with the receptors, beeause in this case the agonist concentration at the receptor region will be strongly dependent on the activity of the inactivating enzyme. A certain fraction of the agonist will normally be destroyed before it can produce an effect and an inhibitor will decrease this. Possible spatial relations between acetylcholinesterase and receptors in atrial tissue have been diseussed (Webb, 1950b) and evidence mesented that such a "sereening" effect is operative in this tissue, where the acetylcholine concentration at the receptors may be only one-hundredth that of the concentration in the medium. It is, therefore, tifficult to develop a quantitative treatment of such inlibition. Equations have been derived by Furchgott (1955) for the situation in which the agonist must diffuse across a membrane to a region where receptors and inactivating enzyme are homogeneously distributed. It is mofortmate that such a direct treatment is probably not justifiable in most cases but the validity must ultimately be determined by accurate experimental data, which at mesent are lacking. It was also assumed that the inactivation is first-order and did not follow enzyme kinetics. Finally, the acetyleholinesterase is a good example of a mutual depletion system (Chapter 3) and to what extent these more complex kinetics must be applied is minnown.

Assuming a constant external concentration of agonist and a homogeneous distribution of receptors and inactivating enzyme within a membrane:

$$
\mathrm{A}_{0} \underset{\mathrm{A}_{i}}{\mathrm{E}_{i}>\text { inactivation }}
$$


the internal concentration of agonist is given by:

$$
\left(\mathrm{A}_{i}\right)^{2}+\left[\left(\boldsymbol{V}_{m} / k\right)+K_{s}-\left(\mathrm{A}_{0}\right)\right]\left(\mathrm{A}_{i}\right)-K_{s}\left(\mathrm{~A}_{o}\right)=0
$$

which is identical with Eq. 7 -11, where $k$ is the diffusion constant of the agonist through the membrane. The behavior of such a diffusion-limited monolinear chain has been discussed. Figure 9-7 shows the variation in

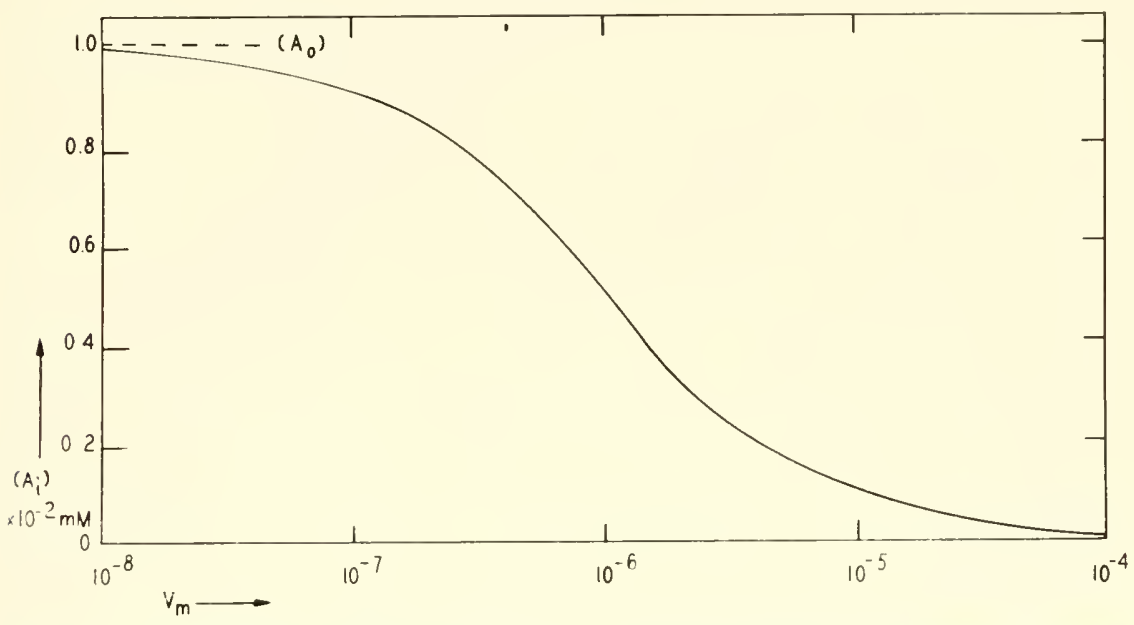

FIG. 9-7. Concentration of an agonist in the receptor region as it depends on the rate of its destruction (scheme 9-16 and Eq. 9-17). $\left(\mathrm{A}_{0}\right)=10^{-3} \mathrm{~m} M, K_{s}=1 \mathrm{~m} M$, and $k=10^{-3}$.

$\left(A_{i}\right)$ with the rate of inactivation and Fig. 9-8 illustrates how the effect of the agonist on the tissue increases with rising inhibitor concentration. As the activity of the enzyme becomes less, the agonist concentration rises in a sigmoidal fashion and eventually becomes equal to $\left(A_{0}\right)$. It is interesting to note that it requires a fair degree of inhibition of the enzyme before there is an appreciable increase in the activity of the agonist. The change in the functional activity occurs mainly between $90 \%$ and complete inhibition of the enzyme. It may be observed that an inhibitor of an inactivating enzyme will have its greatest effect on function when the rates of entrance and destruction of the agonist are comparable: if either one is much larger than the other, such inhibition will have very little effect on the agonist concentration. If the receptors are in the membrane, the partition coefficient of the agonist between medium and membrane wonld have to be introduced; this wonld be the same as assigning different rates to the inward and outward diffusion across the membrane in scheme 9-16.

It is quite possible that the kinetics for the situation in which the enzy'me is forming a screen between the source of agonist and the receptors 
may be relatively simple. In this case, perhaps the structural nature of the enzyme grid allows $\left(\mathrm{A}_{i}\right)$ to equal $\left(\mathrm{A}_{o}\right)\left[\left(k_{1} / k_{-1}\right)\right]$, where $k_{1}$ and $k_{-1}$ are the diffusion constants for the inward and outward directions through the enzyme screen. The enzyme activity would have the effect of reducing the diffusion rate by destroying a certain fraction of agonist molecules; thus $k_{1}$ may be smaller than $k_{-1}$ and $\left(\mathrm{A}_{i}\right)$ smaller than $\left(\mathrm{A}_{0}\right)$. Inhibition of the enzyme would change $k_{1}$ but not $k_{-1}$ since it makes no difference if a

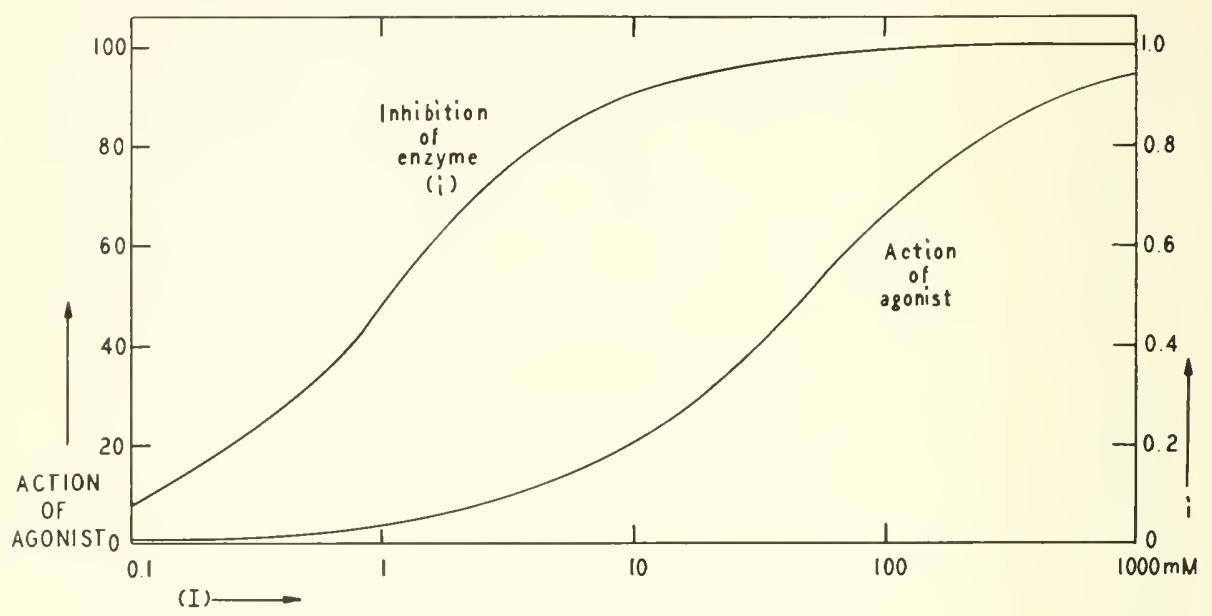

FIG. 9-8. Effect of the inhibition of the inactivating enzyme on the action of an agonist. The agonist action is given in per cent of the action produced by the external concen. tration. Same conditions as in Fig. $9 \cdot 7$ and in addition $K_{a}=10^{-2} \mathrm{~m} M$ and $K_{i}=1 \mathrm{mM}$.

molecule diffusing out is inactivated or not. It can be shown that the internal agonist concentration in the presence of the inhibitor is given by:

$$
\begin{aligned}
& \left(\mathrm{A}_{i}\right)_{i}=\left(\mathrm{A}_{o}\right)\left[\frac{k_{1}}{k_{-1}}(1-i)+i\right] \\
& \left(\mathrm{A}_{i}\right)_{i}=\left(\mathrm{A}_{i}\right)(1-i)+i\left(\mathrm{~A}_{o}\right)
\end{aligned}
$$

When there is no inhibition, $\left(A_{i}\right)_{i}=\left(A_{i}\right)$, and when inhibition is complete, the external and internal concentrations are the same, or $\left(\mathrm{A}_{i}\right)_{i}=\left(\mathrm{A}_{0}\right)$. In case the agonist concentration in the uninhibited state is very low so that it is not affecting tissue function significantly (i.e., when the enzyme is effectively inartivating the agonist almost as rapidly as it is formed), the internal concentration during inhibition will be given approximately by $\left(\mathrm{A}_{i}\right)_{i}=i\left(\mathrm{~A}_{0}\right)$. 


\section{Quantitative Relations between Functional Change and Inhibition}

Very little is known of the mechanisms by which energy is transferred to the functional systems or utilized in the functional acts; indeed, one is generally ignorant of the basic relationship between the level of functional activity and the energy supply. However, we can be reasonably certain that in most cases there are several processes between the generation of the energy and the final cell activity. This may be crudely expressed by an arbitrary series of equations, such as:

$$
\begin{aligned}
\text { Function } & =f_{1}(\mathrm{ATP}) \\
(\mathrm{ATP}) & =f_{2} v_{p}+f_{3} v_{d} \\
v_{p} & =f_{4} v_{0} \\
v_{d} & =f_{5}(\mathrm{ATPase})(\mathrm{X}) \\
v_{o} & =f_{8}(\mathrm{~S})+f_{7}(\mathrm{I})
\end{aligned}
$$

where the $f$ 's indicate the functional dependences, $v_{p}$ is the phosphorylative rate, $v_{d}$ is the rate at which ATP is hydrolyzed by ATPase, $v_{o}$ is the oxidative rate, $(\mathrm{X})$ represents the concentrations of various ions influencing ATPase activity, (S) the concentration of substrate for oxidation, and (I) the concentration of an inhibitor that interferes with oxidation. The dependency of function on the inhibitor concentration would then be given by:

$$
\text { Function }=f_{1}\left\{f_{2} f_{4}\left[f_{6}(\mathrm{~S})+f_{7}(\mathrm{I})\right]+f_{3} f_{5}(\text { ATPase })(\mathrm{X})\right\}
$$

so that it would be extremely fortuitous for any simple relationship to emerge. Thus if one is measuring the functional response to an inhibitor, it is unduly optimistic to expect to be able to elucidate enzymic mechanisms of inhibition from the changes observed; i.e., an inhibition cannot be labeled as competitive on the enzyme level because an increase of substrate concentration tends to overcome the effect of the inhibitor on cell function. The concept that is being emphasized is simply that the relationship between cell function and enzyme inhibition is, in essentially all cases, very complex and this complexity should not be obscured by apparently simple experimental results.

\section{Establishing Correlations between Functional Changes and Enzyme Inhibition}

One of the primary reasons for using inhibitors in living tissue is to determine the specific metabolic basis for functional activity. Thus uncouplers of oxidative phosphorylation are useful in showing a dependence of some cell activity on high energy phosphate compounds, while inhibitors of the tricarboxylic acid cycle (such as fluoroacetate) or glycolysis (such as iodoacetate) are commonly used to demonstrate the presence or absence 
of a conmection of function with these metabolic pathways. It is, however, often very difficult to establish a direct correlation between the metabolic and functional changes for reasons given above; in addition the lack of absolute specificity for most inhibitors complicates the matter still further. Despite the serious problems in such investigation, there are several ways in which a correlation may be made with varying degrees of certainty.

I. Correspondence of inhibitor concentrations used. The concentrations of inhibitor necessary to produce the enzymic and functional changes must be comparable. The use of concentrations far in excess of that inhibiting on isolated preparations, as is often done, eliminates the possibility of correlation, unless evidence can be produced that the intracelhular concentration is lower than the external concentration and similar to that effective in vitro.

II. Demonstration of specificity at the appropriate concentration. When the inhibitor at the concentration causing functional change can be shown to be specific in action on one enzyme or metabolic pathway, and in all probability without nonenzymic action, the relationship between this function and the enzyme attacked is more certain. However, there are very few instances where reasonable specificity can be attributed to an inhibitor.

III. Simultaneous demonstration of functional and metabolic change. Occasionally it is possible to show that during the change in function there is a concomitant inhibition of a particular enzyme or metabolic process, as by the demonstration of the accumulation of some intermediate analytically or spectroscopically, or the failure in formation of some product. Of course, this shows only that the two effects are occurring simultaneously and does not prove that a relation exists betweem them.

IV. Reversal of functional change by reversal of imhibition. One of the best ways, where it is possible, to make correlation is to show that an alleviation of the inhibition is accompanied by restoration of normal function. If the inhibitor is blocking a pathway, the addition of an intermediate past this block may reverse the functional change. The adition of pyruvate during glycolytic inhibition by iodoacetate is commonly used to show that the inhibition is indeed on glycolysis. It is necessary to perform the appropriate controls. particularly the effect of addition of the intermediate without the inlibitor, to establish that the effect is reversal of the block. The reversal of inhibition and functional alteration by adding a substance that combines with and removes the inhibitor reveals nothing.

V. Isolation of the inhibited enzyme. When the enzyme inhibition is reasonably irreversible, it is sometimes possible to extract the enzyme in question from the tissue whose function has been altered, and demonstrate a degree of inhibition on it. This merely shows that both events occur and does not establish cansal relationship. 
VI. Use of several inhibitors or analogs. If the enzyme suspected of connection with the function can be depressed to varying degrees by several inhibitors, a very useful approach is to examine the evidence for parallel effects on enzyme and function. Substances chemically similar to the basic inhibitor, but without inhibitory action on the enzyme, should not alter the function if there is indeed a relationship between the two processes. This technique is particularly valuable when an homologous series of substances can be tested and the degree of inhibition correlated with functional disturbance.

The functional side of the picture may also be complex and the final cellular activity measured may be dependent on both metabolic and nonmetabolic processes. One example may be cited. The dependence of cardiac muscle contraction on high-energy phosphate is being studied by using the uncoupler of oxidative phosphorylation, 2,4-dinitrophenol, and it is found that the contractile force is depressed readily by this substance. The conclusion might be drawn that the cyclic changes in the state of the actomyosin complex are directly controlled by the availability of ATP and that 2,4-dinitrophenol is producing its effect by depressing the formation of ATP to be used by the contractile elements. On the other hand, when the actions of 2,4-dinitrophenol on the cell membrane potentials are recorded simultaneously with the contractile response, it is seen that at least the initial contractile depression is the result of changes brought about in the ionic fluxes across the membrane (Webb and Hollander, 1956). In this case, the membrane processes are more sensitive to 2,4-dinitrophenol than are the contractile events, but the latter, being controlled by the nature of the action potential, are secondarily affected. A method for determining the relative effects of inhibitors on membrane and contractile processes has been suggested (Webb and Hollander, 1959); if this method is valid, it would appear that most inhibitors affect both processes simultaneously but to different degrees. Similar relations between membrane events and the measured function may occur in other types of contractile cells and in cells carrying out various activities, such as secretion or cleavage.

An example of the difficulty in establishing a correlation between the inhibition and the effect produced in a relatively simple situation will be cited for illustration. Many of the modern and most potent insecticides are organophosphorus compounds that are called cholinesterase inhibitors, and it has been generally assumed that the lethal action is the result of the inhibition of this enzyme. However, these substances are known to inhibit other enzymes and recently the question as to the mechanism of their insecticidal action has been argued. In houseflies there are at least two enzymes that are very sensitive to the organophosphorus compounds, a cholinesterase and an aliesterase (hydrolyzing low molecular weight aliphatic esters). Flies were exposed to several insecticides and the inhi- 
bition of each of these enzymes was determined at the time of knockdown (see tabulation). The results show that at the time of death the cholinesterase was inhibited only moderately whereas the aliesterase was in some

\begin{tabular}{l|c|c}
\hline \multicolumn{1}{c}{ Insecticide } & Cholinesterase & Aliesterase \\
\hline Paraoxon & 51 & 95 \\
Parathion & 49 & 95 \\
Diazoxon & - & 91 \\
Diazinon & 32 & 90 \\
DDVP & 27 & 76 \\
Co-Ral & 44 & 75 \\
\hline
\end{tabular}

From Van Asperen (1960).

cases almost completely inhibited. This might be used as evidence that the lethal action is not related to the inhibition of cholinesterase but of aliesterase. However, this conclusion would be completely unjustifiable. In the first place, we do not know how much of the cholinesterase of the fly must be inhibited so that a marked disturbance would occur in the neuromuscular system; thus the moderate inhibitions observed on cholinesterase might well be sufficient to cause death. In the second place, the inhibition of the cholinesterase in the tissue or region involved in the lethal action may be different from the over-all inhibition as determined by extracting whole flies. Differences in the distribution of the insecticides or different susceptibilities of the various tissue cholinesterases could account for this. In the third place, from what little is known of the role of aliesterases, it might be expected that even complete inhibition would not cause such rapid death. The simple fact that an enzyme is strongly inhibited does not necessarily implicate it in the mechanism and the relationship of this enzyme to the function measured or the life of the organism must be established. If the inhibition of a single enzyme is responsible for an effect, this effect should occur at the same inhibition of the enzyme whatever inhibitor is used; this is not true for either enzyme in the flies.

\section{QUANTITATIVE EXPRESSION OFINTRACELLULAR ENZYME INHIBITION}

The problem now to be considered is this: to what extent can the equations derived for the inhibition of isolated enzymes be applied to inhibition within the living cell? As a corollary to this, it might also be asked if it is possible to determine enzyme and inhibition constants from the study of cells or tissues. The discussion will be restricted to inhibition on a single 
enzyme. The problems posed by the possibility that this enzyme is a component of a multienzyme system within the cell and that the intracellular environment may be quite different from an artificial medium have been treated already. The kinetics of enzymes enclosed within membranes that limit the diffusion of substrates have been developed by Best (1955 b) and Blum (1956; Blum and Jenden, 1957) and have been applied to hexokinase and $\beta$-fructofuranosidase in yeast cells (Best, 1955 c). It may be stated generally that when an enzyme is within a cell, the MichaelisMenten kinetics no longer hold in their simple form and that the usual reciprocal plots are often no longer linear or easily interpreted. The bearing of these factors on intracellular inhibition will now be outlined for certain situations.

\section{The Factor of Diffusion into Cells of Different Shapes}

The kinetics of an enzyme enclosed within a membrane through which its substrate must diffuse were presented in the simplest possible form in Chapter 2 and it will be necessary to extend this treatment somewhat to provide a basis for the discussion of inhibition. The reciprocal equation for the situation when diffusion of substrate is a factor in determining the rate of the reaction can be obtained from the expressions for the rate of entry and the enzyme rate (Best, $1955 \mathrm{~b}$ ):

$$
\frac{1}{v}=\frac{1}{V_{m}}+\frac{K_{s}}{V_{m}(\mathrm{~S})\left[1-\frac{v}{k_{0}(\mathrm{~S})}\right]}
$$

where $k_{0}$ is the permeability constant, (S) is the external concentration of substrate, and $K_{s}$ is the substrate constant for the enzyme (as in Chapters 2 and $5, K_{s}$ will be used to express the Michaelis-Menten constant but need not imply that it is a dissociation constant). When $(\mathrm{S})$ is large, the slope of the $1 / v-1 /(\mathrm{S})$ plot will be $K_{s} / V_{m}$ as it would be without the membrane, but when (S) is small, the slope will be greater and equal to $K_{s} / V_{m}+$ $1 / h_{o}$, as shown in Fig. 9-9. It may be easily shown that both competitive and noncompetitive inhibition will increase the slopes and intercepts to the same degree as in homogeneous systems. The determination of $K_{i}$ thus presents no special problems, but if the change of slope is so close to the $1 / v$ axis that a constant slope is assumed, it is possible to obtain an erroneous value for $K_{s}$. A method for calculating the various constants of the uninhibited system, including $k_{o}$, based on the original treatment of Lineweaver and Burk (1934), has been outlined by Best (1955 b).

Extensions of this treatment can be made to cells of different shapes. A long cylindrical cell, e.g. nerve or muscle, in which the enzyme is homogeneously distributed, has been considered by Blum (1956) and two re- 
ciprocal equations derived for saturating and subsaturating substrate concentrations (the second equation appears to be written incorrectly in the original publication):

$$
\begin{aligned}
& \frac{(\mathrm{S})}{K_{s}} \gg 1 \quad \frac{1}{v}=\frac{1}{V_{m}}+\frac{-}{V_{m}\left\{(\mathrm{~S})-V_{m}\left[\frac{r}{2 k_{o}}+\frac{K_{s}}{8 D_{i}}\left(\frac{\alpha}{\alpha+K_{s}}\right)\right]\right\}} \\
& \frac{(\mathrm{S})}{K_{s}} \ll 1 \quad \frac{1}{v}=\frac{\sqrt{\alpha K_{s}}}{2 V_{m}(\mathrm{~S})}\left[\frac{r I_{0}(\sqrt{\alpha} r)}{I_{1}(\sqrt{\alpha} r)}+\frac{D_{i}}{k_{o}}\right]
\end{aligned}
$$

where $r$ is the radius of the cylinder, $k_{0}$ is the permeability constant, $D_{i}$ is the diffusion constant inside the cylinder, $\alpha=V_{m} / D_{i} K_{s}$, and the $I$ 's

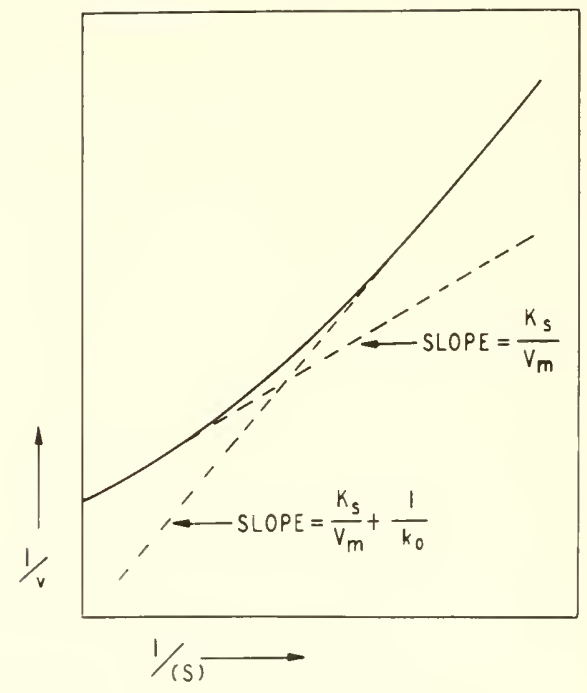

FIG. 9-9. Double reciprocal plot for an enzyme reaction occurring within a membrane of permeability constant $k_{0}$.

are Bessel functions. Furthermore, it follows that in the general case the substrate concentration is not the same throughout the cell, but decreases from the membrane inward in an approximately exponential fashion. Concentration profiles for cylindrical cells were calculated for specific cases and it is seen that they depend on all the various permeability, diffusion, and enzymic factors. The measured rate will be an average of the varying rates in the cell.

Cylinders, spheres, and flat sheets are kinetically analyzed by Blum and Jenden (1957) and equations are given for calculating the concentration 
profiles within such regions. The rate equations are quite complex but the implications for inhibition are clear. For competitive inhibition, since the substrate concentration is not constant throughont the cells, the degree of inhibition varies in characteristic ways from one region of the cell to another. The inhibition in such a case will be greater than predicted from the external substrate concentration, providing the inhibitor penetrates readily into the cells. Noncompetitive inhibitors by reducing the effective enzyme concentration may exert complex effects on the rate when $(S)$ is high relative to $K_{s}$ (see Eq. 9-22), but when (S) is low the increased slope of the $1 / v-1 /(S)$ plot will be identical to that of the enzyme without geometrical constraint. The other methods of plotting will frequently result in nonlinearity but the present state of our knowledge would not justify an accurate analysis of these relationships.

\section{The Factor of Intracellular Concentrations of Substrate and Inhibitor}

The intracellular concentration of an inhibitor may be different from that in the external medium for reasons already discussed. It will now be pertinent to investigate how this may modify the quantitative expression of inhibition. The procedure is simple: the internal concentration. $\left(\mathrm{I}_{i}\right)$. is expressed in terms of the external concentration. $\left(\mathrm{I}_{e}\right)$. and is substituted in the equations of inhibition developed in trevions chapters. The diffculty lies in our ignorance of how to express $\left(I_{i}\right)$ in terms of $\left(I_{e}\right)$. There are actually many possible relations. One immediately thinks of the expression $\left(\mathrm{I}_{i}\right)=r_{i}\left(\mathrm{I}_{e}\right)$ where $r_{i}$ is simply the ratio of concentrations inside and outside the cell. The fractional degree of noncompetitive inhibition would then be given by:

$$
i=\frac{r_{i}\left(\mathrm{I}_{e}\right)}{r_{i}\left(\mathrm{I}_{e}\right)+K_{\imath}}=\frac{\left(\mathrm{I}_{e}\right)}{\left(\mathrm{I}_{e}\right)+\left(K_{i} / r_{i}\right)}
$$

When $1 / v$ is plotted against $1 /(\mathrm{S})$, the intercept on the $1 v$ axis will be

$$
\frac{1}{V_{m}}\left[1+\frac{r_{i}\left(\mathrm{I}_{e}\right)}{K_{i}}\right] \text { and the slope will be } \frac{K_{s}}{V_{m}}\left[1+\frac{r_{i}\left(\mathrm{I}_{e}\right)}{K_{i}}\right] \text {. }
$$

Thus by this method one will obtain not $K_{i}$ but $K_{i} / r_{i}$ by the usual calculation. The type D plot of $1 / v_{i}$ against $\left(\mathrm{I}_{e}\right)$ will be linear with the same intercept as for a noncelhular enzyme (see Fig. 5-3), but the slope will now be

$$
\frac{r_{i}}{V_{m} K_{i}}\left[1+\frac{K_{s}}{(\mathrm{~S})}\right] \text {. }
$$

Dividing the intercept by the slope will not give $K_{i}$ but $K_{i} / r_{i}$. Likewise. the intercept on the (I) axis will now be $-K_{i} r_{i}$ instead of $-K_{i}$. A type F plot will result in a horizontal line but at $K_{i} / r_{i}$ rather than $K_{i}$. as may 
be seen by evaluation of $\left(\mathrm{I}_{e}\right)(1-i) / i$ (see Fig. 5-3). Thus in these methods, and in the others as well, the determination of $K_{i}$ may be seriously in error if the internal and extermal inhibitor concentrations differ appreciably.

The uptake of 2,4-dinitrophenol by yeast cells follows this simple relationship over the range of concentrations generally used, since an increase in the external concentration from $0.0495 \mathrm{~m} M$ to $0.198 \mathrm{~m} M$ (fourfold increase) led to a rise in the intermal concentration from 5.2 to 20.1 (3.9fold increase) (Kiesow, 1959). These concentrations produced a strong inhibition of the respiration and an inhibition of the Pasteur effect. However, the simple situation in which $\left(\mathrm{I}_{i}\right) /\left(\mathrm{I}_{e}\right)$ remains constant for any external concentration of inhibitor is probably rare. This may well hold true over a limited range of concentration but in most cases the internal concentration reaches a maximal level as the external inhibitor concentration is increased. When active transport or exclusion of the inhibitor occurs, the mathematical expression of $\left(I_{2}\right)$ in terms of $\left(I_{e}\right)$ will be impossible, unless the actual concentrations can be determined. For inhibitors that are weak acids or bases, the relationship between inner and outer concentrations may be complex and will, of course, depend on the external and internal $\mathrm{pH}$ (see Chapter 14).

Competitive inhibition will involve in addition the intracellular concentration of the substrate. $\left(\mathrm{S}_{i}\right)$, which may be related to the external concentration, $\left(\mathrm{S}_{e}\right)$, in a manner similar to or different from the inhibitor. The simplest expression would again be $\left(\mathrm{S}_{i}\right)=r_{s}\left(\mathrm{~S}_{e}\right)$. The rate and inhibition equations would then be given by:

$$
\begin{aligned}
& v_{i}=\frac{V_{m}\left(\mathrm{~S}_{e}\right)}{\left(\mathrm{S}_{e}\right)+\frac{K_{s}}{r_{s}}\left[1+\frac{r_{i}\left(\mathrm{I}_{e}\right)}{K_{i}}\right]} \\
& i=\frac{\left(\mathrm{I}_{e}\right)}{\left(\mathrm{I}_{e}\right)+\frac{K_{i}}{r_{i}}\left[1+\frac{r_{s}\left(\mathrm{~S}_{e}\right)}{K_{s}}\right]}
\end{aligned}
$$

to correspond to Eqs. 3-12 and 3-13. The analytical plots may differ even more from the isolated enzyme situation than those discussed above for noncompetitive inhibition. If one could be certain of these simple relationships and the similarity of the extracellular and intracellular enzyme, one might use such methods to obtain an idea of the values of $r_{i}$ or $r_{s}$, but the assumptions are generally too untenable. At least the possibility of distinguishing between competitive and noncompetitive inhibitions within cells is a reality since the usual analytical plots will remain linear and have the characteristics shown in Fig. 5-1 and 5-3 when the relation between internal and external concentrations is a simple function. 


\section{INHIBITION IN TISSUE SLICES AND ISOLATED TISSUE PREPARATIONS}

Tissue slices are used so commonly in metabolic studies that attention will now be turned to some of the special problems encountered when using inhibitors. The abnormality of tissue slices has been frequently emphasized and experimentally investigated, both histologically and biochemically. The outside layers of damaged cells; the possible anoxia of the innermost cells; the leakage of ions, proteins, and enzymes from the slices during incubation; these are problems in any work on such preparations. but here only the points relevant to inhibition will be mentioned.

\section{Penetration of Inhibitors into Slices}

A slice of tissue $0.4 \mathrm{~mm}(400 \mu)$ in thickness may be composed of 10 to 20 layers of cells and an inhibitor, if it cannot penetrate between the cells, must penetrate successively many cell membranes to reach the interior of the slice. Thus it may require a fairly long interval for the inhibitor to be uniformly distributed in all the cells. Another factor may also slow down penetration. This is the binding of certain inhibitors to cell material as they come into contact with it. An inhibitor which reacts readily with protein, such as a heavy metal ion, will be concentrated in the external layers of the slice during the early period of incubation inasmuch as a major fraction that enters the slice will be immediately bound. The inhibition during these early phases can be very inhomogeneous and the measured degree of inhibition only a kinetically meaningless average value. A progressive depression of metabolism might be interpreted in terms of the rate of inhibition of an enzyme system but it could just as well be explained on the basis of a delayed entrance of the inhibitor into the slice. A kinetic analysis of an inhibition in slices, in the manner of the preceding section, presupposes a uniform distribution of the inhibitor.

\section{Binding of the Inhibitor to Nonenzymic Material}

Using isolated enzymes one can often neglect any reduction in inhibitor concentration arising from binding to nonenzymic substances but with tissue slices or strips the amount of this nonenzymic material is much greater relative to the enzymes attacked. The specific inhibitor concentration, $\left(\mathrm{I}^{\prime}\right)=(\mathrm{I}) / K_{i}$, is not sufficient to predict if depletion will occur, because this will now depend on the various dissociation constants and concentrations of all the substances to which the inhibitor may be bound. Let us take an organic arsenical as an example. If its molecular weight is 175 and it is used at $0.01 \mathrm{~m} /$ concentration in a reaction medium of $3 \mathrm{ml}$, the total amount of inhibitor present is $0.00525 \mathrm{mg}$. If the ability of the 
tissue to bind the arsenical is only $0.01 \%(0.1 \mathrm{mg}$ arsenical per gram of tissue) and $100 \mathrm{mg}$ of tissue are present, $0.01 \mathrm{mg}$ of the inhibitor can be bound maximally, and this is approximately twice as much as is present. This factor can then be easily of importance, particularly with the more potent inhibitors. The kinetics in such cases will be similar to those for mutual depletion systems and the calculation of inhibition constants by the use of the classic plotting techniques can lead to very erroneous values.

The uncoupling agent, 2.4-dinitrophenol, is not a substance that is strongly bound to cellular material, and yet when different cell densities of yeast suspension were equilibrated with $0.49 \mathrm{mM} 2$,4-dinitrophenol, the amount taken up per cell decreased with increasing density (Kiesow, 1959). The falling off was particularly evident when the total cell volume reached $100 \mathrm{~mm}^{3}$ and it was calculated that over half the uncoupler could be depleted from the medium. Inasmuch as this uptake was readily reversible and dead cells took up very little 2,4-dinitrophenol, it would seem that binding to the cell components is not the reason for the accumulation in the yeast cells. If the concentration of 2.4-dinitrophenol had been lower, as it usually is in uncoupling experiments, the depletion from the medium would have been more marked. Thus the factor of reduction in the inhibitor concentration must be at least considered in most work done with living cells or tissues.

\section{The Functional State of the Tissue}

It is well known that both the rate and pattern of metabolism vary with the functional activity of a cell or tissue. The problem often arises as to the state of a tissue slice and if results with inhibitors on them can be applier to the intact functioning tissue. Difficulties arise even with more intact tissue preparations, such as isolated atria, ventricular strips, or intestinal segments, unless the contractility can be measured simultaneously with the metabolism or in some manner correlated with it. Kidney slices can perform transport work nucler appropriate conditions but in most cases their activity is far below that of intact renal tissue. The relation between function and metabolism in tissue slices has been studied most carefully on the brain (McIlwain, 1959), where electrical stimulation of the tissue in respirometer flasks results not only in a new pattern of metabolism but alters the sensitivity of the metabolism to various drugs and inhibitors. Iodoacetate. fluoride, and malonate depress stimulated brain slices more readily than resting slices; $10 \mathrm{~m} M$ malonate has no effect on the respiration of resting slices but $1-2 \mathrm{~m} . / \mathrm{m}$ alonate is inhibitory to the extent of $12-40 \%$ when electrical stimulation is induced. It is quite probable that similar behavior wonld be demonstrated by other tissues. Designation of the usual tissue slice as resting is perhaps not always justified. What is the functional state, for example, of a myocardial slice in a Warburg vessel? 
It is not rhythmically contracting but it is not necessarily resting in the sense of existing in its functionally lowest level. Some depolarization of the cell membranes, due to damage or artificial conditions, may occur and this may induce a partial contracture, or alter the metabolism in the absence of any contractile changes since ionic pumps may be more active than in the idealized resting state.

\section{Cell Population in a Tissue Slice}

No tissue slice is composed entirely of a single type of cell. Whatever else may be present, there are always vascular cells or comective tissue. These cells contribute to the total metabolic processes to greater or lesser degrees and occasionally introdnce a different type of metabolism from that of the principal cells being investigated. There is virtually nothing one can do about this but to realize that it can canse deviations in kinetic behavior which make the calculation of inhibition constants more difficult. In some cases a major fraction of the inhibitor-stable metabolism conld be due to a minority population of cells resistant to the inhibitor. The same must apply to any tissue preparation and particularly to complex aggregates of different cells such as one would find in an isolated intestinal segment.

\section{DIFFERENTIAL EFFECTS OF INHIBITORS IN WHOLE ANIMALS}

When an inhibitor is introduced into an animal it will, at proper dosage ranges, affect some tissues more than others and, in rare instances, may even act specifically on one type of cell. It is, indeed, this specificity which is of primary importance in the development of compounds which will depress or kill those abnormal or invasive cells responsible for much disease. The differential actions of inhibitors on tissues will be a fundamental theme running through all the chapters devoted to the individual inhibitors. The question to be considered now is: what are the reasons for the different susceptibilities of tissues to inhibitors?

Inhibitors are distributed inhomogeneously in the body, being concentrated in some tissues and deficient in others, and this is certainly one very important reason for the differential effects usually observed. This has been covered in the previous chapter. The distribution factor, however, cannot by any means explain most cases of specificity and in some instances it would appear that an inhibitor is quite equally distributed between tissues responding very differently.

Each type of cell in the animal body has a characteristic pattern of metabolism, probably distinct from every other type of cell, and for this 
reason one would espect the tissues to respond in different ways to inhibitors of metabolism. Differences become even greater when the microorganisms that invade our tissues are also considered. The concentrations of substrates, intermediates, and coenzymes vary from tissue to tissue, and depend upon the blood supply, the permeability characteristics, and the nature of the metabolism. Marked variations in glucose content of tissues have been demonstrated by Gey (1956) in the rat (see tabulation). The

\begin{tabular}{l|c}
\hline \multicolumn{1}{c|}{ Tissue } & Glucose $(\mathrm{mg} / \mathrm{l} 00 \mathrm{mg})$ \\
\hline & \\
Brain & 8 \\
Testis & 9 \\
Leg muscle & 22 \\
Diaphragm & 25 \\
Spleen & 23 \\
Kidney & 51 \\
Lung & 63 \\
Liver & 82 \\
\hline
\end{tabular}

effects of inhibitors acting on glucose metabolism may differ because of this factor alone, as well as being dependent on the different activities of the metabolism that help determine these variations in glucose. The very high concentration of 2,3-diphosphoglycerate in erythrocytes (1.57 $\mathrm{m} M$ ) which is unique to these cells, could well determine the nature of the response to certain inhibitors (Bartlett et al., 1953) and serves as an example of the great biochemical variety to be expected. The intracellular concentration of a substrate or intermediate is of particular importance in determining the effect produced by a competitive inhibitor.

The most fundamental reason for metabolic divergence between tissues is probably to be sought in the widely varying relative concentrations of the various enzymes. An excellent table of such relative concentrations is given by Dixon and Webb (1958, p. 642). Especially detailed analyses have recently been made of enzymes in the anatomically distinguishable regions of the central nervous system.

The distribution of glutamic- $\gamma$-aminobutyric transaminase in the nervous system of the Rhesus monkey is very interesting and certainly related to the functional differences and susceptibility to metabolic inhibition (Salvador and Albers, 1959). In some regions (optic stalk, lateral pyramids, fasciculus gracilis) none could be detected, while in other regions the values ranged widely: globus pallidus, 52; red nucleus, 62; reticular formation, 113; anterior hypothalamic nucleus, 149; inferior colliculus, 219, and inferior olivary nucleus, 294. There is very little in the peripheral regions, such as the spinal cord or ganglia. Within the cortex the enzyme is present 
in greater amounts in certain cell layers and is found to be very high in the molecular layer of the cerebellar cortex. The distribution of glutamic decarboxylase (Albers and Brady. 1959) is equally inhomogeneous but different from the previous enzyme: lumbar spinal cord. 15, pons and medulla, 34; motor cortex, 62; cerebellum, 79; thalamus, 85; hypothalamus. 96; and midbrain. 100. Cytochrome oxidase and dipeptidase have been determined in the human central nervous system and the relation of the former to the different respiratory rates has been discussed (Pope et al., 1957). The distribution of eight enzymes involved in glucose metabolism and two cycle enzymes has been studied in the cerebellum (Robins et al., 1957). The distribution of certain dehydrogenases between the gray and white matter of human brain perhaps indicates a concentration of these enzymes in the synaptic regions (Tyler, 1960). Thus lactate dehydrogenase concentrations are 3.2-9.2 in gray matter and 2.9-4.5 in white matter. while malate dehydrogenase concentrations are 11.8-23.1 in gray matter and $10-15$ in white matter, the figures being units per milligram of tissue weight. All of these results indicate strongly that the metabolic patterns of the regions within the central nervous system are sometimes quite different. Here one has, then, metabolic differentiation in one type of tissue, so that variations are to be visualized not only between different tissues but between the histologically and functionally separable regions or cells of a single tissue.

When an inhibitor combines with an enzyme in an irreversible or pseudoirreversible fashion, the relative effects on the tissues throughout the body will in part depend on the concentrations of enzyme in these tissues, the fractional inhibition being inversely related to the amount of enzyme (see Chapter 3). When the response of tissue function is being measured, however, the relative effects will be related also to the dependency of the function on the particular enzyme or metabolic pathway inhibited, since the tissue experiencing the greatest metabolic inhibition is not necessarily the one being most functionally disturbed.

The so-called branching points in metabolism may be of special importance in the different responses of tissues to inhibitors. These branching points may be the source of divergent chains or bilinear shunts. Some tissues are undoubtedly resistant to certain inhibitors because they possess a pathway that circumvents the locus of block. Pyruvate is one of the primary branching sites in the cell, since often it may be metabolized in several different ways. Pyruvate in animal tissues can enter the cycle in two different ways; the condensation with $\mathrm{CO}_{2}$ to form dicarboxylic acid which increases the amount of cycle intermediates and allows synthesis, and it can also be oxidatively decarboxylated to acetyl-CoA which can be used as an energy source in the cycle. Injection of $\mathrm{DL}$-alanine-2- $\mathrm{C}^{14}$ into rats and the determination of the labeling in L-glutamate indicates the relative 
amounts entering the cycle by these two routes (Freedman et al., 1958). It was found that in fasted animals the primary route is the former condensation, with fats being used for energy, whereas in animals given glucose there was an appreciable amount of pyruvate oxidatively decarboxylated. In contrast to liver, the Murphy-Sturm sarcoma oxidatively decarboxylated pyruvate even in the fasted animal. Thus the branching point must somehow differ in these two tissues and this difference could serve as the basis for differential response to inhibitors acting on enzymes in this region. These experiments, incidentally, illustrate well the fact that the nutritional state of the animal is sometimes of the ntmost importance in their susceptibility to inhibitors. and examples of this will be cited in the chapters dealing with the inhibitors on which it has been studied.

Finally we must consider the relationship of the response to inhibitors to the height of the functional activity of the tissue. It is commonly stated that those tissues with the greatest activity are more susceptible to inhibition than quiescent tissues. This is not necessarily true in general, since a very active function in one tissue may depend on an entirely different metabolic basis than a less active function in another tissue. However, if one restricts attention to a single function. the statement may have some validity. It is quite obvious that an inhibitor depressing processes connected with cell growth or division will more readily affect those cells that are growing rapidly and can have very little or no effect on nongrowing cells. It is on this principle that some metabolic inhibitors have been used to suppress selectively growing tumor cells or rapidly proliferating microorganisms in the body. It has been noted previously that the functional dependency on the levels of ATP can be the origin of rifferent responses to inhibitors; an activity requiring a high ATP level will be depressed before one capable of proceeding with low concentrations of ATP, when formation of ATP is interfered with. It has also been observed that increasing activity would appear to imply in some cases a progressively greater fraction of a type of metabolism different from that of the resting cell, so that an inhibitor specifically blocking this "activity" type of metabolism would presumably be able to depress more easily cells with high activity. Another factor which has not been consiclered as yet is the possibility that an inhibitor enters an active cell more rapidly than a resting cell. This conld easily be the case with ionic inhibitors since in many cells activity involves a depolarization of the plasma membrane with resultant breaking down of the electrostatic barrier to penetration. So that one might predict on the basis of the above reasoning that the administration of an inhibitor to an animal would primarily cause changes in function in the most active tissues, such as the heart or brain with respect to conduction and contraction, or the kidney with respect to active transport processes, or the hematopoietic system or testes with respect to cell proliferation, and in general 
this prediction is borne out. But there are other instances where unexplainable specific effects are directed towards certain tissues, such as the selective pancreatic effect of alloxan.

\section{ENZYME VARIABILITY}

It is generally accepted that the enzymes of different species or of different tissues of the same species often differ with respect to certain properties, even though the enzymes are all included under the same name. The susceptibility of these enzymes from different sources also varies. But recently a further type of variability has been recognized (Kalow and Da-

$$
\begin{gathered}
\text { Table 9-2 }^{-2} \\
\text { Ixhibition of Humax Serum ('holinesterases }{ }^{a}
\end{gathered}
$$

\begin{tabular}{|c|c|c|c|}
\hline \multirow{3}{*}{ Inhibitor } & \multicolumn{2}{|c|}{$\mathrm{pI}_{50}$} & \multirow{3}{*}{$\begin{array}{c}\text { Ratio } \\
(\mathrm{I})_{50} \text { (atypical) } \\
(\mathrm{I})_{50}(\text { normal })\end{array}$} \\
\hline & It & b th & \\
\hline & Normal ChE & Atypical ChE & \\
\hline Choline & 1.52 & 1.23 & 2.0 \\
\hline Tetramethylammonium & $1.5 \%$ & 1.15 & 2.5 \\
\hline Meperidine & 3.12 & 2.74 & 2.3 \\
\hline Succinylcholine & 4.01 & 2.02 & 99 \\
\hline Procaine & 4.36 & 3.23 & 14 \\
\hline Decamethoniun & 4.88 & 2.74 & $1+1$ \\
\hline Dibucaine & .5 .57 & 4.27 & 20 \\
\hline Diisopropylflıorophosphate & 8.70 & 3.70 & 1 \\
\hline Neostigmine & 6.89 & 5.48 & 25 \\
\hline Physostigmine & 7.84 & 6.57 & 18 \\
\hline
\end{tabular}

a From Kalow and Davies (1958).

vies, 1958). Human sera were selected on a genetic basis and postulated to contain either the usual serum cholinesterase, an atypical variety of cholinesterase, or both enzymes. Inhibition-concentration curves for sixteen different inhibitors showed that generally both enzymes oceurred but in quite different proportions. These two cholinesterases differ very markedly in their susceptibility to inhibitors (Table 9-2). The curves for certain sera and inhibitors showed very clearly the superposition of two curves representing the effect on each enzyme. The active centers of these enzymes must differ significantly. If the occurrence of two different varieties of enzyme were not recognized, inhibition experiments on serum might lead to some very erroneous conchusions as to the properties of the assumed single enzyme. Furthermore, results on the sera from different individuals 
might be interpreted as indicating a spectrum of different cholinesterases instead of variable mixtures of two enzymes. There is no reason to suppose that this phenomenon is restricted to human sera and it is probable that similar genetic variations occur with regard to other enzymes and other species. The over-all effects of inhibitors on animals may thus vary from one individual to another because the susceptible enzyme occurs in different forms and these forms in different proportions. When two or more enzymes catalyze the same reaction and inhibition of each is different, the over-all inhibition is:

$$
i=\frac{i_{1} v_{1}+i_{2} v_{2}+\ldots+i_{n} v_{n}}{v_{t}}
$$

where $v_{t}$ is the total uninhibited rate.

Some readers may believe that an undue amount of emphasis has been placed in this chapter on the complexities of interpretation when inhibitors are used in living systems. It is certainly not the intention to discourage but only to point out some of the reasons for not adopting the naive approach that has often been evident. One cannot with impunity ignore the almost limitless complexity of functioning, metabolizing, and self-perpetuating cells and it is only reasonable to expect that disturbances in the normal state will be beset by an equal degree of complexity in their interpretation. The situation is by no means hopeless but it is only by the realization of the difficulties that theoretical and technical advances can be made, so that the ultimate answers will become penultimate. "Only the most fortunate combination of circumstances will allow one to relate quantitatively the action of an enzyme in an extract and its activity in the intact cell" (Davenport, 1956). One of the aims of the investigator is to ensure the occurrence of this "fortunate combination of circumstances." "An outstanding biochemist once remarked that only uninhibited investigators use inhibitors in complex biological systems" (Racker, 1956); it would, perhaps, be more correct to say that only uninhibited investigators draw dogmatic conclusions from such experiments. 


\section{EFFECTS OF MORE THAN ONE INHIBITOR}

The use of two or more inhibitors simultaneously will often produce results or achieve effects not possible with a single inhibitor, just as the administration of more than one drug will occasionally provide a more effective or selective response. As knowledge of the actions of inhibitors increases, it becomes more and more feasible to apply combinations of inhibitors rationally in the investigation of enzyme mechanisms or patterns of metabolism. It may also be possible with multiple inhibitors to establish effective metabolic blockades in abnormal cells, as has been envisioned by Potter (1951) for the selective depression of neoplastic growth by sequential inhibition. The different inhibitors may act on the same enzyme; on the other hand, entirely different enzymes may be inhibited and these enzymes can be either closely related functionally. as in a multienzyme sequence, or quite separated in the cellular metabolic pattern. Inasmuch as no general term has been used for situations in which two or more inhibitors act simultaneously, it is suggested that this be called multiple inhibition, and this term will be used to include all the various possible types. There are only a few isolated examples of multiple inhibition in the literature and these will be discussed in some detail, but it is likely that this is an approach that will become increasingly more important and therefore a generalized treatment, that may be useful in future investigations, will be given. The kinetics of multiple inhibition have been previously worked out only for some special cases so that it may be valuable to present expressions for situations that have not yet been experimentally demonstrated, in order that both the advantages and disadvantages of multiple inhibition can be visualized in particular instances. It should be obvious that the use of two or more inhibitors introduces complications into an already complex subject, especially when a living cellular system is under investigation, and that the indiscriminate application of multiple inhibitors will be detrimental to progress in both the theoretical and practical aspects. It might be suggested that two or more inhibitors should be used simultaneously only when there is a rational reason for their use, based upon an accurate knowledge of the actions of the individual inhibitors. 


\section{TWO INHIBITORS ACTING ON A SINGLE ENZYME}

When two inhibitors and a substrate react with an enzyme, it is necessary to consider all the possible interactions between the components of the system. The situations conceivably range from the case in which all the reactants bind at the active site and completely interfere with the attachment of each other to the enzyme, so that only the complexes ES, EI, and $\mathrm{EI}_{2}$ are present, to the case in which all the reactants are capable of binding to the enzyme independently, so that in addition to the above complexes, $\mathrm{ESI}_{1}, \mathrm{ESI}_{2}, \mathrm{EI}_{1} \mathrm{I}_{2}$, and $\mathrm{ESI}_{1} \mathrm{I}_{2}$ also occur. The kinetics will thus depend primarily on the type of inhibition - competitive, noncompetitive, uncompetitive, etc. - exerted by each inhibitor. A semigeneralized treatment of single enzyme multiple inhibition will now be presented, the ES complex being considered for simplicity to be the only active form capable of forming the product. The following reaction scheme may be written:

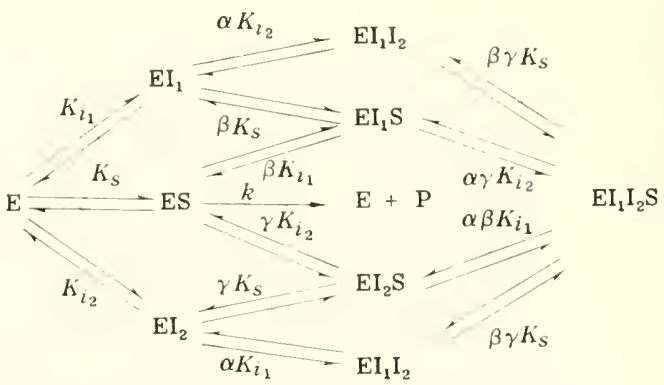

for which the conservation equation takes the form

$$
\left(\mathrm{E}_{t}\right)=(\mathrm{E})+(\mathrm{ES})+\left(\mathrm{EI}_{1}\right)+\left(\mathrm{EI}_{2}\right)+\left(\mathrm{EI}_{1} \mathrm{~S}\right)+\left(\mathrm{EI}_{2} \mathrm{~S}\right)+\left(\mathrm{EI}_{1} \mathrm{I}_{2}\right)+\left(\mathrm{EI}_{1} \mathrm{I}_{2} \mathrm{~S}\right) .
$$

Assuming that $v_{i}=h(\mathrm{ES})$, the rate of the inhibited reaction may be expressed as:

$$
v_{i}=\frac{V_{m}(\mathrm{~S})^{\prime}}{1+\left(\mathrm{I}_{1}\right)^{\prime}+\left(\mathrm{I}_{2}\right)^{\prime}+\frac{\left(\mathrm{I}_{1}\right)^{\prime}\left(\mathrm{I}_{2}\right)^{\prime}}{\alpha}+(\mathrm{S})^{\prime}\left[1+\frac{\left(\mathrm{I}_{2}\right)^{\prime}}{\beta}+\frac{\left(\mathrm{I}_{2}\right)^{\prime}}{\gamma}+\frac{\left(\mathrm{I}_{1}\right)^{\prime}\left(\mathrm{I}_{2}\right)^{\prime}}{\alpha \beta \gamma}\right]}
$$

where specific concentrations have been used for convenience. The constants $\alpha, \beta$, and $\gamma$, giving the degrees of alteration of the binding of each component brought about by the presence of the other components on the enzyme, may be assigned various values depending on the type of inhibition occurring. Taling into consideration only competitive and noncompetitive inhibition, one would expect four situations to be most common. 
Both inhibitors competitive $(\alpha=\beta=\gamma=\infty)$ : if each inhibitor prevents the substrate from binding to the enzyme, it is likely that they will interfere with each other.

$$
\begin{aligned}
r_{i} & =\frac{V_{m}(\mathrm{~S})^{\prime}}{(\mathrm{S})^{\prime}+1+\left(\mathrm{I}_{1}\right)^{\prime}+\left(\mathrm{I}_{2}\right)^{\prime}} \\
i & =\frac{\left(\mathrm{I}_{2}\right)^{\prime}+\left(\mathrm{I}_{2}\right)^{\prime}}{(\mathrm{S})^{\prime}+1+\left(\mathrm{I}_{1}\right)^{\prime}+\left(\mathrm{I}_{2}\right)^{\prime}}
\end{aligned}
$$

Both inhibitors noncompetitive ( $\beta=\gamma=\mathrm{I}$ ): there would be two extreme situations - either the inhibitors react at a similar locus and would thus interfere completely with each other's binding $(\alpha=\infty)$, or the inhibitors may react at different loci independently $(\alpha=1)$.

Binding of inhibitors at the same site $(\alpha=\infty)$ :

$$
\begin{aligned}
v_{i} & =\frac{V_{m}(\mathrm{~S})^{\prime}}{\left[(\mathrm{S})^{\prime}+1\right]\left[1+\left(\mathrm{I}_{1}\right)^{\prime}+\left(\mathrm{I}_{2}\right)^{\prime}\right]} \\
i & =\frac{\left(\mathrm{I}_{1}\right)^{\prime}+\left(\mathrm{I}_{2}\right)^{\prime}}{1+\left(\mathrm{I}_{1}\right)^{\prime}+\left(\mathrm{I}_{2}\right)^{\prime}}
\end{aligned}
$$

Binding of inhibitors at different sites $(\alpha=1)$ :

$$
\begin{aligned}
& c_{i}=\frac{V_{m}(\mathrm{~S})^{\prime}}{\left[(\mathrm{S})^{\prime}+1\right]\left[1 \overline{\left.\left.+\left(\mathrm{I}_{1}\right)^{\prime}+\left(\mathrm{I}_{2}\right)^{\prime}+1 \mathrm{I}_{2}\right)^{\prime}\left(\mathrm{I}_{2}\right)^{\prime}\right]}\right.} \\
& i=\frac{\left(\mathrm{I}_{1}\right)^{\prime}+\left(\mathrm{I}_{2}\right)^{\prime}+\left(\mathrm{I}_{1}\right)^{\prime}\left(\mathrm{I}_{2}\right)^{\prime}}{1-\left(\mathrm{I}_{1}\right)^{\prime}+\left(\mathrm{I}_{2}\right)^{\prime}+\left(\mathrm{I}_{1}\right)^{\prime}\left(\mathrm{I}_{2}\right)}
\end{aligned}
$$

One inhibitor $\left(I_{1}\right)$ is competitive and the other $\left(I_{2}\right)$ is noncompetitive $(\alpha=$ $\gamma=\mathrm{I}, \beta=\infty)$ ): the inhibitors may or may not interfere with each other's binding but it is most likely that they will not since they presumably react at different loci.

$$
\begin{aligned}
i_{i}=\frac{V_{m}(\mathrm{~S})^{\prime}}{\left[(\mathrm{S})^{\prime}+\left(\mathrm{I}_{1}\right)^{\prime}+1\right]\left[\left(\mathrm{I}_{2}\right)^{\prime}+1\right]} \\
i=\frac{\left(\mathrm{I}_{1}\right)^{\prime}+\left(\mathrm{I}_{2}\right)^{\prime}\left[(\mathrm{S})^{\prime}+\left(\mathrm{I}_{1}\right)^{\prime}+1\right]}{\left[(\mathrm{S})^{\prime}+\left(\mathrm{I}_{1}\right)^{\prime}+1\right]\left[\left(\mathrm{I}_{2}\right)^{\prime}+1\right]}
\end{aligned}
$$

Similar expressions can be easily derived for cases in which the inhibitions are partially competitive partially noncompetitive. mixed, uncompetitive. or of other types.

It would be expected that the inhibition observed with two inhibitors would not be the sum of the individual inhibitions when the inhibitors are 
used separately and the equations above provide an estimate of the deviations to be expected. Thus when both inhibitors are noncompetitive and bind at the same site, the sum of $i_{1}=\left(\mathrm{I}_{1}\right)^{\prime} /\left[\left(\mathrm{I}_{1}\right)^{\prime}+1\right]$ and $i_{2}=\left(\mathrm{I}_{2}\right)^{\prime} \mid$ $\left[\left(\mathrm{I}_{2}\right)^{\prime}+1\right]$ which may be written as:

$$
i_{1}+i_{2}=\frac{\left(\mathrm{I}_{1}\right)^{\prime}+\left(\mathrm{I}_{2}\right)^{\prime}+2\left(\mathrm{I}_{1}\right)^{\prime}\left(\mathrm{I}_{2}\right)^{\prime}}{1+\left(\mathrm{I}_{1}\right)^{\prime}+\left(\mathrm{I}_{2}\right)^{\prime}+\left(\mathrm{I}_{1}\right)^{\prime}\left(\mathrm{I}_{2}\right)^{\prime}}
$$

would always be greater than $i_{1,2}$ as given by Eq. 10-6. So that if $\left(\mathbf{I}_{1}\right)^{\prime}=1$ and $\left(\mathrm{I}_{2}\right)^{\prime}=0.4$, the sum of the individual inhibitions would be $i_{1}+i_{2}=$ 0.786 while the inhibition produced by the two acting together wonld be $i_{1,2}=0.583$.

\section{Graphical Analysis of Multiple Inhibition}

The determination of inhibition constants by the plotting methods ontlined in Chapter 5 presents no problem when two or more known inhibitors are used since measmrements with single inhibitors can be made. However, in the presence of an umrecognized inhibitor, such as an added ion or buffer, or an impurity in the enzyme preparation, the calenlated $K_{i}$ may not be the true dissociation constant of the tested inhibitor. For example, the $1 / v_{i}-1 /(\mathrm{S})$ plot for two competitive inhibitors will give a straight line with a slope of

$$
\frac{K_{s}}{V_{m}}\left[1+\frac{\left(\mathrm{I}_{1}\right)}{K_{i_{1}}}+\frac{\left(\mathrm{I}_{2}\right)}{K_{i_{2}}}\right]
$$

and if the presence of $I_{2}$ is not suspected. the ealeulation of $K_{i_{1}}$ will be in error by an amount depending on the magnitude of $\left(\mathrm{I}_{2}\right) / K_{i_{2}}$. Determination of the type of inhibition will not be disturbed by a second inhibitor as long as this inhibitor remains at a constant concentration, except for the special case where the two inhibitors react with one another (see below).

\section{Types of Interaction between Inhibitors}

In the treatment above it was assumed that $I_{1}$ and $I_{2}$ either completely interfere with each other's binding (as when both react with the same active site on the enzyme and $\mathrm{EI}_{1} \mathrm{I}_{2}$ is impossible) or are bound independently (as when they react with sites sufficiently far apart to prechde significant intermolecular forces). However, intermediate sitnations can occur, the binding of the second inhibitor moleenle to the enzyme being either reduced (negative interaction) or facilitated (positive interaction) by the presence of the other inhibitor molecule. Several mechanisms may be visualized to 
explain such interactions. Simple electrostatic forces must be involved where the inhibitors are charged as in the following examples:

Negative interaction:

$(\infty>\alpha>1)$

Positive interaction:

$(\alpha<1)$

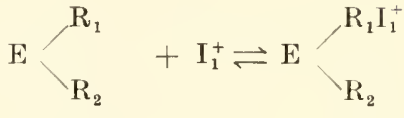

$\mathrm{E} \int_{\mathrm{R}_{2}}^{\mathrm{R}_{1} \mathrm{I}_{1}^{+}}+\mathrm{I}_{2}^{+} \rightleftharpoons \mathrm{E}<\mathrm{R}_{2} \mathrm{I}_{2}^{+}$

$\mathrm{E} \overbrace{\mathrm{R}_{2}}^{\mathrm{R}_{1} \mathrm{I}_{1}^{+}}+\mathrm{I}_{2}^{-} \rightleftharpoons \mathrm{E} \overbrace{\mathrm{R}_{2} \mathrm{I}_{2}^{-}}^{\mathrm{R}_{1} \mathrm{I}_{1}^{+}}$

Furthermore, if $R_{2}$ is an acidic or basic group, the binding of a charged inhibitor molecule on $R_{1}$ could alter the ${ }_{p} K_{a}$ of $R_{2}$ and hence the fraction of these groups that is dissociated. Steric factors could also operate, especially when the first molecule bound extends spatially in such a way as to interfere with the attachment of the second inhibitor, but positive interaction could conceivably result if a greater area of interaction were provided for dipolar and dispersion forces. One must also consider the possibility of inductive effects through the protein structure or an actual change in the configuration of the local protein structure. Finally, the direct reaction of the unbound inhibitors with one another $-I_{1}+I_{2} \rightleftharpoons I_{1} I_{2}-$ can complicate the kinetics considerably, particularly when the inhibitor complex $I_{1} I_{2}$ is also an inhibitor. Indeed, when $I_{1} I_{2}$ is a more potent inhibitor than either $I_{1}$ or $I_{2}$, the inhibition in the presence of both inhibitors may be greater than predicted on the basis of their individual actions.

When the inhibitors react with one another:

$$
\mathrm{I}_{1}+\mathrm{I}_{2} \rightleftharpoons \mathrm{I}_{1} \mathrm{I}_{2} \quad K_{3}
$$

the concentration of $I_{1} I_{2}$ may be calculated from the quadratic equation:

$$
\left(I_{1} I_{2}\right)^{2}-\left(I_{1} I_{2}\right)\left[\left(I_{1}\right)_{t}+\left(I_{2}\right)_{t}+K_{3}\right]+\left(I_{1}\right)_{t}\left(I_{2}\right)_{t}=0
$$

and from this the concentrations of the uncomplexed inhibitors nay be obtained. If $I_{1}, I_{2}$, and $I_{1} I_{2}$ are all inhibitory, and each inhibits noncompetitively, the total inhibition is given by:

$$
i=\frac{\left(\mathrm{I}_{1}\right)^{\prime}+\left(\mathrm{I}_{2}\right)^{\prime}+\left(\mathrm{I}_{1} \mathrm{I}_{2}\right)^{\prime}}{1+\left(\mathrm{I}_{1}\right)^{\prime}+\left(\mathrm{I}_{2}\right)^{\prime}+\left(\mathrm{I}_{1} \mathrm{I}_{2}\right)^{\prime}}
$$

If $I_{1} I_{2}$ were not formed, the inhibition wonld be given by Eq. 10-6; the concentrations of $I_{1}$ and $I_{2}$ would be in this case the total concentrations. Whether the multiple inhibition will be greater or less than in the situation 
in which $I_{1} I_{2}$ is not formed will depend on the relative inhibitory potency of $\mathrm{I}_{1} \mathrm{I}_{2}$ compared to the uncomplexed inhibitors, and the amount of the change in inhibition will depend primarily on $K_{3}$. Thus the reaction between two inhibitors can result in either antagonism or potentiation of the inhibition. If one of the imhibitors is a substance present in the enzyme test medium, its presence will distort the response to the added inhibitor and produce nonlinearity in the usual plotting procedures.

\section{Example of Positive Interaction: Inhibition of Succinic Oxidase by Fluoride and Phosphate}

A very interesting instance of an unexpectedly potent effect produced by a combination of two inhibitors was reported by Slater and Bonner (1952). Both phosphate and fluoride were found to be rather weak competitive inhibitors of cardiac succinic dehydrogenase but in the presence of the two ions the enzyme was depressed much more than would be predicted by the individual inhibitions. The kinetics here are different than those expressed in Eqs. 10-3 and 10-4 for competitive inhibition because both inhibitors can be bound to the enzyme simultaneously and $\alpha$ no longer is infinity. We must now write:

$$
v_{i}=\frac{V_{m}(\mathrm{~S})^{\prime}}{(\mathrm{S})^{\prime}+1+(\mathrm{P})^{\prime}+(\mathbf{F})^{\prime}+\left[(\mathrm{P})^{\prime}(\mathrm{F})^{\prime} / \alpha\right]}
$$

where $(\mathrm{S})^{\prime},(\mathrm{P})^{\prime}$, and $(\mathrm{F})^{\prime}$ are the specific concentrations of succinate, phosphate, and fluoride respectively. Instead of determining the total inhibition produced by phosphate and fluoride acting together, let us rather consider the inhibition by fluoride in various concentrations of phosphate. This will be expressed by $i_{f}$ and is given by:

$$
i_{f}=\frac{(\mathrm{F})^{\prime}\left[1+\frac{(\mathrm{P})^{\prime}}{\alpha}\right]}{(\mathrm{S})^{\prime}+1+(\mathrm{P})^{\prime}+(\mathrm{F})^{\prime}\left[1+\frac{(\mathrm{P})^{\prime}}{\alpha}\right]}
$$

The constants determined by Slater and Bommer are: $K_{m}=0.48 \mathrm{~m} M$ (it has been shown for succinic dehydrogenase that $K_{m}$ is not the substrate dissociation constant), $K_{p}=100 \mathrm{~m} M$ and $K_{f}=20 \mathrm{~m} M$. They also found that when whosphate is bound to the enzyme, the dissociation constant for fluoride is reduced from $20 \mathrm{~m} M$ to $0.067 \mathrm{~m} M$, so that $\alpha=0.00335$, indicating a remarkable and unexplained positive interaction between the inhibitors. When these constants are substituted into Eq. 10-16 the inhibition curves for fluoride shown in Fig. 10-1 are obtained. The marked potentiation is evident: in the absence of phosphate, $10 \mathrm{~m} \boldsymbol{M}$ fluoride inhibits $2.2 \%$ whereas when the phosphate is $50 \mathrm{~m} M$ (which in itself would produce only $2.2 \%$ inhibition), the inhibition by fluoride would be $77 \%$. 
Such potentiation of fluoride inhibition by phosphate must be of significance in the intracellular inhibition of succinic dehydrogenase by flouride, where the phosphate concentration is unknown and may vary under different conditions.

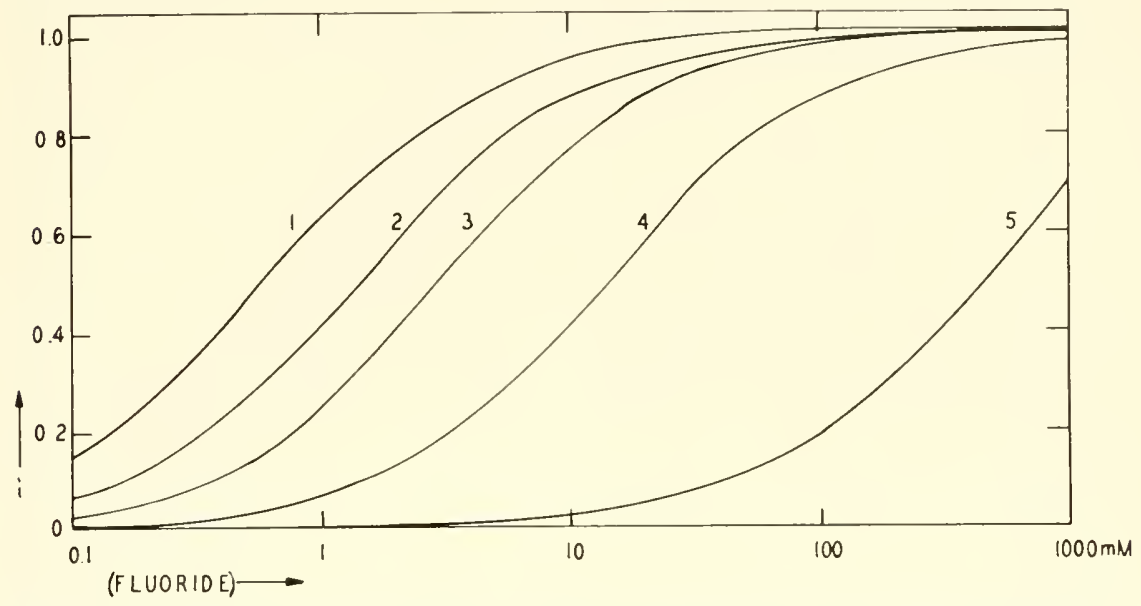

FIG. 10-1. Inhibition of succinic dehydrogenase by fluoride at various concentrations of phosphate. The curves are plotted from Eq. 10-16 using the constants given by Slater and Bonner (1952): $K_{m}=0.48 \mathrm{~m} H, K_{p}=100 \mathrm{~m} H, K_{f}=20 \mathrm{~m} H, K_{p \rho}=0.067$ $\mathrm{m} M,(\mathrm{~S})=10 \mathrm{~m} M$, and $\alpha=0.00335$. Curve $1:(\mathrm{P})=200 \mathrm{~m} \mu$; curve $2:(\mathrm{P})=100$ $\mathrm{m} . I$; curve $3:(\mathrm{P})=50 \mathrm{~m} H$; curve $4:(\mathrm{P})=10 \mathrm{~m} . /$; curve $5:(\mathrm{P})=0 \mathrm{~m} M$.

\section{Other Examples of Multiple Inhibition}

The reversible cholinesterase inhibitors, physostigmine and neostigmine, can protect the enzyme against the organophosphorus compounds (Koelle. 1946), both in vitio and in vivo (Koster, 1946). Butyrylcholine is also a reversible competitive inhibitor of cholinesterase and the quantitative aspects of its effects on inhibition by diisopropylfuorophosphate were studied by Cohen et al. (1951). In one experiment in which the enzyme in the presence of butyrylcholine was incubated for 10-20 min with diisopropylfluorophosphate, the protection provided by the former compound rose with its concentration (see tabulation). In other experiments, the protection

\begin{tabular}{c|c}
\hline $\begin{array}{c}\text { Butyrylcholine } \\
(\mathrm{m} . M)\end{array}$ & ${ }_{0}$ o Protection \\
\hline 50 & 38 \\
100 & 49 \\
150 & 66 \\
200 & 82.5 \\
\hline
\end{tabular}


was occasionally complete. Curves showing the inhibitions produced by different concentrations of diisopropylflnorophosphate in the presence of butyrylcholine are given in Fig. 10-2. The lower the concentration of diisopropylfluorophosphate, the greater the protection, as would be expected.

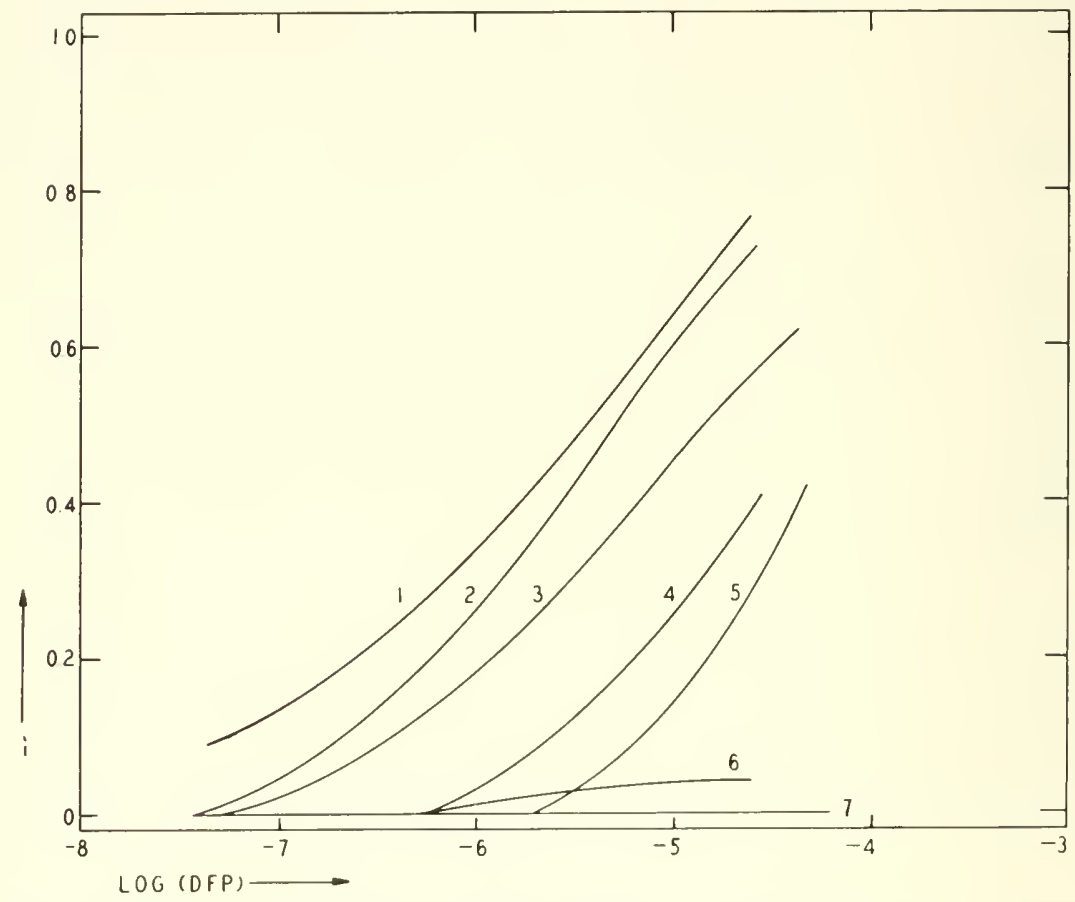

Fia. 10-2. Inhibition of acetylcholinesterase by diisopropylfuorophosphate in the presence of various concentrations of butyrylcholine ( $\mathrm{BuCh}$ ). (From Cohen et al., 1951.) C'urve 1: (BuCh) $=0 \mathrm{~m} H$; curve $:$ : $(\mathrm{BuCh})=0.6 \mathrm{~m} M$; curve 3 : $(\mathrm{BuCh})=1 \mathrm{m.H}$; curve $4:(\mathrm{BuCh})=1.25 \mathrm{~m} M$; curve $5:(\mathrm{BuCh})=2.5 \mathrm{~m} M$; curve $6:(\mathrm{BuCh})=5 \mathrm{~m} H$; curve $7:(\mathrm{BuCh})>12.5 \mathrm{~m} M$.

Once inhibition by the organophosphorus compound has occurred, addition of butyrylcholine will not reverse the inhibition. In such cases, the order of the addition of the inhibitors is very important. The explanation for the protection is simple and the mechanism is much like that involved in protection by the substrate: the reversible inhibitor complexes with the active site and prevents the organophosphorus compound from reaching the site on a certain fraction of the enzyme molecules, so that following dialysis the butyrylcholine will diffuse away from the fraction of sites it has protected. In a similar way, malonate protects succinic dehydrogenase against iodoacetate, heavy metals, and arsenite (Potter and DuBois, 1943; Barron and Singer; 1945; Ackermann and Potter, 1949). 
The inhibitions produced on human serum and erythrocyte cholinesterases by combinations of neostigmine and various tetraalkylammonimm salts are very interesting because they illustrate two different types of interaction (Foldes et al., 1960). The results are shown in Table 10-1 and Fig. 10-3. Marked antagonism was demonstrated with the plasma enzyme. which was interpreted as a blocking off of the anionic site from neostigmine by the tetraalkylammonium ions. The lack of antagonism for the erythrocyte

TABLE $10-1$

Multiple Inhibition of Humax ('holinesterases ${ }^{a}$

\begin{tabular}{|c|c|c|c|c|}
\hline \multirow[b]{2}{*}{ Inhibitor's } & \multicolumn{4}{|c|}{${ }^{\circ}$ Inhibition } \\
\hline & $\begin{array}{c}\text { TMA } \\
(50 \mathrm{~m} . U)\end{array}$ & $\begin{array}{c}\text { TEA } \\
(30 \mathrm{~m} / /)\end{array}$ & $\begin{array}{c}\mathrm{TPA} \\
(3 \mathrm{~m} \cdot \mu)\end{array}$ & 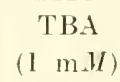 \\
\hline \multicolumn{5}{|l|}{ Plasma cholinesterase } \\
\hline Tetraalkylammoniım & 24.5 & 27.0 & 38.5 & 44.0 \\
\hline Neostigmine & 78.5 & 78.5 & 78.5 & 78.5 \\
\hline Both inhibitors & 36.0 & 31.0 & 47.5 & 36.0 \\
\hline \multicolumn{5}{|l|}{ Erythroeyte cholinesterase } \\
\hline Tetraalkylammonium & 35.0 & $6 \geq .0$ & .56 .0 & 6.5 .0 \\
\hline Neostigmine & 69.0 & 69.0 & 69.0 & 69.0 \\
\hline Both inhibitors & 86.0 & 84.0 & 93.0 & $95.1)$ \\
\hline
\end{tabular}

a TMA, TEA, TPA, and TBA represent the tetramethyl, -ethyl, -propyl, and -butyl ammonium ions. Neostigmine was $0.1 \mathrm{~m} . \mathrm{V}$ for the plasma eholinesterase and $0.2 \mathrm{~m} . \mathrm{I}$ for the erythroeyte eholinesterase. From Foldes et al. (1960).

enzyme may be related to the greater negative charge on the anionic site compared to the plasma enzyme. but it could also be explained by tifferent spatial relations between the anionic and esteratic sites in the two enzymes. The reaction of acetylcholine with the plasma enzyme anionic site must be interfered with less by the tetraalkylammonimm ions than is the case with the erythrocyte enzyme.

\section{Expression of Multiple Inhibition by Isobols}

Isobols are curves showing equieffective combinations of active substances plotted on graphs whose coordinates are the concentrations of the substances (see Chapter 5). Such curves have been used to demonstrate the nature of the interactions of drug pairs on tissues (Loewe. 1957) but can also be applied to enzyme inhibition. Let us first determine the curves 
to be predicted for the various types of inhibition discussed above. The following relationships between $\left(\mathrm{I}_{1}\right)^{\prime}$ and $\left(\mathrm{I}_{2}\right)^{\prime}$ may be derived from Eq. $10-2$ and $i=1-v_{i} / v$.

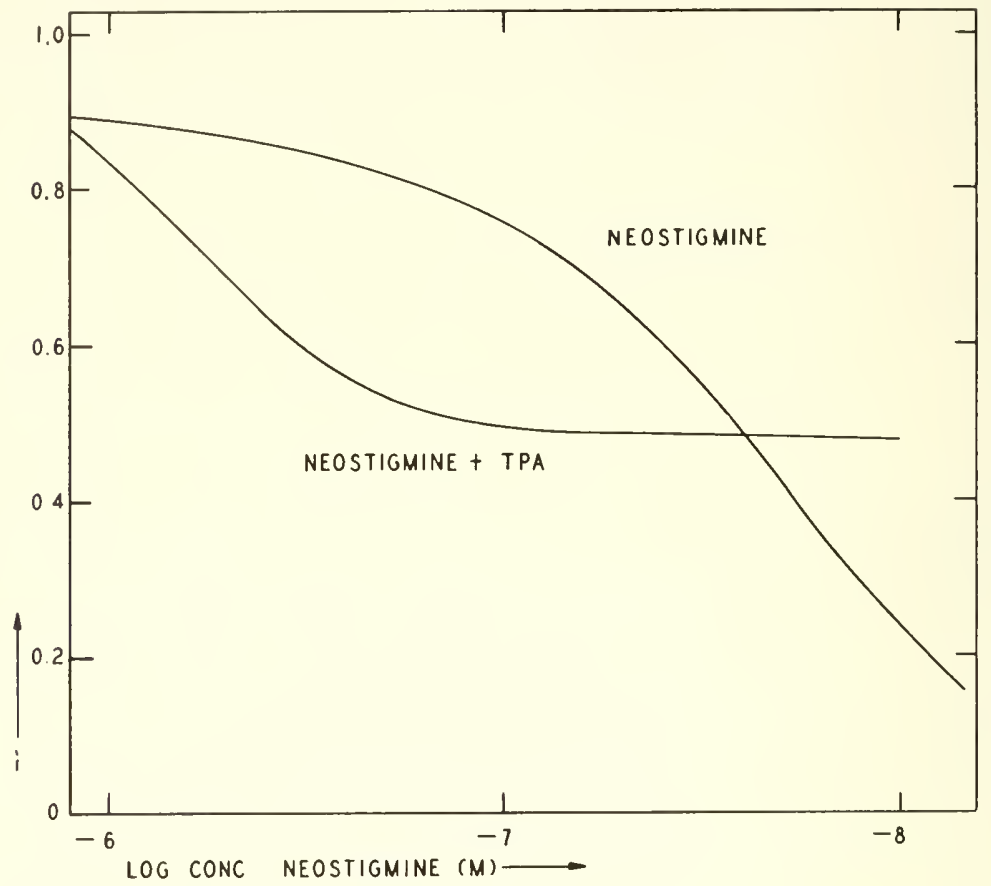

FIG. 10-3. Inhibition of human plasma cholinesterase by neostigmine alone and in the presence of $3.3 \mathrm{~m} M$ tetrapropylammonium ion (TPA).

(From Foldes et al.. 1960.)

Both inhibitors competitive:

$$
\left(\mathrm{l}_{1}\right)^{\prime}=\frac{(i / 1-i)\left[(\mathrm{S})^{\prime}+1\right]-\left(\mathrm{I}_{2}\right)^{\prime}}{1+\left[\left(\mathrm{I}_{2}\right)^{\prime} / \alpha\right]}
$$

Both inhibitors noncompetitive:

$$
\left(\mathrm{I}_{1}\right)^{\prime}=\frac{(i / 1-i)-\left(\mathrm{I}_{2}\right)^{\prime}}{1+\left[\left(\mathrm{I}_{2}\right)^{\prime} / \alpha\right]}
$$

$I_{1}$ competitive and $I_{2}$ noncompetitive:

$$
\left(\mathrm{I}_{1}\right)^{\prime}=\frac{\left[(\mathrm{S})^{\prime}+1\right]\left[(i / 1-i)-\left(\mathrm{I}_{2}\right)^{\prime}\right]}{1+\left[\left(\mathrm{I}_{2}\right)^{\prime} / \alpha\right]}
$$


These equations are plotted for different values of $\alpha$ in Fig. 10-4. The general shapes of the curves are seen to be independent of the type of inhibition involved: e.g.. when the inhibitors prevent each other's binding

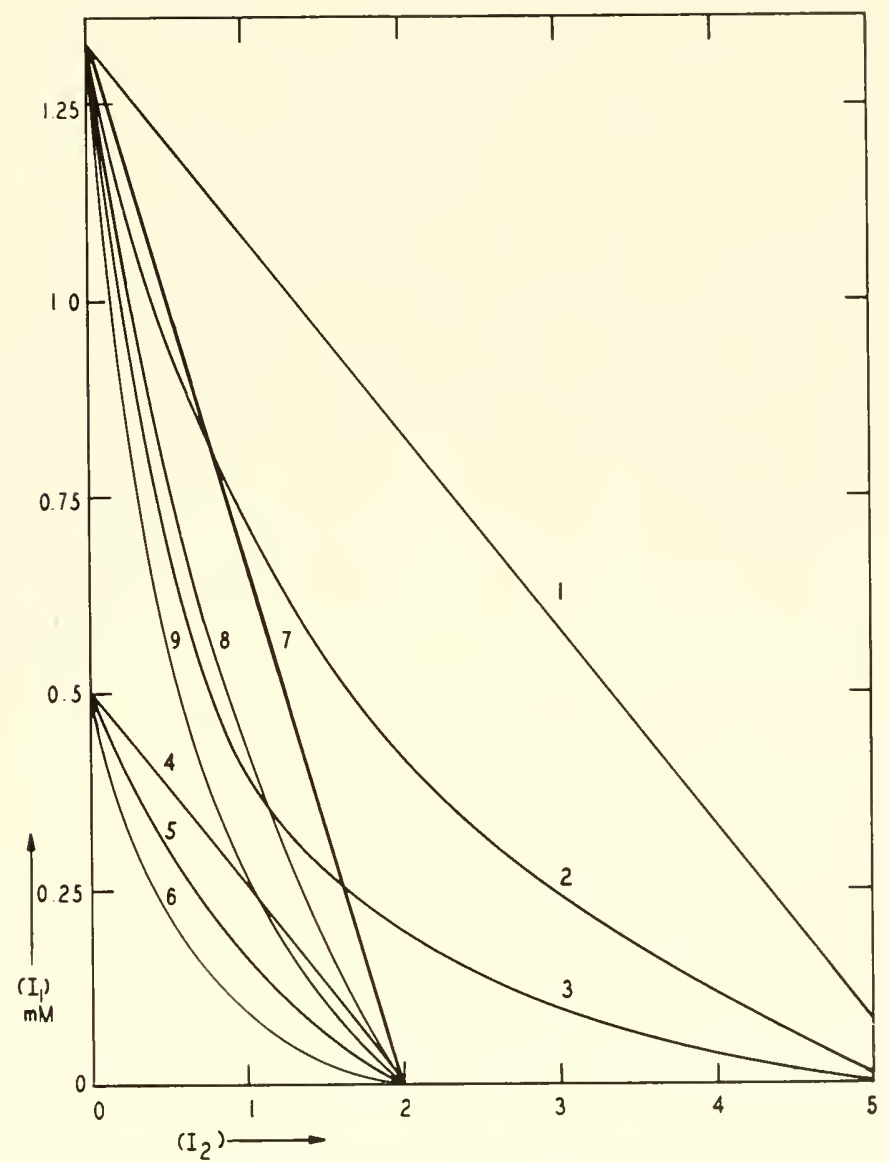

FIG. 10-4. Isobologram for two inhibitors acting on a single enzyme (see Eqs. 10.17 to $10-19$ ), $K_{s}=3 \mathrm{mH}, K_{i_{1}}=0.5 \mathrm{~m} M$, $K_{i_{2}}=2 \mathrm{~m}, H,(\mathrm{~S})=5 \mathrm{~m} M$, and $i=0.5$. Both competitive: curve $1, \alpha=\infty$; curve $2, \alpha=1$; curve $3, \alpha=0.3$. Both noncompetitive: curve $4, \alpha=\infty$; curve $5, \alpha=1$; curve $6 . \alpha=0.3 . \mathrm{I}_{1}$ competitive and $\mathrm{I}_{2}$ noncompetitive: curve $7, \alpha=\infty$ : curve 8 . $\alpha=1$; curve $9, \alpha=0.3$.

$(\alpha=\infty)$. the isobols are linear (curves 1. 4 , and 7$)$ in all cases. As the inhibitors interfere less and less with each other ( $\alpha$ decreasing), the curves become progressively more concave mpwards. 
What information may be obtained from the plotting of isobols? If the isobol is linear, complete binding interference between the two inhibitors is indicated, and the degree of deviation from linearity would be a measure of the effect of each inhibitor on the binding of the other. It is indeed possible to calculate $\alpha$, if the inhibition types for the individual inhibitors are known, by the following equation:

$$
\alpha=\frac{\left(\mathrm{I}_{1}\right)^{\prime}\left(\mathrm{I}_{2}\right)^{\prime}}{x-y\left(\mathrm{I}_{2}\right)^{\prime}-\left(\mathrm{I}_{1}\right)^{\prime}}
$$

where for $50 \%$ inhibition $x=(\mathrm{S})^{\prime}+1$ and $y=1$ when both inhibitors are competitive, $x=y=1$ when both inhibitors are noncompetitive, and $x=y=(\mathbf{S})^{\prime}+1$ when one inhibitor is competitive and the other is noncompetitive. This method could be of value in determining the relative position of binding of inhibitors on an enzyme surface but depends upon an accurate evaluation of $K_{m}, K_{i_{1}}$, and $K_{i_{2}}$. Actually, little information may be obtained directly from these curves but they are one of the most concise and intelligible ways of representing the relations between two inhibitors in both isolated enzyme systems and cellular preparations. Their relation to antagonism and potentiation will be discussed later (page 508).

\section{TWO INHIBITORS ACTING ON A MONOLINEAR CHAIN}

More interesting patterns of inhibition are produced when two enzymes in a multienzyme system are attacked simultaneously and this situation is commonly encountered when two inhibitors are used in mitochondrial, cellular, or whole animal preparations. In this section the simple monolinear enzyme chain will be discussed in some detail as an example of this type of inlribition and in the following section some of the other multienzyme systems will be treated qualitatively. One of the earliest demonstrations of multiple monolinear inhibition is that described by Beerstecher and Shive (1947), who showed that tyrosine depressed the synthesis and $\beta$-hydroxyphenylalanine depressed the utilization of phenylalanine in $E$. coli. A marked potentiation of the inhibition was observed when the two inhibitors were used simultaneously; thus the same degree of growth inhibition was produced by $0.016 \mathrm{~m} M$ tyrosine alone, by $1.6 \mathrm{~m} M \beta$-hydroxyphenylalanine alone, and by $0.005 \mathrm{~m} / \mathrm{t}$ tyrosine and $0.05 \mathrm{~m} / \mathrm{\beta}$-hydroxywhenylalanine together. They postulated that the potentiation was due to the fact that the $\beta$-hydroxyphenylalanine inhibition was competitive and the reduction of the phenylalanine synthesis by tyrosine made this competitive inhibition much more effective. Potter (1951) applied the term "sequential inhibition" to this phenomenon and illustrated it by the simultaneous actions of malonate and fluoroacetate on the tricarboxylic acid cycle. The possible utilization of sequential inhibition in tumor chemother- 
apy was suggested by Potter and extended by Skipper and associates (1954). No treatment of the kinetics of such situations has been made.

Let us consider inhibition to be exerted on each enzyme of the simplest monolinear enzyme chain:

$$
\stackrel{\mathrm{E}_{1}}{\rightarrow} \underset{\mathrm{B}}{\mathrm{E}} \rightarrow \mathrm{C}
$$

A single inhibitor acting on $\mathrm{E}_{1}$ alone will depress the steady-state rate of formation of $\mathrm{C}$ to the same degree as it inhibits reaction 1 , while a single inhibitor acting on $\mathrm{E}_{2}$ alone will not depress the formation of $\mathrm{C}$ unless the system is removed from a steady state. in which case $d(C) / d t$ will be equal to the rate of reaction 2 (see page 323 ). For convenience we shall assume that $I_{1}$ acts specifically on $E_{1}$ and $I_{2}$ on $E_{2}$. If $\left(I_{1}\right)$ is such that reaction 1 is inhibited $50 \%, d(\mathrm{C}) / d t$ is also inhibited $50 \%$; when $\mathrm{I}_{2}$ is added, no further inhibition of $d(\mathrm{C}) d t$ will occur unless the steady state is lost, in which case the over-all inhibition would be no different from that in the presence of $\mathrm{I}_{2}$ alone. Thus in this simple system no greater inhibition is achieved by a combination of the two inhibitors. However, the transition time between steady states may be different if reaction 1 is inhibited simultaneously with reaction 2. Even if $I_{2}$ is competitive. $I_{1}$ will produce the same inhibition with $I_{2}$ as it will alone as long as the steady state is maintained. However, when the physical state of the system prevents sufficient rise in (B) to compensate for the inhibition of reaction 2 (as when $B$ diffuses out of a compartment), addition of $I_{1}$ may decrease (B) and further inhibition can occur.

If reaction 1 is reversible. inhibition of $d(\mathrm{C}) d t$ will be produced with both $I_{1}$ and $I_{2}$ alone and a combination of the two inhibitors will inhibit more strongly than a single inhibitor although less than the smm of the individual inhibitions. This is due to the effect of $(B)$ on the reverse reaction (page 338). In chains of more than two enzymes. the same principles would be demonstrated. Thus in the sequence:

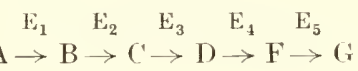

$d(G) / d t$ will depend on the rate of reaction 1 if the system is in a steady state and on the slowest reaction if it is not. Inhibition of $E_{1}$ to the point where $d(\mathrm{G})$ it is reduced makes reaction 4 the limiting reaction; inhibition then on another enzyme would affect $d(G) / d t$ only when the reaction catalyzed by that enzyme approaches the limiting rate. and in this case the over-all inhibition would be the same whether the inhibitor acting on $\mathrm{E}_{4}$ were present or not. Thus multiple inhibition in simple monolinear chains would seem generally to be incapable of producing an effect much greater than a single inhibitor and a marked potentiation of inhibition would be out of the question. 
Nevertheless, one factor must be borne in mind and that is that many multienzyme systems in the cell are prboably not in a steady state, or that at least periods occur when a steady state does not exist. In such nonsteady states the above reasoning may not accurately apply. Also the introduction of two inhibitors may produce an interval of more severe depression before a new steady state can be reached. The complex effects observed upon tissue function or growth may be the result of actions during a nonsteady state period. Celhlar metabolic pathways rarely are completely closed systems so that concentrations of intermediates may be determined by factors other than the relative rates of the enzyme reactions. The operation of an isolated monolinear chain in the cell is probably very uncommon, inasmuch as most intermediates can be formed or metabolized by reactions not in the chain.

In view of the conclusions of this section, it is perhaps necessary to attempt an explanation for the previously mentioned potentiation observed in the assumed simple sequential inhibition of $E$. coli growth by tyrosine and $\beta$-hydroxyphenylalanine (Beerstecher and Shive. 1947). Actually, a metabolic response was not measured here but only the final result on the complex process of growth. In other words. activity in a metabolic monolinear chain was not determined but only the end result of disturbances in such a chain. The growth rate may depend very critically on the utilization rate of phenylalanine, so that up to a certain degree, inhibition could be exerted without a significant effect on the growth, but a marked depression could result from a rather small further increment in the metabolic inhibition. Or in this particular case, the major factor conld be the amount of abnormal $\beta$-hydroxyphenylalanine that is utilized. Depression of the synthesis of phenylalanine may angment the competitive action of $\beta$-hydroxyphenylalanine but the growth response need not be a quantitative measure of the depression of phenylalanine utilization.

\section{TWO INHIBITORS ACTING ON VARIOUS MULTIENZYME SYSTEMS}

Opportunities for potent metabolic depression with two inhibitors often occur when the metabolisn involves two pathways. Comergent chains (Eq. 7-22) offer simple examples of this. Inhibition of either of the converging limbs $\left(\mathrm{E}_{1}\right.$ or $\mathrm{E}_{3}$ ) will depress $d(\mathrm{C})$ dt less than the individual enzymes and never completely. The inhibitions of the formation of $\mathrm{C}$ are given by:

$$
\begin{array}{lll}
\text { Inhibition of } \mathrm{E}_{1} \text { only: } & i_{t_{1}}=i_{1} /(1+r) \\
\text { Inhibition of } \mathrm{E}_{3} \text { only: } & i_{t_{3}}=r i_{3} /(1+r) \\
\text { Inhibition of } \mathrm{E}_{1} \text { and } \mathrm{E}_{3}: & i_{t_{1,3}}=\left(i_{1}+r i_{3}\right) /(1+r)
\end{array}
$$

where $r$ is the ratio between $v_{3}$ and $v_{1}, i_{i}$ and $i_{3}$ are the inhibitions on $\mathrm{E}_{1}$ 
and $\mathrm{E}_{3}$ respectively, and $i_{t}$ is the inhibition of $\mathrm{C}$ formation. It is interesting that this is one of the few situations where the individual inhibitions are additive, i.e., $i_{t_{1}}+i_{t_{3}}=i_{t_{1,3}}$. The inhibition of two or more pathways leading to a final product has been termed "concurrent blocking" (Skipper et al., 1954). This procedure does frequently offer a means of inhibiting the over-all reaction more potently than with either inhibitor alone. especially when it is not possible for some reason to increase the concentration of a single inhibitor sufficiently to produce the required degree of block.

Iultiple inhibition of divergent chains (Eq. 7-25) presents no special problems that have not already been discussed. except for the possibility that a second inhibitor acting on $\mathrm{E}_{3}$ might antagonize the depression of $\mathrm{C}$ formation produced by an inhibitor acting on $\mathrm{E}_{2}$ by diverting more of $\mathrm{B}$ to $\mathrm{C}$. The response of a polylinear system (Eq. 7-30) to multiple inhibition will follow the same rules as for convergent chains and it is in such systems that the use of a second inhibitor will often depress a resistant phase of metabolism. The effects of two inhibitors on a steady-state cyclic system (Eq. 7-35) are complex but the behavior can be treated in general terms. The conservation of intermediates prevents marked changes in their individual concentrations and thus a cycle is usually more inhibitable than a monolinear chain. When $\mathrm{E}_{1}$ is inhibited, the rise in (A) allows the inhibition to be partially antagonized; if $\mathrm{E}_{3}$ is inhibited in addition. this tends to prevent the rise in (A) and the over-all inhibition is augemented. However. two inhibitors will not depress the formation of product additively in most cases and potentiation will not be observed. Another situation is that where a measured product is produced at each step of the cycle (or at certain steps, as $\mathrm{CO}_{2}$ is produced in the tricarboxylic acid cycle). The reduction in the rate of formation of this product will depend on the site of inhibition in the crcle; the additional inhibition produced by a second inhibitor will also depend on where it acts relative to the first inhibitor. Finally, in cycles where the sum of the concentrations of intermediates is not necessarily constant. of which the tricarboxylic acid cycle is an example, inhibition may lead to a disappearance of intermediates (especially if these intermediates participate in other extracyclic reactions) with subsequent reduction in the incorporation of substrate, leading to a progressive deterioration. In such cases, two inhibitors might have a final effect out of all proportion to their individual inhibitions.

Simple regenerative systems (Eq. 7-39) would not be very sensitive to two inhibitors. As has been pointed out. such a system is potently inhibited only when the action is on that reaction which is potentially the slowest and thus further inhibition on the other reaction would not be predicted to increase the inhibition to a great extent. If the reactions are initially of comparable potential rates, inhibition of one will progressively make the other reaction less sensitive to inhibition. 


\section{TWO INHIBITORS ACTING ON CELLULAR AND TISSUE METABOLISM}

There are many possible ways in which the actions of two inhibitors may be related when the system acted upon is as complex as the cell. The purpose of the present section is to outline some of these interrelationships. It is evident that the previously discussed simultaneous actions on enzymes or multienzyme systems may occur within cells but we shall now be concerned with situations usually observed only in living organisms.

\section{Effect of One Inhibitor on the Metabolism or Distribution of Another Inhibitor}

The products from the metabolic alteration of inhibitors may be either active or inactive. The depression of this transformation by a second inhibitor could result in a reduction of inhibition if an active inhibitor is being formed (lethal synthesis) or an accentuation or prolongation of the inhibition if the original inhibitor is being inactivated. Not many of the common enzyme inhibitors are rapidly metabolized and their rate of disappearance is seldom a factor in determining the degree of their action in isolated cell or tissue preparations. However, in the intact animal the eventual fate of the inhibitor often depends upon some enzymic degradation. Not many instances of lethal synthesis have been demonstrated as yet but the principle can be easily illustrated. When flnoroacetate is administered, there is an accumulation of citrate in the tissues due to the block produced on aconitase by the fluorocitrate formed from the fluoroacetate. Potter (1951) showed that malonate conld rednce the amount of citrate formed in the presence of fluoroacetate, presumably by reclucing the amount of oxalacetate available for condensation with the fluoroacetate throngh an inhibition on the succinic dehydrogenase. Thus if citrate accumulation were being used as a criterion for fluoroacetate inhibition. malonate would antagonize this by depressing the metabolism of the fluoroacetate. It is interesting that in the heart this antagonism was not observed, indicating another pathway for the formation of oxalacetate, an example of the metabolic information that may be obtained with the use of two inhibitors. The results in thymus tissue are shown in Fig. 10-5. The final result in a particular case depends usually on the times of addition of the inhibitors relative to each other, their rates of action, and what is being measured. For example, if malonate was given before the fluoroacetate, a block in citrate formation occurred for a couple of hours but if the two inhibitors were given together, some citrate accumulated before the malonate had an opportunity to exert its full effect. It might be expected that arsenite would also antagonize the citrate accumulation by blocking the entry of fluoro- 
acetate into the cycle; yet if oxygen uptake were being measured, no antagonism would be observed.

Renal excretion of an inhibitor makes it possible for a second inhibitor to alter the rate of disappearance from the organism. If the inhibitor is either actively secreted or resorbed by the tubular cells, block of these transport mechanisms would prolong or reduce the stay of the inhibitor in the body. If the inhibitor is passively resorbed with the tubular fluid, a second inhibitor, such as a mercurial, would increase the excretion rate somewhat.

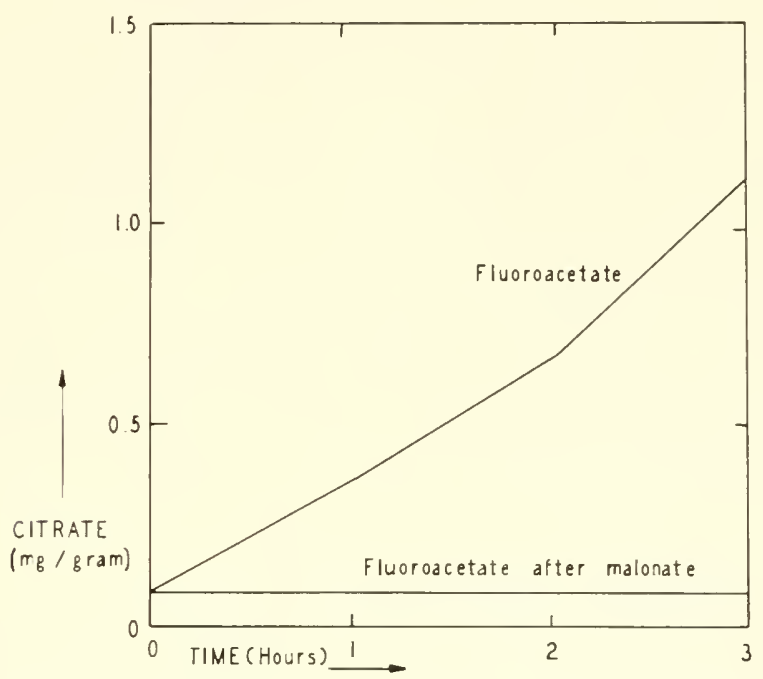

FIG. 10.5. Accumulation of citrate in the thymus following administration of fluoroacetate alone and fluoroacetate after malonate. (From Potter et al., 1954).

It is well known that many inhibitors can change the permeability properties of cells, presumably by modifying the metabolism upon which the structure and function of the membrane depend. It is thus likely that the ability of an inhibitor to penetrate into cells or tissues can be changed in the presence of another inhibitor. An increase in the permeability would particularly affect the response to those inhibitors which normally experience some difficulty in entering cells. The pattern of distribution of an inhibitor in the various tissues of an animal could well be altered by the prior administration of another inhibitor. 


\section{One Inhibitor Alters the Metabolism so That the Response to a Second Inhibitor Is Changed}

The two inhibitors sometimes act on metabolic pathways that are not directly related but because of the complex interrelationships within the living cell their actions will not be independent. A change of intracellular $\mathrm{pH}$ brought about by an inhibitor may modify the effect of another inhibitor, and such $\mathrm{pH}$ changes are by no means uncommon. Likewise, any metabolically dependent components of the cell, such as substrates or intermediates, can rise or fall in response to inhibition, thus altering the conditions upon which the other inhibitor must act. It has been shown in lymph node cells that iodoacetate blocks glucose metabolism and as a consequence the intracellular glucose concentration rises to approximately that in the medium, whereas 2,4-dinitrophenol accelerates glucose utilization and causes a fall in the glucose concentration (Helmreich and Eisen, 1958). A second inhibitor acting on some phase of carbohydrate metabolism might well have its effect modified by these fluctuations in glucose level. Intracellular substrate concentration changes can also be produced by the inhibition of active transport of substrate; depression of amino acid uptake in nerve cells or certain bacteria would create disturbances in metabolism that could increase or decrease the sensitivity to a second inhibitor. Such relationships may be thought of as sequential inhibitions, but truly the actual systems affected can be separated both spatially and functionally in the cell

It has been postulated that proper concentrations of certain inhibitors can reduce the ATP concentration in the cell to levels just sufficient to support growth or function. Under these conditions another inhibitor that also produces a decline in ATP, even though it acts on an entirely different metabolic patlıway, would inhibit those processes that depend on ATP at concentrations much lower than if it were acting alone. The excellent analysis of the inhibition of gastric secretion by Davenport is one of the few competent treatments of a phase of multiple inhibition. As the concentration of 2,4-dinitrophenol was increased, gastric acid secretion began to be inhibited around $0.01 \mathrm{mM}$. It was reasoned that the application of another inhibitor would shift this point of minimal 2,4-dinitrophenol inhibition depending on whether this second inhibitor interfered with ATP generation or with ATP utilization (Davenport et al., 1955). N-Ethylmaleimide shifted the minimal 2,4-dinitrophenol concentration for inhibition to lower concentrations and iodoacetamide shifted it to higher concentrations (Fig. 10-6), from which different sites of action of these two inhibitors were made likely. This type of inhibition analysis could be applied profitably to many other situations and the technique of plotting concentration-inhibition curves for one inhibitor in the absence and presence of another inhibitor is a convenient and often ilhuminating procedure. 
Finally, one must consider relationships of a more physiological nature. The level of metabolic activity. and perhaps even the pattern of metabolism, is related to the functional state of the cells (page 462). An inhibitor depressing by any means the cell functions will secondarily modify metabolism and is thus able to produce an abnormal state that will not be affected in the same way by another inhibitor. If the response to inhibition is measured only at physiological levels (e.g., reflex activity, cardiac rate, intestinal motility, or urine formation), the simple modification of function may alter the response of this function to another agent, as it often does when pairs of drugs are used. Relationships like this must be borne in mind in inter-

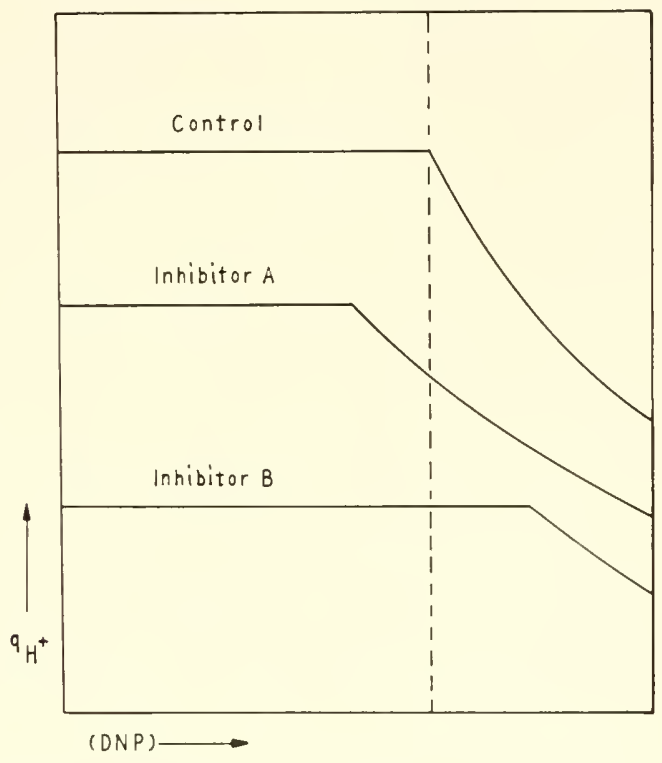

FIG. 10-6. Effects of inhibitors on gastric acid secretion in the presence of 2,4 -dinitrophenol. The control curve shows the effect of 2,4-dinitrophenol alone and the curves for the two inhibitors show the shift in the inflection point. (From Davenport et al., 1955.)

preting the data from multiple inhibition but because of the complex behavior and the almost infinite possibilities of interplay, it is useless to attempt a more quantitative treatment. Hore experimental data will be necessary before theoretical advances may be made. 


\section{Two Inhibitors Acting on the Metabolism of a Pharmacologically Active Compound}

Many active substances (agonists) are formed continuously or intermittently in the tissues and efficient inactivation of these substances is generally neeessary for the proper control of function (page 466). Thus each agonist is formed and destroyed enzymically:

$$
\underset{\mathrm{Y}}{\stackrel{\mathrm{E}_{1}}{\rightarrow} \mathrm{A} \rightarrow \mathrm{E}} \stackrel{\mathrm{E}_{2}}{\rightarrow} \mathrm{X}
$$

where $\mathrm{E}_{1}$ and $\mathrm{E}_{2}$ can represent either single enzymes or sequences. As in any linear system, the agonist concentration will depend on the relative rates of formation and destruction, amongst other things such as diffusion from the region. An inhibitor will alter (A) as described previously (page 468). Two inhibitors, one acting on the formation and the other on the inactivation, will usually counteraet each other with respect to the effect on (A), whereas two inhibitors acting together on either the formation or destruction may augment each other. If the effects of monoamine oxidase inhibitors, such as iproniazid, are primarily due to the rise in certain amine concentrations in the tissues, this action could be increased by the simultaneous use of another inhibitor that blocked the $O$-methylation of some of these amines (since this is another pathway available for inactivation) or decreased by the use of an inhibitor blocking in some manner the synthesis of these amines. In the future it may be possible to potentiate the clinical actions of these monoamine oxidase inhibitors, or to provoke a more specific rise in certain amines, by the administration of a second inhibitor. The possibilities for the useful control of agonist levels are many but at the present time are limited by the ignorance of some of the metabolic pathways involved and the lack of specific inhibitors for pinpoint blocking.

\section{One Inhibitor Prevents the Adaptation of the Cells to Another Inhibitor}

Cells may develop resistance to an inhibitor by several different mechanisms. Most of these meehanisms seem to involve the formation of a new enzyme (either to destroy the inhibitor or to establish an alternate pathway around the block) and thus the ability to adapt is dependent on the whole complex of reactions, exergonic and endergonic, required for protein synthesis. It is not surprising that enzyme adaptation has been found to be susceptible to a variety of inhibitors. Consequently it would appear that in long-term experiments where the development of resistance is an important factor, another inhibitor wonld commonly depress this, not so much by the block of an alternate pathway as by the prevention of the appearance of the alternate pathway. Such phenomena have been taken advantage of in the treatment of tuberculosis where the use of 
$p$-aminosalicylic acid prevents to some extent the development of resistance to streptomycin by the mycobacteria. It is likely that such techniques will have to be used in the chemotherapy of cancer inasmuch as resistance to single agents appears so readily.

\section{ANTAGONISM, SUMMATION, AND SYNERGISM}

The terms antagonism, summation, and synergism have been applied for many years in pharmacology with a variety of meanings. They have been occasionally used in inhibition studies with equal vagueness. If these expressions are to be used, they should have accepted and quantitative meanings; otherwise it is better to state the results and omit the terms. Antagonism is commonly assumed to occur when two agents produce an effect that is less than expected on the basis of their individual actions; likewise, synergism is deduced when the combined effect is greater than expected. The question may be raised as to just what one expects and to the validity of such expectation. For example, if $I_{1}$ depresses respiration $30 \%$ and $\mathrm{I}_{2}$ depresses it $45 \%$, what inhibition would be expected when both $I_{1}$ and $I_{2}$ are present? How would multiple inhibitions of 50, 60, 70, or $80 \%$ be classified? In the examples previously discussed of two inhibitors acting on various types of simple enzyme systems, it was evident that different effects may be expected depending on the particular situation. Before antagonism and synergism (or potentiation) can be defined. some reasonable method of determining the expected result of summation in general cases must be worked out. Actually, it is usually not convenient to approach the subject by comparing the effect of two inhibitors with the effects of each inhibitor separately; it is more profitable to look at the effect of a single inhibitor both alone and in the presence of the other inhibitor.

Let us consider the two inhibitors mentioned above. If the normal respiratory rate is designated as $100, \mathrm{I}_{1}$ alone would depress it to 70 ; if $\mathrm{I}_{2}$ were now added, the respiration would be reduced further to 38.5 if the first inhibitor had no effect on the response to the second. Likewise. if $\mathrm{I}_{2}$ were used alone, the respiration would drop to 55 and the addition of $I_{1}$ would, of course, bring it to 38.5 again. Thus on the basis of independence of action, the expected inhibition by the two inhibitors acting simultaneously would be $61.5 \%$. Such a situation may be called summation. A combined effect of less than $61.5 \%$ inhibition may be called antugonism and an effect greater than this synergism. This may be easily expressed in mathematical form. The fractional respiratory rate in the presence of $I_{1}$ alone is $a_{1}=$ $1-i_{1}$ and the addition of $\mathrm{I}_{2}$ will reduce the activity to $a_{1} a_{2}=\left(1-i_{1}\right)$ $\left(1-i_{2}\right)$. The inhibition produced by both inhibitors will thus be

$$
i_{1,2}=1-a_{1} a_{2}=1-\left(1-i_{1}\right)\left(1-i_{2}\right)=i_{1}+i_{2}-i_{1} i_{2}
$$


Summation:

Antagonism:

Synergism:

$$
\begin{aligned}
& i_{1,2}=i_{1}+i_{2}-i_{1} i_{2} \\
& i_{1,2}<i_{1}+i_{2}-i_{1} i_{2} \\
& i_{1,2}>i_{1}+i_{2}-i_{1} i_{2}
\end{aligned}
$$

when the in hibitors do not influence each other's actions. Stated in another way, antagonism occurs when a second inhibitor produces less effect in the presence of the first inhibitor than it does alone, and synergism occurs when the effect is greater. It should be evident that when antagonism or synergism is observed, it does in no way indicate the mechanism involved nor imply that the two inhibitors act on the same system.

Isobolograms have been used pharmacologically to illustrate antagonism, summation, and synergism (Loewe, 1957). Let us consider the nature of isobols derived from inhibition studies on complex metabolic systems such as respiration. It may be assumed that each inhibitor alone acts according to the equations:

$$
i_{1}=\frac{(\mathrm{I})_{1}}{\left(\mathrm{I}_{1}\right)+K_{1}} \quad i_{2}=\frac{\left(\mathrm{I}_{2}\right)}{\left(\mathrm{I}_{2}\right)+K_{2}}
$$

where $K_{1}$ and $K_{2}$ are simply constants of no physical significance. Combining these equations with the summation Eq. 10-26, the inhibition to be predicted from both inhibitors wonld be given by:

$$
i_{1,2}=\frac{\left(\mathrm{I}_{1}\right)\left(\mathrm{I}_{2}\right)+K_{1}\left(\mathrm{I}_{2}\right)+K_{2}\left(\mathrm{I}_{1}\right)}{\left[\left(\mathrm{I}_{1}\right)+K_{1}\right]\left[\left(\mathrm{I}_{2}\right)+K_{2}\right]}
$$

Relationships between $\left(I_{1}\right)$ and $\left(I_{2}\right)$ may now be obtained for any desired degree of inhibition $i_{1,2}$ and such have been plotted in Fig. 10-7. Summation is therefore represented by a curve concave upwards and not by a straight line. This is actually quite similar to the results on simple enzymes (Fig. I0-4, curves 2,5, and 8 where $\alpha=1$ ). Synergism then would be represented by a curve with greater upward curvature and antagonism by curves of lesser curvature and particularly by curves approaching linearity or concave downwards (Fig. 10-8). A corollary to these considerations is the nonadditivity of inhibitor concentrations: thus if a certain degree of inhibition is produced by $\left(I_{1}\right)$ and $\left(I_{2}\right)$, the two inhibitors together at half their concentrations will not give the same inhibition.

The possibility of antagonism or synergism occurring when two inhibitors are introduced is particularly important in medical applications, as it is in the combined use of any drugs. Many of the results on combination therapy reported in the medical and pharmacological literature are pertinent to inhibition studies, but a thorough and quantitative theoretical approach has never been made. In the pharmacological field. the treatments of Ariëns 


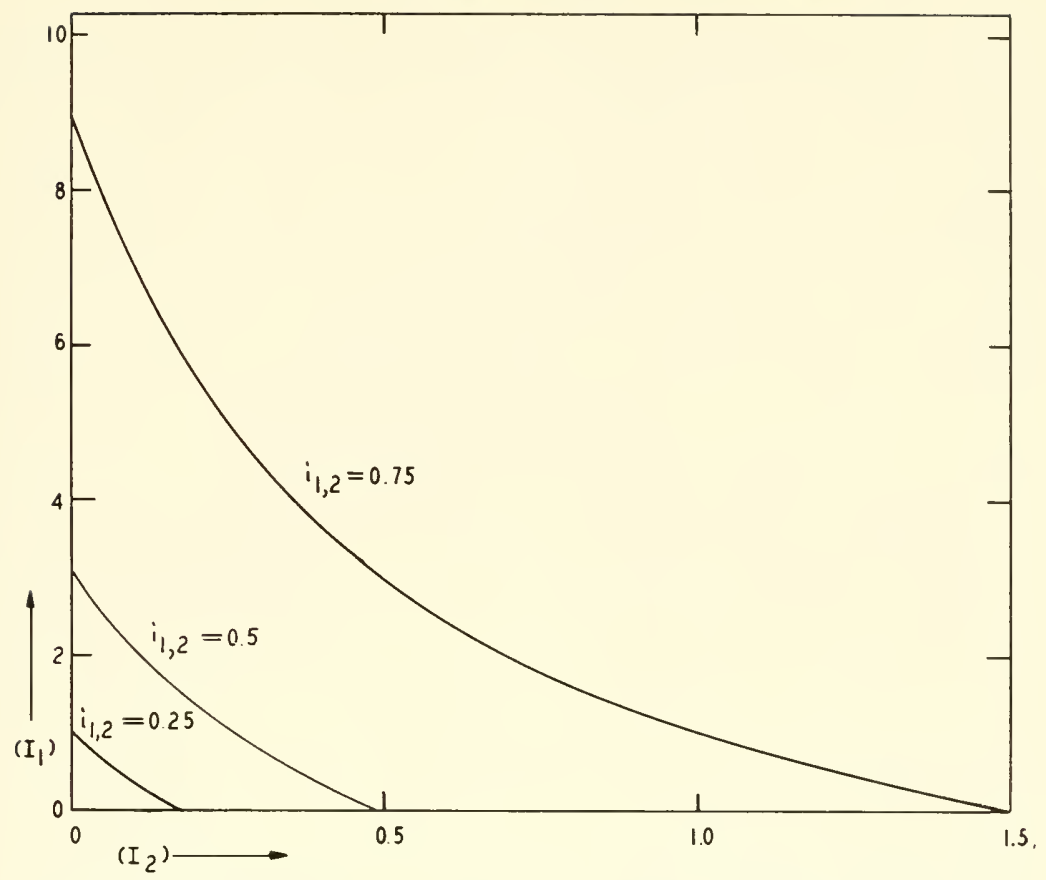

Fig. 10-7. Isobologram for two simultaneously acting inhibitors demonstrating summation (Eq. 10-298).

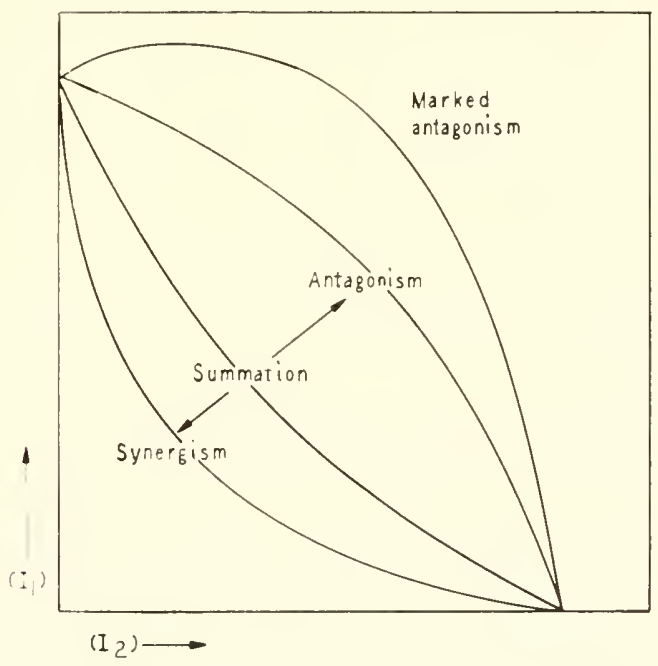

FIG. 10-s. Isobologram for two simultaneously acting inhibitors illustrating the curves to be expected for summation, synergism, and antagonism. 
and associates (1957; Ariëns, 1954) and of Landahl (1958) may be recommended. Nowhere could the cooperation between pharmacology and biochemistry be more fertile than here.

\section{SPECIFICITY OF INHIBITION WITH TWO INHIBITORS}

The general problems of inhibitor specificity will be taken np in Chapter 17 but it is pertinent at this time to consicter the possibility of increasing the selectivity of inhibition by the judicious use of two or more inhibitors. The uninhibited use of multiple inhibitors will generally complicate the situation and reduce the likelihood that a selective block on one enzyme or pathway is being achieved. However, in cases in which the action spectra of two inhibitors are reasonably well known, it is occasionally possible to augment the inhibition of a particular system without simultaneously losing specificity. This sitmation can arise especially when both inhibitors act on a common enzyme but otherwise have different sites of action. The simplest case is where $I_{1}$ acts on $E_{1}$ and $E_{2}$ whereas $I_{2}$ acts on $E_{1}$ and $E_{3}$; a combination of $I_{1}$ and $I_{2}$ will have a greater effect on $E_{1}$ without increase of inhibition on $\mathrm{E}_{2}$ and $\mathrm{E}_{3}$. Of course, the two inhibitors together will inhibit more enzymes than either one alone but the inhibition on these secondary systems will be relatively smaller compared to the primary action on $\mathrm{E}_{1}$. Let us assume that $K_{i_{11}}=K_{i_{12}}=1 \mathrm{~m} . \mathrm{I}$ and $K_{i_{21}}=K_{i_{23}}=$ $3 \mathrm{~m} M$, where the numerical subscripts refer to inhibitor and enzyme in order. It is easy to calculate that for noncompetitive inhibitors, a concentration of $1 \mathrm{~m} I I$ wonk inhibit $\mathrm{E}_{1} 50 \%$ when acting singly, but together at this concentration the inhibition would be $67 \%$. On the other hand, the inhibitions on either $\mathrm{E}_{2}$ or $\mathrm{E}_{3}$ would not be increased, although both would now be depressed $25 \%$. If $50 \%$ inhibition of $\mathrm{E}_{1}$ is desired, it will require only $0.5 \mathrm{~m} . M$ of each inhibitor but now the inhibitions on $\mathrm{E}_{2}$ and $\mathrm{E}_{3}$ will be only around $14 \%$. Thus some improvement in selectively blocking $\mathrm{E}_{1}$ can be achieved with two inhibitors and it would be possible to produce even greater specificity with three or more inhibitors if such exist.

\section{INHIBITION OF BIOLUMINESCENCE WITH SULFANILAMIDE AND URETHANE}

A quantitative investigation of the multiple inhibition of bacterial hminescence was made by Johnson et al., (1943) and a consideration of the complex results obtained may serve to illustrate the problems and difficulties that occur in work with living systems. The results with sulfanilamide and urethane are shown in Table 10-2. By comparing the calculated inhibitions in the last cohms with the experimental imhibitions, it is observed that antagonism betwcen the inlibitors occurs in every case. 
TABLE $10-2$

Effects of Sulfaxilamide and Urethane on Bacterial Luminescence at Different Temperatures ${ }^{a}$

\begin{tabular}{|c|c|c|c|c|c|c|c|c|}
\hline \multirow[b]{2}{*}{$\begin{array}{c}\text { Temp. } \\
\left({ }^{\circ} \mathrm{C}\right)\end{array}$} & \multirow[b]{2}{*}{$\mathbf{I}_{0}$} & \multirow[b]{2}{*}{$\mathrm{I}_{s}$} & \multirow[b]{2}{*}{$\mathrm{I}_{u}$} & \multirow[b]{2}{*}{$I_{s+u}$} & \multicolumn{3}{|c|}{$\%$ Inhibition } & ธี ซีน ธ \\
\hline & & & & & $\begin{array}{l}\text { Sulfanil- } \\
\text { amide }\end{array}$ & $\begin{array}{l}\text { Ure- } \\
\text { thane }\end{array}$ & Both & 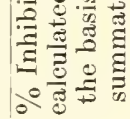 \\
\hline
\end{tabular}

Vibrio phosphorescens

\begin{tabular}{l|r|r|r|r|r|r|r|r}
15 & 15.8 & 4.15 & 12.2 & 9.9 & 73.8 & 22.8 & 27.3 & 79.8 \\
20 & 34.0 & 13.3 & 21.0 & 18.0 & 60.8 & 38.2 & 47.0 & 75.8 \\
25 & 62.0 & 34.5 & 30.5 & 29.0 & 44.5 & 50.8 & 53.2 & 72.7 \\
30 & 100.0 & 65.5 & 30.7 & 29.5 & 34.5 & 69.3 & 70.5 & 79.9 \\
35 & 91.0 & 68.0 & 19.5 & 17.0 & 25.2 & 78.6 & 81.3 & 84.0
\end{tabular}

Photobacterium phosphoreum

\begin{tabular}{r|r|r|r|r|r|r|r|r}
5 & 20.5 & 8.3 & 18.3 & 12.0 & 59.5 & 10.7 & 41.5 & 63.8 \\
10 & 38.0 & 14.5 & 31.5 & 25.0 & 61.8 & 17.1 & 34.2 & 68.3 \\
15 & 61.0 & 29.5 & 48.0 & 40.0 & 51.6 & 21.3 & 34.4 & 61.9 \\
20 & 88.0 & 50.0 & 61.5 & 50.8 & 43.2 & 30.1 & 42.3 & 60.3 \\
$\mathbf{2 5}$ & 99.0 & 67.0 & 62.0 & 50.0 & 32.4 & 37.4 & 49.5 & 57.7 \\
\hline
\end{tabular}

${ }^{a}$ Data from Johnson et al. (1943). $I_{0}$ is the intensity of luminescence in the absence of inhibitors, $I_{s}$ with sulfanilamide, $I_{u}$ with urethane, and $I_{s+u}$ with both inhibitors. Concentrations: sulfanilamicle, $3 \mathrm{~m} . H$ and urethane, $150 \mathrm{~m} . /$. The per cent inhibitions calculated in the last column using the equation $i_{s+u}=i_{s}+i_{u}-i_{s} i_{u}$. The urethane concentration was $75 \mathrm{~m} H$ in the Photobacterium esxperiment.

It is stated by Johnson et al. (1943) that antagonism. no effect, and synergism are all observed depending on the temperature. Their definitions of these terms are quite different than those adopted above, since they use "antagonism" to designate cases in which the combined inhibition is lower than that of either inhibitor alone, "no effect" to indicate a combined inhibition of the same magnitude as that of either inhibitor alone, and "synergism" to indicate a greater combined inhibition than with either inhibitor alone. It may be seen, however, in every case that the presence of one inhibitor reduces the inhibition produced by the other inhibitor and it seems to the author that this would justify classifying the interaction here as antagonism.

It is interesting that the degree of antagonism varies markedly with the temperature, especially in Vibrio where at $15^{\circ}$ the inhibition by the sulfanilamide is almost completely counteracted while at $35^{\circ}$ the situation 
is nearly expressed by summation. This was explained in part by the formation of a complex between sulfanilamide and urethane. The urethane was in much higher concentration than the sulfanilamide and at low temperatures the sulfanilamide was to a large extent complexed and inactive; increase in temperature resulted in a greater dissociation of the complex. This explanation is not entirely satisfactory. In the first place, in Vibrio the combined inhibition followed the inhibition by urethane alone quite closely, at every temperature sulfanilamide contributing little to the inhibition; from this it would appear that temperature had very little effect on the ability of sulfanilamide to inhibit in the presence of urethane. In the second place, the results in Photobacterium are not indicative of complex formation at low temperatures (even though only half the concentration of urethane used with Vibrio was present), since the combined inhibition was closer to that with sulfanilamide alone. If complex formation were the sole origin of the phenomenon, one would expect both organisms to behave similarly. Part of the antagonism of sulfanilamide may be due to complex formation but in addition there would appear to be a metabolic interaction, the nature of which must remain mnknown until the pathways and sites of inhibition are better understood. It is likely that sulfanilamicle and urethane act on different pathways or sites because of the opposite temperature dependence of their inhibitions.

A reduction in metabolic rate might be expected to induce frequently some resistance to an inhibitor. This will be discussed in more detail in Chapter 15 but it is interesting in connection with the inhibition of luminescence to realize that the slower operation of a metabolic pathway can alter the dependence of the rate on enzyme or coenzyme concentrations. During slow activity, the enzymes or coenzymes have greater opportunity to be in excess of what is needed, and thus inhibition or inactivation will not so readily cause a reduction in the rate. Such a factor might be involved in the antagonism between sulfanilamide and urethane. 


\section{LOCALIZATION OF THE SITE OF INHIBITION}

It is commonly found that a substance interferes with some phase of metabolism or produces on cells, tissues, or animals effects whose origin is believed to be biochemical. The basic problem of finding the site or sites of the action then arises. One would prefer to localize the action to a single enzyme or metabolic event and on this basis be able to explain the metabolic disturbance or the functional changes observed in the presence of the substance. The purpose of this chapter is to present some of the principles and techniques by which such analyses may be carried out and to illustrate these methods with a few examples of localization. No one should imagine that this is an easy problem. There are many often-used inhibitors whose exact site of action is not yet known; for example, phlorizin, antimycin, quinones and naphthoquinones. cadmium and lead. diethylithiocarbamate, maleate, dinitrophenols, methane, and alloxan, to name only a few. The action of a new inhibitor may very well not be on a known metabolic system. When fluoride and iodoacetate were first studied on muscle metabolism, the glycolytic pathway had not been elucidated; indeed, these inhibitors played a role in establishing the enzyme sequence in glycolysis. Thus the investigation of a new inhibitor will occasionally bring to light a new pathway or enzyme. Although such a contribution to biochemical knowledge may be important, it makes the eventual determination of the site of action particularly difficult. It is really only under fortuitous circumstances that a satisfactory localization can be readily marle.

\section{METHODS OF LOCALIZATION}

Certain procedures are often useful for localization. It is necessary to inquire into the applicability and validity of these methods, which are based to a large extent on the principles discussed in previous chapters.

\section{Accumulation of Intermediates}

When an inhibitor exerts a blocking action on an enzyme in a metabolic pathway, it would be rredicted that the intermediate acted upon by this 
enzyme would tend to rise in concentration. Some consideration has already been given to the concentrations of intermediates in multienzyme systems (Chapter 7) and it was pointed out that there are several factors which may determine whether accumulation of an intermediate will occur or not. If such an intermediate does indeed accumulate during inhibition and this can be detected by analysis, it provides useful information on the site of the block. Some examples of intermediate accumulation where this phenomenon has been of great importance in the study of the inhibitors might be cited: the rise in hexose phosphates during inhibition by iodoacetate, the appearance of large amounts of keto acids following treatment with trivalent arsenicals, the accumulation of citrate in fluoroacetate poisoning, the rise in toxic aldehyde formed from alcohol in tissues subjected to disulfiram (Antabuse), "the increase in acetylcholine at synaptic junctions when acetylcholinesterase is inhibited, and the progressive accumulations of the catechol amines throughout the body after administration of the monoamine oxidase inhibitors. These and other instances will be discussed in greater detail in those chapters devoted to the inhibitors involved.

Inhibition of an enzyme in a metabolic sequence is not invariably followed by the accumulation of the substrate for this enzyme. There are several possible reasons. An equilibrium may exist between the intermediate whose metabolism is blocked and earlier intermediates in the sequence. Thus triose phosphate does not rise appreciably during inhibition with iodoacetate because the equilibrium conditions favor the hexose phosphates. There may be other pathways for the metabolism of the intermediate so that a block of one path will only shift the pattern of the process. Certain amines in certain tissues do not accumulate even though monoamine oxidase is completely inhibited, presumably because reactions such as $O$ methylation are also available. Sometimes simple diffusion from the region of formation will prevent the accumulation of an intermediate. The relatively low permeability of mitochondria to citrate allows the marked accumulation of this intermediate when aconitase is blocked, whereas other cycle intermediates upon inhibition of their respective enzymes would have a greater tendency to leave the mitochondria. It is thus often important to analyze the medium or perfusate for the intermediate in such cases and not confine the examination to the tissue units. It must also be realized under certain circumstances that the accumulation of an intermediate must be very great for it to be detected, especially when the steadystate level is normally low. Thus a fair degree of inhibition may be exerted without a demonstrable effect upon an intermediate.

Conversely there are several reasons why the accumulation of an intermediate during inhibition does not necessarily imply a block of the enzyme metabolizing that intermediate. This is the case, as we have seen above, when the intermediate is in equilibrium with other substances, and may be 
the case when an alternate pathway becomes more important in the metabolism of the intermediate, because the products of this alternate pathway may increase in concentration. But there are many more complicated situations where, in the closely integrated metabolism of the living cell, a system other than the one directly attacked will be modified and the concentrations of its intermediates altered. A block in any process involved in the formation of high-energy phosphate bonds is apt to induce an accumulation of various precursors for synthetic reactions. Also the reduction in the formation of some product of a sequence may give rise to the accumulation of another compound with which this product reacts. One can easily imagine other circumstances but it should be obvious that in the cell the inhibition of any one process may in a variety of ways affect other processes and the entire steady-state pattern of metabolism be made abnormal. Finally, if the inhibitor is not specific upon a single enzyme, secondary actions may cause rises in the concentrations of intermediates in pathways that are actually not important with regard to the principal mechanism by which the inhibitor produces its effects.

\section{Addition of Various Substrates or Intermediates}

A comparison of the actions of an inhibitor in the presence of various substrates may provide information on the site of action. If an inhibitor has been found to act on the tricarboxylic acid cycle, the sensitive enzyme can usually be found by simply testing the inhibitor against all the substrates and cycle intermediates added individually. Using a reducible acceptor dye it is possible to determine the relative actions of an inhibitor on different dehydrogenases by testing with each substrate alone. Even in intact cells it is often possible to demonstrate different degrees of inhibition when the substrate is changed.

These differences in inhibition may be illustrated by considering some common multienzyme systems. In a monolinear chain:

$$
\stackrel{\mathrm{I}}{\mathrm{A}} \rightarrow \mathrm{B} \stackrel{\mathrm{C}}{\rightarrow} \rightarrow \mathrm{D} \rightarrow \mathrm{E}
$$

if the inhibitor acts on the step $\mathrm{B} \rightarrow \mathrm{C}$, the formation of $\mathrm{E}$ will be depressed when $\mathrm{A}$ or $\mathrm{B}$ are used as substrates, but not when $\mathrm{C}$ or $\mathrm{D}$ are used. In other words, addition of $\mathrm{C}$ or $\mathrm{D}$ will overcome the block. This situation is seen in the differential effects of iodoacetate on glucose and pyruvate metabolism.

Lundsgaard (1932) found that iodoacetate-poisoned yeast would oxidize pyruvate more readily than carbohydrate and Quastel and Wheatley (1932) reported the same situation in guinea pig brain. More recently it has been shown by Heald (1953) that electrically-stimulated brain slices were inhibited more potently by iodoacetate when glucose was the substrate than when lactate was the substrate. The differential effect has been studied 
most thoroughly in cardiac tissue. The depression of the rate and contractility in rabbit atria produced by iodoacetate can be partially overcome by the addition of pyruvate (Webb, $1950 \mathrm{a}$ ). However, this occurred only at lower concentrations of iodoacetate $(0.2 \mathrm{mM})$ and following relatively brief exposures before the depression had advanced too far, while at higher concentrations $(1 \mathrm{mM})$ or after longer exposures, pyruvate had no effect. This can be correlated with the observation that the oxidation of pyruvate by heart slices is completely prevented by $1 \mathrm{~m} M$ iodoacetate (Webb et al., $1949 \mathrm{~b}$ ) and that cardiac mitochondrial oxidation of pyruvate is inhibited very strongly by this concentration of iodoacetate (Yang, 1957). The most complete differentiation of inhibition was achieved in rat ventricle in which iodoacetate $(0.2 \mathrm{~m} M$ for $30 \mathrm{~min})$ completely blocked the contractile stimulation produced by glucose and had little or no effect on the stimulation by pyruvate (Masuoka et al., 1952). The comparison of the effects of glucose and pyruvate in iodoacetate-inhibited preparations will thus give some measure of the selective action of this inhibitor on glycolysis.

When one branch of a convergent chain is blocked, the response to the respective substrates will, of course, be different. Metabolism of $\mathrm{A}$ and $\mathrm{C}$

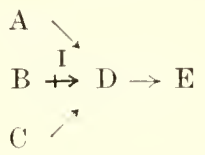

will be unaffected and that of $B$ will be inhibited. In such a situation, some care must be taken not to interpret the overcoming of the inhibition by $\mathrm{A}$ or $\mathrm{C}$ to be a competitive effect with the inhibitor. In a simple cyclic

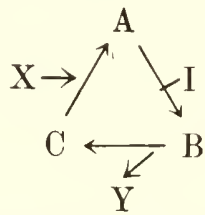

system a block in the step $\mathrm{A} \rightarrow \mathrm{B}$ will be circumvented by addition of $\mathrm{B}$ but not by the addition of $\mathrm{A}$ or $\mathrm{C}$ if the formation of $\mathrm{Y}$ is being determined. These simple principles may be applied to other and more complex systems and form the basis for this method of localization.

\section{Spectroscopic Analysis}

The electron transport systems lend themselves readily to spectroscopic study because of the characteristic absorptions of the nucleotides and cytochromes, and the differences in absorption exhibited by the oxidized and reduced states of these components. An inhibitor blocking the flow of 
electrons at any point in the sequence will modify the steady-state spectral characteristics. Under favorable circumstances these changes may be observed in preparations of living cells. Assuming a generalized electrontransport sequence such as:

$$
\mathrm{SH}_{2} \rightarrow \mathrm{DPN}^{+} \rightarrow \mathrm{FAD} \rightarrow \text { cyt }-\mathrm{b} \rightarrow \text { cyt }-\mathrm{c}_{1} \rightarrow \text { cyt }-\mathrm{c} \rightarrow \text { cyt-a } \rightarrow \text { oxidase } \rightarrow \mathrm{O}_{2}
$$

where the arrows indicate the direction of electron flow, the inhibition of a particular step will slow down or prevent reduction of the components to the right of the block and oxidation of the components to the left of the block. This principle was first applied by Keilin in 1925 in his classic paper on the nature of the cytochromes and extended to more accurate localizations in later reports. More recently this technique has been refined and applied extensively to many systems by Chance (1958; Chance and Williams, 1956; Chance and Hess, 1959).

The typical simple four-banded spectrum of the reduced cytochromes was observed in many tissues by Keilin (1925) and the disappearance of these bands was noted upon oxidation. A yeast suspension under anaerobic conditions thus exhibited the bands of the rednced cytochromes; upon shaking with oxygen these bands faded and disappeared. However, if cyanide were present, the reduced spectrum remained when the cells were exposed to oxygen. It was also found that when cyanide was added to an oxygenated suspension, the bands of the reduced cytochromes appeared rapidly. It was concluded that cyanide acted on some component between oxygen and the cytochromes; 3 years later this was identified with the terminal oxidase (called indophenol oxidase at that time and now known as cytochrome oxidase) (Keilin, 1928). Urethane. on the other hand, inhibited respiration in a different manner because it was shown to block the reduction of the cytochromes without having any effect on their oxidation (Keilin, 1925). A yeast suspension treated with urethane and shaken with oxygen showed the cytochromes to be oxidized; if cyanide was then added, the cytochromes did not become reduced as they would have been in the absence of urethane. Urethane must therefore act on some step between the substrate and the cytochromes. Keilin was able to propose a tentative sequence:

$$
\text { Substrate } \stackrel{\text { (urethane) }}{\longrightarrow} \text { cytochromes } \stackrel{\text { (cyanide) }}{\longrightarrow} \mathrm{O}_{2}
$$

An approximate localization for the sites of inhibition could be made before the actual components of the electron transport system were known and the subsequent elucidation of the components has been aided by the use of these and other inhibitors.

The later analysis of the site of action of cyanide (Keilin and Hartree, 1939) illustrates another but more direct technique. When the component with which the inhibitor reacts possesses a characteristic spectrum, 
this spectrum of absorption is usually altered by reaction with the inhibitor and this constitutes positive evidence for the locus of inhibition. It was possible to demonstrate the absorption bands of another component of the cytochrome system and this was termed eytochrome $a_{3}$. Upon reaction with cyanide or carbon monoxide, these bands changed in a manner indicative of an inhibitor complex, and it was thus possible to equate cytochrome $a_{3}$ with the terminal oxidase and the exact site of action of these inhibitors.

The power and aecuracy of these methods have been markedly improved with the development of rapid difference speetroseopy by Chance. It is now possible to determine preeisely the oxidation-reduction state of each component of the eleetron transport sequence under a variety of conditions. During activity in the respiratory chain, it is now known that every component exists partly in the oxidized and partly in the reduced state, the fractions depending on the relative activities of the enzymes in the sequence and the supply of substrates, oxygen, and phosplrate acceptors (Chance and Williams, 1956). Let us illustrate the behavior of such a system during inhibition by outlining the results with antimyein. When antimycin was added to a mitochondrial suspension in a steacly state of activity, the eytochromes $a_{3}, a$, and $c$ became completely oxidized, while cytochrome $b$ and the nucleotides became completely reduced (Chance and Williams, 1956). The site in the chain where reduction changes over to oxidation is called the "crossover point" by Chance and it is to this loeus that the inhibitory attack is attributed. In this case, the crossover point is between cytochromes $b$ and $c$. Antimyein could thus act either on an unknown component between these eytochromes or it could react with cytochrome $b$ to prevent its oxidation; reaction with cytochrome $c$ is unlikely because it can be reduced readily upon addition of aseorbate. It is possible to localize the action more closely inasmuch as antimycin has been shown to block the reduction of cytochrome $c_{1}$, which operates between cytochromes $b$ and $c$ (Keilin and Hartree, 1955).

Spectroscopic determinations may be combined with additions of specific electron donors or acceptors. In the hypothetical transport chain:

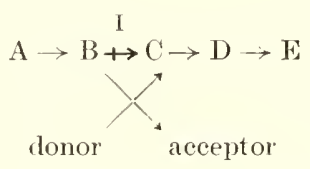

where a block has been established between $B$ and $C$, the addition of a donor from which $\mathrm{C}$ can accept electrons will cireumvent the block; this was the ease in the addition of ascorbate above. Likewise, addition of an acceptor that can take electrons from $B$ will allow the reaction to proceed. There will be, of course, corresponding ehanges in the oxidation-reduction 
state of the components when these donors or acceptors are added. The development of many acceptor dyes, such as phenazine methosulfate, diethylsafranin, 2,6-dichlorophenol-indophenol, and others, acting at different loci in the electron-transport sequence, has increased the versatility of this method.

\section{Distribution of Labeled Substrate Atoms}

A rather laborious but often informative technique for the localization of inhibitor action involves the addition of a substrate labeled at certain positions with radioactive atoms and the determination of the metabolic pathways followed by these atoms in the absence and presence of an inhibitor. The refinement in various types of chromatographic analysis has allowed this procedure to be applied to a great variety of metabolic blockades. The localization achieved is usually restricted to metabolic pathways rather than to individual enzymes but nevertheless may be valuable in the initial mapping out of the action of a new inhibitor. This method is also useful in establishing whether an inhibitor, which has been shown by other techniques to block some enzyme or pathway in vitro, actually does this in the living cell.

Many examples of this procedure will be discussed in connection with the individual inhibitors but one illustration of the method may be taken from studies on the inhibition of photosynthesis by iodoacetate and iodoacetamide. These inhibitors were shown by Kohn (1935) to depress potently the formation of sucrose and indirect evidence indicated that the block was not primarily on the initial photochemical reaction (light reaction) but on the subsequent pathway whereby $\mathrm{CO}_{2}$ is utilized in the formation of carbohydrate (dark reaction). Recently it has been possible to determine the distribution of carbon-14 when plant tissue is illuminated for very brief periods in the presence of $\mathrm{C}^{14} \mathrm{O}_{2}$ and it is apparent that the first stable intermediate is 3-phosphoglycerate, which showed the highest specific activity initially. The formation of hexoses could thus occur through the reversal of the glycolytic pathway, the first step being the reduction of the 3-phosphoglycerate to glyceraldehyde-3-phosphate. If this occurs, iodoacetate and iodoacetamide, which inhibit the phosphoglyceraldehyde dehydrogenase, should block the photosynthesis of carbohydrate in the same mammer as they inhibit glycolysis, and perhaps would cause an accumulation of 3-phosphoglycerate. Calvin and his co-workers (Calvin et al., 1951) treated Chlorella with $0.15 \mathrm{~m} / \mathrm{I}$ iodoacetamide so that $\mathrm{C}^{14} \mathrm{O}_{2}$ fixation was inhibited $90 \%$ and yet it was found that the rate of sucrose formation was not decreased. Also no phosphoglycerate accumulation occurred. It was suggested that enzymes other than phosphoglyceraldehyde dehydrogenase could have been inhibited, blocking other pathways of phosphoglycerate utilization and allowing a more channeled sucrose syn- 
thesis. Arnon (1952) believed that insufficient time had been allowed for the iodoacetamide to act and that the original data pointed to a progressive decline in sucrose formation with time; even if this were true, it is evident that the inhibitor blocks more readily some other enzyme initially. The situation is complicated still more by the determination of carbon-14 distribution in tobacco leaves, infiltrated with $0.15 \mathrm{~m} M$ iodoacetamide and illuminated for periods of 4 and 30 sec, reported by Newburgh and Burris (1954). The fixation of $\mathrm{C}^{14} \mathrm{O}_{2}$ was again depressed strongly and the distribution of the label was markedly altered (Table 11-1). The reduction

TABLE $11-1$

Effect of Iodoacetamide (iani) on the Pattern of Carbon-l4 Incorporation in Tobacco Leaves Exposed to $\mathrm{C}^{14} \mathrm{O}_{2}{ }^{a}$

\begin{tabular}{|c|c|c|c|c|c|c|c|c|}
\hline \multirow{3}{*}{ Compound } & \multicolumn{4}{|c|}{$\begin{array}{l}\% \text { of Total } \mathrm{C}^{14} \mathrm{O}_{2} \\
\text { incorporated }\end{array}$} & \multicolumn{4}{|c|}{ Specific aetivity } \\
\hline & \multicolumn{2}{|c|}{ Control } & \multicolumn{2}{|c|}{ TAAM } & \multicolumn{2}{|c|}{ Control } & \multicolumn{2}{|c|}{ IAAM } \\
\hline & $4 \mathrm{sec}$ & 30 see & $4 \mathrm{sec}$ & $30 \mathrm{sec}$ & 4 sec & $30 \mathrm{sec}$ & $4 \mathrm{sec}$ & $30 \mathrm{sec}$ \\
\hline Phosphoglycerate & 7 & 10 & 2 & 5 & 128 & 118 & 6 & 16 \\
\hline Hexose diphosphate & 6 & 5 & 7 & 5 & 85 & 92 & 28 & 19.3 \\
\hline Glucose phosphate & 2 & 13 & 4 & 24 & 36 & - & 19 & 220 \\
\hline "Phosphate esters" & 2 & - & 23 & - & 30 & - & 68 & - \\
\hline Malate & 18 & 14 & 14 & 11 & - & 7.7 & - & 9.8 \\
\hline Alanine & 41 & 24 & 36 & 14 & 21.6 & 56 & 26 & 139 \\
\hline Serine & 4 & 7 & 7 & 6 & 10 & - & - & 34 \\
\hline Aspartate & 9 & 6 & 2 & 9 & 4.7 & - & 4.5 & - \\
\hline
\end{tabular}

a The time intervals are the durations of exposure to light. Iodoacetamide was $0.15 \mathrm{~m} M$. The specific activity is given as counts/minute/m/lmoles of compound. The "phosphate esters" consisted of several compounds of about equal activity including fructose phosphate and 6-phosphogluconate. The total incorporation of $\mathrm{C}^{14} \mathrm{O}_{2}$ was inhibited about $75 \%$ by this concentration of iodoacetamide.

in the specific activity of phosphoglycerate indicates either an inhibition of the carboxylation reaction or of the supply of ribulose diphosphate for carboxylation. The increased formation of glucose phosphate and the high specific activity at $30 \mathrm{sec}$ point to the reversal of the pentose phosphate shunt pathway; the great rise in "phosphate esters" (which include fructose phosphate and 6-phosphoghnconate) may support this but more detailed analysis of this fraction would be necessary. These experiments in any event have demonstrated multiple sites for the inhibition and have made it unlikely that the effect on phosphoglyceraldehyde dehydrogenase is of major importance. 


\section{Use of a Second Inhibitor}

When several metabolic reactions are proceeding in a preparation, it is occasionally possible to narrow the systems investigated with specific inhibitors. Any effect of the inhibitor that is being studied can then more readily be attributed to the "isolated" pathway or enzyme. The simplest situation is a monolinear chain. The oxidation of $\alpha$-ketoglutarate in mitochondria is followed by the oxidation of the succinate formed and to some extent by further oxidations. By the proper use of malonate, at a concentration that effectively inhibited succinate oxidation but did not interfere with $\alpha$-ketoghtarate oxidation, Slater and Holton (1954) were able to study the properties of the $\alpha$-ketoghtarate oxidase uncomplicated by these subsequent reactions. The blocking of one pathway in a bilinear system will also allow the action of an inhibitor on the other pathway to be studied. An investigation into possible inhibitors of the $O$-methylation of the catechol amines, for example, could be done more quantitatively if the monoamine oxidase were blocked.

Enzyme inhibitors sometimes produce a stimulation of metabolism or cell function (page 451) and the nature of the reactions involved in this may be probed with other inhibitors. Malonate stimulates the respiration of heart slices following an initial depression; the presence of fluoride almost completely prevents this stimulation (Webb et al., 1949 b) suggesting that the rise in respiration is mediated through glycolysis. Malonate also stimulates the contractility of hypodynamic ventricle strips and this is effectively counteracted by low concentrations of iodoacetate (Covin and Berman, 1956), again pointing to the role of glycolysis in the stimulation. These results, of course, did not exactly localize the site of action of malonate but did present some possibilities for further testing, e.g., the elimination of a rate-limiting step in the conversion of glucose to pyruvate.

A comparison of the actions of two or more inhibitors can be of use in making a localization more certain. If a new inhibitor has been shown to act on a particular enzyme or pathway, the demonstration that it produces the same effects as an inhibitor known to act specifically at this site substantiates the localization. Let us assume that a substance has been shown to inhibit the phosphoglyceraldehyde dehydrogenase. If one wishes to be certain that this is its site of action in the cell, the inhibitor should be shown to have at least some of the effects of iodoacetate, e.g., an accumulation of hexosephosphates, a reduction in anaerobic lactate formation, the development of contracture in muscle, or the abolition of retinal activity. In case the actions of the inhibitors differ, it must be concluded that either the new inhibitor is not inhibiting the dehydrogenase specifically or that the classic concept of iodoacetate action is incorrect. The greater the correlation between the over-all effects of a group of imhibitors and the ability of the various members to inhibit a certain reaction, 
the more certain is one that the effects are related to this particular inhibition.

\section{Demonstration of Inhibition on Isolated Enzymes}

Ideally in the localization of action of a new inhibitor, the analysis proceeds from complex metabolic systems to the sensitive enzyme. That is, one first shows that the inhibitor acts on some major process in the cell, then on some particular pathway, then narrows the site to a region of this pathway, and finally shows the necessary inhibition on a single enzyme. In such a procedure it is essential that the nature of the inhibition on the single enzyme or reaction be established if one is to be completely confident of the localization. It is admitted that this is not always immediately possible because, as has been discussed, the site may be a previously unknown component or an enzyme that has never been isolated in an active state. In such cases the final localization will be achieved only with the characterization of this site.

When the approach begins with and restricts itself to a study of isolated enzymes, only very tenuous conchusions can be drawn. Even if all the known enzymes were tested and the spectrum of inhibition established on these, there are reasons why it would be impossible to deduce with validity a site or sites of action in the cell. In the first place, there may be again an unknown enzyme that is more sensitive to the inhibitor than any of those tested. In the second place, isolated enzymes and the same enzymes within the cell do not necessarily behave identieally (page 436). There must be evidence from work on living systems that the metabolism within which the particular enzyme operates is disturbed by the inhibitor. It might be said that the demonstration of inhibition of single enzymes is a neeessary but not sufficient proof of localization.

A very common problem arises when a substance is found to inhibit an enzyme that could well be involved in the action of the substance, but only at a concentration that is appreciably higher than that required to produce the action in a living system. Since few inhibitors are accumulated within cells, the explanation proposed is usually that the enzyme is more sensitive in its intracellular state and enviromment, which is more satisfying than to admit the more likely alternative that this enzyme is actually not involved primarily in the inhibition. Of course. the enzyme may be inlibited more readily in the cell but at least some indireet evidence must be presented that this is so if the hypothesis is to be taken seriously. Nevertheless there are many instances where unwarranted deductions as to the site of action have been taken seriously with the danger that they become eventually incorporated into the structure of demonstrable facts and proved mechanisms. 


\section{Use of Reconstituted Enzyme Systems}

In those instances where an organized multienzyme complex can be broken down into its component enzyme units, these individual units can usually be tested directly for susceptibility to the inhibitor. However, an even more potent method is available if the single enzymes can be put back together in either partial or total reconstitution of the original system. Certain parts of a linear sequence may be reformed and tested with the inhibitor and occasionally artificial or unnatural substrates or intermediates may be introduced. In this manner it is usually reasonably easy to locate the enzyme attacked, providing it is a known and isolatable component. This approach is best illustrated by the recent work of Keilin and King (1960) on the sites of inhibition of several inhibitors of the succinate oxidase system. This electron transport sequence was broken down into its several known enzymes and reconstituted in different ways, and, in addition, electron donors and acceptors were introduced so that it was possible to test almost every individual unit or any partial sequence.

\section{Demonstration of an Uncoupling Site of Action}

When a substance is capable of producing a disturbance in cell function or synthetic processes possibly attributable to an interference with energy flow, and yet does not exhibit a significant depressant action on the respiration in the same concentration range, it is usual to look for the site of inhibition in the reactions whereby high-energy phosphorylations are coupled with oxidations. Substances that dissociate oxidative phosphorylation have been called uncoupling agents or uncouplers. Such inhibitors will be discussed in detail in a later chapter but it is appropriate here to examine critically the evidence that may be presented for an uncoupling site of action. The problem would be clarified if a rigorous and generally acceptable definition of uncoupling existed but the literature contains surprisingly few exact or carefully considered definitions of this phenomenon. The best general discussion of uncoupling and the methods for demonstrating it is perhaps the review by Brody (1955). A brief evaluation of the preparations used in uncoupling studies will illustrate some of the problems in localization and may help to formulate a provisional definition.

Preparations of cells or tissues are unsatisfactory for proving that a substance uncouples oxidative phosphorylation because it is impossible at the present time to determine accurately the rate of ATP formation within the cell where many metabolic reactions involving ATP proceed simultaneously.* The observation that a substance reduces the uptake of

* The immediate product of high-energy phosphorylation will be designated here as ATP for simplicity, since this would appear to be generally the most important substance. Other nucleotides or unknown acceptors, however, may play a role in the conservation of oxidative energy. 
$\mathrm{P}_{i}$ (inorganic phosphate) into cells without a corresponding depression of respiration is, of course, not evidence for uncoupling. Oxidative phosphorylation proceeds normally without appreciable exchange of $\mathrm{P}_{i}$ between the cell and the medium, so that when the classic uncouplers, such as 2,4-dinitrophenol, act upon cells there is no reduction in phosphate uptake unless growth is inhibited. Furthermore, in some cells the uptake of $\mathrm{P}_{i}$ is regulated or metabolically dependent, so that an inhibitor could depress the inward movement of $\mathrm{P}_{i}$ by a mechanism unrelated to the processes of oxidative phosphorylation. Another criterion that has been used to demonstrate uncoupling in cells is the rise in $\mathrm{P}_{i}$ and fall in ATP produced by a substance. This is cortainly suggestive of uncoupling but does not establish it. The levels of $\mathrm{P}_{i}$ and $\mathrm{ATP}$ within a cell depend on many reactions and not solely on the rate of oxidative phosphorylation. An increased utilization or enzymic splitting of ATP may lead to a new steady state in which the ATP is lower and the $\mathrm{P}_{i}$ higher than originally, if the rate of ATP formation is not adequate to maintain the level. Yet another criterion that has been suggested for uncoupling is a stimulation of respiration on the basis that the rate of oxidation is limited by the phosphorylative reaction. However, the dependency of the oxidative rate on phosphorylation varies with the type of cell, the state of the cell, and the nature of the oxidative pathway. A very active tissue utilizing ATP at a high rate may be operating at maximal oxidative capacity and no stimulation would result from uncoupling, whereas in resting cells, in which respiration is limited by phosphate acceptors, a stimulation from uncoupling may be elicited. Uncoupling agents also are able to exert an inhibitory effect on electron transport and this may mask any stimulating action due to uncoupling. Finally, an increase in respiration can be brought about in a variety of ways unassociated with oxidative phosphorylation. These few considerations indicate the difficulties that may be experienced in attempting to establish uncoupling in living cells.

The conclusive proof for an uncoupling site must therefore be derived from experiments on units isolated from cells. These units are of some complexity since as yet no single enzyme carrying out oxidative phosphorylation has been obtained. Mitochondria have been most commonly used but submitochondrial particles obtained by various methods, such as sonic disintegration, are available and perhaps preferable. The results are usually expressed in terms of the $\mathrm{P}$ : $\mathrm{O}$ ratio, which is the number of phosphate groups esterified for each atom of oxygen reduced. It is obvious that if the organic phosphates formed are split by phosphatases or react in any way so that $\mathrm{P}_{i}$ is set free, the experimental value of the $\mathrm{P}: \mathrm{O}$ ratio will be lower than the true value. The phosphate esterified is usually determined from $(a)$ disappearance of $\mathrm{P}_{i}$ from the medium, $(b)$ formation of ATP, (c) formation of glucose-6-phosphate in the presence of hexokinase, or $(d)$ 
formation of creatine phosphate in the presence of creatine phosphokinase. When trapping systems for the esterified phosphate are used, it must be remembered that an inhibitor can also act on the transferring enzymes. For example, when the ATP formed in oxidative phosphorylation is utilized in the formation of glucose-6-phosphate from glucose, an inhibition of the hexolinase will depress the formation of the glucose-6-phosphate and the ATP synthesized may either accumulate or a greater fraction be eventually broken down by ATPase, the experimental P : O ratio decreasing. Also, as in cells, an activation of ATPase by the inhibitor, either directly or indirectly, will tend to reduce the experimental $\mathrm{P}: \mathrm{O}$ ratio.*

Difficulties in interpretation sometimes arise even in mitochondrial studies. There is the possibility of phosphorylating and nomphosphorylating pathways and a shift in the relative activities of these. In such a system,

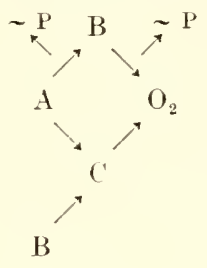

the experimentally determined $\mathrm{P}: \mathrm{O}$ ratio will in general not accurately represent the phosphorylative efficiency of the $\mathrm{A} \rightarrow \mathrm{B} \rightarrow \mathrm{O}_{2}$ pathway. Furthermore, any variation in the oxygen rednced by the $\mathrm{A} \rightarrow \mathrm{C} \rightarrow \mathrm{O}_{2}$ or $\mathrm{B} \rightarrow \mathrm{C} \rightarrow \mathrm{O}_{2}$ pathways will alter the $\mathrm{P}: \mathrm{O}$ ratio. Thus the stimulation of a nonphosphorylating pathway will lower the $\mathrm{P}: \mathrm{O}$ ratio. Whether this should be termed uncoupling or not is sometimes difficult to decide. When the nonphosphorylating pathway is unrelated to the phosphorylating pathway, i.e., with a different substrate or involving different electron transport systems, the effect is not true uncoupling in that no change in the oxidative phosphorylation processes has occurred, but there are instances where the same substrate is involved and it is not certain if a new transport pathway has been opened up or the old pathway uncompled.

A further difficulty, which is actually inherent in much work with inhibitors, exists in the demonstration that the uncoupling, if it does occur, is the result of a direct action of the substance on oxidative phosphorylation or whether it is duc to the disturbances created by the substance acting elsewhere. Mitochondrial oxidative phosphorylation would appear

* If the ATPase activated is not a component of the oxidative phosphorylation pathway, there is little justification for concluding that a drop in the $\mathrm{P}: \mathrm{O}$ ratio is indicative of uncoupling. However, if the ATPase activity measured is an expression of the reversal of the phosphorylation process and involves the same enzymic system, the increase in the ATPase activity will implicate an uncoupling mechanism. 
to be sensitive to structural changes, for example, and any agent that induced disruption of the organized structure could uncouple secondarily. The relationship between mitochondrial swelling and uncoupling is still obscure but in any event swelling probably means an alteration of the intramitochondrial state. Ionic changes, fluctuations in $\mathrm{pH}$, or the release of natural uncompling agents might mediate the effect. The use of submitochondrial particles might eliminate these problems.

A generalized oxiclative phosplorylation system may be represented as:

$$
\left.\mathrm{A}_{o x} \leftarrow \mid \begin{array}{l|l}
\mathrm{A}_{\text {red }} \\
-\mathrm{P}-\mathrm{P}-\mathrm{X} \leftarrow \\
\mathrm{ADP}
\end{array}\right] \rightarrow \mathrm{Y}-\mathrm{P}
$$

where $\mathrm{A}$ is any substrate or coenzyme, $\mathrm{X}$ represents all the possible components that may be involved in phosphate transfer to ADP, and $\mathrm{Y}$ represents any substance that is used to trap the phosphate in the test system. Three basically different types of uncoupling mechanism are possible. (1) The uncoupling agent is able to accept the phosphate from $\mathrm{X}-\mathrm{P}$, competing with ADP, but, being unstable, breaks down rapidly so that little or no ATP is formed and the $\mathrm{P}_{i}$ concentration does not change appreciably. (2) The uncoupling agent is able to replace the phosphate so that complexes such as X-I, ADP-I, or Y-I may be formed, where $\mathrm{I}$ is the inhibitor or uncoupler. It may be that one of these complexes is unstable and the I acceptor is regenerated but in either case there is a reduction of the phosphorylation. (3) The mncoupling agent in some manner (without participating in the reactions) prevents the transfer of $\mathrm{P}_{i}$ to $\mathrm{ADP}$, either by allowing oxidation to proceed without any reactions involving phosphate, or by inducing instability into the $\mathrm{X}-\mathrm{P}$ complex, or by inhibiting the transfer from X-P to ADP. Because knowledge of the intimate details of phosphorylative coupling is lacking, it is impossible at the present time to localize accurately uncouplers of the third type. It is often not too easy to distingnish between the third type and the other two mechanisms mnless the abnormal complexes have lifetimes sufficiently long so that they may be demonstrated.

A definition of uncoupling agents may now be proposed on the basis of these observations. An uncoupling agent interferes directly in one of the reactions between oxidation and the formation of ATP, with the result that the obligatory coupling is abolished and oxidation may proceed without normal ATP synthesis. The definition adopted here implies that the substance acts in some manner between $A_{r e d} \rightarrow A_{o x}$ and $\mathrm{ADP} \rightarrow \mathrm{ATP}$ in scheme 11-6. Substances that act indirectly upon these reactions or that affect systems peripheral to the phosphorylation process act fundamentally in a different way and should not be classified as uncouplers. Evidence for an uncoupling site of action for a substance must therefore include a dem- 
onstration of a direct effect on the coupling process. In addition to this, if the uncoupling is to be made the basis for the effects observed on living cells or tissues, evidence must be presented that the action seen in vitro actually occurs within the cell and that it is the primary action.

\section{GENERAL DEVELOPMENT OF A LOCALIZATION PROGRAM}

Some of the procedures frequently useful in the localization of the site of action of an inhibitor lave been discussed and it is now possible to take up the problem of the over-all design of the investigation. Although it is true that each new inhibitor will usually present a problem that is at least in some respects unique and will require an individual approach, it is possible to formulate some principles of general applicability. The total program may be divided for convenience into four chronological phases: (1) obtaining the preliminary data, (2) planning the attack, (3) sequential anallysis for the inhibition site, and (4) correlation of the action at this site with the original effects observed.

\section{The Initial Phase}

A substance has been found to have an action on living tissue, either isolated or in the whole organism, that is indicative of an interference with metabolism. There are certain things it is often useful to do before starting or even planning a more detailed analysis of the site of action. Easily obtained results at this stage will often save considerable time later. It is suggested that two basic types of data be obtained initially.

(1) A dose or concentration relationship should be established, preferably over the entire range of action of the substance. This will not only provide information on the concentrations to be used in the later work and a basis for comparison of the in vitro and in vivo results, but occasionally will bring out features of the action that will aid in the localization.

A simple example of this may be cited. It was observed originally that low concentrations (0.01-0.03 $\mathrm{m} M /$ ) of 1,10-phenanthroline blocked almost completely the oxidation of pyruvate and $\alpha$-ketoglutarate by heart mitochondria, but during the process of obtaining a concentration-inhibition curve it was found that higher concentrations $(0.1-0.3 \mathrm{~m} M I)$ did not inhibit as strongly, and actually stimulated the oxygen uptake in some instances (Yang et al., 1958). This unexpected result pointed to certain characteristics of the inhibition and later suggested experiments that led to the demonstration of an uncoupling action on the phosphorylation associated with the oxidation of DPNH. However, the basic reason for the reversal of inhibition has not been elncidated and any postulated mechanism in the future must be able to explain this reversal. 
(2) The time relationships of the inhibition should be investigated and this includes both the rate at which the inhibition is developed and the degree of reversibility of the inhibition. A delayed onset of inhibition is sometimes indicative of the metabolism of the substance to an active inhibitor or of a lethal synthesis. The failure of the inhibition to be reversed readily when the substance is removed is also suggestive of mechanisms that may facilitate the localization of the site of action.

\section{Planning the Program of Localization}

It is now of the utmost importance to map ont a plan of attack. One must do this on the basis of the data already obtained, the nature of the inhibitory action, the system that has been found to be affected, and a consideration of the properties of the inhibitor itself. It is well to gather together all of the pertinent information on the physical and chemical characteristies of the inhibitor. Such properties as solubilities, $\mathrm{p} K_{a}$ 's, stability, possible isomerism, complexing abilitics, chemical reactivity, and special structural aspects can be crucial points in cither planning or interpreting the work. Of course, the relationship of the compound, structurally or in other ways, to naturally occurring substances or other inhibitors whose actions are known will often suggest sites of action. In other words, an attack can be made much more efficient and productive when all the available data are at hand to guicle it.

Before outlining the initial phases of the localization, it is necessary to consider all the conceivable mechanisms by which the inhibitor could act to produce the effect observed. in order that the plan will better enable critical experiments to be performed. An ideal plan, perhaps, can be constructed much like a flowsheet or botanical classification key, in that the results of each test should indicate the nature of the subsequent experiment, and so on as the localization progresses. For example, it is usually advisable to begin with a localization to the major pathways of metabolism. If an inhibitor is found to depress bioluminescence, it might act on either the energy-forming systems or the eneruy-utilizing systems; the first step would be to determine which of these is susceptible. If an inhibitor rednces respiration with glucose as a substrate, it should be determined whether the action is primarily on the glycolytic pathways or on the tricarboxylic acid cycle. The general procedure is to go from the major metabolic systems progressively to smaller and smaller segments until the ultimate site is reached. Of course, if the initial action observed is on a tissue function or in a whole animal, the first step should be a pharmacological analysis of the site of action before the biochemical site is sought. 


\section{The Sequence of Experimental Localization}

It is impossible to present any general procedure to follow in all cases because the situations presented by various inhibitors will often have little in common. Some frequently occurring situations will be discussed briefly to emphasize the necessity for a more complete analysis than is usually found in the literature. This approach is particularly to be contrasted to the common type of report wherein a single positive result with an inhibitor is used as the basis for a designation of the site of action. An inhibitor that has been found to depress respiration is immediately tested on the tricarboxylic acid cycle and if inhibition is found, whatever the degree, the cycle is stated to be the site of action; such inferences are invalid in general and tenuous under the most favorable circumstances. A few comments will now be made on the suggested approach as applied to the localization of inhibitors depressing respiration or cell growth.

A depression of respiration by a substance may be observed initially in a variety of ways. Let us assume, for generality, that a substance has been found to reduce the respiration of an intact organ as measured by changes in the arteriovenous oxygen difference, that a dosage or concentration curve has been obtained, that the inhibition has been shown to be readily reversible, and that the nature of the inhibitor gives no clue as to its site of action. What are some of the possible mechanisms by which this respiratory depression could be brought about?

I. Inhibition of some step in electron transport that is common to most oxidation systems (such as one of the cytochromes).

II. Inhibition of an enzyme in the tricarboxylic acid cycle (such as isocitric dehydrogenase or aconitase) or of an enzyme introducing a substance into the cycle (such as the condensing enzyme or pyruvate oxidase).

III. Inhibition of a pathway leading to the tricarboxylic acid cycle (such as glycolysis if glucose or glycogen are the principal substrates or the fatty acid breakdown helix if fatty acids are important substrates).

$I V$. Decrease in permeability or the activity of a transport system for an exogenous substrate.

V. Primary depression of functional activity of the tissue leading to reduction of the respiration.

Althongh the major possibilities listed are few, a survey of the commonly used inhibitors that depress respiration will show that almost all act in one of these ways. It is unnecessary initially to worry about more esoteric mechanisms. 
Perhaps the first preparation to test is a mitochondrial suspension from the tissue using pyruvate as the substrate with priming amounts of malate or oxalacetate. If no inhibition occurs within the proper concentration range of the inhibitor, actions on pyruvate oxidase, on the cycle enzymes, or on electron transport are eliminated, and other major systems must be tested. If inhibition is found, the same preparation may be tested with malate or oxalacetate in equimolar concentrations with the pyruvate, so that now the incorporation of pyruvate into the cycle is not dependent on the complete operation of the cycle. The degree of inhibition will be indicative of the site in the cycle where a block is produced; e. g., if pyruvate oxidase is the susceptible enzyme, the inhibition will be marked whereas if $\alpha$-ketoglutarate oxidase is the site of action, the inhibition will be minimal or even absent, at least during the early period of measurement. The inhibitor is then tested with various of the cycle intermediates as substrates. It is usually possible to determine the blocked site in the cycle quite easily and the localization can then proceed to the electron transport system, if an oxidation step is involved. or otherwise to the isolated susceptible enzyme. If the inhibition is upon the oxidation of only one substrate or intermediate, it is evident that those components of the electron transport sequence that are common to all the oxidation systems are not affected. A comparison of the actions of the inhibitor on succinoxidase and the other oxidation systems is often profitable. If succinate oxidation is preferentially inhibited, this restricts the site of action and may aid considerably in the localization because the succinoxidase can be isolated in a reasonably satisfactory form whereas the other systems must be tested in mitochondria or reconstructed. It is at this point that the use of various electron donors and acceptors, or of difference spectroscopy if available, is helpful.

When a significant inhibition of glycolysis is observed, the subsequent localization may proceed somewhat differently according to the tissue involved or the techniques available. The simplest approach is generally to add the varions glycolytic intermediates and determine at what point along the sequence the pathway is blocked by the inhibitor. Accumulation of intermediates during inhibition may be tested also or the individual glycolytic enzymes, if they are readily available, can be tested directly.

The situation that arises when no inhibition can be demonstrated on the major oxidation pathways calls for a consideration of the last two mechanisms listed (IV and V). Localization to a block in the membrane reducing the entrance of an important substrate is difficult to establish unless the intracellular concentration of that substrate can be determined. For this purpose, it may be possible to block the metabolism of the substrate with another inhibitor so that significant concentrations within the cell can occur. The last mechanism ( V) also presents difficulties for the positive proof of its occurrence. If satisfactory inhibition can be shown on some system 
involved in respiration, the possibility of a direct functional effect is usually ignored, but when no adequate inhibition has been found on any respiratory pathway or transport of substrate into the cell, one is left with at least two possibilities - an action through functional depression or an action on some metabolic system not yet tested. This can be resolved only by finding an inhibition on some system and hence every effort must be expended in testing all possible systems that may be contributing significantly to respiration. Occasionally some investigation of the tissue's particular patterns of respiratory metabolism must be made to determine the major contributing pathways. In rare instances it is possible to "isolate" the functional system and test it directly. Examination of glycerinated muscle fibers may demonstrate an action on the contractile elements unassociated with oxidative reactions. A good example of this problem arose in the study of the effects of drugs and inhibitors on the respiration of electrically stimulated brain slices (McIlwain, 1959). The respiratory depression produced by inhibitors such as cyanide, iodoacetate, malonate, or fluoride (Heald, 1953) could arise either from a general inhibition of respiratory pathways or from a more specific effect on ionic distributions and movements across the cell membranes so that the tissue becomes incapable of responding to the electrical impulses. A functional depression with a secondary effect on metabolism can occur more readily in intact tissues than in slices because conduction of activating impulses could conceivably be blocked more easily than the over-all metabolism of the tissues.

A depression of cellular growth can result from a great variety of inhibitory mechanisms inasmuch as in growing cells a major portion of the metabolism and most of the metabolic pathways are involved in the formation of new cell material. Some of the most likely mechanisms may be listed.

I. Inhibition of the energy-generating systems responsible for the major fraction of the respiration (as discussed above).

II. Inhibition of the synthesis of some coenzyme or growth factor.

III. Inhibition of some step in protein or nucleic acid synthesis.

IV. Incorporation as an abnormal analog into a synthetic pathway resulting in a metabolic block if one of the abuormal substances formed cannot undergo further metabolism or is unable to function as its normal analog.

V. Inhibition of the uptake of precursors for synthetic processes (such as amino acids for the formation of protein).

VI. Direct disturbance of the mitotic sequence (such as inhibition of spindle formation or of the protoplasmic movements associated with cleavage). 
If there is no marked inhibition of respiration at concentrations decreasing growth, an uncoupling of phosphorylations should be tested first because it is relatively easy to do compared to an examination of synthetic and mitotic processes. If uncoupling is found not to be the mechanism, it would be advisable to obtain all the cytological information possible on the nature of the inhibited cells in order to note any unique characteristics not observable when the cells are depressed by respiratory inhibitors, anoxia, or lack of substrate.

Analyses for the total protein or mucleic acid synthesized by the cells are seldom very informative because growth inhibition from whatever cause implies a depression of these basic cell components. Possibly the most helpful technique is the use of labeled compounds to determine the nature of the derangements in synthesis if they occur. The incorporation rates of various amino acids into the cell protein or of nucleotide precursors into nucleic acids can be easily determined, and by chromatographic separation the accumulation of intermediates may be detected. If the structure of the inhibitor is such that it might be participating as an abnormal analog, the inhibitor itself can often be labeled and its metabolic course within the cell traced. Inasmuch as many steps of the synthetic pathways are unknown at present. localization is seldom easy and, indeed, many of the important growth inhibitors act by mechanisms remaining to be discovered, despite a large amount of investigation to elucidate the susceptible sites.

\section{Correlation between the Site of Inhibition and the Effects on Living Tissue}

When a single site of action has been found, it is now necessary to show that inhibition at this locus is responsible for the initial observations. The types of evidence for establishing such a positive correlation have been discussed (page 471). However, it is rare when an inhibitor is specific in its action and blocks at only one site, and therefore one must expect in the course of the localization to find inhibitions of varying chegrees on different pathways or enzymes. There may not be just one site or mechanism reponsible for the depression of complex processes such as respiration, but several actions contributing to various extents. When one ends up with data showing three sensitive enzymes with $50 \%$ inhibition obtained on enzyme $\mathrm{A}$ at $0.25 \mathrm{~mm} /$ inhibitor, on enzyme $\mathrm{B}$ at $0.78 \mathrm{~m} \boldsymbol{M}$ inhibitor, and on enzyme $\mathrm{C}$ at $1.2 \mathrm{mM}$ inhibitor, the question arises as to the relative contributions each of these inhibitions makes to the over-all effect in the complete system. From the in vitro results alone it would be rash to assign contributions at any inhibitor concentration because the enzymes may be either more or less sensitive within the cell. If during the localization the data have not already been obtained, one shonld investigate in the whole system the effects of the inhibitor on intermediate and product concentra- 
tions, using tracer and chromatographic techniques, so that the demonstration of an in vivo block at the sites indicated can be made. The primary sites of action of even some of the longest known and most commonly used enzyme inhibitors when acting on living tissue are uncertain because adequate analysis of their actions has never been made.

It is believed that the planning of a rather rigorous program for localization is advisable but in all problems involving metabolism or living material there frequently arise unforeseen difficulties that block the way to the final solution. At the present time, in any event, a foolproof key for the localization of an inhibitor's action cannot be presented; if this were possible, the problem would lose some of its interest because we would be deprived of the excitement of probing into unknown and complex systems. Indeed, if the situation were as simple as all that, technicians could be relied upon to undertake and solve such problems. Therefore, a final word should be said for those all-important abilities of a scientist, abilities that transcend all keys or formalized systems - the ability to choose the right experiments and the ability to observe accurately and with insight. These abilities might be attributed in part to intuition, which is perhaps the imer and unconscious organization of knowledge and experience in a field, and is allied to the ability of some physicians to diagnose correctly from the same information that leaves $0^{+h}$ ers puzzled, often by mental processes that they themselves cannot explain. The ability to observe extends not only to the actual experiments but to data and the representations of data. In the course of the localization of an inhibition site, it is quite likely that unexpected results will appear at times. These must be recognized and put on an equal status with the data for which the studies were made. It is frequently the accidental observation that eventually leads to the solution. The answer may not lie in what one seeks but in what one finds. 



\section{CHAPTER 12}

\section{RATES OF INHIBITION}

The various formulations of inhibition presented in the previous chapters generally represent or describe systems after equilibrium has been reached with respect to the inhibitor. In other words, it has been assumed that the reaction of an inhibitor with an enzyme has proceeded until the maximum inhibition under the conditions has been achieved. The most important constant characterizing such equilibrium states is the inhibitor dissociation constant, $K_{i}$, which expresses the affinity of the enzyme for the inhibitor. However, from the value of $K_{i}$ it is impossible to deduce the rate at which the inhibition will occur. Some inhibitors react so rapidly with enzymes that equilibrium is reached in times too brief to measure with the usual techniques, while other inhibitors may act very slowly and a steady inhibition will be reached only after several hours, or actually never reached because of secondary factors that reduce or extend the inhibition. The time course of the development of inhibition is often as important a characteristic as the final state reached in studies on either isolated enzymes or living organisms.

The rates at which inhibitors act may be of significance in a variety of ways when complex metabolic systems are investigated. If an inhibitor acts on two or more enzymes but the rate of reaction with one of these enzymes is much faster than with the others, some degree of specificity in the inhibition may be observed over a period of time. It is also possible that the effects on a tissue will be different when an enzyme is inhibited rapidly or slowly. In the latter case, for example, the tissue has greater opportunity to adapt to the inhibition as it progresses. When the response of some cell function is being measured, the rate at which this occurs in the presence of an inhibitor depends not only on the events that transpire between the site of inhibition and the functional process but also on the rate of enzyme inhibition. If one is concerned with the kinetics of functional disturbance, data on the rates of the primary inhibition should be obtained if possible, instead of assuming, as has been done in some cases, that the primary inhibition is practically instantaneous and the delay in depression of function is entirely due to factors frequently designated as "biological lag." With regard to studies on isolated enzymes, it is only by quantitative 
rate determinations of inhibition that certain kinetic data bearing on the nature of the reaction of the inhibitor molecule with the enzyme surface can be obtained. Thus the activation energy of the inhibition, which may be derived from the variation of the rate constant with temperature, can provide some information on the course of the reaction, or the approach of the inhibitor to the active site, and the alteration of the activation energy upon changing the experimental conditions (such as $\mathrm{pH}$ or ionic strength) may indicate the physical nature of the energy barriers that must be crossed. The number of quantitative studies on inhibition rates is, however, regrettably small and the more recent techniques for the rapid recording of enzyme reactions have not been applied as yet to this problem.

The rate of inhibition will be designated by $d i / d t$, the change in the fractional inhibition with time. This is usually not constant throughout an experimental period and therefore when comparing two or more inhibitors or comparing the rates of action of a single inhibitor under a variety of conditions, a rate constant should be calculated if possible, this constant being of such a nature that it relates the inlibition rate with the concentrations of reactants, e.g., $d i / d t=k(\mathrm{E})(\mathrm{S})(\mathrm{I}) \ldots$. The two constants, $k$ and $K_{i}$, the latter characterizing the final equilibrium state and the former the rate at which this is reached, are fundamental and adequately describe the quantitative aspects of an enzyme inhibition.

\section{GENERAL WAYS OF EXPRESSING THE RATE OF INHIBITION}

The time course of inhibition in any system may be expressed either graphically or by equations. The three commonest ways of plotting are shown in Fig. 12-1. Despite the preference of many investigators for plots of type $A$, these may be considered the least satisfactory because it is very difficult by inspection of such curves to visualize the actual inhibition at any time (since this would involve a comparison of the slopes of the curves at a chosen time) and more difficult to trace the time course of the inhibition.* The belief that it is better to plot directly obtained data rather than derived data has no sound basis unless there is a specific reason for indicating the absolute rates. Plots of types $\mathrm{B}$ and $\mathrm{C}$ are both satisfactory

* In a study of the rates at which iodoacetate inlibited respiration of brain tissue, Fuhrman and Field (1943) presented eurves showing the eumulative oxygen uptake against time and stated that this type of plotting has "the further advantage of presenting original rather than derived data" compared to plotting inhibition against time. Additional advantages are evident: (1) there is no necessity to waste time calculating the results, which can be left to others to do, and (2) the data plotted by this method usually look better, espeeially if the results have not been obtained very acenrately, and hence fewer experiments (sometimes only one) need be performed to give the impression that the results are quantitatively reliable. 
but type $\mathrm{C}$ is probably generally preferable, especially when the uninhibited control rate varies during the experimental period. In studies that are directed towards inhibition, it would seem to be best to show directly the variation of the inhibition with time, and yet this method of plotting is actually seldom used. Of course, there are no invariable rules and the plotting technique must always be adapted to the nature of the inhibition and the particular objectives in the investigation.
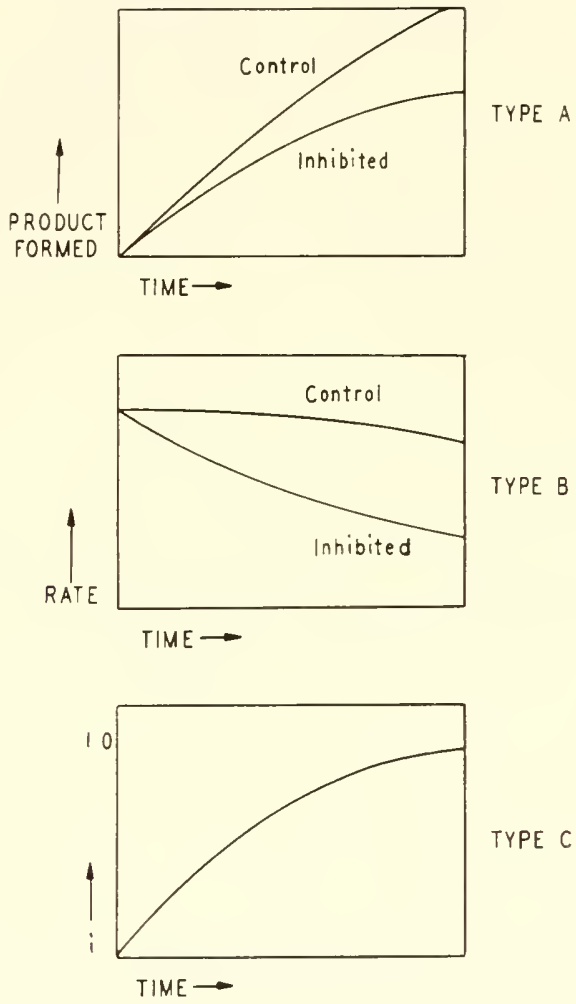

FIG. 12-1. The usual ways of representing the changes of inhibition with time.

Inhibition of a pure enzyme can often be treated on the basis of an assumed mechanism and equations derived by which the data can be interpreted and from which physically significant constants can be calculated. However, in complex systems, such a procedure is usually impossible and more general methods of mathematically expressing the inhibition rate must be used. The object in such cases is to find an equation that will adequately represent the data and which contains a constant or constants 
that may be used to characterize the rate at which the inhibition develops. It is commonly found that the rata of the enzymic or metabolic reaction measured falls exponentially in the presence of the inhibitor, in which case the variation of the rate with time would be given by a curve as in Fig. 12-2 and expressed by an equation of the type:

$$
v_{i}=v_{f}+\left(v_{0}-v_{f}\right) e^{-\alpha t}
$$

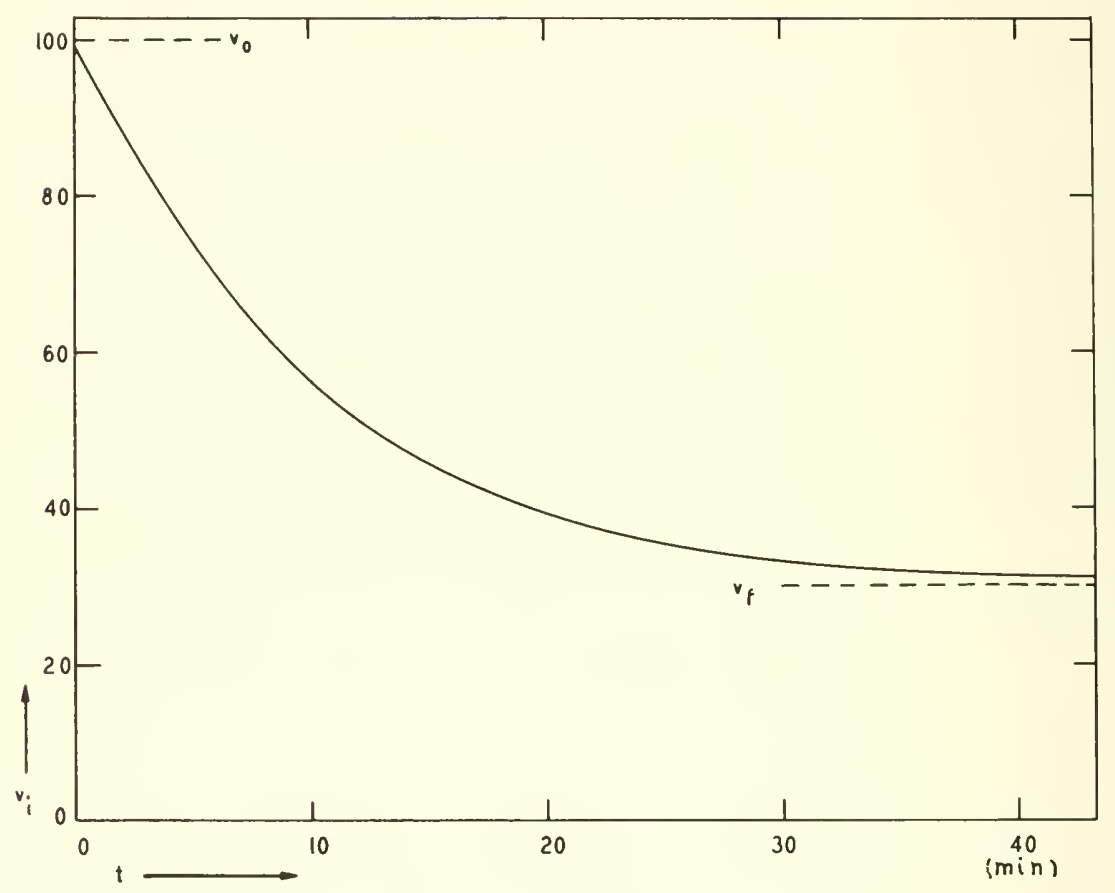

FIG. 12-2. Exponential development of imhibition from the initial uninhibited rate, $v_{0}$, to the final equilibrium rate in the presence of the inhibitor, $v_{f}(\mathbf{E q} \cdot 12-1), v_{o}=100$,

$$
v_{f}=30 \text {, and } \alpha=0.1 \mathrm{~min}^{-1} \text {. }
$$

where $v_{0}$ is the initial minhibited rate, $v_{f}$ the final equilibrium rate, $v_{v}$ the rate at any particular time, and $\alpha$ is a constant. Transposing and taking the logarithm of each side of the equation, it is found that:

$$
\log \left(v_{i}-v_{f}\right)=\log \left(v_{0}-v_{f}\right)-\frac{\alpha}{2.3} t
$$

so that a plot of $\log \left(v_{i}-v_{f}\right)$ against $t$ will give a straight line with a slope of $-\alpha / 2.3$ as in Fig. 12-3. The exponential nature of the inhibition must, 
in fact, be demonstrated initially by the linearity of such a plot. Equation 12-1 may be rewritten in terms of the inhibition as:

$$
i=i_{f}\left(1-e^{-a t}\right)
$$

where $i_{f}$ is the final level of inhibition achieved. Since:

$$
\log \left(i_{f}-i\right)=\log i_{f}-\frac{\alpha}{2.3} t
$$

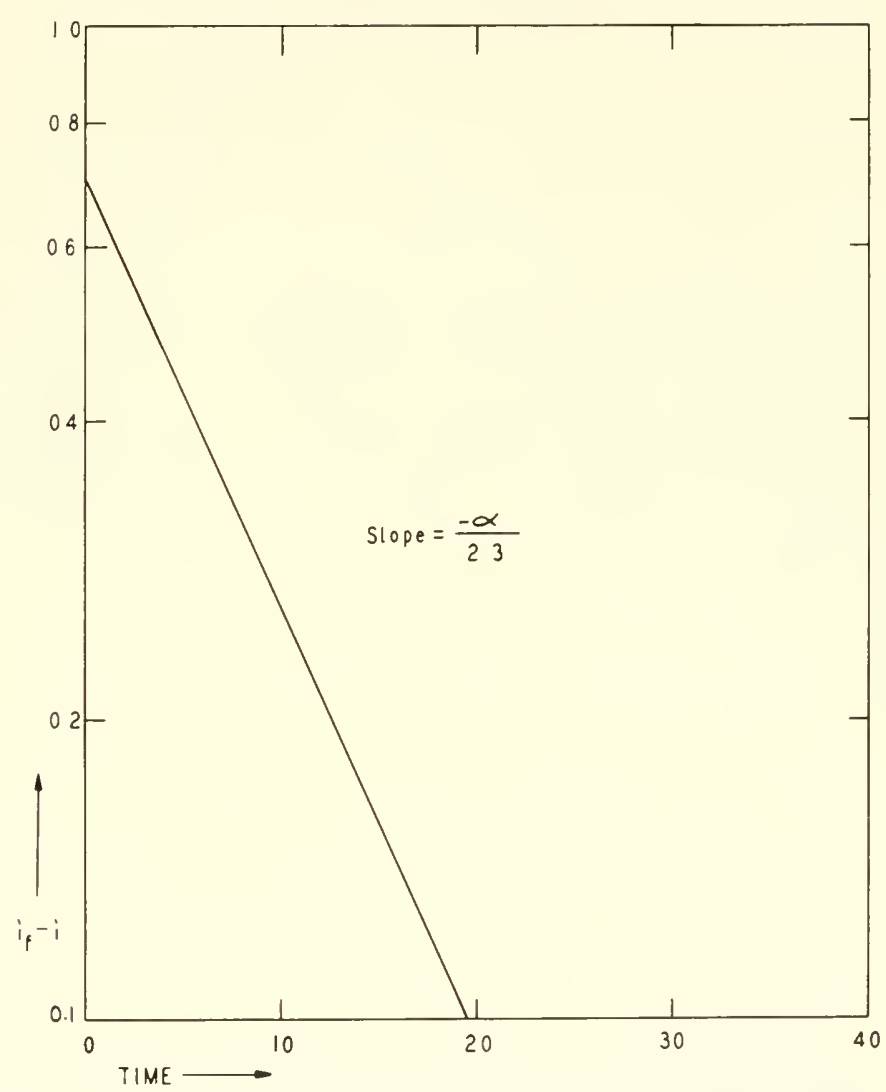

FIG. 12-3. Logarithmic plot of the inhibition shown in Fig. 12-2 (Eq. 12-4).

a plot of $\log \left(i_{f}-i\right)$ against $t$ can also be used to determine $u$, but has no advantage over the direct plotting of the rates. The initial rate of inhibition may be of significance experimentally when it is convenient to take readings over a short period after the inhibitor is added and it is possible 
to derive an expression for the initial rate by differentiating Eq. 12-3 and setting $t=0$, from which it is found that:

$$
(d i / d t)_{0}=\alpha i_{s}
$$

It is worthwhile noting that the initial rate of inhibition is directly proportional to the final equilibrium inhibition reached. Once the total course of the inhibition is established, it is thus possible to obtain useful data from readings taken for a short period after addition of the inhibitor. Indeed, the early portion of the curve is often more accurate to use inasmuch as secondary effects occasionally distort the course of the inhibition later.

An approach such as this was applied to the inhibitions of respiration and glycolysis in mammaliam tissnes by $\mathrm{HgCl}_{2}$ and other heavy metal salts in the early work of Jowett and Brooks (1928). They used the equation $v_{i}=v_{0} e^{-a t}$ and determined the values of $\alpha$ for the various inhibitions. However, it should be pointed out that this equation implies that complete inhibition is eventually reached, so that Eq. 12-1 is generally preferable.

No certain physical meaning can be attached to the constant $\alpha$ in complex systems in which the factors governing the rate of inhibition are unknown, unless it can be proved that the over-all rate measured is proportional to the activity of the enzyme upon which the inhibitor acts, in which case $\alpha$ may be related to the true rate constants for the association and dissociation of the enzyme-inhibitor complex as described in the following section.

If the course of the inhibition is neither logarithmic nor linear with time, and the data cannot be easily represented by any equation, it is necessary to inquire into the best way of presenting the kinetics. The time required to reach a certain chosen degree of inhibition, e.g. $50 \%$, is often given but this is not entirely satisfactory when comparisons are to be made between inhibitors. It is generally true that the early rate of inhibition will bear some relationship to the final inhibition (as shown above for the exponential variation) and thus it is important if two inhibitors are to be compared with respect to the rate of action, that the concentrations of each are chosen so that the final inhibitions produced will be approximately equal. This may be illustrated in Fig. 12-4 showing the experimental curves for two inhibitors acting on the same system. One might be tempted to say that inhibitor 1 acts more rapidly than inhibitor 2 because $d i / d t$ is obviously greater for the former and the time required to reach $50 \%$ inhibition is also shorter. This statement would be true if one were comparing the inhibitors at concentrations that gave quite different final inhibitions but this is a dangerous procedure. Actually it is seen that inhibitor 2 produces half of its final inhibition in a shorter time than does inhibitor 1 and for this reason might be considered to be the more rapidly acting. This can also be expressed mathematically for the case in which the inhibition 
varies exponentially. The time required to produce $50^{\circ} \%$ inlibition is given by:

$$
t_{0.5}=-\frac{2.3}{\alpha} \log \left[1-\frac{0.5}{i_{f}}\right]
$$

whereas the time required to produce an inhibition half that of the final inhibition would be given by:

$$
t_{0.5 i_{f}}=0.69 / \alpha
$$

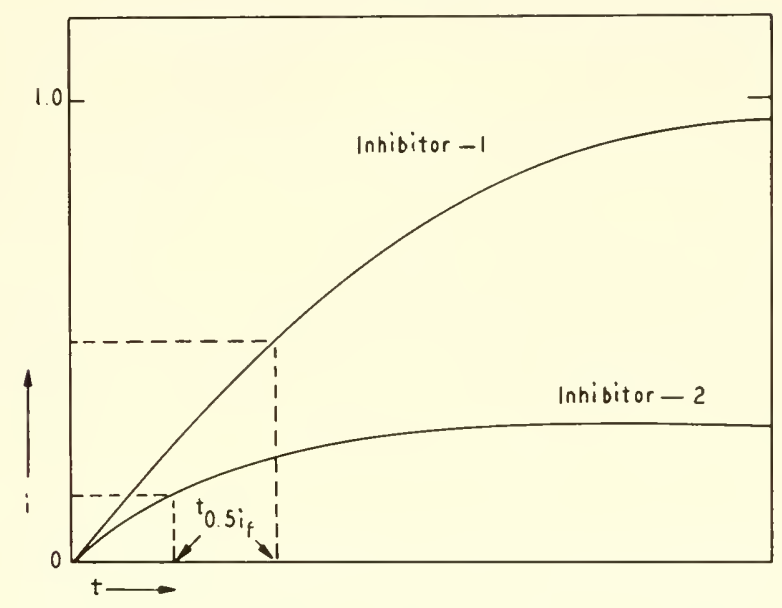

FIG. 12-4. Hypothetical inhibition curves for two inhibitors to illustrate the problems involved in comparing rates of inhibition.

In the former case, $t_{0.5}$ depends on the final inhibition $i_{f}$ but in the latter case it does not and hence Eq. 12-7 is a valid expression of the inhibition rate whatever the eventual inhibition or the concentration of the inhibitor. It is therefore suggested that it would be generally preferable to give the valnes of $t_{0.5 i_{f}}$ rather than $t_{0.5}$.

\section{RATES OF INHIBITION OF PURE ENZYMES}

The kinetic treatment of inhibition rates will vary with the type of inhibition. It will be convenient to start with the simplest situation in which a single inhibitor molecule combines with each enzyme molecule to inhibit noncompetitively. This reaction may be represented as:

$$
\mathrm{E}+\mathrm{I} \underset{k_{-1}}{\stackrel{k_{1}}{\rightleftharpoons}} \mathrm{EI}
$$


The rate of formation of the EI complex will be given by:

$$
\frac{d(\mathrm{EI})}{d t}=k_{1}(\mathrm{E})(\mathrm{I})-k_{-1}(\mathrm{EI})=k_{1}\left[\left(\mathrm{E}_{t}\right)-(\mathrm{EI})\right]-k_{-1}(\mathrm{EI})
$$

and since $i=(\mathrm{EI}) /\left(\mathrm{E}_{t}\right)$ and $K_{i}=k_{-1} / k_{1}$ :

$$
\frac{d i}{d t}=k_{1}(\mathrm{I})-i k_{1}\left[(1)+k_{i}\right]
$$

which upon integration leads to an expression showing the variation of the inhibition with time:

$$
\begin{aligned}
& i=\frac{(\mathrm{I})}{(1)+K_{i}}\left\{1-e^{-k_{1} t\left[(1)+K_{i}\right]}\right\} \\
& i=i_{f}\left\{1-e^{-k_{1} t\left[(1)+K_{i}\right]}\right\}
\end{aligned}
$$

Thus when $t=0$, the inhibition is zero, but the inhibition increases with time until it reaches the final equilibrium value, $i_{f}$. The rate of inhibition at any time may be found by substituting from Eq. 12-11 into Eq. 12-10)

$$
d i / d t=k_{1}(\mathrm{I}) e^{-k_{1} t\left(\langle 1)+K_{i}\right]}
$$

Equation 12-12 may be compared to the general Eq. 12-3, which was derived on the basis of no particular mechanism but only an exponential change, and it is foumd that:

$$
\alpha=k_{1}\left[(\mathrm{I})+K_{i}\right]
$$

The value of a may be found by the plotting procedures described in the previous section and from this the rate constant can be determined.

Equation 12-8 represents opposing reactions of the first and second orders and is applicable ideally over the whole course of the reaction. Occasionally, the dissociation of the complex has been neglected and the secondorder reaction alone considered:

$$
\mathrm{E}+\mathrm{I} \stackrel{k_{1}}{\rightarrow} \mathrm{EI}
$$

in which case it is easily shown that:

$$
i=1-e^{-k_{1}(\mathrm{I}) t}
$$

and that a plot of $\log (1-i)$ against $t$ will give a straight line of slope $-k_{1}(\mathrm{I}) / 2.3$. Such a treatment may be satisfactory when only the initial rates are measured but becomes progressively inaccurate with time; in fact, Eq. 12-16 implies that the inhibition will eventually become complete whatever the inhibitor concentration. 
The variation in the time course of inhibition with the rate constant is shown in Fig. 12-5 where Eq. 12-12 is plotted at constant inhibitor concentration, the final inhibition being the same in each case. The changes in the rate of inhibition with time are presented in Fig. 12-6 in which Eq. 12-13 is plotted. Finally, Fig. 12-7 shows the developments of inhibition when the concentration of the inhibitor is varied, the terminal inhibition now being different in every case. These curves serve to illustrate the typical behavior of a simple bimolecular inhibition reaction.

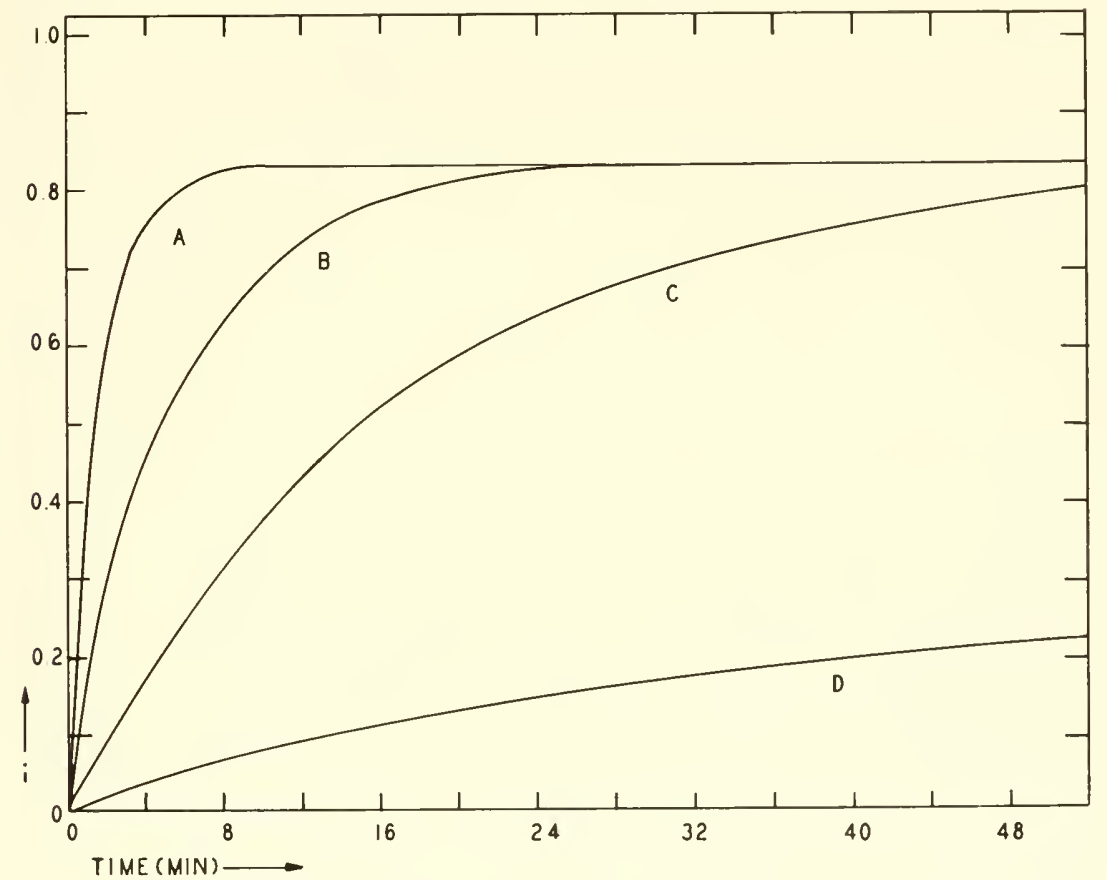

FIG. 12-5. Time courses of inhibition for different rate constants (Eq. 12-12). $K_{i}=1$ $\mathrm{m} . M$ and $(\mathrm{I})=5 \mathrm{~m} . \mu$. Curve $\mathrm{A}, k_{1}=100 \mathrm{~min}^{-1}$; curve $\mathrm{B}, k_{1}=30 \mathrm{~min}^{-1}$; curve C,

$$
k_{1}=10 \mathrm{~min}^{-1} \text {; curve } \mathrm{D}, k_{1}=1 \mathrm{~min}^{-1} \text {. }
$$

When the inhibition progresses slowly, measurements of enzymic activity are frequently made over intervals during which the inhibition is changing significantly. In such cases. an average value for the inhibition over the experimental period is obtained and we may now inquire into the relationship between this average inhibition and the inhibition at a particular time. The mean inhibition over the interval from $t_{1}$ to $t_{2}$ is given by:

$$
\bar{i}=\frac{t_{1}^{t_{2}} i d t}{t_{2}-t_{1}}
$$


and substitution of the value of $i$ from Eq. 12-11, followed by integration, gives:

$$
\bar{i}=i_{f}-\frac{i_{2}-i_{1}}{k_{1}\left(t_{2}-t_{1}\right)\left[(\mathrm{I})+K_{1}\right]}
$$

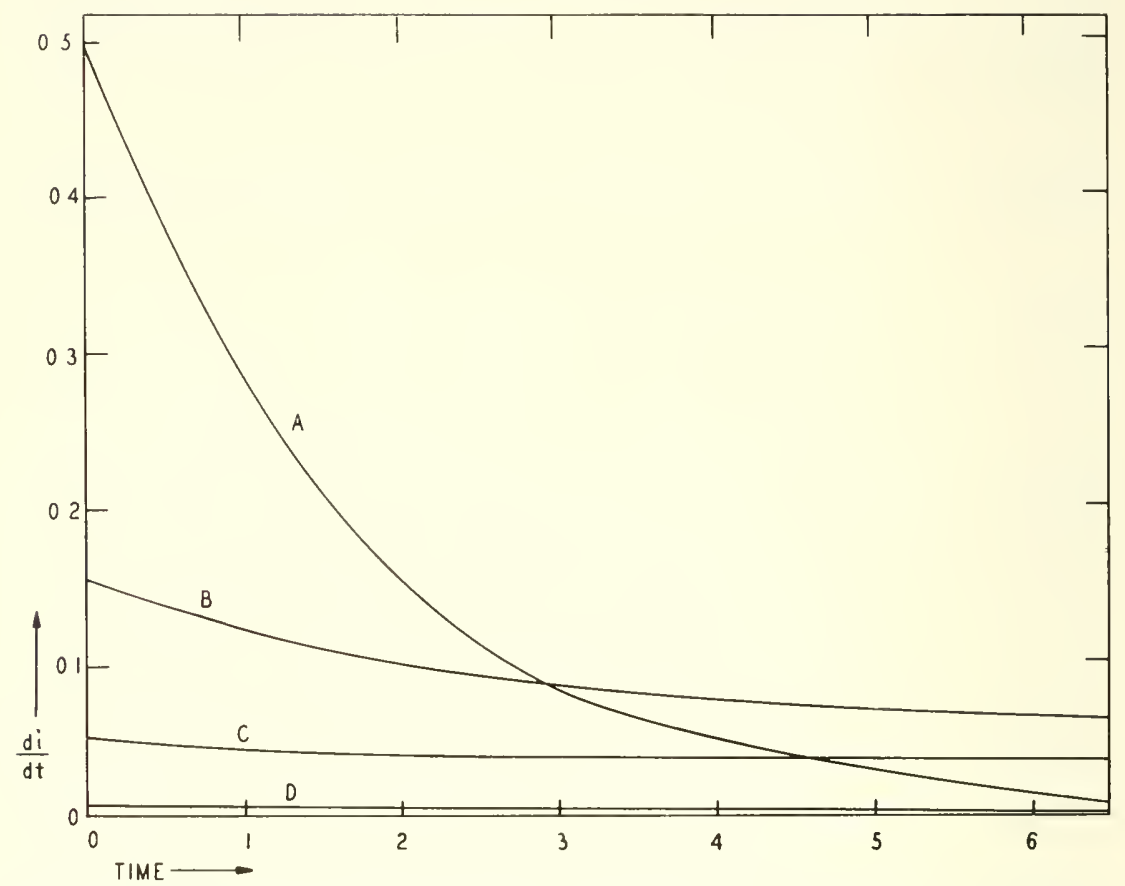

FIG. 12-6. Variation of the inhibition rate with time (Eq. 12-13) for the inhibitions in Fig. 12-5.

where $i_{1}$ is the inhibition at $t_{1}$ and $i_{2}$ is the inhibition at $t_{2}$. When the interval begins with the addition of the inhibitor $\left(t_{1}=i_{1}=0\right)$ and terminates at time $t$, the average inhibition is:

$$
\bar{i}=i_{f}-\frac{i_{t}}{k_{1} t\left[(1)+h_{1}\right]}
$$

where $i$ is the inhibition at the end of the interval. It is possible to calculate $i$, when the average inhibition, the final inhibition, and the slope of the logarithmic plot are known, since:

$$
i_{t}=\alpha t\left(i_{f}-\bar{i}\right)
$$

The experimentally determined average inhibition $\bar{i}$ itself is rather meaning- 
less kinetically and in the construction of inhibition-time curves it is important that the inhibitions at specific times be determined.

Several enzyme inhibitions following an approximately exponential course have been reported. In these cases, the reaction is bimolecular but the kinetics are first order because the inhibitor is much in excess of the enzyme and its concentration does not change appreciably during the reaction.

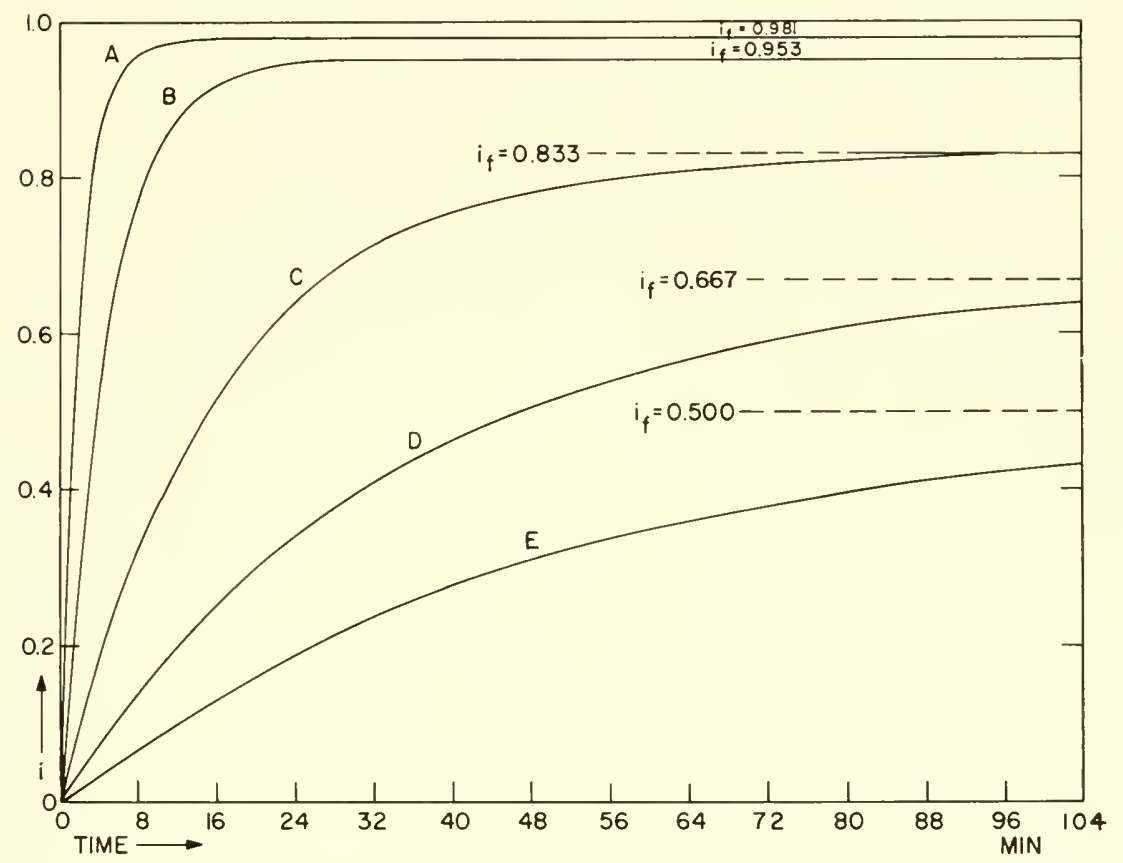

FIG. 12-7. Time courses of inhibition for different concentrations of the inhibitor (Eq. 12-11). $K_{i}=1 \mathrm{~m} M$ and $k_{1}=10 \mathrm{~min}^{-1}$. Curve $\mathrm{A},(\mathrm{I})=50 \mathrm{~m} . /$; curve $\mathrm{B},(\mathrm{I})=$

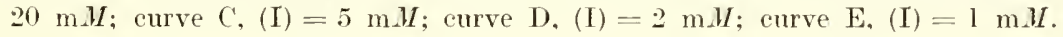

The inhibition of plasma cholinesterase by $\mathrm{HgCl}_{2}$ was studied kinetically by Goldstein and Doherty (1951) and curres such as the one shown in Fig. 12-8 were obtained. A logarithmic plot of this curve gives a reasonably straight line over the initial $60 \mathrm{~min}$ (Fig. 12-9) from which the following values may be estimated: slope $=0.007 \mathrm{~min}^{-1}, \alpha=0.016 \mathrm{~min}^{-1}$, and $k_{1}=$ 1.15 liter's mole $\mathrm{sec}^{-1}$. At higher concentrations of $\mathrm{HgCl}_{2}$ (over $1 \mathrm{~m} . \mathrm{H}$ ), the behavior of the inhibition deviates from this simple mechanism and the logarithmic plots are no longer straight lines. Another example that may be mentioned is the inhibition of monoamine oxidase by iproniazid for which Davison (1957) found an apparent first-order reaction as indicat $\mathrm{d}$ by the logarithmic plots in Fig. 12-10 for three concentrations of the 
inh bitor. For the two lower concentrations, $k_{1}$ may be estimated as approximately 0.094 liters mole ${ }^{-1} \mathrm{sec}^{-1}$ but at the higher concentration of $25 \mathrm{~m} M$, the rate is less than half what would be expeeted. Such a deviation at high inhibitor concentrations is often observed and sometimes it may be due to the fact that the EI complex is not formed directly upon collision of the inhibitor molecule with the active enzyme site but requires a further reaction of some type, which may limit the rate at high eoncentrations of the inhibitor.

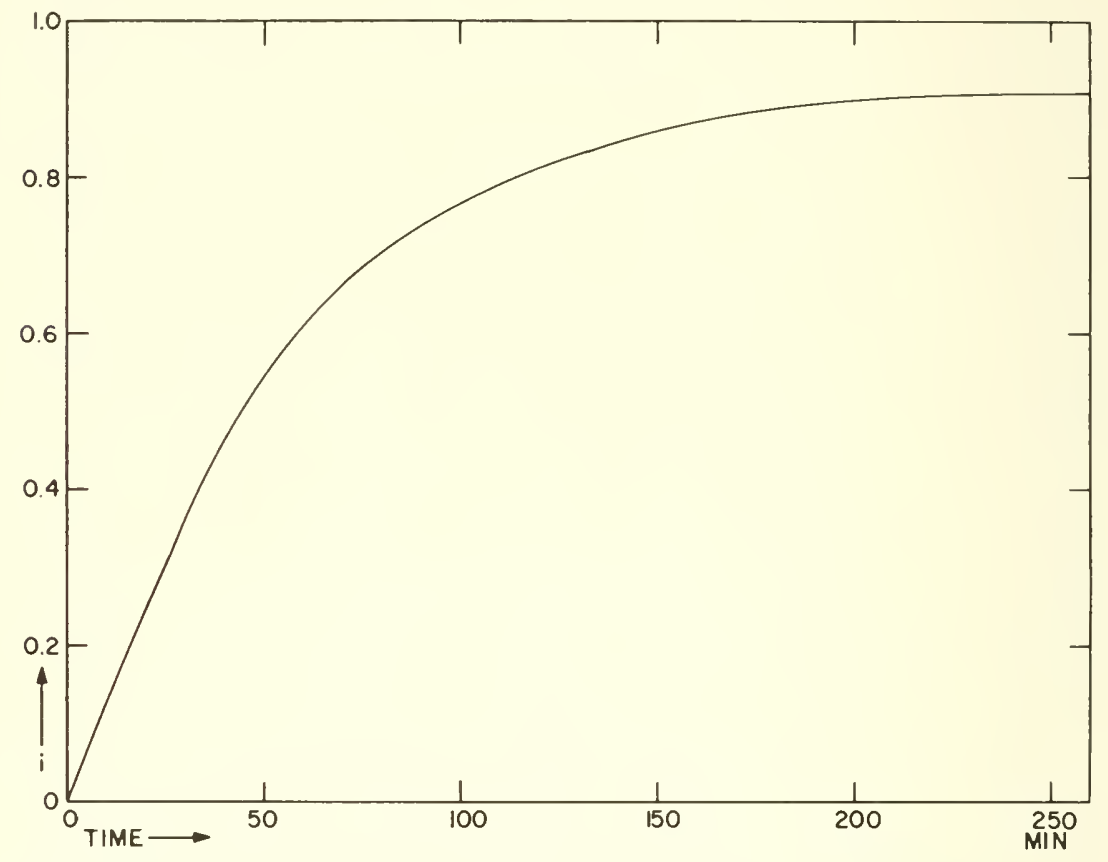

FIG. 12-8. Inhibition of plasma cholinesterase by $\mathrm{HgCl}_{2}(0.2 \cdot 27 \mathrm{~m} . \mathrm{H})$ with time. (From Goldstein and Doherty, 19.51.)

The inhibition of lactate dehydrogenase by $p$-chloromereuribenzoate ( $p$-CMB) presents an interesting sitnation hecause the reaction of the inhibitor with the enzyme can be followed spectrophotometrically by measuring the ehanges in the optical density at $250 \mathrm{~m} \mu$ as the sulfhydryl groups are combined (Neilands, 1954). The time courses of the sulfhydryl reaction and the inhibition are compared in Fig. 12-11 and, although the concentrations of $p$-CIIB were different in the two experiments. comparable rates are evident. The rate of the reaction of $p$-CMB with lactate dehydrogenase is much slower than with most other snsceptible enzymes indicating that the sulfhydryl groups necessily for activity are partially protected in some 
manner. This is also apparent from the fact that iodoacetate, 0 -iodosobenzoate, and $\mathrm{N}$-ethylmaleimide do not inhibit the lactate dehydrogenase. It was tentatively concluded that two sulfhydryl groups per dehydrogenase molecule were reacted with $p$-CIIB after $30 \mathrm{~min}$ of incubation.

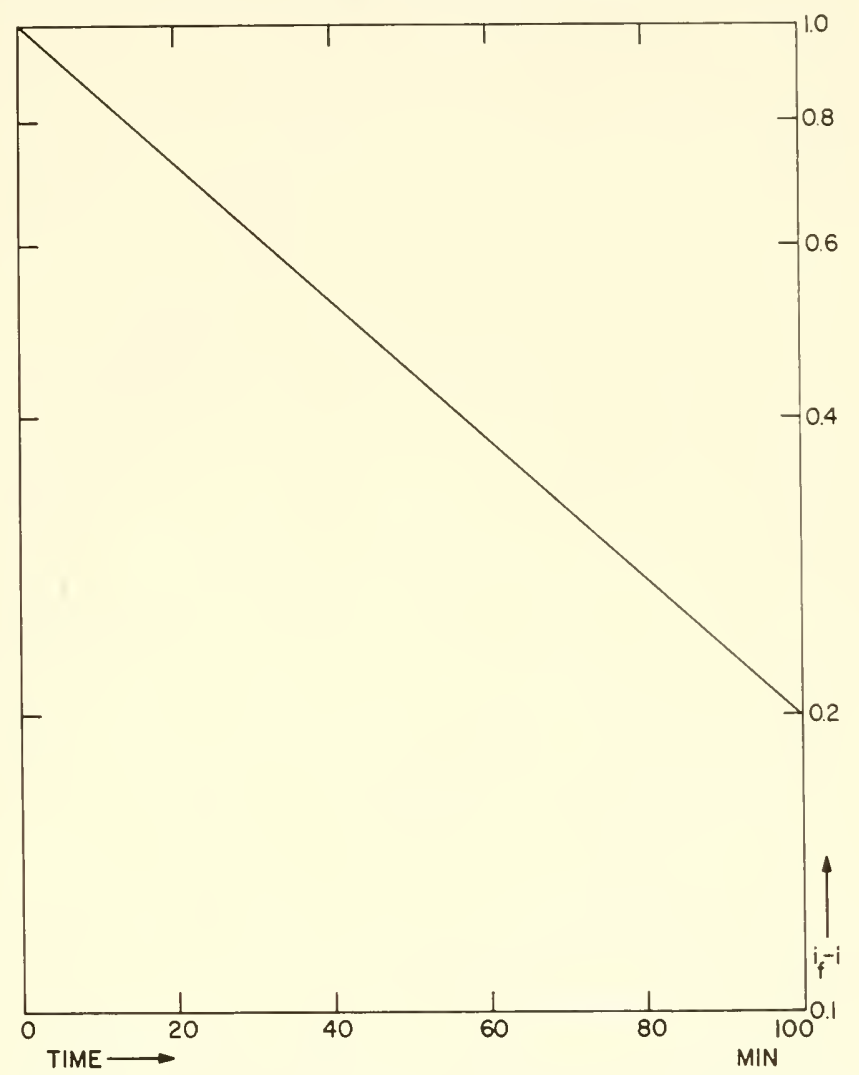

FIG. 12-9. Logarithmic plot of the inhibition of plasma cholinesterase by $\mathrm{HgCl}_{2}$ for the determination of $\alpha$ and $k_{1}$.

A comparison of the rates of inhibition of succinoxidase by a variety of sulfhydryl reagents was made by Slater (1919) and the results are shown in Fig. 12-12. The striking difference between $p$-CMIB and the other inhibibitors should be noted. The reaction of $p$-CIBB with the enzyme was practically instantaneous. whereas the other inhibitors required 30 to $60 \mathrm{~min}$ to reach maximal inhibition. The two substances inhibiting by oxidation of the sulfhyrlryl groups (o-iodosobenzoate and oxidized glutathione) produced their effects particularly slowly. The rate of inhibition with $o$-iodosobenzoate at $16^{\circ}$ (Fig. 12-13) exhibited two phases, a rapid one complete 
after $10 \mathrm{~min}$ and a slower one that was incomplete after $2 \mathrm{~h}$. One might postulate that the rapid reaction involves the oxidation of sulfhydryl groups near, but not at, the active site, and that the slow reaction represents a progressive inactivation or denaturation of the enzyme due to the structural alteration resulting from the formation of $-\mathrm{S}-\mathrm{S}-$ bonds. Such secondary inactivation of enzymes will be discussed in a subsequent section.

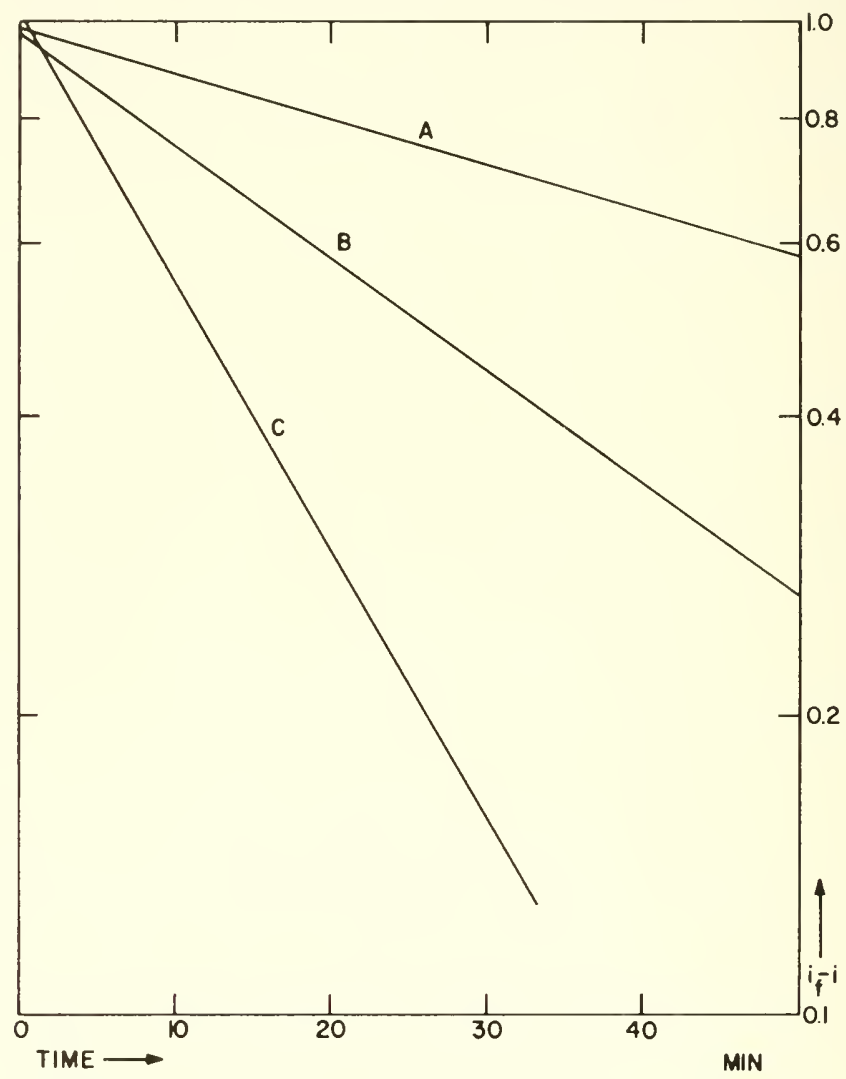

FIG. 12-10. Inhibition of monoamine oxidase by iproniazid plotted logarithmically. (From Davison, 1957). Curve A: $(\mathrm{I})=$ $1.65 \mathrm{~m} H$; curve $\mathrm{B},(\mathrm{I})=5 \mathrm{~m} . I$; curve $\mathrm{C},(\mathrm{I})=25 \mathrm{~m} H$.

\section{Mutual Depletion Systems}

When the concentrations of both enzyme and inhibitor are reduced during the reaction hetween them, not only the final inhibition but also the rate at which this is attained is altered compared to the situation in which the inhibitor is markedly in excess of the enzyme. The initial rate of inhi- 


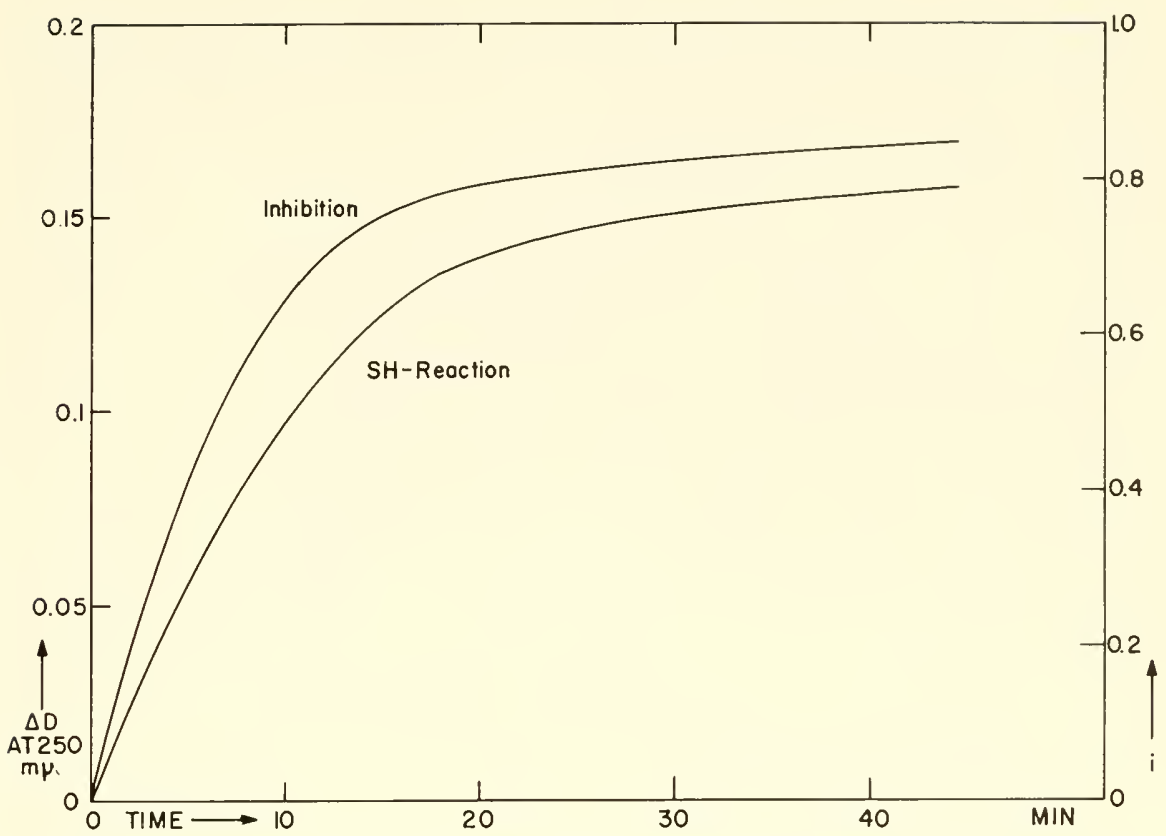

FIG. 12-11. Reaction of lactate dehydrogenase sulfhydryl groups with $p$-chloromercuribenzoate ( $p$-CMB) measured spcetrophotometrically and the time course of the inhibition. For the sulfhydryl reaction, $(p-\mathrm{Cl} \mathrm{B})=0.033 \mathrm{m.} / \mathrm{and}(\mathrm{LDH})=0.01 \mathrm{~m} . \mathrm{I}$; for the inhibition, $(p-\mathrm{CHB})=0.1 \mathrm{~m}, 1 /$. (From Neilands, 1954.)

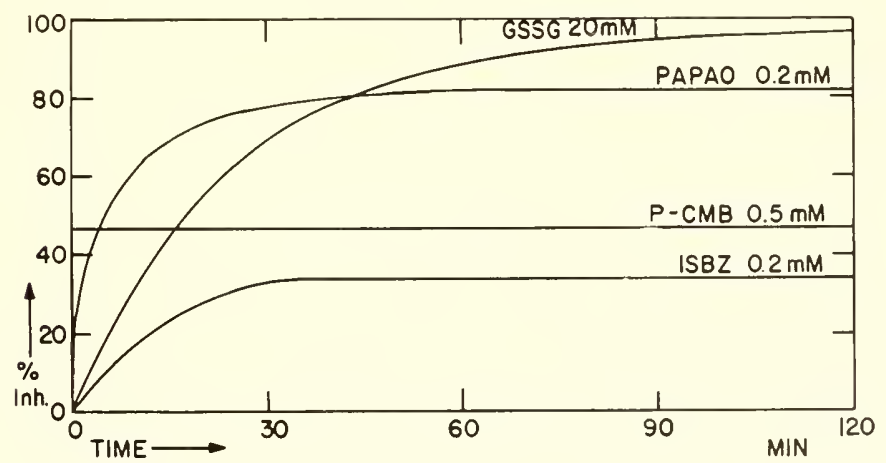

Fig. 12-12. Inhibition of snceinoxidase by several sulfhydryl reagents. GSSG $=$ oxidized glutathione. $\mathrm{PAPAO}=p$-aminophenylarsine oxide, $p$-CMB $=p$-chloromercuribenzoate, and $\mathrm{ISBZ}=$ iodosobenzoate. Temperature $=37^{\circ} .($ From Slater, 1949.) 
bition will depend only on the inhibitor concentration and not on the concentration of the enzyme, but it is obvious that if the enzyme concentration is near that of the inhibitor, the later rates of inhibition will be slower due to the fall in inhibitor concentration. An irreversible mutual depletion reaction may represented as:

$$
\mathrm{E}+\mathrm{l} \stackrel{k_{1}}{\rightarrow} \mathrm{EI}
$$

so that:

$$
\frac{d(\mathrm{EI})}{d t}=k_{1}\left[\left(\mathrm{E}_{t}\right)-(\mathrm{EI})\right]\left[\left(\mathrm{I}_{t}\right)-(\mathrm{EI})\right]
$$

and:

$$
\frac{d i}{d t}=k_{1}\left(\mathrm{I}_{t}\right)-i k_{1}\left[\left(\mathrm{E}_{t}\right)+\left(\mathrm{I}_{t}\right)\right]+i^{2} k_{1}\left(\mathrm{E}_{t}\right)
$$

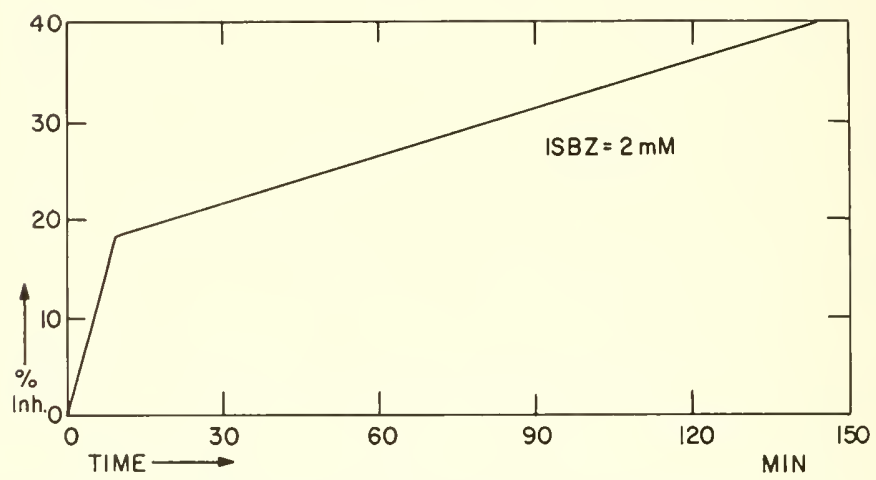

FIG. 12-13. Inhibition of succinoxidase by iodosobenzoate at $16^{\circ}$ and a concentration of $2 \mathrm{~m} \nu$. (From Slater, 1949.)

Integration of this equation leads to:

$$
i=\frac{1-e^{-k_{1}\left[\left(1_{t}\right)-\left(\mathrm{E}_{t}\right)\right]}}{1-\frac{\left(\mathrm{E}_{t}\right)}{\left(\mathrm{I}_{t}\right)} e^{-k_{1}\left[\left(1_{t}\right)-\left(\mathrm{E}_{t}\right)\right]}}
$$

which gives the variation in the inhibition with time for any initial concentrations of enzyme and inhibitor. It may be noted that when $\left(I_{t}\right) \gg$ $\left(\mathrm{E}_{\ell}\right)$, this reduces to the simple expression, $i=1-e^{-k_{1} t\left(\mathrm{I}_{t}\right)}$, which is Eq. 12-16. The changes of inbibition with time for such systems are shown in Fig. 12-14 by plotting Eq. 12-24 for constant concentrations of inhibitor and variable concentrations of enzyme. Curve 1 represents the ordinary logarithmic progression of inhibition when the inhibitor is much in excess of the enzyme and significant depletion of inhibitor does not occur. The other curves show the results of increasing enzyme concentration to levels 
comparable to the inhibitor concentration. In mutual depletion systems, the logarithmic plots of the remaining activity against time deviate from straight lines in a manner shown in Fig. 12-15. Such curvatures are frequently seen in plotting experimental data and in certain cases may be due to the factor of inhibitor depletion. It must be remembered that in enzyme preparations containing nonenzymic material capable of binding the inhibitor. depletion of the inhibitor will occur more readily than in pure enzyme solutions and this can give rise to deviations of this type.

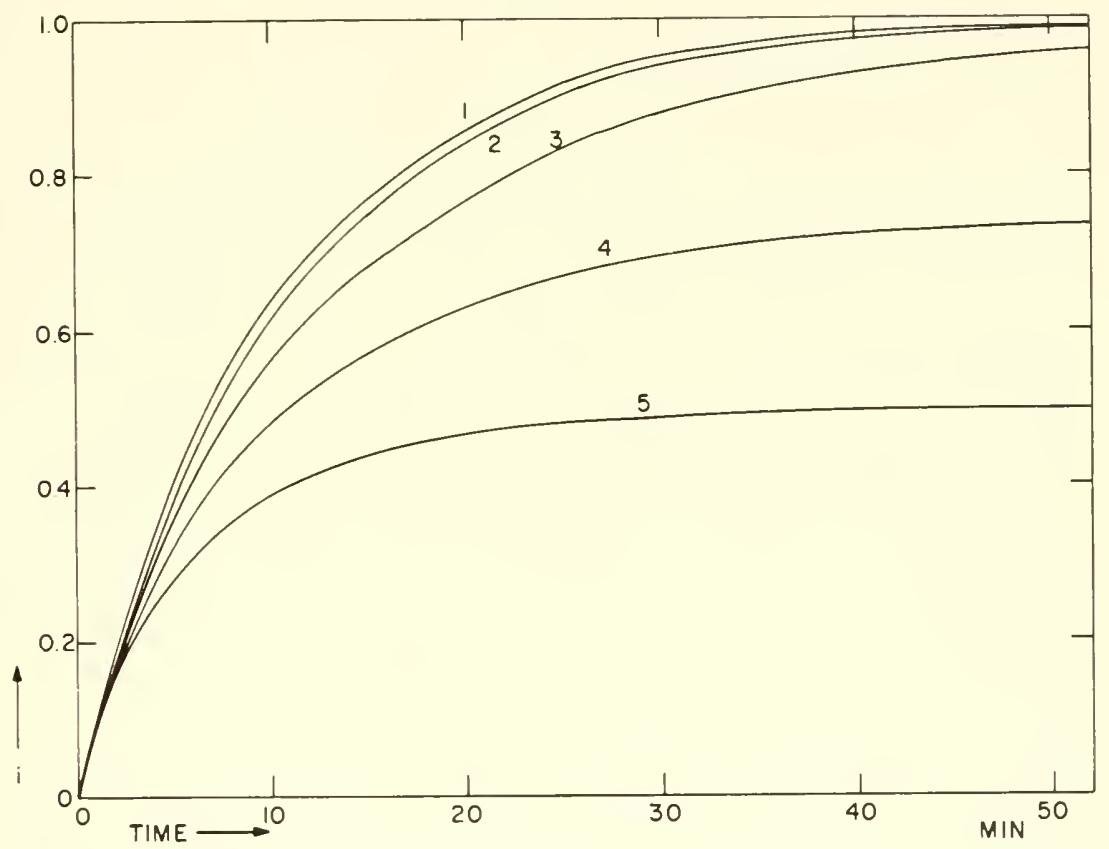

FIG. 12-14. Effect of the enzyme concentration on the rate of inhibition in a mutual depletion system (Eq. 12-24). $\left(\mathrm{I}_{t}\right)=1 \mathrm{mH}$ and $k_{i}=100 \mathrm{~min}^{-1}$. Curve $1,\left(\mathrm{E}_{t}\right)=$ $0.01 \mathrm{~m} H$; curre $2,\left(\mathrm{E}_{t}\right)=0.1 \mathrm{~m} .1$; curve $3,\left(\mathrm{E}_{t}\right)=0.5 \mathrm{~m} . \mathrm{H}$; curve $4 .\left(\mathrm{E}_{t}\right)=1.6 \mathrm{~m} . \mathrm{H}$; curve $5,\left(\mathrm{E}_{t}\right)=\underline{2} \mathrm{~m} . \boldsymbol{H}$.

An irreversible inhibition has been discussed hecanse the kinetics of a reversible mutual depletion system. $\mathrm{E}+\mathrm{I} \rightleftharpoons \mathrm{EI}$, are rather complex. The rate equations:

$$
\begin{aligned}
\frac{d(\mathrm{EI})}{d t} & =k_{1}\left\lceil\left(\mathrm{E}_{t}\right)-(\mathrm{E} \mathrm{I})\right]\left[\left(\mathrm{I}_{t}\right)-(\mathrm{EI})\right]-k_{-1}(\mathrm{EI}) \\
\frac{d i}{d t} & =k_{1}\left(\mathrm{I}_{t}\right)-i k_{1}\left[\left(\mathrm{E}_{t}\right)+\left(\mathrm{I}_{t}\right)+K_{i}\right]+i^{2} k_{1}\left(\mathrm{E}_{t}\right)
\end{aligned}
$$


are integratable but the resultant expressions are unwieldy. However, it is evident that the rate of inhibition will progressively become even less than the irreversible mutual depletion system and the final equilibrium level of inhibition achieved will be less.

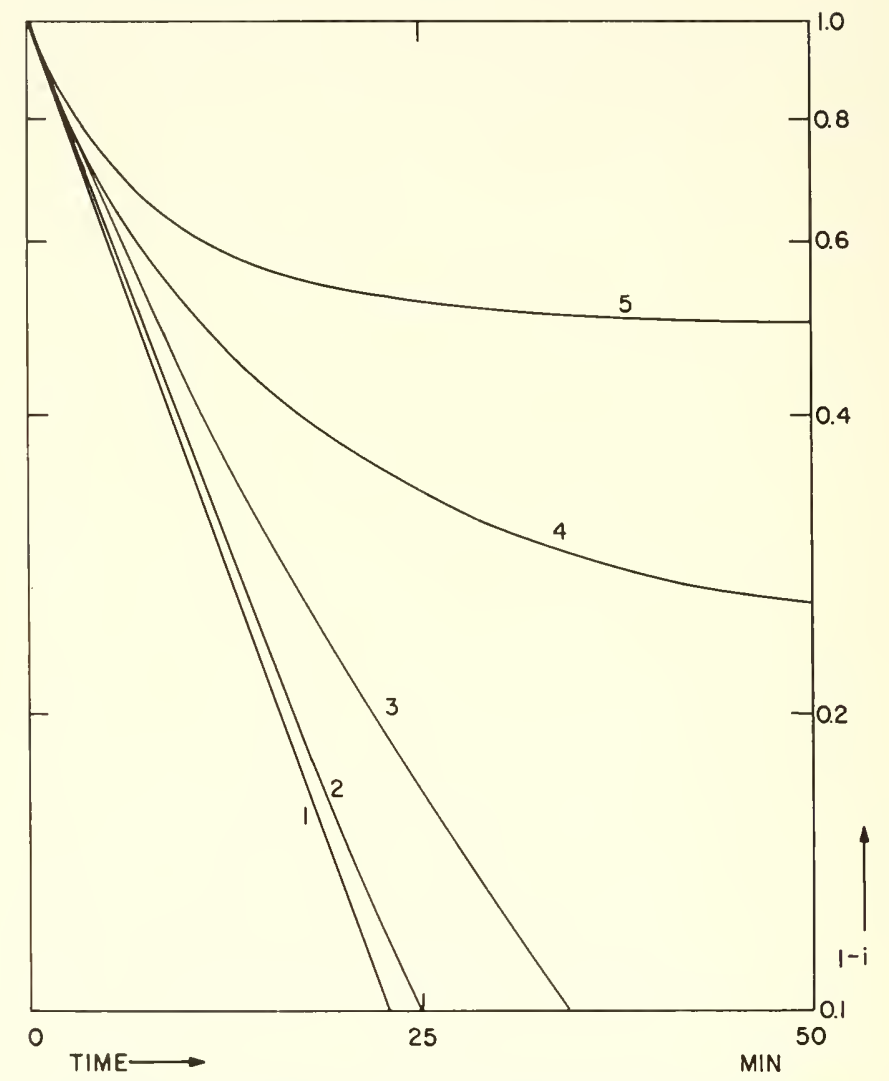

FrG. 12-15. Logarithmic plots of inhibition curves in mutual depletion systems. The curves eorrespond to those in Fig. 12.14.

\section{Rate of Competitive Inhibition}

When the inhibitor and the substrate compete for the active site on the enzyme, the presence of substrate will reduce the rate at which inhibition occurs, as well as decrease the equilibrimm inhibition reached. In such cases, the bound substrate prevents access of the inhibitor to the active site. Thus only that fraction of the total enzyme concentration that represents the free enzyme will be available for reaction with the inhibitor. Of course, the ES complex will dissociate into $\mathrm{E}$ and $\mathrm{S}$ as the reaction 
of $\mathrm{E}$ with I proceeds. It has been said that the inhibitor displaces the substrate from the enzyme, which is what does occur when one considers initial and final states, but this must not be interpreted as a direct displacement, since it is unlikely than an inhibitor molecule could force a substrate molecule from the enzyme surface in most cases.

It might be thought that an expression for the rate of competitive inhibition could be simply derived from that for noncompetitive inhibition (Eq. 12-11) by multiplying $K_{i}$ by the factor $\left[\mathrm{I}+(\mathrm{S}) / K_{s}\right]$, which suffices for the final inhibition, but this is not so. inasmuch as (ES) decreases as (EI) increases and both $d(\mathrm{ES}) / d t$ and $d(\mathrm{EI}) / d t$ must be taken into account. Goldstein (1944) obtained an equation for the development of inhibition in the presence of substrate and applied it to the inhibition of cholinesterase by physostigmine. This treatment will be followed here but modified so that the results may be expressed in terms of inhibition instead of activity. There are now two simultaneous reactions:

$$
\begin{aligned}
& \mathrm{E}+\mathrm{S} \underset{k_{-2}}{\stackrel{k_{2}}{\rightleftharpoons}} \mathrm{ES} \\
& \mathrm{E}+\mathrm{I} \underset{k_{-1}}{\stackrel{k_{1}}{\rightleftharpoons}} \mathrm{EI}
\end{aligned}
$$

where $k_{1}$ and $k_{-1}$ have been retained for the inhibitor reaction to conform to the previous equations. The changes in the concentrations of the complexes would be:

$$
\begin{aligned}
& \frac{d(\mathrm{ES})}{d t}=k_{2}(\mathrm{~S})\left[\left(\mathrm{E}_{t}\right)-(\mathrm{ES})-(\mathrm{EI})\right]-k_{2} K_{s}(\mathrm{ES}) \\
& \frac{d(\mathrm{EI})}{d t}=k_{1}(\mathrm{I})\left[\left(\mathrm{E}_{t}\right)-(\mathrm{ES})-(\mathrm{EI})\right]-k_{1} K_{i}(\mathrm{EI})
\end{aligned}
$$

Eliminating $\left[\left(\mathrm{E}_{t}\right)-(\mathrm{ES})-(\mathrm{EI})\right]$ from these equations:

$$
\frac{d(\mathrm{ES})}{d t}=\frac{k_{2}(\mathrm{~S})}{k_{1}(\mathrm{I})}\left[\frac{d(\mathrm{EI})}{d t}+k_{1} K_{i}(\mathrm{EI})\right]-k_{2} K_{s}(\mathrm{ES})
$$

Since $i=(\mathrm{EI}) /\left(\mathrm{E}_{t}\right)$ and $a=(\mathrm{ES}) /\left(\mathrm{E}_{\ell}\right)=\mathrm{I}-i$, we may write:

$$
\frac{d(\mathrm{EI})}{d t}=\left(\mathrm{E}_{t}\right) \frac{d i}{d t} \quad \text { and } \quad \frac{d(\mathrm{ES})}{d t}=-\left(\mathrm{E}_{t}\right) \frac{d i}{d t}
$$

and hence obtain an equation for di/dt:

$$
\frac{d i}{d t}=\frac{k_{1} k_{2}(\mathrm{I}) K_{s}}{k_{1}(\mathrm{I})+k_{2}(\mathrm{~S})}-i k_{1} k_{2}\left[\frac{(\mathrm{I}) K_{s}+(\mathrm{S}) K_{i}}{k_{1}(\mathrm{I})+k_{2}(\mathrm{~S})}\right]
$$


It should be noted at this point that Eqs. 12-32 limit the treatment to situations in which the enzyme is saturated with substrate so that the total enzyme concentration, $\left(\mathrm{E}_{t}\right)$, is approximately $(\mathrm{ES})+(\mathrm{EI})$, the concentration of free enzyme being very small. This arises from setting $a=$ $(\mathrm{ES}) /\left(\mathrm{E}_{t}\right)=1-i$, which is true only when the enzyme reaction in the absence of inhibitor is proceeding at the maximal rate. This limitation of the treatment was not specifically stated by Goldstein. In his experimental study of cholinesterase inhibition, the substrate concentration was very high, $\left(S^{\prime}\right)$ being approximately 65 , so that the behavior should be adequately represented by this treatment. However, in cases where the enzyme is not saturated with substrate, and $d(\mathrm{ES}) / d t \neq-d(\mathrm{EI}) / d t$, the situation is more complex and the equations are difficult to solve directly.

Equation 12-33 may now be integrated and the following expression for the inhibition is obtained:

$$
i=\frac{(\mathrm{I})}{(\mathrm{I})+\frac{K_{i}}{K_{s}}(\mathrm{~S})}\left\{1-\exp \left[-\frac{k_{1} k_{2}\left[(\mathrm{I}) K_{s}+(\mathrm{S}) K_{i}\right]}{k_{1}(\mathrm{I})+k_{2}(\mathrm{~S})} t\right]\right\}
$$

This is plotted and compared with the noncompetitive rate in Fig. 12-16 for $\left(S^{\prime}\right)=5$ and the marked decrease in rate may be observed. It is true that Eq. 12-34 would not accurately apply to as low a specific concentration of substrate, but the error is not very great. The logarithmic plots of these curves are shown in Fig. 12-17. The slope of the curve for competitive inhibition is the factor multiplied by the time in the exponential part of Eq. 12-34, and it may be seen that values of $k_{1}$ and $k_{2}$ can be calculated from the slopes obtained under different conditions. The data reported by Goldstein (1944) for the inhibition of dog serum cholinesterase by physostigmine are shown in Fig. 12-18 and the curves have been derived from Eq. 12-34 using the constants calculated by Goldstein, except that a slightly lower value of $K_{i}$ was chosen to obtain a closer fit to the experimental points. As was pointed out by Goldstein, the rate of inhibition here in the absence of the substrate would have been too rapid to measure but in the presence of the large amounts of substrate used, the rate was slowed so that it could be easily determined. Thus by this technique it is possible to determine the rate constants of a reaction normally proceeding at an experimentally unmeasurable rate.

With respect to the development of competitive inhibition in such systems, one may conceive of two extreme types. In one case, the substrate rapidly dissociates from the enzyme and the limiting rate of inhibition is that at which the inhibitor reacts with the free enzyme; in the other case, the ES complex dissociates more slowly than the EI complex is formed and thus limits the rate of inhibition. Equation 12-34 
may be rewritten in terms of $k_{-2}$, the rate constant for the dissociation of the ES complex:

$$
i=\frac{\left(\mathrm{I}^{\prime}\right)}{\left(\mathrm{I}^{\prime}\right)+\left(\mathrm{S}^{\prime}\right)}\left\{1-\exp \left[-\frac{k_{1} k_{-2}\left[(\mathrm{I})+\left(\mathrm{S}^{\prime}\right) K_{i}\right]}{k_{1}(\mathrm{I})+k_{-2}\left(\mathrm{~S}^{\prime}\right)} t\right]\right\}
$$

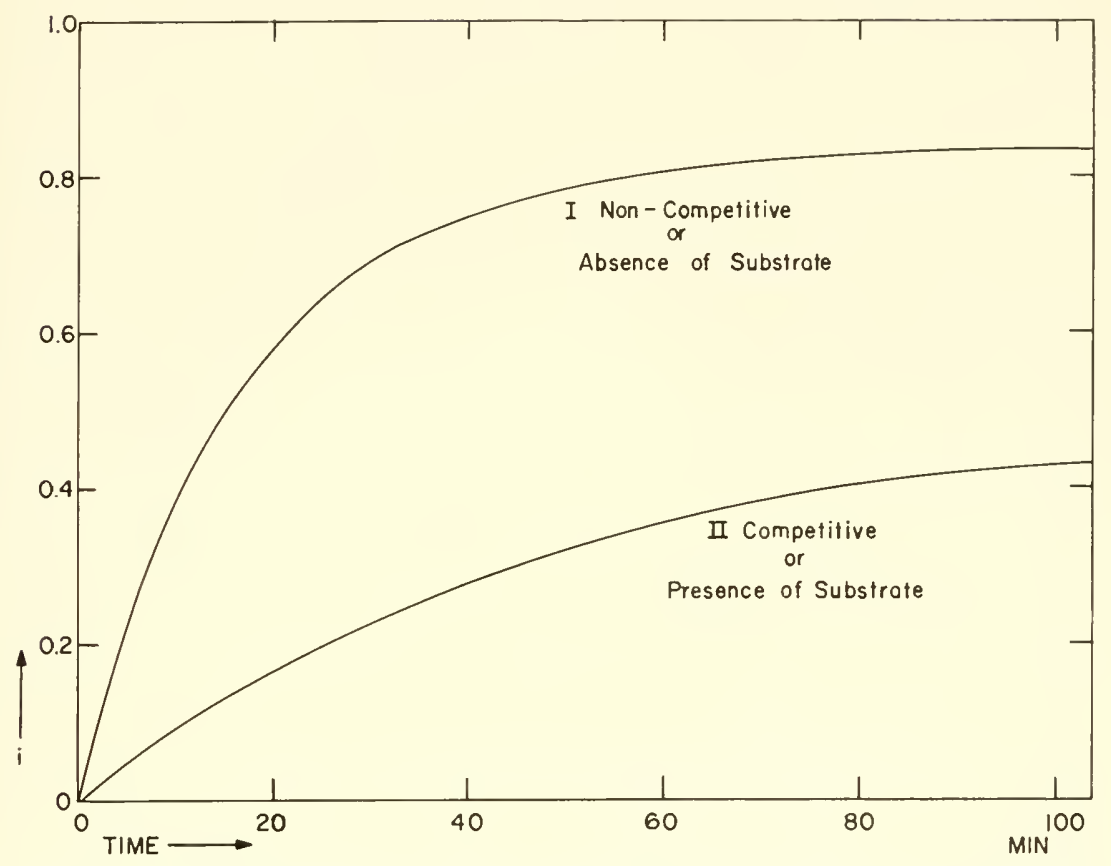

FIG. 12-16. The rate curves for noncompetitive (I) and competitive (II) inhibition. $K_{i}=1 \mathrm{~m} H, k_{1}=10 \mathrm{~min}^{-1}$, and (I) $=5 \mathrm{~m} . \mu$. For the competitive inhibition, Eq. 12-34 is plotted with $K_{s}=2 \mathrm{~m} M$ and (S) $=10 \mathrm{~m} M$. Curve II shows the delaying effect of substrate on the development of inhibition.

The following two situations may be considered:

$$
\begin{aligned}
& k_{1}(\mathrm{I}) \gg k_{-2}\left(\mathrm{~S}^{\prime}\right) \quad i=\frac{\left(\mathrm{I}^{\prime}\right)}{\left(\mathrm{I}^{\prime}\right)+\left(\mathrm{S}^{\prime}\right)}\left\{1-\exp \left[-k_{-2}\left[1+\frac{\left(\mathrm{S}^{\prime}\right)}{\left(\mathrm{I}^{\prime}\right)}\right] t\right]\right\} \\
& k_{-2}\left(\mathrm{~S}^{\prime}\right) \gg k_{1}(\mathrm{I}) \quad i=\frac{\left(\mathrm{I}^{\prime}\right)}{\left(\mathrm{I}^{\prime}\right)+\left(\mathrm{S}^{\prime}\right)}\left\{1-\exp \left[-k_{1}\left[K_{i}+\frac{(\mathrm{I})}{\left(\mathrm{S}^{\prime}\right)}\right] t\right]\right\}
\end{aligned}
$$

Since $\left(\mathrm{S}^{\prime}\right)>(\mathrm{I})$, the value of $k_{1}$ must be much greater than $k_{-2}$ for the first situation to hold, whereas for the second situation the rate constants do not have to differ appreciably. Corresponding to the two types of processes discussed above, in the first case the rate of inhibition depends only on the 
rate constant for dissociation of the ES complex (ES dissociates slowly) and in the second case it depends only on the rate constant for the combination of the inhibitor with the enzyme (ES dissociates rapidly). In the cholinesterase inhibition studies of Goldstein, $k_{1}(\mathrm{I})=0.913$ and $k_{-2}\left(\mathrm{~S}^{\prime}\right)=$ 20.8, so that Eq. 12-37 would be applicable without serious error. The rate

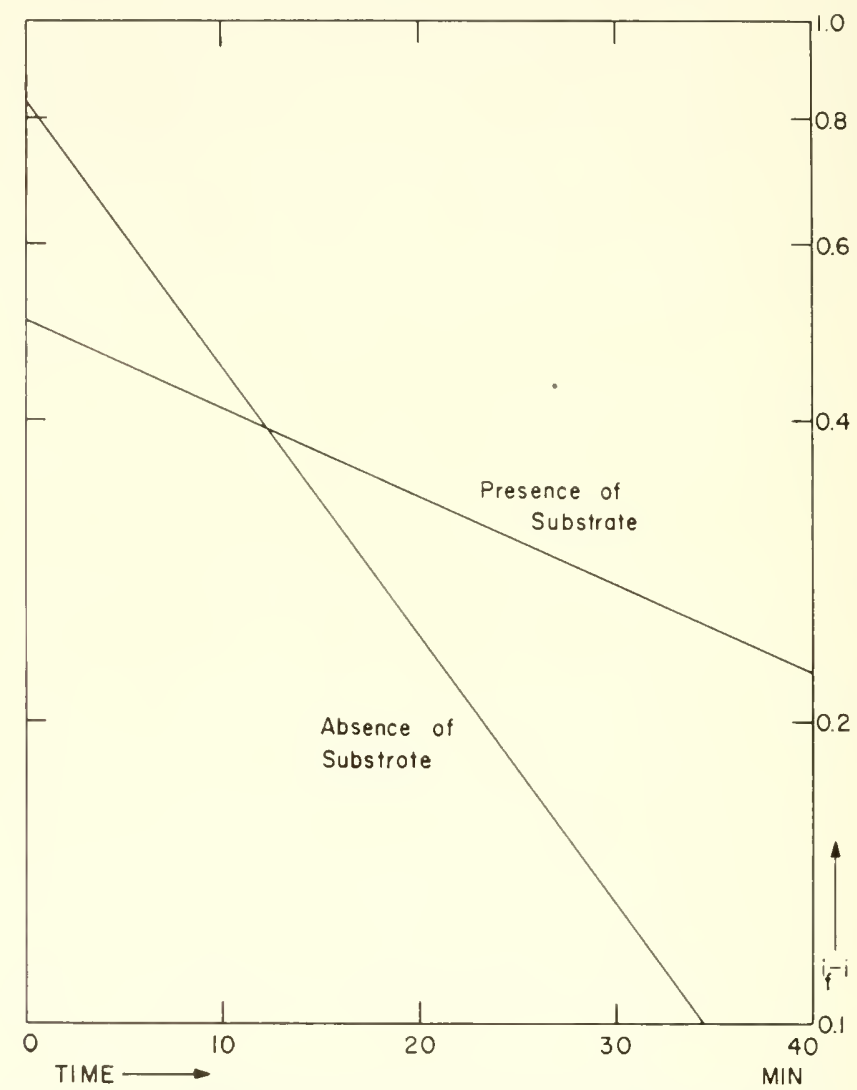

FIG. 12-17. Logarithmic plots of the rate eurves for noncompetitive and competitive inhibition (or for competitive inhibition in the presence and absence of the substrate). Same conditions as in Fig. 12-16.

of inhibition in this case is markedly slowed by the substrate because there is so little free enzyme arailable and not becanse the dissociation of the ES complex is limiting the rate.

Attention must be called to the fact that in Eqs. 12-35 to 12-37, the inhibition approaches the value $\left(I^{\prime}\right) /\left[\left(I^{\prime}\right)+\left(S^{\prime}\right)\right]$ as a final equilibrium state. This shonld actually be $\left(\mathrm{I}^{\prime}\right) /\left[\left(\mathrm{I}^{\prime}\right)+\left(\mathrm{S}^{\prime}\right)+1\right]$ (see Eq. $\left.3-28\right)$. The reason 
for this discrepancy is the assmmption discussed above, namely that the enzyme is either in the form of ES or EI. When this assumption is valid. as it is in the inhilition of cholinesterase under the conditions imposed by Goldstein, (S') must be quite large and much greater than unity, so that the error will be insignificant. However, it is worth pointing out again that when $\left(\mathrm{I}^{\prime}\right)+\left(\mathrm{S}^{\prime}\right)$ is not much larger than unity, these equations will be inaccurate with respect to both the rate of inhibition and the final inhibition that is reached.

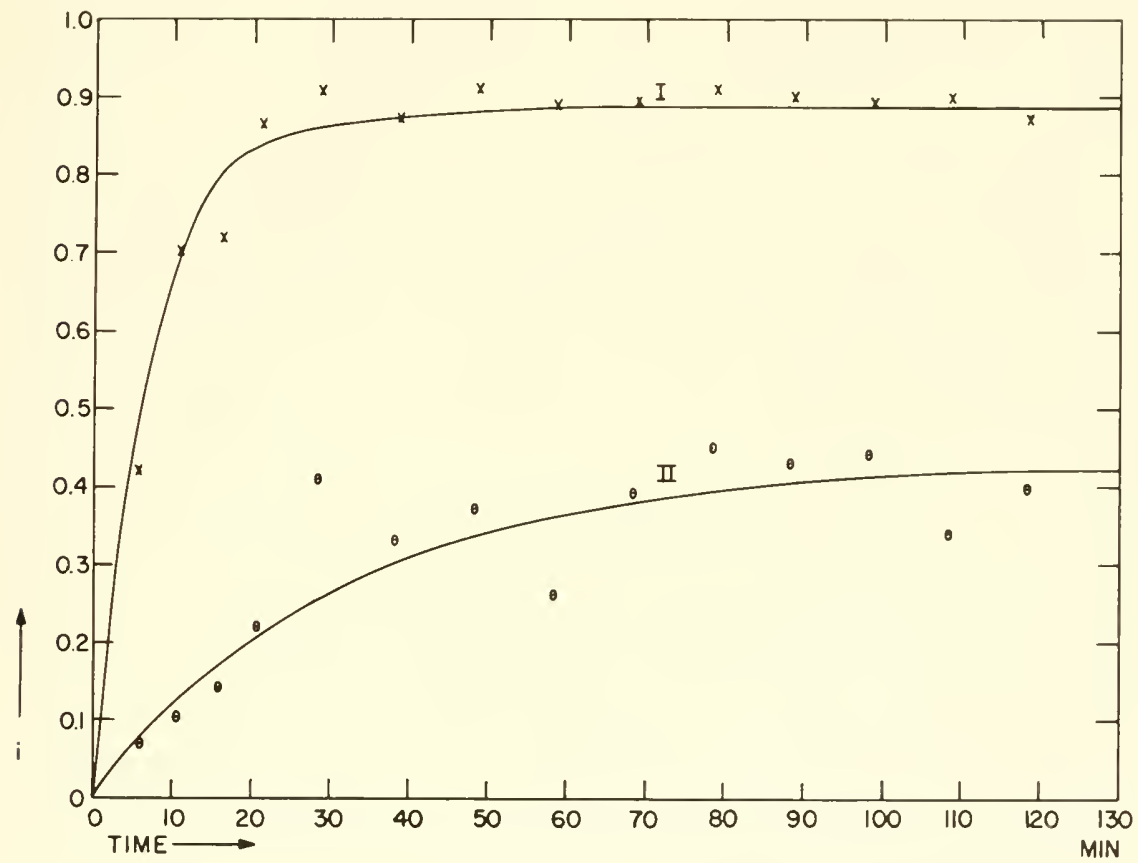

FIG. 12-18. Rate of inhibition of serum cholinesterase by physostigmine. The points represent the data of Goldstcin (1944) and the curves are plotted from Eq. 12-34: $K_{i}=1.9 \times 10^{-8} \mathrm{H}, K_{s}=1.23 \times 10^{-3} \mathrm{H},(\mathrm{S})=8.05 \times 10^{-2} \mathrm{H}$, and $k_{1}=8.3 \times 10^{5}$ liters mole $\mathrm{min}^{-1}$. Curve $\mathrm{I},(\mathrm{I})=1.1 \times 10^{-5} \mathrm{II}$; curve II, (I) $=1.1 \times 10^{-6} \mathrm{M}$.

Kinetic studies of this type can also provide evidence of competitive inhibition and this, as pointed out by Goldstein (1944), is perhaps the most direct method available. The other two methods, which have been discussed in previous chapters. are (1) the plotting of equilibrium inhibition data in varions ways (see Chapter 5) and (2) the demonstration that it requires a greater concentration of inhibitor in the presence of substrate to produce a certain degree of inhibition than in the absence of substrate. The kinetic method would be particularly useful in those situations where, for one 
reason or another, it is difficult to arrive reliably at equilibrium inhibitions.

Many examples of the protection against enzyme inhibition that is afforded by the presence of substrate liave been reported. In most cases the protection has not been studied kinetically. Two further instances of protection by substrate will be mentioned in order to clarify the concept of competition. Yeast $\alpha$-carboxylase is inhibited by various sulfhydryl reagents and the rate at which this inhibition occurs is lower the greater the pyruvate concentration (Stoppani et al., 1953). Protection against $p$-chloromercuribenzoate, $\mathrm{HgCl}_{2}, \mathrm{Cu}^{++}$, arsenicals, o-iodosobenzoate, and iodoacetate was observed. For example, if $p$-chloromercuribenzoate and pyruvate were added together, the inhibition was $48 \%$ after $20 \mathrm{~min}$, but the same concentration of the mercurial in the absence of the substrate inhibited $90 \%$ within 2 min. The substrate tyramine has been shown to reduce the rate of inhibition of monoamine oxidase by iproniazid (Zeller et al., 1955; Davison, 1957). Tyramine at $50 \mathrm{~m} M$ almost completely protected the enzyme against $0.1 \mathrm{~m} M$ iproniazid. For both $\alpha$-carboxylase and monoamine oxidase, these results provide evidence that the inhibitors react with the substrate site on the enzyme. Yet these inhibitions are not truly competitive in the classic sense because when the enzyme is incubated with the inhibitor alone mntil maximal inhibition is achieved, the addition of substrate, even in high concentrations, will not reduce the inhibition or displace the inhibitor from the enzyme. One must then distinguish between kinetic competition, where the rate of inhibition is reduced by substrate but the final degree of inhibition is not affected, and equilibrium competition, where both the rate and the final inhibition are decreased by substrate. In the former case, the usual plotting procedures will indicate noncompetitive behavior when equilibrium inhibitions are measured. However, in this connection, it is obvious that when short experimental periods are used, the inhibition may not have reached an equilibrium value and what one is really measuring is kinetic competition.

\section{Temperature Effects and the Activation Energy of Inhibition}

Changes in the rate of inhibition with temperature may provide information about the thermodynamic properties of the activated complex formed between the enzyme and the inhibitor on the reaction pathway towards the normal complex:

$$
\mathrm{E}+\mathrm{I} \rightarrow \mathrm{EI}^{*} \rightarrow \mathrm{EI}
$$

Knowledge of the energy changes involved during this sequence - and particularly the alterations in these by varying the $\mathrm{pH}$, ionic strength, and other factors - should be interpretable in terms of the physical events 
occurring at a molecuiar level as the inhibitor approaches and contacts the enzyme. Very few data are at present available. The subject will be treated here in a rather cursory fashion for this reason but it is believed that this approach in the future will be necessary to solve some of the problems connected with the forces occurring between enzyme and inhibitor molecules. It is also of some interest to examine the thermodynamic quantities that determine the rate of inhibition.

The inhibition rate constant is given in transition state kinetics as:

$$
k_{1}=\frac{k T}{h} e^{\left.-(\lrcorner F^{*} / R T\right)}=\frac{k T}{h} e^{-\left(.1 B^{*} / R T\right)} e^{\left.(\lrcorner \mathbb{S}^{*} / R\right)}
$$

where $k$ is the Boltzmann constant $\left(1.38 \times 10^{-16} \mathrm{erg} \mathrm{deg}^{-1}\right), h$ is the Planck constant $\left(6.624 \times 10^{-27} \mathrm{erg} \mathrm{sec}\right)$, and $\Delta F^{*}, \Delta H^{*}$, and $1 S^{*}$ all refer to the activated state EI*. The rate constant thus depends upon the change in free energy involved in the formation of the activated complex from the individual components. Therefore, just as in the formation of the normal complex, EI, as discussed in Chapter 6 , the rate will depend upon changes in both enthalpy and entropy. Equation 12-39 may be rewritten as:

$$
\begin{aligned}
& \ln k_{1}=\ln \frac{k}{h}+\ln T+\frac{1 S^{*}}{R}-\frac{\Delta H^{*}}{R T} \\
& \log k_{1}=10.32+\log T+\frac{4 S^{*}}{4.58}-\frac{4 H^{*}}{4.58 T}
\end{aligned}
$$

A plot of $\log k_{1}$ against $1 / T$ will, therefore, not be strictly a straight line but over the limited range of temperatures possible for enzymic study it will show so little curvature that it may be neglected. The slope may be obtained by differentiating Eq. 12-41:

$$
\text { slope }=\frac{d\left(\log k_{1}\right)}{d(1 / T)}=-\frac{\Delta H^{*}}{2.3 R}-\frac{T}{2.3}
$$

and from this the enthalpy of activation. $\Delta H^{*}$, may be calculated. A typical plot is shown in Fig. 12-19 for an inhibition in which $J H^{*}$ is $15 \mathrm{kcal} /$ mole and $1 S^{*}$ is $5 \mathrm{cal} / \mathrm{mole} / \mathrm{deg}$. The linearity over this temperature range is evident and is due to the relatively small contribution of the $T / 2.3$ term.

The relation between the enthalpy of activation and the apparent energy of activation, as determined by the commonly used method of Arrhenius, is easily shown. Arrhenius assumed the rate constant to be expressed by:

$$
k_{1}=A \exp \left(-\frac{E_{a c t}}{R T}\right)
$$


from which:

$$
\log k_{1}=\log A-\frac{E_{a c t}}{2.3 R T}
$$

where $E_{\text {act }}$ is the apparent energy of activation and $A$ is interpreted as the collision frequency of the reacting molecules. The slope of the plot of $\log k_{1}$ against $1 / T$ is used to determine the activation energy:

$$
E_{\text {act }}=-2.3 R \text { (slope) }
$$

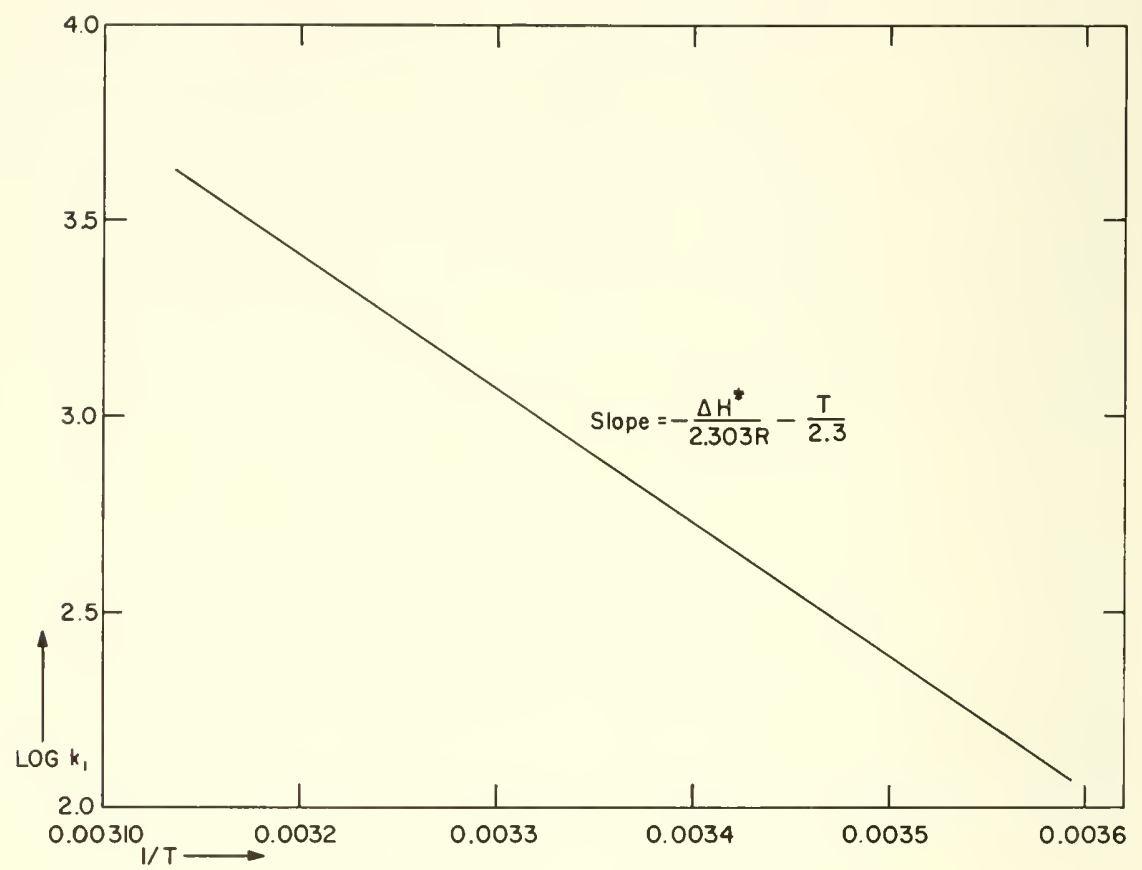

FIG. 12-19. Plot of $\log k_{1}$ against $1 / T$ for an inhibition in which $L H^{*}$ is $15 \mathrm{kcal} / \mathrm{mole}$ and $4 S^{*}$ is $5 \mathrm{cal} / \mathrm{mole} / \mathrm{deg}(\mathrm{Eq} .12-41)$.

The enthalpy of activation from Eq. 12-42 is:

$$
\left..1 H^{*}=-2.3 R \text { (slope }\right)-R T
$$

and therefore:

$$
E_{a c t}=1 H^{*}+R T
$$

The value of $R T$ is around $0.62-0.6+\mathrm{kcal} /$ mole for temperatures in the biological range and since most enthalpies of activation would be between $10-30 \mathrm{keal} / \mathrm{mole}$, the difference between $E_{a c t}$ and $I H^{*}$ is usually not significant. 
The rate constant is determined by the free energy of activation, $.1 F^{*}$, and the enthalpy of activation derived from the plotting procedure is only a part of this, the other part involving the entropy of activation, $1 S^{*}$. The relative contributions of the enthalpy and entropy to the free energy of activation will vary with the nature of the inhibition. The entropy term may well be quite appreciable, becanse not only will the deformation of the inhibitor molecule in the activated complex be a factor, but inasmuch as many inhibitors are ionic, the displacement of water molecules from the ionic groups of both inhibitor and enzyme during the formation of the complex may involve large changes in entropy. Eraluation of the $\exp \left(1 S^{*} / R\right)$ term in Eq. 12-39 leads to the following conchsions about the entropy effect: an increase in entropy of $3 \mathrm{cal} / \mathrm{mole} / \mathrm{deg}$ will increase $k_{1}$ by a factor of 4.53 , an increase of $5 \mathrm{cal} / \mathrm{mole} / \mathrm{deg}$ by a factor of 12.3 , an increase of 10 cal $/$ mole $/ \mathrm{deg}$ by a factor of 151 , and an increase of $20 \mathrm{cal} / \mathrm{mole} / \mathrm{deg}$ by a factor of 22,100 . Thus the entropy change may be a very important factor in determining the rate of inhibition. Some idea of the magnitude of $1 S^{*}$ may be obtained from the values of $k_{1}$ and $J I^{*}$ determined independently. Such data appear to be available for only one inhibition, the inactivation of liver monoamine oxidase by iproniazid. Davison (1957) found the rate constant. $k_{1}$, to be $1.65 \times 10^{3}$ liters mole ${ }^{-1} \mathrm{~min}^{-1}$ at $37^{\circ}$ and the enthalpy of activation to be about $30 \mathrm{kcal}$ mole. Substituting these values in Eq. 12-39. $\lrcorner S^{*}$ is found to be $44.4 \mathrm{cal} / \mathrm{mole} / \mathrm{deg}$, which is surprisingly high. If it were not for this great increase in entropy, the reaction of iproniazid with the monoamine oxidase would be extremely stow. Actually, the free energy of activation, $\mid F^{*}$, is only $16.2 \mathrm{kcal}$ mole and the entrony change may be shown to increase the rate by a factor of $4.7 \times 10^{9}$. Such a high value for $1 S^{*}$ might indicate a change in the enzyme structure upon inactivation, i.e., a partial denaturation.

When the free energy of activation for the binding of an inhibitor to an enzyme is below 5 kcal mole, it is likely that the process is limited by diffusion and that almost every collision of an inhibitor molecule with the reactive site on the enzyme leads to binding. Many ionic reactions, such as the inhibition of succinic dehydrogenase by malonate, probably fall in this category. It is possible that such low free energies of activation are the result of entropy increases attending the rearrangement of hydration water.

\section{Secondary Inactivation of Enzymes during Inhibition}

An enzyme is frequently unstable under the conditions used to determine inhibition. It is well known that many enzymes when extracted from cells and subjected to the abnormal media used to study their activity gradually lose this activity, particularly at $37-38^{\circ}$. This inactivation may be a denaturation of the enzyme protein or it may relate to the loss of some bound cofactor. Determination of the time course of an inhibition must take into 
account such processes of inactivation because the fall in enzymic activity will be due to both the direct action of the inhibitor and the spontaneous inactivation. The presence of the inhibitor on the enzyme may alter the rate of inactivation. A generalized scheme may be written as:

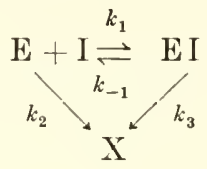

where the free enzyme is inactivated to $\mathrm{X}$ with a rate conswant $k_{2}$ and $\mathrm{EI}$ is inactivated to $\mathrm{X}$ (or a similar derivative) with a rate constant $k_{3}$. We may now outline the various possible situations.

I. Free enzyme is stable: the presence of the inhibitor promotes the irreversible inactivation of the enzyme $\left(k_{2}=0\right)$.

II. Free enzyme is unstable: the free enzyme is inactivated at a certain rate and the inhibitor affects this in the following ways.

A. Inhibitor has no effect on inactivation: E and EI break down at the same rate $\left(k_{2}=k_{3}\right)$.

B. Inhibitor increases instability: EI is more rapidly inactivated than $\mathrm{E}\left(k_{3}>k_{2}\right)$.

C. Inhibitor decreases instability: EI is more stable than $\mathrm{E}\left(k_{2}>k_{3}\right)$.

In the situation IIC, it may be noted that $k_{3}$ could be zero, in which case the inhibitor is exerting a completely protective action. Such a protective effect is quite possible inasmuch as substrate bound to certain enzymes increases the stability markedly. The term "inactivation" will be used here to designate those processes, usually irreversible, that lead to a loss of enzyme activity and are not the direct result of the binding of the inhibitor at the region of the active site.

Detection of inactivation in the presence of an inhibitor is often difficult. Inactivation may be suspected in the case of certain inhibitors when the initial loss of enzyme activity is followed by a progressive fall of activity. Somewhat better evidence for inactivation is a continuing decrease in activity after removal of the inhibitor from the medium surrounding the enzyme molecules, since this cannot now be attributed to a slow phase in the reaction of the inhibitor with the enzyme. In those cases in which complete enzyme activity is not restored upon removal of the inhibitor from the enzyme, assmming that this removal can be experimentally demonstrated, there is justification for suspecting inactivation. It is very important to recognize inactivation when quantitative studies are being pursued 
because the determination of rate and inhibition constants, or even of the type of inhibition, may otherwise be seriously in error.

The mechanisms by which the stability of an enzyme can be affected by the binding of an inhibitor are generally unknown but some suggestions may be made. The secondary and tertiary structures of enzyme proteins are probably often sensitive to the ionic charge distribution on the enzyme surface. The neutralization of such ionic groups or the introduction of new charged groups onto the enzyme (as with iodoacetate) could favor the dissociation of helices or unfolding. The reaction of inhibitors with protein groups that are involved in secondary structure (e.g., inhibitors forming hydrogen bonds with the protein) or tertiary structure (e.g., inhibitors reacting with sulfhydryl groups) may also labilize the enzyme. All the constituent atoms and bonds in a protein molecule are probably in a rather intense electrical field originating from the positively and negatively charged groups distributed in a definite pattern and the interpolypeptide or interhelical linkages maintain the structure within this field. However, if either the electrical field is altered or the linkages are weakened by inhibitors, the delicate equilibrium may be shifted in favor of the so-called denatured or inactive forms. It may also be postulated that an inhibitor could increase the stability of an enzyme if it reacts at the active site in a manner similar to that of the substrate since it is known that enzymes are sometimes more stable in the presence of their substrates, which may by their effects on the local electrical field around the active site favor structural stability.

The question arises as to whether inactivation of an enzyme is associated with general structural changes of the protein or only to local alterations in the region of the active site. Probably both types occur and it is possible that a local disruption of protein structure could spread over the rest of the protein as a wave of instability or denaturation. In any event, it is likely that some instances of inactivation are not accompanied by significant changes in the properties of the protein as a whole. Thus enzyme inactivation need not imply those changes usually associated with denaturation of the protein, for the disturbance may remain localized. In such cases, a degree of reversibility may be inherent in the inactivation and detection of the inactivation may be quite difficult.

The quantitative expression for the loss of enzyme activity with time when both inhibition and inactivation occur simultaneously may be very complex. The kinetics of inactivation have been very well presented by Reiner (1959, p. 275). For a simple first-order denaturation of an enzyme:

$$
\mathrm{E} \stackrel{k}{\rightarrow} \mathrm{X}
$$

the concentration of the inactive form is given by:

$$
(\mathrm{X})=\left(\mathrm{E}_{t}\right)\left[1-e^{-k t}\right]
$$


and the variation in enzyme rate with time by:

$$
v=\frac{V_{m}(\mathrm{~S}) e^{-k t}}{(\mathrm{~S})+K_{s}}
$$

We must now consider how such inactivation may modify the development of true inhibition. It is important to remember that inhibition may be measured in two basically different ways: the decrease in enzymic rate may be based on the initial activity or it may be based at a particular time on the activity of a control enzyme preparation that has not been treated with the inhibitor. In the first case, one is measuring the development of both inhibition and inactivation. whereas in the second case, one attempts as far as possible to separate inhibition from inactivation. Althongh the second procedure may seem valid as a means of eliminating inactivation, it is not necessarily so. If the EI complex is inactivated at a rate either slower or faster than that for the free enzyme $\mathrm{E}$, the concentration of inactivated enzyme $(\mathrm{X})$ will be different in the presence of the inhibitor than in the control. Such a simple correction is valid only when the inhibitor does not affect the inactivation rate (case II $A$ above).

A very cursory treatment of the kinetics of inhibition accompanied by inactivation will now be presented with the aim of illustrating some of the basic characteristics of such systems and the complexities encountered in their analysis.

(I) Free enzyme is stable but the EI complex is unstable. When the binding of the inhibitor to the enzyme is very tight and the dissociation of EI can be neglected. the following simple reaction may be written:

$$
\mathrm{E}+\mathrm{I} \stackrel{k_{1}}{\rightarrow} \mathrm{EI} \stackrel{k_{3}}{\rightarrow} \mathrm{X}
$$

The fractional inhibition is given by:

$$
i=\frac{(\mathrm{EI})+(\mathrm{X})}{\mathrm{E}\left({ }_{t}\right)}
$$

since the inhibited (EI) and inactivated (X) enzyme forms both contribute to the loss in activity. Since:

$$
\frac{d[(\mathrm{EI})+(\mathrm{X})]}{d t}=k_{1}(\mathrm{E})(\mathrm{I})
$$

and from Eq. 12-52:

$$
\frac{d[(\mathrm{EI})+(\mathrm{X})]}{d t}=\left(\mathrm{E}_{t}\right) \frac{d i}{d t}
$$

we can write the differential equation for the inhibition as:

$$
d i / d t=k_{1}(\mathrm{I})(1-i)
$$


which may be integrated to give:

$$
i=1-e^{-k_{1}(\mathrm{I}) t}
$$

It is evident that the development of inhibition is independent of the inactivation reaction since the formation of $\mathrm{X}$ from $\mathrm{EI}$ is of no consequence. The course of the inhibition is plotted in curve 5 of Fig. 12-20 for an arbitrarily chosen set of conditions.

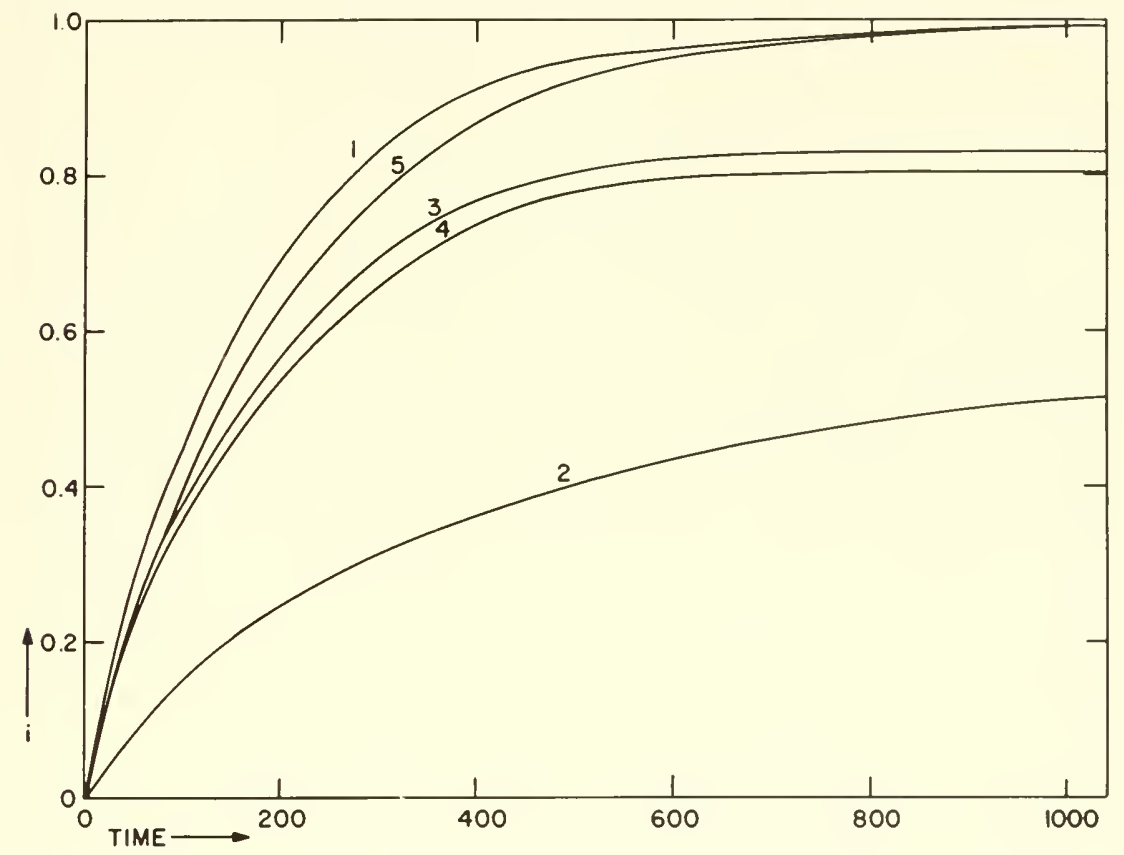

FIG. 12-20. Variation of the inhibition with time in various systems in which inactivation occurs. $K_{i}=1 \mathrm{~m}, \mathrm{H},(\mathrm{I})=5 \mathrm{~m}, \mu, k_{1}=1$, and $k_{2}=10^{-3}$. Curve 1 , E and EI unstable (irreversible); curve $2, \mathrm{E}$ and EI unstable (reversible); curve 3. no inactivation; curve 4, $\mathrm{E}$ stable and $\mathrm{EI} \rightarrow \mathrm{X}$ (reversible); curve 5, $\mathrm{E}$ stable and $\mathrm{EI} \rightarrow \mathrm{X}$ (irreversible).

When the dissociation reaction of the EI complex must be included, the situation is much more complex. We now have:

$$
\mathrm{E}+\mathrm{I} \underset{k_{-1}}{\stackrel{k_{1}}{\rightleftharpoons}} \mathrm{EI} \stackrel{k_{3}}{\rightarrow} \mathrm{X}
$$

for which two differential equations may be written:

$$
\begin{aligned}
d i / d t & =k_{1}(\mathrm{I})(1-i)-k_{1} K_{i}\left[(\mathrm{EI}) /\left(\mathrm{E}_{t}\right)\right] \\
d(\mathrm{EI}) / d t & =k_{1}(\mathrm{I})\left(\mathrm{E}_{t}\right)(1-i)-(\mathrm{EI})\left(k_{1} K_{i}+k_{3}\right)
\end{aligned}
$$


The solution of these simultaneous equations may be obtained by classical methods or by the Laplace transform (see Salvadori and Schwarz, 1954, p. 236) but is very cumbersome unless absolute values are known for the constants and (I). Figure 12-21 shows the variations in the enzyme fractions with time for a typical case. It may be noted that reaction 12-57 is identical in form with the substrate reaction:

$$
\mathrm{E}+\mathrm{S} \rightleftharpoons \mathrm{ES} \rightarrow \mathrm{P}
$$

and the transient-state kinetics for this system have been treated by Chance (1943) and Yang (1954).

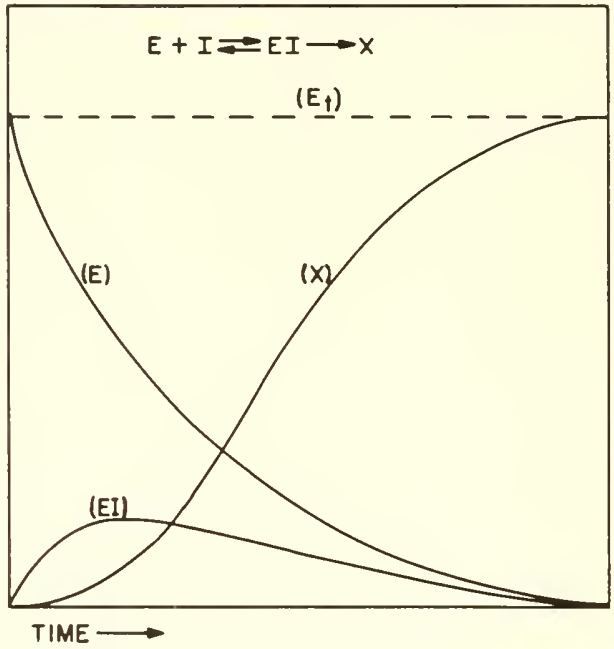

FIG. 12-21. Variations in the concentrations of the enzyme fractions with time (scheme 12-57).

In these cases, the loss of enzyme activity proceeds to completion, but the actual inhibition, exclusive of inactivation, reaches a level which is not necessarily the equilibrium inhibition. The true inhibition is related to (EI) and the anount of enzyme that is not inactivated as follows:

$$
i=\frac{(\mathrm{EI})}{\left(\mathrm{E}_{t}\right)-(\mathrm{X})}
$$

The usual differential equation for EI:

$$
d(\mathrm{EI}) / d t=k_{1}(\mathrm{E})(\mathrm{I})-(\mathrm{EI})\left(k_{1} K_{i}+k_{3}\right)
$$

and the expression for $d(\mathrm{EI}) / d t$ obtained from Eq. 12-60:

$$
\frac{d(\mathrm{EI})}{d t}=\left[\left(\mathrm{E}_{t}\right)-(\mathrm{X})\right] \frac{d i}{d t}-i \frac{d(\mathrm{X})}{d t}
$$


allow the following expression for the rate of change of $i$ to be derived:

$$
d i / d t=k_{1}(\mathrm{I})-\left[k_{1}(\mathrm{I})+k_{1} K_{i}+k_{3}\right] i+k_{3} i^{2}
$$

This may be integrated readily but the general expression is complex and it is more convenient to select values for the constants and (I), which has been done to give curve 4 of Fig. 12-20. This curve may be compared with curve 3 which represents the course of the inhibition when no enzyme inactivation occurs. When EI is unstable, the level of inhibition reached is lower than that for the case when EI is stable (Eq. 12-11). It is impossible, of course, to determine experimentally the true inhibition, unless the inactivation of the enzyme can be followed independently by some means.

An interesting extension of this form of inactivation is provided by the inhibition of yeast alcohol dehydrogenase by the zinc chelator, orthophenanthroline (Williams et al.. 1958). This enzyme is immediately inhibited by $o$-phenanthroline when the inhibitor is added with all the components of the reaction present, but if the enzyme is preincubated with the inhibitor, a time-dependent inhibition is observed. This second inhibition is either irreversible or only partly reversible and thus may be considered an inactivation as in scheme 12-57. The inactivation occurs rather slowly: with $1 \mathrm{mM}$ o-phenanthroline an inhibition of $15 \%$ was reached in about $15 \mathrm{~min}$, while at $5 \mathrm{mM}$ it required about $60 \mathrm{~min}$ to reach an inhibition of $80 \%$. First-order kinetics were followed. The results conform to the theory that the inactivated enzyme is $\mathrm{EI}_{2}$ so that the following reaction series can be written:

$$
\mathrm{E}+\mathrm{I} \underset{k_{-1}}{\stackrel{k_{1}}{\rightleftharpoons}} \mathrm{EI} \stackrel{k_{3}}{\rightarrow} \mathrm{EI}_{2}
$$

From $d\left(\mathrm{EI}_{2}\right) / d t=k_{3}(\mathrm{EI})(\mathrm{I})$ and $\left(\mathrm{E}_{t}\right)=(\mathrm{E})+(\mathrm{EI})+\left(\mathrm{EI}_{2}\right)$, and assuming that $i=\left(\mathrm{EI}_{2}\right) /\left(\mathrm{E}_{t}\right)$, considering only the inactivation, the inhibition is given by:

so that:

$$
i=1-\exp \left[-\frac{k_{3} t(\mathrm{I})^{2}}{(\mathrm{I})+K_{i}}\right]
$$

$$
2.3 \log (1-i)=-\frac{k_{3} t(\mathrm{I})^{2}}{(1)+K_{i}}
$$

A plot of $\log (1-i) / t$ against $0.434(\mathrm{I})^{2} /\left[(\mathrm{I})+K_{i}\right]$ will give a straight line with a slope of $-k_{3}$. The linearity of this plot will provide some confirmation for the mechanism whereby the inactivation is associated with the binding of a second molecule of inhibitor.

(II) Free enzyme and the EI complex are both unstable. We may first consider the case in which the presence of the inhibitor on the enzyme does 
not affect the rate of inactivation, i.e., $k_{2}=k_{3}$ in scheme $12-48$. If the total decrease in enzyme activity is under consideration, the fractional inhibition is given by Eq. 12-52. Since here:

$$
d(\mathrm{X}) / d t=k_{2}\left[\left(\mathrm{E}_{t}\right)-(\mathrm{X})\right]
$$

the concentration of inactivated enzyme may be immediately written as:

$$
(\mathrm{X})=\left(\mathrm{E}_{t}\right)\left[1-e^{-k_{2} t}\right]
$$

so that from the usual differential equation for $d[(\mathrm{EI})+(\mathrm{X})] / d t$ we obtain:

$$
d i / d t=k_{1}(1)+k_{2}+k_{1} K_{i}\left[1-e^{-k_{2} t}\right]-\left[k_{1}(\mathrm{I})+k_{1} K_{i}+k_{2}\right] i
$$

which again does not integrate to a simple expression. However, the course of the loss in activity is quite similar to that for the case in which the free enzyme is stable, except that the activity decreases somewhat more rapidly due to the inactivation of $\mathrm{E}$ as well as of EI. However, if we consider only the true inhibition, it may be easily shown that it develops in an identical fashion to the situation where no inactivation oceurs (Eq. 12-11), which would be the expected result, since $\mathrm{E}$ and $\mathrm{EI}$ are inactivated at equal rates. Put in another way, if experimentally both a control and an inhibited run are made and the inhibition at any time calculated from the relative rates, the true inhibition will be obtained.

Finally, the situation when $k_{2} \neq k_{3}$ may be discussed. Here the presence of the inhibitor on the enzyme alters the rate of inactivation. If the dissociation of EI may be neglected, the kinetics are simple because the reaction, $\mathrm{EI} \rightarrow \mathrm{X}$, is of no consequence, both forms being inactive. From Eq. $12-52$ and:

$$
\frac{d[(\mathrm{EI})+(\mathrm{X})]}{d t}=k_{1}(\mathrm{E})(\mathrm{I})+k_{2}(\mathrm{E})
$$

the rate of change of $i$ is found to be:

$$
d i / d t=\left[k_{1}(\mathrm{I})+k_{2}\right](1-i)
$$

which integrates to:

$$
i=1-e^{-\left[k_{1}(\mathrm{I})+k_{2}\right] t}
$$

This is plotted in curve 1 of Fig. 12-20 and the mereased inhibition compared to curve 5 may be attributed to the inactivation of $\mathrm{E}$. If the reverse dissociation of EI cannot be neglected and the complete scheme 12-48 must be treated, the true inhibition as expressed by Eq. 12-60 may be obtained by the integration of:

$$
d i d t=k_{1}(\mathrm{I})-\left\{k_{1}\left[(\mathrm{I})+K_{i}\right]+k_{3}-k_{2}\right\} i+\left(k_{3}-k_{2}\right) i^{2}
$$


The course of the true inhibition is plotted as curve 2 in Fig. 12-20 for the case in which $k_{3}=5 k_{2}$. The true inhibition is reduced in comparison to curve 3 because the EI complex is inactivated at a rather rapid rate and (EI) does not attain such high levels as when no inactivation occurs.

\section{The Enzyme Is Inhibited and Reactivated Simultaneously}

Certain inhibitors, notably the organophosphorus compounds, bind to the active center and then react chemically with enzyme to inactivate. In some cases the inactivated enzyme may be reactivated by a chemical reaction involving the splitting off of the attached residne. The kinetics for such an inhibition have been treated by van Asperen and Dekuijzen (1958) for the cholinesterases of houseflies and mouse brain. The following reactions occur:

$$
\begin{array}{ll}
\mathrm{E}+\mathrm{I} \rightleftharpoons \mathrm{EI} & k_{i}=(\mathrm{E})(\mathrm{I}) /(\mathrm{EI}) \\
\mathrm{EI} \stackrel{k_{1}}{\rightarrow} \mathrm{EP}+\mathrm{V} & v_{1}=k_{1}(\mathrm{EI}) \\
\mathrm{EP} \stackrel{k_{2}}{\rightarrow} \mathrm{E}+\mathrm{P} & v_{2}=k_{2}(\mathrm{EP})
\end{array}
$$

where the inhibitor is $O, O$-dimethyl-O-2,2-dichlorovinyl phosphate (DDVP), $\mathrm{P}$ is dimethyl phosphate and $\mathrm{V}$ is dichlorovinyl alcohol, $\mathrm{P}$ and $\mathrm{V}$ being the products formed from this particular inhibitor. The phosphorylated enzyme EP is inactive whereas EI, although inactive, may be considered as active since it can give rise to active enzyme. When $v_{2}=0$, the final inhibition will depend only on the relative concentrations or amounts of the enzyme and the inhibitor, but when $v_{2}$ is finite, the inhibition will rise to a level which may remain constant, a steady state in which the formation and breakdown of EP are equal and (E) is constant. Steady inhibition thus occurs when $v_{1}=v_{2}$. Curves are presented for various concentraions of DDVP and it was found that it requires around 30 to 60 min for a steady state to be reached (Fig. 12-22).

The potency of an inhibitor in this situation depends on the rate at which EP is dissociated, i.e., the rate of reactivation, the greater this rate, the higher the concentration of an inhibitor necessary to produce a chosen final level of inhibition. The two cholinesterases from flies and mouse brain differ by a factor of around 50 in their resistance to DDVP becanse of different rates of reactivation.

\section{Preincubation Procedure and Determination of the Component with Which the Inhibitor Reacts}

When the inhibitor and all the components of the enzynie system are mixed simultaneously, or when the inhibitor is added while the enzyme reaction is proceeding, the situation is generally more difficult to deal with 
kinetically than when the inhibitor is preincubated with the particular component with which it reacts. Of course, if the inhibition is for practical purposes instantaneous, the order of addition makes little difference, but if the rate of inhibition can be measured and if one is interested in determining the rate constant, $k_{1}$, or the energy of activation, the preincubation technique is preferable. It is sometimes possible to localize the action of the inhibitor to one component of the enzyme system in this way. For

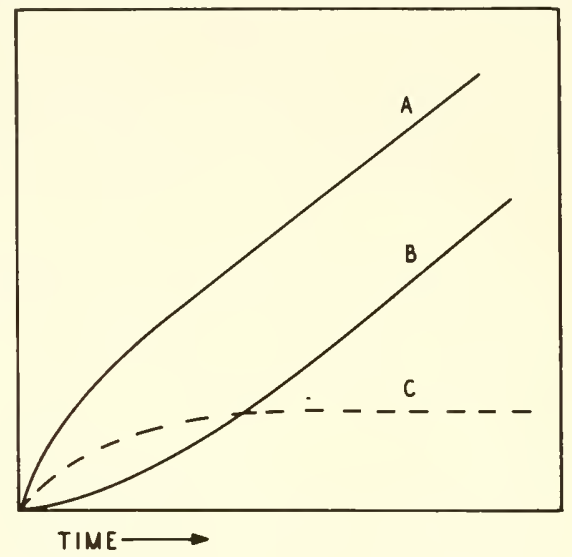

FIG. 12-22. Inhibition of cholinesterase by $O, O$-dimethyl-O-2,2-dichlorovinyl phosphate (DDVP). (From van Asperen and Dekhuijzen, 1958.) Curve A, enzyme that has been phosphorylated. Curve B, enzyme that has been dephosphorylated. Curve $\mathrm{C}$, course of the inhibition showing the achievement of a steady state.

example, if we consider the simplest system, in which the enzyme reaction involves only the enzyme and the substrate as components, the following four experiments should be performed if the nature of the inhibition is unknown:

\begin{tabular}{c|c|c}
\hline Experiment & Preincubation & Reaction started by \\
\cline { 1 - 2 }$(a)$ & $\mathrm{E}+\mathrm{I}$ & $\mathrm{S}$ \\
$(b)$ & $\mathrm{E}$ & $\mathrm{S}$ \\
$(c)$ & $\mathrm{S}+\mathrm{I}$ & $\mathrm{E}$ \\
$(d)$ & $\mathrm{S}$ & $\mathrm{E}$ \\
\hline
\end{tabular}

From $(a)$ and $(c)$ one may be able to show whether the inhibitor reacts with the enzyme or the substrate, while $(b)$ and $(d)$ serve as controls in case 
either the enzyme or the substrate are unstable under the experimental conditions. The preincubation time and the conditions must be identical in each of the procedures. When the locus of inhibition is established, a time course for the inhibition should be determined by using different preincubation intervals. If $K_{i}$ or other equilibrium characteristics are to be obtained, it is essential to know when the reaction of the inhibitor is complete and equilibrium has been achieved.

If an activator or coenzyme is also a component of the enzyme system, the procedure is necessarily more complex and in addition to the four tests described above the procedures given in the tabulation below must be carried out for a complete analysis:

\begin{tabular}{c|c|c}
\hline Experiment & Preincubation & Reaction started by \\
\cline { 1 - 2 }$(e)$ & $\mathrm{A}+\mathrm{I}$ & $\mathrm{S}+\mathrm{E}$ \\
$(f)$ & $\mathrm{A}+\mathrm{S}+\mathrm{I}$ & $\mathrm{E}$ \\
$(g)$ & $\mathrm{A}+\mathrm{E}+\mathrm{I}$ & $\mathrm{S}$ \\
$(h)$ & $\mathrm{S}+\mathrm{E}+\mathrm{I}$ & $\mathrm{A}$ \\
$(i)$ & $\mathrm{E}+\mathrm{S}$ & $\mathrm{A}$ \\
$(j)$ & $\mathrm{E}+\mathrm{A}$ & $\mathrm{S}$ \\
$(k)$ & $\mathrm{S}+\mathrm{A}$ & $\mathrm{E}$ \\
\hline
\end{tabular}

The first test $(e)$ is designed to determine if the inhibitor reacts with the activator or coenzyme; $(f),(g)$, and $(h)$ will show if the inhibitor forms a complex with two components of the system or reacts with the substrate or activator only in the presence of the enzyme; and $(i),(j)$, and $(k)$ are the controls for the three preceding tests. It is suggested that such a series of tests be undertaken before the more quantitative aspects of the inhibition are investigated because the latter can be done efficiently only after the basic mechanism of the inhibition is established.

\section{RATES OF INHIBITION IN MITOCHONDRIA, CELLS, AND TISSUES}

Interpretation of the rate at which an inhibition develops may be very difficult when the enzyme that is attacked by the inhibitor is located within a cell and is separated from the ambient medium by one or more protoplasmic membranes. Actually very little really quantitative work on the kinetics of intracellular inhibition has been done. However, the rates of drug action have been the subject of much study and speculation, and the results and conclusions from this branch of pharmacodynamics are frequently valuable for investigations of metabolic inhibition since fundamentally the same problems are involved. The best discussion of the rates of drug action remains that of Clark (1937) and there he summarizes his 
critical approach in the following words: "The study of the kinetics of drug action is, therefore, of a qualitative rather than a quantitative nature. It is perfectly easy to obtain attractive curves relating time and action, and the ease with which these curves can be fitted by simple formulae is very striking. The difficulties begin when an attempt is made to analyse the factors that must be concemed with the delay." Such a statement is equally valid when applied to the study of inhibition and one must be particularly aware of the dangers of drawing conclusions as to the fundamental mechanisms of inhibition from the fit of the experimental data to some mathematical formulation. Unfortunately many different assumptions as to the mechanism give essentially the same predicted behavior in most cases. It is possible to distinguish between these different mechanisms only by a more direct investigation of the penetration and distribution of the inhibitors and the nature of the metabolic alterations.

\section{Relation of Inhibition Kinetics to the Changes Measured}

Consideration must first be given to the relationship between the primary inhibition and the processes whose changes are detemined in any study of the rates of inhibition in complex systems. If the inhibitor acts on some enzyme within the cell, ideally the activity of this susceptible enzyme would be determined as it changes with time, but this is nsually difficult if not impossible. Unless the inhibition is irreversible, or very slowly reversible, it is not feasible to extract the enzyme from the tissue at intervals to determine the course of the inhibition, so that, if the activity cannot be directly measured within the cells, one must resort to indirect procedures. Most commonly tested is some metabolic process dependent upon the susceptible enzyme (e.g., respiration or glycolysis), but such measurements will not provide an accurate course of the enzyme inhibition unless the rate of the over-all process is immediately and quantitatively related to the activity of this enzyme. In some cases, such as the depression of respiration resulting from the inhibition of cytochrome oxidase, a satisfactory correlation may exist, but in general the metabolic changes will lag behind the enzyme inhibition to varying degrees, and indeed frequently there will be no direct or simple relationship at all. Especially when the uptake of some substrate or the formation of a metabolic proluct is letermined, the kinetics will often relate to factors other than those of the enzyme inhibition. Commonly some biochemical or physiological (functional) change only secondarily dependent on the susceptible enzyme is used as a measure of the development of inhibition and here it is evident that only a very fortuitous set of circumstances will allow quantitative interpretation. The many problems of correlating enzyme inhibition with the functional depression of cells have been discnssed in Chapter 9 with regart to final inhibitions but the same problems arise. perhaps accentuated, in kinetic studies. The 
rate at which an inhibitor alters myocardial contraction, for example, even in isolated preparations to which the inhibitor can be directly applied, will bear little relationship to the primary inhibition rate of the enzyme. Finally, these problems are particularly evident when only the death of cells or organisms is used as an indication of the inhibition because it is likely that in all cases a number of reactions intervene between the susceptible enzyme and the processes upon which the life of the cell depends. The death rate of microorganisms has often been treated in a quantitative fashion and from the data various conclusions as to the mechanism of inhibition have been derived. However. this approach is extremely limited. Justification for such an approach by adducing the principle of limiting reactions must be examined very critically. These problems can best be presented by considering some of the possible factors that affect inhibition rates in cells and by discussing some examples of cellular inhibition studies.

\section{Factors Commonly Affecting Inhibition Rates in Cells}

The lag or latent period between the application of the inhibitor and some metabolic or functional response is generally composed of three phases: (a) the time required for the inhibitor to reach the susceptible enzyme from the site of its application (including the times to reach the cells and to penetrate into the cells). (b) the time for the reaction of the inhibitor with the enzyme, and $(c)$ the time for the cells to respond to the loss of this enzyme activity. These phases may be of any relative duration. In many cases, one phase is much longer than the other two and thus predominantly determines the rate of the inhibition. Occasionally other factors may be of importance, such as the time required for the lethal synthesis of the active inhibitor. The important thing is - mless we know what phase, or phases, is controlling the rate, we cannot interpret kinetic data mechanistically.

(A) Rates of peretration into eells. The rate of development of the inhibition may be determined by the rate at which the inhibitor penetrates into the cells and may have nothing to do with the enzymic process. That the cell membrane can slow down the reaction of a substance with an intracellular component, even in situations where diffusion would be expected to be rapid, is shown by the fact that the oxygenation of hemoglobin in erythrocytes is about eighteen times slower than when the hemoglobin is in solution (Hartridge and Roughton. 1927). If the diffusion of a small uncharged molecule such as oxygen is reduced by the cell membrane, it is evident that most inhibitors will gain entrance to cells relatively slowly and that the combination of the inhibitor with the enzyme would have to be a reasonably slow reaction in order to limit the rate at which the inhibition 
develops. Tissue preparations usually respond to a substance more rapidly when it reaches the cells in the blood supply or perfusion fluid than when it is applied externally in a bath. Thus octyltrimethylammonium ion at $2 \mathrm{~m} M$ produced half-paralysis of gastrocnemius muscle in $0.3 \mathrm{~min}$ when perfused into the muscle but it required $14 \mathrm{~min}$ when the drug was added to the medium in which the muscle was suspended (Ing and Wright, 1931). Diffusion barriers can thus play an important role in kinetics. The major factor is not the absolute rate of penetration into the cells or tissue but the diffusion rate relative to the rate of reaction of the inhibitor with the enzyme.

It is difficult from kinetic measurements alone to determine if the rate at which a substance affects an intracellular component depends primarily on the rate of penetration of the substance into the cell or on the association rate for the formation of the complex. The uptake of dyes by cells has been studied thoroughly and has been found to follow monomolecular kinetics in most cases. Thus Irwin (1925) found that cresyl blue entered the vacuole of Nitella according to such kineties and assumed that the dye combined with something inside the cell; however, the kinetics could be accounted for satisfactorily on the basis of diffusion. The simple diffusion of a substance into a cell:

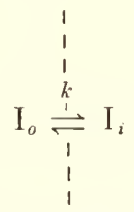

is expressed by the differential equation:

$$
\frac{d\left(\mathrm{I}_{i}\right)}{d t}=k\left[\left(\mathrm{I}_{o}\right)-\left(\mathrm{I}_{i}\right)\right]
$$

which may be integrated to:

$$
\left(\mathrm{I}_{i}\right)=\left(\mathrm{I}_{o}\right)\left(1-e^{-k t}\right)
$$

On the other hand, the reaction of the substance within the cell may be represented as:

$$
\mathrm{I}+\mathrm{E} \stackrel{k}{\rightleftharpoons} \mathrm{EI}
$$

for which the differential equation:

$$
\frac{d(\mathrm{EI})}{d t}=k \cdot(\mathbf{1})(\mathrm{E})-k K_{\imath}(\mathrm{EI})
$$

may be written, where $K_{i}$ is the inhibition constant if the process leads to enzyme inhibition. 
This may be integrated to:

$$
(\mathrm{EI})=\frac{(\mathrm{I})\left(\mathrm{E}_{t}\right)}{(\mathrm{I})+K_{i}}\left\{1-e^{-k\left[(\mathrm{I})+k_{i}\right] t}\right\}
$$

Thus the rate of accumulation of the inhibitor in the cell follows the same kinetics in each case, although the constants, of course, have quite different meanings.

Three different situations may be postulated: (1) the inhibitor enters into the cell much more slowly than it combines with the enzyme so that $\left(I_{i}\right)$ during the development of the inhibition is essentially zero and the rate of the inhibition is dependent primarily on the penetration process; (2) the inhibitor enters the cell much more rapidly than it combines with the enzyme so that $\left(I_{i}\right)$ is approximately equal to $\left(I_{0}\right)$ during the process, the rate of inhibition depending on the reaction with the enzyme; and (3) the rates of entrance and association with the enzyme are comparable so that $\left(I_{i}\right)$ is less than $\left(I_{o}\right)$ but is appreciable. the kinetics being complex. It is interesting that in the first case (1):

$$
\frac{d(\mathrm{EI})}{d t}=h \cdot\left(\mathrm{I}_{o}\right)
$$

where $k$ is a membrane permeability constant, and since $i=(\mathrm{EI}) /\left(\mathrm{E}_{\iota}\right)$ that:

$$
\frac{d i}{d t}=\frac{k\left(\mathrm{I}_{o}\right)}{\left(\mathrm{E}_{t}\right)}
$$

when the affinity of the enzyme for the inhibitor is high. Here the inhibition increases linearly with time providing $\left(\mathrm{I}_{0}\right)$ remains constant. It may be shown that the simnltaneous differential equations expressing the general case where the penetration and association rates are comparable (3) are:

$$
\begin{gathered}
\frac{d i}{d t}=k_{1}\left(\mathrm{I}_{i}\right)-i\left[\left(\mathrm{I}_{i}\right)+K_{i}\right] k_{1} \\
\frac{d\left(\mathrm{I}_{i}\right)}{d t}=k\left(\mathrm{I}_{o}\right)+k_{1} K_{i} i\left(\mathrm{E}_{t}\right)-\left(\mathrm{I}_{i}\right)\left[k+k_{1}\left(\mathrm{E}_{t}\right)(1-i)\right]
\end{gathered}
$$

where $k$ is a permeability constant and $k_{1}$ is the forward rate constant for the formation of the EI complex.*

* It would be relatively easy and perhaps rewarding to test such kinetics on artificial systems wherein an enzyme is contained within a synthetic membrane and the inhibitor is added to the external medium. The permeability of the membrane to the inhibitor and the rate of reaction of the inhibitor with the enzyme could be determined separately. The resulting behavior in the total system could be progressively applied to inhibition within cells as more accurate data become available. 
The most important treatment of diffusion-limited kinetics for the present purpose is that of Ronghton (1960). Equations were developed by Roughton for the diffusion of a substance from medium I through a membrane into medium II, in which the substance diffuses and is immobilized rapidly and irreversibly on a finite number of sites. This morlel would correspond reasonably well to certain situations in which an inhibitor is bound tightly to intracellular components (inchuding the enzyme attacked) following penetration of the plasma membrane. The behavior in such systems depends on spatial factors and the treatment was applied to flat layers, cylinders, and spheres. If the membrane between the media is very thin, the effect of the membrane on the time required for a given per cent uptake in medinm II is linearly related to the ratio of the permeabilities in medium II and in the membrane. This would imply that under these conditions, the rate of the reaction with the sites would be proportional to the membrane permeability.

(B) The $\mathrm{pH}$ of the extracellular medium. Since many inhibitors are weak acid or bases, the rate of inhibition may depend on the $\mathrm{pH}$ of the external medinm because the undissociated and ionic forms penetrate the membrane at different rates. For a simple acidic inhibitor where HI penetrates into the cell but $\mathrm{I}^{-}$does not:

$$
\frac{d\left(\mathrm{I}_{i}\right)_{t}}{d t}=k_{\mathrm{HI}}\left[\left(\mathrm{HI}_{o}\right)-\left(\mathrm{HI}_{i}\right)\right]
$$

where $k_{\mathrm{HI}}$ is the permeability constant for the undissociated form HI. The total concentration of the inhibitor in both forms inside the cell is $\left(\mathbf{I}_{i}\right)_{t}$. The concentration of HI will depend on the $\mathrm{pH}$, both outside and inside the cell, and thus Eq. 12-82 may be rewritten as:

$$
\frac{d\left(\mathrm{I}_{i}\right)_{t}}{d t}=k_{\mathrm{HI}}\left[\frac{\left(\mathrm{I}_{o}\right)_{t}}{1+K_{a} /\left(\mathrm{H}_{o}{ }^{+}\right)}-\frac{\left(\mathrm{I}_{i}\right)_{t}}{1+K_{a}^{r}\left(\mathrm{H}_{i}{ }^{+}\right)}\right]
$$

where $K_{a}$ is the acid dissociation constant of the iuhibitor. The rate of inhibition may thus depend on the intracellular $\mathrm{pH}$ as well as that of the external medium. If the ionized form $\mathrm{I}^{-}$also penetrates into the cell:

$$
\begin{aligned}
\frac{d\left(\mathrm{I}_{i}\right)_{t}}{d t} & =k_{\mathrm{HI}}\left[\frac{\left(\mathrm{I}_{o}\right)_{t}}{1+K_{a} /\left(\mathrm{H}_{o}{ }^{+}\right)}-\frac{\left(\mathrm{I}_{i}\right)_{t}}{\mathrm{l}+K_{a} /\left(\mathrm{H}_{i}^{+}\right)}\right]+ \\
& +k_{\mathrm{I}^{-}}\left[\frac{\left(\mathrm{I}_{o}\right)_{t}}{1+\left(\mathrm{H}_{o}{ }^{+}\right) / K_{a}}-\frac{\left(\mathrm{I}_{i}\right)_{t}}{1+\left(\mathrm{H}_{i}^{+}\right) / \boldsymbol{K}_{a}}\right]
\end{aligned}
$$

where $h_{I^{-}}$is the permeability constant for the ionic species. The usual ex- 
pressions (such as 12-74) must be modified in this manner to inchude the hydrogen ion concentration is such cases. Several instances where the $\mathrm{pH}$ affects the rate of inhibition have been reported (e.g., with the nitrophenols, malonate, iodoacetate, and cyanide) but discussion of these will be postponed to Chapter 14 where the effects of $\mathrm{pH}$ on inhibition will be considered in greater detail.

(C) The state of the membrane as related to functional activity. It is well known that the permeability properties of cell membranes are changed during the fluctuations in electric potential associated with excitation or activity in muscle, nerve, and gland cells. It is likely that most cells have an electric potential across their membranes, as a result of differences in ion concentrations between the cytoplasm and the external medium, and that this potential varies in response to many influences that play upon the cells. The penetration of any ionic inhibitor into a cell will be affected by the distribution of charge across the membrane, not only because of the purely electrostatic effects but perhaps due to the variations in membrane structure brought about by the changes in potential. Inasmuch as excitation of cells generally involves a depolarization of their membranes, it is probable that the permeability to inhibitors would depend on the level of activity or the fraction of the time that the membrane is depolarized. One might expect, therefore, that functionally active tissue would be inhibited more rapidly than resting tissue when the inhibitors are ionic, and perhaps even when they are not ionic. If cellular metabolism or secondary activities are measured, it would be difficult to separate such an effect from the possibly altered susceptibility of the metabolism or function itself, but if the inhibition of a particular enzyme could be followed, it would be possible to test this hypothesis. In any event, the role of fluctuating permeabilities to inhibitors should not be overlooked.

(D) Nonenzymic material and protecting substances. The binding of the inlibitor to nonenzymic substances in a cellular or tissue preparation will lower the effective inhibitor concentration and slow the rate of inhibition. Substances that readily combine with the inhibitor may actually prevent the development of the inhibition completely, as may be observed in the protection afforded by sulfhydryl compounds, such as dimercaprol (BAL), against inhibition by arsenicals. Since cellular preparations contain much material capable of reacting with most inhibitors, this must often be a distorting factor in the kinetics of inhibition.

(E) Metabolism of the inhibitor. Rapid inactivation of the inhibitor by the enzyme systems of the cell is another factor that can reduce the effective concentration within the cell. The kinetics of such systems have been discussed in Chapter 8 and the effective concentrations of inhibitor were there 
expressed in equations applicable to the steady state. The situation can be represented by the following reactions:

$$
\mathrm{I}_{0} \underset{\substack{k \\ \vdots}}{\stackrel{1}{\rightleftharpoons}} \mathrm{I}_{i}+\mathrm{E} \underset{k_{-1}}{\stackrel{k_{1}}{\rightleftharpoons}} \mathrm{EI}
$$

The spatial relationship between the susceptible enzyme and the enzyme inactivating the inhibitor can be an important factor in determining how effectively the inhibitor concentration at the susceptible enzyme is reduced. A rigorous kintic treatment is quite difficult but the behavior of the system will follow the curves in Fig. 12-23.

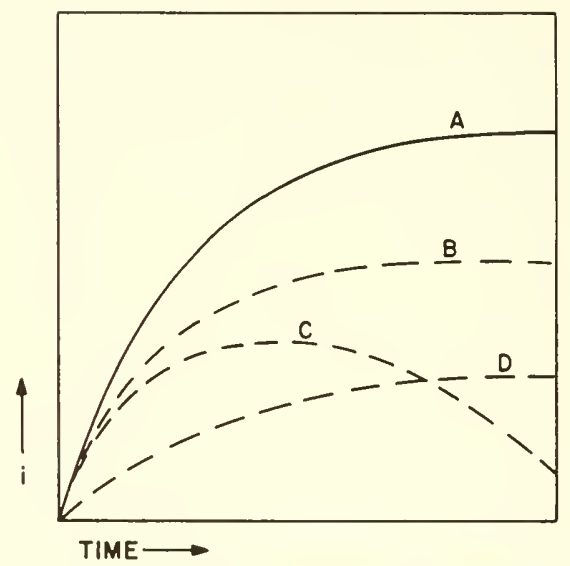

FIG. 12-23. Effect of metabolic inactivation of the inhibitor on the rate of inhibition (scheme 12-85). Curve A, no inactivation occurs. Curve B, inactivation progressively reduces the inhibitor concentration - inhibition irreversible. Curve C, same as B but supply of inhibitor is limited and the inhibition is reversible. Curve $\mathrm{D}$, inactivation prevents the inhibitor from reaching the same initial concentration (as when the inactivating system is effective before the inhibitor attacks the enzyme).

(F) Adaptation of the cell to the inhibition. Adaptation of metabolism to the action of an inhibitor is usually too slow to affect the rate kinetics of the inhibition, but occasionally adaptation is very rapid and shifts in meta- 
bolism occur during the development of the inhibition. Such adaptations are usually not those involved in true tolerance but are only relatively rapid adjustments in the cellular metabolism to the altered enzyme activity. The immediate dependence of electron-transport rates upon phosphate acceptors (such as ADP) would, under certain circumstances, allow for readjustments in respiratory rate in response to an inhibitor. A shift in metabolic pathways to the utilization of another type of substrate could conceivably occur when the pathway of one substrate is blocked. The buffering capacity of many multienzyme systems towards inhibition has been discussed in Chapter 7 and it is evident that such phenomena, usually involving changes in the concentrations of the intermediates, could readily slow down the rate of inhibition. Of course, when the inhibition develops slowly over several hours or longer, other types of adaptation, such as enzyme induction, may occur. The rates at which inhibitors kill microorganisms could well be influenced by this sort of adaptation.

$(G)$ Secondary changes occurring in cells. The primary inhibition on an enzyme may initiate processes that lead to depression of the metabolism or function secondarily. It is known that the inhibition of metabolism or function continues to increase progressively after the inhibitor is removed from the external medium in some cases. This could only be due to the continued development of the secondary changes induced by the inhibitor that is bound within the cells. A good illustration of this is provided by the action of iodoacetate on the heart, where both the respiration (W ebb et al., 1949 b) and function (Webb, 1950a) continue to decline after the iodoacetate is washed out. The action of phenols upon bacteria has been shown (Cooper, 1912) to occur in two phases: an initial absorption or fixation, which is followed by a slower protein denaturation leading to the death of the cells. Since the cell is a closely organized unit, it is quite possible with a strategic interruption of enzyme activity to initiate a sequence of events that will eventually produce total dissolution of the cell.

(H) Multiple actions of the inhibitor. Since inhibitors are unfortunately seldom specific in their actions, the kinetics are often complicated by the simultaneous occurrence of two or more inhibitory processes developing at different rates. To use iodoacetate again as an example, it is clear that this inhibitor can affect various components involved in the total respiratory rate, not only the sulfhydryl enzymes (e.g., phosphoglyceraldehyde dehydrogenase, 6 -phosphofructokinase, pyruvate and $\alpha$-ketoglutarate dehydrogenases, isocitric dehydrogenase, succinic dehydrogenase, and others) but possibly also nonenzymic units functioning structurally in the cell. In such cases, the kinetic data are simply not interpretable in terms of any single reaction.

The study of cellular inhibition kinetics has scarcely begun and a great deal more accurate data must be available before a logical attack can be 
made. Much excellent work has recently been done on the penetration of drugs into cells and tissues, chiefly using radioactively labeled material, but very little information on the commonly used inhibitors has been reported. It is necessary to obtain not only accurate rate data for these inhibitions, but to accumulate evidence for penetration rates and the operancy or inoperancy of the factors discussed above.

\section{Illustrative Examples of Cellular Inhibition}

We shall now present some specific results obtained on varions types of cellular preparations in order to emphasize a few fundamental principles and to indicate how simple analyses of the data have been carried out in the past.

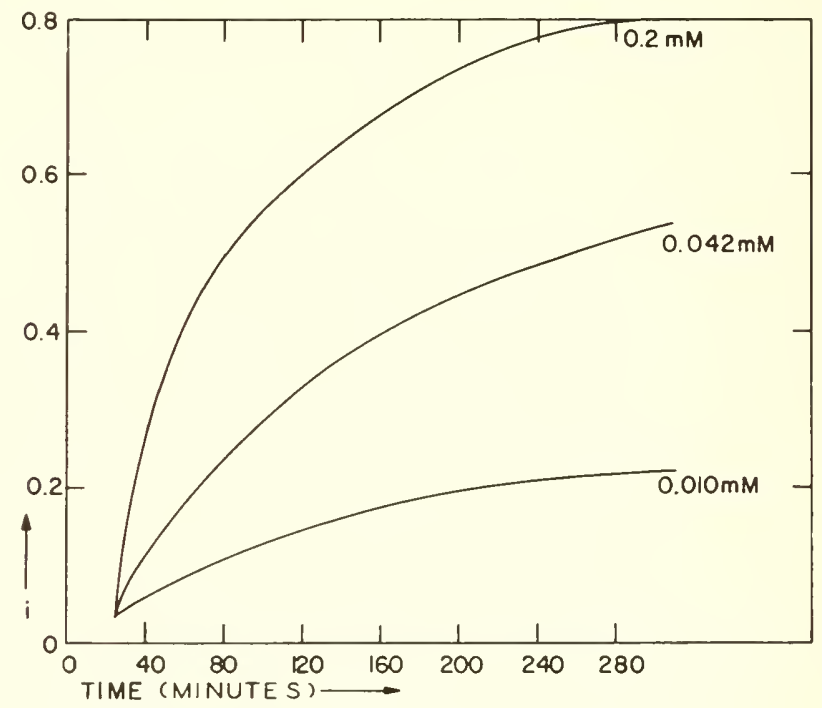

FIG 12-24. Inhibition of glycolysis in rat spleen slices by $\mathrm{HgCl}_{2}$ (From Jowett and Brooks, 1928.)

(A) The inhibition of glycolysis in rut spleen slices by $\mathrm{HgCl}_{2}$. Jowett and Brooks (1928) found that the rate of inhibition varied with $\mathrm{HgCl}_{2}$ concentration in the manner shown in Fig. 12-24. It is obvious that the curves do not all start from the origin and that a short lag period is present, but since no observations were made in less than $25 \mathrm{~min}$. the course of the curves in this region cannot be recorded. This was the first demonstration of an exponential effect of an enzyme inhibitor in which some attempt to analyze the data kinetically was undertaken. They fitted the data to the simple generalized expression:

$$
v=v_{o} e^{-a t}
$$


but found $\alpha$ to vary with the concentration: $\alpha=0.41$ for $0.2 \mathrm{mII}, 0.17$ for $0.042 \mathrm{~m} . \mathrm{H}$, and 0.07 for $0.01 \mathrm{~m} M$. Actually, this equation assumes that the inhibition will eventually be complete, whereas from the shapes of the curves this does not seem likely. When equations of the type 12-1 and 12-3 are used and estimates are made of the probable final inhibition, $\alpha$ does not vary as much with concentration: $\alpha=0.66$ for $0.2 \mathrm{mMI}, 0.38$ for 0.042 $\mathrm{m} M$, and 0.44 for $0.01 \mathrm{~m} M$, with an average of $0.49 \mathrm{~h}^{-1}$. They posed the problem as to whether the observed rate was due to the diffusion into the tissue or to the reaction with the enzymes, and concluded from indirect experiments that diffusion was relatively rapid (equilibrium achieved in 1-2 min) and that the results could best be explained on the basis of a unimolecular reaction of $\mathrm{HgCl}_{2}$ with the enzymes. They finally point out that a disturbing factor is the reduction in $\mathrm{HgCl}_{2}$ concentration due to bind-

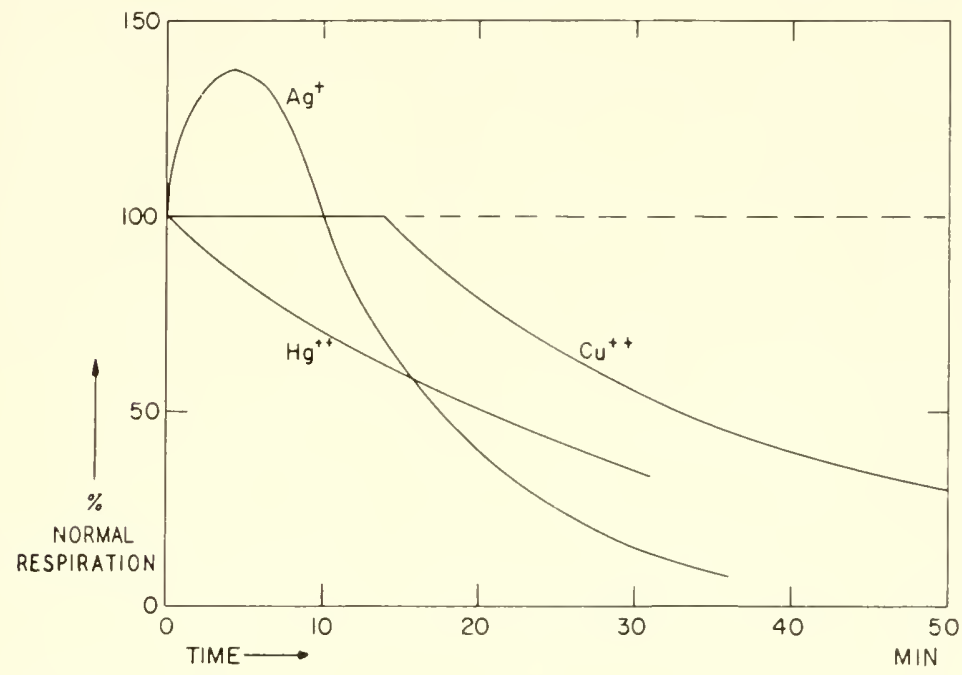

FIG. 12-25. Effects of heavy metal ions on the respiration of Aspergitlus. $\left(\mathrm{Ag}^{+}\right)=0.1 \mathrm{~m} M,\left(\mathrm{Cu}^{++}\right)=7.5 \mathrm{~m} M$, and $\left(\mathrm{Hg}^{++}\right)=0.2 \mathrm{~m} M$. (From Cook, 1926 a.)

ing to the tissue; this would have certainly reduced the rate of inhibition progressively, especially at the lower concentrations.

(B) Effects of heavy metals on the respiration of Aspergillus. An interesting attempt to interpret inhibition rate curves was made by Cook (1926 a), who found a variety of responses in the respiration of the mold. Aspergillus niger, to the heary metal ions (Fig. 12-25). One may notice immediately that these rate curves indicate some differences in the mechanism of action of these heavy metals. Many such differences between inhibitors are prob- 
ably missed by determining the inhibition over a single arbitrary interval; if here the inhibition had been observed only at $30 \mathrm{~min}$, the results would have been unimportant and indicative of nothing fundamental. Secondly, one is struck by the different types of curves obtained for the three heavy metals - the immediate action of $\mathrm{HgCl}_{2}$, the definite latent period of $\mathrm{Cu}^{++}$, and the initial stimulation produced by $\mathrm{Ag}^{+}-$and these differences alone would lead us to expect the kinetics to be complex. The biphasic action of $\mathrm{Ag}^{+}$could be explained on the basis of two kinds of effects on the respiration occurring at different rates, but the mechanism by which the stimulation is produced is unknown. The immediate action of $\mathrm{HgCl}_{2}$ is not unreasonable since a large fraction of the $\mathrm{HgCl}_{2}$ would be un-ionized and would penetrate readily and then the latent period observed with $\mathrm{Cu}^{++}$ might be inferred as due to a delayed penetration into the cells. However, Cook (1926 b) subsequently found that the explanation of the latent period is not quite as simple as this. In the first place, Nitelle and Valonia also demonstrated a definite latent period and here the penetration of $\mathrm{Cu}^{++}$ could be measured; it was found that $\mathrm{Cu}^{++}$enters the cells quite rapidly and this by itself could not be responsible for the lag in inhibition. In the second place, experiments with Aspergillus, wherein the $\mathrm{Cu}^{++}$was washed out at varying times after exposure, indicated that some initial reversible reaction occurred preliminary to the respiratory depression. Cook assumed that there are two consecutive reactions, the first "activating" the $\mathrm{Cu}^{++}$ so that it was then able to block respiration. It was furthermore found that the latent period (LP) was not constant but varied inversely with the concentration of $\mathrm{Cu}^{++}$according to an equation of the type $\mathrm{LP}=a\left(\mathrm{Cu}^{++}\right)^{-b}$ where $a$ and $b$ are constants. Thus the latent period is not merely a "biological lag" requiring certain events to occur before respiration is inhibited. This problem cannot be solved today because the basic mechanism by which $\mathrm{Cu}^{++}$inhibits respiration is unknown.

(C) Effects of iodoacetate on respiration and glycolysis. The inhibition of enzymes by iodoacetate has been known to be a relatively slow process since the earliest work on this inhibitor (Lohmann, 1931). Thus at the commonly used low concentration of iodoacetate $(0.1 \mathrm{mM})$, depression of lactate formation is muscle extracts progressively increased over 1 to 2 hr, although high concentrations (above $1 \mathrm{~m} M$ ) inhibited fairly rapidly. It is now known that the principal reaction of iodoacetate with these enzymes is an alkylation of sulfhydryl groups and that certain of these sulfhydryl groups react more rapidly than others. Inhibition of glycolysis in intact muscle is also slow and the question arose whether this delay was due in part to the penetration of iodoacetate into the tissue. Meyerhof and Boyland (1931) determined the uptake of iodoacetate in frog sartorius muscle simultaneously with measurements of glycolytic inhibition (Fig. 1226). Uptake was almost complete between 20 and $30 \mathrm{~min}$ but glycolysis 
continued to be inhibited progressively for some time afterward at room temperature, whereas at $1^{0}$ both processes were slowed and would appear to run equivalent courses. Iodoacetate reached levels in the muscle about 1.5 times that in the medium and about half of this could be removed by prolonged washing of the muscle. Thus the uptake curves presumably record both reacted and free iodoacetate. It is therefore diffleult to decide if the penetration of the iodoacetate through the cellular membranes is an important factor in the kinetics. It is perhaps safe to conclude with

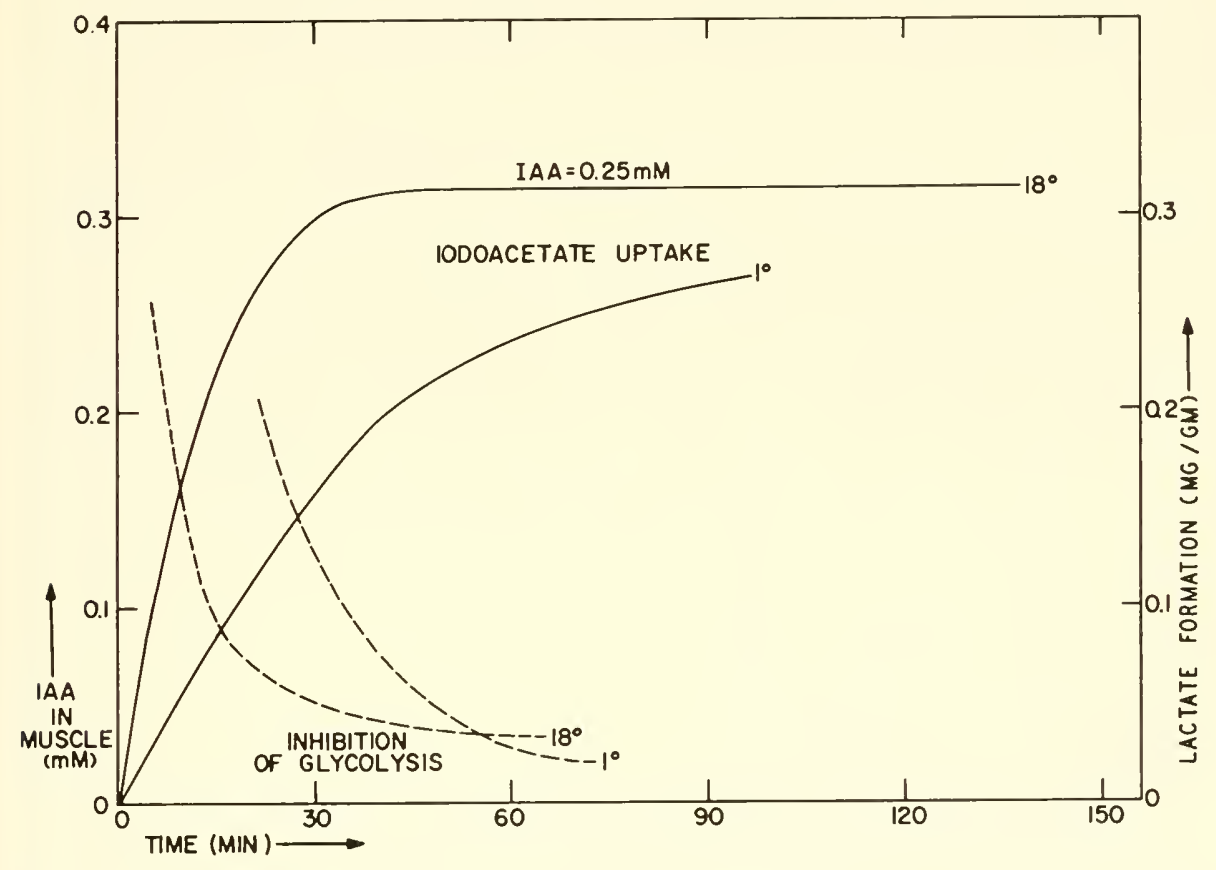

FIG. 12-26. Uptake of iodoacetate by frog sartorius muscle and the resulting inhibition of glycolysis at two different temperatures. The solid lines represent the uptake and the dotted lines the depression of the lactate formation. (From Meyerhof and Boyland, 1931.)

Meyerhof and Boyland that both penetration and reaction with enzymes contribute. Comparing these results with those of Lohmann (1931) on muscle extracts, one would be inclined to attribute the major part of the delay to the slow alkylation of the enzymes. Ghaffar (1935) investigated this question by the determination of the time course of iodide release from iodoacetate during the alkylation reactions in the muscle (Fig. 12-27). It is evident that an appreciable inhibition of glycolysis occurred before much iodide was released; in fact, after complete glycolytic block, iodide 
continued to be released for several hours. The difficnlty of interpretation here lies in the fact, as mentioned above, that there are many different sulfhydryl substances in the cell and their reactivity with iodoacetate varies greatly. As a consequence, it is possible to assume that the course of glycolytic inhibition measures the reaction of iorloacetate with especially susceptible sulfhydryl groups on phosphoglyceraldehyde dehydrogenase and that the bulk of the alkylation results from reaction with "nonglycolytic" sulfhydryl groups.

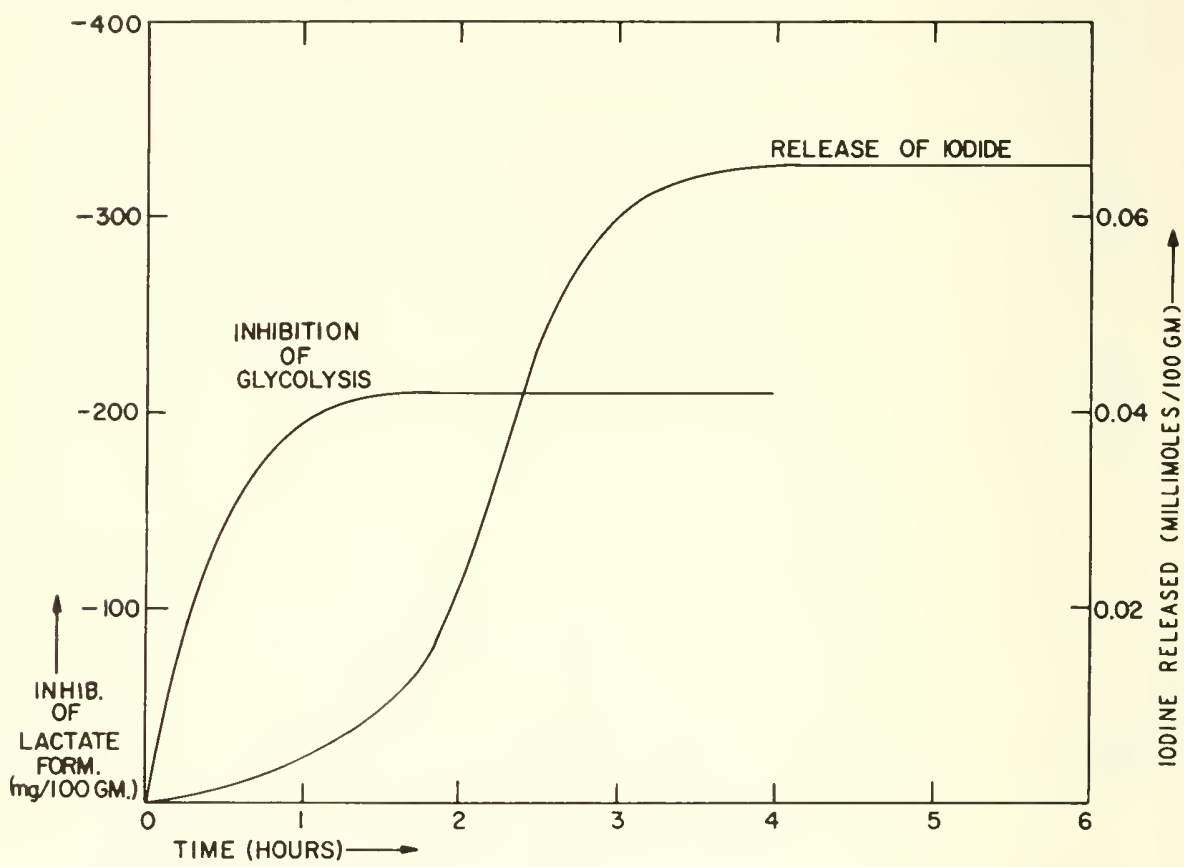

FIG. 12-27. Release of iodide from iodoacetate in frog muscle and the rate of inhibition of glycolysis. Iodoacetate was $1 \mathrm{~m} . \mathrm{I}$ and the temperature was $40^{\circ}$. (From (Ghaffar, 1935.)

The inhibition of alycolysis by iodoacetate in Streptobacterium casei was studied by Field and Fiekl $(1932 \mathrm{a}, \mathrm{b})$ and a definite latent period was demonstrated; at $3.8 \mathrm{~m} . \mathrm{I}$ iodoacetate, no inhibition was noted for $65 \mathrm{~min}$, and at $7.7 \mathrm{~m} . / \mathrm{t}$ the latent period was reduced to $35 \mathrm{~min}$. These results would imply a permeability harrier of some importance in these cells. Once the inhibition begins, the course is as shown in Fig. 12-28, which was interpreted as bimolecular but it could just as well be representative of a monomolecular reaction or a bimolecular reaction with one reactant in excess. Such a prolonged latent period followed by rather sudden development 
of the inhibition might indicate an initial reaction with the membrane leading to a breakdown of the barrier, in which case the curve in Fig. 12-28 would record the rate of reaction with the glycolytic enzymes.

The action of iodoacetate on nerve axons is definitely limited by a slow rate of penetration into the cells. At a concentration of $1.4 \mathrm{~m} . \mathrm{I}$ iodoacetate causes a slow fall in the respiration over $6 \mathrm{~h}$, while concentrations as high as 50-70 m.I have little effect within an hour (Chang and Gerard, 1933). If the nerve sheath was split, the inhibition occurred more rapidly, proving directly that a permeability barrier was present, but even then the rate of inhibition was not very rapid so that some delay resulted from the intracellnlar reactions also. Isolated brain behaves similarly and exhibits a latent period that is inversely proportional to a low power of the iodoacetate concentration. as shown in rat cortex slices by Fuhrman and Field (1943). The tabulation below has been assembled from their curves.

\begin{tabular}{|c|c|c|c|}
\hline \multirow{2}{*}{$\begin{array}{c}\text { Iodoacetate } \\
\text { concentration } \\
(\mathrm{m} . \mathrm{H})\end{array}$} & \multirow{2}{*}{$\begin{array}{l}\text { Latent } \\
\text { period } \\
(\text { min) }\end{array}$} & \multicolumn{2}{|c|}{${ }^{o}$ o Inhibition $3 \mathrm{hr}$ after iodoacetate } \\
\hline & & Respiration & Glycolysis \\
\hline 0.01 & 90 & 12 & 46 \\
\hline 0.05 & 68 & 31 & - \\
\hline 0.1 & 50 & 42 & 61 \\
\hline 0.5 & 45 & 48 & - \\
\hline 1 & 40 & 52 & 68 \\
\hline 5 & 20 & 62 & - \\
\hline 10 & 12 & 67 & 78 \\
\hline 50 & 8 & 72 & - \\
\hline
\end{tabular}

Actually the latent period is somewhat shorter for glycolysis than for respiration, which might be expected on the basis that the respiratory depression follows secondarily to the glycolytic inhibition. When the brain tissue was made into a cell-free brei, the latent period was not reduced; this surprising observation would incicate the unimportance of penetration in determining the rate of inhibition, but certainly such long latent periods in cell-free preparations are contrary to what has been observed in muscle and other tissue extracts. When the $\mathrm{pH}$ was lowered from 7.3 to 5.8 , the latent period was shortened and Fuhrman and Field felt this might implicate permeability factors, but actually all that is done by lowering the $\mathrm{pH}$ is to increase the concentration of the permeant undissociated acid and either penetration or reaction with enzymes would be expected to be increased.

Rate studies of metabolic inhibition have occasionally been used in conjunction with measurements of functional depression in order to determine 
if any correlation exists between the metabolic pathways disturbed and the activity of the cell. Holmes (1933) perfused frogs with media containing iotloacetate at different concentrations and found that the glycolysis was inhibited fairly rapidly (Fig. 12-29) in the brain, followed by a fall in the creatine phosphate levels. However, nerve function, as evidenced by the ability to respond to stryehnime, did not fall appreciably during this phase and began to be depressed only after glycolysis was $90 \%$ blocked, after which it clecreased linearly to a complete loss of function. Nevertheless, it is not justifiable to conchude that function is unrelated to glycolysis or

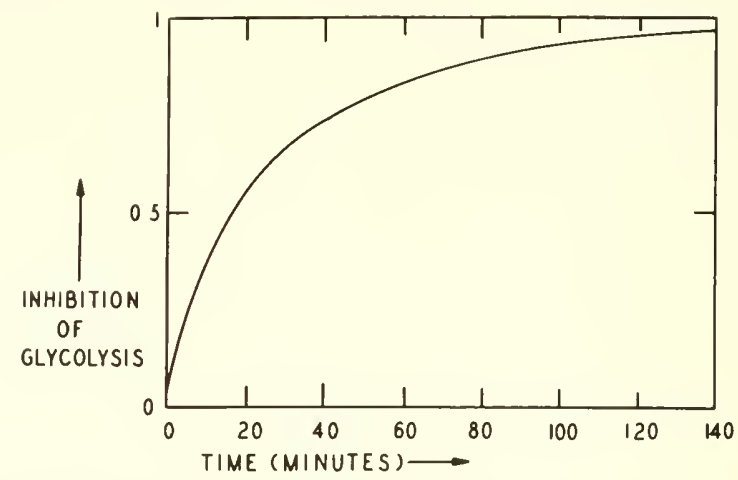

Fra. 12.28 Inhibition of glycolysis in Streptobacterium casei by iodoacetate $(1.1 \mathrm{~m} / \mathrm{H})$. This curve does not include the long latent period before inhibition begins. The bimolecular rate constant, $k$, would be $6.9 \times 10^{-4}$ or the bimolecular rate constant (with one reactant in excess) would be 40 liters mole $\mathrm{min}^{-1}$. (From Field and Field, 1932 b.)

creatine phosphate. In the first place, one does not know just how far it is possible to lower glyeolysis or creatine phosphate without disturbing the function; a fair margin of safety might occur in normal tissue. In the second place, it is now known that it is probably not the ereatine phosphate level which is functionally important, but the level of ATP, which can remain at near normal values until the creatine phosphate has fallen below a critical concentration. In any event, such behavior illustrates well the varying rates at which different processes in the cell can be altered by an inhibitor.

Iodoacetate depresses cardiae function slowly and an attempt was made by Gardner et al. (1954) to elucidate some of the controlling factors. They determined how the rate of inhibition was altered by ehanges in iodoacetate concentration, temperature, and beat frequency (Fig. 12-30). At $37^{\circ}$ the contractility of rabbit atria is more sensitive to iodoacetate than is the spontaneous rate (curves $\mathrm{A}$ and $\mathrm{B}$ ) and a difference is also seen in the 
way an increase in iodoacetate concentration accelerates the inhibition rate. This might be due to different permeabilities of pacemaker and ordinary atrial cells to iodoacetate but is more likely the result of different degrees of dependence of the functions on the susceptible metabolism. Since the lowest concentration used $(0.04 \mathrm{~m} . I)$ stopped anaerobic activity in the atria within 5 to $10 \mathrm{~min}$, one would not expect an increase in concentration above this to increase the rate of inhibition under aerobic conditions. It was suggested that iodoacetate might have a second action in

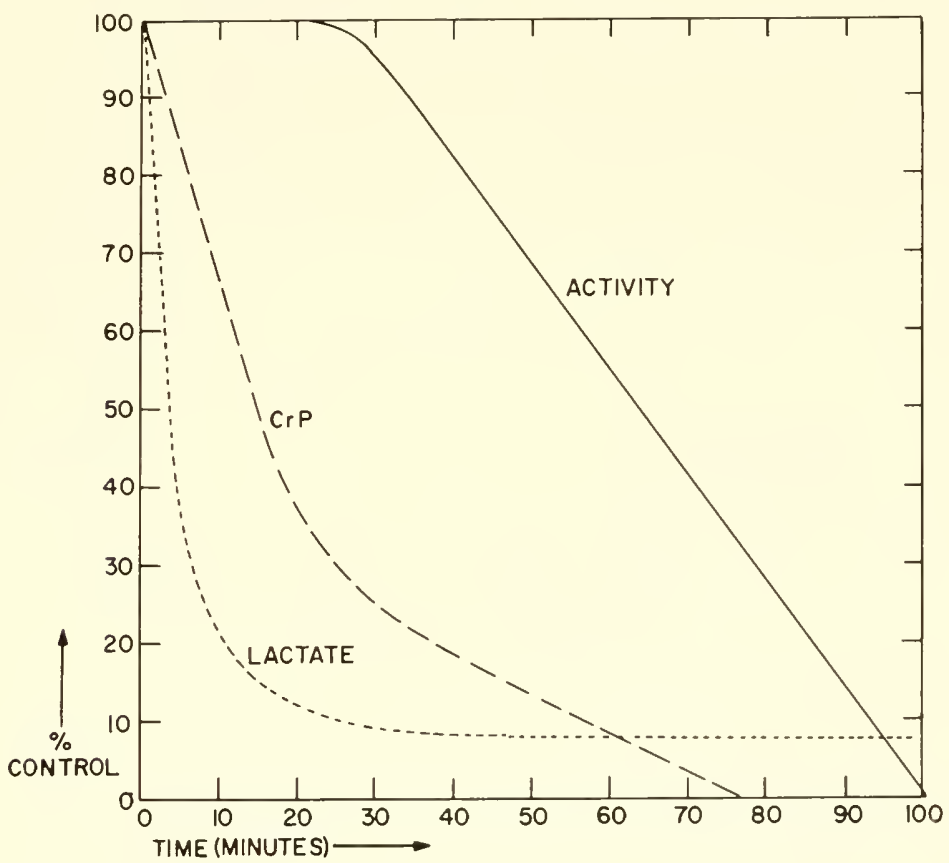

Fig. 12-29. Changes in the frog central nervous system during perfusion with iodoacetate. Inhibition of glycolysis is indicated by the curve for lactate and this is followed by a fall in creatine phosphate (CrP). The activity was measured by the response to strychnine. (From Holmes, 1933.)

addition to the inhibition of glycolysis, and that this second action might play the major role in functional clepression aerobically. Since glycolysis does not appear to be very important in cardiac tissue under aerobic conditions and most of the energy for function is derived from the oxidation of lipids, it is possible that this second action is on fatty acid oxidation or more likely on the tricarboxylic acid eycle. These points will be discussed in detail in the chapter devoted to iodoacetate inhibition. The effect of increasing frequency of the beat on the rate of inhibition can be 
interpreted in two immediately obvious ways. Either the increased number of membrane depolarizations allows the iodoacetate anion to penetrate more rapidly, or the increased functional activit y exerts a greater metabolic demand and consequently depletes the tissue more rapidly of energyrich substances.

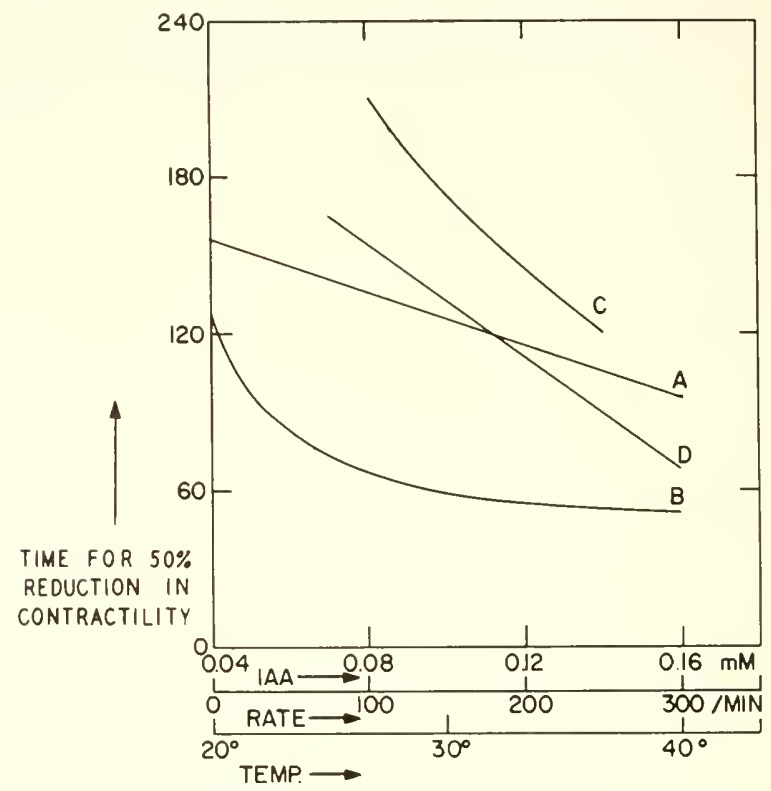

FIg. 12-30. Effects of various factors on the rate of action of iodoacetate on rabbit atria. (From Gardner et al., 1954.) ('urve A. effect of iodoacetate concentration on the atrial rate. Curve $B$, effect of iodoacetate concentration on the amplitude of the contraction. Curve $\mathrm{C}$, effect of stimulus frequency on the response of contraction to iodoacetate. Curve D. effect of temperature on the response of contraction to jodoacetate.

(D) Effects of hany metal ions on diuphragm respriration. One of the most thorongh studies of the factors involved in the kinetics of a celtular inhibition was done by Demis and Rothstein (1955). They found that the nytake of glucose by rat diaphragm muscle was very sensitive to $\mathrm{Hg}^{++}$(it will be convenient to designate mercury in this way although most of the metal in solution exists in complexes with anions), $0.2 \mathrm{~m} .1 /$ inhibiting almost completely in $20 \mathrm{~min}$ (Fig. 12-31). Respiration, on the other hand. was much less sensitive to $\mathrm{Hg}^{++}$; a lag period of over $30 \mathrm{~min}$ was observed before any inhibition occurred and after $2 \mathrm{hr}$ the inhibition had reacherl 
a steady level of aromd $30 \%$. Using different concentrations of $\mathrm{Hg}^{++}$, it was shown that glncose uptake was about 10 times more sensitive to $\mathrm{Hg}^{++}$ than was the respiration. However, the respiration of homogenates of diaphragm was inhibited fairly rapidly, there being no bag period and a maximal effect occmred within 20 min. The lay period and the slow rate of respiratory inhibition would thus indicate a delay in the penetration into the cells. The
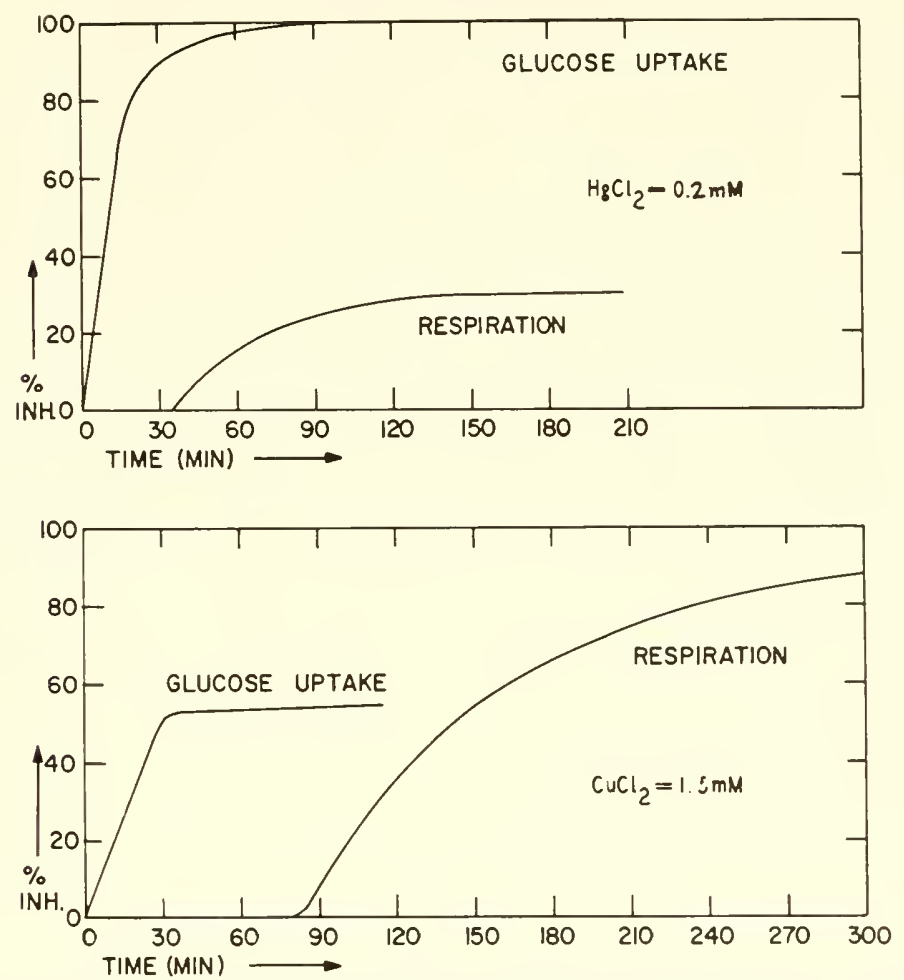

FIG. 12-31. Inhibition of the ghucose uptake and the respiration of rat diaphragm by $\mathrm{HgCl}_{2}$ and $\mathrm{C}^{4} \mathrm{Cl}_{2}$. (From Demis and Rothstein. 1955.)

uptake of ylucose probably involves reactions oceurring at the surface of the cells and thus is inhihited more readily than the respiration. Confirmation for this was obtained by histochenical localization of $\mathrm{Hg}^{++}$after exposure of the tissue for varying periods of time. There was a tendency for the metal to be concentrated initially at the cell boundaries with penetration into the cells occurring later. the rates of these processes corresponding well to the inhibitions of glucose mptake and respiration. The uptake of $\mathrm{Hg}^{203}$ by dianhragm was then determined and foumd to obey first-order kineties for the first 20 to $30 \mathrm{~min}$. The final concentration of $\mathrm{Hg}^{++}$in the 
cells was about 100 times that in the medium indicating that most of the metal in the tissue is bound to some cell components. Of course, in diaphragm, or any other tissue, if the respiration is mainly dependent on exogenous glucose, the oxygen uptake will be inhibiter because of the depression of ghucose uptake into the cells, so that exogeneous glucose respiration should be more sensitive to $\mathrm{Hg}^{++}$than endogenous respiration would be.

The effects of $\mathrm{Cu}^{++}$were different in some resuects from those of $\mathrm{Hg}^{++}$ (Fig. 12-31). The latent period in the inhibition of respiration was even more pronomced, perhaps indicating a slower penetration into the cells, but the respiration eventually was inhibited more strongly than the uptake of glucose. It is quite possible in the case of both of these metals that the early binding to the cell membranes gradually altered the permeability properties until the metals could penetrate more readily into the cells. The lag period might then be interpreted as the time required for the membranes to be sufficiently damaged to allow penetration.

(E) Inhibition of cholinesterase in erythrocytes. The kinetics of the inhibition of acetylcholinesterase in the red blood cells of the goat were studied by Aldriclge (1950), using organic phosphates such as E-600 ( $p$-nitrophenyl diethylphosphate). The inhibition rate followed that expected from a bimolecular reaction where one of the reactants is in excess (Fig. 12-32). There was no evidence at all that the erythrocyte membrane was in any way influencing the course of the reaction, the kinetics being those to be expected if the enzyme were in true solution. When the time to produce $50 \%$ inhibition was plotted against the reciprocal of the inhibitor concentration, a straight line was obtained. An equation related to 12-16 was applied, which is justifiable because of the irreversibility of the inhibition. For $50 \%$ inhibition, this equation may be rewritten as:

$$
k t_{0.5}(\mathrm{I})=-\ln 0.5=0.69
$$

where $t_{v .5}$ is the time to produce this degree of inhibition, so that a plot of $t_{0.5}$ against $1 /(\mathrm{I})$ should give a straight line with slope $0.69 / k$. The rate constant was calculated as $1.1 \times 10^{6}$ liters mole $\mathrm{min}^{-1}$. Thus in this case it was possible to investigate accurately the inhibition of an enzyme within cells, demonstrating that penetration factors do not invariably complicate the kinetics.

\section{RATES OF LETHAL ACTION IN A CELL POPULATION}

When the rate of action of an inhibitor is investigated by determinations of the time course of the lethal effect on a population of cells or organisms, complications are introdnced into the interpretation of the data in terms of the primary inhibitory reactions because of the natural variation in the 
susceptibilities of the cells or organisms to the inhibitor. A curve obtained by plotting the fraction of cells killed (or surviving) against the time of exposure to the inhibitor cannot be interpreted directly as an expression of inhibition rates. With respect to a single cell. one is clealing here with an all-or-none phenomenon. namely the death of the cell, and the course
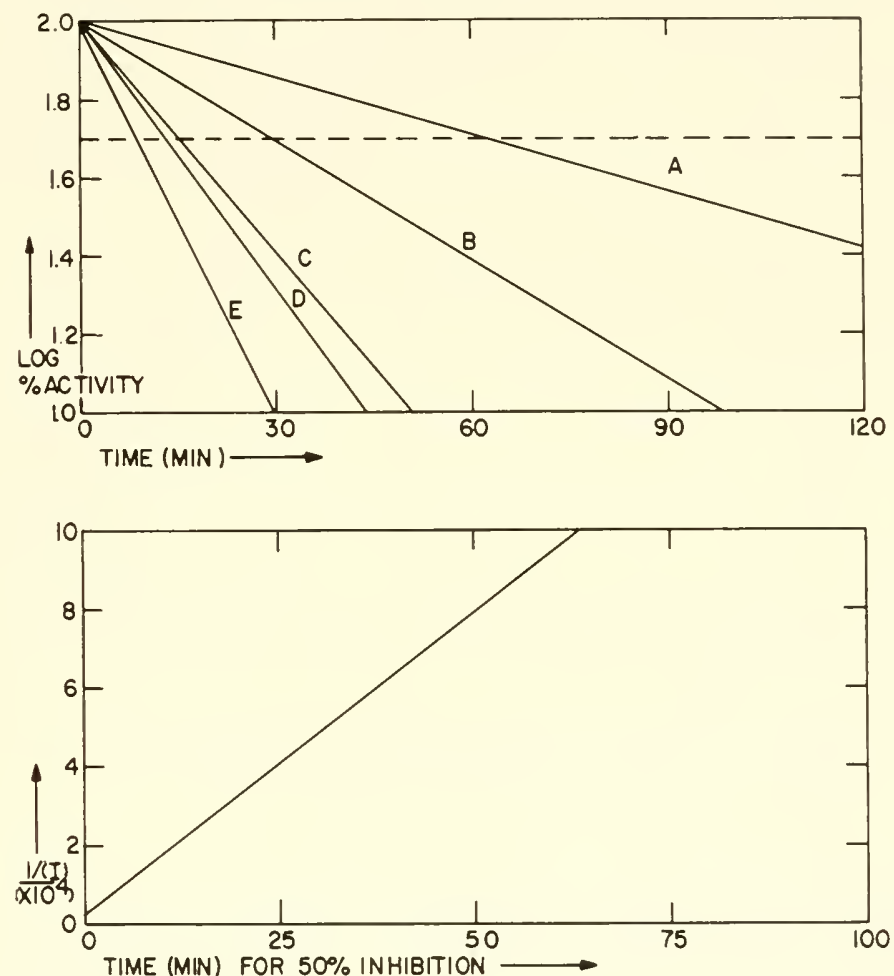

Frg. 12-32. Inhibition of goat erythroeyte acetylcholinesterase by $p$-nitrophenyl diethylphosphate (E-600). The upper curves show the effeet of the inhibitor coneentration on the rate of inhibition. Curve $A, 1.04 \times 10^{-5} \mathrm{~m} . \mathrm{I}$; curve $\mathrm{B} .1 .9 .5 \times 10^{-5} \mathrm{m.I}$; curve C. $3.90 \times 10^{-5} \mathrm{~m} . \mathrm{H}$; curve D. $4.16 \times 10^{-5} \mathrm{~m} . \mathrm{H}$; eurve E. $7.80 \times 10^{-5}$ $m, I$. The lower plot $(\mathrm{Eq}, 1-2-5 \mathrm{~T})$ enables the bimolecular rate eonstant to be determined $\left(k=1.1 \times 10^{6}\right.$ liters mole $\left.\mathrm{min}^{-1}\right)$.

of the effects within the cell leading to death is not measured. To illustrate this let ns take an extreme case. Assume that each cell in the poponlation has the same susceptibility to the inhibitor. In the presence of a lethal concentration of the inhibitor, all of the cells wonk die simnltaneonsly after a latent perior determined by all the rates of the processes induced 
by the inhibitor and progressing to death. However, since it appears that all eell popmlations show some type of variation between the individual cells, the rate at which the cells die will be determined solely by this variation. The absohte latent period before any cell deaths oceur will be the only measmre of the rate at which the inhibitor penetrates into the cells and exerts its final effect.

The problem of the relationship between the nature of the variation in susceptibility and the time conrse of the lethal action will now be considered. If the distribution of susceptibility is symmetrieal around the mean susceptibility (Fig. 12-33, eurve A), a characteristically symmetrical sig-

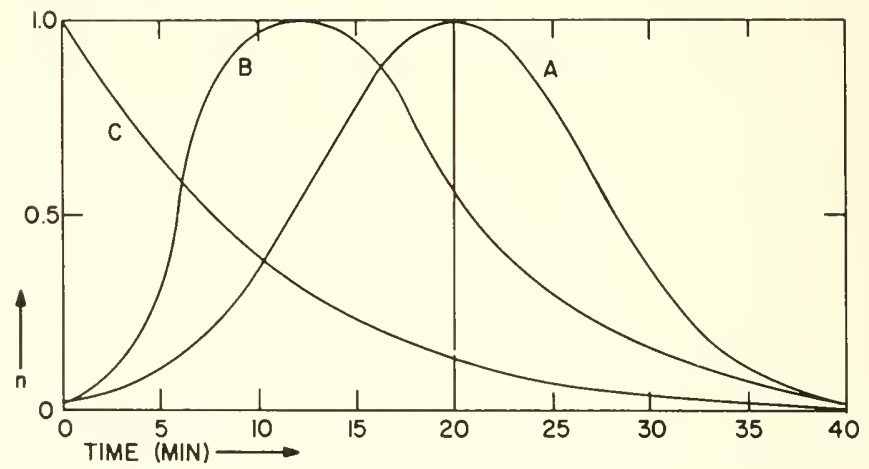

FIG. 12-33. Different distributions of susceptibility in cell populations. Curve A represents a symmetrieal distribution (Eq. 12-88) with $a=1$ and $b=0.1$. Curves $B$ and $C$ show hypothetical skewed distributions.

moid curve for the death rate is obtained (Fig. 12-34, eurve A). It is assumed here that the mean susceptibility is such that the greatest number of cells die at $20 \mathrm{~min}$ after exposure to the inhibitor. and the enrve is plotted aceording to the equation:

$$
n=a e^{-b t^{2}}
$$

where $n=$ number of cells dying at any time $t$, and $a$ and $b$ are constants that determine the form of the distribution curve. This form of equation is nsually accepted as rejresenting most natural or hiological distributions. For the sake of simplicity, it has heem assumed that there is no absolute latent period, i.e., that the most susceptible cells begin to die immediately. If the variation is not symmetrical but is skewed as in eurve B of Fig. 12-32, the death rate curve will he altered (Fig. 12-34. curve B). If the distribution is very asymnetrical as in curve C of Fig. 12-33. where the greatest number of rells die immertiately but more resistant cells also oceur, the death 
rate is no longer sigmoid but is of a hyperbolic or exponential form (Fig. 12-34, curve C). This latter type of curve actually is approached in some cases and most of the published data indicate that the variation curve is indeed generally skewed to the left.

Inch has been written about death rates that obey a so-called "monomolecular" time course and the demonstration of such often has been interpreted in terms of the nature of the reaction of the lethal agent with the cell or the processes that this reaction produces. Such a monomolecular death rate is usually adduced by obtaining a straight line when the logarithm of the fraction of surviving cells is plotted against time. It should

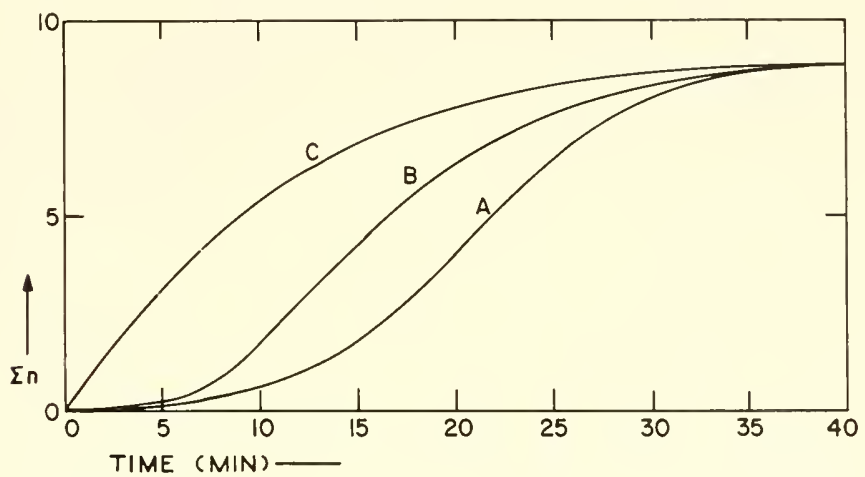

FIt. 12-34. Death rate curves obtained from the distribution curves in Fig. 12-33 by summation.

be evident that such a procedure cloes not provide information about the reaction of the inhibitor at all but only about the rariation between the cells or organisms. Plots of this type are given in Fig. 12-35 for the distributions shown in Figure 12-33. It may be seen that the curves become more linear as the distribution curve is more skewed to the left and that a truly linear curve is obtained only from the extreme distribution of curve C. Linearity of such curves merely implies that the distribution of cells more resistant than the cells of the eommonest susceptibility is exponential.

Perhaps the first work clearly showing that a skew variation in susceptibility to an inhibitor can accomnt for apparent monomolecular behavior was that of Peters (1920). Cultures of Colpidium were exposed to $0.2 \mathrm{mII}$ $\mathrm{Hg}^{++}$. and the percentage of organisms killed after rlifferent time intervals was determined. Figure 12-36 shows the curve and logarithmic plot of the per cent survivors. A straight line was obtained between 10 and $45 \mathrm{~min}$ but this monomolecular behavior was correctly interpreted as arising from a variation curve actually not much different from curve C in Fig. 12-32 with a latent period of $10 \mathrm{~min}$. The forms of these curves thus have no bear- 
ing on the nature or kinetics of the inhibition by $\mathrm{Hg}_{\mathrm{g}}++$ but only show that $\mathrm{Hg}^{++}$kills the individual cells after varying perions of time.

Variations between cells in tissue preparations can likewise modify the rate of response to an inhibitor. Tissnes are generally studied either as slices or strips. or occasionally isolated and intact, but in all cases there is significant inhomogeneity. In the first place, aside from the cells that are

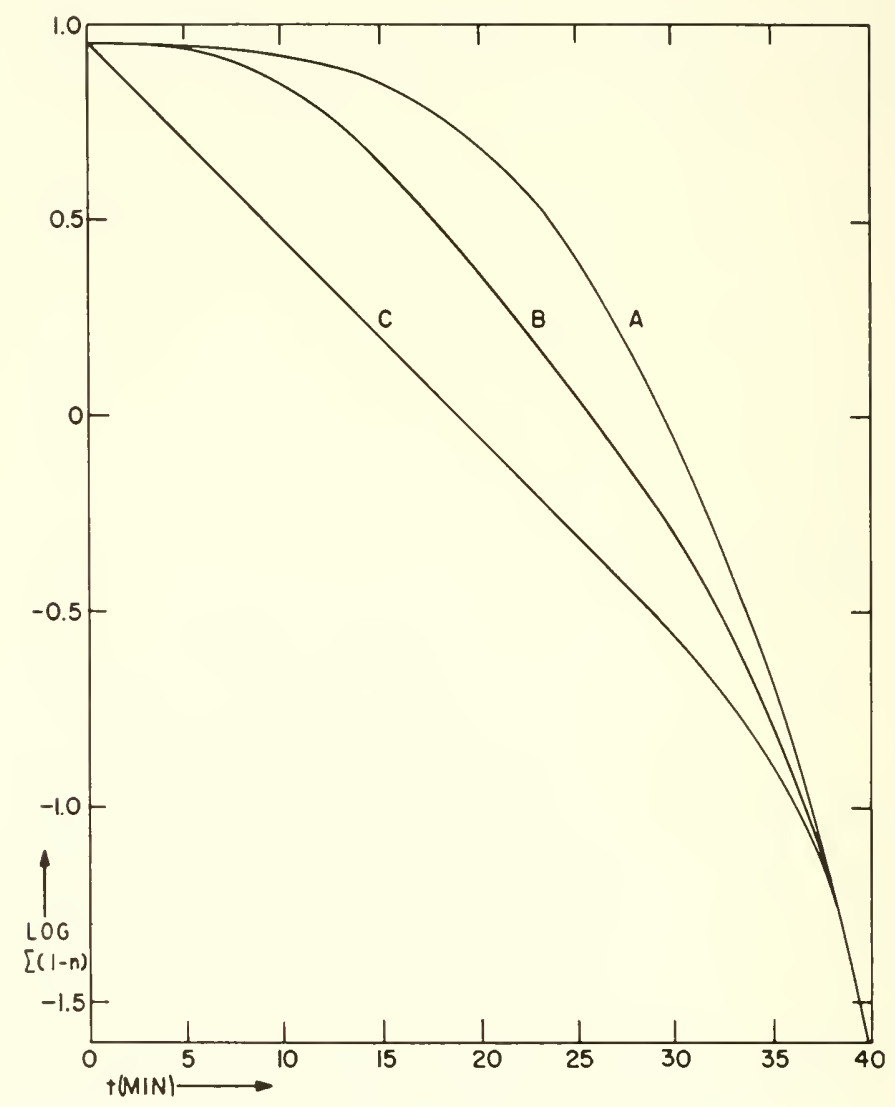

FIG. 12-35. I'lots of the logarithm of the survivors against time for the distribution curves in Fig. 12-33.

primarily being stutied, there are connective tissue cells. blood vessel cells, and often many other types. In the second place, there is very likely to be variation between the primary tissue cells; that is, in isolated nterine or atrial strips, for example, the smooth muscle or myocardial cells are not all alike. In addition to histological demonstrations of differences there is some evidence from cellular membrane potentials that the population 
of cells in a contractile tissue is quite variable (Webb, 1956), indicative perlaps of differences in membrane permeability, ionic distribution, and metabolically-linked active transport. The metabolism of such preparations is thus made up of contributions of varying degree from these different types of cells. The rate at which an inhibitor produces a depression of respiration in a tissue. for example, would also be composite in nature, although here the time course of the effect would depend not only on the variation but also on the true rate of inhibition. In addition to these factors, there is the further problem of the penetration of the inhibitor into

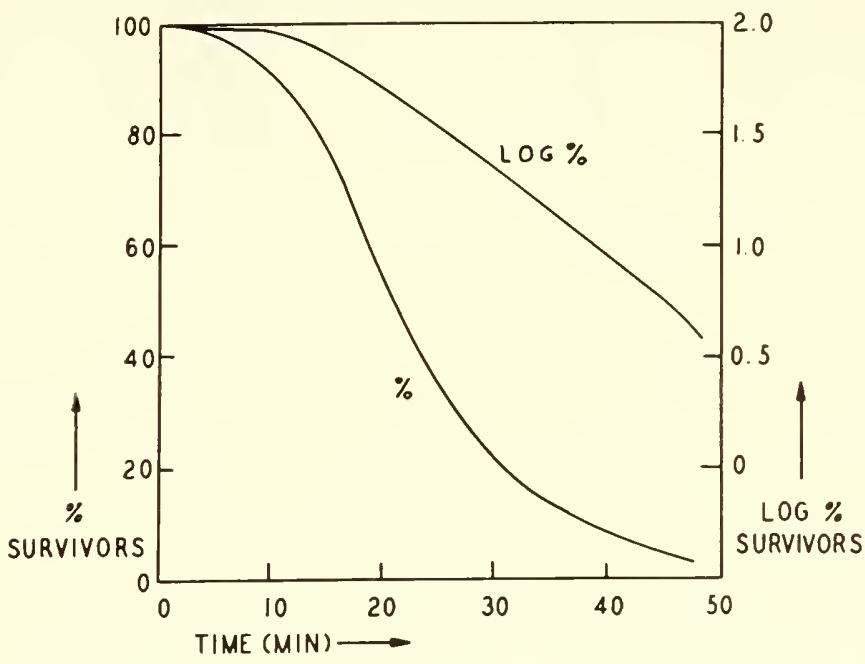

FIG. 12-36. Death rate curve for Colpidium exposed to 0.2 m.1I $\mathrm{HgCl}_{2}$. (From Peters, 1920.)

the inner layers of the tissue. Certainly many inhibitors do not rapidly reach the cells within a tissue or tissue slice when applied in the medium bathing the preparation, and this would, of conrse, make the observed rate deviate even more from the fundamental rate of inhibition in which one is interested.

\section{CONCENTRATION-TIME CURVES FOR INHIBITION}

A common method of expressing inhibition kineties has been to plot the concentration of the inhibitor against the time required to produce a chosen effect. These curves have often been fit by a general equation of the type:

$$
\left[(\mathrm{I})-(\mathrm{I})_{m}\right]^{n}\left[t-t_{m}\right]=C^{\prime}
$$

where $(\mathrm{I})_{m}$ is the minimal inhibitor concentration to prorluce the effect and 
$t_{m}$ is the minimal time in which the effect can be produced whatever the inhibitor concentration (Fig. 12-37). Either (I) $)_{m}$ or $t_{m}$ may be negligible and can be omitted; indeed, the data are occasionally expressed adequately by the simple equation:

$$
(\mathrm{I})^{n} t=\mathrm{C}
$$

Taking the logarithm of each side and transposing:

$$
\log t=\log C-n \log (\mathbf{I})
$$

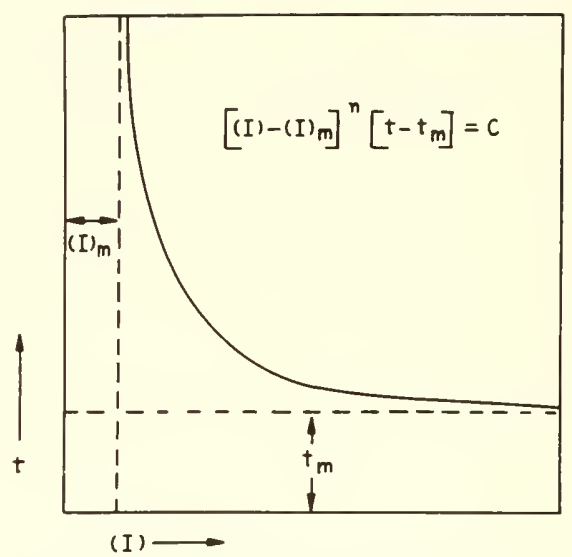

Fic. 12-37. Illustration of a plot of $\mathrm{Eq}_{1}$. $12-89$ where $(\mathrm{I})_{m}$ is the minimal inhibitor concentration to produce an effect and $t_{m}$ is the minimal time in which an effect can be produced.

so that a plot of log $t$ against logr (I) shonlu grive a straight line of slope - $n$ and intercent $\log C$ if this equation is obeyed (Fig. 12-38). The effects of variation in the eonstant $\|$ on the form of the curves is shown in Fig. 12-39, for which the log-log plots are preseuted in Fig. 12-40. As $n$ increases, the curves approach more readily the (I) axis and the minimal radius of curvature decreases. All curves must pass through the point where (I) is unity becanse the exponential factor has no significance here. The value of $n$ is easy to determine, as is the value of $C$; the difficulty arises when an attempt is made to place some physical interpretation on these constants. It is evident that if ( 1$)_{m}$ or $t_{m}$ or both are significant, a plot of $\log t$ against $\log$ (I) will not give a straight line (Fig. 12-41, curve A), so that in orter to test the valiclity of this type of equation in such cases, it is necessary to plot $\log \left[(\mathrm{I})-(\mathrm{I})_{m}\right]$ or $\log \left(t-t_{m}\right)$ (Fir. 12-41, curve B). 


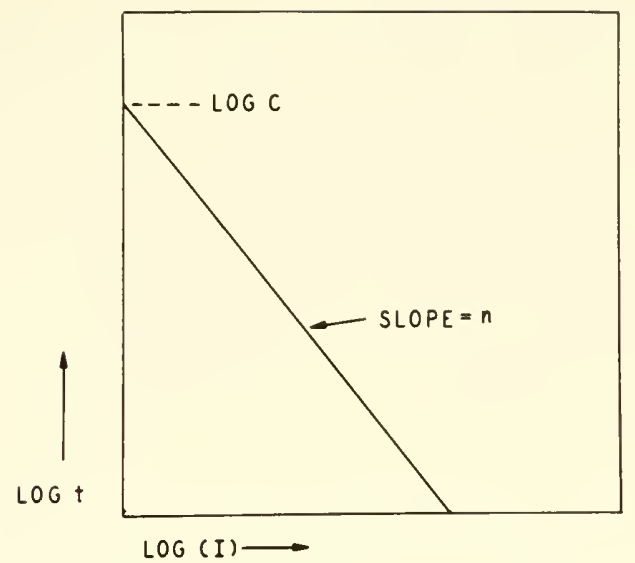

Fig. 12-38. Logarithmic plot of concentrationtime curve (Eq. 12-91).

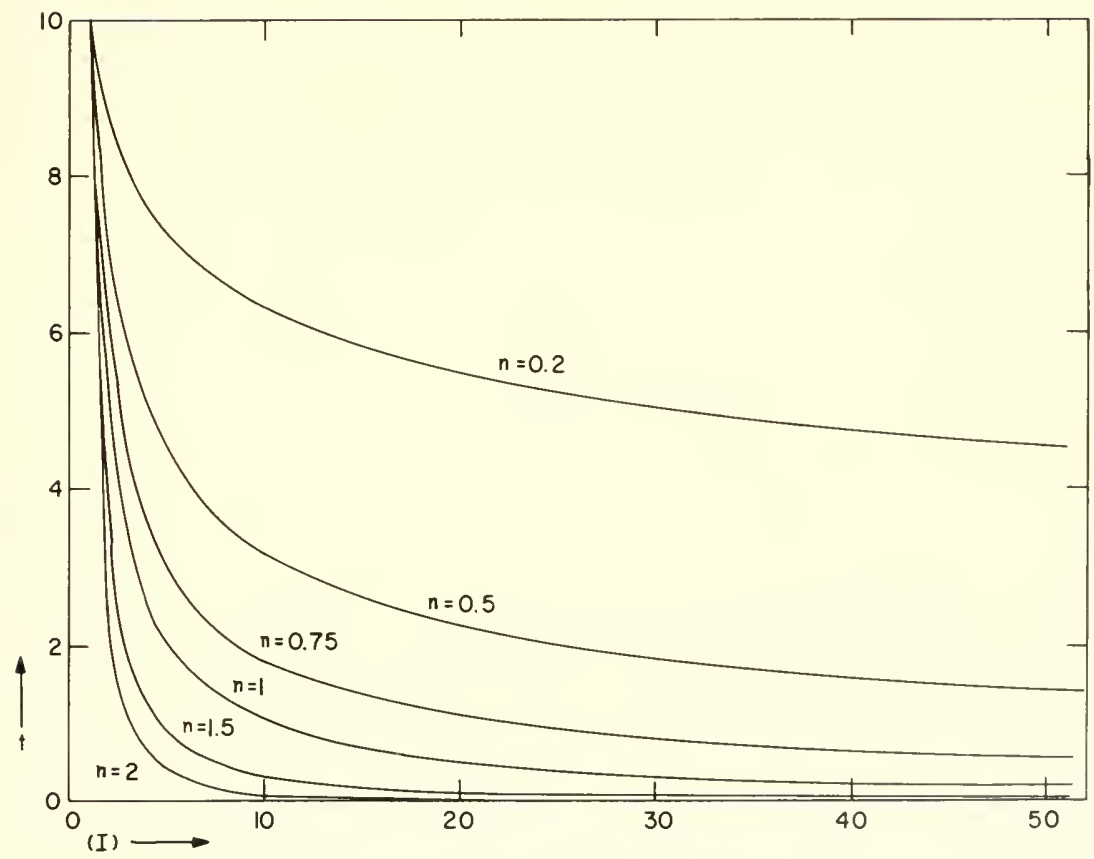

Fig. 12-39. Effect of the variation of the constant $n$ on the form of concentrationtime curves $(\mathrm{Eq}, 1 \geq-90) . C=10$. 


\section{Examples of Concentration-Time Relationships}

The lethal action of $\mathrm{C}_{1}{ }^{++}$on Nitella is expressed fairly well by the equation $\left(\mathrm{Cu}^{++}\right)^{n} t=C$, where $n$ is about 0.33, and the plots of $\log t$ against $\log \left(\mathrm{Cu}^{++}\right)$are straight lines to the first approximation (Cook, $1926 \mathrm{c}$ ). The killing of paramecia by arsenite, however, requires the equation $\left[(\mathrm{I})-(\mathrm{I})_{m}\right]^{n} t=C$, where $(\mathrm{I})_{m}=1 \mathrm{~m} M, n=1.38$, and $C=1.21 \mathrm{M} \mathrm{min}$ (Neuschlosz, 1919), these value being oltained from the linear log-log

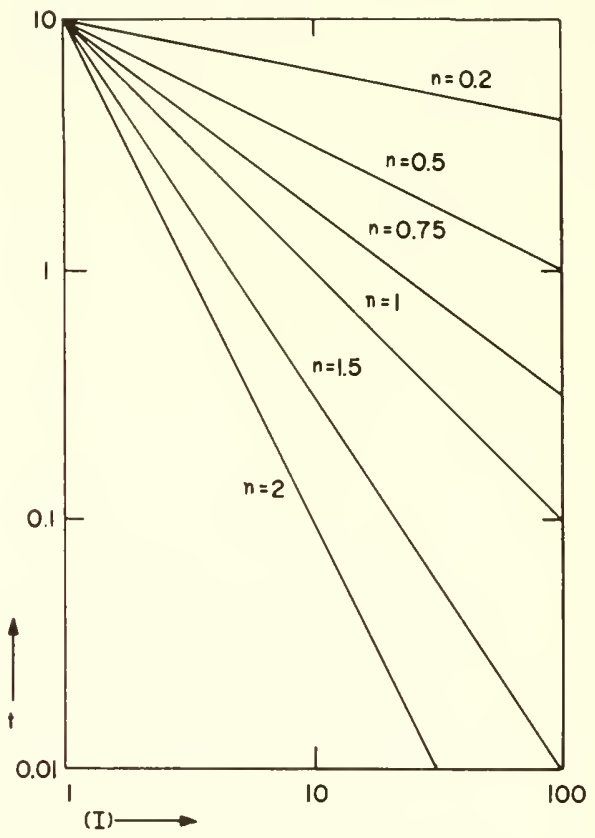

Fig. 12-40. Logarithmic plots of the concentration-time curves in Fig. 12-39 (Eq. 12-91).

plot (Fig. 12-42). It may be noticed that actually the experimental points could be connected somewhat better by a curve concave downwards, so that the ahove equation is only an approximate expression of the kinetics.

Comparison between different members of a series of compounds can also be made on such graphs. The killing of trypanosomes by various arsenicals provided kinetics that gave quite linear log-log plots (Hawkingr. 1938) as shown in Fig. 12-43. The constants calculated are shown in the following tabulation. 


\begin{tabular}{l|c|c|c}
\hline \multicolumn{1}{c|}{ Arsenical } & $(\mathrm{I})_{m}$ & $n$ & $C$ \\
\hline & $(\mathrm{m} / \mathrm{H})$ & & \\
\hline & & & \\
Phenylarsenoxide & $0.36 \times 10^{-5}$ & 1.04 & 0.016 \\
Peduced tryparsamide & $1.7 \times 10^{-5}$ & 0.50 & 2.04 \\
Novarsenobillon & $2.5 \times 10^{-5}$ & 0.60 & 3.55 \\
Arsenite & $177 \times 10^{-5}$ & 0.77 & 21.9 \\
\hline
\end{tabular}

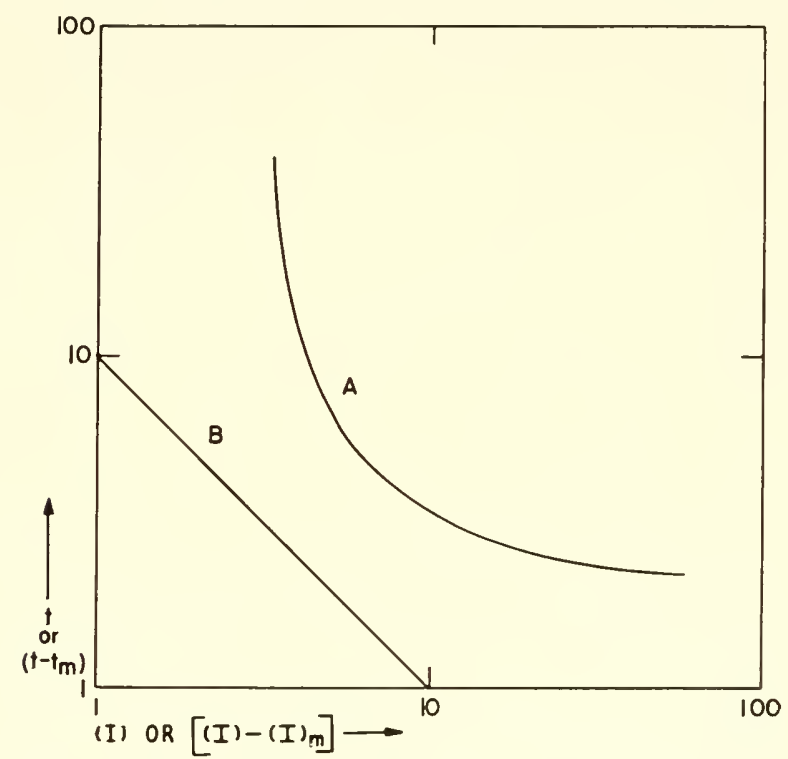

FIG. 12-41. Logarithmic plots of Eq. 12-89. In curve A. $t$ is plotted against (I), and in curve $\mathrm{B},\left(t-t_{m}\right)$ is plotted against $\left[(\mathrm{I})-(\mathrm{I})_{m}\right] .(\mathrm{I})_{m}=3, t_{m}=2$, and $C=10$.

The marked differences in the rates of inhibition and the potencies of these compounds are well illustrated by these constants in an equation of the type $\left[(\mathrm{I})-(\mathrm{I})_{m}\right]^{n} t=C$. It was shown that the delay in the killing action was not due to fixation of the substances. since this was relatively rapid, but to intracellular processes. probably including the time required for the arsenical to react with the susceptible enzymes and the subsequent processes leading to the death of the cells.

\section{Interpretation of Concentration-Time Curves}

There is no question but that concentration-time curves allow kinetic data to be presented in a compact and useful form and that the fitting of 
such eurves to equations of the types discussed provides constants that can be used to characterize inhibitors. However, there is some doubt that much valuable information about the mechanisms involved can be obtained by these means. Let us see first what types of curves would be expected from the simple inhibition of isolated enzymes. An irreversible inhibition (reaction 12-15) rate can be expressed as:

$$
i=1-e^{-k_{1}(1) l}
$$

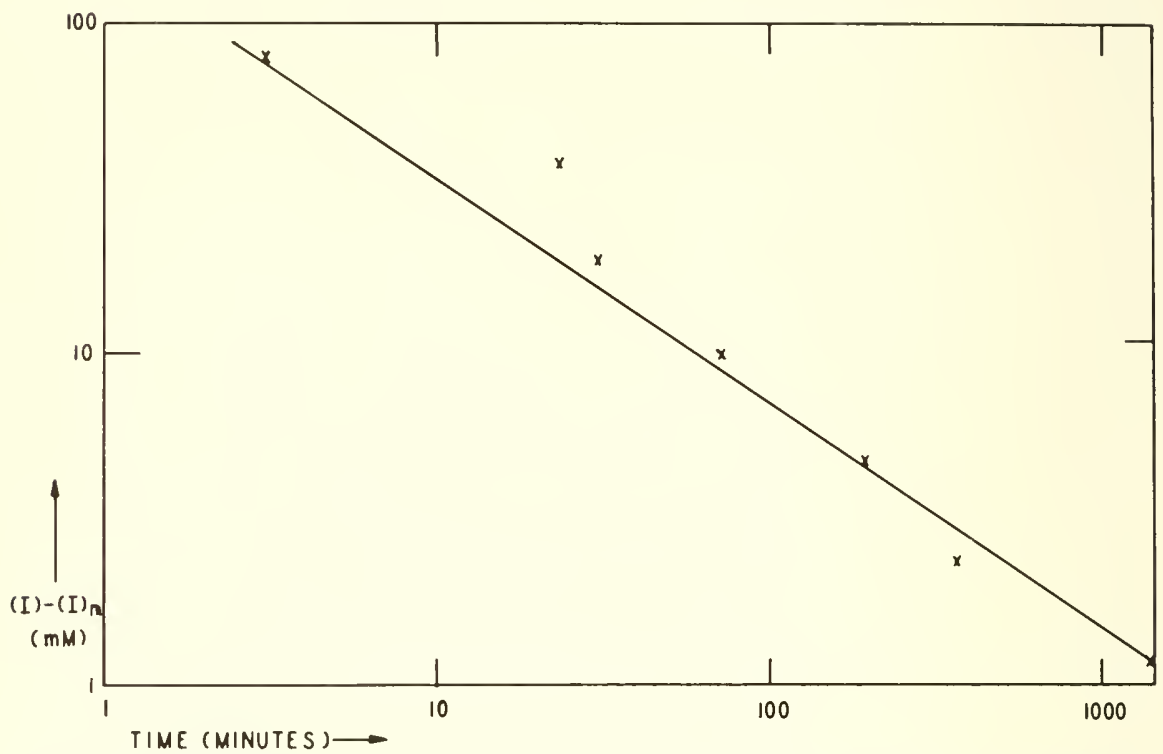

FIG. 12-42. Log- $\log$ plot of the coneentration-time curve for the killing of paramecia by arsenite. $\left[(\mathrm{I})-(\mathrm{I})_{m}\right]^{n} t=C$ where $n=1.38$. ( $\left.\mathrm{I}\right)_{m}=1 \mathrm{~m}, V$ and $C=1.21$ moles liter min. The points are from the data of Neuschlosz (1919).

so that for any degree of inhibition:

$$
\text { (I) } t=\frac{1}{k_{1}} \ln \frac{1}{1-i}
$$

and thus $(\mathbf{I}) t=$ constant. For $50 \%$ inhibition, (I) $t==0.693 k_{1}$. If " molecules of inhibitor reacter with eareh molecule of enzyne to produce the inhibition. $\mathrm{E}+n \mathrm{I} \rightarrow \mathrm{EI}_{n}$, the relation (I)"t = C'should he obeyed. However. a reversible inhibition (reaction $12-8$ ) where the inhibition in given by:

$$
i=i_{f}\left[1-e^{\left.-k_{1} l\left[(1)+k_{i}\right]\right\rceil}\right.
$$


would not conform so simply and:

$$
\text { (I) } t=\frac{1}{k_{1}} \ln \frac{i_{f}}{i_{f}-i}-K_{2} t
$$

Since $i_{f}$ usually is dependent on (I) and $t$ occurs on the right side of the equation, the log-log plot will not be linear. This deviation from linearity

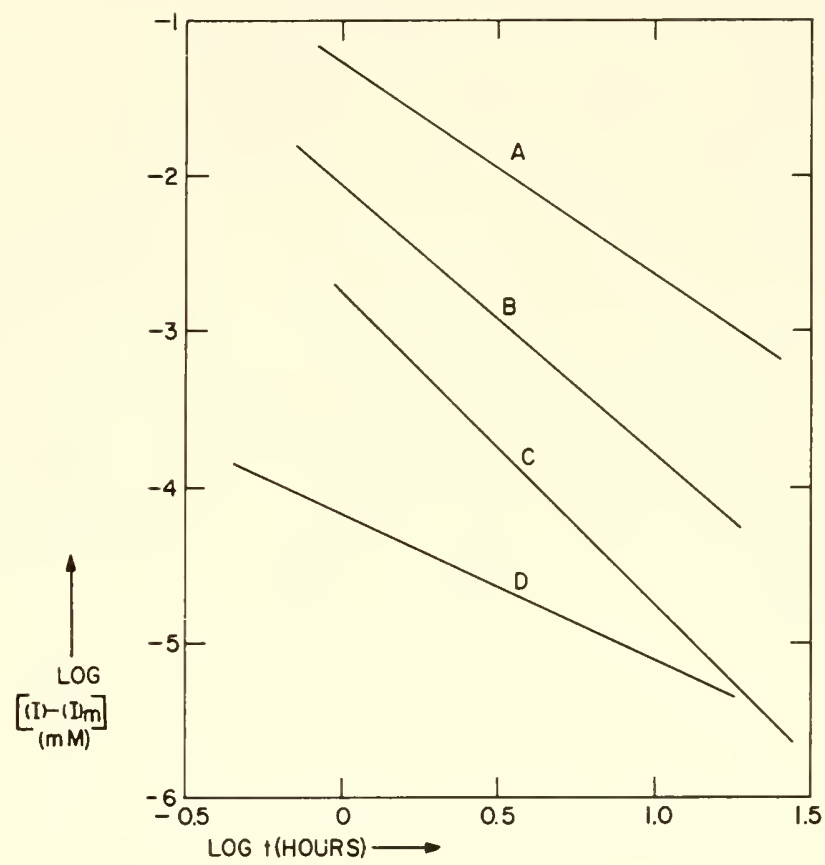

FIG. 1:-43. Log-log plots of the concentration-time curves for the killing for typanosomes by various arsenicals. Time for $90^{\circ}$ o mortality. (From Hawking, 1938.) Curve A, arsenite; curve B. Neoarsphenamine; curve C, reduced tryparsamide: curve D. phenylarsenoxide.

is shown in Fig. 12-44, where curve A represents an irreversibile inhibition and curve $\mathrm{B}$ a similar reversible inhibition. The deviation occurs principally at lower inhibitor concentrations where $K_{\text {; contributes to the exponential }}$ factor in Eq. 12-93 and the final inhibition does not approach completion. In competitive inhibition. further complications arise, and there are many other reasons for the kinetics to deviate from the simple (I)" $t=C$ type of equation. If enzyme inhibition itself is capable of presenting so many different sorts of behavior. it would be expected that cellular inhibition ki- 
netics would be too complex to be interpretable by concentration-time relationships.

The problem here is the same as in the interpretation of any kinetic data - before the results can be related to any mechanism of inhibition, it must be definitely shown that the time intervals measured refer to the primary inhibition. There are several factors that can lead to straight lines when $\log (\mathrm{I})$ is plotted against $\log t$. Penetration of a substance into a cell would be expected to conform to this pattern. Indeed, it has been shown

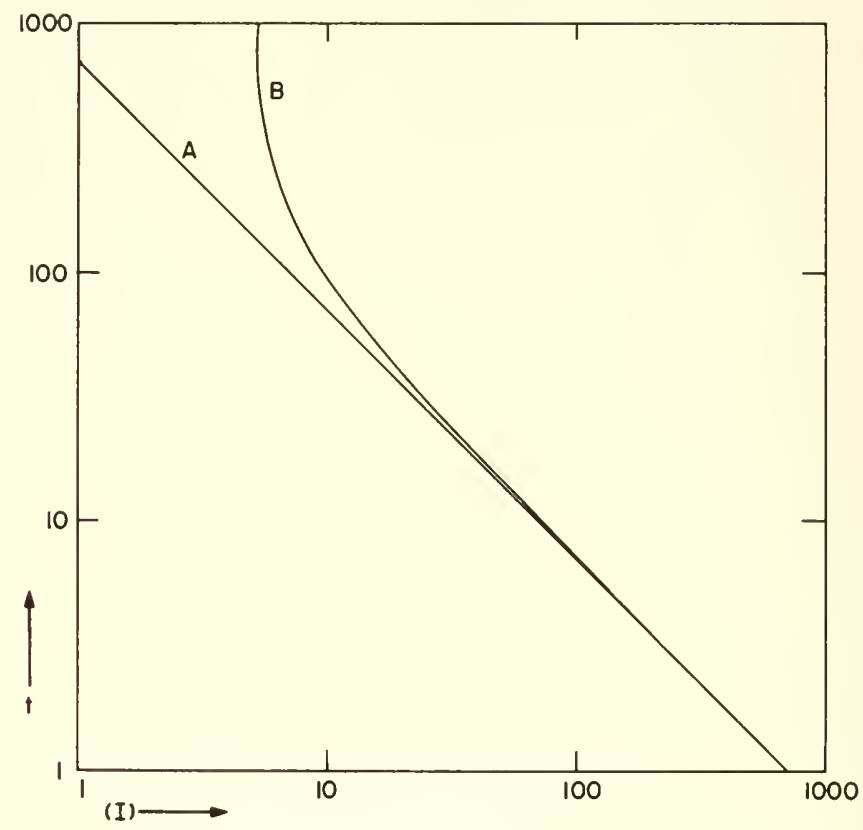

FIG. 12-44. Log-log plots of concentration-time curves for inhibition of enzymes. $K_{i}=5 \mathrm{~m} H, k_{1}=1, C=704$, and $i=0.5$. Curve A, irreversible inhibition (Eq. 12-92); curve B, reversible inhibition (Eq. 12-94).

that the entrance of cresyl blue into Nitella cells is expressed by the Eq. $[($ dye $)-0.7](t-0.6)=102$, where the concentration is in units of $10^{-5} \mathrm{MI}$ and the time is in minutes (Irwin, 1925), and that the log-log plots are linear. Variation in a population of cells could also provide data on killing rates by inhibitors that would fit such a formulation. and it is quite likely that the trypanocidal action of the arsenicals (Hawking, 1938) discussed in the previous section belongs in this category, inasmuch as fixation of the inlibitors seems to be quite rapid.

The value of the exponential $n$ in equations of type (I)" $t=C$ is also not 
directly interpretable in terms of any one rate process or mechanism. For example, if $n$ is found to be less than one, various explanations might be found, including (1) reversible processes of inhibition or penetration, (2) competitive inhibition, (3) nonlinearity of internal inhibitor concentration relative to the external concentration, and (4) delay in action of the inhibitor on the response measured because of intracellular events subsequent to the inhibition and leading to the response. This latter factor is probably of major importance when measurements are made of functional or terminal effects of the inhibitors. It is evident that as the inhibitor concentration is increased, beyond a certain point there will not be a marked reduction in the time required to produce the chosen response, because this time will be mainly dependent on intracellular events whose rates are not directly proportional to the inhibitor concentration or the degree of the primary inhibition. Variations in $n$ could also be produced by adaptations of the cells to the inhibitor in long-term experiments, by metabolism of the inhibitor in the cells, by binding of the inhibitor to nonenzymic material, or by any of the complicating factors considered in this chapter. The expression of inhibition in concentration-time curves or in related equations may eventually be useful when it is combined with a more detailed investigation of the processes involved, but alone it does not lead to reliable conclusions. 



\section{REVERSAL OF INHIBITION}

The rates at which inhibited enzymes may be reactivated are frequently quite important in experimental studies of inhibition and particularly so when the inhibitors are administered to whole animals. This is evident when one considers the problems arising in the clinical use of the irreversible enzyme inhibitors, such as the phosphofluoridates (e.g., diisopropylfluorophosphate or DFP) that inhibit cholinesterases, or certain of the hydrazine derivatives (e.g. iproniazid) that inactivate monoamine oxidase. The degree of reversibility of the inhibition in the tissues of patients determines the frequency with which the inhibitor must be administered to achieve and maintain a desired level of effect, the duration of the action when the administration of the inhibitor is stopped. and often the effectiveness of the inhibitor when it is used for a specific purpose. However, even in work with isolated enzymes or cellular preparations, the readiness with which the inhibition is reversed is important. The kinetics of the inhibition, for one thing. depend on whether the inhibition is rapidly reversible or not, as discussed in previous chapters. Most kineties are formulated on the principle that reversibility occurs and that mass-action equilibria are achieved. When reversal is slow, it may be impossible to reach a state of true equilibrium if the enzyme is unstable and processes of inactivation proceed concurrently with the development or reversal of the inhibition. In any quantitative study of inhibition, it is advisable to determine the reversibility of the inhibition or of the effects measured. In some cases the loss of enzyme activity may be associated with some type of inactivation of the enzyme, as distingnished from simple inhibition due to the binding of the inhibitor to the enzyme surface, and the most straightforward way to test this is to determine the degree to which the activity can be restored by the removal of the inhibitor. Very few studies have been made of the kinetics of inhibition reversal and of the factors that may influence it, and one may look in vain in most of the treatises on enzymes for any discussion of this phenomenon. 


\section{WAYS IN WHICH REVERSAL OF INHIBITION MAY BE ACHIEVED}

Reactivation of an inhibited enzyme may oceur spontaneously or can be brought about by a variety of techniques. Some of the eonditions allowing or favoring restoration of enzyme activity are listed here and later will be discussed in greater detail.

$A$. Replacement of the medium bathing the enzyme or cells with medium free of the inhibitor, or removal of the inhibitor by dialysis.

$B$. Introduction of a substance that binds the inhibitor strongly and removes it from the enzyme.

C. Displacement of the inhibitor from the enzyme with increased concentrations of the substrate, in case the inhibition is competitive.

$D$. Spontaneous reactivation brought about by the chemical alteration and splitting off of the inhibitor through the eatalytic action of the enzyme that is inhibited (in such eases the inhibitor is often structurally related to the substrate so that the reversal oecurs by a reaction similar to that undergone by the substrate but much slower).

$E$. Introduction of a substance that reacts with the EI complex to facilitate the reaction and removal of the inhibitor.

$F$. Metabolism of the inhibitor by enzymes in the preparation or spontaneous inaetivation of an unstable inhibitor.

$G$. Addition of a cofaetor or coenzyme in those cases where the normally present cofactor or coenzyme has been inactivated by the inhibitor.

$H$. Redistribution of the inhibitor from the enzyme to other binding sites which do not pick up the inhibitor rapidly but yet possess a high affinity for it.

I. Synthesis of new enzyme in cells where the inhibition has been truly irreversible and the enzyme permanently inactivated.

There are, of eourse, other ways in which inhibition can be reversed but they are usually relevant to speeial cases only and will be diseussed in relation to the inhibitors exhibiting such behavior. For example, the inhibition of cytochrome oxidase and respiration by earbon monoxide ean be abolished by light which dissociates the EI complex. Volatile inhibitors, such as hydrocyanic acid, can be removed from the enzymes by bubbling a gas mixture through the medium; indeed, in work with cyanide, eare must te taken so that the coneentration of the inhibitor does not decrease during the experimental period due to the eseape of hydrocyanie acid from the liquid 
phase. Reversal of the changes brought about by inhibitors in the complex metabolic and functional processes of cells and tissues will be examined after treatment of the simpler reactivations observed with isolated enzymes.

\section{DISSOCIATION OF THE ENZYME-INHIBITOR COMPLEX}

Reversal of inhibition, either of enzymes in solution or within cellular preparations, can usually be achieved by the removal of the inhibitor from the medium in which the enzymes or cells are studied. If the binding of the inhibitor to the enzyme is reversible, the inhibitor will dissociate from the enzyme and diffuse away along a concentration gradient, the enzyme activity progressively increasing. The rate of the reversal will depend on how effectively the concentration of free inhibitor can be maintained at a low level and on the rate constant for the dissociation of the EI complex.

\section{Dissociation of the El Complex When Free Inhibitor Concentration Is Zero}

The rate of dissociation of the EI complex, according to the reaction:

$$
\mathrm{EI} \underset{k_{1}}{\stackrel{k_{-1}}{\rightleftharpoons}} \mathrm{E}+1
$$

can be expressed as:

$$
\frac{d(\mathrm{EI})}{d t}=-k_{-1}(\mathrm{EI})
$$

if by some means the concentration of free inhibitor is maintained at zero. Since $(\mathrm{EI})=i\left(\mathrm{E}_{t}\right)$, this may also be written as:

$$
\frac{d i}{d t}=-k_{-1} i
$$

which is directly integratable to:

$$
i=i_{0} e^{-k_{-1} t}
$$

where $i_{0}$ is the initial inhibition before reversal begins. Thus if $\log i$ is plotted against time, one will obtain a straight line from the slope of which the rate constant may be determined.

Occasionally it is possible to calculate the rate constant for the combination of the inhibitor with the enzyme even though the reaction cannot be followed directly. If $K_{i}$ is found from the usual graphical procedures, a determination of $k_{-1}$ as described above will provide a value for the association rate constant since $k_{1}=k_{-1} / K_{i}$. The reversal of inhibition is often more readily measured than the development of inhibition because 
the former reaction may be sufficiently slow as to be susceptible to the experimental techniques available. The rate of inhibition reversal depends on the energy of activation for the dissociation of the inhibitor from the enzyme, and since this energy is greater than the association activation energy by an amount equivalent to the over-all free energy change, generally $k_{1}>k_{-1}$, the ratio of the rate constants being given by:

$$
k_{-1} / k_{1}=e^{A F^{\prime} / R T}
$$

For example, if the free energy of binding is $-4 \mathrm{kcal} / \mathrm{mole}$, it is easily caleulated that $k_{-1}$ is only $1 / 645$ of $k_{1}$. Since most inhibitions involve free energy ehanges of several kcal/mole, such relations between the rate constants are usual.

It is perhaps worth noting that, rigorously speaking, it is technically impossible to maintain the free inhibitor concentration at zero throughout the enzyme preparation during the reversal of inhibition. If dialysis is used to remove the inhibitor, the eoncentration of free inhibitor within the dialysis bag is usually significant in ease the dissociation rate of the EI complex is relatively fast, and with any technique it is impossible to prevent some reassociation of released inhibitor molecules. When a substance that binds the free inhibitor is added, the inhibitor concentration cannot, of course, be reduced to zero. Thus reversal experiments invariably involve some assumptions if the simple interpretation outlined above is followed, but frequently these deviations from ideal behavior are quite insignificant and may be ignored. Nevertheless, it is well in studies of this type to consider such factors and determine if they are or are not significant.

\section{Dissociation of the El Complex When Free Inhibitor Concentration Is Not Zero}

When the free inhibitor concentration is suddenly decreased to a lower value, as by dilution of the enzyme solution, the inhibition will drop to a new equilibrium level, providing the inhibition is reversible. In this case, the reverse reaction of association (Eq. 13-1) must be taken into account and the following rate equations may be written:

$$
\begin{gathered}
\frac{d(\mathrm{EI})}{d t}=-k_{-1}(\mathrm{EI})+k_{1}(\mathrm{E})(\mathrm{I}) \\
\frac{d i}{d t}=-k_{-1}\left[1+\frac{\left(1_{f}\right)}{K_{i}}\right] i+k_{-1} \frac{\left(\mathrm{I}_{f}\right)}{K_{i}^{-}}
\end{gathered}
$$

where $\left(I_{f}\right)$ designates the final concentration to which the inhibitor has been reduced. Equation 13-7 may be integrated to give:

$$
i=i_{f}+\left(i_{o}-i_{f}\right) e^{-k_{-1} t /\left(1-i_{f}\right)}
$$


where $i_{0}$ is the initial inhibition and $i_{f}$ is the final equilibrium inhibition, $i_{f}$ depending on $\left(\mathrm{I}_{f}\right)$ in a manner characteristic of the inhibition. This equation reduces to Eq. 13-4 when $\left(\mathrm{I}_{f}\right)=i_{f}=0$. Equation 13-8 is plotted in Fig. 13-1 for various values of $\left(\mathrm{I}_{f}\right)$ and it may be noted that the rate of inhibition reversal depends, as expected, on the magnitude of the decrease in inhibition, $\left(i_{0}-i_{f}\right)$. The time required for the inhibition to fall half

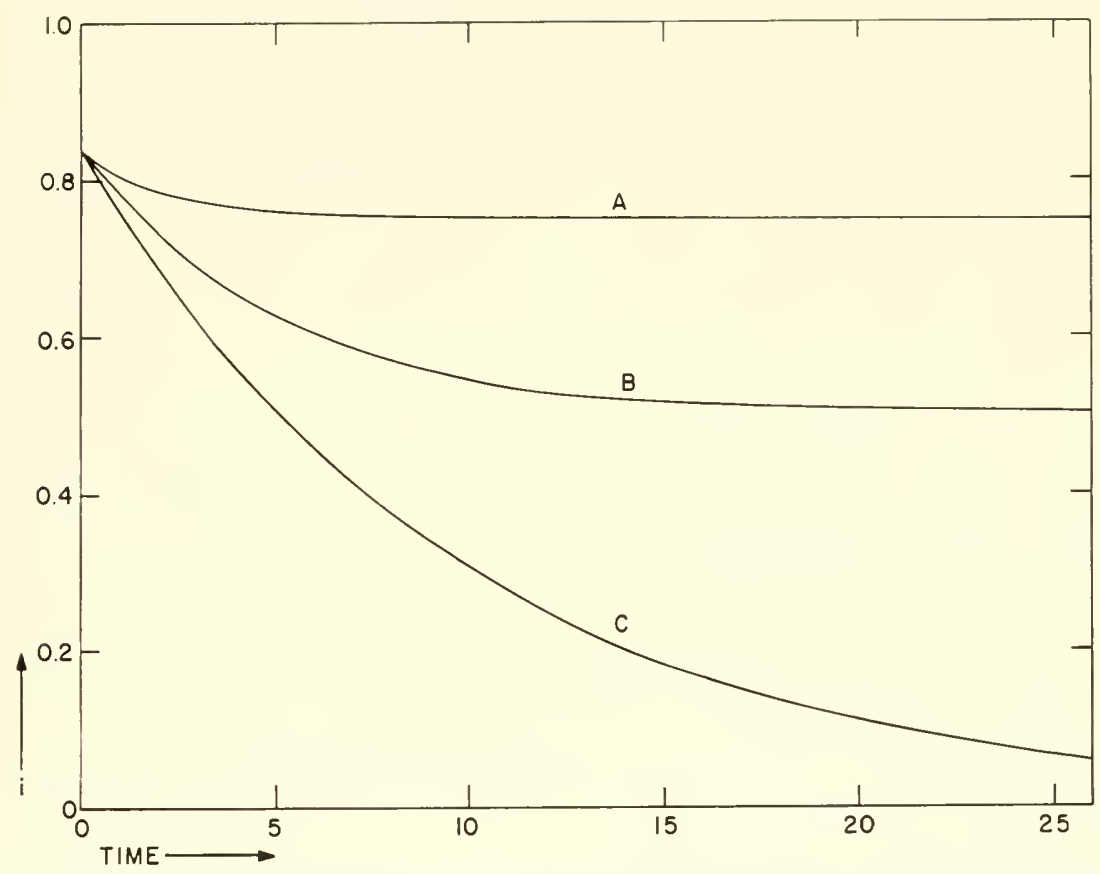

FIG. 13-1. Rates of decrease of inhibition when the inhibitor concentration is suddenly reduced to various levels $(\mathrm{Eq} .13-8) .(\mathrm{I})_{0}=5 \mathrm{~m} H, K_{i}=1 \mathrm{~m} M, k_{-1}=0.1$, and $i_{0}=0.833$. Curve $\mathrm{A}:\left(\mathrm{I}_{f}\right)=3 \mathrm{~m} . M, t_{0.5}=1.73$; curve B: $\left(\mathrm{I}_{f}\right)=1 \mathrm{~m} . H ; t_{0.5}=3.46$; curve $\mathrm{C}:\left(\mathrm{I}_{\boldsymbol{f}}\right)=0, t_{0.5}=6.93$.

the way to its eventual level. $t_{0.5}$, increases as $\left(I_{f}\right)$ is reduced. since from Eq. 13-8:

$$
t_{0 . s}=\frac{0.69}{k_{-1}}\left(1-i_{\rho}\right)
$$

and the time required to reach final inhibition levels varies in a similar fashion. That is, it requires more time to reach equilibrium when the fall in the inhibition is large than when small, even though the rate at which the inhibition falls is greater in the former case.

Let us for a moment consider the effects of dilution upon inhibition in various systems. The results will depend on whether the inhibition is 
competitive or noncompetitive, and on whether we are dealing with mutual depletion systems or not. A dilution factor, $r$, is defined by the equation $r=\left(\mathrm{I}_{0}\right) /\left(\mathrm{I}_{f}\right)$ and thus is the ratio of initial to final inhibitor concentrations. Initial and final inhibitions may then be written as:

$$
\begin{array}{lll}
\text { Noncompetitive: } & i_{0}=\frac{\left(\mathrm{I}^{\prime}\right)}{\left(\mathrm{I}^{\prime}\right)+1} & i_{\rho}=\frac{\left(\mathrm{I}^{\prime}\right)}{\left(\mathrm{I}^{\prime}\right)+r} \\
\text { Competitive: } & i_{0}=\frac{\left(\mathrm{I}^{\prime}\right)}{\left(\mathrm{I}^{\prime}\right)+\overline{1}+\left(\overline{\mathrm{S}^{\prime}}\right)} & i_{\rho}=\frac{\left(\mathrm{I}^{\prime}\right)}{\left(\mathrm{I}^{\prime}\right)+r+\left(\mathrm{S}^{\prime}\right)}
\end{array}
$$

The effects of varying degrees of dilution on the final inhibition are shown for some specific cases in Fig. 13-2. It is particularly interesting to com-

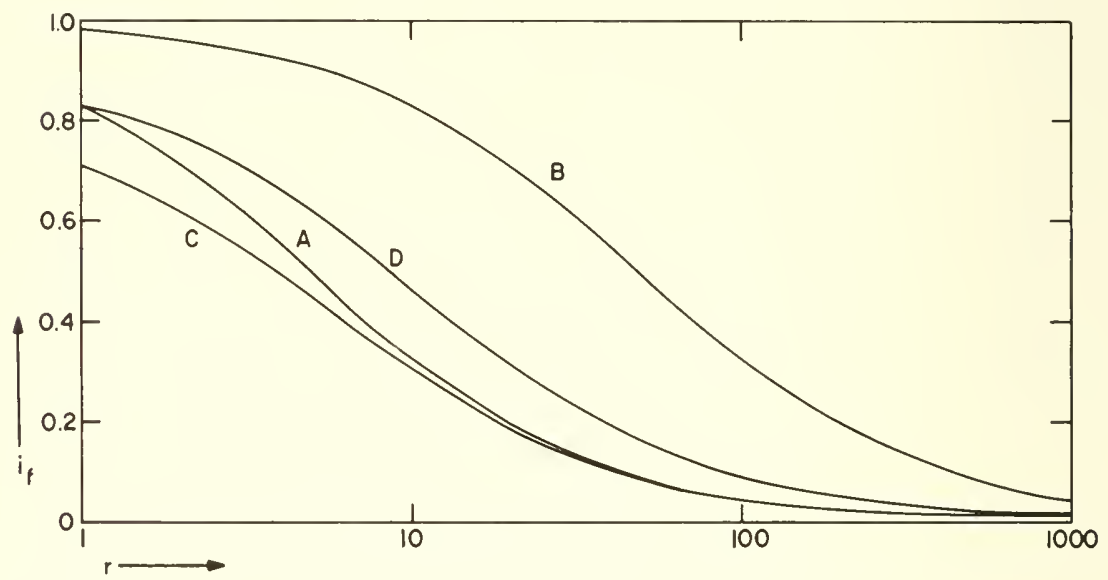

FIG. 13-2. Effects of varying degrees of dilution on the final inhibition (Eqs. 13-10 and 13-11). The dilution factor, $r$, is equal to the ratio of the initial and final inhibitor concentrations, $\left(\mathrm{I}_{0}\right) /\left(\mathrm{I}_{f}\right)$. Noncompetitive: curve $\mathrm{A}, K_{i}=1 \mathrm{~m} M,(\mathrm{I})=5 \mathrm{~m} M$; curve B, $K_{i}=0.1 \mathrm{~m} M,(\mathrm{I})=5 \mathrm{~m} H$. Competitive: Curve C, $K_{i}=0.1 \mathrm{~m} H, K_{s}=1 \mathrm{~m} M,(\mathrm{I})=$ $0.5 \mathrm{~m} M$, and $(\mathrm{S})=1 \mathrm{~m} M$; curve D, $K_{i}=0.0 .5 \mathrm{~m} M, K_{s}=1 \mathrm{~m} M,(\mathrm{I})=0.5 \mathrm{~m} M$, and $(\mathrm{S})=1 \mathrm{~m} M$.

pare curves $\mathrm{A}$ and $\mathrm{D}$ where the initial inhibition is the same; dihution is seen to be more effective in reducing the inhibition in noncompetitive systens than in competitive ones. The reason is that in the competitive case the substrate concentration is also reduced by dihtion and the substrate is increasingly less effective in antagonizing the inhibition. It is also worth noting that the larger $K_{i}$, the more readily will dilution reduce the inhibition, comparing curves $\mathrm{A}$ and $\mathrm{B}$. This means that when one starts out with a relatively high inhibition in a noncompetitive system, it requires 
much dilution before the inhibition is markedly reduced. If the initial inhibition is above $95 \%$, it requires at least a hundred fold dilution to bring inhibition down to $33 \%$. Dilution of inhibited systems in zones B and $\mathrm{C}$ in mutual depletion systems has been discussed in Chapter 3 . This may be summarized by stating that the greater the degree of mutual depletion (i.e., the more the system approaches zone $\mathrm{C}$ in behavior), the less effect will dilution have on the inhibition.

Straus and Goldstein (1943) discussed quantitatively the effects of dilution on equilibrium inhibitions in the system serum cholinesterase-physostigmine and Goldstein (1944) extended this to the linetics of dilution. The experimental data on inhibition reversal were only moderately well described by an equation derived on the basis of simple dissociation. The equation used is equivalent to Eq. 13-8 although it is expressed in quite a different form. However, more than simple dissociation of the EI complex may be involved here, inasmuch as physostigmine is slowly inactivated by the enzyme (Easson and Stedman, 1936). Also the system was not always completely in zone $\mathrm{A}$. Thus some deviation from the monomolecular dissociation kinetics might be expected. It may also be noted that experimental points are not available for the early phases of the reversal, the first readings having been made at $40 \mathrm{~min}$, at which time the inhibitions had been reduced almost to their final equilibrium levels.

\section{Inactivation of the Enzyme During the Experimental Period}

Quantitative determinations of the reverisibility of inhibition should include control tests for possible changes in the activity of the enzyme in the absence of the inhibitor. Complete reversal would not, of course, be anticipated in those cases where the enzyme is unstable and spontaneously inactivated. Unfortmnately this procedure does not reveal secondary inactivation of the enzyme by the inhibitor. For this purpose, it is necessary to determine in some way (e.g., by chemical or radioactivity analysis) if the inhibitor has actually been completely dissociated from the enzyme during the reversal incubation or dialysis. If it can be shown that no inhibitor remains and yet the activity has not returned to the initial value, it is strong evidence for inactivation in addition to the direct effects of the inhibitor. The kinetics of a system in which the enzyme is being simultaneously inactivated as it is recovering from inhibition are complex, but the behavior may be readily predicted. In general, the activity at any time will be the result of the two independent processes and will depend on their relative rates. 


\section{COMPETITIVE DISPLACEMENT OF THE INHIBITOR BY SUBSTRATE}

If an enzyme and a competitive inhibitor are incubated so that a constant equilibrium inhibition is reached and substrate is then added, the inhibition will fall to a new level. The initial inhibition will be given by the usual noncompetitive equation, $i_{0}=\left(\mathrm{I}^{\prime}\right) /\left[\left(\mathrm{I}^{\prime}\right)+1\right]$, since no substrate is present. The final inhibition after the substrate is added will be given by the equation for competitive inhibition, $i_{f}=\left(\mathrm{I}^{\prime}\right) /\left[\left(\mathrm{I}^{\prime}\right)+1+\left(\mathrm{S}^{\prime}\right)\right]$. In the over-all reaction, inhibitor has been displaced from the enzyme. However, this displacement should not be conceived as involving an active participation of the substrate. The substrate molecules do not force the inhibitor from the enzyme but only combine with enzyme molecules that are not reacted with inhibitor. The displacement thus comprises two separate reactions, the dissociation of the EI complex and the formation of the ES complex:

$$
\begin{aligned}
& \mathrm{EI} \underset{k_{1}}{\stackrel{k_{-1}}{\rightleftharpoons}} \mathrm{E}+\mathrm{I} \\
& \mathrm{E}+\mathrm{S} \underset{k_{-2}}{\stackrel{k_{2}}{\rightleftharpoons}} \mathrm{ES}
\end{aligned}
$$

where the numerical subscripts of the rate constants conform to the conventions of this and the last chapter. Since the reaction of the substrate with the enzyme is usually much faster than the dissociation of the EI complex, at least in those cases that can be dealt with experimentally, the rate of the displacement and the time required to achieve the new equilibrium are ordinarily dependent only on the dissociation rate constant $k_{-1}$.

A simple approximate treatment of the displacement rate can be based on the expression for the rate of change of (EI):

$$
\frac{d(\mathrm{EI})}{d t}=\frac{k_{-1}}{K_{i}}\left[\left(\mathrm{E}_{t}\right)-(\mathrm{ES})-(\mathrm{EI})\right](\mathrm{I})-k_{-1}(\mathrm{EI})
$$

Assuming that the equilibrium of the enzyme with substrate is very rapidly achieved and that the rate of breakdown of the ES complex into the product is relatively slow (i.e., when the enzyme conforms to the Michaelis formulation), this equation may be integrated with the boundary conditions defined by the values of $i_{0}$ and $i_{f}$ given above for the beginning and end of the displacement.

$$
i=i_{f}\left\{1+\frac{\left(\mathrm{S}^{\prime}\right)}{\left(\mathrm{I}^{\prime}\right)+1} \exp \left[-\frac{k_{-1} t}{i_{f}} \frac{\left(\mathrm{I}^{\prime}\right)}{\left(\mathrm{S}^{\prime}\right)+1}\right]\right\}
$$


This equation is plotted for different concentrations of added substrate in Fig. 13-3 and the general form of the curves is seen to be similar to that found for dilution (Fig. 13-1). Actually the reversal rates are quite comparable in the two cases when the final inhibition reached is the same, as would be expected since dissociation of $\mathrm{EI}$ is the determining factor in each.

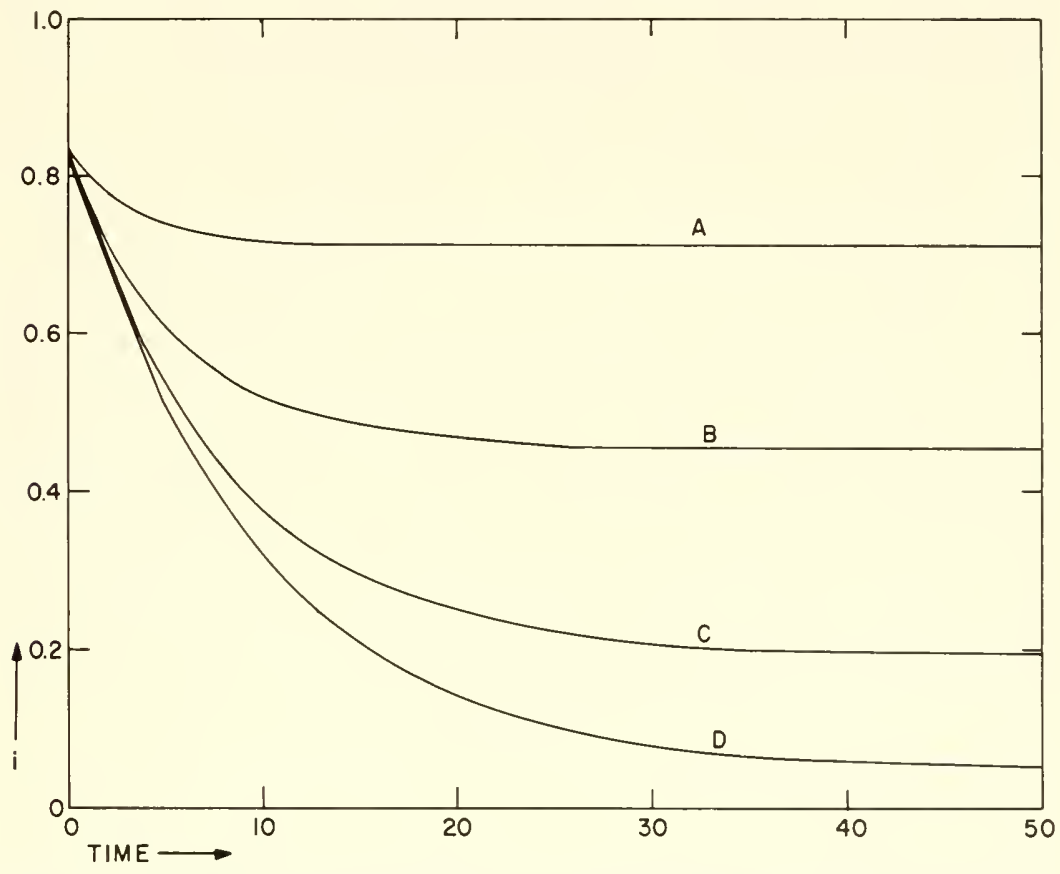

FIG. 13-3. Rates of displacement of the enzyme-bound inhibitor by the substrate in competitive inhibition (Eq. 13-15). $K_{i}=1 \mathrm{~m} .1 \mathrm{I}, K_{3}=3 \mathrm{~m} .1, k_{-1}=0.1$, and $(\mathrm{I})=5 \mathrm{~m} \boldsymbol{H}$. Curve $\mathrm{A}:\left(\mathrm{S}^{\prime}\right)=1$; curve $\mathrm{B}:\left(\mathrm{S}^{\prime}\right)=5$; curve $\mathrm{C}:\left(\mathrm{S}^{\prime}\right)=20$; curve D: $\left(\mathrm{S}^{\prime}\right)=100$.

A more generalized treatment results from the integration of Eq. 12-33 after rewriting it in terms of the dissociation rate constants and setting the inhibition at zero time as $i_{0}$.

$$
i=i_{f}+\left(i_{0}-i_{f}\right) \exp \left\{-\left[\frac{\left(\mathrm{I}^{\prime}\right)+\left(\mathrm{S}^{\prime}\right)}{\left(\mathrm{I}^{\prime}\right) / k_{-2}+\left(\mathrm{S}^{\prime}\right) / k_{-1}}\right] t\right\}
$$

This equation is of the same form as Eq. 13-8 except for the exponential factor. An equation with the same exponential factor was derived by Goldstein (1944) for the displacement of physostigmine from cholinesterase by acetylcholine. However, the rest of his equation differs from that presented 
here because he assumed that the activity was zero at the start of the displacement.

The difference between the assumptions of Goldstein and those made in the present treatment is easily explained. If the rate of combination of the substrate with the enzyme is slow so that the rise in activity is related to this as well as to the dissociation of the EI complex, then the activity must be set equal to zero at the start, as Goldstein did. Here we assume that the reaction of the substrate with the enzyme is rapid and is complete before appreciable loss of the inhibitor from the enzyme has occurred.

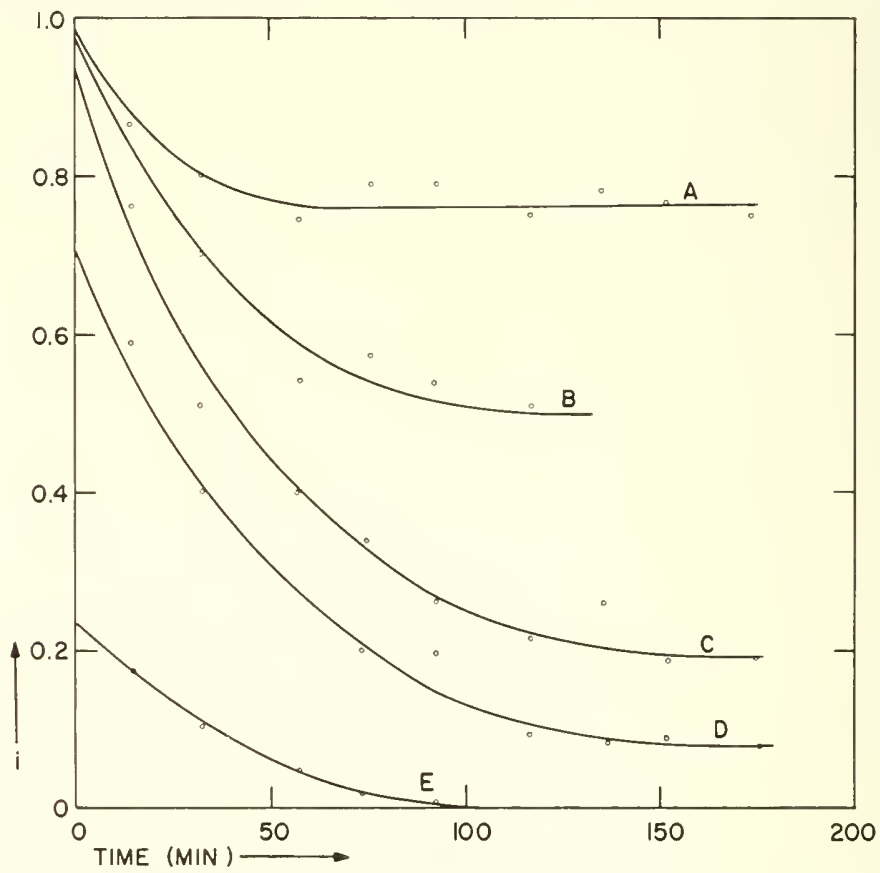

FIG. 13-4. Displacement of physostigmine from serum cholinesterase by acetyleholine. (From Goldstein, 1944.) Curve A: (I) $=5.5 \times 10^{-3}$ $\mathrm{m} H$; eurve $\mathrm{B}:(\mathrm{I})=1.35 \times 10^{-3} \mathrm{~m} H$; curve $\mathrm{C}:(\mathrm{I})=5.5 \times 10^{-4} \mathrm{~m} . H$; eurve D: $(\mathrm{I})=5.5 \times 10^{-5} \mathrm{~m} \boldsymbol{H}$; eurve $\mathrm{E}:(\mathrm{I})=5.5 \times 10^{-8} \mathrm{~m} \boldsymbol{M}$.

The experimental curves for the reversal of the physostigmine inhibition of cholinesterase following the addition of acetylcholine are shown in Fig. 13-4 for different concentrations of physostigmine (Goldstein, 1944). The curves are quite adequately fitted by Eq. 13-16 but not by the equation derived by Goldstein because, in this case, the combination of the acetylcholine with the free enzyme is much more rajid than the dissociation of the physostigmine from the enzyme. The first readings after addition of 
the acetylcholine were taken in 10-15 min whereas equilibration of the substrate with the free enzyme occurred in a matter of seconds probably.

\section{REVERSAL OF INHIBITION BY A SUBSTANCE BINDING THE INHIBITOR}

The presence of a substance having an affinity for the imhibitor and which by reacting with the inhibitor prevents its binding to the enzyme will protect the enzyme. both slowing the development of the inhibition and usually reducing the final equilibrium inhibition. Such a substance will also reverse an established inhibition if the EI complex is dissociable. The outstanding example of this type of protection and reversal is the antagonism exerted by sulfhydryl compounds (such as cysteine, glutathione, and especially dimercaprol or $\mathrm{BAL}$ ) on inhibitions produced by arsenicals, mercurials, and certain other heavy metal ions. The inhibitory effects of some metal ions can also be reduced by appropriate chelating agents, such as ethylenediaminetetraacetate. Advantage has has been taken of this principle in the clinical treatment of poisonings by heavy metals. Actually, for many years the treatment of cyanide poisoning has often involved the administration of a substance (such as a nitrite) that would cause the formation of methemoglobin. which has a high affinity for cyanide and can partially remove it from the tissues and its combination with cytochrome oxidase. These examples cited illustrate such protection and reversal phenomena in a clear-cut form and the agents mentioned are frequently used experimentally in the study of enzyme inhibition. but it must also be remembered that less striking instances of inhibitor binding occur frequently and may go unnoticed. Many inhibitors - fuoride, malonate, parapyruvate, pyrophosphate. and arsenate are a few - form complexes with ions, such as calcium or magnesium, commonly present in the media, and most inhibitors react to varying extents with any nonenzymic proteins in the enzyme preparation. In studies with cellular preparations the possibilities for nonenzymic binding of the inhibitor become so great that care must be exercised in the interpretation of kinetic data. It is important to realize that the substance binding the inhibitor need not have a greater affinity than the enzyme for the inhibitor to produce a significant effect.

\section{Equilibrium Inhibitions in the Presence of a Substance Binding the Inhibitor}

It will be necessary to consider final equilibrium states before rates of inhibition and reversal are treated. The reactions involved are:

$$
\begin{aligned}
& \mathrm{E}+\mathrm{S} \rightleftharpoons \mathrm{ES} \rightarrow \mathrm{E}+\mathrm{P} \\
& K_{s}=(\mathrm{E})(\mathrm{S}) /(\mathrm{ES}) \\
& \mathrm{E}+\mathrm{I} \rightleftharpoons \mathrm{EI} \\
& K_{\imath}=(\mathrm{E})(\mathrm{I}) /(\mathrm{EI}) \\
& \mathrm{R}+\mathrm{I} \rightleftharpoons \mathrm{RI} \\
& K_{\tau}=(\mathrm{R})(\mathrm{I}) /(\mathrm{RI})
\end{aligned}
$$


and the conservation equations may be written as:

$$
\begin{aligned}
& \left(\mathrm{E}_{t}\right)=(\mathrm{E})+(\mathrm{EI})+(\mathrm{ES}) \\
& \left(\mathrm{I}_{t}\right)=(\mathrm{I})+(\mathrm{EI})+(\mathrm{RI}) \\
& \left(\mathrm{R}_{t}\right)=(\mathrm{R})+(\mathrm{RI})
\end{aligned}
$$

For systems in which mutual depletion does not occur (zone A), the term (EI) may be omitted from Eq. 13-18. It will be convenient to treat noncompetitive inhibition since other types of inhibition are generally only extensions of this and the corresponding equations may be easily obtained. The substrate reaction can be neglected for noncompetitive inhibition since the presence of substrate will not modify the inhibition. We are now left with two possibilites: either $\left(\mathrm{R}_{t}\right)$ is much greater than $\left(\mathrm{I}_{t}\right)$ so that the concentration of the reversor is not appreciably reduced by reaction with the inhibitor, in which case $\left(\mathrm{R}_{t}\right)=(\mathrm{R})$, or $\left(\mathrm{R}_{t}\right)$ and $\left(\mathrm{I}_{t}\right)$ are comparable and the reaction reduces the concentrations of both.

In the first case, where $\left(R_{t}\right)=(R)$. it can be easily shown that:

$$
(\mathrm{E})=\frac{K_{i}\left(\mathrm{E}_{t}\right)}{(\mathrm{I})+K_{i}} \quad \text { and } \quad(\mathrm{I})=\frac{K_{r}\left(\mathrm{I}_{t}\right)}{\left(\mathrm{R}_{t}\right)+K_{r}}
$$

Combining these two equations and substituting in $i=1-\left[(\mathrm{E}) /\left(\mathrm{E}_{t}\right)\right.$ :

$$
i=\frac{\left(\mathrm{I}_{t}{ }^{\prime}\right)}{\left(\mathbf{I}_{t}{ }^{\prime}\right)+1+\left(\mathbf{R}_{t}^{\prime}\right)}
$$

Thus the presence of a reversor lowers the inhibition to the same degree as the substrate in competitive inlibition, if the specific concentrations of the reversor and substrate are equivalent. The addition of a reversor to a competitive system would lower the inhibition to:

$$
i=\frac{\left(\mathrm{I}_{t}{ }^{\prime}\right)}{\left(\mathrm{I}_{t}{ }^{\prime}\right)+1+\left(\mathrm{S}^{\prime}\right)+\left(\mathrm{R}_{t}{ }^{\prime}\right)}
$$

The dependency of the inhibition on the concentration of the reversor for different vahes of $K_{r}$ is shown in Fig. 13-5. From such families of curves it is possible to visualize the effectiveness of a particular reversor. To use a reversor properly, so that the resnlts can be accurately interpreted, it is necessary to know at least approximately the dissociation constants, $K_{i}$ and $K_{r}$. The use of a reversor with a $K_{r}$ much higher than $K_{i}$ would not be expected to diminish the inhibition unless its concentration were a good 
deal greater than that of the inhibitor. In those situations where the reversor is not depleted, $\left(R_{t}\right)$ must of necessity be higher than $\left(I_{t}\right)$ if an appreciable reduction in inhibition is to be expected. Thus only those portions of the curves at least above $10 \mathrm{~m} M$ reversor are valid under these conditions. If one wishes to reduce an inhibition to $1 / n$th of the initial value, the concentration of reversor that must be added is given by:

$$
\left(\mathrm{R}_{t}\right)=(n-1) \frac{K_{r}}{K_{i}}\left[(\mathrm{I})+K_{i}\right)
$$

The ratio of the concentration of reversor to that of inhibitor required to produce a specified reduction in the inhibition has often been considered an important factor in describing an inhibition reversal quantitatively,

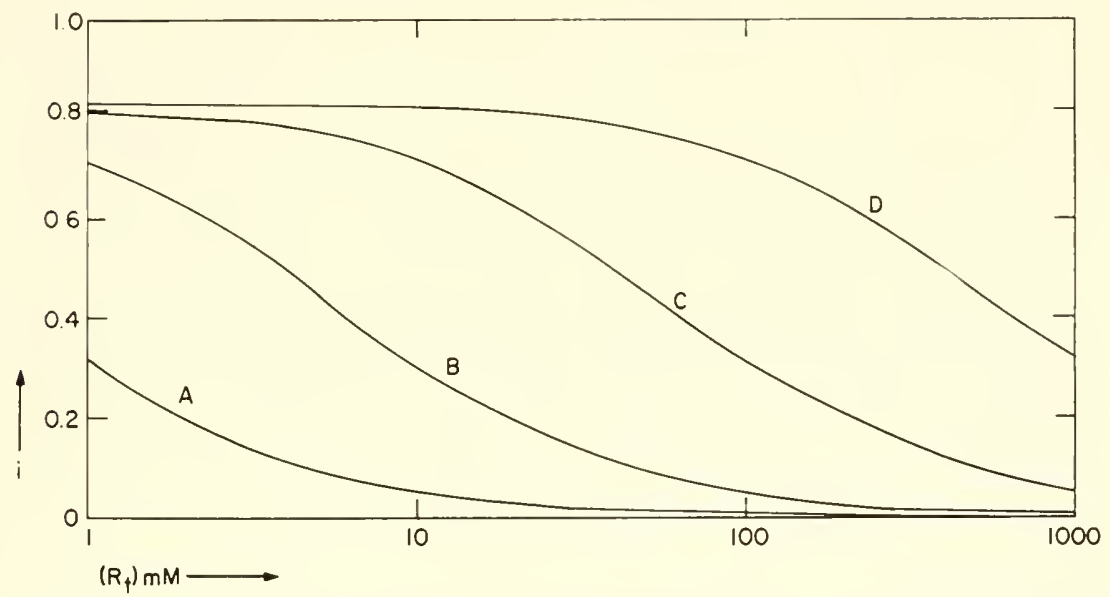

FIG. 13-5. Reversal of the inhibition by a substance binding the inhibitor (Eq. 13-21). $K_{i}=1 \mathrm{~m} M,(\mathrm{I})=5 \mathrm{~m} M$, and $\left(\mathrm{E}_{t}\right)=1 \mathrm{~m} \mu$. Curve A: $K_{r}=0.1 \mathrm{~m} . \mu$; curve B: $K_{r}=$ $1 \mathrm{~m} M$; curve C: $K_{r}=10 \mathrm{~m} M$; curve D: $K_{r}=100 \mathrm{~m} M$.

and in some instances $\left(\mathrm{R}_{t}\right) /\left(\mathrm{I}_{t}\right)$ is specified rather than $\left(\mathrm{R}_{t}\right)$. Under the present assumptions, this ratio is not a constant for each inhibition reversal but depends on the initial degree of inhibition:

$$
\frac{\left(\mathrm{R}_{t}\right)}{\left(\mathrm{I}_{t}\right)}=(n-1) \frac{K_{r}}{K_{i}}\left[1+\frac{K_{i}}{\left(\mathrm{I}_{t}\right)}\right]=\frac{n-1}{i} \frac{K_{r}}{K_{i}}
$$

The less the original inhibition, the greater must be the ratio of reversor to inhibitor for the inhibition to be reduced a chosen amount. A hypothetical example will serve to illustrate this; we shall assume that $K_{r}=K_{i}=1$ and that the inhibition is to be reduced to one-tenth its initial level. 
The following ratios may then be calculated:

\begin{tabular}{c|c|c|c}
\hline \multirow{2}{*}{$\left(\mathrm{I}_{t}\right)$} & $i_{0}$ & $\left(\mathrm{R}_{t}\right) /\left(\mathrm{I}_{t}\right)$ & $i_{f}$ \\
\cline { 1 - 3 } 0.2 & 0.17 & 54 & 0.017 \\
0.5 & 0.33 & 27 & 0.033 \\
1 & 0.50 & 18 & 0.050 \\
5 & 0.83 & 10.8 & 0.083 \\
10 & 0.91 & 9.9 & 0.091 \\
20 & 0.95 & 9.4 & 0.095 \\
\hline
\end{tabular}

On the other hand, if the inhibition in each case is to be reduced to a certain designated value, $i_{f}$, the ratio is given by:

$$
\frac{\left(\mathrm{R}_{t}\right)}{\left(\mathrm{I}_{t}\right)}=\frac{K_{r}}{K_{i}}\left[\frac{1}{i_{f}}-\frac{1}{i_{0}}\right]
$$

The ratio now does not vary so widely with the initial inhibition but changes in the opposite direction compared to the former situation.

\begin{tabular}{c|c|c|c}
\hline$\left(\mathrm{I}_{t}\right)$ & $i_{0}$ & $\left(\mathrm{R}_{t}\right) /\left(\mathrm{I}_{t}\right)$ & $i_{f}$ \\
\hline & & & \\
\hline 0.2 & 0.17 & 14 & 0.05 \\
0.5 & 0.33 & 17 & 0.05 \\
1 & 0.50 & 18 & 0.05 \\
5 & 0.83 & 18.8 & 0.05 \\
10 & 0.91 & 18.9 & 0.05 \\
20 & 0.95 & 18.95 & 0.05 \\
\hline
\end{tabular}

Thus the ratio, $\left(\mathrm{R}_{t}\right) /\left(\mathrm{I}_{t}\right)$, is not proportional to $K_{r} / K_{i}$, as has occasionally been assumed, and is equal to it only when the initial inhibition is high and is reduced $50 \%$ by the reversor.

The more general case, where $\left(\mathrm{R}_{t}\right)=(\mathrm{R})+(\mathrm{RI})$ and mutual depletion of reversor and inhibitor occurs, may now be treated. The simplest approach is to calculate the free inhibitor concentration in the presence of the reversor and use this concentration to determine the inhibition. The quadratic equation for the free inhibitor concentration is:

$$
(\mathrm{I})^{2}+\left[\left(\mathrm{R}_{t}\right)-\left(\mathrm{I}_{t}\right)+K_{r}\right](\mathrm{I})-K_{r}\left(\mathrm{I}_{t}\right)=0
$$

Plots of the inhibitions obtained by such a procedure are shown in Fig. 13-6 for a system with the same inhibition characteristics as in Fig. 13-5. As the affinity of the reversor for the inhibitor increases, the symmetrical sigmoid curves become distorted. There is not so much difference in the effects produced at lower concentrations of the reversor but very marked 
variations appear when $\left(R_{t}\right)$ approaches $\left(I_{t}\right)$. Curve $D$ gives the limiting case where the affinity between inhibitor and reversor is very high, so that $(I)=\left(I_{t}\right)-\left(R_{t}\right)$. Where the affinity is high:

$$
\frac{\left(\mathrm{R}_{t}\right)}{\left(\mathrm{I}_{t}\right)}=1-\frac{i_{f}\left(1-i_{0}\right)}{i_{0}\left(1-i_{f}\right)}
$$

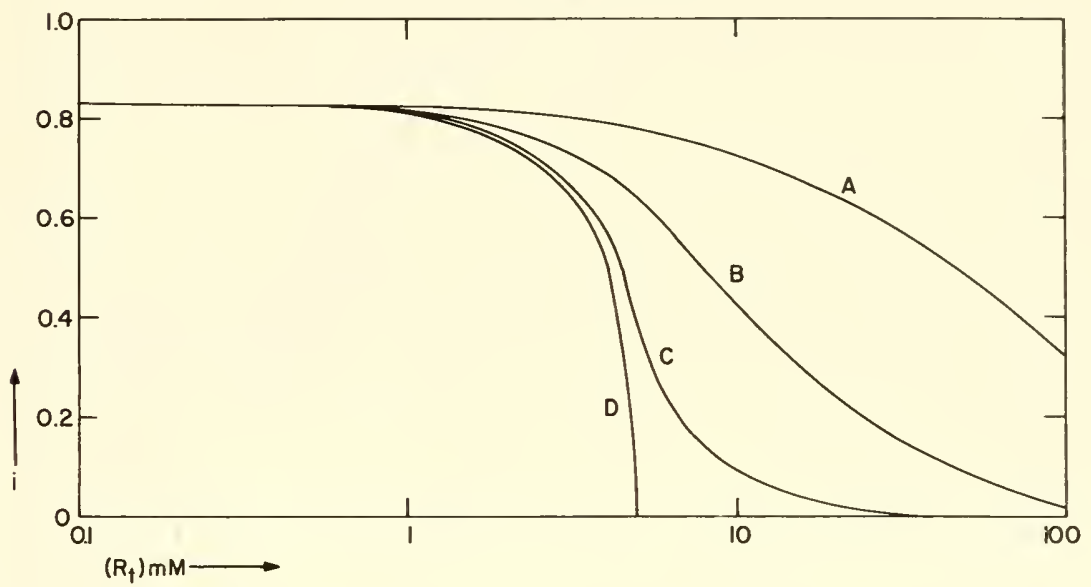

FIg. 13-6. Reversal of the inhibition by a substance binding the inhibitor for the general case in which the concentration of the free reversor is reduced by this binding, i.e., $\left(R_{t}\right)=(R)+(R I)$. The inhibition was calculated from the inhibitor concentrations derived from Eq. 13-26. $K_{i}=1 \mathrm{~m} M,(\mathrm{I})=5 \mathrm{~m} M$, and $\left(\mathrm{E}_{t}\right)=1$ $\mathrm{m} M$. Curve A: $K_{r}=10 \mathrm{~m} M$; curve $\mathrm{B}: K_{r}=1 \mathrm{~m} M$; curve C: $K_{r}=0.1 \mathrm{~m} M$; curve $\mathrm{D}: K_{r}=0$.

so that when the inhibition is reduced to $1 / n$th of the initial level, i.e.. $i_{f}=0.1 \quad i_{0}$ :

$$
\frac{\left(\mathrm{R}_{t}\right)}{\left(\mathrm{I}_{t}\right)}=\frac{n-1}{n-i_{0}}
$$

and thus in this case also the ratio depends on the initial inhibition.

It is possible to visualize the relationships between inhibitors and reversors on a $\mathrm{pI}$ scale. The representation is the same as that used for showing the relationships between acid-base and oxidation-reduction couples (Fig. 13-7). This is possible because one can write the following equations for the reactions of the inhibitor with enzyme and with reversor:

$$
\begin{aligned}
& \mathrm{pI}=\mathrm{p} K_{i}-\log \frac{(\mathrm{EI})}{(\mathrm{E})} \\
& \mathrm{pI}=\mathrm{p} K_{r}-\log \frac{(\mathrm{RI})}{(\mathrm{R})}
\end{aligned}
$$


The ability of a reversor to diminish an inhibition is then related to the position of the I-R curve relative to the I-E curve on the scale. Thus reversor 1 (upper curve) would decrease the inhibition much more effectively than reversor 2 (lower curve).

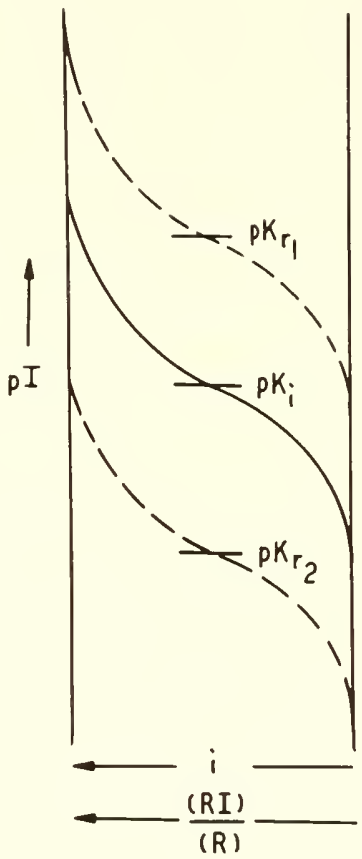

FIG. 13-7. Representation of inhibition reversal on a $\mathrm{pI}$ scale (Eqs. 13-29 and 13-30). The reversor 1 (upper curve) would decrease the inhibition more readily than the reversor 2 (lower curve).

\section{Kinetics of Inhibition Reversal by Substances Binding the Inhibitor}

If it is assumed that the reversor reacts only with free inhibitor and not with inhibitor bound to the enzyme, the first reaction that occurs upon adding the reversor is:

$$
\mathrm{R}+\mathrm{I} \underset{k_{-3}}{\stackrel{k_{3}}{\rightleftharpoons}} \mathrm{RI}
$$


Following reduction of the free inhibitor concentration, the dissociation of the EI complex may occur:

$$
\mathrm{EI} \underset{k_{1}}{\stackrel{k_{-1}}{\rightleftharpoons}} \mathrm{E}+\mathrm{I}
$$

If the former reaction is very rapid relative to the EI dissociation, the kinetics are simply those discussed previously. If the affinity of the reversor for the inhibitor is high and $\left(\mathrm{R}_{t}\right)$ is greater than $\left(\mathrm{I}_{t}\right)$, the inhibitor concentration will be reduced to a very low level and dissociation of the EI complex will proceed at a rate dependent only on $k_{-1}$ (see preceding section), inasmuch as inhibitor released from the enzyme will be immediately bound by the reversor. When the inhibitor concentration is not reduced to near zero but is appreciable and in equilibrium with the reversor, the inhibition will fall to a new level and the kinetics will follow those for dilution (see preceding section). In most experimental studies of reversal, the reversor is added in definite excess over the inhibitor, and the rate of reaction of the two is reasonably rapid. Therefore situations where the rate of reversal is related only to the dissociation of the EI complex are probably quite common.

It is also possible to conceive of cases where the dissociation rate is minch greater than the rate of combination of the reversor with the free inhibitor. Here the rate of change of the inhibition will depend almost solely on $k_{3}$. The differential equation for the inhibitor concentration is:

$$
\frac{d(\mathrm{I})}{d t}=k_{3} K_{r}\left(\mathrm{I}_{t}\right)-k_{3}\left[\left(\mathrm{R}_{t}\right)+k_{r}\right](\mathrm{I})
$$

when the reversor is not depleted during the reaction. This may be integrated to:

$$
(\mathrm{I})=\frac{\left(\mathrm{I}_{t}\right)}{\left(\mathrm{R}_{t}\right)+K_{r}}\left\{K_{r}+\left(\mathrm{R}_{t}\right) e^{-k_{3}\left[\left(\mathrm{R}_{t}\right)+K_{r}\right] t}\right\}
$$

This value of (I) may be substituted in the usual inhibition equations. It may be noted that at $t=0,(\mathrm{I})=\left(\mathrm{I}_{t}\right)$ and $i_{0}=\left(\mathrm{I}_{t}{ }^{\prime}\right) /\left[\left(\mathrm{I}_{t}{ }^{\prime}\right)+1\right]$; when equilibrium has been reached and $t=\infty$, $(\mathrm{I})=\left(\mathrm{I}_{t}\right) K_{r} /\left[\left(\mathrm{R}_{t}\right)+K_{r}\right]$ and $i_{f}=$ $\left(\mathrm{I}_{t}{ }^{\prime}\right) /\left[\left(\mathrm{I}_{t}{ }^{\prime}\right)+1+\left(\mathrm{R}_{t}{ }^{\prime}\right)\right]$, as expected. If the reversor is depleted during the reaction, the differential equation must be modified to:

$$
\frac{d(\mathrm{I})}{d t}=k_{3} K_{r}\left(\mathrm{I}_{t}\right)-k_{3}\left[\left(\mathrm{R}_{t}\right)+K_{r}-\left(\mathrm{I}_{t}\right)\right](\mathrm{I})-k_{3}(\mathrm{I})^{2}
$$

which may also be integrated to provide values for (I) at any time. Since very little quantitative work has been done on reversal kinetics, it is impossible in most instances to relate the changes in inhibition that are meas- 
ured to either one of the possibly limiting reactions. The rates of reactions 13-31 and 13-32 must be determined independently in order to interpret the data obtained when they are proceeding simultaneously.

The most general situation in which the rates of the two reactions are comparable is more difficult to treat rigorously. Two simultaneous differential equations must be solved. One will be either Eq. 13-33 or 13-35, depending on whether the reversor is depleted or not, and the other will be the usual equation for the rate of inhibition change:

$$
d i / d t=k_{-1}\left(\mathrm{I}^{\prime}\right)-k_{-1}\left[\left(\mathrm{I}^{\prime}\right)+1\right] i
$$

The decrease in the inhibition will be slower than in either of the extreme cases discussed above (assuming the same rate constants and conditions) but will follow the same type of curve. It wonld be most difficult to distinguish between these various possibilites from the inhibition reversal data alone.

\section{Examples of Inhibition Reversal}

Most of the studies on reversal have been made with inhibitors that combine with enzyme sulfhydryl groups. It will suffice for the present purpose to mention some of the results obtained on the reversal of inhibitions produced by the mercurials. More detailed discussion will be given in the chapters on the varions inhibitors. One may find instances of complete reversal, partial reversal, or no reversal when sulfhydryl compounds are added to remove the mereurial from the enzymes. For example, complete restoration of activity has been reported in inhibitions of the following enzymes after addition of either cysteine or glutathione: yeast glyceraldehyde-3-phosphate dehydrogenase (Velick, 1954), brain hexokinase (Sols and Crane, 1954), heart laetie dehydrogenase (Neilands, 1951), xanthine oxidase (Harris and Helleman, 1953), and corn leaf ghtumie dehydrogenase (Bulen, 1956). All of these inhibitions had been produced by $p$-chloromercmibenzoate ( $p$-CMB). Partial reversal oceurred when dimercaprol was added to snceinoxidase inhibited with $\mathrm{HgCl}_{2}$ (Barron and Kalnitsky, 1947) or when ghtuthione was added to yeast alcohol dehydrogenase inhibited by $p$ CMB (Barron and Levine, 1952). No reversal was observed with the following enzymes inhibited by $p$-CMB: muscle pyruvate oxidase - eysteine or dimereaprol (Onrust et al., 1954); heart malic dehydrogenase - cysteine or glutathione (Wolfe and Neilands, 1956); Electrophorus acetylcholinesterase - cysteine (Hargreaves, 1955); and crotonase - eysteine or ghtathione (Wakil and Mahler, 1951) to mention only a few. It is likely that these different behaviors do not necessarily reflect different mechanisms of inhibition. It has often been assumed that a lack of reversibility indicates that sulfhyfdryl groups are not involved in the inhibition. Such statements as: "Reactivation is an obligatory condition for testing the specificity 
of the action of a chemical or physical agent that inhibits sulfhydryl groups" (Hargreaves, 1955), are common and misleading. If a series of enzymes is inbibited with the same inhibitor and reactivation is attempted in each case with the same reversor, it might be expected that all degrees of reversibility would be exhibited because the enzymes would show a wide range of affinities for the inhibitor. The results would depend primarily upon the relative dissociation constants of the inhibitor with enzyme and with reversor. Attempting to reverse an inhibition with a substance whose $\mathrm{p} K_{r}$ is much lower than the $\mathrm{p} K_{i}$ for the inhibition is as futile as trying to oxidize or reduce a substance with another whose redox potential is inappropriate. The inhibitions that gave no reversal above probably involve the reaction of sulfhydryl groups as in those cases where complete restoration of activity occurred. The different degrees of reversibility indicate most likely the relative affinities of the various enzymes for the mereurial.

No measurements of the rates of reversal seem to have been reported. In some cases it is surprisingly rapid. The reactivation of $p$-CMIB-inhibited glyceraldehyde-3-phosphate dehydrogenase by cysteine is so rapid that technically it is not possible to measure it (Velick, 1953, 1954). The addition of cysteine in a concentration about a hundred times greater than the $p$-CMB concentration produced immediate and almost complete reversal (Fig. 13-8). Similar results were obtained with brain hexolinase (Sols and Crane, 1954), although the reversal was not as complete. These results show that the reaction of eysteine with $p$-CMB and the dissociation of $p$-CMB from the enzyme are reasonably fast, but no information is available as to which reaction is limiting the rate. Also it is not known how these rates of reversal compare with the rates that would be observed in experiments where the inhibitor was removed by other means, such as dialysis. If it could be demonstrated in any ease that the rate of reversal with a substance binding the inhibitor is greater than the rate found when the inhibitor was removed by physical means, it would inclicate strongly that the reversor is able to react with the inhibitor that is bound to the enzyme and remove it more rapidly than would occur with spontaneous dissociation. It would certainly seem to be very unlikely that such a reaction would occur in most cases, due to steric factors alone, but it is possible, and aceurate comparison of reversal rates might provide some information on this interesting point. Reactions of two substances on the surface of an enzyme commonly oceur but usually the reaction does not involve the groups attaching the substances to the enzyme.

One factor that may be very important in reclucing reversibility is the progressive inactivation of the inhibited enzyme. It has frequently been observed that enzyme inhibitions become increasingly irreversible. In the ease of the inhibition of brain hexokinase with $p$-CMB, which is, under the proper conditions, completely reversible, increasing incubation times with 
the inhibitor led to less and less reactivation from added cysteine (Sols and Crane, 1954). The higher the inhibitor concentration and thus the greater the inhibition, the more likely is secondary inactivation to occur and reversibility be limited. Sometimes this may involve a structural change in the enzyme due to an increased instability in the presence of the inhibitor, or it may be the result of the reaction of the inhibitor with additional less reactive groups on the enzyme, the latter reactions being either irre-

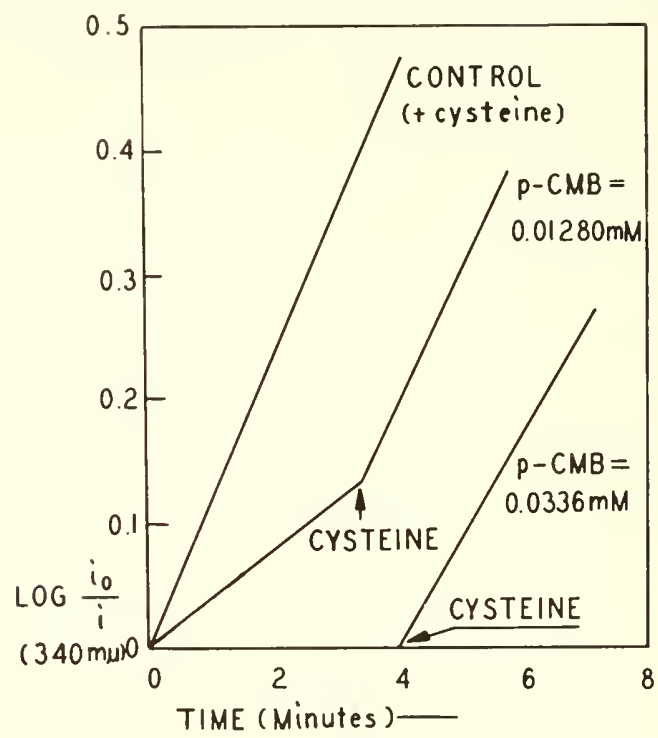

FIG. 13-8. Reactivation of phosphoglyceraldehyde dehydrogenase inhibited by $p$-chloromercuribenzoate $(p$-CMB) with cysteine added at the time indicated by the arrows. The cysteine concentration was $3 \mathrm{~m} .1$. (From Velick, 1953, 1954.)

versible or slowly reversible. In any event, if the reversibility of the primary inhibition is being tested, it is advisable to add the reversor as soon as possible, and it is generally preferable to inhibit the enzyme to the extent of only around $50 \%$ instead of completely. It is quite possible that the partial and total irreversibilities mentioned above were to some degree due to these secondary reactions.

\section{Information Obtained from Protection and Reversal Studies}

Since the majority of the studies with certain inhibitors, especially those reacting with sulfhydryl gromps, include data on the reversibility of the inhibition following the addition of a sulfhydryl compound to remove 
the inhibitor, it is necessary to evaluate critically the information one may obtain from such experiments. There are two minor things one may possibly learn from reversal studies: (1) an estimate of the relative affinities of the enzyme and reversor for the inhibitor, and if $K_{r}$ is known, $K_{i}$ may be calculated approximately; and (2) if complete reversal occurs, no secondary reactions leading to inactivation of the enzyme have taken place. An estimate of $K_{i}$ may be made more readily from the usual graphical procedures and a test for inactivation can be done with less complexity by dialysis. This is not the information that has generally been looked for in such work. Instead, conclusions as to the mechanism of inhibition have often been drawn. There is no relationship between the way in which the inhibitor reduces the activity of the enzyme and the reversibility. From the rate at which an inhibitor can be removed from an enzyme, or from the degree to which the inhibition may be reduced by the addition of an inhibitorbinding substance, one can tell nothing as to the mechanism of the primary inhibition. It has been stated many times that if the inhibition produced by a sulfhydryl agent is reversed by a sulfhydryl compound (such as cysteine, glutathione, or dimercaprol), this is evidence for the reaction of the inhibitor with enzyme sulfhydryl groups as the mechanism of the inhibition. Such a conclusion is not valid and it should be so obviously not valid that no discussion of the problem is warranted.

Let us now consider some problems in the interpretation of partial or complete irreversibility. Failure to restore the total enzyme activity is not certain proof of either an irreversible binding of the inhibitor to the enzyme or of secondary reactions leading to irreversible changes in the enzyme. Homogentisate oxidase is well inhibited with p-CMB but glutathione will not reactivate the enzyme (Crandall, 1955). However, if $\mathrm{Fe}^{++}$ions are added with the glutathione, restoration of activity occurs. The $p$-CMIB by reacting with the enzyme has displaced the $\mathrm{Fe}^{++}$ions necessary for catalytic activity and removal of the $p$-CMB alone, by any means, will not reverse the inhibition. Ferrous ions alone will also not reactivate because they cannot reach the activator site on the enzyme. But removal of the inhibitor followed by addition of the activator abolishes the inhibition. This phenomenon may be rather common. inasmuch as it is known that inhibitors can displace cofactors and coenzymes; the displacement of DPN+ from certain enzymes by the mercurials would be an example.

Another possible explanation for irreversibility would be an effect of the reversor on the enzyme. If the reversor in any way were an inhibitor or inactivator of the enzyme, it would simultaneously remove the original inhibitor and depress the activity of the enzyme. Reactivation of succinoxidase inhibited by $p$-CMB (Slater, 1949) provides an excellent illustration of this. Certain thiols used for reactivation were oxidized in the enzyme preparation (presumably by dissolved oxygen and catalyzed by substances 
in the medium) and in turn oxidized the enzyme sulfhydryl groups. In nitrogen, this reaction does not occur. Glutathione, for example, in air is an inhibitor of succinoxidase by way of its GS-SG form, while simultaneously it will remove the $p$-CMB from the enzyme. It might be suggested that ideally such reversal experiments with thiols be conducted in nitrogen. Some of the failures noted above could well be due to this phenomenon.

Protection studies, where the substance binding the inhibitor is added either before or with the inhibitor, are of even less significance in providing useful or reliable information about the inhibition. If a substance reacts with an inhibitor, it will reduce the concentration of free inhibitor, thereby reducing the rate at which inhibition occurs and decreasing the final inhibition achieved. Results of this type showing protection only confirm the obvious and have no relationship to the mechanism of the inhibition. The only interesting thing that might emerge from protection studies is the demonstration that a substance known to bind or react with the inhibitor does not influence the inhibition in the expected manner, since in such a case it might indicate that the reactive group or groups on the inhibitor, with respect to the inhibition, were not those involved in the reaction with the reversor. The effects of metal chelators on enzymes or metabolism might profitably be compared with the effects of the metal ion-chelator complexes, in order to determine if the inhibition is indeed through chelation of an enzymically important metal ion or, on the other hand, is an action dependent on the total structure of the molecule and unrelated to the chelating activity.

The preceding pessimistic remarks are meant to apply to basic experimental studies on enzyme inhibition. Information on protection and reversal can often be of great pratical importance, particularly as the actions of many poisons and the toxic effects of certain drugs are the result of enzyme inhibitions. It is also not implied that accurate quantitative work on inhibition reversal is valueless; indeed, some very interesting facts might be derived from such work where reversal rates are measured. Finally, protection or reversal by substances binding the inhibitor has been considered here, and other means of modifying the inhibition may be very informative, as will be seen in the following sections.

\section{SPONTANEOUS REACTIVATION OF INHIBITED ENZYMES}

Occasionally it has been observed that the inhibition in pure enzyme systems is not maintained but is slowly lost. The inhibition rises to a maximal value and then dechines with time. This usually means that either the free inhibitor is unstable and is inactivated in solution, or the bound inhibitor is reacted by the enzyme. The former nonenzymic inactivation of 
the inhibitor will be taken up in a later section and here we shall confine ourselves to the latter situation. Chemical modification of the inhibitor by the enzyme usually occurs when the inhibitor in some manner resembles the substrate and attaches to the enzyme in a way characteristic of the substrate.

The most thoroughly documented example of such a spontaneous reversal occurs in the inhibition of cholinesterase by certain organophosphorus compounds (Aldridge, $1953 \mathrm{a}, \mathrm{b}$; Aldridge and Darison, 1953). The time courses of inhibition of rabbit erythrocyte cholinesterase by dimethyland diethyl-p-nitrophenyl phosphates are shown in Fig. 13-9. The inhibition

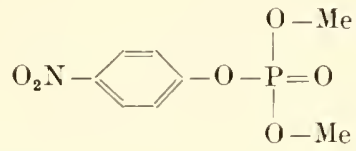

Dimethyl-p-nitrophenyl phosphate

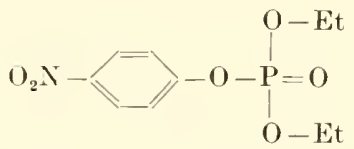

Diethyl-p-nitrophenyl phosphate

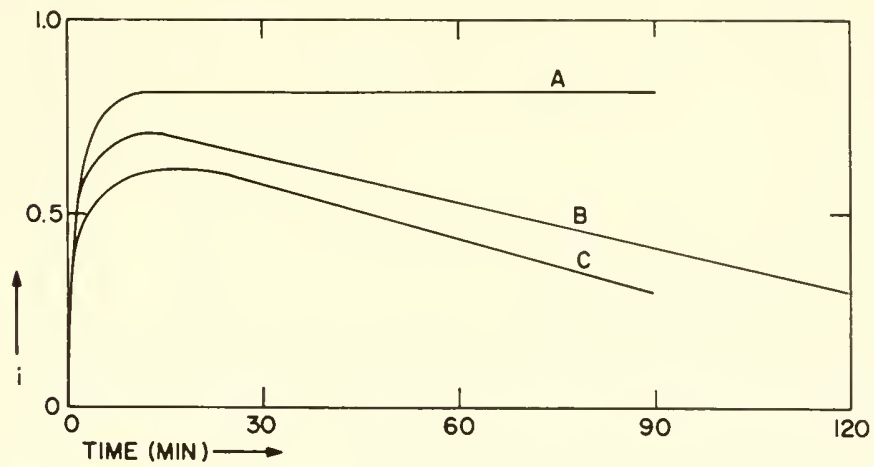

FIG. 13-9. Inhibition of rabbit erythrocyte cholinesterase by p-nitrophenyl phosphates and its spontaneous reversal. (From Aldridge, 1953 b.) Curve A: diethyl-p-nitrophenyl phosphate $\left(4.1 \times 10^{-4} \mathrm{~m} / \mathrm{H}\right)$; curve $\mathrm{B}$ : dimethyl-p-nitrophenyl phosphate $\left(2.4 \times 10^{-3} \mathrm{~m} M\right)$; curve C: dimethyl-p-nitrophenyl phosphate $\left(4.7 \times 10^{-4} \mathrm{~m} M\right)$.

produced by the diethyl compound is stable but that by the dimethyl analog is slowly and spontaneously reversible. The reversal follows first-order kinetics as is shown by the linear relationship between log (per cent inhibition) and time (Fig. 13-10). The first-order rate constant for the reversal reaction was found to be $1.68 \times 10^{-3} \mathrm{~min}^{-1}$ at $17^{\circ}, 4.29 \times 10^{-3} \mathrm{~min}^{-1}$ at $28^{\circ}$, and $8.55 \times 10^{-3} \mathrm{~min}^{-1}$ at $37^{\circ}$, giving an energy of activation of $14.4 \mathrm{kcal} /$ mole. Aldridge postulated the following sequence to explain the inhibition reversal: 


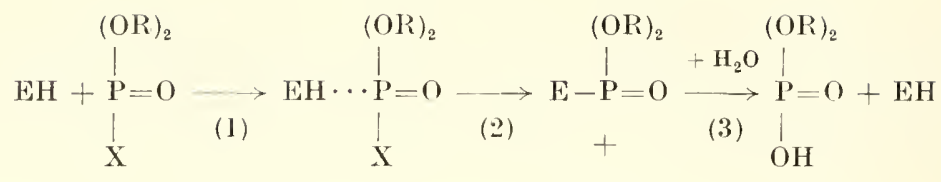

HX

This is very similar to the hydrolysis of acetylcholine by the enzyme and presumably the same active site groups are involved. The inhibition by the dimethyl compound is due to the fact that reaction 3 is very slow and this allows the accumulation of the EI complex. For the diethyl compound, it is apparent that reaction 3 is too slow to measure. The reversal reaction is an hydrolysis of the phosphorylated enzyme. Its rate is independent of

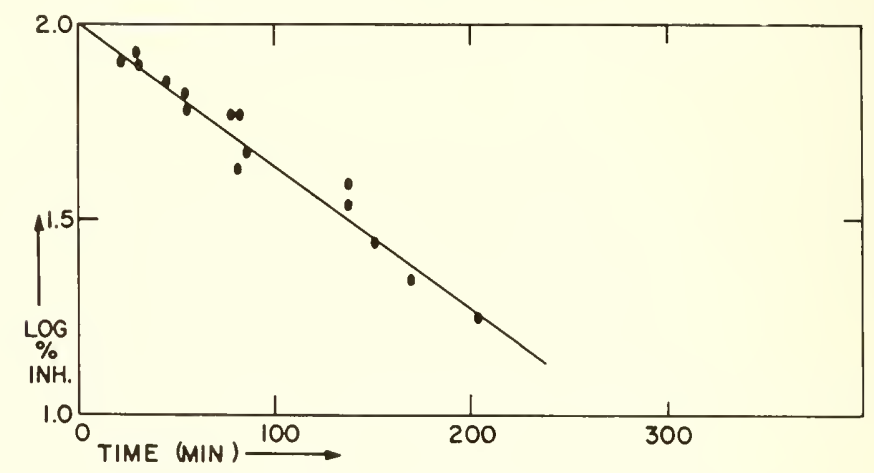

Frg. 13-10. Spontaneous reversal of inhibition of rabbit erythrocyte cholinesterase inhibited by dimethyl-p-nitrophenyl phosphate plotted on a logarithmic scale to show the first-order kinetics. (From Aldridge, 1953 b.)

the group $\mathrm{X}$ but is related to the group $\mathrm{R}$, since $\mathrm{R}$ can modify the electronic configuration and charge aronnd the phosphorus atom and hence alter its reactivity with water.

The maximal inhibition produced in such cases may be expressed easily. The over-all reaction of the inhibitor is:

$$
\mathrm{E}+\mathrm{I} \underset{k_{-1}}{\stackrel{k_{1}}{\rightleftharpoons}} \mathrm{EI} \stackrel{k_{2}}{\rightarrow} \mathrm{E}+\mathrm{Q}
$$

where $Q$ is the product formed by the action of the enzyme on the inhibitor. Thus:

$$
(\mathrm{EI})=\frac{k_{1}\left(\mathrm{E}_{t}\right)(\mathrm{I})}{k_{1}(\mathrm{I})+k_{-1}+k_{2}}
$$


from which:

$$
i=\frac{(\mathrm{I})}{(\mathrm{I})+\left[\left(k_{-1}+k_{2}\right) /\left(k_{1}\right)\right]}
$$

Since $k_{2}$ is so small:

$$
i=\frac{(\mathrm{I})}{(\mathrm{I})+K_{i}}
$$

which is the expression for noncompetitive inhibition. If the substrate is present during the development of the inhibition, it will slow down the rate at which the maximal inhibition is reached and may decrease the maximal inhibition if it can compete significantly with the inhibitor. In those instances where $k_{2}$ is significant, Eq. $13-40$ should be used and the inhibition will no longer relate directly to the inhibition constant. $K_{i}$. The shape of the total curve giving the time course of the inhibition will depend also on the amount of inhibitor present; if $\left(\mathrm{I}_{t}\right) \cong\left(\mathrm{E}_{t}\right)$ the curve will have a sharp maximal peak whereas if $\left(\mathrm{I}_{t}\right) \gg\left(\mathrm{E}_{t}\right)$, the curve will show a plateau since the inhibition will not begin to decrease until there is no more inhibitor to replace that reacted on the enzyme.

A more accurate reaction sequence for the phosphorylation or carbamylation of enzymes might be written as:

$$
\mathrm{E}+\mathrm{I} \underset{k_{-1}}{\stackrel{k_{1}}{\rightleftharpoons}} \mathrm{EI} \stackrel{k_{2}}{\rightarrow} \mathrm{EX} \stackrel{k_{3}}{\rightarrow} \mathrm{E}+\mathrm{X}^{\prime}
$$

where EI is the initial complex of the inhibitor with the enzyme, EX is the chemically inactivated enzyme ( $\mathrm{X}$ representing the inhibitor group remaining on the enzyme), and $\mathrm{X}^{\prime}$ is the inhibitor group after it has been dissociated from the enzyme and perhaps chemically modified (as in a hydrolytic reaction). From the differential equation representing the change in EX concentration:

$$
\frac{d(\mathrm{EX})}{d t}=k_{2}(\mathrm{EI})-k_{3}(\mathrm{EX})
$$

the conservation equation, $\left(\mathrm{E}_{l}\right)=(\mathrm{E})+(\mathrm{EI})+(\mathrm{EX})$, and the equilibrium, $K_{i}=(\mathrm{E})(\mathrm{I}) /(\mathrm{EI})$, the rate of change of $(\mathrm{EX})$ may be determined:

$$
\frac{d(\mathrm{EX})}{d t}=\frac{k_{2}\left(\mathrm{E}_{t}\right)(\mathrm{I})}{(\mathrm{I})+K_{i}}-(\mathrm{EX})\left[k_{3}+\frac{k_{2}(\mathrm{I})}{(\mathrm{I})+K_{i}}\right]
$$

In the steady state, $d(\mathrm{EX}) / d t=0$ so that:

$$
(\mathrm{EX})_{s t}=\frac{k_{2}\left(\mathrm{E}_{t}\right)}{k_{2}+k_{3}\left[1+K_{i} /(\mathrm{I})\right]}
$$


The steady-state concentration of the complex EI may also be similarly determined:

$$
(\mathrm{EI})_{s t}=\frac{(\mathrm{I})\left(\mathrm{E}_{t}\right)}{(\mathrm{I})+K_{i}}\left[1-\frac{k_{2}}{k_{2}+k_{3}\left[1+K_{i} /(\mathrm{I})\right]}\right]
$$

The total inhibition, given by $i=[(\mathrm{EX})+(\mathrm{EI})] /\left(\mathrm{E}_{\ell}\right)$, is thus:

$$
i_{s t}=\frac{(\mathrm{I})}{(\mathrm{I})+K_{i}}\left[1+\frac{k_{2}}{k_{2}\left[(\mathrm{I}) / K_{i}\right]+k_{3}\left[1+(\mathrm{I}) / K_{i}\right]}\right]
$$

This is the total inhibition in the steady state. A slightly different treatment of this system has been given by Wilson et al. (1960) for the carbamylation of cholinesterase and the rate constants for the inhibitors were calculated.

\section{INDUCED REACTIVATION OF INHIBITED ENZYMES}

Inhibitions resulting from a substratelike chemical reaction of a substance with the enzyme active site, in which the breakdown of the EI complex is slow as described in the previous section, may be reversed by compounds reacting with the EI complex and splitting the inhibiting group from the enzyme.* The normal substrates for cholinesterase form acylenzyme intermediates which react with water to complete the hydrolysis; the inhibitors form instead alkylphosphoryl-enzyme complexes which react very slowly or not at all with water. The introduction of a substance that reacts with the EI complex as does water, but much more readily, may split the phosphoryl group from the enzyme and restore the activity. Since the phosphorus atom is electrophilic, it was postulated by Wilson (1951) that strongly nucleophilic groups might attack the phosphorus and dissociate it from the enzyme, and it was indeed fonnd that hydroxylamine would reactivate the acetylcholinesterase of electric tissue after inhibition by tetraethylpyrophosphate (TEPP). Since then many more effective reactivators have been found and most contain the nucleophilic hydroxamate $\left(\mathrm{R}-\mathrm{CO}-\mathrm{NHO}^{-}\right)$or aldoxime $(\mathrm{R}-\mathrm{CH}=\mathrm{NOH})$ groups. The reaction with an hydroxamate may be written as:

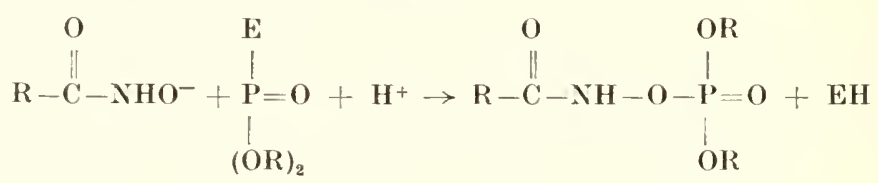

* The inhibited enzyme in such cases will be designated by EI although strictly speaking this is inaccurate, since the inhibitor is chemically modified upon reaction with the enzyme. The inhibiting group on the enzyme is only part of the original inhibitor molecule; in the case of the organophosphorus compounds, the X-group has been lost. However, this simplification should not introduce any confusion into the treatment. 
The most commonly used reactivators have been hydroxamates and aldoximes of $\mathrm{N}$-methylpyridine, such as $\mathrm{N}$-methylnicotinohydroxamate and $N$-methylpicolinohydroxamate, and especially pyridine-2-aldoxime methiodide (2-PAM), which has been found to reactivate inhibited cholinesterase<smiles>C[n+]1ccccc1C=O</smiles>

$N$-Methylpyridine2-aldoxime

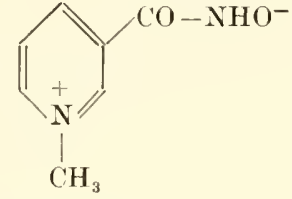

N-Methylnicotinohydroxamate<smiles>C[n+]1ccccc1C(=O)N[O-]</smiles>

$N$-Methylpicolinohydroxamate

in the tissues of poisoned animals. However, the oximes and dioximes of certain bis (pyridinium) compounds have been found to be much more potent reactivators than 2-PAM (Hobbiger et al., 1958). The relationships be-

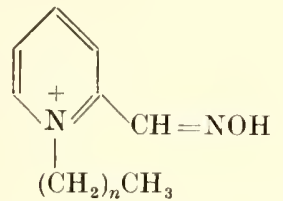

$\mathrm{N}$-Alkylpyridine-

2-aldoximes

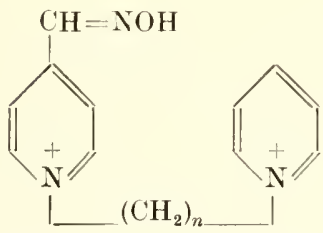

Bis (pyridinium) oximes

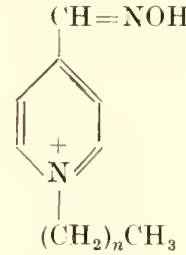

$N$-Alkylpyridine4 -aldoximes

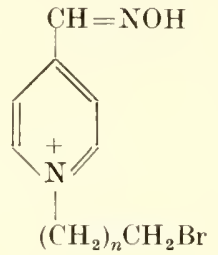

$N$-Bromoalkylpyridine4-aldoximes

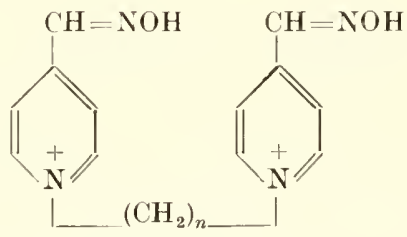

Bis (pyridinium) dioximes

tween structure and reactivating potency are very interesting. In the first place, the presence of a positively charged group adjacent to the nucleophilic group enhances the activity. Comparison of the varions reactivators in their unsubstituted and $N$-methyl forms shows that the latter are always more potent. The reactivation rate constant for pyridine-2-aldoxime is $0.0045 \mathrm{~min}^{-1}$ and for $N$-methylpyridine-2-aldoxime it is $0.14 \mathrm{~min}^{-1}$ (Wilson et al., 1958). In addition, the 2-PAM is bound more tightly to the 
EI complex so that less than one-thousandth the concentration of the unsubstituted analog is required for reactivation. This is believed to be dne to the availability of the anionic site in TEPP-inhibited eholinesterase, the electrostatic attraction between this group and the $\mathrm{N}^{+}$-group facilitating the binding of the reactivator. In cholinesterase inhibited by diisopropylfuorophosphate (DFP), the anionic site is not available and the presence of a positively charged atom on the reactivator is not so important. In the second place, the nucleophilic group should be within a certain distance of the positive charge in the reactivator, and perhaps also in a favorable direction relative to the positive eliarge (if the rest of the pyridine ring is constrained to one orientation on the enzyme surface). The reactivating potency of 2-PAM has been attributed to its ability to react with the enzyme in such a way that the nucleophilic oxygen atom falls about one bond length from and is directed toward the phosphorus atom of the inactivated enzyme (Wilson and Ginsburg, 1958) and the differences between the 2-, 3- and 4 -aldoximes of $N$-methylpyridine have been ascribed to related steric factors (Wilson et al., 1958).

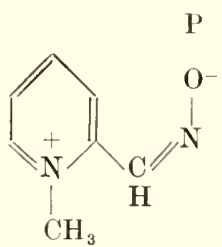

The group attached to the pyridine- $\mathrm{N}^{+}$is also of great importance, as may be seen in the relative potencies given in Table 13-1. This group may either increase binding through its van der Waals interaction with the enzyme surface or interfere with the binding sterically. The reason for the very high activity of the bis(pyridinium) aldoximes is not elear as yet but it could be the result of the tight binding of these compounds to the enzyme surface in a position that is near optimal for the nucleophilic attack on the phosphorus atom. Comparison of large numbers of reactivators has led to the conchusion that the poteney is not correlated with the ability to inhibit or bind to the normal enzyme, nor with the reactivity toward the organophosphorus inhibitors (Hobbiger et al., 1960).

The reactivation reactions and kineties may be expressed in different ways. At least three forms of the enzyme must be assumed in inhibitions by the organophosphorus compounds: the initial reversible EI complex, the phosphorylated enzyme EX, and the final inactivated enzyme after the phosphate group has been shifted to another amino acid. Reactivation seems to be exerted only on the EX form (Sanderson and Edson, 1959). The rate at which EX passes into the irreversibly inactivated form varies with the inhibitor and the enzyme; in those cases where this reaction is 
Table 13-1

Relative Potencies of Reactivators of Human Erythrocyte Cholinesterase Inhibited by Tetraethylpyrophosphate ${ }^{a}$

\begin{tabular}{|c|c|c|}
\hline Compound ${ }^{b}$ & $n$ & $\begin{array}{c}\text { Relative } \\
\text { reactivating } \\
\text { potency }\end{array}$ \\
\hline$N$-Methylpyridine-2-aldoxime & - & 100 \\
\hline$N$-Methylpyridine-3-aldoxime & - & 0.03 \\
\hline$N$-Methylpyridine- 4 -aldoxime & - & 6 \\
\hline \multirow[t]{4}{*}{$N$-Alkylpyridine-2-aldoximes } & 0 & 100 \\
\hline & 1 & 54 \\
\hline & 2 & 63 \\
\hline & 3 & 87 \\
\hline$N$-Ethylpyridine-4-aldoxime & - & 3 \\
\hline \multirow[t]{4}{*}{$N$-Bromoalkylpyridine-4-aldoximes } & 0 & 160 \\
\hline & 2 & 190 \\
\hline & 3 & 25 \\
\hline & 4 & 94 \\
\hline \multirow[t]{3}{*}{ Bis(pyridinium) aldoximes } & 3 & 800 \\
\hline & 4 & 670 \\
\hline & 5 & 750 \\
\hline \multirow[t]{4}{*}{ Bis(pyridinium)dialdoximes } & 2 & 1700 \\
\hline & 3 & 2200 \\
\hline & 4 & 1800 \\
\hline & 5 & 1600 \\
\hline
\end{tabular}

a From Hobbiger et al. (1958).

$b$ The structures of the reactivators are included in text.

rapid, reactivation is difficult. Spontaneous reactivation may proceed along with the reactivation brought about by nucleophilic agents. The expressions for the inhibition are easily obtained for these situations. Spontaneous reactivation:

induced reactivation:

$$
\begin{gathered}
\mathrm{EX} \stackrel{k}{\rightarrow} \mathrm{E}+\mathrm{X}^{\prime} \\
i=i_{0} e^{-k t}
\end{gathered}
$$

$$
\begin{gathered}
\mathrm{EX}+\mathrm{R} \stackrel{k^{\prime}}{\rightarrow} \mathrm{E}+\mathrm{XR} \\
i=i_{0} e^{-k^{\prime}(\mathrm{R}) t}
\end{gathered}
$$


and both spontaneous and induced reactivation:

$$
i=i_{0} e^{\left[-k-k^{\prime}(\mathrm{R})\right] t}
$$

If $\log i$ is plotted against $t$, the slope will provide a means for calculating the rate constants.

Plots of this type were made for the reactivation of acetylcholinesterase carbamylated by dimethylcarbamylcholine and dimethylcarbamylfluoride. These inhibitors react with the enzyme active center in a manner similar to the organophosphorus compounds (Wilson et al., 1960) and the EX form is the same for both the inhibitors. Hydroxylamine reactivates but 2-PAM and other agents effective against the enzyme inhibited by the organophosphorus inhibitors do not. The plots of $\log i$ against $t$ are shown in Fig. 13-11 for both the spontaneous reactivation and that induced by hydroxylamine. The monomolecular spontaneous reactivation rate constant, $k$, was found to be $0.032 \mathrm{~min}^{-1}$.

If the reactivators form a complex with the inactivated enzyme:

$$
\mathrm{EX}+\mathrm{R} \underset{k_{-1}}{\stackrel{k_{1}}{\rightleftharpoons}} \mathrm{EXR} \stackrel{k_{2}}{\rightarrow} \mathrm{E}+\mathrm{XR}
$$

the kinetics of reactivation would be expected to conform to ordinary enzyme kinetics (Green and Smith. $1958 \mathrm{a}, \mathrm{b})$. The rate at which the reactivation proceeds will be given by:

$$
\iota_{r}=\frac{k_{2}\left(\mathrm{EX}_{t}\right)(\mathrm{R})}{(\mathrm{R})+K_{x \tau}}
$$

where $\left(\mathrm{EX}_{\imath}\right)=(\mathrm{EX})+(\mathrm{EXR})$ and $K_{x r}$ is the Michaelis constant, which in the general case is equal to $\left(k_{-1}+k_{2}\right) / k_{1}$. The relative values of the constants are not known and hence it is not certain if $K_{x r}$ is ever a true dissociation constant. The reactivation has been shown to follow first-order kinetics when the reactivator is in excess, and the release of phosphorus from the enzyme has been shown to accompany the reduction in the inhibition (Jandorf et al., 1955).

It is of interest now to express the steady-state inhibition in a system in which a reactivator is present. The following reactions may be written:

$$
\begin{gathered}
\mathrm{E}+\mathrm{I} \underset{k_{-1}}{\stackrel{k_{1}}{\rightleftharpoons}} \mathrm{EI} \stackrel{k_{2}}{\rightarrow} \mathrm{EX} \\
\mathrm{EX}+\mathrm{R} \stackrel{k_{3}}{\rightarrow} \mathrm{E}+\mathrm{XR}
\end{gathered}
$$


and the inhibition may be shown to be:

$$
\left.i_{s t}=\frac{(\mathrm{I})}{(\mathrm{I})+K_{i}} \mid 1+\frac{k_{2}}{k_{2}\left[(\mathrm{I}) / K_{i}\right]+k_{3}(\mathrm{R})\left[1+\frac{(\mathrm{I})}{K_{i}}\right]}\right\}
$$

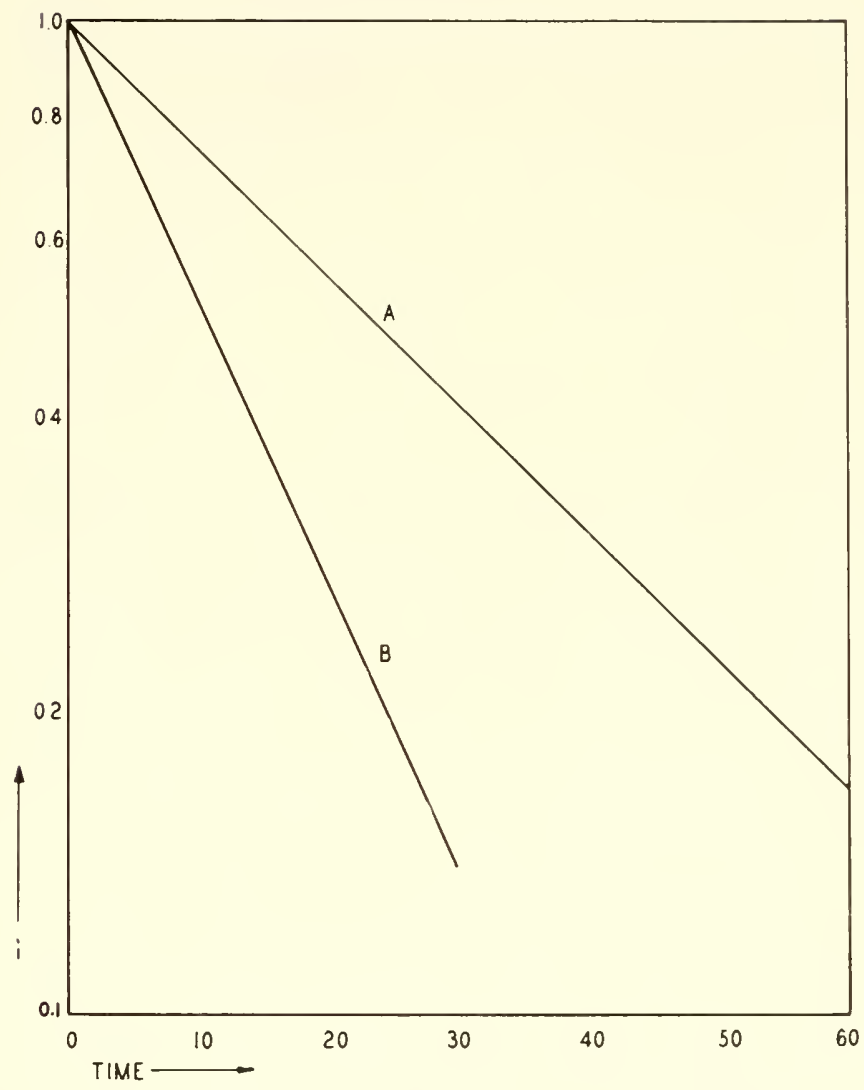

FIG. 13-11. Reactivation of acetylcholinesterase carbamylated by dimethylcarbamylcholine or dimethylcarbamyl fluoride. (From Wilson et al., 1960.) Curve A: spontaneous reactivation: curve B: reactivation with hydroxylamine.

which is the same as Eq. 13-47 except for the dependency on $(\mathrm{R})$, the concentration of the reactivator. It is assumed in this treatment that there is no spontaneous reactivation

The reactivation rate varies with $(a)$ the enzyme that is inhibited, $(b)$ the nature of the inhibitor, $(c)$ the nature of the reactivator, $(d)$ the tem- 
perature, $(e)$ the $\mathrm{pH}$, and $(f)$ the ionic strength of the medium. The effect of temperature is usually quite marked and the apparent activation energies are high. Until the details of the kinetics can be worked out, it will not be possible to attribute these activation energies to any particular step in the reactivation. The entropy of activation for the reactivation of TEPP-inhibited cholinesterase by hydroxylamine is $-37 \mathrm{cal} /$ mole/deg and thus is very high (Wilson, 1952 b). This was interpreted as indicating a very restricted geometric path of attack althongh other factors may certainly contribute to this. A plot of the reactivation rate against $\mathrm{pH}$ shows in every case studied a rather sharp optimal $\mathrm{pH}$, which varies with the enzyme, inhibitor, and reactivator used and is usually between 6.9 and 8.3 (Wilson et al., 1955; Cunningham, 1954; Davies and Green, 1956). The effects of $\mathrm{pH}$ and ionic strength on the reactivation rate are perhaps partially explained on the basis of the electrostatic interactions between the reactivator and the anionic site and partially by the changes induced in the histidine groups that are phosphorylated at the active site (see Chapter 14).

It is interesting in comnection with what was said previously about secondary reactions that proceed during inhibition and reduce the reversibility, that the longer an enzyme is incubated with an organopohsphorus inhibitor, the more irreversible becomes the inhibition. This may be due to the transfer of the phosphoryl group from the primary site of attack, which is probably histidine, to an adjacent serine (Davies and Green, 1956). Reactivation does not occur when the enzyme has reached this final irreversible stage (Sanderson and Edson, 1959). This perhaps explains why the survival of rats poisoned by organophosphorus compounds and treated with reactivators is dependent on the time at which the reactivator is given. If the reactivator is administered within $1 \mathrm{hr}$ following the poisoning, the survival is down to $10 \%$ (Fleisher et al., 1960).

The interest in this type of reactivation is justified not only by the important information it provides on the enzyme active site and the forces and steric factors involved, but also by the practical significance such reactivators have in the treatment of poisoning by the organophosphorus compounds. It has been shown that 2 -PAM and related reactivators are quite effective in protecting animals against these inhibitors and efforts are being made to develop reactivators that are distributed in the body in a satisfactory manner to bring about restoration of cholinesterase activity in the critical tissues. This aspect of the problem will be discussed in a later chapter on cholinesterase inhibitors (Volume 3). Although the inhibition by organophosphorus compounds is the only known example of such reactivation. it may be speculated that other cases will soon be demonstrated, especially in the field of monoamine oxidase inhibitors, since they too presumably react chemically with the active site in a manner related to the amine substrates. 


\section{REVERSAL OF INHIBITION BY DESTRUCTION OF INHIBITOR}

Decline in the enzyme or metabolic inhibition as the result of the destruction of the inhibitor sometimes occurs in pure enzyme preparations but is more common in homogenates or cellular preparations. Destruction of the inhibitor may be enzymic or nonenzymic; if it is enzymic, the reaction may be catalyzed either by the inhibited enzyme or by another enzyme occurring in the mixture. The total time course of inhibition in such cases will follow curves of the type shown in Fig. 13-12. Curves A, B, and C represent

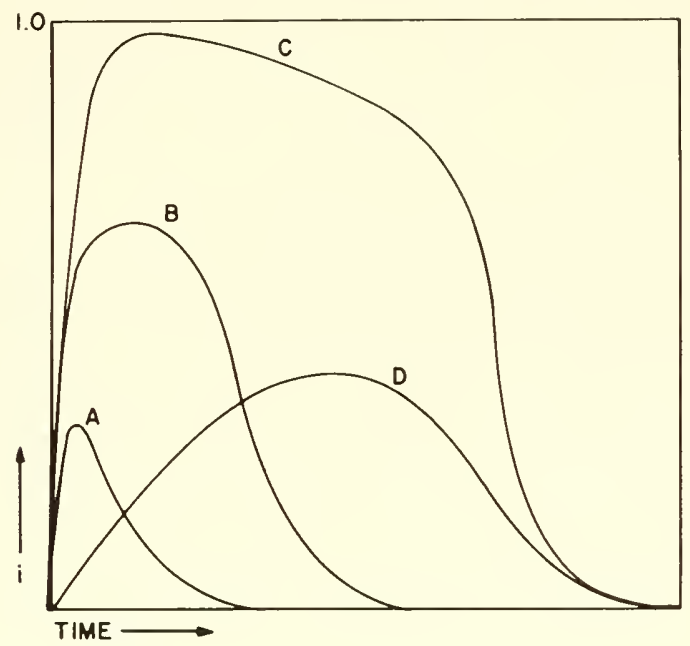

Fig. 13-12. Time courses of inhibition assuming destruction of the inhibitor. Curves A, B, and C show three different concentrations of the inhibitor for a situation in which the rate of inhibition is rapid; the different forms of the curves may be noted. In curve $\mathrm{D}$ the rate of inhibition is slower so that equilibrium inhibition is never reached.

situations in which the inhibitor reacts rapidly with the enzyme whose inhibition is being measured and then is more slowly destroyed, so that the maximal inhibitions reached approximate the equilibrium inhibition expected. These three curves are plotted for different initial concentrations of the inhibitor. Curve D illustrates a situation in which the rate of inhibition and the rate of destruction of the inhibitor are more comparable and here an equilibrium inhibition is never reached due to the appreciable loss of the inhibitor while the inhibition is developing. When this occurs. inhi- 
bition data cannot be interpreted in the usual manner and any calculations of inhibition constants from the results will be incorrect.

\section{Spontaneous Monomolecular Destruction of an Unstable Inhibitor}

The nonenzymic inactivation of an inhibitor is the simplest case of inhibition reversal due to the destruction of the inhibitor and simple expressions for the decline in inhibition with time may be obtained in some instances. The following two reactions proceed simultaneously:

$$
\begin{gathered}
\mathrm{EI} \underset{k_{1}}{\stackrel{k_{-1}}{\rightleftharpoons}} \mathrm{E}+\mathrm{I} \\
\mathrm{I} \stackrel{k}{\rightarrow} \mathrm{Q}
\end{gathered}
$$

where $\mathrm{Q}$ is the inactive form to which the inhibitor is altered. There are three possible situations: the rate of dissociation of the EI complex may limit the rate of reversal, the lestruction of the inhibitor may limit the rate, or the rates may be comparable. When $k \gg k_{-1}$, the concentration of free inhibitor may be kept essentially at zero and the decrease in inhibition will be determined by the EI dissociation rate (Eq. 13-4). When $k_{-1} \gg k$, an approximate equilibrinm will be maintained between the enzyme and the free inhibitor so that the inhibition at any time may be calculated from the concentration of free inhibitor. In this case:

$$
\frac{d(\mathbf{1})}{d t}=-k(\mathrm{I})
$$

and upon integrating, setting $(\mathrm{I})=\left(\mathrm{I}_{0}\right)$ when $t=0$, and replacing (I) by $i K_{i} / 1-i$, the inhibition is found to be:

$$
i=\frac{1}{1+\left[\left(1 / i_{0}\right)-1\right] e^{k t}}
$$

where $i_{0}$ is the initial inhibition. This equation is plotted in Fig. 13-13 for different levels of the initial inhibition. It is characteristic of such curves that at high inhibitions, the inhibition does not change greatly with time, despite the fact that the rate of destruction of the inhibitor is highest then. This results from the nature of the inhibition equation, $i=\left(\mathrm{I}^{\prime}\right)$ $\left[\left(I^{\prime}\right)+1\right]$, in that at high values of $\left(I^{\prime}\right)$, when rednction of $\left(I^{\prime}\right)$ decreases both numerator and denominator comparably, the inhibition is rather insensitive to $\left(I^{\prime}\right)$. Actually each curve in Fig. 13-13 has the same configuration and they are merely shifted along the time axis. This means that at 
any particular level of inhibition, the rate of fall will be the same. The slope at $t=0$ may be obtained by differentiating Eq. 13-60:

$$
\left(\frac{d i}{d t}\right)_{t=0}=-k i_{0}\left(1-i_{0}\right)
$$

Differentiating this again and setting it equal to zero, it is found that the maximal slope, or decrease in inhibition, will occur when $i=0.5$.

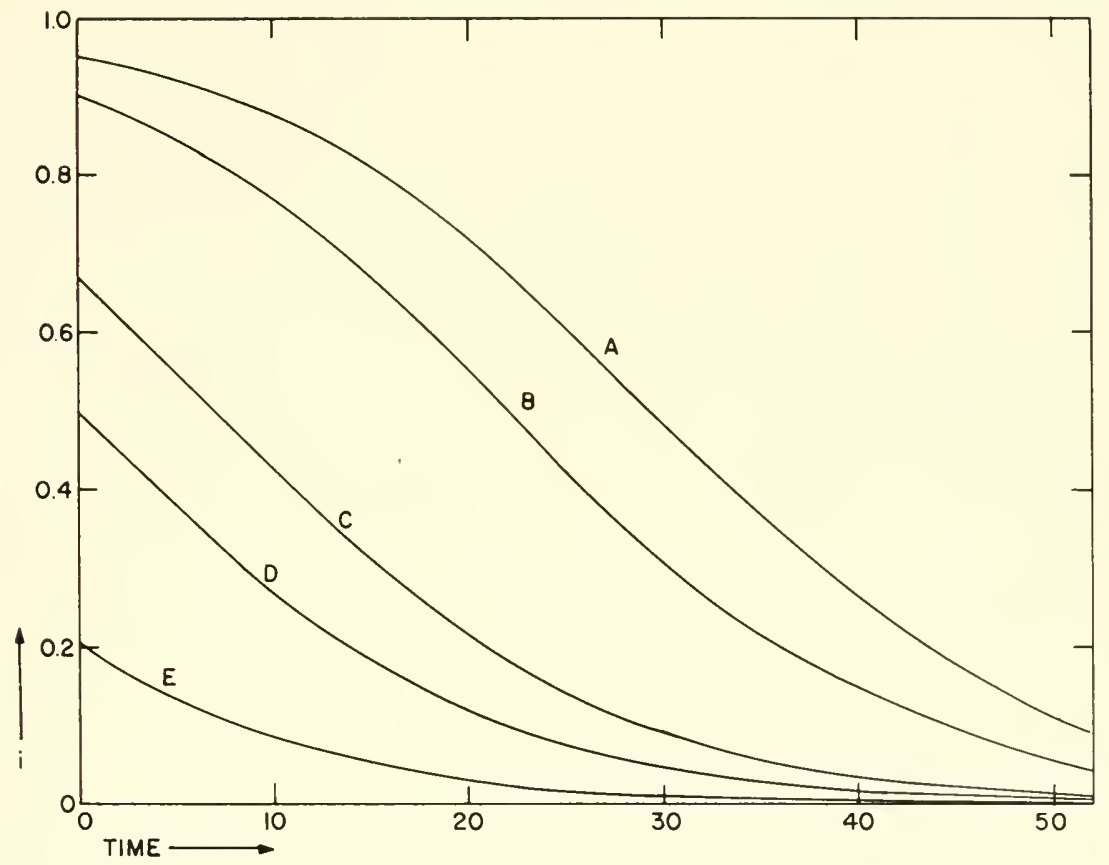

FIG. 13-13. Time courses of inhibition assuming a spontaneous monomolecular destruction of the inhibitor (Eq. 13-60). The different curves represent various initial inhibitions, $k=0.1$. Curve A: $i_{o}=0.95$; curve B: $i_{o}=0.90$; curve $\mathrm{C}: i_{o}=0.67$; curve D: $i_{o}=0.50$; curve E: $i_{0}=0.20$.

When the rates of dissociation and destruction are comparable, it is more difficult to express the decline of the inhibition, since there are now two simultaneous equations, one giving the change in the inhibition due to the dissociation of the EI complex:

$$
\frac{d i}{d t}=\frac{k_{-1}}{K_{i}}(1-i)(\mathrm{I})-k_{-1} i
$$


and the other the rate of change of the free inhibitor concentration:

$$
\frac{d(\mathrm{I})}{d t}=k_{-1}\left(\mathrm{E}_{t}\right) i-\frac{k_{-1}}{K_{i}}\left(\mathrm{E}_{t}\right)(1-i)(\mathrm{l})-k(\mathrm{I})
$$

It may be easily seen that the rate of reversal will be less than that for EI dissociation in the absence of free inhibitor because (I) will always have a significant value. The reversal curves will have a form intermediate between the exponential dissociation type and the sigmoid destruction type (i.e., between curve C in Fig. 13-1 and the curves in Fig. 13-13).

\section{Destruction of the Inhibitor by the Inhibited Enzyme}

A kinetic equation for this situation was derived by Goldstein (1944) in connection with the reversal of sermm cholinesterase inhibited with physostigmine. Expressing his equation in termes of inhibition rather than activity:

$$
\left[\frac{1}{1-i_{0}}+\ln \frac{i_{0}}{1-i_{0}}\right]-\left[\frac{1}{1-i}+\ln \frac{i}{1-i}\right]=\frac{k_{2}\left(\mathrm{E}_{t}\right)}{K_{i}} t
$$

where $k_{2}$ is the rate constant for the dissociation of the EI complex, as in reaction 13-38. Experimental data for the destruction of the inhibitor will provide the means of determining $k_{2}\left(\mathrm{E}_{t}\right) / K_{i}$. If $\left(\mathrm{E}_{t}\right)$ and $K_{i}$ are known, which is seldom the case. $k_{2}$ may be obtained, or $k_{2}$ may be determined by methods such as have been worked out for ordinary substrate reactions (Huemekens, 1953, p. 583). Physostigmine is destroyed slowly by serum cholinesterase and the above equation fits the data obtained by Ellis et al., (1943) with $k_{2}\left(\mathrm{E}_{t}\right) / K_{i}=1.4$ as pointed out by Goldstein, from whon Fig. 13-14 is taken. Curve $\mathrm{B}$ corresponds to the experimental data and curves $\mathrm{A}$ and $\mathrm{C}$ were plotted for other initial inhibitions. The curves are again all of the same configuration and the most rapid decrease in the inhibition occurs now at $i=0.33$.

\section{Destruction of the Inhibitor by an Enzyme Other Than the One Inhibited}

The reactions that occur in this situation are:

$$
\begin{gathered}
\mathrm{E}_{1} \mathrm{I} \underset{k_{1}}{\stackrel{k_{-1}}{\rightleftharpoons}} \mathrm{E}_{1}+\mathrm{I} \\
\mathrm{I}+\mathrm{E}_{2} \underset{k_{-3}}{\stackrel{k_{3}}{\rightleftharpoons}} \mathrm{E}_{2} \mathrm{I} \stackrel{k_{4}}{\rightarrow} \mathrm{E}_{2}+\mathrm{Q}
\end{gathered}
$$

where $E_{1}$ is the inhibited enzyme and $E_{2}$ is the enzyme catalyzing the 
destruction of the inhibitor. If we assume that the dissociation of $\mathrm{E}_{1} \mathrm{I}$ is rapid compared to the destruction of the inhibitor, the inhibition may be calculated from the value of (I) at any time. Since the rate of reaction 13-65 is given by the usual Michaelis-Menten expression:

$$
v=-\frac{d(\mathrm{I})}{d t}=\frac{k_{4}\left(\mathrm{E}_{t}\right)(\mathrm{I})}{(\mathrm{I})+K_{m}}
$$

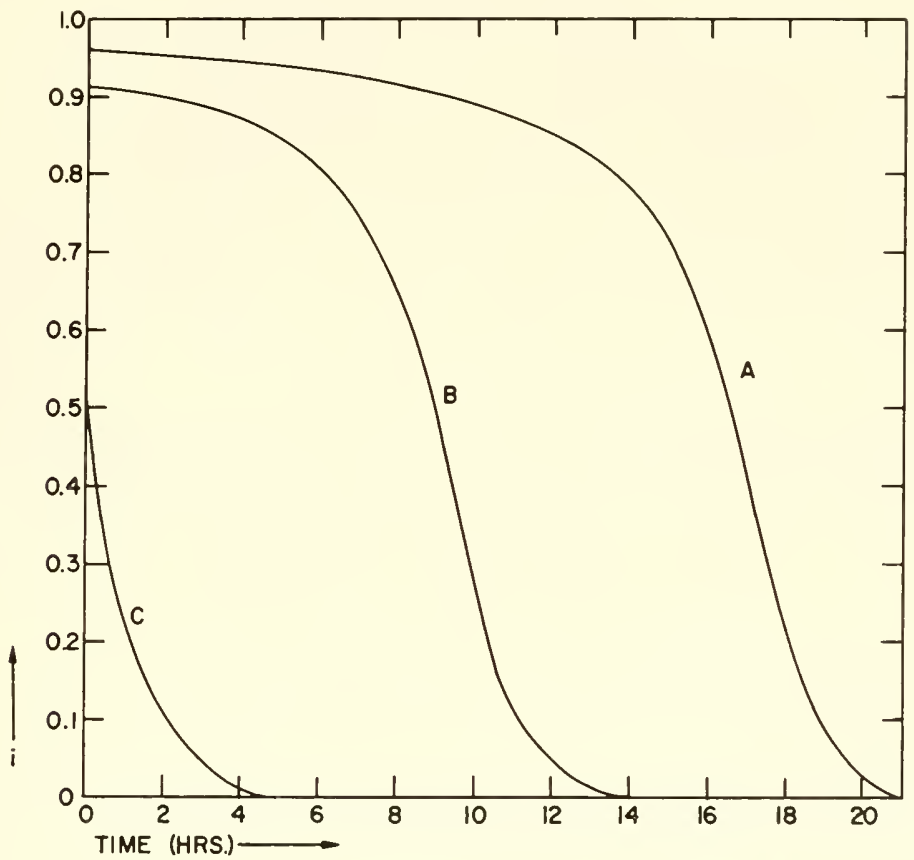

FIG. 13-14. Time courses of inhibition of serum cholinesterase by physostigmine (Eq. 13-64). Curve B fits the experimental data obtained by Ellis et al. (1943) while curves A and C are plotted for the same inhibition constants but different initial concentrations of physostigmine. In all case $k_{2}\left(\mathrm{E}_{t}\right) / K_{i}=1.4$.

the variation of $(\mathrm{I})$ with time may be obtained by integration of this equation with the assumption that $i=i_{0}$ at $t=0$ :

$$
K_{m}\left[\ln \frac{i_{0}}{1-i_{0}}-\ln \frac{i}{1-i}\right]+K_{i}\left[\frac{i_{0}}{1-i_{0}}-\frac{i}{1-i}\right]=k_{4}\left(\mathrm{E}_{t}\right) t
$$

This is similar to Eq. 13-63 but there is a greater possibility of variation in the forms of the reversal curves, arising from different relative values of $K_{i}$ and $K_{m}$. 


\section{Removal of the Inhibitor by an Inactivator}

If the free inhibitor reacts with a substance that is present in the test medium and, as a result, is inactivated, the following reactions may be written for an irreversible inhibition:

$$
\begin{aligned}
& \mathrm{E}+\mathrm{I} \stackrel{k_{1}}{\rightarrow} \mathrm{EI} \\
& \mathrm{I}+\mathrm{A} \stackrel{k_{2}}{\rightarrow} \mathrm{Q}
\end{aligned}
$$

where $Q$ represents a product or products resulting from the inactivation of the inhibitor. Since the inhibitor concentration will decrease with time according to:

$$
(\mathrm{I})=(\mathrm{I})_{0} e^{-k_{2}(\mathrm{~A}) t}
$$

the differential equation for the rate of change of the inhibition is:

$$
d i / d t=k_{1}(1-i)(\mathrm{I})_{0} e^{-k_{2}(\mathrm{~A}) t}
$$

which may be integrated direetly for the situation in which there is sufficient inhibitor present to inhibit the enzyme completely:

$$
i=1-\exp \left[\left(\frac{k_{1}(\mathrm{I})_{0}}{k_{2}(\mathrm{~A})}\right)\left(e^{-k_{2}(\mathrm{~A}) t}-1\right)\right]
$$

If all of the inhibitor is inactivated before the inhibition has reached $\mathrm{I} 00 \%$, the final inhibition will depend on $(\mathrm{I})_{0}$.

\section{REVERSAL OF INHIBITIONS IN CELLS AND WHOLE ANIMALS}

The rise and fall of the intracellular inhibitor concentration during penetration and destruction have been discussed in Chapter 8 and expressed in equations such as 8-8 and 8-9. It was assumed there that the equilibrimm between the enzyme and the inhibitor was achieved rapidly, and thus the variation of the inhibition with time could be directly calculated. If the formation or dissociation of the EI complex lags behind the ehanges in inhibitor concentration, the kineties of inhibition may become quite contplex. Better than following this approach is an examination of the occurrence of the types of reversal discussed above as they are modified in cellular or tissue preparations. It must be borne in mind that there are many possible reasons for the recovery of some cell metabolic process or function that has been depressed by an inhibitor, as listed at the beginning of this chapter, and that such reversal does not necessarily imply recovery of the activity of the primary enzyme inhibited. 


\section{Diffusion of the Inhibitor from the Cells}

Inhibitors that are stable and are not metabolized must eventually diffuse out of the cells if the EI complex is to dissociate and enzyme activity is to be restored. Such occurs most simply when inhibited tissue slices are placed in an inhibitor-free solution, or when an inhibited organ is perfused with an inhibitor-free medium. Diffusion from the tissues also occurs in whole animals as the inhibitor is excreted through the kidneys or the lungs. Just at the rate of entrance of an inhibitor into a cell may modify the rate of inhibition, so the rate of leaving the cell may affect the recovery from the inhibition. The processes involved may be represented as:

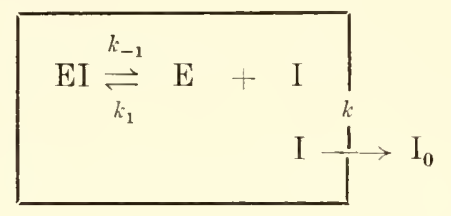

This is kinetically the same as the case in which the inhibitor undergoes monomolecular destruction (reactions 13-59) and the same principles and equations apply here. In other words. the rate of reversal may be limited by either process or may depend on both.

\section{Examples of the Reversal of Cellular Inhibition}

Only a very cursory examination of cellular inhibition reversal will be given. Although the degrees of reversibility have often been reported in the studies of such inhibitions, very little quantitative work that could be submitted to kinetic analysis is available. It will suffice to mention certain examples that illustrate the different types of reversal.

The rate of reversal of inhibition is probably determined mainly by the efflux of the inhibitor from the cells or tissues when most inhibitors are administered to whole animals. This is not primarily because of a low permeability to the inhibitors but to the low concentration gradient across the plasma membranes. The concentration of the inhibitor falls in the serum as the inhibitor is excreted or metabolized and the release of inhibitor from the tissues is consequently dependent on these secondary factors. The fall of inhibitor concentration in the tissues, as in the case of acetazolamide (Table 8-2), is then controlled mainly by the rate of fall of the serum level and is often not related to the dissociation of the EI complex. It has been postulated by Peters (1952) that the prolonged inhibition exerted by fluoroacetate is at least partly related to the inability of the fluorocitrate formed to leave the mitochondria rapidly. The true inhibitor is then in a sense trapped where it is formed. 
Inhibition reversals whose rates are limited by the dissociation of the EI complex probably occur but apparently are not too common. In cell suspensions or tissue slices exposed to mercurials or arsenicals and then resuspended in inhibitor-free media, one might predict that the rate at which metabolic activity is restored would be primarily dependent on the splitting of the inhibitor from the enzymes, but the experiments of Fink and Wright $(1948 \mathrm{a}, \mathrm{b})$ on the binding of oxophenarsine to erythrocytes indicate that the situation may be more complex. Rabbit erythrocytes previonsly incubated with different concentrations of oxophenarsine were resuspended at half-hourly intervals in fresh plasma and the amounts of arsenical released during each period were determined. The results are shown in Table 13-2. The binding of oxophenarsine was mainly reversible

TABLE 13-2

Release of Oxophenarsine from Rabbit Erythrocytes Resuspended in Norital Plasia ${ }^{a}$

\begin{tabular}{c|c|c|c}
\hline \multirow{2}{*}{$\begin{array}{c}\text { Interval } \\
(\mathrm{min})\end{array}$} & \multicolumn{2}{|c}{ Per cent of the arsenical released } \\
\hline & $0.084 \mathrm{mM}$ & $0.21 \mathrm{~m} M$ & $0.42 \mathrm{mM}$ \\
\hline 0.30 & 73 & 67 & 57 \\
$30-60$ & 84 & 70 & 67 \\
$60-90$ & 90 & 76 & 71 \\
\hline
\end{tabular}

a The release is calculated as the percentage of the theoretical amount released assuming complete reversibility. The concentrations of oxophenarsine to which the erythrocytes were previously exposed are given at the heads of the columns. From Fink and Wright (1948a).

but by no means complete at $90 \mathrm{~min}$ and the fraction that was not released increased with the concentration of the arsenical. It is clear that there are different binding sites for the oxophenarsine in the erythrocytes. From some of these sites the arsenical is relatively rapidly dissociated but from other sites only very slowly. No enzymic studies were carried ont and consequently it is not known in what category the enzyme sites should be placed. They then showed that oxophenarsine dialyzed quite readily from serum but much more slowly from blood. However. if the erythrocytes were hemolyzed with saponin, the dialysis of the arsenical was increased; destruction of the cell membrane increased the percentage dialyzing in 6 hr from $33 \%$ to $59 \%$. This conld be interpreted as a barrier to diffusion imposed by the cell membrane. Thus both dissociation and diffiusion factors would be implicated. Certain inhibitors whose actions on cells or tissues are 
developed rapidly, indicating rapid penetration into the cells, may not show nearly as fast a reversal of the inhibition when removed from the extracellular medium. Some of the metal chelators, such as 1,10-phenanthroline or 2.2'-dipyridine, may behave in this manner (Webb et al., 1958) and in these instances the limitation of the rate of reversal may be attributed to the rather slow dissociation of these chelators from metal sites in the cells.

Examples in which the recovery of enzyme activity is dependent on the synthesis of new enzyme by the tissues are provided by the organophos-

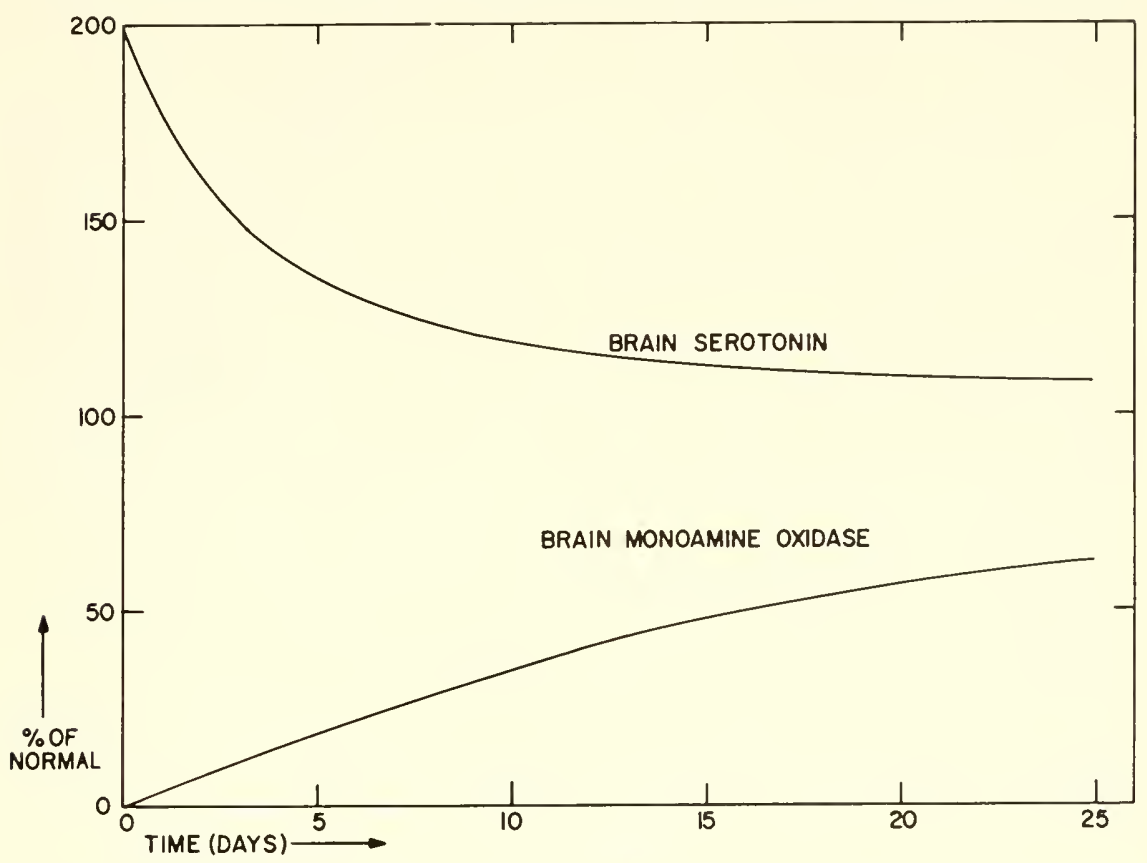

FrG. 13.15. The recovery of monoamine oxidase activity in mouse brain following a single dose of $\beta$-phenylethylhydrazine $(32 \mathrm{mg} / \mathrm{kg}$ intraperitoneally). The fall in the initially elevated brain serotonin is also shown. (From Chessin et al.. 1959.)

phorus inhibitors of cholinesterase and the irreversible inactivators of monoamine oxidase. In these cases it requires many days for restoration of normal enzyme activity. The return of monoamine oxidase in mouse brain after a single dose of $\beta$-phenylethylhydrazine is shown in Fig. 13-15. Correlated with this rise in enzyme activity is the drop in the initially elevated brain serotonin level, which in turn is probably correlated with the return of normal central nervous system function. Single injections of diisopropylfluorophosphate similarly inactivate cholinesterase and modify nervous, 
muscular, and glandular functional activity for long periods of time. Both of these groups of inhibitors contain members that do not produce completely irreversible inactivation and with these the return to activity is dependent on the rate of spontaneous enzyme reactivation as well as resynthesis of the enzyme.

Effective reversal of intracellular inhibition by the addition of a substance binding the inhibitor is well documented and is the basis for the clinical use of dimercaprol in heavy metal poisoning. Walker in 1928 showed that the ciliate Colpidium immobilized by various arsenicals could be rapidly restored by the addition of thioglycolate and in Voegtlin's laboratory (Voegtlin et al., 1931) it was soon demonstrated that glutathione would restore tissue respiration depressed by arsenicals. Since that time many instances of such reversal by a variety of sulfhydryl compounds have been reported, culminating in the discovery of the particular effectiveness of dimercaprol. The injection of dimercaprol into an animal previously treated with an arsenical produces a very rapid increase in the urinary excretion of the arsenical (Eagle et $\iota l ., 1946$ ). This sudden burst of urinary arsenical is accompanied by a rise in plasma arsenic and is due primarily to the withdrawal of the arsenical from the tissues. The dimercaprol probably binds the free arsenical in both the blood and tissue cells since the cells are permeable to this reversor, but it is not necessary for the reversor to penetrate into the cells to reverse the inhibition. Danielli et al., (1947) showed that dimercaprol glucoside (BAL-INTRAV), which presumably enters cells with difficulty, is effective in combatting the toxic actions of arsenicals. This would be expected inasmuch as there is an equilibrium between free inhibitor in the plasma, in the extracellular fluid, in the cytoplasm, and bound arsenical. Reduction of the free arsenical in the plasma to near zero wonld withdraw arsenical from the tissues at the same rate as would be observed if the tissue were placed in an inhibitor-free medium.

\section{Kinetics of Organophosphorus Poisoning and Its Treatment}

The time course of the poisoning resulting from the administration of organophosphorus inhibitors to animals is probably quite complex because of the numerous reactions occurring. A very interesting mathematical treatment of such poisoning was developed by Green (1958) and this can be applied, to varying extents, to other inhibitions or poisonings by irreversible inhibitors. It was assumed that the inhibitor can undergo the following reactions: (1) combination with enzymes, (2) spontaneous inactivation, (3) inactivation by substances present in the body, and (4) reactivation of the inhibited enzyme. The approach used by Green is different from that presented previously in the chapter because his object was to determine the dosages of the inhibitors necessary to produce a lethal level of enzyme inhibition. His treatment will be described for two common situations. 
When the inhibitor reacts with and is inactivated by a substance in the body, and the inhibited enzyme is not reactivated:

$$
\begin{aligned}
& \mathrm{E}+\mathrm{I} \stackrel{k_{1}}{\rightarrow} \mathrm{EI} \\
& \mathrm{I}+\mathrm{A} \stackrel{k_{2}}{\rightarrow} \mathrm{Q}
\end{aligned}
$$

The rates of change of (E) and (I) may be expressed as:

$$
\begin{gathered}
d(\mathrm{E}) / d t=-k_{1}(\mathrm{E})(\mathrm{I}) \\
d(\mathrm{I}) / d t=-k_{1}(\mathrm{E})(\mathrm{I})-k_{2}(\mathrm{I})(\mathrm{A})
\end{gathered}
$$

Dividing these equations, one obtains:

$$
\frac{d(\mathrm{I})}{d(\mathrm{E})}=1+\frac{k_{2}(\mathrm{~A})}{k_{1}(\mathrm{E})}
$$

and integrating:

$$
(\mathrm{I})=\left(\mathrm{I}_{t}\right)-\frac{k_{2}(\mathrm{~A})}{k_{1}} \ln \frac{\left(\mathrm{E}_{t}\right)}{(\mathrm{E})}-\left(\mathrm{E}_{t}\right)+(\mathrm{E})
$$

where $\left(I_{t}\right)$ and $\left(E_{t}\right)$ represent the initial or total concentrations of inhibitor and enzyme. When $\left(\mathrm{I}_{t}\right) \gg\left(\mathrm{E}_{t}\right)$ :

$$
(\mathrm{I})=\left(\mathrm{I}_{t}\right)-\frac{k_{2}(\mathrm{~A})}{k_{1}} \ln \frac{\left(\mathrm{E}_{t}\right)}{(\mathrm{E})}
$$

After all of the inhibitor has reacted and $(\mathrm{I})=0$ :

$$
\left(\mathrm{I}_{t}\right)=\frac{k_{2}(\mathrm{~A})}{k_{1}} \ln \frac{\left(\mathrm{E}_{t}\right)}{(\mathrm{E})_{\min }}
$$

where $(\mathrm{E})_{\min }$ is the final and minimal enzyme concentration reached. If we now require that this minimal enzyme concentration be lethal to the organism, the initial concentration of the inhibitor required to kill is given by:

$$
(\mathrm{I})_{\mathrm{L}}=\frac{k_{2}(\mathrm{~A})}{k_{1}} \ln \frac{\left(\mathrm{E}_{t}\right)}{(\mathrm{E})_{\mathrm{L}}}
$$

where $(\mathrm{E})_{\mathrm{L}}$ is the concentration to which the enzyme must drop for a lethal action. In terms of the enzyme inhibition required for a lethal action:

$$
(\mathrm{I})_{\mathrm{L}}=\frac{k_{2}(\mathrm{~A})}{k_{1}} \ln \left[\frac{1}{1-i_{\mathrm{L}}}\right]
$$


$(\mathrm{I})_{\mathrm{L}}$ may be equated with the lethal dose in moles per liter. This lethal dose must increase as the inhibition required to kill increases.

When a reactivator of the $N$-alkylpyridine aldoxime type is present, the following reaction also occurs:

$$
\mathrm{EI}+\mathrm{R} \stackrel{k_{3}}{\rightarrow} \mathrm{E}+\mathrm{T}
$$

where the distinction between EI and EX has been ignored. A similar treatment leads to an expression for $(\mathrm{I})_{\mathrm{L}}$. If this value for $(\mathrm{I})_{\mathrm{L}}$ in the presence of the reactivator is divided by the value for $(I)_{L}$ in the absence of any reactivator, one obtains what Green called the therapeutic efficiency of the reactivator.

$$
\frac{(\mathrm{I})_{\mathrm{L}, \mathrm{R}}}{(\mathrm{I})_{\mathrm{L}}}=1+1.6\left[\frac{k_{3}(\mathrm{R})}{k_{1}(\mathrm{I})_{\mathrm{L}}} \frac{1}{1-i_{\mathrm{L}}}\right]^{0.8}
$$

where $(\mathrm{I})_{\mathrm{L}, \mathrm{R}}$ is the lethal dose in the presence of the reactivator. This is an approximate expression inasmuch as certain assumptions had to be made to simplify the equation for practical use. For example, it is assumed that the inhibitor and reactivator are homogeneously distributed throughout the body, and that the concentrations of the inactivator and reactivator are constant over a short interval. The expression is valid when $i_{\mathbf{L}}$ is high and the therapentic efficiency is less than 20. Some interesting deductions may be made from Eq. 13-86. The therapentic efficiency depends on the inhibition in such a manner that the efficiency increases as the lethal degree of inhibition increases. Also the therapentic efficiency is proportional to the eight-tenth power of $1 /\left(1-i_{\mathrm{L}}\right)$ whereas without the reactivator, $\langle\mathbf{I}\rangle_{\mathrm{L}}$ is proportional to $\ln 1 /\left(1-i_{\mathrm{L}}\right)$. Therefore, the presence of the reactivator causes the level of required enzyme inhibition, $i_{\mathrm{L}}$, to be very important. If $i_{\mathrm{L}}$ can be changed in any, way, as by the application of atropine to block some of the effects of the rise in acetylcholine concentration, the therapentic efficiency for a particular reactivator may be much improved. Although this approach is, to some extent, qualitative because of the unknown factors that must be involved in the poisoning, it is a first step towards a more accurate and comprehensive treatment based on further experimentation. 


\section{EFFECTS OF pH ON ENZYME INHIBITION}

The degree of enzyme inhibition frequently varies with the $\mathrm{pH}$ and this would be expected inasmuch as the concentrations and constants in the general inhibition Eq. 3-11 may all be $\mathrm{pH}$-dependent. It might also be inferred from the fact that enzymes are proteins and hence possess ionizing groups which determine in part the pattern of electrical charge over the enzyme surface and regulate the extent to which the enzyme interacts with any substance, whether substrate, activator, coenzyme. or inhibitor. The observed variations in enzyme activity with $\mathrm{pH}$, either in the absence or presence of inhibitors, provide data that may be used to demonstrate ionizing groups at the active center, and often allow the acid dissociation constants of these groups to be calculated. Knowledge of these constants makes it possible to speculate on the nature of the ionizing groups and a great deal of what is known about the properties of enzyme active centers has been derived from studies of the effects of $\mathrm{pH}$. Most of the investigations have been concerned with the changes in enzyme activity produced by $\mathrm{pH}$ variations and relatively little has been done with inhibitors. However, for the purpose of characterizing ionizing groups at the active center, inhibition often provides more readily interpretable data than activity studies because the changes in the inhibition constant, $K_{i}$, are directly related to the binding of the inhibitor to the enzyme, whereas in the case of the substrate one must also consider the possibility that $\mathrm{pH}$ affects the rate of breakdown of the ES complex and that the Michaelis constant, $K_{m}$, may not be a simple dissociation constant. Just as it is easier to determine directly $K_{i}$ than $K_{s}$, so it is often more convenient to interpret changes in $K_{i}$ than in $K_{m}$. It should be realized that in any case the effects of $\mathrm{pH}$ may be very complex and that the complexities increase rapidly as one proceeds from single pure enzymes to multienzyme systems and eventually to cells. It is true that many of these effects of $\mathrm{pH}$ are at present beyond our powers of analysis. Nevertheless, if one begins with simple enzymes and progresses to more complex systems, one finds that some general principles appear and that, indeed, pH studies can provide a great deal of information on enzyme and imhibition mechanisms. 


\section{EXPRESSIONS FOR THE PH-FUNCTIONS OF WEAK ACIDS AND BASES}

Some convenience and economy of presentation will be gained by expressing the $\mathrm{pH}$ dependence of the concentration of any species of a weak acid or base in terms of a function. The concentration of the species will then be related through this function to the total concentration of the substance. The simplest case is when a single proton is involved in the dissociation:

$$
\mathrm{H}+\mathrm{B} \rightleftharpoons \mathrm{HB} \quad K_{a}
$$

where $\mathrm{HB}$ is the acid and $\mathrm{B}$ is the conjugate base.

The charges that may exist on these species are intentionally omitted in order to make the treatment more general. Thus the above equation could represent either:

$$
\mathrm{H}^{+}+\mathrm{B} \rightleftharpoons \mathrm{HB}^{+}
$$

or:

$$
\mathrm{H}^{+}+\mathrm{B}^{-} \rightleftharpoons \mathrm{HB}
$$

It is easy to show that the functions to be used will be the same in these cases or in any others, the only requirement being that $\mathrm{HB}$ will have one positive charge more than $B$.

If the total concentration of the substance (acid + conjugate base) is represented by $\left(B_{t}\right)$, the following expressions may be derived:

$$
\left(\mathrm{B}_{t}\right)=\left[1+\frac{(\mathrm{H})}{K_{a}}\right](\mathrm{B})=\left[1+\frac{K_{a}}{(\mathrm{H})}\right](\mathrm{HB})
$$

These may be rewritten simply as:

$$
\left(\mathrm{B}_{t}\right)=f_{b}{ }^{\prime}(\mathrm{B})=f_{h b^{\prime}}(\mathrm{HB})
$$

where $f_{b}^{\prime}$ and $f^{\prime}{ }_{h b}$ are functions relating the concentrations of $\mathrm{B}$ and $\mathrm{HB}$ to the $\mathrm{pH}$.

When the dissociation of two protons is involved:

$$
\begin{array}{ll}
\mathrm{H}+\mathrm{B} \rightleftharpoons \mathrm{HB} & K_{a}{ }^{\prime \prime} \\
\mathrm{H}+\mathrm{HB} \rightleftharpoons \mathrm{H}_{2} \mathrm{~B} & K_{a}{ }^{\prime}
\end{array}
$$

the following expressions may be written:

$$
\left(\mathrm{B}_{t}\right)=\left[1+\frac{(\mathrm{H})}{K_{a}^{\prime \prime}}+\frac{(\mathrm{H})^{2}}{K_{a}{ }^{\prime} K_{a}{ }^{\prime \prime}}\right](\mathrm{B})
$$




$$
\begin{aligned}
& \left(\mathrm{B}_{t}\right)=\left[1+\frac{(\mathrm{H})}{K_{a}{ }^{\prime}}+\frac{K_{a}{ }^{\prime \prime}}{(\mathrm{H})}\right](\mathrm{HB}) \\
& \left(\mathrm{B}_{t}\right)=\left[1+\frac{K_{a}{ }^{\prime}}{(\mathrm{H})}+\frac{K_{a}{ }^{\prime} K_{a}{ }^{\prime \prime}}{(\mathrm{H})^{2}}\right]\left(\mathrm{H}_{2} \mathrm{~B}\right)
\end{aligned}
$$

In terms of the $\mathrm{pH}$ functions:

$$
\left(\mathrm{B}_{t}\right)=f_{b}{ }^{\prime \prime}(\mathrm{B})=f_{h b}{ }^{\prime \prime}(\mathrm{HB})=f_{h_{2} b^{\prime \prime}}\left(\mathrm{H}_{2} \mathrm{~B}\right)
$$

The convention is adopted with respect to these functions that the total number of dissociable protons is represented by the primes and the particular species to which the function applies is given in the subscript. When three or more protons must be taken into account, similar expressions may be formulated.

The values of the various functions may be summarized here for reference. B may refer to the enzyme active site, the substrate, the inhibitor, or to any of the complexes between them; thus $f_{\text {hies }}^{\prime \prime}$ is the function expressing the concentration of HES, the enzyme-substrate complex associated with one proton, when the three forms - ES, HES, and $\mathrm{H}_{2} \mathrm{ES}-$ occur.

$$
\begin{aligned}
& f_{b}^{\prime}=1+\frac{(\mathrm{H})}{K_{a}} \\
& f_{h b^{\prime}}=1+\frac{K_{a}}{(\mathrm{H})} \\
& f_{b}^{\prime \prime}=1+\frac{(\mathrm{H})}{K_{a}^{\prime \prime}}+\frac{(\mathrm{H})^{2}}{K_{a} K_{a}^{\prime \prime}} \\
& f_{h b}^{\prime \prime}=1+\frac{(\mathrm{H})}{K_{a}^{\prime}}+\frac{K_{a}^{\prime \prime}}{(\mathrm{H})} \\
& f_{h_{2} b^{\prime \prime}}=1+\frac{K_{a}{ }^{\prime}}{(\mathrm{H})}+\frac{K_{a}{ }^{\prime} K_{a}{ }^{\prime \prime}}{(\mathrm{H})^{2}} \\
& f_{b}^{\prime \prime \prime}=1+\frac{(\mathrm{H})}{K_{a}^{\prime \prime \prime}}+\frac{(\mathrm{H})^{2}}{K_{a}^{\prime \prime} \bar{K}_{a}^{\prime \prime \prime}}+\frac{(\mathrm{H})^{3}}{K_{a}{ }^{\prime} K_{a}^{\prime \prime} K_{a}{ }^{\prime \prime \prime}} \\
& f_{h b}{ }^{\prime \prime \prime}=1+\frac{K_{a}{ }^{\prime \prime \prime}}{(\mathrm{H})}+\frac{(\mathrm{H})}{K_{a}{ }^{\prime \prime}}+\frac{(\mathrm{H})^{2}}{K_{a}{ }^{\prime} K_{a}{ }^{\prime \prime}} \\
& f_{h_{2} b^{\prime}}{ }^{\prime \prime \prime}=1+\frac{K_{a}{ }^{\prime \prime}}{(\mathrm{H})}+\frac{(\mathrm{H})}{K_{a}{ }^{\prime}}+\frac{K_{a}{ }^{\prime \prime} K_{a}{ }^{\prime \prime \prime}}{(\mathrm{H})^{2}} \\
& f_{h_{\mathrm{g}} b^{\prime \prime \prime}}=1+\frac{K_{a}{ }^{\prime}}{(\mathrm{H})}+\frac{K_{a}{ }^{\prime} K_{a}{ }^{\prime \prime}}{(\mathrm{H})^{2}}+\frac{K_{a}{ }^{\prime} K_{a}{ }^{\prime \prime} K_{a}{ }^{\prime \prime \prime}}{(\mathrm{H})^{3}}
\end{aligned}
$$




\section{VARIATION OF ENZYME ACTIVITY WITH PH}

The effects of $\mathrm{pH}$ variation on the rates of enzyme reactions will be briefly discussed to provide a basis for the understanding of the dependency of inhibitions on the $\mathrm{pH}$. Many excellent theoretical treatments of $\mathrm{pH}$ effects are available and the following are particularly recommended: Laidler (1955 b, c, d, 1958, p. 117), Alberty (1956a,b), Friedenwald and Maengwyn-Davies (1954), Dixon and Webb (1958, p. 120), and Reiner (1959, p. 229). The experimental data can, in most cases, be adequately interpreted at the present time in terms of various ionizing groups assigned to the active center, the substrate or the ES complex, or to any coenzymes or activators that may be involved in the reaction. One aim of this chapter is to apply and extend the results and theories to the problems of enzyme inhibition. It is evident that the experimental determination of the dependence of inhibition on $\mathrm{pH}$ must involve simultaneous measurements of the variations of the minhibited rates in order that control vahues may be obtained and in many cases knowledge of the behavior of the enzyme in the absence of the inhibitor is essential to interpret the mechanism by which the inhibition is modified by $\mathrm{pH}$. In even the most simple enzyme preparations used for the determination of activity, there is often a variety of interacting substances altering the relationships of the enzyme and substrate - activators. coenzymes, inhibitors, physiological and buffer ions - and the mass-action principles of interaction are applicable in all cases. One may consider all such substances as modifiers and very general treatments may be formulated on this basis. However, for practical purposes it is usually more convenient to consider only those substances that are varied in the experimental procedure; thus the effects of the hydrogen ion can be treated separately. Although the interactions of the enzyme with the hydrogen ion are basically quite comparable to the interactions with other modifiers, the results of variation of the $\mathrm{pH}$ are usually more complex because so many of these other modifiers are themselves affected by $\mathrm{pH}$ changes. Therefore, in each case in which the $\mathrm{pH}$ is altered, one must take into account all the possible effects on the dissociable components of the system. The $\mathrm{pH}$-dependent behavior of an enzyme may thus be very complex and although every effort should be made to simplify and isolate the individual effects, this inherent complexity should never be ignored.

\section{Mechanisms by Which pH Effects May Be Exerted}

The rates of enzyme reactions may depend on the $\mathrm{pH}$ for a variety of reasons. The following classification includes most of the important mechanisms. 


\section{Direct alteration of the state of the ionizable groups at the active center}

$A$. Effects on the binding of substrates, activators, or coenzymes to the active center.

$B$. Effects on the rate of dissociation of the ES complex.

$C$. Effects on the catalytic reaction when this involves $\mathrm{H}^{+}$donated or accepted by groups at the active center.

\section{Indirect effects on the ionizable groups at the active center}

$A$. Arising from ionizable groups vicinal to the active center.

$B$. Arising from changes in the total charge on the enzyme protein.

III. Alteration of the states of ionization of the nonemzyme components of the system

A. Components directly involved in the reaction (substrates, activators, or coenzymes).

$B$. Modifiers indirectly affecting the reaction rate (buffers and possible impurities in the enzyme preparation).

IV. Alteration of enzyme protein structure

A. Local changes in structure modifying the configuration of the active center.

$B$. Denaturation processes involving the total protein structure.

C. Association-dissociation reactions of enzyme subunits.

All of these effects relate fundamentally to changes in the ionization states of groups on the components of the total enzyme system. Hence, the primary reactions of the hydrogen ion may be expressed in terms of the classic acid-base dissociation concepts. The relations between these primary reactions and the over-all enzymic rate, however, are often not simple.

The classification of the mechanisms involving enzyme ionization groups according to the location of these groups relative to the active center is similar to that of Alberty (1956 a) who divided the ionizable groups into those that (1) occur at the active center and which must exist in a particular ionized state for catalytic activity, (2) occur in the neighborhood of the active site and have a definite effect on the catalytic activity, and (3) occur over the rest of the surface of the enzyme. The major effects observed upon changing the $\mathrm{pH}$ will depend on the ionizable groups at the active center; the quantitative kinetic treatments have dealt almost exclusively with such groups. However, the vicinal groups and the total enzyme charge can exert effects which are appreciable and they must be considered in more refined theories. Some of the observed discrepancies 
from theories based only on active center groups may well arise in these more indirect influences. The attempts to estimate the effects of these outlying groups will be considered later.

The division of the mechanisms with regard to the steps in the catalytic sequence (as in category I) can be ilhustrated in the reaction:

$$
\mathrm{E}+\mathrm{S} \underset{k_{-1}}{\stackrel{k_{1}}{\rightleftharpoons}} \mathrm{ES} \rightleftharpoons \mathrm{EP} \stackrel{k_{2}}{\rightarrow} \mathrm{E}+\mathrm{P}
$$

where only the forward formation of product is considered. The rate constants, $k_{1}$ and $k_{-1}$, may be sensitive to the $\mathrm{pH}$ and hence $K_{s}=k_{-1} / k_{1}$ may vary with the $\mathrm{pH}$. This means that the affinity of the enzyme for the substrate is $\mathrm{pH}$-dependent because of changes on the groups responsible for the binding (IA). The rate constant, $k_{2}$, for the dissociation into the free enzyme and the product may also be pH-dependent because of the changing electrical fields arising from ionizable groups at or near the active center (IB). The actnal molecular rearrangements oceurring in the transformation of the substrate to the product may also be $\mathrm{pH}$-dependent, most commonly because of the participation of dissociable hydrogen ions in the reaction (IC). In those cases in which the reverse reaction is significant, consideration must also be given to the possible variation of the equilibrium constant between $\mathrm{S}$ and $\mathrm{P}$ with the $\mathrm{pH}$. Reactions that are more complex, involving additional components, will, of course, present other possibilities for $\mathrm{pH}$ effects.

The modifications in enzyme structure brought about by variation in the $\mathrm{pH}$ (IV) are little understood and are not susceptible to quantitative analysis. The structural changes may be reversible or irreversible. In the latter case, it is at least possible to detect such changes and take them into account when subjecting the data to linetic theory. It is important in any study of $\mathrm{pH}$ effects to eliminate these irreversible morlifications in the enzyme (usually termed denaturation). Every enzyme will become unstable outside of a particular and characteristic $\mathrm{pH}$ range. It is necessary to examine, under the conditions of the procedure, the reversibility of the changes induced by the $\mathrm{pH}$ and thus establish that one is working within a $\mathrm{pH}$ range susceptible to kinetic treatment. The return of the enzyme preparation to the initial $\mathrm{pH}$ to demonstrate any loss of activity is the most common method. Sonetimes the instability of an enzyme may be detected by a progressive decline in the rate at abnormal $\mathrm{pH}$ 's, and thus it is advisable to determine the aetivities or inhibitions over different time intervals when a system is first being studied. Eventually it may be necessary to limit the measurements to a short initial period before an appreciable irreversible change has occurred. The evidence for reversible structural changes, particularly if they are localized, is difficult to obtain but it would appear 
likely that proteins are fictile enough to respond structurally to changes in local electrical fields. The distance between two binding or reactive groups at the active center might be varied slightly as the charged groups in the neighborhood appear or disappear. Likewise, little is known of the importance of the dissociation of enzymes into subunits and the effects this would have on activity. It is known that there is little association of the units of $\alpha$-chymotrypsin at $\mathrm{pH} 8$, but that association occurs to some extent at pH 7 and presumably increases at lower pH's (Booman and Niemann, 1957). The enzymic activity seems to be greater in the dissociated form. Since the degree of association depends on the enzyme concentration, the likelihood of significant association, and thus the possibility of modifying it, would be greater in mitochondria or cells where the concentrations of enzymes are often much greater than in studies of isolated enzymes.

\section{General Dependence of Enzymic Rate on $\mathrm{pH}$ : Occurrence of $\mathrm{pH}$ Optima}

A characteristic feature of the rate-pH curves for most enzymes is that the rate is maximal at a certain $\mathrm{pH}$ (or over a range of $\mathrm{pH}$ ) and falls off on either side. The $\mathrm{pH}$ at which the activity is maximal is called the optimal $p H$. The optimal $\mathrm{pH}$ for a particular enzyme will usually vary with the substrate used, the temperatule. the ionic strength, and other factors. The concentration of the substrate may also be important: the ${ }_{\mathrm{p}} \mathrm{H}$ optimum of the alkaline phosphatase of rat kidney shifts to progressively higher values as the substrate concentration is increased (Neumann, 1949). The presence of an inhibitor may modify the $\mathrm{pH}$ optimum and certain information on the mechanism of the inhibition can occasionally be obtained from such an effect. Possible explanations for $\mathrm{pH}$ optima will be presented in the following sections but it is well to emphasize immediately that these optima do not depend on irreversible or denaturation changes, inasmuch as they occur within $\mathrm{pH}$ ranges in which the changes in activity are completely reversible. When denaturation takes place, its effect on activity adds to the reversible changes, and for the purposes of kinetic evaluation this shonld be corrected for. Finally, a common misconception in the interpretation of $\mathrm{pH}$ optima should be pointed ont. It is frequently stated that the existence of a $\mathrm{pH}$ optimum implies two ionizing groups on the enzyme; this is sometimes extended to state that these gromps are actively engaged in the catalytic reaction (e.g., acting as proton donors and acceptors). In the first place, a single dibasic group on the enzyme can account for this phenomenon. In the second place, when two groups are involved, it is not necessary that they both be on the enzyme; the interaction of $\mathrm{E}^{-}$ and $\mathrm{SH}^{+}$, for example, would occur significantly only in the $\mathrm{pH}$ range between the $p K_{a}$ 's of the enzyme and substrate. All that $p^{\mathrm{H}}$ optima imply is that the system involves two or more dissociable protons. 


\section{Expressions for the pH Depencence of Enzymic Rate}

A complete and rigorous steady-state treatment of even relatively simple enzyme system. with respect to their response to $\mathrm{pH}$ changes is laborious and the resultant expressions are too unwieldly to apply to experimental data (Laidler, 1955 b, 1958, p. 125). In most cases it seems that the data can be adequately interpreted by simpler kinetics and for the present purpose it will suffice to outline a basic approach and to illustrate this with some examples. Extensions to cover various complications may readily be made on this basis.

Equilibrim kinetics rather than steady-state kinetics will be used for several reasons. Firstly, as mentioned above, a rigorous steady-state analysis is complex and in the present state of our knowledge it is not profitable. Secondly, proton association and dissociation reaction rates are generally very rapid compared with enzyme reaction rates and hence would seldom be of significance in the over-all rate expression. Thirdly, it is usual to express the results in terms of changes in $K_{m}$ or $V_{m}$; in most cases these cannot be broken down satisfactorily into rate constants at any $\mathrm{pH}$, so it is useless to attempt to interpret $\mathrm{pH}$ effects on these rate constants at present. Lastly, it will be evident later that the extension of the treatment to enzyme inhibition, which is our primary concern, is completely satisfactory.

A further word may be said regarding the rates of proton association and dissociation, inasmuch as these rates, relative to the rates of the other reactions occurring in the inlibition, determine whether ionization rates must be included in the treatment or not. The rate constants for the reaction:

$$
\mathrm{H}+\mathrm{B} \underset{k_{d}}{\stackrel{k_{a}}{\rightleftharpoons}} \mathrm{HB}
$$

for some acids are given by Bell (1959) (see tabulation below). Average values for the carboxyl group wonld be: $\log k_{u}=10.1$ and $\log k_{d}=6.7$.

\begin{tabular}{l|c|c}
\hline \multicolumn{1}{c}{ Substance } & $\begin{array}{c}\text { Log } k_{a} \\
\left.\text { (liters } \mathrm{mole}^{-1} \mathrm{sec}^{-1}\right)\end{array}$ & $\begin{array}{c}\log k_{d} \\
\left(\mathrm{sec}^{-1}\right)\end{array}$ \\
\hline Water & 11.1 & -4.6 \\
Sulfuric acid & 10.6 & 9.0 \\
Acetic acid & 10.8 & 5.7 \\
Formic acid & 9.0 & 4.7 \\
Boric acid & 10.1 & 1.0 \\
Trimethylammonia & 10.9 & 1.1 \\
\hline
\end{tabular}


The formation of water from $\mathrm{H}^{+}$and $\mathrm{OH}^{-}$is very rapid, $k_{a}$ being $1.3 \times 10^{11}$ liters mole $\mathrm{sec}^{-1}$. Since most of these rates are very rapid, one would not expect them to be limiting in ordinary enzyme reactions.

Let us consider initially the simple situation in which the enzyme does not ionize over the $\mathrm{pH}$ range studied; the $\mathrm{p} K_{\alpha}$ of a monobasic substrate, however, lies within this range. It may be assumed that either $\mathrm{S}$ or HS combines with the enzyme. The former case is represented by the reactions:

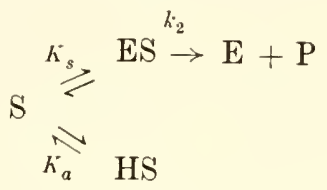

The conservation equations are:

$$
\begin{aligned}
& \left(\mathrm{E}_{t}\right)=(\mathrm{E})+(\mathrm{ES}) \\
& \left(\mathrm{S}_{t}\right)=(\mathrm{S})+(\mathrm{HS})
\end{aligned}
$$

The term (ES) has been omitted in the expression for $\left(\mathrm{S}_{l}\right)$ because this is not a mutual depletion system. The rate is given by $v=k_{2}$ (ES). Solving Eqs. 14-21 and 14-22 for (ES) and substituting this in the rate equation:

$$
v=V_{m} \frac{\left(\mathrm{S}_{t}\right)}{\left(\mathrm{S}_{t}\right)+K_{s}\left[1+(\mathrm{H}) / K_{a}\right]}
$$

or in terms of the functions describeck earlier:

$$
v=V_{m} \frac{\left(\mathrm{S}_{t}\right)}{\left(\mathrm{S}_{t}\right)+f_{s}^{\prime} K_{s}}
$$

Thus one may say in this case that the experimentally determined substrate constant. $K_{s}{ }^{\prime}$, is equal to $f_{s}{ }^{\prime} K_{s}$, where $K_{s}$ is the true substrate constant expressing the association of the active form of the substrate, $\mathrm{S}$, with the enzyme. This relationship can be derived more directly. The apparent substrate constant is given by:

$$
K_{\mathrm{s}}{ }^{\prime}=(\mathrm{E})\left(\mathrm{S}_{t}\right) /(\mathrm{ES})
$$

where $\left(S_{l}\right)$ is the total substrate concentration, i.e.. $(S)+(H S)$. If a mutual depletion system is being dealt with. (ES) must be inchuded and a more complex quadratic expression results. From Eq. 14-3, $\left(\mathrm{S}_{t}\right)=f_{s}^{\prime}(\mathrm{S})$. and thus:

$$
K_{s}{ }^{\prime}=f_{s}{ }^{\prime} \frac{(\mathrm{E})(\mathrm{S})}{(\mathrm{ES})}=f_{s}{ }^{\prime} K_{s}
$$


If only HS combines with the enzyme, it is easy to show that $K_{s}{ }^{\prime}=$ $f_{h s}{ }^{\prime} K_{s}$. Similarly, if the substrate is a dibasic acid and exists in the equilibrimm, $\mathrm{S} \rightleftharpoons \mathrm{HS} \rightleftharpoons \mathrm{H}_{2} \mathrm{~S}$, the apparent substrate constant is given by the true constant multiplied by the appropriate $\mathrm{pH}$ function. Thus, if $\mathrm{HS}$ is the active form and combines with the enzyme, $K_{s}{ }^{\prime}=f_{h s}{ }^{\prime \prime} K_{s}$.

By the active form of any component of the enzyme system, it is meant that this form participates in some way in the enzymic reaction. It is the form that is bound or that undergoes reaction, or both. In the case of an ionizing activator, the active form is that which is bound to the enzyme and is involved in the catalytic transformation of the substrate. In some instances, there is no single active form, the varionsly ionized species participating to different degrees.

If the enzyme ionizes over the $\mathrm{pH}$ range studied but the substrate does not, the following reactions occur:

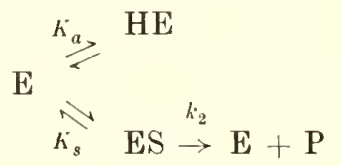

when $\mathrm{E}$ is the active form. The conservation equation is:

$$
\left(\mathrm{E}_{t}\right)=(\mathrm{E})+(\mathrm{HE})+(\mathrm{ES})
$$

and the rate is, as nsual, given by $v=k_{2}(\mathrm{ES})$. This leads to:

$$
v=V_{m} \frac{(\mathrm{S})}{(\mathrm{S})+K_{s}\left[1+(\mathrm{H}) / \overline{K_{a}}\right]}
$$

or, in terms of the $\mathrm{pH}$ function:

$$
v=V_{m} \frac{(\mathrm{S})}{(\mathrm{S})+f_{e}^{\prime} K_{s}}
$$

Thus here, $K_{s}{ }^{\prime}=f_{e}{ }^{\prime} K_{s}$, and when $\mathrm{HE}$ is the catalytically active form, $K_{s}{ }^{\prime}=$ $f_{h e}{ }^{\prime} K_{s}$. If both the substrate and the enzyme ionize within the $\mathrm{pH}$ range investigated, the appropriate $\mathrm{pH}$ functions for both must be used. If the reaction occurs between $\mathrm{E}$ and $\mathrm{HS}$, it may be shown that $K_{s}{ }^{\prime}=f_{\ell}{ }^{\prime} f_{h s}{ }^{\prime} K_{s}$.

Similar relationships in the literature have generally been given in terms of the Michaelis constant, $K_{m}$, instead of the true dissociation constant of the substrate, $K_{s}$. It might be thought that these constants would be interchangeable with respect to the $\mathrm{pH}$ effects, but actually it is $K_{s}$ to which the $p H$ functions may be applied. When $K_{m}$ does not equal $K_{s}$ and is given by $\left(k_{-1}+k_{2}\right) / k_{1}$ (or any more complex combination of rate constants), $K_{m}$ depends on both $K_{s}$ and $K_{2}$. If both the affinity of the enzyme for the substrate aud the rate of breakdown of the ES complex are altered 
by $\mathrm{pH}$ changes, $K_{m}$ will change in a more complex fashion than indicated here. However, it is $K_{m}$ which is usually determined experimentally.

We may now turn to a somewhat more general system in which the enzyme ionizes:

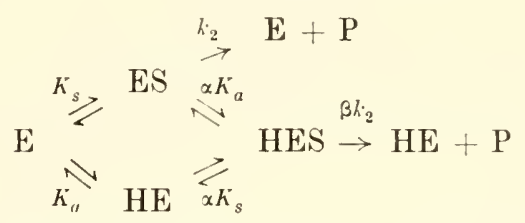

where both complexes, ES and HES, can ocenr and break down into products. The constants $\alpha$ and $\beta$ represent the effects of the association of the enzyme with a proton on $K_{s}$ and $k_{2}$ respectively. This scheme is actually identical with that previously formulated for generalized inhibition (Eq. 3-2), with $K_{r}$ replacing $K_{i}$ and a proton replacing the inhibitor. The relationships between $\mathrm{S}$ and $\mathrm{H}$ may be competitive, noncompetitive. mixed. or coupling. For the competitive situation $(\alpha=\infty)$ :

$$
v=V_{m}-\frac{(\mathrm{S})}{(\mathrm{S})}+f_{e}^{\prime} K_{s}
$$

which means that $\mathrm{H}$ is essentially a competitive inhibitor, since $\mathrm{HE}$ is incapable of binding the substrate. A noncompetitive situation $(\alpha=1$ and $\beta=0$ ) leads to:

$$
v=\frac{V_{m}}{f_{e}^{\prime}} \frac{(\mathrm{S})}{(\mathrm{S})+K_{s}}
$$

in which case $K_{s}$ is not altered but the maximal rate is. In the same manner as with $K_{s}$, one may designate an apparent maximal rate. $V_{m}^{\prime}$, which in this case is equal to $V_{m} / f_{\ell}^{\prime}$. Thus the effect of $\mathrm{pH}$ may be to change either $K_{s}$ of $V_{m}$ or both. If the substrate also ionizes and only one form of the substrate combines with the enzyme. the appropriate $\mathrm{pH}$ functions for the substrate should also be includerl as described above. When more than one form of the substrate combines with the enzyme and the resulting complexes can break down at different rates to form the prodnct, the situation is considerably more complex.

Finally. an extension to enzymes containing two ionizing gromps will be outlined according to the following scheme:

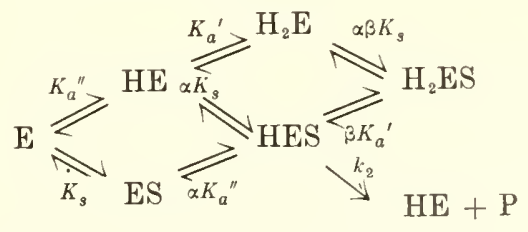


It is assumed that only HES breaks down to form the product. In this case:

$$
v=V_{m} \frac{(\mathrm{S})}{f_{h e s}{ }^{\prime \prime}(\mathrm{S})+\alpha f_{h e}{ }^{\prime \prime} K_{\text {。 }}}
$$

This equation may be rearranged to give:

$$
v=\frac{V_{m}}{f_{h e s}{ }^{\prime \prime}} \frac{(\mathrm{S})}{(\mathrm{S})+\left(\alpha f_{h e} e^{\prime \prime} / f_{\text {hes }}{ }^{\prime \prime}\right) K_{s}}
$$

This allows us to see that the apparent substrate constant, $K_{s}{ }^{\prime}=\alpha f_{h e}{ }^{\prime \prime} K_{s} l$ $f_{\text {hes }}{ }^{\prime \prime}$, is that expected from the above discussion; it must be remembered that $\alpha K_{s}$ is the substrate constant for the product-forming complex. It is also observed that the apparent maximal rate, $V_{m}{ }^{\prime}=V_{m} / f_{\text {hes }}{ }^{\prime \prime}$, is less than the true rate because only a fraction of the enzyme combined with substrate is in the HES form. If $\mathrm{ES}$ and $\mathrm{H}_{2} \mathrm{ES}$ can also form product, the expression for the rate will contain terms for them corresponding to Eq. 14-35. It is evident that this system will exhibit a maximum in the rate-pH curve if HES exchusively forms product (or more rapidly than do ES or $\left.\mathrm{H}_{2} \mathrm{ES}\right)$.

An excellent presentation of the effects of substrate ionization is that given by Frieden (1958). Equations are derived for various situations and some methods for handling the experimental data are discussed. An interesting possibility is brought up by Frieden when he suggests that the form of the substrate that is not catalytically active may be an inhibitor; i.e., if $\mathrm{S}$ is the form that is reacted by the enzyme, SH may not only fail to react but may compete with $\mathrm{S}$ for the substrate site.

\section{Location of $\mathrm{pH}$ Optima}

The $\mathrm{pH}$ at which maximal enzymic activity occurs can be calculated by finding the hydrogen ion concentration necessary to minimize the $\mathrm{pH}$ functions in the denominator of the rate equation. For example, if $\mathrm{E}^{-}$combines with $\mathrm{HS}^{+}$(HE and $\mathrm{S}$ not contributing to the reaction), $K_{s}{ }^{\prime}=f_{e}{ }^{\prime} f_{h s}{ }^{\prime} K_{s}$, and the rate will be highest when $f_{e}{ }^{\prime} f_{h{ }^{\prime}}{ }^{\prime}$ is at its minimum value. Taking the derivative of this product with respeet to $(\mathrm{H})$ and setting it equal to zero:

$$
\frac{d\left(f_{e}^{\prime} f_{h s}{ }^{\prime}\right)}{d(\mathrm{H})}=\frac{1}{K_{a_{e}}}-\frac{K_{a_{s}}}{(\mathrm{H})^{2}}=0
$$

where $K_{a_{e}}$ and $K_{a_{s}}$ are the ionization constants for the enzyme and substrate respectively. Thus:

$$
\mathrm{pH}_{o p t}=1 / 2\left(\mathrm{p} K_{a_{e}}+\mathrm{p} K_{a_{s}}\right)
$$

In such a simple case, the optimal $\mathrm{pH}$ lies midway between the $\mathrm{p} K_{a}$ 's of the 
ionizing groups. If the enzyme ionizes in two steps and $K_{s}{ }^{\prime}=f_{h e}{ }^{\prime \prime} K_{s}$, the same procedure of setting $d\left(f_{h e}{ }^{\prime \prime}\right) / d(\mathrm{H})=0$ gives:

$$
\mathrm{pH}_{o p t}=1 / 2\left(\mathrm{p} K_{a}{ }^{\prime}+\mathrm{p} K_{a}{ }^{\prime \prime}\right)
$$

where $\mathrm{p} K_{a}^{\prime}$ and $\mathrm{p} K_{a}^{\prime \prime}$ refer to the two dissociations of the enzyme.

The more complete scheme shown in Eq. 14-34 may be treated similarly and setting the derivative of the denominator of Eq. 14-35 equal to zero leads to:

$$
\mathrm{pH}_{\text {oDt }}=\frac{1}{2}\left[p K_{a}^{\prime}+p K_{a}^{\prime \prime}-\log \frac{(\mathrm{S})+K_{s}}{[(\mathrm{~S}) / \alpha \beta]+K_{s}}\right]
$$

The $\mathrm{pH}_{\text {opt }}$ in this case will depend on (S) and on the factors, $\alpha$ and $\beta$, that express the effects of $\mathrm{H}$ and $\mathrm{S}$ on each other's binding. If $\alpha=\beta=1-$ i.e,. there is no interaction in the binding of $\mathrm{H}$ and $\mathrm{S}$ - the equation reduces to $14-39$ as expected.

The $\mathrm{pH}$ optimum for $u$-chymotrypsin is 8.3 but the presence of increasing amounts of methanol leads to a progressive fall in this value to 7.8 (Stein and Laidler, 1959). This is shown in Fig. 14-1 for methanol concentrations between 0 and $20 \%$. It may be noticed that the shift in $\mathrm{pH}_{\text {opt }}$ is associated with a depression of the rate. The action of methanol had been previously treated (Barnard and Laidler, 1952) as a dielectric constant effect, but in the more recent report it is felt that the dielectric constant decrease, at least in the lower methanol concentrations, is not sufficient to produce the changes observed. Instead, Stein and Laidler postulate that the methanol may bind to the enzyme and interfere with hydrogen bonding at the active site and thus affect the interaction of the enzyme and substrate. These results demonstrate that the $\mathrm{pH}$ optima for enzymes may vary with the conditions of the experiment and that the inhibition of a reaction may shift $\mathrm{pH}_{\text {opt }}$.

\section{Dixon's Treatment of the Variation of $\mathrm{K}_{s}$ ' with the $\mathrm{pH}$}

A method for utilizing the data obtained from the variation of the $\mathrm{pH}$ for the localization of the ionizing groups was developed by Dixon (1953a). This will be discussed briefly at this point and applied more rigorously to inhibition in a later section. The experimentally determined or apparent substrate constant (not the Michaelis constant) may be expressed as:

$$
K_{s}^{\prime}=\frac{\left(\mathrm{E}_{t}\right)\left(\mathrm{S}_{t}\right)}{\left(\mathrm{ES}_{t}\right)}=\frac{f_{e}(\mathrm{E}) f_{s}(\mathrm{~S})}{f_{e s}(\mathrm{ES})}=\frac{f_{e} f_{s}}{f_{e s}} K_{s}
$$

where here the $\mathrm{pH}$ functions designate the appropriate functions for $\mathrm{E}, \mathrm{S}$, and ES. From this one may write:

$$
\mathrm{p} K_{s}{ }^{\prime}=\mathrm{p} K_{s}-\log f_{e}-\log f_{s}+\log f_{e s}
$$


If $\mathrm{p} K_{s}{ }^{\prime}$ is plotted against $\mathrm{pH}$, the slopes and inflection points will indicate the $p K_{a}$ 's of the ionizing groups and the particnlar form of the component that is ionizing. A similar treatment applies to the apprarent maximal

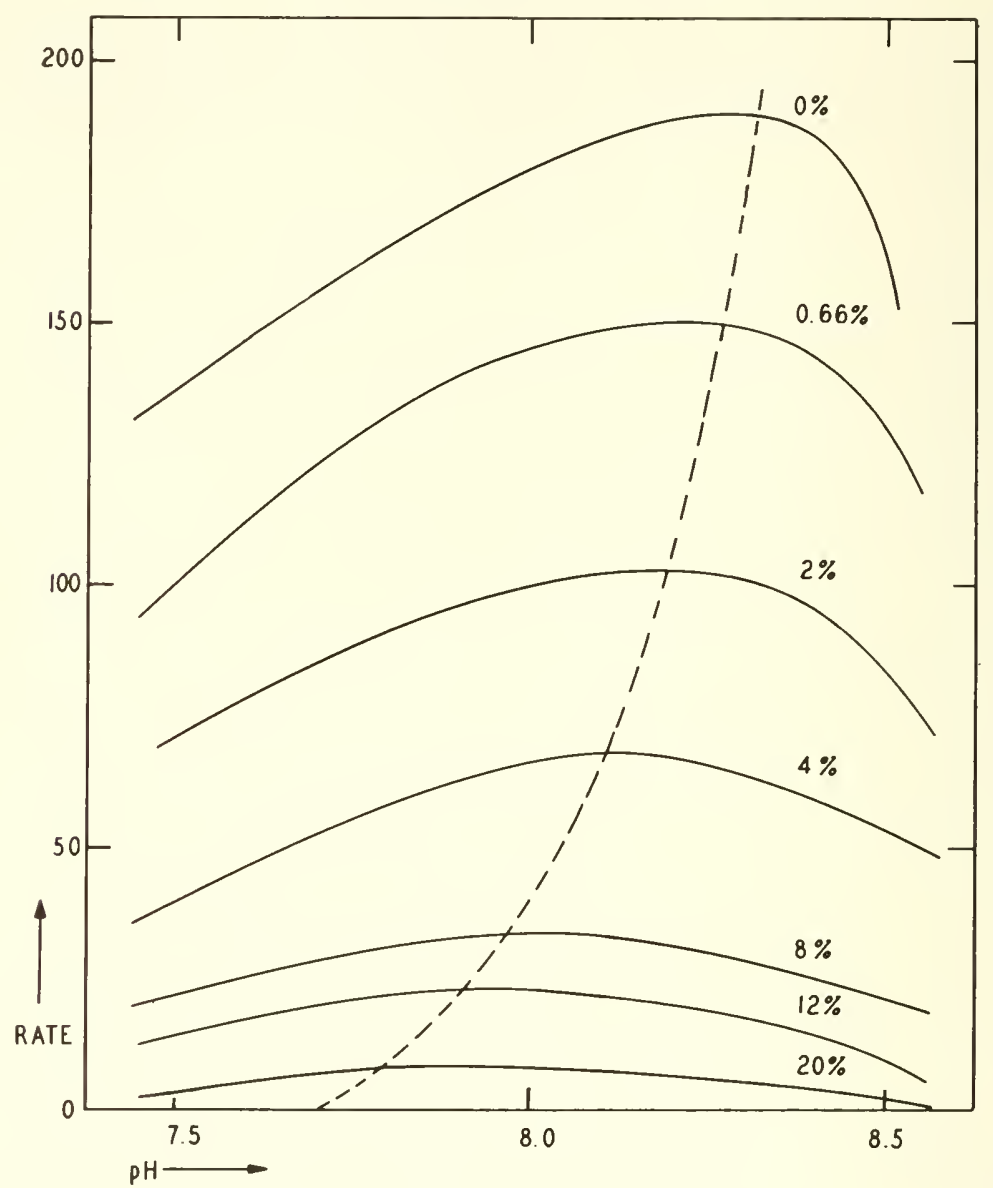

FIG. 14.1. Shifts in the pH optimum for a-chymotrypsin produced by increasing concentrations of methanol. (From Stein and Laidler, 1959.)

rate when it is not equivalent to the true maximal rate and is given by an expression such as:

since:

$$
V_{m}{ }^{\prime}=V_{m} / f_{e s}
$$

$$
\log V_{m}{ }^{\prime}=\log V_{m}-\log f_{e s}
$$

and a plot of $\log V_{m}{ }^{\prime}$ against pH will provide data on the ionization of the ES complex. 
It is now necessary to examine how the $\mathrm{pH}$ functions determine the $\mathrm{p} K_{s}{ }^{\prime}-\mathrm{pH}$ and $V_{m}{ }^{\prime}-\mathrm{pH}$ curves. The variation of the reciprocals of the common functions with $\mathrm{pH}$ is shown in Fig. 14-2. The reciprocals are plotted because the concentrations of the individual ionic species are given by expressions of the type $(\mathrm{B})=\left(\mathrm{B}_{t}\right) / f_{b}$, so that the curves represent the fractions of the varions species that are present. However, it is more important to investigate the behavior of the logarithms of the $\mathrm{pH}$ functions since

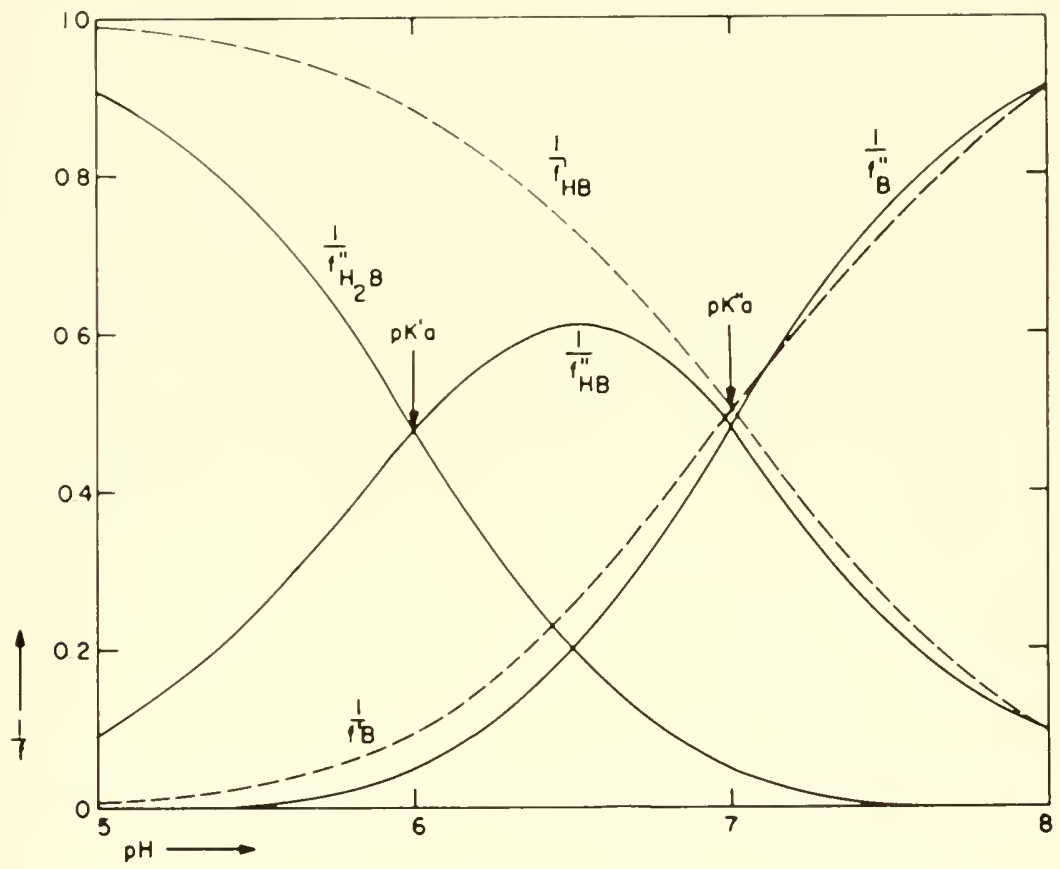

FIG. 14-2. Plots of the reciprocals of the $\mathrm{pH}$ functions. The solid curves are for a dibasic acid with $\mathrm{p} K_{a}^{\prime}=6$ and $\mathrm{p} K_{a}{ }^{\prime \prime}=7$. The dashed curves are for a monobasic acid with $\mathrm{p} K_{a}=7$.

it is these that appear in Eqs. 14-42 and 14-44. Following Dixon (Dixon and Webb, 1958, p. 127), we shall use the expression $\mathrm{p} f=-\log f$ and thus Eq. 14-42 may be rewritten as:

$$
\mathrm{p} K_{s}{ }^{\prime}=\mathrm{p} K_{s}+\mathrm{p} f_{e}+\mathrm{p} f_{s}-\mathrm{p} f_{e s}
$$

Inasmuch as $\mathrm{p} K_{s}$ is assumed to be constant, the changes in $\mathrm{p} K_{s}^{\prime}$ with $\mathrm{pH}$ will be determined by the dependency of the $\mathrm{p} f$ terms on $\mathrm{pH}$. Figures 14-3 and 14-4 show this dependency for the systems plotted in Fig. 14-2. In the case of a dibasic ionization, the form of the curves will depend on the 
separation between the two dissociation constants. In Fig. 14-4 the $\mathrm{p} K_{\alpha}$ 's are only one unit apart and the transitions are not clearly evident, the curves being smooth and scarcely distinguishable from those of a monobasic ionization (Fig. 14-3). The farther the $\mathrm{p} K$, 's are apart, the easier it will be to locate the inflection points and determine the values of the constants. In Fig. 14-5 the difference between $\mathrm{p} K_{a}^{\prime}$ and $\mathrm{p} K_{a}{ }^{\prime \prime}$ is four units and here the inflections in the curves are evident.

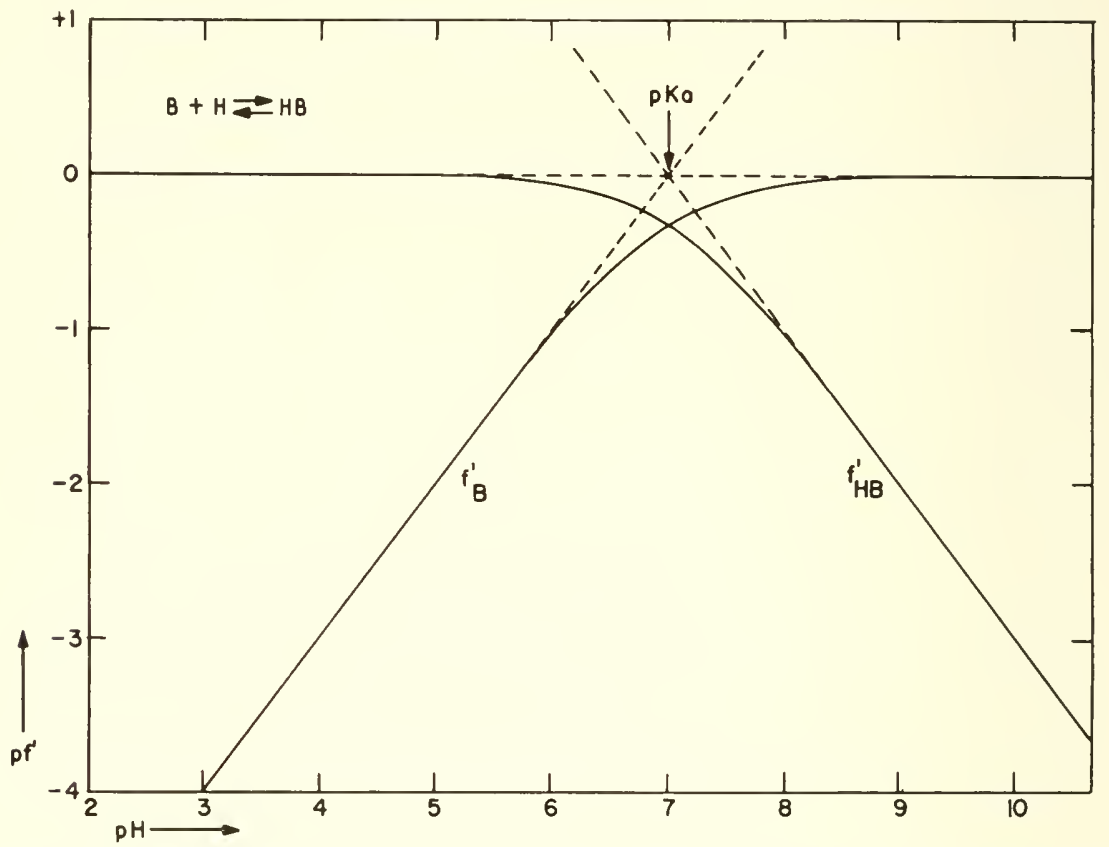

FIG. 14-3. Logarithmic plots of the $\mathrm{pH}$ functions for a monobasic acid with $\mathrm{p} K_{a}=7$ (as in the dashed curves in Fig. 14-2). The intersections of the linear portions of the curves occurs at the $\mathrm{p} K_{a}$.

Let us assume the simple case where $\mathrm{p} K_{s}{ }^{\prime}=p K_{s}+\mathrm{p} f_{b}^{\prime \prime}$. When $\mathrm{p} K_{s}^{\prime}$ is plotted against $\mathrm{pH}$, a curve of the type shown for $\mathrm{p} f_{b}^{\prime \prime}$ will be obtained, but shifted on the vertical axis by the constant amount, $\mathrm{p} K_{s^{*}}$ If we examine the variation of $\mathrm{p} f_{b}^{\prime \prime}=-\log \left\{1+\left[(\mathrm{H}) / K_{a}{ }^{\prime \prime}\right]+\left[(\mathrm{H})^{2} / K_{a}{ }^{\prime} K_{a}{ }^{\prime \prime}\right]\right\}$ with $\mathrm{pH}$, the following may be noted: (1) at low $\mathrm{pH}$ 's, the term $(\mathrm{H})^{2} / K_{z}{ }^{\prime} K_{q}{ }^{\prime \prime}$ will dominate and the logarithm of this is given by $\mathrm{p}_{b}{ }^{\prime \prime}=\mathrm{p} K_{a}{ }^{\prime}+\mathrm{p} K_{a}{ }^{\prime \prime}-2 \mathrm{pH}$; (2) at intermediate values of the $\mathrm{pH}$, the $(\mathrm{H}) / K_{a}{ }^{\prime \prime}$ will dominate and $p f_{b}^{\prime \prime}=$ $\mathrm{p} K_{a}{ }^{\prime \prime}-\mathrm{pH}$; and (3) at high $\mathrm{pH}$, the second and third terms will be insignificant and $p f_{b}^{\prime \prime}=0$. Thus, as one goes from low to high $\mathrm{pH}$ 's, the slope of the curve will initially be 2 , then 1 , and finally 0 ; the transitions between these 
slopes will provide the values of $\mathrm{p} K_{a}{ }^{\prime}$ and $\mathrm{p} K_{a}{ }^{\prime \prime}$, these being given by the intersections of the linear slope lines. If $\mathrm{p} K_{s}^{\prime}=\mathrm{p} K_{s}+\mathrm{p} f_{h l}{ }^{\prime \prime}$, the slope will change from 1 through 0 to -1 as the $\mathrm{pH}$ rises, and for $\mathrm{p} K_{s}^{\prime}=\mathrm{p} K_{s}+$ $\mathrm{p} f_{h_{\mathbf{2}} b}$ " the slope changes from 0 through -1 to -2 . However, it must be remembered that in Eq. 14-45, $p f_{e s}$ is opposite in sign to $p f_{e}$ and $p f_{s}$ so that the curves relating to the former will be inverted with respect to the latter and the inflections will be concave upwards.

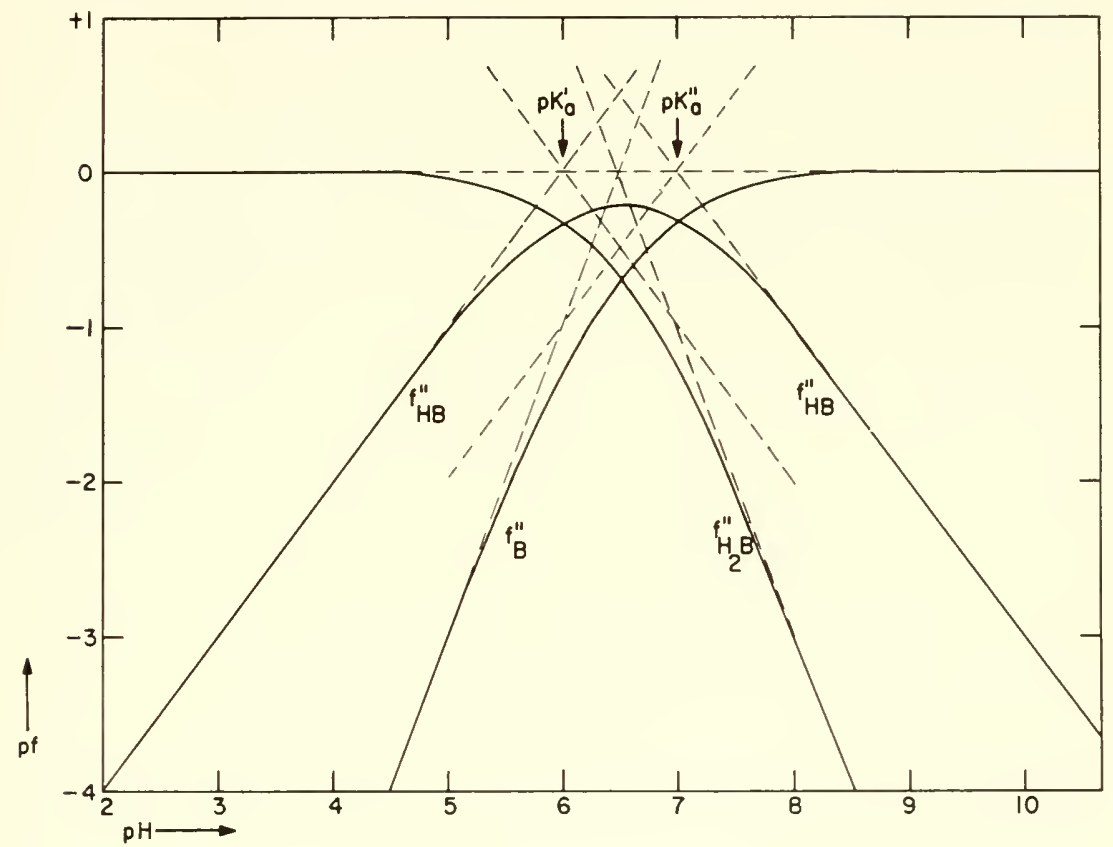

FIa. 14-4. Logarithmic plots of the $\mathrm{pH}$ functions for a dibasic acid with $\mathrm{p} K_{a}{ }^{\prime}=6$ and $p K_{a}^{\prime \prime}=7$ (as in the solid curves in Fig. 14-2).

The rules for interpreting such curves as formulated by Dixon may now be summarized.

I. A curve obtained by plotting $p K_{s}^{\prime}$ against $\mathrm{pH}$ will consist of linear sections (if the $\mathrm{p} K_{2}$ 's are sufficiently separated) and the slopes of these sections will have integral values of $0,1,2,3, \ldots$, either positive or negative.

II. The slope of any linear section is numerically equal to the change of charge upon dissociation of the ES complex in that $\mathrm{pH}$ region.

III. Each inflection of the curve indicates the $\mathrm{p} K_{2}$ of an ionizing group on a component of the enzyme system; continuations of the linear sections intersect at a $\mathrm{pH}$ value corresponding to this $\mathrm{p} K_{\imath}$. 
IV. An inflection that is concave downwards indicates an ionization of the enzyme or the substrate; an inflection that is coneave upwards indicates an ionization of the ES complex.

From the curves alone it is not possible to determine if a particular downward inflection relates to the ionization of the enzyme or the substrate; however, the ionization constants of the substrate are generally known so that experimentally determined $\mathrm{p} K_{/ 2}$ 's that do not correspond to these may

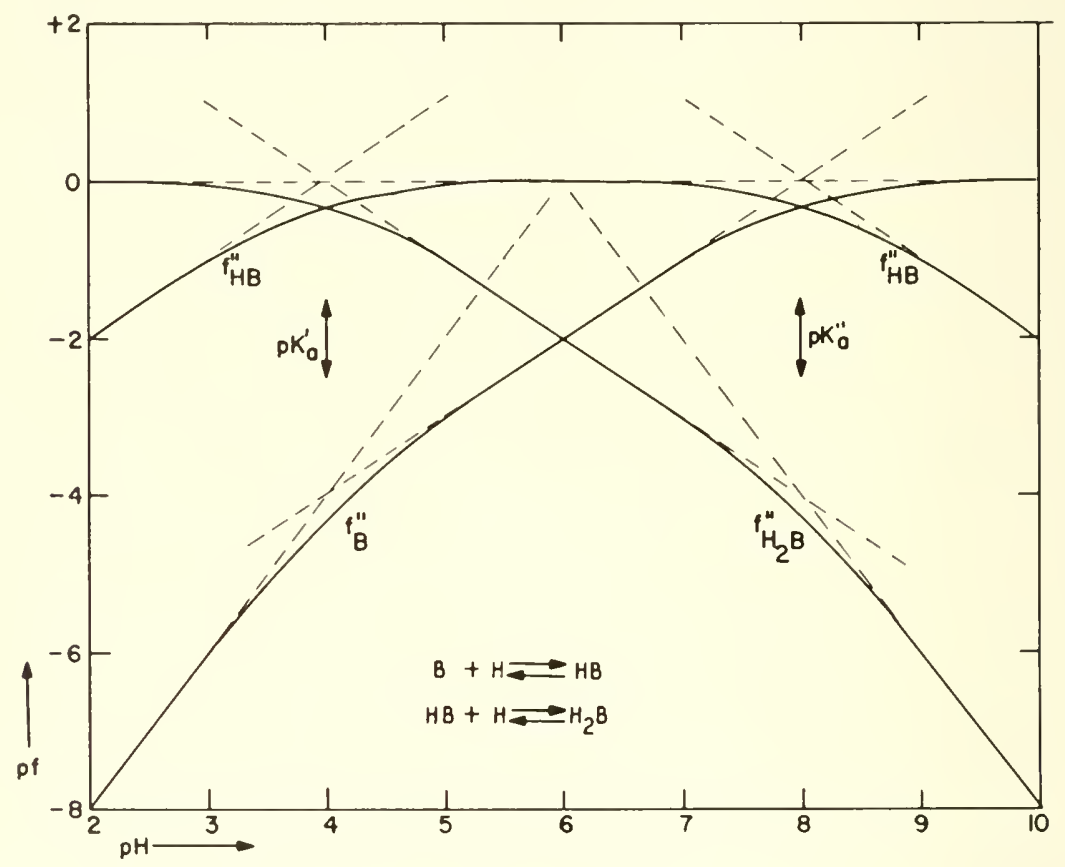

Fic. 14-5. Logarithmic plots of the $\mathrm{pH}$ functions for a dibasic acid with $\mathrm{p} K_{a}^{\prime}=4$ and $\mathrm{p} K_{a}^{\prime \prime}=8$. The intersections of the linear portions of the curves at the $\mathrm{p} K_{a}$ values are shown.

be attributed to the enzyme. It is often the case that there are limitations to the $\mathrm{pH}$ range that may be studied and in such cases only parts of the total $\mathrm{p} K_{s}{ }^{\prime}-\mathrm{pH}$ curves may be obtained. Unless a $\mathrm{pH}$ range of at least 4 to 10 ean be investigated, it is not safe to conclude that all the ionizing groups have been detected. Extensions and criticisms of Dixon's procedure have been made by Reiner (1959, p. 230) and Laidler (1955.c, d, 1958, p. 140). 


\section{VARIATION OF ENZYME INHIBITION WITH $\mathrm{pH}$}

The mechanisms responsible for the alterations in enzyme inhibition when the $\mathrm{pH}$ is changed are generally similar to those outlined with respect to enzyme activity (page 653). The fundamental effects are almost all mediated through the modification in the binding of the inhibitor to the enzyme (or to any other components of the system to which it may be bound) and these effects arise from changes in the ionizations of groups on any of the interacting molecules. In one respect, the interpretation of the results with inhibitors is more straightforward than in studies of enzyme activity: the experimentally determined or apparent inhibition constant $\left(K_{i}{ }^{\prime}\right)$ is either the true dissociation constant $\left(K_{i}\right)$ for the EI complex or is related to it in a simple manner by the proper $\mathrm{pH}$ functions. It is also true that certain factors that alter the enzyme activity when the $\mathrm{pH}$ is changed will not necessarily alter the degree of inhibition if the activity is changed equally in the absence and presence of the inhibitor. A typical curve for the variation of inhibition with $\mathrm{pH}$ may be obtained from the data of Stockell and Smith (1957) for the inhibition of papain by carbobenzoxy-L-glutamate (Fig. 14-6). The decrease in the inhibition with rising $\mathrm{pH}$ was attributed to the ionization of the $\gamma$-carboxyl group, since its $\mathrm{p} K_{a}$ is about 4.4 .

\section{Expressions for the $\mathrm{pH}$ Dependence of Enzyme Inhibition}

Inasmuch as a complete and rigorous treatment of enzyme activity as modified by $\mathrm{pH}$ is too elaborate to be practical, it is obvious that such a treatment of inhibition, which is yet more complex, would be futile. Furthermore, it is likely that most real inhibitions fall into rather restricted categories and that a general treatment would seldom be applicable. Thus we shall consider various simple systems, from which principles that may be applied to more complex inhibitions will emerge. In any inhibited enzyme system, there will be at least three substances that may ionize - the enzyme (active center), the substrate, and the inhibitor. In addition, the ionization of the various complexes with the enzyme must be taken into account when they occur.

Case I: only the inhibitor ionizes. When the only component of the system ionizing within the $\mathrm{pH}$ range studied is the inhibitor, and if mutual depletion of the enzyme and inhibitor cloes not occur, the treatment is very simple. In every case, the experimentally determined or apparent inhibitor constant, $K_{i}{ }^{\prime}$, must be replaced with $f_{i} K_{i}$, where $f_{i}$ is the appropriate $\mathrm{pH}$ function for the form of the inhibitor that is involved and $K_{i}$ is the true inhibitor constant. Thus for the competitive inhibition where only HI binds to the enzyme: 


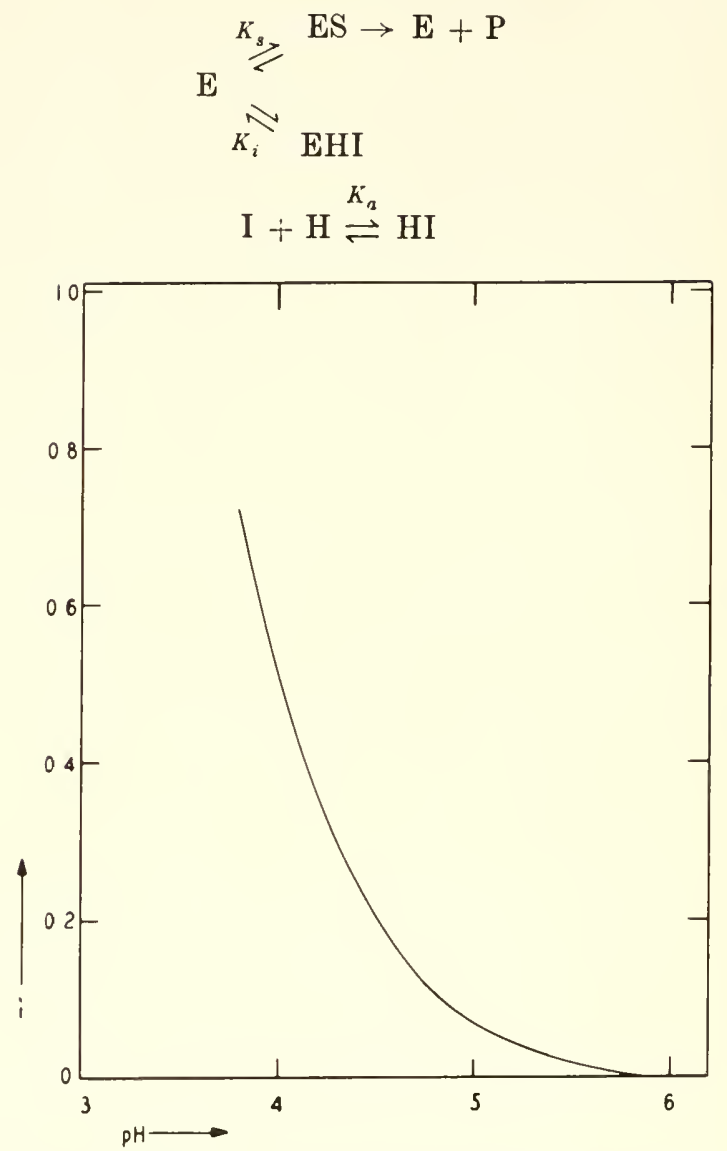

Fig. 14-6. Effect of the $\mathrm{pH}$ on the inhibition of papain by carbobenzoxy-L-glutamate $(20 \mathrm{~m} M I)$. (From Stockell and Smith, 1957.)

the fractional inhibition is given by:

$$
i=\frac{\left(\mathrm{I}_{t}\right)}{\left(\mathrm{I}_{t}\right)+f_{h i}{ }^{\prime} K_{i}\left[1+\frac{(\mathrm{S})}{K_{s}}\right]}
$$

which is identical to the usual expression for competitive inhibition (Eq. 3-13) except for the replacement of the apparent inhibitor constant with $f_{h \imath}{ }^{\prime} K^{\prime}$. If the inhibitor is di- or tribasic, the proper $f^{\prime \prime}$ of $f^{\prime \prime \prime}$ functions may be used. 
If both ionization forms of the inhibitor can bind to the enzyme:

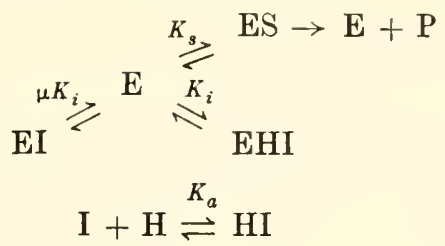

the difference in the dissociation constants being expressed by $\mu$, the inhibition is given by:

$$
i=\frac{\left(\mathrm{I}_{t}\right)}{\left(\mathrm{I}_{t}\right)+\frac{\mu f_{i}^{\prime} f_{h i}{ }^{\prime}}{\mu f_{i}{ }^{\prime}+f_{h i}{ }^{\prime}} K_{i}\left[1+\frac{(\mathrm{S})}{K_{s}}\right]}
$$

If $\mu=\infty$ and EI is not formed, the equation reduces to $14-47$; if $\mu=1$ and both $\mathrm{I}$ and $\mathrm{HI}$ combine with the enzyme equally, $K_{i}{ }^{\prime}=\left[f_{i}{ }^{\prime} f_{h i}{ }^{\prime} /\left(f_{i}{ }^{\prime}+\right.\right.$ $\left.\left.f_{h i}{ }^{\prime}\right)\right] K_{i}=K_{i}$, and the apparent and true inhibition constants are identical.

Case II: only the substrate iomizes. When the substrate concentration is a factor in the inhibition (as in competitive inhibition) and (S) occurs in the expression for the inhibition, the ionization of the substrate may be taken into account by multiplying the true substrate constant by the appropriate $\mathrm{pH}$ function for that form of the substrate that is active. Thus if the substrate is dibasic and only HS reacts with the enzyme:

$$
i=\frac{(\mathrm{I})}{(\mathrm{I})+K_{i}\left[1+\frac{\left(\mathrm{S}_{t}\right)}{f_{h s}{ }^{\prime \prime} K_{s}}\right]}
$$

Case III: both the inhibitor and substrate ionize. In this case both the substrate and inhibitor constants are simply multiplied by the proper pH functions. Indeed, one may write for all the ordinary types of inhibition general equations derivable from Eq. 3-11, from which all special cases may be obtained. Thus, in general:

$$
K_{s}^{\prime}=\alpha f_{s} K_{s}\left[\frac{\left(\mathrm{I}_{t}\right)+f_{\imath} K_{i}}{\beta\left(\mathrm{I}_{t}\right)+\alpha f_{i} K_{i}}\right]
$$

Case IV: only the enzyme (active center) iomizes. Up to this point the results have been simple and expected. However, the ionization of the enzyme introduces some complications which have previously not been realized; in fact most of the interpretations for ionizing groups on the enzyme from work with inhibitors are incorrect. Let us consider a typical enzyme reaction in which the enzyme is active in only one ionization state, either as 
$\mathrm{E}$ or HE. The inhibitor can then combine competitively or noncompetitively with either active form of the enzyme, $\mathrm{E}$ or HE, or with the nonactive form of the enzyme. There are thus six possible situations for this simplest of systems.

(a) $H E$ is active form of enzyme: I combines with $H E$ competitively.

$$
\begin{aligned}
& \stackrel{K_{a}}{\rightleftharpoons} \mathrm{HE} \stackrel{K_{s}}{\rightleftharpoons} \mathrm{HES} \rightarrow \mathrm{HE}+\mathrm{P} \\
& K_{i}^{K_{i}} \text { HEI }
\end{aligned}
$$

In each case, the inhibited rate and the fractional inhibition will be given; the implications will be discussed when the six situations have been presented. It must be remembered that the uninhibited rate is now given by:

$$
v=\frac{V_{m}(\mathrm{~S})}{(\mathrm{S})+f_{h e}{ }^{\prime} K_{s}}
$$

The inhibited rate may be written as:

$$
v_{i}=\frac{V_{m}(\mathrm{~S})}{(\mathrm{S})+K_{s}\left[f_{h e^{\prime}}+\frac{\overline{(\mathrm{I})}}{K_{i}}\right]}
$$

and the fractional inhibition as:

$$
i=\frac{(\mathrm{I})}{(\mathrm{I})+K_{i}\left[f_{h e^{\prime}}+\frac{(\mathrm{S})}{K_{s}}\right]}
$$

(b) $E$ is active form of enzyme: I combines with $E$ competitively.

$$
\begin{aligned}
& \mathrm{HE} \stackrel{\kappa_{a}}{\rightleftharpoons} \mathrm{E} \sum_{k_{1}}^{\kappa_{n}} \underset{\mathrm{EI}}{\mathrm{ES}}-\mathrm{E}+\mathrm{P} \\
& v_{i}=\frac{V_{m}(\mathrm{~S})}{(\mathrm{S})+K_{s}\left[f_{e}{ }^{\prime}+\frac{(\mathrm{I})}{K_{i}}\right]} \\
& i=\frac{(\mathrm{I})}{(\mathrm{I})+K_{i}\left[f_{e}^{\prime}+\frac{(\mathrm{S})}{K_{\mathrm{s}}}\right]}
\end{aligned}
$$


(c) $H E$ is active form of enzyme: I combines with $H E$ noncompetitively.

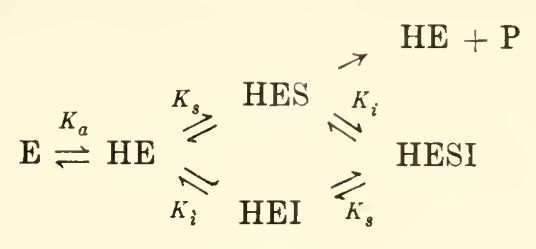

It should be noted that completely noncompetitive inhibition is assumed; i.e., there is no effect of $\mathrm{S}$ on the binding of $\mathrm{I}$ or of $\mathrm{I}$ on the binding of $\mathrm{S}$.

$$
\begin{gathered}
v_{i}=\frac{V_{m}(\mathrm{~S})}{(\mathrm{S})\left[1+\frac{(\mathrm{I})}{K_{i}}\right]+K_{s}\left[f_{h e}+\frac{(\mathrm{I})}{K_{i}^{-}}\right]} \\
i=\frac{(\mathrm{I})}{(\mathrm{I})+K_{i}} \frac{K_{s}}{\left[1+\frac{K_{a}}{(\mathrm{H})} \frac{K_{s}}{(\mathrm{~S})+K_{s}}\right]}
\end{gathered}
$$

(d) $E$ is active form of enzyme: I combines with $E$ noncompetitively.

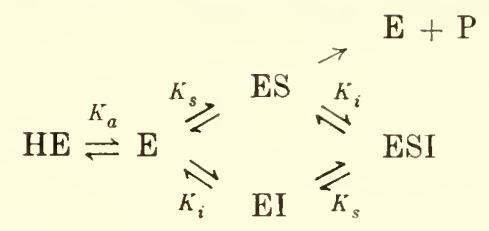

$$
\begin{aligned}
& v_{i}=\frac{V_{m}(\mathrm{~S})}{(\mathrm{S})\left[1+\frac{(\mathrm{I})}{K_{i}}\right]+K_{s}\left[f_{e}^{\prime}+\frac{(\mathrm{I})}{K_{i}}\right]} \\
& i=\frac{(\mathrm{I})}{(\mathrm{I})+K_{i}\left[1+\frac{(\mathrm{H})}{K_{a}} \frac{K_{s}}{(\mathrm{~S})+K_{s}}\right]}
\end{aligned}
$$

(e) $H E$ is active form of enzyme: $I$ combines with $E$.

$$
\begin{gathered}
\mathrm{EI} \stackrel{K_{i}}{\rightleftharpoons} \mathrm{E} \stackrel{K_{a}}{\rightleftharpoons} \mathrm{HE} \stackrel{K_{s}}{\rightleftharpoons} \mathrm{HES} \rightarrow \mathrm{HE}+\mathrm{P} \\
v_{i}=\frac{V_{m}(\mathrm{~S})}{(\mathrm{S})+K_{s}\left[f_{h e}{ }^{\prime}+\frac{K_{a}(\mathrm{I})}{K_{i}(\mathrm{H})}\right]} \\
i=\frac{(\mathrm{I})}{(\mathrm{I})+K_{i}\left[f_{e}^{\prime}+\frac{(\mathrm{H})(\mathrm{S})}{K_{a} K_{s}}\right]}
\end{gathered}
$$


(f) $E$ is active form of enzyme: I combines with $H E$.

$$
\begin{array}{r}
\mathrm{HEI} \stackrel{K_{i}}{\rightleftharpoons} \mathrm{HE} \stackrel{K_{a}}{\rightleftharpoons} \mathrm{E} \stackrel{K_{s}}{\rightleftharpoons} \mathrm{ES} \rightarrow \mathrm{E}+\mathrm{P} \\
v_{i}=\frac{V_{m}(\mathrm{~S})}{(\mathrm{S})+K_{s}\left[f_{e}{ }^{\prime}+\frac{(\mathrm{H})(\mathrm{I})}{K_{a} K_{i}}\right]} \\
i=\frac{(\mathrm{I})}{(\mathrm{I})+K_{i}\left[f_{h e}{ }^{\prime}+\frac{K_{a}(\mathrm{~S})}{K_{s}(\mathrm{H})}\right]}
\end{array}
$$

It may first be noted that in each case when the $\mathrm{pH}$ is in the range where the enzyme is predominantly in the form combining with the inhibitor, the inhibition becomes classically competitive or noncompetitive. In the first four cases, this is the $\mathrm{pH}$ range where the enzyme is in the active catalytic form; in the latter two cases it is when the enzyme is in the inactive form. Secondly, it is seen that when the inhibitor combines with the enzyme noncompetitively (last four cases), the inhibition depends on $(\mathrm{S})$, contrary to what is generally thought to be true for noncompetitive inhibition. Now, in these cases, the inhibition depends on (S) only in a certain range of $\mathrm{pH}$ : in (c) and (f) when the $\mathrm{pH}$ is significantly higher than the $\mathrm{p} K_{a}$ and in $(d)$ and $(\mathrm{e})$ when the $\mathrm{pH}$ is significantly lower than the $\mathrm{p} K_{a}$. As the $\mathrm{pH}$ is changed from one side of the ${ }_{p} K_{n}$ to the other, the character of the inhibition will thus alter. Thirdly, it may be observed that it might have been predicted that $K_{i}{ }^{\prime}=f_{e}{ }^{\prime} K_{i}$ or $K_{i}{ }^{\prime}=f_{\text {he }}{ }^{\prime} K_{i}$, depending on the form of the enzyme with which the inhibitor reacts, but this is only true when the substrate concentration is significantly lower than $K_{s}$. The importance of this will be discussed when the Dixon treatment of inhibition is presented.

This behavior must now be explained on a physical basis. In the first place, why does the inhibition in such cases depend on the $\mathrm{pH}$ at all? One might suppose that since both $v$ and $v_{i}$ are modified by $\mathrm{pH}$ due to shifts in the equilibrium, $\mathrm{E} \rightleftharpoons \mathrm{HE}$, that the inhibition would remain constant. Let us examine the situation depicted in (a) above. From Eq. 14-55, it is seen that when the $\mathrm{pH}$ is sufficiently below the $\mathrm{p} K_{u}$, so that the term in brackets in the denominator becomes essentially $1+\left[(\mathrm{S}) / K_{s}\right]$, the inhibition is independent of $\mathrm{pH}$ change; in a higher $\mathrm{pH}$ range, the hydrogen ion may become a more important factor than the substrate. Now, when the $\mathrm{pH}$ is low, most of the enzyme is in the HE form and the addition of an inhibitor will reduce $(\mathrm{HE})$ in a simple mass-action manner; however, at higher pH's, when the enzyme is predominantly in the E form, the addition of inhibitor will reduce $(\mathrm{HE})$ with the result that $\mathrm{E} \rightarrow \mathrm{HE}$. The concentration of $\mathrm{HE}$ is in a way bufferect by the reserve of $\mathrm{E}$. As long as there 
is a supply of $\mathbf{E}$, the inhibition will not be as great as would be expected from typical competitive inhibition or as great as it is at a lower $\mathrm{pH}$. The other question that might reasonably be asked is: why does the substrate concentration affect the noncompetitive inhibitions in (c)-(f)? The answer lies again in the coupled equilibria. As (S) increases, it tends to deplete the inactive form of the enzyme and it is this that modifies the response to the inhibitor, by a mechanism similar to that described above.

It will be worthwhile to illustrate some of the aspects of these systems by plotting the variation of the inhibition with $\mathrm{pH}$ or (S). All six types of inhibition are compared in Fig. $14-7$ for constant (I) and (S) and the

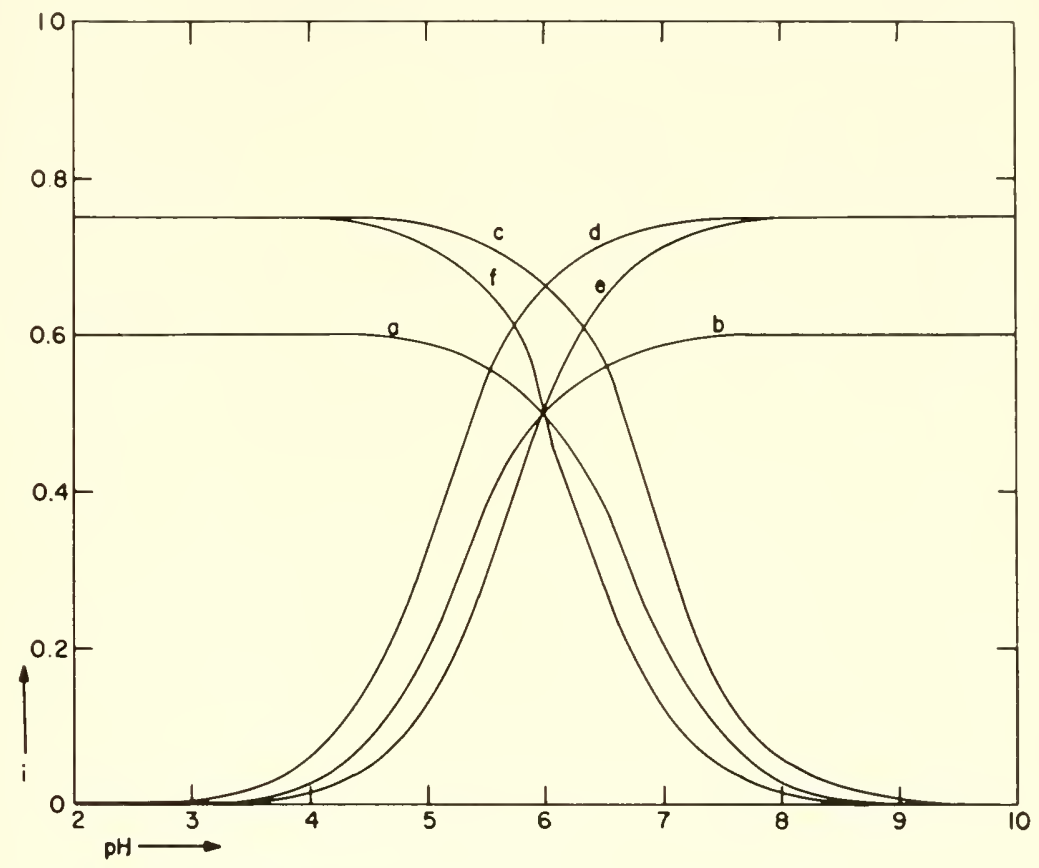

Frg. 14-7. Variation of the inhibition with the $p H$ for the six possible situations in which only the enzyme active center ionizes (case IV). The letters on the curves correspond to those in the text. $K_{i}=1 \mathrm{~m} . \mu, K_{s}=10$ $\mathrm{m} . H, K_{a}=10^{-6} .(\mathrm{I})=3 \mathrm{~m} . H$. and $(\mathrm{S})=10 \mathrm{~m} . H$.

marked $\mathrm{pH}$ dependence may be seen in the region extending about $2 \mathrm{pH}$ mits on either side of the $\boldsymbol{H}_{\text {n }}$ for the enzyme. Figure 14-8 shows the $\mathrm{pH}$ effects on the inhibition at different substrate concentrations for system (a), i.e. competitive inhibition with $\mathrm{HE}$, the active from of the enzyme. Here it may be observed that the $\mathrm{pH}$ region over which the inhibition is independent of $\mathrm{pH}$ varies with (S). This means that the greater the enzyme 
fraction in the HES form, the less able is a rise in $\mathrm{pH}$ by dissociating $\mathrm{HE}$ to form the inactive $\mathrm{E}$ with which the inhibitor does not combine. Finally, it is interesting to see how the noncompetitive inhibitions (c)-(f) vary with (S) and this is shown in Fig. 14-9 for type (c). Type (d) would have the pH values on the curves reversed; type (e) would differ from this in that the inhibition would decrease with rising (S); and type (f) would be like type (e) except that the $\mathrm{pH}$ values would be as in the figure. The figure shows very well how a rise in (S) actually increases the inhibition when there is noncompetitive inhibition on the active form of the enzyme.

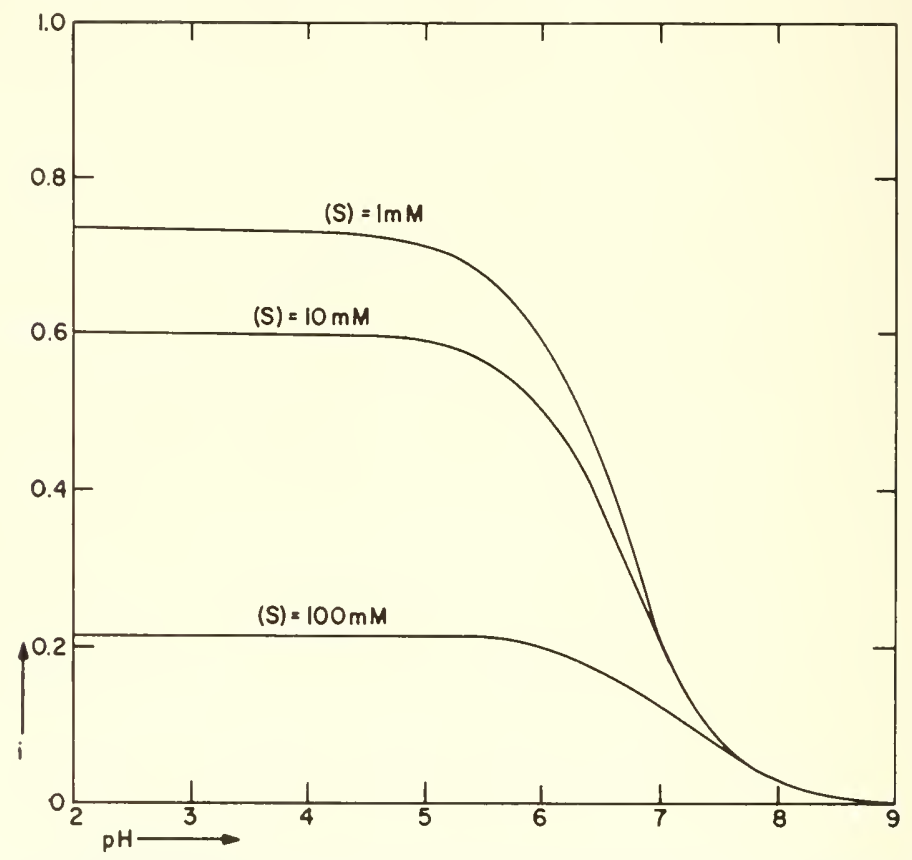

Fig. 14-8. Variation of the inhibition with the $\mathrm{pH}$ at different concentrations of substrate for case IV $a(\mathrm{Eq} .14-55) . K_{i}=1 \mathrm{~m} M$, $K_{s}=10 \mathrm{~m} .1 /, K_{a}=10^{-6}$, and $(\mathrm{I})=3 \mathrm{~m} . \mu$.

Some problems are presented by these systems in the determination of the inhilitor constant by the usual graphical methods and these will be considered after several more inhibition systems have been presented.

A question may be raised as to why in the above formulations no consideration was taken of the ionization of the complexes HES or HEI. It was assumed that $\mathrm{S}$ or I combined only with $\mathrm{HE}$ in these cases and not with $\mathrm{E}$. Thus the dissociation of a proton from the HES or HEI complexes would produce ES or EI, which camnot exist, sinee if EI can arise by dissociation 
of HEI it can also be formed from $\mathrm{E}$ and I. Of course, when I can combine with both $\mathrm{E}$ and $\mathrm{HE}$. a scheme such as:

$$
\begin{aligned}
& \gtrless \mathrm{HES} \rightarrow \mathrm{HE}+\mathrm{P} \\
\mathrm{E} & \searrow \mathrm{HEI} \\
\searrow \mathrm{EI} & \gtrless
\end{aligned}
$$

must be set up. If I combines with both forms equally, there is no effect of the $\mathrm{pH}$ on the inhibition; if the affinities of $\mathrm{E}$ and $\mathrm{HE}$ for I differ. the system will behave in an intermediate fashion between a $\mathrm{pH}$-independent system and the systems discussed above. If the inhibitor is a charged ion, a marked difference in binding to $\mathrm{E}$ and $\mathrm{HE}$ will be the most likely.

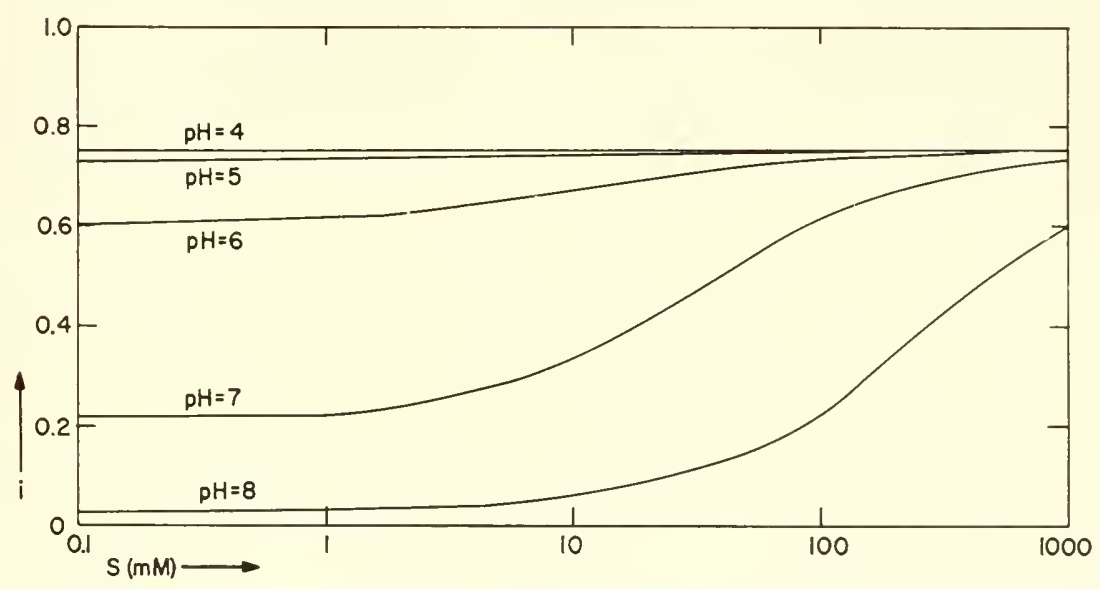

FIG. 14-9. Variation of the inhibition with the substrate concentration at different pH's for case IVc (Eq. 14-61). $K_{i}=1 \mathrm{~m} .1 . K_{s}=10 \mathrm{~m} . \mathrm{H}, K_{a}=10^{-6}$, and (I) $=3 \mathrm{~m} . H$.

Some further discussion must be given to terminology in systems such as 14-71. This reaction scheme is equivalent to:

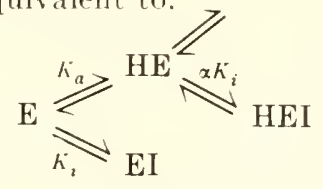

which is usually described by stating that the inhibitor can combine with both ionization forms of the enzyme. It is also equivalent to:

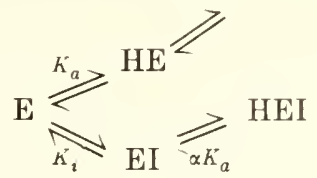


which is usually described in terms of the ionization of the enzyme-inhibitor complex. In all cases, the concentration of HE, and hence of HES, is the same, however the situation is described.

Case V: the enzyme and the inhibitor and/or the substrate ionize. These more complex sitnations actually do not present any problems. If the inhibitor ionizes, the apparent $K^{\prime \prime}$ 's in the equations for the six types of inhibition must be replaced with $K_{i}$ multiplied by the appropriate $\mathrm{pH}$ functions. Likewise, the ionization of the substrate requires only the application of its functions to $K_{s}$. It may only be noted that when both the enzyme and the inhibitor ionize, the possibility arises that there will be a maximum in the $i$-pH eurve, such as has been discussed for uninhibited enzymes in the $v$ - $\mathrm{pH}$ curves. The $\mathrm{pH}_{o p t}$ for the inhibition will not necessarily lie midway between the $\mathrm{p} K_{a}$ 's of the enzyme and inhibitor.

Case VI: the enzyme exhibits a dibasic ionization. This also presents no trouble since only the dibasic pH functions, $f^{\prime \prime}$, for the enzyme have to be substituted in the equations of the type derived under case IV. For example, in an extension of scheme 14-52:

$$
\begin{aligned}
& \mathrm{HES} \rightarrow \mathrm{HE}+\mathrm{P} \\
& K_{a}{ }^{\prime \prime} K_{s} 1 \downarrow \quad K_{a}^{\prime} \\
& \mathrm{E} \rightleftharpoons \underset{K_{i}^{*} 1 \downarrow}{\mathrm{HE}} \rightleftharpoons \mathrm{H}_{2} \mathrm{E} \\
& \text { HEI }
\end{aligned}
$$

to include $\mathrm{H}_{2} \mathrm{E}$, where the inhibitor combines only with IIE. the catalytically active form of the enzyme, the inhibition is given by:

$$
i=\frac{(\mathrm{I})}{(\mathrm{I})+K_{i}\left[f_{h e}{ }^{\prime \prime}+\frac{(\mathrm{S})}{K_{s}}\right]}
$$

which is comparable to Eq. 14-55. The apparent inhibitor constant is given by:

$$
K_{i}{ }^{\prime}=K_{i}\left[\frac{f_{h e}{ }^{\prime \prime} K_{s}+(\mathrm{S})}{K_{s}+(\mathrm{S})}\right]
$$

When the $\mathrm{pH}$ lies between ${ }^{\prime} K_{n}^{\prime}$ and $\mathrm{p} K_{q}{ }^{\prime \prime}$ (assuming they are sufficiently far apart), $K_{i}^{\prime}=K_{i}$ since here $f_{\text {he }}{ }^{\prime \prime} \cong 1$, but on either side of this range, the apparent inhibitor constant will be dependent on $\mathrm{pH}$.

These expressions differ from those given by Alberty (1954), although the formulations of the reactions wonld appear on the surface to be the same. Actually, the systems are quite different. Alberty assumed that the HEI complex conld lose or gain protons, which means that EI and 
$\mathrm{H}_{2} \mathrm{EI}$ can exist, which in turn implies that the reactions $\mathrm{E}+\mathrm{I} \rightleftharpoons \mathrm{EI}$ and $\mathrm{H}_{2} \mathrm{E}+\mathrm{I} \rightleftharpoons \mathrm{H}_{2} \mathrm{EI}$ can occur. The dissociation constant for EI would then, in his system, be given by $K_{i}\left(K_{a_{e i}}^{\prime \prime} / K_{a_{e}}{ }^{\prime \prime}\right)$ and for $\mathrm{H}_{2} \mathrm{EI}$ a related expression would hold. In a system such as assumed by Alberty, the apparent inhibitor constant would be:

$$
K_{i}{ }^{\prime}=K_{i} \frac{f_{h e}{ }^{\prime \prime}}{f_{h e i}{ }^{\prime \prime}}
$$

where $K$, refers to the equilibrium constant for the reaction $\mathrm{HE}+\mathrm{I} \rightleftharpoons \mathrm{HEI}$.

It is quite possible to assume generalized systems wherein all the possible reactions between the components occur. For example, when the enzyme is monobasic and only HES forms the product, it may be useful to set up some such system as the following if one is interested in determining the effects of one component on the interaction of another with the enzyme:

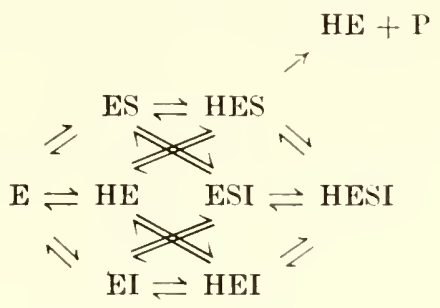

where the equilibrium constants are related through factors expressing the degree of interaction between the bound components. In fact, this system is identical to Eq. 3-57 with the activator replaced by the hydrogen ion, and similar equations would be applicable. Even this system, omitting any ionization of the inhibitor or substrate, presents difficulties in the interpretation of experimental data. A complete system in which the enzyme, substrate. and inhibitor all are dibasic, and all combinations with the enzyme are possible, would comprise forty-two forms of the enzyme and its complexes. Such a system would be the inhibition by malonate of succinic dehydrogenase. However. only a relatively few complexes are actmally possible in most cases; in the inhibition of succinic dehydrogenase, it is likely that the only significant complexes occur between $\mathrm{H}_{2} \mathrm{E}^{++}$and $\mathrm{S}^{=}$ and $I^{=}$. For these reasons the generalized formulations are, at least at present, impractical.

\section{The Determination of $\mathrm{K}_{i}$}

One of the primary purposes of inhibition studies is the determination of the true dissociation constant of the EI complex. It is by means of the values of $K_{i}$ that different inhibitors may be compared or the energies of 
interaction of the inhibitors with the enzyme calculated; it is by the relationship between $K_{i}$ and the apparent inhibitor constant, $K_{i}{ }^{\prime}$, that the $\mathrm{p} K_{a}$ 's of the ionizing groups on the enzyme may be determined with Dixon's treatment. The experimental $\boldsymbol{K}_{i}^{\prime}$ found by the usual graphical methods will generally not be the true $K_{i}$ if ionization of any of the involved components oceurs. It is therefore particularly important to look critically into the methods for the determination of both $K_{i}{ }^{\prime}$ and $K_{i}$.

In the simplest situation, $K_{i}^{\prime}$ differs from $K_{i}$ because only a fraction of the total inhibitor present is active. These fractions are expressed in terms of the $\mathrm{pH}$ functions presented earlier in this chapter. If, for example, the inhibitor exists in the forms I and HI, and only I binds to the enzyme, the true inhibitor constant will be given by:

$$
K_{i}=K_{i}{ }^{\prime} \mid f_{i}{ }^{\prime}
$$

Thus, from the $\mathrm{p} \boldsymbol{K}_{a}$ of the inhibitor and the $\mathrm{pH}$ at which the experiment is rum, it is possible to determine $K_{i}$ after $K_{i}^{\prime}$ has been found. However, when the enzyme also ionizes over the $\mathrm{pH}$ range investigated, the calculation of $K$, may be more complex.

The first matter that must be cxamined is the applicability of the plotting methods described in Chapter 5 to situations in which ionizing gromps are involved in the inhibition. For this purpose we may consider some of the cases presented in the preceding section.

Cases I, II. and III: only the substrate or the inhibitor or both iomize. The apparent inhihitor constant determined by every method of graphical analysis will be $K_{i}$ multiplied by the anpromiate $\mathrm{pH}$ function or functions, as indicated in the expressions for the inhibition (Eqs. 14-47, 14-49, 14-50, 14-51) for each situation. The only eomplication arises in seheme 14-48 where both forms of the inhibitor combine with the enzyme but with different affinities, since here:

$$
K_{i}=\left[\frac{1}{f_{h i}{ }^{\prime}}+\frac{1}{\mu f_{i}^{\prime}}\right] K_{i}{ }^{\prime}
$$

If this type of inlibition is recognized, the value of $\mu$ ma y be estimated from data obtained at different $p H^{\prime}$, and hence $K_{i}$ may he ralculated.

Case $I \mathrm{~T}^{\mathrm{T}}$ : only the enzyme ionizes. Six different situations were discussed for an enzyme with a monobasie ionizing groul at the active center. Six methods of graphieal analysis for the determination of the constants were presented in Chapter 5. It is important to know in each ease how the determined constant relates to $K_{i}$. We shall consider here only three methods of plotting: $1 / v_{i}$ against $1 /(\mathrm{S})$ (type A), $1 / v_{i}$ against (I) (type D), and (I) $(1-i) / i$ against $(\mathrm{S})$ (type $\mathrm{F}$ ). The results obtained by the other methods 
of plotting may be readily obtained in a similar way. We shall write the appropriate equations for each of the six types of inhibition.

(a) Competitive inhibition on the active form HE (scheme 14-52)

$$
\begin{aligned}
& 1 / v_{i}-1 /(\mathrm{S}): \quad \frac{1}{v_{i}}=\frac{1}{V_{m}}+\frac{K_{s}}{V_{m}(\mathrm{~S})}\left[1+\frac{(\mathrm{I})}{K_{i}}+\frac{K_{a}}{(\mathrm{H})}\right] \\
& 1 / v_{i}-(\mathrm{I}): \quad \frac{1}{v_{i}}=\frac{1}{V_{m}}\left[1+\frac{K_{s}}{(\mathrm{~S})}\left(1+f_{h e}{ }^{\prime}\right)\right]+\frac{K_{s}(\mathrm{I})}{V_{m} K_{i}(\mathrm{~S})} \\
& \begin{array}{l}
(\mathrm{I})(1-i) / i-(\mathrm{S}): \\
\frac{(\mathrm{I})(1-i)}{i}
\end{array}=f_{h e}{ }^{\prime} K_{i}+\frac{K_{i}(\mathrm{~S})}{K_{s}}
\end{aligned}
$$

Comparing these equations with those obtained for the nonionizing case (see Eqs. 5-1, 5-5, and 5-7), it is seen that a type A plot will have the same intercept on the $1 / c_{i}$ axis but the slope will be greater due to the term $K_{a} /$ $(H)$. If $K_{i}$ is calculated from the slope $(s)$, the following expressions are obtained:

$$
\begin{array}{ll}
\text { Nonionizing: } & K_{i}=\frac{K_{s}(\mathrm{I})}{s V_{m}-K_{s}} \\
\text { Ionizing: } & K_{i}=\frac{K_{s}(\mathrm{I})}{s V_{m}-f_{h e} K_{s}}
\end{array}
$$

The calculation of $K_{i}$ will thus be in error at pH's near or above the $p K_{a}$ if the ionization is not considered. A plot of type $D$, on the other hand, gives a different intercept but the same slope as the nonionizing case. The calculation of $K_{i}$ from the slope will not be in error since the slope doesn't depend on the pH. A type $\mathrm{F}$ plot will give an intercept of $f_{h e} K_{i}$ and a slope of $K_{i} / K_{s}$ so that a calculation of $K_{i}$ from the intercept, which is usual, must take the ionization into account.

(b) Competitive inhibition on the active form $E$ (scheme 14-56)

$$
\begin{aligned}
& 1 / v_{i}-1 /(\mathrm{S}): \quad \frac{1}{v_{i}}=\frac{1}{V_{m}}+\frac{K_{s}}{V_{m}(\mathrm{~S})}\left[1+\frac{(\mathrm{I})}{K_{i}}+\frac{(\mathrm{H})}{K_{a}}\right] \\
& 1 / v_{i}-(\mathrm{I}): \quad \frac{1}{v_{\imath}}=\frac{1}{V_{m}}\left[1+\frac{K_{s}}{(\mathrm{~S})}\left(1+f_{e}{ }^{\prime}\right)\right]+\frac{K_{s}(\mathrm{I})}{V_{m} K_{i}(\mathrm{~S})} \\
& \begin{array}{l}
(\mathrm{I})(1-i) / i-(\mathrm{S}): \\
i
\end{array} \quad \frac{(\mathrm{I})(1-i)}{f_{e}{ }^{\prime} K_{i}+\frac{K_{i}(\mathrm{~S})}{K_{s}}}
\end{aligned}
$$

What has been said above applies equally here and the only difference is the replacement of $f_{h e}{ }^{\prime}$ by $f_{\ell}^{\prime}$. 
(c) Noncompetitive inhibition on the active form HE (scheme 14-59)

$$
\begin{aligned}
& 1 / v_{i}-1 /(\mathrm{S}): \quad \frac{1}{v_{i}}=\frac{1}{V_{m}}\left[1+\frac{(\mathrm{I})}{K_{i}}\right]+\frac{K_{s}}{V_{m}(\mathrm{~S})}\left[f_{h e^{\prime}}+\frac{(\mathrm{I})}{K_{i}^{-}}\right] \\
& 1 / v_{i}-(\mathrm{I}): \frac{1}{v_{i}}=\frac{1}{V_{m}}\left[1+\frac{K_{s}}{(\mathrm{~S})}\left(1+f_{h e^{\prime}}\right)\right]+\frac{(\mathrm{I})}{V_{m} K_{i}}\left[1+\frac{K_{s}}{(\mathrm{~S})}\right](14-90) \\
& (\mathrm{I})(1-i) / i-(\mathrm{S}): \quad \frac{(\mathrm{I})(1-i)}{i}=K_{i}+\frac{K_{i} K_{a} K_{s}}{(\mathrm{H})\left[(\mathrm{S})+K_{s}\right]}
\end{aligned}
$$

In a plot of type $A$, as in $(a)$ above, the slope depends on the $\mathrm{pH}$ whereas the intercept does not. If $K_{i}$ is determined from the intercept, rather than from the slope, no $\mathrm{pH}$ correction need be mate. The slope of a plot of type $\mathrm{D}$, however, is the same as for the nonionizing case, and depends on $(\mathrm{S})$ and not $\mathrm{pH}$. The higher $(\mathrm{S})$, the greater the deviation from the competitive slope, but no problem is involved in the calculation of $K_{i}$. The type $\mathrm{F}$ plot will be quite different in configuration, compared to the nonionizing case, because ( $S$ ) here occurs in the denominator. but the intercept gives the true $K_{i}$ and does not depend on the $\mathrm{pH}$.

(d) Noncompetitive inhibition on the active form $E$ (scheme 14-62)

$$
\begin{aligned}
& 1 / v_{i}-1 /(\mathrm{S}): \quad \frac{1}{v_{i}}=\frac{1}{V_{m}}\left[1+\frac{(\mathrm{I})}{K_{i}}\right]+\frac{K_{s}}{V_{m}(\mathrm{~S})}\left[f_{e}^{\prime}+\frac{(\mathrm{I})}{K_{i}}\right] \\
& 1 / v_{i}-(\mathrm{I}): \frac{1}{v_{i}}=\frac{1}{V_{m}}\left[1+\frac{K_{s}}{(\mathrm{~S})}\left(1+f_{e}^{\prime}\right)\right]+\frac{(\mathrm{I})}{V_{m} K_{i}}\left[1+\frac{K_{s}}{(\mathrm{~S})}\right] \\
& (\mathrm{I})(1-i) / i-(\mathrm{S}): \quad \frac{(\mathrm{I})(1-i)}{i}=K_{i}+\frac{K_{i} K_{s}(\mathrm{H})}{K_{a}\left[(\mathrm{~S})+K_{s}\right]}
\end{aligned}
$$

The equations here are the same as in $(c)$ except for the reverse dependence on $\mathrm{pH}$ and the calculation of $K_{i}$ presents the same problems.

(e) Noncompetitive inhibition on the inactive form E (scheme 14-65)

$$
\begin{array}{ll}
1 / v_{i}-1 /(\mathrm{S}): & \frac{1}{v_{i}}=\frac{1}{V_{m}}+\frac{K_{s}}{V_{m}(\mathrm{~S})}\left[f_{h e}{ }^{\prime}+\frac{K_{a}(\mathrm{I})}{K_{i}(\mathrm{H})}\right] \\
1 / v_{i}-(\mathrm{I}): & \frac{1}{v_{i}}=-\frac{1}{V_{m}}\left[1+f_{h e}{ }^{\prime} \frac{K_{s}}{(\mathrm{~S})}\right]+\frac{K_{s} K_{a}(\mathrm{I})}{V_{m} K_{i}(\mathrm{~S})(\mathrm{H})} \\
(\mathrm{I})(1-i) / i-(\mathrm{S}): & \frac{(\mathrm{I})(1-i)}{i}=f_{e} K_{i}+\frac{K_{i}(\mathrm{H})(\mathrm{S})}{K_{a} K_{s}}
\end{array}
$$


If $K_{i}$ is calculated from the slope of a type A plot, it will now be given by:

$$
K_{i}=\frac{K_{a}}{(\mathrm{H})} \frac{K_{s}(\mathrm{I})}{s V_{m}-f_{h e} K_{s}^{r}}
$$

which may be compared with 14-84 and 14-85. The slope of a type D plot is also altered by the factor $K_{\alpha}^{\prime} /(\mathrm{H})$. The type $\mathrm{F}$ plot gives $f_{e}^{\prime} K_{i}$ as the intercept and the slope again depends on the $\mathrm{pH}$.

(f) Noncompetitive inhibition on the inactive form $H E$ (scheme 14-68)

$$
\begin{array}{ll}
1 / v_{i}-1 /(\mathrm{S}): & \frac{1}{v_{i}}=\frac{1}{V_{m}}+\frac{K_{s}}{V_{m}(\mathrm{~S})}\left[f_{e}^{\prime}+\frac{(\mathrm{H})(\mathrm{I})}{K_{a} K_{i}}\right] \\
1 / v_{i}-(\mathrm{I}): & \frac{1}{v_{i}}=\frac{1}{V_{m}}\left[1+f_{e}^{\prime} \frac{K_{s}}{(\mathrm{~S})}\right]+\frac{K_{s}(\mathrm{H})(\mathrm{I})}{V_{m} K_{i} K_{a}(\mathrm{~S})} \\
(\mathrm{I})(1-i) / i-(\mathrm{S}): & \frac{(\mathrm{I})(1-i)}{i}=f_{h e}{ }^{\prime} K_{i}+\frac{K_{i} K_{a}(\mathrm{~S})}{K_{s}(\mathrm{H})}
\end{array}
$$

These equations are similar again to those in the previous section (e) with the $\mathrm{pH}$ dependence changed.

These examples well show that the calculation of $K$, for inhibitions on ionizing enzymes will sometimes be straightforward (i.e., as in the nonionizing cases), but in others it will be more complex and will involve terms in $(\mathrm{H})$ or $(\mathrm{S})$. If we define the apparent inhibitor constant, $K_{i}$. as that which would be derived in the graphical methods based on the assumption that no ionization occurs, the relationship between $K_{i}^{\prime}$ and $K_{i}$ will vary with the type of inhibition and the method of plotting. In the literature it has been generally assumed that $K_{i}{ }^{\prime}=f_{e}{ }^{\prime} K_{\text {}}$ or $K_{i}{ }^{\prime}=f_{h e}{ }^{\prime} K_{i}$ when the enzyme ionizes monobasically, but this is not necessarily true. or will be true only over a certain range of $\mathrm{pH}$ or $(\mathrm{S})$.

Case V: enzyme and inhibitor and/or substrate ionize. It can be easily seen, by inspection of the equations given above for the different plots, that in every case in which the inhibitor ionizes. one needs only to multiply $K_{i}$ by the proper $\mathrm{pH}$ function for the inhibitor. Thus, if one wishes to calculate $K_{i}$ for a competitive inhibition on the active form $\mathrm{HE}$ from the intercept of a type $\mathrm{F}$ plot, and only the I form of the inluibitor is effective, $K_{i}=K_{i}^{\prime} / f_{h c}^{\prime} f_{i}^{\prime}$. If the substrate ionizes and its concentration enters into the expression for $K_{i}^{\prime}$. then the $\mathrm{pH}$ functions for the substrate must also be included. For example, the slope of a type $\mathrm{D}$ plot of competitive inhibition is $K_{s} / V_{m} K_{l}(\mathrm{~S})$, but if only one ionization form of the substrate is active, its concentration is not (S) but (S) $/ f_{s}$, so that $K_{i}^{\prime}=f_{s} K_{i}$, or, if the inhibitor 
also ionizes, $K_{i}{ }^{\prime}=f_{s} f_{i} K_{i}$. In most methods of plotting, however, the $\mathrm{pH}$ functions for the substrate need not be considered.

These ilhstrations emphasize that the calculation of $K_{i}$ is not as simple as has often been assumed when the active center possesses one or more ionizing groups, and that the apparent inhibitor constant is not always directly related to the true constant through simple $\mathrm{pH}$ functions.

\section{Ionization of Enzyme-Inhibitor Complexes}

It has been assumed in most of the inhibitions previonsly discussed that only one form of the enzyme binds the inhibitor and therefore that only one enzyme-inhibitor complex occurs. The ionization of this complex in such cases does not enter into the formulations. Before applying the Dixon treatment to inhibition it will be necessary to determine under what conditions ionization of the enzyme-inhibitor complex is important and how it may modify the expressions for rates and inhibitions in the preceding sections. When two substances form a complex, ionizing groups on either substance may be modified and this will be expressed as a shift in the $\mathrm{p} K_{a}$ 's of these groups. Most of these effects arise from changes in the electric field surrounding the ionizing group as a result of the binding of the other substance. Thus the binding of an ionic inhibitor to the active center will superimpose the electric field of the inhilitor onto that of the enzyme; any ionizing groups at the active center or in the vicinity will be modified as a consequence. Thus the $\mathrm{p} \boldsymbol{K}_{z}$ 's of the enzyme-inhibitor complexes are often different from the $\mathrm{pK}_{2}$ 's of the free enzyme or the inhibitor. If the inhibitor is uncharged, the effect upon the $\mathrm{p} K_{a}$ of an enzyme group may be very small, but if the inhibitor is an ion, the enzyme $\mathrm{p} K_{a}$ may be shifted as much as one $\mathrm{pH}$ unit or more.

The two types of competitive inhibition on a monobasic enzyme will be treated to illustrate how the ionization of the enzyme-inhibitor complex may be incorporated into the inhibition equations. If $\mathrm{HE}$ is the catalytically active form of the enzyme:

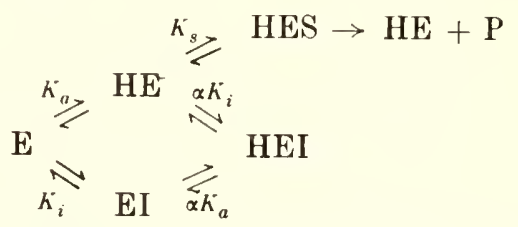

where $\alpha$ indicates the change in $K_{a}$ when the inhibitor is bound to the enzyme. The inhibition is now given by:

$$
i=\frac{(\mathrm{I})}{(\mathrm{I})+\frac{a K_{i}}{f_{h e i}{ }^{\prime}}\left[f_{h e^{\prime}}+\frac{(\mathrm{S})}{K_{s}}\right]}
$$


This is identical with Eq. 14-55 except for the new function $f^{\prime}$ hei, since $\alpha K_{i}$ is equivalent to the $K_{i}$ in the former equation. If $\alpha=1$ and the binding of the inhibitor has no effect on the enzyme $\mathrm{p} K_{\mu}$ :

$$
i=\frac{(\mathrm{I})}{(\mathrm{I})+K_{i}\left[1+\frac{(\mathrm{S})}{f_{h e}^{\prime} K_{s}}\right]}
$$

The inhibition is always somewhat greater when HEI can dissociate to form EI because this will reduce further the concentration of the active complex. HES. If $\mathrm{E}$ is the catalytically active form of the enzyme:

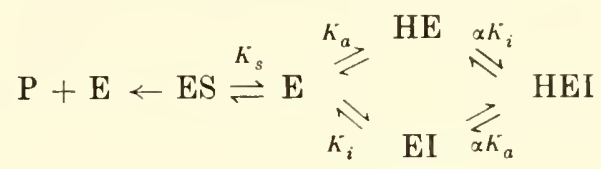

the inhibition is given by:

$$
i=\frac{(\mathrm{I})}{(\mathrm{I})+\frac{K_{i}}{f_{e i}^{\prime}}\left[f_{e^{\prime}}+\frac{(\mathrm{S})}{K_{s}}\right]}
$$

so that again the trme inhibitor constant is divided by the $\mathrm{pH}$ function for the enzyme-inhibitor complex. It should be noted that the inhibitor constant that is applicable is that expressing the binding of the inhibitor to the active form of the enzyme. When $\alpha=1$, a similar equation to 14-104 is obtained with $f_{e}{ }^{\prime}$ replacing $f_{h e}{ }^{\prime}$. In both of the cases given, when $\alpha=1$ the ionization of HEI is not apparent in the inhibition equations as a $\mathrm{pH}$ function for this complex, but nevertheless it increases the inhibition or reduces the apparent inhibitor constant. If the enzyme is dibasic, the proper $f^{\prime \prime}$ functions can be used.

\section{Application of Dixon's Treatment to the Variation of $\mathrm{K}_{i}$ with $\mathrm{pH}$}

Dixon (1953 a) implies that the binding of the inhibitor may be treated analogously to the binding of the substrate and. therefore, that one can write for the general case:

$$
K_{i}=\frac{\left(\mathrm{E}_{t}\right)\left(\mathrm{I}_{t}\right)}{\left(\mathrm{EI}_{t}\right)}=\frac{f_{e}(\mathrm{E}) f_{i}(\mathrm{I})}{f_{e i}(\mathrm{EI})}=\frac{f_{e} f_{i}}{f_{e i}} K_{i}
$$

in conformity with Eq. 14-41. Likewise, we may write:

or

$$
\mathrm{p} K_{i}{ }^{\prime}=\mathrm{p} K_{i}-\log f_{e}-\log f_{i}+\log f_{e i}
$$

$$
\mathrm{p} K_{i}^{\prime}=\mathrm{p} K_{i}+\mathrm{p} f_{e}+\mathrm{p} f_{i}-\mathrm{p} f_{e i}
$$


so that a plot of $\mathrm{p} K_{i}{ }^{\prime}$ against $\mathrm{pH}$ would provide curves, the slopes and inflections of which would lead to information on the ionizing groups.

However, it has been made elear that the experimentally determined $K_{i}^{\prime}$ is not always related to $K_{i}$ in this simple way. Since the $\mathrm{p} K_{a}$ 's of substrates and inhibitors are generally known, or can be determined more readily by other means, the Dixon method is usually designed to provide information on the ionizing groups at the active site, either when the enzyme in free on when it is complexed with substrate or inhibitor. It is particularly in these cases in which the enzyme ionizes, that $K_{i}{ }^{\prime}$ is often not completely given by equations of the type 14-107. One difficulty is that $K_{i}^{\prime}$ can mean different things depending on what graphical method is used for its determination. To take a simple example, if one determined $K_{i}{ }^{\prime}$ from the intercept of a type $\mathrm{F}$ plot (where (I) $(1-i) / i$ is plotted against (S)), for cases of noncompetitive inhibition on the active form of the enzyme, one would obtain the tme $K_{i}$ (Eqs. 14-91 and 14-94); if this were plotted against $\mathrm{pH}$ the slope would be zero and there would be no inflections, so that a lack of ionizing groups on the enzyme might be deduced. If the inhibition were on the inactive form of the enzyme, $f_{e}^{\prime} \boldsymbol{K}_{i}$ or $f_{h e}{ }^{\prime} \boldsymbol{K}_{i}$ would be obtained, and a plot of $p K_{i}{ }^{\prime}$ against $\mathrm{pH}$ would provide information on the ionization constant of the enzyme. One must conclude that the equations for $K_{i}{ }^{\prime}$ and $\mathrm{p} K_{i}^{\prime}$ given above are not generally eorreet. The expressions for $K_{i}^{\prime}$ in terms of $K$, are given in Table 14-1 for most of the inhibitions discussed in this ehapter; the relationship is seldom as simple as in Eq. 14-107.

The types of eurves obtained by plotting $\mathrm{p} K_{i}{ }^{\prime}$ against $\mathrm{pH}$ and the interpretation of such curves must now be examined. Only certain types of inhibition will be discussed to illustrate the problems involved. Actually, the situation is not quite as bad as it would appear from inspection of Table 14-1 sinee even the more complieated expressions for $K_{i}{ }^{\prime}$ generally plot out in a simple manner. The real difficulty lies in the interpretation of the inflections. Nost of the possible expressions for $K_{i}{ }^{\prime}$, as found in Table 14-1, are plotted as $\mathrm{p} K_{i}{ }^{\prime}-\mathrm{pH}$ curves in Fig. 14-10. The fundamental meanings attached to the slopes and inflections are just as valic as in Dixon's rules, e.g., each inflection indieates the $p K_{a}$ of some group, but there are three complications unforeseen in the earlier work.

In the first place, some curves do not show any inflection despite the operation of an ionizing group. This would be true for those expressions for $K_{i}{ }^{\prime}$ in Table 14-1 that do not involve any $\mathrm{pH}$ or $\mathrm{pH}$ functions (and which are not plotted beeanse they are simply straight horizontal lines) but it is also true for inhibition on the inactive form of the enzyme when $K_{i}{ }^{\prime}$ is determined from plots of type A or D (numbers 9, 13, and 14).

In the second place, the directions of the inflections do not correlate with the location of the ionizing group, as specified in Dixon's rules for $\mathrm{p} K_{s}{ }^{\prime}-\mathrm{pH}$ plots. An inflection that is coneave upwards in the $\mathrm{p} K_{s}^{\prime}-\mathrm{pH}$ 
curves indicates an ionizing group on the enzyme-substrate complex. In the $\mathrm{p}_{i}{ }^{\prime}-\mathrm{pH}$ plots this is not necessarily so: ionization of the enzymeinhibitor complex does give an inflection that is concave upwards (numbers $3,4,15$, and 16) but other situations (numbers 5, 6, 7, 8, 10, 11, and 12) also give rise to such inflections. Therefore, an inflection that is concave

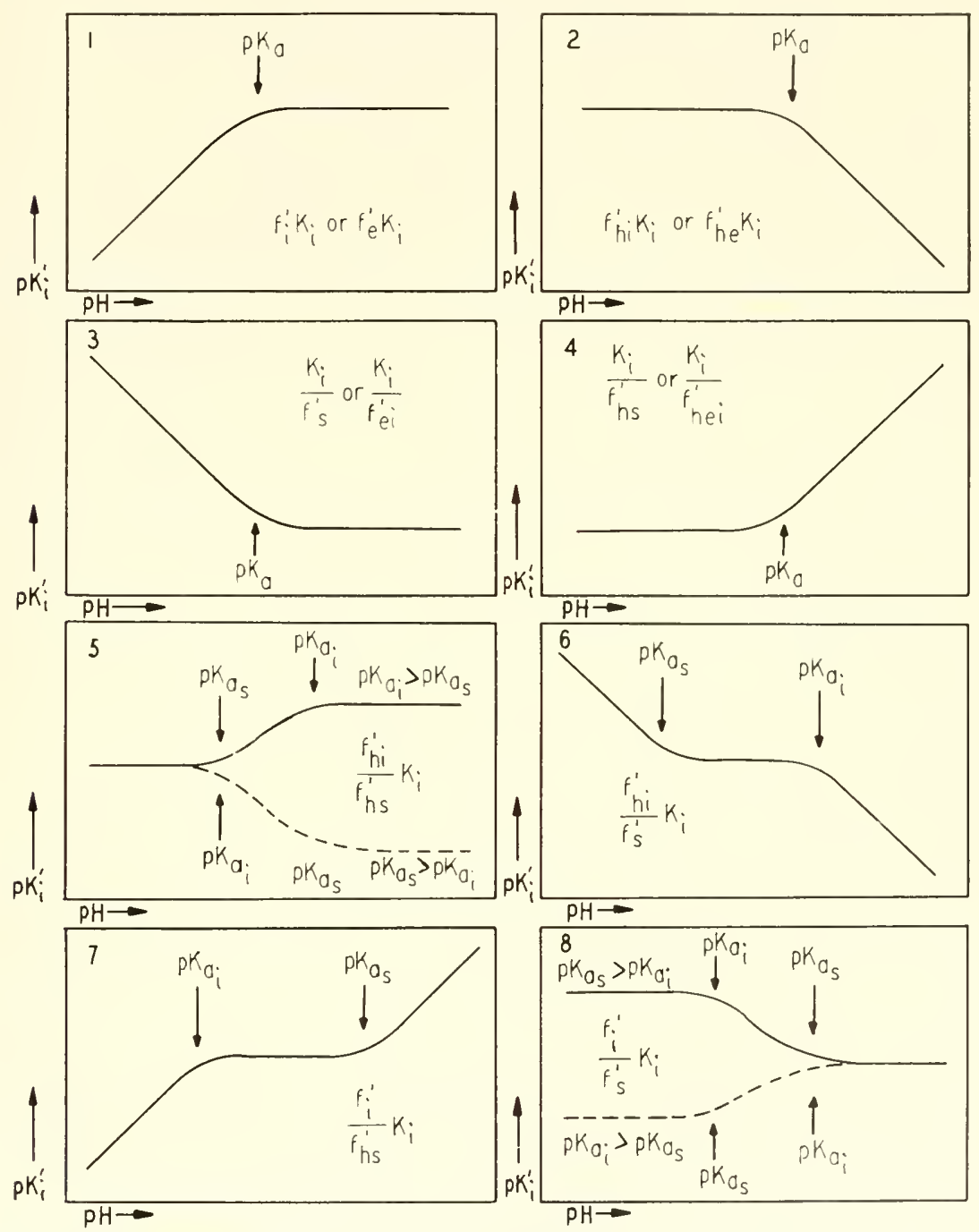

FIG. 14-10. The $\mathrm{p} K_{i}^{\prime}-\mathrm{pH}$ plots for the $K_{i}^{\prime}$ values shown in Table 14-1 to illustrate the interpretation of the inflections when $K_{i}{ }^{\prime}$ is determined from three different types of plotting procedures. 
upwards cannot, in inhibition analysis, be immediately attributed to an jonization of some enzyme-inhibitor complex.

In the thind place, in some types of inhibition, inflections may be attrib-
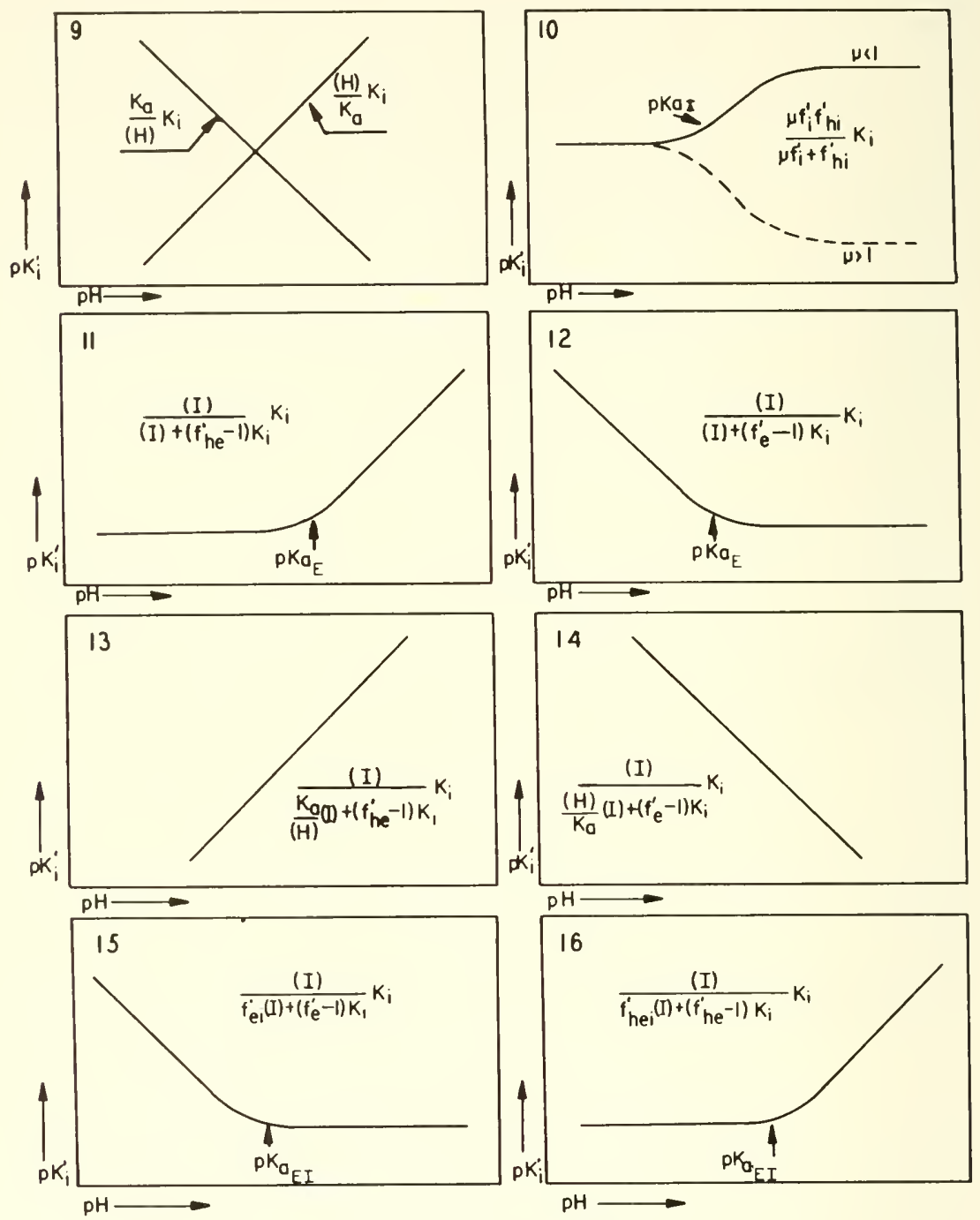

FIG. 14-10 (continued).

utable to ionization of the substrate (number 3 to 8 ) as well as to other components of the system. Therefore, even though the data are derived from inhibition studies and $\mathrm{p} K_{i}{ }^{\prime}$ is plotted, all the inflections observed 
are not necessarily related to ionizations of the enzyme, inhibitor, or the complex between them.

It is usually difficult, if not impossible, to determine the type of inhibition or to locate the ionizing groups from the $\mathrm{p} K_{i}{ }^{\prime}-\mathrm{pH}$ curves alone, since the curves for quite different systems may look alike. It is necessary to elucidate the nature of the $\mathrm{pH}$ dependence of the unimhibited reaction and
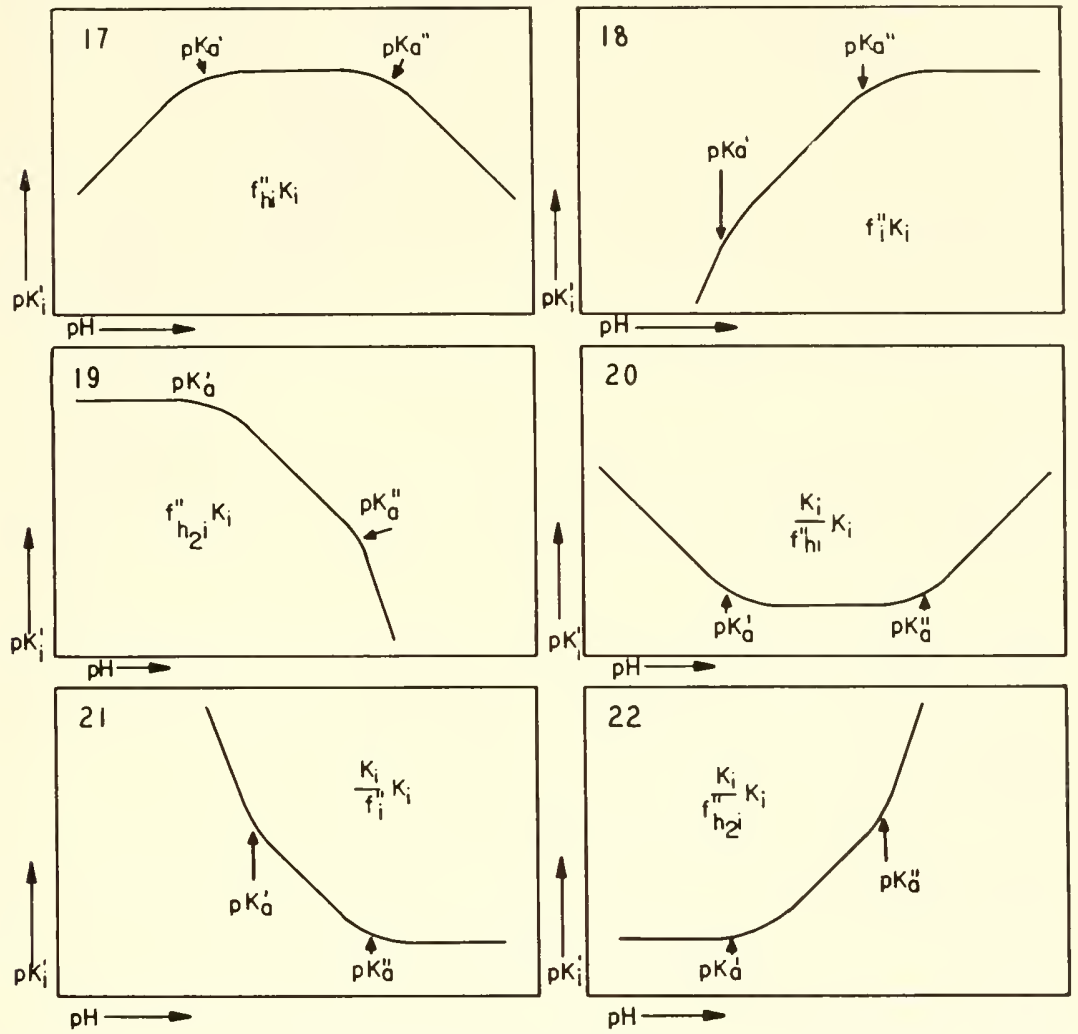

Fig. 14-10 (continued)

the basic mechanism of the inhibition before these curves can be interpreted and the ionizing groups specified. This is not to minimize the importance of the Dixon treatment, which is a very valuable technique when it is applied properly, but only to point out some of the complications inherent in its use in inhibition studies.

Some illustrations of how the curves in Fig. 14-10 are derived from the expressions for $K_{i}^{\prime}$ in Table 14-1 may be given. The simple case in which 
TABLE 14-1

Expressions for $K_{i}{ }^{\prime}$ In Terus of $K_{i}$ for Various Types of Inhibition ${ }^{a}$

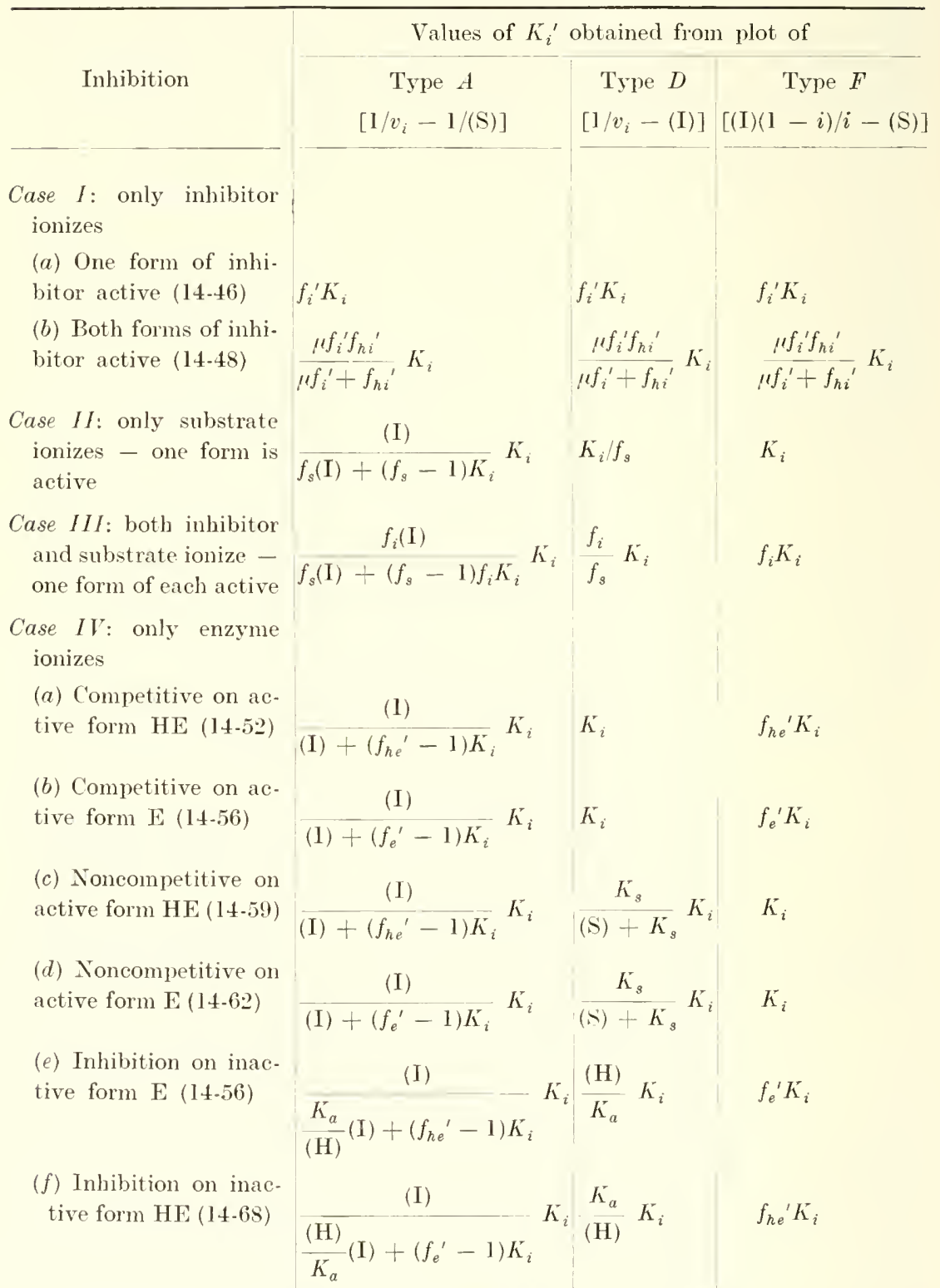

Case V: Enzyme and inhibitor ionize 


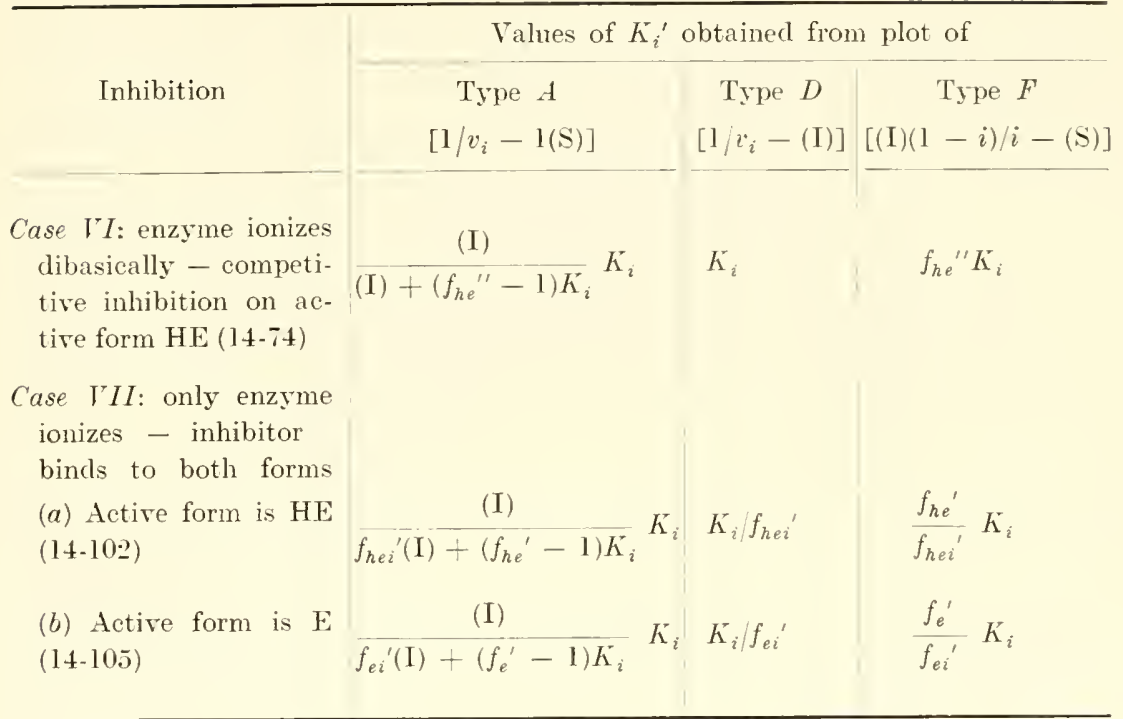

a The types of inhibition are classified as earlier in the chapter and the reaction schemes are indicated where possible. Evaluation of $K_{i}{ }^{\prime}$ is as follows: type A plot, from slope; type D plot, from slope; type F plot, from intercept. It is assumed that $K_{z}^{\prime}$ is evaluated as if no ionization occurred.

only the inhibitor ionizes and the I form is active (Case I $a$ ) provides the expected value for $K_{i}^{\prime}$ :

$$
K_{i}{ }^{\prime}=f_{i}{ }^{\prime} K_{i}=\left[1+\frac{(\mathrm{H})}{K_{a}}\right] K_{i}
$$

Taking the logarithm of both sides:

$$
\mathrm{p} K_{i}{ }^{\prime}=\mathrm{p} K_{i}-\log \left[1+\frac{(\mathrm{H})}{K_{a}}\right]
$$

When the $\mathrm{pH}$ is in a range significantly lower than the $\mathrm{p} K_{\alpha}$, the term $(\mathrm{H}) K_{2}$ will be much greater than one and thus:

$$
\text { Low } \mathrm{pH}: \quad \mathrm{p} K_{i}{ }^{\prime}=\mathrm{p} K_{i}+\mathrm{pH}-\mathrm{p} K_{a}
$$

When the $\mathrm{pH}$ is significantly higher than the $K_{2}$, the term $(\mathrm{H}) K_{2}$ is negligible compared to one and thus:

$$
\text { High } \mathrm{pH}: \quad \mathrm{p} K_{i}{ }^{\prime}=\mathrm{p} K_{i}
$$

Therefore, in the low $\mathrm{pH}$ range the curve will have a slope of +1 but will 
inflect at a $\mathrm{pH}$ equivalent to $\mathrm{p} K_{a}$, at high $\mathrm{pH}$ the slope being zero (number 1 in Fig. 14-10).

When both forms of the inhibitor can combine with the enzyme (Case I $b$ ), the experimentally determined $K_{i}^{\prime}$ is given by:

$$
K_{i}^{\prime}=\frac{\mu f_{i}^{\prime} f_{h i}{ }^{\prime}}{\mu f_{i}^{\prime}+f_{h i}{ }^{\prime}} K_{i}
$$

Taking the logarithm of both sides and writing out the $\mathrm{pH}$ functions:

$$
\begin{aligned}
\mathrm{p} K_{i}{ }^{\prime}=\mathrm{p} K_{i} & -\log \mu-\log \left[1+\frac{(\mathrm{H})}{K_{a}}\right]-\log \left[1+\frac{K_{a}}{(\mathrm{H})}\right] \\
& +\log \left[\mu+1+\frac{\mu(\mathrm{H})}{K_{a}}+\frac{K_{a}}{(\mathrm{H})}\right]
\end{aligned}
$$

Expressions for $p K_{i}{ }^{\prime}$ at low and high $\mathrm{pH}$ values may be derived to give:

Low $\mathrm{pH}: \quad \mathrm{p} K_{i}{ }^{\prime}=\mathrm{p} K_{i}-\log \mu+\mathrm{pH}-\mathrm{p} K_{a}+\log \mu-\mathrm{pH}+\mathrm{p} K_{a}$

$$
\mathrm{p} K_{i}^{\prime}=\mathrm{p} K_{i}
$$

High $\mathrm{pH}: \quad \mathrm{p} K_{i}{ }^{\prime}=\mathrm{p} K_{\imath}-\log \mu+\mathrm{p} K_{a}-\mathrm{pH}-\mathrm{p} K_{a}+\mathrm{pH}$

$$
\mathrm{p} K_{i}{ }^{\prime}=\mathrm{p} K_{i}-\log \mu
$$

In the low $\mathrm{pH}$ range the slope will be 0 but as the $\mathrm{pH}$ is increased there will be either a decrease or increase to a new level of slope 0 ; if $\mu$ is greater than unity, the level will drop, and if $\mu$ is less than unity, it will rise. If the system is known to follow this mechanism, the value of $\mu$ may be calculated from the difference in $\mathrm{p} K_{i}{ }^{\prime}$ at low and high $\mathrm{pH}$ 's. The curves are shown in number 10 of Fig. 14-10.

Finally, the case in which the enzyme ionizes and the inhibition is competitive on the active enzyme form HE (Case IV a) may be examined. By the same procedures as above:

$$
\begin{gathered}
K_{i}{ }^{\prime}=\frac{(\mathrm{I})}{(\mathrm{I})+\left(f_{h e^{\prime}}-1\right) K_{i}} K_{i} \\
\mathrm{p} K_{i}{ }^{\prime}=\mathrm{p} K_{i}-\log (\mathrm{I})+\log \left[(\mathrm{I})+\frac{K_{a}}{(\mathrm{H})} K_{i}\right]
\end{gathered}
$$

Low $\mathrm{pH}: \quad \mathrm{p} K_{i}{ }^{\prime}=\mathrm{p} K_{i}$

High $\mathrm{pH}: \quad \mathrm{p} K_{i}{ }^{\prime}=-\log (\mathrm{I})-\mathrm{p} K_{a}+\mathrm{pH}$

Thus at low ranges of $\mathrm{pH}$ the slope will be 0 but an inflection will occur at $\mathrm{pH}=\mathrm{p} K_{a}$ so that the curve in the high $\mathrm{pH}$ range will have a slope of 1 
(number 11 in Fig. 14-10). Actually the inflection will occur at a $\mathrm{pH}=$ $\mathrm{p} K_{a}$ only if $(\mathrm{I})$ is close to $K_{i}$; if $(\mathrm{I})=10 K_{i}$, the inflection will be shifted one $\mathrm{pH}$ unit ligher and if $(\mathrm{I})$ is much less than $K_{i}$, the inflection will be shifted to lower pH's.

Each method of graphical analysis to determine $K_{i}^{\prime}$ has certain advantages but for the present purpose, the determination of a $K_{i}{ }^{\prime}$ that is simply related to the true constant, $K_{i}$. by the usual $\mathrm{pH}$ functions, it would appear that the single curve method of type $\mathrm{F}$, where $(\mathrm{I})(1-i) / i$ is plotted against (S), is generally the most reliable.

\section{Variation of Fumarase Inhibition with $\mathrm{pH}$}

There are very few reported data on enzrme inhibition to which the Dixon treatment can be applied. However, Massey $(1953 \mathrm{~b})$ conducted an excellent study of the competitive inhibition of fumarase by various organic ions, the results, of which are summarized in Fig. 14-11. It is believed that the inhibition is chiefy bronght about by the binding of these organic anions electrostatically to two properly spaced cationic groups at the active center. This is indicated by the variations with $\mathrm{pH}$ and also by the lack of

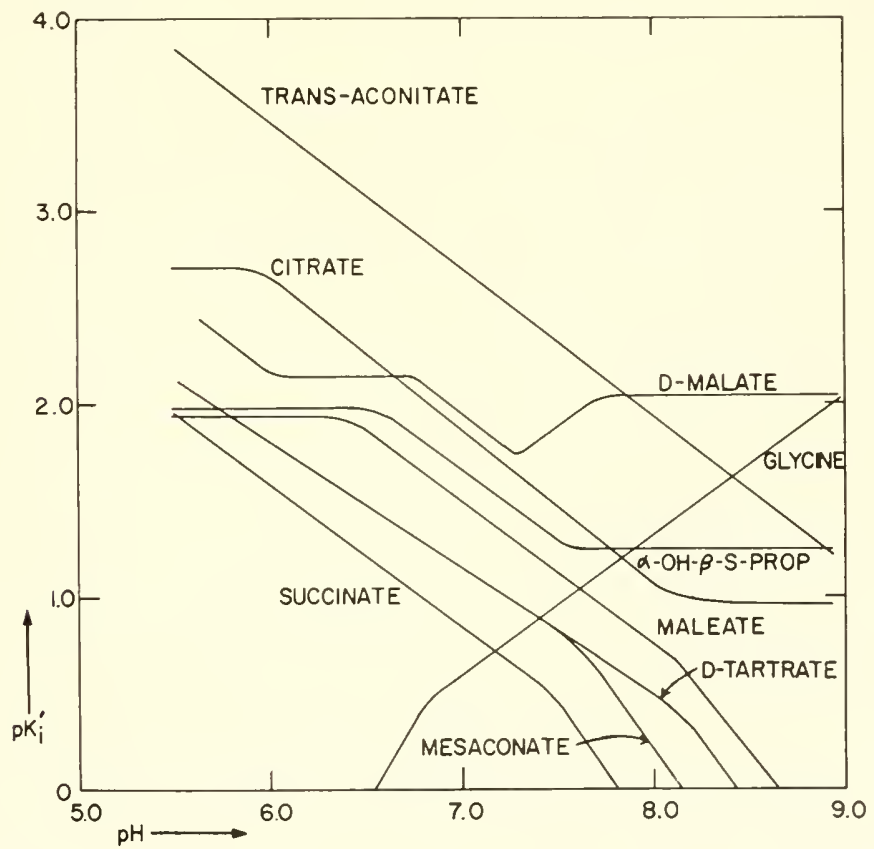

FIG. 14-11. The $p K_{i}{ }^{\prime}-\mathrm{pH}$ curves for the inhibition of fumarase by various organic anions. (From Massey, 1953 b.) 
inhibition when the carboxyl groups on the inhibitors are esterified or replaced by nompolar groups, combined with the retention of the inhibition if the carboxyl group is replaced by a sulfonic acich group. Inasmuch as the range of $\mathrm{pH}$ covered was from 5.7 to 9 , the inflections that would indicate the ionizations of the imhibitor carboxyl groups are not seen.

\begin{tabular}{|c|c|c|c|}
\hline $\mathrm{COO}^{-}$ & $\mathrm{COO}^{-}$ & $\mathrm{COO}^{-}$ & $\mathrm{COO}^{-}$ \\
\hline | & 1 & | & | \\
\hline $\mathrm{CH}$ & $\mathrm{CH}-\mathrm{OH}$ & $\mathrm{CH}_{2}$ & $\mathrm{CH}$ \\
\hline$\|$ & 1 & 1 & $\|$ \\
\hline $\mathrm{HC}$ & $\mathrm{CH}_{2}$ & $\mathrm{CH}_{2}$ & $\mathrm{C}-\mathrm{CH}_{2}-\mathrm{COO}^{-}$ \\
\hline | & | & | & 1 \\
\hline $\mathrm{COO}^{-}$ & $\mathrm{COO}^{-}$ & $\mathrm{COO}^{-}$ & $\mathrm{COO}^{-}$ \\
\hline Fumarate & Malate & Succinate & Aconitate \\
\hline $\mathrm{COO}^{-}$ & $\mathrm{COO}^{-}$ & $\mathrm{COO}^{-}$ & $\mathrm{SO}_{3}^{-}$ \\
\hline $\mathrm{CH}$ & $\mathrm{CH}-\mathrm{OH}$ & $\mathrm{CH}_{2}$ & $\mathrm{CH}_{2}$ \\
\hline$\|$ & & & | \\
\hline $\mathrm{CH}$ & $\mathrm{CH}-\mathrm{OH}$ & $\mathrm{NH}_{3}+$ & $\mathrm{CH}-\mathrm{OH}$ \\
\hline | & | & & 1 \\
\hline $\mathrm{COO}^{-}$ & $\mathrm{COO}^{-}$ & & $\mathrm{COO}^{-}$ \\
\hline Maleate & Tartrate & Glycine & $\begin{array}{l}\alpha \text {-Hydroxy- } \beta \text {-sulfo- } \\
\text { propionate }\end{array}$ \\
\hline
\end{tabular}

The curves for maleate, D-tartrate, mesaconate, and succinate seem to be of a configuration indicating either the active form of the enzyme to be $\mathrm{H}_{2} \mathrm{E}$ or the active form of the inhibitor to be $\mathrm{H}_{2} \mathrm{I}$. Since it is unlikely that the inhibitors would be active in the uncharged form, this would point to two gromps on the enzyme that must be protonated in order to combine with these anions, one with a $\mathrm{p} \boldsymbol{K}_{a}$ of 6.5 or below, the other near 7.5-8.0. Results with other imhibitors and with the substrates, fumarate and L-malate, also point to enzyme groups with $p K_{a}$ 's of similar magnitude. The $\mathrm{p}_{a}$ of approximately 6.5 might correspond to an imidazole gromp in histidine at the active center while the $\mathrm{p} K_{a}$ near 8 could represent an $\alpha$-amino group (or possibly a phenolic group in tyrosine); these two groups would serve to bind the substrates and inhibitors to the enzyme, and in the former case to participate in the transfer of hydrogen atoms. Sulfhydryl groups are exchuled because of the insensitivity of the enzyme to reagents reacting with these groups (iodoacetate, iodoacetamide, iodosobenzoate, $p$-chloromercuribenzoate, and chloroacetophenone).

It is interesting that the trans-aconitate curve shows no inflections at all over the $p H$ range studied. Since there is good evidence that there are ionizing groups on the enzyme, it is remarkable that no alteration of the curve is observed at $\mathrm{pH}$ 's corresponding to the $\mathrm{p}_{\alpha}$ 's of these groups, and the behavior must be considered, for the time being, as anonalous. The in- 
flections that are concave upwards near $\mathrm{pH} 8$ in the curves for citrate and L-hydroxy- $\beta$-sulfopropionate are also difficult to explain in conjunction with the results on the other inhibitors. It is possible. but mexpected, that the mechanism of inhibition differs within this series of inhibitors. It is also disturbing that the slopes of the curves are around 0.7 to 0.8 instead of the theoretical 1.0. This could be attributed in part to the increase in over-all negativity of the enzyme protein as the $\mathrm{pH}$ is increased or possibly to a surface-buffering effect, the $\mathrm{pH}$ on or within the enzyme not changing linearly with the $\mathrm{pH}$ of the bulk phase. Some further explanations for these discrepancies and an extension of the Dixon treatment will be discussed following the next section.

\section{Comparisons of the Relative Potencies of Different Inhibitors}

In relating chemical structure to inhibitory activity, especially in a series of inhibitors acting by the same mechanism, the most informative method is to determine and compare the inhibitor constants. Now, for this purpose, one would be interested only in the true constants, $K_{i}$, since they provide information on the affinities of the enzyme for the varions active forms of the inhibitors. However, it is clear that by the usual methods one determines in most cases an apparent inhibitor constant, $K_{i}{ }^{\prime}$, which may be related to $K_{i}$ in a complex manner involving the $\mathrm{pH}$ and possibly the concentrations of other components of the system. The use of $\mathrm{p} K_{i}$ 's to compare inhibitors may be very erroneous.

It is possible that determinations of inhibitions at a single $\mathrm{pH}$ may give apparent inhibitor constants that are related only to the $\mathrm{p} K_{a}$ 's of ionizing groups, rather than to relative affinities. As an example, let us consider three monobasic inhibitors whose anions are bound equally tightly to the enzyme with a true $\mathrm{p} K_{i}$ of 3 . If the inhibitions on some enzyme are determined at a $\mathrm{pH}$ of 7 , the apparent constants will be given by:

$$
\begin{array}{ll}
\text { Inhibitor } 1\left(\mathrm{p} K_{a}=6\right): & \mathrm{p} K_{i}{ }^{\prime}=\mathrm{p} K_{i}-\log 1.1=2.96 \\
\text { Inhibitor } 2\left(\mathrm{p} K_{a}=7\right): & \mathrm{p} K_{i}{ }^{\prime}=\mathrm{p} K_{i}-\log 2=2.70 \\
\text { Inhibitor } 3\left(\mathrm{p} K_{a}=8\right): & \mathrm{p} K_{i}{ }^{\prime}=\mathrm{p} K_{i}-\log 11=1.96
\end{array}
$$

If these $\mathrm{p} K_{i}$ "s were used to compare binding affinities for the inhibitors, the conclusions would be completely incorrect. and if interaction energies were calculated from these $\mathrm{p} K_{i}^{\prime}$ 's and compared with the structures of the inhibitors, any deductions would be invalid.

Massey (1953 b) pointed ont in his study on fumarase inhibition that a consideration of $\mathrm{pH}$ in such comparisons can be very important and his results well illustrate these principles. Table 14-2 presents the values of $\mathrm{p} K_{i}{ }^{\prime}$ for the different inhibitors at four $\mathrm{pH}$ 's and it is evident that if rela- 
TABLE 14-2

Values of the Experimentally Determined $p K_{i}{ }^{\prime}$ for Inhibitors of Fumarase at Different pH's ${ }^{a}$

\begin{tabular}{l|c|c|c|c}
\hline \multicolumn{1}{c}{ Inhibitors } & \multicolumn{3}{|c}{$\mathrm{p} K_{i}{ }^{\prime}$} \\
\cline { 2 - 5 } & $\mathrm{pH}=5.7$ & $\mathrm{pH}=6.35$ & $\mathrm{pH}=7.0$ & $\mathrm{pH}=8.0$ \\
\cline { 2 - 5 } & & & & \\
Trans-Aconitate & 3.6 & 3.2 & 2.7 & 1.9 \\
Citrate & 2.7 & 2.5 & 2.0 & 1.0 \\
D-Malate & 2.3 & 2.2 & 1.9 & 2.0 \\
D-Tartrate & 2.0 & 1.6 & 1.1 & 0.5 \\
Mesaconate & 2.0 & 1.6 & 1.1 & 0 \\
$\alpha$-Hydroxy- $\beta$-sulfopropionate & 2.0 & 2.0 & 1.7 & 1.3 \\
Maleate & 1.9 & 1.9 & 1.4 & 0.5 \\
Succinate & 1.8 & 1.3 & 0.8 & 0 \\
Glycine & 0 & 0 & 0.6 & 1.2 \\
& & & & \\
\hline
\end{tabular}

a Values estimated from the curves given by Massey (1953 b).

tive potencies were estimated from these $\mathrm{p} \boldsymbol{K}_{i}$ 's, these would vary markedly with the $\mathrm{pH}$ chosen, even for the inhibitors which presumably inhibit in the same manner. It is generally true that the highest value of the $\mathrm{p} K_{i}{ }^{\prime} \mathrm{ob}$ served is the safest to use for comparative purposes since the effect of $\mathrm{pH}$ change is always to reduce the apparent $\mathrm{p} K_{i}{ }^{\prime}$ relative to $\mathrm{p} K_{i}$. The results on fumarase, however, are not available for $\mathrm{pH}$ 's lower than 5.7 and hence the maximal $\mathrm{p} \boldsymbol{K}_{i}$ 's cannot be determined for all the inhibitors.

\section{Variation of the True Inhibitor Constant with $\mathrm{pH}$}

The treatment up to this point has usually been based on the assumption that certain ionization states of the enzyme or the inhibitor are active and that the true inhibitor constant, $K_{i}$, expresses the affinity between these active forms. The apparent inhibitor constant, $K_{i}$, has differed from $K_{i}$ because the concentrations of these active forms vary with the $p H$. In the reaction, $\mathrm{HE}^{+}+\mathrm{I}^{-} \rightleftharpoons \mathrm{HEI}$, it has been assumed that the $K_{i}$ for this complex is independent of the $\mathrm{pH}$ and that changes in the inhibition brought about by varying the $\mathrm{pH}$ are caused by changes in $\left(\mathrm{I}^{-}\right)$or $\left(\mathrm{HE}^{+}\right)$. The Dixon treatment is also based primarily on this assumption. Actually, it is quite probable that the affinity of the enzyme for the inhibitor can also alter with $\mathrm{pH}$ and that $K_{i}$ itself will not remain constant. Enzymes presumably possess ionizing groups surrounding the active center. These groups would not participate directly in the binding of the substrate or the inhibitor, nor would they necessarily play any role in the catalysis, but alterations of the charge on these groups might well significantly affect the binding of 
the substrate or the inhibitor to the active center. especially if the binding involves ionic interactions. One may divide such electrostatic effects into those originating from ricinal gromps near the active center and to those resulting from changes in the total over-all charge of the enzyme protein.

The value of $K_{i}$ is determined by the energy of interaction of the inhibitor with the enzyme. The effect of a ricinal group on $K_{i}$ would then be calculated from the additional interaction energy term derived from the electric field of this group and the position of the inhibitor in this field. A simple system is diagramed in Fig. 14-12. where a positively-charged inhibitor is repelled by a positively-charged vicinal group at a distance $d$ from the bound inhibitor. The affinity of the enzyme for the inhibitor will be less and the $\mathrm{p} K_{i}$ lower than when the vicinal group is not protonated.

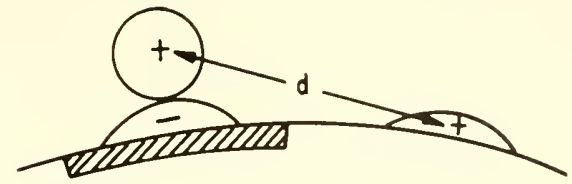

FIG. 14-12. Schematic illustration of the effect of a vicinal ionizing group on inhibitor binding at the active center (crosshatched area). The positively charged inhibitor is repelled by the positively charged vicinal group.

If we start with the $\mathrm{pH}$ above the $\boldsymbol{K}_{\text {, }}$ of the ricinal group, this group will not be charged and the interaction of the enzrme with the inhibitor will be restricted to the active center: when the $\mathrm{pH}$ is lowered, the vicinal group will eventually become protonated so that in the region of $\mathrm{pH}$ around the $\mathrm{p} K_{a}$ there will be a change in $K_{i}$. If $\mathrm{p} K_{i}^{\prime}$ is plotted against $\mathrm{pH}$, there will be a jog in the curve, but not an inflection to a new slope. Some types of expected effects are shown in Fig. 14-13. When the $p K_{i}{ }^{\prime}$ increases due to this mechanism. the charges on the inhibitor and the ricinal group are opposite (curve A) and when it decreases, ther are of the same sign (curve B). A similar jog may occur in those sections of the curves with slope -1 , +1 , etc. (curve $\mathrm{C}$ ). The degree of interaction with the vicinal group is equated with the rertical deriation. $1 p K_{i}$.

For the state in which the ricinal group is uncharged and does not contribute to the interaction, the binding free energy can be written as $1 F_{1}=$ $-2.3 R T \mathrm{p} K_{i_{1}}$, and for the state in which the vicinal gromp is protonated, $1 F_{2}=-2.3 R T \mathrm{p} K_{i_{2}}$. The change in $\mathrm{p} K_{i}$ is then given by:

$$
\Delta \mathrm{p} K_{i}=\mathrm{p} K_{i_{2}}-\mathrm{p} K_{i_{1}}=\frac{\Delta F_{1}-\Delta F_{2}}{2.3 R T}
$$


The effect of the vicinal group can be estimated by the procedure discussed in Chapter 6. The interaction energy between two ionic groups is given by Eq. 6-109 and the changes in $p K_{i}$ due to such interaction are plotted in Fig. 14-14. The following points should be noted about these calculations: (1) the repulsion correction factor, $r=0.92$, for ion-ion interactions was not nsed because the grouns are not in contact at their equilibrium distance, (2) the dispersion energy was omitted since it does not change with the protonation of the vicinal group, (3) the ion-dipole interaction at dis-

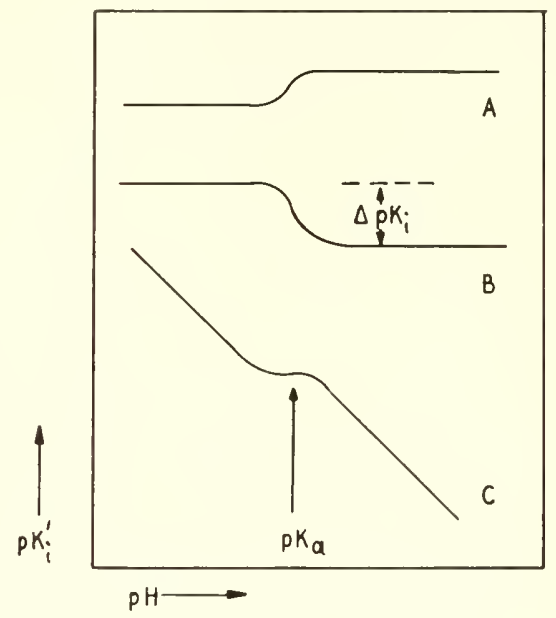

Fig. 14-13. Different types of effects of an ionizing vicinal group on the inhibitor constant. Curve $A$ : charges on inhibitor and vicinal group are opposite. Curve B: charges on inhibitor and vicinal group are the same. Curve C': charges on inhibitor and vicinal group are opposite and $\mathrm{p} K_{i}{ }^{\prime}-\mathrm{pH}$ curve has slope of -1 .

tances above $5 \AA$ can be neglected (Table 6-18), and (t) hydration of the ionic groups has been assumed. Of course such ealculations are only approximate. due primarily to the ambient water molecules and the uncertainty of the value of the dielectric constant, but some confidence in their general validity is found in the experimental data on the effects of charged gromls on acidic dissociations.

The problem of the effects of charged groups on ionization was first treater theoretically by Bjerrum (1923) but this simple approach did not correlate very well with the experimental results. The treatment was refined by Kirkwood and Westheimer (1938) and Westheimer and 
Shookhoff (1939) and the development has been summarized in Edsall and Wyman's recent book (1958, Chapt. 11). The interactions of carboxyl groups in dicarboxylic acids of varying chain length and the interactions between carboxyl and amino groups in amino acids lead to shifts in the $\mathrm{p} K_{a}$ 's of comparable magnitudes to those given in Fig. 14-14. Thus in the series from malonic to azelaic acids, where the inter-carboxyl distance varies roughly from 5 to $12 \AA$, the $1 p K_{n}$ 's vary from 2.26 to 0.26 (Westheimer and Shookhoff, 1939). The microscopic ionization constants

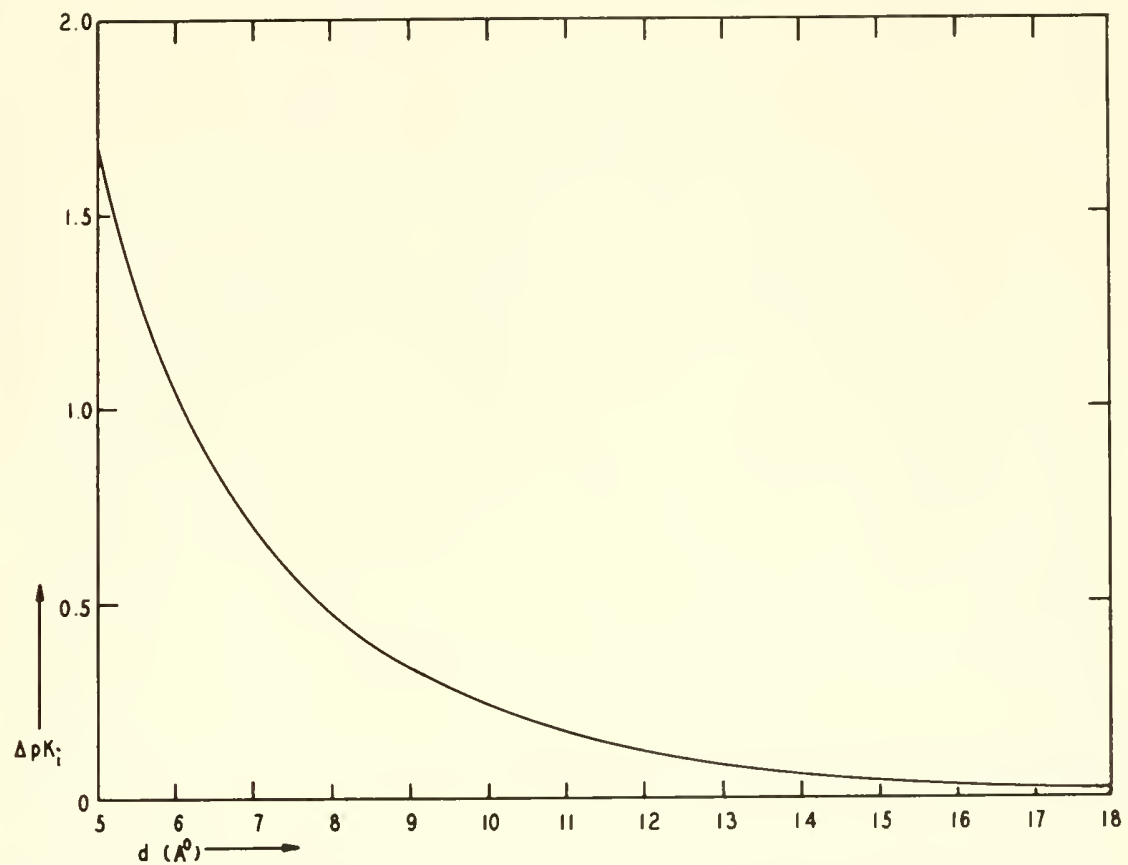

FIG. 14-14. Expected changes in $\mathrm{p} K_{i}$ due to a charged vicinal group at the distance $d$ from the active center. Interaction energy between groups calculated from eq. 6-109 with $r_{0}=10.2 \AA$.

of glutamic acid (Edsall and Wyman, 1958. p. 496), cysteine (Edsall and Wyman, 1958, p. 503), and tyrosine derivatives (Martin et ul., 1958) also indicate that $\mathrm{p} K_{a}$ shifts of 0.4 to 2.1 arise from charged gromps distant 5 to $10 \AA$. Although there is undoubtedly some difference between the dissociation of an inhibitor from the active center and the dissociation of a proton from a single group, one might expect changes in $\mathrm{p} K_{n}$ and $\mathrm{p} K_{i}$ at least of a similar order of magnitude.

It has been shown that the inhibition can be quite sensitive to small changes in the binding energy (Eq. 6-102. Fig. 6-15) and it is clear that 
vicinal groups up to at least $12 \AA$ from the active center ean produce significant effects. Some might consider the ionization of the vicinal group, in its effect upon $K_{i}$, as an ionization of the enzyme-inhibitor complex However, it would seem more usual to consider the EI complex as made up of the inhibitor and the active center, and it is probably preferable to consider ionizing groups outside of the active center as separate from the EI complex. Experimentally, nevertheless, it is sometimes not easy to distinguish between ionizations in the active center and ionizations of groups that are close by, unless a definite jog instead of a change in slope is evident in the $\mathrm{p} K_{i}^{\prime}-\mathrm{pH}$ plot. Such jogs have not been recognized or treated in the enzymes so far subjected to this analysis, but if one examines the experimental points elosely, definite jogs are often observed, usually between 0.1 and $0.4 \mathrm{p} K_{i}^{\prime}$ units in magnitude and thus indieate vieinal groups between 8 and $12 \AA$ distant from the active center.

We have considered the effects of a single vicinal group on the $K_{i}$ but actually there are probably several ionizing groups within an effective distance of the active center on most enzyme proteins. Averaging data from a number of proteins for which the total number of ionizing groups and the molecular size are known, it may be caleulated that a typical protein molecule possesses an ionizing group for each $25 \AA^{2}$ area of the surface. The area of the protein surface within $12 \AA$ of the active center may be esti mated as approximately $500 \AA^{2}$ (since most enzyme proteins are not spherieal, only rough estimates need be made) and henee will contain on the average about twenty ionizing groups. Now, only a fraction of these groups will ionize over the $\mathrm{pH}$ range of interest. Usually not over $25 \%$ of the total groups will have $\mathrm{p} K_{a}$ 's between 5 and 9 and thus we ean assume that around five groups are so situated that they can produce a significant effeet on the $\mathrm{p} K_{i}$. This will elearly vary with the amino acid composition of the enzyme protein but the caleulation shows that usually more than one ionizable group must be expected. If two or more groups have the same $\mathrm{p} K_{a}$, their individual effects will sum; thus three interacting vicinal groups at a distance of $10 \AA$ could change the $p K_{i}$ by as much as one unit. The progressive effects on the $\mathrm{p} K_{i}$ of several vicinal groups with different $\mathrm{p} K_{n}$ 's, when the $\mathrm{pH}$ is varied, may be complex.

Another approach to the effects of protein ionizing groups on the $K_{i}$ is to neglect the interactions of specific groups with the EI complex and consider only the total net charge on the enzyme. The total interaction energy of the protein charge with the EI complex is, of course, the sum of the energies from the individual groups, i.e., $\left\langle F_{\text {total }}=\Sigma_{i=1}^{i=n} \Delta F_{i}\right.$ where $\Delta F_{i}$ is the interaction energy due to the $i$ th group and there are $n$ such groups. Although single groups beyond $15 \AA$ from the active eenter do not affect the $K_{i}$ markedly, there are so many ionizing groups on most proteins that the summed effect may be appreciable. The effects of the total protein 
charge on the $K_{a}$ 's of ionizing groups on proteins have been treated in several different ways and we shall utilize these approaches in discussing the effects on $K_{i}$.

The total free energy for the formation of the EI complex is given by:

$$
\Delta F_{t}=\Delta F_{\text {int }}+\Delta F_{\text {elec }}
$$

where $\Delta F_{\text {int }}$ is the intrinsic association energy when the enzyme is in the isoelectric state and $\Delta F_{\text {elec }}$ is the contribution arising from electrostatic interaction with the net enzyme charge. Tanford (1955), postulated that $\Delta F_{\text {elec }}$ is proportional to the total net charge on the protein and designated the proportionality factor as $2 R T w$. Thus:

$$
\Delta F_{t}=\Delta F_{\text {int }}+2 R T w z
$$

where $z$ is the total number of charged groups and may be either positive or negative. Substituting $R T \ln K_{i}$ for $J F_{t}$ and $R T \ln K_{i}^{o}$ for $J F_{\text {int }}$ and solving for $K_{i}$ :

$$
K_{i}=K_{i}^{0} e^{2 w z}
$$

$K_{i}^{o}$ is the inhibitor constant for the isoelectric enzyme and $K_{i}$ is the inhibitor constant when there is a net charge on the enzyme.

A convention must be adopted with regard to the sign of $z$ in applying these equations to inhibition. For acidic dissociation there is no problem because the proton is always positively charged and the true sign of $z$ will indicate whether $K_{i}$ is larger or smaller than $K_{i}^{o}$. However, an inhibitor may be negatively charged and the opposite effects would be expected. Thus we may formulate the following rule: when the charges on the inhibitor and the enzyme are of the same sign, $z$ is positive, and when the charges are of opposite sign, $z$ is negative. Thus for a negatively charged inhibitor and a positively charged enzyme (on the acidic side of the isoelectric point), $z$ will be negative and, from Eq. 14-125, it is seen that $K_{i}$ will be smaller than $K_{2}^{\circ}$, as anticipated. It may also be noted that the sign convention in Eq. 14-124 is different from that of Tanford because the free energy changes here are related to the formation rather than to the dissociation of the EI complex.

These equations cannot be applied until the electrostatic factor, $w$, is defined and determined in some way. The definition of $w$ is such that $2 R T w z$ is the electrostatic energy involved in bringing a unit charge from infinity up to the enzyme. The value of $w$ was expressed by Scatchard (1949) in terms of the Debye-Hückel theory as:

$$
w=\frac{e^{2}}{2 D k T}\left[\frac{1}{b}-\frac{\varkappa}{1+x a}\right]
$$


where $e$ is the electronic charge, $b$ is the radius of the enzyme protein sphere, $a$ is the distance from the surface of the sphere in which small ions are excluded, and $x$ is the Debye-Hückel constant representing the reciprocal of the thickness of the ionic atmosphere. The value of will depend on the temperature, the ionic strength, and nmmerous other factors. For a given total net protein charge, the larger the protein molecule and the smaller the surface charge density, the smaller $w$ will be. Deviations of the protein from sphericity also will modify $w$. Methods for experimentally determining w have been given by Tanford (1955) and he has reported values of 0.04 for $\beta$-lactoglobulin and 0.021 for bovine sermm albumin at an ionic strength of 0.15 (the valne for $\beta$-lactoglobulin was calculated from a curve based on Tanford's values at other ionic strengths). Many enzyme test media have much lower ionic strengths (see Chapter 2), in which case $w$ will be greater (for $\beta$-lactoglobulin, $w=0.06$ at an ionic strength of 0.019 ).

The variations of the inhibition and the $r K_{i}$ dne to changes in the net protein charge are shown in Fig. 14-15 as calculated from Eq. 14-125 with $w=0.05$. A total of 50 net charges on the enzyme will shift the $\mathrm{p} K_{i}$ by

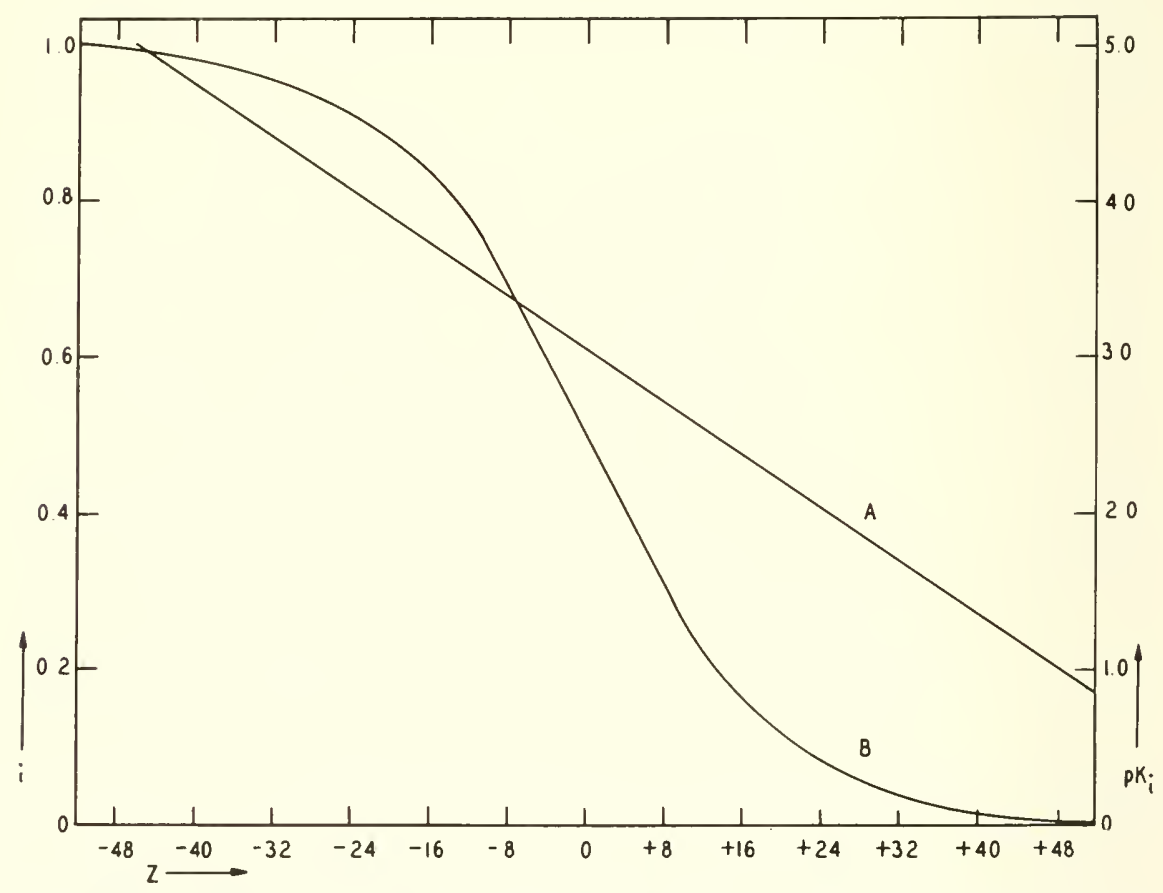

FIG. 14-15. Variation of $p K_{i}$ and the inhibition with the net protein charge on the enzyme (Eq. 14-125). $K_{i}=1 \mathrm{~m} H,(\mathrm{I})=1 \mathrm{~m} H$, and $w=0.05$. Curve $\mathrm{A}: \mathrm{p} K_{i}$; curve $\mathrm{B}$ : inhibition. 
2.17 units here and either abolish the inhibition or make it essentially complete, depending on the sign of the charge. These calculations are, of course, very approximate and nothing is known of the value of $w$ for any enzyme. It is probably valid to conclude that the $\mathrm{p} \boldsymbol{K}_{i}$ can be shifted significantly by a change in $\mathrm{pH}$, either through interactions with vicinal groups or with the net protein charge.

A very interesting observation, which bears on the present problen, was reported by Klotz and Ayers (1957). When the group $(\mathrm{Me})_{2} \mathrm{~N}-\boldsymbol{\varphi}-\mathrm{N}=\mathrm{N}-$ $\varphi-\mathrm{Hg}$ - was attached to cysteine. the $\mathrm{p} \boldsymbol{K}_{2}$ of the dimethylamino group was found to be 3.3 , but when it was linked to serum albumin, the $\mathrm{p} K_{r}$ was 1.8. They interpret this as the result of a "frozen" water structure surrounding the protein polar groups which become less accessible. They speak of the meta structure of the protein, the framework which it imposes on the water in its vicinity, and how this may be altered by $\mathrm{pH}$. On the other hand, in the $\mathrm{pH}$ range below 4 , serum albumin must be strongly positively charged. There are nearly one hundred ionizable gromps that should be positively charged in this region, and below a $\mathrm{pH}$ of 4 the net positive charge must increase rapidly due to the protonation of the carboxyl groups. From the curve for $\mathrm{p} K_{i}$ in Fig. 14-15, a shift of 1.5 units would be brought about by around thirty-five positively charged groups but since $w=0.021$ for serum albumin, a total of eighty-six such groups would be necessary, which is not unreasonable. Therefore, without minimizing the importance of bound water, it would appear that this result would be attributed to the interaction of the group with the net protein charge.

\section{Effects of Charged Inhibitors on the pKa's of Groups at the Active Center}

As a charged molecule approaches an ionizing group on an enzyme, the affinity of this group for protons is altered, and this would be expressed as a change of the $\mathrm{p} K_{a}$. Thus the combination of an enzyme with charged substrate or inhibitor molecules will cause a shift in the $\mathrm{p} K_{a}$ 's of groups at or close to the active center. An experimentally determined $\mathrm{p} K_{\alpha}$ may refer to the ES or the EI complex and this $\mathrm{p} \boldsymbol{K}_{z}$ may be significantly different from the $\mathrm{p} \boldsymbol{K}_{z}$ of the same group on the free enzyme. This may be important in the identification of the nature of the ionizing group. We have seen that in the Dixon method of plotting, the ionizations of free enzyme and enzyme complex groups can often be distinguished by the direction of the inflections in the curves.

The formation of complexes between fumarase and fumarate or malate has been shown to alter the $\mathrm{p} K_{\alpha}$ 's of at least two enzyme groups (Massey and Alberty. 1954; Frieden and Alberty, 1955; Alberty, 1956 a). The results are summarized in Table 14-3 and it is seen that shifts of +0.4 to +1.6 were usually demonstrable. The one discrepancy, the reduction 
Table 14-3

Ionization Constants of Fumarase and Its Complexes witu Substrate ${ }^{a}$

\begin{tabular}{c|c|c|c|c|c}
\hline Buffer & $\mathrm{p} K_{a_{e}}$ & $\mathrm{p} K_{a_{e f}}$ & $\Delta \mathrm{p} K_{a}$ & $\mathrm{p} K_{a_{e m}}$ & $\Delta \mathrm{p} K_{a}$ \\
\hline & & & & & \\
\cline { 1 - 2 } $\begin{array}{c}\text { Phosphate } \\
\text { Group a }\end{array}$ & 5.7 & 5.85 & +0.15 & 7.2 & +1.5 \\
Group b & 7.2 & 7.7 & +0.5 & 8.4 & +1.2 \\
Acetate & & & & & \\
Group a & 6.2 & 5.3 & -0.9 & 6.6 & +0.4 \\
Group b & 6.8 & 7.3 & +0.5 & 8.4 & +1.6 \\
\hline
\end{tabular}

a From Massey and Alberty (1954) and Frieden and Alberty (1955). Phosphate $33.3 \mathrm{~m} M$ and acetate $10 \mathrm{~m} M$.

of the $\mathrm{p} K_{a}$ in the EF complex in acetate, is mnexplained. These results are presented to illustrate the magnitudes of the changes that may occur inasmuch as no EI complex ionization has been worked out in this detail.

Negatively charged inhibitors should increase the $\mathrm{p} K_{n}$ and positively charged inhibitors shonld decrease the $\mathrm{p} \boldsymbol{K}_{n}$. The magnitude of the shift will depend primarily on the amount of inhibitor charge and the distances between the groups. The curve in Fig. 14-14 may be used to estimate the effect, since the ionizing gronp on the inhibitor will have the same effect on the ionizing group on the protein as will the latter on the former. The shifts observed with fumarase indicate the protein groups to be between 6 to $10 \AA$ from the carboxylate ions on fumarate and malate (since the charge on these substrates is -2 , the curve in Fig. 14-14 cannot be read off directly, but inasmuch as the shift in $\mathrm{p} K_{z}$ is proportional to the interaction energy, in this case one-half the observed $l_{p} K_{a}$ 's may be used). It is probable that at least one of these groups on fumarase is involved in the catalytic reaction and not in the binding (perhaps group b which may be an imidazole). One might expect that charged inhibitors would produce similar effects to those reported for the fumarase substrates, i.e., that a single charged group on the inhibitor that is 8 to $10 \AA$ distant from an ionizable group on the enzyme would shift the $\mathrm{p} K_{n}$ around 0.4 units. Factors other than electrostatic may also play a role. An inhibitor molecule might cover or mask an enzyme group so that it would not be readily accessible to protons, or might interfere sterically with it to varying degrees.

The characterization of the chemical nature of an enzyme group from the experimentally determined $\mathrm{p} K_{n}$ sometimes presents problems in that it is impossible to predict accurately what the $\mathrm{p} K_{n}$ of any particular group on an enzyme might be. Ionization constants for some amino acids in different states are shown in Table 14-4 and the marked effects of charged 
TABLE 14-4

Dissociation Microconstants of Three Amro Acids ${ }^{a}$

\begin{tabular}{|c|c|c|c|c|}
\hline Amino acid & Group & $\mathrm{p} K_{a}$ & $\begin{array}{l}\text { Ioniza } \\
\text { of otl }\end{array}$ & $\begin{array}{l}\text { on state } \\
\text { groups }\end{array}$ \\
\hline \multirow[t]{3}{*}{ Glutamic acid } & $a-\mathrm{COOH}$ & $\begin{array}{l}2.15 \\
2.62 \\
4.30 \\
4.74\end{array}$ & $\begin{array}{l}\gamma-\mathrm{COOH} \\
\gamma-\mathrm{COO}^{-}, \\
\gamma-\mathrm{COOH} \\
\gamma-\mathrm{COO}^{-},\end{array}$ & $\begin{array}{l}\mathrm{NH}_{3}{ }^{+} \\
\mathrm{NH}_{3}{ }^{+} \\
\mathrm{NH}_{2} \\
\mathrm{NH}_{2}\end{array}$ \\
\hline & $\gamma-\mathrm{COOH}$ & $\begin{array}{l}3.85 \\
4.32 \\
4.65 \\
5.09\end{array}$ & $\begin{array}{l}\alpha-\mathrm{COOH} \\
\alpha-\mathrm{COO}^{-} \\
\alpha-\mathrm{COOH} \\
\alpha-\mathrm{COO}^{-}\end{array}$ & $\begin{array}{l}\mathrm{NH}_{3}{ }^{+} \\
\mathrm{NH}_{3}{ }^{+} \\
\mathrm{NH}_{2} \\
\mathrm{NH}_{2}\end{array}$ \\
\hline & $\mathrm{N}^{\curlyvee} \mathrm{H}_{3}{ }^{+}$ & $\begin{array}{l}7.04 \\
7.84 \\
9.19 \\
9.96\end{array}$ & $\begin{array}{l}\alpha-\mathrm{COOH} \\
\alpha-\mathrm{COOH} \\
\alpha-\mathrm{COO}^{-} \\
\alpha-\mathrm{COO}^{-}\end{array}$ & $\begin{array}{l}\gamma-\mathrm{COOH} \\
\gamma-\mathrm{COO}^{-} \\
\gamma-\mathrm{COOH} \\
\gamma-\mathrm{COO}^{-}\end{array}$ \\
\hline \multirow[t]{3}{*}{ Cysteine } & $\mathrm{COOH}$ & $\begin{array}{l}1.71 \\
2.79 \\
3.80 \\
4.74\end{array}$ & $\begin{array}{l}\text { SH, } \\
\mathrm{s}^{-} \\
\mathrm{sH} \\
\mathrm{s}^{-}\end{array}$ & $\begin{array}{l}\mathrm{NH}_{3}+ \\
\mathrm{NH}_{3}+ \\
\mathrm{NH}_{2} \\
\mathrm{NH}_{2}\end{array}$ \\
\hline & $\mathrm{SH}$ & $\begin{array}{r}7.45 \\
8.53 \\
9.09 \\
10.03\end{array}$ & $\begin{array}{l}\mathrm{COOH} \\
\mathrm{COO}^{-} \\
\mathrm{COOH}^{\prime} \\
\mathrm{COO}^{-}\end{array}$ & $\begin{array}{l}\mathrm{NH}_{3}+ \\
\mathrm{NH}_{3}+ \\
\mathrm{NH}_{2} \\
\mathrm{NH}_{2}\end{array}$ \\
\hline & $\mathrm{NH}_{3}+$ & $\begin{array}{r}6.77 \\
8.41 \\
8.86 \\
10.36\end{array}$ & $\begin{array}{l}\mathrm{COOH}, \\
\mathrm{COOH}, \\
\mathrm{COO}^{-}, \\
\mathrm{COO}^{-}\end{array}$ & $\begin{array}{l}\mathrm{SH} \\
\mathrm{S}^{-} \\
\mathrm{SH} \\
\mathrm{S}^{-}\end{array}$ \\
\hline \multirow[t]{3}{*}{ Tyrosine } & $\mathrm{COOH}$ & $\begin{array}{l}2.30 \\
2.51 \\
4.25 \\
4.49\end{array}$ & $\begin{array}{l}\mathrm{OH} \\
\mathrm{O}^{-} \\
\mathrm{OH} \\
\mathrm{O}^{-}\end{array}$ & $\begin{array}{l}\mathrm{NH}_{3}{ }^{+} \\
\mathrm{NH}_{3}{ }^{+} \\
\mathrm{NH}_{2} \\
\mathrm{NH}_{2}\end{array}$ \\
\hline & $\mathrm{OH}$ & $\begin{array}{r}9.42 \\
9.63 \\
9.80 \\
10.04\end{array}$ & $\begin{array}{l}\mathrm{COOH} \\
\mathrm{COO}^{-} \\
\mathrm{COOH}^{-} \\
\mathrm{COO}^{-}\end{array}$ & $\begin{array}{l}\mathrm{NH}_{3}{ }^{+} \\
\mathrm{NH}_{3}{ }^{+} \\
\mathrm{NH}_{2} \\
\mathrm{NH}_{2}\end{array}$ \\
\hline & $\mathrm{NH}_{3}{ }^{+}$ & $\begin{array}{l}7.33 \\
7.71 \\
9.28 \\
9.69\end{array}$ & $\begin{array}{l}\mathrm{COOH}, \\
\mathrm{COOH}, \\
\mathrm{COO}^{-}, \\
\mathrm{COO},-\end{array}$ & $\begin{array}{l}\mathrm{OH} \\
\mathrm{O}^{-} \\
\mathrm{OH}^{-} \\
\mathrm{O}^{-}\end{array}$ \\
\hline
\end{tabular}

${ }^{a}$ Values obtained from Edsall and Wyman $(1958$, p. 494) for glutamic acid, from Benesch and Benesch (1955) for cysteine, and from Martin et al. (1958) for tyrosine. 
groups in the vicinity of the ionizing group are evident. Such variation of the $\mathrm{p} \boldsymbol{K}_{\alpha}$ with the enviromment must always be taken into account in interpreting the data. Table $14-5$ presents the $\mathrm{p} K_{a}$ 's of certain important protein groups as determined from the titrations of amino acids, polypeptides, and proteins, and the values are eclectic and somewhat arbitrary.

Table 14.5

Dissociation Constants of Enzymically Important Grodps a

\begin{tabular}{|c|c|c|c|c|}
\hline Group & Type & Amino acids & $\mathrm{p} K_{a}$ & $\mathrm{p} K_{a}$ Range \\
\hline Carboxyl & $\alpha$ & & 3.2 & $2.2-4.7$ \\
\hline Carboxyl & $\beta$ & Aspartic acid & 4.1 & $3.5-4.9$ \\
\hline Carboxyl & $\gamma$ & Glutamic acid & 4.5 & $3.8-5.1$ \\
\hline Imidazolium & & Histidine & 6.1 & $5.8-6.3$ \\
\hline Amino & $\alpha$ & & 8.6 & $7.8-10.4$ \\
\hline Sulfhydryl & & Cysteine & 8.7 & $8.3-10.1$ \\
\hline Amino & $\varepsilon$ & Lysine & 9.8 & $9.5-10.3$ \\
\hline Phenolic & & Tyrosine & 10.1 & $9.6-10.5$ \\
\hline Guanidinium & & Arginine & 12.2 & $12-13$ \\
\hline
\end{tabular}

a The $\mathrm{p} K_{a}$ given is considered to be the most likely value to be found in proteins taking into account the net charge of the average protein in the $\mathrm{pH}$ range near the $\mathrm{p} K_{a^{*}}$. The actual value in a particular protein may vary from this, depending not only on the over-all net charge of the protein but on vicinal ionizing groups and steric factors; the most probable ranges to be found are given in the last column.

\section{Variation of the Rate of Inhibition with $\mathrm{pH}$}

The rate at which the inhibition of an enzyme develops may also depend on the $\mathrm{pH}$. Two basic mechanisms may be involved: changes in the concentration of the active form of the inhibitor and changes in the rate constants for the inhibition. In other words, the situation is the same as in the variation of $K_{i}$ with $p H$. Similarly, we may define a true rate constant, $k_{i}$ or $k_{-1}$, as expressing the rates of formation or dissociation of the EI complex when the enzyme and the inhibitor are in their active forms, and an apparent rate constant, $k_{1}^{\prime}$ or $k_{-1}^{\prime}$, which is experimentally derivable by plotting procedures. Thus, a change in pH may produce a change in the apparent rate constants either by affecting the concentrations of active forms or by directly altering the true rate constants. Of course, the rate constants and $K_{i}$ are closely related and a change in $K_{i}$ implies some modifications of $k_{1}$ and $k_{-1}$. 
If the initial inhibition rate, uncomplicated by the reverse reaction, can be measured, Eq. 12-16 may be rewritten as:

$$
i=1-e^{-k_{1}\left(\mathrm{I}_{t}\right) t f_{2}}
$$

and for the rate of inhibition at any time:

$$
\frac{d i}{d t}=k_{1} \frac{\left(\mathrm{I}_{t}\right)}{f_{i}} e^{-k_{1}\left(\mathrm{I}_{t}\right) t f_{i}}
$$

where $f_{i}$ is the appropriate $\mathrm{pH}$ function for the inhibitor. The value of $k_{i}$ can be determined by plotting $\log (1-i)$ against $t$, since the resulting slope will be $-k_{1}\left(\mathrm{I}_{t}\right) / 2.3 f_{i}$. Therefore, $k_{1}{ }^{\prime}=k_{1} / f_{i}$. It is easy to determine if such a $\mathrm{pH}$ function is involved or whether $k_{1}$ itself is changed. Let us assume that $I$ is the active form of a monobasic inhibitor, so that:

$$
k_{1}{ }^{\prime}=\frac{k_{1}}{1+(\mathrm{H}) / K_{a}}
$$

which may be rewritten in logarithmic form as:

$$
\mathrm{p} k_{1}^{\prime}=\mathrm{p} k_{1}+\mathrm{p} K_{a}-\mathrm{pH}
$$

where $\mathrm{p} k_{1}=-\log k_{1}$, when the $\mathrm{pH}$ is below the $\mathrm{p} K_{n}$, so that a plot of $\mathrm{p} k_{1}{ }^{\prime}$ against $\mathrm{pH}$ will, in this region, be linear with a slope of -1 if the variation is due only to changes in (I). If it does not so conform, it may be suspected that $k_{1}$ varies with $\mathrm{pH}$. If the rate of reversal of the inhibition can be measured at different $\mathrm{pH}$ 's, any changes in $k^{\prime}{ }_{-1}$ must be dne to changes in $k_{-1}$ since the dissociation rate of the EI complex depends only on this rate constant, and if $k_{-1}$ changes it is likely that $k_{1}$ is also altered.

When the reverse reaction (the dissociation of EI) cannot be neglected, Eqs. 12-11 and 12-13 must be used with the substitution of $\left(\mathrm{I}_{t}\right) / f_{i}$ for $(\mathrm{I})$. The value of $k_{i}{ }^{\prime}$ obtained from the slope of a plot of $\log \left(i_{f}-i\right)$ against $t$ will be given by:

$$
k_{i}^{\prime}=-\frac{2.3 s}{\left(\mathrm{I}_{t}\right)+K_{i}}
$$

but the true rate constant is:

$$
k_{1}=-\frac{2.3 f_{i} s}{\left(\mathrm{I}_{t}\right)+f_{i} K_{i}}
$$

so that now $k_{1}^{\prime}$ does not equal $f_{i} k_{1}$.

In the ionic interactions we have been considering, variations in $k_{1}$ or $k_{-1}$ would be clue to the altered electric fields at the active center brought about by changes in the $\mathrm{pH}$. The activation energy for the formation of the 
EI complex would be either raised or lowered depending on whether the change in enzyme charge tends to repel or attract the inhibitor. However, many inhibitions do not involve such clectrostatic mechanisms in the formation of the EI complex, the reaction being frequently chemical, as in the inhibitions produced by alkylating agents, arsenicals, and mercurials on SH-dependent enzymes. It is, of course, possible that electrostatic interactions occur even here if the inhibitor is charged, but the reaction once the inhibitor has reached the surface of the enzyme may vary with $\mathrm{pH}$ for quite different reasons. In these cases. it is possible that the inhibition rate may be increased or decreased. but the final level of inhibition reached remains unchanged by the $\mathrm{pH}$ if $K_{i}$ is sufficiently small so that the inhibition is pseudoirreversible. No really quantitative work has been done on the $\mathrm{pH}$ dependence of the inhibition rates of such reactions and what little has been found will be discussed in the volmmes on individual inhibitors.

\section{Variation of Inhibition with $\mathrm{pH}$ in Mutual Depletion Systems}

When the system is in zone $\mathrm{C}$ (inhibitor almost entirely bound to the enzyme), the inhibition will be independent of $\mathrm{pH}$ since $i=\left(\mathrm{I}_{t}\right) /\left(\mathrm{E}_{t}\right)$ and does not depend on $K_{i}$. If the inhibitor is not all in the active ionic form that binds to the enzyme at the $\mathrm{pH}$ of the experiment. the strong binding will shift the ionization equilibrium until all the inhibitor is combined with the enzyme. In zone B (inhibitor partly bound and partly free), the situation is more complex and one cannot rewrite Eq. 3-32 simply by replacing $K_{i}$ by $f_{i} f_{c} K_{i}$, because the system will be intermediate between zone $\mathrm{A}$ and zone $\mathrm{C}$. In general we may say that zone B inhibition is less sensitive to $\mathrm{pH}$ than a comparable system in zone A wonld be.

One can also assume a system such as:

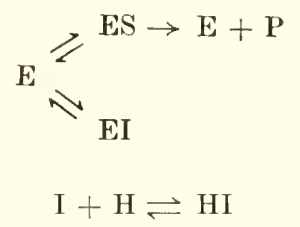

which represents competitive inhibition by an ionizing inhibitor, the conservation equations for which are:

$$
\begin{aligned}
& \left(\mathrm{E}_{t}\right)=(\mathrm{E})+(\mathrm{EI})+(\mathrm{ES}) \\
& \left(\mathrm{I}_{t}\right)=(\mathrm{I})+(\mathrm{HI})+(\mathrm{EI})
\end{aligned}
$$

The concentration of ES is given by a quadratic equation, which may be solved for different pH's to obtain the inhibitions. However, it is difficult to use experimental data from such inhibitions to calculate $K_{i}$ directly. 
It can be shown in the system assumed above, where $I$ is the active form of the inhibitor, that lowering the $\mathrm{pH}$ below the $\mathrm{p} K_{a}$ of the inhibitor will indeed reduce the inhibition, but not to as great an extent as in systems that do not exhibit mutual depletion.

The inhibition of $\alpha$-chymotrypsin by diisopropylfuorophosphate in pure water is reversible and competitive (Stein and Laidler, 1959). This is a mutual depletion system because for inhibitions between $17 \%$ and $88 \%$, $\left(\mathrm{I}_{t}\right) /\left(\mathrm{E}_{\ell}\right)$ was found to be between 0.39 and 3.54 . The variation of $K_{i}$ with

\begin{tabular}{cc}
\hline $\mathrm{pH}$ & \multicolumn{1}{c}{$K_{i}(M)$} \\
7.5 & $14.2 \times 10^{-5}$ \\
7.8 & $19.9 \times 10^{-5}$ \\
8.1 & $20.4 \times 10^{-5}$ \\
8.4 & $9.35 \times 10^{-5}$ \\
8.7 & $5.72 \times 10^{-5}$ \\
\hline
\end{tabular}

the $\mathrm{pH}$ was determined and a maximum around a $\mathrm{pH}$ of 8.1 was demonstrated. Both the uninhibited rate and the inhibition vary with $\mathrm{pH}$ in the same fashion, which led to the conclusion that diisopropylfluorophosphate reacts with the enzyme in the same way as does the substrate. The mechanism of the $\mathrm{pH}$ effects is interpreted in terms of a simultaneons nucleophilic attack by an imidazole nitrogen and an electrophilic attack by a protonated amino group.

\section{The $\mathrm{pH}$ of Inhibitor Solutions and Enzyme Preparations}

Accurate knowledge of the $\mathrm{pH}$ is necessary in all enzyme study and especially so in experiments designed to investigate the effects of varying the $\mathrm{pH}$. Much of the early work on enzymes and inhibition is difficult to interpret because of the failure to control the $\mathrm{pH}$ adequately. In many reports, unfortunately, the $\mathrm{pH}$ at which the experiments were run is not given, but this is becoming progressively less common. Even when the $\mathrm{pH}$ is stated, one can justifiably ask if this is reliable or whether undue faith was put in some buffer system. Perhaps one will be excused for including a few statements on these simple yet important problems.

Many inhibitors are weak acids or bases and solutions of their salts are often at $\mathrm{pH}$ 's far removed from neutrality. Solutions of sodium iodoacetate, sodium malonate, and similar salts at 5 to $10 \mathrm{mII}$ range in $\mathrm{pH}$ from 8 to 9 whereas solutions of the corresponding acids are between 2.5 and 3.5 frequently. Even lower concentrations of the acids can decrease the $\mathrm{pH}$ significantly; e.g., $1 \mathrm{~m} M 2$,4-dinitrophenol has a $\mathrm{pH}$ of 3.85 and $1 \mathrm{~m} . M$ iodoacetic acid a $\mathrm{pH}$ of 3.26 . When such solutions are added to enzyme preparations, the important question is whether in the presence of the buf- 
fers that are usually included, there will be a significant alteration of the $\mathrm{pH}$. Of course, when possible, the $\mathrm{pH}$ of the inhibitor solution should be adjusted previously to the $\mathrm{pH}$ of the enzyme preparation, but sometimes this cannot be done because of the insolubility of the inhibitor at the required $\mathrm{pH}$. When the $\mathrm{pH}$ 's of all the solutions that make up the final test medium are not identical, the only recourse is to determine the $\mathrm{pH}$ after all additions have been made. However, many reports simply state that a buffer of the designated $\mathrm{pH}$ was used and one is left to wonder if the actual $\mathrm{pH}$ was checked.

The $\mathrm{pH}$ of a solution of a weak acid or its salt may be estimated easily by the equations:

$$
\begin{array}{ll}
\text { Solution of HA: } & \mathrm{pH}=\frac{1}{2}\left[\mathrm{p} K_{a}-\log \left(\mathrm{A}_{t}\right)\right] \\
\text { Solution of } \mathrm{NaA}: & \mathrm{pH}=\frac{1}{2}\left[\mathrm{p} K_{w}+\mathrm{p} K_{a}+\log \left(\mathrm{A}_{t}\right)\right]
\end{array}
$$

where $K_{w}$ is the ion product of water and $\left(\mathrm{A}_{\ell}\right)=(\mathrm{HA})+\left(\mathrm{A}^{-}\right)$. Since $K_{w}$ at $37.5^{\circ}$ is approximately $2.5 \times 10^{-14}$, if the measurements are made at physiological temperatures, $\mathrm{p} K_{w}=13.6$. These equations are not exact but are satisfactory for most iulibitors at the concentrations usually used.

The presence of a buffer does not by any means ensure a constant $\mathrm{pH}$. It is especially important to know the exact $\mathrm{pH}$ at which a reaction is run when the experiment is designed to study the effects of $\mathrm{pH}$ variation; it is not enough to set up a series of reaction vessels containing a buffer at different $\mathrm{pH}$ 's and to assume that these values will not change when any addition is made to start the reaction or an inhibitor solution added to one vessel of a pair to compare witl the uninhibited rate. The buffer capacity of a solution depends on the concentration of buffer and the separation of the $\mathrm{pH}$ from the $\mathrm{p} K_{2}$ of the buffer. The enzyme solutions thus will not have the same buffering capacity at different $\mathrm{pH}$ 's and, whereas the addition of an inhibitor at one $\mathrm{pH}$ might not alter the $\mathrm{pH}$ significantly, at another initial $\mathrm{pH}$ the addition would have a marked effect. The $\mathrm{pH}$ of a reaction mixture often changes during the course of the catalysis and the resistance to this change will depend on the initial $\mathrm{pH}$ and the buffering capacity. Errors of $0.1 \mathrm{pH}$ unit or less, or changes of this magnitude during the reaction, can produce mistakes in interpretation and lead to the calculation of incorrect constants. It is, therefore, essential either to measure the $\mathrm{pH}$ after all the components have been mixed or to show by calculation that the buffer capacity is adequate; since there may be several substances involved in the buffer activity in an enzyme preparation, it is usually simpler to determine the $\mathrm{pH}$ experimentally, both at the beginning and the end of the reaction. 
The buffer value of van Slyke is defined as $d X / d_{\mathrm{pH}} \mathrm{where} X$ stands for the moles of strong acid or base added per liter of solution. Its value is given by:

$$
d X / d \mathrm{pH}=2.3 \alpha(1-\alpha)\left(\mathrm{B}_{t}\right)
$$

where $u=\left(\mathrm{B}^{-}\right) /\left(\mathrm{B}_{t}\right)$ and $\left(\mathrm{B}_{t}\right)$ is the total concentration of buffer. Alpha is also given by $K_{a} /\left[\left(\mathrm{H}^{+}\right)+K_{a}\right]$. The maximal buffer value is exhibited when $\mathrm{pH}=\mathrm{p} K_{a}$ and $\alpha=0.5$, at which point $d X / d \mathrm{pH}=0.575\left(\mathrm{~B}_{\ell}\right)$, and it falls off on either side. Let us consider a $0.02 M$ phosphate buffer with ${ }_{\mathrm{p}} K_{a}=7.2$. If the $\mathrm{pH}$ of the solution is $7, \alpha=0.389$ and $d \mathrm{X} / \mathrm{d} \mathrm{pH}=0.011$. This means that $d \mathrm{pH} / d X=90$ so that the addition of a $5 \mathrm{~m} M$ strong acid will shift the $\mathrm{pH} 0.45$ units; actually, the shift will be somewhat greater because as the $\mathrm{pH}$ is lowered, the buffer value decreases. If the $\mathrm{pH}$ had been $6, \alpha=0.0593$ and $d X / d_{\mathrm{pH}}=0.0017$, so that $d \mathrm{pH} / d X=590$ and addition of $5 \mathrm{~m} M I$ strong acid would shift the $\mathrm{pH}$ at least 3 units. The addition of a weak acid will, of course, have less effect, but can be appreciable; for example, the addition of $10 \mathrm{~m} M$ malonic acid to a $0.02 M$ phosphate buffer at $\mathrm{pH} 7$ will lower the $\mathrm{pH}$ to 4.7 . On the other hand, the addition of $10 \mathrm{~m} M$ sodium malonate will affect the $\mathrm{pH}$ negligibly. As a general rule, when the $\mathrm{pH}$ is initially one unit or more from the $\mathrm{p} K_{a}$ of the buffer, the buffering capacity will usually be low and care must be taken to prevent or detect changes in the $\mathrm{pH}$.

Buffer ions occasionally interact with the enzyme or other components of the system, and a change in $\mathrm{pH}-$ by modifying the concentrations of these buffer ions - may exert effects on the binding of the substrate or the inhibitor. We have observed that the $\mathrm{p} \boldsymbol{K}_{a}$ 's of the two enzyme groups on fumarase are different in acetate or phosphate buffers (Table 14-3). Alberty (1954) has examined the rates and constants of the fumarase reaction for their dependence on the concentration and $\mathrm{pH}$ of buffers, and has developed a theory of buffer effects that applies to inhibition. The variation of the apparent inhibitor constant with $\mathrm{pH}$ and concentration of buffer is represented by an equation of the type:

$$
K_{i}{ }^{\prime}=\frac{f_{h e}{ }^{\prime \prime}}{f_{h e i}{ }^{\prime \prime}} K_{i} \frac{1+L_{i}\left(\mathrm{~B}_{t}\right)+\mathrm{L}_{2}\left(\mathrm{~B}_{t}\right)^{2}}{1+\mathrm{L}_{3}(\mathrm{~B})}
$$

where:

$$
\begin{aligned}
\mathrm{L}_{1} & =\frac{f_{h b e}{ }^{\prime \prime}}{f_{h e}{ }^{\prime \prime}} K_{b e}+\frac{f_{h e b}{ }^{\prime \prime}}{f_{h e}{ }^{\prime \prime}} K_{e b} \\
\mathrm{~L}_{2} & =\frac{f_{h b e b}{ }^{\prime \prime}}{f_{h e}{ }^{\prime \prime} K_{b e} K_{b e b}} \\
\mathrm{~L}_{3} & =\frac{f_{h b e i}{ }^{\prime \prime} K_{e i}}{f_{h e i}{ }^{\prime \prime} K_{b e} K_{b e i}}
\end{aligned}
$$


It was assumed that buffer ions could bind to the enzyme competitively and noncompetitively with regard to the substrate, the former being represented by $\mathrm{EB}$ and the latter by $\mathrm{BE}$. The inhibition is competitive and hence BEI may be formed but not EBI. The situation is complex, the data are difficult to interpret, and experimentally there is no way to avoid such effects (unless it is possible to find another buffer with the proper $\mathrm{p} K_{a}$ and which does not interact appreciably with the enzyme).

The complications sometimes introduced by the buffer make it reasonable to reduce the buffer concentration as much as possible consonant with the system used. Some enzymic reactions do not alter the $\mathrm{pH}$ as they proceed and some can be measured before any $\mathrm{pH}$ changes might occur; in such cases, high concentrations of buffer are superfluous. If each of the solutions that must be mixed to give the final enzyme preparation can be adjusted to a single desired $\mathrm{pH}$, the presence of a buffer is not so important. In many cases, 0.01 to $1.0 \mathrm{M}$ buffers are used withont reason, whereas the concentrations could be reduced to minimize any buffer effects.*

\section{Suggestions for $\mathrm{pH}$ Studies of Inhibition}

Certain practical points may be summarized from the discussions covered so far in this chapter. These may be useful in planning investigations of enzymes and their inhibition as related to $\mathrm{pH}$ and thus are itemized here.

$A$. Many reports deal with the characterization of purified enzymes. In most of these studies, the rate is determined at several $\mathrm{pH}$ 's and the $\mathrm{pH}_{\text {opt }}$ given. This is a useful figure but it is not as informative as the $\mathrm{p} K_{a}$ 's of ionizing groups at the active center, since from these some knowledge of the nature of the groups participating in the catalysis can often be obtained. Similarly, when certain types of inhibitors are used, it is not difficult to determine $K_{i}^{\prime}$ at a few $\mathrm{pH}$ 's and, by suitable plotting, to arrive at a better understanding of the chemical make-up of the active center.

$B$. Inhibition studies of an enzyme should be made at a $\mathrm{pH}$ approximating that in the cells from which the enzyme was obtained, if this $\mathrm{pH}$ is known or can be estimated, whatever the $\mathrm{pH}_{o p t}$ of the enzyme. Only data obtained at a physiological $\mathrm{pH}$ can be applied to inhibitions in the living cells.

$C$. The reversibility of $\mathrm{pH}$ effects should be checked in order to recognize and eliminate denaturation and other irreversible inactivation processes; unless this is done, the data cannot be correctly interpreted by the kinetic and plotting procedures discussed above.

* It is interesting that in the Methods in Enzymology (Colowick and Kaplan, 1955) the buffer concentrations used in the standard enzyme assays average $0.057 M$ and in $18 \%$ the buffer concentration is $0 . \mathrm{I} M$ or over. 
$D$. The determination of $K_{i}{ }^{\prime}$ values at different $\mathrm{pH}$ 's should be done with different methods of plotting so that the relationship between $K_{i}{ }^{\prime}$ and $K_{i}$ can be more certainly established.

$E$. In comparing inhibitors on an enzyme, take the $\mathrm{pH}$ effect into account and determine the true inhibitor constant, $K_{i}$, since it is only from $K_{i}$ that the relative affinities and binding energies may be calculated.

$F$. It is better to determine directly the $\mathrm{pH}$ of each enzyme reaction medinm, after all additions and at the beginning and end of the experimental interval, rather than trust a buffer throughout the range of $\mathrm{pH}$ covered.

$G$. In all studies of enzymes, including inhibition, use the lowest concentration of buffer possible under the circumstances so as to avoid buffer effects that may complicate the kinetics. Most buffers are substances that do not occur within cells, or not at the concentrations commonly used, and as a general principle it is advisable to introduce as few unnatural substances as possible and to use media that are as close in composition to the cytoplasm as our information allows.

\section{VARIATION OF MULTIENZYME INHIBITION WITH PH}

The kinetics of multienzyme systems were found in Chapter 7 to be fairly complex and the addition of $\mathrm{pH}$ dependence for each of the steps, dne to ionization of substrates, enzymes, or both, allows the kinetics to be extended in a straightforward manner but often makes the interpretation of experimental results difficult unless the behavior of each enzyme in the system is understood. Yet it is with such problems that one must deal in investigating the response of particulate or cellular metabolic systems to changes in the $\mathrm{pH}$. The individual rates of the various reactions in a multienzyme system will generally show different types of dependence on the $\mathrm{pH}$ and thus any variation in the $\mathrm{pH}$ will alter the steady-state characteristics and the over-all flow pattern. If the substrate, $\mathrm{A}$, and the intermediate, $\mathrm{B}$, in the simple monolinear chain, $\mathrm{A} \rightarrow \mathrm{B} \rightarrow \mathrm{C}$, both ionize, the concentration of the active form of $\mathrm{B}$ will change with $\mathrm{pH}$ not only because of the direct effect on its ionization but also because of the altered rates of the two reactions. In a divergent chain, as in scheme $7-25$, the relative rates at which $\mathrm{C}$ and $\mathrm{D}$ are formed may be dependent on the $\mathrm{pH}$, since $\mathrm{E}_{2}$ and $\mathrm{E}_{3}$ will be affected differently by changes in the $\mathrm{pH}$. It is on the background of such behavior that inhibition in these systems must be considered.

The steady-state kinetics for multienzyme systems are usually obtained by expressing the rates of the various reactions $-v_{1}, v_{2}, v_{3}, \ldots-$ and then relating these rates appropriately in an equation. When the $\mathrm{pH}$ is to be 
taken into account, each rate must now be written with the proper $\mathrm{pH}$ functions included. For example, in a two-step monolinear chain:

$$
\mathrm{A} \stackrel{\mathrm{E}_{1}}{\rightarrow} \mathrm{B} \stackrel{\mathrm{E}_{2}}{\rightarrow} \mathrm{C}
$$

if both $\mathrm{A}$ and $\mathrm{B}$ ionize but the enzyme does not over the range studied:

$$
\begin{aligned}
& v_{1}=V_{1} \frac{\left(\mathrm{A}_{t}\right)}{\left(\mathrm{A}_{t}\right)+f_{a} K_{1}} \\
& v_{2}=V_{2} \frac{\left(\mathrm{B}_{t}\right)}{\left(\mathrm{B}_{t}\right)+f_{b} K_{2}}
\end{aligned}
$$

where the same conventions are used as in Eq. 7-2. The concentration of $\mathrm{B}$ in the steady state will be given by setting $v_{1}=v_{2}$ :

$$
\left(\mathrm{B}_{t}\right)=\frac{f_{b} V_{\mathbf{1}} K_{2}\left(\mathrm{~A}_{t}\right)}{\left(\mathrm{A}_{t}\right)\left(V_{2}-V_{1}\right)+f_{a} V_{2} V_{1}}
$$

which may be compared with 7-3.

If an ionizable inhibitor competitively inhibits $\mathrm{E}_{2}$, the rate of reaction 2 must now be written as:

$$
v_{2}=V_{2} \frac{\left(\mathrm{B}_{t}\right)_{i}}{\left(\mathrm{~B}_{t}\right)_{i}+f_{b} K_{2}\left[1=\frac{(\mathrm{I})}{f_{i} K_{i}}\right]}
$$

so that the change in the intermediate concentration induced by the inhibitor will be given by:

$$
\frac{\left(\mathrm{B}_{t}\right)_{i}}{\left(\mathrm{~B}_{t}\right)}=1+\frac{(\mathrm{I})}{f_{i} K_{i}}
$$

which differs from Eq. 7-8 only in the $f_{i}$ factor. Thus the effect of the inhibitor on $\left(\mathrm{B}_{t}\right)$ will not depend on the ionizations of the substrate or intermediate but only on the ionization of the inhibitor.

When the enzyme also ionizes, the rate expressions for the inhibited step will depend on the type of inhibition, as discussed previously in this chapter. Let us assume that each enzyme conforms to scheme 14-31 and the inhibition on $\mathrm{E}_{2}$ is of the type IV a shown in scheme 14-52. The individual rate expressions will be:

$$
\begin{aligned}
& v_{1}=V_{1} \frac{\left(\mathrm{A}_{t}\right)}{\left(\mathrm{A}_{t}\right)+f_{e} f_{a} K_{i}} \\
& v_{2}=V_{2} \frac{\left(\mathrm{B}_{t}\right)_{i}}{\left(\mathrm{~B}_{t}\right)_{i}+f_{e_{2}} f_{b} K_{2}\left[f_{e_{2}}+\frac{(\mathrm{I})}{f_{i} K_{i}}\right]}
\end{aligned}
$$


Solving for $\left(\mathrm{B}_{\ell}\right)_{i}$, and $\left(\mathrm{B}_{\ell}\right)$ in the uninhibited system, it is found that:

$$
\frac{\left(\mathrm{B}_{t}\right)_{i}}{\left(\mathrm{~B}_{t}\right)}=f_{e_{2}}+\frac{(\mathrm{I})}{f_{i} K_{i}}
$$

Thus the expressions indicating the change in $\left(\mathrm{B}_{\ell}\right)$ upon inhibition are in these cases modified in a simple manner, even when all the components of the system ionize.

The same procedure as outlined here can be applied to the various types of multienzyme systems and their inhibitions to obtain expressions for the concentrations of the intermediates and to precict the behavior of the rates of formation of the products.

\section{VARIATION OF INTRACELLULAR INHIBITION WITH PH}

The various ionic forms of an ionizing inhibitor - I, HI, $\mathrm{H}_{2} \mathrm{I}$, etc. usually penetrate cell membranes at greatly different rates. It is generally assumed that the uncharged form penetrates the most rapidly and the highest charged form the least rapidly (this is discussed in more detail on page 721). It would appear that in some cases, for practical purposes, certain forms of the inhibitor may not penetrate at all. Inhibitors in this respect do not differ from other weak acids or bases and useful information bearing on the entrance of inhibitors into cells may be obtained from results on these nonimhibiting substances. The fundamental problem in the theoretical approach to intracellular inhibition is the calculation of the concentration of the active form of the imhibitor within the cell.

The effects of changes in pH on cellular inhibition prodnced by nonionizing inhibitors must depend on variations in the membrane permeability to the inhibitor or to alterations in the metabolism brought about by shifts in the intracellular pH. It would be predicted - and there is some experimental evidence to support this - that neither the permeability nor the internal $\mathrm{pH}$ vary markedly if the external $\mathrm{pH}$ does not deviate too far from the normal physiological $\mathrm{pH}$ of the medimm. Thus little effect of $\mathrm{pH}$ on inhibition would be expected over this restricted range. However, different types of cells undoubtedly behave in various ways when the external $\mathrm{pH}$ is changed and also the response of the internal $\mathrm{pH}$ will vary with the nature of the buffers in the medim. The $\mathrm{pH}$ effects in such systems camnot be treated theoretically and we shall restrict our discussion mainly to ionizable inhibitors.

Distribution of Ionizable Inhibitors between the Medium and the Cells

The final concentrations of the ionic forms of an inhibitor within a cell will depend on the external $\mathrm{pH}$, the internal $\mathrm{pH}$, the form or forms of the inhibitor that can penetrate the membrane, and the buffer capacity of 
the cell contents. In the following preliminary treatments of the problem, several assumptions are made: (1) the external concentration of the inhibitor remains constant, i.e., the inhibitor in the medium is not depleted significantly because of the penetration into the cells, (2) the external $\mathrm{pH}$ remains constant at its initial value, (3) the $\mathrm{pH}$ and the distribution of the inhibitor within the cells are homogeneous, (4) the system has reached equilibrium, and (5) only the uncharged form of the inhibitor penetrates into the cell. The buffering capacity of the cell is very important in determining the distribution of the inhibitor and we shall first treat the two extreme situations - where the cell is completely buffered so that the internal $\mathrm{pH}$ does not change and where the cell is unbuffered.

Case I: cells completely buffered. The equilibria involved may be represented by the following diagram:

$$
\begin{gathered}
\mathrm{pH}_{o} \\
\mathrm{H}_{o}+\mathrm{I}_{o} \rightleftharpoons \mathrm{HI}_{o} \stackrel{\mathrm{pH}_{i}}{\rightleftharpoons} \mathrm{HI}_{i} \rightleftharpoons \mathrm{I}_{i}+\mathrm{H}_{i}
\end{gathered}
$$

The addition of the inhibitor will result in the entrance of HI into the cells and this HI will dissociate according to the intracellular $\mathrm{pH}$ and the $\mathrm{p} K_{a}$ of the inhibitor. For each molecule of HI that enters the cell, one hydrogen ion is formed upon dissociation and one ion of $\mathrm{I}^{-}$is released if the inhibitor is an acid. If the inhibitor is a weak base, the I form will penetrate and within the cell can pick up a proton to form $\mathrm{HI}^{+}$. The exchange of protons in either case must occur with the cell buffers in order that the $\mathrm{pH}$ will not change. The uncharged form will enter the cell until its external and internal concentrations are equal. The concentration of the charged form within the cell may now be calculated.

The equilibria outside and inside the cell may be written as:

$$
\frac{(\mathrm{H})_{o}(\mathrm{I})_{o}}{(\mathrm{HI})_{o}}=\frac{(\mathrm{H})_{i}(\mathrm{I})_{i}}{(\mathrm{HI})_{i}}=K_{a}
$$

and, since $(\mathrm{HI})_{0}=(\mathrm{HI})_{i}$ :

$$
(\mathrm{I})_{i}=(\mathrm{I})_{o} \frac{(\mathrm{H})_{o}}{(\mathrm{H})_{i}}
$$

$(\mathrm{I})_{0}$ is related to $\left(\mathrm{I}_{t}\right)_{0}$, the total inhibitor concentration in the medium, through the $\mathrm{pH}$ functions used earlier in the chapter, i.e., $(\mathrm{I})_{0}=\left(\mathrm{I}_{t}\right)_{0} /_{0} f_{i}{ }^{\prime}$. The subscript on the left of the $\mathrm{pH}$ function will, in each case, indicate whether the $\mathrm{pH}$ to be used is that outside or inside the cells. The internal 
concentration of $I$ in terms of the total external inhibitor concentration is given by:

$$
(\mathrm{I})_{i}=\frac{\left(\mathrm{I}_{t}\right)_{o}(\mathrm{H})_{o}}{{ }_{0} f_{i}^{\prime}(\mathrm{H})_{i}}
$$

The charged form of the inhibitor is often the active form within the cell and thus its concentration will frequently determine the degree of inhibition. The expression for $(\mathrm{HI})_{i}$ in terms of the total external concentration is simply:

$$
(\mathrm{HI})_{i}=\frac{\left(\mathrm{I}_{t}\right)_{0}}{{ }_{0} f_{h i}{ }^{\prime}}
$$

and the total concentration of the inhibitor in the cell is given by:

$$
\left(\mathrm{I}_{t}\right)_{i}=\frac{{ }_{i} f_{i}^{\prime}\left(\mathrm{I}_{t}\right)_{e}(\mathrm{H})_{o}}{{ }_{0} f_{i}{ }^{\prime}(\mathrm{H})_{i}}
$$

These concentrations are plotted for an hypothetical case in Fig. 14-16.

There are two particularly important points to note in these curves. The first is that the intracellular concentration of the ionic form varies with the

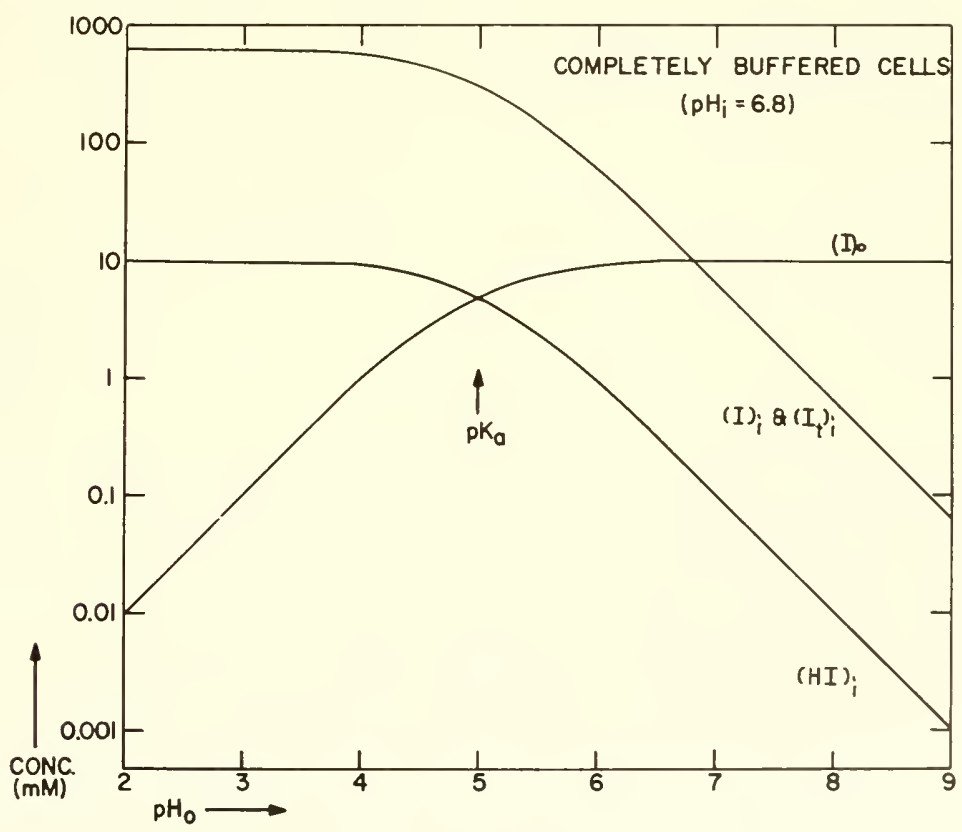

FIG. 14-16. Variation of the intracellular inhibitor concentration with $\mathrm{pH}$ (scheme 14-150). The cells are completely buffered at $\mathrm{pH}_{i}=6.8$. $\left(\mathrm{I}_{t}\right)_{o}=10$ $\mathrm{m} M, K_{a}=10^{-5}$, and $(\mathrm{H})_{i}=1.6 \times 10^{-7} M$. 
$\mathrm{pH}$ in a manner opposite to the external concentration. The $\mathrm{pH}$ dependence of cellular enzyme inhibition may thus be quite different from that of the same enzyme when isolated and in solution. The second point is that the intracellular concentration of the inhibitor may be much higher than in the medium; in the example chosen in Fig. 14-16, it is 63.5 times as great. It is evident that $\left(\mathrm{I}_{t}\right)_{0}=\left(\mathrm{I}_{t}\right)_{i}$ when $\mathrm{pH}_{o}=\mathrm{pH}_{i}$; below $\mathrm{pH}_{i}$ the internal concentration will be greater and above $\mathrm{pH}_{i}$ it will be smaller than outside. The inhibition of an enzyme within the cell may thus be much greater or much less than would be expected from the external inhibitor concentration and studies of the enzyme in vitro.

Case II: cells unbuffered. In addition to Eq. 14-151 and 14-152, there is now a further relationship:

$$
(\mathrm{H})_{i}=(\mathrm{H})_{i}^{0}+(\mathrm{I})_{i}
$$

where $(\mathrm{H})_{i}^{0}$ is the initial hydrogen ion concentration in the cell before the inhibitor is added. This equation results from the fact that for every I released in the cell, a corresponding proton arises. Substituting for $(\mathbf{I})_{i}$ :

$$
(\mathrm{H})_{i}=(\mathrm{H})_{i}^{0}+\frac{K_{a}(\mathrm{HI})_{o}}{(\mathrm{H})_{i}}
$$

and, since $(\mathrm{HI})_{0}=\left(\mathrm{I}_{t}\right)_{0} / f_{h} f_{h i}$ :

$$
(\mathrm{H})_{i}^{2}-(\mathrm{H})_{i}^{0}(\mathrm{H})_{i}-\frac{K_{a}\left(\mathrm{I}_{t}\right)_{0}}{{ }_{0} f_{h i}{ }^{\prime}}=0
$$

When $(\mathrm{H})_{i}^{0}$ is negligible - and we shall see that it usually is - this may be simplified to:

$$
(\mathrm{H})_{i}=\sqrt{\frac{K_{a}\left(\mathrm{I}_{t}\right)_{o}}{{ }_{o} f_{h i}{ }^{\prime}}}
$$

The value of $(\mathrm{I})_{i}$ can be obtained from Eq. 14-156 using $(\mathrm{H})_{i}$ from either Eqs. 14-158 or 14-159 and (HI) ${ }_{i}$ is still given by Eq. 14-154. The concentrations of the forms of the inhibitor and the intracellular $\mathrm{pH}$ are plotted in Fig. 14-17 for the same system as in the previous figure.

The internal concentration of inhibitor does not rise above that in the medium and over most of the $\mathrm{pH}$ range is less. It is, furthermore, interesting to note that the internal $\mathrm{pH}$ is reduced appreciably over a wide range of external $\mathrm{pH}$. That is, even at a $\mathrm{pH}$ far above $\mathrm{pH}_{i}^{0}$, sufficient HI will enter the cell to lower $\mathrm{pH}_{i}$; of course, very little $\mathrm{HI}$ is required when the initial hydrogen ion concentration is as low as it is in most cells. In the example chosen, $(\mathrm{H})_{i}^{0}$ may be ignored up to $\mathrm{pH}_{0}=9$ with less than a $5 \%$ error in using Eq. 14-159. 
These two simple and extreme cases introduce us to the problems that must be encountered when one attempts to formulate the $\mathrm{pH}$ dependence of intracellular inhibition. These basic problems revolve around the mucertainty and variability in the intracellular $\mathrm{pH}$, the nature and the efficiency of the buffers within the cell, and the permeability properties of the cell membrane. Before this preliminary treatment can be extended and applied to experimental data, it will be worthwhile to discuss these problems so that they may point the direction to an improvement of the theory.

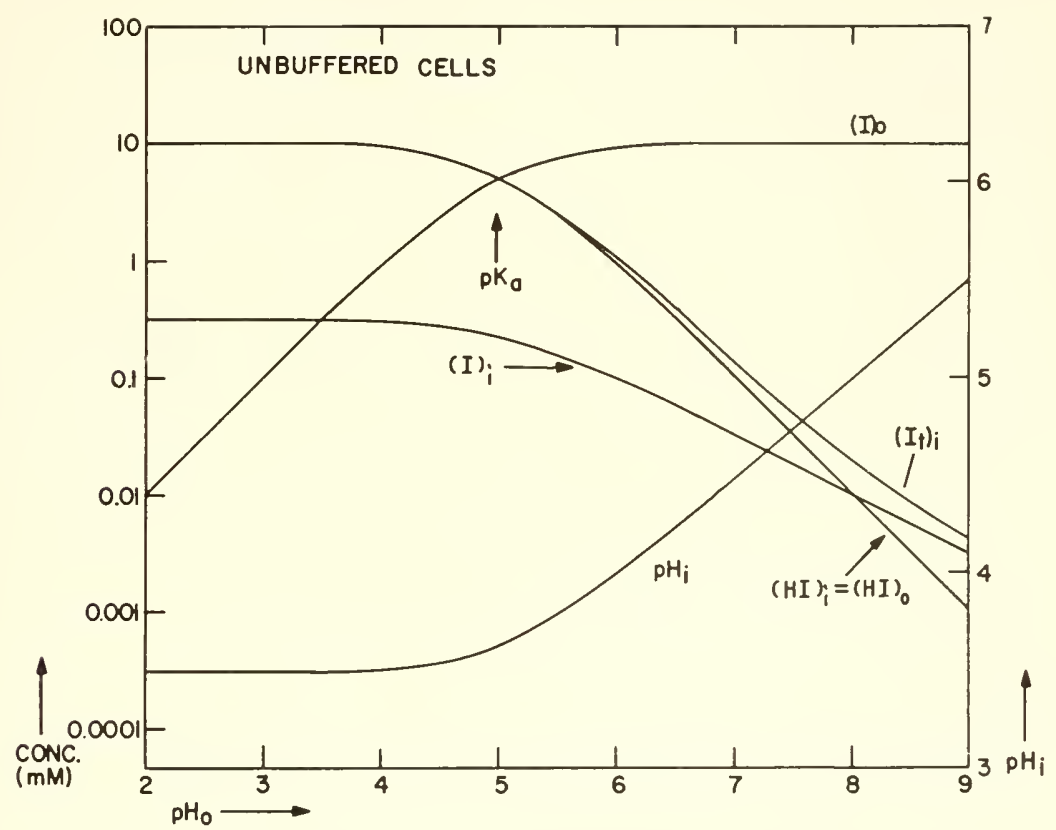

FIG. 14-17. Variation of the intracellular inhibitor concentration and the internal $\mathrm{pH}$ with the external $\mathrm{pH}$ (scheme 14-150). The cells are unbuffered with an initial $\mathrm{pH}_{i}$ of 6.8. $\left(\mathrm{I}_{t}\right)_{0}=10 \mathrm{~m} . \mathrm{I} . K_{a}=10^{-5}$ and $(\mathrm{H})_{i}^{0}=1.6 \times 10^{-7} \mathrm{H}$.

The Bulk Intracellular $\mathrm{pH}$

Determinations of intracellular $\mathrm{pH}$ have been made for the past 50 years with a variety of techniques and under greatly different conditions of the cells. The results of different workers have often shown diserepancies, which is not surprising considering that some of the techniques for measuring the $\mathrm{pH}$ (e.g., the use of dye indieators) are subject to large errors and that any deviation in the state of the tissue from normalcy leads to rapid and marked changes in the $\mathrm{pH}$. The recent development of micro-pH- 
electrodes for intracellular recording will allow more accurate measurements to be made. The thorough review by Caldwell (1956) covers these problems and presents a complete summary of the intracellular $\mathrm{pH}$ values that have been obtained in a variety of cells. Examining the data carefully, eliminating the results from inaccurate methods and from probably abnormal cell preparations, one may arrive at reasonably consistent values for the most likely $\mathrm{pH}_{i}$ 's in important types of cells. It would appear that the following cells generally have a $\mathrm{pH}_{i}$ between 6.8 and 6.9: skeletal muscle, cardiac muscle*, liver, pancreas, kidney, gastric mucosa, tumors (various sarcomas and carcinomas), and amebas. The $\mathrm{pH}_{i}$ in some cells is a little higher - frog and invertebrate nerve (7.05) and erythrocytes (7.2) and in others lower - marine invertebrate eggs (6.65) and yeast (5.8). The vacuolar fluid in large algal cells and the higher plants is usually between 5.8 and 6.0 , but there are no reliable data for the $\mathrm{pH}$ of plant protoplasm. In any event, the majority of cells may be considered to have a $\mathrm{pH}_{i}$ in the neighborhood of 6.8 and this value will be assumed in the examples and calculations of this chapter.

Although the normal $\mathrm{pH}_{i}$ probably does not fluctuate widely under physiological conditions, it drops fairly quickly when the cell is injured. As muscle becomes fatigued and passes into rigor, the $\mathrm{pH}_{i}$ usually falls 0.8 1.0 units, and cells that have sustained enough injury to produce cytolysis will often show a drop of 1.2-1.8 units. The $\mathrm{pH}_{i}$ is closely related to the metabolism for several reasons - the release of protons in oxidations, the formation and utilization of many weak acids, the ionization reactions of nucleotides, etc. - and thus anoxia appears to be capable of altering $\mathrm{pH}_{i}$ readily. In experiments involving living cells with regard to the $\mathrm{pH}$ dependence of inhibition it is necessary to ensure physiological conditions, particularly to provide as normal a medium as possible and to maintain oxygenation of the cells.

\section{Effects of Inhibitors on Intracellular $\mathrm{pH}$}

There are two important ways in which inhibitors can alter the $\mathrm{pH}_{i}$. The first is by entry into the cell of undissociated acid or dissociated base, as illustrated by the case of the unbuffered cells discussed above. The second is through their effects on the cell metabolism. Inasmuch as anoxia readily causes a fall in $\mathrm{pH}_{i}$, one might expect many, inhibitors to do likewise. In part this would be due to the accumulation of acid intermediates, such as lactic acid, and in part due to shifts in the equilibria of the adenine nucleotides and creatine phosphate. If the formation of lactic acid by gly-

* Preliminary and unpublished experiments by Dr. Philip Hollander using a micro$\mathrm{pH}$-electrode in rat atrium and ventricle indicate the normal $\mathrm{pH}_{i}$ for actively contracting tissue to be 6.8 . 
colysis were important in this fall of $\mathrm{pH}_{i}$, one might predict that iodoacetate would prevent this. Actually, Lundsgaard (1930 a) showed that muscle, instead of becoming more acid when inhibited by iodoacetate, exhibited a slight rise in $\mathrm{pH}_{i}$. Voegtlin et al. (1934) found that injections of iodoacetate into rats raised the muscle $\mathrm{pH}_{i}$ whereas cyanide lowered it, although these experiments were complicated by the changes in respiratory function. Dubuisson and Schulz (1938) reported that anaerobic tetanus in frog minscle resulted in a $\mathrm{pH}_{i}$ of 6.93 whereas in the presence of iodoacetate the $\mathrm{pH}_{i}$ was 7.19 . There has been understandably very little work on the changes in $\mathrm{pH}_{i}$ brought about by inhibitors but it is an important aspect of the problem and it is to be hoped that the recent advances in techniques will soon provide some information on this matter. Certainly. some of the actions of inhibitors conld be the result of intracellular changes in $\mathrm{pH}_{i}$ brought about in either of the two ways mentioned.

\section{Heterogeneity of Intracellular $\mathrm{pH}$}

The $\mathrm{pH}_{i}$ is not uniform throughout the cell. There is very little experimental evidence for this statement but our knowledge of the interior of the cell makes it necessary to assume such a heterogeneity. The structural complexity of the cytoplasm becomes more apparent each year and the possibility of many intracellular compartments becomes more likely. The extrusion of cell contents in order to determine the $\mathrm{pH}$ leads to mixing and a disruption of the structure that provides the bulk $\mathrm{pH}_{i}$ values described above. Even the insertion of a micro- $\mathrm{pH}$-electrode, small enough not to damage the cell irreversibly, must modify the cytoplasmic structure in the region of the electrode tip and again can provide only an average cytoplasmic $\mathrm{pH}$. The concept of a continuous bulk phase of cytoplasmic ground substance, as assumed by the early cytologists, is no longer tenable.

Where within cells might the $\mathrm{pH}$ be particularly different from the mean or bulk $\mathrm{pH}$ ? The following come to mind immediately: (a) mitochondria, (b) vacuoles, $(c)$ the region between double membranes, $(d)$ the surfaces and interiors of membranes, especially when an electric potential exists, (e) within gel structures, such as myofibrils or spindle fibers, and $(f)$ sites where certain types of metabolism proceed at a high rate. The $\mathrm{pH}$ near a charged surface, such as that of a protein or nucleic acid, is not that of the medium, due to the attraction or repulsion of such surfaces for protons. Furthermore, each region possessing a structurally fixed constellation of charged groups will have a $\mathrm{pH}$ determined by Donnan equilibria. The occurrence of such multiple Donnan equilibria within cells has been treated quantitatively by Caldwell (1956). The heterogeneity of the $\mathrm{pH}$ is expressed not only in the microscopically observable cell components and structures, but also on the molecular level throughout the cell. 
Early work on the interfacial tension of fihms of the long-chain carboxvlates or amines showed that the inflections produced by varying the $\mathrm{pH}$ did not correspond to the $\mathrm{p} K_{\alpha}$ 's of the substances, indicating that the $\mathrm{pH}$ in the film may not be the same as in the bulk phase. Danielli (1937) extended these observations and from the assumption of a Donnan equilibrium between the film and the bulk phase calculated that the film $\mathrm{pH}$ may be as much as 2 units different than the bulk phase pH. Hartley and Roe (1940) calculated the distribution of ions near particles possessing $\zeta$-potentials and with respect to protons obtained the following equation:

$$
\left(\mathrm{H}^{+}\right)_{s}=\left(\mathrm{H}^{+}\right)_{b} e^{-\varepsilon \zeta / k^{T}}
$$

where $\left(\mathrm{H}^{+}\right)_{s}$ and $\left(\mathrm{H}^{+}\right)_{b}$ represent the concentrations at the surface and in the bulk phase respectively and $\zeta$ is the electric potential between the particle and the medium. This can be rewritten as:

$$
\mathrm{pH}_{s}=\mathrm{pH}_{b}+\frac{\zeta}{62}
$$

where $\zeta$ is in millivolts and the temperature is $37.5^{\circ}$. Since potentials of 50 to 100 millivolts are quite common, it is seen that a significant difference in $\mathrm{pH}$ is produced. They applied the theory to studies of amieroseopie micelles of surfactant compounds and found for triethanolamine cetane sulfonate that $\mathrm{pH}_{b}-\mathrm{pH}_{s}$ was around 1.5 units. The calculations of surface $\mathrm{pH}$ values for proteins by either Donnan equilibria or electrokinetie data were shown by Danielli (1941) to give comparable results. A plot of $\mathrm{pH}_{s}-$ $\mathrm{pH}_{b}$ against $\mathrm{pH}_{b}$ for ovalbumin showed that the surface and bulk phase has the same $\mathrm{pH}$ when $\mathrm{pH}_{b}$ was 4.8; as $\mathrm{pH}_{b}$ is lowered, the $\mathrm{pH}_{s}$ does not decrease as rapidly and hence $\mathrm{pH}_{s}-\mathrm{pH}_{b}$ is positive, and upon raising the $\mathrm{pH}_{b}$, the difference becomes negative. At $\mathrm{pH}_{b}=7$. the difference was about 0.5 unit. The pH difference, of course, depends on many factors, such as the ionic strength, the concentration of buffers, the amphoteric properties of the protein, and the protein concentration, so that such results eannot be directly applied to the cell. However, they do indicate the magnitude of the effects to be expected.

What is the importance of this spatial variation in $p H$ for inhibitor studies? Of greatest significance is the fact that it may lead to a heterogeneous distribution of the inhibitor within the cell, if the inhibitor is a weak acid or base. We have seen that the concentrations of total inhibitor and its ionic forms depend on the $\mathrm{pH}_{i}$ in buffered cells. In the same manner, the concentration of inhibitor within any cell compartment will depend on the $\mathrm{pH}$ of that compartment. The concentration of active inhibitor may vary from region to region at equilibrimm and different degrees of effect will be exerted on the enzymes in these regions. Let us assume that the enzyme with 
which we are concerned is almost totally restricted to the mitochondria; the internal mitochondrial $\mathrm{pH}$ is 5.8 and the experimental bulk $\mathrm{pH}_{i}$ is 6.8. When the cells are allowed to come to equilibrinm with a weakly acidic inhibitor, the concentration of $\mathrm{I}^{-}$, the active form, will vary inversely with the hydrogen ion concentration of the region (Eq. 14-152). In this case, the concentration of $\mathrm{I}^{-}$, in the mitochondria will be one-tenth that calculated on the basis of $\mathrm{pH}_{i}$. Accumulation of an inhibitor in a region within the cell can also occur by this mechanism. What is fundamentally important is the $\mathrm{pH}$ of the region in which the inhibited enzyme is located. When calculations of inhibitor distributions or intracellular inhibitions are made using the bulk cytoplasmic $\mathrm{pH}_{i}$. the results should be considered to be mean values at the best.

The action of an inhibitor is usually not uniform throughout a cell because of its distribution and the fact that the susceptible enzymes are distributed heterogeneously. The entrance of the inhibitor into the cell and its action there will thus not change the internal $\mathrm{pH}$ uniformly. If the inhibitor acted specifically on the mitochondrial enzyme in the above example, the intramitochondrial $\mathrm{pH}$ might be changed to a degree depending on the buffering capacity of the mitochondria, without appreciable immediate changes in the $\mathrm{pH}$ elsewhere. Local changes in $\mathrm{pH}$ could influence markedly the metabolic and functional responses of the cells to inhibitors.

\section{Permeant Forms of Inhibitors}

Weak acids and bases penetrate into cells more rapidly in the uncharged form. This would be expected on the basis of what is known about the permeability of cells to ions and neutral molecules. There is also adequate evidence to support this concept. It has been generally found that the toxicity of weak acids becomes progressively greater as the $\mathrm{pH}$ of the medium is lowered and the concentration of the undissociated form rises. Thus Smith (1925) found that salicylate and benzoate anions are without effect on the development of sand dollar (Echinarachmius) eggs in normal sea water, but that cleavage is depressed when the $\mathrm{pH}$ is lowered to provide sufficient concentrations of the acid forms. The entrance of sulfide into Valonia cells was shown by Osterhout (1925) to be entirely due to the penetration of the membranes by $\mathrm{H}_{2} \mathrm{~S}$. Analyses of the total sulfide concentration within the cells at different external pH's are shown in Fig. 14-18 and may be compared with the curve which gives the calculated concentrations of $\mathrm{H}_{2} \mathrm{~S}$ in the medium. The actual rates of penetration were measured by Jacques (1936) and these were shown to be proportional to $\left(\mathrm{H}_{2} \mathrm{~S}\right)_{0}$ over the 5 -min periods during which the rates were measured. Likewise, the rates of penetration of arsenite into the larvae of the flesh fly were found to rise with a decrease in $\mathrm{pH}$ (Ricks and Hoskins, 1918) as would be expected if arsenious acid were the principal permeant form. Nany other examples from 
inhibition studies point to the same conclusion. The effects of malonate on seven plant tissues (Fig. 14-19) and of iodoacetate on yeast, barley

\begin{tabular}{r|c}
\hline $\mathrm{pH}$ & $\begin{array}{c}\text { Rate of penetration } \\
(\mu \mathrm{g} \text { As/larva/h })\end{array}$ \\
\hline & \\
5.0 & 3.24 \\
9.9 & 1.43 \\
11.5 & 0.26 \\
\hline
\end{tabular}

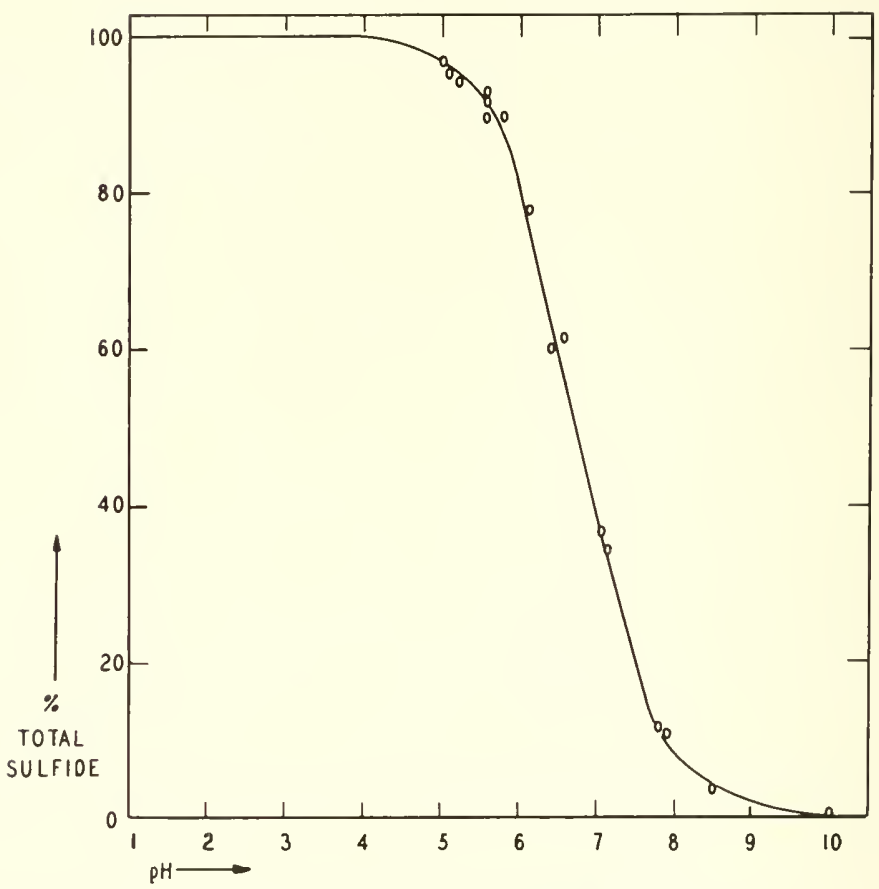

FIG. 14-18. Penetration of $\mathrm{H}_{2} \mathrm{~S}$ into Valonia cells at different external levels of the $\mathrm{pH}$. The circles show the expcrimental points obtained by Osterhout (1925) and the curve shows the per cent of the total sulfide in the medium that is $\mathrm{H}_{2} \mathrm{~S}$.

roots, and oat coleoptiles (Fig. 14-20) vary with external pH in a manner that suggests the undissociated acids to be the permeant forms. The ineffectiveness of malonate in the $\mathrm{pH}$ range above 7 has been noted in work with many tissues (e.g., Webb, 1950 a) and the actions that have been observed with high concentrations are probably due to effects on the mem- 
branes of the cells. Other evidence comes from the work of Simon and Beevers (1952) and this will be discussed in later sections. Finally, similar results have often been obtained in studies on drugs, most of which has been reviewed by Albert (1952). A particularly conclusive study on the barbiturates has been reported recently (Hardman et al., 1959) and it was demonstrated that the depressant action upon isolated cardiac tissue is proportional to the concentration of the uncharged form, the ions not exerting any effects.

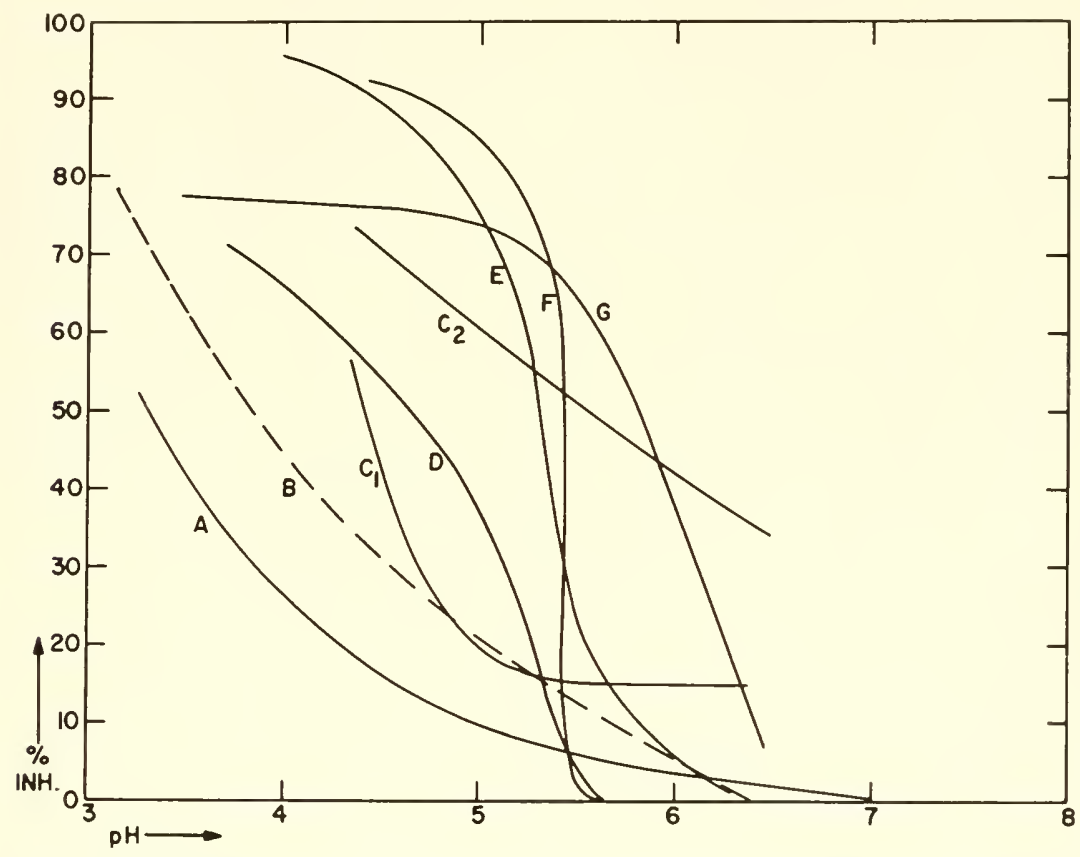

Fig. 14-19. Variations of the inhibition of plant tissue respiration by malonate at different pHs. Curve A: tomato stem slices (Link, et al., 1952). Curve B: maize roots (Beevers, 1952). Curve $\mathrm{C}_{1}$ : rhubarb leaves - malonate $=6.8 \mathrm{mM}$ (Morrison, 1950). Curve $\mathrm{C}_{2}$ : rhubarb leaves - malonate $=34 \mathrm{~m} M$ (Morrison, 1950). Curve D: barley roots (Laties, 1949). Curve E: spinach leaves (Bonner and Wildman, 1946). curve F: Avena coleoptile (Cooil, 1952). curve G: carrot root slices - 136 min after cutting (Hanly et al., 1952).

It is thus established that the uncharged forms of weak acids and bases penetrate into cells more readily than do the ionic forms. The question now arises as to whether the ionic forms penetrate at all or are of any significance in the actions of these substances. The answer to this question is not so easily given. In some cases the results conform closely to the behavior to be expected if only the neutral form penetrates and the ionic form 
is without demonstrable action; that is, the action or accumulation is directly proportional to the concentration of the nentral form and in a $\mathrm{pH}$ range where this form is negligible, the action is undetectable. In Fig. 14-19 it is seen that for most tissnes the inhibiting effect of malonate disappears as the $\mathrm{pH}$ becomes greater than 5.5-7.0. However, the inhibition of the respiration of rhubarb leaves is still apprecialole at these higher

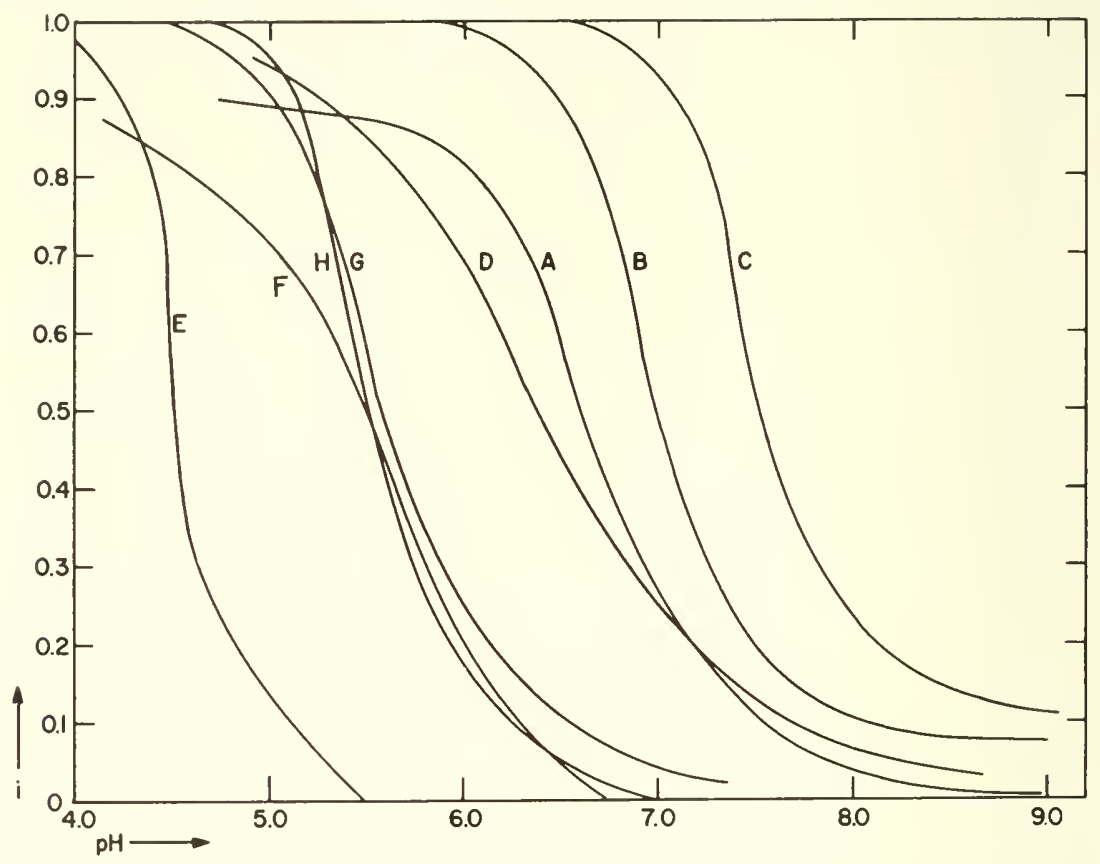

FIG. 14-20. Variations of the inhibitions produced by iodoacetate with the $\mathrm{pH}$. Curve A: yeast fermentation $-(\mathrm{IAA})=1.1 \mathrm{~m} / I$ and time $=20$ min (Sehroeder et $a l ., 1933 \mathrm{a})$. Curve B: yeast fermentation $-(\mathrm{IAA})=1.1 \mathrm{~m} M$ and time $=2 \mathrm{~h}$ (Schroeder et al., 1933 a). Curve C: yeast fermentation $-(\mathrm{IAA})=1.1 \mathrm{~m} M$ and time $=6 \mathrm{~h}$ (Sichroeder et al. . 1933a). Curve D: yeast fermentation $-($ IAA $)=1.1$ $\mathrm{m} M$ (Brïcke, 1933). ('urve E: yeast viability - (IAA) $=1 \mathrm{~m} M$ and time $=30$ min (Aldons, 1948). ('urve $\mathrm{F}$ : barley root respiration $-(\mathrm{IAA})=0.14 \mathrm{~m} M$ (Laties, 1949). Curve G: Avena coleoptile growth - (IAA) $=0.02 \mathrm{~m} / /$ (Cooil, 1952). Curve $\mathrm{H}$ : yeast fermentation $-(\mathrm{TAA})=1 \mathrm{~m} / \mathrm{V}$ and time $=45 \mathrm{~min}$ (Lundsgaard, 1932).

$\mathrm{pH}$ 's; the inhibition of $\mathrm{CO}_{2}$ production actually levels off above $\mathrm{pH} 5.3$. In this case it wonld seem that an ionic form can penetrate. Some cells are undoubtedly more permeable to ions than others and the permeabilities to cations and anions need not be equivalent or even related. Nany types of cells, such as renal tubular cells or erythrocytes, are reasonably permeable to anions. It is not only a matter of membrane permeability. Many cells 
possess a rather fixed intemal anion concentration and for an anion to enter the cell, either a cation must be taken in simultaneously or an internal anion released from the cell in exchange. The amount of exchangeable anion in many cells is very low; for example, the chloride concentration in nerve and muscle is usually less than $10 \mathrm{~m} M$. Of course, some cells have mechanisms for the active transport of organic anions and the entrance of a possible inhibitor anion would depend on how readily it would react with this mechanism.

It is also possible for the the ionic form to be active without penetrating into the cell through an action in some manner on the cell surface. The anions of the tricarboxylic acid cycle substrates exert small but definite actions on the membrane properties of cardiac cells and, through these changes, on the contractility (Webb and Hollander, 1956). These actions are presumably unrelated to their metabolic role and in a similar manner the anions of inhibitors could alter tissue function independently of any enzyme action.

The plotting of equiactive concentrations of inhibitors, or their undissociated forms, against $\mathrm{pH}$ provides a means of testing the activity of the ionic forms and it was concluded by Simon and Beevers (1952), from the results on many types of cells and substances, that the ionic form can be of importance, at least over a certain range of $\mathrm{pH}$. The ion would not have to penetrate well to have a very significant effect on the inhibition in longterm experiments, such as in studies on growth. The penetration of the ion should actnally reduce the inhibition produced by the mucharged form in many cases, becanse the ion, instead of accumulating in the cell as it is formed from the un-ionized inhibitor, will leave the cell. The results will often depend on how long the cells are incubated with the inhibitor and particularly on whether equilibrium has been reached or not. Even if only the un-ionized form penetrates, in a $\mathrm{pH}$ range much above the $\mathrm{p} K_{a}$, the rate or entrance of HI may be very slow due to the low concentration gradient and equilibrium will be achieved only slowly. If HI penetrates more rapidly than $\mathrm{I}^{-}$, HI may enter initially and the concentration of $\mathrm{I}^{-}$build up in the cell, later to be reduced as $\mathrm{I}^{-}$diffuses out of the cell. The best conclusion is probably never to ignore the ionic form until it has been experimentally proved to be impermeant or inactive.

Some confusion has occurred in the past from the use of the term active form when applied to the effects of weak acids or bases on cells. The uncharged form has frequently been called active when it has been found that the cellular action is dependent on its concentration. However, within the cell it may well not be the active form on the enzyme. It would be better to distinguish between the permeant form, that which enters the cell predominantly, and the active form, the form that combines with and inhibits the enzyme. These two forms are often different. Usually it is the ionized 
form that reacts with the enzyme and the un-ionized form that penetrates. The difference is clearly illustrated by the effeets of iodoacetate on yeast (Sehroeder et al., 1933 b). The rate of reaction of iodoacetate with the SH groups of glutathione increases with $\mathrm{pH}$ (Fig. 14-21, curve A) while the inhibition of intracellular fermentation decreases with $\mathrm{pH}$ (eurve B). Although iodoacetate does not depress fermentation by reacting with

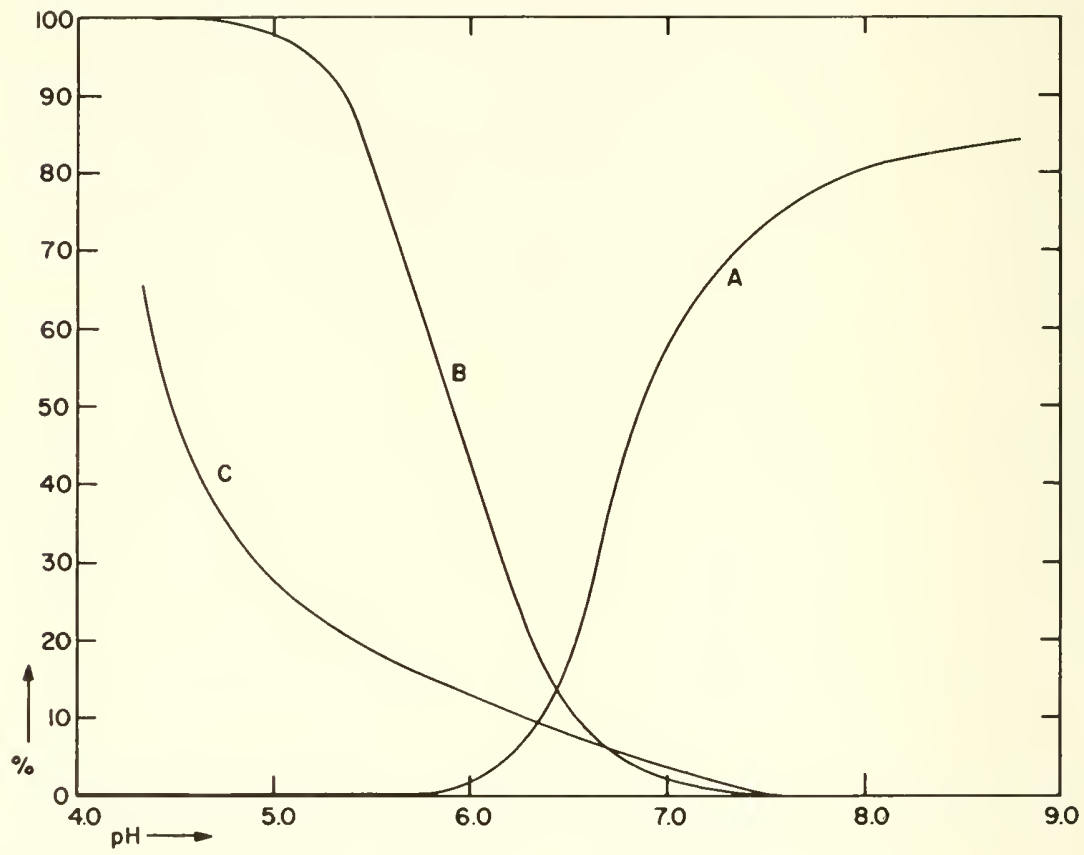

FIG. 14-21. Effect of the pH on the reaction of iodoacetate with glutathione (curve A), on the inhibition of yeast fermentation by iodoacetate (curve B), and on the reaction between iodoacetate and glutathione within yeast cells (curve C). (From Schroeder et al., 1933 b.)

glutathione, the $\mathrm{pH}$ dependence of its inhibition on phosphoglyceraldehyde dehydrogenase is probably similar. The permeant form here is iodoacetic acid and the active form is the iodoacetate anion.

\section{Buffer Capacity of Cells}

The degree of enzyme inhibition that will be produced by an inhibitor that is a weak acid or base will depend on the buffer capacity of the cell interior in many cases. This was seen in the two extreme cases discussed previously. In the completely bufferer cells, the accumulation of $\mathrm{I}^{-}$was much greater than in the unbuffered cells. The greater the buffer capacity 
of a cell, the more HI will penetrate and the greater the concentration of $\mathrm{I}^{-}$or total inhibitor in the cells. Actual cells are neither unbuffered nor completely buffered and so will respond in an intermediate fashion. It would be very useful to know the buffer capacity of cells, particularly those commonly used in inhibition studies, but no adequate quantitative work has yet been done. The newer intracellular methods for determining $\mathrm{pH}$ combined with the use of a radioactively labeled or chemically determinable weak acid or base could provide this information. One must know how much $\mathrm{H}^{+}$has been released within the cell from the $\mathrm{HI}$ that penetrates before accurate estimates of the buffer capacity can be made.

Certainly the intracellular buffer capacity is not great because changes in $\mathrm{pH}_{i}$ occur rapidly and readily with alterations of the activity or the production of injury. The $\mathrm{pH}_{i}$ is particularly sensitive to changes in $\mathrm{CO}_{2}$ tension in the medium. Caldwell (1958) has found that Carcinus muscle and Loligo axons show a drop in the $\mathrm{pH}_{i}$ of 1.1-1.3 units within 3 min following the exposure to sea water saturated with $\mathrm{CO}_{2}$ around a $\mathrm{pH}$ of 5 . The rapidity of penetration of $\mathrm{CO}_{2}$ accounts for this sudden fall in $\mathrm{pH}_{i}$. When the Carcinus muscle cells were exposed to $30 \mathrm{~m} M$ sodium hydrogen phthalate $\left(\mathrm{pH}_{o}=3.4\right)$, the drop in $\mathrm{pH}_{i}$ was much slower $(0.8$ units in $30 \mathrm{~min}$. and 1.4 units in $60 \mathrm{~min}$ ) and with phosphate at $30 \mathrm{~m} M$ and $\mathrm{pH}_{o}=5.7$ the changes were even slower $(0.3$ units in $100 \mathrm{~min})$. These two substances, however. occur at these pH's mainly in the singly ionized state and there is very little of the undissociated acid, so it is not surprising that penetration was slow. The effects of monobasic acids at $\mathrm{pH}_{o}$ 's near or below their $\mathrm{p}_{n}$ 's wonld probably occur with a rapidity comparable to the effects of $\mathrm{CO}_{2}$. Although from this work one cannot calculate the internal buffer capacity, it does provide good evidence that the $\mathrm{pH}_{i}$ does change appreciably when cells are exposed to weak acids.

It is difficult to calculate the buffer capacity of cells because there are so many components capable of playing a significant role. Proteins have rather weak buffering power from $\mathrm{pH} 4$ to 10 , but exert much more efficient buffering outside this range. The inorganic and organic phosphates usually buffer best between $\mathrm{pH} 5.5$ and 7.5 while the organic acids are effective between $\mathrm{pH} 3.6$ and 6 . There are many other substances that can play minor roles. From the titration curves of a number of proteins and the mean protein content of cells $(1.5 \mathrm{~m} M)$, one may estimate that $d \mathrm{pH} / d X$, with respect to protein, is around 80 between $\mathrm{pH} 4$ and 10 and 110 between $\mathrm{pH} 3$ and 4 . Assuming the total intracellular phosphate to be $20 \mathrm{~m} M$ with an average $\mathrm{p} K_{a}$ of $6.7, d \mathrm{pH} / d X$, with respect to phosphates, would vary from 10 to $20 \mathrm{in}$ the $\mathrm{pH}$ range of 6 to 7.5 . Thus the phosphates probably account for the greater portion of the buffer capacity in the neighborhood of the normal $\mathrm{pH}_{i}$. Below a $\mathrm{pH}_{i}$ of $6, d \mathrm{pH} / d X$ would rise, the level to a great extent being determined by the concentration of organic acids in the cell; since this varies widely, all one can say is that $d \mathrm{pH} / d X$ probably ranges from 20 
to 80 in this range. Below a $\mathrm{pH}_{i}$ of $4, d \mathrm{pH} / d X$ would again decrease due to the carboxylic gromps on the proteins. It may well be that some of these values of $d \mathrm{pH} / d X$. especially for phosphate, are too low, since all of the ionizing groups may not be free. It is not improbable that when cells are exposed to an acid solution of a $10 \mathrm{~m} M$ weak acid, between 100 and 200 $m M H A$ can enter the cell and release the equivalent amount of $\mathrm{H}^{+}$. This would drop the $\mathrm{pH}_{i}$ by at least 4 units if the mean $d \mathrm{pH} / d X$ in this range were 40 .

\section{Plotting of Equieffective Concentrations of Inhibitor Against pH (Simon-Beevers Curves)}

Now that certain factors that may play a role in the $\mathrm{pH}$ dependence of intracellular inhibition have been discussed, we may return to the original attempt to formulate these phenomena in a quantitative fashion. Some information may be obtained from plotting the total external inhibitor concentration required to prodnce a standard chosen depression against the external $\mathrm{pH}_{0}$. Althongh this method of plotting was not originated by Simon and Beevers, they applied it generally to the actions of weak acids and bases and have more than anyone developed the theoretical implications (Simon and Beevers, 1952).

Simon and Beevers pointed out that usually the most easily determined effect is a $50 \%$ response in the cells. With regard to inhibitors this means a $50 \%$ depression of the reaction or event measured. Thus one may apply this to individual enzyme effects or to a complex metabolic process or to some cellular activity, such as contraction or growth. It is very important to determine the equieffective concentrations accurately. For this purpose it is necessary to use several concentrations of the inhibitor that produce effects both less and greater than the $50 \%$ reduction at each $\mathrm{pH}_{o}$, and to plot the data to determine the intersection of the curve with the line representing $50 \%$ reduction. It is also necessary to make certain that the $\mathrm{pH}_{o}$ for each concentration of the inhibitor is constant by determining it directly.

We shall assume that the inhibitor is a weak acid and that the active form within the cell is the anion, I. It must also be assumed for the present that any changes in $\mathrm{pH}_{i}$ will not alter the inhibition produced by I. Equation 14-153 for the completely buffered case can be rewritten as:

$$
\left(\mathrm{I}_{t}\right)_{o}=(\mathrm{I})_{i} \frac{(\mathrm{H})_{i}}{(\mathrm{H})_{o}}\left[1+\frac{(\mathrm{H})_{o}}{K_{a}}\right]
$$

Taking the logarithm of both sides:

$$
\log \left(\mathrm{I}_{t}\right)_{o}=\log (\mathrm{I})_{i}-\mathrm{pH}_{i}+\mathrm{pH}_{o}+\log \left[1+\frac{(\mathrm{H})_{o}}{K_{a}}\right]
$$


For a constant degree of inhibition, $(\mathrm{I})_{i}$ must be constant and the $\mathrm{pH}_{i}$ is constant because the cells are completely buffered. At $\mathrm{pH}_{o}$ 's significantly lower or higher than $\mathrm{p} K_{2}$ this equation simplifies to:

Low $\mathrm{pH}_{o}: \quad \log \left(\mathrm{I}_{t}\right)_{o}=\log (\mathrm{I})_{i}-\mathrm{pH}_{i}+\mathrm{p} K_{a}$

High $\mathrm{pH}_{o}: \quad \log \left(\mathrm{I}_{t}\right)_{o}=\log (\mathrm{I})_{i}-\mathrm{pH}_{i}+\mathrm{pH}_{o}$

A plot of $\log \left(\mathrm{I}_{t}\right)_{0}$ against $\mathrm{pH}_{0}$ will be a curve with zero slope at low $\mathrm{pH}_{0}$ 's and with a slope of +1 at high $\mathrm{pH}_{0}$ 's (lower solid curve in Fig. 14-22).

For unbuffered cells, combining Eq. 14-156 and 14-159, neglecting $(\mathrm{H})_{i}{ }^{0}$ and writing the result in terms of $\left(\mathrm{I}_{t}\right)_{0}$, we obtain:

$$
\left(\mathrm{I}_{t}\right)_{o}=(\mathrm{I})_{i}^{2} \frac{1}{K_{a}^{-}}\left[1+\frac{K_{a}}{(\mathrm{H})_{o}}\right]
$$

Putting this into logarithmic form:

$$
\log \left(\mathrm{I}_{t}\right)_{o}=2 \log (\mathrm{I})_{i}+\mathrm{p} K_{a}+\log \left[1+\frac{K_{a}}{(\mathrm{H})_{o}}\right]
$$

Considering again ranges of $\mathrm{pH}_{o}$ significantly lower or higher than $\mathrm{p} K_{a}$ :

Low $\mathrm{pH}_{o}$ :

$$
\log \left(\mathrm{I}_{t}\right)_{0}=2 \log (\mathrm{I})_{i}+\mathrm{p} K_{a}
$$

High $\mathrm{pH}_{0}$ :

$$
\log \left(\mathrm{I}_{t}\right)_{o}=2 \log (\mathrm{I})_{i}+\mathrm{pH}_{o}
$$

Thus again a plot of $\log \left(\mathrm{I}_{t}\right)_{0}$ against $\mathrm{pH}_{0}$. with $(\mathrm{I})_{i}$ constant. will give a curve with zero slope at low $\mathrm{pH}_{0}$ 's and a slope of $+\mathrm{l}$ at high $\mathrm{pH}_{0}$ 's (npper solid curve in Fig. 14-22).

It is, therefore. impossible to distinguish between unbuffered or completely buffered cells by such plotting; it follows that intermediate cases of partial buffering will also give curves of this type. The buffer capacity of the cells will shift the curves along the $\log \left(I_{t}\right)_{0}$ axis, as seen in Fig. 14-22 where the values given on the horizontal portions of the curves are derived from a hypothetical example in which $(\mathrm{I})_{i}=1 \mathrm{~m} M, \mathrm{pH}_{i}{ }^{0}=6.8$, and $K_{a}=10^{-5} M$. If the sensitivity of the inhibited system within the cell is known, and the value of $(\mathrm{I})_{i}$ can be estimated for $50 \%$ inhibition. some idea of the buffer capacity may be obtained from the height of the horizontal portion of the experimental curve.

In a similar manner, $\log (\mathrm{HI})_{0}$ can be plotted against $\mathrm{pH}_{0}$ for the production of a chosen degree of inhibition. The equations in this case do not involve $\mathrm{pH}_{0}$ :

Completely buffered cells: $\log (\mathrm{HI})_{o}=\mathrm{p} K_{a}-\mathrm{pH}_{i}+\log (\mathrm{I})_{i}$

Unbuffered cells:

$$
\log (\mathrm{HI})_{o}=2 \log (\mathrm{I})_{i}+\mathrm{p} K_{a}
$$


The curves will be horizontal over the entire range of $\mathrm{pH}_{o}$ as shown in the dashed lines of Fig. 14-22.

What few experimental data are available do generally conform to the theoretical curves but there are important deviations from the expected behavior. Simon-Beevers' plots for the depression of yeast respiration by four inhibitors are shown in Fig. 14-23. We may conchude that the general theory is correct and that HI is the dominant permeant form of the inhibitor. It may be noted also that the values of $\log \left(\mathrm{I}_{t}\right)_{0}$ at low $\mathrm{pH}_{o}$ 's would indicate at least an appreciable buffering capacity of the yeast cells since

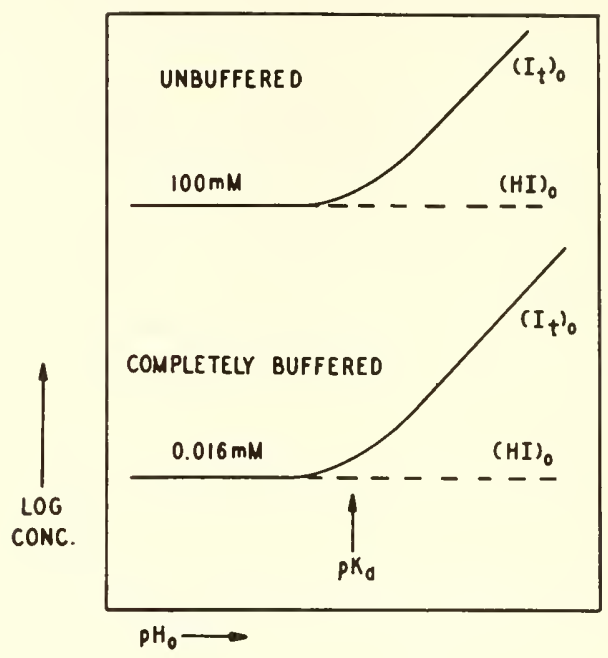

FiG. 14-22. Simon-Beevers plots for unbuffered and completely buffered cellular systems. $(\mathrm{I})_{i}$ $=1 \mathrm{~m} M, \mathrm{pH}_{i}{ }^{\circ}=6.8$, and $\mathrm{p} K_{a}=5$.

the concentrations of the inhibitors required for $50 \%$ inhibition are not much higher than would be expected from work in vitro. Simon and Beevers (1952) produced a generalized curve from the data on all the weak acids studied; from this and from the results with the inlribitors shown in Fig. 14-23, we may observe certain deviations from the theoretical curves. The most important are: $(a)$ the mean slope of the $\log \left(\mathrm{I}_{t}\right)_{0}-\mathrm{pH}_{o}$ curves at high $\mathrm{pH}_{o}$ 's is usually less than unity, and in the generalized curve of Simon and Beevers is $0.56,(b)$ the $\log \left(\mathrm{I}_{t}\right)_{0}-\mathrm{p} \mathrm{H}_{0}$ curve tends to level off at higher $\mathrm{pH}_{o}$ 's, usually when the $\mathrm{pH}_{o}$ is several units higher than the $\mathrm{p} K_{a}$, and $(c)$ the $\log (\mathrm{HI})_{0}-\mathrm{pH}_{o}$ curves almost invariably develop a negative slope in the $\mathrm{pH}_{o}$ range above $\mathrm{p} K_{a}$ [this is, of course, related to the slope of less than unity in the $\log \left(\mathrm{I}_{t}\right)_{0}-\mathrm{pH}_{0}$ curve in this range of $\mathrm{pH}_{0}$ ]. Attempts must now be 
made to explain these discrepancies. It is predicted that a further deviation will be observed when studies are carried to lower $\mathrm{pH}_{o}$ 's than previously, namely that curves in this region will fail to remain horizontal. In very few of the published studies has the $\mathrm{pH}_{0}$ been reduced much below the $\mathrm{p} K_{a}$ of the weak acid tested.

Some of the factors that may modify the configuration of the theoretical

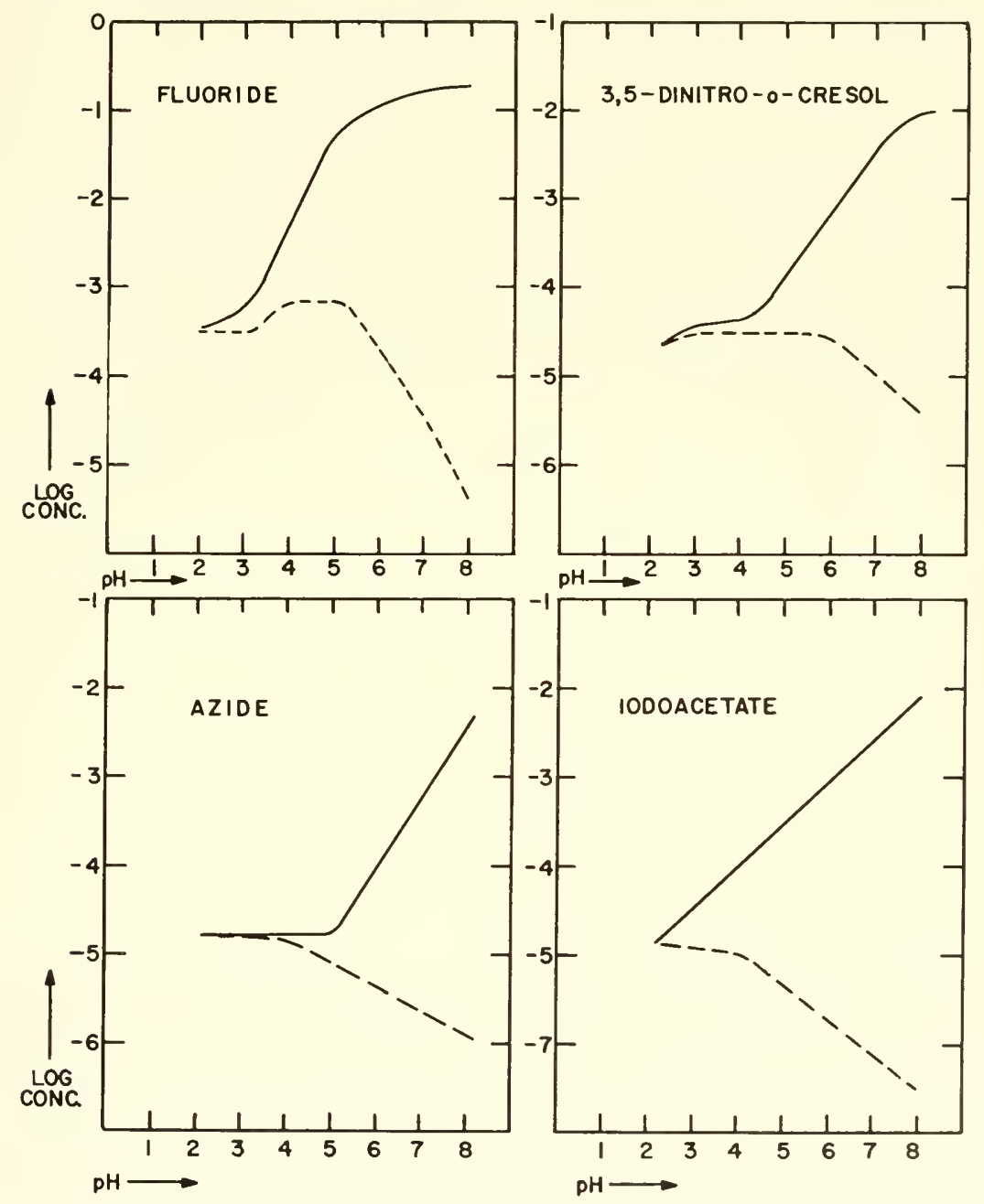

FIG. 14-23. Simon-Beevers plots for the inhibition of yeast respiration by four inhibitors. The solid lines represent the total inhibitor concentration and the dashed lines the concentration of the undissociated forms of the inhibitors. (From Simon and Beevers, 1952.) 
$\log \left(\mathrm{I}_{t}\right)_{0}-\mathrm{pH}_{o}$ curves will be listed and the changes in the eurves are indieated in Fig. 14-24.

(A) Depressant effects due to reduced $p H_{i}$. If the acidification of the cell leads to a depression of the metabolism. in addition to the action of the inhibitor itself, a lower eoncentration of inhibitor than predicted can be used to produce a chosen inhibition. The effeets of this aeidification must always be borne in mind when the $\mathrm{pH}_{i}$ is near the $\mathrm{p}_{a}$ or below. The total inhibition under such circumstances can be expressed as:

$$
i_{t}=i_{\text {inh }}+i_{\mathrm{pH}}
$$

where $i_{\text {inh }}$ is the fraction due to the inhibitor and $i_{\mathrm{pH}}$ is the fraction due to the fall in $\mathrm{pH}_{i}$. The fraction $i_{\mathrm{pH}}$ is not corrected for by rumning a con-

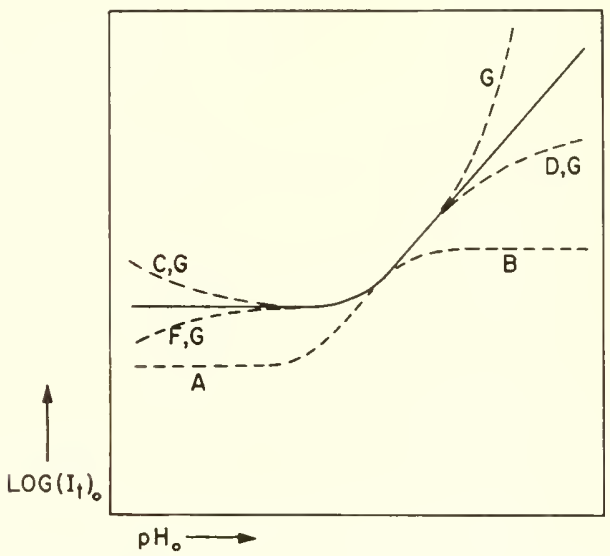

FIG. 14-24. Illustration of the ways in which Simon-Beevers curves can be modified from the theoretical. The letters correspond to the factors discussed in the text.

trol at the lowered $\mathrm{pH}_{0}$ because the drop in $\mathrm{pH}_{i}$ is due to the presence and penetration of the inhibitor. The fact that weak acids damage and kill the cells when the $\mathrm{pH}_{o}$ is lowered. independently of whether the acids are speeifically toxic or not, indieates the importance of this $\mathrm{pH}$ effect. In fact, one possible control to test the occurrence of this $\mathrm{pH}$ effect would be to use a noninhibitory acid that is comparable to the inhibitor in other ways, such as $\mathrm{p} K_{n}$. This factor need not produce a distortion of the shape of the $\log \left(\mathrm{I}_{t}\right)_{0}-\mathrm{pH}_{0}$ curve but may only lower the horizontal portion.

(B) The anion also exerts effects. If the anion contributes to the inhibition in any way - it may not penetrate but act on the cell surface - the curve 
will consist of two major portions, on the acid side of the $p K_{a}$ the action of $\mathrm{HI}$ will be represented and on the alkaline side the action of $\mathrm{I}^{-}$, these being connected near the $\mathrm{p} K_{a}$. Since the anion will often exert much less effect, its part of the curve will be higher than the HI portion. This camnot explain the leveling off of the majority of the experimental curves because this occurs several units above the $\mathrm{p} K_{a}$, whereas the effect of $\mathrm{I}^{-}$would be near maximal one unit above the $\mathrm{p} K_{a}$.

(C) The membrane is somewhat permeable to the anion. Whether or not this will produce a change in the alkaline side of the curve, it will alter the acid portion because $(\mathrm{I})_{i}$ will not rise as high as if the anion were trapped in the cell. The lower the $\mathrm{pH}_{0}$, the smaller is $(\mathrm{I})_{0}$, the more readily will the amion escape from the cell and the lower will be its equilibrium concentration within the cell.

(D) The permeability to the anion becomes appreciable only at high $p H_{0}$. If the anion could penetrate into the cells progressively better at higher $\mathrm{pH}_{o}$ 's, a lower concentration of the inhibitor would be required and the slope of the right-hand portion of the curve would decrease. There would seem to be no evidence for such a phenomenon and, in fact, one wonld predict that the permeability to anions might well decrease at higher $\mathrm{pH}_{0}$ 's because the membrane would become charged more negatively.

(E) Intracellular inhibition varies with $p H_{i}$. In the calculations above, it was assumed that the effect of the anion within the cell was independent of $\mathrm{pH}_{i}$. However, from what has been discussed earlier in the chapter, it is likely that a fall in $\mathrm{pH}_{i}$ will modify the inhibition, first increasing it (due to a greater net positive charge on the enzyme or active center) and then decreasing it (due to the reduction in the concentration of $\mathrm{I}^{-}$). We are concerned with the former effect and this may canse a dropping off of the low $\mathrm{pH}$ portion of the curve.

(F) Lowering of $\mathrm{pH}_{i}$ by buffer penetration leads to altered metabolism. When the external medium contains buffers, the buffers in the undissociated form may penetrate into the cell when the $\mathrm{pH}_{0}$ is lowered and canse an acidification of the cell. This may in turn so alter the metabolism that its sensitivity to the inhibitor will change. In other words, the sensitivity of the metabolic systems to the inhibitor will vary with $\mathrm{pH}_{o}$ independently of the presence of the inhibitor. This may lead to deflections of the low $\mathrm{pH}$ portion of the curve either upwards or downwards, depending on whether the metabolism has been made less or more sensitive to the inhibitor.

(G) Change of $p H_{0}$ produces a change, other than ionization, in the inhibitor. Various properties of the inhibitor may be changed when the $\mathrm{pH}$ is altered from the normal physiological range. The inhibitor may be made more or 
less permeant, or more or less reactive. The inhibition of certain enzymes by hydroquinone depends on the $\mathrm{pH}$ because the equilibrium between hydroquinone and $p$-benzoqninone is sensitive to the $\mathrm{pH}$. It is the $p$-benzoquinone that is active and hydroquinone is oxidized rapidly only in the $\mathrm{pH}$ range near neutrality or above. Thus hydroquinone is rather inactive at a $\mathrm{pH}$ of 5 (Potter, 1942) but active at $\mathrm{pH} 7.4$ by virtue of its rapid oxidation. Changes in structure, reaction with other components in the medium, dimerization, and alteration in general stability can all be brought about by variations in the $\mathrm{pH}$ and such factors can be important in the inhibition. The slope of any part of the $\log \left(\mathrm{I}_{t}\right)_{0}-\mathrm{p} \mathrm{H}_{0}$ curve may be modified because the changes can occur at any $\mathrm{pH}_{o}$ and can be either to increase or decrease the inhibitory potency.

The deviation of the high $\mathrm{pH}$ portion of the curve from a unit slope is the most difficult to understand. Actually, there is much variation between the different weak acids. For the inhibitors in Fig. 14-23, the slopes of the linear sections of the curves are: fluoride, 1.1; azide, 0.75 ; dinitro-o-cresol, 0.73 ; and iodoacetate, 0.47 . The only reasonable explanation is to attribute a slope of less than one to some action of the anion. However, the anion concentration in the medium remains essentially constant as the $\mathrm{pH}_{o}$ rises above $\mathrm{p} K_{a}+2$. An increased penetration or effect of the anion as the $\mathrm{pH}_{o}^{\prime}$ increases wonld explain the curves but would seem unlikely. The yeast cell might be considered as abnormal in view of its relatively large "extracellular" metabolic space, but slopes of less than mnity have been observed in a wide variety of cells (Simon and Beevers, 1952). It may be noted that the same phenomenon is seen in the actions of weak bases, such as phenylhydrazine, except that the slope is now less than one in the low $\mathrm{pH}$ portion of the curve. Thus it is not confined to the alkaline range of $\mathrm{pH}_{o}$. Because the slopes are different with the various weak acids tested on the same type of cell, it must be an effect related to the properties of the active substance and not entirely dependent on the cell response. The low slope of the iodoacetate curve could be explained on the basis of a rise in the rate of reaction with SH groups at or near the cell surface as the $\mathrm{pH}_{o}$ increases, but this would not hold for the other substances. The answer must await further work on a greater variety of substances and cells, and particularly more investigations of the penetration of inhibitors in the higher $\mathrm{pH}_{o}$ range.

\section{Potent and Weak Inhibitors}

The buffer capacity of a cell has been shown to be an important factor in determining the degree of inhibition produced by an ionizing inhibitor. It was also pointed out that actual cells are partially buffered and would be expected to behave in a fashion intermediate between the unbuffered and the completely buffered cells for which equations expressing the distribu- 
tion of inhibitor were derived. The problem of an unknown buffer capacity can sometimes be circumvented when potent inhibitors are used. If a substance is a very active inhibitor, the concentration in the medium need not be high to produce satisfactory inhibition and hence only a small amount of the uncharged form will penetrate into the cell. In such cases, the $\mathrm{pH}_{i}$ would be changed very little even though the buffering was only partial. It may, therefore, be assumed that the cells are completely buffered when sufficiently potent inhibitors are used.

What is the maximum concentration of inhibitor that can be used withont changing the internal $\mathrm{pH}_{i}$ significantly? Let us require that $\mathrm{pH}_{i}$ change not more than $0.2 \mathrm{pH}$ unit and that $d \mathrm{pH} / d X=15$ near the normal $\mathrm{pH}_{i}$ of the cell. Less than $13 \mathrm{~m} M$ HI must enter the cell on this basis. Since in the low $\mathrm{pH}_{o}$ range the maximal concentration of $\mathrm{H}^{+}$released by $\mathrm{HI}$ within the cell is nearly sixty-five times the external inhibitor concentration, it follows that the maximal allowable inhibitor concentration in the medium is $0.2 \mathrm{~m} . I$. Let us then define a potent inhibitor in this connection as one requiring less than $0.1 \mathrm{~m} M$ concentration to produce appreciable inhibition within cells, providing a margin of safety for those cells whose buffer capacity may be less than the assumed value. Since around neutrality, $(\mathrm{I})_{i}$ is approximately equivalent to $\left(\mathrm{I}_{\ell}\right)_{0}$ (see Fig. 14-16), the inhibition must be characterized by a $K_{i}$ of less than $10^{-4} M$. On the other hand, a very weak inhibitor for which the external concentration must be $10 \mathrm{~m} M$ or over can be treated as conforming roughly to the unbuffered situation, especially in the low $\mathrm{pH}_{o}$ range.

\section{Relation between the Inflections of Inhibition Curves and the $\mathrm{pK}_{a}$}

There are two common ways of plotting the $\mathrm{pH}$ dependence of cellular inhibition: the direct plot of the degree of inhibition against $\mathrm{pH}_{o}$ (the $i-\mathrm{pH}_{0}$ plot) and the plot of equieffective inhibitor concentrations against $\mathrm{pH}_{o}$ [the $\log \left(\mathrm{I}_{t}\right)_{0}-\mathrm{pH}_{0} \mathrm{plot}$. The inflections of the latter curves will always occur at $\mathrm{pH}_{0}$ values corresponding to the $\mathrm{pK}_{\alpha}$ 's of the inhibitors. However, this is not necessarily true for the $i-\mathrm{pH}_{0}$ type of plot. It has occasionally been assumed by investigators that the midpoint of an $i$ - $\mathrm{pH}_{0}$ curve should give the $\mathrm{p} K_{\alpha}$ of the inhibitor and some concern has been expressed when the inflection does not coincide with a known $\mathrm{p} K_{n}$. The $\mathrm{pH}_{o}$ at which the midpoint of the inflection occurs is actually dependent on the concentration of the inhibitor in the medium. Inhibition is plotted against $\mathrm{pH}_{0}$ in Fig. 14-25 for a potent inhibitor so that variations in $\mathrm{pH}_{i}$ can be neglected. It is seen that the inflection midpoints are shifted to higher $\mathrm{pH}_{o}$ values when the inhibitor concentration is raised. The inflection occurs near the $\mathrm{pK}_{a}$ only at the lowest concentrations of inhibitor. 


\section{Relation between Inhibitory Activity and the $\mathrm{pK}_{a}$}

When a series of inhibitors is tested on a system at constant $\mathrm{pH}_{o}$, the problem of how to compare the true potencies arises, and, just as in the study of isolated enzymes, the $\mathrm{pH}$ factor must be taken into account. An attempt is often made to relate the different degrees of inhibition to the $\mathrm{p} K_{n}$ 's of the substances tested. The validity of this procedure must now be examined. Let us assume that several inhibitors have been tested at the

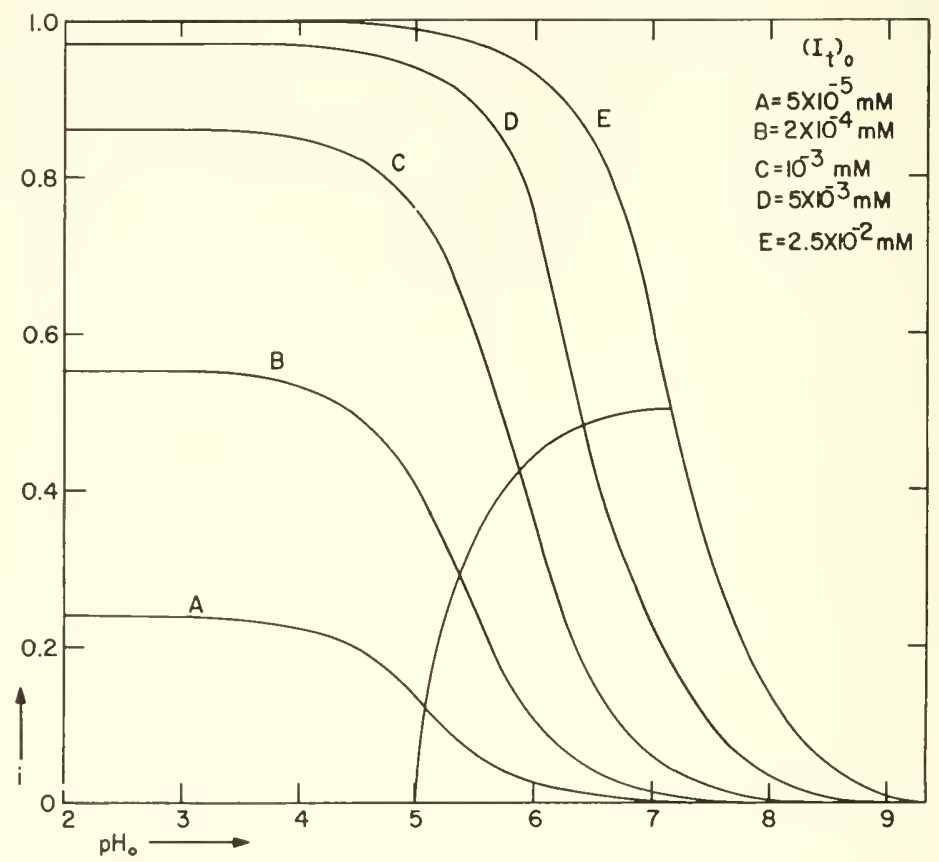

Fig. 14-25. Variation of the inflection points of $i-\mathrm{pH}_{o}$ curves with the concentration of a potent inhibitor. $K_{i}=0.01 \mathrm{~m} H, K_{a}=10^{-5}$, and $\mathrm{pH}_{i}=6.8$.

same concentration in the medium and that a range of inhibitions has been found. The intracellular concentrations of the inhibitors may be related to their $\mathrm{pK}_{a}$ 's by rewriting Eq. 14-162:

$$
\log (\mathrm{I})_{i}=\log \left(\mathrm{I}_{t}\right)_{o}+\mathrm{pH}_{i}-\mathrm{pH}_{o}-\log \left[1+\frac{(\mathrm{H})_{o}}{K_{a}}\right]
$$

For $\mathrm{p} K_{a}$ 's significantly less or more than $\mathrm{pH}_{0}$, this simplifies to:

Low $\mathrm{p} K_{a}: \quad \log (\mathrm{I})_{i}=\log \left(\mathrm{I}_{t}\right)_{0}+\mathrm{pH}_{i}-\mathrm{pH}_{0}$

High $\mathrm{p} K_{a}: \quad \log (\mathrm{I})_{i}=\log \left(\mathrm{I}_{t}\right)_{o}+\mathrm{pH}_{i}-\mathrm{p} K_{a}$ 
Therefore, the curve obtained when $\log (\mathrm{I})_{i}$ is plotted against $\mathrm{p} K_{a}$ will have a zero slope at low values of the $\mathrm{p} K_{a}$ and a slope of -1 when the $\mathrm{p} K_{2}$ 's are above $\mathrm{pH}_{0}$. A plot of the inhibition against $\mathrm{p} K_{z}$ will also have a zero slope in the low $\mathrm{p} K_{a}$ range but the curve at high $\mathrm{p} K_{a}$ 's will have a sigmoid configuration because the inhibition is not proportional to $\log (\mathrm{I})_{i}$. A typical $i-p K_{a}$ curve is shown in Fig. 14-26. It will be observed that the inhibition will not be related to the $\mathrm{p} K_{n}$ of the inhibitor if the values of

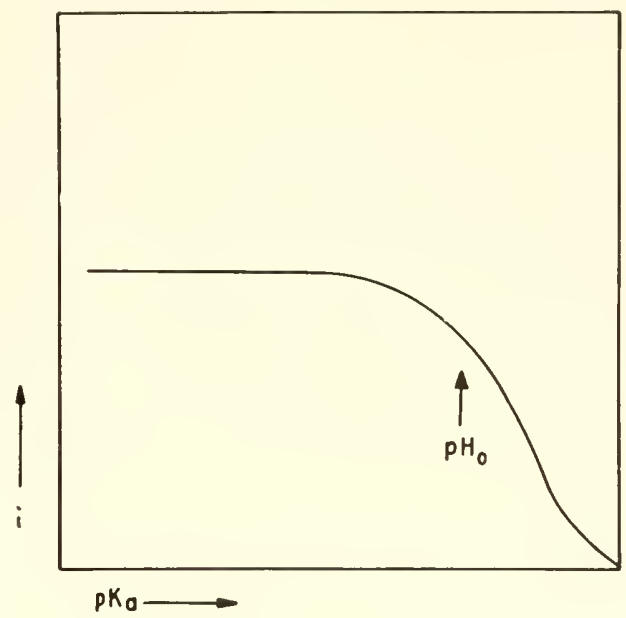

FIG. 14-26. Variation of the inhibition with the $\mathrm{p} K_{a}$ for weak acid inhibitors whenall inhibitors are used at the same total concentration and external pH (Eq. 14-172 and noncompetitive inhibition).

$\mathrm{p} K_{a}$ are much lower than the $\mathrm{pH}_{0}$. In other words, if $\mathrm{pH}_{o}=7.4$, all inhibitors with $\mathrm{p} K$,'s of 6 or less will produce the same inhibition. In the range of higher $\mathrm{p} K_{a}$ 's the inhibition will be related to $\mathrm{p} K_{n}$ in a complex manner.

The most practical way to determine whether the differences in inhibition observed are related only to the differences in $\mathrm{p} K_{a}$ is to solve Eq. 14-174 for the $\log (\mathrm{I})_{i}$ that corresponds to each $p K_{n}$ and then to see if the inhibitions conform to the usual equation expressing $i$ in terms of (I). The comparison between inhibitors would be much more accurate and reliable if a simple $\mathrm{pH}$ curve for each were obtained.

\section{Determination of $\mathrm{K}_{i}$ from Cellular Inhibition}

Nonionizing inhibitors that penetrate into cells readily and reach an intracellular concentration which is equivalent to the external concentration may produce inhibitions which are quantitatively quite comparable 
to those observed on the isolated enzymes. The calculation of $K_{i}$ in such cases may sometimes be done by the usual graphical procedures. However, the true $K_{i}$ cannot be determined if the inhibitor is a weak acid or base, unless the concentrations of inhibitor within the cell can be determined directly. It is clear that it is under only special conditions that $(\mathbf{I})_{i}=\left(\mathbf{I}_{t}\right)_{o}$, namely when $\mathrm{pH}_{o}=\mathrm{pH}_{i}$ for completely buffered cells or when the $\mathrm{pH}_{o}$ is very low for unbuffered cells (see Fig. 14-16 and 14-17). In the latter case, the $\mathrm{pH}_{i}$ will have altered sufficiently so that any $K_{i}$ determined will relate only to the inhibition at this abnormal $\mathrm{pH}$. Furthermore, under the best conditions, the inhibitor constant that is obtained will always be the apparent one, namely $K_{i}{ }^{\prime}$, defined earlier in this chapter. If cellular inhibition data are plotter by one of the usual methods that have been developed for isolated enzymes, the curves may superficially resemble those obtained in vitro, but the constants calculated from the slopes or intercepts will have no predictable relationship to the true constants of the inhibition. It follows from this that a comparison of inhibitor potencies using constants determined in this way must be unreliable. Such apparent constants may be useful in characterizing inhibitions and providing quantitative descriptions of the behavior. but they should not be construed as expressing the affinity of the affected enzyme for the inhibitor.

In certain cases, the calculation of $K_{i}$ from data on cellular inhibition, without too great an error, is possible. An inhibition that is noncompetitive and potent (so that the $\mathrm{pH}_{i}$ will not change appreciably) will depend on the total external concentration of inhibitor in the following manner:

$$
i=\frac{\left(\mathrm{I}_{t}\right)_{o}}{\left(\mathrm{I}_{t}\right)_{o}+K_{i} \frac{(\mathrm{H})_{i}}{(\mathrm{H})_{o}}\left[1+\frac{(\mathrm{H})_{o}}{K_{a}}\right]}
$$

Thus the experimentally determined $K_{i}{ }^{\prime}$ will be related to the true $K_{i}$ in a simple manner. The relation for unbuffered cells is not simple and in cases of competitive inhibition, the ionization and penetration of the substrate must also be considered.

\section{Depletion of Inhibitor from the Extracellular Medium}

Cellular inhibition by inhibitors that are weak acids or bases and the $\mathrm{pH}$ dependence of this inhibition are modified when the total concentration of the inhibitor in the external medium is reduced as a result of the penetration into the cells or to the binding of the inhibitor there. Cowles and Klotz (1948) considered this factor in nitrophenol bacteriostasis and concluded that it would alter the curves resulting from plotting $\log \left(\mathrm{I}_{t}\right)_{0}$ against $\mathrm{pH}_{o}$, accounting for the observed slopes of less than one. Since the depletion of external inhibitor could easily occur under many experi- 
mental conditions, it will be necessary to extend the previons treatment to such situations. The factors that would favor depletion are: a high ratio of the cell or tissue volume to the volume of the medium, a low $K_{i}$ so that small concentrations of the inhibitor are used, the binding of the inhibitor to nonenzymic components of the cells, a $\mathrm{pH}_{0}$ favoring large penetrations of the inhibitor, and a high buffering capacity of the cells, which also favors penetration of larger total amounts of inhibitor. There are various situations, depending on the canse of the depletion, that may be analyzed.

Case I: depletion is caused by penetration into the cell and not to binding. This is represented by diagram 14-150 and Eqs. 14-151 to 14-155 remain valid; however, $\left(\mathrm{I}_{t}\right)_{0}$ does not now remain constant. The cellular fraction of the total reaction volume will be represented by $n$ and thus the fraction that the medium occupies is $1-n$. The convention will be adopted of enclosing over-all concentrations in brackets; thus the concentration of inhibitor in the total volume of medium and cells is $\left[\mathbf{I}_{t}\right]$. The initial concentration of the inhibitor in the medium. before penetration into the cells has occurred, is $\left[I_{t}\right] /(1-n)$, so that the over-all concentration and initial concentration can be easily interconverted; it is, however, easier in some respects to use the over-all concentrations in expressing the behavior.

The over-all concentration in terms of external and internal concentrations is given by:

$$
\left[I_{t}\right]=n\left(I_{t}\right)_{i}+(1-n)\left(\mathrm{I}_{t}\right)_{0}
$$

and in terms of $(\mathbf{I})_{i}$, which is assumed to represent the active form, by:

$$
\left[\mathrm{I}_{t}\right]=(\mathrm{I})_{i}\left[n+\frac{(\mathrm{H})_{i}}{K_{a}}+(1-n) \frac{(\mathrm{H})_{i}}{(\mathrm{H})_{0}}\right]
$$

Let us examine how this depletion will affect a plot of $\log \left[\mathrm{I}_{t}\right]$ against $\mathrm{pH}_{0}$.

Low $\mathrm{pH}_{o}: \quad \log \left[\mathrm{I}_{t}\right]=\log (\mathrm{I})_{i}+\log \left[n+\frac{(\mathrm{H})_{i}}{K_{a}}\right]$

High $\mathrm{pH}_{o}: \quad \log \left[\mathrm{I}_{t}\right]=\log (\mathrm{I})_{i}+\log (1-n)-\mathrm{pH}_{i}+\mathrm{pH}_{o}$

In the low $\mathrm{pH}_{o}$ range the slope will be zero and in the high $\mathrm{pH}_{o}$ range the slope will be +1 . However, the intermediate region will be affected by the depletion and the inflection between slopes will occur at a $\mathrm{pH}_{0}$ different from $\mathrm{p} K_{a}$. Such plots for different values of $n$ are shown in Fig. 14-27. As the relative volume of the cells increases, the over-all concentration of the inhibitor must become larger to produce the same degree of inhibition, which one would expect on the basis of depletion from the medium. Also, 
as $n$ becomes larger, the inflection shifts to higher valnes of $\mathrm{pH}_{0}$. The slopes in the high $\mathrm{pH}_{0}$ range, however, are always +1 .

Case II: depletion is caused by penetration into the cell and to binding to nonenzymic material. If it is assumed that the ionic form binds to some cell component, a new equilibrium must be introdnced:
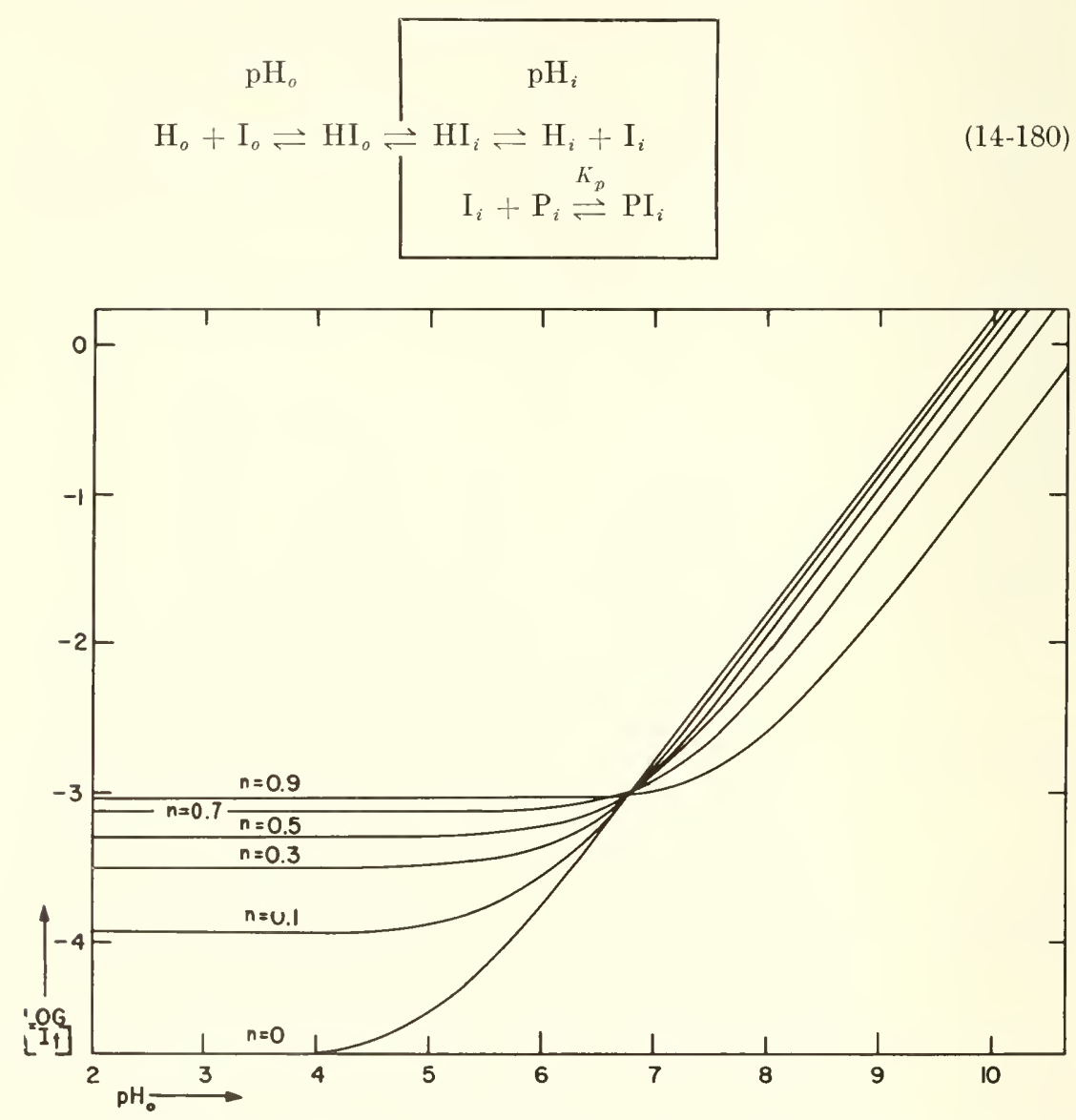

FIG. 14-27. Effect of the depletion of the inhibitor due to its penetration into the cells (Eq. 14-177) for different values of $n$, the cellular fraction of the total reaction volume. $K_{\alpha}=10^{-5},(\mathrm{H})_{i}=1.6 \times 10^{-7} M$, and $(\mathrm{I})_{i}=1 \mathrm{~m} .1$.

The expression for $I_{t}$ now has an additional term:

$$
\left[\mathrm{I}_{t}\right)=(\mathrm{I})_{i}\left[n+\frac{(\mathrm{H})_{i}}{K_{a}}+(1-n) \frac{(\mathrm{H})_{i}}{(\mathrm{H})_{o}}+\frac{n\left(\mathrm{P}_{t}\right)_{i}}{(\mathrm{I})_{i}+K_{p}}\right]
$$


The $\log \left[I_{t}\right]-\mathrm{pH}_{o}$ curves will be similar to those in Fig. 14-27 but the effect of the binding will be to raise the necessary inhibitor concentration in the low $\mathrm{pH}_{0}$ range and to shift the inflections further to the right. The slopes in the high $\mathrm{pH}_{o}$ range will remain +1 .

Case III: depletion is caused by penetration into the cell and to combination with the enzyme. We can simply replace $\mathrm{P}$ by $\mathrm{E}$ and $K_{p}$ by $K_{i}$ in the equation above. However, now we wish to find the over-all concentrations of the inhibitor that will maintain the inhibition constant at different values of $\mathrm{pH}_{0}$. Equation 14-181 may be rewritten as:

$$
\left[\mathrm{I}_{t}\right]=n i\left(\mathrm{E}_{t}\right)_{i}+(\mathrm{I})_{i}\left[n+\frac{(\mathrm{H})_{i}}{K_{a}}+(1-n) \frac{(\mathrm{H})_{i}}{(\mathrm{H})_{o}}\right]
$$

where the $n i\left(\mathrm{E}_{t}\right)_{i}$ term represents the effect of the binding to the enzyme and the other term, as in Eq. 14-177, represents the penetration into the cells. Of course, $(\mathrm{I})$, may be substituted by $i K_{i} /(1-i)$ for noncompetitive inhibition. The $\log \left[\mathrm{I}_{t}\right]-\mathrm{pH}_{0}$ curves will be similar to those for the other cases.

It is clear that the depletion of inhibitor from the medium, from whatever cause, does not change the slope in the high $\mathrm{pH}_{o}$ range from +1 . The reason for this is that at a high $\mathrm{pH}_{0}$ very little $\mathrm{HI}$ is present and the amount of inhibitor entering the cell is so small that depletion is minimized or abolished (see Fig. 14-16). These conclusions are thus in disagreement with those of Cowles and Klotz (1948), who stated that such depletion would decrease the slope in the high $\mathrm{pH}_{o}$ range. However, their equations would actually lead to a slope of +1 at high $\mathrm{pH}_{o}$ and agree with those given above. Their expression for the slope:

$$
\text { slope }=\frac{K_{a}}{K_{a}+(\mathrm{H})_{o}[1+(P / k)]}
$$

where $P$ and $k$ are parameters related to the binding, would provide a slope of +1 when $(\mathrm{H})_{o}$ became sufficiently low. The $1+(P / k)$ factor only shifts the inflection to higher $\mathrm{pH}_{0}{ }^{\prime}$ 's, just as described for the three cases treated in this section. Therefore, one cannot explain the deviation of the slope by the phenomenon of inhibitor depletion. Nevertheless, the effects of depletion are interesting and important in other respects.

\section{Inhibitors That Are Dibasic Acids}

Singly ionizing inhibitors have been treated for convenience and the extension to doubly ionizing inhibitors is straightforward. If only the $\mathrm{H}_{2} \mathrm{I}$ form can penetrate into the cells:

$$
\left(\mathrm{H}_{2} \mathrm{I}\right)_{o}=\left(\mathrm{H}_{2} \mathrm{I}\right)_{i}
$$


and

$$
\frac{(\mathrm{I})_{i}}{(\mathrm{I})_{o}}=\frac{(\mathrm{H})_{o}^{2}}{(\mathrm{H})_{i}^{2}}
$$

so that

$$
\left(\mathrm{I}_{t}\right)_{o}=(\mathrm{I})_{i} \frac{(\mathrm{H})_{i}^{2}}{(\mathrm{H})_{o}^{2}}\left[1+\frac{(\mathrm{H})_{o}}{K_{a}{ }^{\prime \prime}}+\frac{(\mathrm{H})_{o}^{2}}{K_{a}^{\prime} K_{a}^{\prime \prime}}\right]
$$

If the $\mathbf{I}$, or doubly charged ion, is assumed to be the active inhibitor, a plot of $\log \left(\mathrm{I}_{t}\right)_{o}$ against $\mathrm{pH}_{o}$ will have the following characteristics:

$$
\text { Low } \mathrm{pH}_{o}: \quad \log \left(\mathrm{I}_{t}\right)_{o}=\log (\mathrm{I})_{i}-2 \mathrm{pH}_{i}+\mathrm{p} K_{q}{ }^{\prime}+\mathrm{p} K_{a}^{\prime \prime}
$$

Intermediate $\mathrm{pH}_{o}: \log \left(\mathrm{I}_{t}\right)_{o}=\log (\mathrm{I})_{i}-2 \mathrm{pH}_{i}+\mathrm{p} K_{a}^{\prime \prime}+\mathrm{pH}_{o}$

High $\mathrm{pH}_{o}: \quad \log \left(\mathrm{I}_{t}\right)_{o}=\log (\mathrm{I})_{i}-2 \mathrm{pH}_{i}+2 \mathrm{pH}_{o}$

As the $\mathrm{pH}_{0}$ increases, the slope will change from zero, through +1 to +2 . If $\mathrm{HI}$ is the active inhibitor:

$$
\left(\mathrm{I}_{t}\right)_{o}=(\mathrm{I})_{i} \frac{(\mathrm{H})_{i}}{(\mathrm{H})_{o}}\left[1+\frac{(\mathrm{H})_{o}}{K_{a}{ }^{\prime}}+\frac{K_{a}{ }^{\prime \prime}}{(\mathrm{H})_{o}}\right]
$$

and the $\log \left(\mathrm{I}_{t}\right)_{0}-\mathrm{pH}_{o}$ plot will show the same slopes as when $\mathrm{I}$ is the inhibitor. From this type of plot it is impossible to determine the active form of the inhibitor within the cells. If both $\mathrm{H}_{2} \mathrm{I}$ and $\mathrm{HI}$ penetrate into the cells, the inhibitor will behave as if it were monobasic with a $\mathrm{p} K_{a}$ equal to the $\mathrm{p} K_{a}{ }^{\prime \prime}$ of the dibasic acid. It may be noted that if $p K_{a}{ }^{\prime}$ and $\mathrm{p} K_{a}{ }^{\prime \prime}$ are close together, the slope will change from zero to +2 without any intermediate slope of +1 , as long as only the $\mathrm{H}_{2} \mathrm{I}$ form penetrates.

\section{Intracellular Competitive Inhibition}

If both the substrate and the inhibitor are introduced into the medium and both are weak acids or bases, the competitive inhibition on an enzyme within the cells will depend upon the intracellular concentrations of $\mathrm{S}$ and I, and hence upon the degrees to which they penetrate. The usual expression for the inhibition may be written:

$$
i=\frac{\left(\mathrm{I}_{t}\right)_{o}}{\left(\mathrm{I}_{t}\right)_{o}+K_{i}{ }^{\prime}\left[1+\frac{\left(\mathrm{S}_{t}\right)_{o}}{K_{s}{ }^{\prime}}\right]}
$$


where the apparent substrate and inhibitor constants are related to the true constants as follows:

$$
\begin{aligned}
& K_{i}{ }^{\prime}=K_{i} \frac{(\mathrm{H})_{i}}{(\mathrm{H})_{o}}\left[1+\frac{(\mathrm{H})_{o}}{K_{a_{i}}}\right] \\
& K_{s}{ }^{\prime}=K_{s} \frac{(\mathrm{H})_{i}}{(\mathrm{H})_{o}}\left[1+\frac{(\mathrm{H})_{o}}{K_{a_{s}}}\right]
\end{aligned}
$$

When the internal $\mathrm{pH}_{i}$ does not change, an experiment performed at a certain external $\mathrm{pH}_{o}$, using different concentrations of substrate and inhibitor, will give results comparable to competitive inhibition on isolated enzymes. However, the constants determined by graphical methods will be $\mathrm{pH}$-dependent in the manner given above. When $\mathrm{p} K_{a_{s}}$ and $\mathrm{p} K_{a_{i}}$ differ by a significant amount, the behavior is somewhat abnormal. Let us assume that $p K_{a_{i}}<p K_{a_{s}}$. In the $\mathrm{pH}_{o}$ range below $\mathrm{p} K_{a_{i}}$, both the substrate and the inhibitor will penetrate readily, but if the $\mathrm{pH}_{0}$ is raised above $\mathrm{p} K_{a_{i}}$ but is still lower than $\mathrm{p} K_{a_{s}}$, the inhibitor will penetrate less readily while the substrate will enter readily, so that the inhibition will fall in the expected way; however, when the $\mathrm{pH}_{o}$ is raised to $\mathrm{pK}_{a_{\mathrm{s}}}$, the substrate will cease to penetrate readily and the inhibition may not be decreased as much as expected. If $\mathrm{p} K_{a_{s}}<\mathrm{p} K_{a_{i}}$, as the $\mathrm{pH}_{o}$ is raised above $\mathrm{p} K_{a_{s}}$, the substrate will not enter readily and the inhibition may actually increase until $\mathrm{pH}_{o}$ approaches $\mathrm{p} K_{a_{i}}$. The behavior of an hypothetical system where $\mathrm{p} K_{a_{s}}=4$ and $\mathrm{p} K_{a_{i}}=6$ is shown in Fig. 14-28 and the effect of substrate exclusion between $\mathrm{pH}_{0}$ 's 4 and 6 is clearly evident in the rise in the inhibition.

\section{Complex Effects of $\mathrm{pH}$ on Cellular Inhibition}

Effects of changes in $\mathrm{pH}_{0}$ not described or predicted by the simple treatment given in this chapter should be expected occasionally. For one thing, most inhibitions are not specific. The changes of over-all metabolism or function that are measured may depend on actions of the inhibitor on several different systems, each with its particular $\mathrm{pH}$ dependence. Even though the inhibition of each intracellnlar enzyme follows the predicted behavior, the process measured may respond in quite a different manner. The presence of multienzyme systems in most of the important areas of metabolism also must make cellular inhibition $\mathrm{pH}_{0}$ dependence more complex in many instances. Furthermore, we have not considered quantitatively in this chapter the possible effects of the inhibition on the permeability properties of the cell membrane. It is quite possible that metabolic depression would lead to an increased permeability to the ionic forms of the inhibitor and alter the $\mathrm{pH}$ relations. One may imagine many factors that would lead to deviation of the behavior from that described by the basic equations. 
The inhibition of bioluminescence in Achromobacter by 2-methyl-1,4naphthoquinone (menadione) shows a characteristic variation from what wonld be expected on the basis of a simple ionization (Fig. 14-29) (McElroy and Kipnis, 1947). Although more experimental points might be desired, it would appear that there is a rather sudden break in the curve at complete inhibition. The cause for this is not known. In such cases, one possi-

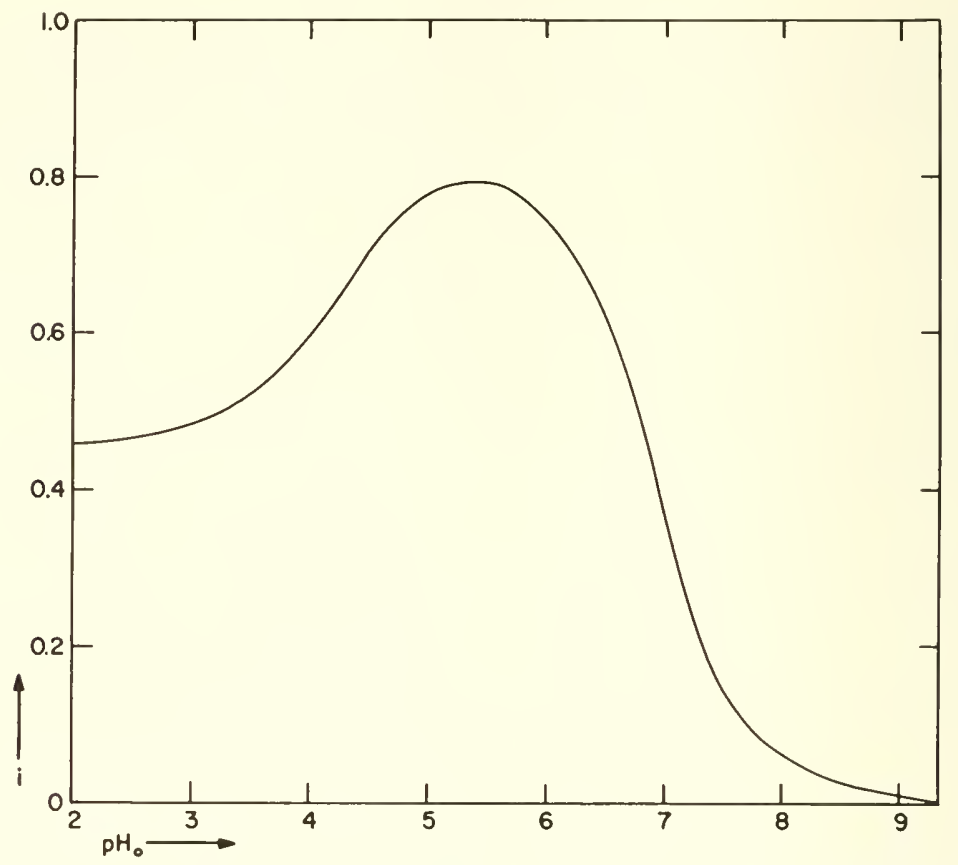

FIG. 14-28. Variation of intracellular competitive inhibition with the external pH (Eq. 14-191). $\left(\mathrm{I}_{t}\right)_{o}=1 \mathrm{~m} . I,\left(\mathrm{~S}_{t}\right)_{o}=0.1 \mathrm{~m} . I, K_{i}=1 \mathrm{~m} \boldsymbol{I}$, $K_{s}=10 \mathrm{~m} M, K_{a_{i}}=10^{-6}$, and $K_{a_{s}}=10^{-4}$.

bility comes to mind. If the metabolism or function is active in proportion to the concentration of some enzyme, or some product of an enzyme reaction, but exhibits a threshold, below which the process fails, the type of curve in Fig. 14-29 might be expected. The curve from zero to complete inhibition may be part of a full sigmoid curve representing the inhibition of an enzyme system. When a certain inhibition has been reached, the process that depends on this enzyme will fail. Looked at in another way, below a threshold $\mathrm{pH}_{o}$ the metabolic process cannot operate because the inhibition on an enzyme is greater than the maximal inhibition allowable for the process to occur. But as the $\mathrm{pH}_{o}$ is increased, when the required amount 
of enzyme or product becomes available, the process will begin to operate, and as the $\mathrm{pH}_{o}$ is further increased the enzyme concentration rises rapidly, so that the inlibition on the measured process falls precipitonsly. From a more general standpoint, the existence of critical thresholds in cell metabolism must be accepted. Up to a certain point the coordinated reactions

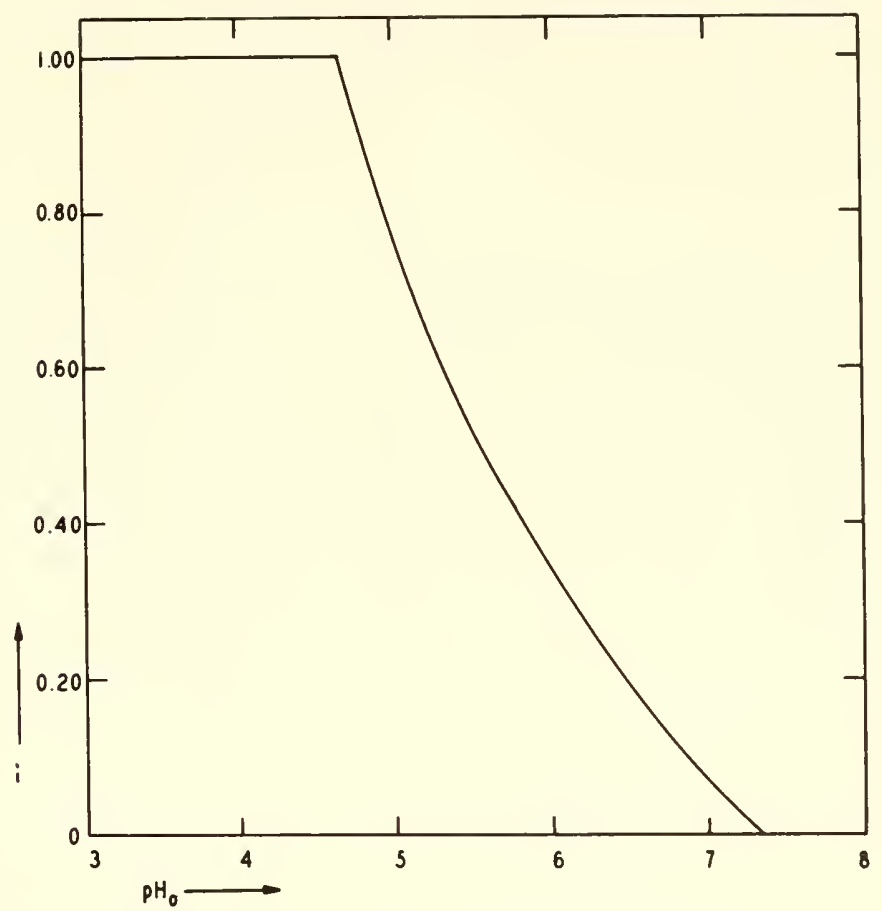

FIG. 14-29. Variation of the inhibition of bioluminescence in Achromobacter by 2-methyl-1,4-napthoquinone with the $\mathrm{pH}$. (From McElroy and Kipnis, 1947.)

can maintain their functional relationship, but beyond this point the system may not only cease to operate but may also disintegrate in the sense of losing irreversibly its organization or necessary components. Cyclical or regenerative systems are especially prone to show such critical operation levels. 



\section{EFFECTS OF VARIOUS FACTORS ON INHIBITION}

The characteristics of enzyme inhibitions can usually be modified by many factors and some of these changes may provide interesting information on the nature of the molecular processes occurring in the interaction of the enzyme with the inhibitor. We have already treated the effects of changes in the concentrations of the reaction components (enzyme, substrate, activator, or coenzyme) and of changes in $\mathrm{pH}$. Four other factors are of particular importance - temperature, pressure. dielectric constant, and ionic strength - and this chapter will be mainly concerned with these. The responses of inhibition to changes in these properties of the system often make it possible to determine important constants that characterize the inhibition and give some insight into the mechanisms involved. Other means may be used to alter the state of the system - as to bombard it with various types of radiation, or to vary the redox equilibrium of the enzyme, or to use different buffers - but these are not so apt to enlarge our knowledge of the fundamental nature of the inhibitions.

\section{EFFECTS OF TEMPERATURE: ENZYMES}

The primary aim of investigations on the temperature dependence of enzyme inhibition is the determination of certain thermodynamic quantities, such as the enthalpy and entropy changes, associated with the formation of the EI complex. and through these quantities to visualize more accurately the energy pathway followed by the reaction. There is also a practical side in certain thermal studies. The actions of insecticides that are enzyme inhibitors on agriculturally important insects depend on temperature and this aspect of the problem is a major factor in the eradication of pests and their possible development of resistance to the agents. Also the actions of drugs that interfere with metabolism may vary in potency during states of fever or hypothermia. Very few thorough studies on the variation of inhibition with temperature have been reported but enough has been done in certain restricted fields to indicate the interesting results that may be obtained. 


\section{General Effects of Temperature on Dissociation Constants}

Temperature changes will alter enzyme reactions or inhibitions by changing the rate constants of each step; for example, the $k_{1}$ and $k_{-1}$ for the formation

$$
\begin{gathered}
\mathrm{E}+\underset{k_{-1}}{\stackrel{\mathrm{S}}{\rightleftharpoons}} \mathrm{ES} \stackrel{k_{2}}{\rightarrow} \mathrm{E}+\mathrm{P} \\
\mathrm{E}+\mathrm{I} \underset{k_{-1}}{\stackrel{k_{1}}{\rightleftharpoons}} \mathrm{EI} \quad K_{i}=k_{-1} / k_{1}
\end{gathered}
$$

and dissociation of the ES or EI complexes, and the $k_{2}$ (or any additional rate constants) for the breakdown of the ES complex into products. The resultant of the effects on $k_{1}$ and $k_{-1}$ is manifested by a change in the substrate or inhibitor constants, $K_{s}$ or $K_{i}$. The Michaelis-Menten constant, $K_{m}=\left(k_{-1}+k_{2}\right) / k_{1}$ may be changed in a more complex fashion. The rate constants may be modified by temperature in two basic ways. The number of reactant molecules capable of smrmounting the activation energy barrier will change, or the activation energy itself may be changed. Usually both will occur. One other possible mechanism must be mentioned, since it has played an important role in inhibition studies. This is the effect of temperature on the equilibrium between catalytically active and inactive (or denatured) enzyme molecules. The applicability and validity of this mechanism will be discussed in a separate section.

Although the value of $K_{i}$ at any temperature is related to the free energy of inhibitor binding, the change in $K_{i}$ with temperature is dependent on the enthalpy change for the formation of the EI complex, since:

$$
\begin{gathered}
\Delta F=R T \ln K_{i}=\Delta H-T \Delta S \\
\ln K_{i}=\frac{\Delta H}{R T}-\frac{\Delta S}{R} \\
\frac{d \ln K_{i}}{d T}=-\frac{\Delta H}{R T^{2}}
\end{gathered}
$$

where $1 H$ and $I S$ are the enthalpy and entropy changes for the binding of the inhibitor to the enzyme. Determination of $K_{i}$ at different temperatures allows the calculation of $1 H$ since a plot of $\log K_{i}$ against $1 / T$ will give a line with a slope of $1 H / 2.303 R$. If the plot is linear over the range of temperatures studied, this indicates that $1 H$ has not changed appreciably. However, if the plot is curved, the slope at any point will give $\Delta H$ for that temperature. If the temperature range is not too great and the $\Delta H$ is reasonably constant, a useful relationship may be derived: 


$$
\begin{aligned}
& \ln K_{i_{1}}=\frac{\Delta H}{R T_{1}}-\frac{\Delta S}{R} \\
& \ln K_{i_{2}}=\frac{\Delta H}{R T_{2}}-\frac{\Delta S}{R} \\
& \ln \frac{K_{i_{2}}}{K_{i_{1}}}=\frac{\Delta H}{R}\left[\frac{1}{T_{2}}-\frac{1}{T_{1}}\right]
\end{aligned}
$$

If $K_{i}$ is determined at one temperature, its value at another temperature may be estimated by this equation if $\angle H$ is known and does not vary markedly with the temperature. Once $J H$ is known it is also possible to calculate $J S$ from Eq. 15-3. Given $K_{i}$, and hence $J F$, at a single temperature, it is impossible to predict what $K_{i}$ will be at another temperature because from $J F$ alone the temperature dependence of $K_{i}$ cannot be established.

A word should be said about the sign conventions in these equations. In the chemical literature the association constant is often used, in which case $\angle F=-R T \ln K$. The signs in Eq. $15-4$ to $15-6$ are then changed. Since $K_{i}$ is usually expressed as a dissociation constant, the sign convention of Eq. 15-3 has been adopted. However, the thermodynamic quantitaties - $J F . \Delta H$, and $\rfloor S$ - refer to the association of the enzyme and inhibitor. The change in the inhibition with temperature in a simple case will thus depend on $J H$ for one thing. The effects of temperature on noncompetitive inhibition, assuming that $1 / I$ does not vary with the temperature, are shown in Fig. 15-1 for varions values of $1 H$ when $K_{i}$ is $1 \mathrm{~m} . I$ at $37.5^{\circ}$. These curves indicate the magnitudes of the effects to be expected under these conditions. In actual inhibitions, however, it is unlikely that $J H$ will remain constant as the temperature is varied, so that this change in $\Delta H$ must be superimposed on the effects indicated by the curves. Since $\Delta H$ may either increase or decrease with a change in the temperature, the variation of the inhibition will be greater or smaller than shown in the figure.

In case the formation of the EI complex involves a chemical reaction with the enzyme and the attachment of the inhibitor by covalent bonds, the effects of temperature are apt to be marked becanse of the highly exothermic nature of most of these reactions. Furthermore, the affinity of the enzyme for the inhibitor will usually fall with rise in temperature, resulting in a decrease in the inhibition. Inhibitions by the sulfhydrylreacting agents probably fall in this category but there is little experimental basis to confirm this. The inhibition of beef heart succinic dehydrogenase by arsenite has been found in the author's laboratory to decrease with rise in the temperature from $17^{\circ}$ to $37^{\circ}$ (Sanyal, 1959). 


\section{Dependence of Inhibition on Temperature}

Noncompetitive inhibition may vary with the temperature because of changes in either the inhibitor concentration (I) or the inhibitor constant $K_{i}$. The possibility of the concentration of active inhibitor being temperature-dependent should not be ignored. If an inhibitor exists in two or more forms of unequal inhibitory potency, the equilibrimm between these forms

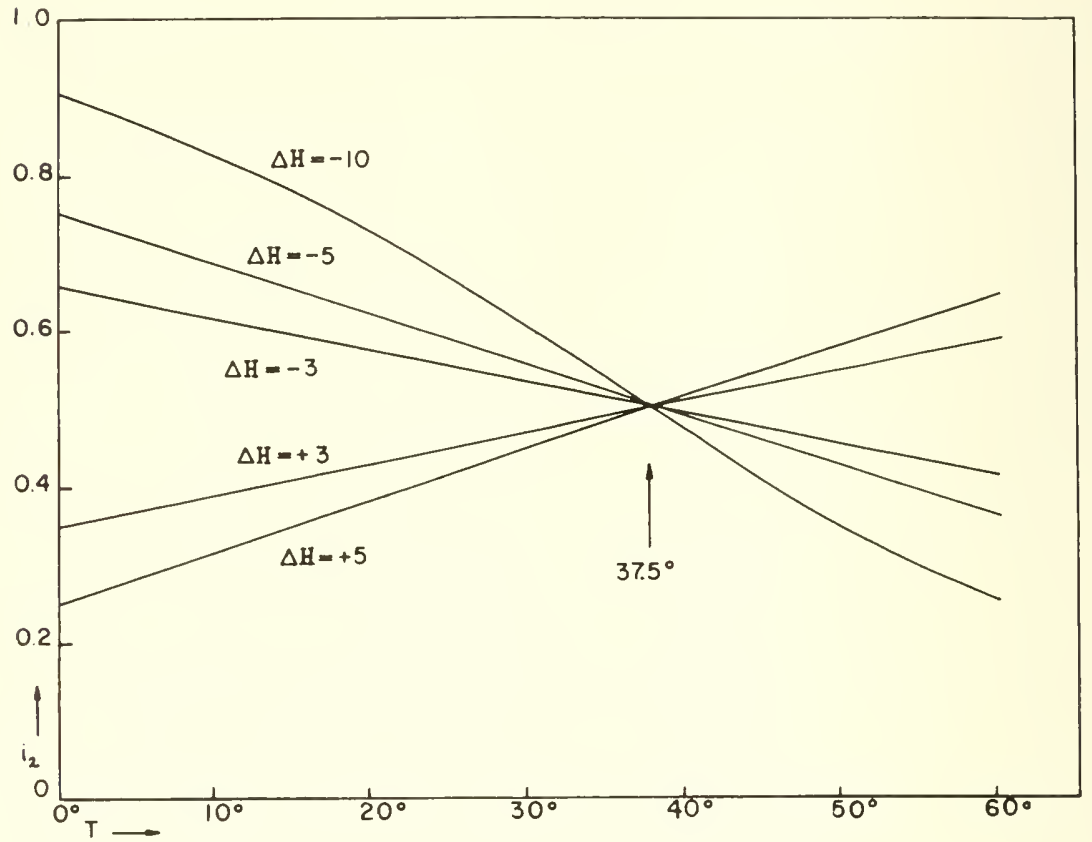

FIG. 15-1. Variation of noncompetitive inhibition with the temperature for various values of $\Delta H$, assuming that $\Delta H$ does not change with the temperature.

$$
K_{i}=1 \mathrm{~m} . H \text { at } 37.5^{\circ} \text {. }
$$

may be shifted by changes in the temperature. The inhibition in competitive situations also depends on the Michaelis constant $K_{m}$ and hence on the variation of this constant with temperature. Since $K_{b}$ and $K_{s}$ will usually vary in the same direction with a change in temperature, due to the fact that substrates and inhibitors react with the enzyme in similar ways in competitive inhibition, the inhibition will generally not be so temperature-dependent as in noncompetitive cases. However, when $K_{m}$ is not equivalent to $K_{s}$, the change in $k_{2}$ may cause $K_{m}$ to vary quite differently than $K_{\imath}$. 
A more general rule may be formulated. We have seen that the inhibition can usually be written in the form:

$$
i=\frac{(\mathrm{I})}{(\mathrm{I})+K_{i}[\mathrm{X}]}
$$

where $[X]$ represents the effects of various factors on the inhibition. In the case of competitive inhibition, $X=1+\frac{(\mathrm{S})}{K_{m}}$, and for the situation in which only the ionized form of the inhibitor reacts with the enzyme, $\mathrm{X}=1+\frac{(\mathrm{H})}{K_{a}}$. In general, $K_{i}^{\prime}=K_{i}[X]$, where, as previously defined, $K_{i}{ }^{\prime}$ is the apparent inhibitor constant which may be obtained from the usual plotting procedures. If the true inhibitor constant $K_{i}$ is determined, then the calculation of the inlibition must take into account the variation of $[X]$ with temperature. On the other hand. if the apparent constant $K_{i}^{\prime}$ is determined, thermodynamic data derived from it cannot be directly applied to the binding of the inhibitor to the enzyme. For the complete description of the temperature dependence of an inhibition, it is necessary to investigate the variations in both $K_{i}$ and $X$. Inasmuch as $X$ often involves two or more dissociation constants when the enzyme or inhibition mechanisms become more complex, the change in the inhibition with temperature may be difficult to estimate.

It is good practice in determining inhibitions at various temperatures to establish the reversibility of the temperature effects and in this way eliminate any irreversible changes that may occur. Raising the temperature can occasionally lead to irreversible denaturation changes in the enzyme and this may affect the inhibition kinetics.

\section{Possible Mechanisms Involved in Temperature Effects on Enzyme Inhibition}

Changes in the equilibrium constants induced by temperature variations and dependent on $1 H$ are common to all chemical reactions and bindings. Interactions of substances with enzymes often involve additional factors that lead to changes in $J H, \perp S$, and $1 F$ with temperature and some of these factors will now be discussed briefly.

(A) Alteration of the water structure. The association between water molecules decreases with rise in the temperature due to the increased thermal movement. It has been shown by $\mathrm{X}$-ray scattering that the water molecules move about $0.02 \AA$ further apart on the average for each $10^{\circ}$ increase in the temperature. There is also a smoothing of some of the scattering peaks and an elimination of others, indicating a greater randomization of the water structure. The relationships between intermolecular forces and the water structure have been discussed in Chapter 6 and it is evident that, 
all enzyme reactions occurring in a milien of water, any modification of this structure can have an influence on the reactions of molecules with the enzyme. This is particularly apt to be expressed in terms of changes in the entropy terms for the formation of the EI complex.

(B) Changes in the dielectric constant. This factor is closely related to the previous one because the variations in the dielectric constant with temperature are mainly the result of changes in the water structure. The dielectric constant is generally proportional to $1 / T$. The variation of the dielectric constant of water with temperature at constant pressure is given by $d D / d T=-0.338$ at physiological temperatures. The interaction energies between charged groups will vary with the temperature for this reason. Since electrostatic interaction energy varies with $1 / D$, it will be proportional to the temperature with respect to this particular factor.

(C) Changes in the hydration of the enzyme and inhibitor. Inasmuch as the hydration of the interacting molecules is a major factor in the consideration of the forces involved, one must take into account hydration changes resulting from variation in the temperature. Unfortunately, very little accurate information on such changes is available. From studies on the viscosity of water and salt solutions, Kaminsky (1957) concluded that certain ions such as $\mathrm{Mg}^{++}$produce a long-range ordering of the water structure, significant orientation occurring beyond the primary hydration layer. As the temperature rises, this secondary hydration is progressively disturbed by thermal motion over a temperature range of $12^{\circ}$ to $42.5^{\circ}$, although the primary shell is not affected. The extent of secondary hydration aromd singly charged ions or ionic groups is nuknown but some ordering probably does occur and would be susceptible to temperature variation. Hasted and Roderick (1958) determined the dielectric constants and relaxation times at different temperatures and frequencies and from such data obtained some idea of the number of water molecules that are prevented from rotating when a solute is introduced into water. It might be predicted that this number would decrease with rise in the temperature, but actually it did not and in some cases actually rose slightly. Indeed, very little difference was found between $3^{\circ}$ and $25^{\circ}$ in any case. Other than ionic types of hydration occur. Sometimes water molecules are attached to inhibitors or enzymes through hydrogen bonding and this type of hydration is probably quite sensitive to temperature. The interaction energy upon the approach of an inhibitor molecule to an enzyme may thus be modified as the temperature changes by differing degrees of hydration of the groups involved.

(D) Changes in the iomization constunts of enzyme groups. Acidic and basic ionization constants are sensitive to temperatures as are all dissociation constants and consequently the ionization state of a protein will vary to some extent with temperature. This is of significance not only with respect 
to groups at the active center but to groups over the entire enzyme since the net charge will be temperature-dependent. The electric field at the active center that results from all the charged groups on the enzyme will thus change with temperature and this will affect the interactions with inhibitors.

(E) Changes in the configuration of the active center. The internal structures of most simple substances undergoing reactions do not change appreciably with temperature in the physiological range. However, proteins are not rigid molecules and it is likely that shape changes occur during variation of the temperature, and not only changes of the over-all shape but changes in the configuration of the active centers of enzymes. More and more evidence for enzyme flexibility is accumulating. Koshland (1959) has presented some very interesting reasons for believing that certain active centers may be sufficiently flexible to adjust their structures to the substrate that is bound. Such self-induced fits would apply to inhibitors as well. If active centers are amenable to structural changes, it is reasonable that changes in temperature could bring about modifications at these regions, or, perhaps, alter the inherent flexibility of the enzyme so that induction of fit by inhibitors could be more or less easy. Another possibility suggested by Sizer (1943) is that some enzymes may exist in two or more forms of differing activities. the equilibrium between these forms being temperaturedependent. These different forms would presumably be susceptible to inhibitors to varying degrees.

\section{Examples of the Effects of Temperature on Enzyme Inhibition}

Before discussing the results obtained with enzymes it may be interesting to mention some of the temperature studies on the binding of small molecules and ions to proteins. In general it has been found that such binding is not markedly affected by changes in the temperature. The binding of the first copper ion to serum albumin is quite tight $(\triangle F=-5.91 \mathrm{kcal} /$ mole) but this is due to a large gain in entropy $(A S=29.2 \mathrm{cal} / \mathrm{mole} /$ degree $)$ so that $1 H$ is only $2.78 \mathrm{kcal} /$ mole (Klotz and Curme, 1948). Thus more $\mathrm{Cu}^{++}$ was found to be bound at $25^{\circ}$ than at $0^{\circ}$ but the change in binding was not very large. The binding of methyl orange and azosulfathiazole to serum albumin showed similar behavior in that $1 H$ was only -2.0 to -2.1 $\mathrm{kcal} / \mathrm{mole}$. the energy for binding being mainly contributed by large entropy increases (Klotz and Urquhart, 1949 a). The binding of chloride ion to serum albumin gave a $J H$ of only $0.43 \mathrm{kcal} /$ mole and was, therefore, very little dependent on temperature (Scatchard et al.. 1950 a). No effect at all of temperature on the binding of thiocyanate to serum albumin could be found (Scatchard et al., 1950 b). The enthalpies of binding are uniformly small and this applies as well to the binding of aromatic sulfo- 
nates, dodecyl sulfate, and other surfactant substances (Klotz, 1953). These results also indicate that the binding may either increase or decrease with a rise in temperature, inasmuch as $1 H$ may be positive or negative.

The $K_{i}$ for the inhibition of beef heart succinic dehydrogenase by malonate was given by Singer et $a l$. (1957) as $0.041 \mathrm{~m} M$ at $38^{\circ}$ and $0.025 \mathrm{~m} M$ at $20-23^{\circ}$. At a constant malonate concentration the inhibition would thus decrease with rising temperature. Assuming that Eq. 15-6 is valid, one may calculate that at $38^{\circ}$ the binding of malonate to the enzyme is characterized by the following thermodymamic quantities: $1 F=-6.26 \mathrm{kcal} / \mathrm{mole}$, $\Delta H=-5.48 \mathrm{kcal} / \mathrm{mole}$, and $\Delta S=2.6 \mathrm{cal} / \mathrm{mole} /$ degree. The enthalpy values for the substrates, succinate and fumarate, are similar in magnitude, supporting the concept that malonate is bound like the substrates to the enzyme active site. The temperature effects on the inhibition of fumarase by malonate and succinate are more complex, in that the formation of the EI complex is exothermic at low temperatures and endothermic at higher temperatures (Massey, $1953 \mathrm{~b}$ ). The plots of $\log K_{i}$ against $1 / T$ show linear portions connected by sharp breaks (Fig. 15-2), $\Delta H$ being

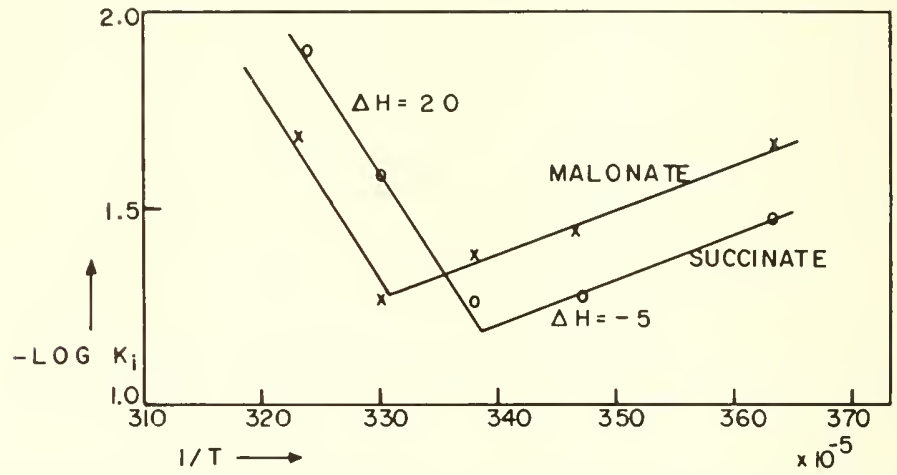

FIG. 15-2. Effects of temperature on the inhibitor eonstants for the inhibitions of fumarase by malonate and suecinate at $\mathrm{pH}$ 6.35. $\Delta H$ given in keal/mole. (From Massey 1953b.)

around $-20 \mathrm{kcal} /$ mole above $27^{\circ}$ and $5 \mathrm{kcal} /$ mole below this temperature. Since $K_{i}$ would be the same for two different temperatures for each inhibitor, and thus the $J F$ the same at these temperatures, the markedly different values of $1 H$ would indicate that the entropies of binding at high and low temperatures are very different. One might readily interpret this striking change with temperature as resulting from a modification of the structure of the active center and supporting the temperature-dependent flexibility of the enzyme. The inhibition of fumarase by thiocyanate is weakly dependent on temperature, the $p K_{i}$ changing from 2.3 to 2.2 as the temperature rises from $25^{\circ}$ to $35^{\circ}$ (Massey and Alberty, 1954). 
The most complete studies of the variation of enzyme inhibition with temperature are those of Gressly and Aebi (1959; Gressly et al., 1959). The actions of azide. hydroxylamime. 2.4-dichlorophenol, and 3-amino-1,2-1 triazole on mouse liver catalase were determined at different concentrations and temperatures. Typical concentration-inhibition curves for azicle are shown in Fig. 15-3 and the marked shift with temperatmre is evident. In

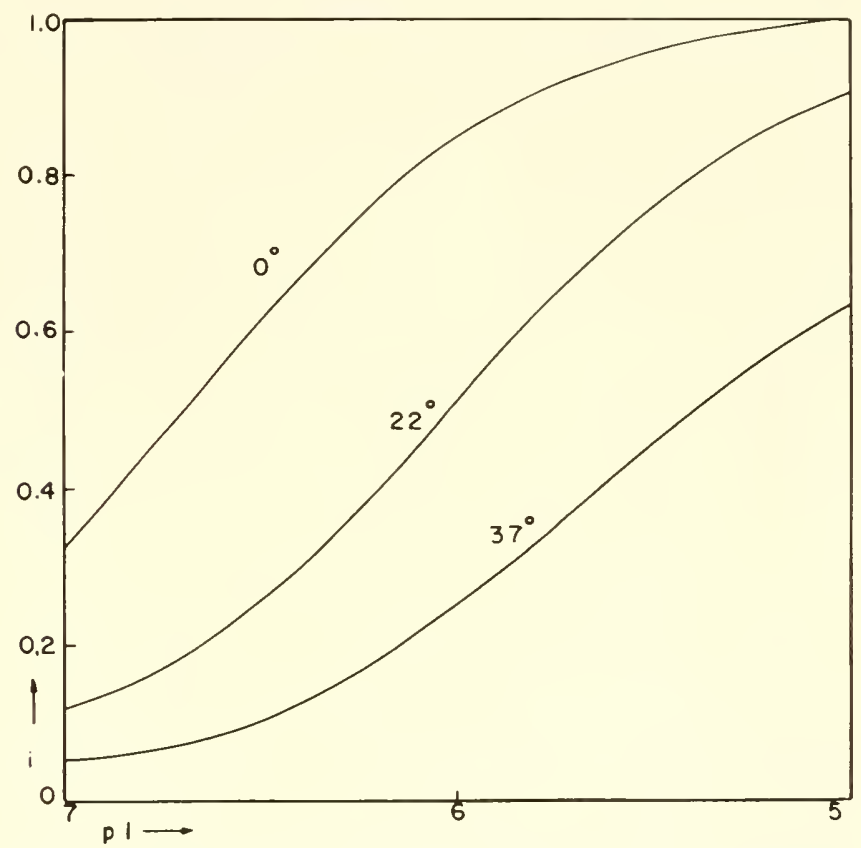

FIG. 15-3. Inhibition of catalase by azide at three different temperatures. (From Gressly et al., 1959.)

Fig. 15-4 the effects of temperature on all the inhibitions are summarized. It is interesting that although three inhibitors follow the usual exothermic pattern, the inhibition by 3-amino-1,2,4-triazole is endothermic. The enthalpy change for azide imhibition is quite high: $1 H=-14.6 \mathrm{kcal}$ mole, $\Delta F=-7.6 \mathrm{keal} / \mathrm{mole}$, and $\angle \mathrm{S}=-2.6 \mathrm{cal} / \mathrm{mole} /$ degree. Another inhibition that increases with rising temperature is that of 1,10-phenanthroline on succinic dehydrogenase, preliminary experiments at the $1 \mathrm{~m} . /$ level showing $13 \%$ inhibition at $17^{\circ} \cdot 14.5^{\%} \%$ inhibition at $27^{\circ}$. and $17.2 \%$ inhibition at $37^{\circ}$ (Sanyal. 1959).

Perhaps the most endothermic inhibition yet reported is that of $500 \mathrm{~m} . I$ urethane on yeast $\beta$-fructofuranosidase between $45^{\circ}$ and $65^{\circ}$, in which range the inhibition increases from $28 \%$ to $97 \%$ (Johnson et al., 1948). It is likely 
that changes in the enzyme structure predispose it to inhibition by urethane at these temperatures and that the rapid rise in the inhibition is not entirely due to an increased affinity. Below $45^{\circ}$ the inbibition is much less dependent on temperature and around $25^{\circ}$ there appears to be no va-
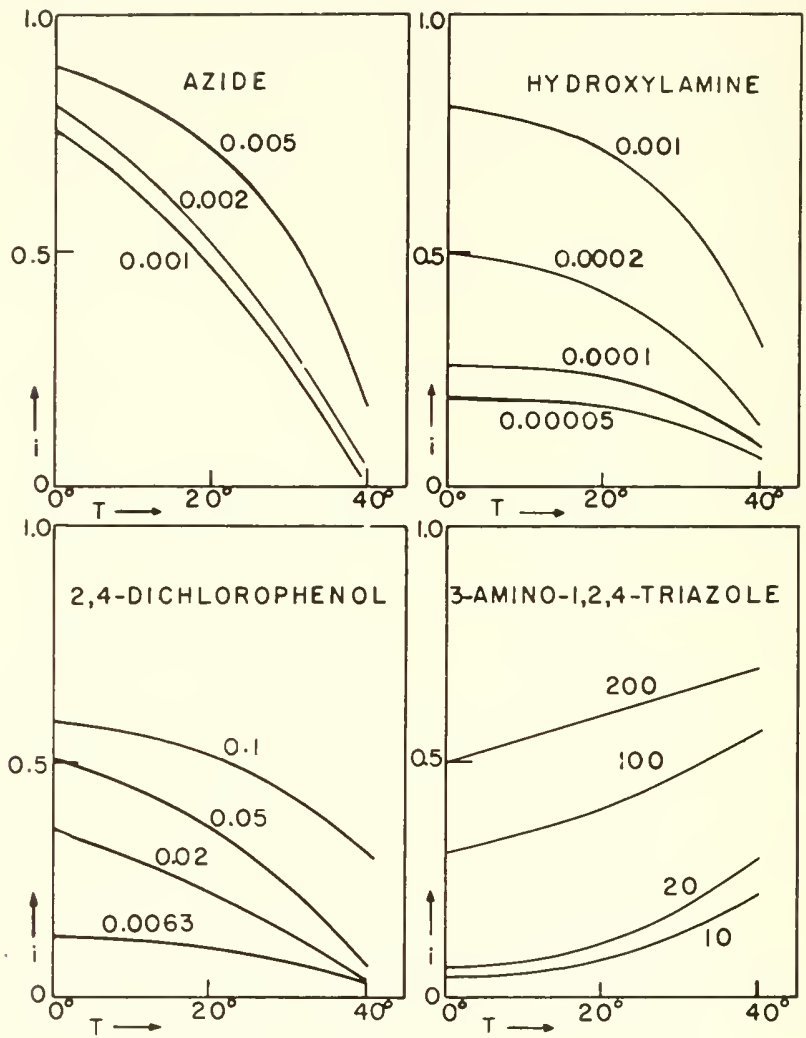

FIG. 15-4. Effects of temperature on the inhibitions of catalase by four inhibitors. Concentrations are given in millimolar. (From Gressly and Aebi, 1959.)

riation with temperature. The mechanisms involved in this inhibition will be discussed in the later section on pressure effects. inasmuch as high pressures antagonize the rise in the inhibition with temperature.

\section{Dependence of Rates of Inhibition on Temperature}

If the rate at which inhibition develops is related to the combination of the inhibitor with the enzyme according to:

$$
\mathrm{E}+\mathrm{I} \stackrel{k}{\rightarrow} \mathrm{EI}
$$


the rate of formation of $\mathrm{EI}$ is given by:

$$
\frac{d(\mathrm{EI})}{d t}=k(\mathrm{E})(\mathrm{I})
$$

Changes in the rates of inhibition with temperature will thus depend on the variations of the rate constant, the enzyme concentration, and the inhibitor concentration with temperature. In most cases the concentrations will not change appreciably with temperature, at least over a physiological range, and only the rate constant need be considered. The rate constant is expressed in transition state theory by:

$$
k=\frac{R T}{N h} e^{-\left(\Delta H^{*} / R T\right)} e^{\left(.1 S^{*} / R\right)}
$$

where $h$ is Planch's constant and $\Delta H^{*}$ and $\lrcorner S^{*}$ are the enthalpy and entropy of activation for the formation of the EI complex. The transmission coefficient $x$ is assumed to be mity and hence is not included in the equation.

In order to determine the change in rate constant with temperature we may write:

$$
\begin{aligned}
& \ln k_{1}=\ln \frac{R}{N h}+\ln T_{1}+\frac{\Delta S^{*}}{R}-\frac{\Delta H^{*}}{R T_{1}} \\
& \ln k_{2}=\ln \frac{R}{N h}+\ln T_{2}+\frac{\Delta S^{*}}{R}-\frac{\Delta H^{*}}{R T_{2}}
\end{aligned}
$$

where $k_{1}$ and $k_{2}$ are the rate constants at temperatures $T_{1}$ and $T_{2}$. Thus:

$$
\ln \frac{k_{2}}{k_{1}}=\frac{\Delta H^{*}}{R}\left[\frac{1}{T_{1}}-\frac{1}{T_{2}}\right]+\ln \frac{T_{2}}{T_{1}}
$$

It may be seen that the temperature dependence of the rate constant is related to $\Delta H^{*}$, the enthapy of activation, just as the temperature dependence of the equilibrium constant is related to $\Delta H$, the over-all enthalpy change in the formation of the EI complex. In the physiological range, an increase in the temperature of $10^{\circ}$ will double the rate constant when $\Delta H^{*}$ is about $12 \mathrm{kcal} /$ mole.

The enthalpy of activation may be obtained by a simple plotting procedure resulting from rewriting Eq. 15-10 in the following form:

$$
\ln \frac{k}{T}=\ln \frac{R}{N h}+\frac{\Delta S^{*}}{R}-\frac{\Delta H^{*}}{R T}
$$

when $\log (k / T)$ is plotted against $1 / T$, a straight line with a slope of - $\left(\Delta H^{*} / 2.303 R\right)$ will be obtained if $1 H^{*}$ does not change appreciably 
with the temperature. This enthalpy of activation is related to the Arrhenius activation energy as follows: $E_{\text {act }}=\Delta H^{*}+R T$. If $1 H^{*}$ ehanges with temperature, the plot will not be linear and the slope at any point will give the $\Delta H^{*}$ at that temperature. The entropy of activation may be obtained from the intercept on the $\log (k / T)$ axis, which is equal to $\log (R /$ $N h)+\left(1 S^{*} / 2.303 R\right)$.

These concepts may be applied to any rate constants, not only for the formation of the EI complex, but also, for example, to $k_{-1}$ for the dissoeiation of the EI complex, or to $k_{2}$ for the dissociation of the ES complex into products. The enthalpies and entropies of activation are actually known for many enzyme reactions, both for the formation and the dissociation of the ES complexes (see Lumry, 1959, Laidler, 1958), but very little has been reported on rate constants related to enzyme inhibition. Information on the course of the binding reaction could be obtained from temperature studies on the rates at which inhibitions develop. As Laidler (1958) points out, an entropy change upon formation of EI* could result from either structural changes in the protein at the active center or in alterations of the state of hydration. If the enzyme unfolds during the formation of the aetivated complex, $1 S^{*}$ will be positive, and there is some indireet evidenee that such may oeeur in the formation of the ES* activated complex. However, 'the entropy changes due to modifieations in the hydration may often mask these structural effects. If eharges on the enzyme and inhibitor are neutralized during the complex formation, water will be set free and $1 S^{*}$ will be positive. Unfortunately the rates of inhibition in many important cases are too rapid to measure with the teehniques that are available and hence the data from which activation energies might be calculated are not available.

More temperature studies on inhibition rates have been done using eholinesterase than any other enzyme, but the extent and aceuracy of the data are often insufficient for thermodynamic eharacterization. The rate of inhibition of human plasma cholinesterase by $\mathrm{HgCl}_{2}$ is markedly dependent on the temperature (Goldstein and Doherty, 1951). For example, a concentration of $0.114 \mathrm{~m} M$ inhibited $76.4^{\%}$ after 4 h at $37^{\circ}$, but at $0^{\circ}$ there was only $18.5 \%$ inhibition after $24 \mathrm{~h}$ and $63.7 \%$ inhibition after 7 days. The inactivation rate at $37^{\circ}$ was about a hundred times greater than at $0^{\circ}$. The kinetics of the inhibition led to the postulate that an initial inhibition (perhaps as the result of reaction with SH groups) is followed by an irreversible inaetivation. The temperature eoefficient is mnch higher for the seeondary inactivation than for the early inhibition, indieating that the former is akin to a denaturation process. The inhibitions of human erythrocyte eholinesterase by urethane (Shukuya. 1953) and salieylate (Shukuya, 1954) also seem to involve two types of inhibition mechanisms, since the temperature coefficients were high when high concentrations of the inhibitors were used, implying a denaturation-type inaetivation, and 
low, or even negative in the case of urethane. when low concentrations were used. Unfortunately the experiments with high and low inhibitor concentrations were done under somewhat different conditions so that comparison camnot be quantitative.

The calculation of thermodynamic values from data obtained on slowly acting and partially irreversible inhibitors has occasionally been ambiguous. When the inhibition is determined after a single time interval, it is difficult to know if this represents a rate process or a partial equilibrium, or both, and, hence, if the calculated enthalpy change refers to an activated complex or to the final complex. If the enthalpy of activation is required, it is preferable to determine the progress of the inhibition and to use the initial rates at different temperatures. The problem of simultaneous inhibition and inactivation has been discussed in Chapter 12 and the formulations presented there may be used as a basis for predicting temperature effects. It may also be pointed out that it is not valid to plot $\log i$ against $1 / T$, as was clone for the inhibition of cholinesterase by urethane (Shukuya, 1953), since the inhibition may be expressed by an equation of the type $i=1-e^{-k(\mathrm{r}) t}$ (see Eqs. 12-12 and 12-16), and $\log i$ is not proportional to $1 / T$ because the rate constant itself is an exponential function of $1 / T$. One must first plot $\log (1-i)$ against $t$ to determine the values of $k$ at different temperatures, and then plot $\log k$ against $1 / T$ to determine the energy of activation, or $\log (k / T)$ against $1 / T$ to determine the enthalpy of activation.

The temperature dependence of the inhibition of cholinesterase by the organophosphorus compounds is complex because at least two steps are involved, the formation of a reversible EI complex and the ensuing chemical reaction of the inhibitor with an enzyme group. Another complicating factor with certain of these inhibitors is the spontaneous hydrolysis of the phosphorylated enzyme, since the rate of this reversal reaction will also be dependent on the temperature. The inhibition of electric organ cholinesterase by diisopropylfuorophosphate has been found by Nachmansohn et al. $(1947,1948)$ to develop more rapidly at higher temperatures, but the difference in the rates is not very large. They stated that the inhibition possessed a $Q_{10}$ of about 2 but Chadwick (1957) recalculated their data and came up with a $Q_{10}$ between 1.22 and 1.44 . Chadwick using fly head cholinesterase found a $Q_{10}$ of 1.58 , which is similar to the $Q_{10}$ of 1.34 for the hydrolysis of acetylcholine by this enzyme. Aldridge $(1953 \mathrm{~b})$ found that the inhibition of erythrocyte cholinesterase by the slowly reversible E-600 was characterized by an energy of activation of $10.6 \mathrm{kcal} /$ mole. This must represent the formation of the activated complex in the phosphorylation of the enzyme. The rate of the reversal of the inhibition by hydrolysis of the phosphorylated enzyme is associated with an energy of activation of $14.4 \mathrm{kcal} /$ mole. A rise in the temperature in such a case may involve an increased rate of formation of the initial EI complex (with a low temperature coeffi- 
cient), an increased rate of enzyme phosphorylation (with a higher temperature coefficient), and a faster hydrolysis of the inhibited enzyme (with perhaps an even higher temperature coefficient). In addition, at the higher temperatures there will be progressive thermal inactivation of the enzyme. Davies (1955) has reported that below $35^{\circ}$ the inhibition of horse serum cholinesterase by choline shows an enthalpy change of $-9.8 \mathrm{kcal} / \mathrm{mole}$ but above $35^{\circ}$ there is a rather sudden change to $-54 \mathrm{kcal} / \mathrm{mole}$, indicating a denaturation-type effect. However, Chadwick has questioned if the accuracy of the $K_{i}$ determinations was sufficient to justify these calculations.

\section{Equilibria between Active and Inactive forms of the Enzyme}

Many temperature studies of enzymes and metabolism have been interpreted on the basis of the effects of temperature changes on an equilibrium between catalytically active and inactive (or denatured) forms of the enzyme, particularly by Johnson and his colleagues (Johnson et al., 1954). There has been some confusion about the kinetics of such systems and, indeed, it is evident that the formulations are enzymically incorrect in most instances. Reiner (1959, p. 267) has pointed out some of the errors and there are others related more specifically to inhibitions that will be mentioned later. Under the circumstances, it will be better to present the kinetic equations for several possible systems first, rather than to discuss erroneous or inapplicable formulations. The fundamental addition that must be made to the systems discussed in earlier chapters is the equilibrium:

$$
\mathrm{E}_{a} \rightleftharpoons \mathrm{E}_{d} \quad K=\left(\mathrm{E}_{a}\right) /\left(\mathrm{E}_{a}\right)
$$

where $\mathrm{E}_{a}$ is catalytically active enzyme and $\mathrm{E}_{d}$ is denatured or inactive enzyme. It must be understood that the inactivating structural change need refer only to the active center and that total enzyme denaturation is not implied; in fact, it might be better if the term "denaturation" were not used in this connection because the structural changes may not be related to what is classically supposed to occur during denaturation.

We shall first consider a simple noninhibited enzyme system:

$$
E_{a} \stackrel{K^{*}}{\rightleftharpoons} \mathrm{E}_{a}+\mathrm{S} \stackrel{K_{s}}{\rightleftharpoons} \mathrm{E}_{a} \mathrm{~S} \stackrel{k_{2}}{\rightarrow} \mathrm{E}_{a}+\mathrm{P}
$$

The rate will be given by $v=k_{2}\left(\mathrm{E}_{a} \mathrm{~S}\right)$ and the conservation equation for the enzyme is $\left(\mathrm{E}_{t}\right)=\left(\mathrm{E}_{a}\right)+\left(\mathrm{E}_{d}\right)+\left(\mathrm{E}_{a} \mathrm{~S}\right)$. The rate in terms of the substrate concentration and the equilibrium constants is then:

$$
v=\frac{V_{m}(\mathrm{~S})}{(\mathrm{S})+K_{s}\left[1+\frac{1}{K}\right]}
$$


When $K$ is very high and the enzyme essentially exists completely in the active form, this reduces to the usual Michaelis-Menten rate expression. The effect of temperature on the rate will now depend on the variation of $K$ with the temperature. It is generally assumed that inactivation of the enzyme increases with temperature and thus $K$ becomes smaller as the temperature is raised, the term in brackets increasing and the rate decreasing. Equation 15-17 can be used to explain the phenomenon of maxima in the rate-temperature curves (see following section).

The inhibitor may combine only with $E_{d}$, or only with $\mathrm{E}_{\alpha}$ (competitive or noncompetitive), or with both $\mathrm{E}_{d}$ and $\mathrm{E}_{\alpha}$ (competitive or noncompetitive).

Case I: inhibitor combines only with $\mathrm{E}_{d}$

$$
\mathrm{E}_{d} \mathrm{I} \stackrel{K_{i}{ }^{\prime}}{\rightleftharpoons} \mathrm{E}_{d} \stackrel{K}{\rightleftharpoons} \mathrm{E}_{a} \stackrel{K_{s}}{\rightleftharpoons} \mathrm{E}_{a} \mathrm{~S} \rightarrow \mathrm{E}_{a}+\mathrm{P}
$$

In all cases $K_{i}{ }^{\prime}$ will indicate the dissociation constant of the complex with inactive enzyme and $K_{i}$ the dissociation constant of the complex with active enzyme. The conservation equation is now $\left(\mathrm{E}_{t}\right)=\left(\mathrm{E}_{\imath}\right)+\left(\mathrm{E}_{d}\right)+\left(\mathrm{E}_{\imath} \mathrm{S}\right)+$ $\left(\mathrm{E}_{d} \mathrm{I}\right)$. The rate is given by:

$$
v_{i}=\frac{V_{m}(\mathrm{~S})}{(\mathrm{S})+K_{s}\left[1+\frac{1}{K}+\frac{(\mathrm{I})}{K K_{i}^{\prime}}\right]}
$$

and the inhibition by:

$$
i=\frac{(\mathrm{I})}{(\mathrm{I})+K_{i}^{\prime}\left[1+K+\frac{K(\mathrm{~S})}{K_{s}}\right]}
$$

It may be noted that when the enzyme is all in the active form and $K=\infty$, the rate will be that of the system in the absence of the inhibitor and the inhibition will be zero, since there will be no $\mathrm{E}_{d}$ with which the inhibitor can combine. When the enzyme is mainly in the inactive form, the rate will be low and the inhibition will approach noneompetitive kinetics. However, in the intermediary range the inhibition will depend on the substrate concentration much as in competitive inhibition. It is difficult to distinguish between this situation and true competitive inhibition. Plots of $1 / v_{i}$ against $1 /(\mathrm{S})$ give straight lines with slopes of

$$
\frac{K_{s}}{V_{m}}\left[1+\frac{1}{K}+\frac{(\mathrm{I})}{K K_{i}^{\prime}}\right]
$$

whose intercepts on the $1 / v_{i}$ axis will not vary with the inhibitor concentration. A plot of $(\mathrm{I})(1-i) / i$ against (S) will likewise give a straight line indistinguishable from true competitive inhibition, but the slope will now be $K K_{i}{ }^{\prime} / K_{s}$ instead of $K_{i}{ }^{\prime} / K_{s}$ and the intercept will be $K_{i}{ }^{\prime}(1+K)$ instead 
of $K_{i}{ }^{\prime}$. In all cases where there is inactive enzyme, the usual determinations of the inhibitor constant will be in error by an amount dependent on the value of $K$. With respect to the effects of changes in the temperature, the inhibition will increase as the temperature rises due to the fall in $K$ and the increase in $E_{d}$ with which the inhibitor can combine. By appropriate graphical analysis it would be theoretically possible to determine the changes in both $K$ and $K_{i}{ }^{\prime}$ but the temperature range would have to be sufficient to allow accurate evaluation.

Case II: inhibitor combines only with $\mathrm{E}_{a}$ - competitive

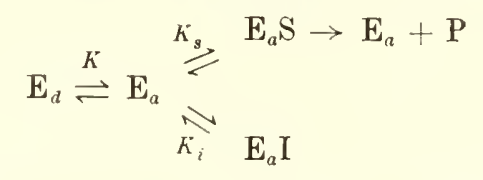

The rate and inhibition equations may be shown to be:

$$
\begin{gathered}
v_{i}=\frac{V_{m}(\mathrm{~S})}{(\mathrm{S})+K_{s}\left[1+\frac{1}{K}+\frac{(\mathrm{I})}{K_{i}}\right]} \\
i=\frac{(\mathrm{I})}{(\mathrm{I})+K_{i}\left[1+\frac{1}{K}+\frac{(\mathrm{S})}{K_{s}}\right]}
\end{gathered}
$$

As $K$ approaches infinity, the inhibition becomes completely competitive, and when $K$ is small. both the minhibited and inhibited rates decrease. Graphical procedures yield plots characteristic of competitive inhibition but from which the calculation of $K_{i}$ will be in error. A rise in temperature will cause a fall in the inhibition.

Case III: inhibitor combines only with $\mathrm{E}_{a}$ - noncompetitive

$$
\mathrm{E}_{d} \stackrel{K}{\rightleftharpoons} \mathrm{E}_{a} \stackrel{K_{s}}{\stackrel{\mathrm{E}_{a} \mathrm{~S}}{\gtrless} \underset{\mathrm{K}_{i} \mathrm{I}}{\stackrel{K_{i}}{\gtrless}} \stackrel{\mathrm{E}_{a}+\mathrm{P}}{\gtrless} \mathrm{E}_{a} \mathrm{SI}}
$$

For completely noncompetitive inhibition the equations take the forms:

$$
\begin{aligned}
v_{i} & =\frac{V_{m}(\mathrm{~S})}{(\mathrm{S})\left[1+\frac{(\mathrm{I})}{K_{i}}\right]+K_{s}\left[1+\frac{1}{K}+\frac{(\mathrm{I})}{K_{i}}\right]} \\
i & =\frac{(\mathrm{I})}{(\mathrm{I})+K_{i}+\frac{K_{i} K_{s}}{K\left[(\mathrm{~S})+K_{s}\right]}}
\end{aligned}
$$


Despite the fact that the inhibition is noncompetitive, the inhibition will depend on the substrate concentration, but in a different manner than in competitive inhibition since here the inhibition will be increased as the substrate concentration rises. As $K$ approaches infinity, the inhibition becomes classically noncompetitive. The inhibition will again decrease as the temperature is increased.

Case IV: inhibitor combines with both $\mathrm{E}_{\gamma}$ and $\mathrm{E}_{d}-$ competitive

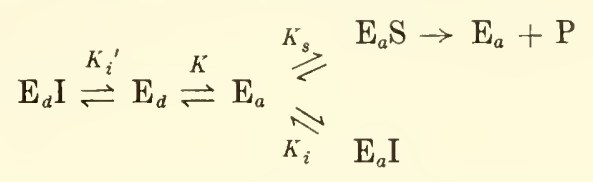

The rate and inhibition equations are somewhat more complex than in the previous situations:

$$
\begin{gathered}
v_{i}=\frac{V_{m}(\mathrm{~S})}{(\mathrm{S})+K_{s}\left[1+\frac{1}{K}+\frac{(\mathrm{I})}{K_{i}}+\frac{(\mathrm{I})}{K K_{i}{ }^{\prime}}\right]} \\
i=\frac{(\mathrm{I})}{(\mathrm{I})+\frac{K K_{i} K_{i}{ }^{\prime}}{K_{i}+K K_{i}^{\prime}}\left[1+\frac{1}{K}+\frac{(\mathrm{S})}{K_{s}}\right]}
\end{gathered}
$$

These more general equations reduce to $15-19$ and 15-20 when $K_{i}=\infty$, and to Eqs. 15-22 and 15-23 when $K_{i}^{\prime}=\infty$, as expected. The variation of the inhibition with temperature will be complex and will depend on the relative values of $K_{i}$ and $K_{i}$.

Case $\mathrm{V}$ : inhibitor combines with both $\mathrm{E}_{a}$ and $\mathrm{E}_{d}$ - noncompetitive

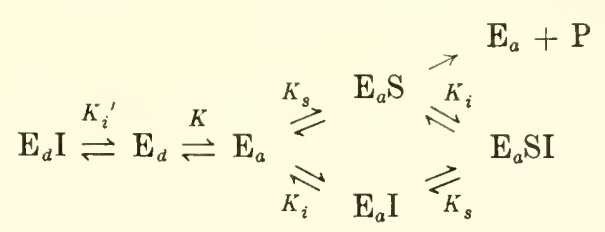

If an enzyme can exist in active and inactive forms. this situation is probably fairly common and leads to the following rate and inhibition equations:

$$
\begin{aligned}
& v_{i}=\frac{V_{m}(\mathrm{~S})}{(\mathrm{S})\left[1+\frac{(\mathrm{I})}{K_{i}}\right]+K_{s}\left[1+\frac{1}{K}+\frac{(\mathrm{I})}{K_{i}}+\frac{(\mathrm{I})}{K K_{i}{ }^{\prime}}\right]} \\
& i=\frac{(\mathrm{I})}{(\mathrm{I})+K_{i}\left[\frac{(\mathrm{S})+K_{s}[1+(1 / K)]}{(\mathrm{S})+K_{s}\left[1+\left(K_{i} / K K_{i}{ }^{\prime}\right)\right]}\right]}
\end{aligned}
$$


When either $K$ is very large or $K_{i}=K_{i}{ }^{\prime}$, the inhibition becomes purely noncompetitive, but generally is dependent on the substrate concentration in a complex fashion. The variation of the inhibition with temperature will depend on the ratio of $K_{i} / K_{i}$.

The variation of the inhibition with the temperature in such systems will thus depend on the form of the enzyme with which the inhibitor combines and whether the inhibition is competitive of noncompetitive. Of course, other types of inhibition, such as uncompetitive or coupling, may occur. The change in inhibition with $K$ for case $V$ has been plotted in Fig. 15-5

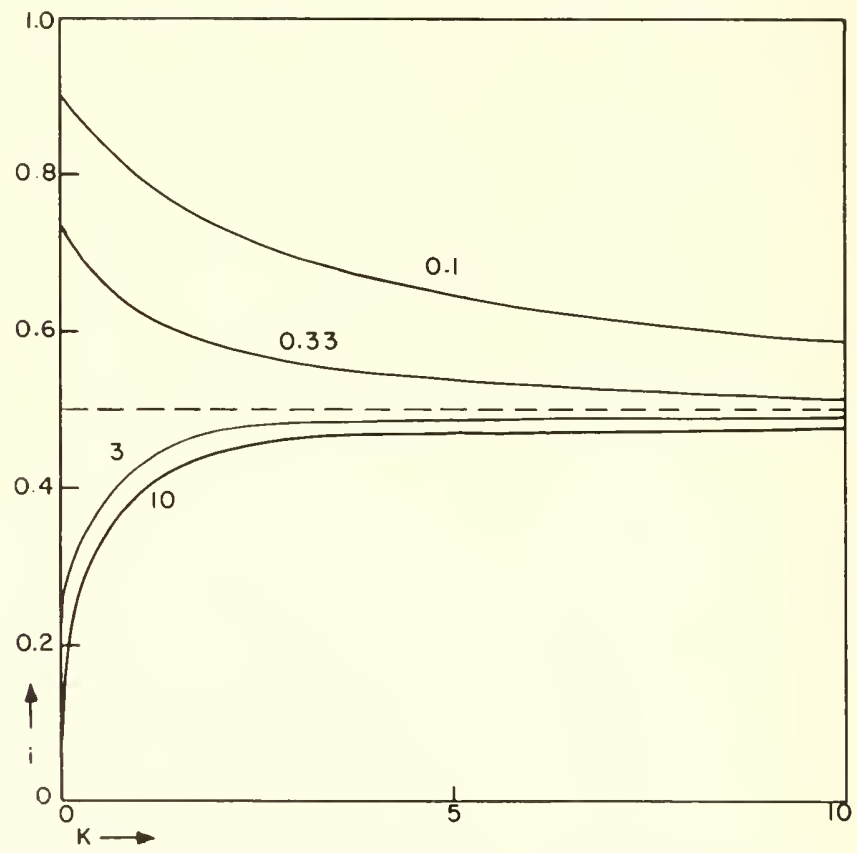

FIG. 15-5. Variation of the inhibition with $K$, the equilibrium constant between active and inactive enzyme, for case $V$ where the inhibitor combines with both $\mathrm{E}_{a}$ and $\mathrm{E}_{d}$ noncompetitively (scheme 15-30 and Eq. $15-32$ ). (I) $=1 \mathrm{~m} \mu$. (S) $=1 \mathrm{~m} M, K_{i}=1$ $\mathrm{m} M$, and $K_{s}=1 \mathrm{~m} \boldsymbol{H}$. The figures on the curves are values of the ratio $K_{i} / K_{i}{ }^{\prime}$.

and it may be observed that when $K$ is high, the inhibition approaches $50 \%$ whatever the ratio $K_{i} / K_{i}{ }^{\prime}$ for the specific situation selected. If increasing the temperature brings about a shift in the equilibrium to form more of the inactive enzyme, i.e., $K$ becomes smaller, the inhibition will either increase or decrease depending on whether the ratio $K_{i} / K_{i}^{\prime}$ is greater than 
unity or less than unity. Changes in the temperature may also alter $K_{s}$ and $K_{i}$ or $K_{i}$ ' so that the variation of the inhibition in most cases would be more complex than represented here. A complete description of an inhibition and its dependency on temperature would require the determination of the constants $K, K_{i}$, and $K_{i}^{\prime}$, but experimentally this would require an exact elucidation of the mechanism of the inhibition and a great deal of very accurate data obtained at different temperatures and concentrations of inhibitor.

The change of $K$ or $\left(\mathrm{E}_{a}\right) /\left(\mathrm{E}_{d}\right)$ with temperature may be roughly estimated if the inactivation process is akin to protein denaturation. The average thermodynamic characteristics for the denaturation of trypsin, chymotrypsinogen, and soy bean trypsin inhibitor are: $J F=-1.2 \mathrm{kcal} / \mathrm{mole}$, $\Delta H=75 \mathrm{kcal} / \mathrm{mole}$, and $1 S=235 \mathrm{cal} / \mathrm{mole} /$ degree. From Eq. $15-6$ it may be calculated that a $10^{\circ}$ rise in temperature in the physiological range would decrease $K$ by a factor of approximately one-fortieth. However, local structural changes at the active center may not follow over-all protein denaturation thermodynamics, so that any estimates on this basis must be considered to be very tentative. The temperature coefficient, nevertheless, would probably be fairly high.

We must now examine as critically as possible this concept of equilibria between active and inactive forms of enzymes and the bearing this has on inhibition and temperature effects. A marked rise in the inhibition of an enzyme upon increasing the temperature is not in itself sufficient evidence for such an equilibrium, since the inhibition reaction may have a high enthalpy change independent of any equilibrium between active and inactive forms. The inhibitor, indeed, may produce a local denaturation of the enzyme and this may occur more readily at higher temperatures. but this does not imply the presence of denatured forms before the inhibitor is added. There is no question about the denaturation of enzymes when the temperature is elevated sufficiently and all enzymes exhibit maxima in their temperature-activity curves. The problem here is: at physiological temperatures between $15^{\circ}$ and $38^{\circ}$. where most enzyme and metabolic work is carried out, is such enzyme inactivation of importance? The optimal temperature for enzyme activity (i.e., the temperature at which the rate is maximal) has been stated to be between $40-50^{\circ}$ for most animal enzymes and between 50-60 for most plant enzymes (Sumner and Somers, 1953). Inasmuch as the temperature coefficient is high. this would indicate that the majority of enzymes do not exist appreciably in the inactive form in the physiological temperature range. However, certain enzymes are particularly unstable even at $37^{\circ}$ when they are isolated from cells, and in such cases an appreciable fraction may occur in the reversibly denatured or inactive form. For most enzymes the situation is probably as shown in Fig. $15-6$ where the experimental temperature range is sufficiently far below the optimal temperature so that only insignificant amounts of inactive 
enzyme are present. Thus it would appear that for an $\mathrm{E}_{a} / \mathrm{E}_{d}$ equilibrium to be assumed to explain the effects of temperature on enzyme inhibition, experimental evidence that such an equilibrium exists in the temperature range studied for the particular enzyme under experimental conditions must be demonstrated.

There is some indirect evidence that $\mathrm{E}_{a} / \mathrm{E}_{d}$ equilibria are not important for the majority of enzymes. We have seen above that noncompetitive

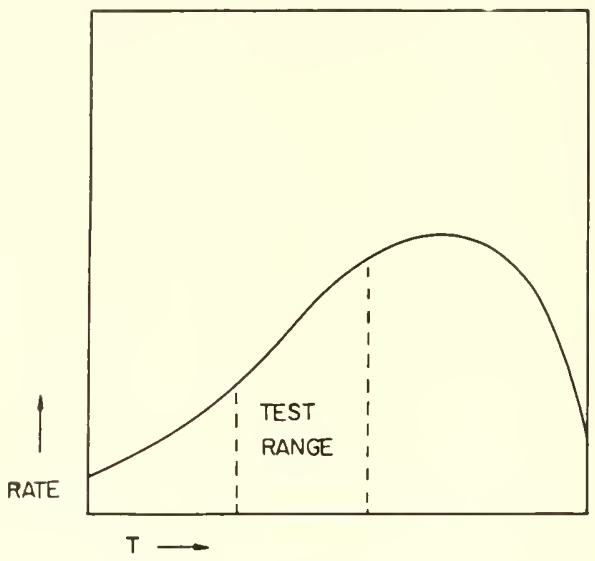

FIG. 15-6. Generalized variation of enzyme rate with the temperature within the common experimental range of temperatures shown.

inhibition would depend to some extent on the substrate concentration if an inactive form of the enzyme occurs, and yet there are many instances where noncompetitive inhibition has been shown to be independent of the substrate concentration in the temperature range studied. Also it may be noted that accorling to Eq. $15-17$ for an uminhibited reaction, the apparent substrate constant would involve $K$, i.e. $K_{s}{ }^{\prime}=K_{s}[1+(1 / K)$. If $K$ varies markedly with the temperature, one would expect the experimentally determined Michaelis constants also to vary strongly with the temperature. which usually does not seem to be the case. The evidence from high pressure studies will be considered later.

A method of calculating $K$, the equilibrium constant for the interconversion of $\mathrm{E}_{\imath}$ and $\mathrm{E}_{d}$, has been snggested by Hnltin (1955) and applied to the hydrolysis of tributyrin by pancreatic lipase at subzero temperatures. Inactivation of the enzyme becomes progressively greater as the temperature is reduced below $-5^{\circ}$ as indicated in the accompanying tabulation where Inltin's $K$ has been altered to fit the convention adopted above. The enthalpy change of the inactivation was calculated to be $-27 \mathrm{kcal} /$ 
mole, which with the rather small change in free energy would imply a large entropy factor. Althongh this sitnation is different from that usually

\begin{tabular}{c|c}
\hline Temperature & $K$ \\
\cline { 2 - 2 }$-5^{\circ}$ & 0.23 \\
$-10^{\circ}$ & 0.014 \\
$-15^{\circ}$ & 0.041 \\
$-18^{\circ}$ & 0.027 \\
$-24.5^{\circ}$ & 0.003 \\
\hline
\end{tabular}

investigated, the principles used in the calculations would be the same for any temperature range.

\section{Effects of Inhibition on the Optimal Temperature}

The temperature at which the rate of an enzyme reaction is maximal is determined usually by a balance between the two major effects of temperatmre, the elevation of the rate with rise in the temperature, due mainly to the increase in $k_{2}$ for the breakdown of the ES complex, and the progressive thermal inactivation of the enzyme. A system such as 15-16 is generally assumed and the variation in the rate, given by Eq. 15-17, with temperature is related to the changes in the constants, $k_{2}, K_{m}$. and $K$. Since the temperature dependence of these constants is determined by the respective enthalpies, these constants may be expressed in terms of the thermodynamic quantities as follows:

$$
\begin{aligned}
& k_{2}=A e^{\left.-(\lrcorner H^{*} / R T\right)} e^{\left(\Delta S^{*} / R\right)} \\
& K_{s}=e^{\left(\Delta H_{s} / R T\right)} e^{-\left(\Delta S_{s} / R\right)} \\
& K=e^{(.1 H / R T)} e^{-(\lrcorner S / R)}
\end{aligned}
$$

and substituted in Eq. 15-17. If the Michaelis constant is not an equilibrium constant, $K_{s}$, each of the rate constants $-k_{1}, k_{-1}$, and $k_{2}-$ must be expressed as in Eq. 15-33. If it is further assumed that the substrate concentration is low so that it may be neglected in the denominator, the rate is given by:

$$
v=\frac{A\left(\mathrm{E}_{t}\right)(\mathrm{S}) e^{\left.(\lrcorner S^{*} / R\right)}}{e^{\left.-(\lrcorner S_{s} / R\right)}}\left\{\frac{e^{\left.-(\lrcorner H^{*} / R T\right)}}{e^{\left(\Delta H_{s} / R T\right)}\left[1+e^{-(\lrcorner H / R T)} e^{(\Delta S / R)}\right]}\right\}
$$

In order to determine the temperature at which the rate is maximal, $d v / d T$ may be set equal to zero. Since the term outside the brackets is 
independent of temperature, it may be disregarded. The optimal temperature is found to be:

$$
T_{\text {opt }}=\frac{\Delta H}{\Delta S+R \ln \left[\frac{\Delta H}{\Delta H_{s}+\Delta H^{*}}-1\right]}
$$

and is thus related to each enthalpy term and the entropy of the inactivation. The relative values of $1 H, \Delta H_{s}$ and $\Delta H^{*}$ so that a maximum rate will occur may be determined from this equation and the conditions for a maximum in the temperature range studied are defined.

The optimal temperature is not a very useful value in characterizing an enzyme, as Dixon and Webb (1958) have pointed out, and is often timedependent, but the effects of inhibition upon it may be of some interest. For example, it has generally been assumed that when an inhibitor lowers $T_{\text {opt }}$, the inhibitor is facilitating the inactivation of the enzyme. However, from the equation above it may be seen that effects on $\Delta H_{s}$ and $\Delta H^{*}$ may also be involved. It is, after all, a matter of shifting the balance of the temperature dependencies of the constants. A decrease in $T_{o p t}$ will occur if either $\Delta H_{s}$ or $\Delta H^{*}$, or both, is decreased by the inhibitor. Most of the work on shifts in $T_{o p t}$ due to inhibitors has been done on multienzyme systems or cell metabolism but to interpret such effects it is necessary to understand what can happen to the individual enzymes. These more complex systems will be discussed later.

\section{EFFECTS OF TEMPERATURE: MULTIENZYME SYSTEMS}

Although no quantitative studies of the effects of temperature on multienzyme systems have been done, it is possible to formulate certain general principles that will be useful later in discussing the work on cellular metabolism. In any multienzyme system there are two or more reactions proceeding simultaneously and the rates of these reactions will generally vary with temperature in different ways. The rates of most enzyme reactions will increase with rising temperature in the physiological range, but to varying degrees, while in some cases the rate of a particular reaction may actually decrease due to the inactivation of the enzyme. The effects of temperature on the behavior of multienzyme systems and on the response of such systems to inhibition will thus depend on the relative changes of the individual rates. It is important to emphasize that the rate changes of enzyme reactions are related not only to the variations in the rate constant $k_{2}$, and hence to $1 H^{*}$, but also to variations in the Michaelis constant $K_{m}$. In general $K_{m}$ will change with temperature according to the values of $1 H_{1}^{*}$, $\Delta H_{-1}$, and $\Delta H_{2}{ }^{*}$, corresponding to $k_{1}, k_{-1}$, and $k_{2}$, but if it is the equilibrium constant for the ES complex, the change will depend on $\Delta H_{s}$, the over-all enthalpy change in the formation of the ES complex. 
The behavior of some of the multienzyme systems treated in Chapter 7 with respect to their responses to variation of the temperature will now be discussed in general terms.

(A) Monolinear chains. In the simple irreversible system:

$$
\mathrm{A} \stackrel{\mathrm{E}_{1}}{\rightarrow} \mathrm{B} \stackrel{\mathrm{E}_{2}}{\rightarrow} \mathrm{C}
$$

the rate of formation of $\mathrm{C}$ will always depend on the rate of reaction 1 if the system is in a steady state, and on the rate of reaction 2 if it is not in a steady state. Therefore, as long as the system remains in a steady state with a change in the temperature, the over-all rate will vary in the same manner as $v_{1}$, the concentration of the intermediate $\mathrm{B}$ adjusting to the new conditions. This concentration of $\mathrm{B}$ depends on $K_{1}$ and $K_{2}$, the Michaelis constants for $\mathrm{E}_{1}$ and $\mathrm{E}_{2}$, and on the ratio $V_{1} V_{2}$ (Eq. 7-3). A rise in (B) will occur if there is an increase in $V_{1} / V_{2}$, an increase in $K_{2}$ or a decrease in $K_{1}$ brought about by the temperature change, which is one way of stating that $(\mathrm{B})$ will rise when reaction 1 is accelerated relatively more than reaction 2 . The change in $(B)$ will thus depend on the values of all the activation enthalpies associated with each rate constant for each enzyme reaction. Thus with regard to $V_{1} / V_{2}$, the change with temperature is given by:

$$
\frac{\left(V_{1} / V_{2}\right)_{a}}{\left(V_{1} / V_{2}\right)_{b}}=\exp \left\{\frac{\Delta H_{2}^{*}-\Delta H_{1}^{*}}{R} \cdot\left[\frac{1}{T_{b}}-\frac{1}{T_{a}}\right]\right\}
$$

where $\mathrm{JH}_{1} *$ and $\mathrm{IH}_{2} *$ are the enthalpies of activation for the breakdown of the ES complexes for reaction 1 and reaction 2 respectively. The difference in the activation enthalpies thus determines the direction and the degree of change in $V_{1} / V_{2}$ and this is a major factor in the alteration of (B) following a variation of the temperature.

If an increase in the temperature speeds reaction 1 sufficiently, relative to the rate of reaction 2. the system may pass out of a steady state. This may occur more readily if a limit is imposed on the degree to which (B) may rise. If the system leaves the steady state. the temperature dependence will be related more to reaction 2 since it is now controlling the rate of formation of $\mathrm{C}$. It is upon this basis that changes in the temperature characteristics have usually been interpreted and this problem will be discussed in greater detail later. However, if the disappearance of $\mathrm{A}$ is being measured. such a shift in the temperature dependence will not be observed.

Care must be taken in those cases where diffusion of the substrate into the multienzyme compartment might be limiting the enzyme rates inasmuch as the observed responses to temperature change may be related more to the diffusion process than to the enzyme reactions. It has often been assumed 
that diffusion has a low temperature coefficient but actually some membrane diffusion rates have high activation energies and are as sensitive to temperature as are enzyme rates. This is particnlarly apt to be the case when the diffusion is slow and can be limiting the rate of formation of some metabolic product.

Inhibition on the first reaction of a linear sequence will be affected by temperature as described above and, since the over-all rate depends only on $v_{1}$ in the steady state, the over-all rate will be affected similarly. If a change of the temperature, however, shifts the limiting step to another reaction, the inhibition may be markedly modified. For example, if a rise in temperature causes the system to leave a stearly state so that reaction 2 controls the formation of $\mathrm{C}$, an inhibition on $\mathrm{E}_{2}$ will be directly reflected in $d(C) / d t$, whereas initially such an inhibition would not affect $d(\mathrm{C}) / d t$ until reaction 2 had been reduced sufficiently to be limiting. Thus temperature changes can alter the sensitivity to inhibition of linear enzyme systems both by effects on the enzyme inhibition itself and by changes in the relative rates of the reactions involved. Conversely, inhibition of a complex system may alter its sensitivity to temperature variation and such changes have been observed in cellnlar studies.

(B) Convergent chains. In treating the system:

$$
\mathrm{X} \underset{\mathrm{E}_{3}}{\mathrm{~A}} \stackrel{\mathrm{E}_{2}}{\rightarrow} \mathrm{C}
$$

we come to one of the serious difficulties in the interpretation of temperatmre effects. If either of the feeding reactions to form $\mathrm{B}$ is speeded relative to the other, the rate of formation of $\mathrm{C}$ will be changed to a degree intermediate between the changes of reaction 1 and reaction 3 . If reaction 1 is changed by a factor of $g_{1}$ and reaction 3 by a factor of $g_{3}$, since $v_{2}=$ $v_{1}+v_{3}$ in the steady state, the factor $g_{2}$ by which the formation of $\mathrm{C}$ is changed will be given by:

$$
g_{2}=\frac{g_{1} v_{1}+g_{3} v_{3}}{v_{1}+v_{3}}
$$

For example, if $v_{1}=10$ and $v_{3}=5$, and a change in temperature brings about changes of $g_{1}=2$ and $g_{3}=4$ in these rates. $g_{2}$ will be found to be 2.67. The temperature characteristies calculated from the data derived in such systems will not apply to any particular enzyme reaction and will be in themselves without physical meaning. Any effect of temperature on $v_{2}$ will not be reflected in the cliange of the rate of formation of $\mathrm{C}$ as long as the system remains in a steady state, because $v_{2}$ is controlled by the sum of the feeding reaction rates. In cellnlar metabolism there are many path- 
ways that are convergent, as well as other types of multienzyme systems which behave in a similarly complex manner, and this frequently makes it impossible to assign the results from temperature studies to any one reaction step.

It is obvious that when $\mathrm{E}_{1}$ is selectively inhibited, the depression of the formation of $\mathrm{C}$ will depend on the relative contributions of reaction 1 and reaction 3 to the production of $B$, and that if temperature changes this relative contribution, the over-all inhibition will be altered (Eq. 7-24). Inhibition of one of the feeding reactions will also modify the response of the system to temperature change, since this response depends on the relative contributions of reaction 1 and reaction 3 .

(C) Divergent chains. Here the result will depend on which product is determined. A change of the temperature may shift the ratio of $v_{2} / v_{3}$, i.e., the fraction of $B$ that goes to each product may be changed. It is

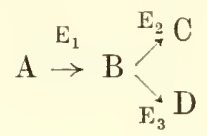

even possible that an increase in the temperature will decrease the rate at which one of the products is formed, due to the other reaction having a relatively high temperature coefficient. If one is primarily interested in reaction 2 , its rate at the control temperature is given by $v_{2}=v_{1}-v_{3}$ and at the altered temperature by $g_{2} v_{2}=g_{1} v_{1}-g_{3} v_{3}$, assuming a steady state. Thus the factor by which reaction 2 is changed by the temperature variation is given by:

$$
g_{2}=\frac{g_{1} v_{1}-g_{3} v_{3}}{v_{1}-v_{3}}
$$

It is true that these relationships are very simple. and perhaps obvious to some, but they have generally been ignored by those studying the effects of temperature on metabolism.

If an inhibitor acts selectively on $\mathrm{E}_{3}$, the fractional stimulation of $v_{2}$ will be $i_{3} r_{3} / r_{2}$. At another temperatme the stimulation of $v_{2}$ will be given by $i_{3} g_{3} v_{3} / g_{2} v_{2}$. Thus the degree to which reaction 2 is stimulated by the inhibitor will depend on $g_{3} / g_{2}$, the ratio of the temperature effects on these two reaction rates, as well as on any alteration of the inhibition of reaction $3 \mathrm{by}$ the temperature change. The steady state concentration of $\mathrm{B}$ depends on $V_{1}, V_{2}, V_{3}, K_{1}, K_{2}$, and $K_{3}$ (Eq. 7-27) in the uninhibited system and. in addition, on $K_{i}$ in the inhibited system, so that the inhibition of any step will alter $(B)$ to a degree dependent on the temperature in a complex fashion. since all of these constants may vary with the temperature. 
(D) Polylinear chains. If an inhibitor acts to varying degrees on the different pathways of a multishmt system, such as:

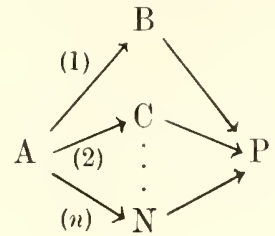

it has been shown in chapter 7 that the over-all inhibition on the rate of formation of the product will be given by:

$$
i=\frac{i_{1} v_{1}+i_{2} v_{2}+\ldots+i_{n} v_{n}}{v_{1}+v_{2}+\ldots+v_{n}}
$$

A change of the temperature will alter the rates of the different pathways to varying degrees indicated by the factor $g$; thus $v_{1}$ will become $g_{1} v_{1}$, etc. In addition, the individual inhibitions may change with temperature, so that $i_{1}$ is now $i_{1}{ }^{\prime}$, etc. The over-all inhibition at this new temperature will then be:

$$
i^{\prime}=\frac{i_{1}{ }^{\prime} g_{1} v_{1}+i_{2}{ }^{\prime} g_{2} v_{2}+\ldots+i_{n}{ }^{\prime} g_{n} v_{n}}{g_{1} v_{1}+g_{2} v_{2}+\ldots+g_{n} v_{n}}
$$

The change in the over-all inhibition with temperature will obviously not be directly related to any single pathway or individual inhibition and no thermodynamically significant characteristics may be obtained from the experimental data.

(E) Cyclic systems. The temperature dependence of rates in cyclic systems is too complex to analyze in detail. Reference to Eqs. 7-37 and 7-38, and the use of the graphical method outlined in Chapter 7 , make possible qualitative predictions as to how the concentrations of the cycle intermediates may change as the temperature is varicd, if the temperature characteristics for the various constants are assumed. In general it may be noted that the rates in cyclic systems are not usually limited by single reactions and hence that the results from the variation of temperature camnot be interpreted in simple terms.

It is mnecessary to deal with any other multienzyme systems because the general conclusions relative to the effects of temperature are clearly illustrated in the cases discussed. Discussions of multienzyme systems in the past have dealt almost exchnsively with monolinear chains. Here it is possible to have a limiting reaction and the effects of temperature can often be related to this reaction. In other types of multienzyme systems this is not true and temperature effects cannot be attributed to single reactions. 
Certainly most metabolic processes involve a variety of multienzyme systems of different types. Respiration normally is the over-all result of enzyme activity in monolinear chains, polylinear chains, branched chains, cycles, and regenerative systems all operating simultaneously. It would appear extremely unlikely that the variation of respiration with the temperature could be interpreted in specific terms, or that temperature characteristics could be assigned to certain enzymes functioning in the total system. The presence of an inhibitor always introduces an additional factor into an already very complex situation and so the effects of temperature on inhibition must also in the general case be considered to be difficult to analyze. However, in some instances the use of a selective inhibitor may simplify the problem to some extent. If certain pathways can be blocked, or their importance in the over-all process minimized, the metabolic system may become more simple and the effects of temperature more readily interpretable.

One more temperature-dependent characteristic of multienzyme systems must be mentioned before proceeding to a consideration of cellular metabolism. When an inhibitor acts on a multienzyme system in the steady state, it requires a definite time for the system to reach a new steady state, if such occurs. This transition time has been discussed in Chapter 7 and it was pointed out there that in a monolinear chain the rate of change of the concentration of the intermediate depends on the difference between the rates of formation and utilization, i.e., $d(\mathrm{~B}) / d t=v_{1}-v_{2}$. When $v_{1}=v_{2}$ in the steady state, $d(\mathrm{~B}) / d t=0$. When reaction 2 is inhibited, $d(\mathrm{~B}) / d t=$ $v_{1}-(1-i) v_{2}$. A change in the temperature will alter $v_{1}$ to $g_{1} v_{1}$ and $v_{2}$ to $g_{2} v_{2}$, so that now $d(\mathrm{~B}) / d t=g_{1} v_{1}-(1-i) g_{2} v_{2}$. The rate at which (B) changes and, hence, the transition time will depend on the relative effects of the temperature change on $v_{1}$ and $v_{2}$. If reaction 1 is speeded relative to reaction 2. the transition time will be less because the rate of change of (B) will be greater; conversely, if reaction 2 is relatively speeded, the transition time will be lengthened. These effects on transition time may well be of importance in the effects of inhibitors on cells and tissues at different temperatures. For example, it is quite possible that more damage will be done to a tissue by an inhibitor if the metabolism cannot reach a new steady state as rapidly.

\section{EFFECTS OF TEMPERATURE: CELLULAR SYSTEMS}

The effects of temperature variations on the activities of cells and their sensitivity to enzyme inhibition are very complex, since not only is the cell a summation of hundreds of enzymes and multienzyme systems, each responsive in a characteristic way to temperature. but the metabolic and functional activities are dependent on a structural organization that is also influenced by temperature changes. A complex system is usually more 
unstable than any of its constituent parts. Thus proteins are much more unstable at elevated temperatures than are the amino acids and, likewise, the cell is more unstable than the enzymes or structural protein complexes as the temperature is raised above the normal levels. Most mammalian cells are seriously disturbed or damaged when the temperature is increased more than $5^{\circ}$ above the physiological value. This instability is due in part to structural alterations but, more importantly, to the imbalance in the overall metabolic pattern. Occasionally it is possible to isolate experimentally some part of the cell metabolism and test its response to inhibition and changes in the temperature, but one must beware of oversimplification, inasmuch as the various activities of the cell are so closely related. For example, it might seem that the bioluminescence system would provide a relatively simple cellular activity to measure, but here one is not only dealing with the enzymes and pathways involved in the production of the radiation, but perhaps also with the systems that supply the energy (respiration and oxidative phosphorylation), the systems regulating the concentrations of enzymes and coenzymes, and possibly with structural factors upon which the organization of the energy flow depends. Despite the inherent complexity of all cellular behavior following temperature changes, the problems are not insuperable if they are approached with a full awareness of this complexity and a knowledge of the temperature dependency of the component systems.

The effects of reduced temperatures on tissues and organs have recently become important as a result of the widespread use of hypothermia in surgery. The responses of the tissues to anoxia, drugs, and metabolic inhibitors at these low temperatures are largely unknown as yet, but must eventually be determined before we can thoroughly understand the hypothermic state and apply it more widely to medical problems. One might say with some justification that there is here a new field of low-temperature biochemistry, physiology, and pharmacology, about which essentially nothing is known at present, relative to our knowledge obtained under normothermic conditions.

\section{Factors Related to Inhibition at the Cellular Level}

When the degree of inhibition produced by a certain concentration of inhibitor on a metabolic or functional activity of a cellular system is found to vary with the temperature, there may be many contributing factors in addition to the primary changes in the inhibition at the enzyme level. In other words, a cellular system cannot be treated as a collection of independent enzyme units laeking spatial organization. The actions of an inhibitor on a eell may be conditioned by a variety of factors imposed by the total structural and metabolic pattern, some of which have been discussed in Chapter 9 , and these factors may be more sensitive to temperature changes 
than are the enzymes themselves. It will suffice here to enumerate some of these factors as a basis for evaluating and interpreting the experimental results.

Sometimes the rate of inhibition may be limited or controlled by the diffusion of the inhibitor to the enzymic site of its action, and the eventual degree of inhibition may be determined by the distribution of the inhibitor between the extracellular and intracellular phases. When penetration of the inhibitor into the cell is slow, relative to the other processes involved in the inhibition, the temperature dependence of the inhibition may reflect changes in the permeability of the plasma membrane and have little to do with the kinetics of the enzyme inhibition. Although simple diffusion rates in water are not altered greatly by changes in the temperature $\left(Q_{10}\right.$ is usually around 1.2 for molecules the size of most inhibitors), the rates of membrane penetration are frequently quite sensitive to temperature $\left(Q_{10}\right.$ often between 2 and 4). It would be predicted that the lower the permeability to a substance, the greater would be the temperature effect, inasmuch as the energy barrier for penetration (akin to the activation energy in a chemical reaction) would he higher than for a rapidly penetrating substance. In addition, the energy barrier may change with the temperature due to structural modifications in the membrane. The temperature characteristics ( $\mu$ values, see page 792 ) for cell membrane penetration by electrolytes or nonelectrolytes lie usually in the range between 15 and $25 \mathrm{kcal} /$ mole (Davson and Danielli, 1952; Bowyer, 1957; Glynn, 1957) and are quite comparable to the values obtained for enzyme rates, cellular metabolism, and tissue function. Thus, if a high $Q_{10}$ or temperature characteristic is found for the rate at which an inhibition develops, one may not conclude from this alone that the process is not limited by a membrane penetration step. It is quite possible that in the case of an inhibitor that enters the cell with some difficulty, a depression of the temperature would soon cause the rate of penetration to be limiting; thus, the temperature characteristics of the inhibition would change as the temperature is lowered. The equilibrium distribution of an inhibitor between the medium and the cell interior is probably not very sensitive to temperature changes. unless the process of uptake is active (metabolically dependent). Thus, the rates at which inhibitions develop in cells will alter more with temperature than will the final equilibrium inhibition levels.

Cellular metabolism is an expression of multienzyme systems and manifold enzymic pathways. What has been said in the previous section on multienzyme systems applies directly to cell processes. The rate that is measured (e.g.. the uptake of oxygen, the formation of lactate, the synthesis of protein, or the disappearance of a substrate) is often a composite of several rates relating to different pathways, with each pathway having its own characteristic response to temperature and inhibition. Much more complex situations can occur. Controlling or homeostatic processes, effective 
in making some adjustment to the presence of an inhibitor, are also sensitive to temperature changes. Depression of terminal electron transport by an inhibitor such as cyanicle brings about a complex sequence of changes in the levels of adenine nucleotides, which in turn accelerates the rates of certain processes that tend to antagonize the respiratory depression. All of these adjustments and their effectiveness will be altered by a variation in the temperature. Both inhibitory and the related adaptive processes change with temperature and the over-all result will not be readily interpretable.

Another factor that must be considered in certain experiments is the effect of temperature on the concentrations of the enzymes and coenzymes in the cells. The enzyme concentration will often depend on the balance between the rates of formation and destruction. since there is a constant tumover of most enzymes. The reactions may be written as:

$$
\mathrm{E}_{p} \stackrel{k_{1}}{\rightarrow} \mathrm{E} \stackrel{k_{2}}{\rightarrow} \mathrm{E}_{i}
$$

where $\mathrm{E}_{p}$ is a precursor of the enzyme, $\mathrm{E}$ is the catalytically active enzyme, and $\mathrm{E}_{i}$ is the inactive product of some process destroying the enzyme. The level of $(\mathrm{E})$ is important in determining the rate of the reaction this enzyme catalyzes and may be altered by temperature if the rates of the two reactions are modified by temperature to different degrees. With regard to inhibition, such changes in (E) would be especially important in the study of very potent inhibitors where the kinetics are those of zone $\mathrm{B}$ or zone $\mathrm{C}$ of mutual depletion systems. The same principles would apply to coenzymes, such as the diphosphopyridine and flavin adenine nncleotides.

Certain metabolic processes appear to be organized on a structural basis, the rapid rates and high efficiency arising from the compartmentalization of the participating enzymes. Almost nothing is known of the effects of temperature changes on such structures, except that the instability increases at both low and high temperatures, and nothing at all is known of how this may be related to the sensitivity to an inhibition. Work with isolated mitochondria has shown that the metabolic characteristics are intimately correlated with the structural integrity, and it is very likely that the response to an inhibitor will likewise be strncture-dependent.

Finally, one may look at the situation in a more general way. The metabolic and functional operation of a cell occurs optimally only over a rather narrow temperature range. Outside this range the cell becomes progressively more deranged or damaged as the temperature is lowered or raised. It is thus possible that in general a cell is most resistant to the detrimental effects of inhibitors in this optimal physiological range. It is here, perhaps, that the cell is most nicely balanced and has the greatest buffer capacity against metabolic disturbance. A cell that is already abnormal because of 
an unfavorable temperature shift will usually be less able to withstand the additional effects of an inhibitor. This applies not to the direct primary actions of the inhibitor but to the eventual over-all changes upon which the survival of the cell depends. A culture of bacteria whose normal temperature range is near $37^{\circ}$ may be very unstable near $47^{\circ}$. A further increase of the temperature to $50^{\circ}$ may produce a rapid drop in the cell count. Now, it would be expected that an inhibitor affecting some vital pathway in these cells would be more effective in killing the bacteria at $47^{\circ}$ than at $37^{\circ}$. Exceptions to this rule would be anticipated, especially at reduced temperatures. becanse other factors, such as a slower rate of inhibition, would also be important.

\section{Dependence of Respiratory Inhibition on Temperature}

The oxygen uptake of most cells is made up of contributions from several different pathways. Endogenous respiration particularly usually involves the oxidation of many substrates, sometimes by the same and sometimes by different electron-transport chains. The utilization of glucose by the Embden-Meyerhof glycolytic pathway and the tricarboxylic acid cycle provides at least six different substrates for oxidation, while the addition of the pentose-phosphate shunt would bring in other oxidations. Almost every type of multienzyme system is operative in normal respiration and, furthermore, operating not in isolation but linked together in varions ways. Not only are there pathways for the degradations of the substrates and the oxidation of the intermediates, but there are also the many reactions involving phosphate that are closely coupled to the total respiratory system. Then there are fatty acids and amino acids and other substrates being broken down and oxidized by pathways that occasionally are confluent with those of carbohydrate metabolism, or which terminate in common channels. The respiratory mechanism alone may be thought of as a kind of supermultienzyme system, the kinetics of which would be inexpressible by our present formulations.

The effects of temperature on cellular respiration have been studied for many years and the results have often been interpreted in terms of single enzyme reactions on the basis of the theory of limiting or master reactions. We have noted that this concept has very limited applicability (Chapters 7 and 9) and when applied to something as complex as respiration, one is justified in maintaining a critical attitude. It has been demonstrated many times by Britton Chance (e.g. Chance and Hess, 1959) that there are multiple controls of respiration and that under various conditions the rates are limited by different reactions, even in single electron-transport chains. The over-all rate of oxygen uptake as a result of tricarboxylic acid cycle activity may likewise be limited by several steps (Montgomery and Webb, 1956). When parallel pathways are simultaneously operating, as is 
usual is respiration, it is extremely difficult to attribute the over-all rate to any one process. The change in the rate with temperature can thus not be interpreted directly.

The presence of an inhibitor will modify the respiratory pattern in a complex fashion, even though the inhibition is specific on a single type of enzyme. The effects of temperature changes on this inhibition may be attributed to a variety of factors, some of which have been discussed above. In many cases, the effect will have little or nothing to do with the primary inhibition. For example, if the inhibitor acts on one of several parallel pathways, a change in the temperature may either increase or decrease the over-all inhibition depending on the relative sensitivities of these pathways to temperature. When a noninhibited pathway has a relatively ligh temperature coefficient, a rise in temperature will lead to a greater contribution by this pathway and the inhibition may be reduced, this effect having nothing to do with the possible changes in the inhibition itself, and, in fact, being superimposed upon it. Such interpretations as have been made of the rise in the inhibition of Rhizobium respiration by urethane as the temperature is increased, this being attributed to a facilitation of enzyme inactivation. must be critically examined (Koffter et al., 1947). Many other factors shonk be eliminated before the results can be related to only a single process. Respiration has been used here as an example of any complex metabolic activity of the cell and the same principles would apply to all.

\section{Naturally Occurring Heat-Labile Enzyme Inhibitors}

It has been found that certain intracellular enzymes become much more active as the temperature is raised, this occurring rather suddenly over a small temperature range and with a temperature coefficient higher than would be expected for any enzyme reaction. Indeed, these enzymes appear to be completely inactive at the normal temperature. The mechanism of this "heat activation" of enzymes was elucidated by Swartz et al. (1957) for the DPN-pyrophosphatases of certain bacteria (Proteus species, Mycobacterium butyricum. Bacillus subtilis. and Staphylococcus aureus). These enzymes when extracted from the cells are inactive and become active when the temperature rises above $40^{\circ}$. This is due to the destruction of a natural inhibitor at these higher temperatures. These inhibitors are probably protein and within the normal cells are bound to the enzymes quite tightly.

It is not known how wilespread this phenomenon may be, nor if it oceurs in cells other than bacteria. Certainly many naturally occurring inhibitors for a wide variety of enzymes have been demonstrated and it is quite possible that these inhibitors. or at least the combination with the enzymes, may be sensitive to temperature changes. Such behavior would be of importance in the response of cells to inhibitors and the variation of this in- 
hibition with temperature. It may be that some inhibitors would become effective only at those temperatures at which the enzymes acted upon were catalytically functional.

\section{Dependence of the Inhibition of Cell Function on Temperature}

It is generally true that the higher the level of functional activity of a cell, the more readily is this function depressed by an enzyme inlibitor (Chapter 9, page 464). Thus a muscle or a nerve will become more sensitive to metabolic depression when it is stimulated more rapidly. It has frequently been assumed that similar relations hold when the cell function is altered by changes in the temperature. A cell or tissue whose functional activity has been depressed by a rednction in the temperature would then be expected to withstand inhibition better than at the nomal temperature because the metabolic energy requirement for the fumction is less. However, the situation is somewhat different from that in which the function has been modified independently of the metabolism. When a muscle is stimulated at a lower frequency, the metabolism and levels of high-energy phosphate are not directly affected; if they change, it is a secondary result of the altered functional activity. Whereas when the temperature is reduced, the metabolic systems concerned with the formation and utilization of high-energy phosphate are simultaneously reduced with the function. In fact, the depression of functional activity by a fall in the temperature may be due to the metabolic slowing. There is some evidence for this in the similarity of the temperature characteristics for both respiration and function in a variety of cells, but this evidence is very tenuous. In any event. when both function and the energy-supplying processes decrease simultaneously, the energy requirement relative to the rate of energy formation need not become less. However, it may well take longer for an inhibition to depress function because the arailable high-energy phosphate will be ntilized more slowly.

An interesting situation may arise when the rate at which a function proceeds is directly correlated with the level of some high-energy compound, such as ATP. Under normal conditions the concentration of ATP may be near the optimal value and an increase will bring about no augmentation of the function. The presence of an inhibitor may reduce the ATP concentration so that the functional activity oceurs more slowly. If the temperature is now increased, the level of ATP may rise and the function may be at least partially restored. The degree of inhibition observed will depend, therefore, on the two relationships - the cellular activity as a function of the ATP concentration and the ATP concentration as a function of the temperature - and the effects of an inhibitor on each. In most cases, however, the functional processes will themselves be sensitive to temperature changes. 
When a function is not directly or immediately dependent on the ATP concentration, as conduction in a nerve axon, the effects of temperature changes will be mainly on the functional system; in the case of the nerve axon, on the rates of movements of ions across the membrane. However, if one is determining the length of time required for the function to fail, this will be dependent to some extent on the rates of formation and utilization of ATP. If the temperature is raised so that the level of ATP is slowly falling, the addition of an inhibitor that depresses ATP formation will accelerate the deeline of the function and shorten the interval before the eventual failure. An inhibitor like this will thus be more effective under conditions in which there is an imbalance in the high-energy metabolism, and such imbalances frequently occur at abnormal temperatures.

These miscellaneous remarks about functional inhibition are meant only to supplement the more detailed discussion in Chapter 9 and to provide some basis for the actual examples to be presented shortly.

\section{Dependence of Anoxic Effects on the Temperature}

The changes produced by hypoxia or complete anoxia of cells are basieally very much like those brought about by certain inhibitors that depress oxidations. A good deal of study has been devoted recently to the effects of anoxia at subnormal temperatures beeause of the use of hypothermic procedures in cardiac surgery. The primary object in hypothermia is to reduce the functional activities of certain tissues in order that short periods of anoxia will not be permanently damaging. If the circulation to the brain is interrupted for a period, it is postulated that the brain will survive this period of anoxia better when the tissue is cooled, and the success of such procedures is good evidence that this can be achieved. It has also been demonstrated in animals that hypothermia reduces the central nervous system damage brought about by periods of ischemia. These studies have bearing on the effects of inhibition at reduced temperatures and a brief discussion of some of the principles involved may not be out of place.

Actually a clear distinction must be made between the functional activities of a tissue and the processes that are responsible for the maintenance of the integrity. In practical hypothermia the effects on brain or other tissue function are not important; it is primarily a matter of the protection against irreversible damage to the tissue. The time element is thus of major importance. When a tissue is deprived of oxygen, a sequence of events occurs that is initiated by the depression of oxidative metabolism. This sequence undoubtedly involves progressively a larger and larger number of cellular events, inchling electrolyte shifts, destruction of certain coenzymes, changes in permeability, and dissolution of cell structure and organization. At some point these processes begin to be irreversible and, 
after an interval that depends on the type of cell and the temperature. the cell will be unable to recover when oxygen is again supplied.

The detrimental processes that occur during anoxia proceed at slower rates when the temperature is lowered and hence the interval required for irreversible damage is lengthened. Fundamentally the same damage will be produced by anoxia under normothermic or hypothermic conditions, but the times necessary to reach a given level of disturbance will be different. It is evident that this may be unrelated to effects on tissue function; that is, hypothermia may protect against anoxic damage but will not necessarily decrease the effects of anoxia on cellular activity. Similar reasoning may be applied to the actions of certain inhibitors.

Both hypothermia and anoxia alone cause a decrease in the $\mathrm{K} / \mathrm{Na}$ ratio of muscle cells (Gollan. 1956.) but together there is essentially no change. In atria the drop of the $\mathrm{K}$ Na ratio is faster under both conditions, and when the tissue is both anoxic and hypothermic the rate of decrease is less than during anoxia alone. Since the $\mathrm{K} / \mathrm{Na}$ ratio is not only indicative of disturbances in ion transport but also is important in determining the physiological changes that occur, these results provide some experimental evidence for the protection afforded by hypothermia. When the $\mathrm{K} / \mathrm{Na}$ ratio falls to a sufficiently low level, the operation of the sodium pump or the selective permeability properties of the membrane are irreversibly altered so that recovery does not occur upon reintroduction of oxygen.

The respiration of several mammalian tissues at $20^{\circ}$ is approximately $30 \%$ of what it is at $37^{\circ}$ (Fuhrman, 1956). The functional activities of tissues or whole organisms often change with temperature to about the same degree as the respiration (Crozier, 1924). However, the situation is sometimes more complex and the various functions of a single tissue may be modified by temperature changes in quite different ways. The heart may be taken as an example (Hoffman. 1956; Brooks. 1956). The rate of the heart beat will usually drop to a level between $15-25 \%$ as the temperature is decreased from $37^{\circ}$ to $20^{\circ}$; for the isolated rabbit atrium the fall is to $18 \%$ of the rate at body temperature (Webb, $1950 \mathrm{~b}$ ). The rate of impulse conduction over the myocardium generally decreases even more. In the rat atrium the conduction rate at $20^{\circ}$ is only $7 \%$ of the normal value ( $\mathrm{Hol}-$ lander and Webb, 1955). On the other hand the contractile tension may rise as much as $45 \%$ as the cardiac tissue is cooled to the same degree, even though the rate of beating is kept constant by electrical stimulation (Hollander and Webb, 1955). The resting membrane potential of rat atrium increases around $28 \%$ upon cooling to $20^{\circ}$, while the magnitude of the action potential is not significantly altered, although the action potential may be greatly prolonged due to slower rates of depolarization and repolarization. In this tissue a change of the temperature thus brings abont a variety of effects and it is obvious that there is no necessary correlation between metabolic and functional variations. One might conclude from 
these results that the myocardim at $20^{\circ}$ while beating spontaneously will not suffer from a significant discrepaney between the energy requirements for function and the netabolic supply, since the rate of beating will decrease mankedly, but that when the heart is driven at a constant rate, there will be a greater energy requirement compled with a smaller smply. In the latter ease the tissue may become more sensitive to anoxia or metabolic inhibitors. while in the former case there would be no necessary change due to these factors alone. These points have been brought out to emphasize that it is dangerous to make generalizations regarding the relative behaviors of metabolism and function, and from these generalizations to draw conchusions as to the responses of tissues to anoxia or inhibitors at different temperatures. It is, perhaps, especially unjustified to state a miori that the sensitivity of a tissue function to metabolic depression will be less at a lower temperature on the basis of a lessened energy requirement.

Very few quantitative studies have been made of the effects of anoxia as they are molified by temperature changes. The work of Fulmman et al. (1950) is interesting in this connection. The respriration of rat ventricle sliees was determined in $100 \%$ oxygen, then in $10 \%$ oxygen, and again in $100 \%$ oxygen, both at $37.7^{\circ}$ and $15^{\circ}$. At $37.7^{\circ}$ the resprintion decreased $45.5 \%$ when the pure oxygen was replaced with $10 \%$ oxygen, while at 150 the decrease was only $17.6 \%$. Recovery in $100 \%$ oxygen at $37.7^{\circ}$ was $54.6 \%$ and at 150 it was $82.3 \%$. Thus anoxia was less effective in depressing the myocarlial respiration at $155^{\circ}$ than at $37.7^{\circ}$, and the ineversible changes that occmred, wherely the respiration could not be l'estored to its original level. were less at the lower temperature. It may be noted that the control respiration at 150 is about $20 \%$ of that at $37.7^{\circ}$. A possible explanation for the less hypoxic depression at $15^{\circ}$ would be that different fractions of the total respiration show difforing sensitivities to lowered oxygen tension, and that a fall in the temperature allows the more resistant type of respiration to become polatively more important. However, as pointed out earlier in this ehapter. the control of respiratory rate may be so complex that it is useless to attempt an explanation without much more knowlerlge than we possess at present.

\section{Examples of Temperature Effects on Cellular Inhibitions}

(A) Respintory inhibition by cyamile. The effect of cyanide on the respilation of frog muscle varies with the temperature in an interesting fashion (Ellinger, 1921). It cyanile concentrations around $1 \mathrm{~m} . \mathrm{l}$, the respuratory inhibition changes very little between 200 and 100 (Fig. 15-7) but as the concentration of eyanide is increased there is a progressively greater fall in the inhibition at the high temperatures. Indered, above $5 \mathrm{~m} . \mathrm{H}$ the inhibition begins to be reversed at all the temperatmes. These results may be 
interpreted on the basis of two antagonistic actions of cyanide: there is the usual block of oxidations, leading to an almost complete suppression of respiration at $4.5 \mathrm{~mL} \mathrm{M}$, and there is the oxidation of the cyanide itself by the muscle preparation. As the cyanide concentration becomes higher, the oxygen consumption due to its own oxidation begins to overshadow the respiratory depression. Furthermore, the temperature coefficient of

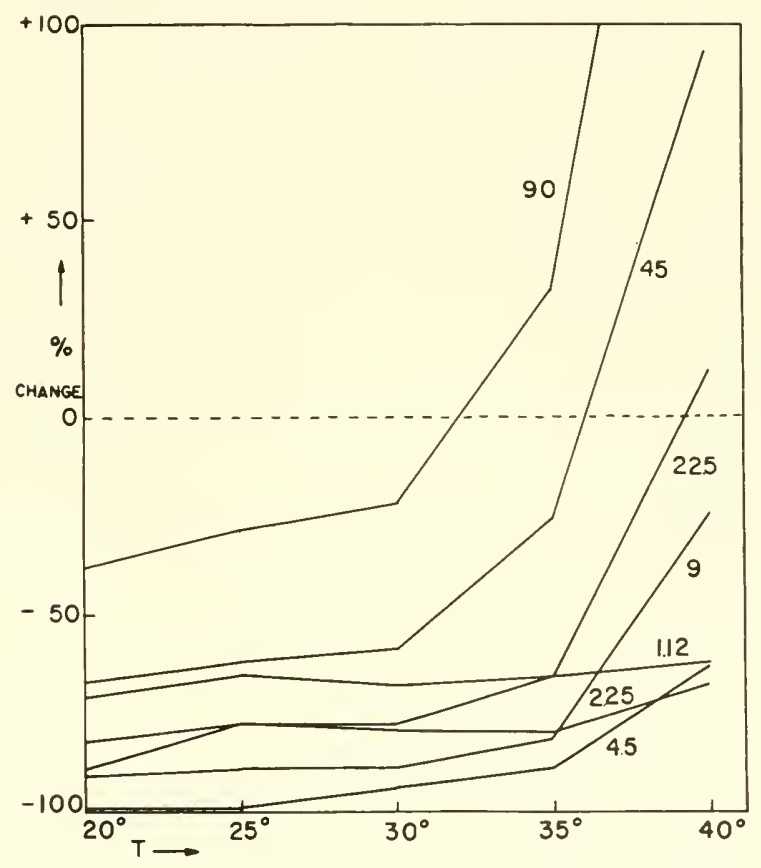

FIG. 15-7. Effects of temperature on the inhibitions of frog muscle respiration prodnced by different concentrations of cyanide. The figures on the curves are cyanide concentrations in millimolar. (From Ellinger, 1924.)

the cyanicle oxidation is presumably high so that this reaction is particularly dominant at the higher temperatures. These experiments well illustrate the complex behavior of a system wherein more than one effect of the inhibitor is observed. They also emphasize the necessity of considering the metabolism of the inhibitor in studies of this type.

The inhibition of trypanosome respiration by eyanide has been claimed to be independent of temperature variations (Brand and Johnson, 1947) but if the results in all the species studied are averaged, it may be seen that there is quite constantly a small increase in the inhibition as the temperature is raised from $28.5^{\circ}$ to $37.5^{\circ}$. At $1 \mathrm{~m} . I$ cyanide the mean increase 
in the inhibition over this range is about $7 \%$. Unfortunately, the concentrations of cyanide used were strongly inhibitory - the average inhibition for five species at $28.5^{\circ}$ was $87 \%$ with $1 \mathrm{~m} / I$ eyanide - so that changes due to temperature variation would not be so marked as they might have been if the inhibition had been around $50 \%$ or lower. In some species only stimulation of the respiration by cyanide was observed and in these cases a rise in temperature usually increased the stimulation significantly; for example, in Trypunosomu equiperdum, $1 \mathrm{~m} . \mathrm{I}$ cyanide stimulated $5 \%$ at $28.5^{\circ}$ and $52 \%$ at $37.5^{\circ}$. Whether this stimulation is the result of the metabolism of cyanide is not known.

It may be noted that a rise in the temperature slightly decreased the cyanide inhibition of frog muscle and slightly increased the inhibition of trypanosomes. The effects of the temperature will depend not only on the temperature sensitivity of the respiratory inhibition, but also on the behavior of the cyanide-resistant fratetion of the respiration. It is quite likely that the ratio between the sensitive and resistant fractions of respiration will change with temperature. The control respiration of the frog muscle preparation rose from 49.1 at 200 to 83.7 at $30^{\circ}$ and then fell to 29.9 at $40^{\circ}$. It would be surprising if the character of the respiration did not also change over this range. That is to say, the changes observed here camot be immediately attributed to the temperature dependence of the inhibition of eytochrome oxiclase by cyanide.

(B) Respirutory stimulation by 2.4-dinitrophenol. The respiratory stimulation induced by -2,4-dinitrophenol (DNP) as a result of its unconpling of oxidative phosphorylation has been found to be related to the temperature. Early work had shown that the calorigenic action of DNP in whole animals was decreased hy exposure to cold (Graja and Dimitrijevic. 1933; Tainter, 193-1) hut it was subsequently demonstrated that DNP depressed shivering and other responses to cold exposme. ant when this was rorrected for, it was found that the calorigenie action of DNP was increased during hypothermia (Hall et al., 1937). In the intact animal there are many factors that may be involved. such as the release of epinephrine and the altered activities of the nervous system. Thus Fuhrman and Field (19:22) investigated the action of DNP on the respiration of tissue slices in order that these other factors might be eliminaterl. The stimulation ratio for cerebral cortex and lidney cortex is plotted in Fig. 15-8 as a function of the temperature, the DNP concentration being kept constant at $0.0335 \mathrm{~m} . /$. In both tissues the stimulation is maximal at a certain temperature - 220 for kidney and 320 for brain - and disapnears as the temperature rises above $40^{\circ}$. The fall with temperature increase might be readily interpreted as resulting from the acceleration of the control respiration to the point where it is not being limited by the phosphorylation reactions. but the decrease at low temperatures is more difficult to understand. However, one cannot 
consider only the electron-transport systems with their associated phosphorylations, but also the many other factors controlling respiratory rate and the level of phosphate acceptors in the cells. It would be very informative to know the P:O ratios for both normal and DNP-treated preparations

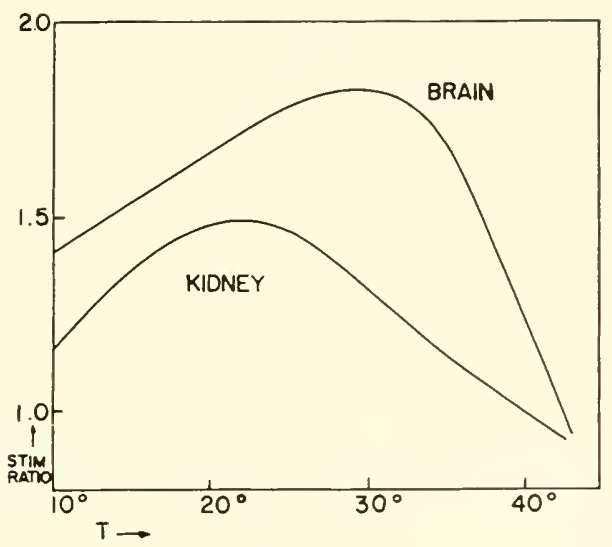

FIG. 15-8. Effects of temperature on the stimulation of tissue slice respiration by $0.0335 \mathrm{~m} M \mathrm{~m} \| I$ 2,4-dinitrophenol. The stimulation ratio is $Q_{\mathrm{O}_{2}}(\mathrm{DNP}) / Q_{\mathrm{O}_{2}}$ (control). The curves are plotted from the data of Furhman and

Field (1942) and are approximate.

at the different temperatures, and also the levels of the various nucleotides. A knowledge of the effects of temperature on DNP uncoupling in simpler subcellular systems would also be useful in understanding the mechanisms involved.

(C) Inhibition of bacterial luminescence. The most quantitative studies of temperature-dependent metabolic inhibitions have been done on the bioluminescence of certain bacteria by Johnson and his co-workers at Princeton during the decade of 1942-1952. The results have been uniformly interpreted on the basis of two major temperature-dependent effects: the usual rise in reaction rates with increasing temperature and the equilibrium between active and inactive enzymes. The maxima in the rate-temperature curves are considered to represent a balance between these two factors - below the optimal temperature the enzymes are mainly in the active forms and the rate varies with the temperature in the classic way, while above the optimal temperature the inactivation or reversible denaturation of the enzymes becomes more important. The relations between inhibition and the temperature are usually ascribed to the effects of the inhibitor on the equilibrium between active and inactive forms. Thus, if the inhibitor 
lowers the optimal temperature, it is believed to combine predominantly with the denatured form of the enzyme or to favor denaturation. Inhibitors are classified into two general types on this basis. Type I inhibitors combine with both $\mathrm{E}_{r}$ and $\mathrm{E}_{d}$ while type II inhibitors combine only with $\mathrm{E}_{d}$. Such types of inhibition and the interpretation of temperature optima for pure enzymes have been discussed earlier in this chapter. It was pointed out there that even for these relatively simple systems it is impossible to conchude from a shift in the $T_{\text {opt }}$ that only the $\mathrm{E}_{q} \rightleftharpoons \mathrm{E}_{d}$ equilibrium has been affected. It is much less likely that in complex cellular metabolism a simple inactivation of an enzyme is determining the $T_{\text {ont }}$. It was also made clear that it is not as easy as some imagine to designate which form of the enzyme combines with the inhibitor from temperature data alone. Hany things might happen within a cell as the temperature rises which would decrease the rate of some metabolic process. and in such cases the $T_{\text {opt }}$ would not be directly related to the denaturation of enzymes, and the shifts in the $T_{\text {opt }}$ brought about by inhibitors wonld not be interpretable as effects on denaturation.

The biolnminescence in Achromobacter is inhibited strongly by $\mathrm{HgCl}_{2}$ and the $T_{o p t}$ is shifted $1^{\circ}$ to 20 lower (Honck. 1942). The inhibition also becomes greater as the temperature increases (Fig. 15-9). These results,

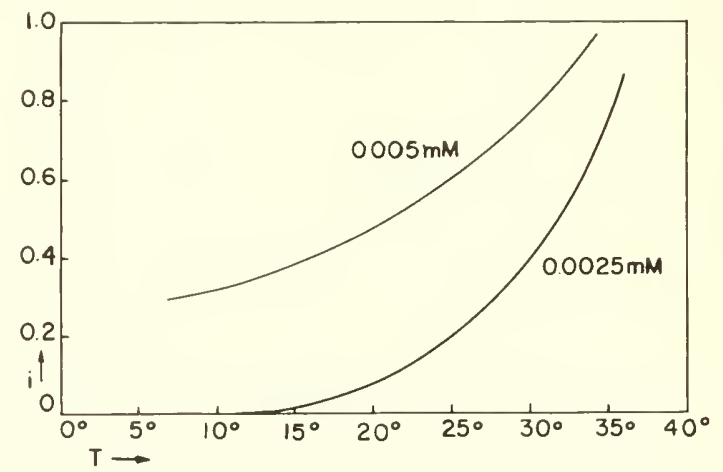

Fic. 15-9. Effects of temperature on the inhibition of bioluminescence in Achromobacter by $\mathrm{HgCl}_{2}$. The curves are calculated from activity curves presented by Houck (1942) and are approximate.

coupled with the known denaturing action of $\mathrm{HgCl}_{2}$, were taken to indicate that the denaturation of some enzyme involved in the hminescence was favored by the $\mathrm{HgCl}_{2}$. Before this explanation conld be accepted. it would have to be shown that the simple inhibition of the enzyme (that is, the reaction of the $\mathrm{HgCl}_{2}$ with the sulfliydryl groups of the enzyme) does not increase with rising temperature to the degree observed. It is often difficult 
experimentally to distingnish between ordinary enzyme inhibition and denaturation of the enzyme by the inhibitor. If the depression of the activity is reversible by some means, the proponents of the theory of denaturation would say that the denaturation is reversible. Inasmuch as combination of the inhibitor with the active center of the enzyme is sufficient for inhubition. it is apparent that the burden of proof for the occurrence of denaturation is on those who propose it is an important factor.

The inhibitions of bacterial luminescence by sulfanilamide and urethane show quite different temperature characteristies (Johmson et al., 1943). The inhibitions observed in Tibrio phosphorescens and Photobacterium phosphoreum are plotted in Fig. 15-10 (the data may be found in Table

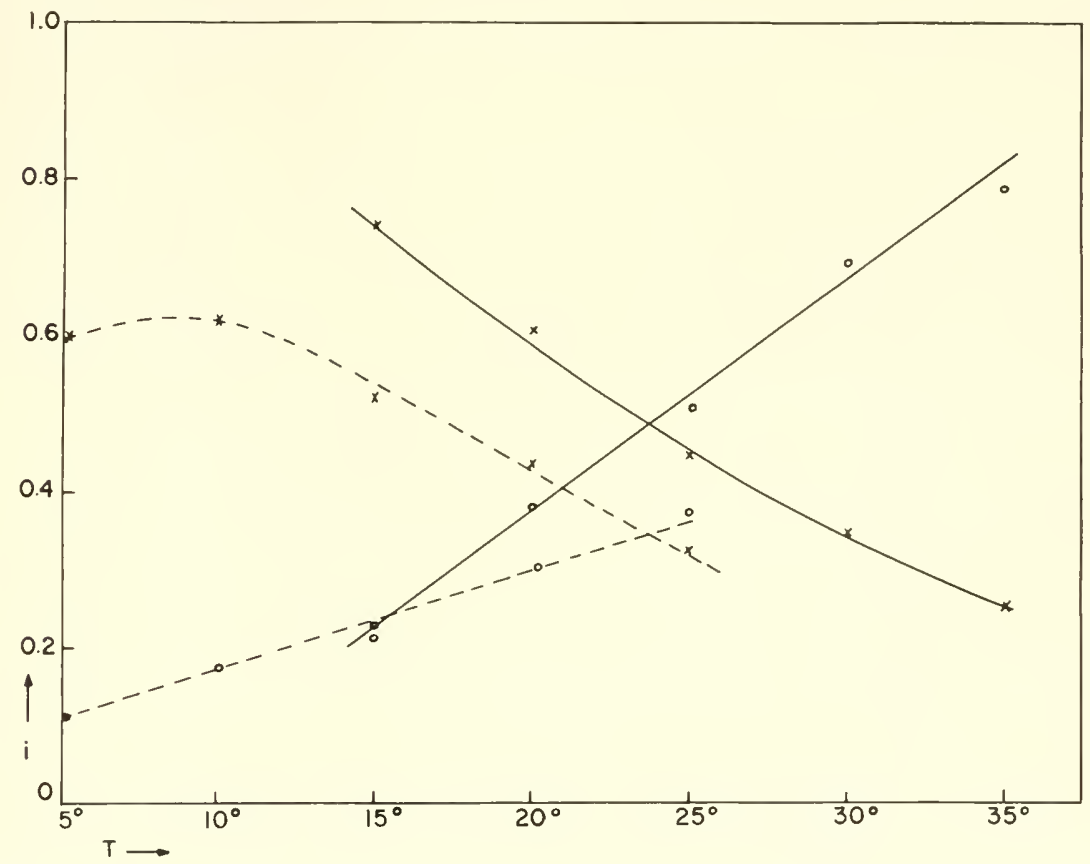

FIG. 15-10. Effects of temperature on the inhibitions of bacterial luminescence by sulfanilamide $(\mathrm{X})$ and urethane $(\mathrm{O})$. The solid curves are for $V$. phosphorescens and the dashed curres for $P$. phosphoreum. Concentrations: sulfanilamide $3 \mathrm{mM}$ and urethane $150 \mathrm{~m} . \mathrm{H}$. (From Johnson et al., 1943.)

10-1) and it is evident that sulfanilamide inhibition falls and urethane inhibition rises with an increase in the temperature. Furthermore, the $T_{o p t}$ for luminescence is shifted upward by sulfanilamide and downward by urethane (Johnson et al., 1945). Urethane at $300 \mathrm{~m} L /$ shifts the $T_{\text {opt }}$ from 320 to $17^{\circ} \mathrm{in} V$. phosphorescens and from $23^{\circ}$ to $17^{\circ} \mathrm{in} P$. phosphoreum 
(Fig. 15-11). These results have led to the hypothesis that sulfanilamide is a type I inhibitor and that urethane is a type II inhibitor. The large changes in heat content ( $1 / H$ between -56 and $-70 \mathrm{kcal} / \mathrm{mole}$ ) and entropy ( $1 S$ between - 165 and $-204 \mathrm{cal} / \mathrm{mole} /$ degree) for the urethane inhibition have been interpreted in terms of the reversible denaturation induced by this substance (Johnson et al., 1942 b).

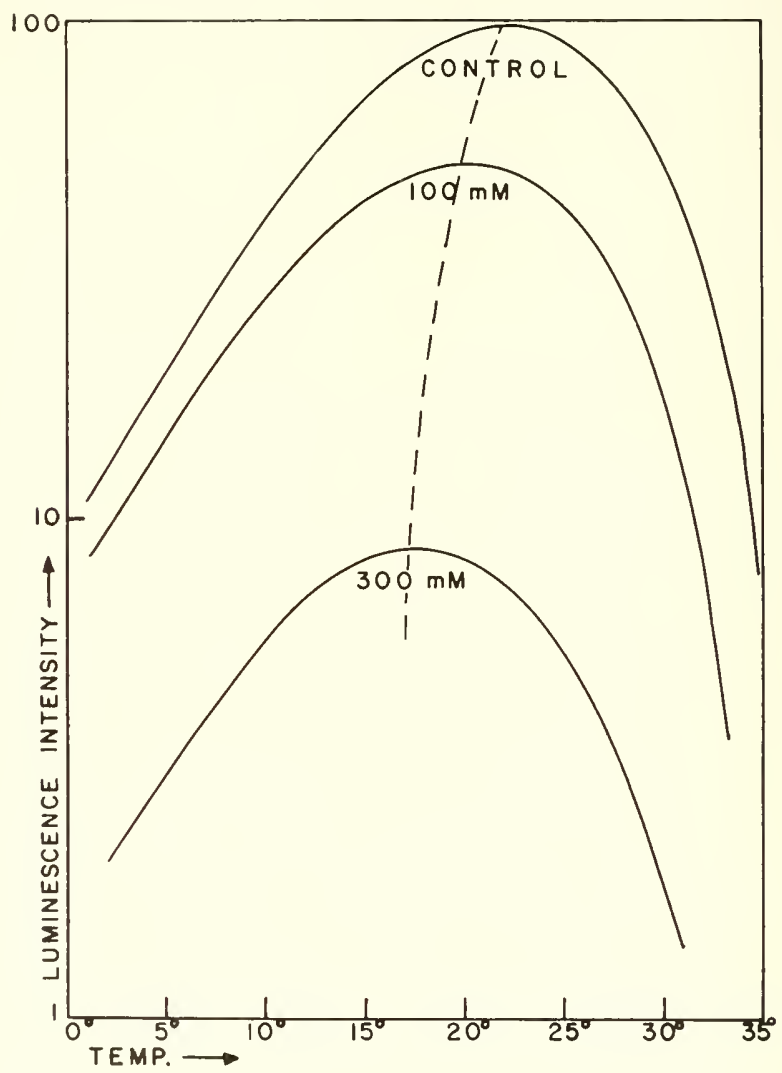

FIG. 15-11. Effects of temperature on the luminescence of $P$. phosphoreum in the absence and presence of urethane, showing the shift in the $T_{\text {opt }}$ produced by urethane. (From Johnson et al., 1945.)

The luminescent system in the living organism is complex and involves not only the enzymes, cofactors, and luminescent substances required directly for the emission of radiation, but also occasionally high-energy phosphate compounds and the oxidative pathways supplying them. Indeed, the bacterial luminescent pathways have not yet been completely delineated. 
It is also not known with which components of the total system inhibitors such as sulfanilamide and urethane react. It is true that urethane and sulfanilanide have been found to inhibit reversibly the Cymmitima lnciferinluciferase reaction in vitro (Johnson and (hase, 1942), but it would seem that this enzyme is generally less sensitive than the in viro systems and it is quite possible that other reactions are depressed in the living cell. It is well known that both urethane and sulfanilamide inhibit pathways other than those of luminescence. In interpreting temperature studies it is important to know whether one system only or several pathways are affected. It is unfortunate that temperature studies have not been done on the inhibition of the purified enzyme system. It would also perhaps be interesting if Johnson and his group had used some of the better known inhibitors whose actions are more specific and characterized. Crethane particularly is quite nonspecific at the high eoncentrations used in the luminescence studies. Of course, denaturing agents are never specific.

The intensity of luminescence in certain species probably depends, for one thing. on the concentration of ATP (Harvey, 1960). which may be modified by inhibition exerted either on the systems forming ATP or utilizing ATP. Temperature optima may occur for the ATP concentration beeause of different temperature coefficients for the forming and utilizing reactions. The presence of an inhibitor may change these optima because of differential effects on these reactions. and consequently the $T_{o p t}$ for the metabolic or functional reactions dependent on ATP may be slifterl. In other words, it is possible in a complex metabolic system to explain a shift in the $T_{o p t}$ by assuming differential effects of the inhibitor on the reactions of the multienzyme system, rather than by the altered action of the inhibitor on a single enzyme component of the system. In the case of bacterial luminescence it is very diffieult to say if the temperature dependence of inhibition is related to a single enzyme or to more complex changes in a multienzyme system. Since the concentration of the intermediate $\mathrm{B}$ in the simple irreversible monolinear chain:

$$
\mathrm{A} \stackrel{\mathrm{E}_{1}}{\rightarrow} \mathrm{B} \stackrel{\mathrm{E}_{2}}{\rightarrow} \mathrm{C}
$$

is given by:

$$
(\mathrm{B})=\frac{K_{2}(\mathrm{~A})\left[V_{1} / V_{2}\right]}{(\mathrm{A})\left[1-\left(V_{1} / V_{2}\right)\right]+K_{1}}
$$

which is modified from Eq. 7-3, and since temperature elanges or inhibitors can alter $K_{1}, K_{2} \cdot V_{1}$, or $V_{2}$ in different ways. it is quite possilbe for (B) to show shifts in the $T_{\text {op }}$ during the inhibition, or to show effects of temperature on the inhibition. The same principles would apply to other more complex multienzyme systems. as has been discussed earlier in this chapter. For example, it may be easily shown from the above equation that 
the effect of an inluhitor on (B) will depend on the initial $V_{1} V_{2}$ ratio, which can be modified by the temprerature.

Examination of an homologous series of carbanates for their effects on the luminescence of Photobucterium phosploremm demonstrated that the temperature relationships changel as the alkyl chain length increased (Johnson et al., 1951). While methyl carbamate and ethyl carbamate (urethane) shift the $T_{\text {opt }}$ downward, n-propyl carbamate and $n$-hexyl carbamate shift it upward, indicating that the inhibiting actions are different in some way. On the enzyme-denaturation hypothesis one must assume then that the higher homologs do not favor denaturation; on the multienzyme basis one must assune that the higher homologs act predominantly on a clifferent pathway. The effects of high pressure on the inhibitions also support a different type of action. It may also be pointed out that ethanol exerts effects similar to urethane in that the $T_{\text {om }}$ is shifted to lower temperatures (Johnson et al.. 1945) and has been shown to inhibit luciferase at high concentrations (it requires about $1, I$ to inhibit $50 \%$ ) (Clase. 1952). A more detailed summary of the study of the inhibition of luminescence and its interpretation may be found in "The Kinetic Basis of Molecular Biology" (Johnson et al., 1954).

(D) Imhibition of Rhizobium respiration by methare. The results obtained by Koffler et al. (1917) for the inhibition of respiration are very similar to those just discussed for luminescence. The inhibition by urethane increases with the temperature; for example, with $300 \mathrm{~m} . \mathrm{l}$ urethane, the following inhibitions were observed. There is no marked effect on the $T_{\text {opt }}$ but it

\begin{tabular}{cc}
\hline Temperature & Tnhibition $(i)$ \\
& \\
$18^{\circ}$ & 0.36 \\
$31^{\circ}$ & 0.56 \\
$40^{\circ}$ & 0.84 \\
\hline
\end{tabular}

is shifted slightly to lower temperatures. These results were interpreted in terms of an equilibrium hetween native and denatured enzymes and the similarity between the inhibitions on respriration and netlyylene blue reduction pointed to the dehyrlyogenases as heing the enzymes involved. Incirlentally. these results make it likely that uretlane in acting on the ATP-forming pathways in addition to heiferase in the studies of bacterial lominescence.

(E) Returlation of cell dirision by niethrne. The cleavage of Aibucia eggs is retarded by urethanes and it has been shown by Cornman (1950. 1957) that the effectiveness is least at $20^{\circ}-21^{\circ}$. The concentrations required for 
a constant retardation effect are shown in Fig. 15-12. It may be noticed that althongh $N \cdot N^{\prime}$-diethylurethane is much more potent than urethane, the temperature of minimal effectiveness is the same. indicating a similar mechanism of action. Also the same cytological changes occur as a response to urethane at all the temperatures and this points to a single mechanism

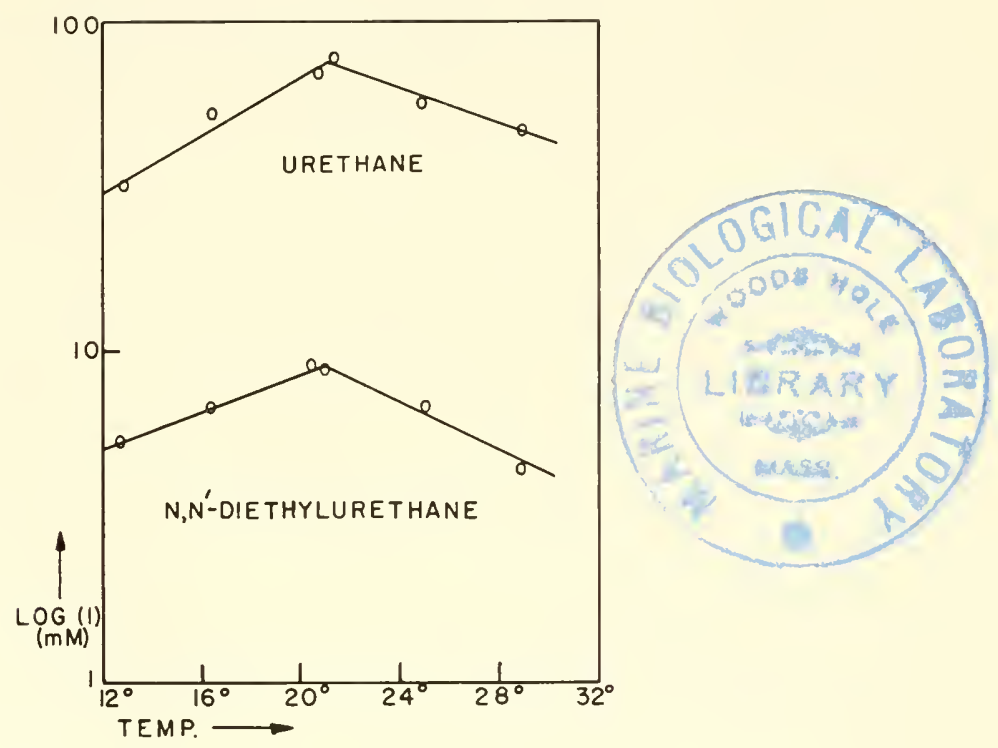

FIG. 15.12. Effects of temperature on the retardation of cleavage of Arbacia eggs by urethanes. The concentrations required for a constant chosen effect are plotted. (From Cornman, 1957.)

of inhibition. These results were provisionally explained as follows: as the temperature rises from 120 to $20^{\circ}$ the binding of the inhibitor becomes less, and at higher temperatures there is progressive acceleration of the denaturation of some important protein or enzyme system. It is possibly significant that the temperature for the minimal effect is the normal biological temperature for Arbacia. In other words, deviation in either direction from the normal temperature leads to a reduction in the resistance to inhibition.

At this point we may summarize some of the principal reasons for the variation of cellular inhibition with the temperature. Let us assume it is found experimentally that the inhibition increases with rising temperature. The following four mechanisms must be considered. 
(1) The inhibitor is bound to the susceptible enzyme more tightly at higher temperatures, i.e., the formation of the $\mathrm{EI}$ complex is endothermic.

(2) The denaturation of the susceptible enzyme by the inhibitor is favored at higher temperatures, the imhibitor either denaturing the enzyme or combining selectively with the denatured form.

(3) Differential effects of temperature and the inhibitor in a multienzyme system occur. For example, in a divergent metabolic chain the increase in temperature may increase the fraction of the total metabolism passing through an inhibitor-sensitive pathway.

(4) A second action of the inhibitor with a high temperature coefficient may lead at higher temperatures to an additional inhibition that is not significant at lower temperatures.

It may be necessary to include other mechanisms in certain cases as, for example, when the active inhibitor is formed metabolically from an added precursor.

\section{Temperature Characteristics of Biological Processes and the Effects of Inhibition}

It has been customary for many years to express the variation of biological rates with temperature in tems of the temperature characteristic, $\mu$, according to the Arrhenius formulation. It was originally assumed that the temperature dependence of the rate is given by the equation:

$$
v=C e^{-\mu / R T}
$$

where $C$ is a constant. The value of $\mu$ may be obtained either from the rates at two different temperatures or by appropriate plotting. Equation 15-49 may be rewritten as:

$$
\log v=\log C-\frac{\mu}{2.303 R T}
$$

Thus a plot of $\log v$ against $1 / T$ should give a straight line with an intercept of $\log C$ and a slope of $-\mu \cdot 2.303 R$ (Fig. 15-13). If only two temperatures are considered, one may write:

$$
\frac{v_{2}}{v_{1}}=\exp \left\{\frac{\mu}{R}\left[\frac{1}{T_{1}}-\frac{1}{T_{2}}\right]\right\}
$$

and the temperature characteristic is given by:

$$
\mu=2.303 R \frac{T_{1} T_{2}}{T_{2}-T_{1}}\left(\log v_{2}-\log v_{1}\right)
$$


The plotting procedure is preferred because linearity cannot always be assumed. If a curve is obtained, the $\mu$ at any temperature can be calculated from the slpoe at that point. When $T_{2}-T_{1}=10^{\circ}: v_{2} v_{1}$ is $Q_{10}$ and thus the relationship between $\mu$ and $Q_{10}$ for temperatures in the neighborhood of $30^{\circ}$ may be written as:

$$
\mu=42,200 \log Q_{10}
$$

It may be noted that $\mu$ is usually given in small calories.

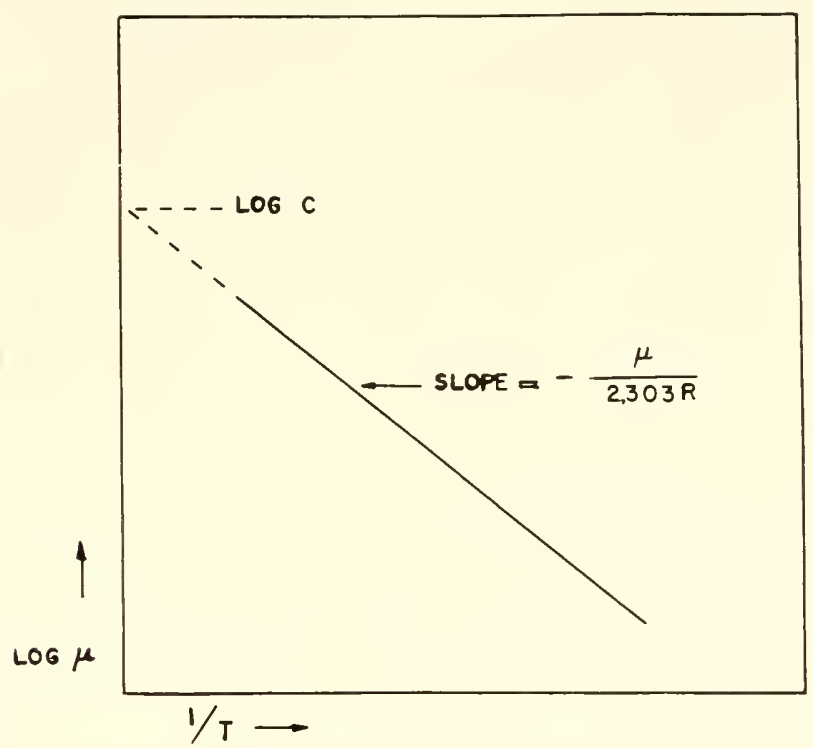

FIG. 15-13. Plot of $\log v$ against $1 / T$ for the determination of the temperature characteristic $\mu$ (Eq. 15-50).

It is possible to obtain $\mu$ values from systems of any degree of complexity and they serve to characterize the temperature dependence of the rates. However. the physical interpretation of $\mu$ becomes more and more difficult as the complexity of the system increases. The principle of limiting reactions has often been called upon to interpret $\mu$ values obtained from cellular systems and it is claimed that $\mu$ relates only to the single limiting reaction that controls the over-all rate. This has given rise to much argument, which is beyond the scope of this book, but reference may be marde to Chapter 7 in which reasons were given for believing that the metabolic or functional rates in living systems are generally not determined by single reactions. Indeed, it is well to consider what $\mu$ means for a single enzyme reaction. It has been frequently stated that $\mu$ is the activation energy for the 
enzyme reaction, which in the general case it is not. Taking the usual expression for enzyme rate:

$$
v=\frac{k_{2}\left(\mathrm{E}_{t}\right)(\mathrm{S})}{(\mathrm{S})+K_{m}}
$$

it may be seen that when the enzyme is saturated with substrate and $v=$ $k_{2}\left(E_{t}\right)$, the changes in the rate with temperature will provide $\mu$ valnes referable to the rate constant $k_{2}$ for the breakdown of the ES complex into free enzyme and products. However, under most conditions $K_{m}$ is not negligible and it also can change with temperature. Usually $K_{m}$ will increase with a rise in the temperature, whether it is the general Michaelis constant or the true substrate constant, and thus the rate will not increase as rapidly with temperatnre as would be expected if only $k_{2}$ were involved. Finally, according to the modern expressions for the rate constant (see Eq. 15-10), a plot of $\log k_{2}$ against $1 / T$ would not be expected to be exactly linear (whereas a plot of $\log k_{2} / T$ against $1 / T$ would be). Most of the $\log v-1 / T$ plots reported in the literature are actually not straight lines if the experimental points are examined, althongh straight lines are frequently used to represent them.

The meaning of $\mu$ in terms of the usual thermodynamic quantities for an enzyme reaction may be derived as follows. Taking the logarithm of Eq. 15-54 and then differentiating with respect to the temperature (assuming that $K_{m}=K_{s}$, one obtains:

$$
\frac{d \ln v}{d T}=\frac{1}{T}+\frac{\Delta H^{*}}{R T^{2}}+\frac{\Delta H}{R T^{2}}\left[\frac{1}{1+(\mathrm{S}) e^{-(.1 H / R T)} e^{(-1 S / R)}}\right]
$$

Since $d \ln v / d T=\mu / R T^{2}, \mu$ is in general given by:

$$
\mu=R T+\Delta H^{*}+\Delta H\left[\frac{1}{1+(\mathrm{S}) e^{-(.1 H / R T)}} e^{(\cdot S / R)}\right]
$$

The temperature characteristic. $\mu$, thus is equal to the enthalpy of activation plus the small $R T$ correction plus the third term which represents the possible ehanges in $K_{s}$ with the temperature. The expression for $d \ln (v / T) / d T$ would be the same with the omission of the $1 / T$ term and $\mu$ would be given by Eq. 15-56 without the RT term.

When multienzyme or cellular systems are considered, it becomes more difficult to understand the meaning of $\mu$ and the significance of its changes. It is true that in some cases (for example, an irreversible monolinear chain) the over-all rate is controlled by one step and the $\mu$ obtained would refer only to that step, but in most instances this is not true. Most $\log v-1 / T$ plots from living systems are curves but sometimes they are represented by a series of two or more linear segments, the transition points between one slope and the next being called critical temperatures (and by some, uncriti- 
cal temperatures). These breaks have been interpreted as indicating a transition between two different limiting reactions with different temperature characteristics. These matters have been much discussed (Burton, 1936, 1939; Sizer, 1943; Morales, 1947; Stearn, 1949; Kavanau, 1950) but general agreement has not been reached.

Dixon and Webb (1958, p. 163) have suggested six explanations for the discontinuities observed in the $\log v-1 / T$ plots for enzyme reactions.

(a) Phase change in the solvent (as the freezing of water at $0^{\circ}$ ).

(b) Two parallel reactions with quite different temperature coefficients so that different reactions are dominant in different temperature ranges (predicting higher $\mu$ values at the ligher temperatures which is usually not the case).

(c) Two successive reactions with different temperature coefficients so that different reactions are limiting in different temperature ranges.

(d) Enzyme can exist in active and inactive forms with a high temperature coefficient for the transformation reaction between them.

(e) Enzyme may be reversibly inactivated, either spontaneously or by some substance in the medium.

$(f)$ Discontinuity affecting the forward reaction only.

Koshland (1959) applied these mechanisms to the myosin ATPase system and concluded that a flexibility in the active center might be involved, this being a variation of explanation $(d)$ above. It may be mentioned that in cellular systems additional explanations may be imagined for the critical temperatures observed.

We may now inquire as to how an inhibitor could alter $\mu$ for an enzyme reaction. Inasmuch as $\mu$ is closely related to the enthalpy of activation for the breakdown of the ES complex, an inluibitor that alters $\Delta H^{*}$ will probably change $\mu$. It would be expected that competitive inhibitors would not produce any change in $\mu$ since they do not interfere with the breakdown of the ES complex but only with its formation. Noncompetititive inhibitors on the other hand modify $k_{2}$ and hence could change $\mu$. Coupling inhibitors and inhibitors binding with the substrate or coenzymes may or may not effect $\mu$, depending on the nature or activity of the complexes formed. In addition, we have seen above that $\mu$ can also depend on $K_{m}$ and the enthalpies and entropies of the ES complex formation, and under certain circumstances these can be altered by inhibitors. There are very few quantitative studies on the effects of inhibitors on enzyme $\mu$ values, but it would appear that this might be a reasonably useful approach in the determination of the interactions of substances on the enzyme surface.

The inhibition of horse serum cholinesterase by quinine is affected by the temperature in an interesting manner (Robert et al.. 1951). The inhi- 
bition decreases with increase in temperature, $T_{o p t}$ is shifted to higher temperatures by the inhibitor, and the $Q_{10}$ decreases with rising temperature in all cases but faster when the inhibitor is present (Fig. 15-14). The $\mu$ values are increased by quinine, particularly in the lower temperature range, giving rise to a discontinnity in the curves which is not present in the case
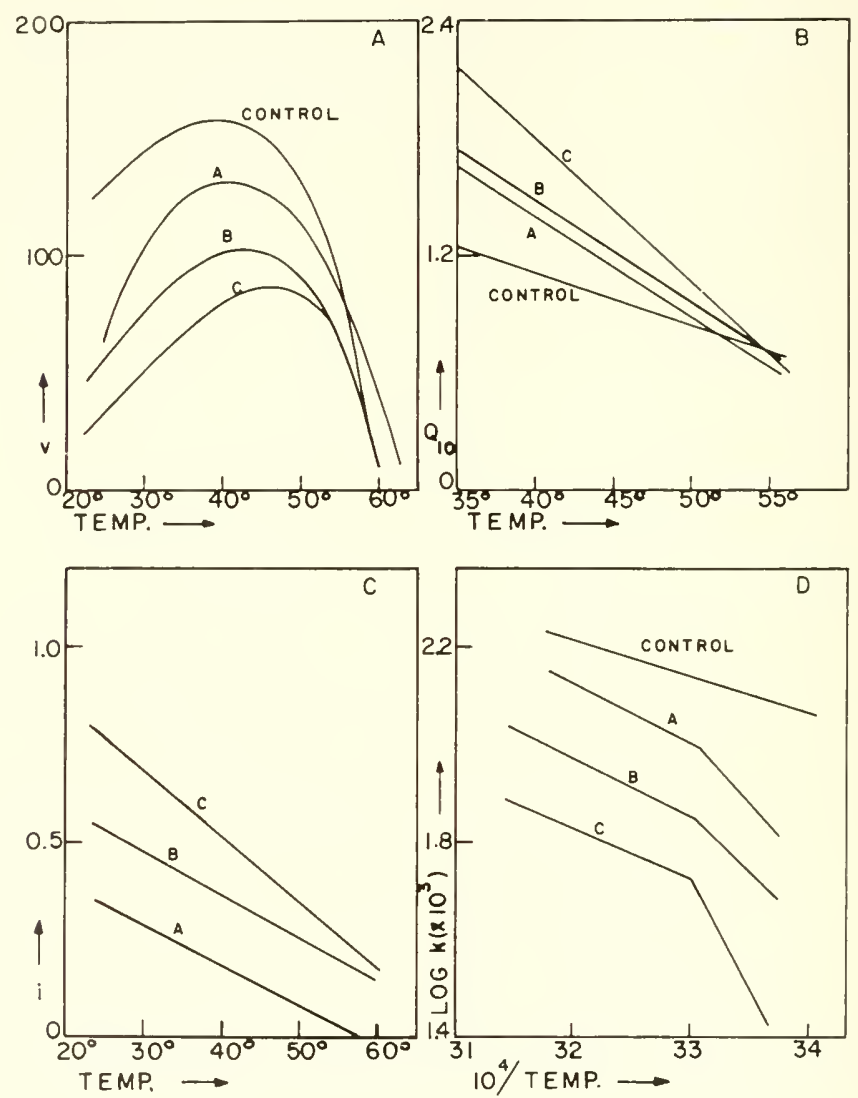

FIG. 15-14. Effects of temperature on the inhibition of horse serum cholinesterase by quinine plotted in four different ways. In all cases, the quinine concentrations are: $\mathrm{A}, 0.025 \mathrm{~m} . L ; \mathrm{B}$, $0.05 \mathrm{~m} . \mathrm{I}$; and C, $0.1 \mathrm{mM}$. (From Robert et al., 1951.)

of the uninhibited enzyme (Table 15-1). It is interesting to speculate if this critical temperature is related to a temperatmre-sensitive active center flexibility. Both quinine and lower temperatures may rednce the flexibility and hence the efficiency of the catalysis. However, the situation may well be more complex than this, as indicated by the reversal of some of the ef- 
TABLE $15-1$

Effects of Quinine ox the Teyperature Characteristics and Theriodranic Properties of Horse Serum Cholinesterase ${ }^{a}$

\begin{tabular}{|c|c|c|c|c|c|}
\hline \multirow{2}{*}{ Quinine } & \multicolumn{2}{|c|}{$\mu($ cal/mole $)$} & \multirow{2}{*}{$\begin{array}{c}\Delta H^{*} \\
(\mathrm{kcal} / \mathrm{mole})\end{array}$} & \multirow{2}{*}{$\begin{array}{c}\Delta S^{*} \\
\text { (cal } / \mathrm{mole} / \\
\text { deg) }\end{array}$} & \multirow{2}{*}{$\begin{array}{c}\Delta F^{*} \\
\text { (kical/mole) }\end{array}$} \\
\hline & $25-30^{\circ}$ & $30-45^{\circ}$ & & & \\
\hline 0 & 4000 & 3500 & 3.4 & -8.76 & 6.1 \\
\hline $0.025 \mathrm{~m}$. & 11,900 & 6000 & 5.4 & -7.16 & 7.6 \\
\hline $0.05 \mathrm{~m} M$ & 12,300 & 6400 & 5.8 & -6.98 & 8.0 \\
\hline $0.1 \mathrm{~m} M$ & 20,200 & 4800 & 4.2 & -8.26 & 6.8 \\
\hline
\end{tabular}

a From Robert et al. (1951).

fects at the highest quinine concentration. The presence of 2,4 -dinitrophenol causes the appearance of a discontinuity in the Arrhenius plot for myosin ATPase when ATP is the substrate (Koshland, 1959). The curve, in fact, becomes quite like that for the hydrolysis of ITP. This was interpreted as resulting from a competition of the phenoxide ion of 2,4-dinitrophenol with the $6-\mathrm{NH}_{2}$ group of $\mathrm{ATP}$ for a group in a protein chain, releasing the constriction normally imposed by the interaction of the $6-\mathrm{NH}_{2}$ group with the enzyme group. The possible effects of inhibitors on enzyme and enzyme-substrate flexibility must therefore be considered in interpretations of changes in the temperature characteristics.

Urease was claimed by Sizer (1939) to show $\mu$ values of either 8700 or 11,700 . In sulfite solution a critical temperature of $23^{\circ}$ was observed, $\mu$ being 11,700 below and 8700 above this temperature. In various mixtures of ferricyanide and ferrocyanide the $\mu$ value was either of these figures and it was suggested that in reducing solutions the configuration of the active center was such that $\mu=8700$ but as certain sulfhydryl groups became oxidized the enzyme activity decreased and $\mu=11,700$, these changes being reversible. Kistiakowsky and Lumry (1949) reported that such a sudden inflection at the critical temperature did not occur in the case of urease and that in sulfite solution $\mu$ rises progressively from 8830 to 15,000 as the temperature is lowered. This change in the temperature characteristic was explained on the basis of an inhibition by sulfite, this inhibition exhibiting an enthalpy change of - $29 \mathrm{kcal} /$ mole. In other words, as the temperature is decreased, the sulfite inhibition becomes important over a rather narrow temperature range and alters $\mu$. They believe that many inflections in biological Arrhenius curves may be explained by the presence of reversible inhibitors whose combinations with enzymes become particularly important at lower temperatures, rather than on the basis of a shift from one lim- 
iting reaction to another. They also point out, with justification, that the experimental accuracy must be a good cleal greater than it usually is in the literature in order to establish sharp inflections and true critical temperatures.

The $\mu$ value for beef heart succinoxidase was found to be changed by certain inhibitors by Hadidian and Hoagland (1939, 1941). Succinoxidase is, of course, a multienzyme system and the results were interpreted in terms of shifts in the limiiing reaction step. Cyanide at concentrations between $1.8 \times 10^{-4}$ and $2.4 \times 10^{-4} \mathrm{~m} M$ (at which the inhibition is around $50-70 \%$ ) raised $\mu$ from a normal value of 11,200 to 16,100 . They assumed that in the normal sequence the dehydrogenase is the limiting step and is characterized by a $\mu$ of 11,200 , whereas in the presence of cyanide the cytochrome oxidase with a $\mu$ of 16,100 becomes limiting. Selenite, which was believed to inhibit the dehydrogenase component, thus should not change the normal $\mu$ value but should bring the $\mu$ of the cyanide-poisoned system back towards the normal value; this was found to occur. Pyrophosphate was found to increase $\mu$ to 17.500 and it was concluded that it inhibited some step between the dehydrogenase and the cytochrome oxidase. The kinetics of regenerative electron-transport chains are complex and it is difficult in the present state of our knowledge to evaltuate accurately such results. For example. it is not easy to understand how an inlibition of only $50-70 \%$ could completely shift the limiting reaction step from the dehydrogenase to cytochrome oxilase. and it is even more difficult to interpret the return of the $\mu$ value to normal brought about by selenite when the inhibition produced was not much greater than with cyanide alone. Much more accurate experimental work must be done with such systems before the sites of inhibition can be localized by temperature studies and the mechanisms involved be established. It is quite possible that this approach might become very useful in the study of cellular inhibitions once a firm foundation is laid.

Before leaving the subject of the temperature effects on inhibition in multienzyme systems it is well to point out that the possibility of critical temperatures and their modification by inhibitors depends on the type of multienzyme system that is being studied. For example, in either convergent or divergent branched chains, the chance of observing a sudden inflection in the Arrhenins plots is very small because one would predict that a gradual change from the $\mu$ value of one branch to the $\mu$ value of the other branch wonld oceur, and that the effect of inhibitors would depend on the degree of inhibition exerted so that any $\mu$ value between the extremes would be obtained. On the other hand, in irreversible (or practically irreversible) monolinear sequences it is possible to envision rather sudden changes in the $\mu$ values and the appearance of true critical temperatures. In the simple chain, $\mathrm{A} \rightarrow \mathrm{B} \rightarrow \mathrm{C}$, the over-all rate of the formation of $\mathrm{C}$ is determined either by the rate of reaction 1 or the rate of reaction 2 . If the 
system is in a steady state, the over-all rate is determined by reaction 1; if it leaves the steady state it is determined only by reaction 2 . The transition from a steady state to a non steady one may be sudden, particularly when some factor limits the rise in the concentration of the intermediate B. Whether the system will or will not pass out of a steady state following a specified rise in the temperature will depend for one thing on how close the initial concentration of $\mathrm{B}$ is to the maximal possible concentration. It will also, of course, depend on the difference in the enthalpies of activation for the breakdown of the ES complexes of each step. The addition of an inhibitor to such a system could easily alter the $\mu$ value from that for one reaction to that for the other. Also it would be predicted that if a critical temperature is observed in the range studied, an inhibitor would shift this critical temperature down if the inhibition is exerted primarily on the second reaction and $u p$ if the first reaction is depressed to a relatively greater extent.

A final word of warning must be spoken for the interpretation of thermal studies on cellular systems. If the rate measured is related solely to a single enzyme or multienzyme process, the effects of temperature on inhibition or the effects of inhibition on temperature characteristics may be attributed directly to these processes. However, if what is measured is a composite rate, the interpretation becomes more mncertain. If there is an appreciable endogenous component or an inhibitor-resistant fraction, the $\mu$ values will depend also on these processes and changes in $\mu$ following inhibition may be complex. Furthermore, in work with living cells one cannot always assume a constancy of enzyme concentration because the processes of enzyme synthesis and inactivation may be modified by inhibitors and changes in the temperature.

\section{EFFECTS OF THE DIELECTRIC CONSTANT}

The magnitude of the interactions between molecules frequently depends on the dielectric constant of the medium separating and surrounding the molecules. The role of the dielectric constant in various ionic and dipolar interactions has been described in Chapter 6 . It is now necessary to inquire into the effects of changes in the dielectric constant on enzyme rates and inhibitions. Inasmuch as the binding of substances to the active sites of enzymes is involved in both enzyme activity and in most inlibitions, it is reasonable to expect that useful information of events on the molecular level might be obtained from experiments in which the dielectric constant is varied. An extensive literature on the effects of the dielectric constant on nonenzyme reactions is available but actually only a small amount of work on enzymes has been reported. However, it is likely that dielectric variation will come to be an important procedure in the investigation of 
enzyme interactions and the nature of the forces between inhibitors and active sites.

Water has a high dielectric constant and this reduces appreciably the interactions between ions, ions and dipoles, and dipoles. If the dielectric constant is lowered, one would expect that these electrostatic interactions would become greater. Not only would two oppositely charged ions associate more readily but repulsion between similarly charged ions would increase. Electrokinetic or dispersion forces would not be significantly altered. Thus the formation of most enzyme-inhibitor complexes would occur at a faster rate upon reduction of the dielectric constant and the binding energy might be increased. The variation of the affinity of the enzyme for the inhibitor, or of the inhibitor constant $K_{i}$, with the dielectric constant may provide some information on the nature of the interaction.

\section{Methods for Reduction of the Dieletric Constant}

The dielectric constant of aqueous media may be decreased by the addition of various organic liquids that are miscible with water. The most commonly used substances for this purpose are dioxane, methanol, ethanol, $n$-propanol, isopropanol, methyl ethyl ketone, acetone, acetonitrile, dimethylacetamide, tert-butanol, ethylene glycol, and glycerol. Although dielectric constants from 2 to 78 may be obtained by such mixtures, one is limited in enzyme work to solutions containing not over $50 \%$ of the organic solvent and thus the dielectric constant can usually not be reduced below 35. Tables giving the dielectric constants of mixtures of water with various solvents are available (Harned and Owen, 1958, p. 161). Plots for three solvents are shown in Fig. 15-15. Over the range used in enzyme study, the relationship between dielectric constant and solvent concentration is linear. One of the most nseful organic solvents is dioxane; it is completely miscible with water, it is nonreactive and electrically neutral, and its low dielectric constant allows rates to be studied over a relatively wide range. It must be remembered that the dielectric constant varies with the temperature. Most of the values given for dielectric mixtures in the chemical literature are for $25^{\circ}$ and if the enzyme reaction is rum at more physiological temperatures the appropriate correction shonld be made. Over a range of dioxane concentration from $10 \%$ to $70 \%$, the temperature correction may be obtained from the approximate relationship:

$$
\frac{d \log D}{d T}=-0.0023
$$

Thus in $30 \%$ dioxane. $D\left(25^{\circ}\right)=51.9$ and $D\left(37^{\circ}\right)=48.6$. 


\section{Meaning of the Dieletric Constant of Solvent Mixtures}

The values of the dielectric constant for solvent mixtures have been obtained from relatively large volumes in which a statistically homogeneous medium may be assumed; they may be referred to as bulk dielectric constants. However, when one is visualizing molecular interactions, the comporrents of the medium may not be so homogeneonsly distributed. Sometimes the affinities of the molecules of each solvent may be greater for their own kind

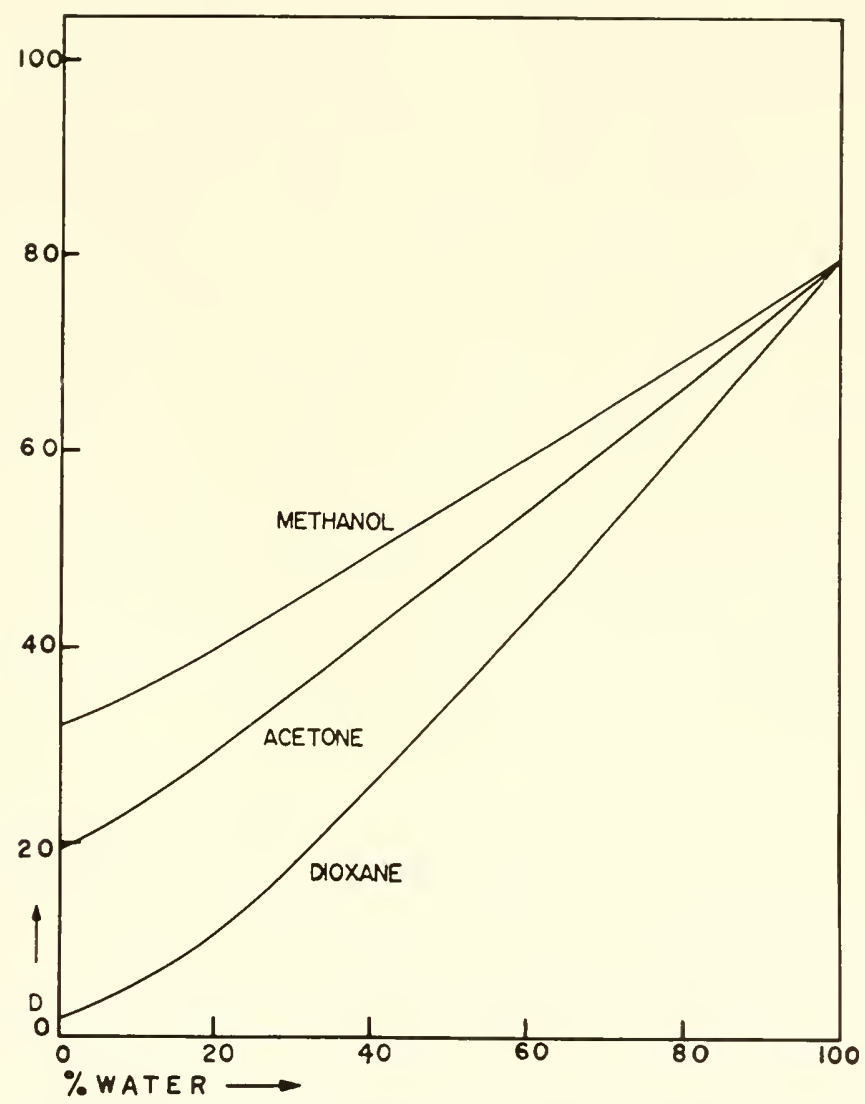

FIG. 15-15. Dielectric constants of water-solvent mixtures at 25. (From Harned and Owen, 1958, p. 161.)

than for the molecules of the other solvent, in which case molecular clusters of varying size may occur. In mixtures of water and dioxane, for example, the water molecules probably retain their tendency to form icelike structures through hydrogem bonding. Furthermore, over short interaction distances the dielectric constant is reduced, as described in Chapter 6, and an 
expression such as $D=6 d-7$ (Eq. 6-72) must be used. We may for convenience call the effective dielectric constants for short-range molecular interactions the microscopic dielectric constants. A problem immediately arises. If the bulk dielectric constant of pure water at $37.5^{\circ}$ is 74.1 and of a $30 \%$ dioxane solution is 48.6 , is the microscopic dielectric constant rednced to the same degree in the dioxane mixture? One might imagine that in the interaction of two ions or ionic groups the ions would be surrounded preferentially by the more polar water molecules and that the presence of the dioxane would not be as important as in the bulk phase. In other words, the interaction energy of two ions up to separation distances around $10 \AA$ might be dependent more upon the dielectric properties of water than would be predicted on the basis of the effect of dioxane on the bulk properties. If the ions are not solvated apureciably by the organic solvent that is added, they must be pictured as primarily hychated as in aqueous media and the interactions between them must still be determined to a great extent by these hydration layers. Of course, when the concentration of the organic solvent becomes high, it is likely that the total hydration of ions is reduced but in mixtures compatible with enzyme work the ions must certainly retain their primary hydration sheaths. Thus some uncertainty is introduced into the nature of the variation of the microscopic dielectric constant with the composition of the medium. However, ionic reaction rates and equilibria have occasionally been shown to vary with the diclectric constant in a predictable manner and the approach must be considered as qualitatively valid. Many discrepancies have also been observed and a nomlinear relationship between the microscopic and bulk diclectric constants may be responsible for these, along with other factors which will be discussed in succeeding sections.

\section{Various Theoretical Dielectric Effects on Enzyme Interactions}

Changing the composition of a medium by the addition of organic solvents may have several possible effects on enzyme reactions and their inhibition. A modification of the interaction energy between a substance and an enzyme due to a simple alteration of the dielectric constant is not the only thing that must be considered in experiments of this type. The rate at which an inhibitor binds to an enzyme and the degree of inhibition will usually be directly affected by the clrange in dielectric properties, but in addition the following factors must be borne in mind in any quantitative treatment.

(a) The p $K_{n}$ 's of acidic or basic groups on the enzyme or the inhibitor may be altered by the change in the dielectric constant. The attraction between a proton and an anionic group will be increased as the dielectric constant is lowered and, hence. the association of the proton will be greater, resulting in a decrease of the acidic dissociation constant. The ionization 
constant of acetic acid, for example, is reduced rery significantly in watermethanol mixtures (Shedlorsky and Kay, 1956). By this effect the total charge on the enzyme, or the charge distribution at or surrounding the active center, or the fraction of the inhibitor molecules in the ionic form, may be changed so that the interaction between the enzyme and the inhibitor is modified.

(b) The interactions of all dipolar and ionic substances in the medium with the enzyme will be changed when the dielectric constant is reduced, and since there is frequently a relationship between the inhibition and these other substances, the inhibition may be altered secondarily. For example, there may be some competition between an ionic imhibitor and similarly charged ions or ionic substances in the medium for a group in the active center, in which case $K_{i}$ will be changed because of the greater ability of these other ions to bind to the enzyme when the dielectric constant is lowered.

(c) The structure of the enzyme or of the active center may be modified, particularly in higher concentrations of the organic solvent. It is well known that most proteins and enzymes are denatured in organic solvents and it is quite possible that minor or localized structural changes could occur before detectable denaturation is evident.

(d) A reduction of the water concentration can slow certain enzyme reactions in which water is one of the components of the reaction, and it will also eventually result in a decreased hydration of both the enzyme and the inhibitor. Thus the concentration of water may play a role in addition to the change in the dielectric constant.

(e) A very important factor may be the interaction of the organic solvent with the enzyme or the inhibitor. Since no solvent that is miscible with water can be completely unreactive or inert, there is always the possibility that the solvent may interfere in the inhibition directly. Most of the organic solvents used can form hydrogen bonds with appropriate groups and also can interact more or less nonspecifically by dipolar and dispersion forces with the enzyme or the inhibitor. Indeed, competition between the solvent and the inhibitor for the enzyme may occur. The alcohols might be said to be particularly prone to affect enzyme reactions and inhibitions in this manner and the examples to be discussed later will provide evidence for this. As mentioned above, dioxane is perhaps less objectionable in this regard than other solvents.

(f) Finally, a question specifically related to the manner in which the water-solvent mixtures are prepared may be mentioned. Enzyme reactions are usually run in media containing various salts. buffers, and cofactors. When additions of an organic solvent are made, should this be done to the normal aqueous medium, resulting in a decrease of the concentrations 
of all the components of the medium, or should the concentrations be adjusted so that they are the same as in the aqueous medium? Although the details are generally not given, it would appear that the former procedure has usually been followed. Such changes in concentrations and ionic strength might have important effects on the reaction studied.

\section{Effect of Dielectric Constant on the Binding of the Inhibitor to the Enzyme}

Lowering the dielectric constant of the medium will increase the electrical interactions between the enzyme and the inhibitor, and if the binding is mainly dependent on ionic and dipolar interactions, the affinity for the inhibitor will increase. A simple approach to the effect of the dielectric constant on the inhibitor constant $K_{i}$ will be ontlined. Let us assume that the inhibitor is bound to the enzyme by ion-ion forces only. We may then combine Eqs. 6-54 and 6-87 to give:

$$
\log K_{i}=214 \frac{z_{1} z_{2}}{d_{e} D}
$$

where $z_{1}$ and $z_{2}$ are the number of charges on the enzyme and inhibitor respectively, $d_{e}$ is the equilibrium interaction distance, and $D$ is the dielectric constant. A plot of $\log K_{i}$ against $1 / D$ should give a straight line with a slope of $214 z_{1} z_{2} / d_{\ell}$. If the inhibition is determined at two different dielectric constants, we may write:

$$
\log \frac{K_{i_{1}}}{K_{i_{2}}}=214 \frac{z_{1} z_{2}}{d_{e}}\left[\frac{1}{D_{1}}-\frac{1}{D_{2}}\right]
$$

The bulk dielectric constants have been used almost exclusively in the studies reported on both chemical and enzymic equilibria, but it is probable that microscopic dielectric constants would be more appropriate. Let us calculate the predicted change in the $K_{i}$ when the dielectric constant is changed from that of pure water to that of $30 \%$ dioxane: we shall assume that $K_{i_{1}}=1 \mathrm{~m} M, D_{1}=74.1, D_{2}=48.6, z_{1}=1, z_{2}=-1$, and $d_{e}=6 \AA$. Application of Eq. 15-59 leads to a value of $K_{i_{2}}$ of $0.56 \mathrm{~m} M$ using the bulk dielectric constants. If we assume that the microscopic diclectric constant for a separation of $6 \AA$ is given by 29 (from Eq. 6-72) in pure water and that the dielectric constant in the dioxane mixture is rednced proportionally, $D_{2}=19$. Using these values, $K_{i_{2}}$ is found to be $0.226 \mathrm{mM}$. Thus it is quite important whether one uses the bulk dielectric constants or attempts to make some correction for the short-range interactions usually occurring in ionic associations.

The general validity of Eq. 15-58 and the decision as to which form of dielectric constant to use may be tested by using the accurate data obtained 
by Fuoss and Kraus (1933) for the dissociation of tetraisoamylammonium nitrate in dioxane-water mixtures. If the logarithm of the dissociation constant is plotted against $1 / D$ (using bulk dielectric constants), the curve shown in Fig. 15-16 is obtained. It may be seen that this is linear over most of the range and deviates only at very low dielectric constants (in mixtures where the water is less than $2 \%$ ). The slope of the linear portion

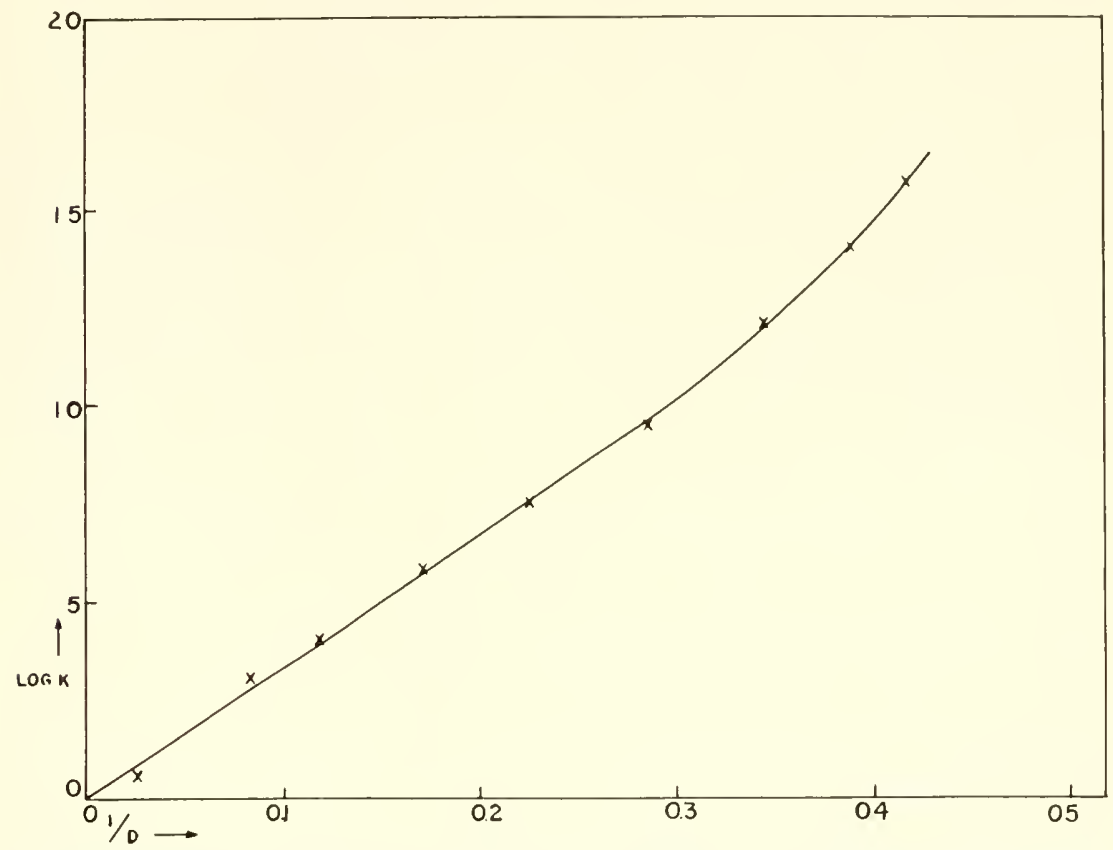

FIG. 15-16. Effects of the dielectric constant (water-dioxane mixtures) on the dissociation constant of tetraisoamylammonium nitrate, using bulk dielectric constants.

(From Fuoss and Kraus, 1933.)

of this curve is approximately 33 and thus $d_{e}$ may be calculated to be $6.45 \AA$. Now this distance is smaller than that calculated on the basis of the closest approach of these two ions. which wonld be between 9-10 $\AA$. It would appear that a more reasonable value for the equilibrium distance would have been obtained if microscopic dielectric constants had been used. In fact, these results indicate that the effective dielectric constant is approximately $70 \%$ of the bulk dielectric constant, which is actually about what one would predict at a separation of $9 \AA$.

For most interactions of an inhibitor with an enzyme involving ionic or dipolar groups one would anticipate that the variation of the inhibitor constant $K_{i}$ with the dielectric constant would be as described above, but 
for other than ion-ion interactions, the slopes of the $\log K_{\imath}-1 / D$ curves would have different values. If dispersion forces play a significant role in the binding, these relationships will be modified as described later.

It is well to remember that as the dielectric constant is reduced, at first there is relatively little effect on $K_{i}$, but because of the exponential relationship, $K_{i}$ will decrease progressively more rapidly. Thus a decrease in the microscopic dielectric constant from 29 to 19. as in the example above, reduces $K_{i}$ by a factor of 4.43 , but a further fall in the dielectric constant to 5 would decrease $K_{i}$ by a factor of 801,000 . In most enzyme work, however, the dielectric constant cannot be reduced far enough to bring about these very marked changes in ion association.

In cases of competitive inhibition involving electrostatic interactions of both the substrate and the inhibitor with the enzyme, a decrease in the dielectric constant will increase the binding of both and the inhibition will not increase as much as in noncompetitive inhibition. It might be thonght that equal changes in $K_{i}$ and $K_{s}$ would lead to no change in the inhibition in competitive systems, but this is not true. Equation 3-13 may be written as:

$$
i=\frac{(\mathrm{I})}{(\mathrm{I})+K_{i}\left[1+(\mathrm{S}) / K_{s}\right]}=\frac{(\mathrm{I})}{(\mathrm{I})+K_{i}+(\mathrm{S})\left(K_{i} / K_{s}\right)}
$$

If $K_{i}$ and $K_{s}$ are decreased equally, the inhibition will become greater because the third term in the denominator will remain unchanged but $K_{i}$ will be less. Thus a decrease in the dielectric constant would be expected to increase competitive inhibition even though the affinities of the enzyme for the substrate and the inhibitor are simultaneously increased to the same degree. However, this increase in the inhibition will not be as great as in a noncompetitive situation. For example. if a noncompetitive inhibition is $33 \%$ with (I) $=1 \mathrm{~m} . M$ and $K_{i}=2 \mathrm{~m} M$, a change of $K_{i}$ to 0.2 $\mathrm{m} M$ will raise the inhibition to $83.3 \%$, whereas a competitive inhibition of $33 \%$ with $(\mathrm{I})=1 \mathrm{~m} M, K_{i}=1 \mathrm{~m} M .(\mathrm{S})=5 \mathrm{~m} M$, and $K_{s}=5 \mathrm{~m} M$ will be increased to $47.6 \%$ if $K_{i}$ and $K_{s}$ are both reduced to one-tenth their original values.

Sometimes additional factors must be taken into account. The inhibition of succinic dehydrogenase by malonate would be an example of the type of competitive inhibition discussed above, since a decreased dielectric constant would increase the affinity of the enzyme for both succinate and malonate. However, the energy required to bend the succinate molecule into the form necessary for binding may be appreciably greater when the dielectric constant is lowered because there will be greater repulsion between the carboxylate groups. This factor is not important for malonate. Thus one would expect that the affinity for malonate would be increased more than for succinate when the dielectric constant is reduced. In other words, every change 
of an electrostatic nature in the over-all reaction must be considered. In the determination of succinic dehydrogenase activity, one must also take into account the effect of the dielectric constant on the acceptor reaction.

\section{Effect of the Dielectric Constant on the Rates of Inhibition}

The rate of a reaction is dependent on the activation energy and this in turn is frequently dependent on electrostatic forces between the interacting molecules. Thus the activation energy can be modified by a change in the dielectric constant. One may visualize these effects simply as the affects of the dielectric constant on the forces between molecules as they approach one another to a distance required for reaction. Reactions between oppositely charged substances should proceed more rapidly as the dielectric constant is decreased, whereas reactions between similarly charged substances should go more slowly. This has been found to be true for many chemical reactions. Rates of inhibition should follow the same laws.

Expressions relating rate constants with the dielectric constant may be derived in several different ways (Laidler and Eyring. 1940; Scatchard. 1932, 1939; Laidler. 1958. p. 205; Amis, 1952; Laidler and Ethier, 1953). We shall consider the dependency of the electrostatic fraction of the free energy of activation on the dielectric constant. The total energy of activation may be divided into electrostatic and nonelectrostatic parts. The energy that is involved in the formation of the activated complex is

$$
\Delta F^{*}=\Delta F_{e s}^{*}+\Delta F_{n e s}^{*}
$$

usually made up of several contributions; not only the different forces operating between the molecules during their approach to one another but also energy terms relating to the structural and electronic alterations necessary for the formation of the complex. In the general case. the electrostatic portion would consist of all ionic and dipolar interactions, but if we consider a simple reaction of ionic groups alone, the treatment is straightforward. The rate constant for the formation of the EI complex may be written as:

$$
k=\frac{R T}{N h} e^{-\left(\lambda F^{*} R T\right)}
$$

according to transition state kinetics. Taking the logarithm of each side and substituting from Eq. 15-61:

$$
\log k=\log \frac{R T}{N h}-\frac{\Delta F_{n e s}^{*}}{2.303 R T}-\frac{\Delta F_{e s}^{*}}{2.303 R T}
$$

The energy required in bringing the ionic groups to within a distance $d$, their separation in the activated complex. is given by (Eq. 6-54):

$$
\Delta F_{s e}^{*}=305 \frac{z_{1} z_{2}}{d D}
$$


and substitution of this in Eq. 15-63 gives:

$$
\log k=\log \frac{R T}{N h}-\frac{\Delta F_{n e s}^{*}}{2.303 R T}-214 \frac{z_{1} z_{2}}{d D}
$$

It must be remembered that $1 F_{e s}^{*}$ is in cal/mole in Eq. 15-63 and in kcal/ mole in Eq. 15-64. A plot of $\log k$ against $1 / D$ should then give a straight line of slope $-214 z_{1} z_{2} / d$ and an intercept on the $\log k$ axis of $\log R T / N h-$ $\Delta F_{\text {nes }}^{*} / 2.303 R T$. If the dielectric constant of the medium is changed from $D_{1}$ to $D_{2}$, the change of the rate constant is given by:

$$
\log \frac{k_{2}}{k_{1}}=214 \frac{z_{1} z_{2}}{d}\left[\frac{1}{D_{1}}-\frac{1}{D_{2}}\right]
$$

The change in the rate constant is thus related only to the electrostatic interactions. The problem arises again of the proper dielectric constants to use. Bulk dielectric constants have been generally used in the past but for the same reasons as given in the section on equilibrium constants, it is likely that the correction for short-range interactions should be applied.

An important result of plotting $\log k$ against $1 / D$ is that the electrostatic interaction energy may be separated from the nonelectrostatic, since one is derivable from the slope and the other from the intercept. Some idea of the distance of separation in the activated complex may also be cbtained if $z_{1}$ and $z_{2}$ are known or can be estimated. From the variation of the free energy of activation with the temperature, the enthalpy and entropy of activation may be calculated, and these quantities will relate to either the electrostatic or nonelectrostatic interactions, depending on whether $\Delta F_{c s}^{*}$ or $\Delta F_{n e s}^{*}$ is used. A similar separation of the free energy of formation of the EI complex into $L F_{e s}$ and $1 F_{\text {nes }}$ may be made by plotting $\log K_{i}$ against $1 / D$.

It must be emphasized that $\angle F_{e s}$ or $\angle F_{e s}^{*}$ may include terms other than those arising from the simple electrostatic interaction of ionic groups. The complex may involve the association or dissociation of a proton and the energy for this will appear in the over-all free energy change. Charged groups on the enzyme or the inhibitor may be moved closer together or farther apart and these energy terms will be dependent on the dielectric constant also. Furthermore, even in interactions of simple ions, the forces arising from induced dipoles may be appreciable in some cases, although in general they are probably less than $1 \%$ of the ion-ion interaction energy (see Table 6-15). When more complex inhibitors are considered, one may have a situation in which $1 F_{e s}$ or $1 F_{e s}^{*}$ is itself made up of several terms, each with a different numerical coefficient. In some instances, energy terms dependent on $1 / D^{2}$ may occur, as in induced dipole interactions, and this may produce some deviation from linearity in the plots. The energy values therefore cannot be directly applied to single simple interactions in 
enzyme inhibition unless there is good evidence that one type of intermolecular force is preponderant.

Lowering the dielectric constant should increase the rates of reaction between oppositely charged ions or between any molecules where electrostatic attraction of any type is important in the formation of the activated complex. This has been tested on many nonenzymic systems, especially the displacement reaction between thiosulfate and bromoacetate (where the rate constant decreases with decreasing dielectric constant since these are both anions) and the reactions of various substances with hydrogen or hydroxyl ions, such as the proton-catalyzed splitting of the azodicarbonate ion (where a marked rise in the rate constant with decrease in dielectric constant is observed), or the reaction of bromophenol blue with hydroxyl ions. Qualitative correspondence to the theoretical expectations is always found but quantitative differences occasionally occur and these may be due to hydration changes in solvent mixtures and to the use of the bulk dielectric constants.

\section{Experimental Studies of Enzyme Reactions}

The effect of changes in the dielectric constant on the rates of enzyme reactions can be attributed to alterations in the affinity of the enzyme for the substrate, or to alterations in the rate of breakdown of the ES complex, or to both. In other words, there may be changes in $k_{2}, K_{s}$. or $K_{m}$. The overall effect on the rate may be complex. For example, in the reaction of a negatively charged substrate with a positively charged active site:

$$
\mathrm{S}^{-}+\mathrm{E}^{+} \rightleftharpoons \mathrm{ES} \stackrel{k_{2}}{\rightarrow} \mathrm{E}^{+}+\mathrm{P}^{-}
$$

in which the product is also negatively charged, a lowering of the dielectric constant will decrease both $K_{s}$ and $k_{2}$, the former tending to increase and the latter tending to decrease the rate. The magnitude of the effect will. of course, depend also on the substrate concentration relative to $K_{s}$ or $K_{m}$, since only changes in $k_{2}$ will be important when the enzyme is substratesaturated but changes in $K_{s}$ or $K_{m}$ will be increasingly significant as the substrate concentration is reduced.

(a) $\alpha$-Chymotrypsin. The addition of methanol up to concentrations around $30 \%(D=64.3)$ progressively slows the rates of hydrolysis of a number of synthetic substrates. The logarithm of the proteolytic coefficient for the hydrolysis of glycyl-L-tyrosinamide and benzoyl-L-tyrosine ethyl ester decreases linearly with the methanol concentration (Kaufman et al., 1949). With acetyl-L-tyrosinamide as the substrate, it was subsequently shown that the rate constant $k_{2}$ is unaltered while $K_{m}$ increases from 0.0326 to 0.0806 , when $20.8 \%$ methanol is present (Kaufman and Neurath, 1949). 
Since $K_{m}$ in this case is $k_{2} / k_{1}\left(k_{-1}\right.$ being negligible compared to $\left.k_{2}\right)$, it was concluded that $k_{1}$ must decrease as the dielectric constant is lowered. The possibility that methanol was acting as a competitive inhibitor was eliminated. The formation of the ES complex is thus slowed by a reduction of the dielectric constant and this would mean that either the substrate and the active site have ionic charges of the same sign or a dipolar interaction involving $\mathrm{H}^{+}$or $\mathrm{OH}^{-}$transfer from the solution occurs. Since the substrate is not ionic, it is likely that the second explanation is correct. However, the possibility of effects of the dielectric constant on the ionization of the imidazole group at the active center should also be considered.

Laidler has used these data and his own to determine the entropies of activation for the formation of the ES complex and for its subsequent reaction (Laidler, 1958, 1. 206; Barnard and Laidler, 1952). Inasmuch as the' variation of the free energy of activation with the temperature allows one to calculate the activation entropy, and since the free energy can be partitioned into electrostatic and nonelectrostatic fractions, it is possible to determine the contributions of these two fractions to the over-all entropy change. These activation entropies are very useful in interpreting the molecular events occurring during the enzyme reaction - changes in liydration or the state of water, separation of charges, structural modification of the enzyme protein, and related events - but Laidler has pointed out the difficulties involved in work with mixed solvents and one really cannot be at all certain that these deductions are quantitatively valid.

A very interesting piece of work - and a disturbing one to lovers of simplicity - on the effects of organic solvents on the hydrolysis of methyl hippurate by $\alpha$-chymotrypsin has been reported more recently by Applewhite and associates (1958) and the results cast some doubt on the interpretation of the rate effects as due entirely to changes in the dielectric constant. A number of organic substances were used and the effects on the kinetic constants often differed (Table 15-2). For example, methanol increases $K_{m}$ and decreases $k_{2}$, whereas acetone increases $K_{m}$ but has no effect on $k_{2}$. A general conclusion is that in all cases there is a rise in the Michaelis constant, $K_{m}$, and this probably reflects the response of $k_{1}$ to the lower dielectric constant. Furthermore, since $k_{2}$ is unchanged by methanol when acetyl-L-tyrosinamide is the substrate (Kaufman and Nenrath, 1949) and is markedly decreased when methyl hippurate is the substrate, different reaction sequences must be postulated. Finally, plots of $\log K_{m}$ against $1 / D$, or $1 / D^{2}$, or $(D-1) /(2 D+1)$, are all nonlinear. There is a rough correlation between $K_{m}$ and the dielectric constant in those cases where $k_{2}$ is unchanged but it is not sufficient to conclude that the dielectric constant is the sole factor involved.

(b) Myosin adenosinetriphosphatase. Inasmuch as ATP is a highly charged anionic substrate, it would be reasonable to expect a large electrostatic 
TABLE 15-2

Effects of Organic Substances on the Hydrolysis of Methyl Hippurate BY $a$-CHYMOTRYPSIN $a$

\begin{tabular}{|c|c|c|c|c|c|}
\hline \multirow{2}{*}{$\begin{array}{l}\text { Nonaqueous } \\
\text { component }\end{array}$} & \multicolumn{2}{|c|}{ Concentration } & \multirow{2}{*}{$\begin{array}{l}\text { Dielectric } \\
\text { constant }\end{array}$} & \multirow{2}{*}{$K_{m}$} & \multirow{2}{*}{$k_{2}$} \\
\hline & Vol. \% & $M$ & & & \\
\hline None & - & - & 78.5 & 7.55 & 2.91 \\
\hline \multirow[t]{2}{*}{ Methanol } & 5 & 1.24 & 76.2 & 17 & 0.76 \\
\hline & 10 & 2.49 & 74.1 & 20 & 0.44 \\
\hline \multirow[t]{4}{*}{ Acetone } & 5.0 & 0.69 & 75.8 & 12 & 2.9 \\
\hline & 10.1 & 1.38 & 73.0 & 21 & 2.8 \\
\hline & 15.2 & 2.08 & 70.0 & 28 & 2.8 \\
\hline & 20.4 & 2.79 & 67.0 & 40 & 2.7 \\
\hline \multirow[t]{3}{*}{ Dioxane } & 5 & 0.59 & 74.1 & 25 & 2.8 \\
\hline & 10 & 1.18 & 69.7 & 50 & 2.8 \\
\hline & 15 & 1.78 & 65.2 & 92 & 2.7 \\
\hline tert-Butanol & 10 & 1.87 & 70.0 & 13 & 2.26 \\
\hline
\end{tabular}

a From Applewhite et al. (1958).

component in the interaction with the enzyme and a greater affinity between the enzyme and the substrate at lower values of the dielectric constant. This has indeed been found by Laidler and Ethier (1953) to be the case. The Michaelis constant decreases progressively as the methanol concentration is raised to $30 \%$ (Table $15-3$ ). Simultaneously the rate constant, $k_{2}$, for the breakdown of the ES complex is slightly increased. The over-all enzyme rate in mixtures of water and dioxane increases as the dioxane con-

TABLE $15-3$

Effects of Methanol on the Kinetic Constants of Mrosin ATPase ${ }^{a}$

\begin{tabular}{c|c|c|c}
\hline $\begin{array}{c}\text { Methanol } \\
\begin{array}{c}\text { concentration } \\
(\text { vol. \%) }\end{array}\end{array}$ & $\begin{array}{c}\text { Dielectric } \\
\text { constant }\end{array}$ & $\frac{K_{m}}{(\mathrm{~m} M)}$ & $\frac{k_{2}}{\left(\mathrm{sec}^{-1}\right)}$ \\
\hline 0 & -18.5 & 0.0128 & 104 \\
10 & 74.1 & 0.0122 & 106 \\
20 & 69.2 & 0.0096 & 113 \\
30 & 64.3 & 0.0078 & 117.5 \\
40 & 59.6 & 0.0082 & 123 \\
\hline
\end{tabular}

${ }^{a}$ From Laidler and Ethier (1953). The dielectric constants were taken from Harned and Owen (1958) and are significantly lower than the values used by Laidler and Ethier. 
centration is raised to $20 \%$ and then declines. The breakdown of the ES complex to inorganic phosphate, ADP, and free enzyme can be visualized as two separate reactions: the splitting off of a negatively charged phosphate from the negatively charged ATP molecule, and the release of the negatively charged ADP from a positively charged active center. The rate of the former reaction would presumably be increased by a low dielectric constant and the rate of the latter reaction decreased. The observed increase in $k_{2}$ might, therefore, be attributed to the effect on phosphate splitting.

Laidler and Ethier used these data to obtain the electrostatic and nonelectrostatic contributions to the entropy change in the formation of the ES complex and to the activation entropy for the breakdown of the complex. Since the Michaelis constant here appears to be the true substrate constant, $K_{s}$, its variation with the dielectric constant provides direct information on the affinity between the enzyme and the substrate. They found that $\Delta S_{e s}$ $=25$ e.u. and from the total entropy change, $1 S=49$ e.u., the nonelectrostatic contribution, $1 S_{\text {nes }}=24 \mathrm{e} .1$. Thus the electrostatic and nonelectrostatic contributions were approximately equal. Now, the calculation of $1 S_{e s}$ involves an expression proportional to $(d D / d T) / D^{2}$. Laidler and Ethier used bulk dielectric constants and the question arises, as it always does, as to the validity of this. Since we do not know the separation distance between the enzyme and the substrate, it is impossible to estimate exactly the microscopic dielectric constant that is applicable. However, if one assumes a separation of around $7 \AA$, which would compare roughly with other ion-ion interactions, the microscopic dielectric constant would be about one-half the bulk dielectric constant; likewise. $d D / d T$ would be about onehalf that for the bulk dielectric constant. Under these assumptions, $\Delta S_{e s}$ would be calculated to be about twice the value obtained by Laidler and Ethier, namely 50 e.u., which would imply that the total entropy change for the formation of the ES complex would be entirely electrostatic. This might actually be more reasonable considering the highly charged nature of ATP and the low binding energies of adenosine and AMP. This large entropy change would perhaps be associated with a marked release of water of hydration. A change in the local configuration of the enzyme must also be considered. In any event, this example illustrates some of the difficulties inherent in the quantitative interpretation of dielectric studies.

(c) Papain. The effects of methanol on the hydrolysis of benzoyl-L-argininamide by papain are shown in Table 15-4 (Smith et al., 1955; Stockell and Smith, 1957). As the methanol concentration is increased, $K_{m}$ becomes larger and $k_{2}$ is relatively unchanged. Since plots of $\log K_{m}$ or $\log k_{2}$ against $1 / D$ are not linear and since an opposite effect on $K_{m}$ was anticipated, in the earlier report it was concluded that the methanol reacted with the papain active site by hydrogen bonding and interfered with the binding of 
TABLE $15-4$

Effects of Methaxol ox the Knnetic Constaxts of Papaix a

\begin{tabular}{c|cc|c}
\hline $\begin{array}{c}\text { Methanol } \\
\text { concentration (vol. \%) }\end{array}$ & $\begin{array}{c}\text { Dielectric } \\
\text { constant }\end{array}$ & $\frac{K_{m}}{(\mathrm{~m} M)}$ & $\frac{k_{2}}{\left(\mathrm{sec}^{-1}\right)}$ \\
\hline
\end{tabular}

Temperature: $5^{\circ} ; \mathrm{pH}: 4.5$

\begin{tabular}{r|rr|r}
0 & 86.2 & 66 & 2.12 \\
10 & 82.6 & 72 & 2.08 \\
20 & 79.0 & 89 & 2.10 \\
30 & 75.3 & 83 & 1.48 \\
50 & 66.5 & 143 & 1.19 \\
\hline
\end{tabular}

Temperature: $38^{\circ} ; \mathrm{pH} 5.2$

\begin{tabular}{r|rrr}
0 & 73.8 & 39 & 11.0 \\
10 & 70.3 & 54 & 11.8 \\
20 & 66.9 & 71 & 9.9 \\
30 & 63.3 & 98 & 9.8 \\
50 & 55.3 & 168 & 9.8 \\
\hline
\end{tabular}

${ }^{a}$ From Stockell and Smith (1957). The dielectric constants may possibly be somewhat too high for the mixtures.

the substrate. However, more complete data and a re-evaluation of the meaning of $K_{m}$ led Stockell and Smith more recently to explain the effects in terms of the dielectric constant. In the first place, $K_{m}$ was found to be $k_{2} / k_{1}$ and not the substrate constant. When $\log k_{2} K_{m}$ was plotted against $1 / D$, a straight line was obtained; this, of course, means that $\log k_{1}$ plotted against $1 / D$ is linear and that the rate of formation of the ES complex does behave as expected with regard to the variation of the dielectric constant. In the second place, the use of isopropanol gave the same dependence of the constants on the dielectric constant and yet isopropanol would not be expected to hydrogen bond to the enzyme as readily as methanol. Since $k_{1}$ decreases with a lowering of the dielectric constant, the formation of the ES complex must involve interactions between ionic groups of like charge or between dipoles oriented in the same direction. How this correlates with the suggested reaction scheme, in which a positively charged substrate group interacts with a negatively charged carboxylate group on the enzyme, is unknown.

(d) Pancreatic carboxypeptidase. The effects of several organic solvents on the hydrolysis of carbobenzoxyglycyl-L-tryptophan by carboxypeptidase were studied by Lumry and Smith (1955) but the results were only 
partially reported. Plots of (S) $/ v$ against (S) at different concentrations of methanol show that the slope is changed but the intercept remains constant. This means that $k_{2}$ is decreased with increasing methanol or decreasing dielectric constant and that $K_{m}$ is relatively unaffected. Since $K_{m}$ for carboxypeptidase is probably $k_{2} / k_{1}$, this would indicate that $k_{1}$ must also decrease in media of lower dielectric constant. However, the other organic substances (ethanol, isopropanol, and dioxane) had pronounced effects on the intercept indicating a marker increase in $k_{1}$. It was pointed out that these results with other solvents make the interpretation of the methanol work more difficult, but might point to electrostatic rearrangements in the activated complex of the rate-limiting step.

(e) Yeast enolase. Methanol, ethanol, and dioxane all depress the rate of the enolase reaction under conditions in which the enzyme is saturated with $\mathrm{Mg}^{++}$and the substrate (Westhear and Malmström, 1957). When the rate is plotted against the concentration of the organic solvent, the points all fall essentially on one curve, althongh the dielectric constant changes produced by these substances are quite different. The decreased rate must be due to either a decrease of $k_{2}$ or of the concentration of active enzyme. Temperature studies showed there is no effect on the activation energies and thus it is unlikely that $k_{2}$ is altered. Denaturation of the enzyme by the solvents or the formation of inactive aggregates by association were eliminated as possible explanations. It was concluded that the solvents acted by displacement of water rather than by a dielectric effect. This decrease in the water concentration might affect the degree of dissociation of proton-transferring groups at the active center. If this explanation is correct, it would emphasize the importance of considering the effects of organic solvents on ionization constants and proton association, factors seldom mentioned in work on other enzymes.

For the useful interpretation of studies in which the dielectric constant is changed, the attitude expressed by Westhead and Malmström is necessary. "While useful information may be obtained from the use of solvent mixtures in studying enzyme kinetics, the situation may be very complex. Results obtained from limited experiments may easily be subject to gross errors in interpretation," This point is particularly important and one is justified in being sceptical of conclusions based on the use of only one organic solvent. Ideally such work should include results from several solvents over as wide a range of concentrations as possible and should test the effects of these solvents on the $\mathrm{pH}$ and temperature characteristics of the reaction studied. Furthermore, all the possibilities by which these solvents may alter the kinetics must be examined and the interpretation must not be based solely on the changes in the dielectric constant.

The effects of solvent-water mixtures on enzyme kinetics have been discussed in some detail because there have been no studies on inhibition. 
The principles brought out above are, however, applicable to inhibition. Indeed, in most instances the investigation of the effects of solvents or dielectric constant on inhibition kinetics would be more straightforward and more readily interpretable than in the case of enzyme kinetics, because one would not have to deal with a sequence of reactions. Changes observed in $K_{i}$ would reflect directly alteration in the affinity of the enzyme for the inhibitor, although the same care would have to be exercised in the attribution of the results to a change in the dielectric constant.

\section{EFFECTS OF SALT CONCENTRATION AND IONIC STRENGTH}

Changes in the electrolyte concentration of media will usually alter the kinetics of enzyme reactions and the susceptibility of the enzymes to inhibitors. As in the variation of the dielectric constant by the addition of organic substances, the effects upon enzymes of adding neutral salts may be due to a variety of mechanisms, some nonspecific (such as brought about by changes in the ionic strength) and some specific (such as result from particular reactions of certain ions with either the enzyme or the other components of the reaction). If one could be certain in any case that the variation of the salt concentration is producing effects upon the kinetics that are entirely attributable to changes in the ionic strength, it would be easier to interpret the results quantitatively and to obtain useful information relative to the molecular events that are involved in the catalysis or the inhibition. Inasmuch as the primary object here is to determine the effects of the ionic strength on enzyme and inhibition linetics, it will first be necessary to outline and discuss briefly the other types of effects that may be observed upon changing the ionic composition of the medium.

\section{General Classification of Salt Effects}

The possible effects of changing the salt concentration on enzymes may be most broadly designated as (1) those producing changes in the solvent water, (2) those directly affecting the interaction between the enzyme and the other components of the system, and (3) those indirectly affecting such interactions.

(a) Changes in the properties of water. Addition of electrolytes alters the bulk dielectric constant of water. This effect is linear with the salt concentration and was expressed by Hasted et al., (1948) as:

$$
D=D_{0}+2 \delta c
$$

where $D$ is the dielectric constant of the salt solution, $D_{0}$ is the dielectric constant of pure water at $25^{\circ}, c$ is the salt concentration, and $\delta$ is the molar 
depression of the dielectric constant. The values of $\delta$ for $\mathrm{KCl}$ and $\mathrm{NaCl}$ are -5 and -5.5 liters/mole, respectively, and thus for most enzymic work Eq. 15-67 may be written simply as $D=D_{0}-10 \mathrm{c}$. Since salt concentrations higher than $0.5 M$ have seldom been used in studies of enzymes, a maximal decrease in the dielectric constant of 5 would be expected. It would appear likely that this factor is a relatively minor one in this range of salt concentrations. Furthermore, the effect of the salt concentration on the microscopic dielectric constant is actually the more important consideration and about this one can say very little. Another effect that might be brought about by the addition of salts is a reduction in the concentration of free water due to the hydration of the ions. However, in the range of concentrations used in enzyme work this is probably a negligible factor; it can be much more important in mixtures of water with organic solvents.

(b) Changes in the activity coefficients of the enzyme reaction components. This is the factor that is responsible for directly affecting the interactions between charged groups. One may look at this effect in a variety of ways. As the electrolyte concentration rises, the ionic atmosphere surrounding each charged molecule and group is increased; i.e., the density of ions of opposite charge to the central ion will increase with the number of ions present. This may be said to reduce the electrical potential of each ion and hence the attraction between ions of opposite sign will decrease. One may also speak of a competition between the nonspecific ions and the substrate (or inhibitor) for the enzyme active site. The deviation from the kinetics of ideal or very dilute solutions is mainly due to this ionic atmosphere and this is the basis for the Debye-Hückel treatment of activity coefficients in real solutions. All of these viewpoints are basically the same. This is the only direct effect dependent on the ionic strength and it will be treated quantitatively and in more detail in a later section.

(c) Changes in the properties of the enzyme or its reaction components. The ionization constants of protein groups vary with the ionic strength and thus a change in the salt concentration could alter the $\mathrm{p} \boldsymbol{K}_{n}$ 's of enzyme groups near the active center, secondarily altering the over-all charge distribution and the affinity between the enzyme and whatever reacts with it. Similar changes on the substrate or inhibitor molecules could also occur. The effects of changes in the ionic strength on the acid dissociation constants of peptides and proteins are discussed thoroughly by Cohn and Edsall (1943). The fundamental reason for a change in the $\mathrm{p} K_{a}$ is the changes in the activity coefficients of the ionic species involved in the acid-base dissociation as discussed many years ago by Brönsted (1928). The extended DebyeHückel equation for the activity coefficient of an ion is:

$$
\log \gamma=-\frac{0.509 z^{2} \sqrt{s}}{1+0.327 r_{o} \sqrt{s}}+K_{s o} s
$$


at $37^{\circ}$, where $\gamma$ is the ionic activity coefficient, $z$ is the charge number for the ions (usually unity for most enzyme work), $s$ is the ionic strength, $r_{0}$ is the closest distance of approach of two oppositely charged ions, and $K_{\text {so }}$ is the salting-out constant (pertaining to the nonionic portions of the molecules and related to the change in the dielectric constant). The acid dissociation constant for a group in a salt solntion can then be written as:

$$
\mathrm{p} K_{a}=\mathrm{p} K_{a_{0}}-\frac{0.509 z^{2} \sqrt{s}}{1+0.327 r_{0} \sqrt{s}}
$$

where $\mathrm{p} K_{a_{0}}$ is the dissociation constant when the ionic strength is zero. If we assume nnit charges on the ions and $r_{0}=4 \AA$, the change in the $\mathrm{p} K_{n}$ upon changing the ionic strength from $s_{1}$ to $s_{2}$ would be given by:

$$
\Delta \mathrm{p} K_{a}=0.509\left[\frac{\sqrt{s_{1}}}{1+1.31 \sqrt{s_{1}}}-\frac{\sqrt{s_{2}}}{1+1.31 \sqrt{s_{2}}}\right]
$$

Addition of $\mathrm{KCl}$ or $\mathrm{NaCl}$ to increase the ionic strength from a physiological value of 0.16 to 0.5 would decrease the $\mathrm{p} K_{z}$ by 0.053 on this theoretical basis. The $\mathrm{p} K_{a}$ of acetic acid in $\mathrm{NaCl}$ solutions at $25^{\circ}$ falls from 4.527 to 4.479 when the ionic strength is increased from 0.16 to 0.5 and thus in this case $\Delta \mathrm{p} K_{a}=0.048$. The $\mathrm{p} K_{a}$ 's of phosphoric acid have been found experimentally to obey an equation of the form, $\mathrm{p} K_{a}=A-B \sqrt{s}$, where the constants $A$ and $B$ have different values for the three ionizations: $A_{1}=2.12$ and $B_{1}=0.467, A_{2}=6.944$ and $B_{2}=0.562$, and $A_{3}=12.185$ and $B_{3}=$ 1.494 (Elliot et al., 1958), and it may be expected that most acidic ionizations will take either this form or that of Eq. 15-69. It is seen that $1 \mathrm{p} K_{z}$ is never very large in the range of ionic strength usually used but if the $\mathrm{p} K_{a}$ is close to the experimental $\mathrm{pH}$ of the enzyme medium, an appreciable alteration of the equilibrium may be induced. Not only will the local charge pattern on an enzyme be changed but the total protein charge will usually be shifted by a modification of the ionic strength, and this can have significant effects on the association of substances with the enzyme as discussed in Chapter 6. It is well known that the electrophoretic behavior of proteins is modified by the salt concentration, reflecting changes in the over-all protein charge. This is an expression of the fact that the isoionic point is a function of the ionic strength. In addition, increase in the ionic strength reduces the mobility by the effect of the ionic atmosphere on the potential.

It is quite possible in certain instances that a change in salt concentration could alter the protein structure by affecting the normal forces between groups on the polypeptide chains or by changing the hydration of the proteins. In those proteins or enzymes where association between units occurs, this may also be modified by salt concentration, and if the enzyme activity or the susceptibility to inhibition varies with this associa- 
tion this can be another way in which salt effects arise. Proteins such as ovalbumin, $\gamma$-globulin, and serum albumin appear to be electrophoretically inhomogeneous and composed of a variety of units differing in total charge (Cann and Phelys, 1957). Equilibrium between these is affected by salt concentration and it was concluded that some structural changes are produced in the proteins. There is no reason why certain enzymes may not behave similarly.

(d) Specific ion binding. When an electrolyte is added to an enzyme preparation, one must always consider the possibility that one or both of the ions reacts specifically with either the enzyme or the other components of the system. By a speeific interaction is meant an association of an ion with a group that is significantly more monounced than wonld be expeeted on the basis of simple electrostatic attraction such as occurs to produce the ionic atmosphere. Ions such as calcimm are particularly prone to associate with groups on enzymes, substrates or inhibitors. The binding of $\mathrm{Ca}^{++}$to serum albumin involves an interaction energy of $4.3 \mathrm{kcal} / \mathrm{mole}$ (Katz and Klotz, 1953) indicating a specifie affinity for some groups on the proteins. Many have assumed, however, that the common ions, $\mathrm{Na}^{+}, \mathrm{K}^{+}$, and $\mathrm{Cl}^{-}$, are not specifically bound. Yet Lewis and Saroff (1957) have demonstrated differential binding of $\mathrm{Na}^{+}$and $\mathbf{K}^{+}$to myosin and Saroff (1957) has reporter different dissociation constants for the complexes of various amphoteric substances with $\mathrm{Na}^{+}, \mathrm{K}^{+}$, and $\mathrm{Li}^{+}$. It was concluded that a certain struetural specificity is required and that a form of chelation may be involved. Thus one camnot be confident of a lack of specific salt effects even when $\mathrm{NaCl}$ and $\mathrm{KCl}$ are used to alter the ionic strength. In this connection, studies designed to determine the effects of ionic strength on enzyme reactions would generally be improved if more than one salt were used. In this way specific effects might be detected and corrections would be possible. It is actually very difficult to draw a distinet line between specific and nonspecific effects in certain instances. It need scarcely be said that in enzyme reactions involving an ionic cofactor, such as $\mathrm{Mg}^{++}$, special attention should be given to the possibility of a direct competition hy the added ions.

\section{Effects of Changes in the lonic Strength on Dissociation Constants}

The dissociation constants of the ES or EI complexes, or of any other complexes that may occur in the enzyme reaction or inhibition, would be expected to vary with the ionic strength. The type of variation will depend on the nature of the forces between the interacting species. We shall first consider ionic interactions because these are treated more readily in a quantitative manner. The approach is based on the variation of the activity coefficients of the interacting molecules with the ionic strength. It has been customary to write the expression for the dissociation constant in terms of 
the thermodynamic activities of the substances. For a simple EI complex:

$$
K_{i_{0}}=\frac{a_{e} a_{i}}{a_{e i}}=\frac{(\mathrm{E})(\mathrm{I})}{(\mathrm{EI})} \frac{\gamma_{e} \gamma_{i}}{\gamma_{e i}}
$$

where the $\gamma$ 's represent the activity coefficients. $K_{i_{0}}$ is the true thermodynamic dissociation constant of the EI complex. The classic or observed dissociation constant is (E)(I)/(EI) which we may designate in the usual manner by $K_{i}$ and thus:

$$
K_{i}=K_{i_{0}} \frac{\gamma_{e i}}{\gamma_{e} \gamma_{i}}
$$

Since an increase in the ionic strength reduces the activity coefficients of ions. it will lead to a rise in the observed $K_{i}$. The activity coefficients are usually expressed in the simple limiting form of the Debye-Hückel equation:

$$
\log \gamma=-0.509 z^{2} \sqrt{s}
$$

where $z$ is the charge on the ion under consideration. Taking the logarithm of each side of Eq. 15-72 and substituting from Eq. 15-73:

$$
\mathrm{p} K_{i}=\mathrm{p} K_{i_{0}}-0.509 \sqrt{s}\left(z_{e}^{2}+z_{i}^{2}-z_{e i}^{2}\right)
$$

Since in most cases, $z_{e i}=z_{e}+z_{i}$ :

$$
\mathrm{p} K_{i}=\mathrm{p} K_{i_{0}}+1.02 z_{e} z_{i} \sqrt{s}
$$

If the charges on the inhibitor and the enzyme active site are opposite, as is usually the case, and are unity:

$$
\mathrm{p} K_{i}=\mathrm{p} K_{i_{0}}-1.021 s
$$

A plot of the observed $\mathrm{p} K_{i}$ against $\sqrt{s}$ would give a straight line, from the slope of which some idea of the charge on the enzyme site might be obtained, knowing the charge on the inhibitor.

Although this treatment leads to simple equations that may be readily plotted, it is not accurate enough when the ionic strength is over 0.05 , as it is in most studies on enzymes; indeed deviations from Eq. 15-73 appear when the ionic strength reaches 0.01 . Therefore, we must use a more refined treatment to express the variation of the activity coefficient. Equation 15-68 affords an expression that is reasonably accurate for ionic strengths up to 1 and will be satisfactory for most enzyme work. Substituting from this equation in Eq. 15-72 and assuming that $r_{0}=4 \AA$, the resulting expression for $\mathrm{p} K_{i}$ is similar to Eq. 15-75 with the corrections for finite ion size and change in the dielectric constant:

$$
\mathrm{p} K_{i}=\mathrm{p} K_{i_{0}}+\frac{1.02 z_{e} z_{i} \sqrt{s}}{1+1.31 \sqrt{s}}+K_{s o} s
$$


When the ionic strength is changed from $s_{1}$ to $s_{2}$, the change in $\mathrm{p} K_{i}$ is then given by:

$$
\Delta p K_{i}=1.02\left[\frac{\sqrt{s_{1}}}{1+1.31 \sqrt{s_{1}}}-\frac{\sqrt{s_{2}}}{1+1.31 \sqrt{s_{2}}}\right]+K_{s o}\left(s_{2}-s_{1}\right)
$$

if the charges on the enzyme and inhibitor are of opposite sign. The ratios of $K_{i}$ to $K_{i_{0}}$ at different ionic strengths are shown in Fig. 15-17 as calcu-

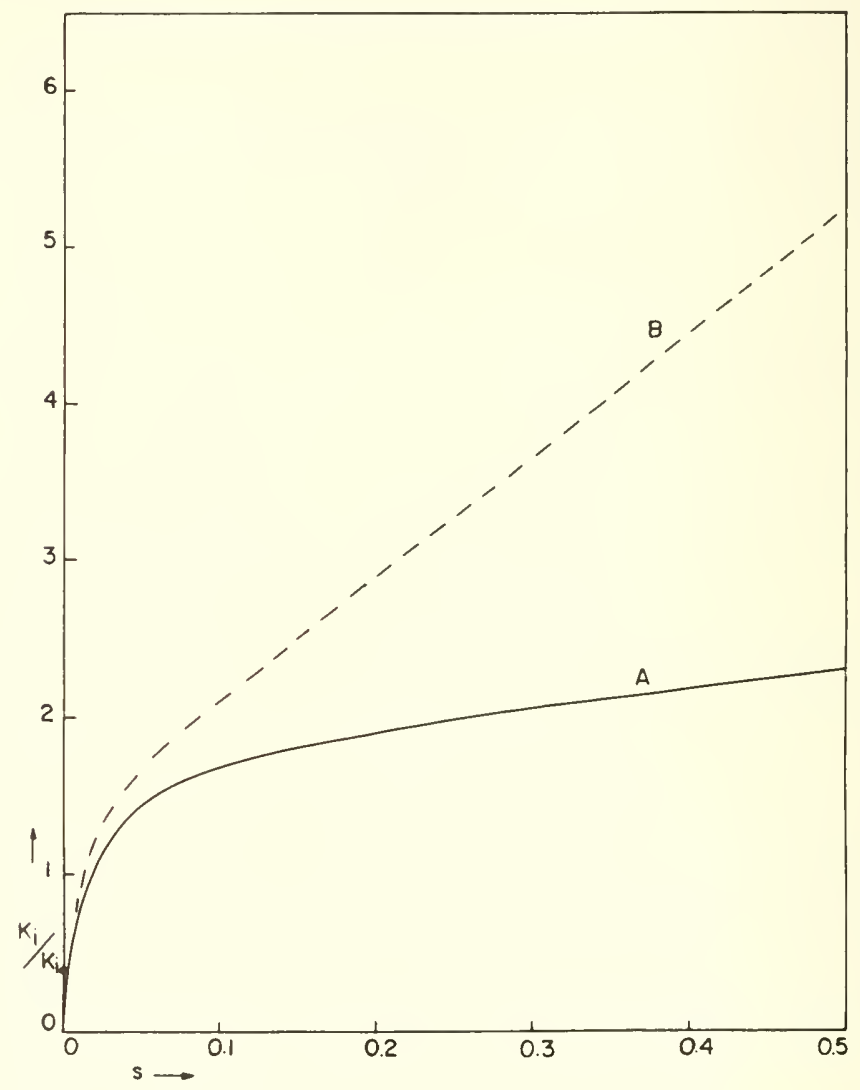

FIG. 15-17. Variation of the ratio $K_{i} / K_{i_{0}}$ with the ionie strength, using the limiting Debye-Hückel equation (A) and the complete Debye-Hückel equation (B).

lated from Eq. 15-77 and compared with the ratios calculated from the simple Debye-Hückel expression and Eq. 15-76. The deviation beyond an ionic strength of 0.01 is easily observed and the difference between the two treatments is very marked at higher ionic strengths. 
A sample calculation will illustrate the magnitude of the effects on inhibition to be expected from variation of the ionic strength. Let us assume that the ionic strength is changed from the normal physiological value of 0.16 to 0.5 by the addition of $\mathrm{KCl}$, for which $K_{s o}=0.0202$. From Eq. 15-78 it is found that $\lrcorner \mathrm{p} K_{i}$ will be -0.10 . The noncompetitive inhibition at $s=0.16$ for a system with $K_{i}=1 \mathrm{~m} M$ and $(\mathrm{I})=1 \mathrm{~m} M$ is $50 \%$. A rise in the ionic strength to 0.5 will increase $K_{i}$ to $1.26 \mathrm{~m} M$ and reduce the inhibition to $44.2 \%$. The effect on competitive inhibition will, in general, be less because there will usually be an increase in $K_{s}$, i.e., a decreased affinity of the enzyme for the substrate. If the above system were competitive and $K_{s}=1 \mathrm{~m} M$ and $(\mathrm{S})=1 \mathrm{~m} M$, the inhibition would be $33.3 \%$; a rise in the ionic strength to 0.5 would lower the imhibition to $30.7 \%$. The effects of even rather large changes in the ionic strength are quite small, particularly when the range of ionic strength investigated is above 0.1 . If the $K_{i}$ is $1 \mathrm{mM}$ when $s=0$ and the ionic strength is increased to 0.16 with $\mathrm{KCl}, \Delta p K_{i}$ may be calculated to be -0.264 and $K_{i}$ would now be $1.84 \mathrm{mM}$, a much greater effect than produced by an increase in the ionic strength from 0.16 to 0.5 .

The question may be raised as to whether the substrate and inhibitor constants determined by the usual plotting procedures are actually the classic dissociation constants expressed by Eq. 15-72. If the derivation of the Michaelis-Menten equation is made using activities and the themodynamic dissociation constants, $K_{s_{0}}$ and $K_{i_{0}}$, the equation for the rate is:

$$
v_{i}=\frac{V_{m}(\mathrm{~S})}{(\mathrm{S})+K_{s_{0}} \frac{\gamma_{e s}}{\gamma_{e} \gamma_{s}}\left[1+\frac{(\mathrm{I})}{K_{i_{0}}} \frac{\gamma_{e} \gamma_{i}}{\gamma_{e i}}\right]}
$$

Thus the constants calculated from the analytical plotting procedures, e.g., the $1 / v$ against $1 /(\mathrm{S})$ plot, are dependent on the activity coefficients in the way indicated above.

From these theoretical considerations, some general principles regarding the optimal approach to ionic strength studies emerge. In the first place, one must be very accurate inasmuch as the changes to be expected are small if the effect is purely related to the ionic strength. Secondly, one should use as wide a range of ionic strengths as is compatible with the enzyme being investigated. Also it is often better to work at the low range of ionic strengths (i.e., below $s=0.1$ ) because the changes in the dissociation constants and the inhibitions are greater in this range (Fig. 15-17). Finally, as pointed out previously, to eliminate specific ionic effects, it is well to use two or more different salts to vary the ionic strength.

We turn now to the problem of the effects of salts on nonelectrostatic interactions between molecules. The decrease in the solubility of most nonpolar substances with increase in the salt concentration is a well-known phenomenon and is often called the salting-out effect. This is an expression 
of the effect of the ions on the solvent water and not directly on the solute molecules. It has been generally found that the activity coefficents of organic nonelectrolytes increase with the salt concentration, and that the relationship is adequately described by the equation:

$$
\log \gamma=k_{m} s
$$

where $k_{m}$ is the salting coefficient. However, the magnitude of the effect is dependent on the salt used and is not related only to the ionic strength; that is, the value of $k_{m}$ varies with the salt used and the nonelectrolyte studied (Harned and Owen, 1958, p. 532). The vahues of $k_{m}$ for addition of $\mathrm{KCl}$ vary from 0.06 to 0.12 in most cases and the mean value for ten nonelectrolytes is about 0.10 . Thus the addition of $\mathrm{KCl}$ to bring about a change of +0.1 in the ionic strength would increase the activity coefficient by a factor of 1.023 , and a change of +0.5 , which is about maximal for most enzyme work, by a factor of 1.122. Thus the effects on interactions of the nonelectrostatic type are small and probably do not contribute greatly to changes in the dissociation constants of EI complexes. What effect there is produces a change in $K_{i}$ in the opposite direction to that occurring in ionic interactions and so if nonelectrostatic forces form a significant fraction of the total interaction energy of an inhibitor with an enzyme, the expected increase in $K_{i}$ based on a purely ionic interaction may be reduced somewhat.

The effects of the ionic strength on dipolar interactions and hydrogen bonding are much more difficult to treat. The activity coefficients of the strongly dipolar amino acids may be either increased or decreased with increasing salt concentration, depending for one thing on the ratio of polar to nonpolar groups in the molecules. Arguments on the possible effects of ionic strength on hydrogen bonding have been presented in a discussion of a paper by Kirkwood (1954, p. 23). No experimental data are available. Boyer and Hammett both feel that there should be very little if any effect of ionic strength while Kirkwood maintains that since the interaction is electrostatic, there should be a small effect. It would seem that one might expect an effect similar to that observed in ion-ion interactions but much smaller, inasmuch as the ionic atmosphere surrounding dipoles is certainly of less electrical density than around ions.

The interactions of many inhibitors with enzymes involve a number of different types of forces and the energy terms corresponding to each of these forces will vary in characteristic ways with the ionic strength. Thus the overall effect of a change in the ionic strength on $K_{i}$ may be complex. In certain protein interactions an increase in the binding with an increase in the ionic strength has been observed and Kirkwood has termed this an inverse ionic strength effect (Kirkwood, 1954). A possible explanation was suggested by Gutfreund in the discussion (p. 21): if the binding depends on a large van 
der Waals' component (due to a structurally favorable complementariness over an extensive area) but is rednced by the presence of oppositely charged groups, an increase in the ionic strength wonld reduce the electrostatic repulsion and hence increase the interaction energy. Actually, in any case in which the binding is impeded by repulsion between ionic groups, even though these groups are not part of the specifically interacting regions. the expected decrease in binding with an increase in the ionic strength will be either reduced or inverted. The intimate nature of the interaction should also be considered because in some instances the opposed groups may be shielded from the influence of ions in the medium and hence the interaction energy between them would not vary in the same way as if they were exposed to the medium.

\section{Changes in the lonic Strength Produced by Additions of Substrate or Inhibitor}

Ionic substrates or inhibitors increase the ionic strength of the medium and. when they are added in increasing concentrations, some of the effects on the enzyme rate may be related to changes in ionic strength. This is particularly true, of course, when the affinities for the enzyme are low and high concentrations must be used. This secondary factor could so modify the kinetics that deviations from linearity would be observed in the analytical plots described in Chapter 5. For example, in the plot of $1 v_{i}$ against (I) (type D), the slope is $K_{m} V_{m} K_{l}(\mathrm{~S})$; if $K_{i}$ becomes larger with increasing inhibitor concentration (due to the increasing ionic strength), the slope will decrease and the curve will become concave downward. Some of the slight activations and inhibitions reported for varions ions, either inorganic or organic, may be due. at least in part. to a nonspecific effect of the ionic strength. One way of minimizing such effects is to use a medium with a relatively high ionic strength, either from buffers or neutral salts. so that ionic strength changes dne to the substrate or the inhibitor will be less effective.

\section{Effects of Changes in the lonic Strength on Rate Constants}

Variations in ionic reaction rates with salt concentration were first put on a firm theoretical basis by Brönsterl (1922. 1928) who introduced the activity coefficients for the reactants into the rate equation. Thus for the bimolecular reaction:

$$
\mathrm{A}+\mathrm{B} \rightarrow \mathrm{X} \rightarrow \mathrm{C}+\mathrm{D}
$$

where $\mathrm{X}$ is the activated complex. the rate is given by:

$$
v=k_{0}(\mathrm{~A})(\mathrm{B}) \frac{\gamma_{a} \gamma_{b}}{\gamma_{x}}
$$


The primary salt effect is the result of the variation of the activity coefficients with the salt concentration. A positive salt effect (increase in the rate on addition of salts) is to be expected when the ions carry charges of the same sign; a negative salt effect when the ions are of opposite signs. The reasoning here is the same as in the previous treatment of the effects of ionic strength on dissociation constants and, incleed, the equilibrium involved in the formation of the activated complex is considered to be the critical factor in the reaction rate. A secondary salt effect that occurs in some reactions was defined by Brönsted as resulting from the effects of the salt concentration on acict-base equilibria whereby the actual concentrations of the reactants or catalysts are changed. Both types of effect can occur in enzyme reactions.

We may define two rate constants, just as in the treatment of dissociation constants; the ordinarily determined rate constant. $k$, and the true rate constant, $k_{0}$, as occurs in Eq. 15-82. It is obvious that:

$$
k=k_{0} \frac{\gamma_{a} \gamma_{b}}{\gamma_{x}}
$$

The relation between these constants in terms of ionic strength may be expressed either by the use of the simple Debye-Huickel Eq. 15-73:

$$
\log k=\log k_{0}+1.02 z_{a} z_{b} \backslash \bar{s}
$$

or by the use of the complete Eq. 15-68:

$$
\log k=\log k_{0}+\frac{1.02 z_{a} z_{b} \sqrt{s}}{1+1.31 \sqrt{s}}+K_{s o} s
$$

The latter equation is more accurate for the ranges of ionic strength usually tested in enzyme reactions. The observed rate constant thus changes with ionic strength to the same degree as the dissociation constant but in the opposite direction. This theory has been found to predict satisfactorily the rate changes of nonenzymic reactions (Benson. 1960, p. 527).

This treatment applies to a bimolecular ionic reaction. Inasmuch as the rate constant $k_{2}$ for the breakilown of the ES complex is important in determining the rate of an enzyme reaction, we must now consider the effects of ionic strength on a dissociation reaction. The recovery from inhibition may also depend on the rate constant $k_{-1}$ for the dissociation of the EI complex. The splitting of a complex AB can be represented in the transition state theory as follows:

$$
\mathrm{AB} \underset{k_{-1}}{\stackrel{k_{1}}{\rightleftharpoons}} \mathrm{X} \stackrel{k_{2}}{\rightarrow} \mathrm{A}+\mathrm{B}
$$

where the rate is proportional to the concentration of the activated complex $\mathrm{X}$, i.e., $v=h_{2}(\mathrm{X})$. The activated complex is assumed to be in equilibrim 
with $\mathrm{AB}$ and thus $K^{*}=(\mathrm{AB}) /(\mathrm{X})$. The thermodynamic equilibrium constant is, however, written in term of the activities:

$$
K_{0}^{*}=\frac{a_{a b}}{a_{x}}=\frac{(\mathrm{AB})}{(\mathrm{X})} \frac{\gamma_{a b}}{\gamma_{x}}
$$

The rate is then given by:

$$
v=\frac{k_{2}}{K_{0}^{*}}(\mathrm{AB}) \frac{\gamma_{a b}}{\gamma_{x}}=k_{0}(\mathrm{AB}) \frac{\gamma_{a b}}{\gamma_{x}}
$$

where the unimolecular rate constant $k_{0}=k_{2} / K_{0}$. The observed unimolecular rate constant may be written as:

Therefore:

$$
k=k_{0} \frac{\gamma_{a b}}{\gamma_{x}}
$$

$$
\log k=\log k_{0}-0.509 \sqrt{s}\left(z_{a b}^{2}-z_{x}^{2}\right)
$$

using the uncorrected Debye-Hückel expression for the activity coefficients. It is evident that when the charges on $\mathrm{AB}$ and the activated complex $\mathrm{X}$ are the same, $k=k_{0}$, and there would be no effect of changing the ionic strength. The charges on $\mathrm{AB}$ and $\mathrm{X}$, however, may not be the same, the formation of the activated complex perhaps involving the dissociation or association of a proton. It is likely that in any case there will be no more than a unit charge difference so that when $\mathrm{AB}$ is neutral, $\log k / k_{0}$ will equal $\pm 0.509 \sqrt{s}$. Thus the rate of dissociation of a complex may be increased, decreased, or unaffected by a change of the ionic strength.

It is probable that in many enzyme reactions, the situation is not quite as simple as this and the decomposition of the ES complex to form products must be written in several steps, such as:

$$
\mathrm{ES} \rightleftharpoons \mathrm{X}_{1} \rightleftharpoons \mathrm{EP} \rightleftharpoons \mathrm{X}_{2} \rightarrow \mathrm{E}+\mathrm{P}
$$

so that the effect of ionic strength upon the enzyme rate may be complex if one step is not limiting the rate. The dissociation of an EI complex is probably straightforward on the other hand. There is also some justifiable doubt as to the validity of using the ordinary Debye-Hückel equations for expressing the activity coefficients applicable to the equilibrium between any complex $\mathrm{AB}$ and its activated counterpart $\mathrm{X}$, especially in enzyme reactions in which the activation process may well occur in a region shielded from the medium.

If the interaction is not between two ionic groups but between an ionic group and a neutral group, Eq. 15-84 would predict that no ionic strength effects woukd be observed, as pointed out by Laidler (1950, p. 131). In this case the additional term, $K_{s o} s$, is important and:

$$
\log \gamma=K_{s o} s
$$


Substituting such values in Eq. 15-83 gives:

$$
\log k=\log k_{0}+\left(K_{s o_{a}}+K_{s o_{b}}-K_{s o_{x}}\right) s
$$

$\log k$ should vary with the first power of the ionic strength instead of with the square root and the effect of ionic strength changes should be much smaller than in ion-ion interactions. Actually, $K_{s o}$ is usually around $5-10 \%$ of the total value of $\log k$ in Eq. 15-85. If the $\left(K_{s o_{a}}+K_{s o_{b}}-K_{s o_{x}}\right) s$ term is small compared to unity, it is possible to write as an approximation:

$$
k=k_{o}\left(1+2.303 K_{s o}{ }^{\prime} s\right)
$$

where $K_{s o}^{\prime}=K_{s o_{a}}+K_{s o_{b}}-K_{s o_{x}}$, and $k$ should be proportional to the ionic strength. These relationships have been found to describe the variations of the rate constants of certain organic reactions, such as the attack by hydroxyl ions on acetals. These types of interactions are probably of fairly frequent occurrence in enzyme catalysis and inhibition, and this is another reason for using the more complete Debye-Hückel equation.

When $K_{m}$ is not the substrate dissociation constant but the kinetic constant, $k_{2} / k_{1}$, the dependence on the ionic strength is quite different from that of $K_{s}$. We may write for the individual rate constants:

$$
\begin{aligned}
& k_{2}=k_{2_{0}}(\mathrm{ES}) \frac{\gamma_{e s}}{\gamma_{x_{2}}} \\
& k_{1}=k_{1_{0}}(\mathrm{E})(\mathrm{S}) \frac{\gamma_{e} \gamma_{s}}{\gamma_{x_{1}}}
\end{aligned}
$$

where the activated complexes may be identified in the following sequence:

$$
\mathrm{E}+\mathrm{S} \rightleftharpoons \mathrm{x}_{1} \rightleftharpoons \mathrm{ES} \rightleftharpoons \mathrm{X}_{2} \rightarrow \mathrm{E}+\mathrm{P}
$$

The Michaelis constant is thus given by:

$$
K_{m}=\frac{k_{2_{0}}}{k_{1_{0}}} \frac{(\mathrm{ES})}{(\mathrm{E})(\mathrm{S})} \frac{\gamma_{e s} \gamma_{x_{1}}}{\gamma_{e} \gamma_{s} \gamma_{x_{2}}}=\frac{K_{m_{0}}}{K_{s_{0}}} \frac{\gamma_{x_{1}}}{\gamma_{x_{2}}}=\frac{k_{2_{0}}}{k_{-1_{0}}} \frac{\gamma_{x_{1}}}{\gamma_{x_{2}}}
$$

Such a Michaelis constant will vary with the ionic strength in a manner depending on the activity coefficients of the two activated complexes. These two complexes can certainly have different charges but the direction of the ionic strength effect is more difficult to predict than in the case of the dissociation constant.

\section{Effects of Changes in the lonic Strength on Enzyme Reactions}

A number of enzyme kinetic studies in which the effects of increasing the salt concentration appear to be due mainly to changes in the ionic strength will be briefly discussed, particularly from the standpoint of the 
modifications in the kinetic constants because these results have bearing on inhibition studies. It should be pointed out, however. that only in rare instances are the salt effects solely due to variations in the ionic strength and the examples discussed here are mostly of a mixed nature with respect to mechanism.

The activity of acetylcholinesterase was shown by Mendel and Rudney (1945) to be decreased by increasing concentrations of $\mathrm{KCl}$ or $\mathrm{NaCl}$ at relatively low concentrations of the substrate (Fig. 15-18). At higher sub-

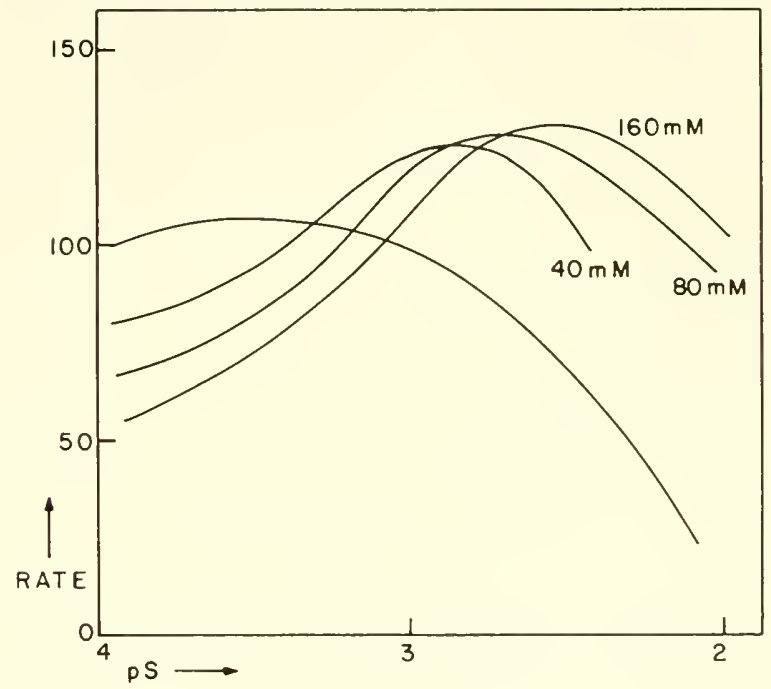

FIG. 15-18. Effects of different concentration of $\mathrm{KCl}$ on the substrate inhibition of mouse brain acetylcholinesterase. The figures on the curves represent the $\mathrm{KCl}$ concentrations in addition to the $25 \mathrm{~m} . \mathrm{MI} \mathrm{NaHCO}_{3}$. (From Mendel and Rudney, 1945.)

strate concentrations the effects are complicated by the substrate inhibition and this will be discussed in a later section. A thorough analysis of human erythrocyte acetylcholinesterase was later made by Myers (1952 c), who determined the varions pertinent constants as the $\mathrm{NaCl}$ concentration was increased to $500 \mathrm{~m} M$ (Table 15-5). With increase in $\mathrm{NaCl}$ there is a marked rise in the first substrate constant indicating a decreased affinity of the enzyme for acetylcholine. which would be expected on the basis of the binding of a positively charged acetylcholine to an anionic group on the enzyme. It is this effect that reduces the rate. However, the rate does not decrease as much as would be expected because there is another effect to increase $V_{m}$ and presumably $k_{2}$. This might indicate that the charge on 
TABLE $15-5$

Effects of NaCl on Erythrocyte Acetylcholinesterase ${ }^{a}$

\begin{tabular}{|c|c|c|c|c|c|c|}
\hline $\begin{array}{l}\mathrm{NaCl} \\
(\mathrm{m} M)\end{array}$ & $\begin{array}{c}K_{s_{1}} \\
(\mathrm{~m} M)\end{array}$ & $\begin{array}{c}K_{s_{2}} \\
(\mathrm{~m} M)\end{array}$ & $K_{s_{2}} / K_{s_{1}}$ & $\begin{array}{l}\left(\mathrm{S}_{0}\right) \\
(\mathrm{m} M)\end{array}$ & $V_{o p t}$ & $V_{m}$ \\
\hline 0 & 0.055 & 11.0 & 200 & 0.78 & 140 & 157 \\
\hline 20 & 0.11 & 12.1 & 110 & 1.15 & 146 & 169 \\
\hline 50 & 0.19 & 13.5 & 70 & 1.60 & 156 & 190 \\
\hline 100 & 0.26 & 15.5 & 60 & 2.05 & 168 & 208 \\
\hline 200 & 0.37 & 18.0 & 50 & 2.70 & 175 & 221 \\
\hline 500 & 0.55 & 22.0 & 40 & 3.65 & 171 & 221 \\
\hline
\end{tabular}

a From Myers (1952 c).

the activated complex for the breakdown of the ES complex is greater than on the ES complex (see Eq. 15-90).

Urease is very sensitive to changes in the ionic strength. Kistiakowsky and Shaw (1953 b) worked with media containing no buffer and hence were able to determine the effects of changes in the ionic strength in the most sensitive range. These data fit the corrected Debye-Hückel equation

\begin{tabular}{c|c}
\hline Ionic Strength & Rate \\
\hline & \\
\hline 0.0003 & 0.425 \\
0.0098 & 0.295 \\
0.0240 & 0.244 \\
0.0478 & 0.202 \\
0.119 & 0.160 \\
0.238 & 0.114 \\
\hline
\end{tabular}

and the rate is inversely proportional to the square root of the ionic strength. It makes very little difference what salt is used; $\mathrm{NaCl}, \mathrm{NaSO}_{4}, \mathrm{KNO}_{3}$, and $\mathrm{KBr}$ all gave the same rate depression at comparable ionic strengths. A dependence of the ionic strength effect on the $\mathrm{pH}$ was also demonstrated, the depression of the rate by salts being greater at a $1 \mathrm{H}$ of 8.95 than at 7-7.5. This is not surprising because the charges on the various components and complexes in the enzyme reaction may be altered by the change in $\mathrm{pH}$. It was concluded that the effect of the ionic strength was mainly on $k_{2}$ since it was independent of the substrate concentration.

The addition of either $\mathrm{NaCl}$ or $\mathrm{KCl}$ to $\alpha$-chymotrypsin leads to an increase in the rate of hydrolysis of chloroacetyl-L-tyrosinamide and the effect is primarily on $k_{2}$ with little if any change in $K_{s}$. The results can be 
represented by the equation: $\log \left(k_{2} / k_{2_{0}}\right)=0.30 \mathrm{~s}$. Thus the dependence on the ionic strength is as expected but the numerical coefficient is less than 0.509, indicating possibly an electrostatic effect from an adjacent group (Shine and Niemam, 1955). One the other hand, later work (Martin and Niemamn, 1958) on the effects of $\mathrm{NaCl}, \mathrm{MgCl}_{2}$, and $\mathrm{CaCl}_{2}$ on the hydrolysis of different acylated $\alpha$-amino acid esters by $\alpha$-chymotrypsin pointed to a decrease in $K_{s}$ with increasing ionic strength, a slight rise in $k_{2}$, and, of conrse, an increase in the rate. These results could not be interpreted easily by the usual theories. The proteinase $\mathrm{C}$ of rat skin with casein as the substrate gave bell-shaped rate curves when the ionic strength was increased by seven different salts (Iartin and Axelrod, 1958). The results with $\mathrm{KCl}$ are shown in Fig. 15-19. It may be noted that the optimal ionic

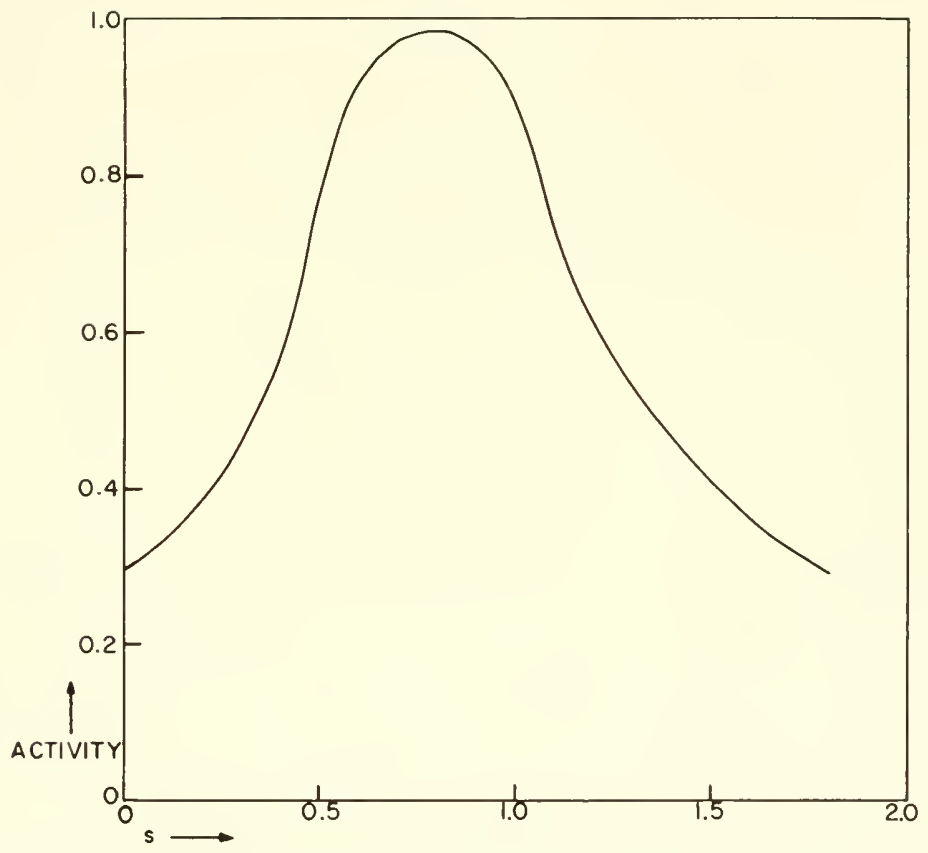

FIG. 15-19. Variation of skin proteinase activity, using casein as the substrate, with the ionic stength (altered by varying the $\mathrm{KCl}$ concentration) at $\mathrm{pH} 7.5$. The activity is plotted on a relative scale.

(From Martin and Axelrod, 1958.)

strength differed somewhat for the different salts. Neither $k_{2}$ nor $K_{m}$ in the case of papain was altered by changes in the ionic stength from 0.05 to 0.3 (Stockell and Smith, 1957), which is surprising in view of the ionic interactions assumed in the mechanism of the catalysis (Kimmel and Smith, 
1957). The rate of the hydrolysis of carbobenzoxyglycyl-L-tryptophan by pancreatic carboxypeptidase increases rapidly with ionic strength (Lumry et al., 1951) up to around 0.25 and then levels off. The salt added makes little difference so that the effect is presumably one of ionic strength. It was claimed that the magnitude of the rate increase is greater than expected from the simple Debye-Hückel theory but unfortunately no data are given. Since the substrate is negatively charged at the experimental $\mathrm{pH}$, the increased rate with higher ionic strengths would indicate that a negative group, or groups, occurs on the enzyme, a decreased $K_{m}$ resulting from a rise in $k_{1}$, since $K_{m}=k_{2} / k_{1}$. The following explanation for the large effects was suggested: a number of negatively charged groups on the enzyme are involved and although individually giving small effects, their total effect may be quite large. A reduction in $K_{m}$ from $20 \mathrm{~m} . I$ to $14 \mathrm{~m} M$ was found for carboxypeptidase by Yanari and Mitz (1957) when the ionic strength was increased from 0.2 to 0.5 , the substrate being carbobenzoxyglycyl-Lphenylalanine. This is not an unreasonably large effect.

It might be expected that ionic strength effects on ribonucleases and deoxyribonucleases would be very marked because of the polyionic nature of both substrates and enzymes. The activity of pancreatic ribonuclease has

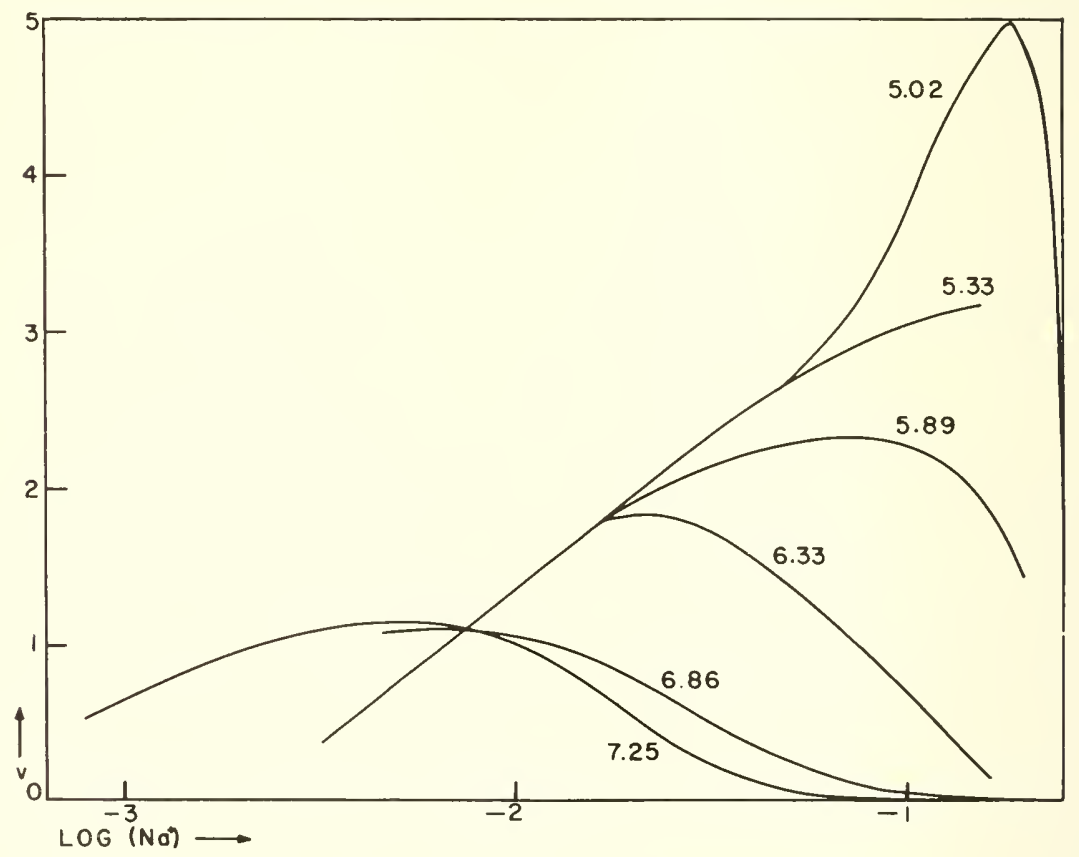

FIG. 15-20. Effects of $\mathrm{Na}^{+}$concentration on the activity of spleen deoxyribonuclease at different $\mathrm{pH}$ values. (From Shack, 1959.) 
indeed been foumd to increase rapidly as the ionic strength is raised to around 0.2-0.3 and there is little further change beyond this (Dickman and Ring, 1958). This effect may be through changes in both the substrate and the enzyme. At least, it was found that increase of the ionic strength altered the substrate RNA in that the absorptivity at $300 \mathrm{~m} \mu$ was markedly decreased. This may be due to an increased dissociation of certain acidic groups and this would perhaps increase the interaction with the enzyme. The effects of $\mathrm{NaCl}$ on spleen deoxyribonuclease are very complex and vary with the $\mathrm{pH}$ (Fig. 15-20) (Shack, 1959). In all cases there are optimal ionic strengths but a decrease in the $\mathrm{pH}$ leads to shifts in these optima to higher concentrations. These interrelationships undoubtedly are due to the effects of the ionic strength on dissociable acidic and basic groups on both enzyme and substrate. However, the shapes of the curves and particularly the slopes point to effects other than mediated through the ionic strength.

The polymucleotide phosphorylase of Micrococcus lysodeiliticus loses most of its activity upon dialysis and the addition of $\mathrm{KCl}$ restores the activity (Beers, 1957). The rate increases very rapidly up to 0.1 ionic strength, reaches a maximum around 0.2 and then falls off at higher salt concentration (Fig. 15-21). The effect is mainly on $K_{m}$ rather than $k_{2}$ and it was concluded

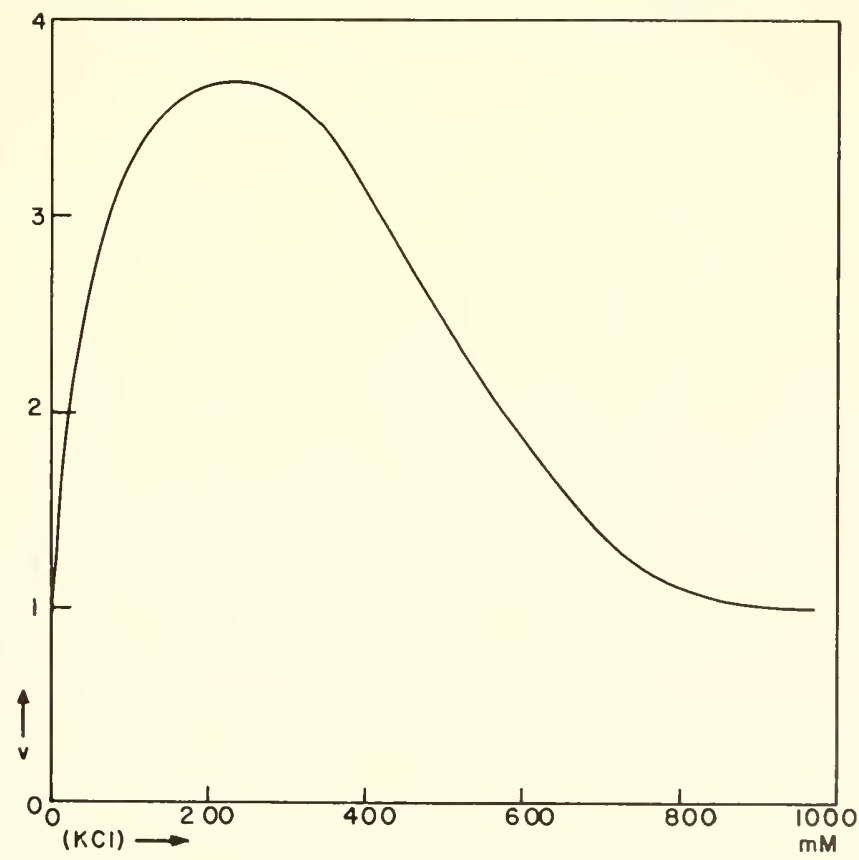

Fig. 15-21. Effects of $\mathrm{KCl}$ concentration on the activity of polynucleotide phosphorylase. The rate is given in terms of inorganic phosphate released ( $\left.\times 10^{-4} M\right)$. (From Beers, 1957.) 
that the principal effect was on the binding of the substrate to the enzyme.

\begin{tabular}{|c|c|c|}
\hline Ionic Strength & $V_{m}$ & $K_{m}$ \\
\hline 0.02 & 1.55 & 1.26 \\
\hline 0.04 & 1.39 & 0.71 \\
\hline 0.1 & 1.46 & 0.563 \\
\hline 0.2 & 1.38 & 0.324 \\
\hline 0.6 & 1.85 & 1.33 \\
\hline
\end{tabular}

The salt concentration also affects the activation of the enzyme by $\mathrm{Ig}^{++}$ and it is possible that this ion is involved in the binding of the substrate. The activity of cytochrome oxidase from spinach and soybean also shows a maximum as the concentration of $\mathrm{NaCl}$ is varied (Fig. 15-22) (Miller and

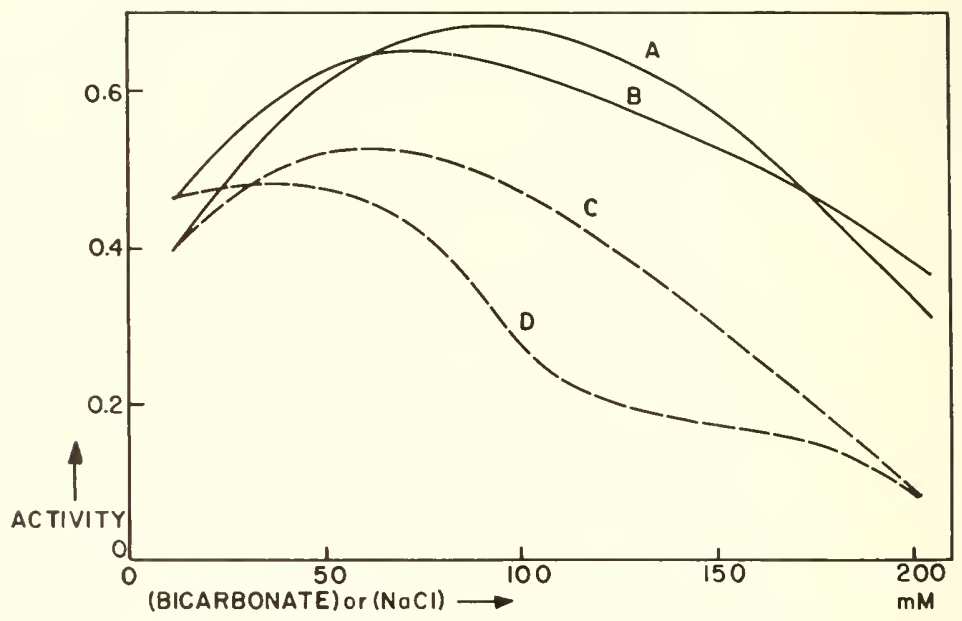

FIG. 15-22. Effects of bicarbonate (dashed curves) and $\mathrm{NaCl}$ (solid curves) on eytochrome oxidase from spinach (A and C) and soybean (B and D). (From Miller and Evans, 1956.)

Evans, 1956). Sodium bicarbonate inhibits this enzyme and part of this effect is due to the $\mathrm{Na}^{+}$, the true inhihition produced by the bicarbonate being the difference between the curves. This is a good illustration of the need for correction when the ordinary physiological ions have effects on the enzyme.

Summarizing the effects observed on various enzymes, it is evident that increasing ionic strength can either decrease, increase, or fail to affect the rate, and that in many cases a biphasic action is evident. i.e., an increase 
in the rate in the low ionic strength range and a decrease at higher salt concentrations. The possible mechanisms responsible for a positive effect of salts on enzyme rates will be outlined and then we shall consider various explanations for the biphasic phenomenon. An increase in rate may be due to the following changes.

(a) Increase of $k_{2}$. This implies changes in charge during the dissociation of the ES complex.

(b) Decrease of $K_{m}$. If $K_{m}=K_{s}$, there is an increased affinity of the enzyme for the substrate and this indicates either the reduction of an electrostatic repulsion or a large salting-out term due to nonpolar forces. If $K_{m}=k_{2} k_{1}$, a difference in the charge on the different activated complexes is suggested, or an increase in $k_{1}$ relative to $k_{2}$.

(c) Increase in the concentration of active enzyme. This could be brought about in a variety of ways: shifts in acid-base equilibria, depolymerization of the enzyme, or altered binding of an ionic cofactor.

(d) Increase in the concentration of active substrate. In most cases this would be restricted to changes in acid-base equilibria.

Both of the first two mechanisms have been demonstrated. Other possibilities may be visualized for more complex enzyme systems.

The "bell-shaped" curves recorded for skin proteinase C, deoxyribonuclease, polynucleotide phosphorylase, cytochrome oxidase, and acetylcholinesterase can all hardly be explained on the basis of ionic strength alone. Activity coefficients begin to rise at higher salt concentrations but this is usually above an ionic strength of 1.0. On the other hand, the maxima in the curves occur at ionic strengths much lower than this in most cases. A dual effect on $K_{m}$ and $k_{2}$, the change in one increasing and a change in the other decreasing the rate. is not feasible because marked effect of ionic strength should occur with both at low salt concentrations. Indeed, the upper limbs of the curves are usnally in ranges of the ionic strength where little effect would be expected. It is more likely that the reduction in the rate is due to an inhibition by the ions superimposed on the ionic strength effect. The jons would be rather weak inhibitors and their actions would be manifest only at higher concentrations. Assuming the simple DebyeHückel formulation for the effect of ionic strength on $k_{2}$ and a competitive inhibition by the added ions (or usually one of the ions), we may write:

$$
v_{i}=\frac{V_{m} e^{1.17 s}(\mathrm{~S})}{(\mathrm{S})+K_{m}\left[1+\frac{s}{K_{i}}\right]}
$$

for a uni-univalent salt. The complete activity coefficient expression can be used and would be preferable, but complicates the following treatment 
and for the present purpose of illustration is unnecessary. Differentiating $v_{i}$ with respect to the ionic strength and setting the derivative $d v_{i} / d s=0$, the ionic strength that is optimal can be calculated from the quadratic equation:

$$
s_{o p t}-1.17 \sqrt{s_{o p t}}+K_{i}\left[1+\frac{(\mathrm{S})}{K_{m}}\right]=0
$$

and the value of the inhibitor constant for the ion can be determined from:

$$
K_{i}=\left(1.17 \sqrt{s_{o p t}}-s_{o p t}\right) \frac{K_{m}}{(\mathrm{~S})+K_{m}}
$$

For a noncompetitive type of salt inhibition the corresponding equations would be:

$$
\begin{gathered}
s_{o p t}-1.17 \sqrt{s_{o p t}}+K_{i}=0 \\
K_{i}=1.17 \sqrt{s_{o p t}}-s_{o p t}
\end{gathered}
$$

If $K_{m}$ also varies with the ionic strength, another term must be introduced into Eq. 15-99; or the ionic strength effect may be on $K_{m}$ only. For optimal ionic strengths around 0.2 , values of $K_{i}$ in the range $200-300 \mathrm{~m} M$ would be expected. The use of the complete Debye-Hückel expression would lower $s_{\text {opt }}$ somewhat.

\section{Specific Ion Effects on Enzyme Reactions}

Because of the importance of recognizing and characterizing specific ion effects in studies of the ionic strength, it may be worthwhile to cite a few examples of such effects so that the varions types of behavior may be appreciated. Phosphogheomutase is inhibited by several common anions, which appear to compete with ribose-1-phosphate for the enzyme site (Klenow, 1955). The inhibitor constants, $K_{i}$, were found to be: chloride, $3.6 \mathrm{mI}$; sulfate, $0.35 \mathrm{~m} . /$; and phosphate, $1.2 \mathrm{~m} / \%$. These valnes show rather tight binding of a magnitude comparable to that of the substrate, for which $K_{m}=0.17 \mathrm{~m} \mu$. Glutathione reductase is inhibited by $\mathrm{NaCl}$ and $\mathrm{KCl}$; $20 \mathrm{mMl} \mathrm{NaCl}$, for example, depresses the rate around $67 \%$ in the complete assay system (Racker, 1955). It is likely that it is the $\mathrm{Cl}^{-}$ ion that is the active inhibitor. Activation of many enzymes with cations has been observed but activation by anions is uncommon. The best known instance is the activation of $\alpha$-amylase by $\mathrm{Cl}^{-}$(Cole, 1904; Myrbäck, 1926). A more recent example is the strong activation of liver arylsulfatase by $\mathrm{Cl}^{-}$and some other umivalent anions when $p$-nitrophenyl sulfate is the substrate (Webb and Morrow, 1959). However, when 2-hydroxy-5-nitrophenyl sulfate is the substrate, $\mathrm{Cl}^{-}$is inhibitory. It was shown that the $\mathrm{Cl}^{-}$has no effect on the affinity of the enzyme for either substrate. Other anions - 
phosphate, sulfate, and fluoride - inhibit with both substrates and a competitive mechanism is likely. Some anions thus interfere with the binding of the substrate and some with the breakdown of the ES complex. A potent activation of fly muscle dehydrogenases by $\mathrm{K}^{+}$at $1 \mathrm{~m} . I$ has been noted (Chefurka, 1957). Both glucose-6-phosphate and 6-phosphogluconic dehydrogenases are activated severalfold by concentrations of $\mathrm{K}^{+}$which preclude any major ionic strength effect. A different sort of effect is seen in the more complex yeast hexolinase system where increases in $\mathrm{Na}^{+}$concentration at constant ionic strength stimulate the rate (Nelchior and Melchior, 1958). Here the active enzyme complex is E-MIg-ATP-glucose and a $\mathrm{Mg}$ ATP complex may be formed independently; $\mathrm{Ig}^{++}$and ATP can also complex separately with the enzyme. Nany ionic interactions are thus involved. The effect of $\mathrm{Na}^{+}$is believed to be due to interactions of the ions with ATP and the E-ATP complex, and this effect is in addition to ionic strength effects which are interpretable in terms of changes in the activity coefficients.

In studies on salt effects, it is often informative to test several salts of different types. This is illustrated by the work of Repaske (1954) on the succinic dehydrogenase of Azotobacter. It was found that $100 \mathrm{~m} M$ $\mathrm{NaCl}$ and $\mathrm{KCl}$ inhibited the enzyme about $33 \%$. From these data alone one might conclude that either it was the $\mathrm{Cl}^{-}$ion that was inhibitory or that the increase in the ionic strength reduced the affinity of the enzyme for succinate. However, $\mathrm{K}_{2} \mathrm{SO}_{4}$ was also used and at $50 \mathrm{mM}$ did not affect the enzyme; thus the ionic strength effect could be eliminated. It is, by the way, rather remarkable that no ionic strength effect was observed in a system where a negatively charged substrate interacts with positively charged enzyme groups. The most likely explanation is that the hydrogen transfer to 2,6-dichlorophenol-indophenol was accelerated by the salts and that this compensated for the reduction in the binding of the succinate to the dehydrogenase.

It may be concluded that the common physiological ions can interact quite specifically with the enzyme, substrate, or cofactors. and that either binding may be altered or the rate of breakdown of the active enzyme complexes may be modified. Activation or inhibition may result and these effects should always be considered in inhibition studies, especially when these ions are added with the inhibitor (as the addition of the sodium or potassium salts of malonate).

\section{Effects of Changes in the Ionic Strength on Enzyme Inhibitions}

Very few accurate studies on inhibition with respect to salt concentration have been reported, but in these few the results are usually interesting and interpretable. Cholinesterase presents an ideal situation for such work because the structure and charge distribution of the active center are to some extent understood. One of the most thorough investigations of ionic 
strength effects was made by Myers (1950) using cholinesterase from human erythrocytes and serum. The inhibitions by physostigmine and neostigmine on both enzymes are reduced by adding $\mathrm{NaCl}$ to the usual bicarbonate media. On the average, the $\mathrm{I}_{50}$ is decreased by around $0.5-0.6$ when the ionic strength is increased by approximately 0.16 . A concentration of $5 \times 10^{-9} \mathrm{M}$ inhibits the erythrocyte enzyme $61 \%$ in the bicarbonate medium and $28 \%$ when $0.125 \mathrm{M} \mathrm{NaCl}$ is added. $\mathrm{KCl}$ produces the same effect and $\mathrm{CaCl}_{2}$ is more effective. The accompanying tabulation shows the effects of varions

\begin{tabular}{r|r|r|r|r|r}
\hline $\begin{array}{r}\mathrm{NaCl} \\
(\mathrm{m} M)\end{array}$ & $\mathrm{pI}_{50}$ & $\begin{array}{c}\mathrm{KCl} \\
(\mathrm{m} M)\end{array}$ & $\mathrm{pI}_{50}$ & $\begin{array}{c}\mathrm{CaCl}_{2} \\
(\mathrm{~m} M)\end{array}$ & $\mathrm{pI}_{50}$ \\
\cline { 1 - 2 } & & & & & \\
\hline 0 & 8.50 & 0 & 8.50 & 0 & 8.50 \\
45 & 8.17 & 125 & 7.94 & 1 & 8.24 \\
125 & 7.94 & & & 4 & 8.01 \\
475 & 7.72 & & & 15 & 7.78 \\
\hline
\end{tabular}

salts on the physostigmine inhibition of erythrocyte cholinesterase. An increase in the ionic strength thus rednces the affinity of the enzymes for the inhibitors, just as it reduces the affinity for acetylcholine, and this is reasonable on the basis of a positively charged inhibitor interacting with a negatively charged group on the active site. The inhibition produced by disopropylfluorophosphate, on the other hand, is unaffected by the ionic strength.

Proteolytic enzymes have been studied rather extensively. Phenylacetate and 3-indolepropionate are competitive inhibitors of chymotrypsin and they are more effective when the ionic strength is increased (Bemhard and Niemann, 1957). The $K_{i}$ for the latter inhibitor is $7.6 \mathrm{~m} M$ in the absence of phosphate and is not changed as the phosphate concentration is increased to $10 \mathrm{mM}$, but then begins to decrease until it is 2.5 at a phosphate concentration of $400 \mathrm{~m} / I \quad(s=1.09)$. These inhibitors are negatively charged and it is postulated that a negative group is sitnated near the active center. An increase in the ionic strength would reduce the repulsion and increase the affinity of the enzyme for the inhibitors. There is evidence that there is also a specific ion effect of phosphate. Certain polyanions such as poly- $\alpha$-L-glutamate, polyeysteate, and heparin, inhibit trypsin and it appears that this is due to a complex formed with the enzyme (Kornguth and Stahmann, 1960). It is postulated that they combine with positively charged groups on either side of the active site, forming a bridge over the site to block off the substrate. The inhibition is markedly decreased by an increase in the ionic strength (Table 15-6) and this supports the electrostatic nature of the binding. It may be noted that the smaller inhibitions 
at high polyglutamate concentration are perhaps due to different molecules binding to the cationic groups so that bridges are not formed and the substrate can reach the active site. Several proteins (serum albumin, $\beta$-lactoglobulin, hemoglobin, and ovalbumin) at low concentrations can inhibit chymotrypsin (Hofstee, $1960 \mathrm{~b}$ ). In the absence of salt, $0.01 \%$ serum albumin inhibits around $80 \%$, giving a $K_{i}$ of approximately $10^{-7} \mathrm{M}$. The addition

TABLE 15-6

Effects of Ionic Strength on the Inhibition of Trypsin by Poly-L-glutamate ${ }^{a}$

\begin{tabular}{c|c|c|c}
\hline $\begin{array}{c}\text { Poly-L-glutamate } \\
(\mathrm{m} M)\end{array}$ & $s=0.005$ & $s=0.13$ & $s=0.27$ \\
\cline { 2 - 3 } & & & \multicolumn{2}{|c}{$\%$ Inhibition } \\
\hline & 60 & 22 & 0 \\
0.09 & 96 & 22 & 0 \\
0.35 & 87 & 22 & 0 \\
1.35 & 74 & 65 & 24 \\
2.70 & 0 & 28 & 0 \\
\hline
\end{tabular}

a The concentration of poly-L-glutamate is given in terms of glutamate residues. From Kornguth and Stahmann (1960).

of $\mathrm{KCl}$ at $100 \mathrm{~m} M$ completely prevents this inhibition indicating a fundamental electrostatic mechanism for the binding. Furthermore, the inhibitions progress slowly and may be completely reversed by $\mathrm{KCl}$ if they have not been allowed to proceed too far. In other words, there is an initial salt-sensitive inhibition, followed by an irreversible inlibition. Similar protection is given by $\mathrm{NaCl}$ and more effective protection by $\mathrm{IgCl}_{2}, \mathrm{SrCl}_{2}$, $\mathrm{CaCl}_{2}$, and $\mathrm{MgSO}_{4}$. The inhibitions by carboxymethylcellulose and nucleic acids are also reversed by increasing salt concentrations. Pancreatic carboxypeptidase is inhibited by several dipeptides (Yanari and Mitz, 1957). The $K_{i}$ for D-leucyl-L-tyrosine is $4 \mathrm{~m} M$ at an ionic strength of 0.2 and falls to $2 \mathrm{mM}$ at an ionic strength of 0.5 . Since this is a competitive inhibitor and $K_{m}$ is also decreased by increasing ionic strength, the inhibition does not rise as much as would be expected from the greater affinity of the enzyme for the inhibitor.

One example of an interesting specific ion effect on enzyme inhibition may be cited. Serum cholinesterase is activated by $\mathrm{Ca}^{++}$and this effect is probably explained by a facilitation on the binding of the enzyme and the substrate. Several phenothiazines inhibit the enzyme and counteract the activating effect of $\mathrm{Ca}^{++}$(Hofstee, 1960 a). It is likely that the phenothiazines and $\mathrm{Ca}^{++}$bind to the same enzyme site. In the absence of $\mathrm{Ca}^{++}$, phenothiazines are completely noncompetitive, but in the presence of $\mathrm{Ca}^{++}$, 
they tend to displace the $\mathrm{Ca}^{++}$from the enzyme and the inhibition is semicompetitive. An anionic group adjacent to the active site is suggested: this interferes in some manner with the interaction of the enzyme and the substrate, so that combination of the group with $\mathrm{Ca}^{++}$favors the formation of the ES complex. The phenothiazines prevent the binding and effect of $\mathrm{Ca}^{++}$. This is actually a case of an inhibitor that is competitive with an activator that happens to be a common physiological ion. Instances in which ions complex with the inhibitor and thus reduce the inhibition are fairly common; the binding of $\mathrm{Ca}^{++}$and $\mathrm{Mg}^{++}$to malonate is a typical example.

Substrate inhibition can also be modified by changes in the ionic strength. This was observed by Mendel and Rudney (1945) in their work on mouse brain acetylcholinesterase (Fig. 15-18). The substrate concentration for the maximal rate, $\left(\mathrm{S}_{0}\right)$, is shifted upward by $\mathrm{KCl} ; 160 \mathrm{mM} \mathrm{KCl}$ increases $\left(\mathrm{S}_{0}\right)$ from $0.25 \mathrm{~m} M$ to $3 \mathrm{~m} M$. Myers $(1952 \mathrm{c})$ found the same behavior with erythrocyte cholinesterase and showed that the binding of the second substrate molecule is weakened at higher ionic strengths (Table 15-5). The effect of $\mathrm{KCl}$ on the rate thus depends on the substrate concentration, a depression of the rate occurring at low substrate concentrations, and an activation at high substrate concentrations. This situation has been discussed in Chapter 4 with respect to the mechanism of substrate inhibition. The substrate inhibition of yeast enolase by 2-phosphoglycerate in the absence of added salts has been shown to be due to an ionic strength effect, the inhibition disappearing when the medium contains $500 \mathrm{~m} \boldsymbol{M ~ K C l}$. This is a good illustration of the danger in interpreting inhibitions in media of low salt content where the addition of the inhibitor can alter the ionic strength and the activity coefficients strongly.

\section{Effects of Salts on Metabolic Systems}

It is at present impossible to interpret ionic strength effects in complex metabolic systems and the likelihood of specific ion effects is increased with the number of enzymes involved. However, it is well known that several types of metabolism are quite sensitive to changes in the salt concentration and one would expect that such changes would alter the responses to certain inhibitors, although no experimental results are available. The marked effects often seen with the common physiological ions are well illustrated in the excellent work of Utter (1950) on the anaerobic glycolysis in brain homogenates. $\mathrm{Na}^{+}$is inhibitory and $\mathrm{K}^{+}$first stimulates and then depresses at higher concentrations. The $\mathrm{Na}^{+}$inhibition is already almost $50 \%$ at $35 \mathrm{mM}$. The mechanisms responsible for the inhibition are several in number. (1) A stimulation of ATP breakdown leading to a fall in the ATP level and a depression of phosphorylations. (2) An increase in the level of AMP which is inhibitory to glycolysis. (3) A decreased rate of phosphorylation of AMP and ADP by inhibition of phosphopyruvate trans- 
phosphorylase, this enzyme being inhibited $41-78 \%$ by $70 \mathrm{mM} \mathrm{Na} \mathrm{Na}^{+} . \mathrm{K}^{+}$ stimulates this transphosphorylase and part of the activation could result from this. The actions of inhibitors on this system would certainly be altered by the concentrations of these ions. Such results indicate how important it is to perform inhibition experiments in as physiologically normal media as possible if conclusions as to the effects in living cells are to be drawn. Effects other than directly on the enzyme systems may also be important. The stimulation of anaerobic glycolysis in slices of guinea pig brain by $\mathrm{Ca}^{++}$was found to be due to actions on the cell membrane (Adams and Quastel, 1956). Mitochondria are also sensitive to salts. The oxidations of acetate and the tricarboxylic acid cycle intermediates are stabilized by $\mathrm{K}^{+}$but not by $\mathrm{Na}^{+}$in heart mitochondria; indeed, $\mathrm{Na}^{+}$is usually inhibitory (Korff et $a l .$, 1954). The soluble acetate-activating enzyme is stimulated by $\mathrm{K}^{+}$but it is not known if this is involved in the stabilization. It may well be that the effects of these ions are on the structure of the mitochondria and it is important in all work with particulate fractions to bear in mind the possibility of such structural clianges.

\section{EFFECTS OF BUFFERS}

Most enzyme and metabolic reactions are studied in media containing buffers of various types. Buffer ions may specifically stimulate or depress enzymes; may interfere or react with substrates, inhibitors, or cofactors; or may exert ionic strength effects. The effects of buffers have been mentioned several times in various connections and the quantitative formulation by Alberty was presented in Chapter 14. Nevertheless, the ubiquity and importance of this problem warrants further discussion and illustration. It is well to emphasize that the buffer concentration should be as low as possible, commensurate with maintenance of a satisfactory $\mathrm{pH}$, and that the media should be made more physiological and the ionic strength normalized by the addition of $\mathrm{KCl}$ or other inorganic or organic ions. It might also be well, wherever possible, first to determine the activity of the enzyme in neutral salt solutions of the appropriate ionic strength and then to determine the effects of various buffers, keeping the ionic strength constant, so that the buffer with the least effect on the enzyme may be chosen.

Examples of inhibitions of enzymes by anions have been known for 50 years and in some cases these are the anions of buffers commonly used. For example, phosphate has been shown to inhibit carboxypeptidase (Smith and Hanson, 1949; Neurath and de Maria, 1950), fumarase (Massey and Alberty, 1954), urease (Kistiakowsky et al., 1952), phosphoghncomutase (Klenow, 1955), carboxylase (Stewart, 1957), arylsulfatase (Webb and Morrow, 1959), and other enzymes. The inhibition of plant cytochrome oxidase by sodium bicarbonate (Fig. 15-22) is partly specific and partly an ionic strength effect (Miller and Evans, 1956). The effect of the bicarbo- 
nate is represented by the differences between the $\mathrm{NaCl}$ and $\mathrm{NaHCO}_{3}$ curves. The use of a neutral salt to correct for specific buffer effects is often a valuable procedure. If the buffer inhibits, the addition of another inhibitor will create a situation of two inhibitors acting simultaneously, as described in Chapter 10, and the inhibition constants calculated for the added inhibitor will not be the true constants for that inhibitor if the buffer effect is neglected.

The inhibitions produced by phosphate, acetate, bicarbonate, and other buffer anions are frequently due to a competition with a negatively charged substrate for a cationic site on the enzyme. Most enzymes that act upon substrates containing phosphate groups will be inhibited to varying extents by phosphate, because an enzyme cationic group will usually be present at the active center for interaction with the phosphate group and this will be susceptible to inorganic phosphate. In other cases these anions may combine with a metal ion incorporated in the enzyme, as in the complex formed between acetate and the iron atoms of catalase (Agner and Theorell, 1946).

The binding of ions to proteins in general is often modified by buffers as has been found in the studies on anion complexes with serum albumin by Klotz (1953) and others. These are mainly competitive reactions. The magnitude of the buffer effect depends on the structure of the buffer anion. Often the greatest interference is introduced by large anions and for this reason buffers such as the barbiturates should be avoided whenever possible. Specific buffer effects on proteins can often be demonstrated by electrophoresis as well as by the indirect experiments on anion binding.

The buffer may affect the ionization of enzyme groups at or adjacent to the active center and thereby alter the binding of substrates or inhibitors. The $\mathrm{p} K_{\text {, ' }}$ 's for the ES complexes of fumarase are generally about 0.5 unit lower in acetate than in phosphate buffer (Frieden and Alberty, 1955) and for the enzyme-malate complexes vary with the phosphate concentration (Massey and Alberty, 1954). The $\mathrm{pH}$ curves for both enzyme activity and inhibition of fumarase are shifted by buffer anions (Massey, 1953 a). As the concentration of phosplrate is increased to $5 \mathrm{~m} M$, stimulation of the rate is observed, but beyond this concentration there is progressive inhibition and an increase in $K_{m}$ (Alberty et al., 1954); in a sense, this might be considered as activator inhibition and treated kinetically in the same way as substrate inhibition.

Particular attention must be paid to the buffer in inhibition studies involving the heavy metal ions (e.g., $\mathrm{Cu}^{++}, \mathrm{Pb}^{++}, \mathrm{Zn}^{++}, \mathrm{Ag}^{+}$, and $\mathrm{Cd}^{++}$) and other substances readily interacting with certain buffers. Not only may the complexes formed reduce the concentration of free inhibitor, but the complexes themselves may possess activity, indeed may be the true inhibitors in some cases. When two or more buffers are used to compare the effects of inhibitors and determine the specific buffer effects, it is im- 
portant to prepare the media at the same ionic strengths to eliminate this factor. Other factors should also be kept constant when possible. The substrate concentration may determine the degree of buffer action; at $66.7 \mathrm{~m} . M$, phosphate inhibits urease $82 \%$ at a urea concentration of $2.2 \mathrm{~m} M$ and only $19 \%$ when the urea is $33.2 \mathrm{mM}$ (Kistiakowsky et al., 1952).

\section{EFFECTS OF PRESSURE}

Whenever any process is accompanied by a change in the total volume of the components, pressure will exert some effect on the rate and equilibrium of the process. The volume changes may be made up of two general parts: one derived from the reactants proper and the other from the solvent water. The simple equilibrium reaction:

$$
\mathrm{A}-\mathrm{W}_{m}+\mathrm{B}-\mathrm{W}_{n} \rightleftharpoons \mathrm{AB}-\mathrm{W}_{p}+(m+n-p) \mathrm{W}
$$

where the subscripts indicate the number of water molecules associated with each species, may involve a volume change which is given by the sum of $\Delta V_{r}$, the volume change of the reactants $\left(V_{\mathrm{AB}}-V_{\mathrm{A}}-V_{\mathrm{B}}\right)$, and $\Delta V_{\mathrm{w}}$, the volume change of the solvent water. If the total volume increases, high pressures will shift the equilibrium to the left because they will oppose the expansion; if the volume decreases, the equilibrium will be shifted to the right at high pressures. The equilibrium constant will thus depend on the pressure. In a like manner, if the complex $\mathrm{AB}$ represents the activated state in the reaction of $\mathrm{A}$ and $\mathrm{B}$ to form products, the rate of the reaction will be either slowed or accelerated depending on the volume change during the formation of the activated complex. In this case, the rate constant depends on the pressure. Studies of the effects of pressure on the rates and equilibria of reactions may be of value in elucidating the molecular mechanisms and the changes in solvent structure.

\section{Molecular Basis for Volume Changes in Enzyme Reactions and Inhibition}

Let us first consider the volume changes related only to the reactants. The bond lengths in the activated complex may be different from those in the normal molecules. This is more apt to occur in chemical reactions than in the simple binding or association of two substances, since in the former situation the transition state may be characterized by unique electronic configuration. Also in chemical reactions, the final products will contain in most cases new bonds and new molecular configurations, so that the sum of the product volumes will not necessarily be equal to the sum of the reactant volumes. Furthermore, in simple association reactions, the two molecules may not fit together accurately and the volume will be increased as a result of the empty space between them. In addition, slight volume changes may arise from the constriction of a reactant in the strong electric 
field of the other reactant. Finally, in enzyme interactions there is always the possibility of alterations in the protein structure, since the active centers of some enzymes appear to be flexible and in other enzymes a certain amount of unfolding or local denaturation may occur upon reaction with the substrates or inhibitors.

With respect to the contributions of the water to the volume changes, the most important effects are probably due to the changes in the ionic character or polarity of the reactants. A charged group is hydrated and the electric field canses the water molecules to occupy less space than they would if they were free, this being termed clectrostriction. The appearance of charged groups thus reduces the solvent volume and the disappearance of charged groups releases water of hydration to increase the solvent volume. Although ionic effects are often dominant, one must also consider the smaller charges associated with dipoles. The electric fields of the reactants may change in quite complex fashion during the formation of the activated complex or the final products and the over-all result will depend on whether there is an increase or decrease in the total polarity. An increase of the total polarity in a reaction will constrict the water and a rise in the pressure will thus favor the reaction. Particularly significant volume changes may occur when oppositely charged ionic reactants form a neutral complex, or when a neutral complex breaks down to ionic products. Solvent volume changes may also occur when neutral molecules react, inasmuch as water molecules may be displaced from the nompolar surfaces and they will be free to enter into the normal water structure, which has somewhat less volume than randomly distributed water molecules.

The over-all volume change may therefore be made up of many contributions. some negative and some positive, but in most simple reactions it is possible to interpret volume changes readily. In reactions occurring in aqueous solution, the major volume changes most commonly relate to the alterations in the water structure. The effects of pressure on the rates of enzyme reactions will, of course, in the general case depend on changes in the binding of the substrate to the enzyme and on the rate of breakdown of the ES complex to products. Thus, both $K_{s}\left(\right.$ or $\left.K_{m}\right)$ and $k_{2}$ may be altered by pressure. In more complex enzyme reactions, pressure effects may be exerted on different steps in the sequence or on the binding of coenzymes or cofactors to the enzyme. The effects of pressure on enzyme inhibition will be usually more easily interpretable. Excellent discussions of the effects of pressure on enzyme reactions have been presented by Laidler (1958, Chapt. VIII) and Johnson et al. (1954, Chapt. 9).

Variation of Equilibrium and Rate Constants with Pressure

The free energy change for a reaction is given by:

$$
\Delta F=R T \ln K=\Delta E+P \Delta V-T \Delta S
$$


where $D E$ is the change in internal energy. Thus:

$$
\ln K=\frac{\Delta E}{R T}-\frac{\Delta S}{R}+\frac{P \Delta V}{R T}
$$

and the variation of $\ln K$ with the pressure is given by:

$$
\frac{d \ln K}{d P}=\frac{\Delta V}{R T}
$$

which was originally obtained by van't Hoff. When the reaction involves an increase in the volume, i.e., $J V$ is positive. $K$ will increase with rise in the pressure, indicating an opposition to the reaction. If we represent the dissociation constant at zero pressure as $K_{0}$ :

$$
\ln K=\ln K_{0}+\frac{P \Delta V}{R T}
$$

when the volume change is independent of the pressure, and:

$$
K=K_{0} e^{(P \cdot A T \cdot R T)}
$$

A plot of $\log K$ against $P$ should then give a straight line with an intercept of $\log K_{0}$ and a slope of $1 \mathrm{~V} / 2.303 R T$. From this slope the over-all volume change may be calculated. Similar reasoning applied to the activation free energy leads to comparable expressions for the variation in the rate constant with pressure:

$$
\begin{gathered}
\ln k=\ln k_{0}-\frac{P \Delta V^{*}}{R T} \\
k=k_{0}-\left(P . V^{*} / R T\right)
\end{gathered}
$$

where $k_{0}$ is the rate constant at zero pressure. Plots of $\log k$ against $P$ thus provide information on the volume change for the formation of the activated complex.

The variation of enzyme rate with the pressure will depend on the substrate concentration relative to $K_{m}$ and on whether $K_{m}=K_{s}$ or not. $K_{m}=K_{s}$ :

$$
\begin{aligned}
& (\mathrm{S}) \ll K_{s} \quad v=v_{0} e^{\left.-P(\lrcorner \mathrm{H}^{\top}+\Delta \mathrm{H}_{2}^{*}\right) / R T} \\
& (\mathrm{~S}) \gg K_{s} \quad v=v_{0} e^{-\left(P_{*} \cdot W_{2}^{*} 2^{*} / R T\right)} \\
& K_{m}=k_{2} / k_{1} \text { : } \\
& (\mathrm{S}) \ll K_{m} \quad v=v_{0} e^{\left.-(P\lrcorner \| Y_{1}^{*} / R T\right)} \\
& (\mathrm{S}) \gg K_{m} \quad v=v_{0} e^{-\left(P . V_{2}^{*} / R T\right)}
\end{aligned}
$$

where $v_{0}$ is the rate at zero pressure (or for practical purposes at normal atmospheric pressure), $J V$ corresponds to the formation of the ES complex, 
$\Delta V_{1} *$ to the formation of the ES* activated complex, and $J V_{2} *$ to the activated complex in the breakdown of the $\mathrm{ES}$ complex to products. When the substrate concentration is close to $K_{m}$ or $K_{m}$ is $\left(k_{2}+k_{-1}\right) / k_{1}$, the relationships are somewhat more complex and intermediate between the extreme situations. These equations may be easily derived by substituting the values for $k_{2}$ and $K_{m}$ obtained from Eq. 15-109 and 15-111.

The gas constant $R=82.05 \mathrm{ml}-\mathrm{atm}$ deg $^{-1} \mathrm{~mole}^{-1}$ and thus this value may be used when the pressure is in atmospheres and the volume change in milliliters. The above equations may be written in the general form:

$$
\log \frac{v}{v_{0}}=-\frac{P \Delta V}{2.30 R T}
$$

where $d V$ represents any specified vohume change. At temperatures around $37^{\circ}$ :

$$
\log \frac{v}{v_{0}}=-\frac{P(\operatorname{atm}) \Delta V(\mathrm{ml})}{58,590}
$$

A sample calculation will illustrate the magnitude of the changes to be expected. The electrostriction is about $10 \mathrm{ml} /$ mole for singly charged ions of the type of $-\mathrm{COO}^{-}$and $-\mathrm{NH}_{3}{ }^{+}$. A reaction involving the appearance of two ionic groups would therefore result in a $20 \mathrm{ml}$-reduction of the solvent volume. If we assume that this is the major volume change and that the pressure is increased to $500 \mathrm{~atm}$ (within a range often used in enzyme studies), the rate is found to increase by a factor of 1.48. The effect of pressure on the binding of an inhibitor to an enzyme may be similarly found, for from Eq. 15-108 we may write:

$$
\mathrm{p} K_{i}=\mathrm{p} K_{i_{0}}-\frac{P \Delta V}{2.303 R T}
$$

If the inhibition involves the appearance of two ionic groups, as above, and the pressure were increased to $500 \mathrm{~atm}$, the $\mathrm{p} K_{i}$ would be increased by 0.17 .

It would be expected that volume changes might be in gencral correlated with entropy changes if disturbances of the solvent structure are of major significance, inasmuch as the transfer of water molecules from a free to a bound state, or vice versa, is accompanied by entropy changes. For example, if ionic groups are produced in a reaction, the volume will decrease dne to the solvent electrostriction; simultaneously there will be a decrease in the entropy because of the immobility imposed on the water molecules. In other words, the volume and entropy changes should be roughly parallel, and this has been found to be true for many reactions, including enzyme catalysis (Laidler, 1958). Pressure studies can, thus, supplement the more classic thermodynamic approaches in probing the movements and fluctuations in the electric field during the various phases of a reaction. 


\section{Effects of Pressure on Enzyme Rates}

Some of the factors that may be involved in bringing about volume changes during enzyme reactions have been discussed. There are a few additional pressure effects that could be important in certain situations. The acidic ionization constants for groups on either the enzyme or the substrate will be altered by pressure increase. The dissociation of a proton from an acidic group will lead to the appearance of two ionic charges which will decrease the solvent volume. A rise in the pressure will thus favor ionization and lead to a decrease in the $\mathrm{p} \boldsymbol{K}_{\alpha}$. On the other hand, the dissociation of a basic group, such as $-\mathrm{NH}_{3}{ }^{+} \rightarrow-\mathrm{NH}_{2}+\mathrm{H}^{+}$, will not so signifieantly alter the water structure and the $\mathrm{p} K_{a}$ 's of such groups will not be changed very much by increases in the pressure. It is evident that any changes in the $p K_{a}$ 's of groups at or near the active center may modify the binding of the substrate or the rate of decomposition of the ES complex.

At very high pressures, perhaps over $5000 \mathrm{~atm}$, enzymes may be irreversibly inactivated, and it is possible that even at lower pressures there may be a shift in the equilibrium between native and denatured forms of the enzyme. It is known that in moderate pressure ranges the rate and extent of protein denaturation are reduced, indicating an increase in volume for the denaturation process, but that at higher pressures the denaturation may be accelerated. It would be well in all pressure work to test the reversibility of the effects to distinguish between the direct effects on rate and equilibrium constants and the denaturation-type changes in the enzyme structure. Actually, the water structure itself is changed to some extent at high pressures, as shown in viseosity studies; for example. a rise in the pressure to $1000 \mathrm{~atm}$ increases the viscosity about $5 \%$ (MoelwynHughes. 1957, p. 1234). It is not certain if such changes could exert significant effects on enzyme reactions but in diffusion-limited systems it is likely they could play a minor role.

Examples of the effects of high pressures on enzyme rates are summarized by Laidler (1958) and calculations of the volume changes for the various steps are presented. In most cases it is not quite certain as to the interpretation of the pressure data because the experiments were not done at widely different substrate concentrations. All enzyme reactions studied are sensitive to pressure changes and in some there are apparently very large volume changes. In general, $1 \mathrm{~T}_{2}{ }^{*}$ is negative $(-5$ to $-30 \mathrm{ml} / \mathrm{mole})$, indicating that the formation of the activated complex in the breakdown of the ES complex to products involves a decrease in volume. High pressures, therefore. usually increase $k_{2}$ and accelerate the enzyme reaction, unless the effects on $K_{m}$ are such as to oppose this. The formation of the ES complex can involve either increases or decreases in the volume, as might be expected from the different factors that may be involved in the binding. The most thorough study is probably that of muscle ATPase 
(Laicller and Beardell, 1955). A rise in the pressure leads to an increase in $k_{2}$ and an increase in $K_{m}$. This means that the binding of ATP to the enzyme involves an increase in the volume, this presumably resulting from the nentralization of ionic charges and the release of water of hydration. This effect alone would decrease the enzyme rate but the increase in $k_{2}$ compensates for this and, especially at high sulstrate concentrations, the rate is increased at high pressures. The increase in $k_{2}$ with pressure and the corresponding decrease in volume on forming the activated complex were attributed to changes in the configuration of the enzyme; however, it is also possible that there is an increase in polarity during the activation and a consequent electrostriction of the water. The high pressure data conform quite well to the entropy changes determined by other means.

\section{Effects of Pressure on Enzyme Inhibition}

There has been, unfortunately, only one study on the response of enzyme inhibition to high pressure. the inhibition of yeast fruetofuranosidase by urethane. The extensive investigations on the inhibition of biolmminescence may relate to the lueiferase system but this is by no means certain so that this work will be discnssed in the following section. Althongh pressure studies will probably never he common. due to the technical problems, it is likely that some enzyme inhibitions will be profitably attacked in this way in the future, so that a brief discnssion of some of the factors that may be involved is not ont of place.

When the inhibition is the result of the simple complexing of the inhibitor with the enzyme. the principal effeet of high pressure will be on the binding affinity as manifested by changes in $K_{\text {. }}$. Many inhibitors are bound to enzymes by electrostatic forces between oppositely charged ionie groups so that the formation of the EI complex in these cases will result in the release of some water of hydration. increasing the volume. The effect of high pressure will then be to reduce the binding and the inhibition. The change in $K_{i}$ may be estimated from Eq. 15-118 or, conversely, pressure data may be used to determine the volume changes upon inhibition. Of course, other factors, such as changes in the $p K_{\text {, }}$ 's of acidic groups or alterations in the enzyme structure. may be important. Also certain inhibitions involve the interaction of nonpolar surfaces and a reduetion in the volume due to the displarement of the water. Just as in the effects of the dielectric constant and the ionie strength. the final result will depend in the case of a complex inhibitor on the balance of the forces participating in the binding.

Competitive inhibition will be affeeted by pressure in a more complex fashion beeause $K_{m}$ will he ehanged along with $K_{\imath}$. Inasmuch as competitive inhibition usually involves an inhibitor strneturally related to the substrate, the forces binding the two substances to the enzyme will often be 
similar. Therefore, $K_{m}$ and $K_{i}$ will generally change in the same way. If the Hichaelis constant is not $K_{s}$, this, of comrse, will not necessarily be true. Competitive inhibition will not be changed as much as noncompetitive inhibition upon changing the pressure because of the similar effects on the binding of both substrate and inhibitor.

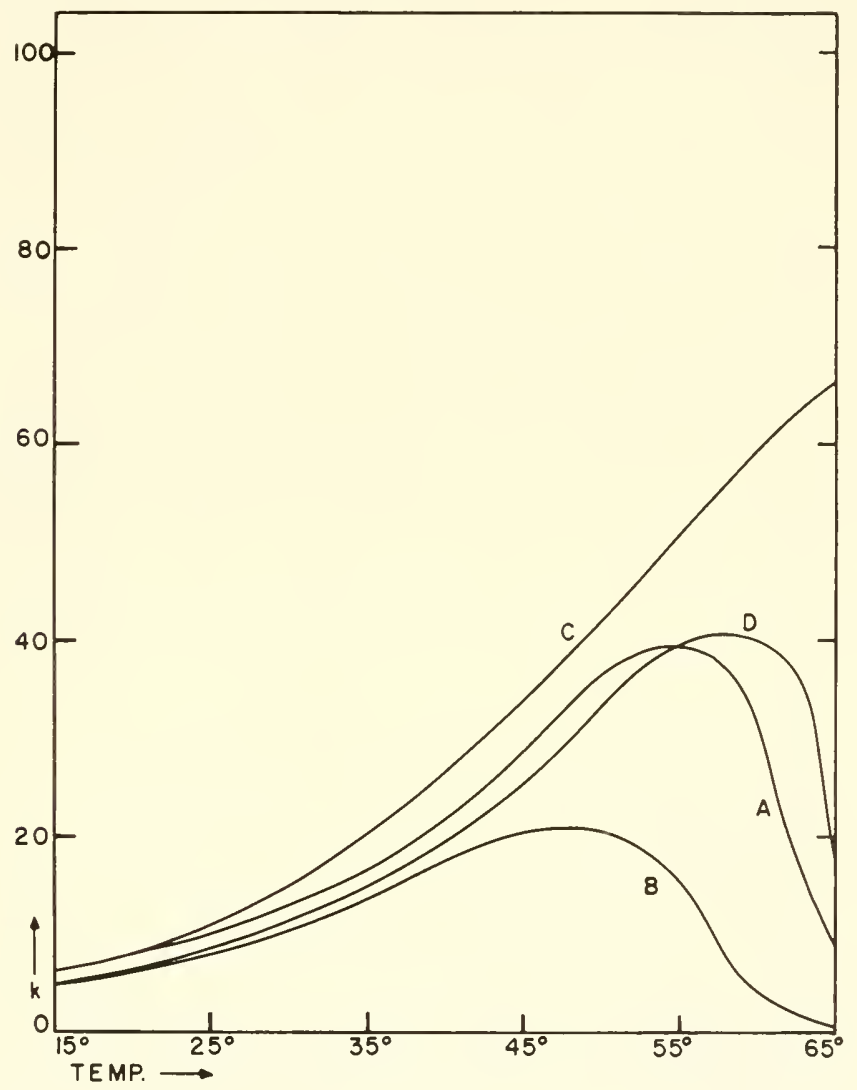

Fig. 15-23. Effects of temperature and pressure on the activity of yeast $\beta$-fructofuranosidase. $A, 1 \mathrm{~atm}$ pressure in the absence of inhibitor; B. $1 \mathrm{~atm}$ pressure and $500 \mathrm{~m} M$ urethane; C. 680 atm pressure in the absence of inhibitor; and D, 680 atm pressure and $500 \mathrm{~m} . Y$ urethane. (From Johnson et al., 1948.)

It would seem that the effects of high pressures on simple enzyme inhibitions would be straightforward and rather easily interpretable. However, the one example we have is not at all simple. The effects of pressure on the uninhibited and urethane-inhibited rates of $\beta$-fructofuranosidase are shown in Fig. 15-23 and on the urethane inlibition in Fig. 15-24 (Jolnnson 
et al., 1948). These curves demonstrate well that the effect of pressure can vary with the temperature; high pressmre reduces the inhibition below $18^{\circ}$ and above $47^{\circ}$. but increases it between these temperatmes. Similar variations wonld probably be seen with changes in the $\mathrm{pH}$, since the pressure effect on the uninhibited rate is pH-dependent. It may be noted that

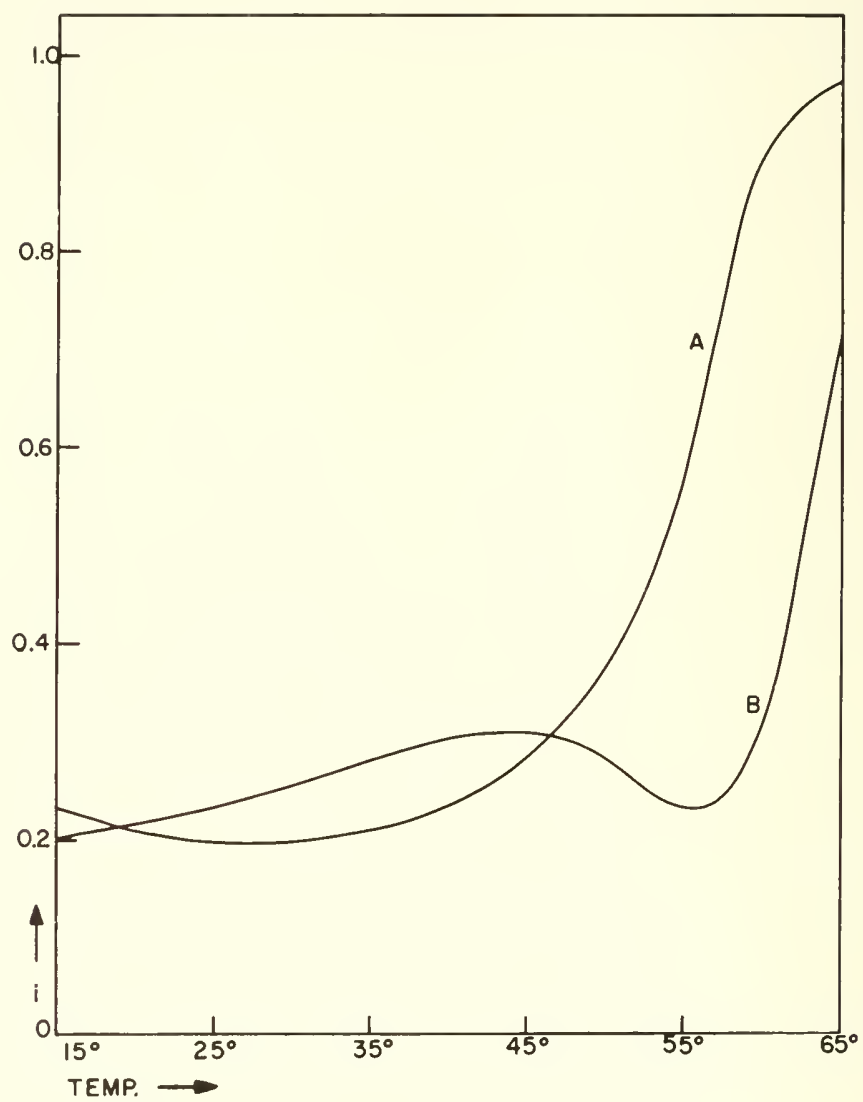

FIG. 15-24. Effects of temperature and pressure on the inhibition of yeast $\beta$-fructofuranosidase by urethane $(500 \mathrm{mM})$. A, $1 \mathrm{~atm}$ pressure and B. $680 \mathrm{~atm}$ pressure. (From Johnson et al., 1948.)

at all temperatures an increase in the pressure accelerates the rate, whether urethane is present or not. The effect of urethane doesn't change with pressure markedly up to $45^{\circ}$ but above this temperature the inhibition is mainly suppressed. Caleulations of the volume changes indieated that in the lower temperature range there is a decrease in the volume (around 
$-10 \mathrm{ml} /$ mole for the formation of the enzyme-urethane complex) but at $50^{\circ}$ this reverses to a volume increase, which becomes quite large aroumd 60-65. Of course, at the higher temperatures one might expect the situation to become more complex because of the instability of the enzyme and the multiple reactions that may be simultaneously proceeding. In the normal temperature range, high pressure increased the inhibition and the volume slightly decreased as mentioned above. What might be the explanation for this effect? The volume change is equivalent to that seen with the appearance of one ionic charge so it is possible that the binding of the urethane to the enzyme brings about an ionization of an enzyme or an inhibitor group, or creates a strong dipole. The pressure effect might also be on an enzyme acidic group to promote ionization and increase the sensitivity of the enzyme to the urethane. However, $\beta$-fructofuranosidase has $\mathrm{p} K_{a}$ 's at 2.6-3.0 and 6.6-6.8, and the experiments were rum at $\mathrm{pH} 4.5$, so that it is unlikely that such a mechanism is correct. If the urethane favored the denaturation of the enzyme, or shifted the equilibrium in favor of a denatured form, one would expect an increase in volume (which is what might occur at higher temperatures). A change in the configuration of the active site due to hydrogen bonding between urethane and the polypeptide chains is a remote possibility. Whatever explanation is correct, this example serves to illustrate the inherent complexity of such studies and issues a warning against the facile interpretation of the results.

\section{Effects of Pressure on Inhibitions in Cellular Systems}

Many studies have shown the marked effects pressure may have on cell motility - for example, muscle contraction, ciliary beating, and cell cleavage - and these effects have been successfully interpreted in terms of changes in the protoplasmic viscosity or shifts in the sol-gel state. Such physical alterations must be important in the responses of some metabolic systems to high pressure but nothing is known about this as yet. It would be most interesting to know what the effects of high pressure are on the tricarboxylic acid cycle in isolated mitochondria. In multienzyme systems, the effects of pressure must indeed be quite complex because each step will be affected to some extent. The only cellular metabolic system studied quantitatively with respect to pressure is the luminescence of certain bacteria by Johnson and his colleagues. These experiments were usually considered to represent the effects on the luciferase reaction but there are other enzymes that are probably involved in determining the intensity of the luminescence, so that it may be safer to envision a multienzyme system. Indeed, it is likely that in bacteria there is no enzyme functioning like luciferase in fireflies. Evidence against the single-enzyme interpretation was present in the early work. For example, the luminescence of different species of bacteria is affected differently by an increase in the pressure: the 
luminescent intensity of Photobacterium phosphoreum is increased, that of Achromobacter fischeri is unaffected except at high pressures, and that of Achromobacter harveyi is depressed (Brown et al., 1942). It might be that the responsible enzymes in these organisms are qualitatively different, but it would seem more reasonable that other factors are the basis for the varying responses. Also the very marked effects of the temperature on the response to high pressures would point to controlling factors more complex than a single enzyme. Work with bacterial extracts has led to the following provisional scheme for the sequence of reactions:

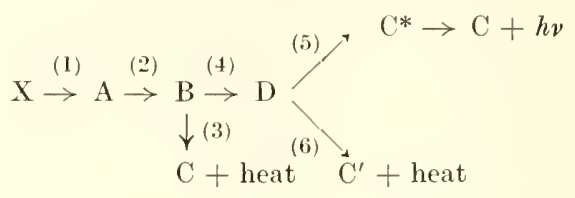

(1) $\mathrm{XH}_{2}+\mathrm{DPN}^{+} \rightarrow \mathrm{X}+\mathrm{DPNH}+\mathrm{H}^{+}$

(2) $\mathrm{DPNH}+\mathrm{FMN}+\mathrm{H}^{+} \rightarrow \mathrm{DPN}^{+}+\mathrm{FMNH}_{2}$

(3) $\mathrm{FMNH}_{2}+1 / 2 \mathrm{O}_{2} \rightarrow \mathrm{FMN}+\mathrm{H}_{2} \mathrm{O}$

$$
\begin{aligned}
& \text { 然 } \\
& \mathrm{FHNH}_{2}+\mathrm{O}_{2}+\mathrm{R}-\mathrm{CHO}-\mathrm{E} \rightarrow \mathrm{FHN}+\mathrm{E}-\mathrm{R}-\mathrm{C}-\mathrm{OOH}_{\mathrm{H}} \\
& \mathrm{E}-\mathrm{R}-\mathrm{C} \underset{\mathrm{H}}{-} \underset{\mathrm{OOH}}{\mathrm{OH}}+\mathrm{FMNH}_{2} \rightarrow \mathrm{E}-\mathrm{R}-\mathrm{CHO}-\mathrm{FMN}^{*} \\
& \mathrm{E}-\mathrm{R}-\mathrm{C} \underset{\mathrm{H}}{-\mathrm{OH}}+\mathrm{OH} \rightarrow \text { nonluminescent products }
\end{aligned}
$$

$\mathrm{XH}_{2}$ represents electron donors. FMN is flavin mononncleotide.

$$
\mathrm{E}-\mathrm{R}-\mathrm{C} \underset{\mathrm{H}}{\stackrel{\mathrm{OOH}}{\mathrm{OH}}}
$$

is an aldehyde-peroxide addition complex, and the asterisks indicate the photochemically activated molecules. The $\mathrm{R}-\mathrm{CHO}$ is a long-chain aldehyde originally obtained from renal cortex and later shown to be palmitic aldeliyde. The enzyme, $\mathrm{E}$, might be considered as the equivalent of luciferase, but the other reactions are also enzymic. It might also be pointed ont that the level of $\mathrm{DPN}^{+}$would be determined by reactions unrelated to the luminescence sequence. The reduction of FMN by DPNH is perhaps the limiting step under certain circumstances but in the living cell it is difficult to know if this is always true. In any event, the effects of pressure may be 
on one or more enzymes, and not necessarily on the one involved in the final emission of light. A study of the effects of sudden pressure changes in such isolated systems has led Strehler (1955) to postulate that high pressure inhibits the DPNH-FIN reaction (indicating a volume increase on activation) and accelerates the oxidation of $\mathrm{FMNH}_{2}$ via the luminescent pathway (indicating an over-all volume decrease). It was pointed out that other interpretations are possible but for our present purpose in discussing the effects of pressure on the inhibition of luminescence it emphasizes that some reevaluation of the earlier conclusions must be made. since in the earlier work the effect of pressure on only one enzyme was assumed. The sites of actions of the various inhibitors in the sequence are not known and this further complicates an analysis of the pressure effects on inhibition. The actions of the inhibitors on the isolated systems with respect to pressure have not been investigated as yet.

The first demonstration that the inhibition of a metabolic system could be reduced or eliminated by an increase in the pressure was in a study of bacterial luminescence by Johnson et al. (1942 a). The inhibitions produced by chloroform, ethyl ether, ethanol, procaine, and certain carbamates are diminished by high pressure, whereas inhibitions by $p$-aminobenzoate. sulfanilamide, barbital, and chloral hydrate are scarcely affected or slightly increased (Fig. 15-25). The explanation for these effects was formulated in terms of shifts between native and denatured forms of some critical enzyme. For example, inhibitors such as ethanol or the carbamates were believed to lower the activation energy for the reversible denaturation of the enzyme; since denaturation involves an increase in the volume, high pressures would tend to counteract the inhibition. Actually. if the interaction of the inhibitor with the enzyme in any way involves an increase in the volume, an antagonism by pressure would be observed. Also. since the luminescence has now been shown to depend upon a multienzyme system, further possibilities for pressure effects are conceivable.

A nore detailed study of the inhibition by ethanol (Johnson et al., 1945) gave the results shown in Fig. 15-26 for the effects of pressure. A consistent depression of the inhibition was seen at all concentrations of the ethanol but the volume changes, obtained by logarithmic plots, were $154 \mathrm{ml} /$ mole at $0.2 \mathrm{M}$ and $60 \mathrm{ml}$ mole between 1 and $1.5 \mathrm{M}$, possibly indicating that somewhat different mechanisms are responsible for the inhibition at different concentrations, or that the actions on two or more steps of the multienzyme system vary relatively. A disturbing factor. which has never been corrected for in studies of this type. is seen at $24.4^{\circ}$ and $0.5 M$ ethanol: increasing pressure accelerates the luminescent reaction equally in both the presence and absence of ethanol, which leads to an apparent decrease in the inhibition. If the stimulation were due to some step unrelated directly to the ethanol inhibition, these results would not actually imply a reduction of this inhibition. It may also be mentioned 
that the antagonism of the ethanol inhibition by high pressure is reduced or abolished at higher temperatures.

Examination of a series of carbamates showed that only the lower members of the series are made more ineffective inhibitors by high pressures,

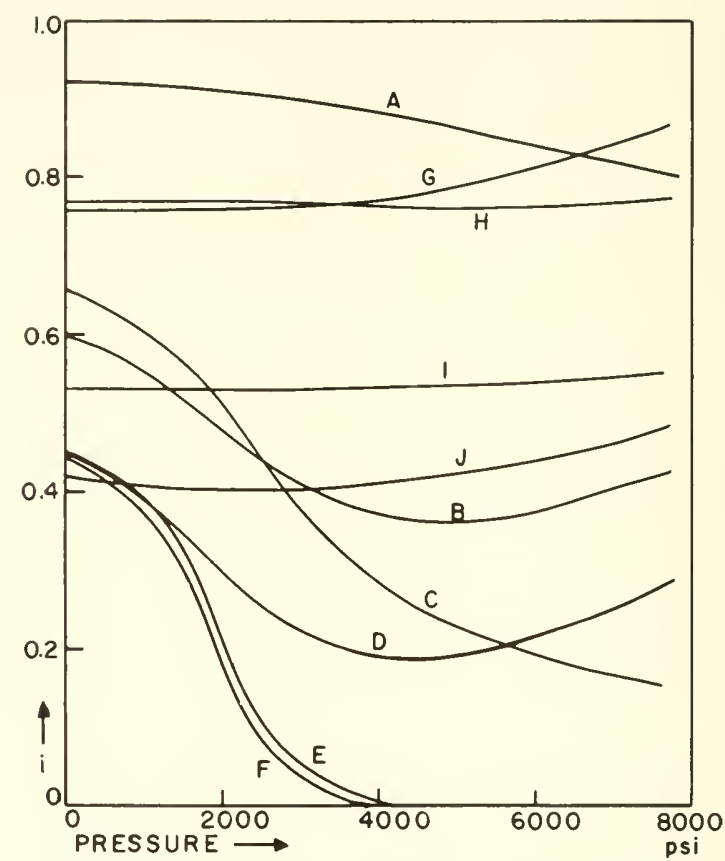

FIG. 15-25. Effects of pressure on several inhibitions of luminescence in $P$. phosphoreum. A, ethyl ether (140 $\mathrm{m} . I)$; B, procaine $(6.4 \mathrm{~m} . H)$; C, ethanol $(500$ $\mathrm{m} M)$; D, phenylearbamate $(4 \mathrm{~m} M)$; E, ethylcarbamate $(780 \mathrm{~m} M): \mathrm{F}$. chloroform $(50 \mathrm{~m} M) ; \mathrm{G}$, chloral hydrate (16 m. $I)$; H. barbital; I, sulfanilamide (3.8 $\mathrm{m} . \boldsymbol{H})$; and $\mathrm{J}, \quad p$-aminobenzoate $(10 \mathrm{~m} \cdot \mathrm{H})$. (From

Johnson et al., 1942a.)

the inhibitions by the hexyl and octyl carbamates actnally increasing with rise in the pressure (Johnson et al., 1951) (Fig. 15-27). The inhibition by methyl carbamate is characterized by a volume increase of around 60$70 \mathrm{ml} /$ mole up to pressures of $3000 \mathrm{psi}, *$ but at higher pressures the calculated volume increase becomes much greater (between 279 and $438 \mathrm{ml} /$ mole). These values are remarkably large and it was suggested were partly

* One $\operatorname{atm}=14.696$ pounds $/$ inch $^{2}$ (psi) $=1.033 \mathrm{~kg} / \mathrm{cm}^{2}$ (bars). 
due to a large experimental error associated with the measurements of small inhibitions. However, in all cases. the calculated rolume changes vary with the pressure, and in the case of ethanol, change in sign at very high pressures. This may be related to the different effects of pressure on denaturation in the different pressure ranges, as mentioned above, but could also be due to changes on a more cellular level, for example a liquefaction of the structural units of the luminescent system.

Completely satisfactory explanations for the effects of pressure on luminescence inhibition must await further experimentation on bacterial

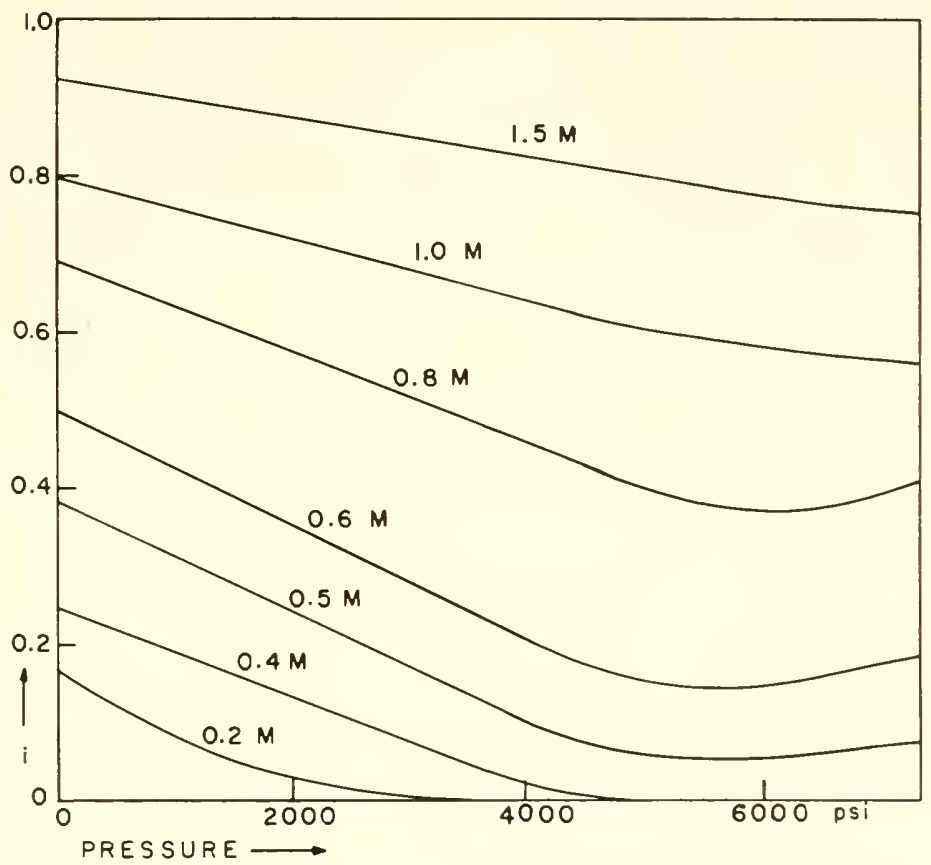

FIG. 15-26. Effects of pressure on the ethanol inhibition of luminescence in Photobacterium at $\mathrm{pH} \quad \boldsymbol{7}$. The figures on the curves show the ethanol concentrations. The curves were replotted from the curves of Johnson et al. (1945).

extracts or otherwise purified systems, with particular attention to the site or sites of inhibitor action and the operation of the system under physiological conditions as far as possible, so that approximately the same steady state as occurs intracellularly can be attained. The results obtained on cellular suspensions indicate to the author that the mechanisms are perhaps more complex than has been assumed. It would also be very interesting to find out how the effects of the more usually used inhibitors are modified 
by pressure. Studies on cyanide, azide, $p$-chloromercuribenzoate, iodoacetate, the naphthoquinones, and other more specific inhibitors on luminescence might be quite informative.

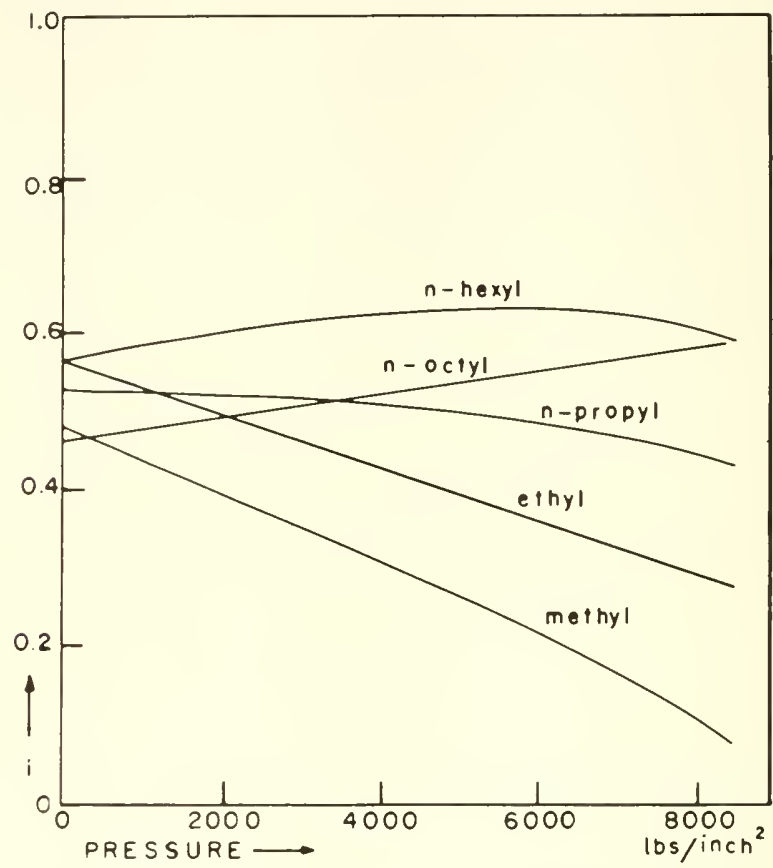

FIG. 15-27. Effects of pressure on the inhibition of luminescence in Photobacterium by a series of carbamate analogs. The concentrations of the inhibitors were such as to give approximately $50 \%$ inhibition at standard pressure, the relative positions of the curves being unimportant. (From Johnson et al., 1951.)

\section{EFFECTS OF EXTRANEOUS BINDING AND IMPURITIES}

It was briefly discussed in Chapter 3 how the binding of an inhibitor to extraneous sites could have important consequences in mutual depletion systems and it was pointed ont that the over-all specific concentration of binding sites is eritical in the determination of the boundary conditions. By extraneous binding sites is meant all the sites and substances, aside from the enzyme active center or immediately adjacent regions, capable of complexing with the inhibitor. In other words, the inhibitor can be bound to noncatalytic gromps on the enzyme, or to other proteins present in the enzyme preparation, or to any other substances present (such as nucleic 
acids, heary metal ions, or polysaccharides). With the increasingly extensive use of more potent inhibitors, this problem becomes of greater significance, and it is necessary to look into the quantitative aspects and the means to detect such binding.

For a system containing $n$ different extraneous binding sites or substances, the conservation equation for the inhibitor in a mutual depletion system may be written as:

$$
(\mathrm{I})_{t}=K_{i} \frac{i}{1-i}+i(\mathrm{E})_{t}+r_{1}\left(\mathrm{P}_{1}\right)_{t}+\ldots+r_{n}\left(\mathrm{P}_{n}\right)_{t}
$$

where $\left(\mathrm{P}_{j}\right)_{t}$ is the total concentration of the $j$ th type of binding site and $r_{j}$ is the fraction of the $j$ th site bound to the inhibitor, i.e., $r_{j}=\left(\mathrm{P}_{j} \mathrm{I}\right) /\left(\mathrm{P}_{j}\right)_{t}$. In comparison with Eq. 3-32. the first term is the concentration of the free inhibitor, the second term the concentration of inhibitor bound to the enzyme active site, and the other terms the concentrations of inhibitor bound to the various extraneous sites. In zone $\mathrm{C}$, where the concentration of the free inhibitor is negligible, the situation is fairly simple, since:

$$
i=\frac{(\mathrm{I})_{t}}{(\mathrm{E})_{t}}-\frac{r_{1}\left(\mathrm{P}_{1}\right)_{t}+\ldots+r_{n}\left(\mathrm{P}_{n}\right)_{t}}{(\mathrm{E})_{t}}
$$

The inhibition will be reduced by the amount indicated in the second term. Let us now assume that the total concentration of each of the extraneous binding sites is related to the total enzyme concentration by equations such as $\left(\mathrm{P}_{j}\right)_{t}=p_{j}(\mathrm{E})_{t}$, so that:

$$
i=\frac{(\mathrm{I})_{t}}{(\mathrm{E})_{t}}-r_{1} p_{1}-\ldots-r_{n} p_{n}
$$

The effect of the extraneous binding sites on the zone $\mathrm{C}$ inhibition will thus depend on (1) the number of different types of binding sites, (2) the concentrations of these sites relative to the enzyme, and (3) the affinities of the sites for the inhibitor as reflected in the $r$ values.

These relationships are particularly important in the deductions made from the studies of pseudoirreversible or titration-type inhibitors, since in these the enzyme concentration is assumed to be proportional to the ammount or concentration of the inhibitor, i.e., $(\mathrm{E})_{t}=(\mathrm{I})_{t} / i$ (see Chapter 3). In the presence of extraneous binding sites:

$$
(\mathrm{E})_{t}=\frac{(\mathrm{I})_{t}}{i+r_{1} p_{1}+\ldots+r_{n} p_{n}}
$$

In any specified condition, $(\mathrm{E})_{t}$ is still proportional to $(\mathrm{I})_{t}$, but when one is determining the relative enzyme concentrations in different preparations or tissues, the term $r_{1} p_{1}+\ldots+r_{n} p_{n}$ may not be constant, i.e., some tissues will have more extraneous binding sites than others and the 
types of sites may also vary. This is why it is important, as pointed out by Potter, in the titration of tissue enzymes with potent inhibitors that the specificity of the inhibitor for the enzyme be very high. This is the specificity not only with respect to other enzymes but to all binding sites or substances. Thus antimycin may block very specifically one step in electron transport and interfere with no other enzyme systems, but this does not mean that it cannot be bound significantly by other substances in the cells. If the affinity of an extraneons binding site for the inhibitor is great, it may not require a very high concentration of this site to reduce the inhibition appreciably.

This treatment applies to zone $\mathrm{C}$ behavior. In zone $\mathrm{A}$ one would not expect any effect of extraneous binding sites on the inhibition while in zone $\mathrm{B}$ the situation wonld be intermediate between zones $\mathrm{A}$ and $\mathrm{C}$. This is one way of looking at it. Another is to assume that the boundary conditions are defined not only by the binding to the enzyme but to all the sites, which is somewhat more reasonable because experimentally the behavior of the inhibition will correspond to a zone determined by the total binding. In other words, the presence of sufficient extraneous binding sites to reduce the free inhibitor concentration will transfer a system from zone A into zone $\mathrm{B}$, or from zone $\mathrm{B}$ into zone $\mathrm{C}$.

Detection of extraneous binding is usmally not easy. If the inhibition is truly in zone $\mathrm{C}$, a plot of the inhibition against the total concentration of the inhibitor will no longer give a straight line passing through the origin (Eq. 15-121). However, in an impure enzyme preparation, if it is found that the inhibition at constant inhibitor concentration varies with the amount of the enzyme present, it is not immediately evident whether the depletion of free inhibitor is dne to the enzyme itself or to extraneous binding snbstances. One can determine the inhibition in preparations of varying purity; if the inhibition becomes greater as the enzyme is purified, it is possible that other substances are binding the enzyme, but on the other hand the enzyme may be made more sensitive by the purification or the extraneons binding sites may be located on the enzyme. In the latter case it is very difficult to determine the extent of such extraneous binding but it is usually not so important to do so as in situations where other substances are interfering with the inhibition. In certain instances, one can directly determine the degree of inhibitor binding in a preparation by equilibrim dialysis or by other techniques and. if the approximate concentration of the enzyme is known, some estimate may be macke of the extent of extraneous binding. The nature of the imhibitor is often the best clue because some inhibitors are obvionsly rather strongly bound to a variety of cell components; for example, the heavy metal ions or the mercurials are bound to many proteins and mucleic acids, so that evidence of free inhibitor depletion would usually be interpreted in terms of extraneous binding. 
Although quite a different phenomenon, it may be mentioned in connection with the presence of impurities, that insufficient attention has often been paid to the possibility of interfering substances being introduced with the inhibitor. Many commercial preparations of inhibitors are not pure and the effects upon enzyme or metabolic systems may be partially or totally dependent on these impurities. An excellent example of this has been provided recently in a study of yeast alcohol dehydrogenase (Hoch et al., 1960). The inhibition of this enzyme by $N^{\prime \prime}$-methylnicotinamide was shown to be due to a contamination of this substance with $\mathrm{Ag}^{+}$ions. The inhibition by semicarbazide could also be attributed to its metal ion content. Complete purification of each substance to remove these metal ions led to an elimination of the inhibition. It was pointed out that in using inhibitors that are not so potent, a dependency of the inhibition on the enzyme concentration should lead one to suspect adventitions inhibition by a potently-acting impurity.

\section{EFFECTS OF ENZYME OR METABOLIC RATE}

The question of whether the degree of inhibition in a particular system will depend on the rate at which the system is operating often arises, and is especially important in considerations of the relative effects of inhibitors on tissues with different levels of activity. There are many ways in which the metabolic rate could be altered: changes in the concentrations of substrate, coenzymes, cofactors, or acceptors such as oxygen; change in the total enzyme concentration; changes in temperature, $\mathrm{pH}$, or ionic strength; elimination of a limiting step, as in the uncoupling of oxidative phosphorylation; the activation of an enzyme, as in the reduction of $-\mathrm{S}-\mathrm{S}-$ groups to - $\mathrm{SH}$ groups; the removal of some product that is slowing the forward reaction; change in the spatial configuration in structurally dependent multienzyme systems; and, in the case of many tissues, a modification of the level of function, as in the stimulation of nerve or muscle. It is, therefore, difficult to formulate any general laws relating inhibition to metabolic rate because in most cases the effect, if any, will depend on the way in which the rate is altered. In every case in which the rate is changed, the direct effect of the rate-altering procedure on the inhibition must be eliminated if the effect of rate alone is to be determined.

\section{Single Enzymes}

An enzyme for which the Michaelis constant is given by the generalized expression, $K_{m}=\left(k_{2}+k_{-1}\right) / k_{1}$, may be inhibited to different degrees by a constant concentration of an inhibitor as the rate is altered. This can occur when some procedure changes $k_{2}$ specifically. If, for example, $k_{2}$ is 
increased, the enzyme rate will rise and $K_{m}$ will increase. Since the inhibition produced by a competitive inhibitor depends on $K_{m}$ :

$$
i=\frac{(\mathrm{I})}{(\mathrm{I})+K_{i}\left[1+(\mathrm{S}) / K_{m}\right]}
$$

as $K_{m}$ increases, the inhibition will become greater. This has been very nicely demonstrated for the inhibition of succinic dehydrogenase by malonate (Thorn, 1953). The rate constant, $k_{2}$, can be altered here by changing the concentration of the hydrogen acceptor, e.g. methylene blue. The $K_{m}$ was found to vary over a twenty fold range with changes in the methylene blue concentration and, as a consequence, the inhibition by malonate was found to increase almost linearly with the rate of succinate oxidation. Noncompetitive inhibition, of course, would not be affected by such rate changes.

It was claimed by Florijn and his colleagues (Florijn et al., 1950) that noncompetitive inhibition in mutual depletion systems is dependent on the rate as influenced by the substrate concentration. However, reference to Eqs. 3-34 to 3-36 shows that the substrate concentration does not enter into the expressions for the inhibition. Actually, a noncompetitive inhibitor inactivates a fraction of the enzyme active sites, although it does not affect the binding of the substrate, and it makes no difference what the substrate concentration is or in what zone with respect to substrate the system happens to be.

It would be well to point out the errors in the treatment of Florijn and his co-workers. In the first place, although noncompetitive inhibition is assumed, only the equilibrium between the substrate and the enzyme is considered, whereas a noncompetitive inhibitor doesn't alter this but only decreases $V_{\eta \|}$ or prevents the substrate from reacting once it has complexed with the enzyme. In the expression for $K_{s}$, the concentration of free enzyme is not $(\mathrm{E})_{t}-(\mathrm{ES})-(\mathrm{EI})$, but only $(\mathrm{E})_{t}-(\mathrm{ES})$, since a noncompetitive inhibitor doesn't reduce the sites for the substrate binding. In the second place, they calculate the inhibition as equivalent to $\left(v-v_{i}\right) / v$ in an equation involving $i$ and in their table showing the variation of inhibition with the substrate concentration, they initially assume that $i=0.2$ and then proceed to calculate an inhibition that varies. The experimental results they obtained. which prompted them to develop the treatment, are, however, very interesting, but will be discussed later since they involve complex metabolic systems.

Obvious relationships between the rate and the inhibition occur in some cases. For example, in mutual depletion systems with respect to the inhibitor, varying the rate by altering the total enzyme concentration will affect the inhibition, because more or less of the inhibitor will be bound to the enzyme. As the enzyme concentration is increased, the rate will go up 
but the inhibition will be diminished. In general, however, enzymes in zone A will be inhibited to the same degrees, whatever the rates as influenced by a variety of factors.

\section{Multienzyme Systems}

The situation here becomes very complex because of the great number of possibilities as to the ways in which the rates may be changed and as to the mechanisms by which the inhibitors can act. Therefore discussion will be limited to specific cases. In simple irreversible monolinear chains, such as $\mathrm{A} \rightarrow \mathrm{B} \rightarrow \mathrm{C} \rightarrow \mathrm{D}$, the over-all rate is determined by the first reaction and will increase with the concentration of $A$ up to saturation of $E_{1}$. Inhibition of an enzyme later in the sequence will depress the formation of product only if the $V_{m}$ of the inhibited step is reduced below the rate of the first step, or it the intermediate that is the substrate for the inhibited enzyme cannot rise in concentration sufficiently to maintain a steady state (see Chapter 7 ). The greater the concentration of $A$ and the more rapid the uninhibited rate, the easier will it be for an inhibitor to reduce the $V_{m}$ of the inhibited enzyme below $r_{1}$. This is seen in Eq. 7-6 where the over-all inhibition in the formation of the product depends on (A).

The opportunities for the inhibition to be affected by the rate are increased in branched chains. A convergent system. such as shown in Eq. 7-22, will be inhibited by an action on $\mathrm{E}_{1}$ or $\mathrm{E}_{3}$ to a degree depending on the fractions of the flow that pass through reactions 1 and 3. For example, if an inhibitor acts on $\mathrm{E}_{3}$, an increase in $(\mathrm{A})$ will increase the fraction of the rate that is resistant to the inhibitor and hence will decrease the inhibition while increasing the rate of formation of $\mathrm{C}$. Such relationships are readily treated and they are mentioned only to emphasize that similar phenomena may occur in cellular metabolism.

One may also generalize that any change in the rates in complicated multienzyme systems that leads to a shift in limiting reactions is very apt to alter the degree of response to an inhibitor, since the sensitivity of the over-all rate to an inhibitor will depend on whether it acts on an enzyme that is limiting. Thus the uncoupling of oxidative phosphorylation may modify the inhibition on electron transport or respiratory systems.

\section{Cellular Metabolism and Function}

The factor of inhibitor-sensitive and inhibitor-resistant fractions in most metabolic systems is very important in connection with the effect of over-all rate on inhibition because the response will depend on whether the fractions remain relatively the same when the total rate changes. If the inhibitor-stable metabolism is always a constant fraction of the total metabolism, whatever the over-all rate, the inhibition will be independent 
of the rate, but otherwise it will vary. If we designate the sensitive fraction by $f_{s}$ and the resistant fraction by $f_{r}$, and if an inhibitor produces an inhibition of degree $i$ on the sensitive fraction, the following may be written:

Uninhibited: $\quad f_{s}+f_{r}=1$

Inhibited: $\quad(\mathbf{1}-i) f_{s}+f_{r}=$ relative rate

$$
i_{t_{1}}=i \frac{f_{s}}{f_{s}+f_{r}}=i f_{s}
$$

If now the total rate is altered and the individual fractions are changed by the factors $n$ and $m$ :

Uninhibited:

$$
n f_{s}+m f_{r}=r
$$

Inhibited: $\quad(1-i) n f_{s}+m f_{r}=$ relative rate

$$
i_{t_{2}}=i \frac{n f_{s}}{n f_{s}+m f_{r}}=i \frac{n f_{s}}{r}
$$

where $r$ is the new rate relative to the initial rate. The ratio of the over-all inhibitions for the two situations is then:

$$
\frac{i_{t_{2}}}{i_{t_{1}}}=\frac{n}{r}
$$

which is the ratio of the change in the inhibitor-sensitive fraction to the change of the total rate. The observed inhibition can thus either increase or decrease. It is probably more common for the inhibitor-resistant fraction to remain at a constant level - i.e., for an increase in over-all rate, $n$ will be greater than $m$, and since $n$ is also greater than $r$, the inhibition will increase. The effect in any case will depend on how the rate is changed, since conceivably any relative change in the individual fractions is possible.

When a tissue becomes more functionally active, the metabolism usually increases, particularly the oxidative and phosphorylative processes, although synthetic reactions may also accelerate. The character of the metabolism is generally modified so that the inhibitor-sensitive and inhibitorresistant portions do not change equally. Consequently, an active tissue is often inhibited differently than a relatively resting tissue, as has been discussed in Chapter 9. The inhibition of such systems may be said to depend on the over-all metabolic rates, but actually it is dependent on the pattern of the metabolism and not directly on the rate.

It has been frequently stated that an active tissue is more readily inhibited or damaged than a tissue that is less active. However, it is not entirely related to the level of activity but to the metabolic requirements also. If a tissue needs a certain rate of metabolism to maintain a specified 
level of function, an inhibitor will depress the function when it has lowered the metabolism to or beyond this required rate. Therefore, if two tissues require the same metabolic activity, but one has a higher metabolic rate than the other, it will be more difficult to depress the function in the former. In a way, it is the margin of safety between metabolic supply and demand that is critical. If a tissue is stimulated to greater activity and its metabolism does not increase proportionally, it will be more easily inhibited. The ability of some tissues to adapt to inhibition in various ways can modify the results very significantly.

Florijn and Smits (1949) explaimed the relatively selective action of urethane and arsenite on tumor tissue by making two assumptions: (1) tumor tissue has a high respiratory rate due to the high energy requirement, whereas normal tissues operate much below the maximal metabolic capacity. and (2) a certain concentration of an inhibitor always blocks the same fraction of the inhibitor-sensitive system. Tumor respiration will thus be depressed more readily than normal tissue respiration; in the latter the residual portion of the inhibitor-sensitive systems can be sufficiently increased to maintain the original respiratory level. In other words, normal tissues can compensate to inhibition by their reserve capacity whereas tumor tissue cannot.

In order to test this hypothesis, studies were done on the inhibition of tissue respiration and yeast fermentation by urethane and arsenite (Florijn et al., 1950). The respiration of rat liver and kidney minces was varied by changing the oxygen tension and adding 2,4-dinitrophenol. The fermentation rate of yeast increases with time in the presence of ammonium sulfate and the inhibition can be tested during varying intervals. The inhibitions observed at these different metabolic rates are shown in Fig. 15-28 and it is seen that the effects of the inhibitors generally increase with increasing rates. It was felt that these results give support to the proposed mechanism for the selective action of the two inhibitors on tumor tissue. It may be noted that in essentially every case, even though the inhibition is greater at higher metabolic rates. that the remaining respiration or fermentation is greater than at low metabolic rates. These interesting results are very difficult to interpret with assurance because it is not known in what way an increase in oxygen tension will alter the patterns of oxidative or fermentative metabolism. If it is assumed that the arsenite acts principally on the keto acid dehydrogenases, the question as to what increased oxygen tension does to the rates of the processes forming and utilizing pyruvate and $\alpha$-ketoglutarate is posed. Since pyruvate oxidation in mitochondrial suspensions is generally accelerated by augmenting the oxygen tension, it might be supposed that in pure oxygen the tricarboxylic acid cycle and the oxidation of pyruvate make up a greater fraction of the total respiration than in air, and thus that an inhibitor, such as arsenite, that blocked pyruvate oxidation would be more effective. However, this explanation 
doesn't hold for yeast fermentation. Indeed, we do not as yet know sufficiently accurately the sites of action of these two inlibitors to enable us to visualize a mechanism. It may be confidently predicted that when different

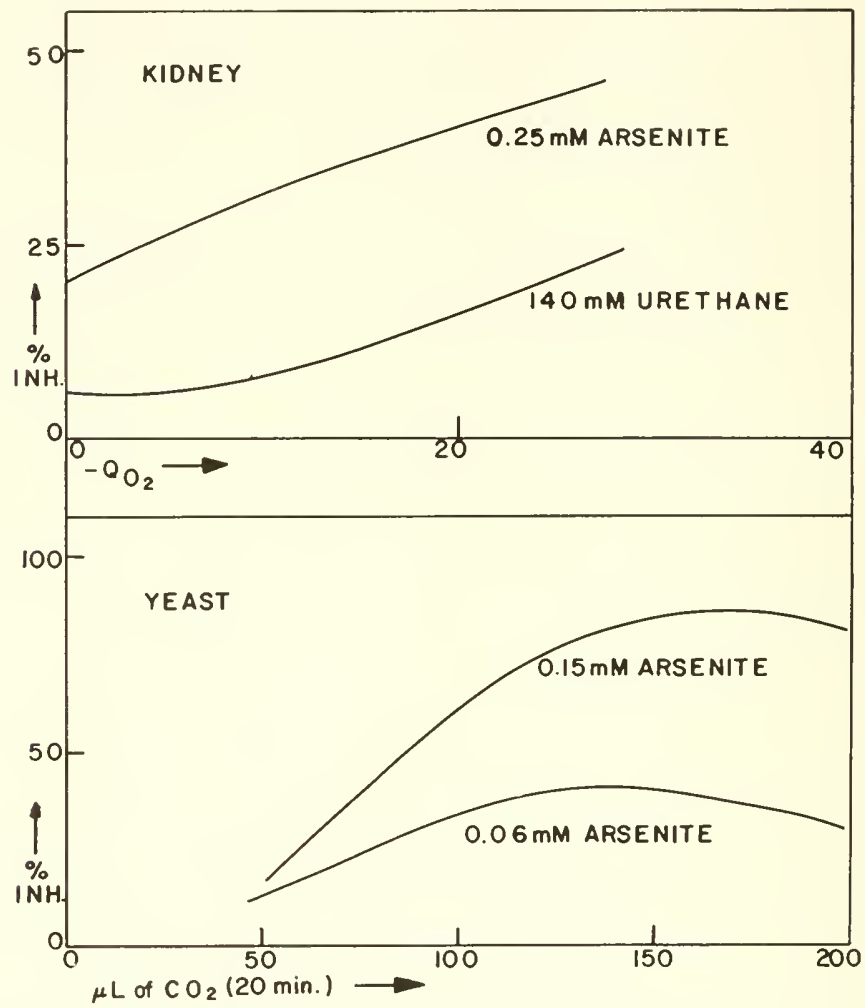

FIG. 15-28. Inhibitions of kidney mince respiration and yeast fermentation by urethane and arsenite at different respiratory rates. (From Florijn et al., 1950.)

tissues, different metabolic processes, different inhibitors, and different ways of altering the metabolism are investigated, many types of effects will be observed, and that one should certainly not conchude that inhibition will generally increase with a rising metabolic rate. 


\section{CHAPTER 16}

\section{SPECIFICITY OF INHIBITION}

Specificity refers in general to the ability of an inhibitor to affect one process more than others. This concept may be applied to any level of organization. For example, the specificity may refer to species, tissues, different functions, metabolic processes, or enzymes. Hany instances of species specificity are known and they are treated in a stimulating manner by Albert in his "Selective Toxicity" (1960). A few examples will serve to illustrate this type of specificity: the suppression or killing of pathogenic organisms with minimal effects on the host (as the selective actions of copper and the dithiocarbamates on fungi parasitic to plants), the killing of certain weeds by dinitro-o-cresol while leaving crop plants unaffected, the ability of urethane and certain naphthoquinones to inhibit tumor cell growth more readily than the growth of normal cells, and the selective effects of mercurials and arsenicals on bacterial and protozoal invaders. Likewise. there are many examples of tissue specificity. The effect of an inhibitor when it is administered to an animal is always selective to some extent, inasmuch as certain organs or functions are altered before others. Noteworthy examples would be the remarkable specificity of alloxan on the pancreatic islet cells, the renal effects of the carbonic anhydrase inhibitors and the mercurials, and the central nervous system changes following fluoroacetate. The cholinesterase and monoamine oxidase inhibitors also show selective effects on those tissues or junctions where these enzymes are functionally important in the determination of the neurohormone concentrations. Such specificity on the organism or cellular level has been discussed in previous chapters in several connections, so that in the present chapter emphasis will be placed on enzyme specificity. Much of species and tissue specificity depends on the differential distribution of the inhibitors and the presence or absence of the enzymes or metabolic systems attacked. It is now necessary to inquire into the selective actions of inhibitors in situations in which several possibly susceptible enzymes are present and the inhibitors are at uniform concentrations.

As Albert has pointed out, specificity of metabolic disturbance is well documented and yet much of the trend during the past decades has been to demonstrate a fundamental similarity in the enzymes and metabolic 
systems of all cells. It is true that most of the basic pathways are the same in different types of cells but the closer one looks into the kinetics and the details of the individual steps, the greater is the divergence. The basis of selectivity is often at the molecular level and not at the metabolic or cellular level. The chemical composition of enzyme active centers, the topography of enzyme surfaces, the patterns of electrical forces, and polarizabilities - these form the true basis for enzyme specificity and are also responsible for much specificity on higher levels.

Inhibitors rarely are specific for a single enzyme. Most often an inhibitor will act upon several enzymes, although not, of course, with the same potency. If an inhibitor is to be used as a tool to investigate the relations between certain enzymes or pathways and the function of a cell or tissue, the question of specificity is of the utmost importance. The more specific the inhibitor, the more valuable will it be to the investigator. Although inhibitors are seldom specific, they may often be used in a manner so that a specific action is exerted or, at least, the maximal speeificity obtained. Theoretically, if one knew the action, or lack of action, of an inlibitor on every enzyme involved in the system studied, including each particular $K_{i}$, one could predict the specificity at any concentration of the inlibitor. This would be true in relatively simple systems, but in studies on the cell or whole animals, the many factors discussed previonsly interfere with the knowledge of exactly what the inhibitor is doing. That is, if one knew from isolated enzyme work the $K_{i}$ of the inlribitor for eacl susceptible enzyme, still in cellular work there would be elements of uncertainty due to concentration factors, unknown intracellular substrate concentrations, altered enzyme environments, and other difficulties. Nevertheless, one should always attempt to reach the ideal of high specificity. One of the things that stands between us and such a goal is the ignorance of the complete spectrum of action of any inhibitor, and, indeed, for most inlibitors we know regrettably little of their ranges of action.

Many investigators appear to look upon certain inhibitors as quite specific for designated enzymes or metabolic pathways; for example, they believe iodoacetate inhibits glycolysis only, or fluoride only enolase, or arsenicals only keto acid oxidases, and so on. This arises in part from an understandable wish for simplicity. in part from unjustifiable statements made repeatedly so that eventually they come to be looked on as true, and in part from inusfficient knowledge of the inhibitor's range of action. If only a few enzymes lave been studied with a particular inhibitor, it is evident that the specificity in this limited sphere is apt to be high. This important fact is well stated in Davenport's law: "The specificity of an inhibitor is inversely proportional to how much is known about it," (verbal communication). Here is meant the apparent specificity because there is an absolute specificity that is untouched by experimental or mental de- 
ficiencies. Another way of stating the problem is that the apparent specificity is always greater than the absolute specificity. However, the more we know about the actions of an inhibitor, the more able are we to use it properly and to achieve true specificity if this is possible. The subject of specificity has been a very neglected aspect of inhibition and the attempt will be made in this chapter to put specificity on a simple quantitative basis that may be a steppingstone for the more detailed analyses that will undoubtedly be made in the future.

\section{Definition of Enzyme Specificity of Inhibition}

It is necessary to express the specificity in some definite way if the phenomenon is to be amenable to quantitative treatment. This is difficult to do in a completely satisfactory manner. The most important element in specificity is the difference in the inhibitions produced by a concentration of a inhibitor on two enzymes, and this we can express simply as:

$$
\sigma=i_{1}-i_{2}
$$

where $\sigma$ is the specificity, $i_{1}$ is the inhibition on the enzyme of interest, and $i_{2}$ is the inhibition on a second enzyme. For absolute specificity, $\sigma=1$, and complete inhibition of one enzyme may be achieved without any inhibition of the other; partial specificity occurs when $\sigma<1$ and there will be some overlap in the inhibitions of the two enzymes. The specificity as thus defined will generally depend on the concentration of the inhibitor.

This definition of specificity is not entirely applicable to all situations because it omits the other factor that is important in the practical use of an inhibitor, namely the requirements with regard to the absolute levels of the inhibitions. As an example we may take a case in which the specificity is designated as 0.7 . Now this degree of specificity could occur with $i_{1}=1.0$ and $i_{2}=0.3$, or $i_{1}=0.9$ and $i_{2}=0.2$, or $i_{1}=0.8$ and $i_{2}=$ 0.1 , or $i_{1}=0.7$ and $i_{2}=0$. Which set of values would be appropriate for a particular situation? In some cases, it is more important to inhibit the enzyme being studied completely, even though the other enzyme is inhibited to some extent, and in other cases, it is more important that the second enzyme is not inhibited at all. Thus a determined specificity does not immediately tell one the correct inhibitor concentration to use, but does provide limits.

Let us first consider two enzymes inhibited noncompetitively, for which the individual inhibitions may be written as:

$$
i_{1}=\frac{(\mathrm{I})}{(\mathrm{I})+K_{i_{1}}} \quad i_{2}=\frac{(\mathrm{I})}{(\mathrm{I})+K_{i_{2}}}
$$


For the enzymes to be inhibited to specified degrees by a certain concentration of the inhibitor, the ratio of the inhibitor constants is given by:

$$
\frac{K_{i_{2}}}{K_{i_{1}}}=\frac{i_{1}\left(1-i_{2}\right)}{i_{2}\left(1-i_{1}\right)}
$$

A few selected results are shown in Table 16-1, from which it may be seen that the requirements for differential sensitivity of the two enzymes depend not only on the specificity, $\sigma$, but on the absolnte values of the inhibitions. It may also be noted that a very large difference between inhibitor constants is required if a high specificity is to be achieved; for example, to inhibit $\mathrm{E}_{1}$ almost completely without in hibiting $\mathrm{E}_{2}$ appreciably, $K_{i_{2}}$ must be about ten-thousand times as large as $K_{i_{1}}$, or the $\mathrm{p} K_{i}$ 's must differ by approximately four units. Finally, it is seen that if $\mathrm{E}_{1}$ is to be inhibited only $50 \%$ without significantly affecting $E_{2}, K_{i_{2}}$ must be about a hundred times as large as $K_{i_{1}}$, so that even to get a low inhibition specifically requires an appreciable difference in the affinities of the inhibitor for the two enzymes (actually around $2.83 \mathrm{kcal} / \mathrm{mole}$ ). Conversely, it is evident that if the $K_{i}$ 's do not differ by more than a factor of 10 , it is impossible to obtain any practically useful degree of specificity. These considerations serve to set some kind of limits to the specificity one may achieve in the simplest type of enzyme inhibition.

\section{The Maximal Specificity for an Inhibitor and Two Enzymes}

The specificity will vary with the concentration of the inhibitor so that it is frequently useful to know the concentration which will give the maximal degree of specificity. From Eqs. 16-1 and 16-2, the specificity for noncompetitive inhibition is:

$$
\sigma=\frac{(\mathrm{I})}{(\mathrm{I})+K_{i_{1}}}-\frac{(\mathrm{I})}{(\mathrm{I})+K_{i_{2}}}
$$

Differentiating and setting equal to zero:

$$
\frac{d \sigma}{d(\mathrm{I})}=\frac{K_{i_{1}}}{\left[(\mathrm{I})+K_{i_{1}}\right]^{2}}-\frac{K_{i_{2}}}{\left[(\mathrm{I})+K_{i_{2}}\right]^{2}}=0
$$

so that the inhibitor concentration for maximal specificity is given by:

$$
(\mathrm{I})_{\sigma=\max }=\sqrt{K_{i_{1}} K_{i_{2}}}
$$

Substituting this value of (I) back in Eq. I6-4 gives the maximal specificity that may be obtained:

$$
\sigma_{\max }=\frac{\sqrt{K_{i_{2}}}-\sqrt{K_{i_{1}}}}{\sqrt{K_{i_{1}}}+\sqrt{K_{i_{2}}}}
$$


TABLE $16-1$

Ratios of the Ixhibitor Coxstants Required for Certain Degrees of Specifictity axd Trdividual Ixhibitioss

\begin{tabular}{|c|c|c|c|}
\hline$i_{1}$ & $i_{2}$ & $\sigma$ & $K_{i_{2}} / K_{i_{1}}$ \\
\hline 0.99 & 0.01 & 0.98 & 9801 \\
\hline 0.99 & 0.0 .5 & 0.94 & 1881 \\
\hline 0.99 & 0.10 & 0.89 & 891 \\
\hline 0.99 & 0.20 & 0.79 & 396 \\
\hline 0.99 & 0.30 & 0.69 & 231 \\
\hline 0.95 & 0.01 & 0.94 & 1881 \\
\hline 0.95 & 0.05 & 0.90 & $36 \mathrm{I}$ \\
\hline 0.95 & 0.10 & $0.8 \tilde{}$ & 171 \\
\hline 0.95 & 0.15 & 0.80 & 108 \\
\hline 0.95 & 0.20 & 0.75 & 76 \\
\hline 0.95 & 0.25 & 0.70 & 57 \\
\hline 0.9 .5 & 0.30 & 0.65 & 44 \\
\hline 0.95 & 0.50 & 0.45 & 19 \\
\hline 0.90 & 0.0 .5 & 0.8 .5 & 171 \\
\hline 0.90 & 0.10 & 0.80 & 81 \\
\hline 0.90 & 0.20 & 0.70 & 36 \\
\hline 0.90 & 0.50 & 0.40 & 9 \\
\hline 0.80 & 0.05 & 0.75 & 76 \\
\hline 0.80 & 0.10 & 0.70 & 36 \\
\hline 0.80 & 0.20 & 0.60 & 16 \\
\hline 0.70 & 0.05 & 0.65 & 44 \\
\hline 0.70 & 0.10 & 0.60 & 21 \\
\hline 0.70 & 0.20 & 0.50 & 9 \\
\hline 0.50 & 0.01 & 0.49 & 99 \\
\hline 0.50 & 0.05 & 0.45 & 19 \\
\hline 0.50 & 0.10 & 0.40 & 9 \\
\hline 0.50 & 0.20 & 0.30 & 4 \\
\hline
\end{tabular}

Finally. if we desire to know the ratio of the inhibitor constants that is necessary to obtain a designated maximal specificity, by rearranging Eq. $16-7$ :

$$
\frac{K_{i_{2}}}{K_{i_{1}}}=\left[\frac{1+\sigma_{\max }}{1-\sigma_{\max }}\right]^{2}
$$


The relationship between this ratio and the maximal specificity is illustrated in Fig. 16-1. The maximal specificity is a characteristic of the system - that is, of the inhibitor and the enzymes involved - and is not dependent on the inhibitor concentration.

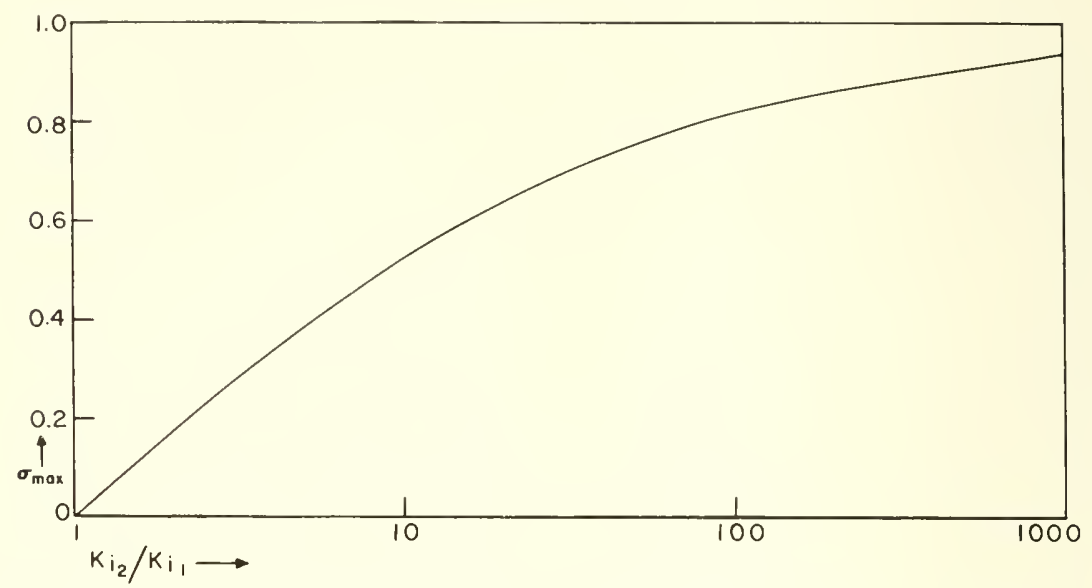

FIG. 16-1. Variation of the maximal specificity possible with the ratio $K_{i_{2}} / K_{i_{1}}$ (Eq. 16-7).

The individual inhibition curves for a situation in which $K_{i_{2}} / K_{i_{1}}=100$ are shown in Fig. 16-2 and the variation of the specificity with the inhi-

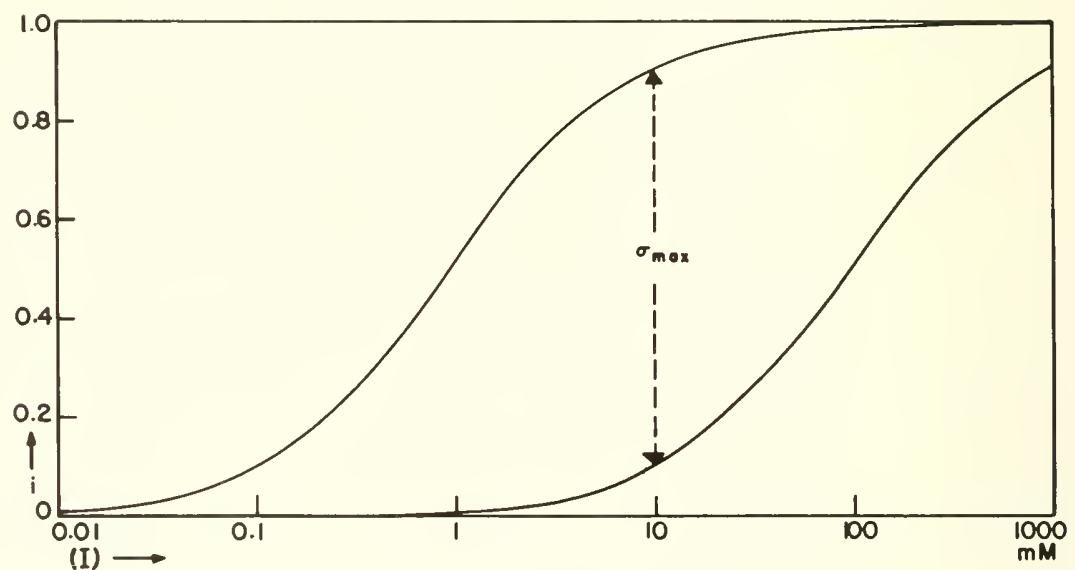

FIG. 16-2. Noncompetitive inhibition curves for two enzymes showing the variation of specificity with the inhibitor concentration. $K_{i_{1}}=1 \mathrm{mM}$ and $K_{i_{2}}=100$ $\mathrm{m} M$. The maximal specificity is 0.818 when $(\mathrm{I})=10 \mathrm{~m} M$. 
bitor concentration in Fig. 16-3. As pointed out above, it is not always advisable to use the concentration of the inhibitor giving maximal specificity. In the situation chosen for illustration. a specificity of 0.7 or greater may be obtained over a range of inhibitor concentration from $2.7 \mathrm{~m} M$ to $37 \mathrm{~m} M$ (see Fig. 16-3). At the former concentration $i_{1}=0.73$ and $i_{2}=$ 0.03 while at the latter concentration $i_{1}=0.97$ and $i_{2}=0.27$. Either concentration or the concentration giving maximal specificity may be experimentally optimal, depending on the systems being tested and the purpose of the investigation. As a general rule, it is perhaps best to work at the lower end of the inhibitor concentration range in order that the inhibition

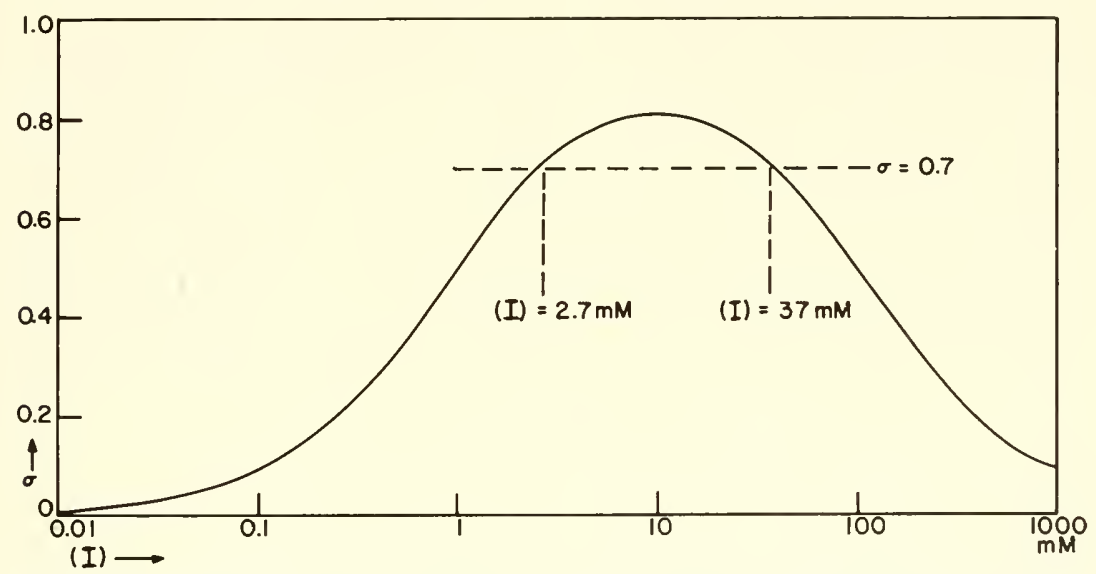

Fia. 16-3. Variation of the specificity with the inhibitor concentration for the case shown in Fig. 16-2.

on the second enzyme be minimal. It is not always necessary for the enzyme that is being studied to be inhibited strongly and correlations between the enzyme and some cell metabolism or function may often be made with inhibitions less than $50 \%$.

\section{Inhibitions Involving Three or More Enzymes}

Many systems commonly studied may contain several enzymes whose susceptibilities to the inhibitor must be consictered. The use of inhibitors on the tricarboxylic acid cycle in mitochondrial suspensions, for example, impels one to take into account at least a dozen possible inhibition sites, unless the conditions are such that only a fraction of the cycle is being studied. In structurally organized multienzyme systems, there are usually more possibilities for inhibition than the number of enzymes involved, because the inhibitor may alter spatial relationships between enzymes by a 
modification of the structure, as well as react with coenzymes or cofactors. With respect to the enzymes themselves, the specificity in systems containing three or more enzymes may be treated by an extension of the formulation given in the preceding section. The over-all specificity may be designated by a series of individual specifirities: $\sigma_{12}=i_{1}-i_{2}, \sigma_{13}=i_{1}-i_{3}$, etc. A situation such as shown in Fig. 16-4 might occur, in which five different enzymes are inhibited to varying degrees by an inhibitor. The problem arises as to the optimal inhibitor concentration to use. This cannot be decided by simply inspecting a diagram of this type, because consideration must also be given to the importance of the various enzymes in what is

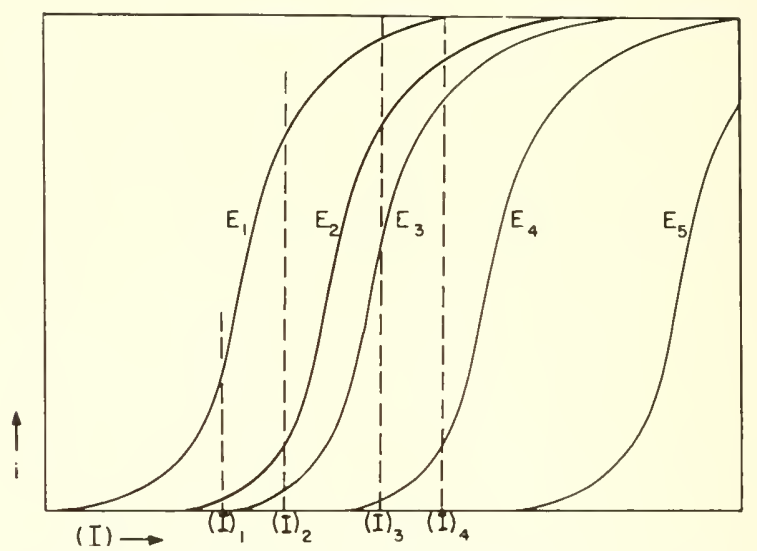

FIG. 16-4. Inhibition-concentration curves for a system of hypothetical enzymes, $\mathrm{E}_{1}$ to $\mathrm{E}_{5}$. See the text for explanation.

being measurect. The concentration (I), will give the maximal specificity for inhibitions of $\mathrm{E}_{1}$ and $\mathrm{E}_{2}$ and if $\mathrm{E}_{2}$ is an important enzyme in the study, inhibitor concentrations above this cannot be readily used; however, if $\mathrm{E}_{2}$ can be neglected due to its obvious nonparticipation in the systemi being investigated, the specificity at this concentration will increase. If both $\mathrm{E}_{2}$ and $\mathrm{E}_{3}$ may be neglected, inhibitor concentrations up to $(\mathrm{I})_{3}$ may be used, which gives the maximal specificity for inhibition on $E_{1}$ and $E_{4}$. If the role of the enzymes in the process beng measured in unknown, a concentration around (I) might well be used for the most certain interpretation of the data. Lastly. if the concentration (I) had been used arbitrarily, or simply because it completely inhibited $\mathrm{E}_{1}$, one can see that $\mathrm{E}_{2}$ and $\mathrm{E}_{3}$ would also be strongly inhibited. while $\mathrm{E}_{4}$ would be partially inhibited, so that no clear interpretation would be possible. Actually, it would appear that such high concentrations are frequently used in complex systems and 
a good many of the conclusions reached from work of this nature will have to be re-evaluated in the light of more extensive knowledge of enzyme susceptibility. In the future volumes covering the individual inhibitors, every effort will be made to present complete surveys of all the enzymes tested so that the specificities through the concentration ranges may be estimated.

\section{Specificity in Competitive Inhibitions}

If one or more of the enzymes is inhibited competitively, the treatment must be modified by the introduction of the $\left\{1+\left[(\mathrm{S}) / K_{s}\right]\right\}$ term in the denominator of the expression for the inhibition. Since the magnitude of this term will usually vary from enzyme to enzyme, the degree of specificity now depends on $K_{s}$ and (S) as well as on $K_{i}$ and (I). In those multienzyme systems in which the concentrations of the intermediates may vary following inhibition, the final specificity will depend on the degrees to which the concentrations of competing substrates can rise. In other words, from the relative values of the $K_{i}$ 's it might be predicted that adequate specificity would be achieved, but if the concentration of the substrate acted upon by the enzyme being studied can rise sufficiently to reduce the inhibition, the actual specificity may be quite low.

\section{Specificity in Intracellular Inhibitions}

The possibility of achieving a specific inhibition in cells or tissues is much less than in cell extracts or enzyme preparations, due not only to the greater number of opportunities for inhibition but also to our lack of knowledge as to intracellular conditions and the behavior of inhibitors within cells. We have seen that the inhibitor concentration is an important factor in the determination of the specificity. The intracellular concentration of an inhibitor is usually not known so that it is difficult to evaluate the specificity even when the in vitro actions of the inhibitor on many enzymes are known. Since the inhibitor concentration in the cells may be either higher or lower than outside, the intracellular specificity may be greater or less than determined on isolated enzymes.

The specificity can be altered by any factors that affect the individual inhibitions, so that $\mathrm{pH}$, temperature, ionic strength, and similar variables can introduce further complications. Unless the in vitio inhibitions are determined at intracellular $\mathrm{pH}$, the specificities in the cell may be significantly different than predicted. Indeed, it has been emphasized that the susceptibilities of enzymes to inhibitors may be quite different depending on whether the enzyme has been isolated and is in an artificial medium or is in its natural environment within the cell. One further factor is in some cases very important: the specificity may vary with the time after introduction of the inhibitor. In the first place, some enzymes are inhibited 
more rapidly than others, particularly when the inhibitor chemically reacts with the enzymes. Thus, during the initial stages an inhibitor such as p-chloromercuribenzoate may possible act specifically on a certain enzyme, whereas later a progressively increasing number of enzymes may be inactivated. In the second place, an initially selective inhibition may give rise to many types of secondary changes that may be confused with the primary inhibition. Specificity for practical purposes may be considered as referring to any secondary or subsidiary effects of an inhibitor, and not merely to the differential actions on enzymes.

Bearing in mind these difficulties inherent in studies on cellular preparations, it is now necessary to say a word about the basic problem that confronts one in attempting to achieve specificity - what range of inhibitor concentrations is optimal? The following suggestions may be offered in the approach to a solution of this problem.

(A) The first thing that must be done is to determine as accurately as possible the sensitivities of all pertinent enzymes. Often much can be obtained from the literature but in most cases the enzymes tested are from a variety of tissues and species so that the data do not necessarily apply to the problem at hand. Therefore, the enzyme or the metabolic system to be attacked should be studied in as pure a form as possible, and a concentration-action curve constructed. All other enzymes or related reactions should be tested in a similar mamner as far as is practicable. If the reactions and inhibitions can be studied directly in the cells, this will be ideal, but is seldom possible. If such studies can be done, at least one knows the effects to be expected at any concentration. Of course, it is always impossible to test all the enzymes or metabolic pathways, so there is inevitably an element of uncertainty, but without such preliminary investigation there is almost complete uncertainty. It is worth emphasizing that these determinations should be made on enzymes and systems from the same tissue and species in which the specific inhibition is desired, because there are many examples of enzymes whose susceptibilities to inhibitors vary greatly with the source.

(B) One should either know or make a reasonable estimate of the intracellular $\mathrm{pH}$ so that the in vitro studies can be as comparable to the protoplasmic conditions as possible. Many enzymes are tested at their optimal pH's rather than at physiological pH's; here we are not interested in the behavior of the enzymes when they are most active but in their relative behavior under constant conditions.

$(C)$ Consideration should be given to the properties of the inhibitor that determine its distribution between the cells and the medium. It is occasionally possible to make an approximate prediction of the ratio of the intracellular to the extracellular inhibitor concentrations, based on the 
differences in $\mathrm{pH}$ and the acidic or basic nature of the inhibitor. In other cases it may be worthwhile to analyze for the inhibitor within the cells. With inhibitors that penetrate through the plasma membrane slowly, it is necessary to provide enough time for equilibrium to occur.

(D) The lowest concentrations of the inhibitor to produce significant effects should be used rather than concentrations aimed at nearly complete inhibition. It is also of value to construct a concentration-action curve for the cellular inhibition and not use only a single concentration of the inhibitor. particularly if correlations between enzyme inhibition and functional disturbance are sought.

One can seldom be absolntely certain of specificity in cellular studies but every effort should be made to approach this goal.

\section{Use of Two or More Inhibitors to Improve the Specificity}

There are two general ways by which the judicious use of additional inhibitors may increase the specificity of the primary inhibitor. The first way is limited to certain types of systems. It is to use a second inhibitor for the purpose of blocking an interfering reaction. For example, in the study of the inhibition of $\alpha$-ketoglutarate oxidation, malonate may be added to block the further oxidation of the succinate formed from the $\alpha$-ketoglutarate, and in this way the first reaction can be in a way isolated and any effects of the inhibitor being tested must be on the $\alpha$-lietoglutarate oxidase. In the same way, the inhibition of certain anaerobic processes can be investigated during a block of the oxidative reactions with cyanide. By this technique the inhibitor is not made more specific but the range of systems on which it can act is reduced.

The other use of two or more inhibitors is more general and theoretically could be used to improve any specificity. This has already been mentioned in Chapter 10 where the actions of multiple inhibitors were treated. If two inhibitors act on one enzyme but their further inhibitions are on different enzymes, the use of them together will increase the specificity with respect to any of these other enzymes. Put simply, if $I_{1}$ acts on $E_{1}$ and $\mathrm{E}_{2}$ whereas $\mathrm{I}_{2}$ acts on $\mathrm{E}_{1}$ and $\mathrm{E}_{3}, \mathrm{I}_{1}+\mathrm{I}_{2}$ will inhibit $\mathrm{E}_{1}$ more potently without inhibiting $\mathrm{E}_{2}$ or $\mathrm{E}_{3}$ more than with the single inhibitors. This is the principle on which drug combinations are used in medicine with the purpose of increasing the beneficial actions while decreasing the side effects. We shall now investigate the conditions under which such a combination of inhibitors can give significant improvement in the specificity and determine the proper concentrations of each inhibjtor to use.

The various inhibitions for a system of two enzymes and two inhibitors of the type described above may be written as follows. 
Inhibition of $\mathrm{I}_{1}$ on $\mathrm{E}_{2}$ :

Inhibition of $\mathrm{I}_{1}$ on $\mathrm{E}_{2}$ :

Inhibition of $\mathrm{I}_{2}$ on $\mathrm{E}_{1}$ :

Inhibition of $\mathrm{I}_{2}$ on $\mathrm{E}_{3}$ :

$$
i_{11}=\frac{\left(\mathrm{I}_{1}\right)}{\left(\mathrm{I}_{1}\right)+K_{i_{11}}}
$$

Inhibition of $\mathrm{I}_{1}$ and $\mathrm{I}_{2}$ on $\mathrm{E}_{1}: i_{121}=\frac{K_{i_{21}}\left(\mathrm{I}_{1}\right)+K_{i_{11}}\left(\mathrm{I}_{2}\right)}{K_{i_{11}} K_{i_{21}}+K_{i_{21}}\left(\mathrm{I}_{1}\right)+K_{i_{11}}\left(\mathrm{I}_{2}\right)}$

where the first subseripts refer to the inhibitor and the last subscripts to the enzyme. This applies to noncompetitive inhibition but the results will be applicable in principle to competitive inhibition as well. To facilitate discussion and interpretation we shall assmme the following valnes for the inhibition constants: $K_{i_{11}}=0.3 \mathrm{~m} M ; K_{i_{12}}=3 \mathrm{~m} M: K_{i_{21}}=1 \mathrm{mM} / K_{i_{23}}=$ $5 \mathrm{~m} M$. The results are not readily plotted in two dimensions and thus the general behavior of the system is shown in Table 16-2, where the inhibitions prodneed by each inhibitor alone and by them acting together have been calculated. The specificities, as defined by Eq. 16-1, are also shown. It may be noted first that certain combinations of the two inhibitors will produce a greater inhibition on $\mathrm{E}_{1}$ compared to the single inluibitors, with no increase in the inhibitions on $\mathrm{E}_{2}$ and $\mathrm{E}_{3}$. In other words, the specificity may be inereased significantly. It is also evident that high concentrations of each inhibitor when they are used together will deerease the speeificity in the sense that both $\mathrm{E}_{2}$ and $\mathrm{E}_{3}$ may be strongly inhibited. It is of some importance, therefore, to inquire into optimal concentrations for inhibitor combinations. The best concentrations to be used will depend in any case on what type of effect is desired. Three general situations may be visualized: (a) it is desired to inhibit $\mathrm{E}_{1}$ to a certain degree with minimal inhibitions on $\mathrm{E}_{2}$ and $\mathrm{E}_{3},(b)$ it is desired to inhibit $\mathrm{E}_{1}$ maximally with certain limits placed on the inhibitions of $\mathrm{E}_{2}$ and $\mathrm{E}_{3}$, or $(c)$ it is desired to achieve the maximal specificity.

If it is required to inhibit $\mathrm{E}_{1}$ to the extent of $i_{121}$, rearrangement of Eq. 16-13 gives the relationship between $\left(I_{1}\right)$ and $\left(I_{2}\right)$.

$$
\frac{\left(\mathrm{I}_{1}\right)}{K_{i_{11}}}+\frac{\left(\mathrm{I}_{2}\right)}{K_{i_{21}}}=\frac{i_{121}}{1-i_{121}}
$$


Thus, if the inhibition on $\mathrm{E}_{1}$ must be $50 \%,\left(\mathrm{I}_{1}{ }^{\prime}\right)=\left(\mathrm{I}_{2}{ }^{\prime}\right)=1$, i.e., the sum of the specific concentrations of the two inhibitors with respect to $\mathrm{E}_{1}$ must be unity. $\left(I_{1}\right)$ and $\left(I_{2}\right)$ must now be chosen so that this equation is obeyed

TABLE $16-2$

Specifictries of Ixhibition by Two Inhrbitors ox a Three-Exzyue System a

\begin{tabular}{|c|c|c|c|c|c|c|}
\hline \multicolumn{7}{|c|}{ Inhibition on } \\
\hline $\begin{array}{c}\left(\mathrm{I}_{1}\right) \\
(1 \mathrm{~m} M)\end{array}$ & $\begin{array}{c}\left(\mathrm{I}_{2}\right) \\
(\mathrm{m} M)\end{array}$ & $E_{1}$ & $E_{2}$ & $E_{3}$ & $\sigma_{12}$ & $\sigma_{13}$ \\
\hline 0.1 & 0 & 0.25 & 0.03 & 0 & 0.22 & 0.25 \\
\hline 0.3 & 0 & 0.50 & 0.09 & 0 & 0.41 & 0.50 \\
\hline 0.6 & 0 & 0.67 & 0.17 & 0 & 0.50 & 0.67 \\
\hline 1.0 & 0 & 0.77 & 0.25 & 0 & 0.52 & 0.77 \\
\hline 3.0 & 0 & 0.91 & 0.50 & 0 & 0.41 & 0.91 \\
\hline 6.0 & 0 & 0.95 & 0.67 & 0 & 0.28 & 0.95 \\
\hline 10.0 & 0 & 0.97 & 0.77 & 0 & 0.20 & 0.97 \\
\hline 30.0 & 0 & 0.99 & 0.91 & 0 & 0.08 & 0.99 \\
\hline 0 & 0.1 & 0.09 & 0 & 0.02 & 0.09 & 0.07 \\
\hline 0 & 0.3 & 0.23 & 0 & 0.06 & 0.23 & 0.17 \\
\hline 0 & 0.6 & 0.37 & 0 & 0.11 & 0.37 & 0.26 \\
\hline 0 & 1.0 & 0.50 & 0 & 0.17 & 0.50 & 0.33 \\
\hline 0 & 3.0 & 0.75 & 0 & 0.37 & 0.75 & 0.38 \\
\hline 0 & 6.0 & 0.86 & 0 & 0.54 & 0.86 & 0.32 \\
\hline 0 & 10.0 & 0.91 & 0 & 0.67 & 0.91 & 0.24 \\
\hline 0 & 30.0 & 0.97 & 0 & 0.86 & 0.97 & 0.11 \\
\hline 0.1 & 0.3 & 0.39 & 0.03 & 0.06 & 0.36 & 0.33 \\
\hline 0.1 & 0.6 & 0.48 & 0.03 & 0.11 & 0.45 & 0.37 \\
\hline 0.1 & 1.0 & 0.57 & 0.03 & 0.17 & 0.54 & 0.40 \\
\hline 0.3 & 0.3 & 0.56 & 0.09 & 0.06 & 0.47 & 0.50 \\
\hline 0.3 & 0.6 & 0.61 & 0.09 & 0.11 & 0.52 & 0.50 \\
\hline 0.3 & 1.0 & 0.67 & 0.09 & 0.17 & 0.58 & 0.50 \\
\hline 0.6 & 0.3 & 0.70 & 0.17 & 0.06 & 0.53 & 0.64 \\
\hline 0.6 & 0.6 & 0.72 & 0.17 & 0.11 & 0.55 & 0.61 \\
\hline 0.6 & 1.0 & 0.75 & 0.17 & 0.17 & 0.58 & 0.58 \\
\hline 1.0 & 0.6 & 0.80 & 0.25 & 0.11 & 0.55 & 0.69 \\
\hline 1.0 & 1.0 & 0.81 & 0.25 & 0.17 & 0.56 & 0.64 \\
\hline 1.0 & 3.0 & 0.86 & 0.25 & 0.37 & 0.61 & 0.49 \\
\hline
\end{tabular}

${ }^{a}$ The assumed values of the inhibitor constants are: $K_{i_{11}}=0.3 \mathrm{~m} M, K_{i_{12}}=3 \mathrm{~m} . I$, $K_{i_{21}}=1 \mathrm{~m} M$, and $K_{i_{23}}=5 \mathrm{~m} M \cdot \sigma_{12}$ is the specificity with respect to $E_{2}$ and $\sigma_{13}$ with respect to $\mathrm{E}_{3}$. 
and the inhibitions on $\mathrm{E}_{2}$ and $\mathrm{E}_{3}$ are minimal. Setting $i_{12}=i_{23}$ it is found that the inhibitor concentrations must be:

$$
\begin{aligned}
& \left(\mathrm{I}_{1}\right)=\frac{K_{i_{11}} K_{i_{12}} K_{i_{21}}}{K_{i_{11}} K_{i_{23}}+K_{i_{12}} K_{i_{21}}} \\
& \left(\mathrm{I}_{2}\right)=\frac{K_{i_{11}} K_{i_{23}} K_{i_{21}}}{K_{i_{11}} K_{i_{23}}+K_{i_{12}} K_{i_{21}}}
\end{aligned}
$$

Substituting the numerical values for the inhibitor constants: $\left(I_{1}\right)=0.20$ $\mathrm{m} M ;\left(\mathrm{I}_{2}\right)=0.33 \mathrm{~m} M ; i_{121}=0.50 ; i_{12}=0.0625 ; i_{23}=0.0625$. If the inhibitors are used singly to inhibit $\mathrm{E}_{1}$ by $50 \%,\left(\mathrm{I}_{1}\right)$ must be $0.30 \mathrm{mM}$ and $\left(\mathrm{I}_{2}\right)$ must be $1 \mathrm{mM}$, the inhibitions on $\mathrm{E}_{2}$ and $\mathrm{E}_{3}$ being $9.1 \%$ and $16.7 \%$ respectively, so that the combination has improved the specificity significantly.

If it is now desired that the inhibitions on $\mathrm{E}_{2}$ and $\mathrm{E}_{3}$ do not exceed $10 \%$ and the inhibition on $\mathrm{E}_{1}$ be maximal; we may set $\left(\mathrm{I}_{1}\right)=0.11 K_{i_{12}}$ and $\left(\mathrm{I}_{2}\right)=0.11 K_{i_{23}}$. In the example chosen, $\left(\mathrm{I}_{1}\right)=0.33 \mathrm{mM}$ and $\left(\mathrm{I}_{2}\right)=$ $0.55 \mathrm{~m} M$, so that this combination of inhibitors would inhibit $\mathrm{E}_{1}$ by $62.5 \%$. To achieve this degree of inhibition on $\mathrm{E}_{1}$ with the inhibitors singly, the inhibitions on the other enzynes would be much higher than $10 \%$.

Finally, if maximal specificity is desired, independent of the absolute values of the individual inhibitions, reference to Table 16-2 shows that the maximal specificity between $\mathrm{E}_{1}$ and $\mathrm{E}_{2}, \sigma_{12}=0.52$, and between $\mathrm{E}_{1}$ and $\mathrm{E}_{3}, \sigma_{13}=0.38$, when the inhibitors are used singly. However, combinations can increase both specificities, $\sigma_{12}$ to as much as 0.61 and $\sigma_{13}$ to as much as 0.69 , but not simultaneonsly. If the maximal specificity with respect to $\mathrm{E}_{2}$ is desired, some sacrifice in the specificity towards $\mathrm{E}_{3}$ must be made, and vice versa. The actual combination chosen will depend on the nature of the work and the relative importance of the subsidiary enzymes in the system being studied.

This approach may be readily extended to combinations of three or more inhibitors and even greater specificity may often be achieved. It may also be applied to inhibitions on multienzyme systems or metabolic sequences, but here it is difficult to express the relationships quantitatively and predict the optimal inhibitor concentrations. For example, if one wished to inhibit the glycolytic pathway with minimal inhibition on the tricarboxylic acid cycle, it might be possible to achieve greater specificity with a combination of iodoacetate, fluoride, and a glucose analog than with any single inhibitor, but the determination of the proper concentrations to use would depend on accurate experimental data using the inhibitors alone. It is worth repeating that in studies attempting to correlate some cellular function with a particular enzyme or metabolic process, it is generally 
advisable to use the lowest inhibitor concentrations commensurate with adequate effects on this enzyme or metabolic process. If another enzyme or sequence is inhibited only $10-20 \%$, it makes any interpretation unreliable.

\section{Examples of Specificity and Lack of Specificity}

Certain inhibitors are really not specific at all in the general sense: mercurials, arsenate, fluoride, chelating agents, heavy metal ions, protein reactants such as diisopropylfluorophosphate, iodoacetate, and others inhibit many systems. Nevertheless, under some conditions, especially in enzyme extracts where the number of significant enzymes is not great, such inhibitors may be used to exert a specific effect. In work with cells or tissues, good specificity is difficult or impossible to achieve. Other inhibitors may be reasonably specific: the monoanine oxidase inhibitors, certain types of cholinesterase inhibitors, fluoracetate, anti-enzymes, and some substrate analogs if used properly can be specific even in cellular preparations. Inasmuch as there are very few complete summaries of inhibition spectra available. every effort will be made in the succeeding volumes to present all the known actions of each inhibitor so that evaluations of specificity may be more readily made.

It is frequently assumed. for example, that iodoacetate inhibits specifically the glyceraldehyde dehydrogenase and can block the glycolytic pathway selectively. Yet a survey of the literature reveals that iodoacetate can significantly inhibit at least 55 enzymes at a concentration of $1 \mathrm{~m} . M$ or below and, of course. not all the enzymes have been tested. Likewise trivalent arsenicals are often considered to block the oxidation of keto acids selectively, but again the literature shows at least 52 enzymes inhibited by $1 \mathrm{~m}, I$ or less. This does not mean that these two inhibitors are useless. These figures are presented to emphasize the wide range of possible action in cellular preparations where hundreds of enzymes are operating, and to point out the necessity of using the lowest concentrations possible. Iodoacetate is often used at $1 \mathrm{~m} M$ when greater specificity could be achieved at a lower concentration. The oxidation of various cycle substrates and intermediates is inhibited from $35 \%$ to $85 \%$ by $1 \mathrm{~m} M$ iodoacetate (Yang, 1957 ) and even $0.1 \mathrm{~m} . / \mathrm{inhibits}$ the oxidation of the keto acids quite strongly. Inasmuch as 0.2 to $0.5 \mathrm{~m} . I$ iodoacetate must usually be used to inhibit glycolysis completely. it is evident that a truly specific effect is difficult to obtain. The oxidation of pyruvate by cardiac tissue is almost completely inhibited by $1 \mathrm{~m} . I$ iodoacetate (Webb et al., 1949 b) so that similar relations apparently hold in intact cells. Some effort has been made to determine the most specific concentration of iodoacetate for cardiac tissue. It was found that rabbit atria whose rates and contractile amplitudes were depressed with $1 \mathrm{~m} . I$ iodoacetate could not be stimulated by pyruvate (Webb, $1950 \mathrm{a}$ ). A concentration of $0.2 \mathrm{~m} . \mathbf{M}$ exerts a pro- 
gressively depressant action on the atria: after 16 min pyruvate can stimulate both rate and amplitude somewhat, after $25 \mathrm{~min}$ only the rate, and later pyruvate is completely inactive. On the other hand, studies with rat ventricle strips have indicated reasonably selective glycolytic block with $0.2 \mathrm{mM}$ iodoacetate (Masuoka et al., 1952; Covin and Berman, 1956). This concentration did not interfere with the positive inotropic action of pyruvate on hypodynamic preparations. If differences in the selectivity of iodoacetate are observed on different cardiac tissues, it is likely that much greater variability would be found in testing a variety of tissues from different species, and this emphasizes the danger of applying results on one tissue to another.

Sometimes a greater faith than is justified is applied to the specificity of certain inhibitors because of categorical statements made in the literature. Thus, Krebs has stated: "If the concentration of malonate is below $0.01 M$, succinic dehydrogenase is the only muscle enzyme affected by this inhibitor; this high specificity makes malonate a most valuable tool." This statement is, in general, true as far as we know and malonate, when used properly, is indeed a very useful inhibitor. However, Das (1937) has reported $50 \%$ inhibition of lactic dehydrogenase from pigeon breast muscle, and a number of other enzymes from other tissues have been found to be inhibited appreciably by $10 \mathrm{~m} \boldsymbol{M}$ or less malonate: oxalacetate decarboxylase from pigeon liver $(100 \%$ by $10 \mathrm{~m} M)$, malic enzyme from cattle lens $(55 \%$ by $8.3 \mathrm{~m} M)$, $\alpha$-ketoglutarate oxidase from pea seedlings $(15 \%$ by $1 \mathrm{~m} M$ ), and others, some of which have not been tested in muscle preparations. Also it must be admitted that many muscle enzymes have never been studied with respect to malonate inhibition so that we simply do not have a complete spectrum of action. One must also consider the possibility of nonenzymic actions by such inhibitors when intact tissues are used. Thus, the above statement may be true for many types of preparation and was undoubtedly made with these reservations in mind, and yet some investigators will interpret it too rigorously and possibly be led astray with a false sense of security. Certainly in our laboratory we have obtained results with $1 \mathrm{~m} M$ to $10 \mathrm{~m} M$ malonate that cannot be readily interpreted in terms of an inhibition of succinic deliydrogenase. The positive inotropic action of malonate on the rat ventricle is apparently unrelated to any action on this enzyme or the tricarboxylic acid cycle (Covin and Berman, 1956) and evidence for a stimulatory action on the glycolytic pathway in brain slices (Heald, 1953) and ventricle slices (Webb et al., 1949 b) has been reported. There is a great need for more emphasis on the important problem of inhibitor specificity and for more energetic attempts to evaluate the specificity in the particular systems being studied. 


\section{SUGGESTIONS FOR PLANNING AND REPORTING INHIBITION STUDIES}

The information that may be obtained from work with enzyme inhibitors is greater in magnitude and reliability when the inhibitors are used correctly. The investigation of metabolic inhibition, like any work with biological systems, is inherently difficult, and anyone who is acquainted with this field will know that in order to achieve significant results, especially in living cells or tissues, much careful experimentation must be done and thorough consideration must be given to the many factors that may complicate the interpretations. Furthermore. a complete and intelligible report of the work is necessary if the results are to be made useful to others. The following practical suggestions may help those who have not worked extensively with inhibitors to obtain the maximal reliable information and are based on personal experience and mistakes, as well as on the consideration of several thousand reports in the literature. Such advice might be taken as presumptuous but even a casual survey of the majority of inhibition studies will demonstrate certain deficiencies and occasionally a rather naive approach. Inhibitors are at present so widely used to characterize enzymes, to plot metabolic sequences, and to correlate cellular functions with the metabolism, that it is perhaps time to suggest that inhibitors should either be used carefully and thoroughly, or not used at all, and that interpretations of the data be made with a full realization of the complexities of the systems studied. The following remarks are partly supplementary to what has already been written in the previous chapters and must, of course, be taken in light of the purpose and nature of the investigations to be undertaken. Specific failures to observe certain simple pre cautions and various types of interpretative errors will be illustrated in the later volumes.

\section{SUGGESTIONS PARTICULARLY APPLICABLE TO INHIBITIONS OF PURIFIED ENZYMES}

(1) Determine initial enzyme rates. It is very important to determine as far as possible the effects of inhibitors on the initial rates following the 
addition of the substrates, inasmuch as the rates may change during the conrse of the reactions due to a variety of factors. If the inhibition is reported over a period of $30 \mathrm{~min}$, for example, one cannot be certain how much of the depression is due to the direct action of the inhibitor and how much to secondary alterations in the enzyme. Unless the effect on the initial rate is determined, the results may not be of quantitative significance and any calculated inhibitor constants may be incorrect. The problem of secondary inactivation of enzymes was discussed in Chapter 12.

(2) Work in the range where the rate is linear with the enzyme concentration. The usual expressions for inhibition kinetics are derived on the basis of a linear dependence of enzyme rate on the enzyme concentration. There are several reasons for nonlinearity under certain conditions, as pointed out by Dixon and Webb (1958, p. 64), so that it is necessary to test the enzyme being investigated under the conditions in which the inhibition is exerted. Nonlinearity may imply limitation by diffusion, relative depletion of coenzymes or cofactors, impurities in the reagents used, and other situations which can easily alter inhibition behavior.

(3) Use as little enzyme as possible. There is some tendency these days with the greater availability of purified enzymes to use quite high enzyme concentrations in the reaction vessels. There are several reasons why these high concentrations may be undesirable. In the first place, there is a greater likelihood for the depletion of free substrate or inhibitor; that is, the system may be in zones B or C, and more complex kinetics must be applied to interpret the data. In the second place, there is more opportunity for impurities or other enzymes to be introduced with the tested enzyme. In the third place, one often wishes to study an enzyme under approximately physiological conditions and within the cell the enzyme may not be so concentrated.

(4) Establish competitive inhibition by quantitative procedures. To demonstrate competitive inhibition, it is not snfficient to show an increased rate of the inhibited enzyme upon increase in the substrate concentration; this would be expected to occur with essentially any type of inhibition. Nor is it enough to show that the inhibition is reduced hy increasing substrate concentration, because this may be brought about by other mechanisms, the reaction of the substrate with the inhibitor for example. It is necessary to show by adequate plotting procedures that competitive kinetics are obeyed and to cover as wide a range of substrate and inhibitor concentrations as possible.

(5) Utilize several analytical plots to determine the inhibition mechanism. It was pointed out in Chapter 5 that the varions types of plotting procedure are not equivalent and that some are more applicable to certain inhibitions 
than others. One should choose that method, or those methods, which are most likely to provide accurate values for the constants characterizing the inhibition, and then to check the determinations using different forms of plotting. In this way certain abnormal types of inhibition may be uncovered because not all the plots will be equally sensitive to deviations from the classic kinds of inhibition. More accurate and reliable values for the inhibition constants may also be obtained. One must always bear in mind that there are many possible inhibition mechanisms, some of which are not easily recognizable in the common plots. It is probably a good idea before interpreting the data, to list all the conceivable mechanisms by which the inhibition might take place and to devise analytical procedures whereby these may be distinguished.

(6) Do not use an unnecessarily high substrate concentration. Many enzymes are inhibited by high substrate concentrations (see Chapter 4 ) and under these conditions the responses to inhibitors will be altered. The enzyme may also be saturated at high substrate concentrations, the rate being independent of the substrate concentration, and under these conditions the effects of inhibitors on the binding of substrates (i.e., changes in $K_{s}$ ) will not be so readily observed.

(7) Compare different inhibitors by determining the true inhibitor constants. In comparing the potencies of several inhibitors on a single enzyme, it is not enough to determine the inhibitions at single concentrations of each inhibitor, especially if one is dealing with a series of homologous compounds, because it is only through the use of the true $K_{i}$ 's that accurate results on relative binding can be obtained. If, for some reason, it is not convenient to determine the values of $K_{i}$, at least several imhibitor concentrations should be used and the concentrations producing a specified degree of inhibition should be presented.

(8) Determine the effects of changing the $p H$ on the inhibition. A great deal of information may frequently be obtained from studies on the variation of the inhibition with the $\mathrm{pH}$. In relation to the suggestion immediately above, it is important in comparing different inhibitors to determine the true $K_{i}$ values. It was shown in Chapter 14 that the inhibition expressions for dissociable inhibitors involve a ${ } H$ term and that the true $K_{i}$ is often quite different from that determined by the plotting procedures. In other words, the values of the apparent or experimental $K_{i}$ for a series of inhibitors may relate principally to the ionization constants of the inhibitors rather than to their relative binding to the enzyme. If one is attempting to correlate inhibition with chemical structure, the relative affinities of the active forms of the inhibitors are desired, not merely the fractions of the inhibitors in the active form at the chosen $p H$. Furthermore, it is clear that information on the nature of the groups at the active centers of 
enzymes can often be obtained by variation of the $\mathrm{pH}$, and the characterization of an enzyme group is more important than a simple demonstration of inhibition.

(9) Consider the possibility of nonspecific effects of inhibitors. In the case of weak inhibitors particularly, where reasonably high concentrations must be used, one must attempt to eliminate such effects as might arise from an increase in the ionic strength. The use of comparable concentrations of otherwise inert salts will often reveal that an observed effect is actually a nonspecific salt effect rather than a direct inhibition.

\section{SUGGESTIONS PARTICULARLY APPLICABLE TO INHIBITIONS IN CELLULAR PREPARATIONS}

(1) Determine and correct for any endogenous metabolism. The presence of a significant degree of endogenous metabolism may influence inhibitions, whether the endogenous metabolism itself is inhibited or not, especially in those cases in which the effects of the inhibitors on the metabolism of a particular added substrate are determined. The means for correcting for endogenous metabolism are discussed in Chapter 9.

(2) Consider the penetration of the inhibitor into the cells. The effect of an inhibitor on an intracellular enzyme will depend in part on the ability of the inhibitor to penetrate to the enzyme and on the concentration of the inhibitor within the cell. Rough predictions of penetrability can often be made on the basis of the chemical nature of the inhibitor but it is always better, whenever possible, to obtain experimental evidence on the intracellular concentration during the inhibition. In this connection, it is frequently of value to vary the $\mathrm{pH}$ of the external medium if the inhibitor is ionizable, for in this way a dependence on the penetration may be demonstrated.

(3) Use as far as possible the optimal inhibitor concentration. Choose that concentration, or range of concentrations, of the inhibitor which will most likely inhibit intracellularly to the degree desired, basing your choice on the in vitro actions of the inhibitor and on whatever information is available on the relationship of intracellular to extracellular concentrations. The greater care that is given to these considerations, the greater will be the chance of a selective and meaningful action.

(4) Use inhibitors, inhibitor concentrations, and conditions which will most likely provide specific effects. One should first consider all the known and possible effects of the inhibitor and choose those conditions, if such are possible, for maximal selectivity of action, as described in Chapter 16 . In addition, all the evidence that it is possible to obtain on the specificity 
of the inhibition in the cells studied should be presented. Only in this way can accurate and reliable correlations be made. It is important to avoid, whenever possible, conclusions based on types of cells other than those being investigated.

(5) Consider the possible metabolic alteration of the inhibitor. An inhibitor may be either changed to a more or less active substance by the enzyme systems of the cells. When there occurs a significant time lag between the addition of the inhibitor and the effect produced, always consider the possibility of a lethal synthesis. Conversely, if the inhibition decreases with time, inactivation of the inhibitor may be occurring. If either occurs, the time over which the inhibition is measured is verv important for the interpretation of the results.

(6) Consider the possible nonenzymic effects of the inhibitor. Effects on cell structure and function may occur independently of any action on enzymes or metabolic processes as pointed ont in Chapter 9. Furtbermore, these effects may be brought about not only by the inhibitor itself but by the other ions introduced with the inhibitor. Also the inhibitor can occasionally alter the ionic balance in the external medium by combining with one or more of the physiological ions. Such effects should, as far as is possible, be distinguished from true inhibition.

(7) Localize the site of inhibition as completely as possible. The useful information that may be obtained from the use of inhibitors, especially new or little known inhibitors, on multienzyme or cellular systems depends on how well the exact site, or sites, of inhibition can be determined, and every effort to provide data for this purpose should be made. The methods of localization are discussed in Chapter 11.

(8) Exercise great care in correlating cellular function with an enzyme or metabolic system. When cellular growth, division, secretion, movement, or other functions are depressed by inhibitors, it is difficult to correlate definitely these changes with a particular enzyme or metabolic sequence acted upon by the inhibitor, for reasons presented in Chapter 9. One should attempt to correlate with respect to inhibitor concentration, the rates of inhibition, the rates of reversal of the inhibition. and the actions of several similarly acting inhibitors. Evidence of specificity is especially important here and must be provided in some manner.

\section{SUGGESTIONS APPLICABLE TO INHIBITIONS OF BOTH PURIFIED ENZYMES AND CELLS}

(1) Provide physiological conditions. As far as is possible, try to create a normal environment for the enzyme. or mitochondria, or cells and tissues, 
by providing a pliysiologically correct ionic balance and $\mathrm{pH}$. Intracellular enzymes should be tested at intracellular $\mathrm{pH}$ 's and cells should be tested at the $\mathrm{pH}$ 's of their usual bathing media if correlations are to be made between the actions of the inhibitors on the experimental preparations and the enzymes or cells under normal conditions. Of course, in many cases the physiological conditions must be varied to obtain pertinent information but the initial and control conditions should be as physiological as possible. It is actually not very important to work under conditions in which the rates are maximal, as is often done. Needless to say, it is never possible to achieve exact physiological conditions when studying intracellular enzymes in isolated preparations, but one should come as close as is feasible.

(2) Control the pH carefully. All solutions to be used in the enzyme test or in cellular inhibition should be adjusted to the same $\mathrm{pH}$ so that mixing will not result in any mnknown shift in $\mathrm{pH}$. If this is impossible for some reason, the $\mathrm{pH}$ should be determined in the final medium. It is also well not to rely completely on buffers to maintain the $\mathrm{pH}$ constant nor is it generally good practice to use high buffer concentrations.

(3) Use a wide range of inhibitor concentrations. It is only by using inhibitor concentrations that give the total range of possible inhibition that an enzyme may be characterized adequately and useful constants obtained. Inhibition data on newly isolated enzymes today are often restricted to the per cent inhibition of each inhibitor at a single concentration but often such results are not very valuable. To say that an inhibitor produces a $95-100 \%$ inhibition at $50 \mathrm{mM}$ means very little; concentrations of 1-5 $\mathrm{mM}$ might very well inhibit quite potently. Because of the configurations of most inhibition-concentration curves, it is more valuable to give the concentration of an inhibitor that produces around $50 \%$ inlibition, if only one concentration is to be reported. It is particularly important in determinations of specificity for a fairly complete inhibition range to be presented.

(4) Use several similarly acting inhibitors for better correlations. If the identification of a particular enzyme group is desired, or if the relationship of some metabolic process to a cell function is being studied, it is occasionally useful to use several inhibitors having common actions. For example, in characterizing the nature of sulfhydryl groups at or near the active center, it is well to use several types of sulfhydryl reagent, such as an arsenical, a mercurial, iodoacetate or iodoacetamide, iodosobenzoate, $N$-ethylmaleimide, a quinone, and other inhibitors. If inhibition is obtained with some but not others, this might indicate that the mechanism of the inhibition is not through reaction with sulfhydryl groups, or it could mean that the particular sulfhydryl group is sitnated at the enzyme surface in such a manner that only certain substances can obtain access to it, in both cases interesting information being obtained. 
(5) Do inhibitor incubation experiments when indicated. It is very useful, especially with a new inhibitor, to incubate the enzyme with the inhibitor for varying times and under various conditions. Not only will this provide important information on the rate of inhibition but can well point to the mechanism of the inhibition. The appropriate techniques are discussed in Chapter 12.

(6) Determine the degree of reversibility of the inhibition. The reversibility of an inhibition will not only give information of a practical nature that can be useful in future work with the inhibitor, but also can indicate inhibition mechanisms, particularly if a chemical reaction with the enzyme is involved. It also serves to demonstrate whether secondary inactivation of the enzyme or damage to the cells has occurred chring the inhibition.

(7) Exercise caution in the interpretation of protection or reversal experiments involving substances reacting with the inhibitor. The protection of an enzyme or a cell preparation from an inhibitor by a substance reacting with or complexing with the inhibitor is not very significant and does not indicate the mechanism by which the inhibition occurs. In such cases, one is merely reducing the concentration of free or effective inhibitor. Reversal experiments may be more informative, but only if analyzed quantitatively and correctly. At the most. such experiments only show the relative affinities of the inhibitor for the enzyme and the protector or reversor.

\section{SUGGESTIONS RELATIVE TO THE REPORTING OF INHIBITION STUDIES}

(1) Report the experimental conditions completely. It is necessary to state accurately "all of the conditions under which the inhibition experiments were run. Some factors that may seem unimportant when the report is written may later be of major significance. Particularly in interpreting early work, it is often very difficult to determine in what manner the experiments were conducted. In studies with purified enzymes the following would be the minimally required information: $(a) \mathrm{pH},(b)$ temperature, (c) ionic composition of the merlium, $(d)$ the gas phase in equilibrium with the medium, $(e)$ the substrates and their concentrations, $(f)$ other substances present, such as buffers, stabilizers, cofactors, etc., $(g)$ the exact source of the enzyme, $(h)$ the intervals of time during which the inhibition was determined, and (i) how the rates were determined. In certain instances, other conditions, such as ionic strength, may be important. Studies on cells or tissues require in addition a complete history of the material, including nutrition of the cells before the experiment, their age, their growth rate or state of functional activity, and any other pertinent information. Unexplained discrepancies between the data of different investigators often result from deficiencies in reporting. 
(2) Present the inhibitions in inhibition studies. It greatly aids the reader if inhibitions are calculated, reported, and averaged if feasible, rather than giving only the experimental data. Of course, the raw data may in certain cases be quite important. The presentation of only rate curves makes it frequently very laborions for the reader to calculate inhibitions and interpret the results quantitatively.

(3) Express concentrations in molar terms. The concentrations in many reports on inhibitors are given in terms of weight per volume. Such concentrations are occasionally ambiguous because one doesn't know the salt used or the assmmed hydration. Furthermore, it is difficult to compare different reports when the units of concentration are not expressed uniformly. Another common practice it to state the amomnt of material in the reaction vessel; e.g., 20 umoles of succinate per vessel, or 3 umoles of ATP in $3.3 \mathrm{ml}$ of the medium. In some cases there is justification for this but nsually one wishes to know the molar concentrations. It makes little difference whether the concentrations are given in $M, \mathrm{~m} M$ or $\mu M$, but it must be remembered that these are abbreviations for concentrations and not for actual quantities, which must be written out as moles, millimoles, or micromoles ( $\mu$ moles).

(4) Compare the relative potencies of different inhibitors on a molar basis. If several inhibitors are to be compared with regard to their actions on an enzyme or metabolic process, the concentrations producing chosen effects must be on a molar basis rather than a weight per vohme basis. This is actually what one does in the determination of different $K_{i}$ 's.

\section{SUGGESTIONS OF A GENERAL NATURE}

(1) Don't use imhibitors without adequate knowledge of their properties. One camnot either use inhibitors properly or interpret the results of their actions if one does not understand the nature of the inhibitions and the ranges of the inhibitor actions. In particular it is important to know the specificity as far as is possible. The initial choice of the inhibitors to be used will, of course, depend on such knowledge and there are many examples where more informative data would have been forthcoming if more care had been devoted to the selection of inhibitors.

(2) Use inhibitors with specific and rational purposes. As in any field of scientific investigation, one should have a good reason for the use of a particular procedure. In many studies involving inhibitors, especially in the characterization of enzymes, it would appear that the inhibitors used are those that happen to be on the shelves and, as a result, the useful information derived is less than one might hope. Naturally there are cases where exploratory testing is necessary because the investigated system is 
poorly understood. However, in general one should not use a particular inhibitor unless consideration of the situation leads to the conclusion that the results, whatever they are, will be meaningful in some way.

(3) Use inhibitors that are as pure as possible. It is important to be able to attribute with certainty the effects observed to the inhibitor used if reliable conchusions are to be drawn and thus it is advisable to have either assurance of the purity of an inhibitor that is purchased or to purify it before use. In many cases the commercially available inhibitors are not pure. It must never be forgotten that enzymes and living cells are often highly sensitive to trace impurities, such as the heary metals, and only very small amounts are required to produce significant effects. Some inhibitors are unstable and alter chemically with time to products that may or may not be active; one is seldom certain of the interval between the preparation of the inhibitor and its purchase, so that in many instances repurification is mandatory. Particular attention must be paid to certain inhibitors such as fluoroacetate, iodoacetate, the quimones, dimercaprol, alloxan, and others, because experience has shown that they are occasionally quite impure as obtained and are unstable. One must, also be cautions in using solutions of inhibitors over several days since many inhibitors are unstable in solution even when stored in the cold. In cases of doubt, it is always better to prepare the inhibitor solutions fresh for each experiment.

(4) Interpret inhibition studies in as quantitative a manner as possible. The attempt has been made thronghout this volume to put the study of inhibition on a quantitative basis because it is believed that a greater amount of information may be obtained by using such an approach. In the case of isolated enzymes it is best to determine the inhibitor constants, $K_{i}$, when possible. and to record changes in these or any other pertiment constants with variations in the experimental conditions. The presentation of some type of rate constants for the development of inhibition and its reversal is also advisable, rather than merely stating that the inhibition proceeds rapidly or slowly. or that the inhibition is readily or difficultly reversible. Whenever possible the thermodynamic characteristics of the inhibition should be determined, especially the binding energies and the entropy terms. Even where absolute binding energies cannot be calculated, the relative binding energies of the inhibitors within a series are often valuable in demonstrating the interaction energies contributed by certain groups. If the aim is to characterize an enzyme active site. a quantitative approach based on physical and chemical primciples is apt to be rewarding. When one turns to the application of inhibitors to cell or tissue preparations, or to whole animals, and especially in the correlation of functional and metabolic changes, it is of the utmost importance to obtain and present 
results that are quantitatively accurate and significant. The importance of the construction of inhibition-concentration curves, both for the complex systems and their enzyme components, has been emphasized. It is hoped that more attention will be given in the future to the rates of cellular inhibition and the problems of the penetration into cells. because the reliability of the interpretations depends very much on these factors. Finally, it is important to remember that quantitatively expressed data obtained from accurately controlled experiments, even though not allowing a complete interpretation immediately, may later, in the light of further work, be very valuable in the final understanding of the phenomena and in the elaboration of comprehensive theories. 


\section{REFERENCES}

Abood, L.G.. Brunngraber, E., and Taylor, V. (1959). J. Biol. Chem. 234. 1307.

Ackermann. T. (1957). Discussions Faraday Soc. Yo. 24, 180.

Ackermann, W.W., and Potter, V.R. (1949), Proc. Soc. Exp. Biol. Med. 72, 1.

Adlams, D.H., and Quastel, J.H. (1956). Proc. Roy. Soc. B145, 472.

Adams, D.H., and Whittaker, V.P. (1950). Biochim. et Biophys. Acta 4, 543.

Agner, K., and Theorell, H. (1946). Arch. Biochem. Biophys. 10, 321.

Albers, R.W., and Brady, R.O. (1959). J. Biol. Chem. 234, 926.

Albert, A. (1952). Pharmacol. Rers. 4, 136.

Albert, A. (1960). "Selective Toxicity," 2nd ed. Wiley, New York.

Alberty, R.A. (1954). J. Am. Chem. Soc. 76, 2494.

Alberty, R.A. (1956 a). J. Cellular Comp. Physiol. 47, 245.

Alberty, R.A. (1956 b). Advances in Enzymol. 17, 1.

Alberty, R.A. (1957). In "Molecular Structure and Biological Specificity" (L. Pauling and H.A. Itano, eds.), p. 155. American Institute of Biological Sciences, Washington, D.C.

Alberty, R.A. (1958). J. Am. Chem. Soc. 80, 1777.

Alberty, R.A., Massey, V., Frieden, C., and Fuhlbrigge. A.R. (1954). J. Am. Chem. Soc. 76, 2485.

Aldous, J.G. (1948). J. Biol. Chem. 176, 83.

Aldridge, W.N. (1950). Biochem. J. 46, 451.

Aldridge, W.X. (1953a). Biochem. J. 53, 62.

Aldridge, W.N. (1953 b). Biochem. J. 54, 44:2.

Aldridge. W.N., and Davison. A.N. (1953). Biochem. J. 55, 763.

Alles, G.A., and Hawes, R.C. (1940). J. Biol. Chem. 133, 375.

Amis, E.S. (1952). J. Chem. Educ. 29, 337.

Amis, E.S. (1953). J. Chem. Educ. 30, 351.

Anfinsen, C.B. (1947). J. Cellular Comp. Physiol. 29, 323.

Anfinsen, C.B. (1956). J. Biol. Chem. 221, 405.

Applewhite, T.H., Martin, R.B., and Niemann, C. (1958). J. Am. Chem. Soc. 80, 1457.

Ariëns, E.J. (1954). Arch. intern. pharmacodynamie 99, 32.

Ariëns, E.J., Simonis, A.M., and Groot, W.M. de (1955). Arch. intern. pharmacodynamie 100, 298.

Ariëns, E.J., van Rossum, J.M., and Simonis, A.M. (1957). Pharmacol. Revs. 9, 218.

Arnon, D.I. (1952). In "Phosphorus Metabolism" (IV.D. McElroy and B. Glass, eds.),

Yol. 2, p. 67. Johns Hopkins Press, Baltimore, Maryland.

Angenstine, L.G. (1959). Proc. Natl. Biophys. Conf. Ist. Conf. Columbus Ohio p. 154.

Augustinsson, K.-B. (1948). Acta Physiol. Scand. 15, Suppl. 52.

Austin. L., and Berry, IT.K. (1953). Biochem. J. 54, 695.

Bailey, K., and Webb, E.C. (1944). Biochem. J. 38, 394.

Ballintine, E.J.. and Maren, T.H. (1955). Am. J. Ophthmol. 40, 148.

Bamann. E., and Schmeller, M. (1929). Z. physiol. Chem. 183, 149.

Barlow, C.F., Schoolar, J.C., and Roth, L.J. (1957). Neurology 7, 820. 
Barnard, M.L., and Laidler, K.J. (1952). J. Am. Chem. Soc. 74, 6099.

Barrier, G.E., and Loomis, W.E. (1957). Plant Physiol. 32, 225.

Barròn, E.S.G., and Kalnitsky, G. (1947). Biochem. J. 41, 346.

Barròn, E.S.G., and Levine, S. (1952). Arch. Biochem. Biophys. 41, 175.

Barròn, E.S.G., and Singer. T.P. (1945). J. Biol. Chem. 157, 221.

Barròn, E.S.G.. Muntz, J.A., and Gasvoda, B. (1948 a). J. Gen. Physiol. 32, 163.

Barròn, E.S.G., Nelson, L., and Ardao, M.I. (1948 b). J. Gen. Physiol. 32, 179.

Bartlett, G.R., Savage, E.. Hughes, L. and Harlow, A.A. (1953). J. Appl. Physiol. 6, 51 .

Beers, R.F., Jr. (1957). Nature 180, 246.

Beerstecher, E., and Shive, W. (1947). J. Biol. Chem. 167, 527.

Beevers, H. (1952). Plant Physiol. 27, 725.

Beinert, H., and Steyn-Parvé, E. (1958). Proc. Intern. Symposium Enzyme Chem. Tokyo and Kyoto 1957 p. 326.

Bell, R.P. (1959). "The Proton in Chemistry," p. 109. Cornell Univ. Press, Ithaca, New York.

Bender, M.L.. and Turnquest, B.WT. (1957). J. Am. Chem. Soc. 79, 1652.

Benesch, R.E., and Benesch, R. (1955). J. Am. Chem. Soc. 77, 5877.

Benson, S.W. (1960). "The Foundations of Chemical Kinetics." McGraw-Hill, New York.

Benson, S.W., Copeland, C.S., Pearson, D., and Newton, P.R. (1957). Sixth Tech. Rep., Phys. Sci. Div.

Bergmann, F. (1955). Discussions Faraday Soc. No. 20, 1:6.

Bergmann, F. (1958). Advances in Catalysis 10, 130.

Berliner, R.W., Earle, D.P., Jr., Taggart, J.Y., Zubrod, C.G., Weleh, W.J., Conan,

N.J., Bauman, E., Sendder, S.T., and Shannon, J.A. (1948). J. Clin. Invest. 27. 98.

Bernhard, R.A., and Niemann, C. (1957). J. Am. Chem. Soc. 79, 4091.

Bernhard, S.A. (1955). J. Am. Chem. Soc. 77, 1966.

Berry, W.K. (1953). Biochem. J. 54, 700.

Bersin, T. (1934). Z. physiol. Chem. 222, 177.

Bertalanffy, L. von (1950). Science 111, 23.

Best, J.B. (1955 a). Federation Proc. 14, 13.

Best, J.B. (1955 b). J. Cellular Comp. Physiol. 46, 1.

Best, J.B. (1955 c). J. Cellular Comp. Physiol. 46, 29.

Biddulph, O., and Cory, R. (1957). Plant Physiol. 32, 608.

Biddulph, O., and Harkle, J. (1944). Am. J. Botany 31, 65.

Bierman, A. (1954). Bull. Wath. Biophys. 16, 203.

Bjerrum, J. (1941). "Metal Ammine Formation in Aqueous Solution." P. Haase, Copenhagen.

Bjerrum, N. (19:3). Z. physik. Chem, 104, 147.

Blaschlio, H., and Himms, J.M. (1955). Brit. J. Pharmacol. 10, 451.

Bloch-Frankenthal, L. (1954). Biochem. J. 57, 87.

Blum. J.J. (1955). Arch. Biochem. Biophys. 55, 486.

Bhum, J.J. (1956). Biochim. et Biophys. Acta 21, 159.

Blum, J.J., and Jenden, D.J. (1957). Arch. Biochem. Biophys. 66, 316.

Bockris, J. O'M. (1949). Quart. Revs. (London) 3, 173.

Bockris. J. O'M., and Conway, B.E. (1954). "Modern Aspects of Electrochemistry." Academic Press, New York. 
Bonner, J., and Wildman, S.G. (1946). Arch. Biochem. Biophys. 10, 497.

Booman, K.A., and Niemann, C. (1957). Biochim. et Biophys. Acta 26, 439.

Borei, H. (1942). Biochem. Z. 312, 160.

Bowyer, F. (1957). Intern. Rev. Cytol. 6, 469.

Brady, G.W. (1958). J. Chem. Phys. 28, 464.

Brady, G.IV., and Krause, J.T. (1957). J. Chem. Phys. 27, 304.

Brand, T. von, and Johnson, E.M. (1947). J. Cellular Comp. Physiol. 29, 33.

Briggs, G. E., and Haldane, J.B.S. (1925). Biochem. J. 19, 338.

Brody, T.M. (1955). Pharmacol. Revs.. 7, 335.

Brönsted, J.N. (1922). Z. physik. Chem. 102, 169.

Brönsted, J.N. (1925) Z. physik. Chem. 115, 337.

Brönsted, J.N. (1928). Chem. Revs. 5, 231.

Brooks, C. MeC. (1956). In "The Physiology of Induced Hypothermia" (R.D. Dripps ed.) p. 287. Natl. Acad. Sci. Washington, D.C.

Brown, A. J. (1902). J. Chem Soc. 81, 373.

Brown, D.E., Johnson, F.H., and Marsland, D.A. (1942). J. Cellular Comp. Physiol. $20,151$.

Brown, G.M. (1959). J. Biol. Chem. 234, 370.

Brown, T.L. (1959). J. Am. Chem. Soc. 81, 3229.

Brücke, F. T. (1933). Biochem. Z. 264, 157.

Bruice, T.C., and Schmir, G.L. (1957). J. Am. Chem. Soc. 79, 1663.

Buckingham, A.D. (1957). Discussions Faraday Soc. No. 24, 151.

Buckinghan, A.D. (1959). Quart. Revs. (London) 13, 183.

Bueding, E., and MacKinnon, J.A. (1955). J. Biol. Chem. 215, 495.

Bulen, W.A. (1956). Arch. Biochem. Biophys. 62, 173.

Burton, A.C. (1936). J. Cellular Comp. Physiol. 9, 1.

Burton, A.C. (1939). J. Cellular Comp. Physiol. 14, 327.

Cafruny, E.J., and Farah, A. (1956). J. Pharmacol. Exptl. Therap. 117, 101.

Cafruny, E.J., Farah, A., and Di Stefano, H.S. (1955). J. Pharmacol. Exptl. Therap. 115, 390.

Cahill, G.F., Ashmore, J., Renold, A.E., and Hastings, A.B. (1959). Am. J. Med. 26, 264.

Caldwell, P.C. (1956). Intern. Rev. Cytol. 5, 229.

Caldwell, P.C. (1958). J. Physiol. (London) 142, 22.

Calvin, M., Bassham, J.A., Benson, A.A., Lynch., V.H., Ouellet, C., Schou, L., Stepka W., and Tolbert, N.E. (1951). Symposia Soc. Exptl. Biol. 5, 284.

Cann, J.R., and Phelps, R.A. (1957). J. Am. Chem. Soc. 79, 4672.

Carr, C.W. (1956), Arch. Biochem. Biophys. 62, 476.

Carr, C.W., and Woods, K.R. (1955). Arch. Biochem. Biophys. 55, 1.

Casimir, H.B.G., and Polder, D. (1948). Phys. Rev. 73, 360.

Chadwick, L.E. (1957). In "Influence of Temperature on Biological Systems" (F.H. Johnson, ed.), p. 45. Am. Physiol. Soc. Washington, D.C.

Chance, B. (1943). J. Biol. Chem. 151, 553.

Chance, B. (1947). Acta Chem. Scand. 1, 236.

Chance, B. (1954). In "The Mechanism of Enzyme Action" (W.D. McElroy and B. Glass, eds.), p. 399. Johns Hopkins Press, Baltimore, Maryland. 
Chance, B. (1956). In "Enzymes: Units of Biological Structure and Function" (O.H. Gaebler, ed.), p. 447. Academic Press, New York.

Chance, B. (1958). Proc. Intern. Symposium Enzyme Chem. Tokyo and Kyoto 1957 p. 9.

Chance, B., and Herbert, D. (1950). Biochem. J. 46, 402.

Chance, B., and Hess, B. (1959). Science 129, 700.

Chance, B., and Neilands, J.B. (1952). J. Biol. Chem. 199, 383.

Chance, B., and Williams, G.R. (1956). Advances in Enzymol. 17, 65.

Chang, T.H., and Gerard, R.W. (1933). Am. J. Physiol. 104, 291.

Chase, A.M. (1952). J. Pharmacol. Exptl. Therap. 105, 371,

Chefurka, W. (1957). Enzymologia 18, 209.

Chessin, M., Dubnick, B., Leeson, G., and Scott, C.C. (1959). Ann. N. Y. Acad. Sci. $80,597$.

Clark, A.J. (1937). "Handbuch der experimentellen Pharmakologie, Heffter's" (W. Heubner and J. Schüller, eds. ), Vol. IV. Springer, Berlin.

Cleland, K.W. (1949). Proc. Linnean Soc. N. S. IJ ales 74, 296.

Cohen, J.A., Warringa, M.G.P.J., and Bovens, B.R. (195I). Biochim. et Biophys. Acta 6, 469.

Cohn, E.J., and Edsall, J.T. (1943). "Proteins, Amino Acids and Peptides." Reinhold, New York.

Cole, S.W. (1904). J. Physiol. (London) 30, 202.

Colowick, S.P., and Kalckar, H.M. (1943). J. Biol. Chem. 148, 117.

Colowick, S.P., and Kaplan, N.O. (1955). "Nethods in Enzymology." Academic Press, New York.

Commoner, B. (1940). Biol. Revs. Cambridge Phil. Soc. 15, 168.

Conway, B.E., Bockris, J. O'M., and Ammar, I.A. (1951). Trans. Faraday Soc. 47, 756.

Conway, E.J. (1954). Symposia Soc. Exptl. Biol. 8, 297.

Conway, E.J. (1957). Physiol. Revs. 37, 84.

Conway, E.J., and Downey, M. (1950). Biochem. J. 47. 347.

Cooil, B.J. (1952). Plant Physiol. 27, 49.

Cook, S.F. (1926 a). J. Gen. Physiol. 9, 575.

Cook, S.F. (1926 b). J. Gen. Physiol. 9. 631.

Cook, S.F. (1926c). J. Gen. Physiol. 9, 735.

Cooper, E.A. (1912). Biochem. J. 6, 362.

Cori, C.F. (1956). In "Currents in Biochemical Research" (D.E. Green, ed.), p. 198.

Interscience, New York.

Cornman. I. (1950). Biol. Bull. 99. 338.

Cornman, I. (1957). In "Influence of Temperature on Biological Systems" (F.H. Johnson, ed.), p. 137. Am. Physiol. Soc., Washington, D.C.

Covin, J.M., and Berman, D.A. (1956). J. Pharmacol. Exptl. Therap. 117, 443.

Cowles, P.B., and Klotz, I.M. (1948). J. Bacteriol. 56, 277.

Crafts, A.S. (1951), Botan. Rev. 17, 203.

Crandall, D.I. (1955). In "Amino Acid Metabolism" (W.D. McElroy and H.B. Glass, eds.), p. 867. Johns Hopkins Press, Baltimore, Maryland.

Crane, R.K., and Sols, A. (1953). J. Biol. Chem. 203, 273.

Crank, J. (1956). "The Mathematics of Diffusion." Oxford Univ. Press, London and New York. 
Crozier, W.J. (1924). J. Gen. Physiol. 7, 189.

Cunningham, L.W., Jr. (1954). J. Biol. Chem. 207, 443.

Dalziel, K. (1957). Biochem. J. 66, 34p.

Danielli, J.F. (1937). Proc. Roy. Soc. B122, 155.

Danielli, J.F. (1941), Biochem. J. 35, 470.

Danielli, J.F., Danielli, M.. Fraser, J.B., Mitchell, P.D.. Owen, L.N., and Shaw, G. (1947). Biochem. J. 41, 325.

Das, N.B. (1937). Biochem. J. 31, 1124.

Davenport, H.W. (1955). Communicated verbally at Symposium on Active Transport at Madison, Wisconsin.

Davenport, H.W. (1956). Am. J. Physiol. 184, 1.

Davenport, H.W., Cliavré, Y.J., and Davenport, V.D. (1955). Am. J. Physiol. 182, 221.

Davies, D.R.. and Green, A.L. (1956). Biochem. J. 63, 529.

Davies, F., Davies, R.E., Francis. E.T.B., and Whittam, R. (1952). J. Physiol. (London) $118,276$.

Davies, J.H. (1955). Ph. D. Thesis, University of Bristol; quoted by Chadwick (1957).

Davies, R.E. (1954). Symposia Soc. Exptl. Biol. 8, 453.

Davison, A.N. (1957). Biochem. J. 67, 316.

Davson, H., and Danielli, J.F. (1952). "The Permeability of Natural Membranes," ?nd ed. Macmillan, New York.

Day, B.E. (1952). Plant Physiol. 27, 143.

de Boer, J.H. (1936). Trans. Faraday Soc. 32, 10.

de Boer, J.H. (1950). Advances in Colloid Sci. 3, 1.

Demis, D.J., and Rothstein, A. (1955). Am. J. Physiol. 180, 566.

Denbigh, K.G., Hicks, M., and Page, F.M. (1948). Trans. Faraday Soc. 44, 479.

Derjaguin, B.V., Abrikosova, T.I., and Lifshitz. E.M. (1956). Quart. Revs. (London) $10,295$.

Derrick, M.V., Miller. R.E., and Sevag, M.G. (1953). Federation Proc. 12, 196.

Dickman, S.R., and Ring, B. (1958). J. Biol. Chem. 231, 741.

Dikstein, S. (1959). Biochim. et Biophys. Acta 36, 397.

Dixon, M. (1949). "Multi-enzyme Systems." Cambridge Univ. Press, London and New York.

Dixon, M. (1953 a). Biochem. J. 55, 161.

Dixon, M. (1953 b). Biochem. J. 55, 170.

Dixon, M., and Thurlow, S. (1924). Biochem. J. 18, 976.

Dixon, M., and Webb, E.C. (1958). "Enzymes." Academic Press, New York.

Dodgson, K.S., Spencer, B., and Williams, K. (1956). Nature 177, 432.

Doherty, D.G., and Vaslow, F. (1952). J. Am. Chem. Soc. 74, 931.

Dolin, M.I. (1956). Arch. Biochem. Biophys. 60, 499.

Doty, P., and Geiduschek, E.P. (1953). In "The Proteins" (H. Neurath and K. Bailey, eds.), Vol. I-A, p. 393. Academic Press, New York.

Doy, C.H., and Pittard, A.J. (1960). Nature 185, 941.

Dubuisson. M., and Schulz, W. (1938). Arch. ges. Physiol. Pflüger's 239, 776.

Eagle, H., Magnuson, H.J., and Fleischman, R. (1946). J. Clin. Invest. 25, 451.

Easson, L.H., and Stedman, E. (1936). Proc. Roy. Soc. B121, 142. 
Ebersole, E.R., Guttentag. C., and Wilson, P.W. (1944). Arch. Biochem. Biophys. 3, 399 .

Edsall, J.T., and Wyman, J. (1958). "Biophysical Chemistry," Vol. I. Academic Press, New York.

Eigen, M. (1957). Discussions Faraday Soc. No. 24, 25.

Eik-nes, K., Schellman, J.A., Lumry, R., and Samuels, L.T. (1954). J. Biol. Chem. 206,411 .

Ellinger, P. (1924). Z. physiol. Chem.136, 19.

Elliot, J.S., Sharp, R.F., and Lewis, L. (1958). J. Phys. Chem. 62, 686.

Ellis, S., Plachte, F.L., and Strans, O.H. (1943). J. Pharmacol. Exptl. Therap. 79, 295.

Ennis, H.L., and Gorini, L. (1959). Federation Proc. 18, 222.

Epstein, S.I., Doty, P., and Boyd, W.C. (1956). J. Am. Chem. Soc. 78, 3306.

Enler, H. von (1942). Ber. deut. chem. Ges. 75, 1876.

Everett, D.H. (1957). Discussions Faraday Soc. No. 24, 220.

Eys, J. van (1958). J. Biol. Chem. 233, 1203.

Eys, J. van, Stolzenbach, F.E., Sherwood, L., and Kaplan. N.O. (1958). Biochim. et Biophys, Acta 27, 63.

Field, J., and Field, S.M. (1932 a). Proc. Soc. Exptl. Biol. Med. 29, 357.

Field, J., and Field, S.M. (1932 b) Proc. Soc. Exptl. Biol. Hed. 29. 733.

Fink, L.D., and Wright, H.N. (1948 a). J. Pharmacol. Exptl. Therap. 94, 455.

Fink, L.D., and Wright, H.N. (1948 b). J. Pharmacol. Exptl. Therap. 94, 465.

Fleisher, J.H., Michel, H.O., Yates, L., and Harrison, C.S. (1960). J. Pharmacol. Exptl. Therap. 129, 31.

Florijn, E., and Smits, G. (1949). Nature 164, 699.

Florijn, E., Gruber, M., Leijnse, B., and Huisman, T.H.J. (1950). Biochim. et Biophys. Acta 5, 595 .

Foldes, F.F., Erdös, E.G., Zsigmond, E.K., and Zwartz, J.A. (1960). J. Pharmacol. Exptl. Therap. 129, 394.

Frank, H.S., and Wen, W.-Y. (1957). Discussions Faraday Soc. No. 24, 133.

Frankenhaeuser, B., and Hodgkin, A.L. (1955). J. Physiol. (London) 129, 51 p.

Fraser, M.J., Kaplan, J.G., and Schulman, J.H. (195.5). Discussions Faraday Soc. No. 20, 44.

Frazier, J.C., Schaff, J.F., and McFarland, R.H. (1956). Botan. Gaz. 118, 122.

Freedman, A.D., and Graff, S. (1958). J. Biol. Chern. 233, 292.

Fridovich, I., and Handler, P. (1958), J. Biol. Chem. 233, 1581.

Frieden, C. (1958). J. Am. Chem. Soc. 80, 6519.

Frieden, C., and Alberty, R.A. (1955). J. Biol. Chem. 212, 859.

Friedenwald, J.S., and Maengwyn-Davies, G.D. (1954.) In "The Mechanism of Enzyme Action" (W.D. MeElroy and B. Glass, eds.). Johns Hopkins Press, Baltimore, Maryland.

Friedheim, E.A.H. (1934). Biochem. J. 28, 180.

Frisell, W.R.. and Mackenzie, C.G. (1955). J. Biol. Chem. 217, 275.

Fuhrman, F.A. (1956). In "The Physiology of Induced Hypothermia" (R.D. Dripps, ed.), p. 50. Natl. Acad. Sci. ,Washington, D.C.

Fuhrman, F.A., and Field, J. (1942). J. Pharmacol. Exptl. Therap. 75, 58.

Fuhrman, F.A., and Field, J. (1943). J. Cellular Comp. Physiol. 21, 307. 
Fuhrman, G.J., Fuhrman, F.A., and Field, J. (1950). Am. J. Physiol. 163, 642.

Fuoss, R.M. (1958). J. Am. Chem. Soc. 80, 5059.

Fuoss, R.M., and Kraus, C.A. (1933). J. Am. Chem. Soc. 55, 1019.

Furchgott, R.F. (1955). Pharmacol. Revs. 7. 183.

Gale, E.F. (1957). Proc. Roy. Soc. B146, 166.

Gamble, J.L., Jr., and Najjar, V.A. (1955). J. Biol. Chem. 217, 595.

Gardner, E.A., Wilson, M., and Farah, A. (1954). J. Pharmacol. Exptl. Therap. 110, 166.

Gavrilescu, N., and Peters, R.A. (1931). Biochem. J. 25, 2150.

George, J.H.B. (1959). J. Am. Chem. Soc. 81, 5530.

George, P., and Lyster, R.L.J. (1957). Federation Proc. 16, 185.

Gey, K.F. (1956). Biochem. J. 64, 145.

Ghaffar, A. (1935). Quart. J. Exptl. Physiol. 25, 61.

Giaja, J., and Dimitrijevic, I.X. (1933). Arch. intern. pharmacodynamie 45, 342.

Giebisch, G., and Dorman. P.J. (1958). Proc. Soc. Exptl. Biol. Hed. 98, 50.

Glynn, I.M. (1957). Progr, in Biophys. and Biophys. Chem. 8, 241.

Goldstein, A. (1944). J. Gen. Physiol. 27, 529.

Goldstein, A. (1949). J. Pharmacol. Exptl. Therap. 95, 102.

Goldstein, A., and Doherty, M.E. (1951). Arch. Biochem. Biophys. 33, 35.

Gollan, F. (1956). In "The Physiology of Induced Hypothermia" (R.D. Dripps, ed.), p. 37. Natl. Acad. Sci., Washington, D.C.

Gordy, W. (1946). J. Chem. Phys. 14. 305.

Gots, J.S., and Gollub, E.G. (1959). Proc. Soc. Exptl. Biol. Med. 101, 641.

Grahame, D.C. (1950). J. Chem. Phys. 18, 903.

Green, A.L. (1958). Biochem. Pharmacol. 1, 115.

Green, A.L., and Smith, H.J. (1958 a). Biochem. J. 68, 28.

Green, A.L., and Smith, H.J. (1958 b). Biochem. J. 68, 32.

Green, D.E. (1936) Biochem. J. 30, 2095.

Green, D.E. (1956). In "Enzymes: Units of Biological Structure and Function" (O.H.

Gaebler, ed.), p. 465. Academic Press, New York.

Greenberg, G.R. (1956). In "Currents in Biochemical Research" (D.E. Green, ed.), p. 537. Interscience, New York.

Gressly, R., and Aebi, H. (1959). Helv. Chim. Acta 42, 1683.

Gressly, R., Erlenmeyer, H., and Aebi, H. (1959). Helv. Chim. Acta 42, 204.

Griswold, R.L., and Pace, N. (1956). Exptl. Cell. Research 11, 362.

Gruber, M., and Wassenaar, J.S. (1960). Biochim. et Biophys. Acta 38, 355.

Gryder, R.MI., Friedenwald, J.S., and Carlson, C. (1955). Arch. Biochem. Biophys. 54, 281 .

Gurd, F.R.N., and Wilcox, P.E. (1956). Advances in Protein Chem. 11, 311.

Gurney, R.IT. (1953). "Ionic Processes in Solution." NcGraw-Hill, New York.

Hadidian, Z., and Hoagland, H. (1939). J. Gen. Physiol. 23, 81.

Hadidian, Z., and Hoagland, H. (1941). J. Gen. Physiol. 24, 339.

Hakala, M.T., Glaid, A.J., and Schwert, G.W. (1956). J. Biol. Chem. 221, 191.

Hakim, A.A. (195̃). Enzymology 18, 241.

Haldane, J.B.S. (1930). "Enzymes." Longmans, Green, New York.

Haldane, J.B.S., and Stern, K.G. (1932). "Allgemeine Chemie der Enzyme." Theo.

Steinkopf Dresden and Leipzig. 
Hall, V.E., Crismon, J.M., and Chamberin, P.E. (1937). J. Pharmacol. Exptl. Therap. 59, 193.

Handley, C.A., and Lavik, P.S. (1950). J. Pharmacol. Exptl. Therap. 100, 115.

Hanly, V.F., Rowan, K.S. and Turner, J.S. (1952). Australian J. Sci. Research Ser. B5, 64 .

Hardman, H.F., Moore, J.I., and Lum, B.K.B. (1959). J. Pharmacol. Exptl. Therap. 126. 136.

Hargreaves, A.B. (1955). Arch. Biochem. Biophys. 57, 41.

Harned, H.S., and Owen, B.B. (1958). "The Physical Chemistry of Electrolytic Solutions," 3rd ed. Reinhold, New York.

Harris, J., and Hellerman, L. (1953). Federation Proc. 12, 215.

Hartley, G.S., and Roe, J.W. (1940). Trans. Faraday Soc. 36, 101.

Hartridge, H., and Roughton, F.J.W. (1927). J. Physiol. (London) 62, 232.

Harvey, E.N. (1960). In "Comparative Biochemistry" (M. Florkin and H.S. Mason, eds.), Vol. II, p. 545. Academic Press, New York.

Hasted, J.B., and Roderick, G.W. (1958). J. Chem. Phys. 29, 17.

Hasted, J.B., Ritson, D.M., and Collie, C.H. (1948), J. Chem. Phys. 16, 1.

Hawking, F. (1938). Amn. Trop. Med. Parasitol. 32, 313.

Heald, P.J. (1953). Biochem. J. 55, 625.

Hearon, J.Z. (1949 a). Bull. Math. Biophys. 11, 29.

Hearon, J.Z. (1949 b). Bull. Math. Biophys. 11, 83.

Hearon, J.Z., Bemhard, S.A., Friess, S.L., Botts, D.J., and Morales, M.F. (1959).

In "The Enzymes" (P.D. Boyer, H. Lardy and K. Myrbäck, eds), Vol I, p 49.

Academic Press, New York

Helmreieh, E., and Eisen, H.N. (1958). Federation Proc. 17, 240.

Henri, V. (1902). Compt. rend. acad. sci. 135, 916.

Heppel, L.A., and Hilmoe, R.J. (I951). J. Biol. Chem. 192, 87.

Hess, S.. Weissbach, H., Redfield. B.G., and Udenfriend, S. (1958). J. Pharmacol.

Exptl. Therap. 124, 189.

Hill, R.L., and Smith, E.L. (1956). Biochim. et Biophys. Acta 19, 376.

Hill, T.L. (1956). J. Am. Chem. Soc. 78, 3330.

Hinshelwood, C.N. (1946). "The Chemical Kinetics of the Bacterial Cell." Oxford

Univ. Press, London and New York.

Hoare, J.P., and Laidler, K.J. (1950). J. Am. Chem. Soc, 72, 2487.

Hobbiger, F., O'Sullivan, D.G., and Sadler, P.W. (1958). N'ature 182, 1498.

Hobbiger, F., Pitman, M., and Sadler, P.W. (I960). Biochem. J. 75, 363.

Hoch, F.L., Williams, R.J.P., and Vallee, B.L. (1958). J. Biol. Chem. 232, 453.

Hoch, F. L., Martin, R. G., Wacker W.E.C.. and Vallee, B.L. (1960). Arch Biochem. Biophys. 91, 166.

Hoffman, B.F. (1956). In "The Physiology of Indueed Hypothermia" (R.D. Dripps, ed.), p. 302. Natl. Acad. Sci., Washington. D.C.

Hoffmann-Ostenhof, O., and Friseh-Niggemeyer, W. (1952). Honatsh. Chem. 83, 1175 .

Hofstee, B.H.J. (1955). J. Biol. Chem. 216, 235.

Hofstee, B.H.J. (I956). Enzymology 17, 273.

Hofstee, B.H.J. (1960 a). J. Pharmacol. Exptl. Therap. 128, 299.

Hofstee, B.H.J. (1960 b). J. Am. Chem. Soc. 82, 5166.

Hollander, P.B., and Webb, J.L. (1955). Circulation Research 3, 604. 
Holmes, E.G. (1933). Biochem. J. 27, 523.

Holzer, H. (1958). Ciba Foundation Symposium on Regulation of Cell Jetabolism.

Holzer, H. (1959). Ann. Rev. Biochem. 28, 171.

Holzer, H., and Goedde, H.W. (1957). Biochem. Z. 329, 175.

Houck, C.R. (1942). J. Cellular Comp. Physiol. 20, 277.

Huennekens, F.M. (1953). In "Investigations of Rates and Mechanisms of Reactions"

(S.L. Friess and A. Weissberger, eds.), p. 535. Interscience, New York.

Hultin, E. (1955). Acta Chem. Scand. 9, 1700.

Humphrey, G.F. (1950). Australian J. Exptl. Biol. Hed. Sci. 28, 1.

Hunter, A., and Downs, C.E. (1945). J. Biol. Chem. 157, 427.

Hurwitz, J. (1953). J. Biol. Chem. 205, 935.

Huzisige, H. (1954). J. Biochem. (Tokyo) 41, 605.

Ing, H.R., and Wright, W.M. (1931). Proc. Roy. Soc. B109, 337.

Irwin, M. (1925). J. Gen. Physiol. 8, 147.

Ito, E., Kondo, S., and Watanabe, S. (1955). J. Biochem. (Tokyo) 42, 793.

Jacques, A.G. (1936). J. Gen. Physiol. 19, 397.

Jandorf, B.J., and MeNamara, P.D. (1950). J. Pharmacol. Exptl. Therap. 98, 77.

Jandorf, B.J., Crowell, E.A., and Levin. A.P. (1955). Federation Proc. 14, ․ㅣ1.

Johnson, F.H., and Chase, A.Ml. (1942). J. Cellular Comp. Physiol. 19, 151.

Johnson, F.H., Brown, D.E.S., and Marsland, D.A. (1942 a). J. Cellular Comp. Physiol. 20, 269 .

Johnson, F.H., Eyring, H., and Williams, R.W. (1942 b). J. Cellular Comp. Physiol. $20,247$.

Johnson, F.H., Eyring, H., and Kearns, W. (1943). Arch. Biochem. Biophys. 3, I.

Johnson, F.H., Eyring, H., Steblay, R., Chaplin, H., Huber, C.. and Gherardi, G. (1945). J. Gen. Physiol. 28, 463.

Johnson, F.H., Flagler, E.A., Simpson, R., and MeGeer, K. (1951). J. Cellular Comp. Physiol. 37, 1.

Johnson, F.H., Eyring, H., and Polissar, M.J. (1954). "The Kinetic Basis of Molecular Biology." Wiley, New York.

Johnson, F.H., Kauzmann, W.J., and Gensler, R.L. (1948). Arch. Biochem. Biophys. 19, 229.

Jowett, M., and Brooks, J. (1928).Biochem. J. 22, 720.

Joyce, B.K., and Grisolia, S. (1959). J. Biol. Chem. 234, 1330.

Kalow, W., and Davies, R.O. (1958). Biochem. Pharmacol. 1, 183.

Kaminsky, M. (1957). Discussions Faraday Soc. No. 24, 171.

Karush, F. (1952). J. Phys. Chem. 56, 70.

Katz, S., and Klotz, I.M. (1953). Arch. Biochem. Biophys. 44, 351.

Kaufman, S., and Neurath, H. (1949). J. Biol. Chem. 180, 181.

Kaufman, S., Neurath, H., and Schwert, G.W. (1949). J. Biol. Chem. 177, 793.

Kavanau, J.L. (1950). J. Gen. Physiol. 34, 193.

Keilin, D. (1925). Proc. Roy. Soc. B98, 312.

Keilin, D. (1928). Proc. Roy. Soc. B104, 206.

Keilin, D., and Hartree, E.F. (1939). Proc. Roy. Soc. B127, 167.

Keilin, D., and Hartree, E.F. (1955). Nature 176, 200.

Keilin, D., and King, T.E. (1960). Proc. Roy. Soc. B152, 163. 
Keilin, D., and Mamn, T. (1937). Proc. Roy. Soc. B 122, 119.

Kielley, W.W., and Kielley, R.K. (1953). J. Biol. Chem. 200, 213.

Kiesow, L. (1959). Z. Naturforsch. 14, 450.

Kimmel, J.R., and Smith, E.L. (1957). Advances in Enzymol. 19, 267.

Kimura, T., and Singer, T.P. (1959). Nature 184, 791.

Kirkwood, J.G. (1939). J. Chem. Phys. 7, 911.

Kirkwood, J.G. (1954). In "The Mechanism of Enzyme Action" (W.D. MeElroy and B. Glass, eds.), p. 4. Johns Hopkins Press, Baltimore, Maryland.

Kirkwood, J.G. (1955). Discussions Faraday Socl. No. 20, 78.

Kirkwood, J.G., and Shumaker, J.B. (1952). Proc. Nat. Acad. Sci. U. S. 38, 863.

Kirkwood, J.G., and Westheimer, F.H. (1938). J. Chem. Phys. 6, 506.

Kistiakowsky, G.B., and Lumry, R. (1949). J. Am. Chem. Soc. 71, 2006.

Kistiakowsky, G.B., and Rosenberg, A.J. (1952). J. Am. Chem. Soc. 74, 5020.

Kistiakowsky, G.B., and Shaw, W.H.R. (1953a). J. Am. Chem. Soc. 75, 866.

Kistiakowsky, G.B., and Shaw, W.H.R. (1953 b). J. Am. Chem. Soc. 75, 2751.

Kistiakowsky, G.B., Mangelsdorf, P.C, Rosenberg, A.J., and Shaw, W.H.R. (1952).

J. Am. Chem. Soc. 74, 5015.

Klein, J.R. (1960). Biochim. et Biophys. Acta 37, 534.

Klenow, H. (1955). Arch. Biochem. Biophys. 58, 288.

Klotz, I.M. (1949). Cold Spring Harbor Symposia Quant. Biol. 14, 97.

Klotz, I.M. (1952). In "Modern Trends in Physiology and Biochemistry" (E.S.G. Barròn, ed.), p. 427. Academic Press, New York.

Klotz, I.M. (1953). In "The Proteins" (H. Neurath and K. Bailey, eds.), Vol. I, Pt. B, p. 727. Academic Press, New York.

Klotz, I.M. (1958). Science 128, 815.

Klotz, I.M., and Ayers, J. (1957). J. Am. Chem. Soc. 79, 4078.

Klotz, I.M., and Curme, H.G. (1948). J. Am. Chem. Soc. 70, 939.

Klotz, I.M., and Urquhart, J.M. (1949 a). J. Am. Chem. Soc. 71, 847.

Klotz, I.M., and Urqubart, J.MI. (1949 b). J. Am. Chem. Soc. 71, 1597.

Koelle, G.B. (1946). J. Pharmacol. Exptl. Therap. 88, 232.

Koffler, H., Johnson, F.H., and Wilson, P.W. (1947). J. Am. Chem. Soc. 69, 1113.

Kohn, H.I. (1935). J. Gen. Physiol. 19, 23.

Korff, R.W. von, Hacpherson, E.H., and Glaman, G.V. (1954). J. Biol. Chem. 209, 151.

Korngutl, S.F., and Stahmam, M.A. (1960). Arch. Biochem. Biophys. 91, 32.

Koshland, D.E.. Jr. (1959). J. Cellular Comp. Physiol. 54, (Suppl. 1), 245.

Koshland, D.E., Jr., and Herr., E.B., Jr. (1957). J. Biol. Chem. 228, 1021.

Koster, R. (1946). J. Pharmacol. Exptl. Therap. 88, 39.

Krebs, E.G., Kent, A.B., Graves, D.J., and Fischer, E.H., (1958). Proc. Intern. Symposium Enzyme Chem. Tokyo and Kyoto 1957 p. 41.

Krebs, H.A. (1957). Endeavour 16, 125.

Krebs, H.A. (1958). Symposium Soc. Gen. Microbiol. Sth p. 104.

Krupka, R.MI., and Laidler, K.J. (1959). Can. J. Chem. 37, 1268.

Kühns, K. (1954). Z. physiol. Chem, 298, 278.

Kuff, E.L., and Hogeboom, G.H. (1956). In "Enzymes: Units of Biological Structure and Function" (O.H. Gaebler, ed.), p. 235. Academic Press, New York.

Laidler, K.J. (1950). "Chemical Kineties." McGraw-Hill. New York. Laidler, K.J. (1955 a). Discussions Faraday Soc. No. 20, 83. 
Laidler, K.J. (1955 b). Trans. Faraday Soc. 51, 528.

Laidler, K.J. (1955 c). Trans. Faraday Soc. 51, 540.

Laidler, K.J. (1955 d). Trans. Faraday Soc. 51. 550.

Laidler, K.J. (1958). "The Chemical Kinetics of Enzyme Action." Oxford Univ. Press, London and New York.

Laidler, K.J., and Beardell, A.J. (1955). Arch. Biochem. Biophys. 55, 138.

Laidler, K.J., and Ethier, M.C. (1953). Arch. Biochem. Biophys. 44, 338.

LaidJer, K.J., and Eyring, H. (1940). Ann. N. Y. Acad. Sci. 39, 303.

Laidler, K.J., and Hoare, J.P. (1949), J. Am. Chem. Soc. 71, 2699.

Laidler, K.J., and Socquet, 1.M. (1950). J. Phys. Chem. 54, 519.

Landahl, H.D. (1958). Bull. Hath. Biophys. 20, 1.

Landau, J., and Marsland. D.A. (1952). J. Cellular Comp. Physiol. 40, 367.

Lardy, H.A., and Phillips, P.H. (1943). J. Biol. Chem. 148. 333.

Laties, G.G. (1949). Arch. Biochem. Biophys. 20, 284.

Latimer, W.M., Pitzer, K.S., and Slansky, C.M. (1939). J. Chem. Phys. 7, 108.

Laurence, D.J.R. (1955). Discussions Faraday Soc. No. 20, 255.

Lee, T.S. (1953). In "Investigation of Rates and Mechanisms of Reactions" (S.L.

Friess and A. Weissberger, eds.), p. 100. Interscience, New York.

Levedahl, B.H., and Bernstein, H. (1954), Arch. Biochem. Biophys. 52, 353.

Levedahl, B.H., and Perlmutter, R. (1956). Arch. Biochem. Biophys. 61, 442.

Levine, S. (195æa). Enzymology 16, 256.

Levine, S. (1954 b). Enzymology 16, 265.

Levine, S., and Wrigley, H.E. (1957). Discussions Faraday Soc. No. 24. 43.

Lewin, J.C. (1955). J. Gen. Physiol. 39, 1.

Lewis, M.S., and Saroff, H.A. (1957). J. Am. Chem. Soc. 79, 2112.

Linder, P.J., Craig, J.C., Jr., and Walton, T.R. (1957). Plant Physiol. 32. 572.

Linderstrøm-Lang, K., and Holter, H. (1931). Compt, rend. trav. lab. Carlsberg 19 (4).

Lineweaver, H., and Burk. D. (1934). J. Am. Chem. Soc. 56. 658.

Link, G.K.K., Klein, R.M., and Barron, E.S.G. (1952). J. Exptl. Botany 3, 216.

Loewe, S. (1957). Pharmacol. Revs. 9, 237.

Loewe, S., and Muischnek, H. (1926). Arch. exptl. Pathol. Pharmakol. Naunyn-Schmiedeberg's 114, 313.

Lohmann, K. (1931). Biochem. Z. 236, 444.

London, F. (1930). Z. Physik 63, 245.

London, F. (1937). Trans. Faraday Soc. 33, 8.

Loomis, W.E. (1955). In "Origins of Resistance to Toxic Agents" (M.G. Sevag, R.D. Reid, and O.E. Reynolds, eds.). p. 99. Academic Press, New York.

Lowenstein, J.1I. (1958). Biochem. J. 70, 222.

Lumry, R. (1959). In "The Enzymes" (P.D. Boyer, H. Lardy, and K. Myrbäck, eds.). Vol. I, p. 15i. Academic Press, New York.

Lumry, R., and Smith. E.L. (195̃)). Discussions Faraday Soc. No. 20, 105.

Lumry, R., Smith, E.L., and Glantz, R.R. (1951). J. Am. Chem. Soc. 73, 4330.

Lundegårdh, H. (1959). Biochim. et Biophys. Acta 35, 340.

Lundsgaard, E. (1930 a). Biochem. Z. 217, 162.

Lundsgaard. E. (1930 b). Biochem. Z. 220, 8.

Lundsgaard, E. (1932). Biochem. Z. 250, 61.

Lynen, F. (1958). Proc. Intern. Symposium Enzyme Chem. Tokyo and Kyoto 1957 p. 25. 
MeElroy, W.D., and Kipnis, D.M. (1947). J. Cellular Comp. Physiol. 30, 359.

Macfarlane, M. G., and Spencer, A. G. (1953). Biochem. J. 54, 569.

McIlwain, H. (1942). Brit. J. Exptl. Pathol. 23, 95.

MeIlwain, H. (1959). "Biochemistry and the Central Nervous System", 2nd ed. Little,

Brown, Boston, Massachusetts.

Mahler, H.R. (1956). Advances in Enzymol. 17, 233.

Mahler, H.R., and Elowe, D.G. (1954). J. Biol. C'hem. 210, 165.

Mahler, H.R., Hübscher, G., and Baum, H. (1955). J. Biol. Chem. 216, 625.

Maley, G.F., and Maley, F. (1959). J. Biol. Chem. 234, 2975.

Manning, D.T., and Niemamn, C. (1958). J. Am. Chem. Soc. 80, 1478.

Marcus, P.I., and Talalay, P. (1955). Proc. Roy. Soc. B 144, 116.

Margenau, H. (1939). Revs. Modern Phys. 11, 1.

Markham, R. (1958). Symposium Soc. Gen. Microbiol. Sth p. 163.

Martin, C.J., and Axelrod, A.E. (1958). Biochim. et Biophys. Acta 27, 52.

Martin, R.B., and Niemann, C. (1958). J. Am. Chem. Soc. 80, 1481.

Martin, R.B., Edsall, J.T., Wetlaufer, D.B., and Hollingworth, B.R. (1958). J. Biol. Chem. 233, 1429 .

Mason, E.A., and Hirschfelder, J.O. (1957). J. Chem. Phys. 26, 756.

Massey, V. (1953 a). Biochem. J. 53, 67.

Massey, V. (1953 b). Biochem. J. 55, 172.

Nassey, V., and Alberty, R.A. (1954). Biochim. et Biophys. Acta 13, 354.

Masuoka, D., Berman. D., and Saunders, P.R. (1952). Am. J. Physiol. 170, 301.

Mealey, J., Brownell, G.L., and Sweet, W.H. (1959). A.M.A. Arch. Neurol. Psychiat. 81,310 .

Mehler, A.H. (1957). "Introduction to Enzymology." Academic Press, New York.

Melchior, X.C., and Melchior, J.B. (1958). J. Biol. Chem. 231, 609.

Nendel, B., and Rudney, H. (1945). Science 102, 616.

Neyerhof, O., and Boyland, E. (1931). Biochem. Z. 237, 406.

Michaelis, L., and Menten, M.L. (1913). Biochem. Z. 49333.

Miller, G. W., and Evans, H.J. (1956). Nature 178, 974.

Millichap, J.G., Woodbury, D.M., and Goodman, L.S. (195̃). J. Pharmacol. Exptl. Therap. 115, 251 .

Mitchell, P. (1957). Nature 180, 134.

Mitchell, P, and Moyle, J. (1956). Biochem. J. 64, 19p.

Moelwyn-Hughes, E.A. (1949). Proc. Cambridge Phil. Soc. 45, 477.

Moelwyn-Hughes, E.A. (1957). "Physical Chemistry." Pergamon, New York.

Monod, J. (1956). In "Enzymes: Units of Biological Structure and Function" (O.H.

Gaebler, ed.), p. 7. Academic Press, New York.

Montgomery, C.M., and Webb, J.L. (1956). J. Biol. Chem. 221. 347.

Morales, M.F. (1947). J. Cellular Comp. Physiol. 30, 303.

Morales, H.F. (1955). J. Am. Chem. Soc. 77, 4169.

Morrison, J.F. (1950), Australian J. Exptl. Biol. Med. Sci. 28, 311.

Morton, R.K. (1955). Discussions Faraday Soc. No. 20, I49.

Mounter, L.A., Dien, L.T.H., and Bell, F.E. (1958). J. Biol. Chem. 233, 903.

Moyed, H.S., and Friedman, M. (1959). Federation Proc. 18, 290.

Müller, A. (1936). Proc. Roy. Soc. A154, 624.

Munck, A., Scott, J.F., and Engel, L.L. (1957). Biochim. et Biophys. Acta 26, 397.

IIurray, D.R.P. (1930). Biochem. J. 24, 1890. 
Myers, D.K. (1950). Arch. Biochem. Biophys. 27, 341.

Myers, D.K. (1952 a) Biochem. J. 51, 303.

Myers, D.K. (1952 b). Biochem. J. 52, 46.

Myers, D.K. (1952 c). Arch. Biochem. Biophys. 37, 469.

Myrbäck, K. (1926). Z. physiol. Chem. 159, 1.

Myrbäck, K.. and Vasseur, E. (1943). Z. physiol. Chem. 277, 171.

Nachmansohn, D., Rothenberg. 11.A., and Feld, E.A. (1947). Arch. Biochem. Biophys. 14, 197.

Nachmansohn, D., Rothenberg, M.A., and Feld, I.A. (1948). J. Biol. Chem. 174, 247.

Neely. W.B. (1958). Nature 182, 1007.

Neely, W.B. (1959). Arch. Biochem. Biophys. 79, 297.

Neilands, J.B. (1954). J. Biol. Chem. 208, 225.

Neilands, J.B., and Stumpf, P.K. (1958). "Enzyme Chemistry," 2nd ed. Wiley, New York.

Nelson, E., and O'Reilly, I. (1960). J. Pharmacol. Exptl. Therap. 129, 368.

Nelson, J.M., and Schubert, M.P. (1928). J. Am. Chem. Soc. 50, 2188.

Neumann, H. (1949). Biochim. et Biophys. Acta 3, 117.

Neurath, H. (1957). Advances in Protein Chem. 12, 1.

Neurath, H., and de Maria, G. (1950). Federation Proc. 9, 208.

Neuschlosz, S.M. (1919). Arch. ges. Physiol. Pflüger's 176, 223.

Newburgh, R.W., and Burris, R.H. (1954). Arch. Biochem. Biophys. 49, 98.

Nilsson, R., Zeile, K., and Euler, H. von (1931). Z. physiol. Chem. 194, 53.

Nisonoff, A., and Pressman, D. (1957). J. Am. Chem. Soc. 79, 1616.

Novikoff, A.B. (1957). Symposia Soc. Exptl. Biol. 10, 92.

Nozaki, Y., Gurd, F.R.X., Chen, R.F., and Edsall, J.T. (1957). J. Am. Chem. Soc. 79, 2123.

Nygaard, A.P. (1956). Acta Chem Scand. 10, 408.

Onrust, H., van der Linden, A.C., and Jansen. B.C.P. (1954). Enzymologia 16, 289. Oster, G., and Kirkwood, J.G. (1943). J. Chem. Phys. 11, 175.

Osterhout, W.J.V. (1925). J. Gen. Physiol. 8. 131.

Oyakawa, E.K., and Levedahl, B.H. (1958). Arch. Biochem. Biophys. 74, 17.

Pardee, A.B. (1959). In "The Enzymes" (P.D. Boyer, H. Lardy, and K. Myrbäck, eds.), Vol. I, p. 681. Academic Press, New York.

Passynsky, A.G. (1958). Proc. Inter. Symposium Enzyme Chem. Tokyo and Kyoto 1957 , p. 350 .

Pauling, L. (1948). Nature 161, 707.

Pauling, L. (1960). "The Nature of the Chemical Bond," 3rd ed. Cornell Univ. Press, Ithaca, New York.

Pauling, L.. and Pressman, D. (1945). J. Am. Chem. Soc. 67. 1003.

Pauling. L., Pressman, D., and Grossberg, A.L. (1944). J. Am. Chem. Soc. 66, 784.

Pease, D.C., and Kitching. J.A. (1939). J. Cellular Comp. Physiol. 14, 135.

Peller, L., and Alberty. R.A. (1959). J. Am. Chem. Soc. 81, 5907.

Peters, R.A. (1920). J. Physiol. (London) 54, 260.

Peters, R.A. (1952). Proc. Roy. Soc. B139, 143.

Peters, R.A. (1955). Discussions Faraday Soc. No. 20, 189. 
Peters, R.A., Sinclair, H.M., and Thompson, R.H.S. (1946). Biochem. J. 40, 516.

Pimentel, G.C., and McClellan, A.L. (1960). "The Hydrogen Bond." W.H. Freeman, New York.

Platzman, R., and Franck, J. (1954). Z. Physik 138, 411.

Plaut, G.W.E. (1957). Arch. Biochem. Biophys. 69, 320.

Polis, B.D., and Meyerhof, O. (1947). J. Biol. Chem. 169, 389.

Pope, A., Hess, H.H., and Allen, J.N. (1957). Progr. in Neurobiol. 2, 182.

Potter, V.R. (1942). Cancer Research. 2, 688.

Potter, V.R. (1951). Proc. Soc. Exptl. Biol. Med. 76, 41.

Potter, V.R., and Blair, D.G.R. (1959). Federation Proc. 18, 304.

Potter, V.R., and DuBois, K.P. (1943). J. Gen. Physiol. 26, 391.

Potter, V.R., and Reif, A.E. (1952). J. Biol. Chem. 194, 287.

Potter, V.R., Busch, H., and Reif, A.E. (1954). Proc. Natl. Cancer. Conf. 2nd Conf. $1954,2,1294$.

Pramer, D. (1954), Ann. Botany (London) 18, 4.63.

Prankerd, T.A.J. (1956). Intern. Rev. Cytol. 5, 279.

Preiss, J.W. (1958). Arch. Biochem. Biophys. 75, 186.

Pressman, D. (1957). In "Molecular Structure and Biological Specificity" (L. Pauling and H.A. Itano, eds.), p. 1. Am. Inst. Biol. Sci., Washington, D.C.

Pressman, D., Pardee, A.B., and Pauling, L. (1945). J. Am. Chem. Soc. 67, 1602.

Pressman, D., and Siegel, M. (1953). J. Am. Chem. Soc. 75, 686.

Pressman, D., and Siegel, H. (1957). J. Am. Chem. Soc. 79, 994.

Pressman, D., Grossberg, A.L., Pence, L.H., and Pauling, L. (1946). J. Am. Chem. Soc. 68, 250.

Pressman, D., Siegel, M., and Hall, L.A.R. (1954). J. Am. Chem. Soc. 76, 6336.

Quastel, J.H., and Wheatley, A.H.M. (1932). Biochem. J. 26, 725.

Quesnel, V.C.J. (1956). Exptl. Cell. Rescarch 10, 575.

Rabin, B.R. (1958). In "Metals and Enzyme Activity" (E.M. Crook, ed.), p. 21. Cambridge Univ. Press, London and New York.

Racker, E. (1955). J. Biol. Chem. 217, 855.

Racker, E. (1956). In "Currents in Biochemical Research" (D.E. Green, ed.), p. 215. Interscience, New York.

Racker, E., and Krimsky, I. (1952). J. Biol. Chem. 198, 731.

Reiner, J.M. (1959). "Behavior of Enzyme Systems." Burgess, Minneapolis, Minnesota. Reiner, L., and Gellhorn, A. (1956). J. Pharmacol. Exptl. Therap. 117, 52.

Repaske, R. (1954). J. Bacteriol. 68, 555.

Ricks, M., and Hoskins. W.M. (1948). Physiol. Zoöl. 21, 258.

Ritson, D.M., and Hasted, J.B. (1948). J. Chem. Phys. 16, 11.

Rittenberg, D., and Krasna, A.I. (1955). Discussions Faraday Soc. No. 20, 185.

Robert, L., Robert, B., and Robert, M. (1951). Bull. soc. chim. biol. 33, 1859.

Robertson, J.D. (1957), Progr. in Neurobiol. 2, 1.

Robins, E., Smith, D.E., and Jen, M.K. (1957). Progr. in Neurobiol. 2, 205.

Roepke, M.H. (1937). J. Pharmacol. Exptl. Therap. 59, 264.

Roth, L.J., Schoolar, J.C., and Barlow, C.F. (1959). J. Pharmacol. Exptl. Therap. $125,128$.

Rothstein, A. (1954). Symposia Soc. Exptl. Biol. 8, 165. 
Roughton, F.J.W. (1960). Trans. Faraday Soc. 56, 1085.

Rydon, H.N. (1955). Discussions Faraday Soc. No. 20, 283.

Ryley, J.F. (1955). Biochem. J. 59, 361.

Salvador, R.A., and Albers, R.W. (1959). J. Biol. Chem. 234, 922.

Salvadori, M.G., and Schwarz, R.J. (1954). "Differential Equitons in Engineering Problems." Prentice-Hall, Englewood Cliffs, New Jersey.

Samoilov, O.Y. (1957). Discussions Faraday Soc. No. 24, 141.

Sanderson, D.M1., and Edson, E.F. (1959). J. Pharm. and Pharmacol. 11, 721.

Sanyal, P.N. (1959). Unpublished work.

Sapirstein, L.A. (1956). Circulation Research 4, 689.

Saroff, H.A. (1957). Arch. Biochem. Biophys. 71, 194.

Scatchard, G. (1932). Chem. Revs. 10, 229.

Scatchard, G. (1939). J. Chem. Phys. 7, 657.

Scatchard, G. (1949). Ann. N. Y. Acad. Sci. 51. 660.

Scatchard, G., Scheinberg, I.H., and Armstrong, S.H., Jr. (1950a). J. Am. Chem. Soc. 72, 535.

Scatchard, G., Scheinberg, I.H., and Armstrong, S.H., Jr. (1950 b). J. Am. Chem. Soc. 72,540 .

Scatchard, G., Hughes, W.L., Jr., Gurd, F.R.N., and Wilcox, P.E. (1954). In "Chemical Specificity in Biological Interactions" (F.R.X. Gurd, ed.), p. 193. Academic Press, New York.

Schachter, D. and Taggart, J.V. (1954). J. Biol. Chem. 211, 271.

Schales, O., Suthon, A.M., Roux, R.M., Lloyd, E., and Schales, S.S. (1948). Arch. Biochem. Biophys. 19, 119.

Schmidt, L.H., Hughes, H.B., and Smith, C.C. (1947). J. Pharmacol. Exptl. Therap. 90, 233.

Schroeder, E.F., Woodward, G.E., and Platt. M.E. (1933 a). J. Biol. Chem. 100, 525.

Schroeder, E.F., Woodward, G.E., and Platt, M.E. (1933 b). J. Biol. Chem. 101, 133.

Schwarzenbach, G. (1936). Z. physik. Chem. 176, 133.

Segal, H.L. (1959). In "The Enzymes" (P.D. Boyer, H. Lardy, and K. Myrbäck, eds.), Vol. I. p. I. Academic Press, New York.

Shack, J. (1959). J. Biol. Chem. 234, 3003.

Shacter, B. (1953). Arch. Biochem. Biophys. 46, 324 .

Shaw, W.N., and Stadie, IV.C. (1959). J. Biol. Chem. 234, 2491.

Shedlovsky, T.., and Kay, R.L. (1956). J. Phys. Chem. 60, 151.

Shine, H.J., and Niemann, C. (1955). J. Am. Chem. Soc. 77, 4275.

Shive, W., and Macow, J. (1946). J. Biol. Chem. 162, 451.

Shukuya, R. (1953). J. Biochem. (Tokyo) 40, 535.

Shukuya, R. (1954). J. Biochem. (Tokyo) 41, 469.

Siebert, G., and Smellie, R.M.S. (1957). Intern. Rev. Cytol. 6, 383.

Simon, E.W., and Beevers, H. (1952). New Phytologist 51, 163.

Singer, T.P., Kearney, E.B., and Massey, V. (1957). Advances in Znzymol. 18, 65.

Single, W.V. (1958). Ann. Botany (London) 22, 479.

Sizer, I.W. (1939). J. Gen. Physiol. 22, 719.

Sizer, I.W. (1943). Advances in Enzymol. 3, 35.

Sizer, I.W., and Gierer, A. (1955). Discussions Faraday Soc. No. 20, 248.

Skipper, H.E., Thomson, J.R., and Bell, M. (1954). Cancer Research 14, 503. 
Slater, E.C. (1949). Biochem. J. 45, 130.

Slater, E.C. (1955). Discussions Faraday Soc. No. 20, 231.

Slater, E.C., and Bonner, W.D., Jr. (1952). Biochem. J. 52, 185.

Slater, E.C., and Holton, F.A. (1954). Biochem. J. 56, 28.

Slater, J.C., and Kirkwood, J.G. (1931). Physiol. Revs. 37, 682.

Smith, E.L. (1958). J. Biol. Chem. 233, 1392.

Smith, E.L., and Hanson, H.T. (1949). J. Biol. Chem. 179, 803.

Smith, E.L., Lumry, R., and Polglase, W.J. (1951). J. Phys. Chem. 55, 125.

Smith, E.L., Finkle, B.J., and Stockell, A. (1955). Discussions Faraday Soc. No. 20, 96

Smith, H.W. (1925). Am. J. Physiol. 72, 347.

Smyth, C.P. (1955). "Dielectric Behavior and Structure." McGraw-Hill, New York. Sollmann, T., and Schreiber, N.A. (1936). A.M.A. Arch. Internal Med. 57, 46.

Solomon, A.K., and Gold, G.L. (1955). J. Gen. Physiol. 38, 371.

Sols, A., and Crane, R.K. (1954). J. Biol. Chem. 206, 925.

Spiegelman, S., and Pieiner, J.M. (1945). J. Gen. Physiol. 28, 385.

Stearn, A.E. (1949). Advances in Enzymol. 9, 25.

Stein, B.R., and Laidler, K.J. (1959). Can. J. Chem. 37, 1272.

Stewart, H.B. (1957). Federation Proc. 16, 256.

Steyn-Parvé, E.P., and Beinert, H. (1958). J. Biol. Chem. 233, 843.

Stockell, A., and Smith, E.L. (1957). J. Biol. Chem. 227, 1.

Stoppani, A.O.MI., Actis, A.S., Deferrari, J.O., and Gonzalez, E.L. (1953). Biochem. J. $54,378$.

Stout, J.W., and Hoagland, D.R. (1939). Am. J. Botany 26, 320.

Stranb, F.B. (1940). Enzymologia 9, 143.

Straus, O.H., and Goldstein, A. (1943). J. Gen. Physiol. 26, 559.

Strehler, B.L. (1955). In. "The Luminescence of Biological Systems" (F.H. Johnson, ed.), p. 209. Am. Assoc. Advance. Sei., Washington, D.C.

Sturtevant, J.M. (1954). In "The Mechanism of Enzyme Action" (W.D. McElroy and B. Glass, eds.), p. 17 and 210. Johns Hopkins Press, Baltimore, Maryland.

Sumner. J.B., and Somers, G.F. (1953). "('hemistry and Methods of Enzymes," 3rd ed. Academic Press, New York.

Supniewski, J.W., Hano, J., and Taschner, E. (1936). Bull. intern. acad polon. sci. p. 33 .

Swartz, M.N., Kaplan, N.O., and Frech, M.E. (1957). In "The Influence of Temperature on Biological Systems", (F.H. Johnson, ed.), p. 61. Am. Physiol. Soc., Washington, D.C.

Syrett, P.J. (1958). Arch. Biochem. Biophys. 75, 117.

Syrkin, Y.K., and Dyatkina, M.E. (1950). "Structure of Molecules and the Chemical Bond." Interscience, New York.

Tabor, H., Mehler, A.H., and Stadtman, E.R. (1953). J. Biol. Chem. 204, 127.

Tainter, M.L. (1934). J. Pharmacol. Exptl. Therap. 51, 45.

Tanford, C. (1955). In "Electrochemistry in Biology and Medicine" (T. Shedlovsky, ed.), p. 248. Wiley, New York.

Theorell, H., and Bonnichsen, R. (1951). Acta Chem. Scand. 5, 1105.

Thorn, M.B. (1953). Biochem. J. 54, 540.

Thorne, G.N. (1958). Ann. Botany (London) 22, 381.

Topper, Y.J., Mehler, A.H., and Bloom, B. (1957). Science 126, 1287. 
Turner, R.B. (1954). In "Chemical Specificity in Biological Interactions" (F.P.N. Gurd, ed.), p. 29. Academic Press, New York.

Tyler, H.R. (1960). Proc. Soc. Exptl. Biol. Hed. 104, 79.

Ulrich, F. (1960) Am. J. Physiol. 197, 997.

Umbarger, H.E., and Brown. B. (1958). J. Biol. Chem. 233, 415.

Utter, M.F. (1950). J. Biol. Chem. 185, 499.

van Asperen, K. (1960). Biochem. Pharmacol. 3, 136.

van Asperen, K., and Dekhnijzen, H.H. (1958). Biochim. et Biophys. Acta 28, 603. van Liere, E.J. (1942). "Anoxia, Its Effect on the Body." Univ. Chicago Press, Chicago, Illinois.

van Overbeek, J. (1956). Ann. Rev. Plant Physiol. 7, 355.

Vaslow, F., and Doherty, D.G. (1953). J. Am. Chem. Soc. 75, 928.

Velick, S.F. (1953). J. Biol. Chem. 203, 563.

Velick, S.F. (1954). In "The Mechanism of Enzyme Action" (W.D. McElroy and B. Glass, eds.), p. 491. Johns Hopkins Press, Baltimore, Maryland.

Voegtlin, C.. Rosenthal, S.M., and Johnson. J.M. (1931). Public Health Repts. (U.S.) 46,339 .

Voegtlin, C., Fitch, R.H., Kahler, H., and Johnson, J.M. (1934). Am. J. Physiol. 107, 539 .

Wakil, S.J., and Mahler, H.R. (1954). J. Biol. Chem. 207, 125.

Walker, E. (1928). Biochem. J. 22, 292.

Webb, E.C., and Horrow, P.F.W. (1959). Biochem. J. 73, 7.

Webb, J.L. (1950 a). Brit. J. Pharmacol. 5, 87.

Webb, J.L. (1950 b). Brit. J. Pharmacol. 5, 335.

Webb. J.L. (1956). Science 124, 1209.

Webb, J.L., and Hollander, P.B. (1956). Circulation Research 4, 618.

Webb, J.L., and Hollander, P.B. (1959). Circulation Research 7. 131.

Webb, J.L., Saunders, P.R., and Thiencs, C.H. (1949a). Arch. Biochem. Biophys. 22, 444.

Webb, J.L., Saunders, P.R., and Thienes. C.H. (1949b). Arch. Biochem, Biophys, 22, 458

Webb, J,L., Hollander, P.B., and Yang, W.C. (1958). Proc. I'estern Pharmacol. Soc. $1,16$.

Weil. L., and Buchert, A.R. (1952). Federation Proc. 11, 307.

Weil-Hatherbe, H., and Bone, A.D. (195I). Biochem. J. 49, 339.

Westhead, E.W., and Malmström, B.G. (1957). J. Biol. Chem. 228, 655.

Westheimer, F.H., and Kirkwood, J.G. (1938). J. Chem. Phys. 6. 513.

Westheimer, F.H., and shookhoff. H.W. (1939). J. Am. Chem. Soc. 61, 555.

Wheland, G.W. (1955). "Resonance in Organic Chemistry." Wiley, New York.

Wilhelmi, A.E., Russell, J.A., Engel, F.L., and Long, C.N.H. (1944 a). Am. J. Physsiol. 144, 669 .

Wilhelmi, A.E., Russell, J.A., Engel, F.L., and Long. C.N.H. (194t b). Am.J. Physiol. 144, 674 .

Withelmi, A.E., Pussell, J.A., Long, C.N.H., and Engel, F.L. (194t c). Am.J. Physiol. 144, 683 . 
Wilkes, B.G., and Palmer, E.T. (1932). J. Gen. Physiol. 16, 233.

Williams, R.J.P., Hosch. F.L., and Vallec, B.L. (1958). J. Biol. Chem. 232, 465.

Wilson, I.B. (1951). J. Biol. Chem. 190, 111.

Wilson, I.B. (1952 a). J. Biol. Chem. 197, 215.

Wilson, I.B. (1952 b). J. Biol. Chem. 199, 113.

Wilson, I.B. (1954). In "The Mechanism of Enzyme Action" (W.D. McElroy and B.

Glass, eds.), p. 642. Johns Hopkins Press, Baltimore, Maryland.

Wilson, I.B. (1957). In "Molecular Structure and Biological Specificity" (L. Pauling and H.A. Itano, eds.). p. 174. Am. Inst. Biol. Sci., Washington, D.C.

Wilson, I.B., and Bergmann, F. (1950). J. Biol. Chem. 185, 479.

Wilson, I.B., and Cabib, E. (1956). J. Am. Chem. Soc. 78, 202.

Wilson, I.B., and Ginsburg, S. (1958). Biochem. Pharmacol. 1, 200.

Wilson, I.B., and Quan. C. (1958). Arch. Biochem. Biophys. 73, 131.

Wilson, I.B., Ginsburg, S., and Meislich, E.K. (1955). J. Am. Chem. Soc. 77, 4286.

Wilson, I.B., Ginsburg, S., and Qnan, C. (1958). Arch. Biochem, Biophys. 77, 286.

Wilson, I.B., Hatch, M.A., and Ginsburg, S. (1960). J. Biol. Chem. 235, 2312.

Wilson, P.W. (1949). In "Respiratory Enzymes" (H.A. Lardy, ed.), p. 16. Burgess,

Minneapolis, Mimnesota.

Winer, A.D., and Schwert, G.IT. (1958). J. Biol. Chem. 231, 1065.

Winzler, R.J. (1943). J. Cellular Comp. Physiol. 21, 22.29.

Wolfe, R.G., and Neilands, J.B. (1956). J. Biol. Chem. 221, 61.

Wu, R. (1959). J. Biol. Chem. 234, 2806.

Yagi. K., Okuda, J., Ozawa, T., and Okada, K. (1959a). Biochim. et Biophys. Acta 34,372 .

Yagi, K., Ozawa, T.. and Okada, K. (1959 b). Biochim. et Biophys. Acta 35, 102.

Yanari, S., and Mitz, M.A. (1957). J. Am. Chem. Soc. 79, 1154.

Yanari, S., Snoke, J.E., and Bloch, K. (1953). J. Biol. Chem. 201, 561.

Yang, C.-C. (1954). Arch. Biochem. Biophys. 51, 419.

Yang, J.T., and Doty, P. (1957). J. Am. Chem. Soc. 79, 761.

Yang, W.C. (1957). Science 125, 1087.

Yang, W.C. (1960). Biochem. Biophys. Research Communs. 2, 22.2.

Yang, W.C., Yanasugondha, D., and Webb, J.L. (1958). J. Biol. Chem. 232, 659. Yasnoff, D.S., and Bull, H.s. (1953). J. Biol. Chem. 200, 619.

Yos, J.M., Bade, W.L., and Jehle, H. (1957). In "Molecular Structure and Biological Specificity" (L. Pauling and H.A. Itano, eds.), p. 28. Am. Inst. Biol. Sci., Washington, D.C.

Zannoni, V.G., and La Du, B.X. (1959). J. Biol. Chem. 234, 2925.

Zeller, E.A., and Bissegger, A. (1943). Helv. Chim. Acta 26, 1619.

Zeller, E.A., Schär, B., and Staehlin, S. (1939). Helv. Chim. Acta 22, 837.

Zeller, E.A., Barsky. J., and Berman, E.R. (1955). J. Biol. Chem. 214, 267.

Ziff, M., Jahn, F.P., and Renshaw, R.R. (1938). J. Am. Chem. Soc. 60, 178. 


\section{AUTHOR INDEX}

A

Abood, L. G., 433, 889

Abrikosova, I. I., 227, 893

Ackerman, T., 258, 889

Ackerman, W. W., 78, 81, 494, 889

Actis, A. S., 558, 904

Adams, D. H., 286, 288, 289, 434, 839, 889

Aebi, H., 755, 756, 895

Agner, K., 840, 889

Albers, R. W., 482, 483, 889, 903

Albert, A., 106, 723, 863, 889

Alberty, R. A., 24, 42, 111, 133, 191, $202,652,653,676,701,702,709,754$, $839,840,889,894,900,901$

Aldous, J. G., 724, 889

Aldridge. W. N., 590, 627, 628, 889

Allen, J. N., 483, 902

Alles, G. A., 126, 889

Amis, E. S., 249, 807, 889

Ammar, I. A., 246, 247, 892

Anfinsen, C. B., 194, 452, 889

Applewhite. T. H., 810, 811, 889

Ardao, M. I., 452, 890

Ariëns, E. J., 106, 508, 510, 889

Armstrong, S. H., Jr., 753, 903

Arnon, D. T., 520, 889

Ashmore, J., 344, 891

Augenstine, L. G., 194, 889

Augustinsson, K, B., 128, 139, 146, 152, 889

Austin, L., 138, 139, 889

Axelrod, A. E., 829, 900

Ayers, J., 701, 898

B

Bade, W. L., 228, 906

Bailey, K., 111, 132, 889
Ballintine, E, J., 398, 889

Bamann, E., 111, 123, 889

Barlow, C. F., 396, 397, 402, 889, 902

Barnard, II. L., 661, 810, 890

Barrier, G. E., 409, 890

Barròn, E. S. G., 452, 463, 494, 622,

$723,890,899$

Barsky, J., 558, 904

Bartlett, G. R., 482, 890

Bassham, J. A., 519, 891

Baum, H., 452, 900

Bauman, E., 395, 890

Beardell, A. J., 846, 899

Beers, R. F., Jr., 831, 890

Beerstecher, E., 498, 500, 890

Beevers, H., 723, 725, 728, 730, 731, 734, 890,903

Beinert, H., 19, $890,90 t$

Bell, F. E., 112, 130, 900

Bell, M., 499, 500, 903

Bell, R. P., 656, 890

Bender, M. L., 196, 890

Benesch, R., 703, 890

Benesch, R. E., 703, 890

Benson, A. A., 519, 891

Benson, S. W., 240, 824, 890

Bergmann, F., 202, 285, 286, 288, 890, 906

Berliner, R. WV., 395, 890

Berman, D., 516, 521, 878, 900, 892

Berman. E. R., 558, 906

Bernhard, R. A., 836, 890

Bernhard, S. A., 191, 286, 890

Bernstein, H., 316, 899

Berry, W. K., 138, 139, 889, 890

Bersin, T., 45\%, 890

Bertalanffy, L., von, 320,890

Best, J. B., 34, 434, 475, 890

Biddulph, O., 409, 410, 890 
Bierman, A., 320, 378, 890

Bissegger, A., 122, 125, 906

Bjerrum, J., 315, 890

Bjerrum, N., 696, 890

Blair, D. G. R., 369, 902

Blaschko, H., 309, 310, 890

Bloch, K., 145, 906

Bloch-Frankenthal, L., 112, 132, 890

Bloom, B., 19, 904

Blum, J. J., 35, 145, 295, 475, 476, 890

Bockris, J. O'H., 246, 247, 250, 251, 890, 892

Bone, A. O., 144, 905

Bonner, J., 723, 891

Bonner, W. D., Jr-21, 49:2, 904

Bonnichsen, R., 19, 904

Booman, K. A., 655, 891

Borei, H., 452, 891

Botts, D. J., 191, $\$ 96$

Bovens, B. R., 493, 494, 892

Bowyer, F., 775, 891

Boyd, W. C.. 266, 267, 894

Boyland, E.. 582, 583, 900

Brady, G. W., 25.2, 253, 891

Brady, R. O., 483, 889

Brand, T. von, 783, 891

Briggs, G. E., 20, 891

Brody, T. M., 523, 891

Brönsted, J. N., 816, 823, 891

Brooks, (. HcC, 781, 891

Brooks, J., 540, 580, 897

Brown, A. J., 17, 891

Brown. B., 368, 369, 905

Brown, D. E., 389, 390, 850, 851, 85:2. 891,897

Brown, G. M., 345, 891

Brown, T. L., 299, 891

Brownell, G. L., 395, 900

Brücke, F. T., 724, 891

Bruice, T. C., 196, 891

Brumngraber, E., 433, 889

Buchert, A. R., 196, 905

Buckingham, A. D., 229, 253, 258, 891

Bueding, E., 144, 891

Bulen, W. A., 622, 891

Bull, H. S., 19, 906

Burk, D., 35, 149, 150, 191, 475, 899

Burris, R. H., 520, 901
Burton, A. C., 320, 383, 795, 891

Busch, H., 81, 503, 902

Cabib, E., 45, 906

Cafruny, E. J., 405, 891

Cahill, G. F., 344, 891

Caldwell, P. C., 430, 718, 719, 727, 891

Calvin, 11., 519, 891

Cann, J. R., 818, 891

Carlson, C., 46, 89, 895

Carr, C. W., 313, 315, 891

Casimir, H. B. G., 226, 891

Chadwick, L. E., 759, 891

Chamberlin, P. E., 784, $\$ 96$

Chance, B., 19, 21, 365, 367, 379, 389, $449,461,517,518,566,777,891$

('hang, T. H., 585, 892

Chaplin, H., 787, 788, 790, 851, 853, 897

Chase, A. M., 789, 790, 892, 897

Chavré, V. J.. 504, 505, 893

Chefurka, W., 835, 892

('hen, R. F., 314, 901

Chessin, 1I., 645, 892

('lark, A. J., 571, 892

Cleland, K. W., 452, 892

Cohen, J. A., 493, 494, 892

('ohn, E. J., 295, 816, 892

Cole, S. W., 89 ?

Collie, C. H., 815, 896

Colowick, s. P., 144, 429, 710, 892

Commoner, B., 447, 892

Conan, N. J., 395, 890

Conway, B. E., 246, 247, 251, 430, 431, 890,892

Conway, E. J., 434, 892

Cooil, B. s., 723, 724, 892

Cook, S. F., 581, 582. 598, 892

Cooper, E. A., 579, 892

Copeland, (. S., 240, 890

Cori, C. F., 432, 89?

Cormman, I., 790, 791, 892

Cory, R., 409, 890

Covin, J. M., 521, 878, 892

Cowles, P. B., 738, 741, 892

('rafts, A. S., 408, 409, 892

Craig, J. C., Jr. 409, 899

(randall, D. I., 625, 892 
Crane, R. K., 144, 148, 622, 623, 624, 892,904

Crank, J., 373, 892

Crismon, J. M., 784, 896

Crowell, E. A., 634, 897

Crozier, W. J., 781, 893

Cunningham. L. W. Jr., 636, 893

Curme, H. G., 753, 898

\section{D}

Dalziel, K., 4:2, 893

Danielli, J. F., 646, 720. 775, 893

Danielli, M., 646, 813

Das, N. B., 878, 893

Davenport, H. W., 486, 504, 505, 893

Davenport, Y. D., 427, 504, 505. 893

Davies, D. R., 636, 893

Davies. F., 431, 893

Davies, J. H., 760, 893

Davies, R. E., 431, 893

Davies, R. O., 485, $89 \%$

Davison, A. ㅊ., 545, 548, 558, 561, 627, 889,893

Davson, H., 775, 893

Day, B. E.. 407, 893

de Boer, J. H., 244, 298, 893

Deferrari, J. 0., 558, 901

Dekhuijzen, H. М., 569. 570, 905

de Maria, G., 839, 901

Demis, D. J., 588, 589, 893

Denbigh, K. G., 387, 388, 893

Derjaguin, B. V., 227, 893

Derrick, M. V.. 434, 893

Dickman, S. R., 111, 12.2, 831, 893

Dien, L. T. H., 112, 130, 900

Dikstein, s., 눅, 893

Dimitrijevic, I. N., 784,895

Di Stefano, H. S., 405, 891

Dixon, II., 13, 16, 20, 39, 42, 47, 60, $61,65,111,116,117,132,133,153$, $191,196,319,346,347,355,373,457$. 482, 652, 661, 663, 683, 768, 795, 880. 893

Dodgson, K. S., 59, 60, 893

Doherty, D. G., 19, 198, 893, 905

Doherty, M. E., 545, 546, 758, 895

Dolin, M. T., 19, 893
Dorman, P. S.. 396. 405. 895

Doty, P., 195, 266, 267, 277, 893, 894, 906

Downey, M., 434, 892

Downs, C. E., 153, 191, 897

Doy, C. . . 369,893

Dubnick, B., 645, 892

Du Bois, K. P., 494, 902

Dubuisson, M., 719, 893

Dyatkina, M. E., 244, 908

Eagle, M., 646, 893

Earle, D. P. Jr., 395, 890

Easson, L. H., 67, 71, 73, 611, 893

Ebersole, E. R., 59, 894

Edsall, J. T., 96, 워, 295, 313, 314, 315, 697, 703, 816, 892, 894, 900, 901

Edson, E. F., 632, 636, 903

Eigen, М., $271,89.1$

Eik-nes, K.. 317, 894

Eisen, H. N.. 504, 896

Ellinger, P., 782, 783, 894

Elliot, J. S., $517,89 t$

Ellis, S., 640, 641, $\$ 94$

Elowe, D. G.. 201, 900

Engel, F. L., 298, 90.5

Engel, L. L., 458, 900

Ennis, H. L., 369, $89 t$

Epstein. S. I.. 266, 267, 894

Erdös, E. G., 495, 496, 897

Erlenmeyer, H., 75.5, 89.5

Ethier, M. C.. 265, 267, 294, 807, 811, 899

Euler, H. von 104, 542, 894, 901

Evans, H. J., 83:. 839, 900

Everett, D. H., 257, 894

Evring, H., 263, 510, 511, 760, 787, 788, $790,807,84 \%, 851.853,897,899$

Eys, J. van 19, 60, 196, 894

\section{F}

Farah, A., 405, 586, 588, 891, 895

Feld, E. A., 759, 901

Field, J., 536, 584, 585, 586, 782, 784, $785,894,895$

Field, S. MI., 584, 586, 894

Fink, L. D., 64t, 894 
Finkle, B. J., 812, 904

Fischer, E. H., 344, 898

Fitch, R. H., 719, 905

Flagler, E. A., 790, 852, 854, 897

Fleisher, J. H., 636, 894

Fleishmann, R., 646, 893

Florijn, E., 858, 861, 862, 894

Foldes, F. F., 495, 496, 894

Francis, E. T. B., 431, 893

Franck, J., 252, 902

Frank, H. S., 256, 257, 894

Frankenhauser, B., 435, 894

Fraser, J. B., 646, 893

Fraser, M. J., 436, 894

Frazier, J. C., 408, 894

Frech, M. E., 778, 90.1

Freedman, A. D., 341, 484, 894

Fridovich, I., 121, 894

Frieden, C., 111, 660, 701, 702, 840, 889, 894

Friedenwald, J. S., 46, 54, 59, 82, 89,

$118,131,191,652,894,895$

Friedheim, E. A. H., 452, 894

Friedman, M., 369, 900

Friess, S. L., 191, 896

Frisch-Niggemeyer, W., 452, 896

Frisell, W. R., 202, 295, 296, 894

Fuhlbrigge, A. R., 111, 840, 889

Fuhrman, F. A., 536, 585, 781, 782,

$784,785,894,895$

Fuhrman, G. J., 782, 895

Fuoss, R. M., 273, 805, 895

Furchgott, R. F., 466, 468, 895

\section{G}

Gale, E. F., 434, 895

Gamble, J. L. Jr., 144, 895

Gardner, E. A., 586, 588, 895

Gasvoda, B., 463, 890

Gavrilesco, N., 49, 895

Geiduschek, E. P., 277, 893

Gellhorn, A., 412, 902

Gensler, R. L., 755, 847, 848, 897

George, J. H. B., 252, 271, 895

George, P., 198, 895

Gerard, R. W., 585, 892

Gey, K. F., 482, 895
Ghaffar, A., 583, 584, 895

Gherardi, G., 787, 788, 790, 851, 853, 897

Giaja, J., 784, 895

Giebisch, G., 396, 405, 895

Gierer, A., 30, 903

Ginsburg, S., 630, 631, 632, 634, 635, 636,906

Glaid, A. J., 111, 122, 125, 895

Glaman, G. V., 839, 898

Glantz, R. R., 111, 120, 830, 899

Glynn, I. M., 775, 895

Goeddc, H. W., 345, 897

Gold, G. L., 431, 90.1

Goldstein, A., 23, 45, 67, 68, 69, 72, 313, $545,546,553,554,557,611,613,614$, $646,758,895,904$

Gollan, F., 781, 895

Gollub, E. G., 369, 895

Gonzalez, E. L., 558, 904

Goodman, L. S., 399, 900

Gordy, W., 215, 895

Gorini, L., 369, 894

Gots, J. S., 369, 895

Graff, S., 34l, 484, 897

Grahame, D. C., 246, 247, 895

Graves, D. J., 344, 898

Green, A. L., 634, 636, 646, 893, 895

Green, D. E., 372, 452, 895

Greenberg, G. R., 319, 380, 895

Gressly, R., 755, 756, 895

Grisolia, S., 112, 897

Griswold, R. L., 431, 895

Groot, W. M. de 106, 889

Grossberg, A. L., 108, 247, 279, 281, 284, 901,902

Gruber, M., 180, 858, 861, 862, 894, 895

Gryder, R. I., 46, 89, 895

Gurd, F. R. N., 313, 314, 895, 901, 903

Gurney, R. W., 245, 895

Guttentag, C., 59, 894

\section{$\mathrm{H}$}

Hactidian, Z., 798, 895

Hakala, M. T., 111, 122, 125, 895

Hakim, A. A., 19, 895

Haldane, J. B. S., 20, 24, 117, 152, 891, 895 
Hall, L. A. R., 282, 902

Hall, V. E., 784, 896

Handler, P., 121, 894

Handley, C. A., 405, 896

Hanly, V. F., 723, 896

Hano, J. 452, $90 t$

Hanson, H. T., 839, 904

Hardman, H. F., 723, 896

Hargreaves, A. B., 622, 623, 896

Harned, H. S., 800, 801, 811, 822, 896

Harris, J., 622, 896

Harrison, C. S., 636, 897

Hartley, G. S., 720, 896

Hartree, E. F., 517, 518, 897

Hartridge, H., 573, $\$ 96$

Harvey, E. N., 789, 896

Hasted, J. B., 246, 247, 752, 815, 896 , 902

Hastings, A. B., 344, 891

Hatch, M. A., 630, 634, 635, 906

Hawes, R. C., 126, 889

Hawking, F., 589, 601, 602, 896

Heald, P. J., 515, 531, 878, 896

Hearon, J. Z., 191, 378, 896

Hellerman, L., 622,896

Helmreich, E., 504, 896

Henri, V., 17, 896

Heppel, L. A., 112, 132, 896

Herbert, D., 19, 892

Herr, E. B. Jr., 43, 898

Hess, B., 365, 461, 517, 777, 892

Hess, H. H., 483, 90?

Hess, S., 405, 406, 896

Hicks, M., 387, 388, 893

Hill, R. L., 194, 896

Hill, T. L., 264,896

Hilmoe, R. J., 112, 132, 896

Himms, J. M., 309, 310, 890

Hinshelwood, C. N., 319, 380, 389, 896

Hirschfelder, J. O., 241, 242, 900

Hoagland, D. R., 408, 904

Hoagland, H., 798, 895

Hoare, J. P., 43, 111, 145, 896, 899

Hobbiger, F., 631, 632, 633, $\$ 96$

Hoch, F. L., 84, 857,896

Hodgkin, A. L., 435, 894

Hoffman, B. F., 781, 896

Hoffmann-Ostenhof, O., 452, 896
Hofstee, B. H. J., 111, 120, 124, 137, 152, $191,837,896$

Hogeboom, G. H., 433

Hollander, P. B., 434, 466, 473, 645. $725,781,896,905$

Hollingworth, B. R., 699, 703, 900

Holmes, E. G., 586, 587, 897

Holter, H., 432, 899

Holton, F. A., 452, 521, 904

Holzer, H., 345, 379, 390, 897

Hosch, F. L., 567, 906

Hoskins, W. M., 721, 902

Houck, C. R., 786, 897

Huber, C.. 787, 788, 790, 851, 853, 897

Hübscher, G., 452, 900

Huennekens, F. M., 640, 897

Hughes, H. B., 395, 399, 903

Hughes, L., 48:, 890

Hughes, IV. L., Jr. 313, 903

Huisman, T. H. J., 858, 861, 862, 894

Hultin, E., 766, 897

Humphrey, G. F., 452, 897

Hunter, A., 153, 191, 897

Hurwitz, J., 145, $\$ 97$

Huzisige, H., 346, 897

Ing, H. R., 574, 897

Irwin, М., 574, 60:2, 897

Ito, E., 98, 897

\section{J}

Jacques, A. G., 721, 897

Jahn, F. P., 146, 906

Jandorf, B. J., 395, 705, 634, 897

Jansen, B. C. P., 622, 901

Jehle, H., 228, 906

Jen, M. K., 483, 902

Jenden, D. J., 35, 475, 476, 890

Johnson, E. M., 783, 891

Johnson, F. H., 2263, 389, 390, 510, 511 ,

$755,760,778,787,788,789,790,842$,

$847,848,850,851,852,853,854,891$.

897,898

Johnson, J. M., 646, 719, 905

Jowett, M., 540, 580, 897

Joyec, B. K., 112, 897 
$\mathrm{K}$

Kahler, H. 719, 905

Kalckar, H. M., 144, 892

Kalnitsky, G., 622, 890

Kalow, W., 485, 897

Kaminsky, M., 256, 752, 897

Kaplan. J. G., 436, 894

Kaplan, N. O., 19, 60, 429, 710, 778, 892, 894,904

Karush, F., 317, 897

Katz, S., 266, 818, 897

Kaufman, S., 809, 810, 897

Kauzmann, W. J., 755, 847, 848, 897

Kavanau, J. L., 795, 897

Kay, R. L., 803, 903

Kearney, E. B., 345, 754, 903

Kearns, W., 510, 511, 787, 897

Kejlin, D., 19, 517, 518, 523, 897, 898

Kent, A. B., 344, 898

Kielley, R. K., 145, 898

Kielley, W. W., 145, 898

Kiesow, L., 478, 480, 898

Kimmel, J. R., 202, 829, 898

Kimura, T., 346, 898

King, T. E., 523, 897

Kipnis, D. MI., 744, 745, 900

Kirkwood, J. G., 210, 211, 224, 263, 264, $267,696,822,898,901,904,905$

Kistiakowsky, G. B.. 38, 107, 111, 797, $828,839,841,898$

Kitching, J. A., 389, 901

Klein, J. R., 10.5, 898

Klein, R. M., 7:23, 899

Klenow, H., 834, 839, 898

Klotz, I. M., 203, 258, 263, 266, 300, 313, $314,316,317,701,738,741,753,754$, $818,840,897,898$

Koelle, G. B., 493, 898

Koffler, H., 778, 790, 898

Kohn, H. I., 519, 898

Kondo, S., 98, 897

Korff, R. W. von 839,898

Kornguth, S. E., 836, 837, 898

Koshland, D. E. Jr. 44, 753, 795, 797, 898

Koster, R., 493, 898

Kraus, C. A., 805, 895
Krause, J. T., 253, 891

Krasna, A. I., 902

Krebs, E. G., 344, 898

Krebs, H. A., 459, 898

Krimsky, I., 19, 902

Krupka, R. M., 186, 187, 188, 898

Kühns, K., 431, 898

Kuff, E. L., 433, 898

La Du, B. N., 112, 113, 904

Laidler, K. J., 13, 22, 30, 43, 111,128 , $145,186,187,188,191,198,263,265$, $266,267,294,652,656,661,662,666$, $707,758,807,810,811,825,842,844$, $845,846,890,894,898,904$

Landahl, H. D., 510, 899

Landau, J., 389, 899

Lardy, H. A. 45:2, 899

Laties, G. G., 723, 724, 899

Latimer, W. M., 251, 899

Laurence, D. J. R., 43, 899

Lavik, P. S., 405, 896

Lee, T. S., 373,899

Leeson, G., 645, 892

Leijnse, B., 858, 861, 862, $89 t$

Levedahl, B. H., 316, 317, 899, 901

Levin, A. P., 634, 897

Levine, S., 197, 274. 622, 890, 899

Lewin, J. C., 452, 899

Lewis, L., 817,894

Lewis, M. S., 262, 818, 899

Lifshitz, E. M., 227, 893

Linder, P. J., 409, 899

Linderstrøm -Lang, K., 432, 899

Lineweaver, H., 35, 149, 150. 191, 475, 899

Link, G. K. K., 723, 899

Lloyd, E., 60, 903

Loewe, S., 183, 495, 508, 899

Lohmann, K., 582, 583, 899

London, F., 223, 899

Long, C. N. H., 458, 905

Loomis, W. E., 407, 409, 890,899

Lowenstein, J. M., 198, 899

Lum, B. K. B., 723, 896 
Lumry, R., 111, 120, 202, 217, 292, 293, $317,758.797,813,830,894,898$, 899,901

Lundegärdh, H., 372, 899

Lundsgaard, E., 452, 515, 719, 724, 899

Lynch, Г. H., 519, 891

Lynen, F., 365, 389, $\$ 99$

Lyster, R. L. J., 198, 895

\section{M}

Me Clellan, A. L., 215, 902

Mc Elroy, W. D., 744, 745, 900

Mac Farland, R. H.. 408, 894

IIac Farlane, M. G. 431, 900

Mc Geer, K., 790, 85:2, 85.4, 897

Mcllwain, H., 106, 480, 531. 900

Mackenzie, C. G., 202, 295, 296, 894

Mackinnon, J. A., 144, 891

Mc Namara, P. D., 395, 405, 897

Macow, J., 106, 903

Mac Pherson, E. H., 839, 898

Maengwyn-Davies. C. D., 56, 59, 8:2. 118, 131, 191, 891

Magnuson, H. J., 646. 893

Mahler, H. R., 201, 345. 452, 622, 900 , 90.5

Maley, F., 369, 900

Maley, G. F., 369, 900

Malmström, B. G.. 111, 131, 133, s14, 905

Mangelsolorf, P. C.. 839, 841, 898

Mann, T., 19,898

Manning, D. T., 309, 311. 900

Marcus, P. I., 111, 12.2, 128, 299, 900

Maren, T. H., 398, 889

Margenan, H., 228, 244, 900

Markham, R., 437, 900

Markle, J., 410, 890

Marlow, A. A., 482, 890

Marsland, D. A., 389, 390, 850, 851. \$52, $891,897,899$

Jartin, C. J.. 829, 900

Martin, R. B., 697, 703, 810, 811, 829, 889,900

Martin, P. G., 857, 894

Mason, E. A., 241, 242, 900

Massey, V., 111, 345, 691, 693, 694, $701,702,754,839,840,889,900,903$
Masnoka, D., 516, 878, 900

Mealey, J., 395, 900

Mehler, A. H., 19, 145, 319, 900, 904

Meislich, E. K., 636, 906

Melchior, J. B., 835. 900

Melchior, N. C., 835, 900

Mendel, B., 827, 838, 900

Menten, M. L.. 14, 900

Heyerhof, O., 452, 58:, 583, 900, 902

Michaelis, L., 14. 900

Michel, H. O., 636, 891

Miller. G. W., 832, 839, 900

Miller, R. E., 434, 89.3

Millichap, J. G., 399, 900

Mitchell, P.. 434, 900

Mitchell. P. D., 646, 893

Mitz, M. A., 830, 837, 906

Moelwyn-Hughes, E. A., 21:2, 22.5, 229. $251,253,373,845,900$

Ilonod, .J., 434, 900

Montgomery, C. M., 777,900

Moore, J. I., 7:23, 896

Morales, M. F., 61, 191, 795, 896, 900

Morrison, J. F., 723, 900

Norrow, P. F. W., 834, 839, 905

Morton, R. K.. 198, 900

Mounter, L. A.. 112. 130, 900

Hoyed, H. S., 369, 900

Movle, J., 434, 900

Müller, A., 900

Muischnek. H., 183, 899

Munck, A.. 298, 900

Muntz, J. A., 463, 890

Murray. D. R. P., 111, 135, 137. 900

Myers, D. K., 71, 73, 75, 111, 122, 124. $127,827,828,836,838,901$

Myrbäck. K., 434, 834, 901

\section{N}

Nachmansohn, D., 759, 901

Najjar, V. A., 144, 895

Neely, W. B., 112, 196, 901

Neilands, J. B., 19, 191, 546, 549. 622?, $89: 901,906$

Nelson, E., 419, 901

Nelson, J. M., 43, 111, 133, 901

Nelson, L., 452, 890 
Neumann, H., 655, 901

Neurath, H., 197, 809, 810, 839, 897, 901

Neuschlosz, S. M., 598, 600, 901

Newburgh, R. W., 520, 901

Newton, P. R., 240, 890

Niemann, C., 309, 311, 655, 810, 811 , 829 , $836,889,890,891,900,903$

Nilsson, R., 452, 901

Nisonoff, A., 278, 280, 283, 901

Novikoff, A. B., 433, 901

Nozaki, Y., 314, 901

Nygaard, A. P., 125, 901

Okada, K., 90, 906

Okuda, J., 90, 906

Onrust, H., 622, 901

O'Reilly, I., 419, 901

Oster, G., 211, 901

Osterhout, W. J. V., 721, 722, 901

O'Sullivan, D. G., 631, 633, 896

Ouellet, C., 519

Owen, B. B., 800, 801, 811, 822, 896

Owen, L. N., 646, 893

Oyakawa, E. K., 317, 901

Ozawa, T., 90, 906

\section{P}

Pace, N., 431, 895

Page, F. M., 387, 388, 893

Palmer, E. T., 433, 906

Pardee, A. B., 147, 280, 901, 902

Passynsky, A. G., 336, 901

Pauling, L., 108, 197, 202, 215, 224, $247,259,263,277,278,279,280,281$, 284, 901, 902

Pearson, D., 240, 890

Pease, D. C., 389, 901

Peller, L., 24, 901

Pence, L. H., 247, 279, 281, 284, 902

Perlmutter, R., 317, 899

Peters, R. A., 49, 436, 452, 593, 595, $643,895,901$

Phelps, R. A., 818, 891

Phillips, P. H., 452, 899

Pimental, G. C., 215, 902
Pitman, MI., 632, 896

Pittard, A. J., 369,893

Pitzer, K. S., 251, 899

Plachte, F. L., 640, 641, 897

Platt, M. E., 724, 726, 903

Platzman, R., 252, 902

Plaut, G. W. E., 452, 902

Polder, D., 226, 891

Polglase, W. J., 292, 293, 904

Polis, B. D., 452, 902

Polissar, 11. J., 263, 760, 790, 842, 897

Pope, A., 483, 902

Potter, V. R., 78, 80, 81, 369, 487, 494, $498,502.503,734,889,902$

Pramer, D., 408, 902

Prankerd, T. A. J., 434, 902

Preiss, J. W., 434, 902

Pressman. D., 108, 202, 224, 247, 259, $263,277,278,279,280,281,282,283$, $284,901,902$

\section{Q}

Quan, C.. 290, 291, 631, 632, 906

Quastel, J. H., 434, 515, 839, 889, 902 Quesnel, V. C. J., 107, 902

\section{R}

Rabin, B. R., 200, 902

Racker, E., 19, 486, 834, 902

Riedfield, B. G., 405, 406, 896

Reif, A. E., 80, 81, 503, 902

Reiner, J. M., 14, 24, 60, 88, 120, 191, $319,443,444,453,456,563,652,666$, $760,902,901$

Reiner, L., 412, 891

Renold, A. E., 344, 891

Renshaw, R. R., 146, 906

Repaske, R., 835, 902

Ricks, M.. 721, 902

Ring, B., 111, 122, 831, 893

Ritson, D. M., 246, 247, 815, 896, 902

Rittenberg, D., 902

Robert, B., 795, 796, 902

Robert, L., 795, 796, 902

Robert, M., 795, 796, 902

Robertson, J. D., 434, 902 
Robins, E., 483, 902

Roderick, G. W., 752, 896

Roe, J. II., 720, 896

Roepke, M. H., 146, 902

Rosenberg, A. J., 38, 839, 841, 898

Rosenthal, S. M., 646, 905

Roth, L. J., 396, 397, 402, 889, 902

Rothenberg, II. A., 759, 901

Rothstein, A., 434, 588, 589, 893, 902

Roughton, F. J. IT., 573, 576, 894, 903

Roux, R. M.., 60, 903

Rowan, K. S., 723, 896

Rudney, H., 827, 838, 900

Russell, J. A., 458, 905

Rydon, H. N., 436, 903

Ryley, J. F., 45?2, 903

\section{S}

Sadler, P. W., 631, 632, 633, 896

Salvador, R. A., 482, 903

Salvadori, M. G., 566, 903

Samoilov, O. Y., 257, 903

Samuels, L. T., 317, 894

Sanderson, D. M., 63:, 636, 903

Sanyal, P. N., 749, 755, 903

Sapirstein, L. A., 400, 903

Saroff, H. A., 262. 313, 818, 899, 903

Saunders, P. R., 458, 516, 521, 579, 877. $878,900,905$

Savage, E., 48:. 890

Scatchard, G.. 313, 699, 753, 807, 903

Schachter, D., 145, 903

Schär, B., 111, 906

Schaff, S. F., 408, 894

Schales, O., 60, 903

Schales, S. S., 60, 903

Scheinburg, I. H., 753, 903

Schellman, J. A., 317, 894

Schmellen, M., 111, 123, 889

Schmidt, L. H., 395, 399, 903

Schmir. G. L., 196, $\$ 91$

Schoolar, J. C.. 396, 397, 402, 889, 902

Schou, L., 519, 891

Schreiber, N. A., 395, 901

Schroeder, E. F., 724, 726, 903

Schubert, M. P., 43, 111, 133, 901

Schulman, J. H., 436, 894
Schulz, W., 719, 893

Schwarz, R. J., 566, 903

Schwarzenbach, G., 246, 247, 903

Schwert, G. W., 111, 122, 124, 125, 895, 897,906

Scott, C. C.. 645,892

Scott. J. F., 298, 900

Scudder, S. T., 395, 890

Segal, H. L., 19, 903

Sevag. M. G., 434, 893

Shack. J., 830, 831, 903

Shacter, B., 45:, 903

Shannon, J. A.. 395, 890

Sharp, R. F., 817, 894

shaw, G., 646, 893

Shaw, W. H. R., $107,111,828,839$, 841,898

Shaw, W. ‥, 107, 434, 903

Shedlovsky, T., 803, 903

Sherwood, L., 19, 60, 89.4

shine, H. J., 8:9, 903

Shive, W., 106, 498, 500, 890, 903

Shookhoff, M. W., 697, 905

Shukuya, R., 758, 759, 903

shumaker, J. B., 263. 898

Siebert, G., 433, 903

Siegel, 11., 278, 279, 283, 284, 902

Simon, E. W., 723, 725, 728, 730, 731, 734, 903

Simonis, A. M., 106, 508, 889

Simpson, R., 790, 852, 854, 897

Sinclair, H. M., 45̃?, 90 ?

Singer, T. P., 345, 346, 494, 754, 898 , 898,903

Single. WV. V., 408, 903

Sizer, I. W., 30, 753, 795, 797, 903

Skipper, H. E., 499, 500, 903

Slansky, C. M., 251, 899

Slater, E. C., 21, 127, 105, 191, 224, 452, 492. 521, 547, 549,550, 625, 904

Smellie, R. M. S., 433, 903

Smith, C. C.. 395, 399, 903

Smith, D. E., 483, 903

Smith, E. L., 111, 120, 194, 196, 202, $292,293,667,668,812.813,8 \geq 9,830$, $839,896,898,899,904$

Smith, H. J., 634, $\$ 96$

Smith, H. It., 721, 904 
Smits, G., 861, 894

Smyth, C. P., 216, 221, 222, 904

Snoke, J. E., 145, 906

Socquet, I. M., 22, 899

Sollman, T., 395, $90 t$

Solomon, A. K., 431, 901

Sols, A., 144, 148, 622, 623, 624, $\$ 92,901$

Somers, G. F., 765, 904

Spencer, A. G., 431, 900

Spencer, B., 59, 60, 893

Spiegelman, S., 456, 904

Stadie, W. C.., 434, 903

Stadtman, E. R., 145, 904

Staehlin, S.. 111, 906

Stahmann, 11. A., 836, 837, 898

Stearn, A. E., 795, 901

Steblay, R., 787, 788, 790, 851, 853, 897

Stedman, E., 67, 71, 73, 611, $\$ 93$

Stein, B. R., 661, 662, 707, 904

Stepka. W., 519, 891

Stern, K. G., 152, 895

Stewart, H. B., 839, 904

Steyn-Parvé, E., 19, 890, 904

Stockell, A., 667, 668, 812, 813, 829, 904

Stolzenbach, F. E., 19, 60, 894

Stoppani, A. O. M., 558, 904

Stout, J. IV., 408, 904

Straub, F. B., 45.5, 904

Strans, O. H., 23, 65, 67, 68, 69, 611, $640,641,894,904$

Strehler, B. L., 851, $90 t$

Stumpf, P. K., 191, 901

Sturtevant, J. M., 141, 267, 904

Sumner, J. B., 765, 904

Supniewski, J. W., 452, 904

Suthon, A. 11., 60, 903

Swartz, M. N., 778, 904

Sweet, IV. H., 395, 900

Syrett, P. J., 390, 904

Syrkin, Y. K., 244, 904

\section{$T$}

Tabor, H., 145, 904

Taggart, J. V., 145, 395, 890, 903

Tainter, M. L.. 784, 904

Talalay, P., 111, 122, 128, 299, 900

Tanford, C., 700, 904
Taschner, E., 452, 904

Taylor, M., 433, 889

Theorell, H., 19, 840, 889, 904

Thienes, C. H., 458, 516, 521, 579, 877, 878,905

Thompson, R. H. S., 452, 902

Thomson, J. R., 499, 500, 903

Thorn, M. B., 104, 182, 183, 858, 904

Thorne, G. N., 409, 904

Thurlow, S., 111, 132. $\$ 93$

Tolbert, N. E., 519, 891

Topper, Y. J., 19, $90 t$

Turner, J. S., 723, 896

Turner, R. B., 317, 905

Timnquest, B. W.. 196, 895

Tyler, H. R., 483, 905

\section{$\cup$}

Udenfriend, S., 405, 406, 896

Ulrich, F.. 431, 90j

Umbarger, H. E., 368, 369, 905

Urquhart, J. M., 203, 263, 317, 753, 898

Utter, M. F., 838, 905

\section{V}

Vallee, B. L., 84, 567, 857, 896,906 , van, Asperen, K., 569, 570, 905

van der Linden, A. C.. 6:2

van Liere. F. J., 457, 905

van Overbeek, J., 408, 905

van Rossum. J. 1I.. 508, 889

Vaslow, F., 19, 198, 893, 905

Vassenr, E., 434, 901

Velick, S. F., 62:2, 623, 624, 905

Voegttlin, C., 646, 719, 905

\section{W}

Wacker. W. E. C., 857, 894

Wakil, S. J., 622, 905

Walker, E., 646, 905

Walton, T. R., 409, 899

Warringa, M. G. P. S., 493, 494, 892

Vassenaar, J. S., 180, $\$ 95$

Watanabe, S., 98, 897 
Webb, E. C., 13, 20, 39, 42, 47, 60, 61, 65. 111, 116, 117, 132, 133, 191, 196. $347,373,482,652,663,768,795$. $834,839,880,889,893,905$

Webb. J. L., 431, 434. 458, 466, 468, 473 . $516,521,527,579,595,645,722,725$, $777,781,877,878,896,900,905,906$

Weil, L., 196, 905

Weil-Malherbe, H., 144, 905

Weissbach, H., 405, 406, 896

Weleh, W. I., 395, 890

Wen, W. Y., 256, 257, 891

Westhead, E. W., 111, 131, 133, 814, 905

Westheimer, F. H., 696, 697, 905

Wetlaufer, D. B., 697, 703, 900

Wheatley, A H. M., 515, 903

Wheland, G. W.. 215, 216, 2200, 238. 308, 309, 905

Whittaker, V. P., 286, 288, 289, 889

Whittam, R., 431, 893

Wilcox, P. E., 313, 896903.

Wilchman, S. G., 723, $\$ 91$

Wilhelmi, A. E., 458, 905

Wilkes, B. G., 433, 906

Williams, G. R., 367, 389, 449, 517, 518,892

Williams, K., 59, 60, 893

Williams, R. J. P., 84, 567, 896, 906

Williams, R. W'., 788, 897

Wilson, I. B., 45, 202, 285, 286, 290. 291, 630, 631, 632, 634, 635, 636, 906

Wilson. M., 586, 588, 895

Wilson, P. W., 59, 191, 778, 790, 895, 898,906
Winer, A. D., 124, 125, 906

Winzler, R. J., 60, 906

Wolfe, R. G., 622, 906

Woodbury, D. M., 399, 900

Woods, K. R., 313, 315, 891

Woodward, G. E., 724, 726, 903

Wright, H. N., 64t, 894

Wright, W. MI., 574, 897

Wrigley, H. E.. 군, 899

Wu, R., 382, 906

Wyman, J., 96, 202, 313, 315, 697, 703,897

\section{Y}

Yagi, K., 90, 906

Yanari, S., 145. 830, 837, 906

Yanasughonda, D., 527, 906

Yang, С.-С.. 566, 906

Yang. J. T., 195, 906

Yang. W. C., 432, 516, 527, 645, 877, 905

Yasnoff, D. S., 19, 906

Yates, L., 636, $89 t$

Yos, J. M., 228, 906

\section{Z}

Zannoni. T. G., 112, 113, 906

Zeile, K., 452, 901

Zeller, E. A. $111,122,125,558,906$

Ziff. M., 146, 906

Zsigmond, E. K.. 49.). 496. 89.1

Zubrod, C. E., 395, 890

Zwartz, J. A., 495, 496, 894 



\section{SUBJECT INDEX}

\section{A}

Accumulation.

of inhibitors, during repeated adminis-

trations, $421-424$

of intermediates,

in localization of action, 513-515

in monolinear chains, 329. 336-337

reasons for failure to occur, 514

Acetaldehyde,

inhibition of pyrurate decarboxylase.

180 of sarcosine oxidase. 296

Acetazolamide (Diamox),

distribution in central nervons system. $396-397,399-400$

in ocular tissues, 398

rate of fall in tissues, 643

Acetone, use in lowering dielectric constant. 801

Acetylcholine,

inhibition of formation, $466-467$

of inactivation, $467-470$

Acetylcholinesterase, see Cholinesterase

Achromobacter, inhibition of luminescence by $\mathrm{HgCl}_{2}$. 786-787

Acid-base catalysis. enzymic, 30

Acids, proton association constants, 656

Aconitase, inhibition by flnorocitrate, 436

trans-Aconitate. inhibition of fumarase, effect of $\mathrm{pH}$ on. $691-692$

Activation energy, effect of dielectric constant on, 807

Activation of substrate, definition of, 28

Activator.

definition of, 45

effects on enzyme kinetics, 45-47 inhibition by reaction with, $8 \mathbf{2 - 8 5}$

in systems involving, types of plot:

obtained. 165

reaction of inhibitor with, $89-90$

Activator inhibition. 131

Active center,

area of, 194

composition of, 194-197

definition of, 194

examples of, 200-201

flexibility of, 199-203

heterogeneity of, 108-109

nature of, 194-204

of acetylcholinesterase, 200

of carboxypeptidase. 201

of cytochrome $c$ reductase, 200

of fumarase, 201

of papain. 201

of proliclase, 200

of sarcosine oxidase. 201

reactions occurring at, 13

relation of structure to inhibition.

$203-204$

terminology of, 50

topography of, 197-198

two on an enzyme, 36-39

types of, 199

Activity coefficients,

dependence on ionic strength, 816

use in enzyme kinetics, $26-27$

Activity metabolism, inhibition of, 484

Acyl dehydrogenases, enzyme-substrate complexes of, 19

Adaptation.

of cells to inhibitors.

effect on rate of inhibition, 578-579 prevented by second inhibitor, 506507 
Addition reactions, kinetics of, 39-43

Adenosinetriphosphatase (ATPase). binding energy of terminal ATP phosphate to, 295

effect of dielectric sonstant on, 294 , $810-812$

of pressure on, 845-846

energy of binding of ATP to, 294-295

entropy in formation of ES complex, $266-267$

of ATP binding, 294

evidence for a water site, 44

inhibition by dinitrophenol,

temperature characteristies of, 797

stimulation by phenylmercuric compounds, 452

Adenosinetriphosphate (ATP), dependence of cellular function on, 464-166

Administration, see Ronte of administration

Agonists,

concentration of,

effects of two inhibitors on, 506

inhibition of enzymes forming, $466-467$

of enzymes inactivating, $467-470$

Alcohol dehydrogenase,

complexes with DPN, 19

inhibition by $p$-chloromereuribenzoate. reversal with GSH, 6:2

inhibition by o-phenanthroline, 84 inactivation of enzyme during, 567 nature of $\mathrm{H}$ transfer in, 30

of $\mathrm{K}_{m}, 2 \mathrm{l}$

Aldolase. Es complex, 19

Aldoximes,

reactivation of inhibited cholinesterases, $630-633$

kinetics of, 648

Aliesterase, inhibition by inseeticides, $473-474$

Amine oxidases, see also Monoamine oxidase

inhibition by diamidines, 309-310

Amines, tissue concentrations of, effects of multiple inhibition on, 506
D-Amino acid oxidase, inhibition by chlortetracycline, 90

L-Amino acid oxidase, substrate inhibition, 111, 133

Amino acids, bond angles in, 240

Aminoacylase I, substrate inhibition, 112. 130

$p$-Aminobenzoate,

inhibition of $\mathrm{D}$-amino acid oxidase, 90 of bioluminescence, effect of pressure on, $851-852$

Amino group,

binding energy to sarcosine oxidase, 297

configuration of, 235

dipole angle of, 2.22

dipole moment of, 222

hydration of, 251,255

interaction with carboxylate group, $270-273$

molar refraction of, 222

polarization of benzene ring by, 298 van der Waals radius of, 234

$m$-Aminophenol, inhibition of $\mathrm{D}$-amino aeirl oxidase. 90

3-Amino-1, 2, 4-triazole, inhibition of catalase,

effect of temperature on, $755-756$

Ammonia ,

dipole moment of, 244

dispersion electrons of, 225

interaction energy contributions of, $\mathbf{2 4 4}$

molar refraction of, 2.25

optical eonstants, of, 225

polarizability of, 225

a-Amylase, activation by $\mathrm{Cl}^{-}, 834$

Amytal, on electron transport, 346

Anions, inlibition of enzymes, 839-840

Aniline, inhibition of D-amino acid oxidase, 90

Anoxia,

differential damage to central nervous system, 457

to embryos, $457-458$ 
effects of temperature on responses to, $780-782$

effects on brain slice respiration, 458 on heart slice respiration, 458

on liver metabolism, 458

\section{Antagonism,}

between urethane and sulfanilamide on bioluminescence, 510-511

definition of, 507.508

isobolograms for, 508-509

of fluoroacetate by malonate, 502-503

Anticompetitive inhibition, see Coupling inhibition

Antigen-antibody reactions, dilatation of antibody cavities, 202,263 entropy changes in, 266-267

liberation of water in, $\mathbf{2} 67$

thermodynamic constants of, 266

Antimycin A.

on electron transport, 346,518

on tumor succinate oxidase, 81

respiration resistant to, 346

spectroscopic changes produced by, 518 titration of succinate oxidase, 80

Antiphosphatase, inhibition of phosphatase in intact yeast, 434

Apoenzyme, definition of, 50

Arbacia eggs,

inhibition of cleavage by urethane.

effects of temperature on, 790-791

stimulation of respiration by quinones, 452

Aromatic compounds, interactions of, $297-299$

Arsenicals, inhibition of Colpidium. reversal by thioglycolate, 646

of keto acid oxidation, 10

specificity of, 877

of pyruvate oxidation, 5

of succinate oxidase, rate of, 547,549

of sulfhydryl enzymes, 9

lethal action on trypanosomes,

kinetics of, 598.599, 601

poisoning by, reversal by dimercaprol, 646 resistant pathways of pyruvate metabolism, 449

role of permeability in action of, 403

Arsenite,

concentrations in tissues, 395

entrance into fly larvas, effect of $\mathrm{pH}$ on, $721-722$

inhibition of erythrocyte respiration, 8 of fermentation, effect of fermentative rate on, 861-862

of respiration, effect of respiratory rate on, $861-862$

of succinate dehydrogenase, effect of temperature on, 749

of tumor growth, 861

lethal action on paramecia, kinetics of, 600

stimulation of diatom respiration, 452

of hexose diphosphatase, 452

of malate dehydrogenase, 452

of papain, 452

of $\mathrm{P}_{i}$-ATP exchange, 452

of spermatozoal respiration, 452

of trichomonal respiration, 452

of uricase, $45^{2}$

of yeast respiration, 452

Arylamine transacetylase, product inhibition, 145

Arylsulfatase, activation by anions, 834

inhibition by phosphate, 839

Ascites tumor cell, metabolic controls of, 461

Aspergillus niger, respiration of, rate of inhibition by heavy metals, $581-582$

Atabrine, see Quinacrine

Atomic charge, of bonded atoms, 220

Atomic polarization, definition of, 217

Atomic refraction, 219

ATP, see Adenosinetriphosphate

ATPase, see Adenosinetriphosphatase

ATP-P $P_{i}$ transphosphorylation, effect of enzyme on, 198 
Azide,

inhibition of eatalase, effect of temperature on, 755-756

of respiration, effect of $\mathrm{pH}$ on, 731 stimulation of yeast respiration, 452

Azosulfathiazole, binding to seralbumin, effect of temperature on, 753

\section{B}

\section{BAL, see Dimercaprol}

Barbiturates, see also Amytal effects of $\mathrm{pH}$ on actions of, 723 inhibition of bioluminescence, effect of pressure on, 851-852

Benzene,

dispersion electrons of, 225

dispersion energy of,

between two rings, 298

in reaction with carboxypeptidase, 294

with cholinesterase, 290

interaction with alkyl groups, 299

molar refraction of, 2.25

optical constants of, 2.25

polarizability of, 255

polarization by ionic groups, 298

van der Waals radii of, 234

Benzoate, inhibition of cleavage. effect of $\mathrm{pH}$ on, 721

Bicarbonate, concentration in tissues, 430

Biochemical lesion, definition of, 49

Bioluminescence,

effects of pressure on, 849-854

transitions between steady states, 389-390

inhibition of, effect of pressure on, 851 -

. 854

inhibition by $\mathrm{HgCl}_{2}, 786-787$

by menadione, 744-745

by sulfanilamicle, 510-512

effect of temperature on, 787-788

by urethane, $510-512$

effect of temperature, on, 787-788

multiple inhibition of, 510-5 I2

sequence of reactions in, 850
Biphasic action,

of $\mathrm{Ag}^{+}$on Aspergillus respiration, 581. 582

of inhibitors, 452

$2,2^{\prime}$-Bipyridine, rate of inhibition reversal, 645

Blood-brain barrier, effect on inhibitor distribution, 403-404

Blood supply of tissues, effect on inhibitor distribution, $400-402$ table of, $400-401$

Bond angles, table of, 240

Bond energies, table of, 220

Bond lengths, table of, 2:20

Bond moments, table of, 216

Boundary conditions. competitive inhibition, 75 noncompetitive inhibition, 69-71

Branched chains, convergent, 340-341

divergent, $341-345$

explanation of inhibitor-stable metabolism, 449

multiple inhibition of, $500-501$

Bromoacetate, on muscle lactate formation, 10

Buffer capacity, definition of, 331 of cells, effects on inhibition, 714-717, 726.728

of cyclic systems, 35:

of diffusion-limiter monolinear chains, 335

of divergent chains, $3+3$

of monolinear chains, $331-332,338$

of regenerative systems, 361

of self-regulatory (feedback) systems, $370-372$

Buffers.

effect on bindling, of ions to proteins, 840 of methyl orange to seralbumin, 314 effect on fumarase, 709-710, 840

on ionization of enzyme groups, 840 on $\mathrm{pK}_{a}$ 's of fumarase, $701-702$ 
Buffer value, definition of, 709

Bulk dielectric constant, definition of, 801

Butyrylcholine, inhibition of cholinesterase, 493-494

Calcium.

activation of cholinesterase, 837

binding to seralbumin, 818

distribution in the cell, 431

effect on substrate inhibition of cholinesterase, $127-128$

Carbamates, inhibition of bioluminescence,

effect of pressure on, 851-85:

of temperature on, 790

Carbon dioxide,

dispersion electrons of, 225

energy constants of, 237

energy curve for interaction of, 236

interaction energy of, 237

molar refraction of, 225

optical constants of, 225

polarizability of, 225

rarbon monoxide,

dipole moment of, 244

dispersion electrons of, 225

inhibition of cytochrome oxidase, 9-10 interaction energy contributions of, 244 molar refraction of, 22.5

optical constants of, 225

polarizability of, 2.25

Carboxylase, see Pyruvate decarboxylase

Carboxylate group,

binding to sareosine oxidase, 295,297

bond angle in, 240

configuration of, 235

dispersion energy in hapten-antibody

reactions, 278

hychration of, 251,255

interaction with amino group, 270-273

molar refraction of, 222.2

polarization of benzene ring by, 298

rate of proton association, 656

van der Waals radius of. 234
Carboxypeptidase,

active center of, 201

bindig energies of inhibitors of, 292-294

effect of dielectric constant on, 813-814

of ionic strength on, 830

inhibition by dipeptides, effect of ionic

strength on, 837

by phosphate, 839

nature of $\mathbf{K}_{m}, 21$

substrate inhibition, 111, 120

Catalase.

adsorption at interphases, 436

complex with acetate, 840

enzyme-substrate complex, 19

heme in protein crevice, 198

inhibitions of, effect of temperature on, $755-756$

Cavity radii, for ions, $240-241$

Cellular function, changes in, relation to enzyme inhibition, $462-474$

dependence on ATP, 464-467

effect on inhibitions, $484-485$

on penetration of inhibitors, 484

level of,

effect on inhibitions, 480-481

in tissue slices, $\mathbf{4 8 0 - 4 8 1}$

metabolic basis studied with inhibi. tors, 6

methods of establishing correlations, $471-474$

nonenzymic effects of inhibitors, 463.

464

quantitative relations in, 471

Cellular inhibition, 472-486

effects of $\mathrm{pH}$ on, $713-718$

of temperature on, 782-792

rates of, $57 \mathrm{I}-590$

reversal of, $642-646$

rellular variations, in tissue slices, 481

('hloral hydrate, inhibition of bioluminescence,

effect of pressure on, 851-852

Chlorella.

glucose effect on respiration and ATP level, time relations of, 389 
photosynthesis in, inhibition by iodoacetamide, $519-520$

Chlorguanide (Paludrine), concentration in tissues, 395, 399

Chloride,

binding to seralbumin, effect of temperature on, 753

concentration in tissues, 430

Chlormerodrin (Neohydrin),

correlation of inhibition with renal concentration, 405

kiclney-plamsa ratio of, 396-397

Chloroform, inhibition of bioluminescence,

effect of pressure on, 851-852

$p$-Chloromercuribenzoate,

displacement of $\mathrm{Fe}^{++}$from homogentisate oxidase, 625

effect on muscle contractility, 462-463 inhibition of lactate dehydrogenase, rate of, 546,549

of pyruvate decarboxylase, protec-

tion by substrate, 558

of succinate dehydrogenase, rate of, 547,549

reversal of inhibitions due to, 622

Chlortetracycline, inibition of D-amino acid oxidase, 90

Choline, inhibition of cholinesterase, effect of temperature, on, 760

Cholinesterase,

active center of, 200

boundary conditions for noncompeti-

tive inhibition of, 71

carbamylation of, 634-636

determination of serum concentration of, $71-72$

different types in serum, 485-486

dispersion interaction,

of benzene ring, 290

of halogen groups, $288-290$

of trimethylammonium ion, 245

effect of ionic strength on, 8:7-8:8 of substituent position on inhibitors, 290.291 of temperature on inhibition of, 758 760

inhibition at junctions, $467-470$

by alkylammonium ions, 285-288

by $p$-chloromercuribenzoate, failure of cysteine to reverse, 622

by E-600, 590-591

by $\mathrm{HgCl}_{2}, 545-547$

by insecticides, $473-474$

by organophosphorus compounds, induced reactivation, 630-636

kinetics of reversal, 646-648

reactivation during, 569

spontaneous reactivation, $627-630$

by phenothiazines, effect of $\mathrm{Ca}^{++}$on, 837

by phenyltrimethylammonium ions, $290-291$

by physostigmine,

dilution effect on, 611

effect of ionic strength on, 836

rate of, $554,557.558$

reversal by destruction of inhibitor, 640-641

reversal rate after adding substrate, 613-615

by quinine, temperature characteris tics of, $795-796$

interaction of substrates and inhibitors with, 284-292

ion-ion interactions of, $284-288$

multiple inhibition of, 493-495

product inhibition of, $\mathbf{1 4 6}$

role of water in kineties, 45

substrate inhibition, $111,121-122,125$.

$128,138-139$

effect of ionic strength on, 838

synthesis of new enzyme following irreversible inhibition, 645

$a$-Chymotrypsin.

association of units of, 655

effect of dielectric constant on, 809-811

of ionic strength on, 828-829

entropy change in formation of ES complex, 266

enzyme-substrate complex, 19

formation from chymotrypsinogen, 197 
inhibition by diisopropylfuorophosphate, 187

effect of $\mathrm{pH}$ on, 707

inhibition by proteins, effect of ionic strength on, 837

by D-tyrosinamides, 309, 311

inhibitions of, effect of ionic strength on, 836

nature of $K_{m}, 21$

$\mathrm{pH}$ optima of, effect of methanol on, $661-662$

Cilia, of Mytilus gill, effect of pressure on. 389

Citrate, inhibition of fumarase, effect of $\mathrm{pH}$ on, 691-69:

Cleavage, inhibition by urethane, effect of temperature on, 790-791

inhibition of, effect of $\mathrm{pH}$ on, 721

Closed systems, definition of, 320

Coenzymes,

inhibition by reaction with. 8.-8.5 reaction of inhibitor with, 89-90

Colpidium.

inhibition by arsenicals, reversal by thioglycolate, 646

rate of killing by $\mathrm{HgCl}_{2}, 593-595$

Compartmentalization.

effect on transition between steady states, 391

on transit time, $373-379$

in glycolytic pathway, 365

of intracellular enzymes. 432-435

('ompetitive displacement, of inhibitor by substrate.

rate of, $612-615$

Competitive inhibition, by substrate, 116, 118

definition of, 54-55

dilution effect on. 76

discovery of, 9

distinction between kinetic and equi-

librium competitions, 558

effect of adding substrate on, 61:-615

of dielectric constant on, 806

of endogenous metabolism on, 445 of $\mathrm{pH}$ on, 659, 668-670, 682-683

of pressure on, $846-847$

of substrate diffusion into cells on. 35

of temperature on, 750-751

equations for, 57

impossibility in zone C, 74

in diffusion-limited systems, 475-477

influence of substrate inhibition on,

134-139

intracellular, 475-478, 742-744

isobolograms. for, 184-185

methods of plotting, 150-153

mutual depletion systems. 72-75

of forward and backward reactions. 101

of monolinear chains, 332-334

of substrate-inhibited enzymes, 178

rate of, $552-558$

of reversal of, 610

ratio $K_{s} / K_{i} .104-105$

specificity of, 871

two inhibitors on single enzyme, 489 . 496

variation of $K_{i}$ with substrate, $105-106$ with respect to activator, 83-84

types of plots obtained 168-169. $171-172$

Concentration factor, in inhibitor distribution, 399

Concentration of inhibitors in tissues, see also Distribution of inhibitors, methods of expressing. 394

Concentration-time curves, for inhibitions. 595-603

examples of, 598-599

interpretation of, 599-603

methods of plotting. 596-597

Concurrent blocking of convergent chains, 501

Constants of inhibition, generalized equations for, 154-157 methods for determination of, 149-191

Convergent chains, 340-341

effects of temperature on, 770

multiple inhibition of, 500-501 
Copper,

binding to imidazole, 314

to seralbumin, $266,314-315$

effect of temperature on. 753

complexes with ammonia, 313

hydration of $\mathrm{C}^{+++}, 25 \mathrm{I}$

inhibition of respiration, rate of, 581582, $588-590$

lethal action on Nitella, concentration-time curves of, 598

Coupling inhibition (uncompetitive inhibition),

by substrate, $118-119$

definition and equations of, 59-60

types of plots obtained, 160, 166

Covalent bonds, nature of, 205-206

Covalent bond lengths, table of, 238

Covalent radii, of atoms, 233

Critical temperatures,

definition of 794-79

in inhibition of cholinesterase by quinine, 796

possible reasons for, 795

Crossover points, definition of, 518

Crotonase, inhibition by $p$-chloromercuribenzoate,

failure of cysteine and GSH to reverse. 622

Cumulation, see Accumulation

Cyanide,

diversion of electron flow, 454

effect on ademine nucleotides and phos-

phates in yeast, time relations of, 389

on spectra of yeast cytochromes, 517 inhibition of eytochrome oxiclase, 9 of respiration, effect of temperature on $782-784$

of sea urchin respiration, 8

of succinate oxidase, effect on tem-

perature characteristics, 798

resistant fraction of metabolism, 447 . 448

stimulation by removal of inhibitory metal ions, 453

treatmant of poisoning by forming methemoglobin, 615 (yclic systems, 348-354

effect of cycle intermediates on, 351 of substrate concentration on, 350 . 3.51

of temperature on, 772

multiple inhibition of, 501

Cyclohexane ring, dispersion energy in reaction with carboxypeptidase, $\mathbf{2 9 4}$

Cysteine,

ionization microconstants for, 703

reversal of inhibitions by mercurials, 622

Cytochrome c reductase, active center of. 200

Cytochrome oxidase,

effect of ionic strength on, 832

inhibition by bicarbonate, 832,839

Deacylases, function in divergent chains, 344

Debye, definition of, 210

Denaturation, effects on enzyme inhibition, 760-768

Deoxyribonnclease, effect of ionic strength on, 830-831

Destruction of inhibitor, effect on inhibitions, $637-642$

DFP, see diisopropylfuorophosphate

Diamidines, inhibition of amine oxidases, 309-310

Diamine oxidase, substrate inhibition, 111

Diamox, see acetazolamide

Diaphragm, rate of $\mathrm{Hg}^{203}$ uptake by, 590 . 591

Diatoms, respiration of, stimulation by arsenite, 45:

2,4 -Dichlorophenol, inhibition of catalase.

effect of temperature on, 755-7.56

Dielectric constant. definition of, 245

effect of ionic strength on, 815-816 
of temperature on, 75 ?

on ATPase, 810-812

on carboxypeptidase, 813-814

on $\alpha$-chymotrypsin, 809-811

on enolase, 814-815

on enzyme inhibition, 799-815

on formation of EI complex, 804-807

on ion-ion interactions, $248-249$

on papain, $812-813$

on rate of inhibition, $807-809$

examples of effects on enzymes, 809 . 815

for different types of interactions, $\mathbf{2} 48$. 249

interpretation in solvent mixtures, 801 . 802

methods for changing, 800

possible mechanisms involved, 802-804

variation with distance, $246-248$

with temperature, 245

Diethyl-p-nitrophenyl phosphate, inhibition of cholinesterase, $6 \geq 7-628$

Differential inhibition, in whole animals, 481-485

Diffusion,

effect on intracellular kineties, 475-477

into cells of different shapes, 475-477

limiting enzyme reactions, 34-35

of inhibitor from cells, 643

of substrates into compartments, 34-35

Diffusion-limited inhibition, rate expressions for, $575-576$

Diffusion-limited monolinear chains, 335337

Diffusion-limited reactions, transit time of, $373-379$

Diisopropylfluorophosphate, concentration in tissues, 395

inhibition of cholinesterase, 493-494 effect of temperature on, 759-760 in different tissues, 405

in vivo, 645-646

reactivation with aldoximes, 632

inhibition of $\alpha$-chymotrypsin, 187

effect of $\mathrm{pH}$ on, 707

use in determining amino acids at active center, 195
Dilution,

of enzyme-inhibitor systems, $76-77$

on rate of inhibition reversal, 609-611

Dimercaprol (BAL),

reversal of heavy metal poisoning, 646 of inhibitions by mercurials, 622

Dimercaprol glucoside, reversal of arsenical poisoning, 646

Dimethylcarbamylcholine, inhibition of cholinesterase, 634-636

Dimethyl-p-nitrophenyl phosphate, inhibition of cholinesterase, 627-628

3,5-Dinitro-o-cresol, inhibition of respiration,

effect of $\mathrm{pH}$ on. 731

2.4-Dinitrophenol (DNP),

effect on adenine nucleotides and phosphates in yeast, time relations of, 389 on cardiac function, 473

on glucose concentration in lymph cells, 504

inhibition of ATPase, temperature characteristics of, 797

of gastric secretion, effects of other inhibitors on, 504-505

$\mathrm{pH}$ of solutions of, 707

secondary effects of, 440-441

stimulation of respiration, 10

effect of temperature on, 784-785

uncoupling in regenerative systems, 367 uptake by yeast cells, 478, 480

Dioxane, use in lowering dielectric constant, $800-801$

Diphosphoglyceromutase, substrate inhibition, 112

Diphosphopyridine nucleotide (DPN), complexes with steroids, $298-299$

Dipolar molecules, interaction energy contributions, in, 244

Dipole angles, 22:2

Dipole-dipole interactions, 211-214

contributions of various forces, 243

dependence on orientation, 211-213

effect of dielectric constant on, 248 . 249 
of freely rotating dipoles, 213-214 potential energy of, 212-214

Dipole-induced dipole interactions, 222. 223

Dipole moments, definition of, 208

dependence on inductive and resonance effects, 308

induced, 218

in interaction of halogen groups with cholinesterase, 288-289

of bonds, 216, 220

of molecular groups, 22:2

of molecules in solution, 210-211

of rotating molecules, 208.209

Dipyridyl, see $2,2^{\prime}$-bipyridine

Diseases, mimicked by inhibitors, 7

Dismutation, kinetics of, 47.48

Dispersion electrons, number of, 2.25. 226

Dispersion energy, of benzene ring and alkyl groups, 299 of steroids and adenine nucleotides, 298.299

of two benzene rings. 298

Dispersion forces, see electrokinetic interactions

Dissociation constants. effect of ionic strength on, 818-8.3

of pressure on, $84-843$

of temperature on, 749.750

Distortion energy, 202-264

of enzyme charge distribution, 263

of enzyme side-chains. 263

of enzyme structure, 263

of inhibitor structure, 263

Distribution of enzymes, intracellular, $432 \cdot 435$

Distribution of inhibitors, 393-4.5

acetazolamide, 396-400, 402

arsenite, 39.5

chlorguanide, 294-395. 399

chlormerodrin, 396-397

concentrations in tissues, 394-398

correlation with enzyme inhibition, 405 dependence on binding to plasma proteins, 404

on cell permeability, $402-403$

on dose of inhibitor, $399-400$

on route of administration, 398-399,

406-407

on tissue composition, 404

on tissue vascularity, $400-402$

diisopropylfuorophosphate. 395

during repeated administration, 421 . 424

effect of second inhibitor on, 503

in plants, $405-410$

intracellular, 410-412

isoniazid, 396-398, 402

mercuric chloride, 395

quinacrine, 395

relation to affects produced, 425

time course of, 413-421

Distributive systems, 346-348

Divergent chains.

effects of temperature on, 771

multiple inhibition of, 501

nature of $341-345$

stimulation of one branch by inhibitor, $4.54-4.5$

DNP, see 2.4-Dinitrophenol

Dose of inhibitor, effect on tissue distribution. 399-400

DPN, see Diphosphopyridine nucleoticle

DPNH-peroxidase, complex with DPNH, 19

DPN-pyrophosphatase, natural inhibitors of. 778

Dye reduction, inhibition of, 101.104

\section{E}

EDTA, see Ethylenediamine tetracetate

Effective concentration of inhibitor, definition of. 411

Ehrlich ascites tumor cell, see Ascites tumor cell

Electrical field strength, surrounding an ion, 206 
Electrical potential, of ions, 207

Electrokinetic interactions, 223-2.28 carboxypeptidase inhibition, $292-294$ London formulation of, 224 of benzene rings, 299 of steroids and adenine nucleotides, 298-299 potential energy of, 224

Slater-Kirkwood formulation of, 224 2.25

specificity of, 228

Electron displacement, role in enzyme reactions, 30

Electronegativity, definition of, 206

of biologically important atoms, 215

Electronic polarization, definition of 217

Electron transport, inhibition of dye reduction in, 101-104 localization of site of inhibition in, $517-518$

Electrostriction,

definition of, 842

role in pressure effects, $841-842$

Endogenous metabolism, 441-447

effects of added substrate on, 442-443

effects on inhibition kinetics, 444-447 necessity of correction for, 441-442

Energy constants, for nonpolar molecules, 237

Energy contours, of enzyme reactions, 212.2, $28-29$

Energy flow, modification by inhibitors, $1-3$

Energy of activation of enzyme inhibition, 559-561

Energy of interaction, see Interaction energy

Energy, potential, of two ions, 207-208

Enolase,

effect of dielectric constant on, 814. 815

substrate inhibition, 111, 131

effect of ionic strength on, 838
Enthalpy,

determination of changes in, 265-266 of hydration, 251

Entropy,

determinations of changes in, $265-\mathbf{2 6 6}$ electrostatic and nonelectrostatic components, 265

formation of ES complexes, 266-267 of activation, of enzyme inhibition, $5.59-561$

of hydration, 251

relation to changes in water structure, 266

Enzyme-inhibitor complex, ionization of, $682-683$

rate of dissociation of, 607-611

Enzyme-product complex, effect on kinetics of, $23-24$

Enzymes,

charge on, effect on $\mathrm{K}_{i}, 694-701$

determination of concentration of, 71 . 72

development of concepts of, $7-8$

intracellular distribution, $432-435$

intracellular state, 436

reaction rates, factors determining, $31-32$

synthesis of, inhibition of, 440-441

variability of, response to inhibitors 485.486

Enzyme-substrate complex, entropy for formation, 266 evidence for, 19 nature of, $27-31$

Equation of fit, applied to enzyme interactions, 30-303

Equilibrium competition, definition of, 5.58

Equilibrium distance, determination from $\mathrm{K}_{i}, 270-274$ expressions for, $230-240$ of carboxylate and amino groups, 270 273

Equilibrium interaction energy, 230-232

Erythrocytes, respiration of, stimulation by quinones, 452 
Eserine, see Physostigmine

Esterases, substrate inhibition, 123, 135

Ethane, optical constants of, 225

Ethanol,

dipole moment of, 244

inhibition of bioluminescence, effect of pressure on, 851-853

interaction energy contributions of, $\mathbf{2 4 4}$

Ethylenediamine tetraacetate, stimulation by removal of heavy metal jons, 453

Ethyl ether, inhibition of bioluminescence,

effect of pressure on, 851-852

$N$-Ethylmaleimide, inhibition of gastric secretion,

effect of 2,4-dinitrophenol on, 504-505

Ethyl urethane, see Urethane

Exaltation,

definition of, 219

table of values for, 221

\section{$\mathrm{F}$}

False start, in transition between steady states, $387-388$

Fate of inhibitors, 413.421

Feedback systems, see Self-regulatory systems

Ficin, nature of $\mathrm{K}_{m}, 2 \mathrm{l}$

Field strength, electrical, surrounding an ion, 206

Fit, of inhibitor to enzyme, equation of fit, 302-303

index of fit. 303

means for expression of, $302-303$

Flavin-adenine dinucleotide, complexes of substances with, 90

Flexibility of cnzymes, 199, 263 effect of temperature on, 753

Fluctuation, in transition between steady states, 386-388

Fluoride, hydration of, 251 inhibition of respiration, effect of $\mathrm{pH}$ on, 731

of stimulated brain slices, 480

of succinate oxidase, 492-493

stimulation of yeast respiration, 452

Fluoroacetate,

accumulation of citrate, antagonism by malonate, 502-503

inhibition of sarcosine oxidase, 296

Fluorocitrate, inhibition of intracellular aconitase, 436

Forces, intermolecular, classification of, $\mathbf{2 0 5}$

Formate dehydrogenase, localization in staphylococcal membrana, 434

Fractional inhibition, definition of, 57

Free energy, of hydration, 2.51

$\beta$-Fructofuranosidase (invertase), inhibition by urethane,

effect of pressure on, $846-849$

of temperature on, $755-756$

intracellular kinetics of, 475

kineties of, 14

location relative to membrane in yeast, 433-434

rate as a function of water concentration, 43

substrate inhibition, 111, 133

Fumarase,

active center of, 201

effect of buffers on ionization of, 840 inhibition by malonate, effect of temperature on, 754

by phosphate, 839

inhibition of, effect of $\mathrm{pH}$ on, 691-694

$\mathrm{pK}_{a}$ 's active center groups, 701-702

substrate inhibition, 111

Function, see Cellular function

\section{G}

Gastric secretion, multiple inhibition of, $504-505$

Glucose oxidase, complexity of kinetics of, 27 nature of $\mathrm{K}_{m}, 21$ 
Glucose-6-phosphate dehydrogenase, activation by $\mathrm{K}^{+}, 835$

Glutamate $\gamma$-aminobutyrate transaminase, distribution in nervous system, $482-483$

Glutamate decarboxylase, distribution in nervous system, 483

Glutamate dehydrogenase, inhibition by $p$-chloromercuribenzoate, reversal by cysteine and GSH, 6:22

Glutamic acid, ionization microconstants of, 703

Glutathione, inhibition of succinate oxidase,

in reactivation experiments, $625-626$ rate of, 547,549

reversal of inhibitions by mercurials. 622

Glutathione reductase, inhibition by salts, 834

Glutathione synthetase, product inhibition, 145

Glyeine,

inhibition of alkaline phosphatase, 90 of fumarase, effect of $\mathrm{pH}$ on, 691692

Glycine transacetylase, product inhibition, 145

Glycolysis,

effects of ions on, 838-839

example of regenerative system, 364365

product inhibition controlling, 148

rate of action of $\mathrm{HgCl}_{2}, 580-581$

Graphical analysis

determination of activation energy of inhibition, 559-560

of activation enthalpy of inhibition, $757-758$

of inhibition rate constants, 539,

$542,547-548,552,556$

of reactivation rate constants, 633 -

634

general methods of, 150-153

of multiple inhibition, 490
pH effects, 678-682

relation between inhibition and $\mathrm{pH}$, 735, 737

suggestions for determination of constants, 188-191

Growth, localization of site of inhibition on, 531.532

\section{H}

Hapten-antibody reactions, 274-284 determination of group interaction energies, $275-278$

dispersion energy of, equation for, 277 table of, 278

effect of group position on, 282-284 of hydration on, 284

of ionic group size on, 281-282

energy terms involved in, $\mathbf{2 7 5}-\mathbf{2 7 6}$

experimental procedures, 275

ion-ion interactions in, $279-280$

specificity of ionic groups, 280-281 water displacement during, $276-277$

Heart cells, of frog,

effect of pressure on, 389

Heparin, inhibition of trypsin. effect of ionic strength on, 836

Heterogeneity, of active centers, 108. 109

Hexokinase,

activation by $\mathrm{Na}^{+}, 835$

inhibition by $p$-chloromercuribenzoate, reversal by cysteine and GSH, 622.624 intracellular kinetics, 475 product inlibition of, 144,148

Hexose diphosphatase, stimulation by arsenite, 452

Histidine, occurrence at active centers, 196

Homogentisate oxidase, inhibition by $p$-chloromercuribenzoate, reversal by GSH and $\mathrm{Fe}^{++}, 625$

Homologous series, comparison of members, 311-312 inhibitory potencies in $309-312$ 
Hydration, 250-259

differences between cations and anions. 255

effect of temperature on, 752

effect on hapten-antibody binding, 284

on inhibition of cholinesterase, 288

energy, 250-255

definition of, 250

example of calculation of, $252 \cdot 25.5$

of common ions, 251

enthalpy, $25 \mathrm{l}$

entropy, 251

free energy, 251

importance in enzyme interactions, 2.59

in mixed solvents, 802

numbers, table of, 251

of fatty acids, 257

of proteins, 258

orientation of water around ions, 258

primary, definition of. 250

role in ion-ion interactions, 250,272 274

secondary, definition of, 250

Hydrocarbon chain,

bond angles, in, 240

configuration of, 235

interactions between, 300

Hydrocyanic acid, see also Cyanide

dispersion electrons of, 2.25

molar refraction of, 2.55

optical constants of, 225

polarizability of, 2025

Hydrogen,

dispersion electrons of, 225

energy constants of, 237

energy curve for interaction, 236

molar refraction of, 225

optical constants of, 2.25

orientation on interaction energy, 241-

242

polarizability of, 225

Hydrogen bonding, 214-217

Hydrogen sulfide,

dispersion electrons of, 2.25

molar refraction of, 225

optical constants of, 2.25

polarizability of, 2.25
Hydroquinone, effect of $\mathrm{pH}$ on reactivity, 734

Hydroxylamine,

inhibition of catalase, effect of temperature on, $755-756$

reactivation of cholinesterase inhibited by TEPP, 630,636

of cholinesterase inhibited by carbamylating agents, $634-636$

Hydroxyl group,

bond angle in, 240

configuration of, 235

dipole angle of, 222

dipole moment of, $2 \cdot 2 \cdot 2$

dispersion energy in hapten-antibody reactions, 278

hydration of, 258

interaction with cholinesterase, $290-291$

molar refraction of, $2 \cdot 2 \cdot 2$

van der Waals radius of, 234

$\beta$-Hydroxyphenylalanine, inhibition of phenylalanine utilization in $E$, coli, 498,500

p-Hydroxyphenylpyruvate oxidase, substrate inhibition, 112-114

$\beta$-Hydroxysteroid dehydrogenase, complex of steroids and DPN, 299 substrate inhibition, 111, 121-122, 128. 130

Hypothermia, effects on response to anoxia, $780-782$

\section{।}

Inactivation,

of enzymes,

effect on rate of inhibition, 561-569

progressive during inhibition, 98-100

of inhibitor, progressive during inhibition, 100

Index of fit, applied to enzyme interactions, 303

3-Indolepropionate, inhibition of a-chymotrypsin,

effect of ionic strength on, 836

Induced polarization, definition of, 217 
Inhibitor constants $\left(\mathbf{K}_{i}\right)$, determination of, $677-682,684$ from intracellular inhibition, 475. 478. $737-738$

in multienzyme systems, 382-383 suggestions for. 188-191

effect of dielectric constant on, 804.807 of ionic strength on, 818-8.23

of pressure on, 844

of temperature on, $748-749$

variation with substrate, 105-106

Inhibitor-resistant metabolism. due to polylinear chains, 345 explanations of, $\mathbf{4 4 9 - 4 5 1}$

for cyanide, $447-448$

for iodoacetate, 449

Inhibitors,

as drugs, 6-7

history of, 7-11

reaction with substrate,

isobolograms for, 186

types of plots obtained, 165, 173

Inhibitor titer, determination of relative enzyme concentrations. 80

Inhibition index, definition of, 106

Insecticidal action, enzyme basis for, $473 \cdot 474$

Interaction.

between inhibitors on enzyme surface, 490- 493

constants,

determination of, 181-182

effect on inhibitions, 61.64

definition of, 51

energy,

dependence on fit, 301-304

general equations for, 232

of methyl group in a cavity, $301-302$

relation to inhibitor constant, 264

forces,

in colloids, 244

relative contributions of, $242-245$

Intermolecular forces, classification of, 205

Intracellular concentrations, of enzymes, 482.483 of inhibitors, $477-478$

maximal level of, 415

relation to external concentration, 411

variation with time, 413-4-3

of substrates, $477-478,482$

Intracellular enzymes, differences from extracted enzymes. 4.2

Intracellular equilibria, effect of inhibitors on. $456-458$

Intracellular inhibition, determination of $\mathrm{K}_{i}$ from, 475-4i7 effect of diffusion on. 475-477 quantitative expression of, 474.478

Intracellular media, ionic composition of. $430-431$

Intrinsic constants, see Microscopic constants

Invertase, see $\beta$-Fructofuranosidase

Iodoacetamide, effect on dinitrophenol inhibition of gastric secretion, $504-505$

Iodoacetate.

accumulation of hexose phosphates, 514

effect on glucose concentration in lymph cells, 504

on heart,

continuation of inhibition after removal of inhibitor, 579

rate of, $586-598$

on muscle $\mathrm{pH}, 718-719$

or photosynthesis, 519

inhibition of cardiac metabolism, 516 of fermentation, effect of $\mathrm{pH}$ on, 726 of glycolysis.

early work on, li

pyruvate addition to localize, 472 rate of. 582.558

specificity of, 877-878

of pyruvate oxidation, $87 \overline{7}-878$

of respiration,

effect of $\mathrm{pH}$ on, 731

rate of, $582 \cdot 588$

of sareosine oxidase, 296

of stimulated brain slices, 480 
inhibition by, effect of $\mathrm{pH}$ on, 722.724 $\mathrm{pH}$ of solutions of, 707

pyruvate utilization during inhibition by, 515.516

rates of inluibition of glycolysis, $582-588$ reaction with glutathione, effect of $\mathrm{pH}$ on, 726

resistant fraction of glycolysis, 449 specificity of, $877-878$

stimulation of yeast respiration, 452

o-Iodosobenzoate, inhibition of succinate oxiclase,

rate of, $547,549-550$

Ion-dipole interactions, 208-211

contributions of various forces to, 243 dependence on orientation 209

for rotating dipoles, 208-209

potential energy of, 208.210

Ionic atmosphere, 260-262

effect on ion-ion interactions, 260

energy for displacement of, 262

Ionic radii, table of, 251

Ionic refractions, 2.21

Ionic strength,

classification of effects of, 815-818

effect of substrate concentration on, 113

effect on activity coefficients, 816

on carboxypepticlase, 830,837

on cholinesterase, $827-828$

of $\alpha$-chymotrypsin, $8: 8-829, \quad 836$ 837

on cytochrome oxidase, 832

on dielectric constant, $815-816$

on dissociation constants, 818-8:3

on enzyme inhibitions, 835-838

on enzyme reactions. 826-834

on inhibition of carboxypeptidase, 837

on inhibition of cholinesterase, 836

on inhibition of $\alpha$-chymotrypsin, 836 -

837

on inhibition of trypsin, 836-837

on ionization constants of enzymes, 816-817

on polynucleotide phosphorylase,

$831-832$ on proteinase C, 829

on rate constants. $823-826$

on ribonuclease, $830-831$

on substrate inhibition, 126-128, 133, 838

on urease, 828

Ion-induced dipole interactions, 217-222 polarization of benzene ring by ionic group, 298

potential energy of, 218-219

Ion-ion interactions,

contributions of various forces, 243

displacement of water during, 271, 279

effect of dielectric constant on, 248-249, 804

of hydration on, 250

entropy of, 265

index of fit. 303-304

in hapten-antibody reactions, 279-280

potential energy of, 207

Ionization constants,

effect of buffers on, 840

of active center groups, effect of charged inhibitors on, 701-704

Ionization potential, dispersion energy and, 2204

Ion-nonpolar interactions, contributions of various forces, 243

Ions,

binding in the cell, 431

cavity size of, $240-241$

concentrations in tissues, 430-431

electrical potential of, 207

field strength of, 206

potential energy between, $207-208$

specific binding to proteins, 818

Iproniazid, inhibition of monoamine oxidase,

entropy of activation of, 561

in different tissues, 405-406

protection by tyramine, 558

rate of, $5 \mathbf{4 5}, 548$

time course in liver, 406

Irreversible inhibition, definition of, 52. 54 
Isobolograms,

competitive inhibition, 185

expression of antagonism, summation, and synergism, 508-509

of inhibitions, 183-184

reaction of inhibitor with substrate, 186 two inhibitors acting simultaneously, 495-498

Isoniazid, distribution in central nervous system, 396-398, 402

Isoxanthopterin, inhibition of xanthine oxidase, 120, 124, 137-138

\section{K}

a-Ketoglutarate utilization, stimulation by malonate, $\mathbf{4 5 2}$

Kinetic competition, definition of, 5.58

Kinetic constant, definition of, 20

Kinetic energy of molecules, effect on interaction distance, $238-240$

Kinetics,

complex enzyme reaction types, $32-48$ diffusion of substrate to enzyme, 34-35 Michaelis-Menten formulation of, 14 of enzyme reactions.

cyclic reactions, $47-48$

effect of activator on, 45-47

of EP complex on, 23-24

of number of substrate molecules bound, $25-26$

of $\mathrm{pH}$ on, $652-666$

of reversibility, $24-25$

of substrate depletion on. 2-2-23

enzyme concentration greater than substrate, 35-36

participation of solvent, 43-45

two different substrates, 39-43

two interdependent active centers, 36-39

use of activities in, $26-27$

of inhibition,

dependence on inactive forms of enzyme, $760-\overline{7} 6 \overline{7}$

destruction of inhibitor on, 100

development of, 9 effect of endogenous metabolism, $444-447$

of enzyme inactivation on, 561569

of nonenzymic binding on, 615-620 of $\mathrm{pH}$ on, $667-677$

of substrate and inhibitor concentrations on, 61-64

generalized equations for, 55.59

influence of substrate inhibition on, 134-139

inhibition of forward and backward reactions, 100-101

inhibitor also a substrate, 96-98

more than one inbibitor molecule per enzyme molecule, 90-96

progressive inactivation of enzyme, 98.100

reaction with coenzyme or activator, 89-90

with coenzyme or activator sites, $8 \cdot-85$

with substrate, $85-88$

reduction of acceptor, 101.104

reversible and irreversible, 5.2-54

substrate diffusion into cells, 35

two inhibitors on a single enzyme, $488 \cdot 492$

Krupka-Laidler plot, determination of $\mathbf{K}_{i}$ in mutual depletion systems, 188

\section{L}

Lactate dehydrogenase, complexes with DPN, 19 distribution in nervous system, 483 inhibition by $p$-chloromercuribenzoate, rate of, 546,549

reversal by cysteine and $\mathrm{C}, \mathrm{SH}, 622$ inhibition by excess apoenzyme. 455

by substrate analogs, 297

localization in staphylococeal membrane, 434

substrate inhibition, 111, 121-122, 124125

Lag period, see Latent period

Latent period, of inhibition, 573

of Aspergillus respiration by $\mathrm{C}^{++}, 582$ 
Lethal synthesis, definition of, 437

effect of a second inhibitor on, 502-503 examples of, 437

kinetics of, $437-439$

Limiting reactions, concept of, 380-38:

definition of, 320

limiting rare coefficient, 381 role in complex enzyme kinetics, 48

Lipase, effect of temperature on, 766-767 substrate inhibition, 111

Lipid content of tissues, table of, 401

Liver, respiration of, stimulation by quinones, 452

Localizations, of site of inhibition, 513533

accumulation of intermediates, $513-515$ addition of substrates or intermediates, 515-516

distribution of labeled atoms, 519-521 general program of localization, $527-533$ reconstructed enzyme systems, 523 spectroscopic analysis, 516-519 uncoupling wechanism, 523-527 use of second inlibitor, 521-522

London forces, see Dispersion energy, Electrokinetic interactions

\section{M}

Magnesium,

concentration in tissues, 430

distribution in cells, 431

Malate dehydrogenase, distribution in nervous system, 483 inhibition by $p$-chloromereuribenzoate. failure of cysteine nad GSH to reverse, 622

localization in staphylococcal membrane, 434

stimulation by arsenite, 452

Halate, inhibition of fumarase, effect of $\mathrm{pH}$ on, 691-692

Maleate, inhibition of fumarase, effect of $\mathrm{pH}$ on, 691-692
Malonate,

antagonism of citrate accumulation due to fluoroacetate, 502-503

inhibition of bacterial dehydrogenases, 9

of fumarase, effect of temperature on, 754

of respiration, effect of $\mathrm{pH}$ on, 722 723

of stimulated brain slices, 480

of succinate dehydrogenase, 104

dependence on rate of reaction, 858

determination of $\mathrm{K}_{s} / \mathbf{K}_{i}, 183$

distortion of structures, 263

effect of dielectric constant, 806

effect of temperature on, 754

$\mathrm{pH}$ of solutions of, 707,709

protection of succinate dehydro-

genase against SH-agents, 494

resistance to, 450

specificity of, 878

stimulation of ATPase, 453

of cardiac respiration, 5: I

of $\alpha$-ketoglntarate oxidation, 5, 521

of $\alpha$-ketoglutarate utilization, 452

of pyruvate incorporation into cycle, 452

use to studly $\alpha$-ketoglutarate oxidation, 5,521

Mechanisms of inhibition, classification of, $50-51,60-61$

Media, for enzyme experiments, composition of, 428-432

Nembranes, localization of enzymes in, 433.435

Menadione, inhibition of bioluminescence, effect of $\mathrm{pH}$ on, 744-745

Mepacrine, see Quinacrine

Mereurials, see Chlormerodrin, $p$-Chloromereuribenzoate, Mercuric chloride

Mercuric chloride,

concentrations in tissues, 395

inhibitions of bioluminescence, effect of temperature on, 786-787 
of cholinesterase, effect of temperature on, 758

of glycolysis, rate of, $580-581$

of respiration, rate of, 580-581, 588590

of succinate oxidase, partial reversal by dimereaprol, 622

rate of killing of Colpidium, 593-595

Mercuric dimer of seralbumin, thermodynamics of, 267

Mesaconate, inhibition of fumarase, effect of $\mathrm{pH}$ on, 691-692

Metabolic differentiation, detection with inhibitors, 5-6

Metabolic rate, effect on inhibitions, 857862

Metabolic variation, between different tissues, 481-484

Metabolism of inhibitor, effect of a second inhibitor on, 502-503 effect on rate of inhibition, $577-578$

Methane, dispersion electrons of, 225 energy constants of, 237 energy curve for interaction, $\mathbf{2} 36$ interaction energy of, 237 molar refraction of, 225 optical constants of, 225 polarizability of, 225

Methanol, dipole moment of, 244 effect on $\mathrm{pH}$ optimum of $\alpha$-chymotrypsin, 661 interaction energy contributions of, 244

use in lowering dielectric constant, 801

Methoxyacetate, inhibition of sarcosine oxidase, 295 - 297

Methylene blue, inhibition of rednction by dehydrogenases, 101-104

Methylene group, binding energy to carboxypeptidase, 292

to lactate dehydrogenase, 297

to sarcosine oxidase, 297 configuration of, 235

dispersion energy for interaction with protein, 300

van der Waals radius of, 234

Methyl group,

binding energy to sareosine oxidase, 297

configuration of, 235

dipole angle of, $2 \cdot 2 \cdot 2$

dipole moment of, 222

dispersion energy in hapten-antibody reactions, 278

index of fit in protein cavity, 303-304 interaction energy in protein cavity, 301-302

interaction with benzene ring, 299

molar refraction of, 222

polarizability of, 299

van der Waals radius of, 234

$N^{\prime}$-Methylnicotinamide, inhibition of alcohol dehydrogenase, due to contaminating $\mathrm{Ag}^{+}, 857$

Methyl orange

binding to seralbumin, 314-315 effect of temperature on, 753

binding to various proteins, 317

Michaelis constant $\left(K_{m}\right)$ definition of, 15,17

deternination of, 16

effect of pressure on, 843

of temperature on, 748-749

energy contours and, 21

expression for, 20

table of values of, 18

Michaelis-Menten kineties, validity of, $17 \cdot 27$

Microscopic constants, applied to $\mathrm{K}_{i}$, 95-96

Miotine, inhibition of cholinesterase, 71

Mixed inhibition,

detection of, 160

equations for, 58

types of plots obtained, 162

Molar refraction,

definition of, 218

double bond conjugation and, 219 
of atoms, 219

of bonds, 220

of ions, 221

of molecular groups, 222

of protein sites, 277

Molecular group refractions, 222

Molecular structure, alterations of, classification of, 305

effect on inhibitions, 306-307

on properties, $304-312$

examples of, 308-311

on type of inhibition, 310-311

optical isomers, 309-310

Monoamine oxidase, inhibition by iproniazid,

entropy of activation of, 561

protection by tyramine, 558

rate of, 545,548

synthesis of new enzyme following, 645

Monolinear chains,

irreversible,

buffer capacity of, 331-332

concentrations of intermediates, 323 -

$324,327-331,333-335$

diffusion-himited, 335-337

effect of temperature on, $769-770$

of two inhibitors on, 498-500

inhibition of, $327-335$

of several steps, 337

over-all rate factors, $323-327$

reaction of inhibitor with interme-

diates, 334-335

reversible,

concentrations of intermediates, 338 .

340

noncompetitive inhibition of, 338 340

Multienzyme systems,

branched linear chains, 340-345

classification of, $321-322$

compartmentalization of, 377

cyclic systems, $348-354$

definition of, 319

determination of $\mathrm{K}_{s}$ and $\mathrm{K}_{i}$ in, 382. 383

distributive systems, $346-348$

effect of $\mathrm{pH}$ on inhibition of, 711-713 of steady-state rate on inhibition of, 859

of temperature on, 768-773

limiting rate concept, $380-382$

monolinear chains, 322-340

multiple inhibition of, 500-501

network systems, 37 ?

polylinear chains (shunts), 345-346

product inhibition in, 147-148

regenerative systems, 354-368

self-regulatory (feedback) systems, 368 .

372

structural disruption of, 379

terminology of, $320-322$

transition time between steady states, 383-391

transit time, $373-377$

use of inhibitors in, 5

Multiple inhibition, 487-512

antagonism, sunmation, and synergism

$507-510$

expression by isobols, 495

of biolmminescence, $510-512$

of cholinesterase, $493-495$

of succinate oxidase, $492-493$

specificity of, $5 \mathrm{I} 0$

two inhibitors.

on cellular metabolism 502-507

on monolinear chain, 498-500

on one enzyme, 488.498

on various multienzyme systems, $500-501$

Multipoint attachment,

of substrate to active center, 28

relation to catalysis, 197-198

role in substrate inhibition, 113

Multipoles, 228-229

Muscle, respiration of, stimulation by quinones, 452

Mutual depletion systems, 66-78 boundary conditions in, 69-71, 75 cellular, $77-78$

competitive inhibition in, 72-75

dilution effects in, $76-77$

effect of nomenzymic binding of inhi-

bitor, 854-857

of $\mathrm{pH}$ on inhibitions in, 706-707 
extraneous substances, role $\mathrm{in}, 78$ noncompetitive inhibition $\mathrm{in}, 66-72$ of inhibitor and reversor, 618-620 rate of inhibition in, $\mathbf{5 4 8 - 5 5 2}$ steady-state kinctics in, 75 types of plots obtained, 184-188

Myosin, binding of $\mathrm{K}^{+}$and $\mathrm{Na}^{+}, 262,818$

\section{N}

Naturally occurring inhibitors, effect of temperature on, 778-779

Neohydrin, see Chlormerodrin

Neostigmine, inhibition of cholinesterase, effects of tetraalkylammonium ions on, 495

Network systems, 372

Nitella

lethal action of $\mathrm{Cu}^{++}$, concentrationtime curves for, 598

penetration of cresyl blue into, 602

Nitric oxide, optical constants of, $\mathbf{2} 2 \mathbf{2 5}$

Nitrogen,

dispersion electrons of, $2: 25$

energy constants of. 237

energy curve for interaction, 236

interaction energy of, 237

molar refraction of, 225

optical constants of, 2.25

polarizability of, 225

Nitro group,

bond angle of, 240

dipole angle of, 2-2:2

dipole moment of, 222.2

dispersion energy in hapten-antibody reactions, 278

molar refraction of, 22.2

Nitrous oxide, optical constants of, 225

Noncompetitive inhibition,

by products, 143-144

by substrate, 114, 118-1:0

definition of, 54.55

dilution effect on, 76

discovery of, 9

effect of endogenous metabolism on, $445-447$ of ionic strength on, 821

of $\mathrm{pH}$ on, 659, 671-673

of temperature on, 749-751

equations for, 58

in diffusion-limited systems, 475-477

influence of substrate inhibition on, 134

intracellular, $475-477$

more than one inhibitor combines with enzyme, $90-96$

mutual depletion systems, 66-72

of cyclic systems, 351-362

of divergent chains, 343

of forward and backward reactions, 100-101

of monolinear chains, 327-332 338-340

of regenerative systems, 364-366

of self-regulatory (feedback) systems, $370-372$

of substrate-inhibited enzymes, types of plots obtained, 177, 179

progressive destruction of inhibitor during, 100

progressive inactivation of enzyme during, 98-100

rate of, $541-548$

of reversal of, 610

specificity of, 866.870

transition time between steady states, $384-386$

two inhibitor molecules bound to enzyme, types of plots obtained, 174, 489 . 496

types of plots obtained, 158

with respect to activator, 83 ,

to substrate, 54-55, 58

Nonenzymic binding of inhibitors, 615 . $624,854-857$

effect on inhibitions, $615-620$

examples of, $622-624$

reversal of inhibition by, $620-622$

Nonenzymic effects of inhibitors, on cellular function, $463-464$

Nonenzymic material,

binding of inhibitors to, effect on rates of inhibition. 577

in mutual depletion systems, 78

Nu 683, inhibition of cholinesterase, 71-72 
Open systems, definition of, 320

Optical constants, for simple molecules, 225

Optical isomerism, importance in group effeets on inhibition, 309-310

Optimal temperature, effect of inhibition on, 767.768

Organophosphorus compounds, see also Diisopropylfluorophosphate inhibition of cholinesterase, effect of temperature on, 759-760 induced reactivation, $630-636$ spontaneous reactivation, 627-630 inseeticidal action, 473-474 poisoning by,

kineties of reversal, 646-648

treatment with reactivators, 636

Organ weights, table of, 401

Orientation polarization.

definition of, 217 of permanent dipole, 208

Overshoot, in transition between steady states, 386-388

Oxidation-reduetion reactions, kineties of. 47.48

Oxidative phosphorylation, example of regenerative system. 366-368

Oxophenarsine, binding to erythrocytes, reversal of, 644

Oxygen,

diffusion rate into erythroeytes, 573 dispersion electrons of, 225

molar refraction of, 225

optical constants of, 225

polarizability of, 225

\section{$\mathrm{P}$}

Pacemakers of metabolism, see also Limiting reactions

definition of, 459

examples of, 459

in ascites tumor cells, 461

restriction of eoncept, 460
Paludrine, see Chlorguanide

Papain,

active center of, 201

degradation by leneine aminopeptidase, 195

effeet of dielectrie constant on, 812. 813

inhibition by earbobenzoxy-L-glutamate, effeet of $\mathrm{pH}$ on, 667-668

nature of SH group at active center, 196

stimulation by arsenite, 452

Paramecia, lethal aetion of arsenite, kineties of, 600

Partially competitive inhibition, detection of, 160

types of plots obtained, 159

Partially noncompetitive inhibition, types of plots obtained, 161

Penetration of inhibitors into tissues, see also Permeability to inhibitors effect of fumetional aetivity on, 577, 588

of membrane depolarization on, 484 on rate of inhibition, 573-576 malonate, 402-403 rough classification of, 403

Pepsin.

entropy changes in formation of $\mathrm{ES}$ complex, 266

enzyme-substrate complex, 19

Permeability to inhibitors, see also Penetration of inhibitors into tissues effeet on distribution, 402-403 relation to ionization, 721-726

Permeases, concept of, 439

Peroxidase,

diffusion-limited steady-state system of, $335-337$

enzyme-substrate complex, 19

heme in protein erevice, 198

nature of $\boldsymbol{K}_{m}, 21$

$\mathrm{pH}$,

effect on enzyme inhibition, 649-745 on inhibition in multienzyme sys. tems, $711-713$ 
on inhibition in mutual depletion systems, 706-707

on inlubition of papain, $667-668$

on intracellular imhibitions, 713-718

on rate of inlibition, $576-57 \overline{7}, 704$ -

706

on reactivation rate of choline.

sterase, 636

on substrate inhibition of lactate de-

hydrogenase, 124-125

functions, formulation of, 650-65l

intrecellular,

bulk values of, $717-718$

effects of inhibitors, on, 718-719

heterogeneity of, 719-721

of inhibitor solutions. $707-710$

optima,

definition of, 655

location of, $660-661$

o-Phenanthroline,

inhibition of alcohol dehyclrogenase, 84 inactivation of enzyme during, 567 inhibition of pyruvate oxidation, 527 of succinate dehydrogenase,

effect of temperature on, 755

of tetrazolium dye reduction, 102 rate of inhibition reversal, 645

stimulation by removal of inhibitory metal ions, 453

Phenols,

inhibition of D-amino acid oxidase, 90 phases of action on bacteria, 579

Phenylacetate, inhibition of a-chymotryjsin,

effect of ionic strength on, 836

Phenylalanine,

synthesis in $E$. coli, inhibition by tyrosine, 498

utilization by $E$. coli, inhibition by $\beta$-hydroxyphenylalanine, 498, 500

$\beta$-Phenylethylhydrazine, inhibition of brain monoamine oxidase, reversal of, 645

Phenylmercuric compounds, see also $p$-Chloromercuribenzoate

stimulation of ATPase, 452

of succinate oxidase, 452
Phlorizin, on renal phosphorylations, 10

Phosphatase,

activation by $\mathrm{Zn}^{++}, 46$

competition between various sub.

strates, 98

function in divergent chains, 344

inhibition by glycine, 90

localization in staphylococcal mem-

brane, 434

in yeast membrane, 434

$\mathrm{pH}$ optimum of, 655

Phosphate,

concentration in tissues, 430

inhibition of enzymes, 839

of suceinate oxidase, $492-493$

Phosphoglucomutase, inhibition by anions, 834

by phosphate, 839

6-Phosphogluconate dehydrogenase, activation by $\mathrm{K}^{+}, 835$

Phosphoglyceraldehyde dehydrogenase, complexes with DPN, 19

inhibition by $p$-chloromercuribenzoate. reversal by cysteine and GSH, 622-624

Phosphorylase, function as an ATPase, 344-345

Photobacterium phosphoreum, inhibition of luminescence in, 511

effect of temperature on, $787-790$

Physostigmine,

displacement from cholinesterase by substrate, $613-615$

inhibition of cholinesterase.

effect of ionic strength on, 836

reversal by destruction of inhibitor, $640-6+1$

pI scale, application to inhibition reversal, 619-620

Plants,

absorption of inhibitors through roots of, $406-407$

distribution of inhibitor's in, $405-410$

foliar application of inhibitors, 407. 409.410

translocation of substances in, $407-410$ 
Plasma membrane,

inhibition of transport in, $439-440$ localization of enzymes in, 433-435

Plasma, proteins, binding of inhibitors to, 404

Plotting, see Graphical analysis

Polarizability, asymmetry of, 219-220

definition of, 217

of atoms, 219

of bonds, 220

of molecular groups, 222

Polarization,

atomic, definition of, 217

electronic, definition of, 217

induced, definition of, 217

orientation,

definition of, 217

of permanent dipole, 208

role in induced dipole interactions, 217-218

Polyanions, inhibition of trypsin, effect of ionic strength on, 836-8.37

Polyglutamate, inhibition of trypsin, effect of ionic strength on, 836-837

Polylinear chains (shunts), 345-346

effect of temperature on, 772

multiple inhibition of, 501

Polynucleoticle phosphorylase, effect of ionic strength on, 831-832

Potassium, concentration in tissues, 430 intracellular binding of, 431

Potential, electrical, of ions, 207

Potential energy, of two ions, $207 \cdot \underline{2} 08$

Potentiation, see Synergism

Preincubation procechures, for determining site of inhibitor action, 569-571

Pressure, effects of, $841-854$

as transitions between steady states, $389-390$

mechanisms of, 841-842

on cellular inhibitions, 849-854

on dissociation constants, 842-843 on enzyme inhibitions, 846-849

on enzyme rates, $845-846$

on rate constants, $843-844$

Primary hydration, definition of, 250

of common ions, 251

Primary salt effect, definition of, 824

Product inhibition, 140-148

by formation of inactive enzyme complex, 143-144

by reversal of enzyme reaction, 140-143

examples of, 144-147

in multienzyme systems, 147-148

of arylamine transacetylase, 145

of cholinesterase, 146

of glutathione synthetase, 145

of glycine transacylase, 145

of hexokinases, 144, 148

of pyridoxal phosphokinase, 145

of urease, 145-146

types of plots obtained, 180

Proflavine, bacterial adaptation to, as transition between steady states, 389

Proliclase, active center of, 200

Prosthetic group, definition of, 50

Proteinase (', effect of ionic strength on, 829

Proteins,

charge on, effect of binding to ions, 315-316

hydration of, 258

interactions with small molecules, competition for binding sites, 313314

effect of temperature on, 316

of total protein charge on, 315-316

multiple binding sites, $314-315$

specificity of, $316-317$

Proton association, rates of, 656

Pseudo-irreversible inhibition, 78-82, 855

Pseudo-steady state systems, 32l

Pseudo-substrate inhibition, illustration of, 113-114

Pyridine-2-aldoxime methiodide (2-PAM), reactivation of cholinesterase, 631-633 
Pyridoxal phosphokinase, product inhibition, 145

Pyrophosphatase, substrate inhibition, 111,132

Pyrophosphate, inhibition of succinate oxidase,

effect on temperature characteristics, 798

Pyruvate decarboxylase, inhibition by phosphate, 839

by $\mathrm{SH}$-agents, protection by substrate, 558

product inhibition, 180

Pyruvate incorporation into cycle, stimulation by malonate, 45 른

Pyruvate oxidase, inhibition by $p$-chloromercuribenzoate,

failure of cysteine and dimercaprol to reverse, 622

Pyruvate pathways of metabolism, 483481

Quinacrine,

concentration in tissues, 395

intracellular distribution of, 413

Quinine, inhibition of cholinesterase.

temperature characteristics of. $795-796$

Quinones,

stimulation of Arbacia egg respiration. 45 는

of deoxyribonuclease, 452

of erythrocyte respiration, 452

of liver respiration, 45?

of spermatozoal respiration. 452

\section{R}

Rate constants,

effect of dielectric constant on, 808 of ionic strength on, 8:3-826

of pressure on, $842-843$

of temperature on, 757

Rate equations, general procedure for derivation, $32-33$
Rate of inhibition, 535-603

competitive, 552-558

concentration-time curves, 595-603

effect of dielectric constant on, 807-809

of $\mathrm{pH}$ on, $704-706$

of population variation on. $590-595$

of secondary inactivation on, 561-

569

of temperature on, 558-561, 756-760

expressions for, 536-541

in mitochondria and cells, 571-590

in mutual depletion systems, 548-552

of cholinesterase,

by E-600, 590-591

by $\mathrm{HgCl}_{2}, 545-546$

by physostigmine, $557-558$

of glycolysis,

by $\mathrm{HgCl}_{2}, 580-581$

by iodoacetate, $582-588$

of lactate dehydrogenase by $p$-chloromercuribenzoate, 546

of monoamine oxidase by iproniazid, $545-546$

of pure enzymes, 541-548

of respiration by heavy metal ions, $581-582,588-590$

Ratio of $\mathrm{K}_{s} / \mathrm{K}_{i}$, determination of, 18:-18:3

interpretation of, 104-105

Reactivation, see also Reversal of inhibition

effect on kinetics of cholinesterase inhibition, 569

Refraction,

anisotropy, due to asymmetric polarizability, 219

dependence on polarizability, 218

double bond conjugation and, 219

molar, see Molar refraction

Regenerative systems, 354-368

buffer capacity of, 361

concentration of regenerant $35 \overline{7}$

effect of reactant concentration, 358 . 359

of regenerant concentration, 358-359 examples of, $355-356$

inhibition of, $360-361$ 
oxidation-reduction reactions, 363-365 phosphate transfer, 365-368 steady-state conditions of, 359-360

Relative concentrations, see Speeific concentrations

Relative interaction energies, determination of, $268-270$

Repeated administration of inhibitors, 421.425

Repulsion, correction factors for, $231-232$ interaetions in, 229-231 orientation faetors in, $241-242$

Resistant metabolism, see Inhibitorresistant metabolism

Respiration,

hypoxic depression of, effect of temperature on, 782

inhibition by cyanide, effect of temperature on, 782-784

by heavy metal ions, rate of, 581 . 582. $588-590$

by urethane, effect of temperature on, 790

inhibition of,

effect of $\mathrm{pH}$ on, 722-724, 730-731 of temperature on, $777-778$

localization of site of, 529-531 stimulation by 2,4-dinitrophenol, effect of temperature on, 784-785

by various inhibitors, 452

Retardation, factor in dispersion forces, $226-227$

Reversal of inhibition, 605-648 by destruction of inhibitor, 637-64: by substance binding the inhibitor. 615-622

competitive displacement of inhibitor by substrate, 612-615

dissociation of EI complex, 607-612 examples of, $622-624$

in cells and whole animals, 642-648 induced reactivation of inhibited enzymes, $630-636$ information from, 624-626 spontaneous reversal of inhibited enzymes, 626-630

teehniques for demonstration, 606-607

Reversible enzyme reactions, kinetics of, 24.25

Ribonnelease, effect of ionie strength on, 830-831 enzyme-substrate complex, 19 inactivation by pepsin, 195 substrate inhibition, 111, 122

Route of administration, effect on inhibitor distribution, 398-399, 406-407

\section{S}

Salicylate,

inhibition of $\mathrm{D}$-amino acid oxidase, 90 of eholinesterase, effeet of temperature on, 758-7.59 of eleavage, effect of $\mathrm{pH}$ on, $72 \mathrm{l}$

Salt effeets, see also Ionie strength specifie ion effects on enzymes, 834-835

Salting-out effeet, definition of, 821

Sarcosine oxidase, active center of, 201 binding energies of inhibitors, 295-297 Secondary effects of inhibitors, 4,57-458 Secondary hydration, definition of, 250 Secondary inactivation, of enzyme during inhibition, 561-569

Sccondary salt effect, definition of, 824

Selenite, inhibition of snecinate oxidase, effect on temperature characteristies, 798

Self-regulatory (feedback) systems. 368372

effect of substrate concentration on, $369-370$

examples of, $368-369$

inhibition of, $370-372$

Sequential inhibition, of cycle by malonate and fluoroacetate, $498,502-503$ of phenylalanine metabolism in $E$. coli, 498,500 
Seralbumin, binding oi,

$\mathrm{Ca}^{++}, 266,314,818$

$\mathrm{Cl}^{-}, 313$

$\mathrm{Cu}^{++}, 266,314$

dodecyl sulfate, 314

ions, effect of temperature on, 753

methyl orange, 314-315

$\mathrm{Ig}^{++}, 314$

optically isomeric dyes, 317

phenylbutyrate, 314

surfactants, 300

testosterone, 314, 317

$\mathrm{Zn}^{++}, 314-315$

Serotonin, levels in brain following monoamine oxidase inhibition, 645

Shunts, see Polylinear chains

Silver, inhibition of respiration. rate of, $581-58$.

Simon-Beevers curves, 728-734

Sodium,

concentration in tissues, 430

intracellular binding of, 431

Solvent, participation in enzyme reactions, $43-45$

Spatial factor, in inhibitor distribution, 398

Srecific concentrations, definition of, 65-66

Specificity of inhibition, change with time of, 535

definition of, 510

examples of, $877-878$

in cellular systems, 871-873

in competitive systems, 871

in systems of three or more enzymes. 869-871

maximal for two enzymes, 866-869

multiple inhibition, 510

with two or more inhibitors, 873-877

Spermatozoa, respiration of,

stimulation by arsenite, 452

by quinones, 452

spontaneous reversal of inhibited enzymes, $626-630$

Steady state,

definition of, 320 in yeast metabolism, effect of cyanide and dinitrophenol on, 389

kinetics, 20

derivation of rate equations, $32-33$

mutual depletion systems. 75

relation to type of inhibition, 61

of convergent chains, 341

of divergent chains, $341-342$

of monolinear chains, 323-330

transitions between, 383-391

Steroids,

binding to proteins, 317

complexes with adenine nucleotides, 298- 299

Stimulation of metabolism, by inhibitors, $451-456$

examples, of, 452

possible mechanisms of, $453-456$

Streptobacterium casei, glycolysis in, rate of inhibition by iodoacetate, 584 585

Structural alteration, of multienzyme systems, $379-380$

Substrate, concentration of, effect on enzyme rates, $14,35-36$ on inhibitions, 61-64

reduction by enzyme binding, $22-23$ intracellular concentrations of, 482 protection against inhibition, 558 reaction of inhibitor with, $85-88$

Substrate constant $\left(K_{s}\right)$, definition of, 17 variation with $\mathrm{pH}, 661-666$

Substrate inhibition, 111-139

by an ionic strength effect, 133

by attachment outside active center, $118-130$

by combination with activator, 130 131

by competition with substrate-activator complex, 132

by interference with acceptor binding, 132-133

by partial attachment to substrate sites, 114-117 
by reduction of water concentration, 133

effect of ionic strength on, 838

on other inhibitions, 134-139

maximal rate $i n, 117,135$

of acetylcholinesterase, 111, 121, 122, I25-I28, 138-139

of L-amino acid oxidase, 111,133

of aminoacylase I, 112,130

of carboxypeptidase, 111, 120

of dianine oxidase, 111

of diphosphoglyceromutase, 112

of enolase, 111, 131

of esterases, 123, 135

of $\beta$-fructofuranosidase, 111, 133

of fumarase, 111

of $p$-hydroxyphenylpyruvate oxidase,

112,114

of $\beta$-hydroxysteroid dehydrogenase,

$111,121-122,128-130$

of lactate dehydrogenase, 111, 121-122,

$124-125$

of lipase, 111

of pyrophosphatase, 111, 132

of ribonuclease, 111,122

of sucrose transglucosylase, 112

of urease, 111

of xanthine oxidase, 111, 120-121, 124.

132-133, 137-138

possible mechanisms of, 112-114

types of plots obtained, 174, 177

Succinate,

inhibition of fumarase,

effect of $\mathrm{pH}$ on, $691-692$

of temperature on, 754

nature of binding to succinate dehydrogenase, 29

Succinate dehydrogenase, see also Succinate oxidase

binding of succinate to, 29

distortion of during malonate inhibition, 263

inhibition by arsenite, effect of temperature on, 749

by malonate, 104

determination of $\mathrm{K}_{s} / \mathrm{K}_{i}, 183$

effect of temperature on, 754 by mercurials in kidney, 405

by $o$-phenanthroline, effect of temperature on, 75.5

by salts, 835

localization in staphylococcal membrane, 434

nature of $\mathrm{K}_{m}, 2 \mathrm{l}$

Succinate oxidase, see also Succinate dehydrogenase

effects of inhibitors on temperature characteristics, 798

inhibition by arsenical, rate of, 547 , 549

by $p$-chloromercuribenzoate, rate of, 547,549

by fluoride and phosphate, $492-493$

by $\mathrm{HgCl}_{2}$, reversal by dimercaprol, 622

by $o$-iodosobenzoate, rate of 547 , $549-550$

by oxidized glutathione, $625-626$

rate of, $547-549$

site of inhibition based on reconsti. tution of system, 523

stimulation by phenylmercuric compounds, 4.52

Sucrose transglucosylase, substrate in. hibition, 112

Sulfanilamide, inhibition of bioluminescence, 510-512

effect of pressure on, $851-852$

of temperature on, 787.788

Sulfhydry] group,

bond angle of, 240

configuration of, 235

dipole angle of, 222

dipole moment of, 222

molar refraction of, $\mathbf{2 2 2}$

van der Waals radius of, 234

Sulfide, entrance into Valonia cells, effect of $\mathrm{pH}$ on, 721.72.2

Sulfisoxazole, variation of body content with time, 419-421

Sulfite, inhibition of urease, effect on temperature characteristics, 797 
Summation,

definition of, 507-508

isobolograms for, 508-509

of inhibitions on convergent chains, 501

Synergism,

between fluoride and phosphate on succinate oxidase, $492-493$

definition of, $507-508$

inhibition of phenylalanine metabo-

lism in $E$. coli, 498,500

isobolograms for, 508-509

Synthesis of enzymes, inhibition of, 440

\section{$T$}

Tartrate, inhibition of fumarase. effect of $\mathrm{pH}$ on, 691-692

Temperature,

effect on binding of small molecules to proteins, 316

on cellular inhibitions, $773-780,782$ -

792

on dissociation constants, $748-749$

on enzyme inhibition, $750-756$

on inhibition of cell function, $\mathbf{7 7 9}$. 780

on multienzyme systems, $768-773$

on rate of inhibition, 558.561, 756-

760

on respiratory inhibition, $777-778$

on response to anoxia, $780-782$

on sulfanilamide inhibition of bio-

luminescence, 511.512

on urethane inhibition of bioluminescence, 511-512

equilibria between active and inactive forms of enzyme, $760-767$

optimal for enzyme reactions, $767-768$

Temperature characteristics, 792-798

definition of, 792

determination of, 793

for inhibition of ATPase by dinitrophenol, 797

for inhibition of cholinesterase by quinine, 795-796

for inhibitions of succinate oxidase, 798 interpretation of, 793-794

of urease, 797

Temporal factor, in inhibitor distribution, 398-399

Testosterone, binding to seralbumin, 314, 317

Tetraalkylammonium ions, inhibition of cholinesterase,

effects of neostigmine on, 495

Tetraethylpyrophosphate (TEPP), inhibition of cholinesterase, reactivation by aldoximes, 630-633

Thermal cavity size, relation to ionic size. 241

Thiocyanate,

binding to seralbumin, effect of temperature on, 753

inhibition of fumarase, effect of tem. perature on, 754

Tissue slices,

binding of inhibitor to nonenzymic material, $479-480$

functional state of, $480-481$

inhibitions in. $479-481$

penetration of inhibitors into, 479

variation between cells in, 481

effect on rate of inhibition, 594-595

Titration inhibitors, see Mutual depletion systems. Pseudo-irreversible inhibition

Tobacco leaves, photosynthesis in, inhibition by iodoacetamide, 5:0

Transacetylases, in distributive systems, 347

Transacetylation, energy contours for, 29

Transaminase,

distribution in nervous system, 482483

in distributive systems, 347

Transfer reactions, kinetics of, 39-43, $47-48$

Transition,

between steady states, 383-391

examples of, $389-390$

false start, $387-388$ 
fluctuation, 386-388

in metabolic systems, 390-391

in monolinear chains, 384-386

overshoot, 386-388

time required, see Transition time

time, 383-386

importance in enzyme reactions,

383-384

variation with inhibition, 385

variation with substrate concentration, 386

Transit time, $373-379$

definition of, 373

effect of compartmentalization on, 377 379

on enzyme rates, $373-375$

Translocases, concept of, 439

Translocation, of inhibitors in plants, 407 . 410

Transmethylases, in distributive systems. 347

Transmitters, see Agonists

Transphosphorylase, inhibition by $\mathrm{Na}^{+}$. 838.839

Transphosphorylation, energy contours for, 29

Transport systems, inhibition of, 439. 440

Trichomonas, respiration of, stimulation by arsenite, 452

Trimethylammonium group, dispersion energy in interaction with cholinesterase, 245, 287

in hapten-antibody reactions, 279,281 interaction with carboxylate group, 279 van der Waals radius, 234

Triose phosphate dehydrogenase, see Phosphoglyceraldehyde dehydrogenase

Trypanosomes, lethal action of arsenicals. kinetics, of, 598-599, 60I

\section{Trypsin,}

area of active center, 194

binding of soybean inhibitor, thermodynamic constants for, 267 effect of ionic strength on inhibition of, 836-837

entropy change in formation of ES complex, 266

formation from trypsinogen, 196

nature of $\mathrm{K}_{m}, 21$

Tryptophanase, intracellular $\mathrm{K}_{m}$ compared with that of extracted enzyme, 436

Tyrosine,

inhibition of phenylalanine synthesis in E. coli, 498, 500

ionization mieroconstants for, 703

\section{U}

Uncompetitive inhibition, see Coupling inhibition

Uncoupling,

of oxidative phosphorylation, 523-527

criticism of methods for demonstrating, 523-524

definition of, 523-526

in regenerative systems, 367-368

Uranium,

inhibition of transport systems in yeast, 434

nonenzymic effects on yeast, 463

Urease,

effect of ionic strength on, 828

entropy change in formation of ES complex, 266

evidence for a water site, 4.3

inhibition by phosphate, $839,84 \mathrm{I}$

by thiourea, 108

kineties indicating two active centers, 38

product inhibition, 145-146

substrate inhibition, 43-44, III

temperature characteristics of, 797

Urethane,

effect on spectra of yeast cytochromes, 517

inhibition of bioluminescence, 510-512 effect of temperature on, 787-788, 790 
inhibition of cholinesterase, effect of temperature on. $758-759$

of cleavage, effect of temperature on, $790-791$

of fermentation, effect of fermentative rate on, $861-86$ ?

of $\beta$-fructofuranosidase,

effect of pressure on, $846-849$ of temperature on, $755-756$

of respiration,

effect of respiratory rate on. 861 . $86: 2$

of temperature on. 778,790

of tumor growth, 861

Uricase, stimulation by arsenite, 45:2

\section{V}

Van der Waals forces.

definition of, 233

in carboxypeptidase inhibition, 292

Van der Waals radii,

of atoms, 233

of molecular groups, $234-235$

Variability in cell populations, effect on rates of inhibition. $590-595$

Vibrio phosphorescens, inhibition of luminescence in, 511

effect of temperature on, $787-790$

\section{W}

Water, see also Hydration

bond angle in, 240

concentration of, reduction by high substrate concentrations, 113, 133

configuration of, 235

content of tissues, 401

dielectric constant of, $\mathbf{2 4 5}$

dipole moment of, 244 dispersion electrons of. 22.5

interaction energy contributions of, 244

liberation in interactions. $266-267,298$.

299,313

molar refraction of, 2.25

optical constants of, 2.25

participation in enzyme reactions, 43 -

45

polarizability of, 2225

structure of. 25.5-258

around fatty acids, $25 \%$

cages surrounding nonpolar mole-

cules, 256

effect of solute on, $256-258$

of temperature on. 256

formers and breakers, 256

van der Waals radii, 234

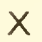

Xanthine oxidase,

inhibition by $p$-chloromercuribenzoate, reversal by cysteine and GSH, 622 substrate inhibition, 111, 120-121, $124,132-133.137-138$

\section{Y}

Yeast, respiration of,

stimulation by arsenite, 4.5.2

by azide, 452

by fluoride, 4.52

by iodoacetate, 452

Z

Zine,

binding to seralbumin. 314-315

hydration of, 251

in substrate binding of phosphatase, 46

Zones, in mutual depletion systems, 67, 74 






